

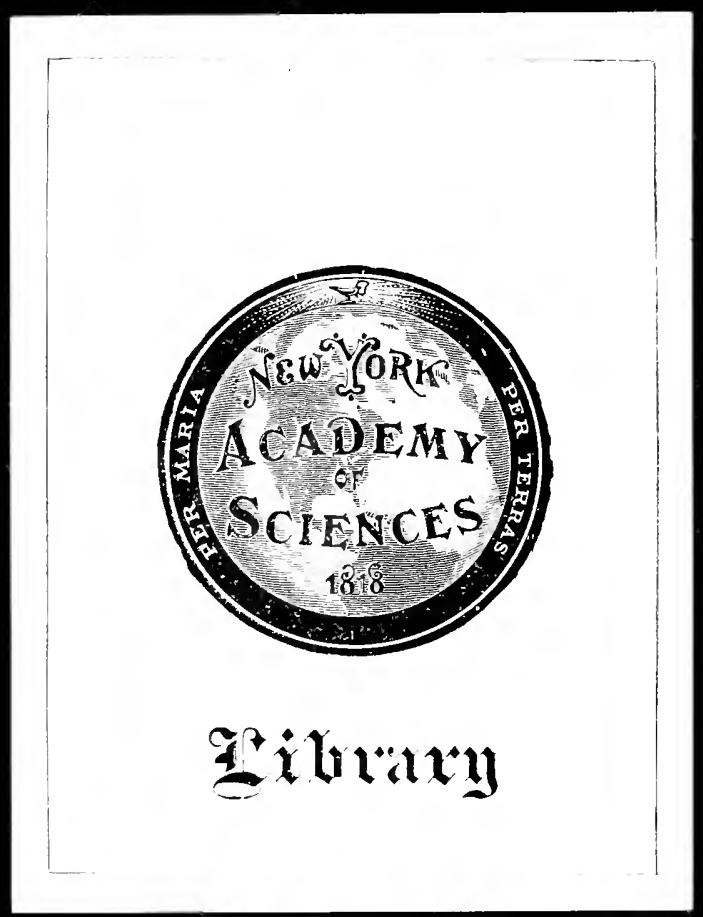




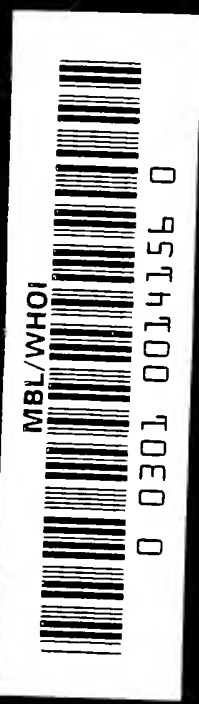


THE NEN YORK

ACADEMY OF SGIENCES.

SYNOPSIS OF THE

\section{BRITISH BASIDIOMYCETES}

A Descriptive CATAlogue of THE DRAWINGS AND SPECIMENS IN THE DEPARTMENT OF BOTANY BRITISH MUSEUM

WORTHINGTON GEORGE SMITH, F.L.S.

\section{LONDON}

PRINTED BY ORDER OF THE TRUSTEES OF THE BRITISH MUSEUM

г 908

(All rights reserved) 


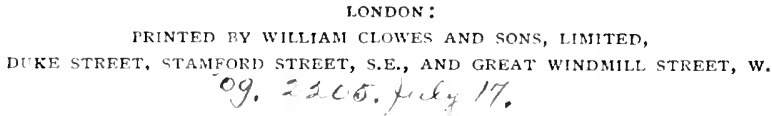

$A=180$ 


\section{P R E F A C E}

IN 1905 the Trustees acquired the manuscript descriptions drawn up by Mr. Worthington G. Smith, when preparing the fine series of coloured drawings of British Fungi which are exhibited in the Public Gallery of the Department of Botany. The descriptions were accompanied by line drawings illustrating the characters of each genus. In 1907 the Trustees gave permission for their publication in the form of the present Handbook, which it is hoped will prove a useful introduction to the study in the field of the larger British Fungi. Of the numerous introduced species all that have appeared in the open air are included; but those occurring in greenhouses and stoves are omitted, except those which continually reappear, and those which stray into gardens.

Mr. Smith himself and Mr. W. E. St. John Brooks, Assistant in the Department, have given valuable help in the preparation of the book for the press.

A. B. Rendle.

DePaRtMent of BOtany,

British Museum.

October, 1908. 



\section{SYNOPSIS}

OF

\section{BRITISH BASIDIOMYCETES}

INTRODUCTION

THE British Basidiomycetes include nearly all the larger British Fungi. They comprise all the Mushroom-like Fungi, practically all the larger woody corky and gelatinous fungi of trees stumps and fallen branches (Hymenomycetes), the Phalli, Puff-balls and their allies, and a small number of Truffle-like subterranean species (Gasteromycetes). The term Basidiomycetes is used in the sense in which

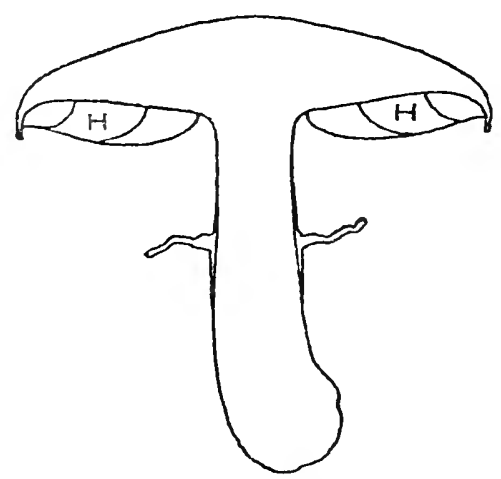

Fig. I.-Section through Mushroom, showing exposed hymenium $(\mathbf{H})$. One-haif natural size.

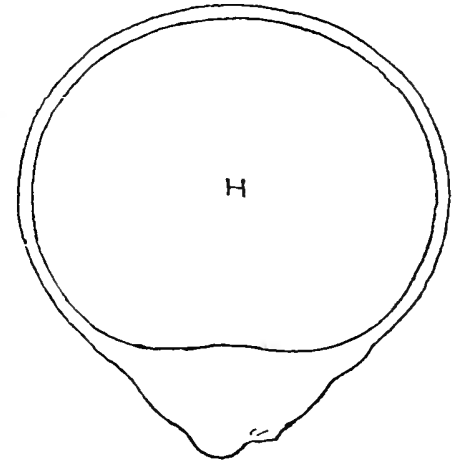

Fig. 2.- Section through Puff-ball, showing hymenium $(\mathbf{H})$ closed in. One-quarter natural size.

it was employed by De Bary, and not with the larger meaning assigned to it by Brefeld, which includes the Rust-fungi or Uredinea.

In the Hymenomyctes the spore-bearing surface or hymenium is exposed during development, as in the Mushroom (fig. r); in the Gasteromy'ctes the hymenium is closed or hidden within the substance of the fungus until maturity, as in the Puff-ball (fig. 2). 
The larger Fungi not described in this work are a few of the Ascomycetes, including the Morel and its allies, the true ascusbearing Truffles and a limited number of Cup-fungi.

The microscope is unnecessary for the determination of the greater number of the Basidiomycetes; nearly all are large and can be satisfactorily examined by the unaided eye or with the assistance of a hand-lens. A few forms found under Family iv Thclephoracia, as Solenia and Cyphella, superficially resemble certain of the Ascomycetes, as $P_{\text {ecisa }}$; but with a little experience even obscure forms

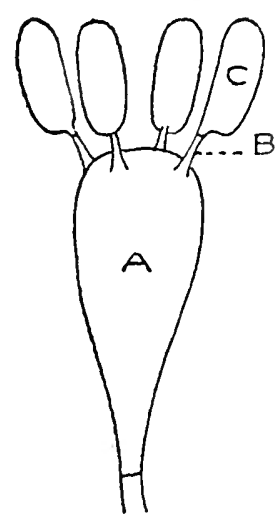

Fig. 3.-Pleurotus ostriatus Quél.

A, basidium; B, sterigma ; c, spore. $\times 750$. may be easily determined with the aid of a simple lens. In some genera of the Thelephoracea a microscopic examination of the hymenium is sometimes desirable.

The Basidiomycetes are highly plastic and variable. No one species is constant in all its characters, and a single example seldom wholly accords with any other single example of the same species. Examples which appear to be intermediate between allied, and sometimes between not allied, species are frequently met with. About one species in ten is perhaps fairly well and distinctly marked, but all species will at times present aberrant characters. Any one character is liable to fail ; in the determination of species, therefore, all the characters must be studied together.

The Basidiomycetes are so named on account of the spores being borne on more or iess club-shaped cells named basidia (fig. 3). They are commonly borne in fours, on slender usually short threads or supports named sterigmati. In rare instances, however, the basidium bears two spores or perhaps only one; in these cases the normal number of four is sometimes reverted to in well-dereloped examples. In some species of the Tremellinacece the spores are septate, and in some Gasteromycetes six or even more spores are produced on a basidium.

The spores are usually smooth and simple or composed of one cell; they are sometimes echinulate or warted. The commonest form is oval or round; a few are multiangular. They are of nicroscopic size, varying from about $18 \mu$ to $3 \mu$ in length, and of various colours.

The character of the basidium with its four naked spores is one of great importance, as it separates the Basidiomycetes from the Ascomycetes. In the latter the spores are borne usually in a series of eight, within microscopic transparent elongated colourless sacs named asci (fig. 4); the asci when ripe open at the apex and the spores escape.

In an examination of the spore-bearing surface or hymenium of 
the Basidiomycetes three kinds of cells are met with,- the ordinary barren cells of the hymenium, the basidia with the spores, and certain other large cells named cystidia. The last-named are sometimes very small or practically absent, as in Clacitariar; in other genera they are very large, as in Coprinus (fig. 5), Hymenochete (fig. 6) and Pentiophore.

The more highly developed Basidiomycetes as a rule bear white or slightly tinted spores; the lower or dung-borne species usually produce black spores. In most instances the spore-colour is permanent, but in some species it vanishes and leaves the spores hyaline. The colour of the spores is often used as a generic or subgeneric character; the tint often, but not invariably, gives the distinctive hue to the mature hymenial surface. It is readily seen by placing examples with the hymenium downwards upon a piece of glass on which the spores will be deposited.

At maturity, the spores of the Hymenomycetes drop from the sterigmata and are free in the air, but in the Gasteromycetes the outer coats become naturally ruptured before the spores can escape. In the Phatloidacece the sporiferous surface is at first enclosed in a veil (volva), and even after the elongation of the stem, the cap (pileus) is

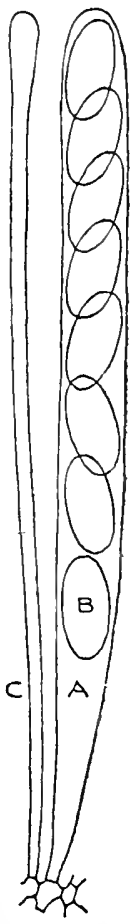

covered by a very thin, almost invisible Fig. 4.-Morchella esculinta Pers. membrane; when the spores are ripe they $A$, ascus; $B$, spore; $C$, paraphysis. are enveloped in mucus which runs down from the upper parts of the fungus to the lower, where there is a gelatinous stratum; the spores may possibly germinate in the decomposing gelatine. In the fetid species the odour attracts swarms of flies and sometimes small beetles to the sporiferous mucus, which they eagerly devour. On flying away the insects disseminate the spores, which have been seen to germinate after having passed through flies. In the Lycoperlacece the spores are at first enclosed in a peridium, and it is only when this is ruptured that they are set free. 'The Hymenogastracece are subterranean, or nearly so, and the spores must either germinate in the decaying substance of the mother plant or be carried away by larve, insects, etc.

In germination the spores open at one or both ends; a transparent germinal tube emerges (fig. 7) and forms strands, threads or hyphre of cellular mycelium. The strands branch and coalesce 
with other hyphæ belonging to other spores of the same species. In rare instances, as in the Tremellinacea, the spores germinate and

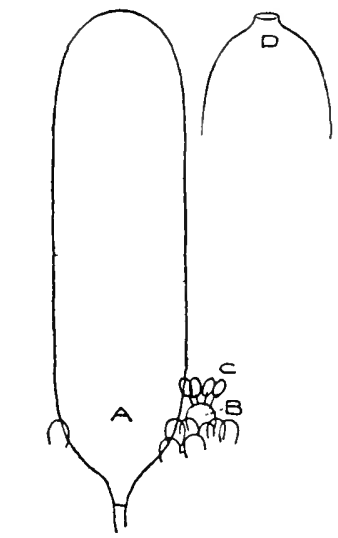

Fig. 5.-Coprinus atramentarius $\mathrm{Fr}$. $A$, cystidium, orifice open at $D$; B, basidium; C, spores. $\times 200$.

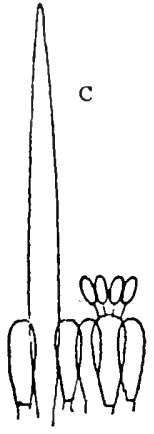

Fig. 6.-Hymenochate nigrescens Cooke.

Part of hymenium with long cystidium, c. $\times 300$.

produce a rudimentary mycelium (fromycclium) bearing spores, from which only is a true mycelium produced. The mycelium

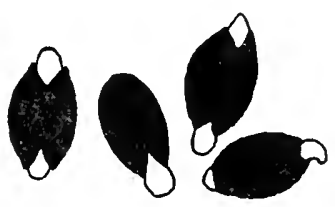

Fig. 7.-Psathyrella prona Gill. Spores germinating. $\times 350$. or finely matted growth produced by germinating spores is the "spawn" of gardeners; it is usually white in colour, but is sometimes tinted; in one or two instances it is blood-red. If the mycelium or spawn be placed upon a suitable matrix the fungus will, under favourable circumstances, be reproduced. The spores of some Basidiomycetes very speedily die after falling from the hymenium. No sexual organs are known in the Basidiomycetes.

The season of growth is usually the autumn, but a considerable number appear in the spring and summer, and some continue to grow till late in the autumn or even early winter. Many species of Marasmizs will, after being quite parched in dry weather, revive in rain, and several of the late-growing species of Clitocybe and Collybia revive after being frozen. There are two crops of some Basidio. mycetes every year, one vernal and the other autumnal; this is the case with Cofrinus atramentarius, of which the first crop appears after the rains of April and May, the second in September or October. If vernal examples are gathered and buried with decayed wood or beside decaying posts a crop of perfect fungi will appear in September or October. There are sometimes three crops of Coprinus micaceus in one year. If living plants of Coprinus 
radiatus are placed upon manure heaps the spores will germinate at once and reproduce the plant in a day or two.

Some of the perennial Polyporace continue to increase in size for many years. The years of growth can be counted in the annual fleshstrata, which are easily seen when sections are made. Mycelium will continue to live in wood or in the ground for an apparently unlimited time. I have known a "fairy-ring" of Clitocybe geotropa on Dunstable Downs for forty or more years, which under favourable conditions of light can be seen at a distance of more than a mile. The diameter has not altered much during the time I have known it, for sometimes it grows inwardly for several years and then again outwardly.

Most species are terrestrial and grow on or just below the ground; a large number grow on dead leaves and twigs; others on dead wood, bark, branches and trunks. It is unusual to see fungi on healthy trees; the majority of the larger fungi so commonly seen growing on tree-trunks in parks, woods and forests are "wound parasites," which germinate upon a damp injured place and grow parasitically upon the living host. Rootlets are often attacked by mycelium which at length develops as a perfect fungus at the tree's base, as in Fomes annosus, or in other cases travels further up the trunk as in $F$. igniarius, and other species. Polyporus Schucinitzii is well known to be highly destructive to conifers; Pims Strobus, $P$. syluestris and Larix curopea especially suffer. Many other species of Polyporus are more or less destructive. Amongst the Agaricacere, Armillaria mellea is one of the most destructive; it often grows upon living roots, not uncommonly under the shade of large trees, the growth of the fungus being limited to the extent of the root-growth of the trees. One form of its mycelium, named Rhizomorpha, lives perennially beneath the bark of trees. It destroys tree-roots, chiefly those of conifers, but sometimes attacks plum and other trees; it also appears on squared timber, when used for bridges, in mines, etc.

Certain species grow parasitically upon other Basidiomycetes. Volvaria Lovciana grows upon the pileus of Clitocybe ncbularis; two species of Nyctalis grow upon the pileus or stem of species of Russula and Lactarins; and Bolctus parasiticus grows from the base or side of Scleroderma z'ulgare. Some species are almost peculiar to human habitations: Coprinus radians grows upon plastered walls, $C$. aphthosns commonly appears in kitchens and cellars, Merulius lacrymans (Dry Rot), Poria hybrida and Polyporus destrnctor are almost entirely confined to the interior of houses and ships, and Merulius corium often grows in dwelling-houses. Coprinus domesticus is usually seen in or close to human dwellings; $C$. comatus and Cortinarius urbicus commonly grow close to houses or to places where human beings resort. Many species grow on burnt wood and earth, as Clitocybe fumosa, Collybia atrata, C. ambustu, Flammula decipiens, $F$. sapinea, F. carbonaria, Lepiota Fricsii, Cantharellus radicosus, Polystictus cinnamomeus, and many others. 
The taste of the majority of the Basidiomycetes when raw is watery-insipid; many are practically tasteless or mild. Some when uncooked have a pleasant nutty flavour, as Psalliota campestris (mushroom) and Lepiota procerc. Several are bitter, as Boletus felleus; others are bitter-nauseous, as Hypholoma fasciculare, and many species of Lactarius and Russilliz are very acrid or bitter acrid.

The odours are most diverse. Clitocybe fragrans is very sweet and recalls Melilot, as does also Hydnum grazcolens; $C$. odora is fragrant of Woodruff or Vernal Grass; Trametes suaveolens, $T$. odora, Lactarius glyciosmus and Clavaria stricta are also very sweetscented. One variety of Cantharallus cibarius smells strongly of apricots, and Clitocybe geotropa is almond-scented. Many smell strongly of onions or garlic, the best known examples being different species of Marasmius. Fetid and disgusting odours are common; a familiar instance is that of Ithyphallus impudicus. The odours possibly serve some purpose at present unknown. The carrion-scented species attract swarms of carrion-feeding insects which greedily devour the highly fetid, soft, sporiferous material of the Phalloidacee.

The exudation of fluid, the so-called milk, when the stem is broken, is a remarkable character of some species. Mycena galopus and $M$. lacter contain a white, $M$. crocata and $M$. chelidonia a yellow, M. homatopus a dark purple-red, and $M$. sanguinolenta a red juice; Lacturins deliciosus exudes an orange-coloured and L. sanguiffuns a deep blood-red juice which, on exposure to the air, quickly becomes green. The milk of L. chrysorrheus and L. theiogalus is sulphur-yellow, that of $L$. acris is at first white, then reddish. The milk of L. uvidus is white and quickly changes to violet; that of $L$. scrobiculatus is first white, then sulphur; that of $L$. fuliginosus is white, then saffron. 'The sten of Marasmius araricosus is filled with dark blood-red juice, which flows when the stem is bruised or broken.

Some species of Asaricacece, as Hypholoma lacrymabundum and H. velutinum, have "weeping gills"; in mature examples drops of fluid may be seen sprinkled all over the surface of the gills which, when examined under the microscope, are seen to be charged with spores and cystidia. The hymenium of the dry rot fungus, Merulins lacrymans, is usually covered with globules of exuded moisture; Polyporus dryadeus is often seen in the same condition.

The Basidiomycetes as a rule do not exhibit brilliant colouring, but there are remarkable exceptions, the most striking being scarlet and crimson, as in Amanita muscaria. Yellow, orange, blue, purple, and white also occur, also rarely green and black, but the majority are pallid, watery-brown, brown, greyish or buff. The brown and buff colours of Agarics often cause the fungi to be overlooked when growing amongst dead leaves.

The Mushroom is a highly nitrogenous plant and contains a con- 
siderable amount of fat. The following are its chief constituents, as given by Professor A. H. Church :-

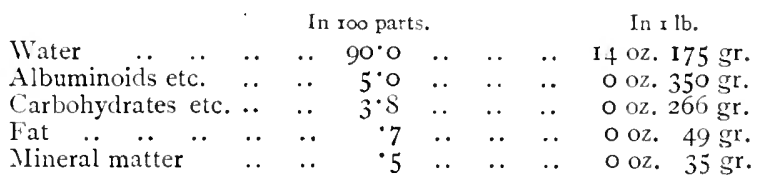

The chemical composition of the Champignon, Marasmius oreades, is nearly the same. Several species are known to be highly poisonous in all stages of growth, both cooked and uncooked; those without unpleasant taste are the most dangerous, as Amanita plualloides.

Specimens of fungi for determination must be quite perfect and fresh ; examples past maturity are useless. Young specimens should be secured whenever possible, as some characters which are then very obvious vamish as the plant advances to full growth.

In the descriptions which follow the sequence is almost identical with that of Fries's Hymenomycetes Europiei, 1874. 'This sequence has been almost universally approved, and has been followed in this country by Berkeley, Cooke, Stevenson, and in my supplementary volume to Berkeley's Outlines of British Fungology. The large collection of drawings, prints and dried examples of British Basidiomycetes in the Herbarium of the British Museum is arranged on the same plan.

The number before each species corresponds with the number in my series of coloured drawings of British Fungi exhibited in the Public Gallery of the Department of Botany, British Museum.

The letters $a b c$ used in the text refer to the drawings and specimens in the British Museum. The letter $a$ indicates that there is a drawing of the species in the Public Gallery, $b$ that there is a drawing and $c$ that there is a specimen in the Herbarium.

The specific descriptions contain the salient distinctive characters, which must be used with the generic and sectional characters. If fuller descriptions of the Hymenomycetes are desired, Fries's Hy'menomycotes Europai ( I 874) and his Monographiar Hymenomycetum Suecice (I 557) may be consulted; the Gasteromycetes are described in greater detail in Massee's Monograph of the British Gasteromycetes (1889) and the same author's British Finnglls Flora, vol. i. (I892). Saccardo's Sylloge Fungorum (i $882-$ i go8) may also be consulted.

The names of species obviously introduced are printed in small capitals ; introduced genera are distinguished typographically.

The text was prepared and checked at the time when the drawings were made, when living and dried examples, drawings, plates, printed descriptions, and notes were before me.

The dimensions give the typical size in inches of well-developed examples as found in Britain, for which those given by Fries are 
sometimes very misleading. The numerals represent the diameter of the pileus, the height from the base of the stem to the top of the pileus, and the diameter of the stem near the middle. The stem in most of the stem-bearing Basidiomycetes is cylindrical or slightly attenuated upwards; this character is not reiterated in the descriptions, where departures from the type only are given. In stemless species, which are generally excentric, the longer diameter of the pileus is given.

The notes as to taste and odour are generally original, supplemented by the observations of others; those as to colour have in most instances been drawn up from living examples, supplemented by printed descriptions and by reference to characteristic coloured drawings and plates.

The habitats and dates of appearance are from my own notes, and from observations of other British botanists; in a few instances no habitat or date is on record.

Spore measurements have been omitted as untrustworthy; in many cases authors have measured immature spores from young plants, in others it is certain that authors have misnamed the fungus from which the spores have been taken.

The following abbreviations are employed:-

HYMENOMYCETES.

$\begin{array}{ll}\text { A. } & \text { Annulus. } \\ \text { Br. } & \text { Bristles. } \\ \text { Cl. } & \text { Clubs. } \\ \text { Co. } & \text { Cortina. } \\ \text { G. } & \text { Gills. } \\ \text { Gr. } & \text { Granules, } \\ \text { Hym. } & \text { Hymenium. } \\ \text { H. } & \text { Hymenophore. } \\ \text { Myc. } & \text { Mycelium. } \\ \text { P. } & \text { Iileus. } \\ & \text { circ. circumference } \\ & \text { gr. granules } \\ & \text { marg. margin } \\ & \text { mid. middle } \\ & \text { sc. scales } \\ & \text { sq. squamules } \\ & \text { um. umbo } \\ & \text { z. zones. }\end{array}$

$\begin{array}{ll}\text { Pa. } & \text { Papillæ. } \\ \text { Pi. } & \text { Pileoli. } \\ \text { Po. } & \text { Pores. } \\ \text { Scl. } & \text { Sclerotium. } \\ \text { Se. } & \text { Setæ. } \\ \text { Sp. } & \text { Spines. } \\ \text { St. } & \text { Stem. } \\ \text { Sub. } & \text { Subiculum. } \\ \text { T. } & \text { Tubes. } \\ \text { Te. } & \text { Teeth. } \\ \text { Tub. } & \text { Tubercles. } \\ \text { V. } & \text { Veil. } \\ \text { Ve. } & \text { Veins. } \\ \text { Vo. } & \text { Volva. } \\ \text { Z. } & \text { Zone. }\end{array}$

The stem is supposed to be cylindrical or nearly so unless otherwise described, and simple if without volva and annulus.

\section{GASTEROMYCETES.}

Cap. Capillitium.

Col. Columella.

Cor. Cortex.

End. Endoperidium.
Ex. Exoperidium.

Fun. Funiculus.

GI. Gleba.

Pe. Peridiun.

Ped. Pedicel.
Per. Peristome.

Peri. Peridiolum.

Rec. Receptacle.

Vo. Volva. 


\section{ORDER I. HYMENOMYCETES}

Basidia and spores exposed in the mature plant (fig. 8).

Families I-VI.

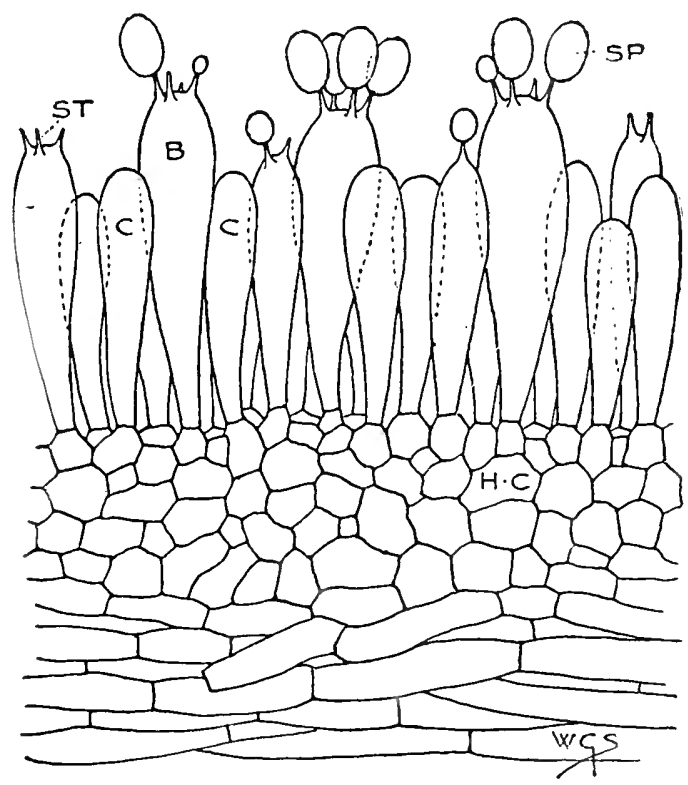

Fig. 8.-Basidia and spores of Mushroom (Fsalliota campestris Quél.). B, basidia; sp, spores; C, cystidia or barren basidia :

ST, sterigmata; H.C, hymenial cells. $X$ ı000.

I. Agaricacee. Hymenium spread over the surface of radiating gills or lamella.

Genera 1-58

II. Polyporaceæ. Hymenium lining the surface of small pores or tubes.

Genera 59-69

III. HydnaceÆ. Hymenium spread over spines or protuberances. Genera 70-79

IV. Thelephorace.. Hymenium unilateral, spread over a firm smooth or corrugate under or upper face. Genera 80-90

V. Clavariaces. Hymenium spread over the surface of smooth, simple or branched clubs; in Sparurssis over a large spongelike mass of coalescing branches.

Genera 91-96

VI. TremellinaceE. Hymenium spread over a smooth gelatinous surface. 


\section{ORDER II. GASTEROMYCETES}

Hymenium concealed within a peridium until the spores are mature (fig. 9).

Families VII-XI

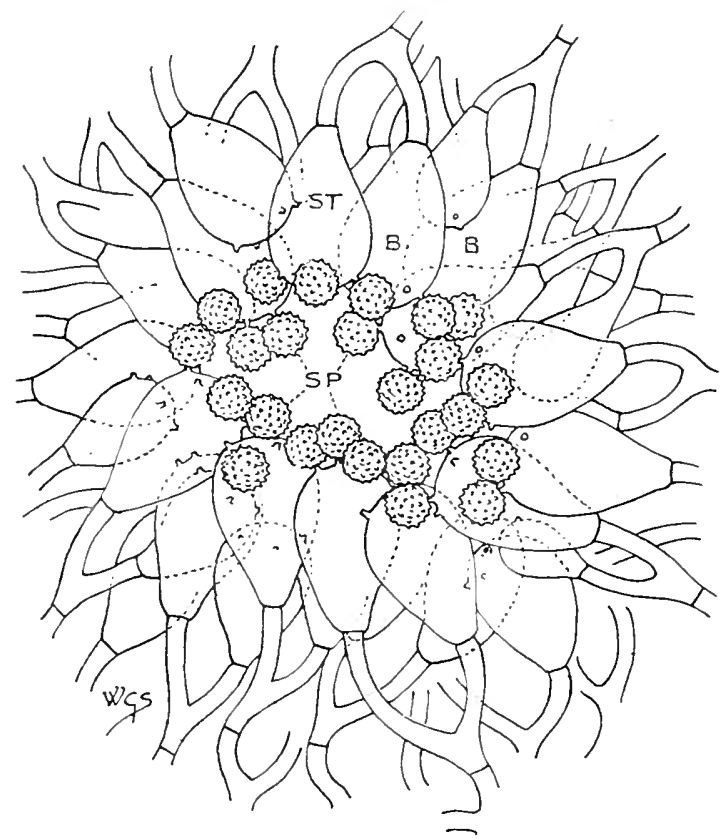

Fig. 9.-Basidia and spores of Scleroderma vul gare $\mathrm{Fr}$. B, basidia; SP, spores; $\mathrm{sT}$, sterigmata. $\times 400$.

VII. Phalloidacee. Pileus and stem or latticed receptacle at first enclosed in a globular peridium with a gelatinous stratum. Peridium at length opening at the apex and the gleba extended on a stem or lattice.

Genera 107-110

VIII. LyCOPERDACEÆ. P'eridium globular, with a capillitium; at length opening for the dispersion of the spores.

Genera 111-116

ix. Scleroinermacee, Peridium globose, thick, without a capillitium, dehiscing irregularly.

Genera 117-118

I. Nimulariaceæ. Peridium bell- or cup-shaped, containing compressed or globose peridiola which become free at maturity.

Genera 119-122

XI. Hyaenogastraceæ. Subterranean, peridium without a capillitium, indehiscent.

Genera $123-128$ 


\section{ORDER I. HYMENOMYCETES}

\section{FAII. I. AGARICACE $Æ$}

Hymenium spread over the surface of radiating gills, the axis a stem, rarely a point, usually central, sometimes lateral. In some forms the young fungus is entirely enclosed in a primary or universal reil (fig. Io B). During growth, as the stem elongates, the veil is ruptured, the lower portion called the volia (Vo.) remaining as a sheath round the base of the stem, while the upper portion, still attached to the pileus, is torn into fragments as the latter expands (U.v.). In some species a secondary or partial z'eil is also present in the earlier stages, spreading from the upper part of the stem to the edge of the pileus. This veil is finally ruptured, and partly persists as a ring or anmulus (A), encircling the stem. Contains two-thirds of the whole Basidiomycetes. Species 1-1455

\section{Key to The Genera.}

In using the key for the determination of the genus the following characters must be ascertained :- $r$. Colour of the spores. 2. Nature of the substance, whether fleshy, putrescent, deliquescent or coriaceous. 3. Whether the hymenophore is distinct from the stem, or confluent and homogeneous with it. 4. Nature of the pileus, whether dry, viscid, silky, etc. 5. Nature of the stem, whether simple, annulate or volvate, and whether it is homogeneous with the pileus, or heterogeneous with a cartilaginous bark. 6. Nature of the gills and their attachment to the stem.

I. Spores white.

A. Plant fleshy, more or less firm, not deliquescent or coriaceous.

Hymenophore distinct from the fleshy stem.

Pileus bearing warts or patches free from the cuticle. Stem volvate and annulate ...................... Stem volvate...................................

Pileus pulverulent, smooth or scaly; scales, when present, concrete with the cuticle. Stem annulate

I Amanita.

2 Amanitopsis.

Stem simple

3 Lepiota.

4 Hiatula.

Hymenophore confluent and homogreneous with the fleshy stem.

Stem without cartilaginous bark.

Stem central (some of the plants under 50 are stemless).

Annulate 
Simple.

Gills sinuate

Gills decurrent

edge acute......

edge swollen

separating from hymenophore.

Gills adnate.

Parasitic on Agarics

Not parasitic.

Milky

Not milky.

Rigid and brittle

Waxy......

Stem lateral or absent....

Stem with cartilaginous bark.

Gills usually adnate.......................

Gills sinuate ...............................

Gills decurrent

6 TRICHOLOMA.

7 Clitocybe.

50 Cantharellus.

46 Paxillus.

(Group I. Lepista.)

B. Plant tough, coriaceous or woody.

Stem central.

Gills simple

5 I Nyctalis.

48 Lactarius.

Gills branched

49 Russula.

47 HrGROPHORUS.

I I Pleurotus.

Stem lateral or absent.

Gills toothed...

8 Collybia.

9 MrCena.

io Omphalia.

Gills not toothed

Gills channelled longitudinally or crisped...

Gills splitting longitudinally

Gills anastomosing

52 Marasmius.

55 Xerotus.

53 Lentinus.

54 PaNus.

56 Trogia.

57 SCHIZOPHYLLUM.

58 Lenzites.

II. Spores rosy or salmon-colour.

A. Stem without cartilaginous bark.

Hymenophore distinct from the fleshy stem.

Stem volvate.

12 Volvaria.

Stem annulate

13 AnNularia.

Stem simple

14 Pluteus.

Hymenophore confluent and homogeneous with the fleshy stem.

Sten central.

Gills adnate or sinuate.............................

Gills decurrent ......................................

Stem lateral or absent.

I5 ENTOLOMa.

i6 Clitopilus.

20 Claudopus.

B. Stem with cartilaginous bark.

Gills decurrent

Gills not decurrent.

Pileus scaly

19 Eccilia.

Pileus papillose, subcampanulate.

Gills membranous, persistent

I7 LEPTONIA.

Gills subdeliquescent

IS NolnNeA.

43 Bolbitius.

III. Spores brown, reddish- or yellowish-brown.

Stem without cartilaginous bark.

Stem central.

Stem annulate.

Annulus membranous.

Terrestrial

22 TOGARIA.

Growing on wood.

Annulus arachnoid, filamentous or exanescent.

Gills adnate, terrestrial

24 Pholiota.

Gills decurrent or adnate, growing on wood... 
Stem simple.

With a volva

2 I Acetabularia.

Without a volva.

Gills adhering to the hymenophore and sinuate.

Cuticle fibrillose or silky

Cuticle smooth, viscid.

Gills separating from the hymenophore and decurrent, stem sometimes obsolete ......

(iills decurrent

Gills not decurrent.

Margin of pileus at first incurved

Margin of pileus always straight.

Hymenophore free from the stem

Stem lateral or absent.

Hymenophore confluent with the stem...

25 INOCYBE.

26 Hebeloma.

46 Paxillus.

(Group 2. Tapinia.)

30 Tubaria.

$2 S$ Naucoria.

23 Pluteolus.

29 Galera.

3i Crepidotus.

IV. Spores purple, brownish-purple, dark purple, or dark brown.

A. Stem without cartilaginous bark.

Hymenophore distinct from the fleshy stem.

Stem volvate...

32 Cintonia.

Stem without a volva.

Stem annulate

33 Psalliota.

Stem simple

Iymenophore confluent with the stem.

Veil normally ring-like on the stem .

$V$ eil normally fibrillose and adhering to the margin of the pileus.

B. Stem with cartilaginous bark.

Margin of pileus at first incurved

Margin of pileus at first straight

34 Pilosace.

35 Stropharia.

36 IIypholoma.

37 Psilocibe,

$3 \mathrm{~S}$ Psathyk.

V. Spores black or nearly so.

Gills deliquescent

42 Coprinus.

Gills not deliquescent.

Gills decurrent

45 Gompindius.

Gills not decurrent.

Stem annulate....

39 Anellaria.

Stem simple

40 PANEOLUS.

Pileus striate.

4 I Pathirella.

Genera 1-41 represent the genus Agaricus as understood by Fries who regarded the modern genera as of subgeneric rank only. They include species 1-920, and are arranged in five series defined by the colour of the spores as illustrated in the five diagrams (Plates I.-T.).

The genera which are marked in corresponding positions on the different diagrams are structurally identical; the only difference is in the colour of the spores. For instance, Amanitopsis, on the first diagram, has white spores; it corresponds in structure with Volvaria, with pink spores, on the second diagram, with Acitabularia, with brown spores, on the third, and with Chitonia, with purple spores, on the fourth. There is no genus structurally identical with Amanitopsis 
in Britain with black spores, consequently this position on the fifth diagram is left vacant.

White-spored species represent the highest types and black-spored species the lowest, the pink-, brown-, and purple-spored species are intermediate. Amanita is the highest type of the white-spored Agarics. The majority of the white-spored species are terrestrial and more or less persistent, whilst the black-spored species commonly grow on dung and are evanescent.

\section{Series I. LEUCOSPORI Fr.}

(From the white spores; Gr. lcukos, white, sporos, a seed.)

Spores white, rarely with a faint shade of ashy-grey, a faint tint of ochre, pale brown or pale greenish; usually oval or round and smooth, rarely slightly spinulose.

Genera I-XI.

\section{AMANITA Pers.}

\section{(Galen's name for certain fungi.)}

Veil universal, clothy, distinct from the cuticle of the pileus, after rupture, as maturity is reached, remaining as a volva at the base of the stem, with broken patches of its substance frequent on the pileus. Hymenophore distinct from the fleshy stem. Pileus convex,

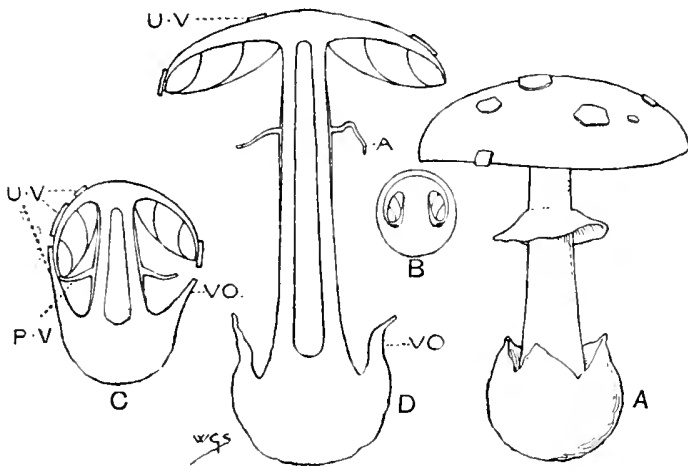

Fig. IO.-Amanita phalloides Link. One-third natural size.

$A$, perfect plant; $B$, section of young plant showing universal veil: C, section of half-grown example showing rupture of universal veil; D, section of nature example; v.v, universal veil; P.v, partial veil, becoming annulus; $\mathrm{A}$, annulus: vo, volva.

then expanded, not decidedly fleshy. Stem central, annulate and volvate. Gills free or nearly so, white or whitish. (Fig. Io.)

All the species grow on the ground in woods and uncultivated places. A few are edible, others are poisonous or suspicious. 
Volz'a bulb-like, large, exhibiting a free expanded border in splitting. Pileus naked or nearly so, and viscid, except $4 \mathrm{a}, 4 \mathrm{~b}$, and $4 \mathrm{c}$; margin striate, except $4 \mathrm{a}, 4 \mathrm{~b}$, and $4 \mathrm{c}$. Gills free or adnexed.

$1-4$

Voli' $a$ splitting regularly, its margin closely adpressed to the stem and the detached upper part forming patches or warts on the pileus. Gills free.

Volva friable, broken into small scales or warts, not persistent at the somewhat bulbous base of the stem.

$10-13$

Volva rudimentary, floccose, disappearing. Stem slightly enlarged at the base, not truly bulbous.

$14-17$

1. A. virosa Quél. (virus, poison) a $b c$. Wholly white.

$P$. marg. lobed, floccose with V. St. somewhat scaly. A. large. Vo. lax, wide.

Poisonous, fetid. Woods; uncommon. Aug.-Oct. $5 \times 7 \frac{1}{2} \times \frac{5}{8} \mathrm{in}$. Compare 3. This and the next when young greatly resemble the edible mushroom.

2. A. phalloides Link (from the volva as in Phalloidacce) $a b c$.

$P$. white, yellowish or light, or dark olivaceous. St. somewhat smooth, white.

Poisonous, narcotic, acrid. Odour when young, strong, not disagreeable, at length fetid. Woods; frequent. Apr.-Nov. $3 \frac{1}{2} \times 4 \frac{1}{2} \times \frac{5}{5}$ in. Many fatal cases of poisoning on record.

3. A. verna Quél. (z'r, spring) a b. Wholly white.

$P$. marg. not lobed, not floccose. St. floccose. $A$. not large. $V o$. somewhat adpressed to stem.

Probably poisonous. Odour not strong, but unpleasant. Woods; uncommon. Nay-Oct. $3 \times 5 \times \frac{3}{8}$ in. Probably a var. of 2 . Compare 1 .

4. A. mappa Quél. (from the resemblance of the volva to a napkin, mappa) a bc.

$P$. dry, patched with remains of Vo., white to pale yellowish-buff. St. somewhat smooth, white.

Poisonous, fetid. Woods; frequent. Usually small. Aug.-Nov. $3 \times 3$ 妾 $\times \frac{1}{2}$ in. Compare 12 .

4a. A. porphyria A. \& S. (from the colour of the pileus) $a b$.

$P$. warm brown or purplish-brown; marg. smooth. St. slightly scaly below, whitish. $A$. distant.

Odour none. Woods, pine. Sept. $9 \frac{1}{1} \times 3 \frac{1}{2} \times \frac{5}{8}$ in.

4b. A. recutita Gill. (from the smoothly cut edge of the volva) $a$.

$P$. sometimes umbonate, dry, glabrous, pale or vinous-umber; marg. smooth. St. slightly scaly below, white, becoming faintly brownish. $A$. distant. $V o$. more or less adpressed.

Woods, pine. Sept. $6 \frac{1}{4} \times 4 \frac{5}{8} \times \frac{5}{8}$ in.

4c. A. aureola Gill. (from the golden colour of the pileus) a b $c$. $P$. slightly umbonate, bright yellow or orange-yellow; marg. 
smooth. St. floccoso-squamose, white. $A$. superior, large, spreading.

Woods, under birches. Sept. $3 \frac{1}{2} \times 7^{\frac{1}{2}} \times \frac{1}{2}$ in.

4d. A. citrina Pers. (from the lemon-coloured pileus) $b$.

$P$. convex, obtuse, brassy-yellow, with white warts. St. hollow, white. $A$. large. $V o$. imperfect. $G$. free, broad, white.

Woods. Oct. $4 \frac{3}{8} \times 4 \frac{1}{2} \times \mathrm{I}$.

5. A. muscaria Pers. (from its former use as a fly-poison; masca, a fly) a $b c$.

$P$. scarlet or orange, rarely yellow, brown or liver-colour; marg. slightly striate. St. white, rarely yellowish. Vo. bulb-like, large. Poisonous, narcotic, acrid. Woods, birch, fir. July-Nov, $7 \times 9 \frac{3}{4} \times 1$ in. Compare brown form with 6.

6. A. pantherina Quél. (from the markings of the pileus) a b $c$.

$P$. viscid, pale or dark brown; marg. striate. St. whitish.

Poisonous. Woods and pastures; frequent. July-Oct. $4 \times 5 \times \frac{1}{2}$ in. Compare 11.

7. A. excelsa Gonn. \& Rab. (excelsus, tall) a bc.

$P$. viscid, papilloso-wrinkled, brownish; marg. striate. St. brownish-white.

Solitary. Poisonous. Taste not unpleasant. Woods, chiefly beech and birch; frequent. July-Oct. $4 \frac{3}{4} \times 5 \frac{1}{2} \times \frac{3}{4}$ in.

8. A. strobiliformis Quél. (from the resemblance of the scales of the pileus to the scales of certain fir-cones; strobilus, a pine cone) $a b c$.

$P$. white, then ashy-brown, covered with large sc.; marg. even. St. whitish.

Said to be edible. Taste and odour at first slight, becoming disagreeable. Open places in and near woods; rare. Pileus sometimes $\mathbf{I} 2$ in. in diam. July-Sept. $S \times 6 \frac{1}{4} \times \mathbf{r} \frac{3}{8}$ in.

9. A. solitaria Secr. (from its solitary habit) $a b$. White or buff-white. $P$. marg. even. St. brownish-white. $V o$. forming an obconic bulb; base rooting.

Solitary. Taste insipid. On the ground, damp places. Sept. $6 \times 5 \frac{1}{2} \times I_{\frac{1}{8}}^{\frac{1}{8}}$ in.

10. A. rubescens Pers. (from the flesh becoming sienna-red when broken or bruised) $a b c$.

$P$. reddish-sienna, light to dark. St. pale sienna-red.

Edible; Golmelle of continental markets. Taste not unpleasant; odour strong. Woods; common. Commonly much eaten by larva. There is a white variety. June-Nov. $5 \frac{1}{2} \times 5 \times \mathrm{I}$ in. Compare 14 .

11. A. spissa Opiz (from the small crowded warts; spissus, crowded) $a b$.

$P$. smoky-brown or grey, with ash-coloured warts. St. concentrico-squamulose, obconic at base, rooting, whitish.

Woods, mixed ; uncommon. July-Oct. $4 \times 4 \frac{1}{1} \times \frac{5}{8}$ in. Compare 6 .

11a. A. cariosa Gill. (from the carious stem) $a b$.

$P$. soft, even, brown, whitish-brown, or dark grey, unequally clad with thin, mealy, white patches and clouds. St. fragile, 
somewhat enlarged below, white, pale umber and brown, scaly at base. $G$. adnate then seceding and free.

Suspected poisonous. Woods. Sept. $4 \frac{7}{5} \times 5 \frac{7}{8} \times \frac{3}{7}$ in. Closely allied to 11 .

12. A. nitida Fr. (from the shining pileus; nitidus, shining) $a b$.

$P$. whitish, cream or whitish-buff, rarely yellowish or olive, warts brownish. St. squamulose, white to buff-white.

Woods. Aug. $4 \times 4 \frac{7}{4} \times \frac{5}{8}$ in. Compare 4.

13. A. aspera Pers. (from the rough pileus) $a b c$.

$P$. whitish- or dusky-olive, crowded with small, pointed warts. St. white.

Poisonous. Taste not unpleasant; odour strong. Woods, beech, fir ; uncommon. June-Nov. $3 \times 3 \frac{1}{2} \times \frac{3}{5}$ in.

14. A. magnifica Sacc. (magnificus, splendid) $a$.

$P$. whitish, pallid-tan or sienna-brown, floccoso-spotted; marg. sulcate. St. reddish-white. G. adnato-decurrent. Flesh white, tawny when broken.

Woods, fir, beech ; rare. July-Oct. $4 \times 4 \frac{1}{2} \times \frac{1}{2}$ in. Compare 10.

15. A. arida Gill. (aridus, dry) a. Greyish.

$P$. soon plane; marg. sulcate. St. subglabrous, white. $A$. distant. $G$. adnexo-free or adnexed. Flesh white.

Plantations, birch. Sept.-Oct. $3 \times 3 \frac{1}{2} \times \frac{3}{8}$ in.

15a. A. junquillea Quél. (from a fancied resemblance in the colour of the pileus in some examples to the flowers of the jonquil) $b$.

$P$. campanulato-convex, viscid, buff with paler marg., clad with the greyish remains of the V.; marg. deeply striate, white. St. hollow, villous-scaly, white, whitish or pale yellowish, pale olive at base. $A$. superior, membranous, partly appendiculate at marg. of $\mathrm{P}$. in young examples. Vo. marginate, at length torn, white, becoming brown at the edge. $G$. crowded, adnate with a tooth or adnexed, broader in front, white to faint yellowish-ivory.

Woods. Spring and autumn. $4 \frac{3}{4} \times 5 \frac{1}{4} \times \frac{5}{8}$. Compare 20.

16. A. megalodactyla Sacc. (from its tall stem; Gr. megras, great, dactulos, a finger) $a c$.

$P$. reddish-grey or brownish; marg. not sulcate. St. solid, white. $A$. large. $G$. free, at length red, not olive-tinged.

Odour strong. Woods. Oct.-Nov. $4 \times 5 \frac{1}{2} \times \frac{3}{8}$ in. Allied to 17 and to Lefiota.

17. A. lenticularis W. G. Sm., Agaricus lenticularis Lasch. (from its lentil-shaped stem) a.

$P$. tan or buff; marg. not sulcate. St. solid or slightly stuffed, tan-white. $A$. large. $G$. free, sometimes faintly olive.

Odour mouldy. Plantations, mixed. Oct. $4 \frac{1}{2} \times 6 \times \frac{7}{8}$ in. Allied to 16 and to Lefiota. 


\section{AMANITOPSIS Roze}

\section{(From its relationship to Amanita.)}

Vill universal, clothy, distinct from the cuticle of the pileus. After its rupture, as maturity is reached, it remains as a volva at the base of the stem and as broken patches on the pileus. Hymenophore

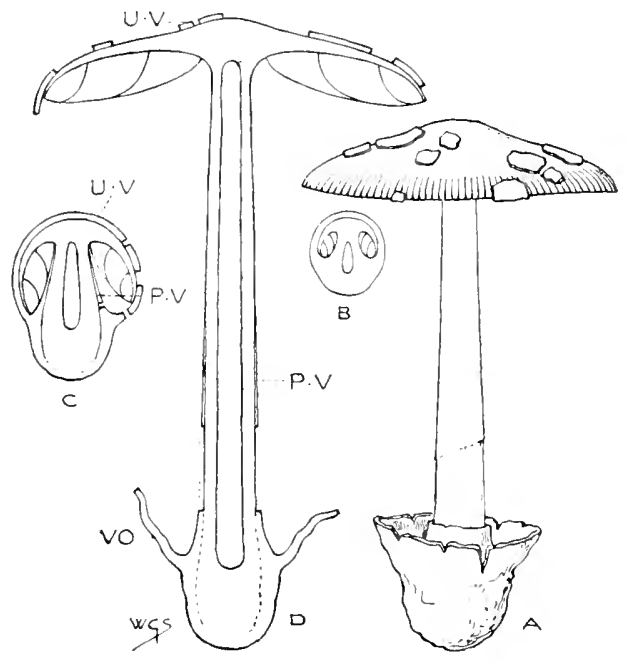

Fig. 11.-Amanitopsisvaginata Roze. One-third natural size. $A$, perfect plant; $B$, section of young example showing universal veil; $c$, section of half-grown example showing rupture of universal veil: I, section of mature example: U.v, universal veil; $P . v$, partial veil, adnate to stem; vo, volva.

distinct from the fleshy stem. Pileus convex, then expanded, not decidedly fleshy. Stim central, volvate, simple. Gills free, adnate in 20 , white or whitish. (Fig. I I.)

All the species grow on the ground.

Species $18-20$

18. A. vaginata Roze (from the volva; a'agina, a sheath) a $b c$.

$P$. brown; marg. sulcate. St. white or pale brown. Vo. large, free, lax, sheathing within.

said to be edible, but sometimes produces perspiration and illness. Tasteless or insipid when raw, but cooking brings out a strong meat-like flavour; odour none. Woods; common. Sept.-Nov. $4 \frac{7}{2} \times 6 \frac{1}{2} \times \frac{5}{8}$ in. There are several varieties; mizalis W. G. Sm. (Amanita mivalis Grev.) is white.

18a. A. fulva W. G. Sm., Agaricus fulz'us Schæeff. (from the bright reddish-tawny colour) $b$.

$P$. sienna; mid. dark, sometimes almost black; marg. striate or sulcate. St. squamulose, pale sienna. $V^{\prime}$ o. large, free, lax, sienna.

Poisonous. Woods, birch. Iune-Sept. $3 \frac{1}{1} \times 6 \frac{1}{4} \times \frac{3}{8}$ in. Amanita spadicia l'ers. is a brown form of this. 
19. A. strangulata Roze (from the transverse constrictions of the stem) $a b c$.

$P$. mouse-colour, brown or yellowish-brown; marg. sulcate. St. brownish-white. Vo. not large, adpressed to St. Fles/ whitish, tawny when broken.

Taste sweet; odour none. Woods; uncommon. May-Oct. $6 \frac{1}{2} \times S \times \frac{7}{8}$ in.

20. A. adnata Sacc. (from the adnate gills) $a b c$.

$P$. firm, not brittle, buff-yellow; marg. not sulcate. St. minutely fibrillose, buff-white. $G$. crowded. $V_{0}$. not large, lax, irregularly torn, pubescent.

Woods and plantations, oak, holly; uncommon. Oct. $3 \times 3 \frac{3}{4} \times \frac{1}{2}$ in. Compare 15a.

\section{LEPIOTA Quél.}

(From the scaly pileus and stem; Gr. lepis, a scale.)

$V_{c i l}$ universal, concrete with the cuticle of the pileus, breaking up in the form of scales, granules or silkiness. Hymenothore distinct from the fleshy stem. Pileus never compact, often very thin, at first plane,

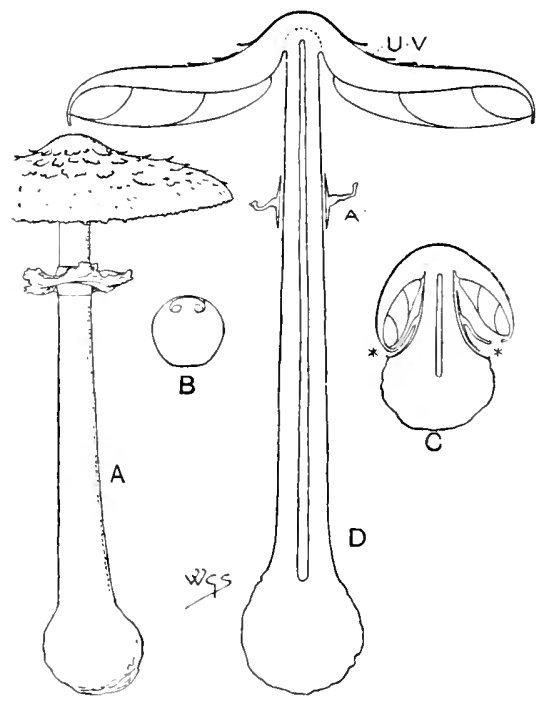

Fig 12.-Lifiota trocera Quél. One-quarter nat. size. A, perfect plant : $B$, section of young example showing universal veil; $c$, section of half-grown example showing at ** rupture of veil ; 1 , section of mature example showing at $\mathrm{A}$ annulus free from the stem; U.v, remains of universal veil.

then scaly, granular, silky, smooth or viscid. St:m central, annulate, somewhat cartilaginous, firmer in texture than the pileus, Gills free 
or nearly so, except 46,47 , and 50 . Spores normally white, sometimes yellowish, greenish or green. (Fig. I 2.)

The species grow mostly in rich grassy places, more often in fields than woods; some occur in stoves and greenhouses. Nearly all are autumnal. Some are edible.

Species 21-59

A. Epidermis dry.

a. Procerce. Scales of pileus brown or brownish on a white ground. Stem bulbous. Annulus more or less movable. Gills remote. Edible. The species are nearly allied; intermediate forms occur between all. $\quad 21-25$

b. Clypeolurice. Pilens and usually the stem scaly or covered with granules. Ammulus fixed. Gills free. Odour, except 29, strong, disagreeable, pungent, sometimes weak. 26-37 c. Annulose. Annulus superior, fixed, somewhat persistent; fugacious in 41 and 42.

$38-43$

d. Gramulose. Pileus and lower part of stem covered with granules. Anmulus inferior. Gills approaching stem, adnate in 47.

c. Mesomorpha. Pileus dry, entire, not torn, not usually granular, small, slender.

B. Pileus smooth, viscid.

f. Viscide.

$55-59$

\section{a. Procere.}

21. L. procera Quél. (procirus, tall) a $b c$.

$P$. umbonate, finely scaly. St. minutely scaly. $G$. subremote. Flesh unchanging white.

Solitary or in troops. Edible. Taste and odour pleasant, delicate. Pastures, parks, gardens, heaths, among bracken, etc.; common. July-Oct. $8 \times 10_{4}^{3} \times \frac{7}{8}$ in.

22. L. rachodes Quél. (from the ragged pileus; Gr. rakos, a ragged garment) $a b c$.

$P$. subglobose, coarsely scaly. $G$. subremote, sometimes reddening when bruised. Flesh changing to saffron-reddish when broken.

Gregarious or crespitose. Edible. Taste and odour pleasant, strong, varying to rank. Woods and hedges, fir-plantations, greenhouses; common. July-Dec. $5 \frac{1}{2} \times 6 \times \mathrm{I}$ in. The form puellaris is smaller and shining white. Bears the same relation to 21 as the horse-mushroom does to the pasture-mushroom. There is a white variety.

22a. L. prominens Sacc. (from the prominent umbo) $a b$.

$P$. sphærico-expanded, strongly and subacutely umbonate; sc. imbricate on um.; brownish or dull pale ochreous. St. abruptly bulbous at the base.

Edible. Fields. Oct. $8_{4}^{3} \times 4 \frac{3}{8} \times \frac{1}{2}$ in.

22b. L. permixta Barla (from its mixed characters) $a$.

$P$. campanulato-convex, slightly subumbonate, then expanded, smooth then fibrillose; sc. opaque, somewhat pale brown on a brownish-ivory ground; marg. sublacerate, white. St. hollow, 
enlarged at base, irregularly scaly, colour as P. Flesh becoming faintly rufous. $A$. membranous, fibrillose, lax. $G$. free, ventricose, crowded, ivory or faintly flesh-coloured.

Odour weak. Hilly pastures. Autumn. $7 \times 4 \frac{1}{8} \times \frac{1}{2}$ in. With the mixed characters of 21,22 , and 23 .

23. L. excoriata Quél. (from the cracked membrane of the pileus) a b $c$. $P$. semiglobose, slightly scaly, pale buff. $A$. less movable than in 21 and 22. Flesh unchanging white.

Said to be edible. Taste and odour usually delicate and agreeable, but varying to rank and unpleasant. Pastures and hedge-sides; less common than 21 and 22. May-Oct. $4 \times 4 \times \frac{5}{8}$ in.

24. L. gracilenta Quél. (from its slender stem) $a b c$.

$P$. subumbonate, slightly scaly; sc. broad, adpressed, pale brown. $A$. slightly movable. Flesh unchanging white.

Often crespitose. Edible. Pastures, under beeches; uncommon. JuneNov. $4 \times 7 \times \frac{3}{8}$ in.

25. L. mastoidea Quél. (from the resemblance of the pileus to a breast; Gr. mastos) a $b c$.

$P$. acutely umbonate, whitish; sc. minute, dark brown, varying whitish. St. brownish-white.

Weods. Sept.-Oct. $2 \times 3 \frac{1}{3} \times \frac{3}{15}$ in.

\section{b. Clypeolarice.}

26. L. Friesii Quél. (after Elias Fries) $a b c$.

$P$. tomentoso-scaly, rich dark brown. St. pale brown. A. superior. $G$. crowded, branched.

Odour heavy, strong. Pastures, grassy places, gardens, sawdust, tree-roots, cinder-heaps. A ug.-Oct. $4 \frac{1}{4} \times 4 \times \frac{5}{8}$ in.

27. L. acutesquamosa Gill. (from the sharp pointed scales of the pileus) $a b c$.

$P$. lobed, ochreous buff-brown; sc. dark brown. St. pale brown. $A$. large. $G$. free, but close to stem, branched.

Sometimes cæspitose. Said to be edible. Taste mild, insipid; odour slighi, mephitic, disagreeable. Gardens, yards, hot-beds, melon-frames, churchyards, greenhouses. Sept.-Nov. $4 \frac{1}{2} \times 4 \frac{3}{4} \times \frac{3}{x}$ in.

28. L. Badhami Quél. (after C. D. Badham) $a b c$.

$P$. pale crimson-brown; sc. small velvety, dark purple-brown. St. crimson-brown or deep purple-brown below, paler above. $G$. at length black edged. Flesh changing to saffron-crimson when broken.

Taste none or insipid; odour strong, usually unpleasant. Under yews, hawthorns, hedges. Sept.-Nov. $3 \frac{3}{4} \times 5 \times \frac{3}{8}$ in.

29. L. emplastrum Sacc. (from the plaister-like scales) $a b$.

$P$. yellow-buff; sc. large, smooth, brown. St. dull pale buffyellow. Flesh pink when broken.

Gregarious. Taste and odour none. Amongst grass under trees. Oct. $3 \times 3 \frac{1}{1} \times \frac{1}{2}$ in. 
30. L. MELEAGR1s Quél. (from its markings, as of the guinea-fowl, meleagris) a $b c$.

$P$. pale salmon; sc. minute, almost black. St. bulbous-rooting, black-spotted, blackish at base. $G$. white or lemon-yellow. Flesh bright red when broken, reddish when dry.

Hothouses, greenhouses, on spent tan; rare. May-Oct. $2 \frac{1}{4} \times 2 \frac{1}{2} \times \frac{3}{16}$ in.

31. L. Blorvata Sacc. (from its twofold ornamentation) $a c$.

$P$. whitish; sc. dot-like, dark red. St. enlarged below, rooting, red-spotted. $A$. red-spotted at marg. Flesh white, reddish in St., dark when dry.

In hot-beds, stoves, melon and cucumber frames. July. $2 \frac{1}{1} \times 4 \frac{1}{4} \times \frac{5}{16} \mathrm{in}$.

32. L. hispida Gill. (from the rough pileus; hispidns, rough) $a b$.

$P$. velvety, rich brown. St. woolly-scaly, brown below A. Flesh unchanging white.

Odour disagreeable, somewhat pungent of radishes. Woods, chiefly pine. July-Oct. $3 \times 3 \times 3$ in.

33. L. elypeolaria Quél. (from the shield-like pileus; clypcus, a shield) $a b c$.

$P$. floccoso-scaly, sometimes pale buff, rosy or rufescent. St. scaly, whitish. Flesh white.

Odour weak or none. Woods, stoves, greenhouses. Oct.-Nov, $2 \frac{3}{t} \times 3 \frac{3}{t} \times \frac{1}{\text { in }}$ in. Colour variable, sometimes wholly white. Sometimes very small.

Var. alba Bres. (from the dead-white colour) $b$.

$P$. fleshy, convex, then expanded, broadly umbonate, silkywhite; mid. glabrous; marg. fibrilloso-flocculose, then squamulose, becoming yellowish with age. St. equal or attenuate downwards, white, somewhat fuscous at base, white-floccose and yellow-spotted below the distant, floccose, fugacious white $A$. $G$. free, crowded, white then ochreous. Flesh white, somewhat yellowish in stem. Often forming a spurious second $A$.

Edible. On the ground. Nov.

33a. L. felina Karst. (from the markings on the pileus; felis, a cat) $a b$.

$P$. subumbonate, black when young, then black-scaly on a white ground. St. white, often black-scaly below. A. large, often black-dotted.

Woods, fir, amongst moss. May-Sept. $1 \frac{3}{4} \times 3 \times \frac{3}{15}$ in.

33b. L. nigromarginata Nlass. (from the black-edged gills) a $b$.

$P$. campanulato-expanded, subumbonate; sc. umber on a pale sienna-ochre ground. St. hollow, attenuate upwards, smooth, salmon-white. A. persistent, membranous, white. G. broader in front, whitish; edge bordered with dark umber. Flesh thin.

Amongst grass. May. $2 \times 2 \frac{3}{4} \times \frac{1}{10}$ in. Allied to 33 , and especially to 33 , but differing from both in the dark-edged gills. 
34. L. metulæspora Sacc. (from its obelisk-shaped spores; metula, an obelisk) $a b c$.

$P$. umbonate, white; um. and sc. pallid; marg. sulcate. $r$. appendiculate. St. pallid or lemon-colour within and without.

Odour weak or none. Woods, fir, amongst moss and leaves; rare. Sept.Oct. $\quad 1 \frac{1}{2} \times 2 \frac{1}{2} \times \frac{3}{16}$ in.

35. L. cristata Quél. (from the scaly-crested pileus) $a b c$.

$P$. white; sc. and mid. bright dark brown. St. not scaly, brown at base. Sometimes red when broken.

Suspected poisonous. Taste strong, disagreeable; odour pungent, mephitic, sulphurous, or like radishes, but noxious. Fields, orchards, gardens, lawns, etc.; common. Aug.-Nov. $2 \frac{1}{2} \times 3 \frac{1}{2} \times \frac{3}{16}$ in. Sometimes red when broken.

36. L. erminea Gill. (from the ermine-like pileus) $a b c$. Wholly white. Very fragile.

$P$. silky.

Taste radish-like or none; odour slight. Grassy places. Sept.-Nor. $2 \times 3 \frac{1}{8} \times \frac{3}{16}$ in.

37. L. Micropholis Sacc. (from the minute scales on the pileus; Gr. mikros, small, pholis, a scale).

$P$. white; sc. radiating, erect, dark grey or black; marg. substriate. St. white.

Stoves on coco-nut fibre. $\stackrel{3}{\frac{3}{x}} \times \mathbf{I} \times \frac{1}{16}$ in.

\section{c. Anmulosa.}

38. L. Vittadinii Quél. (after Carlo Vittadini, Italian mycologist) a $b$. Wholly white or whitish.

$P$. rough with pointed sc. St. concentrically scaly. $A$. large.

Suspected poisonous. Groves, pastures, downs. June-Aug. $5 \frac{1}{2} \times 6 \frac{3}{4} \times \frac{7}{8}$ in. Allied to Amanita.

38a. L. nympharum Karst. (from its beauty, as of a wood-nymph) a $b$.

$P$. umbonate, covered with large revolute scales, white. St. white, or faintly buff-stained below. $G$. crowded, ventricose, white.

Grassy places. Oct. $4 \times 4 \frac{3}{4} \times \frac{1}{2}$ in.

39. L. holosericea Gill. (from the silky pileus; Gr. holos, entire, serikos, silken) a $b c$. Buff-white. Substance very soft.

$P$. fibrilloso-silky, then even. St. longitudinally fibrillose, then even. $A$. large.

Edible. Odour none. Gardens, cultivated places. Sept.-Oct. $3 \frac{3}{4} \times 2 \frac{3}{4} \times \frac{5}{5}$ in.

40. L. naucina Quél. (from the nut-like flavour; macinus, of a nut) $a b c$. Buff-white.

$P$. granular; marg. not plicate, appendiculate with $\mathrm{V}$. $A$. large. $G$. becoming faintly rosy. Flesh white, usually becoming pale salmon or salmon-brownish when cut.

Somewhat cæspitose. Edible. Taste mild, pleasant; odour strong. Fields; rare. July-()ct. $5 \times 3 \frac{1}{2} \times \frac{5}{8}$ in. Compare 790 . 
41. L. Cepeastipes Quél. (from the onion- or chive-shaped stem; cepa, an onion, stipes, a stem) a $b c$. White, pale lemon, or sulphur.

$P$. floccose; marg. plicate. St. floccose. A. fugacious.

Gregarious. Stores, frames, pine-pits, on tan and leaves, melon-beds, etc.; common. Mar.-Sept. $\quad 2 \frac{3}{4} \times 4^{\frac{3}{4}} \times \frac{3}{8}$ in. Sometimes becomes vinous or claret-colour in drying.

Var. cretacea Sacc. $P$. white; sc. darker.

42. L. citrophylla Sacc. (from the lemon-tinted gills) a $c$. Lemonyellow.

$P$. sc. rufous; um. rich-brown; marg. not crenate or sulcate St. squamulose. $A$. fugacious. Flesh and $G$. sometimes change to green.

On the ground; rare. Oct. $1 \frac{1}{4} \times \mathbf{I}_{ \pm}^{3} \times \frac{1}{8}$ in.

43. L. Licmophora Sacc. (from the sulcate pileus: a fancied resemblance to a winnowing fan, Gr. likmos) $a b c$.

$P$. lemon-yellow. $A$. small. $G$. remote.

Hothouses, conservatories, etc., on the ground, on coco-nut fibre. Aug.-Oct. $2 \frac{1}{8} \times 4 \frac{3}{4} \times \frac{3}{16}$ in. Resembles a Bolbitius or a yellow Hygrophorns. Sometimes wholly sulphur-white. At one time regarded as a var. of 41 .

\section{d. Granulosa.}

44. L. carcharias Karst. (from a fancied resemblance in the granules of the pileus to the teeth of a dog-fish, Gr. karcharias) $a b c$.

$P$. Flesh-colour; gr. darker. $A$. granulose.

Taste disagreeable, bitter or none; odour faint to strong of meal. Woods, fir. May-Nov. $2 \times \mathbf{I}_{\frac{3}{4}}^{\frac{3}{4}} \times \frac{3}{16}$ in. Sometimes very small.

45. L. cinnabarina Karst. (from the cinnabar or vermilion colour) a b c.

$P$. granular, often coarsely so; marg. fimbriate with V. St. granular below $\mathrm{A}$, orange-vermilion.

Taste mild. Woods, fir, grassy places. Sept.-Nov. $2 \frac{1}{2} \times 2 \frac{1}{2} \times \frac{5}{16} \mathrm{in}$. Agaricus Terreyi B. \& Br. is a form of this species.

46. L. granulosa Quél. (from the granular pileus) $a b c$.

$P$. floccoso-granular, red-brown. St. granular-scaly, red-brown below. $G$. slightly adnexed, sometimes adnate.

Woods, fir, grassy places; very common. July-Nov. $2 \times 2 \frac{1}{2} \times \frac{3}{16}$ in. There is a white var. mufiscens Sacc., which becomes red in drying.

46a. L. atrocrocea Mass. (ater, black, croceus, saffron) a b.

$P$. expanded, slightly depressed, bright salmon-orange, more or less covered with purple-brown almost black granular flocci. St. hollow, attenuate upwards, bright salmon-orange; salmonbrown-squamulose. $A$. fugacious. $G$. broadly adnate, salmonwhite. Flesh thin; salmon-orange-brown.

Oct. $\quad \mathrm{I} \frac{1}{2} \times 1 \frac{1}{4} \times \frac{1}{5}$ in. Allied to 46 and 47 . 
47. L. amianthina Karst. (from its pure colour; Gr. amiantos, undefiled) $a b$.

$P$. ochraceous; mid. orange. St. squamulose, yellow. $A$. fugacious. $G$. adnate or slightly decurrent, becoming light yellowish. Fles/2 wholly or partially yellow.

Woods; uncommon. Aug.-Nov. $1 \frac{1}{2} \times 2 \times \frac{1}{8}$ in. A form occurs intermediate between this and 46 .

Var. Broatzoodice Sacc. P. hemispherical, tomentose, yellow; G. white.

48. L. polysticta Gill. (from the many-spotted pileus; Gr. polus, many, stiktos, spotted) $a b c$.

$P$. fleshy, livid-brownish; sc. small, red-brown. $A$. fugacious. $G$. faintly yellowish.

Taste insipid ; odour none or mephitic, like 35. Grassy places, roadsides, etc. ; uncommon. Sept. - Nov. $2 \times I_{4}^{3} \times \frac{3}{8}$ in.

\section{e. Mesomorpha.}

48a. L. parvannulata Gill. (from the small annulus) $a$.

$P$. umbonate or gibbous, at first slightly pruinose, white to faintly yellowish. St. fibrillose below A., white. A. spreading, subpersistent.

Pastures. Autumn. $\frac{7}{8} \times 1 \frac{1}{2} \times \frac{1}{10}$ in.

49. L. sistrata Quél. (from a fancied resemblance to a rattle, sistrum) a ó.

$P$. shining-atomate, whitish, becoming light yellowish or fleshcolour; marg. appendiculate with V. St. pruinoso-silky, white. $A$. fibrillose.

Sandy ground amongst grass, gardens, etc. Oct. I $\times 2 \times \frac{1}{8}$ in.

50. L. seminuda Gill. (from the partially mealy pileus and stem; scmi, half, mudus, naked) a $b c$. Whitish or flesh-coloured.

$P$. floccoso-mealy then naked, usually white. St. mealy and flesh-coloured below, naked and white above. $G$. adnate

Odour none. Woods. May-Nov, $\frac{5}{3} \times 1 \frac{1}{2} \times \frac{1}{16}$ in.

51. L. Bucknalli Sacc, (after Cedric Bucknall) a $c$. White.

$P$. and St. minutely granular, lilac.

Odour strong of gas-tar. Oct. $1 \frac{1}{8} \times 2 \frac{1}{8} \times \frac{1}{8}$ in.

52. L. mesomorpha Gill. (from its intermediate characters; Gr. mesos, middle, morphe, form) a $b c$. Whitish, yellowish, brownish or brown.

$P$. and St. smooth. A. entire, persistent.

Woods. Oct.-Dec. $\mathbf{I} \frac{1}{8} \times \mathbf{I} \frac{3}{4} \times \frac{1}{16}$ in.

53. L. MARTIALIS Sacc. (from its red colour) $a b$.

$P$. fibrillose, ochreous-red; marg. striate. St. ochreous above, rufous below.

Conservatories, tree-fern stems. March. $\mathbf{I} \frac{1}{8} \times \mathbf{I} \frac{3}{8} \times \frac{1}{16}$ in.

54. L. IAnthina Sacc. (from the violet pileus; Gr. ion, a violet) $a$.

$P$. fibrillose; mid. darker; marg. not striate.

Stoves. March. $\mathbf{I} \times \mathbf{I} \frac{1}{8} \times \frac{1}{8}$ in. 
54a. L. submarasmioides Sacc. (from its slight resemblance to a species of Marasminis) a $b$.

$P$. umbonate, tough, arachnoid-squamulose, white to pale buff; um. rufous; marg. subplicate, cracking. St. at first arachnoidpruinose, white. A. superior, movable. $G$. membranoustough. Flesh tough, rufous within towards base.

Low ground at Worcester. Sept. I $\times 2 \frac{1}{8} \times \frac{1}{8} \mathrm{in}$.

\section{f. Viscidie.}

55. L. medullata Gill. (from its pith-bearing stem ; medulla, pith) $a b$. White or grey-white.

$P$. umbonate; marg. appendiculate with V. St. with a removable pith.

Odour of radish or mephitic like 35. Woods, fir. Aug.-Oct. $2 \times 3 \frac{1}{3} \times \frac{1}{4}$ in. Compare 58. Resembles $\mathbf{1 1 8 8}$ in stature, colour and viscidity of pileus.

56. L. glioderma Gill. (from the clanmy pileus ; Gr. gloios, clammy, derma, skin) $a c$.

$P$. brownish-red. St. dry, floccoso-scaly below, white or buff-white. Woods, thickets, pine; uncommon. July-Sept. $I_{\frac{3}{4}}^{\frac{3}{4}} \times 3 \frac{1}{5} \times \frac{1}{4} \mathrm{in}$.

57. L. delicata Gill. (delicate) $a b$.

$P$. thin, subumbonate, rufescent, yellowish, pale rose or brownish. St. dry, floccoso-scaly below, whitish.

Taste and odour sometimes rank, like Polyporns squamosus. Hothouses, woods, stumps, etc. Sept. $1 \frac{1}{4} \times \mathbf{I} \times \frac{1}{8}$ in.

58. L. illinita Quél. (from the glutinous pileus; illinitus, besmeared) a b.

$P$. umbonate white, varying to ochreous or clay-colour with a brown um. St. glutinous below. $A$. well developed on St., or appendiculate at edge of $P$.

Woods. Aug.-Sept. $2 \frac{1}{ \pm} \times 3 \frac{3}{ \pm} \times \frac{5}{16}$ in. Compare 55 and 1188.

59. L. Georginæ Sacc. (after Georgina E. Johnstone) $a b$.

White, all parts becoming bright crimson when touched. $P$. viscido-pruinose. Brown when dry.

Taste and odour none. On sphagnum in greenhouses. May-Nov. $1 \frac{1}{1} \times 2 \frac{1}{8} \times \frac{1}{8}$ in.

\section{HIATULA Mont.}

(From the furrows on the pileus; Gr. hio, to gape.)

Hymenophore distinct from the stem. Veil universal, manifest in the granular or powdery covering, and sometimes in the appendiculate margin of the pileus. Pilezs fleshy to membranous. Stim central, simple. Gills free. Spores normally white, sometimes greenish or green as in Lepiota. (Fig. I3.)

Differing from Lepiota in the absence of an annulus, and agreeing in structure with Phutus, Pluteolus, and Pilosace.

Pileus somewhat fleshy.

$60,60 \mathrm{a}$

Pilcus membranous, sulcate. $60 \mathrm{~b}, 60 \mathrm{c}$. 
60. H. Eyrei W. G. Sm., Schulzeria Eyrei Mass. (after Rev. W. L. W. Eyre) $a b c$.

$P$. broadly umbonate, smooth, pallid; mid. minutely granular ochreous; marg. incurved, appendiculate with V. St. smooth,

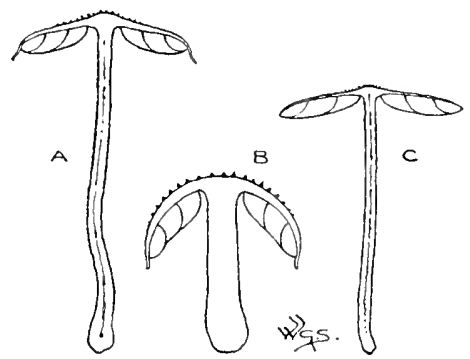

Fig. 13.-A, section through Fiatula Eyrei

W. G. Sm. : B, ditto, H. lycopertoides WV.

G. Sm. ; c, ditto, H. Wymice B. \& Br.

One-half natural size.

even, glabrous, minutely mealy above, pallid. G. free, narrowed behind, thin, crowded then distant, pale green then deep bluishgreen. Spores same colour as G.

Gregarious. Taste and odour none. Under spruce-firs. Autumn. $1 \frac{1}{2} \times 2 \frac{7}{8} \times \frac{1}{5}$ in.

60a. H. lyeoperdoides W. G. Sm., Schulzeria lycoperdoides Cooke \& Mass. (from a resemblance in the pileus to certain species of Lycoperdon as 2063 and 2064) $a b$.

$P$. convex, then expanded, soft, buff-ivory, beset with pyramidal silky-fibrous dark brown warts; marg. appendiculate. St. solid, equal, minutely fibrillose, whitish; faintly buff-ivory below. $G$. somewhat crowded, white. Flesh white, pale buff-brown under cuticle of $\mathrm{P}$.

Taste and odour none. Under cedars. Autumn. $\quad 1 \frac{1}{4} \times 1 \frac{1}{2} \times 1 \frac{1}{4}$ in.

60b. H. Wynneæ B. \& Br. (after Mrs. Lloyd Wynne).

$P$. campanulate then plane, subumbonate, pulverulent, white; mid. brownish. St. hollow. $G$. free, at first very slightly adnexed, somewhat distant, with a shrivelled appearance as in 41. Has been observed emitting a greenish phosphorescent light.

Stoves, on wood.

60c. H. grangei W. G. Sm., Schulzeria grangci Eyre (from the original locality, Grange Park, Hants).

$P$. flatly umbonate, cracking into dark green fibrous scales on a white ground. St. squamose, sq. tipped with colour of $\mathrm{P}$. $G$. widest in front, minutely denticulate.

Caspitose. On soil amongst beech-leaves. Nov. $1 \frac{3}{8} \times 2 \frac{3}{8} \times \frac{1}{5}$ in. 


\section{ARMILLARIA Quél.}

(From the annulus; armilla, a ring.)

Veil universal, imperfect, remaining as minute, concrete warts or flocci on the pileus and as the scales of the stem. Hymenophore confluent and homogeneous with the fleshy stem. Pileus usually

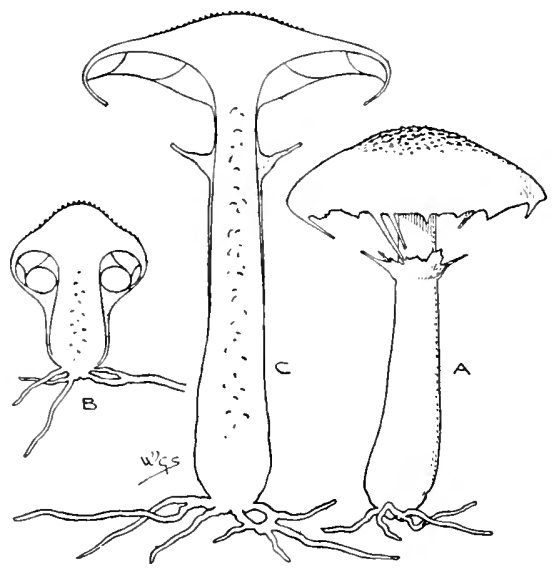

Fig. 14.-Amillaria melléa Quél. One-third natural size. A, perfect plant: B, section of young example showing universal veil; $c$, section of mature example.

fleshy, the surface broken up into small scurfy warts or floccose scales. Stem central, of the same substance with the pileus, annulate. Gills sinuato-adnexed, adnate or decurrent. Spores white, elliptical, smooth. (Fig. I4.)

The species grow on the ground or on or about stumps.

Nust not be confounded with the last section of Amanita.

Species $61-72$

Stem furnished with a fugitive annulus. Gills sinuato-adnexed.

Ste'm furnished with a sometines fugitive annulus. Gills more or less decurrent.

Stem annulate. Gills adnate, varying rounded, sinuate or sinuatodecurrent.

61. A. bulbiger Quél. (from the bulbous stem) $a b c$.

$P$. subumbonate, nearly smooth, pale yellowish or buff. $S t$. strongly bulbous, whitish, longitudinally black fibrillose. $G$. becoming pale yellowish. Flesh whitish.

Taste and odour none. Woods, pine. Oct.-Nov. $3 \frac{1}{2} \times 3 \frac{1}{2} \times \frac{1}{2}$ in. 
62. A. focalis Gill. (from the thick clothy annulus; focale, a neckcloth) $a b$.

$P$. umbonate, silky-fibrillose, rich yellowish-red, ochre or livid yellow. Flesh pale yellowish-brown.

Woods, pine, heaths, bare ground under old laurels. Aug.-Oct. $4 \frac{1}{2} \times 3 \frac{1}{2} \times \frac{7}{8}$ in. The dimensions of the variety called Goliath by firies are $606 \frac{3}{4} \times 1 \frac{1}{4} \mathrm{in}$.

63. A. robusta Gill. (from its sturdy growth) $a b c$.

$P$. convex, nearly smooth, rich brown. St. attenuate downwards, not scaly, brownish-white. Fle'sh hard.

Odour of new meal, but nauseous. Woods and plantations, pine, maple; rare. Oct. $2 \frac{1}{2} \times 2 \times \frac{3}{4} \mathrm{in}$. The form major is twice the size of minor, the form illustrated.

63a. A. ealigata Gill. (from a fancied resemblance in the stem to a leg with a soldier's shoe, caliga) $b$.

$P$. compact, convex, then plane, tawny, weasel-colour, spotted with adpressed silky sq. of the same colour. St. solid, zoned with brown sq. below the persistent membranous A. $G$. emarginate, white.

Odour strong. On the ground in woods, pine. Autumn. $3_{4}^{3} \times 3^{\frac{3}{8}} \times 1 \frac{1}{8} \mathrm{in}$. Agaricus causetta Barla, is a form of this species.

64. A. aurantia Quél. (from its orange colour) $a b$.

$P$. convex, innato-squamulose, viscid. St. very scaly. Flesh yellowish.

Woods, pine. Oct. $2 \frac{3}{4} \times 3 \frac{3}{4} \times \frac{1}{2}$ in.

65. A. ramentacea Quél. (from a fancied resemblance in the pileus to wood-shavings, ramenta) $a c$.

$P$. floccose becoming revolute, whitish, then yellowish or brownish; sc. and punctate mid. darker. St. white, clad with adpressed brown sc. below A. G. becoming yellowish. Flesh white.

Odour unpleasant. Under pines; uncommon. Sept. $2 \frac{1}{2} \times 2 \frac{1}{2} \times \frac{5}{16}$ in. Allied to Lipiota.

66. A. hæmatites Sacc. (from the colour of the pileus; Gr. haima, blood) $a$.

$P$. hemispherical, red liver-colour. St. liver-colour. Flesth pale liver-colour, darker at base of St.

Amongst fir-leaves. Nov. $1 \frac{3}{8} \times 1 \frac{5}{8} \times \frac{1}{8}$ in. Allied to Lepiota.

67. A. Jasonis Sacc. (from a fancied resemblance in the pileus to the golden fleece of Jason) $a b$.

$P$. campanulate, granular, golden-yellow; mid. reddish. St. goldenyellow.

Cæspitose. Taste none; odour ustally none, but sometimes strong. Stumps. Sept. $2 \times 2 \frac{1}{4} \times \frac{5}{16}$ in. Resembling Pholiota.

68. A. constricta Gill. (from the compact substance; constrictus, compact) $a$. White.

$P$. convex, silky-smooth. Flesh becoming pale yellow when bruised.

Odour of new meal. Pastures, sunny and urine-scorched places. Sept.-Oct. $2 \times \mathrm{I}_{4}^{3} \times \frac{3}{5}$ in. 
69. A. mellea Quél. (from the honey-coloured pileus; mel, honey) a bc.

$P$. smoky yellow-olivaceous, usually dark-scurfy or squamulose; marg. striate. St. often sulphur-yellow at base. $A$. usually large and white-floccose, sometimes small or absent. $G$. usually yellowish or pale salmon.

Solitary, gregarious, or crespitose. Taste acrid and disagreeable; odour strong, foxy. Sold as an esculent in Continental markets; said by Mrs. Hussey to be poisonous. On or near stumps, tree-roots or root-fibres, squared timber. July-Nov. $4 \frac{1}{2} \times 5 \times \frac{5}{8}$ in. Extremely variable. Compare 222. Collybia tabescino Sacc. is a ringless form.

Var. versicolor W. G. Sm., Agaricus versicolor With. (from the change of colour in the stem and gills).

St. bulbous, white then brown. G. yellow-white, then deep redbrown.

Amongst grass. July. I-4 $\times 2 \times \frac{1}{\text { sin. }}$ in.

Var. laricina WV. G. Sm., Agariaus laricinus Bolt. $P$. flesh-colour, glabrous; marg. smooth. G. white. On larch.

69a. A. denigrata Gill. (from the very dark or blackish pileus; denigro, to colour black) a.

$P$. subviscid, guttato-punctate; marg. even. St. solid, pallidfuscous, whitish above. $A$. superior, entire fugitive, white. $G$. pale brown, becoming darker.

Solitary or gregarious. On the ground, damp, shady places, bases of trunks. $2 \frac{1}{2} \times 2 \frac{1}{2} \times \frac{5}{8}$ in. Sometimes confused with 560 , which has brown, not white spores.

70. A. citri Gill. (from the citron-colour of pileus) a $b$.

$P$. subumbonate, smooth, sulphur-yellow ; marg. crenulate, becoming white. St. whitish, reddish-brown below.

Crespitose. Odour of new meal. Stumps. Sept. $2 \times 3 \times \frac{3}{16}$ in.

71. A. subeava Gill. (from the partially hollow stem; caz'us, hollow) $a$. White.

$P$. umbonate, thin ; mid. brownish.

Woods, pine. Nov. $2 \times 3 \frac{1}{2} \times \frac{3}{16}$ in. Allied to Lefiota.

72. A. mucida Quél. (from the slimy pileus) a bc. White.

$P$. hemispherical, radiato-wrinkled; mid. sometimes faintly brownish. St. externally cartilaginous. $G$. broad, mucid.

On beeches. Aug.-Nov. $3 \frac{1}{2} \times 6 \times \frac{1}{4}$ in.

\section{TRICHOLOMA Quél.}

(From the hairy, silky, or downy covering of the pileus, best seen in young examples; Gr. thrix, a hair, loma, a fringe.)

I' eil universal, imperfect, manifest in scales, flocci, fibrils or down, which adhere to the margin of the pileus in infancy, sometimes obsolete or nearly so. Hymenophore confluent and homogeneous with the fleshy stem. Pilus usually convex and fleshy, never umbilicate. Stom central, fleshy, simple, with cartilaginous bark. Gills sinuate, sometimes separating from the hymeno-phore. (Fig. I 5.) 
The species, except 91, 98 and 149, grow on the ground, the larger usually grow in upland woods, the smaller in pastures. Nostly autumnal ; some late; section Guttate is usually vernal. Many have a pleasant odour of meal, a few have a disagreeable odour; some are edible, a few suspicious or poisonous.

Species $73-152$

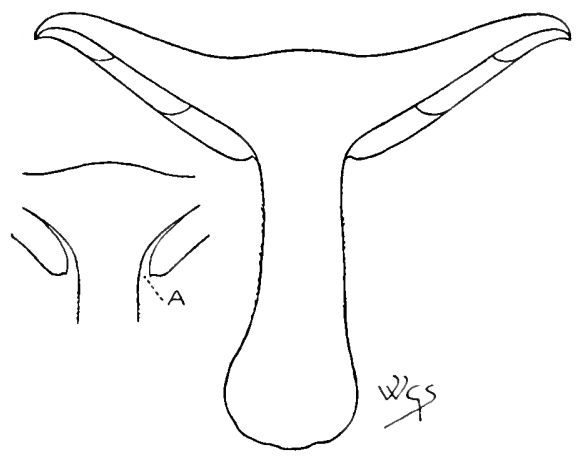

Fig. 15.-Section of Tricholoma personatum Quél. One-third natural size.

The separation of the stem from the hymenophore is shown at A.

A. Pileus viscid when damp, pubescent, fibrillose or scaly, not scaly.torn. Stem fibrillose. Flesh, except 77, not hygrophanous.

a. Limacine. Pileus fleshy. Stem solid, except 73a, 76, and 79. Gills not changing colour or becoming rufescent. $\quad 73-79$ Gills changing colour usually to rufescent, commonly redspotted.

$80-89$

b. Gonnine. Pilens torm into scales or fibrils, not viscid or hygrophanous, moist in 94. Taste sometimes bitter and disagreeable. Odour, except 93, generally not unpleasant or none.

Gills, except 94, not changing colour, or becoming rufous or black-spotted.

$90-95$

Gills rufescent or becoming grey, usually marked with rufous or blackish spots.

$96-101$

c. Rigidce. Pileus, except 106, punctato-granulate or broken up into smooth squamules, not viscid, scaly-floccose or fibrillose. Flesh in stout species cartilaginous-hard, in thin species fragile. Odour of the majority of the species unpleasant.

Gills unchanging white or becoming hyaline-whitish, except 103, which often becomes reddish. $102-108$

Gills changing colour, rufescent, or becoming ashy or spotted. 
d. Sericelle. Pileus smooth, dry, silky, never scaly, moist or viscid, flocculose in 121.

Gills broad, somewhat thick, subdistant, except 115. Odour strong, fetid.

Gills narrow, thin, crowded. Odour none, except 119.

$117-121$

B. Pileus smooth, moist in wet weather, not viscid, downy or scaly. Flesh soft, spongy, watery, hygrophanous.

c. Guttatie. Pileus thick, fleshy. Stem obese, solid. Odour pleasant. Usually vernal; often growing in troops, or cæspitose or forming large rings.

Gills whitish.

$122-124$

Gills changing colour, rufescent or smoky.

$125-127$

f. Spongiose. Pileus obtuse, smooth, moist, except 133 ; not hygrophanous. Gills sinuato-decurrent. Often late autumnal. Usually growing in troops. Must not, on account of the sinuato-decurrent gills, be confounded with Clitocybe.

Gills whitish.

$128-134$

Gills changing colour.

$135-142$

g. Hygrophana. Pileus hygrophanous, changing colour on parting from its absorbed water.

Gills white or faintly brownish, unspotted.

$143-148$

Gills becoming violet, grey, or smoky.

$149-152$

\section{a. Limacine.}

73. T. equestre Quél. (from its being the handsomest of the group; cques, a knight) $a b c$.

$P$. gibbous, then expanded, sulphur-olive or ochraceous, streaked olive-brown or olive-purplish. St. sulphur-olive, sulphur-white within. $G$. sulphur-olive.

Taste pleasant or none; odour none. Woods, fir ; uncommon. Aug.-Nov. $4 \times 2 \frac{3}{4} \times 1$.

73a. T. coryphæum Gill. (from its position as type of group; Gr. koruphaios, a leader).

$P$. white, shaded sulphur-white or sulphur, punctato-rirgate with dull purplish scales. St. equal to obclavate, white, shaded sulphur-white. $G$. crowded, white, edged or clouded pale sulphur.

Under beeches. Nov. $2 \frac{1}{2} \times 4 \times \frac{5}{5} \mathrm{in}$.

74. T. sejunctum Quél. (from the gills, separated from the stem; sejunctus, separated) $a b c$.

$P$. at first umbonate, buff or yellow, streaked brownish or purplesooty. St. white, clouded pale yellow. $G$. white.

Taste disagreeable, bitter; odour strong of rancid meal. Woods, chiefly pine; uncommon. Sept. - Nor. $4 \frac{1}{1} \times 4 \frac{3}{x} \times \frac{7}{5}$ in. 
75. T. portentosum Quél. (from its variability; portintosus, strange) a bc.

$P$. unequal, slaty-brown, black-fibrillose; mid. darker. St. white or greyish. $G$. somewhat distant, white, pale grey or yellowish.

Solitary, gregarious or cæspitose. Taste mild or somewhat nauseous; odour none. Woods, fir ; frequent. Aug.-Nov, $4 \frac{1}{2} \times 3 \frac{1}{2} \times \frac{7}{8}$ in. Pileus sometimes $\mathrm{S}$ in. in diameter. Sometimes springing from an involved mass of thick cord-like mycelium.

76. T. fucatum Gill. (from the dyed appearance of the pileus; fuco, to dye) $a b c$.

$P$. yellowish or palest yellow, streaked brownish-buff or pale slate. St. yellowish, sometimes white above and below, greyor blackish-squamulose or fibrillose. $G$. whitish.

Woods, chiefly pine; frequent. Aug.-Nov. $3 \frac{1}{4} \times 3 \frac{3}{4} \times \frac{5}{8}$ in.

77. T. quinquepartitum Gill. (from the pileus being supposititiously five-Iobed; the character does not however belong to this species) $a b$. Somewhat hygrophanous.

$P$. yellowish-buff; mid. darker, unstreaked. St. ivory-white, not squamulose. $G$. white.

Taste mild; odour none. Woods, pine; rare. Oct. $3 \frac{3}{1} \times 4 \frac{1}{ \pm} \times \frac{5}{8} \mathrm{in}$.

78. T. resplendens Karst. (from its brightly shining appearance) $a b$. Shining white.

$P$. sometimes very faintly stained yellowish or brownish.

Gregarious. Taste and odour pleasant. Woods, beech, hazel, etc.; frequent. Sept.-Oct. $2 \frac{3}{4} \times 2 \frac{5}{8} \times \frac{1}{2}$ in. Resembles 1188 in general appearance.

79. T. spermaticum Gill. (derivation obscure) a b. White.

$P$. sometimes cream or faintest ochre-white. $G$. coarsely notched.

Poisonous. Odour strong like tallow, varying to cucumber, but stinking. Woods, fir. Oct. $3 \frac{3}{4} \times 2 \frac{1}{4} \times \frac{3}{4}$ in. Resembling 78.

80. T. colossum Quél. (from its great size) $a b$.

$P$. rich brown, paler or whitish at marg. St. bulbous, reddishbrown with a white floccose apex. $G$. white or dull fleshcolour. Fles/l hard, changing to tawny when broken.

Odour none or cheese-like. Under Scotch firs, elms. Oct. $8 \times 4 \frac{1}{2} \times 1 \frac{3}{4} \mathrm{in}$. Resembling 1490 in size and colour. A form occurs apparently between this and 83. Boudier considers this species to belong to Armillaria.

81. T. nictitans Gill. (from its sporadic appearance; nictitans, winking) $a b$.

$P$. rich brown ; mid. darker. St. enlarged below. $G$. yellowish.

Taste usually mild or sweet, slightly of mushrooms, sometimes clisagreeable, but not bitter; odour none or sweet. Woods; rare. Sept. $5 \frac{1}{ \pm} \times 3 \frac{1}{2} \times \frac{5}{8}$ in. Resembling 1458 in form, colour and viscidity.

82. T. fulvellum Gill. (from the tawny pileus ; fulcus, tawny) a $c$.

$P$. dotted-wrinkled, rich tawny-brown; mid. darker. St. not enlarged below. $G$. white.

Odour none. Woods, beech. Oct.-Nov. $3 \frac{1}{2} \times 3 \times \frac{1}{2}$ in. 
83. T. flavobrunneum Quél. (far'us, light yellow, brunnezs, brown) a b $c$.

$P$. streaked, tawny or rich brown; mid. darker. St. slightly enlarged below, rich brown or brownish. $G$. light jellowish. Flesh white or partially pale yellowish.

Gregarious, crespitose or in troops. Odour strong of rank meal or cheese. Deciduous woods, especially birch, beech ; amongst gorse. Sept.-Nov. $3 \frac{1}{2} \times 2 \frac{1}{4} \times \frac{3}{4}$ in. A form occurs apparently between this and 80 .

84. T. albobrunneum Quél. (alluss, white, brunneus, brown) a b $\mathrm{c}$.

$P$. streaked, rich ochreous to pale brown; mid. darker. St. not enlarged below, yellowish-brown, white above and below. $G$. white. Flesh permanently white.

Said to be edible. Taste usually mild, sometimes bitter; odour none. Plantations, fir. Sept.-Dec. $4 \times 2 \times \frac{3}{4}$ in.

85. T. ustale Quél. (from its scorched appearance ; ustus, burnt) a $b c$. $P$. dotted-wrinkled, rich-or purple-brown, becoming black-brown, unstreaked. St. slightly attenuate downwards, brownish-white. $G$. white. Flesh sometimes changing to faintest rose.

Ołour wone. Woods, chietly pine; rare. Aug.-Nov. $3 \times 2 \frac{3}{5} \times \frac{3}{8}$ in. Intermediate between 83 and 86 .

86. T. pessundatum Quél. (from the foot-trodden appearance in Sterbeek's illustration) $a b c$.

$P$. guttato-spotted, not streaked, brown. St. equal, pale ochrebrown. $G$. white.

Said to be edible, but suspected poisonous. Taste mild ; odour strong of new meal. Woods, fir; rare. Sept.-Nov. $4 \frac{1}{8} \times 2 \frac{1}{2} \times 1$ in.

87. T. stans Gill. (from its standing distinct from 86) $a b c$.

$P$. reddish-brown. St. equal, white. $G$. white. Fles/l reddish under cuticle.

Woods. Sept.-Oct. $3 \frac{5}{8} \times 4 \times$ I. Compare 106.

88. A. Russula Gill. (from its resemblance to certain of the red Russule $a$ a b c.

$P$. rose, or crimson-granulate, or rose-liver-purplish, variegated rose and ivory-white. $G$. white, palest rose or clouded as P.

Eaten in Austria. Taste and odour mild, pleasant. Under trees. Oct. $4 \frac{5}{8} \times 2 \frac{1}{2} \times \frac{7}{8}$ in. Perhaps allied to 1192 .

89. T. frumentaceum Quél. (from its odour of meal; frumentum, corn) ac.

$P$. whitish-buff; mid. brownish-buff. St. equal. G. white. Flesh white.

Odour strong of new meal. Sept.-Oct. $3 \frac{1}{2} \times 1 \frac{7}{5} \times \frac{5}{5}$ in.

\section{b. Gonuine.}

90. T. rutilans Quél. (rutilo, to be reddish) a $b c$.

$P$. dark purple on a yellow ground. St. enlarged below, light sulphur, purple-floccose. $G$. bright yellow. Flesh sulphur to yellow.

Suspected poisonous. Odour none. Woods, pine, birch; base of trees; common. Aug.-Nov, $5 \frac{1}{2} \times 4 \frac{1}{4} \times \mathrm{I}$ in. Pileus sometimes $7 \mathrm{in}$, or more in diam. 
91. T. variegatum Gill. (From the variegated pileus) $a b$.

$P$. reddish-purple on a yellow ground. St. slightly enlarged below, reddish-purple-floccose on a yellowish-white ground. $G$. yellowish-white. Flesh whitish to palest sulphur.

Rotten wood. June-Oct. $2 \frac{3}{2} \times 2 \frac{3}{4} \times \frac{3}{8}$ in. Resembling a small, attenuate, dull-coloured state of 90 .

92. T. lupidum Quél. (from its lurid colour) a $b c$.

$P$. whitish-brown-livid, or ashy-yellowish; mid. darker. St. white. $G$. very crowded, whitish.

Taste mild; odour of new meal. IVoods, pine, etc. ; common. Sept.-Oct. $2 \frac{1}{2} \times 2 \frac{1}{2} \times \frac{1}{2}$ in. Compare 74 with distant gills and 103 with odour of soap.

93. T. guttatum Gill. (from its spotted pileus; grtta, a drop) $a$.

$P$. pale buff, mid. darker, clad with large brown granular sq.; marg. remotely sulcate, white or faint rose-floccose. $G$. white. Flesh white, pale rose under cuticle and at base of St.

Somewhat crespitose. Taste and odour acid-bitter. Woods. Oct. $3 \times 2 \times \frac{5}{8}$ in. Resembling 99.

94. T. columbetta Quél. (from its dove-colour; columla, a dove) a $b c$.

$P$. moist, white or faintly clouded buff-white; mid. sometimes brownish-grey. $G$. separating from stem.

Solitary. Said to be edible. Taste mild; odour none. Pastures, woods, birch, fir, beech. Aug. -Nov. $4 \times 3 \times \frac{3}{4}$ in. Sometimes rell- or bluespotted; sometimes exuding a deep bright permanent violet stain.

95. T. scalpturatum Quél. (fronı its scratched appearance: scalpte, to scratch) $a c$.

$P$. livid- or grey-brown on a whitish ground. St. and Flesh whitish. $G$. white to yellowish.

Woods, fir ; uncommon. Sept.-Nov. $3^{\frac{1}{4}} \times 3^{\frac{1}{4}} \times \frac{2}{3}$ in. Differs from $101 \mathrm{in}$ the yellowish, not grey gills.

96. T. imbricatum Quél. (from the scales of the pileus; imbricatıs, overlapping) $a b c$.

$P$. rich reddish- or crimson-brown. St. crimson-brown, whitepulverulent above. $G$. crowded, at first white.

Scattered or in troops. Said to be edible. Taste when raw disagreeable. Woods, pine, oak. Sept.-Nov. $4 \frac{1}{2} \times 2 \frac{3}{4} \times \frac{3}{4}$ in.

97. T. vaecinum Quél. (from the reddish-brown colour; z'acar, a cow) a $b c$.

$P$. warm-rose to crimson-brown. St. crimson-brown, not white above. $G$. somewhat distant, separating from St.

In troops. Taste bitter, disagreeable; odour strong, not unpleasant. Woods, fir. Aug.-Oct. $2 \frac{3}{4} \times 2 \frac{1}{2} \times \frac{5}{8}$ in.

98. T. immundum Quél. (immundus, discoloured) a $b c$.

$P$. grey-buff. St. greyish-buff. $G$. pearly-grey.

Crespitose. Taste bitter, disagreeable; odour mealy to offensive. On sheepdung; rare. Sept.-Oct. $3 \times 2 \times \frac{3}{8}$ in. 
99. T. inodermum Gill. (from the fibrils of the pileus; Gr. is, inos, a nerve, derma, the skin) a $b$.

P. pink-buff; mid. brownish, brown-scaly. St. white, then partiaily reddish, mealy above. $G$. white, red-spotted when bruised. Flesh pale pink-buff.

Damp, dense pine-woods. $2 \frac{3}{4} \times 2 \frac{1}{1} \times \frac{1}{2}$ in. Resembling 93 .

100. T. gausapatum Quél. (from the shaggy pileus; gausapa, a shaggy woollen cloth) $a b$.

$P$. bullate, obtuse, wavy, woolly-fibrillose, somewhat dark-ashygrey; edge at first incurved, white-woolly. St. solid, white, fibrillose. $G$. adnate, pale grey.

Taste mild; odour none. Woods; grassy places. Sept.-Oct. $3 \times 2 \times \frac{5}{8}$ in.

101. T. terreum Quél. (from its earthy colour ; ter $a$, the earth) $a b c$.

$P$. umbonate, dark purplish-grey or pale slate. St. grey, whitepruinose above. $G$. white to grey.

Solitary, gregarious or cæspitose. Taste slightly rancid; odour none. Woods, chiefly beech, pine; common. Aug. - Nov. $3^{\frac{1}{4}} \times 2 \frac{3}{9} \times \frac{5}{8}$ in. Often becoming black in drying. Sometimes springing from rosecoloured mycelium or from rhizomorphoid threads. Var. orimtbens MIass. G. recl-edred. Var. argyraceum Mass. Wholly silvery-whitish. Var. virescens W. G. Sm., Agaricus scalpturatus Fr. var. zirescens Cooke. Becoming yellowish-green. Var. atrosquamosum Mass. Black, scaly. Var. chrysites Mlass. Becoming yellow.

101a. T. squarrulosum Bres. (squarmlosis, scaly) a.

$P$. becoming striato-hairy, fuscous to lurid tan; mid. blacksquamulose. St. broadly striato-rough, dark grey-umber on a pale dull salmon ground. $G$. grey-white as in 101 .

Under oaks, hazel. Sept. $3 \frac{1}{5} \times 3 \frac{1}{5} \times \frac{5}{8} \mathrm{in}$.

101b. T. horribile Rea (horribilis, rough) $a$.

$P$. dark-fuscous-squamulose, the ground pink at maturity. St. enlarged below, white, smooth, hollow. $G$. emarginate, crowded; marg. uneven, whitish, becoming pinkish. Flesh white, becoming pinkish and fuscous-spotted.

Under beech. $4 \frac{5}{8} \times 3^{\frac{3}{4}} \times 1$ in.

\section{c. Rigida.}

102. T. macrophizum Sacc. (Gr. makros, long, rhiza, a root) a b.

$P$. smooth, then tessulato-cracked, ochreous-buff. St. whitishochreous. Flesh slightly yellowish.

Taste mushroom-like or unpleasant; odour strong like 113, cadaverous, like Lilium auratum, like carrion or fetid-sweet, varying pleasant to unpleasanl. Nov. $4 \frac{1}{4} \times 5^{\frac{3}{4}} \times \mathrm{I}_{\frac{1}{4}}$ in. Pileus sometimes $8 \mathrm{in}$. or more in diam. with a long rooting stem in proportion.

103. T. saponaceum Quél. (from its soapy odour) $a b c$.

$P$. smooth, then olive-brown scaly. St. pale yellowish-grey, often minutely black scaly. $G$. distant, yellowish-grey-white.

Woods, hedgesides, etc., amongst leaves; common. Aug.-Nov. $3 \frac{1}{2} \times 2 \frac{3}{4} \times \frac{5}{8} \mathrm{in}$. Often reddish when bruised. Nust not be confounded with the scentless $112 \mathrm{~b}$. 
104. T. cartilagineum Quél. (from its gristly substance) a $c$.

$P$. black-dotted on a brownish ground; mid. darker. St. smooth, whitish. Flesh white. G. crowded, white or pale greyish.

Odour none. Grassy places in woods and pastures, amongst pines; uncommon. Aug.-Nov. $3 \frac{1}{2} \times 2 \frac{1}{ \pm} \times \frac{5}{8}$ in. Compare 112a. An abnormal growth of this, $4 \mathrm{ft}$. I in. $\times 2 \mathrm{ft}$. 1 in., with many pilei, raised the pavement of Goswell Road, London, in IS65.

105. T. tenuiceps Mass. (from the thin flesh of the pileus; tenuiceps, thin-headed) $a$.

$P$. conico-hemispherical, granular, dark smoky-brown. St. punctato-granulose, ochreous-white. $G$. subdistant, ventricose, white.

Solitary or in clusters of two or three, springing from thick cord-like mycelium. Amongst grass under trees. July, $2 \frac{1}{3} \times \mathrm{I}_{4}^{3} \times \frac{3}{4} \mathrm{in}$. Resem. bling 227 (var. repenss).

106. T. loricatum Gill. (from the leathery cuticle; loriat, a leather cuirass) $a c$.

$P$. unusually lobed, moist or subviscid, the thick cuticle peeling off, rich brown; marg. paler. St. reddish-buff. $G$. whitish straw-colour.

Odour strong, pleasant or nauseous. Woods, mixed. Sept. $2 \frac{3}{8} \times 2 \frac{1}{4} \times \frac{1}{2}$ in. Compare with 87.

107. T. atrocinereum Quél. (ate\%, black, cinereus, ash-coloured) a. $P$, ashy-brown or blackish; mid. darker. St. whitish. Flesh hygrophanous.

Odour of new meal. Grassy ground. Oct. $1 \frac{3}{4} \times \mathbf{I} \frac{3}{4} \times \frac{1}{4}$ in.

108. T. cuneifolium Gill. (from the wedge-shaped gills; cuncus, a wedge, folium, a leaf) a $b c$. Fragile.

$P$. smooth, brittle, often concentrically cracked, rich brown to livid. St. slightly attenuate downwards, splitting, pale brownish, white above. $G$. distant.

Said to be edible. Taste disagreeable, rancil ; odour strong of new meal. l'astures; frequent. Sept.-Nov, $\mathbf{I}_{\frac{3}{4}} \times \mathrm{I}_{\frac{1}{1}} \times \frac{3}{8}$ in. A Araricus cinereorimosum Batsch, a form of this, is British.

109. T. erassifolium Sacc. (from its thick gills; crassus, thick, folium, a leaf) $a$.

$P$. ochraceous or somewhat buff; mid. umber. St. pale ochreous.

Gregarious or subcrespitose. Odour strong. Woods, fir ; uncommon. Oct. $2 \frac{1}{2} \times I_{\frac{3}{4}}^{3} \times \frac{3}{8} \mathrm{in}$.

109a. T. sudum (quél. (sudus, dry) a.

$P$. ashy-rufous or blackish-umber; mars. paler. St. punctate with minute squanules, pallid, shaded rufous. $G$. whitish, edge rufescent.

Woods. Amongst grass. $3 \frac{3}{4} \times 3 \frac{1}{2} \times \frac{5}{8}$ in. Compare 131 . 
110. T. tumidum Gill. (tumidus, swollen) $a b$.

$P$. irregular, often cracked, ashy-livid; mid. darker. St. often swollen and attenuate below, white, often red-tinged within.

Taste and odour weak, not unpleasant. Woods, pine, moist places. Oct.Nov. $3^{\frac{1}{2}} \times 3_{\frac{3}{4}} \times \frac{5}{8}$ in. Var. Keithii Sacc. (after the Rev. Dr. J. Keith) the whole plant becomes partially rufescent.

111. T. murinaceum Gill. (from its mouse-colour; mus, a mouse) $a b c$. Ashy-or lilac-greyish.

$P$. cracked, streaked grey-silky-scaly; sc. sometimes blackish. St. more or less scaly like P. G. grey.

Taste very disagreeable, bitter ; odour strong, unpleasant, sometimes nitrous. Open woods and pastures; rare. Aug.-Nov. $4 \frac{1}{5} \times 3 \frac{1}{2} \times \frac{3}{7}$ in. Must not be confounded with 1244 .

11la. T. hordum Quél. (perhaps from hordeum, barley, on account of the hard substance).

$P$. subumbonate, soon cracking, in dry weather squarrose, mousegrey ; mid. darker. St. glabrous, whitish, shaded greyish. $G$. subdistant, white to greyish.

Taste mild; odour none. Under beeches. $3 \frac{1}{2} \times 4 \frac{3}{8} \times \frac{5}{8}$ in.

112. T. virgatum Gill. (from the streaked pileus; airgra, a stripe) a $c$. $P$. pale ashy-grey, finely-streaked black-fibrillose. St. subbulbous. $G$. crowded, becoming hoary. Flesh cinereous-whitish.

Usually solitary. Taste when young very bitter, becoming tasteless; odour usually none, sometimes sweet like melilot. Woods, mixed, oak, pine. Aug.-Oct. $3 \frac{1}{ \pm} \times 3 \frac{5}{8} \times \frac{1}{2}$ in.

112a. T. elytroides Karst. (from the appearance of the pileus, like the wing-cases of certain beetles; Gr. clutron, a beetle's wingcase, cidos, appearance) $i b$.

I. expanded, obtuse, scabrous, with minute linear black-brown scales on a yellowish or pale brown ground, caused by the cracking of the cuticle. St. soft, densely brownish-fibrillose. $G$. ashy-grey or brownish-white. Flesh white.

Odour faint of new meal. Oct. $3 \frac{1}{2} \times 3^{\frac{1}{4}} \times \frac{5}{3}$ in. Compare 104.

112b. T. opicum Gill. (opicus, clownish) $a$.

$P$. somewhat thin, obtusely umbonate, even, soon squamulose, at length unusually upturned and split, very dry, becoming minutely squamulose, grey. St. stuffed, fibrillose, becoming almost glabrous, pallid to greyish. $G$. arcuato-adfixed, somewhat thick, hoary. Flesh greyish.

Ociour none. Woods, pine, amongst moss. $1 \frac{1}{2} \times 3 \times \frac{1}{4}$ in. MLust not be confounded with 103, which smells strongly of soap.

\section{d. Siricellic.}

113. T. sulphureum Quél. (from its sulphur-colour) $a b c$.

$P$. sulphur-yellow, sometimes rufescent-clouded. St. striate, sulphur. $G$. light sulphur. Flesh sulphur.

Gregarious. Probably poisonous. Odlour strong and penetrating of gas-tar, fetid, like Himerosallis flaza, sometimes pleasant. Woods, mixed; common. Aug.-Nor. $3 \frac{1}{2} \times 4 \times \frac{1}{2}$ in. 
114. T. bufonium Gill. (from a fancied resemblance in the pileus to a toad's back; bufo, a toad) a $b c$.

$P$. dotted-wrinkled, yellowish-tan or buff; mid. rufescent. St. flocculose, yellow or yellow rufescent. $G$. yellow-tan.

OHlour same as 113 , but fainter, sometimes not unpleasant. Woods, under pines. Sept.-Nor. $3 \times 2 \frac{3}{4} \times \frac{1}{2} \mathrm{in}$.

115. T. lascivum Gill. (from its many affinities; lascious, playful, wanton) $a b$.

$P$. not umbonate, becoming depressed, cream to pale buff; mid. darker. St. ivory-white. G. separating from stem, crowded, white.

Taste strong, disagreeable, pungent, somewhat acid; odour like gas-tar, varying to fried mushrooms, but disagreeable, more or less like 113. Mixed woods; frequent. May-Nov. $3 \frac{1}{4} \times 2 \frac{3}{4} \times \frac{1}{2}$ in. Not unlilie 1188 in general appearance.

116. T. inamœnum Gill. (from its unpleasant odour; inamonns, unpleasant) a $c$.

$P$. subumbonate, ivory-white. $G$. white or buff-white. Spores ochre-white.

Suspected poisonous. Odour, especially when young, as in 113, but stronger and more fetid. Woods, amongst pine-leaves. Sept.-Nov. $2 \frac{1}{4} \times 3 \frac{1}{3} \times \frac{3}{8} \mathrm{in}$. Sometimes confounded with 115 . Resembling 1188 in general appearance.

117. T. cerinum Quél. (from its colour, like yellow wax; cara, wax) ac $c$.

$P$. yellow, then fuscous-brown. St. yellow, base often brownish, or wholly fuscous. $G$. deep yellow.

Pine-woods, lawns. June-July. $1 \frac{5}{3} \times 2 \frac{1}{8} \times \frac{1}{4} \mathrm{in}$.

118. T. fallax Sacc. (from its resemblance to a dwarf form of 114 or 117 ; fallax, deceitful) $a$.

$P$. yellow; mid. sometimes rufous. St. yellowish. $G$. white, then yellowish.

Under firs. Autumn. $1 \frac{1}{5} \times 1 \times \frac{3}{16}$ in.

118a. T. onychinum Gill. (onychimus, of the colour of the human nail) $a$.

$P$. fleshy, subumbonate, convexo-plane, glabrous, purplish or siemna-umber; marg. striate, orange sienna or silky-purple. St. solid, almost equal, fibrilioso-silky, pallid or dull orangesulphur, reddish or sienna pulverulent above, brownish below. $G$. rounded, at length free, crowded, yellow. Flesh yellowishwhite, or pale olive-sulphur.

Amongst pines, mossy places. Autumn. $2 \frac{3}{4} \times 3 \times \frac{1}{4}$ in.

119. T. ionides Quél. (from its violet colour; Gr. ion) a $c$.

$P$. lilac or violaceous, sometimes livid-reddish. St. pale lilac. Flesh lilac under membrane of P., and at base of St.

Olour faint, agreeable, like 1280 . Woods, moist places, open pastures, under firs, cedars. Aug.-Nov. $2 \frac{1}{4} \times 2 \frac{1}{5} \times \frac{1}{4}$ in. Kesembles 298 in greneral appearance. 
120. T. earneum Quél. (from its flesh-colour; caro) $a b c$.

$P$. becoming whitish. St. attenuate downwards, paler than $P$. $G$. white. Flesh white.

Fields and waysides in woods; frequent. May-Oct. $\mathrm{I}_{\frac{3}{4}} \times 1 \frac{1}{2} \times \frac{1}{4} \mathrm{in}$.

121. T. cælatum Gill. (from its umbilicus, chiselled out; calum, a chisel) a b $c$.

$P$. becoming flocculose, brownish, then pale grey. St. pale brown. $G$. dull white or grey.

Woods, downs. April-Sept. $1 \frac{1}{4} \times 1 \frac{1}{4} \times \frac{3}{16}$ in. Resembling certain species of Omphatict.

\section{c. Guttate.}

122. T. gambosum Gill. (from the hoof-like pileus; samba, a hoof) a b c. Buff-white.

$P$. hemispherical. $G$. crowded.

Solitary or 2-3 connate; in large rings or troops. Edible if gathered in dry weather and not rank smelling. Odour strong of new meal, pleasant, varying to rank as in Polyporzes squamosus. Fields and downs. AprilJuly. $3 \frac{5}{8} \times 2 \times 1 \frac{1}{8}$ in. "St. George's mushroom." Must not be confounded with 133 or the solitary form of 123 .

123. T. albellum Quél. (alhus, white) a b c.

$P$. conical, then convex, pale dull yellowish-white. St. ovatobulbous, colour as P. $G$. very crowded.

Solita1y or connate. Taste pleasant; odour weak. Woods. April-Oct. Solitary- $3 \times 2 \times 1$ in. Connate- $1 \frac{3}{8} \times 1 \frac{1}{2} \times \frac{1}{3}$ in. The connate form must not be confounded with connate 153 .

124. T. boreale Karst. (from its growing chiefly in northern countries; borcalis, northern) a b $c$.

$P$. subumbonate, flesh-colour. St. attenuate downwards, pale flesh-colour. $G$. crowded.

sail to be edible. Odour of fresh meal. Grassy places. $2 \frac{3}{4} \times 2 \frac{1}{4} \times \frac{1}{2}$ in. Not unlike 648 in general appearance.

125. T. amethystinum Gill. (from its colour) $a$.

$P$. livid lilac or grey-purple. St. livid.

Woods, pine. Sept. $2 \frac{3}{8} \times \mathbf{2} \times \frac{1}{2}$ in.

126. T. tigrinum Quél. (from the markings on the pileus) a $b$.

$P$. pallid brown, spotted-brown. St. white.

Solitary or crespitose. Odour usually none, sometimes fetid. Woods, fir, open places; rare. June-July. Allied to 129 and 142.

127. T. pes-capræ Quél. (from a fancied resemblance in the stem to a goat's foot, pes-afpece ac.

$P$. umbonate, fragile, grey or brownish; marg. cracked. St. brownish-white. $G$. becoming ashy, rarely olive.

Odour of meal. Open places, under oaks. Oct.-Nov. I $\frac{7}{8} \times 2 \frac{3}{4} \times \frac{3}{3}$ in. Var. multiforme Mass. Smaller and flesh of pileus thinner than type, gregarious. 


\section{f. Spongiosa.}

128. T. Schumacheri Gill. (after Christian Friedrich Schumacher) a b. $P$. livid grey or faint brownish-lilac; mid. darker; marg. exceeding G. St. ivory-greyish or white, base villous. $G$. subdecurrent, very crowded, white or brownish-white.

Woods, hothouses. Autumn. $3 \frac{3}{8} \times 3 \frac{3}{8} \times \frac{3}{4}$ in. Not unlike 153.

128a. T. amicum Gill. (from its being well known; amicus, a friend) $a$. $P$. fleshy, convexo-expanded, broadly umbonate, even, glabrous, dusky- or olive-brown; membrane of $\mathrm{P}$. extending beyond $\mathrm{G}$. St. solid, firm, bulbous, white or slightly suffused with colour of P. G. rounded, almost free, broad, distant, white. Flesh white.

Solitary. Odour none. Amongst pines, mossy places. Autumn. $3 \times 3 \frac{1}{2} \times \frac{1}{2}$ in.

129. T. circumtectum Sacc. (from the encircling margin of the pileus, circumtectus) a b.

$P$. olive or dusky; mid. tawny. St. white. $G$. emarginate, distant.

Taste mild ; odour none. Sept. $2 \frac{1}{2} \times 1 \frac{3}{8} \times \frac{3}{4}$ in. Elastic. Compare 126 and 142 .

130. T. patulum Quél. (from the spread out pileus, patulus) a $b c$.

$P$. ivory-brown to pale, dull lavender-brown or whitish, becoming pale yellowish. St. white. $G$. rounded, almost free to distant, whitish.

Solitary, crespitose or in troops. Odour none. Wuods, under birches. Only appearing in very wet seasons. Oct. $4 \times 3 \times 1 \frac{1}{3}$ in. The crespitose form must not be confounded with 179.

131. T. arcuatum Quél. (from its arched gills) a $c$.

$P$. dark-brown; mid. almost black, becoming lighter. St. subbulbous, white, brown at base. $G$. sinuate, white or buff-white. Flesh beconiing yellow or brownish.

Gregarious. (irassy ground. Oct.-Nov. $2 \frac{3}{4} \times 1 \frac{1}{2} \times \frac{7}{16}$ in. Sometimes confounded with 109a and 144 .

132. T. oreinum Gill. (from its usual habitat; Gr. oreinos, hilly) $a$.

$P$. livid brown; marg. exceeding $G$. St. subbulbous, white above, ochreous below. $G$. rounded, free. Flesh white.

Solitary. Odour none. Heaths and open ground. Autumn, $I_{\frac{1}{2}} \times I_{\frac{1}{5}} \times \frac{1}{4} \mathrm{in}$. Sometimes confoanded with 144.

133. T. album Quél. (albus, white) a b c. Ivory-white, sometimes becoming faintly yellowish or slightly buff, Heshy.

$P$. dry. St. attenuate upwards. $G$. sinuate.

Perhaps poisonous. Taste unpleasant, bitter, acrid; odour weak or none. Woods; fretuent. Aug.-Nov. $4^{\frac{1}{2}} \times 3^{\frac{3}{4}} \times \frac{3}{4}$ in. Var. casariatum Quél. $P$. thin. St. thin. G. almost free. Must be carefully distinguished from other white species. 
134. T. leucocephalum Quél. (Gr. leukos, white, cephale, head) a bc. Unchanging white.

$P$. silky-downy. St. attenuate downwards. $G$. rounded.

Odour strong and pleasant of new meal. Woods, amongst leaves, lawns, numerous specimens growing through the flattened remains of a dead hedgehog. Aug.-Oct. $2 \frac{1}{4} \times 2 \frac{5}{8} \times \frac{3}{8}$ in.

135. T. acerbum Quél. (accrbus, bitter) a b $c$.

$P$. viscid when moist, buff or brown; marg. involute brownwrinkled. St. solid, squamulose, light buff, darker below. $G$. crowded, pallid, then spotted or rufescent.

Taste usually bitter, sometimes none; odour strong and unpleasant, sometimes pleasant or none. Woods, shady places; frequent. Aug.-Dec. $6 \times 3 \frac{1}{4} \times 1 \frac{1}{4}$ in.

136. T. militare Gill. (from its appearance as contrasted with 137 ; miles, a soldier) $a$.

$P$. very fleshy, viscid, cinnamon; marg. white-floccose. St. pallid. $G$. somewhat crowded, becoming livid-spotted.

Crespitose or in rows. Taste and odour unpleasant. Woods. Ocl. $5 \frac{1}{5} \times 3^{\frac{1}{4}} \times \mathrm{I}$ in.

137. T. eivile Gill. (from its appearance as contrasted with 136; cizis, a citizen) a $c$.

$P$. subfleshy, subviscid, pale yellowish; mid. darker. St. whitish. $G$. becoming pale yellow, rarely spotted.

Odour none. Woods, pine. Oct.-Nov. $3 \times 2 \frac{1}{4} \times \frac{1}{2}$ in. Resembling an exannulate 17.

138. T. personatum Quél. (from its mask - the velvety edge of the pileus and the scales on the stem as contrasted with 139; porsona, a mask) a b c.

$P$. very fleshy, somewhat pale livid-brown. St. brownish, bright purple above, brittle below. $G$. livid, sometimes becoming purplish.

Solitary or gregarious. Edible in dry weather, soddened and unwholesome in wet. Pastures and downs, sometimes woods; common. June-Nov. $5_{\frac{1}{4}} \times 3^{\frac{1}{4}} \times$ I in. The "bluette," "blewitt," or "blue-leg." Often confounded with 139 and 1039.

138a. T. glaucocanum Bres. (slancus, bluish-grey, camıs, greywhitish) a $b$.

$P$. fleshy, somewhat soft, convexo-expanded, moist; marg. involute, somewhat flocculoso-pruinose. St. solid, bulbous, fibrillosostriate, subsquamulose above, whitish-grey. $G$. emarginate, very crowded, separating from hymenophore, purple-greyish.

Edible. Taste mild; odour strong of new meal. Under conifers. Autumn. $2 \frac{7}{3} \times 3 \times \frac{3}{4}$ in. At length wholly hoary. Intermediate between 138 and 139.

138b. T. sævum Gill. (from its aspect; saz'us, sinister) $a$.

$P$. convex, then plane, pallid brownish. St. somewhat shaggysquamulose, bright lilac-purple, lighter above, white at base. $G$. crowded, pallid whitish.

On the earth, in woods, pasturages. Nov. $3 \frac{5}{5} \times 2 \frac{3}{8} \times \frac{7}{8}$ in. 
139. T. nudum Quél. (from the naked edge of the pileus and smooth stem when contrasted with 138) a $b c$.

$P$. somewhat thin, purple, rufescent. St. purple. $G$. purple to rufescent.

Odour acid, pleasant. Woods, often amongst firs; frequent. Sept.-Dec. $3 \frac{1}{3} \times 2 \frac{7}{8} \times \frac{1}{3}$ in. Often confounded with 138, 149, 160, and 1039 . There is a large form twice the size of type.

140. T. cinerascens Gill. (from its ashy colour; cinis, ashes) $a b$.

$P$. white to ashy-grey. St. whitish. $G$. rounded, free, white, then darker than $\mathrm{P}$.

Gregarious, cæspitose. Odour somewhat disagreeable, pungent, sometimes like new meal. Woods; frequent. Sometimes becomes blackish when bruised. Aug.-Oct. $3 \times 3^{\frac{1}{4}} \times \frac{1}{3}$ in.

141. T. panæolum Quél. (Gr. fanciololos, variegated) a $b$.

$P$. convex, then flat, sooty-grey pruinose, or brown-yellow blotched different shades of umber. St. naked, whitish-grey. $G$. sinuatorounded, then subdecurrent, white, then grey to dusky or brownish.

Said to be edible and delicions. Odour not unpleasant. Grassy places, Sept.-Nov. $3 \frac{1}{4} \times 1 \frac{3}{4} \times \frac{1}{2}$ in.

141a. T. enista Gill. (perhaps from Gr. Knisa, odour of roasting fat) $a$. $P$. convex, obtuse, plane, even, glabrous, moist, pale tan or whitish; disc darker; marg. incurved, even, naked. St. solid, equal, even, glabrous, tough, white. $G$. adnexo-rounded, crowded, transversely veined, inclined to separate from hymenophore, white, pallid yellow or reddish when bruised. Flest rather thick, soft, white.

Grassy open places. Autumn. $3^{\frac{3}{4}} \times 3^{\frac{1}{4}} \times \frac{1}{3}$ in. Similar to 141 , but white, not becoming grey.

142. T. duracinum Sacc. (durus, hard, acimus, a berry) a. Ashy-grey. $P$. very fleshy, broadly umbonate. St. attenuate upwards, reticulato-squamose above, paler than P. or white. $G$. cinereous.

Under cedars. Oct. $2 \frac{7}{5} \times 2 \frac{1}{4} \times 1 \frac{1}{8}$ in. Allied to 126 .

\section{g. Hy'rophance.}

143. T. grammopodium Quél. (from the striate stem; Gr. gramme, a line, pous, a foot) a b $c$.

$P$. depressed, umbonate, livid, opaque, brownish. St. whitish brown, enlarged and white-villous below. $G$. whitish or livid.

Taste unpleasant ; odour mouldy, unpleasant. Pastures and grassy places in woods; frequent. June-Nov. $5 \frac{1}{4} \times 4 \times \frac{5}{3}$ in. The pileus is frequently clouded with white mycelium as in 153 . There is a wholly white varity.

144. T. melaleucum Quél. (from the dark pileus and white stem: Gr. melas, black, lenkos, white) a bc.

$P$. flat, subumbonate, dark, smoky, olive- or slate-brown, varying to lavender. St. white, often buff below. $G$. white, sometimes becoming yellowish. Flesh unchanging white.

Taste strong and disagreeable or none; odour pleasant or none. Grassy places, orchards, gardens; uncommon. March-Oct. $3 \frac{5}{8} \times 2 \frac{1}{2} \times \frac{1}{2}$ in. 
Sometimes confounded with 131 and 132. Var. poliolcucum Gill. P.livid, then grey. Var.forphyroleucum Gill. Firmer. P. fuliginous or fuscous, then rufescent, with evanescent um.

145. T. brevipes Quél. (from its short stem ; brezils, short, pes, a foot) $a b c$.

$P$. broadly subumbonate, livid pale- or red-brown or dull lavender. St. bulbous, white to brownish. $G$. whitish to fuscous. Flesh brown, white when dry.

Solitary or somewhat connate. Odour none. Woods, open places, fields, etc. June-Nov. $3 \frac{3}{4} \times 1 \frac{3}{8} \times \frac{1}{2}$ in.

146. T. humile Quél. (humilis, low, dwarf) a b c.

$P$. livid ashy- or warm-brown, pale dull buff or pale slate, sometimes faintly zoned darker. St. pulverulento-villous, ashywhite. $G$. whitish. Flesh whitish or ashy-brown.

Gregarious or crespitose. Taste not unpleasant; odour none. Woods, open places, gardens, cinder-heaps; frequent. Aug.-Nov. $2 \frac{1}{4} \times 1 \frac{5}{8} \times \frac{2}{4}$ in.

147. T. exscissum Quél. (from the linear gills, as if cut away; excinde, to tear out) $a b c$.

$P$. umbonate, brownish-white, pale mouse-colour or somewhat buff, varying dark brown. St. smooth, white. Flesh white. Pastures. May-Nov. $3 \times 2 \frac{1}{2} \times \frac{3}{8}$ in.

148. T. subpulverulentum Karst. (from the minute hoary powder frequent on the pileus; fulvis, dust) a $b$.

$P$. broadly subumbonate, pale livid-brown, greyish or whitish. St. smooth, slightly striate, white. $G$. white. Flesh white.

Sometimes in large rings, twenty or more feet in diameter. Taste at first agreeable, at length astringent. Woods, pastures, gardens. Aug.-Oct. $2 \frac{1}{6} \times 1 \frac{5}{5} \times \frac{3}{8}$ in.

149. T. sordidum Quél. (sordidus, dirty) a b c .

$P$. broadly subumbonate, pale livid to purplish pale brown. St. colour as P. G. sinuato- or adnato-decurrent, becoming distant, purplish or pale brownish.

Gregarious, somewhat cæspitose. Odour none. Pastures, manure, dung heaps. July-Nor. $3 \frac{1}{5} \times 2 \frac{3}{8} \times \frac{3}{8}$ in. Sometimes mistaken for a pale form of 139 .

150. T. pædidum Quél. (padidus, nasty, mean) a.

$P$. tough, flaccid, umbonate, smoky-grey or dull brown, at first radiato-streaked with fibrils. St. paler than I. $G$. crowded, rounded, whitish, then grey.

Odour none. Woods, grassy places, gardens, on dunghills. Aug. $\mathrm{I} \frac{3}{4} \times 1 \frac{1}{4} \times \frac{1}{4}$ in.

151. T. lixivium Karst. (from its watery-ashy colour; lixizium, ashes and water) a $b c$.

$P$. umbonate, umber, ashy-brown when dry. St. floccosopruinose, white, then as P. $G$. distant, adnexo-rounded, grey.

Woods, pine. Nov. $3 \times 2 \frac{5}{8} \times \frac{1}{2} \mathrm{in}$. 
152. T. putidum Karst. (putidus, stinking) a $c$.

$P$. subumbonate, olive-grey or brown, hoary or sprinkled whitesilky. St. grey, white pruinose. $G$. crowded, adnexo-free, ashy-grey.

Odour strong, rancid. Woods, pine. ()ct. - Nov, $2 \times 2 \frac{1}{5} \times \frac{1}{4}$ in.

\section{CLITOCYBE Quél.}

(From the decurrent gills; Gr. klitos, a declivity, kube, a head.)

I'il universal, imperfect or obsolete, manifest as pruina, flock, silkiness or squamules on the pileus and stem. Hymenthore confluent and homogeneous with the fleshy stem. Pilus varying

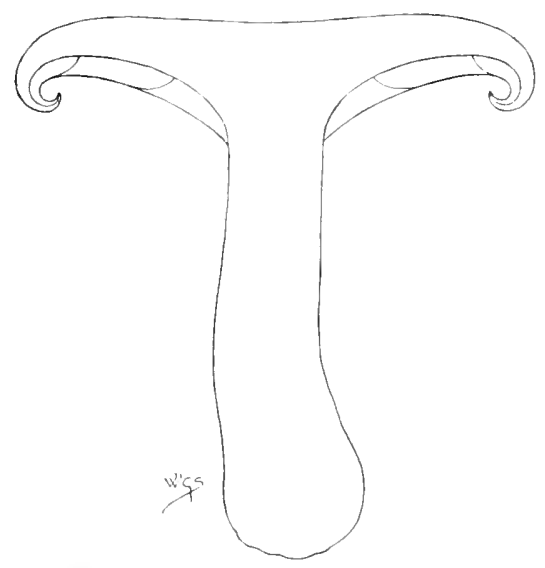

Fig. 16.-Section of Clitocybe nebularis Quél.

One-third natural size.

infundibuliform to plano-depressed, usually fleshy at the disc, margin at first involute. St'm central, simple, without cartilaginous bark. Gills decurrent, rarely adnate, with an acute edge. Spores elliptical or subglobose, smooth. (Fig. I6.)

The species usually grow on the ground, but exceptions occur in $185,201,208,212$, and 223 , which rarely grow on logs, stumps or rotten wood; they commonly grow in clusters, many are fragrant and appear in the late autumn or early winter; a few are edible.

Must not be confounded with Hygrophorus, where the gills are thick and often of a wax-like substance, or with Canthurellus, where the gills are very thick and fold-like, or Lentimus, Pamus or Xerotus, where the substance is leathery and the habitat usually stumps. 
A. Pileus fleshy, not hygrophanous, often becoming pale and silky in drying.

a. Disciformes. Pilens convex, then plane or depressed, regular. Gills adnate or adnato decurrent.

Pileus ashy or brownish.

Pileus violaceous, rufescent.

$153-159$

Pilius becoming yellow.

$160-163$

Pilits green, except 170, then pale ; odour sweet. $\quad 168-170$

Pileus white.

$171-177$

b. Difformes. Pilens at first umbonate, then expanded or depressed. Gills unequally decurrent. Generally cæspitose, sometimes solitary.

$178-189$

c. Infundibuiliformes. Pileus at length deeply depressed. Gills truly decurrent. Often losing colour in drying.

Piteus coloured, becoming hyaline, minutely flocculose or

silky. Hygrophanous but not externally moist. 190-195

Pilcus varied in colour. Moist in rainy weather. 196-204. Pileus shining whitish, slightly flocculoso-sprinkled or smooth.

$205-207$

B. Hygrophanous.

d. Cyathiformes. Pilius cup-shaped. Gills at first adnate, then decurrent. Colours dull when moist. (Must not be confounded with the Hydrogramma, under Omphalia.)

$208-214$

c. Orbiformes. Pileus convex, then plane or depressed, shining, not mealy or squamulose. Gills adnate or adnato-toothed. Colours dull or watery-hyaline.

Pileus at first dark. Gills becoming ashy. 215-217 Pileus becoming pale. Gills whitish. 218-221

f. Versiformes. Pileus tough, more or less squamulose or furfuraceous. Gilis adnate or decurrent, somewhat thick, distant. Stem tough.

Pilius dull or dusky with darker squamules.

222,223 Pileus bright in colour.

\section{a. Disciformes.}

153. C. nebularis Quél. (from the frequent clouds of white mycelium on the pileus; nebulat, a cloud) $a b c$.

$P$. smoky-brown, grey, or pale slate. St. spongy-elastic, whitish or grey. G. crowded, shortly decurrent, white. Flesh opaque white.

Laxly gregarious, rarely connate as in 123 . Edible. Taste somewhat peppery ; odour of curd cheese. Woods, fir, beech, hedge-bottoms, etc, amongst leaves; common. Aug.-Dec. $6 \times 4 \frac{1}{2} \times 1 \frac{1}{4} \mathrm{in}$. There is a white variety. Must not be confounded with 128 . The mycelium on pileus gives rise to 451. A similar mycelium occurs on 143. 
154. C. clavipes Gill. (from the club-shaped stem; clire't, a club, fes, a foot) $a b c$.

$P$. flat, sooty-brown or grey; marg. often white. St. smoky-livid, somewhat fibrillose. $G$. subdistant, deeply decurrent, white. Flesh hyaline-whitish.

Odour mild, pleasant. Woods, spruce-fir, pine, beech; common. July-Nov. $2 \frac{7}{8} \times 2 \frac{3}{8} \times \frac{2}{2}$ in. There is a white variety. The pileus is sometimes 4 in. or more in diam.

154a. C. comitialis Gill. (perhaps from its being one of a number of allied forms which meet at this position in Clitocy be $) a$.

$P$. obtuse, soon plane, even, glabrous, somewhat moist, not hygrophanous, sooty-brown, almost black. St. stuffed, attenuate upwards, obconic, glabrous, sooty-brown. $G$. subdecurrent, horizontal, thin, crowded, white. Fles/l white or whitish.

Odour none. Pine-woods, damp places amongst moss. Autumn. $I^{\frac{3}{8}} \times 3^{\frac{1}{4}} \times \frac{5}{16}$ in. Firmer and smaller than 154.

155. C. gangrænosa Gill. (from its ulcerous appearance; gangranc, an ulcer) $a b c$.

$P$. livid, at first white-pulverulent. St. slender to stout, sootygrey. $G$. sub- or sinuato-decurrent.

Taste insipid, unpleasant; odour sometimes strongly fetid, meal-like but nauseous, sometimes strong and not unpleasant. Woods and plantations, larch; rare. Oct.-Nov. $3 \frac{3}{8} \times 3 \frac{1}{4} \times \frac{3}{8}$ in. More or less changing within and without to slate-colour or black; the flesh changes from livid to indigo, then black; sepia-black when dry. Must not be confounded with 1168.

155a. C. polia Karst. (Gr. polios, grey) a b.

$P$. glabrous, not hygrophanous, pale grey or pale olive-brownish; marg. whitish. St. solid, slightly attenuate upwards, white, pale brownish below. $G$. somewhat deeply decurrent, closely crowded, very narrow, white. Flesh white, pale brown in P.

Cæespitose, usually in small clusters, but sometimes I ft. 4 in. in dian. Woods. Autumn. $2 \times 3 \frac{1}{\frac{1}{4}} \times \frac{3}{8}$ in.:

156. C. inornata Gill. (inornatus, unadorned) $a b c$.

$P$. with a separable livid-brownish pellicle, which frequently cracks from the marg. upwards and peels up, unchanging livid. St. greyish or brownish. $G$. decurrent, colour as St. Flesh grey.

Odour mushroom.like. Fields, woods; under cedars; rare. Cct.-Nov. $4 \times 2 \frac{1}{4} \times \frac{1}{2}$ in.

156a. C. luseina Karst. (from the often small eye-like pileus and the solitary habit ; luscinus, one-eyed) a.

$P$. somewhat fleshy, even, edge spreading, glabrous, grey on a pale reddish or buff ground, purple or slate-brown when young; marg. striate. St. solid, attenuate downwards, greyish or faintly brownish, white pulverulent. $G$. subdecurrent, crowded, yellowish- or salmon-whitish, shaded grey.

In pastures. Autumn. $2 \times 2 \times \frac{3}{16}$ in. 
157. C. curtipes Gill. (from the short stem; curto, to shorten, pes, a foot) $a$.

$P$. expanded, oblique, obtuse, olive-ochre to olive- or flesh-brown. St. rigid, thickened upwards, olive-brown, subpruinose. $G$. adnate, closely crowded, ivory-white.

Grassy places. Autumn. $\mathbf{I} \frac{3}{8} \times \mathbf{I} \times \frac{1}{8}$ in.

158. C. hipneola Quél. (from a fancied jug-like shape; hirnea, a jug) a b $c$.

$P$. umbilicate, minutely silky-hoary, whitish, grey, tan or olive shaded. St. colour as P., hoary above. G. decurrent, crowded, lighter than $\mathrm{P}$., becoming hoary.

Amongst moss and grass, roadsides. Oct. $\quad \mathbf{I}_{\frac{1}{2}} \times \mathbf{I}_{\frac{3}{8}} \times \frac{1}{8}$ in. Must not be confounded with members of Omphatia.

159. C. zygophylla Sacc. (from the vein-connected gills; Gr. zy'gon, a yoke, phullon, a leaf) a $b$.

$P$. whitish; mid. pale buff; marg. rugose or plicate, becoming white. St. whitish, downy at base. $G$. decurrent, subdistant, pale clay-colour.

Amongst leaves. Oct.-Nov. $4 \times 1 \frac{3}{4} \times \frac{1}{2}$ in. Resembling in general appearance 202 and 1278. In the whitish-tawny pileus and clay-coloured gills it resembles 570 .

160. C. cyanophæa Sacc. (Gr. kumos, blue, phaios, dusky) a.

$P$. pale slate-blue; mid. brown. St. paler than P., becoming yellowish, abruptly white above. $G$. deeply decurrent, pale slate-blue.

Woods. $2 \frac{3}{4} \times 2 \frac{3}{3} \times \frac{1}{2}$ in. Must not be confounded with 139 .

161. C. opipara Gill. (opiparus, splendid) $a b$.

$P$. shining, bronzy flesh-colour. St. pale brownish. $G$. adnatodecurrent, white to pale yellowish.

Taste and odour pleasant. Mossy places under trees, beech. Oct. $3 \times 3 \times \frac{7}{8}$ in.

162. C. amara Quél. (amarus, bitter) a b c .

$P$. flat, dull buff, then whitish. St. white. $G$. adnato-decurrent, crowded, white.

Odour of new meal. Woody places. Oct. $3 \frac{1}{5} \times 1 \frac{7}{8} \times \frac{3}{8}$ in.

163. C. socialis Gill. (from its gregarious habit) $a$.

$P$. acutely umbonate, reddish-buff. St. brownish, strigose at base. $G$. sinuato-decurrent, scarcely crowded, becoming yellowish.

Odour none. Amongst fir-leaves. Sept.-Oct. $1 \frac{5}{8} \times I_{\frac{3}{8}}^{\frac{3}{8}} \times \frac{1}{4}$ in. MIust not be confounded with 165 .

164. C. amarella Quél. (amarns, bitter) a $b$.

$P$. subumbonate, pallid fawn to whitish. St. solid, colour as P. $G$. subdecurrent, crowded, pallid.

Taste very fetid-bitter; odour strong of prussic acid or very disagreeable.

Woods. Oct. $2 \frac{1}{5} \times \mathbf{I} \frac{3}{4} \times \frac{1}{4} \mathrm{in}$. 
165. C. vernicosa Gill. (from the shining pileus; zcrnis, varnish) a $b$. $P$. yellowish or buff. St. stuffed, yellow. $G$. adnato-decurrent, subdistant, sulphur-yellow. Fles/e whitish to pale sulphur.

Odour none. Woods, fir, larch; rare. Autumn. $3 \frac{1}{8} \times 1 \frac{3}{4} \times \frac{3}{8} \mathrm{in}$. Somewhat resembles a yellow Hygrophorus.

166. C. venustissima Karst. (i'cmustus, beautiful) $a b$.

$P$. thin, subumbilicate, orange-vermilion ; marg. crenate. St. orange-yellow, base white. $G$. decurrent, subdistant, yellow.

Odour none. Amongst larch and pine leaves. Autumn, $2 \times 1 \frac{1}{2} \times \frac{1}{4}$ in. Resembles a yellow Hy'srophorus.

167. C. subalutacea Gill. (from its colour; aluta, soft leather) $a c$.

$P$. thick, subumbonate or depressed, pale tan. St. pale straw. $G$. adnato-decurrent, distant, pallid white.

Odour as in 168, or weak of anise, sometimes strong and disagreeable of rancid meal or none. Under trees, hollies. Nov. $2 \times 1 \frac{3}{4} \times \frac{3}{8} \mathrm{in}$.

168. C. odora Quél. (odorus, fragrant) a $b c$.

$P$. subumbonate, dull greenish or bluish. St. greyish, whitish, greenish or pale brownish. $G$. paler than P.

Single or subcrespitose. Odour strong and pleasant of melilot, anise, almonds, meadow-sweet or new-mown hay, stronger in recently dried examples. Woods, grassy places, roadsides. Aug. - Nov. $3 \times \mathbf{I} \frac{3}{4} \times \frac{3}{8}$ in.

169. C. Trogii Sacc. (after Jacob Gabriel Trog, Swedish botanist) $a$. $P$. subumbonate, ashy-white to buff-grey. St. solid, white. $G$. white.

Said to be edible. Odour fragrant and spicy as in 168 . Woods, amongst leaves. Oct.-Nov. $3 \frac{1}{4} \times I_{\frac{1}{4}}^{\frac{1}{4}} \times \frac{1}{2}$ in. The colour approaches 215 .

170. C. rivulosa Quél. (from the rivulose cracks on the pileus) a $b$.

$P$. whitish, very pale or brownish flesh-colour or brownish-ochre with no shade of green, appearing at first as if whitishvarnished, the cracking of this coat giving the rivulose appearance. St. colour as P. G. flesh-colour.

Solitary, gregarious or cæespitose. Taste mild; odour pleasant. On leaves, amongst moss, pastures, roadsides, etc. Oct.-Nov. $1 \frac{3}{4} \times 1 \frac{1}{8} \times \frac{3}{16}$ in. Some small forms, as the var. neptunea Mass. approach 171 .

171. C. cerussata Quél. (from its colour; cerıssa, white-lead) a $b c$.

St. solid. $G$. adnato-decurrent, very crowded, never yellow.

Often in troops. Taste mild, pleasant, sweet, or like 1394, sometimes rancid; odour pleasant or none. Woods, mixed, fir ; frequent. AprilOct. $3 \frac{3}{8} \times 2 \times \frac{5}{8}$ in. There are three forms :-I. Stout; like 122 and 123 . 2. Slender; like 172 . 3. Large, stout, irregular. The var. difformis (iill. is British. Sometimes springs from dense, creeping mycelium. A close ally of 188.

172. C. phyllophila Quél. (Gr. fhullon, a leaf, philos, loving) a b c.

$P$. tan or white. St. stuffed or hollow, sometimes pale brownish. $G$. slightly decurrent, subcrowded, sometimes buff-white or yellowish.

Caspitose and larger, solitary and smaller. Odour mealy or none. Wonds, chiefly amongst beech-leaves, one form on pine-leaves. Sept.-Dec. $3 \times 2 \frac{1}{2} \times \frac{3}{8}$ in. Must not be confounded with 367 . 
173. C. pithyophila Gill. (Gr. pitus, pine, philos, loving) a b $c$. Persistently white.

$P$. plano-umbilicate, thin, hygrophanous. St. somewhat hollow, tomentose at base. $G$. adnato-decurrent, very crowded.

Gregarious or subcaspitose. Odour pleasant. Pine-woods; frequent. Sept. - Nov. $2 \frac{3}{8} \times \frac{5}{8} \times \frac{3}{15}$ in. Must not be confounded with 206.

174. C. tornata Quél. (from its shape: torno, to turn on a lathe) a.

$P$. gibbous; mid. fleshy. St. stuffed, pubescent at base. $G$. adnate with a decurrent tooth, very crowded.

Odour none. Woods, amongst grass, by clecayed stumps, elm. Oct. $2 \times 2 \times \frac{1}{4}$ in. Always small.

175. C. candicans Quél. (candicans, shining-white) a $b c$.

$P$. mid. not fleshy. St. somewhat hollow. $G$. adnato-decurrent, crowded.

Odour of new meal. Woods, amongst damp leaves; common. July-Nov. I $\frac{7}{3} \times I_{\frac{1}{2}} \times \frac{1}{8}$ in. Must not be confounded with 176 .

176. C. dealbata Gill. (déalbatus, whitewashed) a b $c$.

$P$. not Heshy. St. stuffed or hollow. $G$. adnate or adnatodecurrent, crowded.

Odour sweet, weak, pleasant, mealy but fungoid. Woods and pastures, sometimes on old mushroom-beds; common. July-Nov. $2 \frac{1}{2} \times 1 \frac{3}{8} \times \frac{3}{8}$ in. Some small, delicate forms, var. minor Sacc., are at times confounded with 175.

177. C. gallinacea Gill. (from its colour, like a hen's egg; gallina, a hen) a $b c$.

St. solid. $G$. adnato-decurrent, crowded.

In troops, sometimes in rings, amongst grass. Taste slightly fetid-acrid; odour strong, earthy. Woods and pastures, amongst grass and moss; common. Sept. - Nov. $\mathrm{I} \frac{3}{4} \times 1 \frac{7}{5} \times \frac{1}{4} \mathrm{in}$.

\section{b. Difformes.}

178. C. ampla Gill. (amplns, large) a b.

$P$. fleshy, somewhat silky, sooty or ochreous-brown. St. white or brownish-white. $G$. decurrent or sinuato-adnate and toothed. Flesh fibrous.

Tasteless; odour none. Woods, amongst moss. Sept.-Nov. $6 \frac{3}{4} \times 3 \frac{3}{4} \times I_{8}^{3}$ in.

178a. C. molybdina Gill. (from the dark, lead-coloured pileus; Gr. molubdaina, lead-ore) a.

$P$. fleshy, campanulate, expanded, umbonate, even, glabrous; marg. thin, somewhat dark sooty-umber; mid. sooty-buff. St. solid, stout, fibroso-striate, mealy-squamulose above, pallid or yellowish salmon-white, white at base. $G$. adnate, broad, pale yellowish-salmon.

Cæspitose. Woods. Autumn. $5 \frac{1}{2} \times 6 \frac{3}{4} \times \frac{3}{\frac{3}{4}}$ in. With the general appearance of 458 (var. eximins), but the adnate gills and white spores very different. 
179. C. decastes Quél. (from its growing in clusters of about ten; Gr. dekas, a company of ten) a $b$.

$P$. very thin, of two easily separable strata, tan-grey or tan. St. whitish. $G$. adnato-decurrent, subdistant, white.

Crespitose, in clusters of about one foot in diam. Woods, oak, beech, bypaths, plantations, gardens, orchards, on sawdust. Oct.-Nov. $9 \times 4 \frac{1}{2} \times \frac{1}{2}$ in. 1lust not be confounded with 130 .

180. C. subdecastes Sacc. (from its affinity with 179) $a b$.

$P$. very thin, campanulate, smooth, even, ivory-white; mid. ochreous. St. silky-fibrous, shining-white. $G$. rounded, crowded, thin, white.

Crespitose. Taste and odour none. On the ground. Sept. $3 \frac{1}{t} \times 5 \frac{1}{2} \times \frac{1}{2}$ in.

181. C. aggregata Gill. (from its growing in clusters; aggregatus, collected together) a $c$.

$P$. gibbous, then depressed, not fleshy, often excentric, grey or dull ochreous. St. whitish. $G$. subdecurrent, crowded, pale ochreous or flesh-colour.

Odour strong. Woods, oak, on sawdust, mushroom-beds. July-Oct. $3 \frac{1}{2} \times 3 \frac{1}{2} \times \frac{5}{8}$ in. Becoming rufous-stained.

182. C. elixa Karst. (from its sodden condition in wet weather; clixus, soaked) $a b$.

$P$. gibbous, very fleshy, hygrophanous, sooty-brownish or greyish, becoming pale. St. pallid brownish. $G$. decurrent, distant, white.

In troops. Woods; frequent. Oct. - Nov. $4 \frac{3}{4} \times 2 \frac{1}{2} \times \frac{3}{4}$ in. Very bibulous, becoming saturated with moisture; heavy and brittle in wet or frosty weather, breaking with its own weight.

183. C. fumosa Quél. (from its smoky colour ; fumus, smoke) $a b c$.

$P$. subgibbous, then plane, greasy-looking brown to livid whitish; mid. sometimes slightly scurfy as in 69 . St. whitish or brownish. $G$. rounded, adnate, or decurrent, crowded, greyish-or brownish-white, or yellowish-olive-white.

Gregarious or caspitose in groups a foot or more in diam. Suspected poisonous. Tasteless or insipid ; odour strong, fungoid, or none. Woods, often on wood-ashes, old sawdust, amongst grass, on rubbish-heaps, on earth where trees have been felled; uncommon. June-Nov. $4 \frac{1}{4} \times 2 \frac{3}{4} \times \frac{5}{8}$ in. Often in company with 277 and 1374.

184. C. tumulosa Sacc. (from the mound-like appearance of the connate groups; tumulus, a mound) $a b$.

$P$. gibbous, umber becoming pale. St. floccoso-pruinose, white. $G$. subdecurrent, crowded, white to livid grey.

Larger forms laxly cæespitose, smaller, densely cæspitose. Woods. Sept. $3 \frac{1}{3} \times 3 \frac{1}{2} \times \frac{3}{4}$ in.

185. C. pergamena Mass. (from its colour, like parchment, pergamena) $a$.

$P$. umbonate, at first brown. St. solid, with a brittle cartilagenous bark. $G$. broadly sinuato-decurrent.

Cæspitose. Stumps. Oct. $2 \times 5 \times \frac{5}{16}$ in. The habitat and cartilaginous bark point to Collybia rather than Clitocybe. 
186. C. cryptarum Mass. (from its occasional appearance in cellars; crypta, a vault) a b.

$P$. subconical, then depressed, floccose, pale buff or brown, spotted. St. hollow, paler than P., mottled within. G. subdecurrent, white.

Densely crespitose. Taste insipid ; odour none. Sawdust. Oct. $2 \frac{1}{5} \times 3 \frac{1}{2} \times \frac{7}{5}$ in. With the varying habit and size of 184.

187. C. monstrosa Gill. (monstrosns, strange) a b. White.

$P$. convexo-umbonate. St. solid, white-farinose above. $G$. rounded, crowded.

Often densely crespitose. Probably edible. On the ground. Sept.-Oct. $2 \frac{7}{3} \times 1 \frac{1}{2} \times \frac{7}{8}$ in. May be an autumnal form of 122 or a variety of 133 , or a form of 94. It may be a Tricholoma, as suggested by Berlieley, but cannot be a form of 124 as suggested by Fries. It is more probably a form of 123 ; the spores are the same in size. Stem sometimes 6 in. long.

188. C. opaca Gill. (from its opaque colour) $a b c$. White with a silvery glare.

$P$. convexo-plane, subumbonate then repand, floccose. St. stuffed. $G$. adnato-decurrent, very crowded. Flish pale brownish.

Single or connate. Woods. Oct.-Nov. $3 \times 2 \frac{3}{8} \times \frac{5}{16}$ in. A close ally of 171 .

189. C. occulta Mass. (from its doubtful characters; occultus, hidden) $a$. Cartilaginous, tough.

$P$. convexo-plane, then slightly depressed, innato-virgate; mid. livid-smoky; marg. whitish. St. solid, paler than P. or white. $G$. subdecurrent, subdistant, white.

Gregarious. Charred ground. Nov. $3 \times 2 \frac{1}{8} \times \frac{3}{8}$ in.

\section{c. Infundibuliformes.}

190. C. maxima Quél. (maximus, the greatest) a b. Tan-whitish.

$P$. thin, umbonate; marg. even. St. solid. $G$. subcrowded, not branched. Flesh white.

Odour weak and pleasant, sometimes strong. Grassy places, hedge-banks, woods, pastures; frequent. July-Nov. $10 \frac{3}{4} \times 4 \frac{7}{8} \times 1$ in. Sometimes a foot or more high and broad. () ften confounded with 1172; the latter has no umbo, has a sulcate margin, is less infundibuliform, has branched gills, and a short stem.

191. C. infundibuliformis Quél. (infundibulam, a funnel, forma, form) a $b c$.

$P$. thin, typically umbonate, pale buff then white. St. spongystuffed, ivory-white. $G$. subcrowded, ivory-white.

Odour usually weak and pleasant, often like anise, sometimes none. Woods, grassy places, near trees, beeches, rarely on rotten wood: common. June-Dec. $3 \frac{1}{8} \times \mathbf{I} \frac{\pi}{8} \times \frac{1}{4}$ in. A variable species. There is a wholly white variety. Var. membranacia Gill. P. membranous; brick-resl. Woods, pine. 
192. C. trullæformis Karst. (trulla, a ladle, forma, form) a c. $P$. thin, flocculoso-villous, dry, brown grey to brown. St. spongystuffed, ashy-grey. $G$. distant, comnected by veins, white.

Borders of fir-woods, amongst grass and bushes. Oct. $21 \times 1 \frac{7}{3} \times \frac{1}{4} \mathrm{in}$. Must not be confounded with 208.

193. C. incilis Gill. (from its incised or crenate pileus; incilis, incised) $a b$.

$P$. plano-depressed, silky-flocculose, reddish. St. hollow, attenuate downwards, colour as P. $G$. distant, veined, white.

Odour strong of meal, sometimes none. Woods, under firs. Nov. $2 \frac{1}{5} \times \frac{3}{4} \times \frac{3}{4}$ in. Resembles a small 1297.

194. C. sinopica Gill. (from Sinope in Patagonia, where red-lead is found-from the vermilion-brownish pileus) $a b c$.

$P$. slightly depressed, floccoso-rivulose, dry. St. stuffed, colour as P. or buff. $G$. subdecurrent, very crowded, white, then yellowish.

Woods, chiefly on burnt places. Oct. $\quad \mathbf{I}_{5}^{\frac{3}{5}} \times \mathbf{I} \frac{1}{\frac{1}{4}} \times \frac{3}{16} \mathrm{in}$. Resembling a sirall 1300 .

195. C. parilis Gill. (from its symmetrical growth) $a b c$.

$P$. subdepressed, atomate or flocculose, brownish, mottled darker, then white-grey. St. stuffed, pale yellowish-brown or browngrey. $G$. very crowded, ivory-grey.

Woods, sides of plantations. Sept.-Oct. $2 \frac{1}{5} \times 1 \frac{1}{2} \times \frac{1}{4}$ in. Resembling a Lactarius. Must not be confounded with Tricholoma or Omphatia.

196. C. gilva Quél. (gili'us, pale yellowish-brown) a b $c$.

$P$. subdepressed, sometimes reddish, guttate-spotted. St. paler than P. $G$. pallid, then ochreous.

Woods, pine. Oct.-Dec. $3 \times 2 \frac{1}{6} \times \frac{1}{2}$ in. Staining paper umber when dried. Resembles in form Faxillus and Lactarius.

197. C. subinvoluta W. G. Sm., Agaricus subinzolutus W. G. Sm. (from the subinvolute margin of the pileus) $a b$. Wholly pale buff, at length slightly spotted or clouded buff or yellow.

$P$. fleshy, plane, subdepressed; mid. darker guttate-spotted; marg. at first subinvolute. St. solid, subbulbous, darker below. $G$. decurrent, broad. Flesh buff-white, darker below.

Under firs. Oct. $3 \frac{5}{3} \times 2 \frac{1}{2} \times \frac{3}{4}$ in. Not Agaricus subinvolutus Batsch, which is a form of 1181 .

198. C. geotropa Quél. (from the often strongly deflected margin of the pileus; Gr. ge, the earth, tripo, to turn) a $b c$.

$P$. depressed, typically umbonate, pale brown, at first guttatespotted. St. attenuate upwards, tan-brownish. $G$. white.

Sometimes in fairy-rings of great size. Odour of almonds. Woods, pastures. Sept.-Dec. $6 \frac{1}{4} \times 6 \times \frac{7}{8}$ in. Pileus sometimes 9 in. in diam. Varies with a short stem and exumbonate. Intermediate forms occur between this and 197. 
199. C. spinulosa Sacc. (from a mistaken reference to the supposed spinulose spores) $a b$.

$P$. plane, subdepressed, umbonate, leathery, pale buff to rufescent flesh-colour. St. red-buff above, paler below, concentrically cracked white, as in $21 . G$. white, then light yellowish. Flesh white.

In troops or two or three connate. Odour sweet, pleasant. Amongst grass by roadsicles. Nov, $4 \times 3_{\frac{3}{4}}^{3} \times \frac{3}{4}$ in.

200. C. splendens Gill. (splendens, shining) a b.

$P$. depressed, pale shining metallic-yellowish, shaded reddishbrown. St. solid, attenuate upwards, colour as P. G. white, then light yellow.

Solitary. Woods, amongst dead pine-leaves, etc. Sept. $4 \times 1 \frac{7}{5} \times \frac{5}{8}$ in. Intermediate between 196 and 202.

201. C. inversa Quél. (from the down-turned margin of the pileus) $a b c$.

$P$. depressed, somewhat fragile, oily-smooth in appearance, not guttate, yellowish-buff to dull crimson-brownish. St. stuffed or solid, lighter than P. $G$. whitish or yellowish. Flesh as P. but lighter.

Solitary, sulggregarious or cæspitose. Taste same as mushroom ; odour acid. Woods, chiefly fir, sometimes on logs; frequent. Aug.-Dec. $3 \frac{1}{2} \times 3 \times \frac{5}{16}$ in. Not unlike 1299 in seneral appearance. A luxuriant form is Aguricus lobatus Sowerby.

202. C. flaccida Quél. (from the limp pileus; flaccidus, limp) a b $c$.

$P$. broadly umbilicate, sienna or buff. St. subhollow, pale sienna. $G$. obconico-decurrent, crowded, whitish, becoming yellow. Flesh white to brownish.

Solitary, sometimes gregarious, forming rings. Taste insipid. Plantations, fir, hills. Sept.-Jan. $3 \times \mathrm{I} \frac{1}{2} \times \frac{3}{5}$ in. 201 and 202 are sometimes undistinguishable; the colours vary yellowish-brown to brick-red. II ust not lee confounded with 159 . Resembles 1278.

203. C. vermicularis Quél. (from its colour; vermis, a worm) a b.

$P$. broadly umbilicate, flesh-colour or light brownish. St. yellow to white. $G$. arcuato-decurrent, colour as St. Flesh lighter than $\mathrm{P}$.

Fir-woods. Sept.-Oct. $2 \frac{1}{8} \times 2 \frac{1}{8} \times \frac{3}{8}$ in. Resembling a small 1288 .

204. C. senilis Gill. (from the wrinkled aspect of the pileus; senilis, old) a $b$.

$P$. infundibuliform, concentrically cracked, olive-brown or tanbrownish. St. solid, paler than P. G. decurrent in a straight line, paler than St.

Gregarious. Odour none. Woods, pine, lawns, etc. Autumn. $3 \frac{1}{ \pm} \times \mathrm{I}_{\frac{8}{4}} \times \frac{5}{15}$ in.

205. C. catinus Quél. (from its bowl-shaped pileus; catinus, a bowl) $a$.

$P$. depressed, becoming flesh-colour. St. spongy-stuffed, attenuate upwards. $G$. colour as P.

Odour pleasant. Amongst dead leaves. Autumn. $2 \frac{3}{4} \times 2 \times \frac{3}{8}$ in. 
206. C. tuba Gill. (from its trumpet-shaped pileus; tuba, a trumpet) a $c$.

$P$. narrowly umbilicate, sometimes pale blue-grey. St. stuffed or hollow, colour as P. $G$. deeply decurrent, very crowded, white.

Gregarious. Taste mild of radish ; odour of meal or horseralish or none. Voods, pine, on dead leaves. Oct.-Nov. $2 \frac{1}{4} \times 2 \frac{1}{4} \times \frac{3}{16}$ in. Must not be confounded with 173.

207. C. ericetorum Quél. (from its habitat; cricctum, a heath) $a b c$.

$P$. deeply depressed, arid. St. solid, attenuate downwards. $G$. subdecurrent, subdistant.

Odour pleasant. Sept.-Nov. $1 \frac{1}{x} \times \mathbf{I} \times \frac{1}{\frac{1}{x}}$ in. Resembling 1214 in appearance.

\section{d. Cyathiformes.}

208. C. eyathiformis Quél. (cyatlucs, a cup, forma, form) a b c.

$P$. broadly umbilicate, deep brown, pale when dry. St. attenuate upwards, paler than P. $G$. distant, pale brownish-white. Flesh colour as G.

Woods, pastures, etc., rarely on rotten wood and tan-heaps: common. Aug.-Feb. $2 \frac{1}{4} \times 2 \frac{1}{4} \times \frac{3}{8}$ in. Must not be confounded with 192 .

209. C. cinerascens W. G. Sm., Agraricus cincrascens Batsch (from its colour ; cinis, ashes) $a$.

$P$. plano-depressed, lavender-brownish. St. equal, striate, pale greyish or brownish. $G$. adnato-decurrent, white, then yellowish or grey.

Amongst moss. Autumn. $1 \frac{1}{\frac{1}{x}} \times 1 \frac{1}{8} \times \frac{3}{16}$ in.

210. C. expallens Quél. (expallens, growing pale) a b.

$P$. plano-depressed, very watery, white or pale brown. St. hollow-flocculose, paler than P. $G$. adnato-decurrent, white or greyish.

Woods, mixed, pastures, etc. Aug.-Dec. $2 \times 1 \frac{1}{8} \times \frac{3}{16}$ in. Smaller, usually earlier, paler, less depressed and with less distant gills than 208.

211. C. obbata Quél. (from the cup-shaped pileus; oblecr, a cup) a $c$.

$P$. deeply depressed, submembranous, blackish-brown or blackishgrey; marg. striate. St. hollow, ashy-brown. $G$. distant, dull grey.

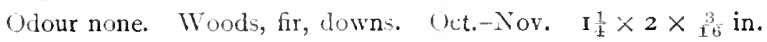

212. C. pruinosa Quél. (from its pruinose appearance) a $c$.

$P$. broadly and somewhat deeply depressed, sometimes squamulose, olive-brown, then grey. St. paler than P. G. crowded, white, then dull yellowish.

Odour none. Pine-wools amongst moss, sometimes on rotten woxl. Nov.Dec. $3 \times 1 \frac{5}{8} \times \frac{1}{4}$ in. 
213. C. concava Gill. (from the concave pileus) $a c$.

$P$. deeply umbilicato-depressed, very thin, tough, grey- or olivebrown; marg. not striate. St. ashy-grey. $G$. very crowded, colour as P.

Often gregarious. Odour none. Plantations, woods, fir, pastures; rare. Sept. $2 \frac{1}{8} \times \mathbf{I} \times \frac{3}{15} \mathrm{in.}$ Must not be confounded with Omphalia.

214. C. brumalis Quél. (from its usual time of appearance; bruma, winter) a $b c$.

$P$. broadly plano-umbilicate, brown or whitish. St. hollow, paler than P. $G$. crowded, livid to yellowish-white.

Taste sometimes slightly nauseous; odour weak, earthy, not unpleasant, evanescent or none. Woods, on pine-leaves, amongst heather, on commons, on dirt-heaps; common. Oct.-Jan. $2 \times 2 \frac{1}{4} \times \frac{1}{4} \mathrm{in}$.

\section{e. Orbiformes.}

214a. C. orbiformis Gill. (orbis, a circle, forma, form) a $c$.

$P$. convex, plane, obtuse, not truly depressed, glabrous, hygrophanous, smoky-grey; marg. spreading, even. St. stuffed, elastic, somewhat striate, attenuate and naked upwards, grey, base downy. $G$. adnato-decurrent, somewhat distant, greyishwhite.

Odour none. Grassy places in pine-wo ds. Autumn. $2 \times 3 \times \frac{3}{16}$ in. Analogous with, but not allied to 208.

215. C. metachroa Quél. (Gr. metachroos, changing colour) a $b c$.

$P$. umbonate, then plano-depressed, ochreous-whitish to brownishgrey; marg. at length striate. St. white. $G$. adnato-decurrent, ashy-whitish.

Odour none. Woods, pine, dry places, grassy woods, on leaves, rotting fern, turf, earth, dirt-heaps. May-Nov. $1 \frac{3}{t} \times 2 \frac{1}{5} \times \frac{3}{4}$ in.

216. C. pausiaca Gill. (from its olive-coloured gills; pansia, an olive) a $c$.

$P$. plano-depressed, subumbonate, tan, brown or grey. St. ashygrey, white above. $G$. obtusely adnato-rounded.

Odour weak of meal. Woods, pine. Oct. $1 \frac{3}{8} \times 1 \frac{3}{4} \times \frac{1}{8}$ in.

217. C. ditopus Gill. (from the habit, often growing in twos; Gr. dittos, twofold, pous, a foot) $a b c$.

$P$. broadly umbilicate, horny, dark ashy-grey, or dull brown- or fuscous-ochre; marg. clouded-sooty. St. hollow, pale ashygrey. $G$. adnate, crowded, colour as P.

Odour strong of new meal. Woods, pine, dead leaves. Oct. $3 \times 2 \times \frac{1}{4} \mathrm{in}$.

218. C. diatreta Quél. (from its perforate stem; Gr. diatritos, perforate) $a$.

$P$. plano-depressed, flesh-colour, then tan. St. pallid fleshcolour. $G$. adnato-decurrent, colour as St.

Odour almost obsolete. Woods, pine. Autumn. $1 \frac{1}{1} \times 1 \frac{1}{4} \times \frac{1}{8}$ in. 
219. C. fragrans Quél. (fracrans, sweet-scented) $a b c$.

$P$. broadly depressed, pallid white; marg. substriate. St. colour as P. G. adnato-decurrent, somewhat crowded.

Odour strong of anise. Woods, pastures. July-Jan. $2 \frac{1}{x} \times 3 \times{ }_{16}^{3}$ in. Often attacked by the mould, Sporodinia Astergilluts.

220. C. angustissima Gill. (from its very narrow gills; angustus, narrow) $a b$. Pallid white.

$P$. broadly umbilicate, very thin, firm. St. fibrous, Hexuous. $G$. decurrent, very crowded.

Odour none. Woods, amongst leaves. Oct. $2 \times 2 \frac{1}{2} \times \frac{1}{8} \mathrm{in}$.

221. C. obsoleta Quél. (from its pallid tint; obsoletus, faint) a c. Hygrophanous.

$P$. plano-depressed, pale grey or flesh-colour, soon white. St. not flexuous. $G$. adnato-decurrent, somewhat broad, pale grey. Gregarious. Slightly fragrant of bitter almonds or anise. Amongst grass and leaves. Uct.-Nor. $I_{\frac{1}{4}} \times I_{\frac{3}{4}} \times \frac{1}{8} \mathrm{in}$.

\section{f. Versiformes.}

222. C. ectypa Gill. (from the projecting sooty squamules in the middle of the pileus; Gr. cktufos, worked in relief) $a b$.

$P$. fleshy; plano-depressed, yellowish or brownish; marg. striate. St. attenuate upwards, dull light yellow, becoming black at base. $G$. decurrent, white, then rufous-spotted.

Gregarious, often caspitose. Odour pleasant of anise, becoming fetid. Meadows, damp places. Dec. $2 \frac{3}{4} \times 5 \frac{1}{\frac{1}{1}} \times \frac{9}{16}$ in. Often greatly resembles 69 .

223. C. bella Gill. (bellus, beautiful) $a c$.

$P$. convex, umbilicato-depressed, orange; sq. rufescent. St. pale orange. $G$. adnato-toothed, broad, yellow, rufescent or pale Hesh-colour.

Subcrspitose. Fetid. Plantations, fir, sometimes on rotten fir-wood; uncommon. Sept. $1 \frac{5}{8} \times I_{4}^{\frac{3}{4}} \times \frac{3}{16}$ in.

224. C. laccata Quél. (from the red colour--as of gum-lac-of the pileus) $a b c$.

$P$. convex, plano-umbilicate, then repand, mealy-squamulose, rufescent, becoming ochreous. St. usually attenuate downwards, fibrillose, colour as P. G. adnato-toothed, Hesh-colour.

Odour sometimes of garlic. WVods, forests, commons; common. May-Dec. $2 \times 3 \times \frac{3}{8}$ in. The pileus is sometimes brilliant or blackish-purple, becoming lavender, with stem and gills bright purple (Agaricus amethy'stimus Bolt.). A very small form with a hair-like stem occurs.

Agaricus Sadleri B. \& Br. placed in this position by Stevenson is probably the same as 824. Berkeley has proposed a new genus, Laccaria, for the reception of 224 and its allies.

224a. C. proxima Boud. (from its being like the brown form of 224) $a$.

$P$. convex, then plane, glabrous, yellowish-brown. St. even, smooth, paler than P. $G$. adnato-decurrent, somewhat pink.

Woods. Oct. $1 \frac{3}{4} \times 2 \frac{1}{\frac{1}{4}} \times \frac{3}{16}$ in. Differs from 224 in the glabrous P. and warted spores. 


\section{COLLYBIA Quél.}

(From the pileus, which is frequently small and regular in form like a small coin ; Gr. kolluhos.)

Vill almost obsolete, when present represented by a fibrillose, floccose, or pruinose covering. Hymenophore confluent with, but heterogeneous from the cartilaginous stem. Pilcus slightly fleshy to

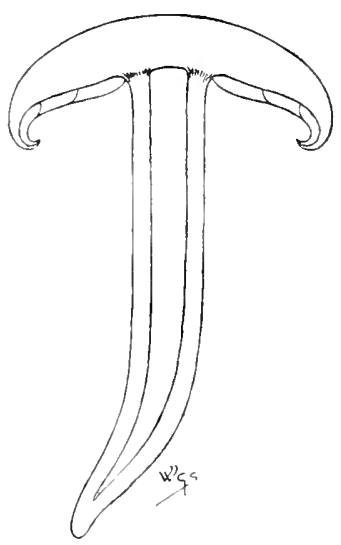

Fig. 17.-Section of Collybia macu. lata Quél. One-third natural size.

thin, margin at first involute. Stem central, wholly cartilaginous, or with a cartilaginous bark and stuffed with a pith ; often rooting, simple. Gills membranous, soft, free or adnexed. Spores smooth. (Fig. I 7.)

Often small, frequently somewhat tough, maturing more slowly and lasting longer than other Agarics. The species grow on stumps, branches and leaves, sometimes on other fungi or on the ground. Must be carefully distinguished from members of Marasmius, where the species are less fleshy, less putrescent, more leathery, and revive with moisture after becoming dry.

Species 225-286

A. Gills white or brightly coloured, not ashy. Flesh white.

a. Strichedes. Stem stout, hollow, or with a spongy pith, longitudinally striate, except 225a.

Gills broad, except 228, somewhat distant. 225-229 Gills crowded, narrow. $\quad 230-236$

b. I'estipedes. Stcm thin, equal, fistulose or with a pith, even, velvety, floccose, or pruinose.

Gills broad, somewhat distant.

Gills very narrow, very crowded. 
c. Lacipides. Stem thin, equal, fistulose, naked, smooth except at base, not conspicuously striate.

Gills usually broad, lax, commonly more or less distant.

$250-260$

Gills narrow, crowded except 270.

B. Gills becoming cinereous. Hygrophanous.

d. Tephrophance. Colour fuscous, becoming cinereous.

Gills crowded, somewhat narrow.

$271-278$

Gills very broad, except 285 , more or less distant. 279-286

\section{a. Stricepdes.}

225. C. radicata Quél. (from the rooting stem; radix, a root) a b c. $P$. glutinous, umber ; mid. radiato-rugose. St. rigid, brittle, attenuate downwards, deeply rooting, white. $G$. adnesed or adnate, sometimes with a tooth.

Solitary. On and about stumps, amongst fir-leaves; common. June-Nov. $4 \frac{3}{4} \times 1-2 \frac{1}{2} \times \frac{3}{4}$ in. There is a white variety.

225a. C. Henriettæ Sacc. (after Henrietta Smith, who first found it) $a b$.

P. dry, even, somewhat downy, somewhat yellowish-umber. St. attenuate upwards, even, slightly rooting, subpruinose, pale, pallid yellowish-brown within and without, somewhat darker below. $G$. broad, broadly adnate, distant, slightly rounded near St. Flesh very thin.

On and about trees, stumps, etc. Sept. $4 \times 7 \frac{3}{8} \times \frac{1}{4}$ in. Intermediate between 225 and 226 .

225b. C. retigera Bres. (from the net-like veining of the pileus ; rete, a net, gero, to carry) $a b$.

$P$. thin, campanulate, expanded, subumbonate, dry, glabrous, with a network of anastomosing veins best seen when old, pallid; mid. shaded tawny-brown; marg. striate. St. equal, solid to stuffed, pallid, minutely downy at base, somewhat rooting. $G$. somewhat broad, ventricose; edge fimbriate, pallid.

Bases of stumps, ash, beech. Dec. $2 \times 3 \frac{1}{2} \times \frac{1}{4} \mathrm{in}$.

226. C. longipes Quél. (from the long stem; longus, long, piss, a foot) $a b c$.

$P$. dry, velvety, livid brown. St. attenuate downwards, deeply rooting, villous, dark brown, varying paler. $G$. rounded, adnexed, white.

Stumps, etc, ; uncommon. Aug.-Oct. $2 \frac{1}{4} \times 9 \frac{1}{2} \times \frac{1}{2}$ in.

226a. C. eriocephala Rea (from the velvety pileus; Gr. crion, wool, kcphale, a head) $a$.

$P$. convex, expanded, velvety, fulvous-tawny. St. stuffed, then hollow, fusiform, extended into abundant long branched rhizomorphoid proliferous brown mycelium, striate; micl. 
slightly velvety, colour as P. above but paler, brown below. $G$. sinuato-adnate, distant, pale to deep ochre. Flesh thick at mid., thin at marg., yellow-white, then yellowish, rufous externally at base.

Cxspitose. Interior rotten elm. Jan. $2 \frac{1}{4} \times 3 \frac{3}{4} \times \frac{3}{8}$ in. Rooting base $4 \frac{1}{2}$ in.

227. C. platyphylla Quél. (from the broad gills; Gr. platus, broad, phullon, a leaf) $a b c$.

$P$. Hat, ashy-or livid-yellow-brown to sepia, streaked fibrillose. St. not attenuate or rooting, colour as P. $G$. adnate or adnexed.

Woods, amongst leaves, on rotten logs; rare. May-Oct. $4 \frac{3}{4} \times 4 \frac{3}{4} \times \frac{5}{8}$ in. Frequently springs from a villous cord-like mycelium. The var. repens (iill. resembles 105 .

228. C. semitalis Quél. (from its growing in footpaths, semita) $a b c$. $P$. plane, hygrophanous, sooty-brown, yellowish or grey when dry. St. attenuate upwards, greyish. $G$. sometimes narrow, adnato-rounded, sometimes with a tooth, white, brown-spotted.

Sometimes crespitose. On the ground after much rain. Oct.-Nor. $3 \frac{1}{x} \times 4 \frac{1}{4} \times \frac{1}{2}$ in. Sometimes turns black. Compare 227.

229. C. fusipes Quél. (from the spindle-shaped stem; fusus, a spindle, pes, a foot) $a b c$.

$P$. convex, unbonate, brown. St. attenuate downwards, rooting, often split, yellowish. $G$. adnexed, pale brown, pinkish, faintly brown-spotted.

Caspitose. Considered edible by some persons. Stumps; common. JuncNov. $2 \frac{1}{4} \times 4 \frac{1}{\frac{1}{4}} \times \frac{1}{2} \mathrm{in}$. Var. codcmatopus has a stout ventricose St.

229a. C. lancipes Gill. (from a fancied resemblance in a longitudinal section of the stem to the blade of a spear; lancia, a spear, pis, a foot) a b.

$P$. convex, expanded, umbonate, radiately rugose from mid., dry, glabrous, pale flesh-colour, becoming pallid; marg. striate. St. solid or stuffed with twisted filaments, striate, gradually attenuate to the rooting, downy base, whitish or pale fleshcolour. G. adnexed, emarginate, broad behind, distant, thick, firm, connected with veins, pale flesh-colour. Flish thick, firm, not watery.

Solitary, scattered, rarely clustered. On the ground. Autumn. $2 \frac{1}{2} \times 5 \times \frac{3}{4}$ in. Every part firm and rigid.

230. C. maculata Quél. (from its foxy stains; macula, a spot). a b c. White or pale brown, spotted-sometimes wholly-rufescent.

$P$. convexo-plane. St. hard, subventricose, attenuate downwards, sometimes deeply rooting. $G$. rounded free.

Taste slightly acid, unpleasant, odour not unpleasant. Woods, chiefly pine, beech, alder, moist places. May-Nov. $3 \frac{1}{2} \times 4 \frac{1}{2} \times \frac{3}{4}$ in. There is a permanently whitish or white var. immaculata Mass., with $G$. sometimes abnormal, labyrinthiform, merulioid. Var. siorsonerea Gill. Small, yellow; St. long-rooting; G. yellow. 
231. C. fodiens W. G. Sm., Agraricus fodicns Kalch. (from the rooting stem; fodiens, digging) $\alpha$.

$P$. convex, faintly yellowish; mid. ochreous-flesh-colour. St. ventricose, attenuate downwards, rooting, white. $G$. rounded or emarginate-free, yellowish-white.

Odour of meal. Grass-borders. Oct. $3 \frac{1}{8} \times 5 \frac{1}{4} \times \mathbf{I}$ in.

232. C. prolixa Gill. (from the stretched out appearance of the pileus; prolixus, stretched out) a $b$.

$P$. campanulate, subumbonate, rufescent-brown; mid. darker. St. solid or imperfectly hollow, often contorted, paler than P. $G$. rounded-free, whitish, unspotted.

In dense clusters on leaf-heaps. Aug.-Sept. $4 \frac{3}{x} \times 4 \frac{1}{4} \times \frac{1}{2}$ in.

233. C. distorta Quél. (from the twisted stem) $a b c$.

$P$. convex, subumbonate, buff-brown; mid. darker, usually with darker blotches. St. attenuate upwards, pale brown. $G$. rounded-free, livid white, stained brown if brtised.

Gregarious or fasciculate. Taste insipid; odour strong, mephitic, like 35. On tree-roots, pine, rotten pine-trunks, heaps of leaves. Oct.-Nov. $2 \frac{3}{4} \times 4 \times \frac{3}{8}$ in. Somewhat resembling 230 .

234. C. butyracea Quél. (from the pileus, buttery to the touch; hutyrum, butter) a $b c$.

$P$. plane, subumbonate, pale brown with a darker marginal zone; mid. almost black. St. attenuate upwards, pale rufescent or ochreous-brown. $G$. adnesed, white. Flesh pale brown.

Woods, fir. Jan.-Dec. $3 \frac{1}{2} \times 2 \frac{1}{2} \times \frac{1}{2}$ in. Becomes whitish when dry.

234a. C. bibulosa Mass. (from the bibulous substance) $a b$.

$P$. fleshy, subglobose, obtuse, expanded, moist, very smooth, even, deep olive- or blackish-green when moist, pale grey to whitish when dry. St. cartilaginous, spongy within, stuffed to imperfectly hollow, attenuate upwards, minutely striate, pale clear warm-brown, often rooting into the wood. $G$. adnexorounded, thin, edge subundulate, dingy.

In groups of three or four. Stumps. Autumn, $2 \times 3 \times \frac{3}{8} \mathrm{in}$.

235. C. stridula Quél. (stridéo, to creak, derivation obscure), a b. $P$. broadly umbonato-plane, dark brown. St. fibrous, paler than P. $G$. adnexed, pale brown.

On the ground. Oct. $2 \frac{1}{1} \times 2 \frac{1}{2} \times \frac{1}{4}$ in.

235a. C. pulla Gill. (frompullus, dark-coloured) $a b$.

$P$. fleshy, thin, fragile, campanulate, then expanded, obtuse, smooth, even, glabrous, hygrophanous, shining dark rich brown, purplish-bay, livid purplish-slate or nearly black, paler when dry. St. hollow, twisted, somewhat striate, soft, naked, sometimes slightly rooting, whitish. $G$. adnexed, somewhat broad, crowded, transversely pellucid-striate, whitish. Flish white.

Under birch-trees, amongst Polytrickum. (Oct. $2 \frac{1}{3} \times 3 \frac{7}{3} \times \frac{1}{4}$ in. 
236. C. xylophila Gill. (Gr. xulon, timber, philos, loving) a.

$P$. campanulate, subpapillate, very thin, ivory-white; mid. ivorybuff. St. ivory-buff, pale yellowish-brown within. $G$. adnate with a tooth, white.

Gregarious, cæspitose. Stumps and rotten trunks of elm, etc. Sept. $3 \frac{1}{2} \times 3 \times \frac{1}{ \pm}$ in.

\section{b. Vestipedes.}

237. C. velutipes Quél. (from the velvety stem; z'llus, fleece, pes, a foot) $a b c$.

$P$. plane, fleshy, viscid, yellow ; mid. brown. St. yellow above, brown and almost black towards base, or wholly brown. $G$. adnate or adnexed, pallid white or yellowish.

Crespitose. Taste agreeable. Trunks, stumps, sometimes on timber; common. Aug.-April. $2 \frac{5}{8} \times 2 \frac{3}{4} \times \frac{5}{16}$ in. Sometimes springs from a luxuriant golden byssoid mycelium (Ozontum auricomum Link), sometimes from thick cord-like, branching and deeply rooting mycelium or from a sclerotium. See 1569. The var. rubescens Sacc. becomes foxyspotted.

238. C. laxipes Quél. (from the lax stem; laxus, lax, pes, a foot) $a b$.

$P$. convexo-plane, moist, white. St. white above, rich brown below. $G$. adnexo-free, white.

On wood, chips, twigs. Feb. $\frac{7}{8} \times 3 \frac{1}{2} \times \frac{1}{16}$ in.

239. C. mimica Sacc. (from its resemblance to 709) $a b$.

$P$. flat, thin, smooth, with a separable cuticle, pale yellow-buff; mid. buff-brownish. St. solid, apex subpruinose, with brownish bark, attenuate downwards, fibrilloso-strigose at base, yellowbuff above, deep brown below. $G$. adnate with a tooth.

Taste and odour strong of fish. On deal-shavings. Nov. $1 \frac{5}{8} \times 2 \times \frac{1}{8}$ in.

240. C. floccipes, Gill. (from the floccose-woolly stem; floccosus, floccose, pes, a foot) $a b$.

$P$. plane, umbonate, deep umber. St. hollow, black punctatofloccose on a white ground. $G$. adnexed, white.

On the ground and about trunks. Sept. $I_{5}^{\frac{1}{5}} \times 2 \times \frac{1}{16}$ in.

241. C. vertirugis Sacc. (from the rugose pileus; vertix, the top, ruga, a wrinkle) $a \cdot b c$.

$P$. convexo-plane, radiato-wrinkled, minutely pulverulent, brownashy or indigo-lead colour. St. strigose at base, brown, paler above. $G$. adnate, whitish.

Rootstocks of dead ferns, twigs, stumps, etc. Sept.-Oct. $\frac{7}{8} \times 3 \frac{1}{8} \times \frac{1}{16}$ in.

242. C. stipitaria Gill. (from its habitat; stipes, a stem) $a b c$.

$P$. umbilicato-plane, velvety or fibrillose; mid. pale ochre to sienna; marg. whitish. St. tough, fibrilloso-shaggy, light to dark sienna. $G$. adnexo-free, white.

Frequent. July-Nov. $\frac{5}{8} \times 1 \frac{5}{5} \times \frac{1}{16}$ in. 
243. C. hariolorum Quél. (from its fancied use by soothsayers; hariolus, a soothsayer) $a b$.

$P$. campanulato-convex, smooth, pale ochreous-white; mid. ochreous. St. colour as P. or gradually dark fuscous below, naked and pallid above. G. adnato-free, whitish.

Densely gregarious, somewhat crespitose. Odour strong. Woods, amongs: (lead leaves, beech, sometimes on rotten wood. Sept. $\mathbf{I}_{\frac{3}{4}}^{\frac{3}{4}} \times 2 \frac{3}{8} \times \frac{1}{8} \mathrm{in}$. Closely allied to Mfarasmius.

244. C. confluens Quél. (from the confluent stems) $a b c$.

$P$. convexo-plane or subumbonate, tough, white-buff. St. compressed, pulverulento-woolly, white or pale brownish-lavender or purplish.

In troops, or connate confluent ruws. Amongst fir-leaves; frequent. June-()ct. $2 \times 3 \frac{1}{5} \times \frac{1}{t}$ in. Closely allied to Marasmius.

245. C. ingrata Quél. (from the unpleasant odour; ingratus, unpleasant) $a b$.

$P$. umbonato-expanded, very thin, pale livid buff. St. brownishpurple, white mealy above. $G$. free, colour as $P$.

Crespitose. Taste none, or slightly sweet ; odour slight, mouldy, fungoid, sweetish-unpleasant. Woods, pine, damp places; uncommon. Aug.-Oct. $2 \frac{3}{4} \times 3 \frac{1}{2} \times \frac{1}{4} \mathrm{in}$. There is a smaller form one-half the size of type.

246. C. conigena Quél. (from its habitat; conts, a cone, gigno, to bear) $a . b c$.

$P$. convexo-plane or slightly umbonate, smooth, whitish-ochre or brownish. St. strigose at base, white. $G$. adnexo-free, white.

Usually gregarious. Under conifers on the cones and amongst the leaves. Iune-Dec. I $\times 2 \frac{1}{t} \times \frac{1}{8}$ in. Must not be confounded with 259 and 260 .

247. C. cirphata Quél. (from the twisted base of the stem ; cirrıs, a curl) $a b c$.

$P$. convexo-plane, slightly silky, white; mid. pale sienna. St. flexuous, attenuate and twisted at the smooth or strigosofibrillose base. $G$. adnate, white.

In troops. Amongst leaves of fir, elc., and decaying fungi. Aug.-Nov. $\frac{5}{8} \times 3 \times \frac{1}{16}$ in. NI Ist not be confounded with 1424a.

248. C. tuberosa Quél. (from the tuberous base of the stem) a bc.

$P$. convexo-plane, subumbonate, slightly silky, white; mid. faintly ochreous. St. slightly pulverulent, base smooth, sometimes fibrillose, occasionally proliferous, attached to a sclerotiun. $G$. adnate, white.

Gregarious. On dead Agarics, Russulu (1306, 1307), Lactarii, on Polyporns squamosus, fir-leaves, rotten fir-twigs, amongst moss, etc. Aug.-Nov. $2 \frac{3}{4} \times 4 \frac{1}{1} \times \frac{9}{16}$ in. Fries says the summer form has no sclerotium; this is very doubtful. The autumnal forms may have no sclerotium as the floccose mycelium condenses in winter and forms a summer sclerotum.

249. C. racemosa Quél. (from the glandular hairs round the stem; racemus, a cluster) $a b$.

$P$. expanded, papillate, subtomentose, white or faintly greyish. St. racemose, apparently proliferous with minute hairs bearing 
glands or abortive pilei, colour as P. Attached to Sclerotizm lacunosum. $G$. adnate, white.

Terrestrial and on putrid fungi, chiefly Agarics; very rare. Autumn. $\frac{3}{8} \times 1 \frac{3}{4} \times \frac{1}{32}$ in. Turns black in drying.

\section{c. Laripedes.}

250. C. collina Quél. (from its frequent habitat; collinus, a hill) a $b c$. $P$. expanded, subumbonate, somewhat viscid, pale yellowishbrown. St. colour as P. G. adnexo-toothed, white.

Usually gregarious. Grassy slopes, about beech-stumps; uncommon. Oct. $2 \times 4 \frac{1}{4} \times \frac{3}{16}$ in.

251. C. thelephora Sacc. (from its bearing a small umbo on the pileus; Gr. thcle, a nipple, phcro, to bear) a $b$.

$P$. campanulate, brown or yellow-brown, paler at marg. St. brown above and whitish below, or the reverse. $G$. adnatotoothed, white to yellowish.

Gregarious. Inodorous. Peat-bogs, partly dry sphagnum-bogs. $1 \frac{5}{8} \times 3^{\frac{1}{2}} \times \frac{1}{8}$ in. A near ally of 250 .

252. C. ventricosa Gill. (from the enlarged lower part of stem; z'enter, the belly) $a b c$. Pale warm-brownish.

$P$. campanulato-convex, slightly umbonate. St. attenuate upwards and attenuato-rooting, darker below. $G$. broadly adnatotoothed.

Woods. Oct. $2 \frac{3}{8} \times 3 \frac{5}{8} \times \frac{3}{16}$ in.

253. C. Stevensonii Sacc. (after the Rev. Dr. Stevenson, mycologist) a. $P$. semi-ovate, viscid, pallid yellow. St. attenuate downwards, deeply rooting, whitish-yellow above, brownish below. $G$. very broad, adnato-toothed, white.

Old pastures. Aug. $\frac{5}{8} \times 2 \frac{3}{8} \times \frac{1}{16}$ in. Resembling in habit members of Psathyra.

254. C. psathyroides Sacc. (from its resemblance to members of Psathyra) a. Ivory-white.

$P$. semi-ovate or conic, subviscid. St. not rooting. $G$. broadly adnato-toothed.

Oct. $\quad \frac{7}{8} \times 3 \frac{3}{4} \times \frac{1}{3}$ in. Greatly resembles a MIycena. Allied to 253 .

255. C. xanthopus Quél. (from the yellow stem; Gr. xanthes, yellow, pous, a foot) $a b c$.

$P$. campanulato-convex, subumbonate, whitish to deep biscuit; um. sienna. St. strigose at base, yellow to sienna-biscuit. $G$. adnexo-free, whitish.

Pine-woods, amongst cones, leaves and twigs. July-Nov. $I_{\frac{3}{4}} \times 2 \frac{1}{2} \times \frac{1}{5} \mathrm{in}$. Not unlike 1394 in general appearance.

256. C. nitellina Gill. (from its colour; nitella, a dormouse) $a b$.

$P$. convexo-plane, smooth, reddish-tan or brown, sometimes pale. St. yellow above, brown below, or tan. $G$. adnate, attenuate in front, tan.

Solitary or sregrarious. Odour none. By roals and paths in woods. MayOct. $2 \frac{1}{4} \times 3 \frac{1}{4} \times \frac{3}{16}$ in. There is a very small umbonate variety. Allied to Clitocylo. 
257. C. succinea Quél. (from its colour; succinum, amber) a $b c$.

$P$. plano-convex, smooth, deep rich brown. St. colour as $\mathrm{P}$. $G$. adnate, white.

Solitary. Odour none. Woods, mixed, grassy places, under trees. NIayDee. $15 \times 1 \frac{5}{8} \times \frac{1}{4}$ in.

258. C. nummularia Gill. (from a fancied resemblance in the pileus to a small coin, nummulus) a $c$. White, sometimes faintly shaded ochreous or reddish.

$P$. expanded, slightly depressed, somewhat umbonate. St. attenuate downwards. $G$. adnexo-free.

In troops, exespitose. Woods, mixed, amongst leaves and twigs. July-Nov. I $\frac{3}{4} \times 2 \frac{1}{8} \times \frac{1}{5}$ in.

259. C. esculenta Quél. (from its edible qualities) a b c.

$P$. plane, dull ivory-ochreous to umber. St. attenuate downwards, deeply rooting, yellow to pale reddish-brown. $G$. adnexed to adnato-toothed, whitish.

Gregarious. Edible. Taste bitter, agreeable to some, unpleasant to others. Pastures, grassy places near plantations, pine-woods, sometimes on hotbeds; common. April-MIay. I $\times 5 \times \frac{1}{1}$ in. Sold in Austrian markets as food under the name Nagelschwämme.

260. C. tenacella Quel. (tinax, tough) a $b c$.

$P$. convexo-plane, subumbonate, brown or brownish to white. St. attenuate and fibrillose at base, whitish above, rufescent below. $G$. adnexo-toothed, whitish.

Solitary or in troops. Taste pleasant. On leaves and cones in pine-woods, and under cedars. April-Dec. $1 \frac{1}{8} \times 3^{\frac{3}{4}} \times \frac{1}{\frac{1}{5}}$ in. The var. stolonifer Quél. roots for four or more inches. It is difficult to distinguish 260 from 259, and neither must be confused with 246 .

260a. C. planipes Sacc. (from the plane stem; planus, plane, pes, a foot) $a$.

$P$. orbicular, expanded, somewhat viscid, bay. St. rooting, colour of P. $G$. free.

Caspitose. Wools. Sept. $\frac{7}{8} \times 1 \frac{3}{8} \times \frac{1}{10}$ in.

261. C. acervata Gill. (from its growing in dense clusters; acrous, a heap) $a b$.

$P$. hemispherical, then flat, tan or brown. St. tan- to deep rufous-brown. $G$. adnexo-free, salmon.

Woods, fir, dead wood ; frequent. Sept.-Oet. $2 \frac{1}{2} \times 3 \frac{1}{2} \times \frac{1}{4}$ in.

262. C. dryophila Quél. (Gr. drus, oak, fhilos, loving) a b c.

$P$. plane, tan, brown or brownish when soddened; mid. faintly ochreous. St. villous at base, tan to brown. $G$. adnexo-free, whitish. Flish white.

Dangerous when cooked according to a case cited by Dr. Badham (Esculent Funsuses, is63, p. I07). The taste when raw leaves a burning sensation on the fauces and a sense of suffucation. Amongst leaves, voods, hedige. bottoms, gardens, grassy places; common. Nay-Oct. $3 \frac{1}{4} \times 4 \frac{3}{4} \times$ J in. Variable, gilis sometimes sterile. Var. funicularis Karst. Large, tutted, decumbent ; $G$. sulphur. 
263. C. aquosa Gill. (from its watery substance; aqua, water) $a b c$. $P$. plane to subdepressed, tan-ochreous to ochreous. St. fibrillose at base, ochreous-brown. $G$. adnexo-free, pale yellowish.

Woods, damp places, amongst moss. Autumn. $\mathrm{I}_{\frac{3}{4}} \times 2 \frac{3}{4} \times \frac{1}{8} \mathrm{in}$.

264. C. extuberans Quél. (from the broad umbo; cxtuberans, swelling) $a b c$.

$P$. olive-brownish. St. attenuate downwards, pale grey-brownish. $G$. adnexo-toothed, white.

Usually solitary when on the ground, cæspitose and smaller when on trunks. Odour none. Amongst grass, hillsides. Nay-Sept. $1 \frac{3}{4} \times 3 \frac{1}{4} \times \frac{3}{10} \mathrm{in}$.

265. C. exseulpta Gill. (from the cut-out, sinuato-rounded gills) $a b c$. $P$. plane, subumbilicate, dull ochreous-orange or umber, pale whitish-buff when dry; mid. darker. St. equal or subbulbous, ochreous or sulphur, darker below. $G$. adnato- or adnexotoothed, subdecurrent, sulphury.

Gregarious. Taste and odour strong, mushroom-like, but with a suggestion of tar. Trunks, dead wood, on the ground. May-Oct. $1 \frac{1}{2} \times 2 \times \frac{1}{8} \mathrm{in}$. Lry, tough, reviving with moisture after the manner of a Marasmizs.

266. C. macilenta Gill. (macies, leanness) $a$.

$P$. convexo-plane, subumbonate, deep yellow. St. yellow. $G$. adnexo-free, bright yellow. Flesh pallid yellowish.

Troods, pine, on leares. Autumn. $1 \frac{1}{8} \times 2 \frac{1}{8} \times \frac{1}{16}$ in. Resembles some of the slender yellow Hystothori.

267. C. clavus Quél. (from the nail-like shape, clavus) a c.

$P$. hemispherical, orange-vermilion. St. substrigose at base, whitish, brownish below. $G$. adnexed, whitish.

On fir, twigs, leaves, moss; rare. Oct. $\frac{1}{4} \times \frac{3}{4} \times \frac{1}{32}$ in.

268. C. ocellata Quél. (from the eye-like umbonate umbilicus ; occllus, a little eye) $a b c$.

$P$. white; mid. pale sienna. St. attenuate downwards, rooting, fibrillose, brownish-white, or buff above and white below. $G$. adnate, white.

Pine-woods, amongst leaves, grassy places, on Fungermannia ; uncommon. Sept. Oct. $\frac{5}{8} \times 2 \times \frac{1}{10} \mathrm{in}$.

269. C. muscigena Quél. (from its habitat; muscus, moss, signo, to bear) $a$. White.

$P$. hemispherical, pellucid, thin. $G$. adnate.

Amongst moss and grass, near trunks. Aug. $\frac{3}{8} \times \mathbf{1} \frac{1}{3} \times \frac{1}{16}$ in.

270. C. leucomyosotis Sace. (from its resemblance to a pale form of 736, Nancoria Mrosotis; Gr. liukos, white) a b.

$P$. convex, broadly umbonate, pale translucent umber; marg. striate. St. brittle, subpruinose above, colour as P. G. adnato-sinuate, thick, somewhat distant, whitish.

Strong-scented, somewhat fragrant. On Sphagnum in wet places in bogs. May. $\quad 1 \frac{3}{8} \times 3 \frac{7}{5} \times \frac{1}{4}$ in. Whitish when dry. 


\section{d. Tephrophance.}

271. C. rancida Quél. (from its rancid odour) $a b c$.

$P$. convex, umbonate, sometimes viscid, dark umber, slate or slate-grey, or slate with mid. umber. St. subfusiform, rooting, sometimes deeply, colour as P. $G$. adnexo-free, slaty fleshcolour, grey-white or almost white.

Taste disagreeable; odlour strong and offensive when cut or broken, like rancid meal. VVoods, amongst trunks. Oct.-Nov. $I \frac{1}{2} \times 4 \times \frac{1}{5}$ in.

272. C. eustygia Sacc. (Stygian, from its becoming black) $a$.

$P$. convexo-plane, subdepressed, white or white-brownish. St. attenuate downwards, rooting, white above, grey and punctatoscaly below. $G$. adnexo-rounded, dark grey.

Odour of rancid meal. On the ground. Oct. $2 \frac{1}{5} \times 3 \frac{1}{5} \times \frac{5}{16}$ in. Black when dry.

273. C. coracina Gill. (from its colour ; Gr. korax, a raven) $a c$.

$P$. convexo-expanded, black to dark- or grey-umber. St. attenuate upwards, not rooting, white above, brown below. $G$. adnate, greyish or yellowish.

Odour strong of new meal. Grassy places, fir-plantations. Nov. $\mathbf{I} 3 \times \mathbf{I} \frac{1}{2} \times \frac{3}{10} \mathrm{in}$.

274. C. ozes Karst. (Gr. ozo, to smell) $a$.

$P$. convexo-plane, subumbonate, grey-or clay-brown. St. slightly attenuate upwards, white above, sooty-grey below. $G$. adnate, smoky-olivaceous.

Odour of new meal. On the ground and on pine-leaves. Feb. $I_{5}^{\frac{1}{5}} \times 3 \frac{1}{4} \times \frac{1}{5}$ in.

274a. C. mephitica Karst. (from the offensive mephitic odour, sometimes, however, like new meal) $a$.

$P$. Heshy, convex, subumbonate, hygrophanous, smooth, dry, silky, greyish-ochre, becoming whitish. St. equal, enlarged below, stuffed, grey, white-floccose or pruinose-velvety. $G$. obtusely adnate, separating from stem, attenuate in front, crowded, grey. Flish yellowish.

Amongst fir-needles. Oct. $1 \frac{3}{5} \times 2 \frac{3}{5} \times \frac{3}{16}$ in.

275. C. inolens Quél. (inolens, scentless) a.

$P$. convexo-plane, umbonate, tan, greyish or rufous. St. attenuate upwards, slightly rooting at the white, strigose base. $G$. adfixed, almost free, whitish- or ochreous-grey.

Woods, chiefly pine. Sept. - Nov. $2 \frac{1}{5} \times 2 \frac{3}{4} \times \frac{1}{4}$ in.

276. C. plexipes Gill. (from the twisted stem ; plecto, to twist, pes, a foot) $a b c$.

$P$. hemispherical, umbonate, pale umber or greyish ; mid. darker. St. attenuate downwards, rooting, pale umber. $G$. narrowly adnate, whitish-grey. Flesh pale umber.

Odour none. Woods, beech, amongst grass; uncommon. Sept.-Nov. $1 \frac{1}{4} \times 6 \times \frac{1}{8}$ in. 
277. C. atrata Quél. (atcr, black) a b c.

$P$. plano-depressed, greasy feeling, blackish-umber. St. fuscous within and without. $G$. adnato-decurrent, livid-brownish.

Scattered or in groups. Odour strong, disagreeable. On burnt ground often in company with 183, 685 and 1374 . Sept.-Dec. $1 \frac{1}{2} \times 1 \frac{1}{2} \times \frac{1}{8}$ in. Nust not be confounded with 369 .

278. C. ambusta Quél. (from its habitat, burnt ground; ambustus, burnt) $a b c$.

$P$. convexo-plane, papillate, umber. St. paler than P. $G$. adnate with a tooth, pale umber. Flesh colour as G.

Odour of meal. July-Nov. $\frac{3}{4} \times 1 \frac{1}{5} \times \frac{1}{15}$ in.

279. C. lacerata Gill. (from the torn pileus) $a$. White-grey or slightly ochreous-brown.

$P$. convex, shining, often cracked, sometimes brown-fibrillose. St. twisted. $G$, adnexed, thick.

Somewhat cæspitose. Woods, pine; rare. Autumn. $2 \times 3 \times \frac{3}{4} \mathrm{in}$.

280. C. murina Gill. (from its mouse-colour; mus, a mouse) $a$.

$P$. convex, subdepressed, brown, becoming paler. $G$. attenuatoadnexed.

Woods, under oaks. Oct. $1 \frac{1}{8} \times 2 \frac{3}{8} \times \frac{1}{8}$ in.

281. C. protracta Gill. (from the long stem; protractus, drawn out) $a b$.

$P$. convexo-plane, slightly umbonato-depressed, grey-brown or slaty flesh-colour. St. attenuate downwards, rooting, strigose, colour as P., dark slate-colour below. $G$. adfixed, ventricose, grey, white-pruinose.

Mossy ground, near stumps of pine, etc., amongst dead leaves. Aug.Nov. $I_{\frac{1}{2}} \times S \times \frac{1}{8}$ in.

282. C. tesquorum Gill. (from its growing in waste places, tesqua) $a$.

$P$. convex or umbonate, fuscous-black or olive-brown. St. white above, brown below. $G$. adnexo-free, ashy-fuscous or pale olive-brown.

Scattered. Amongst grass, sunny banks. $\frac{3}{4} \times 1 \frac{1}{2} \times \frac{1}{16}$ in.

283. C. clusilis Gill. (chusilis, easily closing, derivation obscure) a $b c$. $P$. convexo-plane, depressed, whitish tan or greyish; mid. faintly ochreous. St. shining, colour as P., or paler. $G$. adnatotoothed, colour as St.

Amongst moss, grass, often with 597. Sept.-Oct. $1 \frac{3}{8} \times 2 \frac{1}{2} \times \frac{1}{8}$ in.

284. C. tylicolor Gill. (from its colour, that of a small worm; Gr. tulos) $a b c$.

$P$. plano-umbonate, ashy-grey or brown; mid. darker. St. wavy, whitish-pulverulent on a grey-brown ground. $G$. adnato-free, varying in attachment, livid-brownish or grey. Flcsh pale brown.

Taste slightly rank; odour weak or none. Shady woods amongst grass. Oct. $\quad 1 \frac{1}{4} \times 2 \frac{1}{8} \times \frac{1}{8}$ in. 
285. C. Dorotheæ Sacc. (after Lady Dorothy Neville) $a$.

$P$. globose, then flat, slightly umbilicate, very thin, dark brown ; marg. denticulate. St. slightly swollen at base, minutely velvety, white, yellowish or rufous. $G$. adnexed, narrow, white.

On dead fern-stems in hothouses. $1 \frac{1}{5} \times 3 \frac{1}{4} \times \frac{1}{15}$ in.

286. C. CALDARI Sacc. (from its habitat, a caldarium or hot-house) $a$. $P$. hemispherical, slightly umbonate, brown. St. paler than $P$. $G$. adnato-decurrent, somewhat ashy.

On Sphagnum in orchid-pot. $\frac{5}{8} \times 2 \frac{1}{8} \times \frac{1}{16} \mathrm{in}$.

\section{M Y CENA Quél. \\ (Gr. muk's, a fungus.)}

$V$ iil almost obsolete, only seen in fibrilla, pile, flock, tomentum or gluten. In Section h, Basipides, the edge of the pileus is commected with the circumference of the basal disc in infancy, and

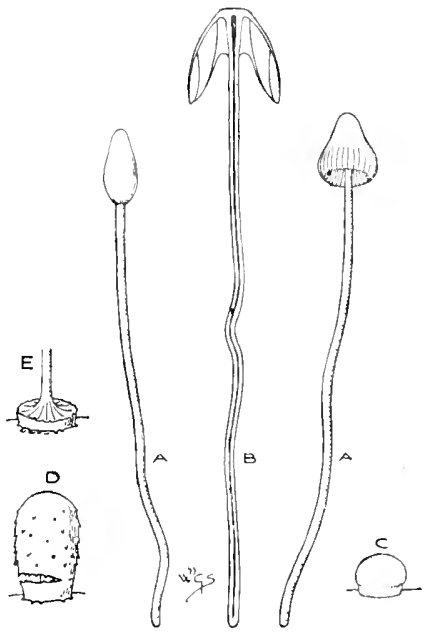

Fig. 13.-A, A, Hycenar cpifte'y'yzia Quél. ; B, ditto in section. One-half natural size. C, M. te'terrima ( luél., young state showing basal disc and veil ; $0,1 /$. stylobatés Quél., young state, showing basal disc and veil; E, basal disc ; C, D, E, enlarged.

so forms a kind of universal veil. Hymenophore confluent with but heterogeneous from the cartilaginous stem. Pileus at first conicocylindrical, becoming more or less campanulate, submembranous, not cartilaginous, never truly umbilicate, margin more or less 
striate, at first adpressed to the stem, never involute. A few species as the next gemus is approached have the pileus slightly depressed. Stem simple, slender, central, fistulose (except 310) and cartilaginous, sometimes hair-like, sometimes rooting amongst dead leaves. Gills adnate or adnexed, often sinuate, varied in attachment, not truly decurrent, often minutely denticulate or fringed at the edge. (Fig. 18.)

Most of the species grow on twigs, stumps, dead branches and dead leaves; some grow on the ground. They are usually slender; small, beautiful and scentless, some have a strong alkaline or mephitic odour; some contain a coloured juice termed milk. They appear after rain in summer and autumn; a few remain till early winter.

Species $287-366$

a. Calodontes. Stem juiceless, base not dilated into a disc. Gills saw-edged or fimbriate, darker edged.

$287-297$

b. Adonidece. Colour pure and bright, not becoming fuscous or ashy, except 300. Stem juiceless, base not dilated into a disc. Gills of one colour, including the edge. Single, terrestrial, except 306 and sometimes 305. 298-306

c. Rigidipedes. St'm firm, tough, juiceless, somewhat strigose and rooting. Gills white, changing to grey or reddish. Persistent, odourless, usually growing on stumps and very cæspitose, but single examples occur on the ground.

$307-316$

d. Fragilipcdes. Pilcus hygrophanous. Stem fragile, juiceless, not dilated into a disc at the base. Gills changing colour. Normally terrestrial and single, a few cæspitose and growing on wood. Strong smelling.

317-334

e. Filipedes. Not hygrophanous. Stem juiceless. Gills changing colour. Terrestrial amongst grass; usually single.

$335-342$

f. Lactipedes. Whole plant exuding a white or coloured juice.

$343-349$

s. Glutinipedes. Stem glutinous. Must not be confounded with species with smooth, moist or slippery stems.

$350-356$

h. Basipcdes. A small dilated base, bulb or disc at the base of the dry, equal, stem. Usually solitary.

i. Insititice. Stem apparently inserted or grafted into dead leaves, etc. Soon flaccid, very tender.

362-366

\section{a. Calodontes.}

287. M. pelianthina Quél. (from the livid pileus; Gr. pcliaino, to make livid) $a b$.

$P$. umbonato-convex, pale brown, dull flesh-colour or lavender, whitish when dry. St. ochreous-whitish or whitish-lavender. 
$G$. adnate, purplish or brownish-white, minutely black-dotted at edge. Flesh white to faintly purplish.

Woods, beech, oak, stumps and dead leaves. Aug.-Oct. $\mathbf{1}_{5}^{5} \times 3 \frac{5}{8} \times \frac{3}{16} \mathrm{in}$. Somewhat resembles 224 .

287a. M. carneosanguinea Rea (from the change of colour in the flesh-on section-from white to blood-red; caro, flesh, sanguis, blood) a.

$P$. convex, subumbonate, smooth, livid-grey; mid. tinging to rufous, paler when old. St. grey, yellowish below, base clad with short mycelial threads. $G$. adnate, dull purplish-brown with a crimson-red edge. Flesh moderately thick at mid. of P. and apex of St., white, changing above to bright rose or blood-red.

Woods. Aug. $1 \frac{1}{4} \times 1 \frac{3}{4} \times \frac{1}{4}$ in.

288. M. balanina Karst. (from its frequent habitat, beech-mast; Gr. balanos) a b.

$P$. convexo-umbonate, granulose, umber-ochreous. St. attenuate downwards, satiny, white above, deep brown below. $G$. rounded, free, sprinkled and fringed with dull purple spiculæ.

Beech-mast, acorns, oak-leaves; rare. Sept. $1 \frac{7}{8} \times 3 \frac{7}{5} \times \frac{1}{8}$ in.

289. M. iris Quél. (from its rainbow tints ; Gr. iris, the rainbow) $a b c$. $P$. hemispherical, evanescent blue-fibrillose on a pale umber, lead-coloured or greenish ground; marg. sometimes cobalt. St. pilose, pale umber above, bright blue-fibrillose at base. $G$. adnexo-free, forming a collar behind, sometimes denticulate, ashy.

On decayed twigs, stumps, chips, sticks and cones of fir, often deeply rooting amongst pine-leaves. Sept.-Nov. $\frac{5}{8} \times 3 \frac{1}{4} \times \frac{1}{16}$ in.

290. M. mirabilis Quél. (mirabilis, wonderful) $a$.

$P$. conical, dull lavender-blue; mid. ochreous. St. floccose, subrooting, bluish below. $G$. adnexed, white, edge dotted with dark particles.

Fir-trunks, amongst $H_{y}$ f $^{\prime}$ um. Aug. $\frac{1}{2} \times 3 \times \frac{1}{16}$ in. Perhaps the same as 291 .

291. M. marginella Quél. (from the minute fringe at the margin of the gills) $\alpha$.

$P$. conico-convex, floccose, pallid; marg. dull bluish. St. tomentose and bluish below. $G$. adnate or adnexed, white, edge bluish- or reddish-flocculose.

Fir-trunks amongst Hypulm cufressiforme. Iug.-Sept. $\quad \frac{2}{ \pm} \times 2 \frac{1}{10} \mathrm{in}$.

292. M. aurantiomarginata Quél. (from the orange margin of the gills) $a$.

$P$. hemispherical, umbonate, olive-brown. St. attenuate upwards, pale yellowish. $G$. attenuato-adnexed with a tooth, whitish, floccose at edge.

Odour strong. Woods, silver fir. Nor. $1 \frac{5}{8} \times 1 \frac{5}{8} \times \frac{3}{16}$ in. 
293. M. elegans Quél. (eleģans, neat) $a b c$.

$P$. campanulate, livid yellowish; mid. fuscous. St. floccose at base, livid-yellowish. $G$. adnato-decurrent, whitish or yellowish, edge orange.

Odour weak of fennel or none. Woods, chiefly pine, fir-leaves, stems of Rubus; frequent. Aug.-Nov. $\frac{3}{4} \times 2 \frac{5}{8} \times \frac{1}{16}$ in.

294." M. rubromarginata Gill. (from the red margin of the gills) $a c$. $P$. hemispherical, subumbonate, white or pale reddish. St. white. $G$. adnato-decurrent, whitish or grey, edge rose or brownpurple.

Odour none. On branches, leares and rotten twigs in woods, chiefly pine and larch; frequent. July-Nov. $\frac{5}{8} \times 1 \frac{1}{3} \times \frac{1}{16}$ in. A variety, fuscopurpurea Mass., with a dusky purple pileus, grows on willows.

295. M. olivaceomarginata Mass. (from the olive margin of the gills) $a b$.

$P$. conico-hemispherical, subumbonate, honey-colour. St. colour as P., base tinged rufous. $G$. uncinato-adnexed, broad, subdistant, pallid with a brownish-olive edge.

Woods, lawns. Oct. $\frac{3}{ \pm} \times 1 \frac{7}{8} \times \frac{1}{16}$ in.

296. M. strobilina Gill. (from its usual habitat, fir-cones, strobilus) $a b$.

$P$. campanulate, acutely umbonate, scarlet, paler at marg. St. scarlet, white-strigose at base. $G$. adnato-decurrent, paler than P., edge blackish-scarlet. Flesh crimson.

Cones, leaves and twigs of Scotch fir, sometimes in beech-woods; rare. Oct. $\frac{3}{ \pm} \times 2 \times \frac{1}{15}$ in.

297. M. rosella Quél. (roseus, rose-coloured) $a b c$.

$P$. campanulate, subumbonate, deep or pale rose. St. colour as $P$., seldom strigose at base. $G$. adnate, pale rose, edge dark purple.

Laxly gregarious. Amongst leaves, twigs, and decaying cones in fir-woods ; uncommon. June-Dec. $\frac{5}{5} \times 1 \frac{3}{9} \times \frac{1}{16}$ in.

\section{b. Adonidia.}

298. M. pura Quél. (from its pure colour) a b $c$.

$P$. campanulate, broadly umbonate, brittle, flesh-colour, variable in shade. St. often rooting, flesh-colour, pale ochreous below. $G$. sinuato-adnate, veined, whitish to pale flesh-colour.

Taste and odour strong, hot, disagreeable, pungent of radish or cabbagestalk. Woods, meadors; common. June-Nov. $2 \times 4 \times \frac{1}{4}$ in. There is a white variety. Varies larger and smaller, sometimes very dark when dry. Sometimes attacked by Mucor macrocarpus.

299. M. pseudopura Sacc. (from its resemblance to 298 ; Gr.pseudos, false) $a$.

Structure and colour same as 298 , of which it is probably one of the smaller forms. Colour rosy. $G$. whitish.

Odour none. Woods. Autumn. I $\times 2 \frac{3}{4} \times \frac{1}{16} \mathrm{in}$. 
300. M. zephira Quél. (from its coming with the rain-wind or west wind Zephyros) a $c$.

$P$. hemispherical, diaphanous, white flesh-colour; mid. often fuscous. St. white to flesh-colour. $G$. adnate, veined, white.

Decayed fir, amongst dead leaves in woods. Sept.-Dec. $1 \frac{3}{8} \times 3 \frac{1}{3} \times \frac{1}{8}$ in. $G$. and $P$. sometimes foxy-stained.

301. M. Adonis Quél. (from its beauty-like Adonis) $a b$.

$P$. conico-campanulate, white, yellow, greenish, orange or crimson. St. lighter than P. $G$. ascending, adnexo-toothed or adnate, colour as St.

Woods, pastures; uncommon. Sept.-Nov. $\frac{5}{8} \times 2 \frac{1}{1} \times \frac{1}{16}$ in.

302. M. lineata Quél. (from the lined or sulcate pileus) $a$ b.

$P$. plane, subumbonate, membranous, pale yellowish. St. colour as P. $G$. adnate, white. Flesh pale brownish.

Amongst moss. Sept.-Oct. $I \times 2 \frac{1}{1} \times \frac{1}{16}$ in. Colour variable.

302a. M. farrea Quél. (from the pileus, the colour of ripe wheat and powdered as if with flour ; farreus, made of spelt) $a$.

$P$. very thin, campanulato-expanded, subumbonate, pale buffyellowish, becoming pale, shining white pulverulent; marg. sulcate, crenulate. St. somewhat rooting, silky-striate, pale steel-grey, white pulverulent above. $G$. adnate, thin, connected with veins, somewhat fimbriate, white.

Mossy and grassy places. $I \times 3 \frac{1}{8} \times \frac{1}{10}$ in. This and 336a are the only species of the genus with pulverulent pilei.

303. M. luteoalba Quél. (lutezus, yellow, albus, white) a bc.

$P$. expanded, umbonate, pale yellow. St. white, faint yellowish at base. $G$. adnato-toothed, shining white.

Woods, fir, amongst moss ; uncommon. Aug. -Nov. $\frac{5}{8} \times \mathrm{I}_{\frac{3}{4}}^{\frac{3}{16}} \times \frac{1}{16}$ in.

304. M. flavoalba Quél. (flaz'us, light yellow, albus, white) a $b c$.

$P$. expanded, broadly umbonate, ochre-biscuit, paler at marg. St. faint yellowish at base. $G$. adnexed or adnate, distant, white.

In troops. Woods and meadows, under firs; frequent. July-Dec. $I \times 1 \frac{3}{8} \times \frac{1}{16}$ in.

305. M. lactea Quél. (from the milk-white colour ; lac, milk) $a b c$.

$P$. campanulate, umbonate, varying faint biscuit. St. attenuate upwards, fibrillose at base. $G$. adnato-rounded or sinuate, crowded.

Scattered, rarely crespitose. Woods, chiefly amongst and on pine-leaves; common. July-Dec. $\quad 1 \times 2 \frac{3}{4} \times \frac{1}{15}$ in. Must be carefully distinguished from other species of the genus which become white.

306. M. gypsea Quél. (from its colour, like gypsum) $a b$.

$P$. conico-campanulate, umbonate; mid. faintly biscuit. St. attenuate upwards, strigose at the faintly biscuit base. $G$. adnexo-ascending or adnate.

Cæspitose. Tasteless; odour alkaline, varying strong to weak. Stumps. June-Nov. $\frac{7}{8} \times 3 \frac{3}{\frac{3}{4}} \times \frac{1}{5}$ in. 


\section{c. Rigidipedes.}

307. M. eohærens Gill. (from the adherent stems; cohero, to stick together) a $c$.

$P$. campanulate, broadly umbonate, pale umber, cinnamon or orange-brown; mid. darker ; marg. striate. St. even, smooth, shining whitish above, downy and ochreous-whitish to dark brown below. $G$. rounded-free, distant, white to yellowish or greyish. Flesh brown.

Sometimes solitary. Brambles, pine-leaves, etc, on the ground. Oct.-Feb. $1 \frac{5}{5} \times 4 \frac{1}{ \pm} \times \frac{1}{8}$ in. Resembles 313 (var. calopzts).

307a. M. Berkeleyi Mass. (after the Rev. M. J. Berkeley) $a$.

$P$. campanulate, expanded, subumbonate, hygrophanous, dull brown ; um. darker and brighter, paler when dry ; marg. striate. St. with a tapering, rooting base, somewhat striate, dull pale brownish with a purple tinge. $G$. adnato-sinuate with a decurrent tooth, subdistant, ventricose, thin, tinged purplish or flesh-colour. Flesh pale flesh-colour, tinged purplish.

Solitary or subcrespitose. Tree-trunks. $3 \frac{1}{1} \times 5 \times \frac{1}{\frac{1}{4}}$ in. Rooting base, $2 \frac{1}{\frac{1}{4}} \mathrm{in}$. May be the same as 309 .

308. M. prolifera Gill. (from the stem, sometimes densely proliferous with small individuals at the base) $a b$.

$P$. campanulato-expanded, broadly umbonate, pale umber; marg. sulcate. St. ventricose, splitting, white. $G$. adnexed or adnate, white.

Inodorous, at length nauseous. Woods and gardens, on gravel, near bushes. Sept. Uct. $\quad 1 \frac{7}{8} \times 6 \times \frac{3}{16}$ in. There is a white variety.

309. M. excisa Gill. (from the cut-out gills) a $c$.

$P$. expanded, umbonate, brown; mid. darker. St. as in 308 , pale brown, white above. $G$. adnexed or sinuato-free, thick, distant, hoary, veined, white, pale brown or purplish.

Woods, trunks, sometimes on the ground. Sept.-Oct. $2 \frac{3}{8} \times 4 \times \frac{7}{4}$ in. Var. fagetorum Karst. Small, amongst beech-leaves.

310. M. psammicola Sacc. (from its habitat; Gr. psammos, sand) $a$. $P$. hemispherical, sprinkled with minute particles, brown: marg. whitish. St. solid, rooting, white pulverulent on a brownish ground. $G$. adnate, white.

Sandy banks amongst moss. Sept. $\frac{3}{8} \times 1 \frac{1}{2} \times \frac{1}{32}$ in.

311. M. rugosa Quél. (from the wrinkled pileus; rugra, a wrinkle) a $b$. $P$. expanded, subumbonate, pale grey-brown; mid. brownish. St. subventricose, colour as P. G. adnato-toothed, whitish.

Tasteless or at length slightly bitter. ()n and near stumps; common. July-Dec. $2 \frac{1}{2} \times 3 \frac{3}{8} \times \frac{1}{8}$ in. Very tough and dry.

312. M. sudora Gill. (from the riscid pileus; sudor, sweat) $a b c$.

$P$. campanulate, umbonate, whitish, livid-biscuit or brownish. $S t$. colour as P. $G$. adnate, white, flesh-colour or yellowish-white.

Usually solitary. Stumps, beech. Aug.-Nov. $1 \frac{7}{8} \times 5 \frac{1}{4} \times \frac{1}{3}$ in. Colour often like 350 or 1228 . 
313. M. galericulata Quél. (from the shape of the pileus: galiriculum, a little cap) a $b c$.

$P$. expanded, broadly umbonate, pale brown; mid. darker. St. colour as P. $G$. adnato-toothed, veined, white to pale rose.

Usually tasteless. Stumps, bramble, willow, and on the ground; common. July-Jan. $2 \frac{3}{8} \times 4 \frac{1}{2} \times \frac{3}{16}$ in. Var. calofus Karst. has an umber pileus and dark sienna stem. Inucor macrocarfus is frequent on this species.

314. M. polygramma Quél. (from the longitudinally striate stem ; Gr. polus, many; sramme, a stroke) a $b c$.

$P$. campanulate, umbonate, pale to dark brown. St. steel-grey. $G$. adnato-toothed, attenuate near St., pale salmon.

Gregarious. (dlour disagreeable, or none. Stumps ; common. Sept.Jan, $2 \times 5 \frac{5}{8} \times \frac{1}{8}$ in.

315. M. parabolica Quél. (from the parabolic curves of the pileus) a b c.

$P$. conico-campanulate, sides incurved, slate; mid. umber. St. attenuate upwards; colour as P., paler below. $G$. adnatoascending, white or faint grey. Flesh brownish-grey.

Gregarious or cæespitose. Rotten wood, stumps, especially fir, willow, fern ; uncommon. Sept.-Dec. $\quad 1 \frac{7}{8} \times 2 \frac{1}{2} \times \frac{1}{8}$ in.

316. M. tintinnabulum Quél. (from the bell-shaped pileus; tintinnabulum, a bell) $a b$.

$P$. plane, subumbonate, umber, varying bluish, yellow-brownish and whitish. St. white. $G$. adnate with a tooth, white or pale flesh-colour.

Gregarious, sometimes cæspitose. Fallen trunks, beech. April-Dec. $\mathbf{I} \frac{3}{8} \times \mathbf{I} \times \frac{1}{8}$ in.

\section{d. Fragilipedes.}

317. MI. Codoniceps Sacc. (from the bell-shaped pileus; Gr. kollon, a bell ; kephale, a head) $a b$.

$P$. campanulate, obscurely sulcate and rugulose, hispid, umber; marg. recurved. St, attenuate downwards, moist, smooth, white above, umber below. $G$. vertically ascending, somewhat thick, distant, adnate with a tooth, white.

Single or crespitose. Tree-fern stems. June. $\frac{1}{16} \times \frac{\frac{5}{2}}{2} \times \frac{1}{6 \pm}$ in.

318. M. flavipes Quél. (from the yellow stem; flazus, yellow, pes, a foot) $a b$.

$P$. hemispherical, rose or flesh-colour. St. tough, villous at base. $G$. adnate, paler than P.

Odour of radishes. Stumps. Autumn. $\frac{3}{4} \times 2 \frac{3}{4} \times \frac{1}{16}$ in.

319. M. atroalba Gill. (ater, black, albus, white) a $b c$.

$P$. broadly campanulate, dark slate-grey; marg. whitish. St. with a large strigose bulb, apt to split as in 320 , grey and white. $G$. free, white, then glaucous.

Woods, amongst moss. July-Nov. $I_{8}^{3} \times 3 \frac{1}{3} \times \frac{1}{8}$ in. Shape and colour of 315 , habit different. 
320. M. dissiliens Quél. (from the stem splitting when broken; dissilio, to burst asunder) $a b$.

$P$. campanulato-flat, sulcate, ashy-brown; marg. whitish. St. ashy. $G$. rounded-free, white.

Odour insipid, sometimes strong, rancid rather than alkaline. Trunks, branches, on the ground amongst grass; frequent. July-Nov. $1 \frac{3}{4} \times 2 \frac{3}{4} \times \frac{1}{8}$ in.

321. M. atrocyanea Gill. (from the blackish-indigo pileus; ater, black, cyaneus, dark blue) a b $c$.

$P$. campanulato-conical, umbonate, sulcate. St. slightly attenuate upwards, colour as P. G. ascending, attenuato-adnate. Flesh pale slate.

Solitary or caspitose. Odour none. Amongst fir-leaves, on the ground. Sept.-Nov. $\frac{5}{8} \times 3 \frac{5}{8} \times \frac{1}{16}$ in. Becoming black when dry.

322. M. pullata Sacc. (pullus, dark-coloured) $a$.

$P$. campanulate, umbonate, sulcate, dark umber, becoming paler with age. St. slightly attenuate upwards, colour as P., base white-floccose. $G$. adnexed, white.

Odour slightly nitrous. Amongst dead leaves. Oct.-Nov. $\frac{7}{5} \times 3 \frac{5}{8} \times \frac{1}{16}$ in.

323. M. paupereula Sacc. (from its appearance ; panper, poor) $a b$.

$P$. hemispherical, white or faint tawny. St. attenuato-rooting, white. $G$. adnexo-free, white.

Odour of fresh meal, or sweet. Inside decayed stumps, oak; uncommon. July-Sept. $\frac{3}{16} \times \frac{3}{8} \times \frac{1}{32}$ in.

324. M. leptocephala Gill. (from the delicate pileus; Gr. leptos, fine, kephale, a head) a.

$P$. expanded, umbonate, sulcate, ashy; mid. darker. St. colour as P. $G$. emarginate, ashy-white.

Solitary. Odour strong of nitre. On the ground and on trunks. Autumn. $\frac{1}{4} \times 2 \frac{1}{8} \times \frac{1}{16}$ in.

325. M. alkalina Quél. (from its alkaline odour) $a b c$.

$P$. campanulate, umbonate, brown or ashy; mid. darker. St. brown or yellow. $G$. adnato-ascending, white, or ashy with a white edge.

Solitary or caspitose. Odour strong, disagreeable. Stumps, etc., bramble, privet, fir ; uncommon. Jan.-Dec. $1 \frac{5}{8} \times 5 \times \frac{1}{8}$ in. Varialle in colour, often exhibiting shades of yellow or pale rose. There is a white variety.

326. M. ammoniaca Quél. (from its ammoniacal odour) $a b$.

$P$. conico-campanulate, papillate, deep brown to greyish; marg. ashy. St. whitish-grey or brownish. $G$. adnate, whitish or grey.

Solitary. Odour strong. On the ground, amongst grass. Sept.-Nor. I $\times 3 \frac{5}{8} \times \frac{1}{5}$ in.

327. M. metata Quél. (from the pileus, like a mitu, the conical column at the end of the Roman Circus $a b c$.

$P$. conico-campanulate, cream-grey, flesh-colour or livid. St. colour as P. $G$. adnato-ascending, whitish.

Gregarious. Odour alkaline, strong to weak or none. Woods, amongst moss. Oct.-Nov. $\frac{7}{8} \times 3 \frac{1}{8} \times \frac{1}{8}$ i.. 
328. M. plicosa Gill. (from the sulcate pileus; plico, to fold) a $b c$. $P$. expanded, umbonate, often split, pale tan; mid. sienna; marg. greyish. St. colour as P., white at base. $G$. adnate, greyish.

Woods, on the ground; rare. Sept.-Oct. $1 \frac{1}{x} \times 1 \frac{7}{5} \times \frac{1}{5}$ in.

329. M. peltata Gill. (from the shield-like pileus; pelta, a small shield) $a b$.

$P$. plane, with a central projecting ring, fuscous-blackish or greyish. St. pale umber. $G$. sinuate, pale grey.

Woods, commons, etc., amongst moss; rare. Aug.-Oct. $\frac{1}{1} \times 2 \frac{3}{4} \times \frac{1}{16}$ in.

330. M. consimilis Sacc. (from its likeness to various other species of the genus) $a$.

$P$. campanulate, umbonate, splitting, pale grey; mid. umber. St. pale umber. $G$. adnato-ascending, pale grey.

Odour none. Amongst grass. Autumn. $1 \frac{3}{8} \times 2 \times \frac{1}{8} \mathrm{in}$.

331. M. ætites Quél. (from the colour; Gr. atites, iron ore) a bc.

$P$. expanded, umbonate, sulcate, opaque brown-ochre. St. paler than $P$. or pale livid-umber. $G$. adnato-sinuate, veined, white or whitish-grey. Flesh pale brown-ochre.

Scattered or loosely gregarious. Odour bitter or none. Woods, amongst moss and grass. June-Oct. $1 \frac{1}{4} \times 1 \frac{7}{8} \times \frac{1}{8}$ in.

332. M. stannea Quél. (from the colour; stammum, tin) a b.

$P$. expanded glistening, grey, sometimes umber. St. pale tan. $G$. adnato-toothed, whitish-grey.

Scattered or loosely gregarious. Odour none. Woods, amongst grass, on the ground. Sept.-Oct. $\frac{3}{4} \times 3 \frac{1}{8} \times \frac{1}{16} \mathrm{in}$.

333. M. vitrea Quél. (from its fragility ; z'itrum, glass) $a b$.

$P$. campanulate, subumbonate, umber; mid. darker. St. glistening, paler than P. $G$. sinuato-adnate, whitish.

In troops. Odour none. Woods, fir, moist places; uncommon. Sept.-Oct. I $\frac{3}{8} \times 4 \frac{1}{4} \times \frac{1}{8}$ in.

334. M. tenuis Gill. (temuis, thin) $a b c$.

$P$. campanulato-convex, white; mid. pale brown; marg. toothed. St. white. $G$. adnate, whitish.

Woods, shady moist places, often on the root-fibres of trees; rare. Sept. $\frac{3}{4} \times 5 \times \frac{1}{16}$ in. Watery, delicate, fragile, wavering.

\section{e. Filipedes.}

335. M. filopes Quél. (from the thread-like stem; flum, a thread, pes, a foot) $a b c$.

$P$. expanded, pale livid-brown or whitish. St. rooting, pilose at base, white. $G$. adnexo-free, white or greyish.

Singly or in troops. Woods, amongst leaves. Aug.-Nov. I $\times 6 \frac{1}{42} \times \frac{1}{32}$. 
336. M. amicta Quél. (from the pulverulent pileus and stem; amicio, to clothe) $a$.

$P$. conico-campanulate, subumbonate, greenish, pale slate or livid. St. rooting, livid. $G$. adnexo-free, pale grey with lighter edge.

Gregarious. Odour none. Amongst moss and leaves, about roots of Nardus stricta. Sept. $\frac{3}{8} \times 3 \frac{1}{2} \times \frac{1}{32}$ in.

336a. M. plumbea Karst. (from the colour of the pileus; plumbus, leaden).

P. membranous, convexo-flattened, obtuse, sulcate, sprinkled white-pruinose. St. fragile, ashy-pulverulent, white-hyaline above, strigose below. $G$. adnate, horizontal, colour as $\mathrm{P}$.

Odour none. Mossy pastures. Autumn. $\frac{5}{8} \times 4$ in.

336b. M. cinerea Mass. \& Crossl. (from its colour; cinis, ashes). Wholly grey.

$P$. slightly gibbous or obtuse, then expanded; marg. striate, paler and silky when dry. St. glabrous, base white-downy. $G$. adnate, subdistant; edge paler.

Amongst short grass. Odour strong of radishes. I $\times 2 \frac{3}{3} \times \frac{1}{15}$ in. Differs from 324 in sulcate $\mathrm{I}$. ; from 327 in white G.; from 336a in absence of odour.

337. M. debilis Quél. (dibilis, weak) a c.

$P$. conico-campanulate, subumbonate, livid-whitish or brownishsalmon. St. colour as P., fibrillose at rooting base. $G$. broadly adnate, whitish.

Gregarious. Woods, chestnut, fir, amongst moss. Oct. $\frac{3}{8} \times 2 \frac{1}{2} \times \frac{1}{32}$ in.

338. M. vitilis Quél. (from the deep strix of the pileus; vitilis, plaited) $a b$.

$P$. conico-campanulate, sometimes papillate, brownish, becoming whitish. St. shining, livid. $G$. attenuato-adnate or slightly sinuate, whitish or grey, or dark ashy with a white edge.

Solitary or gregarious. Odour none. Woods, mixed amongst leaves; frequent. Sept.-Nov. $\frac{1}{2} \times 4 \frac{3}{8} \times \frac{1}{3}$ in.

339. M. collariata Quél. (from the union of the gills in a minute collar at the top of the stem) a $c$.

$P$. campanulato-convex, subumbonate, greyish-white, rose-tinged; mid. fuscous. St. smooth, whitish-brown. $G$. adnate, hoary white or faintly salmon.

Woods, amongst grass and oak-bark. Oct.-Nov. $\frac{5}{8} \times 2 \frac{1}{2} \times \frac{1}{16}$ in.

340. M. speirea Gill. (from the twisted base of the stem ; Gr. speira, a coil) $a$.

$P$. plane, umbonate, pallid grey or whitish; mid. dark fuscous. St. attenuato-rooting, fibrillose and brown below, white above. $G$. adnate, thin, deeply decurrent, white.

In troops during very rainy weather. Woods, mixed, mossy trunks; uncommon. Aug.-Oct. $\frac{1}{2} \times 1 \frac{7}{8} \times \frac{1}{32}$ in. 
341. M. tenella Quél. (tencr, tender) a. White or livid Hesh-colour. $P$. campanulato-convex, pellucid. $G$. adnato-decurrent.

Cæipitose. Decayed trees. Autumn. $\frac{1}{3} \times \frac{7}{8} \times \frac{1}{3}$ in.

342. M. acicula Quél. (from its resemblance to a small pin, aciculc) a b c .

$P$. conico-campanulate, papillate, orange-vermilion. St. attenuate and fibrilloso-rooting, deep yellow. $G$. adnate or adnexorounded, yellow with a whitish edge or wholly white.

Wood, leaves, twigs, etc. ; frequent. July-Oct. $\frac{3}{8} \times I_{t}^{\frac{3}{3}} \times \frac{1}{32}$ in.

\section{f. Lactipides.}

343. M. hæmatopus Quél. (from the blood-red juice of the stem ; Gr. haima, blood, pous, a foot) $a b c$.

$P$. conico-campanulate, subumbonate, whitish Hesh-colour. St. pulverulent, colour as P. G. adnate, whitish. Milk deep brown-crmmson.

Cæspitose. Taste slightly disagreeable. Stumps, old timber, alder, birch ; frequent. Aug.-Nov. $1 \frac{5}{5} \times 3 \times \frac{1}{8}$ in.

344. M. eruenta Quél. (from the blood-like juice; cruor, gore) a $b$.

$P$. conico-campanulate, subumbonate, brown. St. tense, villousrooting, paler than P. $G$. adnate, whitish. Milk deep brownish-crimson.

Solitary or subcrespitose. Woods, pine-cones, etc.; rare. Sept. I $\times 3 \frac{1}{3} \times \frac{1}{16}$ in.

345. M. sanguinolenta Quél. (from the blood-red juice; sanguinolentus, bloody) a $b c$.

$P$. hemispherical, brown or purplish-brown. St. flaccid, fibrillose at base, colour as P. G. pale purplish-brown; edge sometimes dark purple. Milk brownish-crimson.

Gregarious. Woods, chips, twigs, fir-leaves, moss, etc., tree-fern stems in greenhouses. May-Nov. $\frac{1}{2} \times 3 \frac{5}{8} \times \frac{1}{32}$ in. Sometimes wholly pale brown and varying $\frac{1}{2}$ to $\frac{1}{2}$ in. high.

346. M. erocata Gill. (from the saffron-coloured juice; crocus, saffron) a b.

$P$. convex, olivaceous, ashy, yellowish or white; mid. reddish. St. tense, deep yellow, orange-crimson at fibrillose base. $G$. adnato-ascending or adnexed, white.

Scattered. Woods, beech, amongst leaves; uncommon. Autumn. $\frac{7}{8} \times 4 \frac{3}{\frac{3}{4}} \times \frac{1}{16}$ in. A small var. appears on beech-trunks.

347. M. chelidonia Quél. (from the yellow juice, like that of Chelidonizm majus) a $b c$.

$P$. hemispherical, yellowish tiesh-colour; mid. ochreous. St. firm, yellow at the villous-rooting base. $G$. adnate, white or yellowwhite. Milk, saffron to vinous-red.

Subcrspitose. Odour somewhat alkaline. Stumps, beech, alder; rare. Oct. $\frac{5}{8} \times 2 \frac{7}{8} \times \frac{1}{16} \mathrm{in}$. 
348. M. galopus Quél. (from the white juice; Gr. grala, milk, pous, a foot) $a b c$.

$P$. hemispherical, subumbonate, dark then pale purplish-fleshcolour; mid. blackish. St. colour as P., base villous. $G$. adnexed or adnate, white or glaucous. Milk white.

In troops. Almost tasteless or like radishes. Woods, amongst moss and leaves; common. July-Nov, $\frac{5}{8} \times 2 \frac{7}{8} \times \frac{1}{16}$ in. Sometimes wholly dark in colour.

349. M. leucogala Sacc. (from the milk-white juice; Gr. leukos, white, gala, milk).

$P$. campanulate, umbonate, purple-brown. St. lighter than P., white tomentose at base. $G$. adnate with a tooth, ashy.

Cæspitose. Amongst grass, rotten stumps. Oct. $\frac{7}{8} \times 3 \times \frac{1}{16}$ in.

\section{g. Glutinipedes.}

350. M. epipterygia Quél. (from its frequent growth on brake-fern, Pteris aquilina a $b \mathrm{c}$.

$P$. conical with a separable viscid pellicle, dark olivaceous then pale livid-sulphur. St. greenish-sulphur. G. sinuato-ascending, whitish or greyish. Flesh pale sulphur.

Solitary, gregarious or cespitose. Odour none. Woods, amongst grass, leares, dead lracken, on manure-heaps; common. Aug.-Dec. $\frac{3}{4} \times 5 \frac{1}{2} \times \frac{1}{16}$ in. There is a white var.

351. M. clavicularis Gill. (from its shape; clacus, a nail) $a b$.

$P$. expanded, subumbonate, dry, pelliculose, white or light yellowish. St. tough, slightly viscid, whitish. $G$. adnatodecurrent, whitish.

IVoods, fir, grassy places. Oct. $\frac{3}{4} \times 2 \frac{1}{8} \times \frac{1}{16} \mathrm{in}$.

352. M. pelliculosa Quél. (from the thin separable pellicle of the pileus) $a b c$.

$P$. at first umbonate then plane, viscid, brownish; mid. darker. St. rigid, livid grey-whitish. $G$. adnato-decurrent, thick, white.

In troops during very rainy weather. Taste none. Heaths, heathy woods; frequent. Sept.-Dec. $\frac{7}{8} \times 2 \frac{1}{2} \times \frac{1}{16}$ in.

353. M. vulgaris Quél. (vulgraris, common) a $b c$.

$P$. convex, depressed, papillate, subviscid, brownish or ashy-grey. $S t$. attenuate and slightly strigose below, ashy-grey. $G$. adnatodecurrent, white.

Gregarious. Odour strong of new meal, or none. Woods, fir, amongst moss, on dead twigs, larch and pine leaves; common. July-Nov. $\frac{1}{2} \times 1 \frac{3}{4} \times \frac{1}{32}$ in.

354. M. citrinella Quél. (from its colour; citrus, lemon) a $b c$.

$P$. hemispherical, subviscid. St. pale brown villous at base. $G$. adnato-ascending, at first white.

Gregarious. Woods, pine, rotten trunks; uncommon. Oct. $\frac{5}{16} \times \frac{1}{8} \times \frac{1}{32}$ in. The var. candida (iill. is at first white, sulphur in drying. 
355. M. plicatocrenata Gill. (from the folded and margin-notched pileus; plica, a fold, crena, a notch) $a$.

$P$. conic, subumbonate, white then light yellow. St. pale pallidreddish. $G$. adnato-ascending, white.

Amongst heath and moss, under larches. Oct. $\frac{3}{8} \times 1_{\frac{3}{4}}^{3} \times \frac{1}{32} \mathrm{in}$.

356. M. rorida Quél. (from the dripping jelly of the stem; roridus, dewy) $a b c$.

$P$. convex, whitish-ochre, pale buff, faintly ashy or white. St. white. $G$. arcuato-decurrent, white.

Taste none; odour somewhat rank, strong. Dead sticks, twigs, bramble; rare. June--Sept. $\frac{1}{2} \times 1 \frac{5}{8} \times \frac{1}{32}$ in.

\section{h. Basipedes.}

357. M. stylobates Quél. (from the shape of the stem; Gr. stulos, a pillar, basis, a base) a $b c$. White or faint grey.

$P$. campanulate, sprinkled with minute hairs. St. pruinose above. $G$. free.

Dead grass, leaves, rushes, fern-stems, twigs, bark, chips, in wet places; uncommon. June-Oct. $\frac{3}{8} \times 2 \frac{1}{5} \times \frac{1}{32}$ in. In infancy the edge of the pileus is attached to the edge of the basal disc and forms a veil.

358. M. tenerrima Quél. (tenerrimus, very tender) a $b c$. White.

$P$. hemispherical, pruinose. St. pubescent below. $G$. free.

Gregarious. Mossy bark of trees, willow, dead bramble, fir-cones, dead wood, sticks, chips, etc.; uncommon. Aug.-Mar. $\frac{1}{16} \times \frac{3}{8} \times \frac{1}{64}$ in. Edge of pileus and disc as in 357.

359. M. discopus Gill. (from the basal disc of the stem ; Gr. diskos, a disc, pous, a foot) $a b$. White.

$P$. conical, subumbonate, pulverulent. $G$. adnate.

Twigs, acorns, dead wood, etc., in moist places. Autumn. $\frac{1}{16} \times \frac{3}{8} \times \frac{1}{64}$ in.

360. M. saccharifera Gill. (from its sugary appearance under a lens; saccharon, sugar, firo, to bear) a $b c$. Whitish.

$P$. hemispherical, covered with minute subglobose granules. $G$. subdecurrent.

Dead bramble, rose, furze, nettle. Nov.-Mar. $\frac{1}{16} \times \frac{3}{16} \times \frac{1}{66}$ in. In var. electica MIass, the $P$. is sulcate and $G$. adnate.

361. M. pterigena Quél. (from its habitat; pteris, a fern, gigno, to bear) $a b c$.

$P$. hemispherico-oblong, pale rose with orange marg. St. paler than P. $G$. adnate, pallid with bright orange edge.

Gregarious, sometimes fasciculate. Dead Lastrea, veins of dead leaves, etc. ; uncommon. Sept.-Nov. $\frac{3}{16} \times 1 \frac{3}{4} \times \frac{1}{32}$ in.

\section{i. Insititice.}

362. M. corticola Quél. (from its habitat; cortex, bark, colo, to inhabit) $a b c$.

$P$. hemispherical, depressed, brownish, varying grey. St. slightly furfuraceous, paler than P. $G$. sinuate, colour as St.

Amongst moss, on living ash, bramble, oak, willow, apple, pear; common. June-Jan. $\frac{5}{16} \times 1 \frac{1}{1} \times \frac{1}{32}$ in. 
363. M. hiemalis Quél. (from its time of growth; hicms, winter) a $c$. Whitish or flesh-colour, rarely brownish or slate.

$P$. hemispherical. St. slightly downy below. $G$. adnato-ascending, narrow.

Scattered. Trunks, oak, willow; rare. Sept.-Mar. $\frac{5}{8} \times 1 \frac{1}{8} \times \frac{1}{16}$ in.

364. M. setosa Gill. (from the hairy stem ; seta, a bristle) $a b$. White, becoming brownish.

$P$. hemispherical. $G$. adnate.

Densely gregarious. Dead beech-leaves; rare. Oct.-Nor. $\frac{1}{15} \times \frac{3}{7} \times \frac{1}{1} \frac{1}{2} 8$ in.

365. M. capillaris Quél. (from the hair-like stem; cupillus, a hair) $a b c$. White.

$P$. plane, slightly depressed. St. smooth, attenuate downwards. $G$. adnato-decurrent.

Woods, on herbaceous stems, moss and dead leaves, chiefiy beech, oak. Sept.-Nov. $\frac{1}{15} \times \frac{3}{4} \times \frac{1}{12}$ in. Chiefly seen in very rainy weather.

366. M. juncicola Gill. (from its habitat; juncus, a rush, colle, to inhabit) $a$.

$P$. acutely conic, rose-colour, rufescent, blood-red or tawny. St. brownish. $G$. adnate, white or pale yellowish.

Rushes in bogs, twigs ; rare. June-July. $\frac{1}{16} \times \frac{5}{8} \times \frac{1}{1 \frac{1}{2} 5}$ in.

\section{OMPHALIA Quél.}

(From the umbilicus; Gr. omphales.)

Til almost obsolete, only seen in pruina, squamules or tomentum of the pileus and stem, and rarely in a basal disc to which the edge

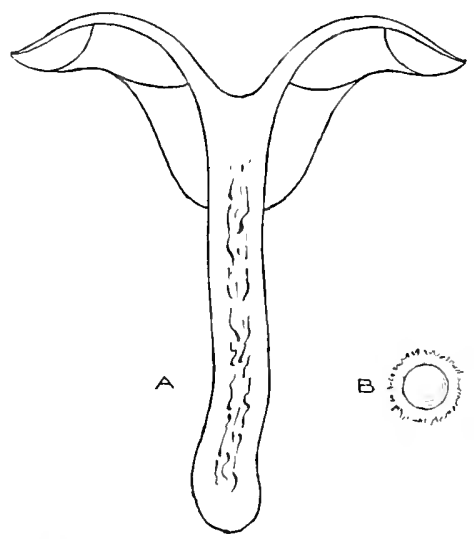

Fir. 10. $-\mathrm{A}$, section of Omphatia muratis Quél. $\times$ 3. B, O. psendodirecta W. G. Sm. : young state seen from above to show volva-like base.

of the pileus is attached in infancy. Hymenophore confluent with, but heterogeneous from the cartilaginous stem. Pileus somewhat 
membranous, umbilico-infundibuliform, hygrophanous. Stem simple, central, cartilaginous, fistulose or stuffed, more or less lustrous, sometimes hair-like. Gills decurrent. Spores more or less elliptical, smooth. (Fig. I 9.)

Many of the species are small, beautiful and generally epiphytal. Odour obsolete or nearly so. Mostly peculiar to hilly positions, preferring damp, woody situations and rainy seasons.

The species must not be confounded with the thick-gilled species of similar structure found amongst the Cantharelli.

Species $367-406$

Collybiarle (from the resemblance to Collybia). Pilezs with a deflexed margin.

a. Hydrogramme. Comparatively large. Gills narrow, very crowded, arcuate, from the first truly decurrent. $\quad 367-370$

b. Pyxidate. Intermediate in size. Gills narrow, slightly distant, attenuate at both ends. 371-382

c. Umbelliferce. Gills broad, commonly thick, very distant. Must not be confounded with Xerotus.

383-392

Mycenarie (from the resemblance to Mycena). Pilens at first companulate, margin straight, pressed to the stem.

d. Campanilla. Gills broad, perfect, unequal.

$393-403$

c. Intrgrelle. Gills fold-like, narrow.

$404-406$

\section{COllybiarie.}

\section{a. Hyidrogrammae.}

367. 0. hydrogramma Quél. (from its watery streakings; Gr. Iuddor, water, grelmme, a line) a $b$.

$P$. subconvex, striate, broadly umbilicate, tan. St. subattenuate and villous below, tan. $G$. livid whitish or tan.

Somewhat crespitose. In woods amongst dead leaves, beech. Oct. $2 \frac{5}{8} \times 3 \frac{3}{8} \times \frac{1}{1}$ in. Resembles 172 in appearance.

367a. 0. detrusa Gill. (from the umbilicus-as if pushed in ; detrudo, to pusb down) a $b$.

$P$. somewhat fleshy, convex, expanded, at length umbilicate, even, glabrous, indistinctly zoned, dark grey or whitish-umber. St. stuffed to hollow, glabrous, dark grey or dull lavender. $G$. subdecurrent with a tooth, thin, crowded, whitish or somewhat pale ochreous.

Woods, amongst moss. Autumn. $1 \frac{7}{5} \times 2 \frac{1}{1} \times \frac{3}{10}$ in.

368. 0. umbilicata Gill. (from the umbilicate pileus) $a$.

$P$. plane, deeply umbilicate, tan to fuscous. St. silky striate above, villous below, whitish-buff. $G$. whitish.

Somewhat crespitose. Woods, chiefly pine, amongst moss. Autumn. $\mathbf{I} \frac{5}{5} \times I_{\frac{1}{2}}^{\frac{1}{2}} \times \frac{3}{16}$ in. 
369. 0. maura Gill. (from its swarthy appearance; maurus, a Moor) $a b$.

$P$. plane, striate, deeply umbilicate, sooty-brown. St. sometimes rooting, livid to dark brown. $G$. white.

Odour of new meal. Lawns and moist places. Oct.-Nov. $1 \frac{3}{4} \times 1 \frac{1}{2} \times \frac{1}{8}$ in. Nust not be confounded with 277 .

370. 0. offuciata Gill. (from its appearance; offucic, paint) $a b$.

$P$. deeply depressed, rarely plane, with a small papilla, tan to brownish or flesh-colour. St. colour as P., brown within at base. $G$. colour as P.

Solitary or slightly fascieulate. Under beeches. Oct.-Nov. $1 \frac{3}{8} \times 1 \frac{3}{8} \times \frac{1}{8}$ in. The salmon-colour of the gills must not cause this to be confounded with Series II. Hyporhodii.

\section{b. Pjixidate.}

371. 0. chrysophylla Gill. (from the golden-yellow gills; Gr. chrusos, gold, phullon, a leaf) $a b$.

$P$. plane, broadly umbilicate, ochreous-rufescent, tan or greyhoary when dry. St. colour as G.

Gregarious. Decayed pine, stumps, chips, sawdust. Aug.-Oct. $2 \frac{1}{4} \times 2 \times \frac{3}{16}$ in. The colour of gills and stem must not cause this to be confounded with Flammula.

372. 0. Postii Karst. (after H. von Post) $a b$.

$P$. plane, broadly umbilicate, striate, bright orange or rufescent. St. light yellow to whitish. $G$. whitish to grey-white.

Charcoal beds, swampy places. July-Oct. $2 \frac{1}{8} \times 2 \frac{1}{4} \times \frac{1}{8}$ in. The var. aurea Mass. bright golden-yellow, anongst Sphagnum in swamps.

373. 0. pyxidata Quél. (from the shape of the pileus; pyxis, a small box) $a b c$.

$P$. infundibuliform, striate, rufous-fuscous to umber or pallid. St. colour as P. G. white to brown. Flesh pale brown.

Lawns and woods amongst grass and moss; frequent. July-Nov. $1 \frac{1}{2} \times 1 \frac{3}{8} \times \frac{1}{8}$ in. Sometimes $\frac{1}{5} \times \frac{1}{4}$ in.

374. 0. leucophylla Gill. (from the white gills; Gr. lcukos, white, phullon, a leaf) $a b c$.

$P$. plane, broadly depressed, at length subrimose, dark ashy to pale or dark umber. St. colour as P., usually white at top and bottom.

Taste insipid ; odour somewhat strong. Woods, moist places, amongst short grass. April-Sept. $\quad 1 \frac{5}{8} \times \mathrm{r} \frac{3}{4} \times \frac{1}{8} \mathrm{in}$.

375. 0. striæpileus Gill. (from the striate pileus) a b c .

$P$. plane, depressed, rarely with a small papilla, livid olivaceous or brownish; mid. brown. St. pale livid umber, becoming fuscous. $G$. whitish.

Woods, amongst moss and grass. Oct.-Nov. $1 \frac{3}{8} \times 1 \frac{1}{2} \times \frac{1}{5}$ in. 
376. 0. telmatiæa Sacc. (from its habitat; Gr. telmatiaios, occurring in marshy places) $a b$.

$P$. infundibuliform, minutely virgate, dark brown or fuscous to livid. St. slate or tan flesh-colour. $G$. livid or pale brown.

On Sphagnum. Aug. $2 \frac{3}{8} \times 1 \frac{1}{2} \times \frac{1}{4}$ in. (Agaricus affricatus $\mathrm{B}$. \& Br., not Fr.)

376a. 0. epichysium Quél. (from a fancied resemblance to an ancient Greek wine-vessel, epichusis).

$P$. membranous, plane, umbilicate, livid umber, pale livid-reddishumber, or mid. slate-lavender and marg. dull salmon, silky floccoso-squamose and pallid when dry; marg. striate when moist. St. somewhat hollow, glabrous, ashy, grey or slate, dull salmon above and below. $G$. plano-decurrent, ashy or salmon-brown-white.

On wet rotten wood, rarely in bogs. Autumn. $\mathbf{I} \frac{1}{8} \times \mathbf{I} \frac{3}{4} \times \frac{1}{10}$ in. Wholly soft and watery.

377. 0. sphagnicola Karst. (from its habitat; sphagntum, bog-moss, colo, to inhabit) $a b c$.

$P$. infundibuliform, substriate, brownish-tan or dull pale ochreous. St. tan-white or ashy. $G$. thick, colour as P. or whitish.

In swamps, on Sphagnum acutifolium; uncommon. June-Sept. $\mathrm{I}_{\frac{1}{4}} \times$ $1 \frac{5}{8} \times \frac{1}{8}$ in.

378. 0. philonotis Quél. (Gr. philos, loving, notis, moisture) a. Smoky-ashy or brown-white.

$P$. infundibuliform, fragile. St. floccose at base.

On Sphagnum, swampy places in fir-woods. Aug. $1 \frac{1}{4} \times 2 \frac{3}{8} \times \frac{1}{8}$ in. Fries says this is one of the vernal species, with 377 and 380 .

379. 0. onisea Gill. (from its colour, like a codfish; Gr. oniskos) a $b c$. Dark ashy to pale umber, becoming grey-hoary.

$P$. broadly umbilicate, striate. $G$. shortly decurrent.

Not cæspitose. Woods, swampy places; rare. Dec. $1 \frac{1}{8} \times \frac{7}{8} \times \frac{1}{8}$ in.

379a. 0. Luffii Mass. (after Mr. John Luff).

$P$. convex, undulate, then depressed, even, glabrous; marg. usually upturned at extreme edge, pallid, white when dry. St. solid, thickened above, cartilaginous, often crooked, glabrous, polished, pallid. $G$. decurrent, crowded, colour as St. Flesh very thin, white.

Gregarious. Odour fragrant, spicy, like 219. $1 \frac{1}{1} \times \frac{1}{4} \times \frac{1}{12}$ in. Allied to 379.

380. 0. eæspitosa Sacc. (from its cæspitose habit) $a b c$.

$P$. hygrophanous, plano-depressed, livid buff, yellowish-white or olive-shaded, at length white; marg. crenate, sulcate. St. slightly enlarged below, colour as P. G. very distant, sometimes branched, whitish or yellowish-buff.

Moors, by boggy water-courses under pines, damp peaty soil. May-Oct. $\mathbf{I} \frac{1}{4} \times \mathbf{I} \times \frac{1}{8}$ in. Whole plant sometimes becomes bright yellow in drying. 
381. 0. glaucophylla Gill. (from the olive-grey gills; Gr. glaukos, the bluish-grey of the olive, phullon, a leaf) $a c$.

$P$. plano-umbilicate, mouse-colour; marg. striate. St. colour as $\mathrm{P}$.

Woods, mossy places. Sept. $\frac{1}{2} \times \frac{5}{8} \times \frac{1}{16}$ in. Pileus sometimes $\frac{7}{8}$ in. in diameter.

382. 0. rustica Quél. (from its habitat; rus, the country) $a b$.

$P$. plano-umbilicate, fuscous to grey, or whitish-umber, shaded lavender; marg. striate. St. colour as P., or dull rather pale lavender. $G$. thick, olivaceous or olive-grey.

Woods, grassy avenues, heathy ground, charcoal heaps. Aug.-Sept. $\frac{1}{2} \times \frac{1}{2} \times \frac{1}{16}$ in.

382a. 0. alutacea Sacc. (from its colour; aluta, tanned leather) $a b$. Tan-colour, or dull pale ochreous.

$P$. membranous, convex, then umbilicate, smooth ; marg. incurved, even. St. hollow, smooth, paler than P. $G$. somewhat narrow, crowded, arcuate, colour as St.

Amongst grass and moss. Sept. $\frac{3}{4} \times \mathrm{I}_{\frac{3}{4}} \times \frac{1}{16}$ in.

\section{c. Umbellifere.}

383. 0. demissa Karst. (dcmissus, drooping) a b.

$P$. infundibuliform, fuscous-rufescent to tan-brown. St. liverrufescent to pale tan-brown. $G$. becoming purple.

Waste places, banks in woods, burnt wood; rare. Aug.-Oct. $\frac{5}{5} \times \frac{1}{2} \times \frac{1}{16}$ in. Agaricus mufulus B. \& Br. Not unlıe some forms of 224 .

384. 0. hepatica Gill. (from its colour; Gr. hepar, the liver) a b c. $P$. infundibuliform, tough, rufous flesh-colour to tawny and tan. St. fuscous flesh-colour. $G$. whitish.

Lawns, mossy pathsides in woods, etc. ; rare. Nov.-Dec. $1 \frac{1}{4} \times 1 \frac{3}{8} \times \frac{1}{8}$ in. Often in company with 373.

385. 0. muralis Quél. (from one of its habitats; murus, a wall) a bc. $P$. infundibuliform, rufous-brown to tan-umber; marg. striate. St. paler than P. G. brownish-white.

Old mossy walls, amongst moss in woods, on turf walls, sanily banks, etc. Jan. - Nov. $\quad 1 \times \frac{5}{8} \times \frac{1}{8}$ in. Sometimes only $\frac{1}{5} \times \frac{1}{4}$ in. There is a white var. Several other species of Omphalia occur on old mossy walls.

386. 0. umbellifera Quél. (from the umbrella-like pileus; umbilla, an umbel or umbrella, fero, to bear) $a b c$.

$P$. plano-depressed, deep brown to ochreous-whitish; marg. striate. St. slightly attenuate downwards, colour as P. G. grey-whitish, umber or dusky flesh-colour, edge whitish.

Taste none; odour at first somewhat disagreeable, evanescent. Boggy ground, stumps, rotten wood, old walls, heathy banls, etc. Mar.-Dec. $\frac{7}{8} \times \frac{7}{8} \times \frac{1}{16}$ in. Var. abiegna Mass. gruws on firwood. Var, ziridis sacc. is verdigris-green. There is also a wholly deep yellow variety. 
386a. 0. velutina Quél. (vilutinus, velvety) $a$.

$P$. convex, umbilicate, striate, greyish or yellowish-grey. St. solid, colour as P., white at base. $G$. arcuate, wide, distant, colour as P. Flesh dark grey.

In heaths. Sept. $\frac{1}{2} \times 1 \frac{1}{8} \times \frac{1}{2}$ in. Has characters between 386 and 391 .

387. 0. infumata Sacc. (from its colour; infumo, to smoke) $a b$.

$P$. obtuse, at first green. St. dilated at base, tomentose, yellow. $G$. yellow.

Moss on bark. Autumn. $\frac{5}{16} \times 1 \frac{3}{8} \times \frac{1}{2}$ in. Colours of 350 .

388. 0. retosta Gill. (from its appearance; retostus, scorched) a $c$. Umber within and without.

$P$. plano-depressed. St. equal or attenuate below. $G$. somewhat darker than flesh.

Lawns, etc., amongst dead leaves. Oct. $1 \frac{1}{8} \times 1 \times \frac{1}{8}$ in. Firmer than 386.

389. 0. abhorrens Sacc. (from its fetid odour) $a$.

$P$. plano-depressed, fuscous to warm ochreous. St. brown-ochre above, dark brown below. $G$. colour as P.

Crespitose. Odour dung-like. On lawns, under yews. Oct. $\frac{1}{2} \times \frac{3}{4} \times \frac{1}{32}$ in. A close ally of 388 ; much like 1411.

390. 0. pseudoandrosacea Gill. (from its resemblance to 1420 Marasmius androsacius Fr.; Gr. pseudos, false) a c. White, whitish-grey, or umber-white.

$P$. plano-depressed; marg. striato-plicate, crenulate.

Mossy lawns. Oct.-Nov. $\frac{1}{2} \times 1 \frac{1}{8} \times \frac{1}{16} \mathrm{in}$.

391. 0. griseopallida Quél. (from its colour; griscus, grey, fallidus, pallid) $a b c$.

$P$. infundibuliform, squamulose, fuscous-grey or pale greyishumber; marg. sometimes slightly striate. St. paler than P. $G$. fuscous- or slate-grey.

On the ground, gardens. Sept.-Oct. $\frac{7}{5} \times \frac{5}{8} \times \frac{1}{5}$ in.

392. 0. stellata Quél. (from the star-like, radiate base) $a b c$. White. $P$. plano-depressed, diaphanous-striate.

In woods, on sticks, decayed stems of herbaceous plants, wood ; uncommon. Feb.-Nov. $\frac{5}{16} \times I \times \frac{1}{6 \pm}$ in. Must not be confounded with the Basipedes section of Mycena.

\section{Mycenarie.}

\section{d. Campanclla.}

393. 0. campanella Quél. (from the shape of the pileus; campanclla, a little bell) a b $c$.

$P$. campanulato-plane, umbilicate, yellow-ferruginous, orange or brown. St. brown, usually dark strigose at base. $G$. dull ochre.

Crespitose. Woods, pine, trunks. Aug.-Nov. $\frac{3}{4} \times 1 \frac{1}{2} \times \frac{1}{16}$ in. Sometimes completely covering stumps. There is a var. with a small acute papilla in the umbilicus. 
394. 0. picta Gill. (from its varied colours ; pictus, painted) $a$.

$P$. hood-shaped, subdepressed, fuscous; marg. striate; mid. yellow or brown. St. horny, brown; blackish at strigose base. $G$. adnate, broader than long, subdecurrent, whitish or yellowish.

Woods, mixed, on sticks, chips, dead wood, sawdust, etc. Aug.-Oct. $\frac{3}{8} \times 1 \frac{1}{4} \times \frac{1}{16}$ in.

395. 0. eamptophylla Sacc. (from the curved gills; Gr. kampto, to curve, phullon, a leaf) $a b$.

$P$. convexo-depressed, brown or brown-white; marg. striate, grey. St. yellow to white above, rufescent to pale brown below. $G$. adnate, subdecurrent, white.

Sticks, chips, etc.; rare. Aug.-Oct. $\frac{1}{2} \times 2 \times \frac{1}{32}$ in.

396. 0. umbratilis Gill. (from its growing in the shade; umbratilis, remaining in shade) $a c$.

$P$. convexo-plane, broadly depressed, black-fuscous, hoary when dry. St. fuscous-dead-black. $G$. adnato-decurrent, becoming fuscous-white.

Gregarious. Amongst grass. Autumn. $\frac{7}{8} \times \frac{7}{3} \times \frac{1}{16}$ in.

397. 0. grisea Quél. (griscus, grey) a b 6 .

$P$. conico-campanulate, subumbonate, then expanded and umbilicate, livid grey to umber, becoming hoary. St. attenuate downwards, longitudinally cracking, ashy-whitish. $G$. sinuatodecurrent, whitish-grey, dull lavender with a white edge or livid umber.

Taste and odour somewhat unpleasant. Woods, pine, mixed, dead sticks, leaves; rare. Sept.-Nov. $\frac{7}{8} \times 1 \frac{3}{4} \times \frac{1}{16}$ in.

398. 0. fibula Quél. (from its shape; fintla, a pin) a $b c$.

$P$. plano-depressed, sometimes papillate, orange-yellow or vermilion. St. colour as P. $G$. decurrent, yellow or orange. Flesh orange.

Mossy ground, shady banks, moist woods, moss, sedge, grass, stumps, etc. Mar.-Dec. $\frac{1}{2} \times I_{\frac{3}{2}}^{\frac{3}{2}} \times \frac{1}{32}$ in. Var. Swartsii Karst. $P$. $\tan$ or white-purple; mid, deep purple-brown. The variation from orange to purple agrees with 224 and its purple form. There is a white variety.

399. 0. directa Sacc. (from the long hairs at right angles to the stem ; directus, straight) a $c$. White.

$P$. plano-depressed. St. sometimes faintly rufous. $G$. subdistant, decurrent.

Dead leaves. May-Nov, $\frac{1}{16} \times \frac{3}{4} \times \frac{1}{1 \frac{1}{5}}$ in.

400. O. PSEUDODIRECTA W. G. Sm. (from its being mistaken by Dr. M. C. Cooke for 399 ; Gr. pseudos, false) a b.

$P$. plano-depressed, white-pruinose. $S t$. white, mealy-granular below, springing from a white floccose disc or volva which becomes obsolete as the stem lengthens. $G$. few, adnate, white then saffron, pruinoso-sparkling.

On Encephalartos cone at Messrs. Veitcl's nursery, Chelsea. May. $\frac{1}{15} \times \frac{1}{2} \times \frac{1}{12}$ in. Stems bristle-like, often non-pileate. 
401. 0. Belliæ Karst. (after the Misses Bell of Coldstream) a $b$.

$P$. infundibuliform, pale livid-brown; marg. waved and furrowed. St. whitish above, pale brown below, springing from a slightly enlarged, floccose base. $G$. wrinkled, veined, white.

Dead reeds. Oct. $\frac{1}{2} \times 1 \frac{3}{4} \times \frac{1}{16}$ in.

401a. 0. graeilis Quél. (gracilis, slender) a. Wholly white.

$P$. membranous, campanulate, papillate, striate, smooth, transparent. St. filiform, fistulose, transparent, pruinose, fibrillose at base. $G$. distant, very decurrent, thin.

Dead grass. Oct. $\frac{1}{5} \times 1 \frac{1}{2} \times \frac{1}{30} \mathrm{in}$.

402. 0. gracillima Quel. (gracillimus, very slender) $a b$. White.

$P$. convex, subumbonate then plane and umbilicate. St. springing from a floccose base. $G$. fold-like, disappearing short of marg. of P.

Scattered. Dead sticks, herbaceous stems. Ang. $\frac{5}{16} \times \mathbf{I} \times \frac{1}{32}$ in.

403. 0. bullula Sacc. (from its watery appearance; lullulla, a watery vesicle) a. White.

$P$. hemispherical, diaphanous.

Scattered. Dead sticks, twigs. Autumn. $\frac{7}{ \pm} \times \frac{5}{5} \times \frac{1}{3} 5$ in.

$$
\text { e. Integrilla. }
$$

404. 0. integrella Quél. (from its perfect structure; integer $r$ whole) a b c. White.

$P$. hemispherical, depressed or plane. St. pilose downwards to a minute villous disc at base. $G$. adnato-decurrent disappearing short of marg. of $P$.

Commonly fasciculate. Shady places, woods, on decayed sticks, rotten wood, grass, twigs, etc. May-Oct. $\frac{3}{16} \times \frac{7}{8} \times \frac{1}{32}$ in.

405. 0. buccinalis Sacc. (from its trumpet-shaped pileus; buccina, a trumpet) $a$. White.

$P$. fleshy, plano-depressed. St. with a stellate disc at base. $G$. vein-like as in Cantharellus.

Twigs, stems, etc. ; common. $\frac{1}{4} \times \frac{7}{8} \times \frac{1}{32}$ in. Sometimes branching as in 1437 on a small scale.

406. O. Neville Sacc. (after Lady Dorothy Neville) $a$.

$P$. hemispherico-depressed, granulate, striate, brown becoming pale at marg. St. very thin, brown, rough with black gr., villous, subdilated at base. $G$. veined at sides and interstices.

On Sphagnum in orchid-pot. $\frac{1}{2} \times 1 \times \frac{1}{16}$ in.

\section{PLEURotuS Quél.}

(From a fancied resemblance in many of the species to an ear; Gr. pleuron, a side, ous, an ear.)

Veil universal, seen in the squamules, scurf and appendiculate margin of the pileus and in the fugitive annulus, sometimes developed 
as gluten, or obsolete or nearly so. Hymenophore confluent and homogeneous with the fleshy stem, when present. Pileus usually excentric, compact, spongy to fleshy or membranous, sometimes resupinate. Stem usually excentric, lateral, annulate, simple or

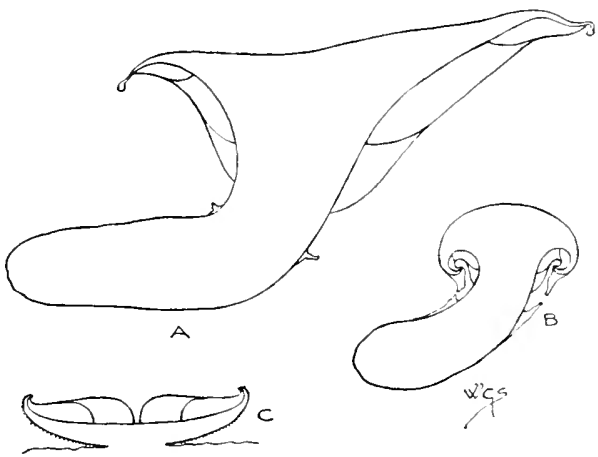

Fig. 20.-A, section of Flcurotus dryinus Quél. One-third natural size. $B$, young state, section, showing veil; $c$, section of $P$. applicatus Quél. $\times 2$.

none; when present without cartilaginous bark; when the largerstemmed species grow vertically on prostrate trunks, the stem is sometimes central or nearly so. Gills sinuate, adnate or decurrent, in some of the Resupinatie often starting from an excentric point. Stores more or less elliptical, smooth. (Fig. 20.)

The majority of the species grow on trunks, branches, twigs, moss, etc., a few are terrestrial; many are large, handsome and polymorphic; some are small and resupinate. They generally appear late in the year, 421 and 422 commonly grow in the spring and reappear year after year on the same habitat. All are harmless, and a few are considered by some to be edible. All become putrid when old, and none are leathery or woody-in this they differ from members of Lentimus, Pamus, Xerotus, Trogia, Schizophyllum and Lenzites.

Species $407-448$

a. Excentrica. Pileus laterally extended; stem excentric.

$V$ til forming an annulus to the stem and hanging as a ragged fringe at the margin of the pileus.

$407-409$

I cil obsolete. Gills sinuate or obtusely adnate. $\quad 410-417$

Veil obsolete. Stcm distinct, somewhat vertical. Gills deeply decurrent.

$418-420$

Vill obsolete. Pilius lateral, sessile or extended behind into a short, oblique, stem-like base. Gills deeply decurrent.

$421-425$

b. Dimidiatc. Pileus definitely lateral, immarginate behind, not at first resupinate. 
c. Resupinatic. Pilius at length reflexed, sessile. Gills starting from an excentric point.

Pilius fleshy, uniform.

436,437

Pilcus fleshy, striate, with an upper gelatinous stratum or viscid pellicle.

Pilcus membranous, not viscid.

$438-444$

$445-448$

\section{a. Excontrice.}

407. P. corticatus Quél. (from the thick covering of the pileus and stem; cortex, bark) a b.

$P$. fleshy, floccose, pale buff and grey. St. squamuloso-fibrillose white to pale buff. $A$. silky-floccose. $G$. decurrent, anasto mosing near St., yellowish when old. Flesh white or faintly yellowish.

Solitary or crespitose. Tasteless; odour pleasant, rather strong. Trunks, elm, ash, apple, etc. Sept.-Oct. $9 \times 3 \frac{1}{2} \times 1 \frac{3}{8} \mathrm{in}$.

408. P. dryinus Quél. (from its frequent habitat; Gr. drus, oak) $a b c$. $P$. fleshy, plane, whitish with fuscous sc.; mid. darker. St. white or yellowish-white, fuscous at base. $A$. floccose. $G$. decurrent, white, yellowish when old.

Taste pleasant, mushroom-like. Trunks, oak, elm, apple, willow, walnut, ash, poplar. Sept. - Feb. $6 \times 4 \times 1 \frac{1}{3}$ in.

409. P. spongiosus Sacc. (from its spongy substance) $a b c$.

$P$. pulvinate, subumbonate, downy, white, tan or ashy. St. whitetomentose, sometimes almost obsolete. $A$. evanescent. $G$. decurrent, anastomosing on stem, whitish.

Stumps, beech, apple, poplar, hombeam; rare. Oct.-Nov. $3 \frac{1}{2} \times 1 \frac{1}{4} \times \frac{5}{8}$ in. Solt and spongy, shrinking to almost half its original size in drying.

410. P. ulmarius Quél. (from its usual habitat; ulmus, elm) $a b c$. Whitish.

$P$. pulvinate, broadly umbonate, smooth. St. villous at base, rarely central. $G$. adnate.

Solitary or crespitose. Saicl to be edible. Odour mealy or shightly acid. Trunks, elm, wych elm, beech, oak, poplar, willow, sometimes parasitic on 1587. Sept.-Dec. $6 \frac{1}{2} \times+\times \mathrm{i}_{\frac{3}{4}}$ in. Sometimes proliferous, with many small growths at base of stem.

411. P. tessulatus Gill. (from the tesselated markings of the pileus) a.

$P$. convex, subumbonate, pale tawny, brown-tesselated. St. smooth, white. $G$. sinuate, white or faintly brownish.

Solitary or cespitose. Odour of new meal. Trunks. $4 \frac{1}{\frac{1}{1}} \times 2 \times \frac{5}{5}$ in.

412. P. subpalmatus Gill. (from a fancied resemblance to the shape of the hand) $a b c$.

$P$. convex, with a thick, gelatinous, reticulato-corrugate pellicle, rufescent-salmon. St. whitish above, salmon below. $G$. sinuate with a decurrent tooth or adnato-rounded, sometimes adnate with a collar, paler than P. Flesh salmon-streaky.

Taste bitter-aromatic. Squared timler, trunks, etc., willow, elm, oak; rare. Sept.-Nov, $4 \frac{1}{2} \times 2 \frac{1}{2} \times \frac{1}{2} \mathrm{in}$. 
413. P. craspedius Gill. (from the thick stem; crassus, thick, pes, a foot) $a b$.

$P$. pulvinate or subumbonate, pale buff; marg. crenato-lobed. St. and Flesh white. $G$. rounded or adnate.

Cæespitose. Strong odour of cucumber. Trunks, rotten wood, poplar. Sept.-Oct. $4 \frac{3}{8} \times 2 \times 1 \frac{3}{8}$ in.

414. P. fimbriatus Gill. (from the fringed margin of pileus; fimbrice, fringe) $a$.

$P$. infundibuliform, hygrophanous, whitish; marg. lobed and reflexed. St. central or lateral, somewhat attenuate downwards, whitish. $G$. adnato-ascending, narrow, thin, crowded, white. Flesh remarkably thin.

Gregarious, somewhat cæspitose. Trunks, dead; rare. Aug. -Jan. $4 \frac{3}{8} \times 1 \frac{5}{8} \times \frac{1}{4}$ in. Habit not unlike Lentinus.

415. P. Ruthæ Sacc. (after Ruth Berkeley) a.

$P$. plano-depressed, fan-shaped, tan-whitish; marg. striate. St. lateral or excentric, salmon-reddish. $G$. adnato-decurrent, veined, paler than St.

Sawdust. Oct. $2 \frac{3}{8} \times \frac{5}{8} \times \frac{7}{4}$ in. Sometimes twisted and contorted like 1158, for the white variety of which with similar habitat it might be readily mistaken.

416. P. lignatilis Gill. (from its habitat and hardness; lignum, wood) a $b c$. Whitish-tan.

$P$. convexo-plane, slightly depressed, flocculoso-pruinate; mid. pale buff. St. stuffed, woody, pruinato-villous. G. adnate or sinuate. Flesh whitish, faintly sienna at base of stem.

Cæspitose or single. Otlour strong of meal. Beech, growing on 1532 and 1593. $3 \times 2 \frac{1}{2} \times \frac{1}{2}$ in. A variety has $P$. ashy with mid. black and marg. white.

417. P. circinatus Gill. (from the orbicular pileus; circino, to make round) $a b c$. White.

$P$. plane, silky-lustrous. St. smooth, attenuate downwards. $G$. subdecurrent.

Odour weak and pleasant, not mealy. Rotting stumps, birch. Sept.-Oct. $2 \frac{5}{8} \times 1 \frac{3}{4} \times \frac{3}{16}$ in.

418. P. sapidus Sacc. (from its taste ; sapidus, savoury) a b $c$.

$P$. plano-depressed, fleshy, smooth, white, pale ochreous, brown or lavender shaded. St. connato-branched, smooth, white. $G$. subdistant, reticulate on stem, white. Spores pale lilac.

Cæspitose. Trunks, elm. July-Aug. $4 \frac{1}{ \pm} \times 2 \frac{1}{\frac{1}{4}} \times \frac{5}{8}$ in.

419. P. pantoleucus Gill. (from its colour; Gr. pas, all, ienkos, white) a b c .

$P$. convex, dimidiate, spathulate, smooth. St. excentric. $G$. broad.

Solitary. Trunks, branches, willow. Oct. $4 \frac{1}{ \pm} \times 2 \frac{1}{\frac{1}{1}} \times \frac{5}{8}$ in. Sometimes small with a depressed pileus. 
420. P. mutilus Gill. (from its being changeable in form; mutilus, maimed) $a c$. White.

$P$. excentric, lateral, reniform or spathulate, depressed, silkysmooth. St. excentric or lateral with a villous base.

Dead wood, rotting elm, fir-plantations, on a grassy hillside. Aug.-Dec. I $\frac{5}{8} \times \frac{7}{8} \times \frac{1}{8}$ in. Might be mistaken for a small Clitocybe. Sometimes appears on mushroom-beds.

421. P. ostreatus Quél. (from the shape and colour of the pileus; ostrea, an oyster) $a b c$.

$P$. convexo-depressed, subexcentric, slate or brownish, when young sometimes almost black. St. connato-branched, excentric, rarely central, sometimes obsolete, white. $G$. reticulate below, whitish.

Crespitose-imbricate. Said to be edible. Odour strong. Trunks and logs, elm, poplar, walnut, beech, laburnum, apple, WVistaria; once on dry cerebral matter in skull of a stranded whale. Jan.-Dec. $4 \frac{5}{8} \times 2 \times \frac{7}{8}$ in. Var. columbinus Quél. has a purplish pileus and bluish or greenish marg. Var. glandulosizs IIass. has tear-like drops on the gills.

422. P. euosmus Sacc. (Gr. euosmus, sweet-smelling) $a b c$.

$P$. convexo-depressed, tan to olivaceous-brown. St. varying long, short or obsolete. $G$. reticulate below. Spores tan, lilac or salmon-shaded.

Cæespitose-imbricate. Said to be poisonous. Odour of tarragon. Stumps, logs, poplar, elm. April-Oct. $5 \frac{1}{4} \times 3^{\frac{1}{4}} \times \mathrm{I}$ in. Intermediate forms between this and 421 occur.

423. P. revolutus Gill. (from the ultimately revolute margin of the pileus) $a$.

$P$. plane, smoky, lead- or mouse-colour; mid. darker. St. whitish. $G$. serrulate, whitish.

Solitary or subcrespitose. Trunks, poplar, beech. Autumn. IO $\frac{1}{2} \times 2 \frac{1}{4} \times 2$ in.

424. P. salignus Quél. (from one of its habitats; Salix, willow) a $b c$. $P$. pulvinate, plane, ochreous, smoky slate-colour or almost black. St. almost obsolete, tan. Spores dull whitish.

Solitary, rarely crespitose or imbricate. Trunks, willow, ash, elm, sycamore, alder. Sept.-Feb. $5 \frac{1}{2} \times \frac{1}{2} \times \mathbf{I}_{\frac{1}{4}}$ in.

425. P. acerinus Gill. (from one of its habitats; Acer; maple) $a b$. White.

$P$. tough, fleshy, thin, silky-villous, pale rufous when dry. St. sublateral or obsolete, villous. $G$. very crowded, thin, white then yellow; livid-vinous when dry.

Trunks, maple, asb, plane. Autumn. $3 \times \mathbf{I} \frac{1}{8} \times \mathbf{I}$ in.

\section{b. Dimidiata.}

426. P. petaloides Quél. (from a fancied resemblance to a leaf; Gr. petalon, a leaf, eidos, appearance) a $b c$.

$P$. plane, subumbonate, fuscous, brown, ochreous or purplish. St. sublateral, villous, whitish. $G$. decurrent, white, yellowish or ashy.

Gregarious, crespitose or imbricate. Taste bitter. Terrestrial and on stumps, pine; rare. Aug.-Jan, $3 \frac{3}{4} \times I \times I$ in. 
427. P. pulmonarius Quél. (from its lung-like texture; pulmo, a lung) $a c$.

$P$. plane, smooth, ashy or rufous. St. lateral, whitish. $G$. decurrent or adnato-decurrent, whitish or ashy.

Solitary, rarely cæspitose. Trunks, beech. Sept. $2 \frac{5}{3} \times \frac{3}{4} \times \frac{1}{2}$ in. Var. juglandis Karst. grows on walnut.

428. P. serotinus Gill. (from its late appearance: serotinus, late) a b $c$. $P$. reniform, olivaceous. St. lateral, brilliant deep yellow, squamulose, spotted near gills, somewhat rich brown. $G$. crowded, sinuato-decurrent, cream ochre to bright yellow or orange.

Taste insipid; odour apple-like. Trunks, posts, ash, birch, hornbeam, alder, beech; rails. Oct.-Dec. $2 \times \frac{3}{4} \times \frac{1}{2}$ in.

429. P. mitis Quél. (from its taste; mitis, mild) $a b c$.

$P$. reniform, gristly, without a viscid pellicle, pale flesh-colour or tan. St. lateral, minutely scaly, whitish flesh-colour or tan. $G$. somewhat rounded, white.

Taste and odour almost obsolete. Dead trunks, sticls, fir, larch. Oct.Dec. $\frac{7}{8} \times \frac{3}{8} \times \frac{3}{8}$ in. Sometimes $I_{\frac{3}{4}}^{3}$ in. in diam. Somewhat like 1444 in general appearance.

429a. P. rufipes Sacc. \& D. Sacc. (from the reddish stem; milus, red, $p^{\prime}$ 's, a foot) $a b$.

$P$. dimidiate or reniform, convex, membranous, very glutinous when moist, white; mid. salmon; marg. incurved. St. becoming recurved, vinous-reddish, salmon-red within, white in centre, base white-downy. $G$. adnate, broad, distant, white, interstices pale salmon.

On wood. Autumn. $\frac{1}{8} \times \frac{3}{16} \times \frac{1}{40}$ in.

430. P. GADinioldes Sacc. (from its resemblance to the bivalve Gadinia; Gr. eidos, appearance) a b c. White.

$P$. convex, hygrophanous, smooth or clothed with adpressed flocci, no gelatinous upper stratum. St. minute, lateral or none. $G$. adnate, slightly branched.

Tree-fern stems. IIay. Diam. $\frac{3}{4}$ in. Reniform as seen from below.

431. P. limpidus Gill. (from its pellucid substance; limpidus, clear) $a b c$.

$P$. reniform, plane, subumbonate, whitish. St. sublateral, rudimentary or obsolete. $G$. sinuato-decurrent, white or strawwhitish.

Taste mild. Stumps, ash, willow, beech. Dec. Diam. $1 \frac{1}{2}$ in.

432. P. reniformis Karst. (from the kidney-shaped pileus; renis, a kidney, forma, form) a.

$P$. plane, whitish, or greyish-brown. St. lateral, rudimentary. $G$. sinuato-decurrent, paler than P.

Branches, silver fir, on the ground amongst moss. Aug.-Oct. Diam. I in. 
433. P. Laurocepasi Sacc. (from its habitat, stems of cherry-laurel, Prumus Leturoceresus) a b.

$P$. convex, oyster-shaped, sulcate, pale brownish-salmon. St. obsolete. $G$. very broad, adnate, veined, salmon-white.

Oct. Diam. $\mathbf{I}^{\frac{3}{3}}$ in.

434. P. tremulus Quél. (from its tremulous habit) $a b c$.

$P$. reniform, depressed behind, fuscous-grey, pale slate or brown. St. attenuate downwards, grey. $G$. adnato-decurrent, whitishumber or greyish.

Solitary. On the ground, on moss, fungi, e.g. 1790, when growing on moss; rare. Aug.-Dec. $1 \frac{1}{4} \times \frac{3}{16} \times \frac{1}{1} \mathrm{in}$.

435. P. acerosus Quél. (from its habitat, amongst dead pine-leaves; acersosus, full of chaff or husk) $a b c$.

$P$. plane, reniform, membranous, whitish, brownish or greyish. St. faintly umber-whitish. $G$. adnate, branched, colour as P. or paler.

Taste insipid, somewhat disagreeable. Lawns, wood, gravel, leaves, pinewood, pine-leaves, Shluggnum; rare. Aug.-Dec. $1 \frac{7}{5} \times \frac{1}{1} \times \frac{1}{8}$ in. Resembles 1388.

\section{c. Resupinate.}

436. P. porpigens Gill. (from its stretched out habit; forrigo, to stretch out) a $b c$. White.

$P$. at length plano-depressed, undulate. $G$. thick, sometimes forming pores. Flesh thin, subcartilaginous.

Imbricate. Odour pleasant or insipid. Stumps, chiefly pine. June-Nov. Diam. $6 \frac{1}{2}$ in. Base villous.

437. P. septicus Quél. (from its habitat, rotten wood; scpticus, putrifying) a $b c$. White.

$P$. convex, villous. St. lateral, villous, at length disappearing. $G$. rounded-adnate, subdistant.

Decayed wood, branches, twigs, pine, bramble, straw, dung, fungi. Mar.Nov. $\frac{3}{8} \times \frac{1}{16} \times \frac{1}{16} \mathrm{in}$.

438. P. mastrucatus Sacc. (from its somewhat woolly coat; mastruar, a sheepskin) a $b c$.

$P$. ear-shaped, plane, squarrose-scaly, greyish- or whitish-brown. $G$. adnato-decurrent. Flesh mouse-colour.

Imbricate. Old trunks, logs, beech, amongst moss and lichens; rare. Diam. $3 \frac{3}{3}$ in.

439. P. atrocæruleus Gill. (from the colour of the pileus; ater, black, caruleus, azure-blue) a b c.

$P$. plane, reniform; marg. ochreous-sienna. $G$. whitish strawcolour. Flesh with an olive middle stratum.

Gregarious, subimbricate. Odour pleasant. Trunks, birch, elm, poplar, beech, etc. (Ict.-Dec. Liam. $1 \frac{5}{3} \mathrm{in}$.

440. P. Leightonii Sacc. (after the Rev. W. A. Leighton) $a b c$. $P$. at first obliquely conical, furfuraceous, umber or lead-colour. $G$. thick, distant, forked at base, pallid tan.

Wood, rotten rails. Dec. Diam. $\frac{1}{2}$ in. Might be mistaken for 444 . 
441. P. algidus Quél. (from its frequent growth in northern countries; algidus, cold) $a b c$.

$P$. plane, subreniform, pruinose when young, viscid, brown. $G$. adnate, becoming pallid yellow.

Usually cæespitose and imbricate. Trunks, mountain-ash, willow. Aug.Oct. Diam. $2 \frac{1}{8}$ in.

442. P. fluxilis Gill. (from the gelatinous, upper stratum of the pileus; fluxilis, fluid) a.

$P$. plane, reniform, umber. $G$. adnato-rounded, whitish. Fles/ a gelatinous stratum without a membrane.

Solitary. Wood, amongst moss on trunks, sawdust, mosses. Oct. Diam. $\frac{1}{8}$ in. Not usually resupinate.

443. P. cyphellæformis Sacc. (from its resemblance to a Cyphclla) a bc.

$P$. cup-shaped then conico-dependent, greyish or brownish. $G$. radiating from a central point, white. Flesh, upper stratum dark brown, lower white.

Gregarious. Dead herbaceous stems, Faniculum, Efilobium; rare. Feb.Oct. Diam. $\frac{3}{16}-\frac{3}{8}$ in.

444. P. applieatus Quél. (from its close attachment to branches, etc.; applicatus, closely attached) a $b c$.

$P$. cup-shaped, then expanded or reflexed, dark grey. $G$. radiating from a central point, lighter than $P$.

Dead branches, twigs, bramble, oak, ash, pine, Ribes, Vitis. Sept.-June. Diam. $\frac{3}{8}$ in. Sometimes proliferous.

445. P. Hobsonii Sacc. (after Lieut. Julian C. Hobson) $a$.

$P$. plano-convex, reniform, downy, pale grey. $G$. subdistant, adnate, pallid.

Stumps, larch. Sept. Diam. $\frac{3}{8}$ in.

446. P. striatulus Quél. (from the slightly striate pileus) a $c$.

$P$. cup-shaped, not usually reflexed, fuscous or grey. $G$. few, radiating from a central point, lighter than $P$.

Gregarious. Stumps, twigs, etc., fir, hazel, elm. May-Dec. Diam. $\frac{1}{4}$ in.

447. P. hypnophilus Sacc. (from its frequent habitat, $H_{y}$ m $и m$; Gr. philos, loving) ac. White.

$P$. at length plane, subreniform. St. villous, at length vanishing. $G$. narrow, radiating from a central point.

On the larger mosses, leaves, leaves and stems of grass. Sept.-Dec. Diam. $\frac{3}{8}$ in. Resembles 553 in habit.

448. P. chioneus Gill. (from its colour ; Gr. chion, snow) $a b c$.

$P$. subresupinate, convex, villous. St. lateral, villous, at length vanishing. $G$. very distant, broad, radiating from a central point.

Wood, decorticated elder, leaves, dung, bone ; rare. Sept.-Dec. $\frac{1}{4} \times \frac{1}{32} \times$ $\frac{1}{3^{2}}$ in. 


\section{SERIEs II. HYPORHODII Fr.}

(From the rose-coloured gills; Gr. hupo, beneath, rhodon, a rose.)

Spores white when young, then pale rose-colour, salmon, yellowishsalmon, salmon-whitish, nankeen, whitish-vinous or salmon-brownish, never lilac, elliptical and smooth or globose or nodulose and angularly warted.

There is no analogue of Amanita amongst the British Hyporhodii, but an analogous genus, Metraria, occurs in Australia.

It is remarkable that Sowerby has modelled an example of $\mathbf{4 5 0}$ with an ample annulus; if correct this plant would be a Metraria.

Genera XII-XX。

\section{VOLVARIA Quél.}

(From the volva.)

Veil universal, entirely enveloping the young plant. Hy'menophore distinct from the fleshy stem. Stcm central, simple, solid, volvate,

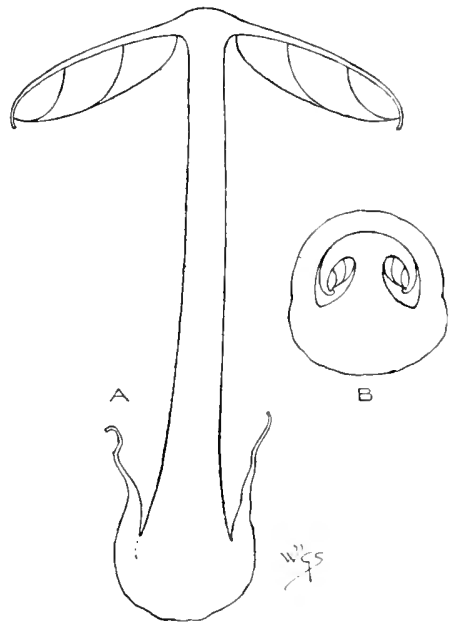

Fig. 21.-A, section of lotaratia voluacea Quél. one-third natual size; $B$, of young plant showing universal veil; half natural size.

without cartilaginous bark. Gills free, at first white, then rose or salmon, sometimes becoming brownish. Spores smooth, salmon or rose-colour. (Fig. 2 I.)

All the species are beautiful, some appear in spring and early summer. They grow in gardens, hothouses and pastures; and on dung, manured ground and rotten wood; 451 grows on the pileus 
of 153. They are almost all tasteless or insipid; 455 is fetid and said by Letellier to be poisonous. None are known to be edible.

Volvaria agrees in structure with Amanitopsis, Acetabularia and Chitoniar.

Pileus dry, silky or fibrillose.

Species $449-457$

Pilens more or less viscid, smooth.

449. V. bombycina Quél. (from the silky pileus; bombyx, silk) $a b c$. $P$. broadly umbonate, white or pale brownish. St. colour as $\mathrm{P}$. $V o$. large, lax, ochre-sienna or umber. $G$. becoming rufous.

Solitary or cæspitose. Decayed wood, stumps, hollow trees, ash, elm, birch. June-Sept. $8 \frac{1}{8} \times 7 \times \frac{3}{4}$ in.

450. V. volvacea Quél. (from the large volva) a $b c$.

$P$. campanulate, subumbonate, black-fibrillose on a brown ground or brown-fibrillose on a white ground. St. white. Vo. large, lax, umber.

Gregarious. Reputed poisonous. Stoves, on tan, roadsides. $5 \times 7 \times \frac{1}{2}$ in. Sowerby has modelled an example of this with a large annulus.

451. V. Loveiana Gill. (after the Rev. R. T. Lowe) $a b c$. $P$. convex, white-silky. St. white. Vo. lax, whitish. $G$. thick.

Gregarious, subcæspitose. On 153 and other species of Clitocybe. $2 \frac{7}{8} \times 2 \times$ $\frac{3}{8}$ in.

452. V. Taylorii Gill. (after Michael A. Taylor) $a b c$.

$P$. expanded, subumbonate, rimose, whitish; marg. faint umber. St. white. Vo. lax, small, white or umber. G. sinuato-free, irregular, attenuate near St.

Odour none. On the ground, gardens. July-Oct. $2 \frac{3}{8} \times 2 \times \frac{1}{x}$ in. Variable in size, sometimes as small as 453 and smaller than 457 .

453. V. TEMPERATA Sacc. (from its growing in temperate greenhouses) $a b$.

$P$. campanulate, umbonate, pulverulent, pale ochreous-sienna; marg. striate. St. white. $V_{0}$. ample, faintly ochreous.

Greenhouses. Feb. $\quad \frac{5}{5} \times \frac{7}{8} \times \frac{1}{16}$ in.

454. V. speciosa Gill. (speciosus, handsome) a $b c$.

$P$. campanulate, subumbonate, whitish, greyish or brownish; mid. darker. St. attenuate upwards, white. Fo. bulb-like, white.

Odour strong. Roadsides, rubbish heaps, manure heaps; rare. June-Oct. $4 \times 6 \frac{1}{2} \times \frac{1}{2}$ in.

455. V. gloiocephala Gill. (from its glutinous pileus; Gr. gloios, sticky, kephale, a head) a b c.

$P$. convex, subumbonate, whitish; marg. striate, greyish or brownish; mid. darker. St. subfibrillose, pale brownish or tawny. $V_{0}$. bulb-like, adpressed, brown. G. serrulate, becoming reddish.

Probably poisonous. Taste disagreeable; odour strong, offensive, like putrid broad beans. On the ground. June-Nov. $4 \frac{1}{2} \times 6 \frac{3}{4} \times \frac{1}{2}$ in. Fragments of $\%$. often left on $P$. 
456. V. media Gill. (from its intermediate position between 455 and 457) $a b c$.

$P$. convexo-plane, subumbonate, white; mid. pale brownish; marg. pale rose. St. white. Vo. small, lax, spreading; whitish.

Woods, on the ground. Aug.-Oct. $2 \frac{1}{4} \times 3 \times \frac{1}{4} \mathrm{in}$.

457. V. parvula Quél. (parvulus, small) a $b c$. Whitish.

$P$. convexo-plane, subumbonate. $V o$. large, lax.

Gregarious. Pastures, gardens, fir-woods, dead wood; frequent. May-Oct. $1 \frac{1}{5} \times I \frac{1}{4} \times \frac{1}{8}$ in. A minute form of this occurs in stoves.

\section{ANNULARIA Gill.}

(From the annulate stem.)

Veil universal, concrete with the cuticle of the pileus. Hymenophore distinct from the fleshy stem. Pilcus plane or broken up into minute squamules. Stem central, annulate, hollow. Gills free. (Fig. 22.)

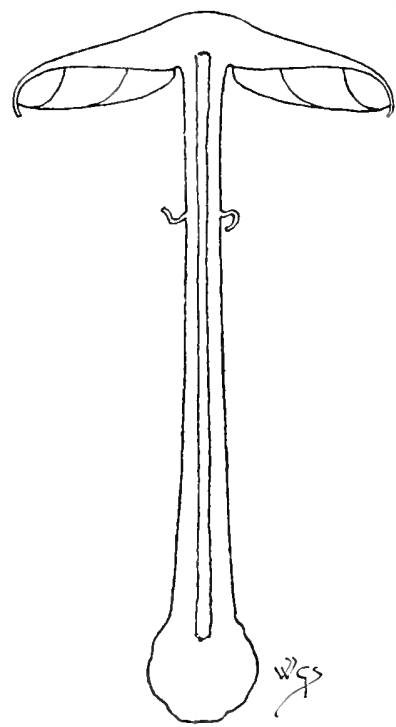

Fig. 22.-Section of Annuluria lavis Gill.
One.half natural size.

Terrestrial. Annularia agrees in structure with Lepiota, Togaria and Psalliota.

457a. A. lævis Gill. (from the usually smooth pileus; levis, smooth) a b.

$P$. convex, expanded, obtuse to subumbonate, smooth, glabrous or minutely squamulose, white or pallid. St. slightly attenuate 
upwards, even, smooth, silky, bulbous, colour as P. $A$. somewhat distant, large, becoming free. $G$. free to distant, thin, somewhat crowded, white, then salmon. Flesh somewhat firm white.

Bushy places, amongst grass. Aug.-Oct. $3^{\frac{1}{4}} \times 5_{\frac{3}{4}}^{\frac{3}{4}} \times \frac{3}{3}$ in.

\section{PLUTEUS Quél.}

(From a fancied resemblance in the pileus to the roof of a turret or sentry box, pluteus.)

Veil represented by the fibrils, flock or pruina of the pileus. Hymenophore distinct from the fleshy stem. Stem central, simple. Gills free, white, then usually salmon, or with a shade of yellow.

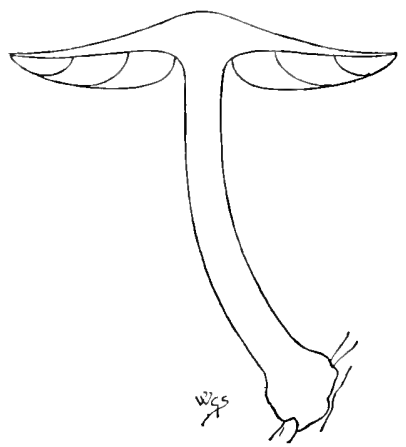

Fig. 23.- Section of Iuturus cervinus Quél. One-third natural size.

Spores smooth or warted, pale rose, salmon or somewhat reddish. (Fig. 23.)

Mostly growing on or close to tree-trunks, appearing from the spring to late autumn. They are all beautiful and tasteless; none are edible.

Phatcus agrees in structure with Hiatula, Pluteolus and Pilosace.

Cuticle of pileus separating into fibrils or flocci.

458. P. cervinus Quél. (from the fawn-brown pileus; cirnts, a deer) a b c.

$P$. expanded, broadly umbonate; marg. entire. St. solid, whitish, black-fibrillose, lighter above. Flesh silky-white.

Tasteless, insipid, or subacid. Trunks, fir, pear, sawdust, earth, straw, dung; common. April-Nov. $4 \frac{3}{4} \times 4 \frac{3}{4} \times \frac{1}{2}$ in. Sometimes mistaken for the mushroom. lar. eximizs Mlass. rufous-black. $6 \frac{1}{4} \times 5 \times 1 \frac{1}{2}$ in. Some- 
times $P . \mathbf{2} 2$ in. in diam. Var.petasa/us Mass., $P$. greyish-white. $6 \times S \times \frac{3}{4}$ in. Var. patricius Mass., $P$. scaly. Var. Bullit Mass., $P$. deep-brown. $6 \frac{1}{2}$ in. in tiam.

459. P. umbrosus Gill. (from its habitat; umlrosus, shady) $a b$.

$P$. convex, broadly subumbonate, wrinkled, umber; marg. fimbriate with V. St. solid, villous-scaly; umber-whitish. $G$. fuliginous-fimbriate at marg.

Dead wood, fir, oak. Sept. $2 \frac{3}{3} \times 2 \frac{1}{2} \times \frac{5}{15}$ in. This has less claim to specific rank than var. eximizs of 458 .

460. P. ephebius Gill. (from the down on the pileus; ephebins, like a jouth) a $b$.

$P$. convex, tomentose, pale slate-blue or lilac, sometimes slate. St. stuffed, whitish or bluish. $G$. salmon.

Rotten wood, trunks. $2 \frac{5}{8} \times 2 \frac{1}{2} \times \frac{1}{4} \mathrm{in}$.

461. P. salicinus Quél. (from its usual habitat, willow, Salix) $a b$.

$P$. convex, subumbonate, subpilose, pale blue-grey; mid. slightly wrinkled. St. colour as P., or clouded greenish.

Willow, bramble, alder. Feb.-Dec. $\mathrm{I} \frac{3}{4} \times 2 \frac{1}{2} \times \frac{1}{4}$ in. Var. beryllus Karst., $P$. greenish-streaked ; alder. Var. floccosa Sacc., two or three times smaller than 460 , to which it is allied.

462. P. hispidulus Gill. (from the slightly hispid pileus) $a b$. $P$. convex, grey or purplish-grey. St. hollow, silvery-white.

Stumps, beech, earth in hothouses. $\frac{5}{8} \times \mathbf{1} \frac{1}{2} \times \frac{1}{16} \mathrm{in}$.

463. P. pellitus Quél. (from the separable membrane of the pileus; pellitus, clad in skins) a b.

$P$. hemispherical, silky-white. St. stuffed, white.

Odour disagreeable. Trunks, earth, rotten branches, twigs, chips, roots of trees, amongst grass in pastures. July-Sept. $\quad I_{5}^{5} \times I_{\frac{3}{8}} \times \frac{1}{8} \mathrm{in}$.

464. P. nanus Quél. (from its small size; namus, dwarf) a $b c$.

$P$. convex, umber or slate-brown; mid. wrinkled. St. solid, whitish.

Thatch, rotten wood, rotten sawdust, earth, sticks, leaves and in gardens. Aug.-Oct. $\quad 1 \frac{5}{8} \times 2 \frac{7}{8} \times \frac{3}{16}$ in. Var. lutescens Karst. has an orangeyellow St., sulphur-yellowish flesh and salmon or sulphur G. Var. major Mass. is larger than the type.

465. P. spilopus Sacc. (from the spotted stem; Gr. spilos, a spot, pons, a foot) a $b c$.

$P$. convex, subumbonate, rugulose, brown. St. stuffed, tan-white or greyish, black-dotted.

Stumps, wood. Oct. $2 \frac{1}{5} \times I_{\frac{7}{3}}^{7} \times \frac{1}{4}$ in. Sometimes small, $\frac{7}{8} \times \mathrm{I}$ in. or less.

466. P. semibulbosus Gill. (from the slightly enlarged base of stem) $a b$.

$P$. hemispherical, sulcate, meals, white. St. hollow, white, base sienna and white cottony. $G$. distant; marg. entire.

Wood, branches, rotten wood. $\frac{5}{5} \times \frac{7}{5} \times \frac{1}{8} \mathrm{in}$. 
467. P. violarius Sacc. (from its bright violet exudation) $a b$.

$P$. expanded, smooth, even, spongy, dry, purple, crimson-purple or sienna. St. hollow, silky-white, black-fibrillose on a pale umber ground below. $G$. serrulate, crowded. Flesh grey.

Gregarious. Taste pleasant; odour none. Stumps, rotten wood. $\mathbf{1} \frac{1}{8} \times \mathbf{I} \times \frac{1}{3} \mathrm{in}$.

468. P. roseoalbus Gill. (from the rosy-white or salmon pileus) $a b$.

$P$. expanded, subumbonate. St. solid, pale rose or white. Flesh pale salmon.

Trunks, elm. Sept.-Nov. $3 \frac{1}{8} \times 3 \frac{1}{\frac{1}{4}} \times \frac{5}{16}$ in.

469. P. leoninus Quél. (from the colour of the pileus, often liontawny) a b $c$.

$P$. convex, striate, lemon, yellow, orange, tawny or scarlet; mid. darker; or orange with sulphur marg. St. solid, yellowish. $G$. often with a yellowish edge.

Caspitose Wood; rare. Sept.-Jan. $2 \frac{3}{\frac{3}{4}} \times 2 \frac{1}{2} \times \frac{1}{\frac{1}{4}}$ in. Var. cuccineus Mlass., $P$. crimson-orange or vermilion.

470. P. chrysophæus Quél. (from the dusky-golden stem ; Gr. chrnsos, gold, phaios, dusky) a $b c$.

$P$. plane, cinnamon; marg. striate. St. sulphur, sulphur-white or yellowish. $G$. colour as St., then salmon.

Taste insipid; odour none or subacid. Rotten wood, rotten sawdust, rotten ash, beech-stumps, boards, earth, hollow trees. May-Nov. $2 \frac{7}{8} \times 2 \frac{1}{8} \times \frac{3}{8}$ in.

471. P. phlebophorus Gill. (from the strongly veined pileus; Gr. phleps, a vein, phcro, to carry) a bc.

$P$. convex, subumbonate, umber; marg. plain. St. hollow, white. Fles/ greyish-white.

Dead stumps, rotten wood, sawdust, sticks; rare. June-Oct. $2 \times 2 \frac{3}{\frac{3}{4}} \times \frac{1}{\frac{1}{4}}$ in.

472. P. umbrinellus Gill. (from its umber pileus) $a b$.

$P$. submembranous, expanded, sometimes subumbonate; marg. fimbriate. St. hollow, fibrous, shining, whitish becoming brownish.

On the ground. Oct. $1 \frac{1}{1} \times 2 \frac{7}{3} \times \frac{1}{3}$ in.

\section{ENTOLOMA Quél.}

(From the potential, rather than definite veil ; Gr. cntos, within, loma, a fringe.)

I cil universal, faintly indicated by silkiness, flock, or scaliness. Hymenofhore confluent and homogeneous with the fleshy stem. Piletes somewhat fleshy, margin at first incurved. Stem central, simple, fleshy, fibrous, soft or sometimes waxy. Gills sinuatoadnexed, sinuate or adnate often separating from stem, at first white then usually salmon. Spores elliptical and smooth or subglobose and coarsely warted, rosy or salmon. (Fig. 24.) 
All the species grow on the ground in fields and woods chiefly after heavy rain, several appear in spring and summer; none are known to be edible, some are poisonous, many smell of meal.

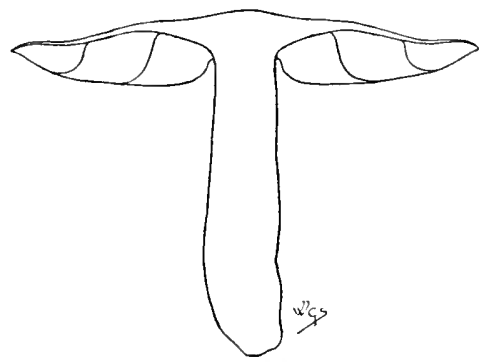

Fig. 24.-Section of Entolonta rhodopolium Quél. One-quarter natural size.

Entoloma agrees in structure and habit with Tricholoma and Hibcloma, and to a great extent with Hypholome. It is necessary to carefully distinguish the rose-and salmon-coloured spores of Entoloma from the sometimes pale clay-coloured spores of Hebeloma.

Species $473-502$

a. Gimine. Pileus fleshy, when full grown; smooth, often viscid but not hygrophanous, not innato-floccose or squamulose.

$473-483$

b. Liptonider. Pilcus dry, except 484, flocculose, somewhat scaly.

$484-490$

c. Volanidere. Pileus thin, commonly irregular and repand, for the most part scissile, hygrophanous, smooth, with a silky appearance when dry.

$491-502$

\section{a. Genuine,}

473. E. sinuatum Quel. (from the sinuate margin of the pileus) $a b c$. $P$. expanded, then repand, white or shaded tan, lavender, pale yellowish or brownish. St. solid, whitish. $G$. at first lividwhitish, then pinkish, at length brownish-salmon.

Gregarious. Poisonous. Odour strong, pleasant, somewhat of burnt sugar. Woods, mixed. July.-Oct. $7 \times 5 \times 1$ in. $P$. sometimes $S$ to $\mathbf{I}$ in. in diam. Young examples are sumetimes mistaken for the mushroom.

474. E. lividum Quél. (from the livid colour of the pileus) a $b$. $P$. plane, broadly subumbonate, livid tan; mid. pale yellowishbuff. St. subhollow, whitish. $G$. adnexo-rounded, salmon.

Poisonous. Odour of new meal or none. Woods. April-Oct. $4 \frac{3}{4} \times 3 \frac{1}{1} \times \frac{7}{8}$ in. Sometimes mistaken for the mushroom. Var, roscum sacc., $P$. slightly rose-tinted ; logs. 
475. E. prunuloides Quél. (from its odour being like that of 503 Clitopilus prunulus Quél.) a b c.

$P$. convex, broadly subumbonate, whitish, yellowish, greyish or brown; marg. striate. St. solid, white. G. emarginate, salmon.

Scattered. Odour of new meal, after maturity like decaying turnips. Woods, amongst moss and grass; uncommon. May-Sept. $2 \frac{3}{4} \times 2 \times \frac{1}{2}$ in.

475a. E. porphyrophæum Karst. (Gr. porthurcos, purple, phaios, dusky) $a$.

$P$. campanulate, expanded, subumbonate, not hygrophanous, even, almost glabrous, opaque sooty-brown or deep umber, becoming paler and mouse-colour when dry; marg. wavy, often incised and lobed. St. solid, attenuate upwards, fibrous, soft, opaque sooty-purple or brownish; base white-downy. $G$. truncate behind, almost free, subdistant, ventricose, greyish-white to reddish-grey or salmon. Flesh white.

Amongst grass. Autumn. $3 \frac{3}{8} \times 4 \frac{13}{15} \times \frac{5}{16}$ in. Must not be confused with 486 .

476. E. repandum Gill. (from the pileus being occasionally repand), $a b c$.

$P$. expanded, umbonate, whitish or faintly ochreous; marg. lobed. St. solid, colour as P. $G$. emarginate, salmon or faintly ochreous.

Odour of new meal. Amongst grass; rare. June-Oct. $3 \times 2 \frac{1}{4} \times \frac{3}{8}$ in. Not unlike some forms of the mushroom.

476a. E. erophilum Karst. (from its frequent appearance in spring; Gr. cros, spring, philos, loving) $a b$.

$P$. convex, obtuse to umbonate, venoso-striate, dry, pale ashy or pale olive-brownish; mid. somewhat darker. St. solid, equal or attenuate downwards, farinose, white, shaded ivory. $G$. rounded-adnate, broad, subdistant, pallid or pale salmon. Flesh white.

Woods, amongst grass, often in large circles. Sept. $I_{\frac{3}{4}}^{3} \times 2 \frac{1}{4} \times \frac{5}{16} \mathrm{in}$.

477. E. placenta Quél. (from the shape of the pileus; placenta, a flat cake) $a b$.

$P$. flat, umbonate, brownish or brown. St. solid, tan-brownish. $G$. emarginate, pallid flesh-colour. Flesh thin.

Solitary. Taste at length acrid; odour none. Hedgre-rows, damp places; rare. April-Oct. $2 \frac{1}{4} \times 3 \times \frac{1}{4}$ in.

478. E. helodes Gill. (from its frequent habitat; Gr. helos, a marsh) $a$.

$P$. umbonate, ashy or sooty-purplish. St. hollow, ashy or greyish. $G$. adnate, salmon. Flesh thin, pallid.

Taste watery; odour of new meal. Grassy places, heathy pastures, turfy swamps, moors. Autumn. $2 \frac{3}{8} \times 1 \frac{1}{2} \times \frac{3}{8}$ in.

479. E. Batschianum Karst. (after Aug. Jo. Georg. Car. Batsch) a $c$. $P$. convex, subumbonate, dark fuscous. St. hollow, grey. $G$. adnate, subsinuate, ashy or sooty. Flesh colour as I', white when dry.

Odour none. Woods, moist places, amongst grass in damp places. Oct. $\quad \mathbf{I}_{8}^{3} \times 3 \frac{3}{8} \times \frac{1}{4}$ in. Resembles one of the Pratelli as 804 . 
480. E. Bloxamii Sacc. (after the Rev. A. Bloxam) $a b c$.

$P$. convex, umbonate or papillate, waved, splitting, dull dark purple. St. solid, lighter than P. $G$. adnexed, pale salmon.

Downs and exposed pastures. Sept.-Nor. $4 \frac{1}{4} \times 2 \frac{3}{x} \times \frac{5}{8}$ in. Compare 482a.

480a. E. Farrahii Mass. \& Crossl. (after Mr. John Farrah) $a$.

$P$. cylindrico-ovate, then campanulate and somewhat repand, umbonate, smooth, fibrilloso-silky, blackish-blue ; marg. pallid. St. solid, ventricose, glabrous, colour as $\mathrm{P}$. with a white base. $G$. sinuato-adnexed, broad, ventricose, somewhat distant, salmon-colour.

Gregarious. Taste mild ; odour none. Amongst short grass. $2 \frac{1}{4} \times 2 \frac{1}{2} \times \frac{3}{5}$ in. Differs from its allies in its smooth, elliptical spores.

481. E. ardosiacum Quél. (from its slate-coloured pileus; ardosia, a slate) $a c$.

$P$. convex, steel-blue, shaded greenish, brownish or purplish. St. hollow, steel-blue, white at base. $G$. free, grey then salmon. Flesh dull pale purplish.

Odour none. Neadows, pastures, moist grassy places and under pines. $3 \frac{3}{1} \times 4 \times \frac{5}{16}$ in. Might be mistaken for one of the Pratelli as 802 .

482. E. liqueseens Sacc. (from the gills becoming liquescent) $a b$.

$P$. campanulate, broadly subumbonate, ochreous; mid. sienna. St. hollow, white. $G$. free, crowded, pale slate.

Gregarious. Under trees. April. $2 \frac{5}{8} \times 2 \bar{g} \times \frac{1}{4}$ in. Deliquescent like a Coprinus, which character with the dull pink spores and time of growth points to Bolbitius. Resembles one of the Pratelli.

482a. E. madidum (Gill. (from the moist pileus; madidus, moist) $a b$. $P$. campanulate, convex, obtuse, even, glabrous, moist, often viscid in rainy weather, shining when dry, not hygrophanous; when young blackish-violet, purple-slate, dull smalt or dull deep cobalt : sooty when old; mid. umber and whitish-yellow; marg. thin, inflexed, substriate. St. hollow, sometimes solid, fleshy-fibrous, slightly attenuate upwards, fibrillose, apex naked, colour as P., base whitish. $G$. slightly adnexed, ventricose, greyish-white to salmon. Flesh white.

Suspected poisonous. Odour strong, pungent, like 1341. Amongst moss, leaf-heaps, etc. $2 \frac{3}{8} \times 2 \frac{3}{8} \times \frac{3}{8} \mathrm{in.}$ Much like $480 \mathrm{in}$ appearance.

483. E. ameides Sacc. (from its odour of starch) $a b$.

$P$. convex, broadly subumbonate, pale reddish-grey; mid. darker. St. stuffed, paler than P. or almost white. $G$. emarginatoadnexed, pale pink.

Tasteless; odour of hay, apples, meal, linseed, orange-flower-water and starch. Pastures. Sept.-Nov, $2 \times 2 \frac{1}{4}, \times \frac{1}{2}$ in. Changing to yellowish or reddish when bruised. Must not be mistaken for 1222 . 


\section{b. Leptonidere.}

484. E. Saundersii Sacc. (after W. Wilson Saunders) $a b$.

$P$. expanded, broadly subumbonate, then repand, glutinous, white, becoming fuscous. St. solid, silky-fibrous, whitish. $G$. emarginato-adnexed, salmon.

Caspitose. On earth, river-sand, sawdust ; rare. June-Oct. $3 \frac{1}{2} \times 4 \times \frac{5}{8}$ in.

485. E. fertile Gill. (from its large, fruitful appearance) $a b$.

$P$. expanded, broadly subumbonate, pinkish-buff. St. solid, fibrillose, whitish. $G$. emarginato-adnexed, salmon.

Woods; rare. Aus.-Sept. $6 \frac{1}{4} \times 4 \frac{1}{4} \times 1_{\frac{1}{2}}^{\frac{1}{4}}$ in.

485a. E. Rozei Quél. (after M. E. Roze, French mycologist) a $c$.

$P$. convexo-plane, thin, pallid grey, or somewhat pale, dull slate-grey, paler when dry, minutely white-velvety or silky shining. St. solid, tough, silky-fibrillose, white. $G$. adnate, crowded, narrow, whitish flesh-colour.

Amongst pines. Oct. $1 \frac{1}{2} \times 2 \frac{3}{8} \times \frac{3}{16}$ in. Wholly dark umber when dry.

486. E. jubatum Karst. (from the fibrillose pileus; jubatus, having a mane) $a b$.

$P$. expanded, umbonate, mouse-colour. St. hollow, colour as P. $G$. adnexed, salmon or dusky purplish.

Single or crespitose. Taste watery, disagreeable. Old pastures and mossy places. Nay-Dec. $3 \frac{1}{\frac{1}{4}} \times 4 \frac{1}{4} \times \frac{5}{16} \mathrm{in}$. Must not be confused with 475a.

487. E. resutum Karst. (from the fibrillose pileus; resutus, ripped open) $a$.

$P$. convex, subumbonate, fuscous. St. stuffed, smooth, greyish. $G$. adnexed, grey or reddish-grey.

Odour none. Old pastures, woods. Oct. $1 \frac{7}{8} \times 3 \frac{3}{\frac{3}{4}} \times \frac{1}{5} \mathrm{in}$.

488. E. griseocyaneum Quél. (griseus, grey, cyaneus, dark blue) a $c$. $P$. campanulate, steel-grey, lilac shaded. St. hollow, fibrillose, colour as P. or whitish. $G$. adnexed or adnate, ventricose, salmon.

Sunny pastures, grassy places, open woods; rare. Oct. $1 \frac{3}{4} \times 3 \times \frac{3}{16} \mathrm{in}$.

488a. E. pulvereum Rea (from the mealy stem ; pulzereus, dusty) $a$.

$P$. campanulate then flat, minutely scaly, fuliginous; marg. at first striate. St. stuffed then hollow, velvety, covered with reddish meal. $G$. thick, sinuato-adnate, veined, somewhat distant, exceeding marg. of P., edge irregular, pale or pinkish, then deep rose.

Amongst oak-leaves, under holly. Oct. $1 \frac{1}{4} \times 2 \frac{1}{8} \times \frac{1}{10}$ in.

489. E. sericellum Quél. (from the slightly silky pileus; scriceus, silky) $a b c$.

$P$. plane, whitish. St. subhollow, smooth, white. G. adnate, salmon.

Gregarious. Odour none. Woods, grassy llaces; frequent. July-Oct. $\frac{7}{8} \times I_{5}^{5} \times 1 \frac{1}{1}$ in. 
489a. E. Cookei Ch. Richon (after Dr. M. C. Cooke) $a$.

$P$. convex, expanded, pale reddish- or sienna-orange or brickred, reticulate all over with raised pale yellowish ribs; marg. incurved. St. solid, often slightly incurved, almost central, whitish, shaded pale yellowish. $G$. adnexed, subdistant, thickish, pale salmon. Flesh thick at disc of P., thin elsewhere, colour as $\mathrm{G}$.

Stumps. Autumn. $1 \frac{1}{2} \times \mathbf{I} \times \frac{3}{16}$ in. Cooke described this as a form of 471, but Ch. Richon and Massee have made it an Entoloma. I have followed them with great doubt, thinking it more probably an abnormal form of 412 .

490. E. Thomsonii Sacc. (after Dr. Thomson) $a$.

$P$. Hat, umbonate, velvety, grey; mid. reticulato-ribbed. St. subhollow, tomentose, paler than P. G. adnexed, salmon.

Amongst grass in plantations. Autumn. $\quad 1 \frac{7}{5} \times 1 \frac{7}{8} \times \frac{3}{16}$ in.

\section{c. Nolanidere.}

491. E. elypeatum Quél. (from the shape of the pileus; clypeus, a shield) $a b c$.

$P$. flat, umbonate, umber or grey-brown, streaked darker. St. stuffed or hollow, paler than P. or almost white. $G$. adnexorounded, salmon or shaded greyish or brownish.

Solitary or crespitose. Woods, gardens, waste places; frequent. April-Oct. $3 \frac{1}{4} \times 4 \frac{3}{4} \times \frac{3}{8}$ in.

492. E. nigrocinnamomeum Sacc. (from the blackish-cinnamon colour of the pileus) $a b$.

$P$. flat, umbonate, moist, shining, silky when dry. St. hollow, fibrillose, grey-brown. G. emarginato-rounded, reddish-salmon.

Gregarious. Odour of new meal. Pastures, amongst grass and heather. Oct. $3 \frac{1}{5} \times 2 \frac{1}{2} \times \frac{3}{8}$ in.

493. E. rhodopolium Quél. (from its occasionally rosy-grey colour ; Gr. rhodon, a rose, polios, grey) a $b c$.

$P$. expanded, subumbonate, varying pale to dark fuscous, livid, brown or purplish-brown. St. stuffed or hollow, smooth, whitish. $G$. sinuate, rose.

Odour rank of new meal, of 1527 , of nitric acid or none. Woods; frequent. NIay-Oct. $2 \frac{1}{8} \times 3 \frac{3}{4} \times \frac{1}{4}$ in.

494. E. pluteoides Karst. (from its resemblance to Pluteus) $a b$.

$P$. convexo-plane, subumbonate, pale purplish-slate. St. hollow, fibrillose, whitish. $G$. emarginato-adnexed, crowded, salmon. Stumps. $2 \frac{1}{5} \times 3 \frac{3}{4} \times \frac{\frac{1}{4}}{4}$ in.

495. E. majale Karst. (from its appearance in May) $c$.

$P$. campanulate, subumbonate, dark ochreous or brown, becoming pale. St. hollow, whitish. G. free, salmon. Flesh very thin.

Subcrespitose. Meadows, open woods, mossy places in fir-woods; rare. April-May. $3 \frac{1}{4} \times 3 \frac{3}{8} \times \frac{3}{8}$ in. 
496. E. Wynnei Sacc. (after Mrs. Lloyd Wynne) $a b$.

$P$. plane, smooth, sometimes umbilicate, pale umber or smoky; marg. crisped, striate, at first smooth, then breaking up into minute sc. St. hollow, dusky grey. $G$. sinuate, reined, pallid salmon.

Taste insipid; odour of bugs or like 1278. Woods, fir. Sept.-Nov. $2 \frac{1}{4} \times 3 \times \frac{3}{16}$ in. Allied to 497 , but with $G$. less veined, less undulate and less distant.

497. E. costatum Gill. (from the transverse ribs or veins on the gills; cost $a$, a rib) $a b c$.

$P$. plane, fuscous-livid or greyish. St. hollow, colour as P. $G$. very broad, almost free, salmon.

Solitary or crespitose. Odour none. Damp meadows, pastures, swampy ground, grass-covered stumps: common. Aur.-Nov. $2 \frac{3}{8} \times 2 \times \frac{5}{16}$ in.

498. E. sericeum Quél. (from the silky pileus; sericeus, silky) $a b c$.

$P$. plane, umber; mid. darker. St. hollow, pale greyish-umber. $G$. emarginate, greyish then rufescent.

Gregarious. Odour strong of new meal. Meadows, open woods; frequent. May-Uct. $2 \times 2 \frac{1}{8} \times \frac{3}{15}$ in. This and 499 may be one species.

499. E. Persoonii Sacc. (after C. H. Persoon) $a b c$.

$P$. campanulate, subumbonate, silky-fibrous, splitting, membranous, white; mid. faintly ochreous; marg. striate to disc. St. hollow above, abruptly bulbous, cartilaginous, white. $G$. adnexoadnate, pale rose or flesh-colour, then darker.

Grassy places. Autumn. $2 \times 2 \frac{7}{8} \times \frac{3}{16}$ in.

500. E. nidorosum Quél. (from its strong odour; nidor, reeking) $a b c$. $P$. plane, ashy fawn-colour, whitish or purple-brown. St. stuffed, whitish. $G$. emarginate, salmon.

Odour nitrous, alkaline, sometimes obsolete. Lawns, open woods; common. Aug.-Nov. $3 \times 3 \frac{3}{ \pm} \times \frac{5}{16} \mathrm{in}$.

501. E. speculum Quél. (from the shining pileus; speculım, a mirror) ac.

$P$. plane or depressed, umbonate, whitish-straw or whitish ; marg. striate. St. stuffed or hollow, paler than P. G. adnexo-free, ventricose, salmon.

Solitary or subcæspitose. Odour none. Amongst grass and twigs. Oct. $2 \frac{1}{4} \times 2 \frac{3}{4} \times \frac{1}{4}$ in.

502. E. tortipes Mass. (from the twisted stem ; tortus, twisted, pcs, a foot) a $b$.

$P$. hygrophanous, thin, splitting, plane, depressed, umbonate, livid brown. St. solid, with a bark, striate above, pale brown within and without. $G$. sinuate, salmon-white.

On dung. Autumn. $3 \frac{5}{9} \times 2 \frac{1}{2} \times \frac{5}{15}$ in. 


\section{CLITOPILUS Quél.}

(From the decurrent hymenophore; Gr. klitos, a declivity,

$$
\text { pilos, a cap.) }
$$

Veil indicated by silkiness and flock. Hymenophore confluent and homogeneous with the fleshy stem. Pilcus more or less depressed or umbilicate, margin at first involute. Stem central, simple, fleshy

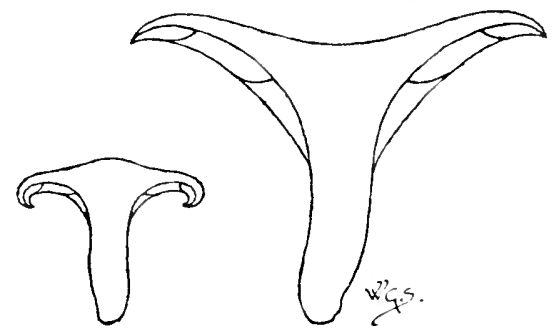

Fig. 25.-Sections of Clitopilus prunulus Quél.

One-half natural size.

or fibrous. Gills decurrent, white, except 506, then salmon. Spores smooth or warted, salmon, sometimes very pale. (Fig. 25.)

All the species grow on the ground. They have a more or less mealy odour. Some are tasteless, others edible.

Clitopilus agrees in structure with Clitocybe and in part with Flammula.

Species 503-513

a. Orcelle. Pilcus irregular, somewhat excentric, flexuous, somewhat hygrophanous, margin at first flocculose. Gills deeply decurrent.

$503-509$

b. Sericilla. Pilezs regular, silky or hygrophanous-silky, margin involute, naked. Gills adnate, slightly decurrent. 510-513

a. Orcella.

503. C. prunulus Quél. (from the pruinose appearance of the pileus) abc.

$P$. plane or convex, broadly subumbonate; when young faintly grey, then whitish. St. solid, frequently central, paler than P. $G$. subdistant, flesh-colour.

Edible. Odour strong of new meal, or like 1527. Woods and open places; common. June-Oct. $3 \frac{1}{4} \times 2 \frac{3}{4} \times \frac{1}{2}$ in. This and 504 are not insect eaten.

504. P. orcella Quél. (from the old Italian popular name; orgella, like an ear) $a b c$.

$P$. plano-depressed, lobed, undulate, white. St. solid, flocculose, often excentric, colour as P. $G$. crowded, whitish flesh-colour.

In troops. Edible. Odour as in 503 or of Syringa leaf or cucumber, or fresh meal and cucumber. Open grassy places, sometimes in rings 20 feet in diam. ; frequent. June-Oct. $4 \frac{3}{8} \times \frac{7}{8} \times \frac{1}{2}$ in. Intermediate forms occur between this and 503 . 
505. C. mundulus Gill. (mundus, neat) a $b c$.

$P$. convex, then depressed, white. St. stuffed, sometimes excentric, white, becoming black internally at base. $G$. crowded, whitish salmon. Flesh white.

Taste bitter; odour none. Woods, amongst leares; uncommon. Autumn. I $\frac{3}{8} \times \frac{5}{8} \times \frac{3}{16}$ in. In var. nigrescens Sacc. the flesh becomes wholly black.

505a. C. Sarnicus Mass. (after the place of finding-Sarnia, the Roman name for Guernsey) $a$.

$P$. campanulate, plane, subumbonate, often more or less depressed round the um., slightly striate when moist, even when dry, minutely silky-flocculose, mouse-colour or grey, paler with a ruddy tinge when dry. St. minutely fistulose, equal, subflexuous, even, glabrous, white. $G$. subcrowded, plane nearly to the stem, then suddenly decurrent, pinkish-salmon. Flish very thin.

On the ground, in Guernsey. $\quad \mathrm{I} \frac{1}{1} \times 1 \frac{1}{x} \times \frac{1}{10}$ in. Allied to 505 .

506. C. popinalis Gill. (from its edible qualities; popina, a cookshop) a $b c$.

$P$. convex, subumbonate or depressed, ashy or dull slate. St. stuffed, paler than P. $G$. crowded, grey or brown, becoming reddish.

Solitary or gregarious. Odour of new meal or rank and strong. Downs, fields, grassy roadsides. Aug.-Oct. $2 \frac{3}{4} \times \mathbf{1} \frac{7}{3} \times \frac{1}{2}$ in.

507. C. undatus Gill. (from the undulate pileus) a $b c$.

$P$. expanded, depressed, sometimes much lobed, sometimes with a papilla, dull ashy or tan-ochreous. St. hollow, colour as $\mathrm{P}$. or paler, faintly salmon-shaded. $G$. not crowded, salmon, sometimes reddish.

Odour none. Downs, etc. Oct. $\quad \mathrm{I} \frac{5}{5} \times \mathbf{I} \frac{3}{4} \times \frac{1}{5}$ in.

508. C. cancrinus Quél. (from the frequently cracked pileus; cancer; a lattice) $a b c$.

$P$. hemispherical, depressed, dry, at length shining and silky fibrillose, whitish. St. stuffed or fistulose, colour as P. $G$. distant, flesh-colour.

Odour none. Pastures. July-Oct. I $\frac{1}{8} \times I_{\frac{3}{8}}^{3} \times \frac{1}{5}$.

509. C. cretatus Sacc. (from the colour of the pileus; creta, chalk) a b $c$.

$P$. lobed, umbilicato-depressed. St. excentric, tomentose, colour as P. G. rose.

Solitary or gregarious. Woods, pastures; uncommon. Oct. $\frac{3}{4} \times \frac{1}{x} \times \frac{1}{16}$ in. Resembles a small 504 .

\section{b. Sericelle?.}

510. C. carneoalbus Gill. (from the flesh-coloured gills and white pileus ; arro, flesh, albus, white) a b c.

$P$. convexo-plane, subdepressed, white, faintly greyish or rufescent. St. stuffed or hollow, white. $G$. subdistant, flesh-colour.

Gregarious. Odour none. Woods, heathy places. Sept. $I_{8}^{3} \times 2 \times \frac{3}{10}$ in. 
511. C. vilis Gill. (zrilis, insignificant) a b c .

$P$. convex, depressed, grey. St. fistulose, colour as P. $G$. crowded, pale salmon.

Downs, amongst moss. July-Aug. $1 \frac{1}{2} \times 2 \frac{3}{8} \times \frac{1}{8}$ in.

512. C. stilbocephalus Sacc. (from the sparkling pileus; Gr. stilbo, to glisten, kephale, a head) a $b$.

$P$. campanulate, obtuse or umbonate, whitish or greyish; um and marg. sometimes ochreous. St. hollow, pale greyish, pallid below. $G$. sinuate, veined, salmon.

Odour of new meal. Aug.-Oct. $\mathrm{I}_{3}^{\frac{2}{3}} \times 3 \frac{1}{6} \times \frac{1}{5} \mathrm{in}$.

513. C. straminipes Sacc. (from the straw-coloured stem; strame'n, a straw, pes, a foot) $a b$.

$P$. expanded, depressed, subumbonate, glabrous, shining-silky, whitish or faintly ochreous. St. hollow, white-farinose above. $G$. salmon.

Among grass. Sept. $2 \times 2 \times \frac{1}{5}$ in. Very fragile.

\section{LEPTONIA Quél.}

(From the slender growth; Gr. lepos, slender.)

Veil indicated by fibrilla, scales, dots on stem, etc. Hymenophore confluent with but heterogeneous from the cartilaginous stem.

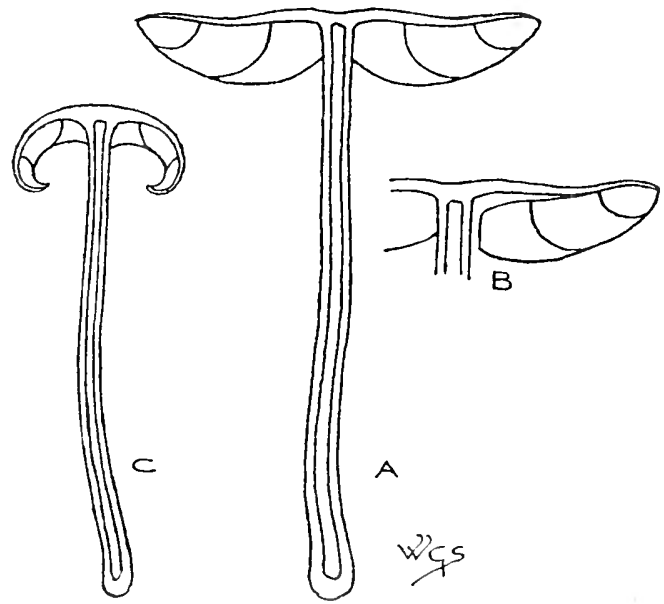

Fig. 26.-A, section of Leptonia lampropus Quél., showing at B separation of gills from hymenophore; c, section of $L$. chalyiciat Quél. Natural size,

Pileus thin, umbilicate or with a dark middle, cuticle fibrillose or separating into darker scales; margin at first incurved. Stcm central, 
simple, cartilaginous, tubular or stuffed, glabrous, sublustrous. Gills adnexed or adnate, readily separating from their attachment to the stem, not decurrent, variable in colour. Spores smooth or rough, salmon. (Fig. 26.)

Many of the species are gregarious and grow in troops, they are mostly small, inodorous, except 517 and 524, and often beautiful in colour, shades of light or dark blue or green being frequent.

All grow on the ground except 521; they chiefly grow in mossy pastures and marshy places. One non-British species-L. parasitica Quél.-grows on the hymenium of 1370 . They are most common in rainy seasons. The species generally are suspected to be poisonous.

Leptonia agrees in structure with Collybia, Nancoria, Psilocybe and Pancolius.

Species 514-528

Fileus slightly fleshy, without striæ, hygrophanous. Gills whitish.

Gills light or dark blue or bluish.

$514-519$

Gills pallid, becoming yellow or green.

Pileus somewhat striate, hygrophanous. Gills grey or glaucous.

$520-523$

$524-526$

527,528

514. L. placida Karst. (from its pleasing appearance) $a$.

$P$. campanulate, broadly subumbonate, dusky brown or duskyslate-squamulose on a tan ground. St. stuffed, slaty-blue, black-dotted above. $G$. adnexed or somewhat adnate, salmonwhite. Flesh brownish or lavender.

Usually solitary. By beech-trunks. Autumn. $2 \times 2 \frac{1}{2} \times \frac{3}{16}$ in.

515. L. anatina Quél. (from the colours; anatinus, pertaining to a duck) a b $c$.

$P$. campanulate, broadly subumbonate, umber-squamulose. St. stuffed, jurple-bluish or smalt above, brown and whitish below. $G$. adnexed or somewhat adnate, pale salmon.

Fields. Oct. $\mathbf{I}_{\frac{5}{8}} \times \mathbf{I} \frac{1}{2} \times \frac{1}{4} \mathrm{in}$.

516. L. lappula Quél. (from the prickly pileus; lappa, a burr) $a$.

$P$. convex, umbilicate, grey or purple-slate; mid. black-punctate. St. hollow, lilac, shaded brownish. $G$. subadnate, broad, white, faint greyish or whitish-salmon.

Odour none. Amongst beech-leaves. Autumn. $1 \frac{1}{2} \times 2 \frac{7}{8} \times \frac{3}{16}$ in.

517. L. lampropus Quél. (from the lustrous stem; Gr. lampros, shining, pous a foot) a b $\mathrm{c}$.

$P$. expanded, depressed, mouse-colour, steel-blue, pallid yellowish, brownish or brown. St. fistulose, steel-blue, pale slate or purplish, brownish below, white at base. $G$. adnexed or subadnate, broad, pale salmon.

Taste and odour somewhat strong, disagreeable. Pastures; common. July-Nov. $\quad 1 \frac{3}{8} \times 2 \frac{1}{4} \times \frac{1}{5}$ in. 
518. L. æthiops Gill. (from the black pileus and stem; Gr. aithiops, an Ethiopian) a.

$P$. plano-depressed, smooth. St. stuffed, fuscous-blackish. $G$. subadnate or adnexed, narrow, pale salmon-white.

Woods, grassy places. Sept. $1 \frac{1}{1} \times 2 \frac{1}{2} \times \frac{1}{8}$ in.

519. L. solstitialis Gill. (from its sometimes appearing in the summer solstice-June) $a$.

$P$. plane, umbonate, fuscous, brown- or olive-shaded. St. fistulose, dusky, whitish above or as P. $G$. emarginate, pale salmon.

Amongst stones, grassy ground, woods. Aug.-Sept. $1 \frac{1}{8} \times 2 \times \frac{1}{8} \mathrm{in}$.

520. L. serrulata Quél. (from the black-toothed edge of the gills, contrasted with 528 ; serrular, a saw) $a b c$.

$P$. convex, depressed, blackish-blue. St. fistulose, paler than P. $G$. adnexed or adnate, slate-whitish, then grey-salmon, varying slate-purplish. Fl'sh slate-white.

Sometimes crespitose. Pastures and grassy woods. June-Oct. $I_{2}^{5} \times 2 \frac{1}{2} \times \frac{1}{8}$ in.

521. L. euchroa Gill. (from its fine colour, Gr. cuchroos) $a b c$.

$P$. convex, subumbonate, purple-blue or bright smalt-purple. St. stuffed, colour as P. or lighter, or bluish flesh-colour. $G$. adnexed or subadnate, pale grey-blue, blue, purple-blue or slate.

Solitary, gregarious or caspitose. Probably poisonous. Woods, trunks, stumps, branches, alder, hazel ; rare. Aug.-Oct. $\mathbf{I}_{\frac{3}{4}}^{\frac{3}{2}} \times 2 \times \frac{1}{8} \mathrm{in}$. Pileus sometimes $\frac{1}{4}$ in. or less in diam.

522. L. chalybæa Quél. (from its steel-blue colour; Gr. chalups, steel) $a b c$.

$P$. plane, subumbonate, separable, dark violaceous, brownish-slate or slate with satin-like threads from mid. to marg. St. stuffed, colour as P. G. adnate, bluish-grey-whitish, pale olive-salmon, salmon or purplish.

Taste somewhat musliroom-like, but strong and unpleasant. Pastures; frequent. July-Oct. $\quad \mathbf{I}^{3} \times 1 \times 1 \frac{7}{8} \times \frac{1}{8}$ in.

523. L. lazulina Quél. (from the lapis lazuli colour of the stem) $a b$.

$P$. expanded, dusky-black, steel-slate, dark-brown-streaky. St. fistulose, light or dark blue. $G$. adnate, colour as St., then shaded salmon. Flesh slate-steel.

Gregarious. Amongst moss and grass. Sept. $\frac{7}{8} \times 2 \frac{1}{8} \times \frac{1}{16}$ in.

524. L. incana Gill. (incentus, hoary) a $b c$.

$P$. plane, umbilicate, striate, olive- or bronzy-green, streaked. St. fistulose, colour as $\mathrm{P}$. or verdigris or greenish-blue. $G$. adnexed or adnato-toothed, whitish-green then salmon.

Gregarious. Odour strong of mice. Woods, pastures, downs; frequent. July-()ct. $\quad 1 \frac{5}{5} \times 2 \times \frac{1}{16}$ in.

525. L. formosa Gill. (formosus, beautiful) a $b$.

$P$. plane, broadly umbilicate, yellow or brownish, minutely sootysquamulose. St. fistulose, yellow. $G$. adnato-toothed, light salmon-yellow, then pale flesh-colour.

Amongst Equisetum, grass, commons, pine woods. Sept. $2 \frac{1}{2} \times 3 \frac{3}{3} \times \frac{1}{6}$ in. Var. suavis Sacc. St. steel-bluish. 
526. L. ehloropolia Gill. (from the pale green-grey pileus; Gr. chloros, greenish-yellow, polios, hoary) $a$.

$P$. flat, slightly subumbonate, livid or sulphur-livid; mid. blacksquamulose. St. fistulose, colour as P. above, pale grey-bluish below. $G$. adnate, salmon-white.

Grassy places, woods. Oct. $1_{6}^{5} \times 2 \frac{5}{6} \times \frac{1}{6}$ in.

527. L. asprella Quél. (asper, rough) $a b c$.

$P$. expanded, broadly umbilicate, striate, smoky or mouse-colour; mid. villous-squamulose. St. fistulose, grey-white. G. adnatorounded, at length grey-salmon.

Open pastures, grassy places; uncommon. Oct. $\mathbf{I}_{\frac{3}{8}} \times \mathbf{I}_{\frac{3}{4}} \times \frac{1}{8} \mathrm{in}$.

528. L. nefrens Quél. (from the edge of the gills being without teeth, contrasted with 520 ; ne, not, frendo, to bite) $a$.

$P$. flat, depressed, smoky, yellowish-umber or olivaceous. St. fistulose, lighter than P., or fuscous-livid. $G$. adnexed or adnate, at length light red with a blackish edge.

Grassy places. Autumn. $\mathbf{I}_{\frac{5}{8}} \times \mathbf{1} \frac{3}{8} \times \frac{3}{16}$ in.

\section{NOLANEA Quél.}

(From a fancied resemblance in the pileus to a little bell, nola.)

$V$ eil indicated by fibrils, squamules, silkiness or flock; Mr. George Massee has observed a rudimentary annulus in 534. Hymenophore

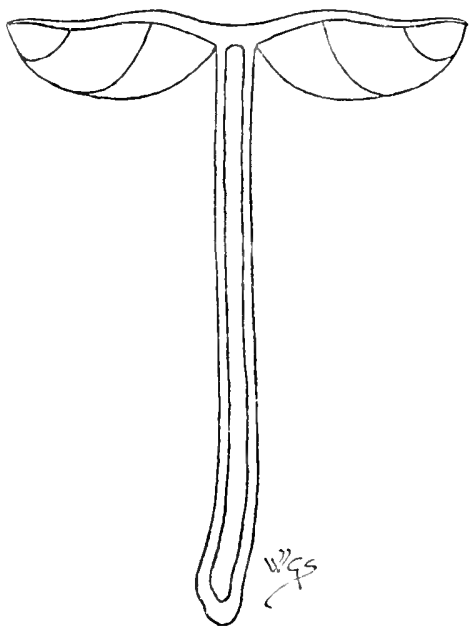

Fig. 27. - Section of Nolanca pascua Quét. Natural size.

confluent with but heterogeneous from the cartilaginous stem. Pileus submembranaceous, more or less campanulate, subpapillate or 
subumbonate, not umbilicate, except 536 and 542, striate, even or floccose, margin straight, at first adpressed to the stem, not involute. St'm central, simple, fistulose or stuffed, cartilaginous. Gills free or adfixed, not decurrent, usually whitish at first, then salmon or rose. Spores rose-coloured or salmon. (Fig. 27.)

The species are thin, slender, and usually brittle, some are tough. They are terrestrial except 541, and appear in summer and autumn on grassy hills and in damp places in woods. The group generally is suspected to be poisonous.

Nolanea agrees in structure with Mycena, Galera, Psathyra, and Psathyrella.

Species 529-543

Pilcus dark-coloured, hygrophanous. Gills grey or fuscous.

$529-533$

Gills becoming yellow or rufescent.

$534-537$

Pile'us hygrophanous. Gills shining white then rosy. 538, 539

Pileus not hygrophanous. Gills whitish.

$540-543$

529. N. pascua Quél. (from its habitat, pastures) $a \cdot b c$.

$P$. flat or subumbonate, silky, grey-or pinkish-brown. St. striate, faintly brownish or ochreous, or almost white. $G$. adnatoadnexed, pale salmon.

Odour none. Fields, open woods; common. May-Nov. $1 \frac{7}{8} \times 2 \frac{3}{8} \times \frac{3}{16}$ in.

530. N. Babingtonii Sacc. (after Prof. C. C. Babington) $a$.

$P$. conico-campanulate, ashy-grey; mid. dark-brown-squamulose. St. equal, downy, dark brown. $G$. adnato-ascending, ashy-grey.

Woods; rare. Oct.-Nov. $\frac{1}{2} \times 1 \frac{1}{8} \times \frac{1}{16}$ in.

531. N. mammosa Quél. (from the shape of the pileus; mamma, a breast) $a b c$.

$P$. umber or smoky. St. smoky white-brown. G. adnexed, grey then salmon.

Lawns, woods. Feb. $2 \frac{5}{8} \times 3 \frac{1}{2} \times \frac{3}{16}$ in. Often grows in company with 1238.

531a. N. papillata Bres. (from the papillate pileus).

$P$. submembranaceous, subcampanulate, convex, expanded, striate, fuscous-bay becoming cinnamon. St. glabrous, shining, obsoletely white-mealy at apex, white-tomentose at base. $G$. sinuato-adnate, somewhat crowded, livid white becoming fuscous flesh-colour. Flesh colour as P. but paler.

Odour pleasant, sometimes absent. In grassy or woody places. Sept. $1 \frac{1}{4} \times 2 \frac{1}{4} \times \frac{1}{10}$ in.

532. N. juncea Quél. (from its usual habitat, rushy places; juncus, a rush) $a b$.

$P$. hemispherical, papillate or subumbilicate, smoky or umber. St. smooth, reddish-brown. $G$. adnato-ascending, grey- or brownish-salmon.

Odour none. Woods, commons. Oct. $1 \frac{1}{4} \times 2 \frac{3}{4} \times \frac{1}{8}$ in. 
533. N. fulvostrigosa Sacc. (from the tawny strigose stem).

$P$. hemispherical, subwrinkled, grey. St. furfuraceo-squamulose, reddish. $G$. adnate, grey.

Woods. Sept. $\frac{3}{ \pm} \times \frac{1}{2} \times \frac{1}{16}$ in.

534. N. pisciodora Gill. (from its fishy odour; piscis, a fish, odor, a smell) $a b c$.

$P$. campanulate, velvety, umbonate, tawny-cinnamon, salmonbrownish or dark brown. St. rarely with a rndimentary A., chestnut-brown or black. $G$. adnexed, toothed, pale yellowish-tawny, then flesh-colour or dull salmon. Flesh warm brown, dark at base of stem.

Odrur strong of rotten fish, varying to cucumber. Woods, amongst decayed leaves, on sticks; frequent. Oct. $1 \frac{3}{8} \times 2 \frac{1}{2} \times \frac{1}{5}$ in. This may be only a form of 709. See 239, 535 and 538. Spores may possibly vary white, salmon and brown.

535. N. nigripes Gill. (from the black stem; niger; black, fes, a foot) a $b$.

$P$. hemispherical, floccose, fuscous or umber. St. equal, twisted, somewhat wavy, hollow. $G$. adnexed, yellow-flesh-colour or salmon.

Odour as in 534. Swamps. Nov. $\mathbf{I}_{\frac{3}{4}}^{3} \times 3 \frac{7}{8} \times \frac{1}{8}$ in. Perhaps a form of 534.

536. N. rufocarnea Sacc. (from its colour; rufus, reddish, caro, flesh) $a b c$.

$P$. hemispherical, subumbilicate, red-brown. St. minutely fibrillose, pale rufous. $G$. adnate, rose-colour.

Taste somewhat bitter. Grassy heaths; frequent. Sept. $1 \frac{1}{2} \times 2 \times \frac{1}{8}$ in.

537. N. ieterina Quél. (from its colour; Gr. ikteros, jaundice) a b.

$P$. hemispherical, sometimes papillate, slightly silky, greenishsulphur or olive-brassy. St. stuffed, colour as P. or full ochreous. $G$. adnexed, adnate or decurrent, salmon-white to salmon, saffron in decay. Flesh sulphur in P., white with a buff bark in St.

Woods, gardens. Oct. $1 \frac{1}{4} \times 1 \frac{1}{8} \times \frac{1}{8}$ in.

538. N. picea Gill. (from the more or less pitch-black colour ; piccus, pitch-black) $a b$.

$P$. campanulate, papillate, smooth, at first umber; marg. lighter, shaded slate. St. pruinose, colour as P., or dull salmon, blotched and shaded slate. $G$. ascending, emarginate, fleshcolour.

Odour of decaying fish and cucumber. Grassy places, gardens. $1 \frac{3}{4} \times 2 \frac{1}{2} \times \frac{1}{8}$ in. Perhaps the same as 534, 535 and 709.

539. N. infula Gill. (perhaps from the lustrous gills, at first dazzling white then rose; infula, a fillet or ornament) $a b$.

$P$. plane, papillate, fawn or smoky when damp, ochreous-tan when dry. St. smooth, colour as P. or grey-brown. G. varied in attachment.

Odour none. Lawns, woods, burnt ground. Sept.-Oct. $1 \times 2 \frac{1}{2} \times \frac{1}{5}$ in. 
540. N. verecunda Gill. (from its appearance ; a'crecundus, modest) $a$. $P$. convex, umbonate, watery-reddish. St. faintly ochreous. $G$. adnate, salmon-white. Spores reddish.

Densely gregarious or in troops. Amongst short grass. Sept. $\frac{3}{4} \times \frac{7}{8} \times \frac{1}{16}$ in.

541. N. cœlestina Gill. (from the colour of the pileus, sometimes azure; coxlum, the sky) $a$.

$P$. convex, umbonate, pale slate. St. colour as P. G. adnate, salmon-grey or hoary-white. Spores faintly rosy.

Wools, pine; on wood, rotten oak. Oct. $1 \frac{1}{2} \times 2 \frac{1}{4} \times \frac{1}{\frac{1}{4}}$ in.

541a. N. exilis Quél. (exilis, small).

$P$. membranous, conical, expanded, glabrous, striate, livid grey to lilac; mid. papillose, darker. St. hollow, glabrous, tough, apex naked, greyish-green. $G$. adnexed, subcrowded, whitish with a tinge of pink.

Amongst grass, leaves, etc., under beeches, pines; in swamps. $\frac{2}{3} \times 3 \times \frac{1}{2}$ in. Allied to 526, but more slender and delicate.

542. N. RUBid Sacc. (from its frequent reddish tinge) $a b c$.

$P$. convex, subumbilicate, greyish or faintly ruddy. St. attenuate below, colour as $\mathrm{P}$ or paler. $G$. adnate, rose.

Odour of new meal. Amongst grass in a conservatory. Mar.-Nov. $\frac{1}{2} \times \frac{1}{2} \times \frac{1}{8}$ in.

543. N. RHODOSPORA Sacc. \& D. Sacc. (from the rose-coloured spores) $a b c$.

$P$. convex, sooty-fibrillose or rufescent-pilose. St. subbulbous, white. $G$. sinuate or free, salmon or rose.

On earth and wooden borders in stoves. May-Sept. $\quad \mathbf{I} \times \mathbf{1} \times \frac{1}{16}$ in.

XIX. ECCILIA Quél.

(From the hollowed out or depressed pileus; Gr. ckkoiloo, to hollow out.)

$V_{c}$ il represented by pruina, fibrillæ or dot-like scales. Hymenofhore confluent with but heterogeneous from the cartilaginous stem.

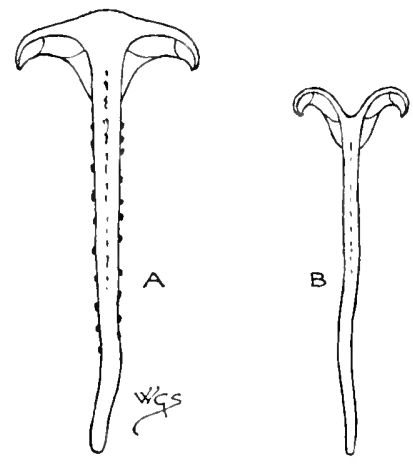

Fig. 28.-A, section of Eccilia atropuncta Gill. $\mathrm{B}$, section of E. acus Sacc. Natural size. 
Pileus somewhat membranous, margin at first incurved, umbilicate, except 548. Stem central, simple, fistulose, stuffed in 550. Gills decurrent, not separating from their attachment to the stem as in Nolanea. Spores smooth or warted, salmon or rose-colour. (Fig. 28.)

Most of the species grow on the ground, one grows on rotten wood and on walls, another amongst leaves.

Eccilia agrees in structure with Omphalia and Tubaria.

Species $\mathbf{5 4 4 - 5 5 2}$

544. E. parkensis Quél. (from its being first noted in the King's park, Upsala) a $c$.

$P$. plano-convex, fuscous or blackish, not pale when dry; marg. substriate. St. attenuate downwards, not dotted, fuscous. $G$. dull flesh-colour.

Grassy places. July-Aug. $\frac{3}{4} \times 1 \frac{1}{8} \times \frac{1}{16}$ in.

545. E. carneogrisea Gill. (from the colour of the pileus; caro, flesh, griseus, grey) $a b$.

$P$. plane; marg. striate. St. colour as P. G. distant, rosy, edge darker.

Gregarious. Amongst fir-leaves, grass. Aug.-Oct. $1 \frac{1}{8} \times 1 \frac{1}{2} \times \frac{1}{8}$ in.

545a. E. Smithii W. G. Sm., Agaricus Smithii Mass. (after Worthington George Smith) a $b$.

$P$. soon plane, orbicular, umbilicate, even, atomate, yellowish- or buff-white; marg. somewhat undulate, becoming salmon-striate. St. equal or slightly attenuate upwards, surface undulate, fibrillose, salmon, paler above, white downy below. $G$. broadly adnato-decurrent, salmon-rose. Flesh somewhat thick except at marg., pale salmon-white.

On the ground. Oct. $\mathrm{I}_{\frac{3}{4}} \times 2 \frac{1}{8} \times \frac{1}{8}$ in. Differs from 512 in the plane pileus, coloured stem, different spores, etc.

546. E. griseorubella Quél. (from the reddish-grey pileus) a.

$P$. hygrophanous, plane, brown or grey-brown; marg. striate. St. smooth, colour as P. $G$. subdistant, flesh-colour.

Gregarious. Suspected poisonous. Inodorous. On earth and wood. Autumn. $1 \frac{1}{8} \times 2 \times \frac{1}{8}$ in.

547. E. atrides Quél. (ater, black) a.

$P$. plane, fuscous, black-streaked, becoming pale ; marg. striate. St. pallid, black-dotted upwards. $G$. distant, slightly serrulate, pallid with a black edge.

Gregarious. Woods, moist places. Autumn. $1 \frac{1}{2} \times \mathbf{1}_{\frac{3}{8}}^{3} \times \frac{1}{8}$ in. Perhaps a form of 520 .

548. E. atropuneta Gill. (from the black-dotted stem; ater, black, functum, a dot) $a b c$.

$P$. convex, pale ashy or dull umber; marg. lighter. St. umber, dark or pale, black-punctato-scaly. $G$. distant, ashy flesh-colour.

Taste disagreeable. On the ground amongst moss. Sept.-Oct. $\frac{7}{8} \times 1 \frac{1}{2} \times \frac{1}{8}$ in. 
549. E. nigrella Gill. (from the blackish pileus ; niger, black) $a b$.

$P$. smooth, deep umber. St. glaucous, pale salmon-brown, not black-punctate. $G$. flesh-colour or subashy, not serrulate or with a black edge.

Sometimes cæspitose. Pastures. Autumn. $\frac{1}{2} \times 2 \times \frac{1}{1 E}$ in.

550. E. rhodocylix Gill. (from the resemblance of the pileus to a rose-coloured cup; Gr. rhodon, a rose, kulix, a cup) $a b$.

$P$. convex, fuscous, grey or tan; marg. striate when moist. St. stuffed, ashy, tan, or white. $G$. very distant, flesh-colour.

Rotten wood, stumps, alder, wall-tops. Sept. $1 \frac{1}{8} \times \mathrm{I} \times \frac{1}{16}$ in. In the young state when the gills are white this resembles 386 .

551. E. Flosculus Sacc. (from its likeness to a small flower) $a b$.

$P$. slightly irregular, pruinoso-crystalline, pink-tan; mid. dark brown. St. pruinose or innately fibrillose, pinkish-tan, dark at base. $G$. slightly wavy, bright rose.

In conservatories, on the ground and on tree-fern stems. June. $\frac{7}{8} \times \frac{5}{8} \times \frac{1}{15}$ in.

552. E. Acus Sacc. (from the needle-like stem; acus, a needle) $a b$.

$P$. deeply umbilicate, pruinose, white; marg. incurved, striate. St. smooth, white. $G$. thick, distant, deeply decurrent, rose.

Coco-nut fibre in conservatories. Aug. $\frac{1}{2} \times \frac{1}{1} \times \frac{1}{32} \mathrm{in}$.

\section{CLAUDOPUS Gill.}

(From its dwarfed stem; claudus, lame, Gr. pous, a foot.)

$V$ il represented by tomentum or down. Hymenophore confluent and homogeneous with the fleshy stem when the latter is present. Pileus excentric, lateral or resupinate. Stem, when present, lateral,
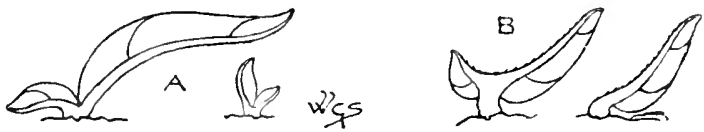

Fig. 29.-A, sections of Claudopus variabilis Gill. ; B, sections of C. byssisedus Gill. Natural size.

simple, without cartilaginous bark. Gills reaching to the excentric stem or axis. Spores smooth or warted, rubiginous. (Fig. 29.)

The species are small. They grow on wood or on the ground.

Claudopus agrees in structure and habit with Plezurotus and Crepidotus.

Species 553-555

553. C. variabilis Gill. (from its variability) a $b c$.

$P$. resupinate, then reflexed, white-tomentose. St. minute, excentric or none. $G$. salmon-pink or salmon-brownish.

Sticks, stumps, leaves, oak, beech, maple, bramble, apple, sedsre, moss, living chickweed; common. May-Mar. Diam. $1 \frac{1}{2}$ in. Sometimes sterile with white gills. Sometimes $\frac{1}{16}$ in. or less in diam. 
554. C. depluens Gill. (from its watery substance; depluo, to rain) a $b c$.

$P$. resupinate, then reflexed, hygrophanous, rufescent-hoary. St. small, lateral, rarely central, or none, white villous. $G$. adnato-decurrent, rufescent.

On the ground, amongst moss, sawdust, wood-ashes, sometimes in stoves; rare. Oct. Diam. I in.

555. C. byssisedus Gill. (from the fibrils on the stem; byssus, fine linen thread, sedeo, to sit) $a b c$.

$P$. resupinate, then horizontal and reniform, villous, pale grey. St. small, white-villous. $G$. adnato-decurrent, salmon.

On the ground, rotten wood, beech; rare. Sept.-Oct. Diam. $\frac{7}{8}$ in.

\section{SERIES III. DERMINI Fr.}

(From the membrane of the pileus; Gr. derma, a skin.)

Spores various shades of reddish-brown, brown, red, yellowishbrown or dull yellow: Genera XXI-XXXI.

XXI. ACETABUlaRIA Nass.

(From the cup-like volva; acctabulum, a vinegar-cup.)

Veil universal, forming a volva. Hymenophore distinct from the fleshy stem. Stem central, simple, volvate. Gills adnexed. Spores pallid-tawny or brownish.

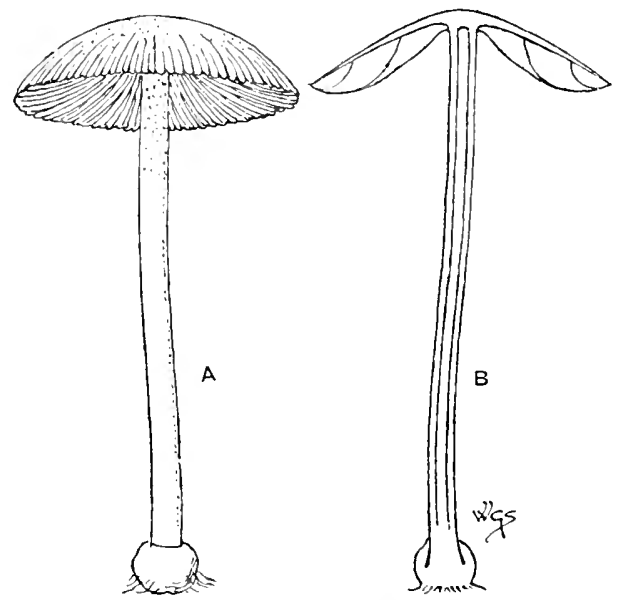

Fig. 3o.-Acetabularia acetabulosa Mass. Natural size.

$A$, perfect plant : $B$, plant in section.

Acetabularia agrees in structure with Amanitopsis, Volvaria and Chitonia. 
Saccardo (Sylloge Fungorum, vol. v. p. 76r) makes Berkeley's Acetabularia analogous with Gillet's Locellina. The two genera are quite distinct ; Locellina is not represented in Britain.

\section{Locellina.}

Pileus smooth, glabrous.

Gills broadly adnate.

St'm fibrilloso-annulate.

\section{Acetabularia.}

Pilicus sulcate, white-furfuraceous. Gills adnexo-free.

Stem simple.

556. A. acetabulosa Mass. (from the cup-like volva; acetabulum, a vinegar-cup) $a b$.

$P$. convex, salmon ; mid. sienna-reddish, white-furfuraceous ; maro. striate, denticulate, splitting. St. hollow, whitish, white-mealy above. $G$. adnexed, subdistant, glandular, pale brownishsalmon with a lighter edge.

By the Thames at Millbank. May. $I_{8}^{3} \times 2 \times \frac{1}{8}$ in. The glands on the gills, as illustrated by Sowerby, may be cystidia.

\section{TOGARIA IV. G. Sm.}

(From the annulus, like a cloak, well seen in the young state of 557 ; toga, a Roman garment.)

$V_{\text {eil }}$ universal, manifest in the membranous annulus and in the

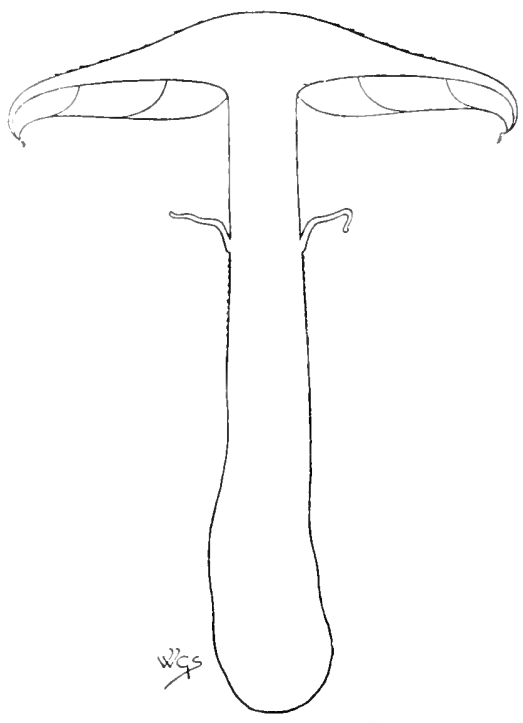

Fig. 3t.-Section of Togaria aura W. G. Sm.

One-quarter natural size.

squamules, fibrillæ and flock of the pileus; the squamules, etc. being sometimes appendiculate at the margin. Hymenophore not 
truly distinct from the fleshy stem, except in 564, although in the majority of cases it is nearly so. Pileus fleshy. Stem central, fleshy, annulate. Gills adnato-decurrent, adnate, adnexed, or roundedfree. Spores ferruginous. (Fig. 3I.)

All the species grow on the ground.

Togaria agrees in nearly all points of structure and habit with Lepiota, Anmilaria and Psalliota.

In 564 the gills are normally rounded-free, and in several other species the gills are frequently adnexed. In Lcpiota one species47-has adnate gills and several others have approximate gills; in Psalliota several species have approximate gills. Species 557-566

a. Endermina. Spores ferruginous.

$557-564$

b. Phaote. Sporis fuscous-ferruginous.

565,566

\section{a. Eudermina.}

557. T. aurea IV. G. Sm., Agaricus alureus Mattusch. (from its golden colour ; aurum, gold) $a b c$.

$P$. convex, velvety, squanulose; marg. partially appendiculate with V. St. solid, striate and scurfy below A. A. superior, large. $G$. adnato-decurrent to adnexo-free, ochreous then sienna. Flesh whitish-sulphur above, golden-sienna below.

Subcæspitose. Plantations, cedar; rare. Aug.-Nov. $10 \times \mathbf{I} \mathbf{I}_{\frac{3}{4}}^{\frac{3}{4}} \times \mathbf{I} \frac{3}{\frac{3}{4}}$ in. Var. Vahliii W. (ᄎ. Sm. (Agaricus Vahlii Schum.). P. even, smooth. Var. herefordensis W. G. Sm. (Agaricus aurcus Mattusch. var. herefordensis Renny.) St. granulate, tuberculate.

558. T. caperata W. G. Sm., Agaricus caperatus Pers. (from the wrinkled pileus; capero, to wrinkle) $a b c$.

$P$. convex, white-floccose on a deep yellow or buff-tan ground; marg. deeply wrinkled or lobed. St. solid, white, shaded dull salmon; white-squamulose above A. $A$. median, somewhat large. $G$. adnate or adnexed, dull ochreous or clay-cinnamon, sometimes colour of iron-oxide.

Solitary. Woods. Aug.-Dec. $4 \frac{1}{ \pm} \times 4 \frac{1}{2} \times \frac{3}{4}$ in. Sometimes there is an obscure volva. Pale forms resemble 789 .

559. T. terrigena IV. G. Sm., Agaricus tirrigenus Fr. (from its habitat; torra, the earth, gigno, to bear) $a$.

$P$. convex, silky-fibrillose, yellowish ; marg. partially appendiculate with V. St. colour as P., clad with ferruginous-yellow warts. $A$. apical. $G$. adnate with a decurrent tooth, yellowish then brownish.

Woods, damp places, old earthy stumps. Aug.-Nov. $2 \frac{3}{8} \times 2 \frac{5}{8} \times \frac{5}{16}$ in. Perhaps 589 is a form of this. Small forms must not be confounded with 1085.

560. T. erebia W. G. Sm., Agaricus erebius Fr. (Gr. erebos, dark) a bc.

$P$. convex, at first viscid, dark ferruginous brown or umber, becoming paler; marg. often streaky-wrinkled. St. hollow, smooth, pale salmon-brownish, darker at base and white above, 
white squamulose below. $A$. apical, membranous, white. $G$. adnate, sometimes with a decurrent tooth, somewhat pale lividbrown. Fles/l pale brownish.

Gregarious. Taste fungoid, insipid; odour slight or obsolete. Woods, shrubberies, grassy places, under birches. Aug.-Oct. $2 \times 2 \frac{3}{4} \times \frac{1}{4}$ in. Not unlike some forms of 792. Nust not be confounded with 69a.

561. T. ombrophila W. G. Sm., Agaricus ombrophilus Fr. (Gr. ombros, rain, philns, loving) a $b$.

$P$. convex, gibbous, smooth, clay-colour, sometimes shaded ferruginous ; marg. with traces of the white V. St. hollow, paler than P. or almost white. $A$. distant, white. $G$. adnate with a tooth or adfixed, pallid brownish, then ferruginous-brown.

Grassy places after heavy rain. Oct. $2 \frac{1}{8} \times 2 \frac{1}{2} \times \frac{5}{16}$ in. Var. brunnecla W. G. Sm. (Agaricus ombrophilus var. brunneolus Fr.). $P$. brown. $1 \frac{1}{2} \times 1 \frac{1}{2}$ in.

562. T. molliscorium W. G. Sm., Agaricus molliscorium Cooke \& Mass. (from the skin of the pileus; molliscorium, with a soft, leather-like skin) $a b$.

$P$. convex, smooth, polished, dry, subumbonate, tawny yellow; mid. ochreous or sienna. St. hollow, equal, pale ochreous, silky fibrillose, broken up into minute sq. above A. A . deciduous, brownish. $G$. adnate or uncinate, crowded, ferruginous.

Taste and odour none. June. $2 \frac{5}{8} \times 2 \frac{3}{4} \times \frac{1}{4}$ in.

563. T. togularis IV. G. Sm., Agaricus togularis Bull. (from the annulus, like a little cloak, togula) a $b$.

$P$. convex, pallid ochreous; mid. sienna or umber. St. hollow, whitish, yellowish or brownish. $G$. adnate or adnexed, yellow then pallid ferruginous. Fles/h colour of exterior.

In troops. Grassy woodlands, gardens, wood-yards, amongst chips; rare. May-Nov, $2 \frac{1}{4} \times 3 \frac{1}{8} \times \frac{3}{16}$ in.

564. T. blattaria W. G. Sm., Agaricus blattarius Fr. (from the colour of the pileus; blatta, a cockroach) $a$.

$P$. flat, subumbonate, ferruginous; mid. darker. St. fistulose, whitish, brownish at base. $A$. white. $G$. rounded-free or slightly adnexed, watery cinnamon.

Gardens and cultivated places. Autumn. $1 \frac{1}{8} \times 1 \frac{1}{2} \times \frac{1}{8}$ in. Like an annulate Galera.

\section{b. Plicota.}

565. T. dura W. G. Sm., Agraricus durus Bolt. (durus, hard) a $b c$.

$P$. convexo-plane, kid-leather-like, becoming cracked, tawny, pale ochreous or almost white; marg. partially appendiculate with V. St. paler than P., sometimes with root-like fibres at base. $A$. ample, fugitive. $G$. adnate with a decurrent tooth, livid brown to deep ferruginous.

Possibly poisonous. Fields, gardens; uncommon. May-Oct. $2 \frac{3}{4} \times 3 \frac{1}{4} \times \frac{3}{8} \mathrm{in}$. Sometimes gathered for $\mathbf{7 9 2}$. 
566. T. præcox W. G. Sm., Agaricus pracox Pers. (pracox, early) $a b c$.

$P$. convex, obtuse, smooth, whitish or whitish-ochre. St. hollow above, mealy-floccose, white, sometimes with root-like fibres at base. $A$. ample, fugitive, white. $G$. rounded-adnexed or subadnate, at length fuscous.

Said to be edible. Taste rank; odour strong, not unpleasant. Gardens, pastures, road-sides; common. Mlay-Oct. $3 \times 4 \times \frac{3}{8}$ in. Sometimes wholly whitish. May not be distinct from last. Sometimes gathered for 792.

566a. T. sphaleromorpha IV. G. Sm., Agaricus sphaleromorphus Bull. (from the difficulty of distinguishing this from the two last species; Gr. sphaleros, delusive, morphe, form) $a b$.

$P$. globose, convex, plane, obtuse, even, smooth, expanded, white or pale buff to ochre. St. stuffed, then partially hollow, incrassate and villose below, when young clavate, silky, pale buff to yellowish. $A$. entire, median to distant, ample, membranous. $G$. arcuato- or adnato- to sinuato-decurrent, yellowishwhite, becoming ochre, ferruginous-tan or brownish.

Leaf-soil. Oct. $4 \frac{1}{8} \times 3_{\frac{3}{8}}^{3} \times \frac{1}{2}$ in. Mlust not be confounded with 565 and 566.

\section{PLUTEOLUS Gill.}

\section{(Diminutive of Pluteus, Genus XIV.)}

Veil indicated by gluten, fibrillæ and meal. Hymenophore distinct from the fleshy stem. Pileus conical or campanulate, then expanded,

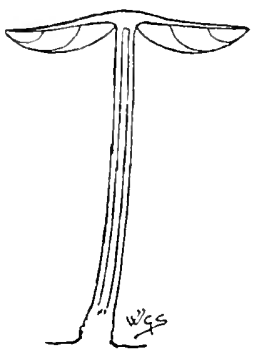

Fig. 32.-Section of Pluteolus reticulatus Gill. One-half natural size.

slightly fleshy, viscid, margin striate, at first straight and adpressed to the stem. Stem fistulose, simple. Spores ferruginous or saffron. Gills rounded-free. (Fig 32.)

The species grow on dead wood, rotten sticks, etc.

Plutcolus agrees in structure with Hiatula, Pluteus, and Pilosace. 
567. P. reticulatus Gill. (from the network of veins on the pileus; retc, a net) $a b$.

$P$. expanded, subumbonate, violaceous, fuscous or greyish; mid. faintly ochreous. St. fibrillose, whitish. $G$. dull cinnamon. Spores dark ferruginous.

Dead wood ; rare. Sept. $1 \frac{7}{8} \times 2 \frac{1}{4} \times \frac{1}{8}$ in.

568. P. aleuriatus Karst. (from the flour-dusted appearance of the stem; Gr. aleuron, flour) $a$.

$P$. convexo-plane, bluish-grey, rose-colour or tan ; mid. darker. $G$. saffron-ochraceous.

Rotten sticks. Oct. I $\times \mathrm{I} \times \frac{1}{16}$ in.

XXIV. PHOLIOTA Quél.

(From the scaly pileus; Gr. pholis, a scale.)

leil partial in the form of an annulus, or universal as scales and gluten on the pileus and stem. Hymenophore confluent and homogeneous with the fleshy stem. Pilius fleshy, viscid, dry, naked or

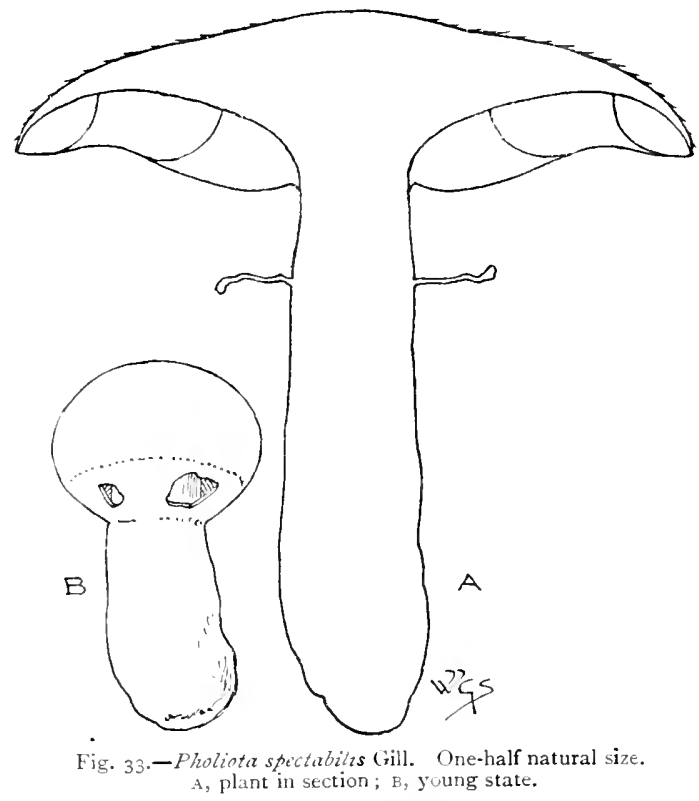

scaly. Stem central, annulate. Anmulus persistent, membranous, spreading, not arachnoid or fibrillose. Gills usually adnate, with or without a decurrent tooth, variously attached, at first pale or tawny, then usually becoming dark fuscous, but not the bright 
ferruginous colour of Cortinarius. Spores sepia-brown, bright yellowish-brown, or light red. (Fig. 33.)

All the species except 596 and 597 grow on trees, stumps and branches.

Pholioti agrees in structure and habit with Armillaria and Stropharia.

Pholiota must not be confounded with Cortinarius, where all the species are terrestrial, have an arachnoid veil and spores the colour of iron-oxide.

Species $569-597$

TrunCigexe. Mostly cæspitose, growing on trunks and stumps.

a. Egeritina. Pileus naked, not scaly, but here and there rimoso-rivulose. Gills pallid, then rufescent, becoming fuscous.

$569-574$

b. Squamosa. Pileus scaly, not hygrophanous. Gills changing colour.

Gills pallid, then fuscous, olivaceous, clay-coloured, not purely ferruginous. $\quad 575-579$

Gills yellow, then purely ferruginous or tawny. $\quad 580-586$

c. Hy,rophana. Gills cinnamon, not at first light yellow. Hygrophanous.

$587-595$

Muscigexe. Hygrophanous, resembling annulate Galerce; mostly growing on moss.

596,597

TRUNCIGENE.

a. Egeritine.

569. P. radicosa Quél. (from the rooting stem) $a b c$.

$P$. convexo-plane, viscid, pale tan-brownish, clouded darker. St. spongy-solid, enlarged below and fusiform rooting, whitishtan. $A$. distant, fugitive. $G$. adnato-toothed or rounded.

Solitary or gregarious, rarely cæspitose. Taste pleasant or obsolete; odour strong, like prussic-acid or sweetish, not unpleasant. Woods, about stumps, on wood; frequent. Aug.-Oct. $4 \frac{1}{2} \times 10^{\frac{3}{4}} \times \frac{5}{8}$ in. P. sometimes 6 in. in diam.

570. P. pudica Gill. (from its modest colour ; fudicus, modest) $a b c$. $P$. convex, moist, hygrophanous, rivuloso-wrinkled, tan; mid. sienna. St. solid, firm, rigid, paler than $\mathrm{P}$. $A$. persistent. $G$. adnato-rounded, colour as St. Flesh pallid white.

Single or cæspitose. Odour strong, penetrating, disagreeable. Stumps, elder, elm, rarely on lawns, downs. May-Nor. $2 \frac{5}{8} \times 2 \frac{1}{2} \times \frac{3}{8}$ in. Sometimes mistaken for 792 .

571. P. leochroma Sacc. (from its lion-tawny colour; Gr. leon, a lion, chroma, colour) $a b$.

$P$. convex, then depressed; mid. yellowish-white or pale sienna; marg. pale. St. solid, whitish above, brown below. $A$. per- 
sistent. $G$. adnato-rounded, dark cimnamon. Flesh pale livid-brownish, darker in St.

Cæspitose. Said to be edible. Stumps, elm, fir, Iarch. July-Sept. $3 \frac{1}{2} \times 4 \frac{7}{4} \times \frac{1}{x}$ in. Perhaps a form of 570 .

572. P. capistrata Sacc. (from a fancied resemblance in the annulus to a halter, capistrum) a b.

$P$. Hat, subviscid, tan ; mid. pale sienna ; marg. rivuloso-wrinkled. St. stuffed, squamulose, tan-white. $A$. large, inrolled, persistent. $G$. decurrent or rounded, thin, tan-white.

Crespitose. Said to be edible. Taste varying pleasant to unpleasant. stumps, elnı. Aug.-Nov. $4 \times 4 \times \frac{5}{5}$ in. Intermediate forms occur between this and $\mathbf{5 7 0}$ and 571.

573. P. ægerita Quél. (from its frequent habitat, poplar trunks; Gr. aigciros, the black poplar) $a b$.

$P$. convex, rivuloso-wrinkled, pale buff to bright full tawny. St. stuffed, sometimes substriate from the decurrent base of G., tan-white, white-mealy above. $A$. superior, swollen. $G$. serrulate, adnate with a tooth, fuscous.

Cæspitose. Odour pleasant. Stumps, Populus fastigiata, willow, ash, elm. July-Nov. $4 \times 5^{\frac{1}{4}} \times \frac{1}{2}$ in. The "Pioppini" of Italy.

574. P. luxurians Gill. (from its frequent luxuriant growth) $a b$.

$P$. flat, silky, squamulose, yellowish-white then rufous. St. stuffed, subsquamulose, colour as $\mathrm{P}$. $A$. apical, fugacious. $G$. adnate with a tooth, fuscous.

Crespitose. Stumps, elm, poplar, oak, asl.. July. $3 \times 2 \frac{3}{4} \times \frac{3}{8}$ in.

\section{b. Squanosa.}

575. P. comosa Quél. (from the hairy scales of the pileus; coma, a hair) $a b$.

$P$. convex, obtuse, viscid, tawny ; sc. paler ; marg. appendiculate with V. St. solid, bulbous, fibrillose, brassy-white. A superior, subpersistent. $G$. adnate or adnato-decurrent, fuscous.

Subcæspitose. Odour faint. Stumps, beech, poplar, birch; rare. Oct.Nov. $7 \times 4 \frac{1}{2} \times 1 \frac{1}{2}$ in.

576. P. heteroclita Quél. (from the curved stem; Gr. heteros, other, klino, to lean) a $b c$.

$P$. convex, obtuse, viscid when old and wet, whitish, yellowish or brownish; marg. appendiculate with V. St. solid, bulbous, fibrillose, whitish-brown at base. $G$. adnate or rounded-adnexed.

Solitary. Odour strong and pungent of horse-radish. Trunks, birch, poplar ; small examples on chips. Sept. - Nov. $5 \frac{1}{2} \times 3^{\frac{3}{4}} \times \mathbf{I}$ in.

577. P. aurivella Quél. (from a fancied resemblance in the pileus to a golden fleece; aurnm, gold, reilus, fleece) $a b c$.

$P$. convex, subumbonate, sulphur or deep ferruginous yellow. St. stuffed, fibrillose, colour as $\mathrm{P}$. A. superior. $G$. adnate with a tooth or sinuato-adnexed, brown.

Caspitose or solitary. Odour faint. Trunks, ash, willow, beech; uncommon. Sept.-Nov. $4 \frac{5}{5} \times 4 \times \frac{1}{2}$ in. 
578. P. squarrosa Quél. (from the rough-scaly pileus; squarrosus, rough) $a b c$.

$P$. convex, gibbous, dry, ochreous-sienna ; marg. appendiculate with V. St. stuffed, attenuate downwards, brown-squarrose below A. $G$. adnate with a decurrent tooth, olivaceousferruginous. Flesh light yellow.

Cæspitose, sometimes in fascicles of 50 or more. Sometimes eaten. Odour strong and nauseous of putrid cheese or none. On or near stumps, beech, oak, ash, laburnum, acacia, apple, plum; common. July-Dec. $3 \frac{7}{8} \times 4 \frac{1}{2} \times \frac{5}{5}$ in. Sometimes twice the size of type. Var. verruculosa Gill. $P$. glutinous when wet; maple. Var, refexa Gill. P. umbonate. Var. Miilliri Gill. small, wholly sulphur.

579. P. subsquarrosa Quél. (from the pileus, less scaly than 578) $a c$. $P$. plane, viscid, brown; marg. appendiculate with $T$. St. subsquarrose below A., yellow-ferruginous. $G$. adnate with a decurrent tooth, or almost emarginato-free, pale brown. Fles/l pale ochreous-brownish.

Subcæspitose. Odour almost obsolete. Trunks, fir, and on the ground. Sept. $2 \times 2 \frac{5}{8} \times \frac{5}{16}$ in.

579a. P. grandis Rea (grandis, large) $b$.

$P$. convex, then expanded, broadly gibbous, dry, fulvous-tawny, covered with small innate deeply tawny sq. at the circ., revolute at mid. St. solid, fusiform, slightly squamulose and tawny below the distant, almost fugacious A., deeply striate above. $G$. broad, attenuate in front, deeply sinuato-decurrent behind, somewhat crowded, pallid, then fuscous. Flesh light yellow, ferruginous in St.

Caspitose. Taste and odour very pleasant. Trunks, ash, oak. Oct. $9 \times 8 \frac{1}{4} \times 2 \frac{1}{4}$ in.

580. P. spectabilis Gill. (spectabilis, notable) a $b c$.

$P$. convex, obtuse, dry, rich sienna-yellow. St. solid, squamulose or smooth, ventricose, rich yellow. $G$. adnate with a tooth or subdecurrent. Flesh sulphur.

Crespitose. Taste, bitter-aromatic. Stumps, oak, beecl, hornbeam, hawthorn; frequent. Aug. - Dec. $5 \frac{5}{8} \times 5 \frac{5}{8} \times 1 \frac{1}{8}$ in. Stem sometimes 9 in. Resembles 1049 in appearance.

581. P. adiposa Quél. (from the pileus, as if covered with fat) $a b c$.

$P$. plane, subdepressed, very glutinous, sulphur; sc. Hoccose, ferruginous; mid. deep sienna-yellow; marg. appendiculate with V. St. squarrose, viscid, colour as P. A. small, floccose. $G$. adnato-rounded.

Cospitose. Trunks, beech, ash, plum, silver-fir, birch; frequent. July-Oct. $7 \times 5 \times \frac{3}{4} \mathrm{in}$.

581a. P. lucifera Quél. (from the flame-like colours; lux, light, fero, to carry) $a$.

$P$. fleshy, convex, almost plane becoming umbonate, viscid, covered at first with darker adpressed sc. which soon disappear, yellow, or orange with sulphur marg. St. stuffed to hollow, 
sometimes solid, equal, somewhat squamulose, becoming smooth, whitish above, brown below without and within. $A$. distant. $G$. emarginato-adfixed, narrow, crenulate, yellow-white, luminous yellow, then olive or ferruginous.

Trunks, branches, straw, almost terrestrial. Odour strong. Autumn. $2 \frac{1}{2} \times 2 \times \frac{3}{8}$ in.

582. P. flammans Karst. (from its fiery colours) $a b c$.

$P$. convex, subumbonate, yellow or orange; sc. sulphur. St. squarrose, sulphur or yellow. $A$. superior. $G$. sulphur, then brown. Flesh sulphur.

Gregarious or in clusters. Odour none. Woods, pine, pine-leaves, dead pine-branches. July-Oct. $2 \times 2 \frac{7}{8} \times \frac{5}{16}$ in. Mycelium often deep orange.

583. P. Junonia Karst. (from its beauty, belonging to Juno) $a b$.

$P$. convex-plane, subumbonate, yellow to deep sienna-orange. St. even, solid, yellow above, sienna below. $A$. distant. $G$. adnate.

Usually solitary. Trunks. Oct. $3 \frac{3}{8} \times 3 \frac{1}{2} \times \frac{3}{8}$ in. Must not be confounded with 695 .

584. P. tuberculosa Gill. (from the bulbous base of the stem) a $b$.

$P$. convexo-plane, obtuse, tawny or pale sulphur; mid. brown. St. hollow, subbulbous, attenuato-rooting, fibrilloso-scaly, light yellow or ochre. $A$. fugitive. $G$. adnate or emarginate, serrulate.

Solitary or crespitose. Trunks, birch; sawdust. Oct. $2 \times 1 \frac{5}{8} \times \frac{1}{4}$ in.

585. P. curvipes Quél. (from the frequently curved stem; curad, a curve, pes, a foot) $a b c$.

$P$. expanded, subumbonate, tawny or orange; marg. at first appendiculate with white V. St. fistulose, fibrillose, light yellow. $A$. floccose, fugitive. $G$. adnate with a white edge. Flesh light yellow.

Gregarious, subcæspitose. Taste somewhat pleasant-insipid; not strongly scented. Trunks, sawdust, deal boards in greenhouses. Oct. $2 \frac{1}{8} \times 1 \frac{7}{8} \times \frac{1}{4}$ in.

586. P. cruentata Sacc. (from the blood-coloured scales of the pileus ; cruentutus, blood-stained) $a b$.

$P$. convex, obtuse, yellow; sc. crimson-brown. St. solid, attenuato-rooting, subsquamulose below A., colour as $\mathrm{P}$. $A$. fibrillose. $G$. adnate or emarginate, brown, somewhat olive. Fiesh pale yellow.

Taste insipid. Trunks, oak; burnt ground. Aug.-Sept. $1 \frac{3}{8} \times 1 \frac{1}{2} \times \frac{1}{4}$ in.

\section{c. Hygrophance.}

587. P. Paxillus Gill. (from its resemblance to 1181, Paxillus intolutus Fr.) a.

$P$. expanded, subumbonate, ochreous-buff; mid. darker. St. solid, smooth, colour as P., brown at base. A. narrow, spreading. $G$. subdecurrent, crowded.

Trunks, Autumn. $6 \times 6 \frac{3}{4} \times \frac{3}{4}$ in. Sometimes wholly cinnamon. 
588. P. dissimulans Sacc. (from its appearing like, but differing from, 560 ; dissimulo, to dissemble) $a c$.

$P$. convex, subümbonate, buff-brown, becoming whitish. St. fistulose with transverse dissepiments, attenuate upwards, white. 4. fragile. $G$. adnexed or adnate with a tooth, clay-brown.

Sticks, hawthorn, sloe. Oct.-Nov. $\mathrm{I}_{8}^{\frac{3}{8}} \times \mathrm{I}_{\frac{7}{8}} \times \frac{1}{16}^{3} \mathrm{in}$.

589. P. Cookei Sacc. (after Dr. M. C. Cooke) $a b c$.

$P$. convex, obtuse, viscid, dull yellowish; sc. adpressed, faintly brownish. St. solid, whitish above, fibrillose and yellowishbrown at base. $A$. narrow. $G$. adnate.

Damp places, amongst grass, stumps. Sept.-Oct. $2 \frac{1}{2} \times 2 \frac{1}{4} \times \frac{1}{4}$ in. Perhaps the same as $\mathbf{5 5 9}$.

590. P. sublutea Karst. (sublutcus, yellowish) $a c$.

$P$. flat, subumbonate, squamulose, dull brownish-yellow; sc. darker. St. stuffed, smooth, attenuate upwards, paler than P. $A$. narrow, spreading. $G$. decurrent, crowded.

Subcrespitose. Grassy ground. Oct. $2 \frac{1}{2} \times 4 \frac{1}{4} \times \frac{1}{4}$ in.

591. P. confragosa Karst. (confragosus, rough) $a b$.

$P$. convexo-plane, obtuse, densely furfuraceous, red, cinnamonrufous or umber, tawny when dry; flocci fugitive, white. St. hollow, shining pale warm-umber; flocci fugitive. G. adnate or subdecurrent.

Somewhat crespitose. Fallen elm. Oct. $1 \frac{7}{8} \times 2 \times \frac{3}{16}$ in. Resembles 917 .

592. P. mutabilis Quél. (mutabilis, changeable) a $b c$.

$P$. convex, obtusely umbonate, cinnamon, pale when dry, or mid. deep cinnamon, with marg. straw-colour; sometimes appendiculate with V. St. pale above, cimnamon below, subsquarrose below A. A. narrow, spreading, fugitive. $G$. adnatodecurrent, crowded.

Sometimes eaten, taste of gingerbread. Stumps, lime, ash, sawdust. AprilDec. $2 \times 3^{\frac{3}{4}} \times \frac{1}{4}$ in.

593. P. marginata Quél. (from the striate margin of the pileus) $a b c$. $P$. expanded, subumbonate, honey-colour, tan when dry. St. fistulose, fibrillose, paler than P. $A$. spreading, fugitive. $G$. adnate or somewhat rounded.

Solitary, gregarious, crespitose. Woods, fir, beech, pine, chips; frequent. Sept.-Dec. $\quad I_{\frac{1}{8}} \times 2 \times \frac{1}{8}$ in.

594. P. mustelina Quél. (from its colour; mustclla, a weasel) a.

$P$. convex, dry, yellow or reddish. St. fistulose, whitish. $A$. superior, reflexed. $G$. adnate.

Solitary, subgregarious. Woods, pine, stumps. Sept. $\frac{5}{8} \times \frac{3}{4} \times \frac{1}{15}$ in.

595. P. unicolor Gill. (from its being wholly of one colour) $a c$. Bay-brown then ochreous.

$P$. convex, subumbonate. St. fistulose, somewhat smooth. $G$. adnexed, adnate or subdecurrent.

Subgregarious, subcæspitose. Trunks, branches, larch. Sept.-Nov. $1 \frac{1}{2} \times 2 \frac{1}{8} \times \frac{1}{8}$ in. 


\section{MusCigente.}

596. P. pumila Gill. (pumilus, diminutive) $a b c$.

$P$. hemispherical, smooth, ochreous; marg. substriate. St. fistulose, smooth, colour as P. or paler; base often darker. $A$. floccose. $G$. adnate with a tooth, subdistant, pallidbrownish.

Solitary, gregarious. Moss, walls, earth, sticks, sawdust, firs; common. Aug. - Jan. $\frac{3}{4} \times 2 \frac{3}{8} \times \frac{1}{8}$ in.

597. P. mycenoides Quél. (from its resemblance in stature to a Mycena) a $b c$.

$P$. hemispherical, ferruginous, tawny pale or medium; marg. striate. St. fistulose, smooth, colour as P. A. persistent, white. $G$. adnate with a decurrent tooth.

Amongst moss in damp places, lawns. Sept.-Oct. $\frac{1}{2} \times 1 \frac{7}{8} \times \frac{1}{35}$ in. Often accompanied by 283 .

\section{INOCYBE Quél.}

(From the fibrous pileus; Gr. is, inos, a fibre, kube, a head.)

Veil concrete with the cuticle of the pileus, fibrillose or somewhat arachnoid, often appendiculate at the margin of the pileus and sometimes forming an indefinite annulus. Hymenophore confluent

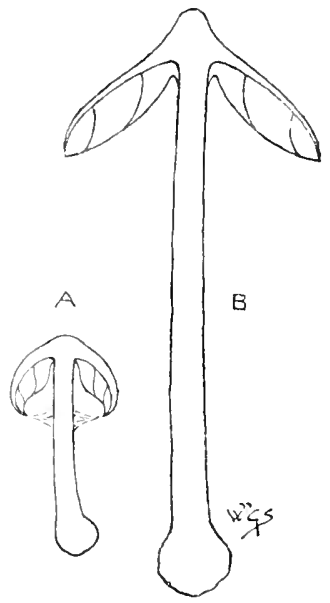

Fig. 34-A, section of Inocybe scabra Gill.; B, ditto $I$. fastigiata Quél. One-half natural size.

and homogeneous with the fleshy stem. Pileus squarrose, fibrillose or velvety; in a few species becoming smooth or viscid. Stem central, scaly, fibrillose or smooth, simple or imperfectly squarroso-annulate. 
Gills usually sinuate, sometimes adnexed, adnate or decurrent, adhering to the hymenophore, except 602 and 621, at first pale, changing colour, but not to fuscous-ferruginous. Spores often angular; pale dull tan, brownish or brown. (Fig. 34.)

All the species, except 644, grow on the ground; they are somewhat small; none are edible, and the majority have a somewhat nauseous odour.

Inocybe as defined by Fries (Hymenomycetes Europai, p. 226) has no genus corresponding wholly with it in structure amongst the white-, pink-, purple- or black-spored groups. Certain of the fibrouspileated species of Tricholoma, Entoloma and Stropharia might, however, be separated as analogues. Inocybe was formerly included in the next genus, Hebcloma.

Species 598-646

Mr. George Massee, in his monograph of the genus (Annals of Botany, XVIII. (1904), p. 459), has rearranged the species in two groups, viz.: I. Spores rough, and II. Spores smooth; these groups he has again divided into-A. Cystidia present, and B. Cystidia absent, with minor distinctions as to a whitish or coloured stem and brownish or olive-tinted gills.

a. Squarrose. Pileus squarrose, somewhat fuscous. Stem scaly, smooth above, same colour as pileus, solid except 204.

$598-607$

b. Lacerce. Pileus torn into scales or fibres, not split into cracks. Stem fibrillose, paler than the pileus. 608-619

c. Rimose. Pileus longitudinally fibrous, soon cracked, partially adpressed-scaly. Stim fibrillose, whitish, slightly tinged with the colour of the pileus. Many become yellow when old.

$620-634$

d. Velutine. Pileus not cracked, the cuticle fibrilloso-woven, becoming even or adpressedly-scaly. Stem shining, smooth, whitish, mealy above.

$635-642$

e. Viscide. Pileus viscid, even.

$643-646$

\section{a. Squarrosa.}

598. I. hystrix Karst. (from the scaliness; hystrix, a porcupine) a bc.

$P$. plane, subgibbous, mouse-colour to brown. St. attenuate downwards; sc. fuscous, brownish or mouse-colour. $G$. adnate, fuscous.

Woods, beech. Oct. $3 \times 2 \frac{1}{8} \times \frac{3}{8}$ in. Resembling a small 578 in appearance.

599. I. relicina Quél. (from the recurved floccose scales of the pileus ; riclimus, curled backwards) a $c$.

$P$. expanded, obtuse, fuliginous or rufescent livid-brown. St. slightly attenuate downwards, floccoso-scaly, colour as P. $G$. adnexed, olivaceous.

Odour almost obsolete. Wcods, fir, marshy places, amongst Sphagnum. JulyOct. $2 \frac{7}{8} \times 3 \frac{1}{4} \times \frac{5}{16} \mathrm{in}$. 
599a. I. mimica Mass. (from its resemblance to 26 as figured in Cooke's Illustrations, p. 94I).

$P$. campanulate, obtusely umbonate, fibrillose, yellow-brown, covered with large adpressed fibrous slightly darker sc. St. solid, equal, fibrillose, paler than P. G. deeply sinuate, broad, yellow-brown.

Woods. Autumn. $3 \times 3 \times \frac{3}{8}$ in.

600. I. ealamistrata Gill. (from the curled scales of the pileus; calamister, a curling iron) $a b c$.

$P$. campanulate, fuscous-scaly on a tan ground. St. squarrose, somewhat bulbous, at first pale, then as P., tinged with dull blue at base. $G$. adnexed, ferruginous with a white edge. Flesh becoming red when cut.

Sometimes subcæspitose. Odour strong, not unpleasant. Woods, pine, by paths and ditch-sides; rare. Aug.-Oct. $2 \times 2 \frac{1}{4} \times \frac{3}{16}$ in.

601. I. hirsuta Quél. (from the bristly pileus; hirsutus, bristly) $a b$.

$P$. campanulate, acutely umbonate; mid. fuscous or greenish; marg. pale tan. St. fibrillose, pale tan above, greenish at base. $G$. adnexo-ascending, umber with a white edge. Flesh faintly rose when cut.

Odour fainter than 600. Woods, beech, damp places, pastures; rare. Oct. $1 \frac{1}{8} \times 2 \frac{1}{4} \times \frac{1}{16}$ in.

602. I. lanuginosa Quél. (from the woolly pileus; lanuginosus, woolly) $a b c$.

$P$. hemispherico-convex, obtuse, umber then yellow. St. squamuloso-fibrillose, whitish-fuscous. $G$. separating-free, denticulate, pallid clay-colour.

Odour faint or none. Wcods, mixed, near paths, sandy places, near firs. July-Nov. $\quad 1 \frac{3}{8} \times 1 \frac{5}{8} \times \frac{1}{8}$ in.

602a. I. calospora Quél. in Bres. (from the beautiful spores; Gr. kalos, Deautiful) $a$.

$P$. convex or campanulate, then expanded and umbonate, fibrillose with darker sq. at mid., yellowish-brown or tawnygrey ; edge paler. St. pale then reddish, or as P. G. sinuatofree, tawny-ochre or brownish.

Woods, shady places. Autumn. I $\times 2 \frac{1}{2} \times \frac{1}{8}$ in.

603. I. dulcamara Quél. (from the taste-sweet, becoming bitter; dulcis, sweet, amatrus, bitter) a c.

$P$. campanulato-convex, umbonate, olivaceo-fuscous. St. squamuloso-fibrillose below, paler than P. $G$. adnexed, olivaceous.

Gregarious. Woods, plantations, fir, mixed. Aug.-Oct. $1 \frac{5}{8} \times 1 \frac{7}{8} \times \frac{1}{8}$ in.

604. I. plumosa Quél. (from the downy tufts on the pileus; plumosus, feathery) $a b$.

$P$. hemispherico-convex with squarrose flocci in erect fascicles, mouse-colour or shaded rufous. St. stuffed or hollow, floccoso- 
scaly, paler than P. or smoky. $G$. subadnate, pallid mousecolour.

Odour faint, not umpleasant. Woods, pine, moist places; rare. Auy. $1_{13}^{3} \times 2 \frac{5}{8} \times \frac{1}{8}$ in. The colours are not unlike those of Series II., Hyporhodii.

605. I. cincinnata Quél. (from the curved scales of the pileus; cincimnus, a curl) $a$.

$P$. convexo-plane, usually subumbonate, villoso-scaly, fuscous. St. fibrilloso-scaly, fuscous-violaceous or lavender above, brownish below. $G$. adnexed, fuscous-violaceous or lavender.

Subgregarious. Odour none. Woods, beech. Autumn. I $\times 2 \times \frac{1}{8}$ in. Compare 618 and 641a.

606. I. hæmacta Sacc. (from the change of colour to blood-red when broken; Gr. haimaktos, mingled with blood) $a b$.

$P$. plano-convex, floccoso-fibrillose, umber; mid. dark; marg. pallid. St. scaly-fibrillose, whitish above, dull verdigris below inside and out. $G$. adnate, clay-umber. Flesh crimson in P., greenish-blue below.

Subcrespitose. Lawns. Aug.-Oct. $\mathbf{I}_{\frac{3}{4}}^{\frac{3}{4}} \times 2 \times \frac{1}{4}$ in.

607. I. fasciata Sacc. (from its growing in bundles; fasciatus, bound together in bundles) $a b$.

$P$. convex, obscurely acuto-umbonate, minutely brown-squarrososcaly on a pale ground. St. solid, attenuate below, pallid above, vinous within and without at base. $G$. adnato-sinuate, crowded, thin, soft, pallid.

Densely crespitose. Taste and odour none. Grassy places. $3 \times 3 \frac{1}{3} \times \frac{5}{16}$ in.

\section{b. Lacera.}

608. I. pyriodora Quél. (from its odour of pears ; py'rus, a pear, odor, a smell) $a \cdot b c$.

$P$. convex, subumbonate, fuscous to tan-ochreous. St. solid, whitish. G. adnato-emarginate, fuscous, often olive-shaded, edge whitish. Flesh becoming pale reddish.

Taste none; odour strong, sweet, pleasant of pears, decaying pears, violets, cinnamon, of Muscuri racemosum. Woods, gardens. May-Dec. $2 \frac{7}{8} \times 3 \frac{3}{3} \times \frac{5}{16}$ in. Sometimes $P$. and St. whitish-tan, G. tan-rufescent.

609. I. incarnata Bres. (from the flesh-coloured pileus; incarnatus) $a b$.

$P$. expanded, broadly umbonate, sometimes tinged with pale orange-scarlet, crimson or purple ; marg. appendiculate with $\mathrm{V}$. St. solid, base enlarged, colour as P. $G$. sinuate, whitish to olive-brownish, often spotted orange, scarlet or crimson, or wholly rufescent, edge crenulate, white. Flesh pale orange or pale purple-hyacinth.

Odour strong of meal or pears. Woods, pine, etc. June-Oct. $3 \times 3 \frac{1}{4} \times \frac{1}{2}$ in. Red when bruised or broken. Closely allied to 608 . 
609a. I. adequata Sacc. (from its claim to equal specific rank with 610).

$P$. campanulate, then expanded, squarrose and fibrous-scaly, yellow and red-brown or pale, somewhat vinous-umber with darker umber sc. St. whitish- or reddish-fibrous, or as P. $G$. adnexed, thick, somewhat distant, pale greyish-white or yellowish-umber, becoming greyish-brown, always with a white edge.

Odour none. Woods. Summer and autunn. $3 \frac{1}{2} \times 4 \frac{1}{10} \times \frac{9}{16}$ in. Allied to 610 .

610. I. seabra Gill. (scaber, rough) a b $c$.

$P$. convex, subgibbous, pale fuliginous, tan or umber. St. solid, whitish. $G$. adnexed, often separating from stem, dull whitish-brown. Flesh white.

Suspected poisonous. Woods, plantations, fir, mixed; frequent. Iune-Oct. $\mathbf{1} \frac{3}{4} \times \mathbf{1} \frac{3}{x} \times \frac{1}{\frac{1}{4}}$ in. Var. firma Mass. fuscous-tan with fuscous scales.

611. I. maritima Karst. (from its being first found on the sea-shore) $a b c$.

$P$. hygrophanous, expanded, obtuse or umbonate, fuscous or mouse-colour, hoary when dry. St. solid, paler than P. $G$. adnexo-rounded, or somewhat sinuate, grey, then ferruginous.

Sometimes cæspitose. Odour faint. Woods, pine, sandy places, damp sand by sea-shores. Sept.-Oct. $1 \frac{1}{4} \times 1 \frac{5}{5} \times \frac{3}{16}$ in.

612. I. lacera Quél. (from the scaly-torn pileus; lacerus, torn) a $c$.

$P$. expanded, subumbonate, mouse-colour, pale and yellow when old. St. stuffed, paler than P., white and mealy. $G$. attenuatoadnexed, mouse-colour. Flesh reddish.

Commonly gregarious. Woods, pine, mixed. July-Oct. $I \frac{1}{2} \times I \frac{3}{4} \times \frac{3}{16}$ in.

613. I. floceulosa Sacc. (from the small flocci on the pileus) $a b c$.

$P$. convex, umbonate, rufescent-sienna. St. paler than P., solid, stuffed or hollow. $G$. adnato-ascending, colour as St., then chocolate with a white edge.

Odour of new meal, but nauseous. Amongst grass, woods. Sept.-Oct. $I \times 2 \frac{1}{4} \times \frac{1}{8}$ in. Compare 602 and 612 .

614. I. Bongardii Karst. (after H. G. Bongard, a Russian botanist) $a b c$

$P$. campanulate, obtuse, greatly to slightly scaly, often cracking between the sc. and showing the flesh, fuscous, pale when dry. St. solid, subbulbous, lighter than P., sometimes dark below. $G$. adnexed, ventricose, reddish, cinnamon or umber, edge white. Flesh reddish.

Usually solitary. Odour pleasant of ripe pears or bergamot. Sandy ground, sand-hills, pine-woods, pastures. Nay-Oct. $2 \frac{1}{8} \times 4 \times \frac{3}{8} \mathrm{in}$.

615. I. mutica Karst. (from the at first obtuse pileus; muticus, curtailed) $a b$.

$P$. obtuso-convex, plane, then subdepressed, whitish, tan or straw. St. hollow, attenuate downwards, colour as P. G. adnate, white or tan, then subfuscous.

Woods, shady places, roadsides. Sept.-Oct. $2 \frac{1}{4} \times 2 \times \frac{1}{4}$ in. 
616. I. carpta Quél. (from the torn woolly pileus; carpo, to tear) $a b c$.

$P$. flat or depressed, dark fuscous or umber. St. hollow, attenuate downwards, paler than P. G. adfixed to adnate, ventricose, brown-fuscous with a pale edge.

Taste bitterish-sweet. Woods. Aug. $\mathrm{I}_{\frac{1}{8}} \times \mathrm{I}_{\frac{3}{4}} \times \frac{1}{8} \mathrm{in}$.

617. I. deglubens Gill. (from the fibrils of the pileus which peel off; deglubens, peeling off) $a b$.

$P$. expanded, obtusely umbonate, date-brown-rufescent, becoming yellowish. St. solid, colour as P. above, paler and yellowish below. $G$. adnate, ventricose, grey then cinnamon.

Woods, pine, moist places; rare. Aug.-Sept. $1 \frac{5}{8} \times 2 \frac{1}{2} \times \frac{1}{8}$ in.

617a. I. cervicolor Quél. (from its dark tawny colour, as of a deer; cri'lls, a stag) $a$.

$P$. campanulate, covered with brown recurved fibrils. St. firm, whitish, fibrillose, with brown recurved filaments throughout its length. $G$. emarginate, ventricose, distant, rusty brown; marg. denticulate, whitish. Flesh white, tinged purplish when cut.

Odour strong, unpleasant. Amongst grass, woods. Autumn. $\frac{7}{8} \times 3 \times \frac{1}{8}$ in. Compare 614.

618. I. obscura Gill. (from the dark scales of the pileus; obscurus, dark) $a b c$.

$P$. expanded, umbonate, purplish-brown or shaded lavender or faint crimson. St. stuffed, lilac or purplish above, yellowbrownish below, lilac within. $G$. sinuato-adnexed, olivaceous, then brown.

subcæspitose. Odour strong, somewhat bitter. Amongst pines, damp places, woods; rare. July-Nov. $2 \frac{1}{2} \times \mathbf{1}_{\frac{3}{4}}^{\frac{3}{4}} \times \frac{1}{1}$ in. Var. rufa Sacc., $P$. brownish-rufous, $G$. violet.

619. I. Rennyi Sacc. (after James Renny, mycologist) $a b$.

$P$. hemispherical, fawn-colour; mid. brown. St. equal, whitish at top and bottom, yellow-brown in mid. $G$. adnexo-ascending with a tooth, whitish-brown then cinnamon.

Woods, fir. Nov. $1 \frac{1}{8} \times 2 \frac{5}{8} \times \frac{1}{8}$ in. The var. major Mass. is illustrated in the Public Gallery, the type is little more than half this size.

\section{c. Rimose.}

620. I. schista Sacc. (from the clefts in the pileus; Gr. schistos, a cleft), $a b$.

$P$. campanulate, obtuse, shining bay-brown; mid. sienna. St. solid, twisted, splitting, paler than P. G. adnato-ascending with a tooth, separating, sienna-cinnamon; edge serrate, white.

Taste pleasant, somewhat insipid. Lawns. May. $2 \frac{3}{\frac{3}{4}} \times 3 \times \frac{1}{2}$ in. 
621. I. fibrosa Gill. (from the fibrous pileus) $a b c$.

$P$. campanulato-expanded, umbonate; mid. foxy-red, lighter at marg. St. solid, splitting, pale ochreous. $G$. adnexo-sinuate, separating, foxy.

Odour fetid. Woods, fir. May-Sept. $5 \frac{7}{8} \times 4 \frac{7}{ \pm} \times \frac{7}{8}$ in.

621a. I. proximella Karst. (from its superficial resemblance to 628) $a$.

$P$. conico-convex, then expanded and umbonate, even, then longitudinally fibrosely cracked, pallid; mid. and um. rustybrown or bay. St. stuffed, sometimes wavy, subfibrillose, pallid. $G$. adnate, crowded, broad, ventricose, pallid, tan, then brown. Flesh white.

Woods. Sept. $1 \frac{3}{8} \times 2 \frac{3}{4} \times \frac{1}{4}$ in. Distinguished by its ventricose gills.

622. I. perlata Sacc. (from the pileus; perlatus, very broad) $a$. $P$. expanded, broadly umbonate, straw-ochreous; mid. blackfuscous; marg. pale. St. solid, straw-pallid, black-fuscous at base. $G$. adnexed, subrounded or slightly sinuate, umber.

Under trees, hornbeam. Aug. $4 \frac{3}{\frac{3}{4}} \times 2 \frac{7}{8} \times \frac{1}{2}$ in.

623. I. phæocephala Sacc. (from the dusky pileus; Gr. phaios, dusky, kcphale, a head) a.

$P$. conico-campanulate, umbonate, bay-brown, fuliginous or rufous. St. solid, subbulbous, pale brown above, white below. $G$. adnexo-free, olive-brown.

Woods, pine, mixed. Oct. $3_{\frac{3}{4}}^{\frac{3}{4}} \times 4^{\frac{3}{4}} \times \frac{3}{8}$ in. Perhaps not belonging to Inocybe.

624. I. fastigiata Quél. (from the pileus, pointed like a gable, fastigium) $a b c$.

$P$. conico-campanulate, acutely umbonate, dull yellow-brownish. St. solid, paler than P. G. adnexo-free, crowded, olive-brown.

Gregarious, sometimes cæespitose. Woods, grassy places; uncommon. June-Oct. $3 \frac{5}{8} \times 5 \times{ }_{15}^{5}$ in.

625. I. hiulea Gill. (from the furrow-like cracks of the pileus; Gr. hio, to gape) $a c$.

$P$. expanded, umbonate, fuscous, olivaceous or ochreous. St. stuffed, whitish. $G$. adnexo-free, subdistant, olive-brown.

Woods, pine ; uncommon. Aug.-Nov. $2 \frac{1}{x} \times 2 \frac{3}{4} \times \frac{5}{16}$ in. Young examples sometimes wholly white. Rufescent when bruised.

626. I. Curreyi Sacc. (after Frederick Currey, mycologist) $a b$.

$P$. expanded, dull yellowish-brown. St. solid, pale rufescentbrown. $G$. free, olive-brownish. Flesh pale olive-brown.

Odour none. Woods. Aug. $2 \frac{7}{8} \times 3 \frac{3}{8} \times \frac{3}{8}$ in.

627. I. rimosa Quél. (from the cracked pileus; rima, a crack) $a b c$. $P$. campanulate, subumbonate or obtuse, rufescent- or olive-brown ; mid. darker; cracks yellowish. St. solid, slightly marginatobulbous, pallid above, yellowish and rufescent below. $G$. attenuato-adnexed, olivaceous-brown or fuscous.

Subgregarious. Odour earthy. Woods and open places amongst trees. June-Oct. $2 \frac{7}{8} \times 3 \frac{1}{4} \times \frac{5}{16}$ in. 
627a. I. subrimosa Sacc. (from the slightly" cracked pileus; rima, a crack) $a$.

$P$. conico-campanulate, then expanded, prominently umbonate, even, smooth, longitudinally fibrillose, at length cracked, pale ochre, brown or pallid ferrugineo-ochreous; mid. brown. St. solid, equal, shining, base somewhat marginately bulbous, white-pruinose. $G$. attenuate behind, adnexed or free, slightly ventricose, whitish clay-colour, then ferruginous.

Taste insipid; odour none. Grassy places, gardens. Sept. $\quad I_{\frac{1}{2}} \times I_{\frac{3}{4}}^{3} \times \frac{1}{8}$ in.

628. I. asterospora Quél. (from the star-shaped spores; aster, a star) $a b$.

$P$. expanded, umbonate, shining, sometimes viscid, sooty-brown, brown-striate. St. marginato-bulbous, whitish, often reddish at base, $G$. emarginato-adnexed to adnate, cinnamon.

Taste none; odour mouldy or none. Woods and open places amongst trees. Aug.-Oct. $2 \frac{7}{8} \times 3^{3} \times \frac{5}{16}$ in.

628a. I. brunnea Quél. (from its brown colour) $a$.

$P$. campanulate, umbonate, fibrilloso-silky, then cracked, chestnutbrown. St. stuffed, thickened below, fibrilloso-striate, light brown, apex white-pruinose. $G$. emarginate, broadest in front, ivory then umber, edge white, indented.

Amongst grass, near pines. Oct. $1 \frac{3}{8} \times I \frac{7}{5} \times \frac{1}{5}$ in.

629. I. eutheles Quél. (from the well-formed umbo; Gr. en, well, thele, a teat) $a b c$.

$P$. expanded, umbonate, fawn-colour, with darker stria. St. solid, subbulbous, whitish above, brownish below. $G$. adnate, subattenuate, tan- or olive-brownish.

Taste not unpleasant; odour of new meal, but disagreeable, or none. Amongst fir-leaves, woods. Aug.-Oct. $2 \frac{1}{2} \times 3 \frac{1}{2} \times \frac{1}{4}$ in.

630. I. margarispora Sacc. (from the pearl-like spores; Gr. margarites, a pearl) a.

$P$. expanded, broadly umbonate, fawn-colour or olive tan-brown. St. solid, whitish or shaded tan-olive, darker below. $G$. ascending, adnexo-adnate, faintly rufescent-pallid.

On the ground. Oct. $2 \times 3^{\frac{3}{4}} \times \frac{3}{4} \mathrm{in.}$

630a. I. prætervisa Quél. in Bres. (from the fact that it had been previously confused with 624 ; preter, before, rider, to see) $a$.

$P$. conico-campanulate, then expanded, umbonate, fibrillosovirgate, slightly viscid, fawn. St. solid, bulbous, pubescent, white then pale straw. $G$. attenuate behind, adnexed, white, then fawn with a white elge. Flesh white.

Taste mild; odour somewhat earthy. Woods, pine. Aug. $\mathbf{I}_{4}^{3} \times 2 \frac{1}{4} \times \frac{1}{4} \mathrm{in}$. Distinguished by its viscid pileus. Related to 624 and 627 , but distinct in its white or straw-coloured stem. 
630b. I. Bucknallii Mass. (after John Bucknall, mycologist) $a$.

$P$. campanulato-convex, fibrillose ; mid. subsquamose, brownish. St. fibrillose, brownish. $G$. adnexed, thick, subdistant, rustybrown, edge minutely fimbriate.

Under bushes. Autumn. $\frac{5}{8} \times I_{\frac{1}{2}} \times \frac{1}{16}$ in. Distinguished by the large size of the basidia, spores and periphyses.

630c. I. duriuscula Rea (from its firm substance) $a$.

$P$. fleshy, campanulate, expanso-gibbous, floccose, soon longitudinally fibrillose, tawny-ochreous ; mid. paler ; marg. at length revolute. St. solid, firm, apex and base slightly enlarged, striate, white. $G$. sinuato-adnate, decurrent as ribs on apex of St., somewhat crowded, white, then brown. Flcsh white.

Open places in woods. Sept. $2 \frac{3}{8}-2 \frac{3}{4} \times 3^{\frac{1}{4}} \times \frac{5}{5}$ in.

631. I. destricta Quél. (from the fibres of the pileus stripping off; destringo, to strip off) $a b c$.

$P$. flat, umbonate, whitish to vinous or rich sienna-brown, fibres stripping off and often showing white cracks. St. solid, sometimes subbulbous, light or dark rufescent or whitish above or below. $G$. uncinato-adnate, grey-brown or olive-shaded. Flesh rufescent.

Odour unpleasant. Woods, pine, oak. July-Oct. $2 \frac{1}{2} \times 2 \frac{1}{8} \times \frac{1}{4}$ in. Sometimes 4 inches or more high.

632. I. perbrevis Gill. (from the comparatively short stem ; perbrizis, very short) $a b$.

$P$. expanded, undulate, umbonate, dry, silky, fibrillose, tawny or rufous, becoming yellowish. St. stuffed, attenuate downwards, colour as P. G. uncinato-adnexed or sinuate, subdistant, tawny to cinnamon.

Woods, shady places. Autumn. $2 \times 1 \frac{1}{4} \times \frac{3}{16}$ in.

633. I. descissa Quél. (from the split pileus; scissus, split) a $b c$.

$P$. campanulate, umbonate, whitish to pallid fuscous or ochreoussiemna. St. hollow or solid, white or pallid, darker below. $G$. adnexo-free, fuscous.

Suspected poisonous. Woods, pine; rare. Sept.-Nov. $\mathbf{I}_{3}^{\frac{7}{8}} \times \mathbf{I} \frac{3}{4} \times \frac{1}{4}$ in. Var. auricoma Gill. smaller, $P$. golden-fibrillose. Somewhat resembling 640 , but differing in colour of $\mathrm{P}$. and absence of odour.

633a. I. Godeyi Gill. (after Dr. Godey, French botanist) $a$.

$P$. campanulate, obtusely umbonate, silky, fibrillose, rimose, whitish, then tinged rosy, sometimes rosy and ochreous. St. equal, subbulbous, colour of P., apex white-pruinose. $G$. adnexo-free, narrowed behind, whitish, then dusky cinnamon with an olive tinge, edge minutely flocculose, white.

Autumn. $\quad \mathrm{I}_{\frac{1}{4}}^{3} \times 2 \frac{3}{4} \times \frac{1}{4} \mathrm{in} . \quad$ Rosy-red and ochreous-rosy when bruised.

634. I. Trinii (after Carl Bernard Trinius, Russian botanist) $a b$.

$P$. convex, obtuse, whitish-rufescent, tawny or pale brown-reddish. St. stuffed, colour as $\mathrm{P}$., or paler. $G$. rounded-adnexed, 
ventricose, cinnamon with a white slightly jagged edge. Flesh pale salmon.

Odour strong of clove-pinks, evanescent. Grassy places, woods. Aug.Sept. $\frac{3}{4} \times 1 \frac{7}{8} \times \frac{1}{16}$ in. Sometimes $1 \frac{1}{2} \times 1 \frac{1}{2}$ in.

\section{d. Velutina.}

635. I. sambucina Quél. (from its being first noted under elder, sambucus) $a$.

$P$. convex, obtuse, often repand, whitish. St. solid, striate, colour as P. $G$. adnexed, colour as P. or pale tan-brownish.

Solitary. Odour strong, disagreeable. Woods, pine, grassy places. $2 \frac{1}{8} \times 1 \frac{5}{8} \times \frac{1}{2}$ in. Compare 638 , which has narrower gills.

636. I. cæsariata Karst. (from the woven-hairy pileus; cesariatus, covered with hair) $a b c$.

$P$. expanded, subumbonate, dull tawny. St. solid, pale brownishochreous. $G$. adnexo-adnate, dull whitish to brown.

Gregarious, subcæespitose. Odour disagreeable. Under beeches. Sept.-Oct. $2 \frac{3}{8} \times 1 \frac{7}{8} \times \frac{5}{16}$ in.

637. I. lucifuga Gill. (from its habit, apparently shumning light; lux light, fugio, to Hee) $a b c$.

$P$. plane, subumbonate, brownish-olivaceous or fawn. St. solid, paler than P., or pallid. $G$. adnexed, yellowish or olivaceous, then dark olive.

Odour strong, pleasant or unpleasant, somewhat of radish. Woods, pine ; frequent. Sept.-Oct. $\quad \mathbf{I}_{\frac{3}{8}} \times 2 \frac{3}{4} \times \frac{1}{8}$ in.

638. I. sindonia Karst. (from the woven surface of the pileus; Gr., sindon, muslin) a b $c$.

$P$. convex, gibbous or subumbonate, whitish or faintly ochreous; marg. when young fibrillose with V. St. hollow, whitish. $G$. attenuato-adnexed, whitish-fuscous.

Mixed woods, damp shady places; rare. Sept.-Oct. $2 \frac{1}{8} \times 2 \frac{1}{2} \times \frac{3}{16}$ in. Resembling 640, but differing in the hollow stem and absence of odour.

639. I. Clarkii Sacc. (after J. Aubrey Clark) $a$.

$P$. campanulate, obtuse, whitish. St. stuffed to solid, flocculose, colour as P. G. ascending, adnexed, whitish-fuscous with a white edge.

On the ground, shady places in woods. Oct. $\quad \mathbf{I} \times \mathbf{I} \frac{3}{4} \times \frac{3}{16}$ in.

639a. I. corydalina Quél. (from its odour of Corydalis ca'a) a.

$P$. campanulate, then expanded, fibrillose, white, the prominent um. glaucous-green. St. fragile, pruinose, white, then fuscous. $G$. adnato-emarginate, narrow, brown, with a white edge. Fles/ white, sometimes tinged violet.

Under oaks. Sept. $2 \times 2 \frac{5}{8} \times \frac{3}{8}$ in.

640. I. geophylla Quél. (from the earthy colour of the gills; Gr. ge, the earth, phullon, a leaf) $a b c$.

$P$. expanded, umbonate, never truly squamulose, white, lilac or pale or dark bright purplish-blue; mid. sometimes brown; 
or whole P. brown. St. stuffed, sometimes with slight trace of A., white, or as P. $G$. adnexo-free, separating from hymenophore, umber.

Odour usually faint, sometimes very strong and unpleasant. Woods, under trees, hedges. Autumn. $\frac{5}{8} \times 2 \times \frac{1}{16}$ in. Var. lateritia W. G. Sm., Agaricus geophylllus Sowerb., var. lateritius Stev. P. red, varying yellow or white, sometimes white-scaly. There is a large form, twice the size of type or larger.

641. I. scabella Quél. (from the roughish pileus; scabcr, rough) a $b$.

$P$. expanded, umbonate, scaly-torn, fuscous. St. white, or as P. $G$. adnato-adnexed, yellow-brown or fuscous.

Gregarious. Odour none. Woods, amongst short grass. Sept.-Nov. $\mathrm{I}_{2} \times \mathrm{I}_{4}^{\frac{3}{4}} \times \frac{1}{8} \mathrm{in}$.

641a. I. fulvella Bres. (fulz'us, yellowish-brown).

$P$. subhygrophanous, conico-campanulate, then expanded and umbonate, floccosely silky, yellowish, then brownish-olive; mid. tawny. St. stuffed, narrowed downwards, glabrous, lilac, then rufescent, apex white-pruinose. $G$. subdistant, ventricose, rounded behind and nearly free, pale lilac, then ochraceouscinnamon; edge fimbriate. Flesh yellow, rufescent-lilac at apex of stem.

Shady places. Autumn. $\frac{1}{2} \times 1 \frac{1}{8} \times \frac{1}{16}$ in. Allied to 641 .

642. I. violaceofusca Sacc. (from the dusky-violet colour of the stem and gills) $a$.

$P$. expanded, obtusely subumbonate, squamose, umber; marg. fimbriate with V. St. solid, pale violaceous above, pallid below. $G$. adnate or emarginate, pale dull violaceous, then umber.

Subcrespitose. Amongst grass in open places. Autumn. $2 \frac{1}{8} \times 1 \frac{3}{4} \times \frac{1}{4}$ in.

\section{e. Viscide.}

643. I. trechispora Karst. (from the rough spores; Gr. trachius, rough, spora, a seed) $a b c$.

$P$. expanded, umbonate, whitish with mid. tawny. St. stuffed, white with a mass of white mycelium at the base. $G$. emarginate, or attenuato-adnexed, pinkish-grey, then brown.

Wood, damp places, amongst ferns. July-Oct. $\quad 1 \frac{1}{4} \times 2 \frac{5}{8} \times \frac{1}{5}$ in. Sometimes fatal to pheasants.

644. I. vatricosa Karst. (from the stem, twisted below; vatricosus, with bad feet) ac $c$.

$P$. plane, broadly subumbonate, whitish or shaded light-yellowish. St. hollow. colour as P., white-pulverulent. $G$. adnexed, broadly emarginate, ochreous-fuscous.

Odour none. Woods, pine, bare places, on dead stumps, twigs, chips. Sept. $\quad 1 \frac{3}{4} \times 2 \frac{5}{8} \times \frac{3}{16}$ in.

645. I. Whitei Sacc. (after Dr. F. Buchanan White, botanist) a $c$.

$P$. hemispherico-campanulate, obtuse, tawny; marg. whitish. St. solid, white, becoming tawny. $G$. adnexed, tawny.

Woods, pine. Oct. $\mathrm{I} \frac{1}{5} \times \mathrm{I}_{\frac{3}{4}} \times \frac{1}{5} \mathrm{in}$. Allied to 640 . 
646. I. Tricholoma Sacc. (from its resemblance to species of Tricholoma) a $b c$.

$P$. convexo-plane, subdepressed, ivory-whitish, shaded pale buff; marg. fringed with V. St. stuffed, fibroso-scaly above, colour as P., or faintly shaded salmon. G. adnato-decurrent, crowded, pale clay-fuscous or faintly olive-shaded.

Woods, mixed. Nov. $1 \frac{1}{2} \times 1 \frac{1}{2} \times \frac{3}{16}$ in.

\section{HEBELOMA Quél.}

(From the fringe-like veil, seen in certain young examples; Gr. hebe, youth, loma, a fringe.)

I'eil partial - in 648, 654a and 655 forming an imperfect annulus - or universal, indicated by gluten, squamules, fibrillæ or silkiness. Hymenophore confluent and homogeneous with the fleshy stem. Pileus fleshy, cuticle continuous, smooth, damp, subviscid or

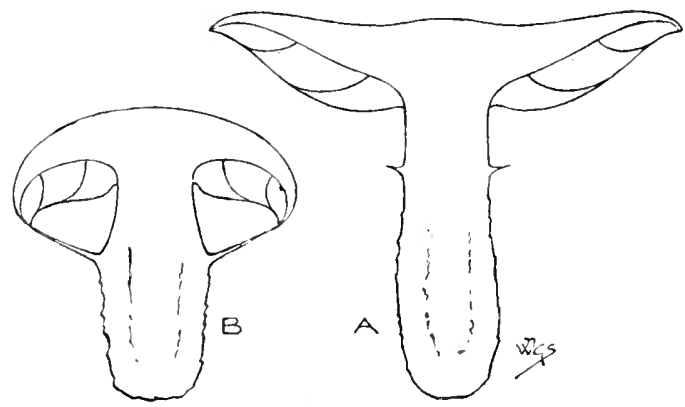

Fig. 35.-A, section of Hebcloma fastibile Quél. : B, ditto half-grown example showing veil. One-half natural size.

glutinous, margin at first incurved, sometimes silky from veil. Stem central, simple, fibrous-coated, submealy at the apex, simple or rarely imperfectly annulate. Gills sinuato-adnate or adnexed, at first pallid-whitish or clay-colour, then darker. Spores dull tan or somewhat clay-colour or brown. (Fig. 35.)

All the species, except 649 , grow on the ground; they are gregarious and strongly scented; some are poisonous, none are edible ; they appear early in autumn and last till late in the season.

Hikloma corresponds with the non-fibrillose species of Tricholoma, Entoloma and Hypholoma.

Species 647-669

a. Indusiatce. Pileus furnished with a cortina from the veil, by which the margin is often superficially silky. Stem usually white-pruinate or white-mealy above or minutely whitescaly.

b. Denudate. Pileus smooth; cortina obsolete. 
c. Pusille. Pileus scarcely an inch broad; stature that of Nancoria, but without the cartilaginous bark.

668,669

\section{a. Indusiate.}

647. H. mussivum Sacc. (from its uncertain position amongst the Indusiater; mussivus, undecided) a.

$P$. plane, obtuse or subumbonate, pale primrose-yellowish ; mid. sienna. St. stuffed or hollow above, fibrillose, light yellow. $G$. emarginate or somewhat rounded adnate, light yellow then subferruginous. Flesh becoming yellow.

Odour faint, not unpleasant. Woods, pine. Oct. $4 \frac{3}{4} \times 5 \frac{1}{\frac{1}{4}} \times \mathrm{r}$ in.

647a. H. sinuosum Quél. (from the frequently irregular pileus) a $b$.

$P$. fleshy, convex, expanded, obtuse, even, almost glabrous, viscid, then dry, yellowish-red to pale buff, sometimes whitish; marg. wavy. St. stuffed to hollow, equal, fibrilloso-striate, at first flocculoso-squamulose above, soft, biscuit. $G$. slightly adnexed, broadly emarginate to enarginato-free, dry, crowded, dull white to brownish-salmon and ferruginous. Flesh white.

Odour faint, pleasant. Woods. Aug. $3 \frac{5}{8} \times 5 \times \frac{7}{8}$ in.

648. H. fastibile Quél. (from its disagreeable odour) a $b c$.

$P$. convexo-plane, viscid in wet weather, pale yellowish-tan; mid. darker, sometimes sienna. St. solid, fibrillose, scaly or white silky, sometimes with an imperfect silky-floccose A. $G$. emarginate, subdistant, dull whitish-clay-colour or tan-brownish.

Subcrespitose, often in large dense rings. P'oisonous. Taste like radishes, but disagreeable and bitter; odour disagreeable, fetid. Woods, mixed pastures, near trees and bushes, sometimes on mushroom-beds; common. July-1)ct. $4 \times 2 \frac{3}{8} \times \frac{3}{4}$ in. Often mistaken for the mushroom and sold in markets with mushrooms. Often slug-eaten. Var. elegans Mass., $P$. purple-brown.

649. H. glutinosum Sacc. (from the glutinous pileus) $a b c$.

$P$. plane, obtuse, yellow-white or pale buff with mid. rich siennabuff, white-squamulose. St. stuffed, attenuate upwards, white, dull faint ochreous below, clad with fugitive white sc. $G$. sinuato-adnate, light yellowish then clay-cinnamon. Flesh pale reddish in stem.

Taste and odour mild, not unpleasant of radish. Woods, amongst leaves and branches, oak, beech ; frequent. Sept.-I)ec. $3 \frac{1}{2} \times 3 \frac{3}{8} \times \frac{1}{2}$ in. Sometimes one-third the size of type. Compare 678.

650. H. testaceum Quél. (from the colour of the pileus; testa, a brick) $a b c$.

$P$. convex, obtuse or subumbonate, crimson-sienna through red and yellowish to tan. St. hollow above, fibrillose, colour as $\mathrm{P}$. or paler. $G$. attenuato-sinuate or almost free, clay-colour then subferruginous or crimson-sienna.

Taste disagreeable, sickly.nauseous; odour faint of radish or none. Woods, fir, pastures; frequent. Sept.-Nov. $2 \frac{3}{4} \times 3 \frac{1}{4} \times \frac{5}{16}$ in. 
651. H. firmum Gill. (from its firm substance) $a$.

$P$. flat, subumbonate, subsquanulose, yellowish-brown or reddish; mid. darker. St. solid, brownish, darker below, whitesquamulose. $G$. adnexed or adnate, rounded, ferruginous, edge white, serrulate.

Odour faint. Woods, fir. Feb.-Oct. $2 \frac{3}{8} \times 3 \times \frac{3}{8}$ in.

652. H. claviceps Quél. (from its resemblance, when young, to a nail ; clavns, a nail, caput, the head) $a$.

$P$. hemispherical, gibbous-expanded, viscid, whitish or faintly yellowish; mid. sienna; marg. fibrillose with V. St. stuffed, whitish, fuscous below. $G$. emarginate, pallid brown.

Woods, mixed; uncommon. Sept.-Oct. $\mathrm{I}_{\frac{3}{4}} \times 3 \frac{1}{8} \times \frac{1}{4}$ in.

653. H. punctatum Quél. (from the viscid-dotted pileus; functus, a point) $a$.

$P$. plano-gibbous, pale sienna-brown, whitish at marg. St. hollow, silky-fibrillose, faintly brownish. $G$. sinuato-adnate, brownish.

Gregarious, in troops. Odour faint, not unpleasant. Woods, pine; uncommon. Sept. $2 \times 3 \frac{1}{4} \times \frac{1}{4}$ in.

654. H. versipelle Gill. (from its changeable appearance; zerto, to turn, pellis, skin) $a b c$.

$P$. flat, silky-agglutinate, sienna-reddish or dull tan. St. hollow, tan-white, fuscous below. $G$. rounded, serrulate, whitish-fleshcolour.

Subcæspitose. Odour faint, not unpleasant, but sometimes strong, fetidoily or like bugs. Grassy places, amongst fir-leaves. Aug. $2 \frac{1}{8} \times 3 \times \frac{3}{16}$ in

654a. H. strophosum Sacc. (from a fancied resemblance in the annulus to a sword-belt, Gr. strophos) $b$.

$P$. convex, plane, slightly subumbonate, somewhat viscid, bay or vinous-sienna, clouded deep claret-brown; marg. whitesilky. St. hollow, equal, silky, whitish, shaded brown or redsalmon within and without near base. $A$. superior, silkywhite. $G$. adnexo-free, crowded, ventricose, cream to dull flesh-colour and dull cinnamon. Flesh white.

On the ground. Oct. $1 \frac{1}{5} \times I_{\frac{3}{4}} \times \frac{3}{16}$ in.

655. H. mesophæum Quél. (from the dusky centre of the pileus: Gr. mesos, the middle, phaios, dusky) $a b c$.

$P$. plane, subgibbous, pale yellowish or pale pinkish-brown. St. fistulose, fibrillose, whitish or faintly ochreous, ferruginous below, sometimes with an imperfect fugitive $A . G$. rounded or emarginate, pale clay-ferruginous.

In troops, gregarious. Odour faint. Wvods, pine; uncommon. Sept.-Oct. $1 \frac{3}{4} \times 2 \frac{33}{46}$ in. 
656. H. subcollariatum Sacc. (from the gills which form an imperfect collar at the apex of the stem) $a b$.

$P$. convex, subviscid, pallid; mid. fuscous ; marg. at first white and scaly. St. stuffed or fistulose, brown at base. G. sinuatoadnate, clay-colour with white edge.

Subcrespitose. Taste and odour strong and rank, like 1527. On the ground. Mlay-Oct. $1 \frac{1}{2} \times 2 \frac{1}{8} \times \frac{3}{16}$ in.

657. H. senescens Sacc. (from the hoary margin of the pileus and the white apex of the stem; senseco, to grow old) $a$.

$P$. obtuse or subdepressed, ochreous-tawny. St. solid, squamulose, white above, fuscous below. $G$. rounded-adnexed, crowded, cinnamon.

Odour acrid. Amongst firs. Sept. $4 \times 3 \frac{3}{8} \times \frac{3}{4}$ in.

\section{b. Denudate.}

658. H. sinapizans Gill. (from its odour; sinapi, mustard) $a b$.

$P$. expanded, undulate, subdepressed, subviscid, pale claycolour; mid. yellowish. St. somewhat solid, whitish. $G$. emarginate, crowded, unspotted, not distilling drops, claycinnamon.

Solitary. Odour strong, usually of radish. Under trees, fields, woods; uncommon. Sept.-Oct. $5 \frac{3}{8} \times 2 \frac{7}{8} \times \mathbf{I}_{\frac{1}{2}}$ in. $P$. sometimes 9 in. in diam. Compare with 473.

659. H. erustuliniforme Quél. (from the shape of the pileus; crustulum, a small pie) $a b c$.

$P$. expanded, obtuse or subumbonate, subviscid, whitish; mid. pale reddish-ochreous. St. whitish. $G$. adnexed, crowded, distilling drops, clay-colour or brown.

sometimes forming large rings. Poisonous. Odour very strong of radish, but fetid or likie laurel flowers. Woods, mixed, lawns, pastures, on heavy soil; common. Aug.-Nov. $4 \frac{1}{1} \times 2 \frac{1}{x} \times 1$ in. Variable in size, Often mistaken for the horse-mushroom, 789 .

659a. H. subsaponaceum Karst. (from the somewhat soapy odour; sapo, soap).

$P$. fleshy, obtuse, pallid, darker when dry. St. equal, somewhat wavy, adpressedly fibrillose, apex somewhat mealy. $G$. adnate.

Taste bitter ; odour strong of soap. Woods, mixed, fir. Aug. $1 \frac{1}{1} \times 1 \times \frac{1}{8}$ in.

660. H. elatum Gill. (elatus, tall) $a b$.

$P$. expanded, subviscid, tan; mid. faintly ochreous, slightly reddish or shaded brown. St. stuffed, twisted, whitish or faintly ochreous. $G$. adnato-rounded with a tooth, pale cinnamon, becoming cinnamon-salmon, sometimes with a white edge.

Odour very strong of radish. Woods, pine, commons, under oaks. Sept.Nov. $2 \times 4 \frac{1}{4} \times \frac{1}{4}$ in. 
661. H. longicaudum Quél. (from the long stem; longus, long, cauda, a tail) $a b$.

$P$. expanded, sometimes umbonate, viscid, clay-colour: mid. darker. St. stuffed, subbulbous, fibrous-elastic, white above, tawny below within and without. $G$. emarginate, crowded, serrulate, cinnamon.

Odour faint, not unpleasant. Woods. Sept.-Nor. $5 \times 7_{4}^{3} \times \frac{5}{8}$ in. Var. radicalum Sacc. St. fusiform-rooting.

662. H. lugens Gill. (from its sombre colour ; lugeo, to mourn) $a$.

$P$. convexo-plane, subviscid, brown or yellowish. St. solid, shining, fibrilloso-striate, whitish. $G$. attenuato-adnexed or almost free, ferruginous.

Odour strong, not radish-like. Under beeches. $2 \frac{3}{x} \times 2 \frac{1}{2} \times \frac{3}{8}$ in.

663. H. truncatum Sacc. (from the short stem; truncus, maimed) $a b$.

$P$. obtuse, repand, irregular, ochreous-rufous or tan-ochreous, faintly shaded rose. St. solid, pruinate, white. $G$. emarginate or emarginato-free, watery-ferruginous.

Subcæspitose. Odour faint, not unpleasant. Grassy places in woods. Sept. $3 \frac{1}{4} \times 2 \frac{1}{5} \times \frac{3}{4}$ in.

664. H. nudipes Karst. (from the naked stem; midus, naked, pes, a foot) $a b$.

$P$. expanded, subumbonate, viscid, paie clay or oclireous-white; marg. thin, exceeding G. St. solid, smooth, whitish. $G$. emarginate, sienna-tan.

Odour faint, not unpleasant, somewhat of meal. Woods. Oct. $2 \frac{3}{s} \times 3 \times \frac{1}{4}$ in.

665. H. ischnostylum Sacc. (from the thin stem; Gr. ischnos, thin, stulos, a pillar) a $b$.

$P$. expanded, broadly subumbonate, subviscid, whitish; mid. pallid. St. naked, colour as P. G. adnate, clay-colour.

Odour none, or faint of meadow-sweet. Fields, plantations, woods, amongst grass. Sept.-Oct. $\mathrm{I}_{4}^{\frac{3}{4}} \times 2 \times \frac{1}{8}$ in.

666. H. nauseosum Sacc. (nauseosus, rank-smelling) $a b$.

$P$. conrex, gibbous, subviscid, ochreous-white; mid. sienna-tan. St. solid, silky-fibrillose, white-mealy above, base at length black. $G$. sinuate, broad, subdistant, salmon then ferruginous, sometimes with a purplish shade.

Odour very strong and offensive. Woods, mixed. Oct. $2 \frac{1}{4} \times 3 \frac{1}{\frac{1}{x}} \times \frac{1}{2} \mathrm{in}$.

667. H. capniocephalum Gill. (from the sooty-coloured pileus; Gr. kapnos, smoke, kephale, the head) ac.

$P$. convexo-plane, riscid, pale yellowish; mid. siemna or sooty; marg. at length black. St. stuffed, attenuate below, rufescent, fibrilloso-striate on a whitish ground. $G$. emarginate, subcrowded, brown-ferruginous.

Woods, mixed, elm-stumps. Oct.-Nov. $3 \frac{1}{8} \times 2 \frac{1}{4} \times \frac{3}{4}$ in. 


\section{c. Pusilla.}

668. H. magnimamma Karst. (from the large umbo; magnıs, large, mamma, breast) a.

$P$. plane, ochreous-red, then pale yellowish. St. stuffed or fistulose, smooth, naked, colour as P. G. adnate, subsinuate, ferruginous.

Amongst grass, under apple-trees. Sept. $\mathrm{I} \times 1 \frac{1}{4} \times \frac{1}{5} \mathrm{in}$.

669. H. petiginosum Quél. (from the scurfy-hoary pileus; petigo, scab) $a b c$.

$P$. convex, subumbonate, hoary-silky, brown or shaded ochreous, rufescent, slate or purplish. St. stuffed, rufescent, paler above, white-pulverulent. $G$. slightly adnexed, or free, crowded, olivaceous-brown or shaded rufescent.

Woods, beech. Oct. $\mathbf{I} \times \mathbf{I} \frac{7}{8} \times \frac{1}{8} \mathrm{in}$.

\section{FLAMMULA Quél.}

(From the frequent flame-like colours ; flamma, a flame.)

$V_{\text {eil }}$ fibrillose, fugitive or obsolete. Hymenophore confluent and homogeneous with the fleshy stem. Pileus fleshy, margin at first involute. Stem central, subannulate or simple, fleshy-fibrous, not

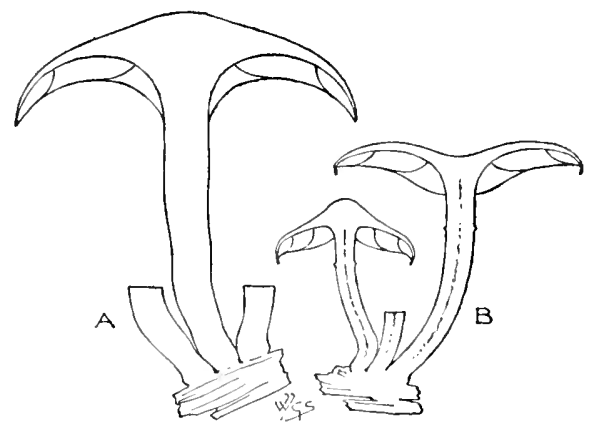

Fig. 36.-A, section of Flammula gymnnpodia Quél. ; B, ditto $F$. flavida Quél. One-third natural size.

mealy above. Gills decurrent or adnate, without a sinus, commonly entire and of one colour, at first whitish, clay-colour or yellowish, then coloured by the spores. Spore's mostly pure ferruginous, sometimes fuscous-ferruginous or tawny-ochraceous. (Fig. 36.)

The species usually grow on wood, some grow on the ground, many are bright yellow, orange or orange-brown in colour. Some agree in structure with Clitocybe and Clutopilus, those growing on wood approach Pholiota, and agree with Armillaria and in part with Hibcloma and Stropharia. 
a. Gymnote. Pileus dry, most frequently squamulose. Veil none, except 673 and 676. Spores ferruginous, fuscousferruginous in 675 .

$670-677$

b. Lubrica. Pileus covered with a continuous, subseparable, smooth, viscid pellicle. Veil fibrillose. Gills usually not truly sinuate. Spores ferruginous, fuscous-ferruginous in 685 , not tawny. Gregarious, terrestrial, rarely growing on wood. Allies of Hebeloma.

$678-685$

c. Ude. Pileus with a continuous, non-separable cuticle, slightly viscid in rainy weather. Veil appendiculate at the margin of the pileus, or forming an imperfect fibrillose annulus. Spores not tawny or ochraceous. Cæspitose. Growing on wood. Allies of Pholiota.

$686-694$

d. Sapinere. Pilcus scarcely pelliculose, flesh scissile or torn above into scales, not viscid. $V_{c}$ il fibrilloso-adpressed to the stem, not appendiculate at the margin of the pileus. Gills light yellow or yellow, then tawny. Subcrespitose. On pines, or terrestrial amongst pine-branches. 695-698 c. Sericelle. Pileus dry, or at first viscid, slightly silky. Veil fibrillose.

$699-702$

\section{a. Gymnotie.}

670. F. gymnopodia Quél. (from the naked stem; Gr. gumnos, naked, pous, a foot) $a$.

$P$. convex, gibbous, squamulose, ochreous-sienna; mid. darker. St. solid, paler than P. $G$. deeply decurrent, crowded, colour as P., cinnamon or orange.

Crespitose. Pine sawdust, on the ground. Autumn. $4 \frac{3}{ \pm} \times 3 \times \frac{1}{2}$ in.

671. F. Aldridgei Mass. (after Miss Emily Aldridge) $a$.

$P$. infundibuliform, velvety, rich orange; mid. sienna. St. hollow, colour as P., black below. $G$. deeply decurrent, rich orange.

Gregarious. Woods, amongst moss. Sept. $2 \frac{1}{4} \times 2 \frac{3}{4} \times \frac{1}{4}$ in.

672. F. vinosa Gill. (from its colour ; zinum, wine) $a c$.

$P$. infundibuliform, flocculose, umber, pink-shaded. St. solid, attenuate upwards, flocculose, paler than P. G. decurrent, colour as St., faintly pinkish at base.

On the ground; rare. Oct. $2 \frac{3}{4} \times 1 \frac{1}{2} \times \frac{5}{16}$ in.

673. F. purpurata Sacc. (from its purple colour) $a b$.

$P$. expanded, subumbonate, minutely flocculose, purple to brownish-purple. St. solid, pallid above, purplish-brown below. $A$. superior, red-fibrillose. $G$. adnate, lemon-yellow then bright ferruginous, purple-brown where touched.

Taste very bitter. Tree-fern stems. May. $2 \times 1 \frac{3}{8} \times \frac{3}{16}$ in. 
674. F. floceifera Sacc. (from the white fibrils on the pileus; flocils, a flock of wool, fero, to carry) a.

$P$. expanded, tawny. St. hollow, attenuate downwards, whitesilky, pale ochreous below. $G$. adnate, wrinkled, ferruginous with a white edge.

Crespitose. Lime-stumps. Oct. $2 \times 1 \frac{1}{2} \times \frac{1}{4}$ in.

675. F. decipiens Sacc. (from its likeness to 685 ; decipio, to deceive) a b c .

$P$. convex, subumbonate, sienna; mid. almost white. St. stuffed, attenuate downwards, striate, tawny. $G$. decurrent, crowded, sienna. Flesh yellowish.

Subfasciculate. Burnt gorse-stumps; in company with 685 . June. $1 \frac{1}{4} \times$ I $\frac{3}{4} \times \frac{1}{4}$ in.

676. F. clitopila Sacc. (from the depressed pileus; Gr. klitos, a declivity) $a b$.

$P$. expanded, dry, pale purplish-brown, becoming whitish. St. hollow, subventricose, attenuate downwards, slightly fibrillose towards base, at first white then paler than P. $A$. imperfect, fibrillose. $G$. sinuato-adnexed, crowded, colour as St.

Amongst firs. Nov. $2 \frac{1}{8} \times 2 \frac{1}{4} \times \frac{3}{8}$ in.

677. F. nitens Sacc. (from the shining pileus; nitens, shining) $a b$.

$P$. expanded, obtuse, dry, silky, deep purple-brown or deep bright chestnut. St. solid, fibrillose, reddish-salmon. $G$. adnate, crowded, clay-umber. Flesh salmon-white.

Crespitose. Taste and odour none. On the ground. Aug.-Sept. $1 \frac{3}{4} \times 2 \frac{1}{2} \times \frac{3}{5}$ in.

\section{b. Lubrice.}

678. F. lenta Gill. (lentus, tough) $a b c$.

$P$. plane, obtuse, whitish, clay-colour to lemon-white. $V$. white, stretching from marg. to St. in infancy. St. substuffed, paler than P., pale rufous below, white-scaly. $G$. adnate, sinuate or decurrent, ferruginous. Flesh white to pale sulphur, brownish at base of St.

Sometimes ciespitose. WVoods, mixed, beech, oak, fir, on wood, leaves, on the ground, meadows. Sept.-Dec. $2 \frac{1}{2} \times 2 \frac{1}{2} \times \frac{1}{4}$ in. See 649 .

679. F. lubrica Quél. (from the slimy pileus; lubricus, slimy) a.

$P$. flat, obtuse or depressed; mid. deep sienna; marg. orangeyellow. St. solid, laxly fibrillose, white above, fuscous below. $G$. adnate or with a decurrent tooth, clay-colour.

Almost scentless. On or near trunks. Autumn. $2 \frac{1}{2} \times 2_{4}^{3} \times \frac{5}{16} \mathrm{in}$.

680. F. lupina Karst. (from the colour of the pileus; lupus, a wolf) $a c$.

$P$. obtuse, then plano-depressed, tawny. St. stuffed, whitish above, ferruginous and adpressed-fibrillose below. $G$. adnatodecurrent, light yellowish. Flesh white.

Taste bitter; odour mild to strong and pungent. l'astures. Aug.-Oct. I $\frac{7}{8} \times 2 \frac{1}{\frac{1}{4}} \times \frac{1}{4}$ in. 
681. F. mixta Karst. (from its position amongst its allies; mixtus, mixed) $a b c$.

$P$. plane, subdepressed, tan or shaded-ochreous; mid. slightly sienna. St. white-ochreous above, laxly fibrillose and rufous below, minutely fibrilloso-annulate. $G$. adnate or adnatodecurrent, separating from stem, clay-colour.

Subcaspitose. Taste disagreeable; odour slight or none. Woods, pine, mixed ; rare. Aug.-Nov, $2 \frac{1}{8} \times 3 \times \frac{3}{16}$ in.

682. F. juncina Sacc. (from its habitat; juncus, a rush) $a b c$.

$P$. convex, sulphur-ochreous : mid. brown. St. stuffed, attenuate downwards, subfibrillose, colour as P. $G$. adnate, crowded, very thin, red-brown. Flesh sulphur-whitish or livid-brownish.

Taste nauseous, disagreeable, somewhat bitter. Dead bulrushes. Nov. I $\frac{5}{8} \times 3 \frac{3}{4} \times \frac{3}{16}$ in. A close ally of 681 .

683. F. gummosa Quél. (from the viscid-gummy pileus) a $b c$.

$P$. flat, obtuse or depressed, light yellowish or olive-shaded; marg. appendiculate with V. St. silky-fibrillose, paler than P., ferruginous below. $G$. adnate, crowded, cinnamon.

Crespitose. Snspected poisonous. Taste not bitter; odour none. On and about stumps. Oct.-Dec. $2 \frac{1}{2} \times 2 \frac{7}{8} \times \frac{5}{16}$ in.

684. F. spumosa Karst. (from the frothy-riscid pileus; spuma, froth) a $b c$.

$P$. Hat, obtuse or depressed, light yellowish or olive-shaded; mid. sienna; or whitish with mid. sulphur and yellow; marg. appendiculate with V. St. silky-fibrillose, paler than P., ferruginous below within and without. $A$. slight, fibrillose, evanescent. $G$. adnate, crowded, pale olive-brown to cinnamon.

Solitary, gregarious or subcæspitose, sometimes in rings. Taste not bitter; odour none. Woods, fir, stumps, sawdust. Sept.-Dec. $2 \frac{5}{8} \times 2 \frac{7}{5} \times \frac{5}{16}$ in.

685. F. carbonaria Quél. (from its habitat; arbbo, charcoal) a $b c$.

$P$. plane, sometimes depressed, tawny. St. fibrilloso-squamulose, brownish-white above, brown below. $G$. adnate, fuscous, claycolour. Flesh brownish-white. Spores fuscous-ferruginous.

Single or subcæspitose. Taste sweetish. Burnt wood, earth and leaves in and near woods; frequent. Sept.-Dec. $2 \frac{1}{2} \times 2 \frac{3}{4} \times \frac{1}{4}$ in. Sometimes $4 \times 4$ in.

\section{c. Ude.}

686. F. filia Mass. (filia, a daughter; derivation obscure) a $b$.

$P$. expanded, subumbonate, buff; mid. sienna; marg. whitezoned with V. St. stuffed, fibrilloso-striate, rooting, partially annulate, whitish above, buff or salmon-buff below. $G$. sinuatodecurrent, tan-buff or olive-shaded.

Odour none. Woods, logs, on the ground. Oct. $4 \times 6 \times 2$ in. 
687. F. fusus Gill. (from the spindle-shaped stem ; fusus, a spindle) $a$. $P$. plane, depressed, whitish-brown ; mid. sienna. St. stuffed, whitish above, pale brown below. $G$. subdecurrent, ferruginous.

Solitary, gregarious, sometimes crespitose. Taste mild. IVoods, stumps, on the ground. Sept.-Nov. $4 \times 2 \frac{1}{4} \times \frac{5}{8}$ in. Var. superba Mass., bright decp orange ; $G$. bright yellow.

688. F. astragalina Quél. (from its colour ; Gr. astragalinos, a goldfinch) $a b$.

$P$. expanded, blood-saffron; mid. almost vermilion; marg. yellow, with fibrillose sulphur-white V. when young. St. hollow, saffron ; base darker. $G$. sinuato-adnate, white, sulphur, then yellowish-brown or olive-shaded. Flesh saffron.

Taste and odour bitter, nauseons, as in 69. Stumps, pine, fir. Aug.-Oct. $1 \frac{7}{8} \times 3 \frac{1}{2} \times \frac{1}{4}$ in. Sumetimes turns black when cut or bruised.

688a. F. rubicundula Sacc. (from the vinous stains; rubicundulus, somewhat reddish) a $b$.

$P$. fleshy, convex, plane, viscid and innato-fibrillose, then smooth, yellow, becoming vinous-shaded; marg. paler. St. solid, whitish then red-shaded, white-mealy above. $G$. sinuatoadnate to adnato-decurrent, crowded, light ochre then ferruginous; edges reddening with age. Flesh bright yellow, then lighter.

Taste acrid; odour none. Woods, under scrub-oak. July-Sept. $3 \times 4 \frac{3}{4} \times \frac{9}{16}$ in. Allied to 688.

689. F. alnicola Quél. (from its frequent habitat; almus, alder) a $b c$.

$P$. Hat, sulphur-yellow; mid. sienna; marg. clouded sulphurgreenish. St. attenuato-rooting, sulphur-yellow above, sienna below, sometimes with an imperfect fibrillose A. $G$. adnate with a small tooth, bright ferruginous. Flesh sulphur-yellow. Spores umber.

Usually fasciculate. Taste and odour strong, dry-insipid, bitter or acrid. On or near stumps, alder, willow, oal, thorn, birch, sallow; rare. Sept.-Oct. $2 \frac{1}{4} \times 4 \frac{1}{4} \times \frac{1}{4}$ in.

690. F. flavida Quél. (faz'us, light yellow) a $b c$.

$P$. expanded; mid. darker yellow. St. colour as P., ferruginous at base. $A$. slight, fibrillose, fugitive. $G$. adnate, tawnyferruginous. Flesh light-yellowish.

Cæespitose, sometimes in large clusters. Tasteless or insipid. Woods, stumps, fir, lime, on the ground ; frequent. Aug. - Nov. $3 \frac{1}{2} \times 3 \frac{1}{2} \times \frac{5}{16}$ in.

691. F. inaurata Sacc. (from the colour; inauro, to gild) a $b c$.

$P$. expanded, sulphur-ochreous; marg. appendiculate with V. St. lemon-white, ferruginous within at base. $V$. slight, fibrillose, fugitive. $G$. adnate with a tooth, pale yellowish clay-colour, then vinous-clay.

Single or crespitose. Tasteless or insipid. Willows. Nov. $1 \frac{1}{4} \times 1 \frac{3}{8} \times \frac{3}{16}$ in. 
692. F. conissans Gill. (from its abundant dust-like spores; Gr. konis, dust) $a b c$.

$P$. flat, subdepressed, light yellowish-tan or pale bronzy-ochre. St. attenuate downwards, lighter than $P$., ferruginous below, base white-villous. $G$. adnate with a tooth, crowded, fuscousferruginous. Flesh white.

Densely crespitose. Taste and odour none or acid. Woods, willows. Oct.Nov. $2 \frac{1}{4} \times 4 \times \frac{1}{4}$ in. Often passed over as 826 .

693. F. inopus Karst. (from its fibrillose stem; Gr. is, incs, a fibre, pous, a foot) $a b c$.

$P$. expanded, obtuse, pale yellowish-tan or sulphur ; mid. sienna. St. fistulose, attenuato-rooting, pallid above, sienna-brown below within and without, sometimes with a fibrillose A. when young. $G$. adnate, yellowish-white, pale olive-brown or sulphur-greenish, sometimes fuscous. Flesh paler than P.

Fasciculate. Woods, stumps, fir, larch, rooting amongst pine-leaves, on the ground; rare. Vernal and Aug.-I)ec. $3 \times 8 \frac{1}{2} \times \frac{5}{16}$ in. Nust not be confounded with 824 .

694. F. apicrea Gill. (from its taste; Gr. apikros, not bitter) a.

$P$. expanded, pale ochreous-tan ; mid. sienna. St. hollow, colour as P., fibrillose, ferruginous below. $G$. adnate or adnatodecurrent, ferruginous.

Subcrespitose. Odour mild. Woods, pine, trunks. Sept.-Oct. $4 \frac{1}{2} \times 3 \frac{1}{1} \times \frac{7}{16}$ in.

\section{d. Sapinece.}

695. F. hybrida Gill. (from its hybrid characters) $a b c$.

$P$. flat, obtuse or subdepressed, tawny-buff, orange or sienna. St. attenuate upwards, tan-brownish, brownish below. $G$. adnate, tawny or rufous, unspotted. Flesh yellowish or brownish.

Subcrespitose or in troops. Fir, stumps, branches, under beeches, on saw. dust and wood ; rare. Aug.-Dec. $2 \frac{1}{5} \times 2 \frac{1}{5} \times \frac{1}{4}$ in.

696. F. sapinea Quél. (from its growing on pine-wood; sapinus, a pine) $a b c$.

$P$. flat, golden-tawny or orange-vermilion; mid. darker; marg. appendiculate with white V. in young examples. St. sulcate, orange or fuscous-white above, white or brown below. $A$. fibrillose, fugitive. $G$. adnate, colour as $P$. or tawnycinnamon.

Single or cæespitose. Taste unpleasant, bitter ; odour strong, not unpleasant. Fir-stumps, branches, sawdust, wood, in and near woods, sometimes on the ground, under beeches and larches. Aug.-Jan. $2 \frac{5}{5} \times 3 \frac{1}{2} \times \frac{1}{4}$ in. Var. terrestris Sacc., fusiform-rooting.

697. F. liquiritiæ Quél. (from its taste of liquorice) $a c$.

$P$. flat, subumbonate, bay-brown or orange-tawny; mid. darker. St. hollow, attenuate upwards, striate, tawny or ferruginous. $G$. adnate or sinuate, golden or tawny.

Gregarions or cæspitose. Taste sweet; odour acid. Firs. Oct. $1 \frac{7}{8} \times 2 \frac{7}{8} \times \frac{3}{16}$ in. 
698. F. picrea (Gr. pikros, bitter) $a b c$.

$P$. convex, obtuse, rufous; traces of white $\mathrm{V}$. at marg. in young examples. St. fistulose, umber, darker at base; when young wholly white-pulverulent. $G$. adnato-ascending or decurrent, ferruginous.

Crespitose. Pine-stumps, old deal boards; rare. Oct.-Dec. $2 \frac{1}{4} \times 4 \frac{3}{8} \times \frac{1}{4}$ in.

\section{c. Sericella.}

699. F. Filicea Sacc. (from its habitat, tree-fern stems; flix, a fern) $a b c$.

$P$. flat, subumbonate, golden-yellow, appendiculate with V. St. stuffed or hollow, fibrillose, colour as P., ferruginous below. $r$. annulate, fibrillose, fugacious, reddish. $G$. adnate, tawny.

Tree-ferns in greenhouses. Spring and summer. $\quad 1 \frac{7}{8} \times 1 \frac{3}{4} \times \frac{1}{5} \mathrm{in}$.

700. F. ochrochlora Karst. (from the colour of the pileus; Gr. ochros, yellow, chloros, green) a $b$.

$P$. plane, subumbonate, subviscid, pale dull olive-ochre, minutely white-squamulose. St. fistulose, paler than P., ferruginous at base, white-squamose. $\quad I$. slight, seen in young examples. $G$. adnate, olivaceous, then olive-brown.

Crespitose. Old trunks, furze-roots, hazel-sticks. Aug.-Nov. $2 \frac{1}{8} \times 2 \frac{3}{4} \times \frac{1}{4}$ in.

701. F. helomorpha Quél. (from its resemblance to a nail ; Gr. helos, a nail, morphe, form) a $c$.

$P$. convex, gibbous or obtusely umbonate, viscid, white or claywhite. St. solid, smooth, tan-white. $G$. plano-decurrent, clay-white. Spores pale brown.

Scattered. Wood, fir. Nov. $\frac{3}{4} \times 1 \frac{5}{8} \times \frac{1}{8}$ in.

702. F. scamba Sacc. (from the frequently curved stem; Gr. skambos, bow-legged) $a b$.

$P$. plane, subdepressed, viscid in wet weather, whitish or faintly ochreous. St. stuffed, flocculose or mealy, colour as P.; base sometimes faintly rufescent. $G$. adnate, light clay-yellowish. Flesh brownish.

Gregarious. Suspected poisonous. Odour strong like 1527. Woods, pine, rotten wood, larch, on the ground; uncommon. Sept.-Oct. $1 \frac{3}{8} \times I_{8}^{3} \times \frac{1}{8}$ in.

\section{NAUCORIA Quél.}

(From the almost obsolete veil; nuncum, a trifle.)

Veil squamulose, fugacious or obsolete. Hymenophore confluent with, but heterogeneous from the cartilaginous stem. Pilius more or less fleshy, convex, conical or convexo-plane, becoming flat, 
naked, flocculose, squamulose, silky or atomate; margin at first incurved, not usually striate. Stem normally central, cartilaginous, fistulose or spongy-stuffed, simple or imperfectly annulate. Gills free or adnate, not decurrent. Spores ferruginous. (Fig. 37.)

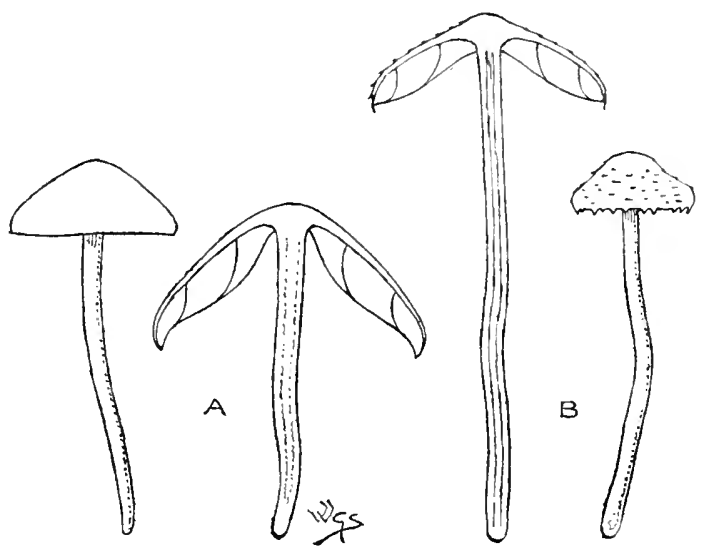

Fig. 37.-A, Naucoria Cucunis Gill. ; B, N. porriginosa Karst. Entire and in section; one-half natural size.

The species grow on the ground or are epiphytal, sometimes rooting; they are mostly small in size and brown in colour.

Nancoria corresponds in structure with Collybia, Leptonia, and partially with Psilocybe and Panceolus.

Species $703-746$

a. Gymmoter. Pileus smooth. I'́il obsolete, except 711 and 717. Stores ferruginous, not becoming fuscous-ferruginous, except 712.

Gills usually free or slightly adnexed.

Pileus convexo-plane. Gills usually adnate.

$703-716$

Pileus campanulate, then expanded, except 722 and 723.

Gills usually adnate.

$722-728$

b. Phaoter. Pileus naked. Veil potential, but rarely manifest; when visible, in the form of a fugacious cortina. Gills and spores fuscous-ferruginous.

Growing in fields and on plains, except 730 .

$729-734$

Growing in moist uncultivated woody places.

$735-738$

c. Lepidote. Pileus flocculose or squamulose. Veil manifest. Spores ferruginous, except 744.

Pileus with separating, superficial squamules.

739,740

Pileus with innate squamules.

$741-744$

Pileus without scales, silky or atomate.

745,746 


\section{a. Gymnote.}

703. N. lugubris Sacc. (from its sombre colours; lugeo, to mourn) $a$. $P$. expanded, gibbous or subumbonate, sometimes glutinous, ochreous-tan to ferruginous. St. fusiform-rooting, whitish above, ferruginous below. $G$. ferruginous.

Often crespitose. Mountainous fir-woods. Autumn, $2 \times 3 \frac{7}{8} \times \frac{3}{8}$ in. Resembling 229.

704. N. festiva Bres. (festiz'lls, handsome) a $b$.

$P$. expanded, umbonate, glutinous, brownish-olive, whitish when dry. St. attenuate downwards, buff-white. $G$. ventricose, crowded, salmon-ferruginous.

Woods, amongst dead leaves and grass. Sept. $1 \frac{5}{8} \times 4 \times \frac{3}{16}$ in.

705. N. obtusa Sacc. (from the obtuse pileus) $a b$.

$P$. campanulate, sienna-reddish, becoming pale; marg. substriate. St. paler than P. $\quad l$. obsolete. $G$. adnato-ascending, edge serrulate, colour as P. or St.

(on the ground. Autumn. $1 \frac{3}{5} \times 2 \frac{1}{2} \times \frac{3}{16}$ in.

706. N. subglobosa Sacc. (from the shape of the pileus) a $c$.

$P$. subviscid, yellowish or pale brassy-yellow. St. striate, paler than P. $G$. ascending, ventricose, ochreous flesh-colour. Spores pale, pallid.

On the ground. Autumn. I $\times 2 \frac{1}{5} \times \frac{1}{8} \mathrm{in}$.

707. N. hamadryas Sacc. (from the habitat ; Gr. hamadruas, a woodnymph) a $b$.

$P$. hygrophanous, expanded, gibbous or umbonate, bay-brownferruginous, becoming buff or ochreous-tan. St. shining silkyfibrillose, whitish-tan, with slight A. low down in some young examples. $G$. sinuato-adnexed, crowded, edge eroded, ferruginous.

Woods, on the ground. Nov, $2 \frac{1}{8} \times 3 \frac{1}{4} \times \frac{1}{4}$ in.

708. N. cidaris Sacc. (from a fancied resemblance in the pileus to a tiara, Gr. kidaris) a.

$P$. campanulate, clay-cimnamon to tan. St. attenuate downwards, colour as P. above, deep sienna-blackish below. $G$. adnexoascending, honey-colour.

Odour none. Woods, pine. Nov, $2 \frac{1}{8} \times 3 \frac{1}{8} \times \frac{3}{6}$ in.

709. N. Cucumis Gill. (from its frequent odour of cucumber, Cucumis). a $b c$.

$P$. campanulate, deep chestnut-umber; marg. tan-buff ; or wholly tan-buff. St. attenuate downwards, bay or rich purple-brown. $G$. adnexo-ascending, or strongly sinuate, ventricose, tan-buff. Flesh purple-brown.

Odour strong, rank and penetrating of stale or rotten fish, sometimes of cucumber or a combination of fish and cucumber as in the odour of the smelt. Woods, fir, grassy places, garlens, sawdust, fragments of wood; frequent. Sept.-Nov. $2 \frac{1}{4} \times 2 \frac{3}{4} \times \frac{3}{56}$ in. See 534. 
710. N. Echinospora Sacc. (from the spinulose spores; echimis, a hedgehog) a $b c$.

$P$. flat, subumbonate, moist hygrophanous, slightly furfuraceous, buff, then pale; marg. substriate. St. brownish-salmon, paler above, rufescent below, white-flocculose. $G$. sinuate, subdistant, ochre, olive-shaded.

Greenhouses. Aug. $\frac{7}{2} \times \frac{7}{8} \times \frac{1}{16}$ in.

711. N. anguinea Sacc. (from the stem, spotted like a snake, anguis) $a b$.

$P$. expanded, gibbous, ochreous to tan; marg. white-zoned with patches of V. St. attenuate upwards, $\tan$ above, bay-brown below, white-fibrillose with V. $G$. adnexo-ascending, pale ferruginous. Flesh brownish.

Odour none. On the ground. May-Nov, $2 \frac{1}{2} \times \frac{3}{3} \times \frac{3}{16}$ in.

712. N. centuncula Gill. (from the change of colour in the pileus; cento, patchwork) $a$.

$P$. plane, often excentric, fuscous-olive to light ochreous-greenish or ashy. St. attenuate upwards, ashy-light-yellowish. $G$. adnate, thick, colour as St. Spores ochraceous.

Gregarious or crespitose. Rotten wood, beech ; rare. Oct. $\mathbf{I} \times \mathbf{I} \frac{3}{8} \times \frac{1}{8}$ in.

713. N. horizontalis Quél. (from the partially horizontal stem) $a b c$. Watery-cinnamon or rufescent.

$P$. convexo-plane, obtuse, subexcentric, splitting. St. curved. $G$. rounded-free, broad.

In troops. Branches, $\log s$, felled trees, elm; rare. Dec. $\frac{3}{4} \times \frac{1}{2} \times \frac{1}{8}$ in.

714. N. rimulincola Sacc. (from its growing in small cracks of bark; rimula, a snall crack, incolo, to inhabit) a $c$.

$P$. hemispherical, umbilicate, plicate, subexcentric, deep cinnamon. St. curved, colour as P. G. broad, crenulate, whitish-cinnamon. Spores cinnamon.

Twigs, branches, elm, pear. Oct.-Dec. $\frac{1}{2} \times \frac{1}{2} \times \frac{1}{16}$ in.

715. N. semiflexa Sacc. (from the slightly curved stem; semi, half, flicto, to bend) a.

$P$. convex, subexcentric, chestnut. St. tan. G. adnexed, distant, tawny.

On the ground, on branches. Oct. $\frac{1}{2} \times \frac{1}{2} \times \frac{1}{16} \mathrm{in}$.

716. N. Pubricata Sacc. (from the white pileus tinged with red; rubrico, to colour red) a.

$P$. expanded. St. tan. $G$. adnexed, whitish to brownish. Spores pale.

Twigs, bramble. Autumn. $\frac{1}{4} \times \frac{1}{2} \times \frac{1}{32}$ in.

717. N. abstrusa Sacc. (from its secluded habitat; abstrustus, hidden) $a$.

$P$. viscid, ferruginous clay-colour: mid. brown. St. lustrous, colour as P., darker at base. G. crowded, cinnamon. Flesh pallid ferruginous.

On the ground, woods, sawdust. Oct. $I \frac{1}{1} \times 2 \frac{1}{1} \times \frac{1}{8} \mathrm{in}$. 
718. N. innocua Sacc. (imnocuns, harmless) $a$.

$P$. striate, pale ochreous-rufous to rufous, pale when dry. St. tan-flesh-colour, white-fibrillose, base woolly. $G$. light yellowochreous. Spores rubiginous.

Damp places. Autumn. $\mathbf{I} \frac{1}{8} \times \mathbf{I} \frac{1}{8} \times \frac{1}{8}$ in.

719. N. cerodes Quél. (from its waxy appearance; Gr. keros, wax) ac $c$.

$P$. depressed, striate, watery-cinnamon to tan. St. pallid above, brown below. $G$. adnate or adnexed, cinnamon.

Gregarious. Woods, amongst moss, on the ground, on burnt earth. MaySept. $\quad 1 \frac{3}{8} \times 1 \frac{3}{8} \times \frac{1}{8}$ in.

720. N. melinoides Quél. (from its resemblance to honey in colour; Gr. mel, honey, cidos, appearance) a $b c$.

$P$. obtuse, striate, shining, ochreous to sienna-ochreous. St. paler than P., whitish at top and bottom. G. crowded, somewhat tawny.

Lawns, pastures, roadsides. June-Nor. $1 \frac{1}{8} \times 1 \frac{5}{5} \times \frac{1}{16}$ in.

721. N. pusiola Gill. (from its small size; pusus, a little boy) $a$. $P$. subviscid, shining, yellow, tawny or lemon. St. subviscid, lemon. $G$. crowded, watery-cinnamon.

On the ground, amongst moss, grass. Autumn. $\frac{1}{2} \times 1 \frac{1}{2} \times \frac{1}{32}$ in.

722. N. nucea Sacc. (from the shape and colour; mux, a nut) $a$.

$P$. globose, lobed, umbilicate, pale chestnut; marg. strongly incurved. St. bulbous, silky-fibrillose, then even, white. $G$. adnexo-free, often waved, cinnamon.

Woods, pine, amongst heath and furze ; rare. Autumn. $\frac{3}{4} \times 3^{\frac{1}{2}} \times \frac{1}{1^{16}}$ in.

723. N. glandiformis Sacc. (from the shape of the pileus; glans, an acorn) $a b$.

$P$. at length hemispherical, obtuse or slightly depressed, lobed, tan, sienna-shaded; marg. strongly incurved. St. equal, striate, twisted, splitting, pale brownish-tan. $G$. adnexoascending, broad, crowded, serrate, whitish-umber.

On the ground. Oct. $1 \frac{1}{8} \times 3 \frac{1}{8} \times \frac{1}{8}$ in.

724. N. scolecina Quél. (from the flexuous stem; Gr. skolex, a worm) $a b$.

$P$. sometimes umbonate, bay-brown-ferruginous; paler at the striate marg. St. whitish above, fuscous below, at first wholly white-mealy. $G$. subdistant, ferruginous.

Dead leaves, moist ground under alders. Sept. $\frac{3}{4} \times \mathbf{I}_{4}^{\frac{3}{4}} \times \frac{1}{15}$ in.

725. N. striæpes Sacc. (from the striate stem; $p e s, a$ foot) $a b$.

$P$. obtuse, sometimes rugulose or lacunose, ochreous. St. brittle, white. $G$. adnexo-ascending, serrate, crowded, tawnyferruginous.

Gregarious or cæspitose. Lawns, amongst grass on soil-heaps. Nov. $1 \frac{5}{8} \times 3 \frac{5}{16} \times \frac{3}{16}$. Perhaps a var. of 723. 
726. N. sideroides Gill. (from the colour of the stem, like oxide of iron; Gr. sideros, iron, cidos, appearance) a $c$.

$P$. subumbonate, subviscid, pale yellowish to cinnamon; marg. slightly striate. St. slightly attenuate downwards, white above, becoming yellowish or ferruginous. $G$. with a tooth, somewhat cinnamon.

Stumps, trunks, chips, ash; rare. Oct.-Nov. $1 \frac{1}{ \pm} \times 2 \frac{1}{2} \times \frac{1}{16}$ in.

727. N. badipes Sacc. (from the brown stem; badius, bay-brown, pes, a foot) $a b$.

$P$. umbonate, tan to cinnamon; mid. darker; marg. striate. St. ferruginous, fuscous-blackish below, base white-floccose. $G$. ventricose, pale yellowish-ferruginous.

Under larches. Oct.-Nov. $1 \frac{1}{8} \times 3 \times \frac{1}{16} \mathrm{in}$.

728. N. nasuta Sacc. (from the shape of the umbo; nasutus, with a conspicuous nose) $a b$.

$P$. acutely umbonate, ochreous to citron- or greenish-ochreous; marg. striate; mid. orange or sienna. St. equal, flexuous, smooth, even, yellow, ferruginous below. $G$. broadly adnate, then rounded adnate, subcrowded, pallid, then brownish. Flesh thin, citron-lemon, ferruginous in St.

Gregarious. Swampy places under trees. Autumn. $1 \frac{1}{4} \times 1 \frac{7}{8} \times \frac{1}{15}$ in. Massee regards the British form as a distinct species, $N$. hydrophila.

\section{b. Pliceta.}

729. N. vervacti Quél. (from its habitat; zera'actum, fallow ground) a $c$. $P$. plane, subviscid, light yellow; mid. darker. St. smooth, whitish. G. adnate, toothed, ferruginous-fuscous. Flesh white. Pastures, gardens; uncommon. May-Nov. $1 \frac{1}{8} \times 1 \frac{3}{8} \times \frac{1}{8} \mathrm{in}$.

730. N. triscopus Sacc. (from the hair-like stem; Gr. thrix, a hair, pous, a foot) a.

$P$. convexo-plane, umbonate, bay-brown to ochreous. St. Hexuous, ferruginous. $G$. adnate or adnexed, subcrowded, dark ferruginous.

Copses, rotten wood, old wood in cellars. Autumn. $\frac{1}{2} \times 1 \frac{1}{8} \times \frac{1}{32}$ in.

731. N. pediades Quél. (from its frequent habitat; Gr. fedion, a plain) $a b c$.

$P$. plane, obtuse, rimoso-rivulose, yellow or somewhat buff. St. with a small basal bulb, colour as P., paler above and below. $G$. adnexed or adnate, subdistant, dull cinnamon. Flesh whitish. Pastures, lawns, roadsides, etc.; frequent. July-Nov. $I_{2}^{\frac{1}{2}} \times 2 \frac{5}{8} \times \frac{1}{10}$ in.

732. N. arvalis Quél. (from its habitat; arz' $u m$, a cultivated field) a bc. $P$. plane, subviscid, subrugose, tan-buff. St. enlarged downwards, rooting, or branched-rooting, colour as $\mathrm{P}$., furnished with an arachnoid A., when young. $G$. adnexo-sinuate, subdistant, warm-umber.

Taste disagreeable. On the ground, sea-sands. July. $\quad 1 \frac{1}{2} \times \mathbf{r} \frac{5}{5} \times \frac{1}{5}$ in. Rooting base $1 \frac{1}{2}$ in. 
733. N. semiorbicularis Quél. (from the hemispherical pileus; semi, half, orbicularis, round) a $b c$.

$P$. smooth, viscid, corrugate when dry, tawny-ferruginous to tan. St. pallid-ferruginous or tan, rarely with trace of A. G. adnate, subrounded or sinuate, ferruginous.

Pastures, roadsides, etc., examples in Brit. Mus. from interior of skull of a stranded whale; common. June-Nov. $1 \frac{1}{8} \times 2_{4}^{\frac{3}{4}} \times \frac{1}{8}$ in.

734. N. tabacina Gill. (from the colour of the pileus as of tobacco, tabacum) $a$. Bay-brown-fuscous.

$P$. plane, subobtuse, hygrophanous, traces of V. at marg. St. lighter above, darker below. $G$. adnate or adnexed.

Subcrespitose. Waysides, amongst short grass. Autumn. $\frac{3}{4} \times \mathbf{I} \frac{1}{4} \times \frac{1}{16} \mathrm{in}$.

735. N. tenax Gill. (tenax, tough) $a b c$.

$P$. expanded, obtusely umbonate, viscid, cinnamon or shaded olive, fuscous or pale fuscous-slate. St. adpressedly fibrillose, light-yellowish to slate-white. $G$. adnate, subcrowded, whitishfuscous.

Fields, amongst sticks. Oct. $1 \times 2 \frac{1}{4} \times \frac{1}{16}$ in.

736. N. Myosotis Quél. (from its growing in wet places with forgetme-not, Myosotis) a b.

$P$. expanded, umbonate, viscid, olivaceous or olive-brownish, becoming light; marg. striate, cortinate with white $\mathrm{V}$. St. often flexuous, fibrillose, white above, brownish below, sometimes with a fugitive $\mathrm{A}$. $G$. adnate with a tooth, subdistant, brown-ferruginous, with a serrate white edge.

Bogs with Myosotis and Fotentilla Comarum. July-Aug. $2 \frac{1}{4} \times 5 \frac{5}{8} \times \frac{1}{4}$ in. Very variable.

737. N. temulenta Quél. (from the bibulous substance and flexuous stem ; tcmulentus, drunken) $a b$.

$P$. expanded, subumbonate, moist, ferruginous or reddish, ochreous or whitish when dry; marg. striate. St. pale dull orange or reddish, white at base. $G$. adnate, subdistant, attenuate in front, reddish.

Woods, open moist places. Aug.-Sept. $\mathbf{1} \frac{1}{2} \times \mathbf{2} \frac{1}{2} \times \frac{1}{3}$ in.

738. N. latissima Sacc. (from the very broad gills; latissimus, very broad) a $b c$.

$P$. hemispherical, umber, orange-sienna-shaded, or orange-yellow ; mid. flat. St. greatly attenuate downwards, tan with a brownish base. $G$. rounded adnate, separating, greyish then brown.

Amongst grass. Sept. $1 \frac{1}{8} \times 2 \frac{1}{8} \times \frac{3}{16}$ in. Pileus sometimes $I_{4}^{3}$ in. in diam. The mycelium forms an earthy ball at base of stem.

\section{c. Lipidote.}

739. N. porriginosa Karst. (from the scurfy pileus; forriginosus, full of scurf) $a b$.

$P$. expanded, obtuse or subumbonate, dull yellow, clad with saffron flocci; marg. appendiculate with V. St. silky, then 
smooth, whitish above and below; mid. ochreous. $G$. adnate, cinnamon. Flesh lemon-white.

Amongst twigs and rubbish. Oct. $2 \times 4 \times \frac{3}{16}$ in.

740. N. sobria Gill. (from its not being hygrophanous; sobrizıs, sober) a $c$.

$P$. convex, obtuse or subumbonate, honey-colour. $V$. appendiculate at marg., fugacious. St. tan above, ferruginous below, sometimes white spotted with V. $G$. adnate, paler than $\mathrm{P}$.

Woods, mixed, on the ground. July-Oct. $\frac{7}{8} \times 1 \frac{3}{8} \times \frac{1}{8}$ in. Var. disfersa Sacc. is half the size of type.

741. N. erinacea Gill. (from the bristly pileus; erinaceus, a hedgehog) $a b c$.

$P$. expanded, depressed or umbilicate, umber or siemna. St. shaggy, deep sienna, lighter above and below. $G$. adnate with a tooth, clay-umber.

Solitary. Taste insipid. Dead branches; rare. Jan.-Dec. $\mathbf{I} \times \frac{1}{2} \times \frac{1}{8}$ in. Often difficult to see, being exactly the colour of the bark-matrix to which it is frequently adpressed.

742. N. siparia Gill. (from the covering formed by the veil ; siparium, a small curtain) $a$. Rufous-ferruginous, upper part of St. and G. somewhat paler.

$P$. convex, densely villoso-squamulose. $V$. appendiculate at marg. St. villoso-downy, except upper part. $\quad G$. adnate.

Wood, earth, dead fern-stens, caddis-worm cases, twigs; rare. July-Oct. $\frac{1}{2} \times 1 \frac{1}{8} \times \frac{1}{16}$ in. Often differs greatly in length of stem from 741 .

743. N. conspersa Quél. (from the besprinkled scales of the pileus; conspersus, besprinkled) $a b c$.

$P$. convex, hygrophanous, bay-brown or crimson-shaded. St. fibrillose, cinnamon or ochreous. $G$. adnate, crowded, dark cinnamon.

Gregarious. On the ground or amongst dead leaves and Sthagmum in woods; uncommon. Sept.-Oct. $\frac{7}{8} \times \frac{1}{2} \times \frac{1}{16}$ in.

744. N. escharoides Quél. (from the scurfy pileus; Gr. eschara, a scar, cidos, appearance) $a b$.

$P$. campanulate, obtuse, umbonate or umbilicate, tan or whitish ; mid. ochreous or fuscous, with an evanescent white V. St. adpressedly fibrillose, then smooth, pallid or pale ochre to fuscous. $G$. adnate with a tooth or emarginato-free, somewhat cinnamon, with a pale edge.

Gregarious, subcrespitose. Bare damp ground, under alders. Aug.-Oct. $\frac{7}{8} \times 1 \frac{1}{2} \times \frac{1}{16}$ in.

745. N. carpophila Quél. (from its growing on fruit, beech-mast, etc. ; Gr. karpos, fruit, philos, loving) $a$.

$P$. convex, obtuse, furfuraceous, atomate, tan-whitish. St. furfuraceous, then naked, pallid. $G$. adnexo-rounded or adnate, ochreous.

Beech-mast, leaves, etc.; rare. Sept.-Oct. $\frac{3}{16} \times \frac{3}{4} \times \frac{1}{32}$ in. 
746. N. graminicola Gill. (from its habitat; gromen, grass, colo, to inhabit) a $c$.

$P$. convex, sometimes papillate, shaggy-tomentose, fuscons to fawn-ochraceous. St. hirsute, fuscous. $G$. slightly adnexed, pallid ochreous

Decaying rushes, straw, grass, twigs. Oct. $\frac{3}{8} \times 1 \frac{1}{2} \times \frac{1}{32}$ in. The stem is rarely branched.

\section{GaLERA Quél.}

(From the shape of the pileus; grilirus, a cap.)

$V_{t}$ il fibrillose or obsolete. Hymcnofhore confluent with, but heterogeneous from the cartilaginous stem. Pilezs more or less membranous, conical or semi-ovate, sometimes expanding; margin striate, at first straight and adpressed to the stem. Stem central,

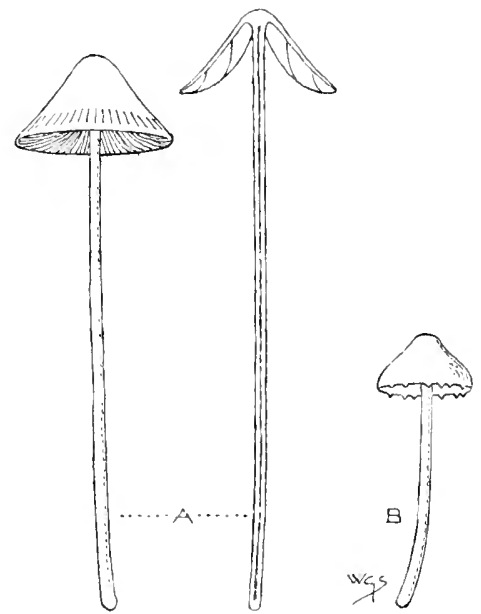

Firg. 33.-A, Galera tenera Quél., entire and in section. B, G. raviala Quél.

One-half natural size.

somewhat cartilaginous and, except 752, fistulose, simple or imperfectly annulate. Gills adnate or with a decurrent tooth, or adnexed and becoming almost free. Spores elliptical, smooth, ochreous-ferruginous or tawny-ochreous. (Fig. 38.)

The species are usually small, slender, tender and fragile; they grow on the ground or amongst moss. Mostly autumnal.

Gateri agrees in structure with Mycona, Nolanca, Psathyra and Psathyrella. 
a. Conoccphale. Veil obsolete. Pileus usually conico-campanulate, hygrophanous, somewhat even, when dry dotted with soft particles. Stcm tense and straight. Gills ascending, inserted at the top of the cone, somewhat crowded.

$746 a-754$

b. Bryogena. Veil cortinate, fugacious. Pilens membranous, campanulate, striate, smooth, hygrophanous, even when dry, opaque, very slightly silky. Stem thin, lax, flexile, slender. Gills broadly and planely adnate, broad, somewhat denticulate. Habitat amongst moss except 758 .

$755-759$

c. Eriodermea. Veil manifest, superficial, separating, at first chiefly round the margin, silky and squamulose. somewhat membranous.

\section{a. Conocepluale.}

746a. G. apala Quél. (from its habitat, rich pastures, not marshes as in some allied plants; $a$ without, palies, a marsh) $a c$.

$P$. obtusely campanulate, submembranous, smooth, hygrophanous, somewhat pale warm brown, or pallid-livid to white. St. hollow, attenuate upwards, fragile, velvety, white. $G$. almost free, somewhat narrow, ventricose, pale whitish-ochre.

In rich grassy places. Siept. $7 \frac{1}{8} \times 2 \frac{1}{4} \times \frac{3}{16}$ in. The form Stharobasis has a smooth stem and bulbous base.

747. G. lateritia Quél. (from its reddish-brick-colour ; lat'r, a brick) $a b c$.

$P$. conico-cylindrical, membranous, pale yellowish to ochreousbrown; marg. striate when moist. St. shining-whitish or paler than P., white-pruinose. $G$. adnexo-ascending, cinnamon.

Rich pastures, grassy places; rare. June-Oct. I $\times 4 \frac{1}{2} \times \frac{1}{8}$ in.

748. G. tenera Quél. (tcncr, tender) $a b c$.

$P$. submembranous, deep buff or ferruginous to whitish ; marg. striate when moist. St. shining, striate above, colour as $\mathrm{P}$. $G$. adnato-ascending, cinnamon.

Pastures, gardens, flower-pots, roadsides, grassy places in woods ; common. April-Dec. $\quad 1 \frac{1}{4} \times 4 \frac{1}{2} \times \frac{1}{8}$ in. Var. filosella Karst. $P$. pubescent; rotten wood.

749. G. siliginea Quél. (from the colour, like bread; siliginens, wheaten) $a b$.

$P$. campanulate, smooth, membranous, whitish to sienna-bay; when dry whitish and atomate-sparkling; mid. sometimes brown. St. subpruinose, tan-whitish, lilac or purplish, darker below. $G$. adnato-ascending, subdistant, ochreous then bright cinnamon.

Taste none; odour strong. Amongst short grass, road scrapings. Oct. I $\times 2 \frac{1}{2} \times \frac{1}{16}$ in. G. campanulata Mass, is a form of this. 
750. G. ovalis Gill. (from the semi-oval pileus) $a b c$.

$P$. ferruginous to dull yellowish. St. colour as P., at first imperfectly annulate. $G$. adnexo-ascending or free, ferruginous. Amongst grass, on dung; uncommon. Sept.-Oct. $\quad \mathbf{I}_{\mathrm{s}}^{\frac{5}{8}} \times 4 \frac{1}{4} \times \frac{1}{x}$ in.

751. G. antipus Quél. (from the sinuous, rooting base differing from the straight stem ; (ir. anti, opposite, pous, a foot) a b c.

$P$. expanded, hygrophanous, atomate, deep ochreous to whitish; marg. paler. St. attenuate below, the rooting base long, tortuous, tan-ochreous, sometimes with an imperfect $A . G$. adnexo-ascending, cinnamon. Spores rubiginous.

Single. Gardens, dung, newly made lawn; rare. Mar.-Aug. $1 \frac{3}{8} \times 2 \frac{1}{4} \times \frac{1}{15}$ in. Rooting base 2 in.

752. G. CONFERTA Gill. (from the crowded habit; confertus, crowded) $a$.

$P$. somewhat acutely pointed, white; mid. yellowish-brown. St. solid, shining white. $V$. downy, fugitive. $G$. ascending, adnexed or free, crowded, pale brownish. Spores fuscousferruginous.

Crespitose. Amongst tan in hothouses. Nov. Withering and becoming like soft paper in decay. Resembles 41 in habit.

752a. G. spicula Quél. (from the atomate pileus; spiculum, a little hard point) $\dot{b}$.

$P$. membranous, conico-campanulate, then expanded, smooth, hygrophanous, striate when moist, flocculose and atomate when dry, brown-ochre. St. hollow, thickened below, densely whitefloccose. $G$. adnate, ventricose, ochreous then cinnamon.

Coco-nut fibre. Nov. $\frac{3}{4} \times I \times \frac{1}{16}$ in.

753. G. spartea Quél. (from the rigid grass-like stem; spartum, esparto-grass) $a b$.

$P$. expanded, ferruginous-cinnamon, becoming paler; marg. striate when moist. St. smooth, whitish. $G$. adnate, darker than $\mathrm{P}$.

Gregarious. Amongst short grass and moss. Sept. $\frac{1}{2} \times 2 \frac{7}{3} \times \frac{1}{32}$ in. Sometimes wholly whitish-orange.

754. G. pygmæoaffinis Quél. (from its resembling Naucoria pygmaa, but differing in the elongate stem) $a b$.

$P$. flat, reticulato-wrinkled fuscous, honey-colour or tan. St. shining-white. $G$. adnexo-free, ferruginous-ochreous.

Tree-shaded walks, amongst grass, in a cucumber house, base of trunks. Nov. $13 \times 2 \frac{1}{2} \times \frac{1}{16}$ in.

\section{b. Bryegence.}

755. G. vittæformis Quél. (from a fancied resemblance in the striate margin to a head-band or chaplet, vitta) $a$.

$P$. brown; mid. sometimes paler. St. bright rusty-brownish. $I$. almost obsolete. $G$. adnato-ascending, colour as St.

Amongst moss. Nov, $\frac{3}{4} \times 2 \frac{3}{4} \times \frac{1}{10}$ in. 
756. G. rubiginosa Gill. (from the dark ferruginous stem; rubige, rust) $a c$.

$P$. wholly striate, cinnamon or honey-colour to tan ; mid. darker. $G$. adnato-ascending, distant, ochreous.

Amongst moss. Sept.-Oct. $I_{\frac{1}{4}}^{1} \times 2_{x}^{3} \times \frac{1}{8}$ in.

757. G. hypnorum Quél. (from its growing on moss; Hypmum, a genus of mosses) $a b c$.

$P$. campanulato-expanded, obtuse or umbonate, pale ochre or pale sienna to $\tan$; mid. darker. St. sienna below, paler and whitepruinose above. $G$. ventricose, distant, cinnamon-tawny.

Odour very strong, alkaline. Amongst mosses and hepatics in wools. May-Nov. I $\times 4 \times \frac{1}{16}$ in. Var. bryorum Gill. Larger than tyle. Var. sphagnorum Quél. On Sthagnum in swamps.

758. G. mniophila Gill. (from its growing on moss ; Gr. mion, moss, philos, loving) $a$.

$P$. subpapillate, fuscous-light-yellowish to clay-colour. St. fibrillose, mealy above, floccose below, yellow. $G$. adnatoascending, fuscous-clay-colour.

On moss. Sept.-Oct. $\frac{5}{8} \times 3 \frac{1}{4} \times \frac{1}{16}$ in.

759. G. minuta Quél. (from its very small size) $a$.

$P$. tan-ochreous. St. tawny; base forming a small white disc.

$G$. triangular, adnate, clay-colour, edge whitish.

Decayed wood, amongst moss. Sept.-Oct. $\frac{1}{8} \times \frac{3}{8} \times \frac{1}{32}$ in.

\section{c. Eriodermece.}

760. G. ravida Quél. (rac'us, grey) $a$.

$P$. tan-greyish or tan-umber; marg. appendiculate with white $V$. St. pallid, white-silky. $G$. adnexed, ventricose, ochreoussalmon.

Gregarious. On the ground, amongst chips and rotten wood. Autumn. $1 \frac{3}{4} \times 2 \frac{1}{5} \times \frac{1}{5}$ in.

761. G. mycenopsis Quél. (from its resemblance to a Myctua: Gr. opsis, resemblance) $a$.

$P$. expanded, umbonate, pale honey-colour ; marg. appendiculate with white V. St. white-yellowish, white silky. G. adnexed, ventricose, yellowish.

On Sthagnum, marshy ground. Aug.-Oct. $1 \frac{1}{4} \times 4 \frac{3}{8} \times \frac{1}{5}$ in. Somewhat like 350 .

XXX. TUBARIA Gill.

(From the trumpet-shape of typical species; tuba, a trumpet.)

Fil universal, floccose at margin of pileus. Hymenophore confluent with, but heterogeneous from the cartilaginous stem. Pileus submembranaceous, sometimes umbilicate or depressed, margin--except 
770-striate. Stem central, subcartilaginous, fistulose, simple or imperfectly annulate. Gills subdecurrent, broadest behind and
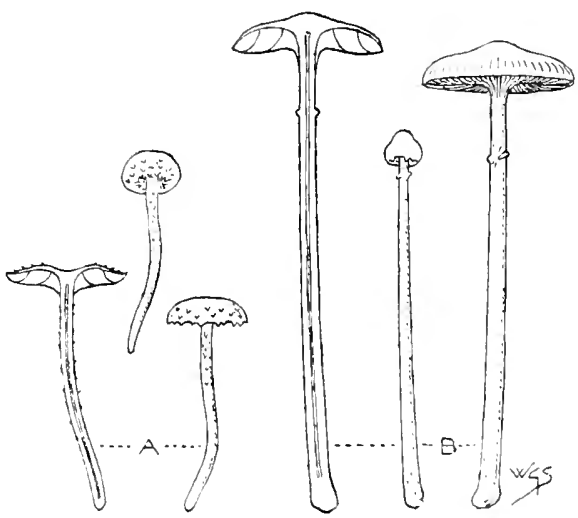

Fig. 39.-A, Tubaria furfuraca Gill.; B, T. taludosa Karst. Entire and in section. ()ne-half natural size.

somewhat triangular. Sfores ferruginous or fuscous-ferruginous. (Fig. 39.)

The species are all small; some grow on the ground, others on mosses, twigs, chips, leaves, etc.

Tubaria agrees in its chief structural characters with Omphalin and Eccilia.

Species 762-771

a. Ginnine. Stores ferruginous.

$762-768$

b. Pheota. Spares fuscous-ferruginous.

$769-771$

\section{a. Gimuince.}

762. T. eupularis Gill. (from the shape of the pileus ; cupularis, cup)shaped) $a$.

$P$. plano-depressed, hygrophanous, smooth, rufescent to light yellowish. St. whitish or faintly ochreous. $G$. serrulate, reddish.

Nountainous heaths, amongst grass. Aug.-Sept. $\quad I_{\frac{1}{5}}^{\frac{1}{5}} \times 2 \times \frac{1}{16 j} \mathrm{in}$.

763. T. furfuracea Gill. (from the scurfy pileus; furfur, bran) a $\dot{v} \mathrm{c}$. $P$. Hat, umbilicate, cinnamon to tan-whitish; marg. substriate when moist, appendiculate with white-squamulose V. St. floccose, colour as P., villous at base. $G$. subdecurrent, bright cinnamon or clay.

Gregarious. Tasteless or pleasant. Thatch, chips, twigs, wood, shavings, ivy, humus. Jan.-Dec. $\mathbf{I} \times \mathbf{I} \frac{3}{8} \times \frac{1}{5}$ in. Polymorphous. Var. trigonophylla Sacc. Smaller than type ; $G$. triangular. Var. heterosticha Karnt. I'. depressed, umbonate. 
764. T. paludosa Karst. (from its habitat ; palus, a marsh) $a b$. $P$. expanded, papillate, sometimes striate, pale yellowish-fuscous to whitish. St. colour as P., flocculose above, white-villous below. $G$. adnato-decurrent, paler than P.

Amongst Sthagnum. May-Sept. $\quad I_{\frac{1}{4}}^{1} \times 3 \frac{3}{4} \times \frac{1}{8}$ in.

765. T. stagnina Gill. (from its habitat; stagnum, a swamp) a $b$.

$P$. expanded, brown, or somewhat pale and ochreous; marg. white-scaly with V. St. attenuate below, rubiginous. $G$. adnato -decurrent, broad, ferruginous.

Amongst Sthagnum in bogs. Sept. $\mathbf{I} \times 4 \times \frac{1}{16}$ in.

766. T. pellucida Gill. (from the translucent pileus; pellucidus, transparent) $a b$.

$P$. campanulate, umbonate, cinnamon to ochreous-tan; marg. striate, silky-squamulose with V. St. shining, paler than P., pruinose above. $G$. adnato-decurrent, subtriangular, colour as P., faintly sienna-tinted.

Roadsides, amongst leaves. Autumn. $\frac{7}{8} \times \mathrm{I}_{8}^{3} \times \frac{1}{15}$ in.

767. T. embolus Karst. (from the wedge-shaped gills; Gr. cmbolos, a wedge) a $b c$.

$P$. expanded, obtuse, pale yellowish-tawny to ochreous-tan. St. attenuate downwards, smooth, naked, paler than P. G. thick, very distant, separating from hymenophore, bright tawnycinnamon.

Damp heathy ground, amongst moss and grass; rare. June-Nov. $\frac{7}{8} \times 2 \times \frac{1}{16}$ in. Stem sometimes drawn up abnormally amongst grass.

768. T. autochthona Sacc. (from its terrestrial habit; Gr. autos, self, chthon, the earth) $a b c$.

$P$. flat, ochreous-white; marg. flocculose with V. St. paler than P., base white. $G$. adnato-toothed, honey-colour.

On the ground, amongst grass. Iuly-Oct. $\quad \mathbf{I} \times \mathbf{1} \frac{1}{8} \times \frac{1}{15}$ in.

\section{b. Pheote.}

769. T. muscorum Gill. (from its growing on moss, muscus) a $b$.

$P$. expanded, subdepressed, tawny-yellow; mid. sienna. St. colour as P. $G$. horizontal, adnate with a decurrent tooth, paler than P.

Amongst mosses on heaths, on tree-trunks. July. $\frac{7}{8} \times 2 \frac{1}{8} \times \frac{1}{16}$ in.

770. T. erobulus Karst. (from a fancied resemblance in the pileus to a knot of hair on the crown of the head, Gr. krobulos) $a$.

$P$. expanded, obtuse, sienna- to whitish-tan, white-floccose with V., then naked. St. white-floccose, sometimes subannulate. $G$. adnato-decurrent, crowded, fuscous-ferruginous.

Amongst sticks, chips. Sept.-Oct. $I \times I \frac{1}{4} \times \frac{1}{8}$ in.

771. T. inquilina Gill. (from its growing on other substances; inquilinus, a lodger) $a b c$.

$P$. plane, depressed, broadly umbonate, viscid when moist, yellowish-sienna, becoming pale; marg. striate, sienna. St. 
attenuate downwards, paler than P., white-fibrillose, base whitefloccose. $G$. adnato-decurrent, subdistant, umber.

Gregarious. Grass, ferns, stalks, chips; common. May-Oct. $\frac{7}{8} \times 1 \frac{1}{8} \times \frac{1}{16}$ in. II ust not be confounded with 858 or 860 . Var. ecbolit karst. Stem rooting; roots of grasses.

\section{CREPIDOTUS Quél.}

(From the shape of some of the species; Gr. krepis, a slipper.)

Vill obsolete. Hymenophore confluent and homogeneous with the fleshy stem. Pileus excentric, lateral or resupinate, commonly thin and with soft flesh. Stem lateral, simple or absent. Gills
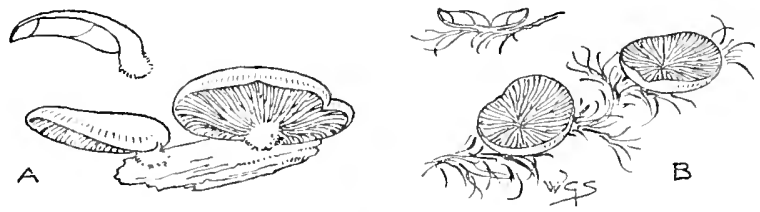

Fig. 40.-A, Crepitotus applanatus Karst. One-half natural size. B, C. cpibryus Quél. Twice natural size.

Entire and in section.

more or less decurrent, reaching the stem or axis, or radiating from an excentric point. Spores pale ferruginous or cinnamon. (Fig. 40.)

The species, except 784, grow on wood, trunks, twigs, grass, moss, etc., they are mostly small in size.

Crepidotus agrees in structure with Pleurotus and Claudopus.

Species $\mathbf{7 7 2 - 7 8 5}$

772. C. palmatus Gill. (from a fancied resemblance in the pileus to the palm of the hand) $a c$.

$P$. Heshy, convex, expanded, smooth, ferruginous to warm brownish-buff. St. solid, excentric or lateral, curved, smooth, whitish. $G$. rounded, subdistant, brownish-buff.

Trunks. Oct. $5 \times \mathbf{1}_{4}^{\frac{3}{4}} \times \frac{1}{2}$ in.

773. c. alveolus Quél. (from a fancied resemblance, when inverted, to a little trough; alveus, a trough) a $c$.

$P$. obovate, dimidiate, ochreous-fuscous. St. a villous base. $G$. subdecurrent on base, clay-fuscous.

Stumps, oak; rare. Aug.-Nov. Diam. 2 in.

774. C. mollis Quél. (mollis, soft) a $b c$.

$P$. obovate, dimidiate, pale yellowish-tan ; marg. at first incurved, then undulate. St. a strigose base. $G$. rounded on base, sometimes broad, cinnamon.

Stumps, poplar, willow, pear, sticks, logs, sawdust, squared timber, liumus; common. May-Dec. Diam. $2 \frac{3}{\mathrm{t}}$ in. 
775. C. applanatus Karst. (from the plane pileus) $a c$.

$P$. reniform, watery, pale cinnamon to whitish; marg. substriate when moist. St. when present white-tomentose. $G$. subrounded, watery-cinnamon or ochreous.

Decaying wood. Oct. $1 \frac{1}{4} \times \frac{1}{4} \times \frac{1}{4}$ in. Much like 1444 in appearance.

776. C. calolepis Karst. (from the beautiful scales of the pileus; Gr. kalos, beautiful, lepis, a scale) a $b$.

$P$. reniform, pale dull-ochreous, rufescent-scaly. St. a small villous base. $G$. rounded on base, pale brown then fuscous-ferruginous. Dead wood, poplar. Oct. Diam. I⿳亠口冋口, in., sometimes $\mathbf{2}_{\frac{3}{t}}^{3}$ in.

777. C. haustellaris Karst. (from its watery substance; haustellaris, a drawer of water) a.

$P$, obovate or reniform, pale yellowish-tan, then paler. St. excentric, slightly attenuate upwards, white. $G$. rounded, adnexed, fuscous-cinnamon.

Gregarious. Dead trunks, poplar; rare. $1 \times \frac{3}{8} \times \frac{1}{8}$ in.

778. C. Rubi (from its habitat; Rubus, bramble) $a c$.

$P$. obovate or reniform, crystalline-mealy, yellow to livid-grey, then pallid. St. excentric, whitish, strigose at base. G. subdecurrent, brown. Spores brown.

Gregarious; uncommon. July-Oct. $\frac{5}{16} \times \frac{1}{5} \times \frac{1}{16}$ in.

779. C. chimonophilus Sacc. (from its time of growth; Gr. cheimon, winter, philos, loving) a.

$P$. circular, convex, white, villous-downy. St. excentric, almost obsolete, white. $G$. subdecurrent on base, few, distant, pale yellow-brown. Spores pale yellow-brown.

Dead leaves, Pyrus torminalis, logs. Dec. Diam. $\frac{1}{t}$ in.

780. C. epibryus Quél. (from its frequent habitat, moss; Gr. cpi, upon, bruon, moss) a $c$.

$P$. resupinate, at first cup-like, sessile, membranous, white. $G$. rounded to a central or excentric axis, palt: yellowish.

Mosses, grass, leaves, holly, Vaccinium. Oct. Diam. $\frac{3}{16}$ in.

781. C. Phillipsii Sacc. (after IVilliam Phillips, mycologist) a b $c$.

$P$. ovate, expanded, smooth, pale umber; marg. striate. St. curved, colour as P. $G$. rounded-adnate, paler than P.

Deal grass. May-Oct. $\frac{7}{4} \times \frac{1}{3} \times \frac{1}{32}$ in.

782. c. proboscidens Gill. (from the trunk-like stem; Gr. proboskis, a snout) $a b$.

$P$. obovate, pendulous, scabrous-spotted, pale sepia; marg. incurved. St. attenuate to base, inserted on and darker than P., shaded umber-sienna. $G$. inferior, adnate, rounded to an excentric axis, flesh-colour.

Pendulous from a wooden ceiling. Dec. $\frac{5}{8} \times \frac{5}{8} \times \frac{1}{16}$ in.

783. C. pezizoides Gill. (from its resemblance! to certain cup-fungi of the genus $P_{c z i z a)}$ a.

$P$. resupinate, saucer-shaped, then reflexed, sessile, mealy, whitish. $G$. radiating from a central axis, tawny.

Gregarious. Rotten branches; rare. Dian. $\frac{1}{4}$ in. 
784. C. epigæus Sacc. (from its growing on the ground; (ir. cpigaios, upon the earth) $a$.

$P$. subreniform, becoming horizontal, fragile, reddish-grey. St. a villous lateral base. $G$. rounded on base, watery, rufescent.

On marlstone clay. Nov. Diam. $\mathbf{I}_{\frac{1}{2}}$ in.

785. C. Ralfsii Sacc. (after J. Ralfs) $a b c$.

$P$. reniform, horizontal, slightly hispid-furfuraceous, yellow. St. almost obsolete. $G$. rounded to a laterai base, clay-colour, edge whitish.

Decaying wood, dead branches. March. Diam. $\frac{1}{2}$ in.

785a. C. putrigenus Sacc. (from its habitat, rotten logs; putcr, putrid, signo, to bear) $a c$.

$P$. membranous, dimidiate or reniform, pubescent, whitish; marg. substriate, base villose. $G$. broad, irregular, unequal, whitish, then coloured by the ferruginous-brown spores.

(iregarious, or imbricate, in close clusters, closely sessile. Damp or putrid logs, beech. Sept. T. $\frac{3}{4} \times \frac{3}{8}$ in.

SERIES IV. PRATELLE Fr.

(From the habitat; pratum, a meadow.)

Spores typically black-purple or fuscous-purple, often fuscous, brown-black or dark brown.

Genera XXXII-XXXVIII.

\section{CHITONIA Karst.}

(From the veil which in infancy covers the plant like a cloak; Gr. chiton, a tunic.)

Icil universal, separate from the pileus and remaining as a volva at the base of the stem. Hymenophore distinct from the fleshy

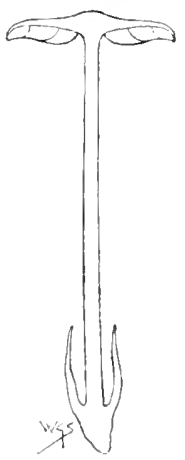

Fig. 41.-Section of Chitonia rubriccts Sacc.

One-half natural size.

stem. Pileus subfleshy. Stim central, simple, hollow, volvate. Gills free. Spores fuscous-purple. (Fig. +I.)

Represented only by one introduced species. 
Chitonia agrees in structure with Amanitopsis, Volvaria and Acetabularia.

786. C. RUbriceps Sacc. (from the red pileus; ruber; red, caput, the head) $a$.

$P$. expanded, umbonate, smooth, even, brick-red. St. rooting, dull pale reddish-ochreous, paler below. $V o$. large, sheathing, saccate, torn at edge. $G$. subcrowded, purplish-brown.

Soil in an aroid house. Dec. $1 \frac{3}{8} \times 3^{\frac{1}{2}} \times \frac{1}{8}$ in.

\section{PSALLIOTA Quél.}

(From the annulus; Gr. psalion, a ring.)

Vill universal, concrete with the cuticle of the pileus and forming an annulus on the stem. Hymenophore distinct from the fleshy stem. Pilens fleshy. Stem central, fibrous-fleshy, annulate. Gills

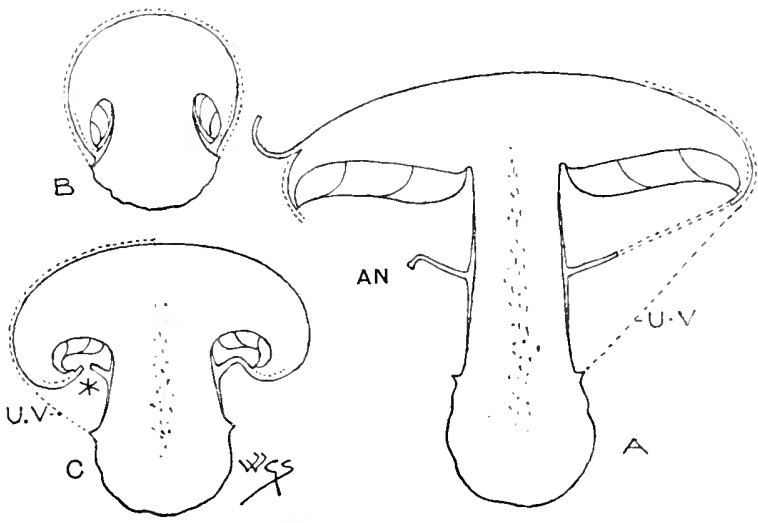

Fig. 42.-P'salliota campestris Quél. In section. Half natural size.

A, full grown example; AN, annulus; $U v$, universal veil ; $B$, young example, showing universal veil; $\mathrm{C}$, half-grown example, showing rupture of veil at $*$.

free, at first whitish, then frequently salmon-colour, at length deep purple-brown. Spores dark brownish-purple, brown or reddishpurple. (Fig. 42.)

All the species grow on the ground and are late æstival or autumnal, rarely vernal; they mostly grow in rich pastures and on manured ground, sometimes in woods; a non-British species, Agaricus geniculatus Briz., grows on wood.

Several species are valued for their esculent qualities.

Psalliota agrees in structure with Lcpiota, Anmularia and Togaria. Species $787-800$

a. Edules. Larger, fleshy. Edible.

$787-794$

b. Minores. Usually smaller, thinly fleshy. Not used as food. 


\section{a. Edules.}

787. P. augusta Quél. (from its appearance; augustus, noble) a b.

$P$. expanded, fibrilloso-squamose, rich ochreous-brownish ; marg. almost white, sometimes appendiculate with V. St. solid, enlarged below, tan, faintly siemna below. $G$. crowded, faintly brownish.

An inferior esculent. Orchards, woods, under spruce-firs, on ant-hills. Aug.-Sept. $7 \times 6 \frac{1}{2} \times \mathrm{I}_{\frac{1}{4}}$ in. Might when young be mistaken for a Lepiota.

788. P. Elvensis Henn. (from its place of growth when first named, near the River Elwy, N. Wales, I 863 ) $a b$.

$P$. expanded, fibrilloso-scaly, warm brown with darker scales. St. solid, equal or attenuate downwards, fibrillose, paler than $\mathrm{P}$. $A$. large. $G$. brownish flesh-colour. Flesh changing to red or pale rose when cut.

Sometimes crespitose. Under oaks, beech, fir. Aug.-Sept. $6 \frac{1}{2} \times 5 \times 1 \frac{1}{\frac{1}{4}} \mathrm{in}$. A drawing in Brit. Mus. is dated I 847 .

789. P. arvensis Quél. (from its habitat; arvuım, a cultivated field) $a b c$.

$P$. at length flat, floccose, silky or squamulose, whitish; mid. faintly yellowish; marg. ragged with V. St. hollow, attenuate upwards, even, smooth, white. $A$. large. $G$. ventricose, at length dark purple-brown. Flesh white, changing to pale yellowish or brownish.

Spring to winter. $7 \frac{3}{4} \times 4 \frac{1}{4} \times 1^{\frac{1}{4}}$ in. The cultivated mushrooms of gardeners belong to this species. There are several named varieties. I. purpurascens $\mathrm{WV}$. G. Sm., Asaricus arvensis Schaeff., var. purpurascens Cooke, $P$. purple. 2. albosquamosa IV. G. Sm., $P$. white-scaly. 3. vaporaria IV. G. Sm., d. vaporarius Otto, $P$. even, brown-pilose. 4. hortinsis $\mathrm{W}$. G. Sn., A. campestris L., var. hortensis Cooke, P. brownish fibrillose or squamulose. 5. Buchananii IV. G. Sm., A. campestris L., var. Buchananii Berk., $P$. smooth as if whitewashed, $A$. slight, fugacious. 6. cryptarum IV. G. Sm., A. cryptarum Letell, the name applied to the form grown in caves, grey-white or brownish-white.

789a. P. perrara Magnus (perrarus, very rare) $a b$.

$P$. fleshy, globoso-hemispherical, then expanded, scaly, whitishochre; sc. concentric, floccose, brownish-yellow to siemna. St. stuffed, then hollow, equal or attenuate upwards and submarginately-bulbous, smooth above, squamose below, white to pale yellowish or salmon-shaded; sc. salmon-brown. $A$. large, superior, membranous, floccose, persistent. $G$. free to remote, crowded, whitish, then salmon to full brownish-slate or fuscous. Flesh white, becoming yellowish, faint reddish-ochre or salmon when broken.

Taste somewhat sweet; odour somewhat of anise. Damp ground, under oaks, near conifers. Aug. $5 \frac{1}{2} \times 4 \times \frac{7}{8}$ in. Analogous with 21, 33 and 792a. 
789b. P. xanthoderma W. G. Sm., Agariaus xanthodermus Genev. (from the yellow-stained outer surface and flesh; Gr. xanthos, yellow, derma, skin) $a$.

$P$. fleshy, convex, eren, greyish-white, becoming yellowish when touched. St. stuffed to hollow, white, yellow at base. $A$. membranous. $G$. sinuato-free, pale rose, becoming purplebrown. Flesh becoming yellow.

Pastures. Autumn. $3 \frac{1}{2} \times 4 \times \frac{3}{4}$ in.

790. P. cretacea Quél. (from its chalky-white colour; cretcr, chalk) $a b c$.

$P$. convexo-flattened, obtuse; marg. even, rivulose or squamulose. St. hollow, attenuate upwards, smooth. $G$. becoming fuscous. Fles/ unchanging white.

Pastures, stoves; rare. Feb.-Oct. $3 \frac{3}{4} \times 4 \times \frac{5}{8}$ in. Resembling 40.

791. P. pratensis Quél. (from its habitat; pratum, a meadow) a $c$. $P$. expanded, obtuse, floccoso-farinose, then smooth, whitish, then greyish. St. hollow, colour as P. G. arcuate, brown.

Taste and odour pleasant. Pastures, woods. Oct. $3 \frac{1}{2} \times 3 \times \frac{5}{8}$ in.

792. P. campestris Quél. (from its habitat ; campus, a field or plain) $a b c$.

$P$. expanded, silky-even, sometimes squamulose, whitish. St. stuffed, even or squamulose, whitish. $G$. crowded, bright salmon, then umber-fuscous. Flesh white.

Odour pleasant. Nlay-I)ec. $5 \times 3 \frac{3}{4} \times \mathbf{I}$ in. Variable in size and colour. The common edible mushroom. Sometimes the flesh becomes faintly reddish or brownish when cut or broken. Sometimes the odour is strong and somewhat unpleasant. The following varieties have been described: -1. alba W. G. Sm., Agariaus camfestris L. var. albus Viv., P. silkywhite; St. short. 2. praticola Magnus, $P$. rufous-squamulise; flesh rufescent when cut. 3. rufescens WV. G. Sm., A. camfestris L., var. mfescens Fr., $P$. rufous-squamulose, St. long. 4. zmbrina Mlagnus, $P$. at length even; umber. 5. fulvastera IV. G. Sm., A. campestris L., var. fulvister Viv., $P$. ochreous-tawny; St. solid. 6. costata W. G. Sm., A. campestris L., var. costatus Viv., $P$. sulcate. 7 . silvicola Magnus, $P$. sm oth, shining: St. elongate, somewhat bulbous, hollow ; A. large. S. elongata V. G. sm., $A$. campestris L., var. clongatus Berk., $P$. at length even, shining-white; marg. permantntly appendiculate with veil ; St. elongate, bulbous. 9. exannulata W. G. Sm., A. campestris L., var cxanmulatus Cooke, A. fugitive or obsolete. The gills of cultivated mushrooms are frequently thickened or obliterated by disease; this is caused by a white parasitic fungus, Mycogone perniciosa. Diseased examples are not fit for food. Sound examples of true mushrooms sometimes cause diarrhoea and sweating.

792a. P. villatica Magnus (from its frequent habitat, near houses in the country; villa, a country house) $a b$.

$P$. convex then expanded, silky, soon squamose, ground pale yellowish or brownish straw-colour then vinous-brownish; sc. vinous-brown. St. solid, somewhat ventricose, paler than P., clouded pale vinous-brown. $A$. superior, large, tomentose beneath. $G$. free, very crowded, not at first bright salmon, 
becoming vinous-brown. Flesh white, pale reddish-yellow when broken.

Edible. Taste of almonds, odour somewhat rank. Rich pastures, lawns, manured fields, in cellars, on mushroom-beds in and out of doors; rare. Ift. 2 in. $\times 7 \frac{1}{2} \times 3 \frac{1}{8}$ in. This is the "gigantic mushroom" of newspapers; it atlains a diam. of $1 \mathrm{ft} .5$ in. or more and a weight of $4 \frac{1}{2}$ lbs. By some authors considered a var. of 792 or 789 , but probably distinct. Badham, "Esculent Funguses of England," p. Ioo, mentions an example with a circumference of 43 ins., and weight of 5 lbs. 6 ozs.

793. P. silvatica Quél. (from the habitat; silz' $a$, a wood) a $b$.

$P$. at length flat, subumbonate, floccoso-squamulose, pale rustybrown or rufescent to pale ashy-l)rown. St. fibrillose below $\Lambda$., much paler than P. or white. $G$. thin, arid, reddish to umberfuscous.

Edible, with a delicate flavour. Woods, under cedars; frequent. July-Sept. $3 \frac{5}{8} \times 3 \frac{1}{ \pm} \times \frac{3}{x}$ in. Usually thinner than its allies. St, varying long and short.

794. P. hæmorphoidaria Magnus (from the flesh changing colour to blood-red when bruised; Gr. haimorrhoides, veins which discharge blood) $a b$.

$P$. at length expanded, obtuse, adpressed-scaly, rufous-fuscous. St. hollow, fibrillose, slightly attenuate upwards, whitish. $G$. crowded, bright salmon to umber-purple.

Suspected sometimes poisonous. Woods, parks. Aug. - Nov. $4 \frac{5}{8} \times 4 \frac{3}{8} \times \frac{7}{8} \mathrm{in}$.

794a. P. peronata W. G. Sm., Agaricus peronatus Mass. (from the scaly stem; peronatus, sheathed) a.

$P$. hemispherical then expanded, pale dull ochreous, densely scaly ; sc. small, silky, brown. St. hollow, equal, marginately bulbous, smooth above A., white-scaly below. $A$. large, spreading. $G$. very distant, crowded, pink then pale purplebrown. Flesh white, brownish in St. when cut.

Edible. Yine-woods. Oct. $3 \frac{1}{2} \times 6 \frac{1}{2} \times \frac{1}{2} \mathrm{in}$. Attaining a size of $4 \frac{1}{2} \times 8$ in. Allied to 787 (but differing in the hollow, peronate stem) and 794 .

\section{b. Minores.}

795. P. comtula Quél. (from the beautifully coloured gills; comptus, gaily adorned) a $b$.

$P$. flat, adpressedly fibrilloso-scaly, yellowish-white; marg. appendiculate with V. St. hollow, colour as $\mathrm{P}$. or paler. $A$. fugacious. $G$. crowded, fuscous-flesh-colour. Flesh colour as $\mathrm{P}$.

Edible. Taste and smell strong of anise. Woods. Miay-()ct. $1 \frac{1}{2} \times 1 \frac{3}{8} \times \frac{3}{16}$ in. Might be mistaken for a small 792 .

796. P. subgibbosa Henn. (from the somewhat obtuse pileus; subgibbosus, somewhat gibbous) a $c$.

$P$. plane, smooth, sienna-tan: marg. silky-fibrillose. St. hollow, tan-white. $A$. fugacious. $G$. ashy-brown.

Woods. Sept. $4 \times 3 \frac{1}{x} \times \frac{1}{2}$ in. Might be mistaken for 792 . 
797. P. sagata Henn. (from the pelliculose pileus, clothed with a cloak; saga, a mantle) a b.

$P$. plane, smooth, bright yellowish-brown. St. hollow, colour as P., ochreous or reddish. $A$. distant, white. $G$. pale purplebrown or brown-salmon.

Taste and odour of 792 . Under beeches. Oct. $2 \frac{3}{8} \times 2 \frac{5}{8} \times \frac{1}{4}$ in.

798. P. rusiophylla W. G. Sm., Agaricus rusiophyllus Lasch. (from the rose-coloured or reddish gills; rusents, reddish, Gr.phullon, a leaf) $a b$.

$P$. somewhat fleshy, convex then plane, subumbonate, fibrillose, pale flesh-colour or pale purple, becoming pallid. St. imperfectly hollow, attenuate upwards, silky fibrillose, white, shaded faint ochre. $A$. persistent, drooping. $G$. free, crowded, thin, dry, rosy, then pale purple, pinkish-sienna and brownish. Flesh whitish, unchangeable.

On the ground. Autumn. $2 \frac{1}{8} \times 2 \frac{1}{8} \times \frac{1}{4}$ in.

799. P. dulcidula Henn. (from its taste ; dulcis, sweet) $a$.

$P$. plane, subgibbous, glabrous, dry, whitish to pale ochreous. St. hollow, sometimes subbulbous, colour as P. $A$. persistent. $G$. crowded, slate-colour.

Odour mild. Heaths. Oct. $2 \frac{1}{2} \times 2 \times \frac{1}{4}$ in. Might be mistaken for 792.

800. P. echinata Quél. (from the rough pileus; cchinus, a hedgehog) $a b c$.

$P$. expanded, obtuse, floccoso-pulverulent, dark brown; mid. darker; marg. appendiculate with V. St. fistulose, usually attenuate downwards, floccose, light or dark dull-brownish, crimson, purple or grey-brown. $G$. crowded, rose, cinnamoncrimson, crimson or purple. Flesh brownish or reddish. Spores very pale whitish-dull-purple.

Gregarious or crespitose. Odour strong and disagreeable, mephitic or like cucumber or like 35. Woods, beech, greenhouses. May-Nov. $\frac{1}{\frac{7}{8}} \times 3 \times \frac{1}{4}$ in. Springing from a compart ball of earth or mycelium. Becomes jet-black when dry. The vesiculose meal on the pileus is transparent yellow. Sometimes placed in Inocybe, but retained here on account of the colour of the spores when fresh.

\section{PILOSACE Quél.}

(From the pelliculose pileus; Gr. pilos, felt, sakos, a garment.)

Vill obsolete. Hymenophore distinct from the fleshy stem. Pilcus fleshy. Stem central, simple, without cartilaginous bark. Gills free or remote. Spores purple-brown. (Fig. 43.)

The only British species grows on the ground. 
Pilosace agrees in structure with the fleshy species of Hiatulu, Plutelus and Pluteolus.

Species 801

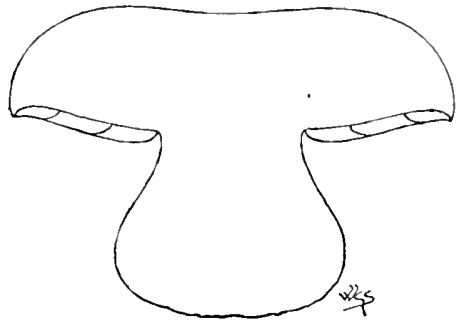

Fig. 43,-Pilosace algeriensis Quél.

In section. Half natural size.

801. P. algeriensis Quél. (from its being first found in Algeria) $a b$.

$P$. plane, gibbous or subumbonate, smooth, white, becoming brownish above when fully developed. St. solid, silky, white. $G$. narrow, salmon to rose, becoming dark purple-madder.

Edible. On the ground. Aug. $3^{\frac{3}{4}} \times 1 \frac{1}{2} \times 1 \frac{1}{4}$ in. Intermediate between Psalliota and Siropharia with the aspect of an exannulate 789.

\section{STROPHARIA Quél.}

(From a fancied resemblance in the annulus to a sword-belt, Gr. strophos.)

Vil annular on stem. Hymenophore confluent and homogeneous with the fleshy stem. Pilus with or without a pellicle, even, scaly

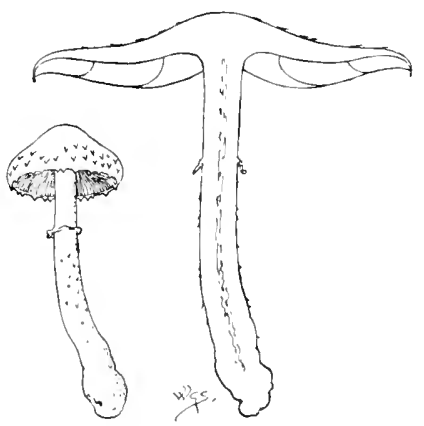

Fig. 44.-Stroplazria amsinosa Quél. ; entire and in section. One-third natural size.

and viscid, or innato-fibrillose and dry. Stem central, fleshy, annulate. Gills more or less adnate, at first whitish, or pale clay-brownish. (Fig. 44.) 
Most of the species are epiphytal, some grow on the ground. Stropharia agrees in structure with Armillaria and Pholiota.

Species $802-820$

a. Viscipelles. Pileus with an even or scaly, usually viscid pellicle.

Not usually growing on dung.

Growing on dung; annulus often imperfect.

$811-814$

b. Stintrigere. Pileus without a pellicle, innato-fibrillose, not viscid.

$815-820$

\section{a. Viscipelles.}

802. S. æruginosa Quél. (from its verdigris colour; arugo, verdigris) $a b c$.

$P$. expanded, subumbonate, brownish-yellow, covered with transparent blue gluten, clad with white scales; marg. appendiculate with V. St. hollow, white scaly and viscid below A., blue or verdigris, often furmished with stringy root-like growths. $G$. purple.

Sometimes crespitose. Odour strong of rancid ointment. WWoods, meadows, damp thatch, wood, stumps, hazel, amongst stones; common. July-Nor. $3 \frac{1}{2} \times 4 \frac{1}{4} \times \frac{1}{2}$ in.

802a. S. squamulosa Mass. (from the small scales of the pileus; squama, a scale)

$P$. soon plane, dry, silky, deep verdigris-green with darker, innate, floccose squamules; mid. yellow-orange; marg. white with veil. St. at length hollow, paler than P., white at base. $A$. fragmentary in the form of large hard white sq. at apex. $G$. crowded, brown, with a vinous tinge. Flesh white in P.. pale verdigris in St.

Amongst stones, Ripon. Sept. IS95. $2 \frac{3}{8} \times 2 \frac{\pi}{8} \times \frac{5}{8} \mathrm{in}$.

803. S. albocyanea Quél. (from its colours; albus, white, cy'ancus, blue) a $b c$.

$P$. plane or subumbonate, bright blue or verdigris from the gluten, becoming dull yellowish or white. St. hollow, not viscid, white. $A$. fibrillose, purple. $G$. fuscous-salmon. Flesh white, pale verdigris-green or pale blue.

Sometimes crespitose. Pastures, woods, sometimes on dung; uncommon. Sept.-Nov. $1 \frac{1}{2} \times 3 \frac{3}{1} \times \frac{1}{5}$ in.

804. S. inuneta Quél. (from the besmeared pileus; inungo, to besmear) a $b c$.

$P$. convex, subumbonate, light yellowish or brownish, slate-purple from the gluten. St. fistulose, not viscid, white. $A$. fibrillose. $G$. adnate, dull fuscous-purple. Flesh white.

(iregarious, sometimes crespitose. Taste and odour like radishes, but disagreeable. Rich pastures, graveyards, lawns, stumps, sticks. Sept.Nov. I $\frac{3}{4} \times 3 \frac{3}{8} \times \frac{3}{16}$ in. Form lundensis Sacc., $P$. convex, expanded ; St. stuffed. Form upsaliensis sacc., P. convex, plane, umbonate; St. hollos. Var. pallida IV.G. Sm., Agrricus inunctus Fr., var. pallidius B. \& Br.. pale. 
805. S. coronilla Quél. (from a fancied resemblance to a crown in the whitish-floccose margin of the pileus; corona, a crown) $a b$. $P$. convexo-expanded, obtuse, light yellow; mid. sub- or whollyvinous. St. stuffed, fibrillose, white. $G$. crowded, vinousbrownish.

Waysides, parks. May-Oct. $I_{1}^{\frac{1}{4}} \times \frac{7}{3} \times \frac{1}{8}$ in. Perhaps the same as 807 .

806. S. obturata Quél. (from the stuffed stem; obturo, to stuff) a bc.

$P$. very fleshy, convex, obtuse, often rimoso-squamulose, pale ochreous; mid. shaded sienna. St. not scaly, white, with a membranous collapsing A. $G$. crowded, dull purplish-brown.

On the ground, amongst grass; rare. Sept.-Oct. $I_{\frac{1}{2}} \times I_{\frac{5}{3}}^{\frac{5}{3}} \times \frac{3}{16}$ in. Might be mistaken for $\mathbf{7 9 2}$.

807. S. melasperma Quél. (from the blackish spores; Gr. melas, black, sperma, a seed) a c.

$P$. expanded, biscuit-colour or pale buff, or white with a yellowish marg. St. hollow, white. $G$. rounded, ventricose, purplish, then deep purple-brown. Flis/ white, yellowish in St.

IIeadows and borders of thickets; rare. Aug.-Oct. $23 \times 2 \times \frac{3}{16}$ in. Might be mistaken for 792 .

808. S. squamosa Quél. (from the scaly pileus; squama, a scale) a bc.

$P$. expanded, obtuse, ochreous or brownish; mid. darker, clad with small whitish or brown scales. St. hollow, viscid-scaly below distant A., pallid above, sienna below. $G$. roundedadnate, blackish, with a whitish edge.

Woods, fields, amongst chips, sometimes from interior of beech-nuts. Sept.Nov. $2 \frac{1}{t} \times 5 \frac{5}{5} \times \frac{3}{16}$ in. Var. thrausta Mass. $P$. soon glabrous, more slender than type. Var. aurantiaca Mass. $P$ '. orange or brick-red.

809. S. Percevalii Sacc. (after Cecil H. Spencer Percival) a $b c$.

$P$. expanded, subumbonate, warm brown-ochreous; marg. appendiculate with floccose white V. St. hollow, attemuate upwards, fusiform-rooting, pallid above, pale brownish and squarrose below distant A. $G$. distant, pallid umber.

Sawdust, rotten wood. Nov. $2 \frac{5}{8} \times 3 \frac{5}{3} \times \frac{3}{8}$ in. Possibly the same as S. defilata Karst., but that has a solid stem. Might be mistaken for a Flammula. Allied to 808 .

810. S. Worthingtonii Sacc. (after Worthington G. Smith) $a b$.

$P$. hemispherico-conical, smooth, golden-yellow. St. fistulose, cobalt, or turquoise-blue. $A$. imperfect. $G$. adnato-ascending, pallid brown. Flesh pale sulphur-yellow in P., pale bright blue in St.

Pastures, woods. Nov.-Dec. $\frac{7}{5} \times 2 \frac{3}{ \pm} \times \frac{1}{15}$ in.

811. S. luteonitens Quél. (from the shining yellow pileus; lutizs, yellow, nitens, shining) $a$.

$P$. expanded, umbonate, sometimes whitish, with a few small sienna scales. St. hollow, even, pruinose above distant A., 
colour as P., sienna at base. $G$. adnexed, ventricose, pale purplish-brown.

Dung, sawdust, earth. Sept. $22_{4}^{3} \times 2 \frac{1}{ \pm} \times \frac{1}{4}$ in. Not unlike 818 .

812. S. merdaria Quél. (from its habitat; merda, dung) $a b c$.

$P$. convexo-plane, smooth, hygrophanous, yellow or brownish then straw-colour; marg. appendiculate with white V. St. stuffed, floccose, dry, straw-white or brownish, base white-villous. $G$. adnato-rounded or subdecurrent, purplish-fuscous.

Gregarious, subcrespitose. Dry dung, road scrapings. Aug. - Oct. $I_{\frac{1}{2}} \times I_{\frac{1}{2}} \times \frac{1}{5}$ in. 817 is perhaps form major Fr. of this species. Easily overlooked, colour of pileus exactly that of dry horse-dung.

813. S. stercoraria Quél. (from the habitat; stercus, dung) a $b c$.

$P$. hemispherical, smooth, yellow ; mid. darker ; marg. substriate. St. stuffed, yellowish. A. viscid. $G$. very broad, subdistant, purple-slate.

Dry dung in pastures. May-Nov. $1 \frac{1}{2} \times 3 \frac{1}{4} \times \frac{1}{5} \mathrm{in}$.

814. S. semiglobata Quél. (from the half-globose pileus) $a b c$.

$P$. yellowish. St. fistulose, smooth, colour as P., thickly covered with transparent gluten below the glutinous A. $G$. very broad, pale purplish, clouded purple-black.

Gregarious. Dung, rablit, horse, in pastures; common. April-Nov. $\mathrm{I} \frac{1}{4} \times 5 \frac{1}{2} \times \frac{1}{8}$ in. Usually smaller than 813 , but sometimes twice the size of type.

\section{b. Spintrigera.}

815. S. caput-medusæ Karst. (from a fancied resemblance in the pileus to the head of Medusa ; caput, a head) a $b c$.

$P$. expanded, obtusely umbonate, scaly-squarrose, soon naked, pale white umber; mid. umber; sc. sepia. St. hollow, white above, squarrose and white umber below superior $\mathrm{A}$., if present. $G$. ventricose, at length separating free, pallid umber or oliveshaded, spotted ferruginous, edges white.

Subcaspitose. Woods, pine, about fir-stumps, base of elm ; rare. Sept.Oct. $3 \times 3 \frac{1}{3} \times \frac{1}{2}$ in. Becoming darker when dry.

816. S. scrobinacea Sacc. (from the appearance of the pileus as if sprinkled with sawdust, scrobis) $a b$.

$P$. expanded, obtuse, tan; nid. ochreous-tan, or grey or brown shaded, clad with minute, fugitive sepia squamules. St. hollow, fibrillose, whitish, squamulose below the fugitive A. G. crowded, purple.

Sulcrespitose. On or near stumps, ash; rare. Aug.-Oct. $I \frac{7}{5} \times 3 \frac{3}{8} \times \frac{1}{4}$ in. Thinner and more graceful than 815 .

817. S. ventricosa Mass. (from the ventricose gills) $a$.

$P$. expanded, broadly gibbous, even, smooth, subriscid in wet weather, tawny-ochreous, becoming paler. St. hollow, ventricose below with a rooting base, minutely squamulose, pale yellow. $A$. large, persistent. $G$. slightly adnexed, ventricose, subcrowded, purplish-brown.

Caspitose. On the ground. $1 \frac{1}{2} \times 3 \times \frac{1}{4}$ in. l'erhaps only a form of 812 form major $\mathrm{Fr}$. 
818. S. Jerdonii Sacc. (after Archibald Jerdon) $a$.

$P$. expanded, obtusely umbonate, minutely rivulose, pale ochreous; mid. slightly darker; sc. white. St. hollow, attenuate upwards, white above A., subscaly, tan-ochreous and brownish below. $G$. transversely striate, purple-brownish.

Stumps, fir ; rare. Sept.-Nov. $2 \times 2 \frac{1}{2} \times \frac{1}{4}$ in.

819. S. spintrigera Sacc. (from the annulus; spinthicr, a bracelet, sire, to carry) $a$.

$P$. flat, obtuse, even, smooth, faintly ochreous or brownish. St. hollow, attenuate upwards, floccoso-fibrillose below A., white. $A$. distant, fugacious. $G$. crowded, fuscous-brown.

Crespitose. Stumps, beech. Sept. $3 \frac{1}{4} \times 3 \frac{1}{5} \times \frac{3}{16} \mathrm{in}$.

820. S. hypsipus Karst. (from the long stem ; Gr. hupsipons, highfooted) $a b$.

$P$. plane, subobtuse, even, smooth, hygrophanous, pallid, pale brownish, or shaded dull flesh-colour; marg. striate. St. hollow, smooth, white. A. distant. G. subdistant, brown or dull purplish-slate.

Swampy places, amongst grass, sometimes on moss and sticks. Oct. $3 \frac{1}{2} \times 6 \frac{1}{2} \times \frac{5}{16}$ in.

\section{XXYVI. HYPHOLOMA Quél.}

(From the web- or fringe-like veil; Gr. luphe, a web, loma, a fringe.)

$V$ il fringe-like, adhering to the margin of the pileus, sometimes forming a fibrillose annulus. Hymenophore confluent and

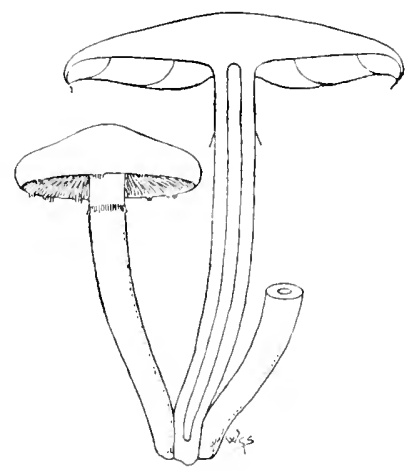

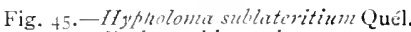
Entire and in section.

i) ne-third natural size.

homogeneous with the fleshy stem. Pilus more or less fleshy, the margin at first incurved. Stom central, simple, or slightly 
fibrilloso-annulate. Gills adnate or sinuate. Spores slate-purple. (Fig. 45.)

The species grow on wood and have a cæespitose habit. None are edible, some are nauseous-bitter.

Hypholoma agrees in structure with Tricholoma, Entoloma and Hebeloma.

Species 821-844

a. Fasciculares. Pileus tough, smooth, dry, except 821 and 828 , brightly coloured, not hygrophanous. Gills adnate.

b. Viscide. Pilius naked, viscid.

c. Velutine. Pileus silky with innate fibrils or streaked.

$831-834$

d. Flocculoser. Pilizus with floccose superficial separating scales.

835,836

c. Appendiculate. Pileus smooth, hygrophanous.

$837-844$

\section{a. Fiasciculates.}

821. H. silaceum Quél. (from its colour; sil, yellow-ochre) a.

$P$. subexpanded, obtuse, viscid, brown-ochreous ; marg. cracked, whitish. St. bulbous, fibrilloso-striate, shining, colour as P'. $G$. purplish-brown or grey-olivaceous.

Solitary to cæspitose. Taste mild ; odour mealy. Old pastures, under firs. Auy.-Nov. $2 \times 3 \frac{1}{2} \times \frac{1}{\frac{1}{4}} \mathrm{in.}$ Not unlike some forms of 822 ; intermediate forms occur.

822. H. sublatepitium Quél. (from the colour of the pileus; sub, somewhat, later, a brick) a b $c$.

$P$. convexo-plane, obtuse, tawny brick-red; marg. paler, appendiculate with V., sometimes with sc. St. stuffed, attenuate downwards, scaly-fibrillose, pale yellow above, ferruginous below. $G$. sinuate, arcuate or decurrent, dull yellow-greenish to pale slate-purple. Flesh sulphur-white.

Cxspitose to solitary, sometimes in rings near trees. Probably poisonous. Taste usually bitter-nauseous. Forests, hedgerows, stumps, rails, old pine wood, oak; common. April-Dec. $33^{\frac{1}{2}} \times 4^{\frac{3}{2}} \times \frac{1}{2}$ in. Polymorphic, P. sometimes $7 \mathrm{in}$. in diam. Var. Schaeffiri sacc. $G$. deeply decurrent. Var. squamosum Sacc. P. brown-squamulose.

823. H. capnoides Quél. (from the smoky-tinted gills; Gr. kafnos, smoke, cidos, appearance) a b c .

$P$. subexpanded, obtuse, shining, pale ochreous with mid. siennared, or wholly orange. St. hollow, silky, even, elastic, pale ochreous, or whitish above and brownish below. $G$. subcrowded, arid, grey to fuscous-purple.

Taste and odour mild. Woods, pine, pine-stumps; uncommon. April-Dec. $2 \frac{7}{5} \times 5 \times \frac{3}{8}$ in.

824. H. epixanthum Quél. (from the yellowish-brown colour; Gr. efixanthos, yellowish-brown) a $b c$.

$P$. expanded, obtuse, silky, apt to split; marg. lobed, undulate. St. hollow, floccoso-fibrillose, reddish-sienna, darker below. 
$G$. crowded, light yellow, sometimes cinereous. Flesh white, becoming light yellow, sienna in St.

Taste acrid ; odour strong, acicl. Stumps, fir, fallen firs, on the ground; frequent. Aug.-Dec. $33^{3} \times 6 \frac{1}{ \pm} \times 3$ in. Sometimes wholly bright yellow; often sterile. One form of this is Clitocybe Sadleri sace.

825. H. elæodes (iill. (from the olive colour of the gills ; Gr. claios, olive, cidos, appearance) a b $c$.

$P$. plane, subumbonate, tan or buff. St. fibrillose, colour as P., becoming ferruginous. $G$. crowded, thin, white to olivaceous. Flesh pale buff.

Taste bitter; odour sour. Stumps, on the ground. Oct. $2 \frac{5}{8} \times 2 \frac{1}{8} \times \frac{1}{4} \mathrm{in}$. Sometimes wholly ivory-whitish. Sometimes regarded as a form of 826 .

826. H. faseiculare Quél. (from the fasciculate habit) a $b c$.

$P$. Hat, subobtuse, sulphur-yellow; mid. sienna. St. hollow, fibrillose, sulphur above, sienna below or wholly sulphur-yellow, often fibrilloso-annulate. $G$. crowded, sulphur to green. Flesh sulphur.

Gregarious, densely cæespitose. Probably poisonous. Taste and odour acrid, nauseous, bitter, sickening. Stumps, on the ground; very common. April-Dec. $2 \frac{5}{4} \times 4 \frac{5}{8} \times \frac{1}{4} \mathrm{in}$. There are many varieties. Recorded from Glyncorrwg collieries with stems 4 feet long.

827. H. instratum Mass. (from the naked, uncovered pileus; instratus, uncovered) a.

$P$. convex, broadly umbonate, rugose, rich dark-brown. St. hollow, smooth and white above, fibrillose and pale brown below. $G$. adnato-ascending, brown to purple-brown, edge paler. Flesh pale sienna-brownish.

Stumps. Autumn. $1 \frac{3}{8} \times 2 \frac{5}{8} \times \frac{3}{15}$ in.

828. H. dispersum Quél. (from the scattered habit of growth; dispergo, to scatter) $b$.

$P$. expanded, viscid, obtuse, tawny-yellowish to purplish-umber. St. fistulose, sometimes rooting, silky-fibrillose, faintly ferruginous above, rich fuscous-brown below. $G$. distant, thick, brownish flesh-colour or shaded olive, with a white edge. Flesh pale yellow-tawny.

Woods, pine, dry to marshy places, on the ground, on sawdust. April-Nov. $I_{\frac{5}{8}} \times 4^{\frac{1}{2}} \times \frac{1}{8}$ in. Variable in colour and habit.

\section{b. Viscida.}

829. H. incomptum Mass. (from its simple, unadorned appearance; incomptus, rough, without ornament) a.

$P$. expanded, broadly gibbous, even, deep bay-brown, or orangetawny. St. hollow, equal, pale above, dark ferruginous below, brown-fibrillose and yellow tomentose. $G$. adnato-rounded, crowded, deep olive, purple-clouded.

Stumps. Autumn. $4 \times 3 \frac{1}{2} \times \frac{1}{2} \mathrm{in.}$ 
830. H. œdipus Sacc. (from the swollen base of the stem; oidipous, with a swollen foot) $a$.

$P$. hemispherical ; mid. umber ; marg. pallid, appendiculate with V. St. solid, semibulbous, fibrillose below the distant A., whitish, shaded pale sienna. $G$. adnate, umber.

Solitary or in groups of two or three. Sticks and dead leaves. Feb. $\frac{7}{8} \times 1 \frac{1}{2} \times \frac{1}{5}$ in.

\section{c. Velutince.}

831. H. pseudostorea Rea (from its being at first considered the same as $H$. Storea) a $b$.

$P$. convex, obtuse or subumbonate, then expanded, fleshy, at first purplish-brown with mid. darker, soon breaking up into large fibrillose purplish-brown scales, silky-white to pale fawn beneath; marg. appendiculate and entire when young. St. hollow, equal or attenuate downwards, at first even, then silkybrown-fibrillose, white above, pale yellowish below. $G$.adnate, at first white, then rose, becoming brown to purple-brown, not exuding drops of water.

Usually cæspitose, sometimes solitary. Taste insipid, somewhat disagreeable; odour strong, disagreeable. Woods, plantations, under larches. Sept.-Nov. $2 \frac{7}{3} \times 4 \frac{1}{2} \times \frac{1}{2}$ in. This is the plant referred to Agaricus Stone Fr. by Berkeley, and afterwards named A. Stora, var. cespitosa by Cooke. Sometimes confounded with 832.

832. H. lacrymabundum Quél. (from the tear-like drops on the gills; lachrymabundus, tearful) $a b c$.

$P$. expanded, obtuse, innato-pilose, whitish, then fuscous; sc. darker; marg. appendiculate with V. St. hollow, fibrillososcaly, fuscous-white. $G$. adnato-rounded, whitish to whitepurple-brownish, studded with drops of water.

Crspitose. Stumps or on the ground, yards. Sept.-Dec. $3 \times 3 \frac{1}{2} \times \frac{5}{16}$ in. Offen confounded with 831 and 834. Used for inferior ketchup. Sometimes sold for the mushroom.

833. H. pyrotrichum Quél. (from the fiery-red fibrils of the pileus: Gr. fur, fire, thrix, a hair) $a$.

$P$. expanded, obtuse, pale bright vermilion-ochre ; marg. appendiculate with $V$. St. hollow, fibrillose, whitish to pale orange, sometimes imperfectly annulate with V. $G$. adnate, dull purplish-brown, studded with drops of water.

Crespitose. Woods, pine, beech, about roots. Aug.-Oct. $3 \times 3 \frac{1}{3} \times \frac{5}{16}$ in. Var. egregium Mass, with a densely shaggy stem.

834. H. velutinum Quél. (from the velvety pileus of young examples; vellus, fleece) $a$ b $c$.

$P$. expanded, obtusely umbonate, tawny, umber or sienna ; marg. appendiculate with V. St. hollow, fibrillose, whitish above, sienna or umber below. $G$. adfixed, brown-fuscous, darkspotted, studded with drops of water.

Subcespitose. Taste insipid. Stumps; common. July-Oct. $4 \times 5 \times \frac{1}{2}$ in. Variable in stature; pileus sometimes 6 in. in diam. Often confused with 
832. Intermediate forms occur between this and 832 and 833 . Sometimes sold for the mushroom. Used for inferior ketchup. Var. leiocephalum Mass. I'. mid. rugged, mich smaller than type.

\section{d. Flocculose.}

835. H. cascum Quél. (from its grey and white colours; cascus, old) $a b$. Fragile.

$P$. hemispherical, obtuse, dull greyish-ochre or ochre; marg. paler, appendiculate with white V. St. hollow, fibrillose, white. $G$. ascending, adnexo-rounded, arid, at first pale dovecolour then blackish-brown, edge whitish.

Gregarious or crespitose. Pastures. July. $\mathbf{I}_{2}^{\frac{1}{2}} \times 3 \frac{1}{1} \times \frac{3}{15}$ in.

836. H. punctulatum Mass. (from the squamuloso-punctate pileus) $a$.

$P$. convex, pallid, shaded yellowish, brownish or white sulphur; sc. dot-like, pale. St. stuffed, punctiform-squamulose from base to A., colour as P.; sc. faintly rufescent. $G$. sinuatoadnate, subcrowded, pale umber to whitish-sienna. Flesh colour as P. Spores brown.

Densely crespitose. Chips, sawdust, rotten twigs. $1 \frac{3}{5} \times \mathbf{I}_{3}^{3} \times \frac{1}{4} \mathrm{in}$. Ijerkeley, Cooke and Massee assign this plant to the present position, Kalchbrenner places it in Pholiota near 578. Fries includes it in Stropharia after 816.

\section{e. Aptindiculatie.}

837. H. LANARIPES Sacc. (from the woolly stem; lanla, wool, pis, a foot) $a b c$.

$P$. expanded, obtuse, thin, tough, apt to split, somewhat hairyscaly, atomate, rugose, tawny or brownish ; marg. appendiculate with white V. St. hollow, fibrous, striate above, subfibrillose or tomentoso-squarrose below. $G$. adnato-ascending, at first white, then pale-brown, at length purplish-brown.

Subcrespitose. Soil in conservatories. June-July. $3 \frac{1}{\frac{1}{4}} \times 4 \frac{1}{4} \times \frac{1}{4} \mathrm{in}$.

838. H. Candolleanum Quél. (after Alphonse de Candolle) a $b c$.

$P$. flat, oltuse, depressed, smooth, apt to split, thin, brown, becoming white; mid. ochreous. St. hollow, striate above, white. $G$. adnexo- or adnato-rounded, ventricose, serrulate, fuscous-cinnamon. Flesh white.

Gregarious, crespitose. Taste slightly styptic, disagreeable or none; odour strong. Stumps, elm, on the ground; frequent. Aprit-Nov. $3 \times 3 \frac{1}{2} \times \frac{1}{4}$ in. Firmer than 839 .

839. H. appendiculatum Quél. (from the appendiculate veil at the margin of the pileus) a b $c$.

$P$. hygrophanous, expanded, rugose, thin, apt to split, atomate, faintly ochreous-white; mid. ochreous. St. fistulose, pruinate above, white. $G$. adnato-rounded, purplish flesh-colour or brownish.

Caspitose. Stumps, sticks, under elm, on the ground; common. June-Oct. $3 \times 3 \frac{3}{4} \times \frac{1}{5}$ in. Thinner and more fragile than 838 . 
840. H. felinum Sacc. (from the tawny pileus, coloured like members of the cat genus, Felis) a b.

$P$. expanded, smooth, ochreous, biscuit-colour when dry. St. fistulose, shining, striate above, white-floccose at base. $V$. annulate. $G$. dove-colour, shaded brown.

Gregarious, subcæspitose. Parks, roadsides, etc, on the ground. Sept. $1 \times 1 \frac{1}{2} \times \frac{1}{8}$ in.

841. H. leucotephrum Sacc. (from the colour of the gills; Gr. leukos, white, tchlros, ash-coloured) $a b c$.

$P$. convexo-expanded, wrinkled, whitish or faint-salmon or ochreous-shaded. St. hollow, striate above, silky-fibrillose below, whitish. $A$. large. $G$. adnato-ascending, deep slateblack, edge whitish.

Caspitose. Base of ash-trees. Sept.-Oct. $2 \times 4 \frac{3}{\frac{3}{4}} \times \frac{1}{\frac{1}{4}} \mathrm{in}$.

842. H. egenulum Sacc. (from its poor appearance; egenulus, poor) $a$. $P$. hemispherical, broadly umbonate, smooth, white; marg. finely striate. St. fistulose, minutely adpressedly scaly, white, faintly sienna-ochreous below. $G$. adnate with a tooth, ventricose, purple-umber, edge white.

Solitary. On the ground amongst grass. MIay. $1 \frac{3}{8} \times 1 \frac{3}{4} \times \frac{1}{5} \mathrm{in}$.

843. H. hydrophilum Quél. (from its hygrophanous habit; Gr. hudor, water, philos, loving) a $b$.

$P$. flat, brittle, smooth, rugose, deep ferruginous-sienma or tawnyochreous, paler when dry. St. hollow, fibrilloso-mealy, shining, whitish above, faintly sienna below. $G$. adnexed or adnatorounded, crowded, exuding drops of water, brown or siemna.

Crespitose. About and on trunks and stumps. Oct.-Nov. $25 \times 4 \frac{1}{1} \times \frac{1}{4}$ in. P'ileus sometimes 5 in. in diam. Sometimes placed in Bolbitizs.

844. H. pilulæforme Gill. (from the appearance of the pileus, like a little ball, pilula) $a$.

$P$. even, smooth, fuscous, ochreous when dry. St. fistulose, white. $V$. annulate in young examples. $G$. adnexo-ascending, fuscous.

Crespitoso-crowded. Mossy trunks; rare. Autumn. $\frac{3}{4} \times \mathrm{I} \frac{1}{8} \times \frac{1}{16} \mathrm{in}$.

\section{PSILOCYBE Quél.}

(From the naked pileus, owing to the usually obsolete veil ;

Gr. psilos, naked, kube, a head.)

Fil very fugacious, usually obsolete. Hymonophore confluent with, but heterogeneous from the cartilaginous stem. Pilins more or less fleshy, smooth; the margin at first incurved. Stem central, fistulose or stuffed, subcartilaginous, rigid or tough, often rooting, simple. Gills becoming fuscous, brownish or purplish. (Fig. 46.) 
Nearly all the species grow on the ground and are gregarious and sometimes crespitose, a few grow on dung; they are inodorous and none are edible.

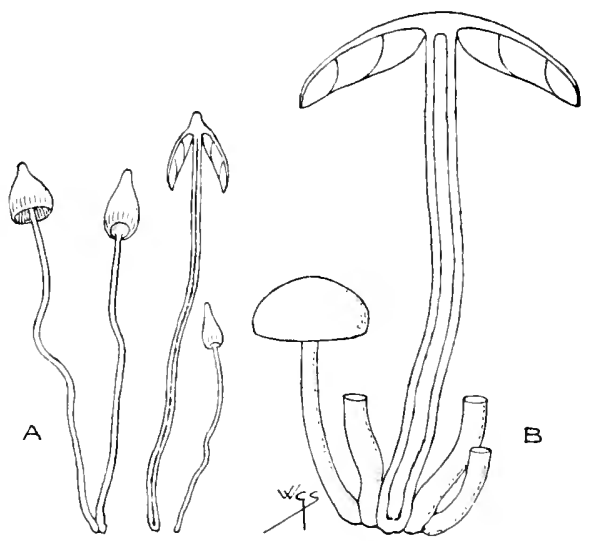

Fig. 46.-A, Fsilocy éc semilanceata Quél. : B. S. spadicea Quél. Entire and in section. One-half natural size.

Psilocybr agrees in structure with Collybia, Leptonia, Nancoria and Pancolus. Species $845-871$

«. Tenaces. Titil rarely conspicuous. Pilcus pelliculose, slightly viscid in wet weather, bright in colour, becoming pale. Stcm with a thick tough bark, flexile, usually coloured.

Gills ventricose, not decurrent.

$845-854$

Gills plane, very broad behind, subdecurrent

$855-860$

Gills ascending sublinear.

861,862

0. Rigida. Téil obsolete. Pileus scarcely pelliculose, flesh usually scissile, hygrophanous. Stcm rigid. Gills adnexed, rarely adnate.

$863-871$

\section{a. Tinaces.}

845. P. sarcocephala (iill. (from the fleshy pileus; Gr. sarx, flesh, kiphale, the head) a b.

$P$. expanded, obtuse, dry, pale tawny, salmon or pale ferruginous. St. whitish to rusty. $G$. adnato-rounded, salmon, then sooty. Flesh white, pale salmon in stem.

Gregarious, subcæespitose. Woods, grassy places, usually about or near stumps, elm. Sept.-Nov. $4 \times 5 \frac{3}{4} \times \frac{3}{4}$ in. Resembles 230 .

845a. P. helvola Mass. (from the yellowish colour; heliolus, pale yellow) $a$.

$P$. campanulate or conical, convex, then expanded, obtuse, even, glabrous, tawny-ochreous; mid. darker; cuticle often cracked into patches near mid. St. equal, hollow, fibrillose, often 
twisted, white or tinged ochreous, base thickened, white-downy. $G$. adnate, then separating, broad, subdistant, purplish-umber, clouded, edge white.

On the ground amongst grass. Sept. $\quad 1 \frac{1}{2} \times 2 \frac{3}{4} \times \frac{3}{15}$ in.

846. P. ericæa Quél. (from the habitat; erica, heath) a $b$.

$P$. flat, obtuse or subumbonate, tawny-ferruginous or umber, ochre when dry. St. smooth, subsilky, tan above, brownish below, base white-villous. $G$. adnate, subdistant, blackish with a light edge.

Gregarious. Woods, pastures; rare. May-Oct. $\quad 1 \frac{1}{4} \times 4 \frac{1}{2} \times \frac{1}{8}$ in. A smaller form occurs in wet places.

847. P. subericæa Sacc. (from its partial resemblance to 846) a b.

$P$. plane, obtuse or subumbonate, tawny or whitish-yellow, paler when dry. St. pallid or pale yellowish. $G$. adnato-sinuate, blackish.

Taste acid. Fields, under firs. June-Aug. $2 \frac{1}{8} \times 2 \frac{3}{8} \times \frac{3}{16}$ in.

848. P. uda Gill. (from the moist pileus ; udus, moist) $a b c$.

$P$. flat, obtuse, smooth or wrinkled, brick-tawny or yellowish. St. fibrillose, tan above, pale tawny-ferruginous below. $G$. adnato-sinuate, broad, fuscous-purple.

Scattered. Swampy places amongst Sphagnum. Oct.-Nov. $\quad 1 \frac{1}{2} \times 3 \frac{3}{2} \times \frac{1}{8}$ in. Gills sometimes remaining pallid and sterile.

849. P. eanofaciens Mass. (from the hoary appearance; anofaciens, making hoary) $a b$.

$P$. expanded, obtuse, umber; mid. subareolate, sienna; marg. darker with feeble traces of a fibrillose or arachnoid $V$. St. equal, hollow, brittle, umber-greyish, sooty below, white scaly. $G$. adnato-ascending, broad, livid-brownish, purple clouded.

Crespitose. Rotten straw and rubbish. May. $\quad 1 \frac{5}{8} \times 2 \frac{7}{5} \times \frac{3}{16} \mathrm{in}$.

850. P. areolata Sacc. (from the cracked-areolate pileus) $a b c$. Not brittle.

$P$. subexpanded, minutely fibrillose, white, then ochreous or fuscous. St. fibrillose, striate, $\tan$ and pulverulent above, sienna below. $\quad V$. fibrillose, evanescent. $G$. adnato-rounded, dull purple with a white edge.

Densely crespitose. Gardens, on wood. May-Oct. $\quad \mathbf{I}_{x}^{3} \times 2 \frac{1}{2} \times \frac{1}{4}$ in.

851. P. virescens Mass. (from the green tints; virescens, green) $a b$.

$P$. expanded, at first minutely silky, cracking, bright dark-brown, interstices of cracks clear pale verdigris-green, then yellowish. St. downy, apex striate, pale green above, ferruginous below. $G$. adnexed, subcrowded, smoky-purple with paler edge.

Solitary. Taste and odour none. Rotten stumps and chips. Autumn. $\frac{1}{2} \times 2 \frac{1}{2} \times \frac{1}{4}$ in.

852. P. agraria Karst. (from the habitat; ager, a field) $a$.

$P$. flat, obtuse or umbonate, white or ashy. St. smooth, white. $G$. adnate, ashy to fuscous. Flesh white.

Near stumps. Sept.-Oct. $1 \frac{3}{4} \times 2 \frac{3}{8} \times \frac{3}{16} \mathrm{in.}$ 
853. P. ehondroderma Sacc. (from the parchment-like pellicle of the pileus; Gr. chondros, cartilage, derma, skin) $a$.

$P$. conico-campanulate, smooth, cracked, dark date-brown; marg. jagged-appendiculate with V. St. fibrillose, pale sienna, darker below. $G$. ascending, adfixed, purplish-brown, edge white.

Woods, fir. Sept. $I_{\frac{1}{4}}^{\frac{1}{4}} \times I_{\frac{1}{2}}^{\frac{1}{2}} \times \frac{3}{16}$ in. Stains paper etc., yellow.

854. P. scrobicola Sacc. (from its habitat; scrobis, sawdust) a.

$P$. convex, umbilicato-depressed, whitish; marg. undulate. St. attenuate downwards, fibrillose, colour as P. G. arcuatoadnate, brown, with a red tinge.

Pine sawdust, branches. Nov. $\mathbf{I}_{\frac{3}{x}}^{\frac{3}{4}} \times \mathbf{1}_{8}^{3} \times \frac{3}{16}$ in.

855. P. ammophila Gill. (from the habitat; Gr. ammos, sand, philos, loving) $a b$.

$P$. expanded, subumbonate, sienna-buff; marg. striate. St. base clavate, apex striate, white to pale buff, half buried in sand. $G$. adnate or with a decurrent tooth, pale purplish, becoming slate-pulverulent with spores.

Sea-shores, amongst sand. Aug.-Sept. $\quad \mathbf{I}_{8}^{3} \times 2 \times \frac{1}{8}$ in.

856. P. coprophila Quél. (from the habitat; Gr. kopros, dung, philos, loving) a $c$.

$P$. expanded, obtuse or subumbonate, downy, white, then rufescent. St. shaggy-flocculose, whitish. $G$. adnate, very broad, crowded, fuscous.

Amongst grass, rabbits'-dung, on dung-heaps; rare. Oct.-Jan. $1_{\frac{3}{5}}^{3} \times I_{\frac{3}{7}}^{\frac{3}{7}} \times \frac{1}{8}$ in. Sometimes infesting mushroom-beds.

857. P. bullacea Quél. (from a fancied resemblance in the pileus, when young, to a knob, bulla a a $b c$.

$P$. expanded, subumbonate, dark tawny, tan when dry; marg. appendiculate with V. St. subfibrillose, tawny or yellow, base fuscous-ferruginous, fibrilloso-annulate when young. $G$. adnate with a small sinus, very broad, crowded, fuscous-ferruginous.

Gregarious. Dung, rich soil, amongst Polytrichum. April-Sept. $\mathbf{I} \frac{1}{8} \times \mathbf{I} \times \frac{1}{8}$ in.

858. P. physaloides Quél. (from a fancied resemblance in the pileus to a bladder; Gr. phusalis, a bladder, cidos, appearance) a $b c$.

$P$. flat, umbonate, fuscous-purple. St. adpressedly fibrillose, date-brown below, paler above. $G$. adnato-decurrent, crowded, ventricose, pallid ferruginous-fuscous.

Rich grassy places, woods, on moss, horse-dung; old walls; uncommon. Feb.-Sept. $\quad$ I $3 \times \frac{1}{3} \times \frac{1}{15}$ in. Must not be confounded with 771 .

859. P. atrorufa Quél. (from the blackish-red pileus; atcr, black, rufus, red) $a$.

$P$. expanded, obtuse, black-rufous or purple-fuscous; mid. darker; marg. striate. St. at first subfibrillose, subpruinate 
above, colour as P., base darker. $G$. adnate with a small tooth, umber to purple-umber.

Woods, mixed. Nov. $1 \frac{1}{\frac{1}{x}} \times 2 \frac{1}{8} \times \frac{1}{15}$ in.

860. P. nuciseda Mass. (from the frequent habitat, nuts; $n u x$, a nut, sedeo, to sit) $a$.

$P$. expanded, subumbonate, subsilky when dry, light yellow or shaded sienna; mid. sienna. St. slightly attenuate downwards, fuscous, or white below. $G$. adnate, broad, ventricose, fuscous to umber-black.

Beech and hazel nuts, chips, wood, $I \frac{1}{8} \times 1 \frac{1}{8} \times \frac{1}{15}$ in. Habit of 771 .

861. P. compta Sacc. (from the adorned pileus, sprinkled with minute shining spots ; comptus, ornamented) $a$.

$P$. expanded, obtuse, pallid to ochreous; marg. striate, subcrenulate. St. flexuous, silky-shining, tan, faintly rufous below. $G$. adnato-ascending or adnexed, distant, rosy-umber.

Woods, amongst grass; rare. Sept.-Oct. $1 \frac{1}{5} \times 2 \frac{1}{1} \times \frac{1}{16} \mathrm{in}$.

862. P. semilanceata Quél. (from a fancied resemblance in the pileus, as seen in vertical section to a lance-head; semi, half, lancea, a spear) $a b c$.

$P$. acutely conical, whitish-ochre; mid. pale; marg. slightly striate, slate, sometimes fuscous-shaded. St. flexuous, smooth, fibrilloso-annulate when young, colour as P., base pale sienna. $G$. adnexo-ascending, crowded, purplish to purple-black.

Gregarious. Reputed poisonous. Pastures, roadsides; common. Stem sometimes 6 in. high. Aug.-Nov. $\frac{1}{2} \times \frac{3}{8} \times \frac{1}{16}$ in. Var. cerrulescens S. Sacc. $P$. subobtuse. St. more or less distinctly linged with biue.

\section{b. Rigida.}

863. P. canobrunnea Quél. (from the greyish-brown pileus; canus, hoary, brunneus, brown) a $b$.

$P$. plane, subviscid when moist, flesh-brown, pale clay when dry. St. rooting, squamulose, whitish. $G$. adnexed, crowded, broad, ventricose, brownish-purple.

Solitary or laxly gregarious. On the ground in sunny places, on scorched ground. Autumn. $2 \times \mathrm{I}_{8}^{3} \times \frac{3}{16} \mathrm{in.}$

864. P. spadicea Quél. (from the colour of the pileus; sfadiculus, date-brown) $a b c$.

$P$. plane, usually cracked, dark to ochreous-brown, becoming paler in patches. St. smooth, whitish, base pale sienna. $G$. adnexo-rounded, adnate or adnate with a tooth, crowded, brown.

Laxly clustered. Stumps, earth, manure-heaps, amongst short grass; common. Feb.-Nov. $25 \times 4 \times \frac{3}{\mathrm{i}} \mathrm{in}$. Var. hygrophila Karst. larger than iype. Var. folycephala Gill. densely crowded. 
865. P. squalens Karst. (from its dirty colours ; squalins, dirty) $a$.

$P$. flat, pale livid-ferruginous, pale dull clay-colour when dry. St. striate above, colour as P. G. adnato-decurrent, crowded, cinnamon-umber.

Solitary or caspitose. On and near trunks; rare. $1 \frac{3}{4} \times 2 \frac{1}{4} \times \frac{1}{5} \mathrm{in}$.

866. P. cernua Quél. (from the frequently drooping pileus; cermuo, to fall head foremost) $a b c$.

$P$. expanded, slightly wrinkled when dry, whitish. St. smuoth, flexuous, colour as P. G. adnato-ascending, cinereous-blackish or slate-sprinkled.

Solitary or gregarious, usually cæespitose. Dead wood, chips, leaves, lawns; uncommon. Aug.-Dec. $\quad \mathbf{1} \frac{1}{2} \times 3 \frac{1}{2} \times \frac{1}{8}$ in.

867. P. hebes Karst. (from the obtuse pileus; hcbes, blunt) a b.

$P$. becoming flat, striate when moist, lurid, obscure olive, pale when dry. St. naked, whitish. $G$. adnexo-rounded, broad, crowded, brownish or brown.

Usually solitary. Amongst grass and leaves, near chestnut-trees, trunls. Nor. $1 \frac{1}{4} \times 1 \frac{1}{4} \times \frac{1}{8}$ in.

868. P. fœnisecii Quél. (from its habitat, meadows ; fonisicia, hayharvest) a $b c$.

$P$. campanulate, obtuse, pale ferruginous-fuscous to deep umber, becoming paler in patches. St. pulverulent, then naked, rufescent, siemna-tan or flesh-colour. $G$. adnato-ascending, ventricose, separating from stem, umber to black-purple.

Gregarious. Fields, lawns, roadsides, manure, horse-dung ; common. Feb.Dec. $\frac{3}{4} \times 2 \frac{3}{4} \times \frac{1}{16} \mathrm{in}$.

869. P. callosa Quél. (from the tough stem ; callosus, haring a hard skin) $a b c$.

$P$. conico-campanulate, obtuse, pale buff. St. smooth, paler than P. $G$. adnato-ascending, ventricose, sooty or purpleblack.

Scattered or in small groups. Fields, lawns, roadsides. Aug.-Oct. $\frac{5}{16} \times 2 \frac{3}{8} \times \frac{1}{32}$ in.

870. P. elivensis Sacc. (from the place where it was first observed, King's Cliffe, Northamptonshire) a $b$.

$P$. expanded, shining-atomate, striate, pallid brown or ochre to white. St. silky, whitish. $G$. adnate, widely emarginate, rentricose, umber, edge white.

Amongst grass, on downs. June-Oct. $1 \frac{1}{4} \times \mathrm{I}_{4}^{3} \times \frac{1}{5}$ in.

871. P. catervata Mass. (from its fasciculate habit; cateri'd, a troop) a.

$P$. campanulate, obtuse, satiny, brittle, white. St. white. $G$. slightly adnexed, somewhat lroad, crowded, purple-shaded or cimnamon, edge white.

On the ground. Oct. $\frac{5}{8} \times 2 \frac{5}{8} \times \frac{1}{15}$ in. 


\section{PSATHYRA Quél.}

(From the friable substance; Gr, psathuros, friable.)

Veil when present universal, floccoso-fibrillose, not annulate, sometimes obsolete. Hymcnophore confluent with, but heterogeneous from the cartilaginous stem. Pileus conical or campanulate, then expanded, submembranous, margin at first straight and adpressed to
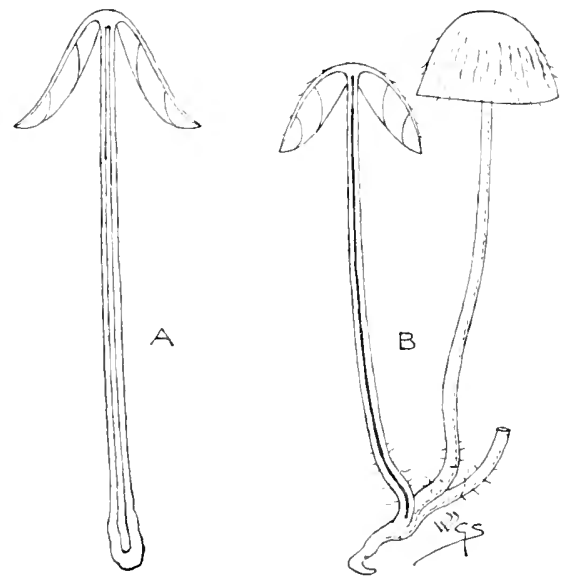

Fig. 47.-A, section of F'satlyyra conopizea Quét. ; B, $P$. bifrons Sacc. entire and in section.

One-half natural size.

the stem, except 884. St'm central, somewhat cartilaginous, fistulose, lustrous, fragile, simple. Gills at first pale, becoming purple or fuscous. Stores purple, brownish-purple, dark purple or dark brown. (Fig. 47.)

The species grow on the ground, or on rotten wood, they are slender, fragile, hygrophanous and with fugitive colouring.

Psathyra agrees in structure with Mycona, Nolanea, Galera and Psathyrilla.

Species $872-893$

a. Conopilar. Itil obsolete. Pilcus conico-campanulate. Stcm tense and straight, fistulose. Gills usually adnexoascending, often free. On the ground in grassy or damp places.

$872-877$

b. Obtusate. Vil obsolete. Pilius campanulato-convex, becoming flat; smooth or atomate. Gills plano- or arcuatoadfixed.

$878-880$

c. Fibrillosa. Pilcus-except 884 and 888 -and stem at first fibrillose or floccose from the veil. St'm fistulose. 


\section{a. Conopiler.}

872. P. elata Nass. (from its long stem ; clatus, tall) $a b$.

$P$. even, glabrous, atomate, dark brown, pale ochreous when dry. St. hollow, silky-shining, white. $G$. broadly adnate, crowded, purplish-brown.

Amongst grass, on hedge-banks. Aug. $2 \times 7 \frac{5}{8} \times \frac{1}{ \pm} \mathrm{in}$.

873. P. conopilea Quél. (from the conical pileus) $a b c$.

$P$. even, smooth, whitish, greyish-brown or umber with marg. tan. St. attenuate upwards, whitish, silvery-lustrous. $G$. slightly adnexed, subventricose, dark slate or fuscous-purple.

Grassy places, gardens, ditch-bottoms, under beeches; rare. Sept.-Nov. $1 \frac{5}{8} \times 4 \frac{3}{8} \times \frac{1}{5}$ in. Stem sometimes $6 \frac{1}{2}$ in. long. Var. sufterth W. G. Sm., Agaricus suferbus Jungh. larger. $P$. chestnut-brown to buff.

874. P. mastigera Sacc. (from the breast-like shape; Gr. mastos, the breast, gero, I bear) a $b$.

$P$. acutely umbonate, dark chestnut-brown, umber-tan when dry. St. fibrillose, furfuraceous or smooth, white. $G$. narrow, umber. Flish pale brown.

Amongst grass, roadsides. July-Nov, $\frac{7}{5} \times 3 \frac{5}{5} \times \frac{1}{8}$ in.

875. P. glareosa Sacc. (from the habitat, gravelly soil; glarcosus, gravelly) $a$.

$P$. obtuse or umbonate, minutely tomentose, atomate, striate, grey; mid. pale chestnut. St. brown, white fibrillose. $G$. broadly adnate, umber. Flesh brown.

Gravelly soil after wet weather. June. $\frac{3}{4} \times 2 \frac{1}{4} \times \frac{1}{16}$ in.

876. P. corrugis Quél. (from the pileus, at length wrinkled; corrugis, having folds) $a b c$.

$P$. subumbonate or obtuse, smooth, substriate, whitish, pale rose, ochreous-tan or pale umber. St. smooth, colour as P. G. adnato-asccnding or somewhat sinuate, pale, then purple-black or dark slate.

Pastures, grassy places, open places in woods, amongst grass and sticks, old manure patches; frequent. April-Jan. $1^{\frac{3}{4}} \times 3_{\frac{1}{8}}^{\frac{1}{8}} \times \frac{3}{16}$ in. Stem sometimes $5 \mathrm{in}$. long. Var, zinosa IIass. $P$. rosy-vinous half-way up. Var. gracilis Mass. more slender than type.

876a. P. pellosperma W. G. Sm., Agaricus pellospermus Cooke (from the dark coloured spores; Gr. pellos, dark coloured, sperma, a seed) a.

$P$. subcampanulate or subovate, even, then striate, sometimes rugose, white or ochreous-white, becoming fuliginous with age. St. fistulose, naked, nearly equal, white or as P. G. broad, free, much narrowed at the tips, cinereous, then fuliginous, at length black. Flesh thin, white.

On the ground. Autumn, I $\times 4 \frac{7}{8} \times \frac{1}{8}$ in. 
877. P. gyroflexa Quél. (from the weak flexuous stem; gyrus, a circle, flexus, bent) $a b$.

$P$. atomate, striate, whitish or pale grey; mid. pale buff or rufescent. St. white, silky-shining. $G$. adnato-ascending, ventricose, greyish-purple to slate.

Scattered or subcespitose. Margins of woods, amongst rubbish. Aug. $1 \frac{1}{6} \times 2 \times \frac{1}{16}$ in.

\section{b. Obitusatie.}

878. P. spadiceogrisea Quél. (from the brown-grey pileus; spadix, bay-brown, sriseus, grey) a b c. Watery, fragile.

$P$. even, pellucid-striate to mid., date-brown, greyish and buff when dry. St. subpulverulent, striate above, whitish. $G$. adnexed, crowded, narrow, umber-fuscous or brown.

Solitary or gregarious, subcrespitose. On trunks, old boards, chips, on the ground. Aug.- -Nov, $1 \frac{5}{3} \times 3 \frac{1}{5} \times \frac{1}{8}$ in.

879. P. obtusata Gill. (from the obtuse pileus) a $b c$.

$P$. even, brown-fuscous or pale umber, paler and striate at marg. St. fibrilloso-silky, not pulverulent, eren above, whitish. $G$. adnate, broad, suldistant, ashy-brown or umber.

Single or cæspitose. Woods, on the ground, on wood, trunls, oak; rare. June-Dec. $\quad I^{3} \frac{3}{8} \times 2 \frac{7}{5} \times \frac{1}{8}$ in. Stem sometimes rooting.

880. P. neglecta Mass. (from its having been overlooked) $a b$.

$P$. almost plane, even, glabrous, atomate, pale rufous, almost white when dry ; mid. pale clay. St. somerwhat wary, pellucid, pruinose above, white, tinged pale rufous below. $G$. slightly attached, ventricose, crowded, pink then purple-brown.

On the ground, gardens. Oct.-Nov. $\frac{3}{8} \times \mathbf{I} \times \frac{1}{32} \mathrm{in}$.

\section{c. Fibrillose.}

881. P. frustulenta Sacc. (from its fragility, breaking at a touch; frustulentus, full of pieces) $a$.

$P$. hygrophanous, glabrous, substriate, siemna-tan, tan when dry. St. subwavy, whitish. $G$. adnato-ascending, crowded, brownish. Gravel in damp places. Sept. $1 \frac{1}{t} \times 2 \frac{1}{t} \times \frac{1}{15}$ in.

882. P. bifrons Sacc. (from the two-coloured pileus; bifrons, with two faces) a $b$.

$P$. obtuse, subwrinkled, ochreous-brown tinged with red, whitish when dry, evanescent white-fibrillose. St. subrooting, minutely satiny, fibrillose below, white. $G$. adnate, broad, ventricose, pink-cinereous; edge white.

Sometimes two or three connate. Woods, amongst sticks, twigs, chips; rare. Aug.-Sept. $1 \frac{1}{5} \times 3 \frac{7}{3} \times \frac{1}{16}$ in. Tar. semitincta Sacc. P. vinouspurple ; mid. pale ochre. 
883. P. semivestita Sacc. (from the pileus white-fibrillose halfway to the middle; semi, half, vestio, to clothe) a $b$.

$P$. obtuse, chestnut-brown, tan when dry. St. fibrilloso-silky, whitish. $G$. adnato-ascending, grey, becoming umber.

Solitary and large, or cæespitose and smaller. Rich pastures, waysides, woods. Oct. Solitary $2 \frac{1}{4} \times 4 \frac{3}{4} \times \frac{1}{8}$ in. Cæspitose $\frac{7}{8} \times 3 \frac{1}{4} \times \frac{1}{16}$ in.

884. P. Loscosii Sacc. (after Francisco Loscos) $a b$.

$P$. flat, radiato-pitted, sulcato-wrinkled, moist, cracking, atomate, tan-umber. St. striate, twisted, subfibrillose, elastic, tan, white-pruinose above, rufescent below. $G$. adnate, ventricose, thin, purple-umber with pale edge. Flesh sienna.

Crespitose. Taste and odour slight, fungoid. Gardens, on mushroom-bed. Nov. $2 \times 7 \frac{1}{ \pm} \times \frac{3}{16}$ in.

885. P. fatua Quél. (from its resemblance to its allies; fatuus, foolish) $a$.

$P$. rugose, at length smooth, dingy-ochreous, then whitish; mid. sienna. St. striate and mealy above, becoming smooth, white. $G$. adnato-ascending, crowded, brown.

Gardens. Autumn. $1 \frac{1}{8} \times 3 \frac{3}{\frac{1}{4}} \times \frac{1}{5}$ in.

886. P. fibrillosa Quél. (from the fibrillose covering) a $b$.

$P$. conical then flat, striate, becoming smooth, livid or whitish, the white fibrils becoming black with age or disappearing. St. white squamulose on pale steel-grey ground, or spreading whitefibrillose. $G$. adnate, pale slate then purple-black.

Solitary. Woods, damp places, rotten branches, sticks and leaves; rare. Oct. $2 \frac{1}{5} \times 2 \frac{1}{8} \times \frac{3}{16}$ in.

887. P. Gordonii Gill. (after the Marchioness of Huntly) $a$.

$P$. sulcato-striate, pale cinereous to white. St. transversely undulate, white-pruinose above, then shining, smooth, white. $G$. ascending, narrowly adnate, cinereous.

Densely cæspitose. Odour faint to strong and nauseous. Stumps, amongst chips. Oct. $\quad 1 \frac{5}{8} \times 2 \frac{1}{2} \times \frac{1}{8}$ in.

888. P. helobia Sacc. (from its habitat; Gr. helos, a marsh, bios, life) a $c$.

$P$. flat, subumbonate, radiato-rugose; mid. with concentric elevated ridges, sooty-brown, becoming ochreous-salmon or reddish. St. reddish-umber, salmon when dry. $G$. adnate, sooty-brown. Flesh pale pink.

Woods. Sept. $2 \frac{1}{4} \times 5_{\frac{3}{4}}^{\frac{3}{4}} \times \frac{3}{T^{6}}$ in. Allied to 884 .

889. P. pennata Quél. (from the feathery scales; pcnna, a feather) a $b c$.

$P$. livid, whitish or pale brown ; mid. sienna ; marg. appendiculate with large white sc., then naked. St. villous, white pulverulent above, white. $G$. adnexo- or adnato-ascending, ventricose, fuscous-blackish.

Gregarious. Gardens, scorched ground, earth, burnt sawdust ; rare. JuneOct. I I $\frac{3}{8} \times 2 \frac{3}{3} \times \frac{1}{8}$ in. 
890. P. gossypina Gill. (from its cotton-like fibrils; gossypizım, cotton) $a b c$.

$P$. expanded, ochreous-clay; mid. pale sienna; marg. striate. St. whitish-tomentose. $G$. adnexed, ventricose, white, then fuscous-black.

Subcrspitose. Woods, twigs, on the ground. May-Oct. $2 \frac{1}{2} \times 2 \frac{5}{8} \times \frac{3}{16}$ in.

891. P. noli-tangere Quél. (from its fragility, breaking at a touch; noli tangere, do not touch) $a b$.

$P$. expanded, obtuse, hygrophanous, pale umber when large, dark fuscous when small, becoming pale; marg. striate, appendiculate with sc. St. subnaked, even, grey-white above, fuscous or slate below. $G$. adnexo- or adnato-rounded, fuscous. Flesh pale brownish.

Gregarious. Oak chips, amongst moss, damp sandy ground. Sept.-Dec. I $\times 1 \frac{3}{8} \times \frac{1}{16}$ in.

892. P. microrrhiza Sacc. (from the small rooting stem ; Gr. mikros, small, rhiza, a root) $a b c$.

$P$. ochreous or rufous, at first yellow-pilose, then pale, atomate. St. attenuato-rooting, whitish-scaly. $G$. adnexo-ascending, black-brown.

Gregarious. Gardens, on the ground. Sept. $1 \frac{3}{8} \times 4 \times \frac{1}{16}$ in.

893. P. urticæcola Sacc. (from its habitat, nettle-roots; urtica, a nettle, colo, to inhabit).

$P$. white-flocculent; marg. striate. St. white-flocculent. $G$. adnexo-ascending, rich chocolate.

At the base of old nettle-stems. Aug. $\frac{5}{16} \times \mathbf{I} \times \frac{1}{15}$ in.

\section{SERIES V. COPRINARII Fr.}

(From the habitat; Gr. kopros, dung.)

Spores black. Gills from the first separate, not cohering and becoming fluid as in Coprimus.

Intermediate between the preceding series and Cofrimus.

Genera XXXIX-XLI.

\section{ANELLARIA Karst.}

(From the annulus; ancllus, a little ring.)

Veil appendiculate at the margin of the pileus. Hymenophore confluent with, but heterogeneous from the cartilaginous stem. Pilus subfleshy, conico-campanulate, without striæ, shining when dry, not expanding, the margin exceeding the gills. Stcm central, lustrous, somewhat firm, fistulose, annulate. Gills ascending, black or variegated slate-blackish, not deliquescent. (Fig. 48.) 
The species usually grow in rich grassy places or on dung, from summer to late autumn, often near towns and villages.

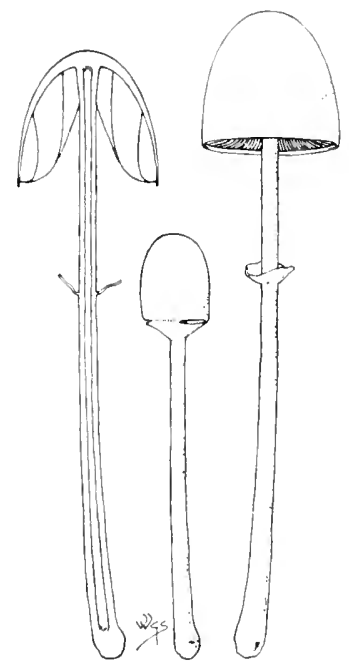

Fig. 48.-Anellario separata Karst.

Entire and in section.

One-third natural size.

The nearest structural allies are found in Amanita, and the nonBritish genera Metraria and Locellina.

Species 894-896

894. A. separata Karst. (from its appearance, distinct from species placed near it) $a b c$.

$P$. whitish to tan-ochreous; mid. sienna-tinted. St. attenuate upwards, colour as P. $A$. distant, persistent, white. $G$. adnatoascending, broad, dark purplish-slate.

In pastures on dung; common. April-Dec, $2 \frac{1}{2} \times 66^{3} \times \frac{1}{4}$ in. Stem sometimes 8 in.

895. A. scitula Sacc. (scitullus, handsome) a $b$.

$P$. dull pale ochre. St. white, base peronate, ending as a persistent $A$. below mid. $G$. adnexo-ascending, ashy-grey to black. Flesh white.

On cow-dung, on soil in a flower-pot, Scarborough, $1885 . \quad \frac{7}{16} \times 1 \frac{1}{4} \times \frac{1}{16}$ in.

896. A. fimiputris Karst. (from its habitat, rotten dung ; fimus, dung, putris, rotten) $a b c$.

$P$. innato-silky, pale umber to dark lead-colour; marg. appendiculate with white $\mathrm{V}$. St. colour as P., umber below. $A$. superior, small, imperfect. $G$. adfixo-ascending.

Solitary, rarely cæspitose. Taste insipid. Fields, gardens, horse-dung; common. April-Dec. $\mathrm{I}_{\frac{1}{2}} \times 5_{\frac{3}{3}} \times \frac{1}{8}$ in. Examples with a wrinkled pileus must not be confounded with 900 . 


\section{PANÆOLUS Quél.}

(From the variegated gills; Gr. panaiolos, all variegated.)

Veil appendiculate at the margin of the pileus. Hymenophore confluent with, but heterogeneous from the cartilaginous stem. Pileus subfleshy, conical without stria, not expanding, the margin exceeding the gills, viscid when moist, lustrous when dry. Stem central, lustrous, somewhat firm, fistulose except 905-8, simple.

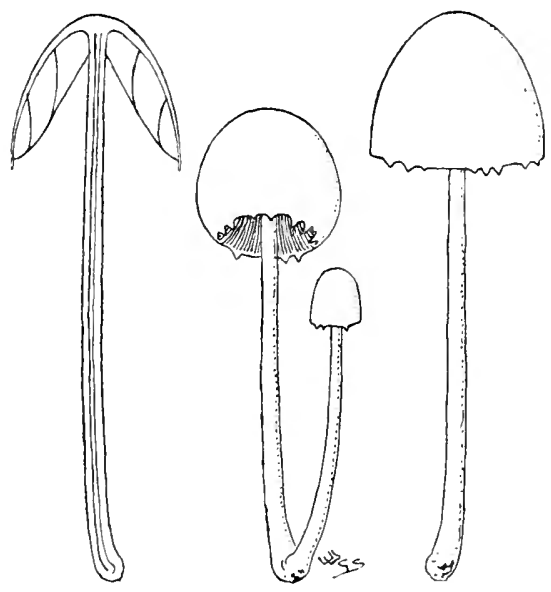

Fig. 49.-Pancolus campanulatus Quél.

One-half natural size. Entire and in section.

Gills ascending, black or variegated slate-blackish, not deliquescent. (Fig. 49.)

The species usually grow in rich grassy places or on dung from summer to late autumn, often near torvns and villages.

The nearest structural allies are found in Collybia, Leptonia, Nancoria and Psilocybe.

Species $897-908$

Pileus conico-campanulate with a gelatinous-liquescent cuticle, viscid, shining when dry. Stem fistulose, minutely so in 898.

$897-899$

Pileus hemispherical to conico-campanulate, moist or damp, opaque, bibulous, somewhat flocculose when dry. Stem fistulose.

900,901

Pilcus hemispherical to conico-campanulate, dry, smooth, slightly shining, not zoned. Stcm fistulose.

$902-904$

Pileus conical to campanulate, dry, smooth, zoned round the " margin. 
897. P. leucophanes Sacc. (from its white appearance; Gr. leukos, white, phaino, to appear) $a b$.

$P$. expanded, obtuse, innato-silky, white or faintly ochreous; marg. appendiculate with V. St. mealy, fibrillose, white. $G$. adnato-ascending, black with white edge.

Pastures, grassy places, manure-heaps. Aug.-Nov. $1 \frac{1}{4} \times 3 \frac{3}{8} \times \frac{1}{8}$ in.

898. P. egregius Sacc. (egregins, distinguished) $a b$.

$P$. slightly agglutinato-downy, watery, virgate when dry, bright orange-brown; mid. darker. St. solid, attenuate upwards, fibrous, fibrillose, splitting longitudinally, warm umber, base white-cottony. $G$. adnexo-ascending, ventricose, crowded, brownish- or purple-black. Flesh ochreous.

Solitary. Odour none. On the ground, on horse-dung in fields. Jan. $2 \frac{1}{4} \times 4 \frac{3}{4} \times \frac{3}{8}$ in.

899. P. phalænarum Quél. (from the variegated gills, like the wings of certain moths; Gr. phallaina, a moth) $a b c$.

$P$. obtuse, clay-white; marg. appendiculate with fugacious $V$. St. subnaked, tan-rufescent. $G$. adnexo-ascending, broad, ventricose, ashy-black.

Rich pastures, on dung. Sept.-Oct. $I \frac{3}{4} \times 4 \frac{7}{8} \times \frac{1}{8}$ in.

900. P. retirugis Gill. (from the net-like wrinkles on the pileus; rcte, a net, ruga, a wrinkle) a $b c$.

$P$. obtuse, atomate, fleshy-tan ; mid. ochreous-tan or dark umber with paler marg., appendiculate with V. St. pruinose, fleshcolour, then purplish, rufescent or cinereous. $G$. adfixoascending, cinereous-blackish.

Sometimes fasciculate. Dung, cow, manure-heaps, woods; uncommon. Feb.-Nov. $1 \frac{1}{2} \times 4 \frac{3}{4} \times \frac{1}{8}$ in.

901. P. sphinctrinus Quél. (from the pileus, contracted, never expanded; Gr. sphigktir, a muscle naturally contracted) a $b c$.

$P$. opaque, silky or fibrillose when dry, fuliginous or greyishshaded, livid when dry; marg. appendiculate with V. St. smooth, pruinose above, fuliginous-grey. $G$. adnato-ascending, crowded, cinereous-blackish.

Pastures, on dung and on garden soil. April-Nov. $I \times 3 \frac{1}{4} \times \frac{1}{16}$ in.

902. P. papilionaceus Quél. (from the speckled pileus, as in some butterflies; papilio, a butterfly) a b $c$.

$P$. rimoso-scaly when dry, dull tan whitish or greyish; mid. faintly ochreous. St. white pulverulent above, whitish or faintly ochreous. $G$. adnato- or adnexo-ascending, broad, ventricose, blackish.

Pastures, lawns, manured ground, dung; common. July-Nov. $I_{\frac{3}{4}}^{3} \times 4 \frac{1}{1} \times \frac{1}{8}$ in.

903. P. campanulatus Quél. (from the bell-shaped pileus; campanula, a little bell) $a b c$.

$P$. sometimes umbonate, not cracked, fuliginous-fuscous, or somewhat umber-slate with umber mid., sienna and umber or grey when dry. St. smooth, pruinose and grey above, rufescent 
or purplish below. $V$. fugacious or obsolete. $G$. adnatoascending, crowded, grey and black, edge whitish.

Pastures, rich ground, horse-ång, amongst grass; common. June-Nov. I $\frac{3}{8} \times 4 \frac{1}{4} \times \frac{1}{8}$ in.

904. P. caliginosus Gill. (from its gloomy colour; caligo, gloom) $a b c$. $P$. obtuse, even, smooth, ochreous-brown or grey. St. naked, rufescent-brown, lighter above. $V$. feeble or obsolete. $G$. adnato- or adnexo-ascending, ventricose, fuliginous-black.

Rich pastures, lawns. Oct.-Nov. $\frac{9}{16} \times 2 \frac{3}{4} \times \frac{1}{16}$ in.

905. P. subbalteatus Sacc. (from the somewhat zoned margin of the pileus; balteus, a band) a.

$P$. obtuse or subumbonate, hygrophanous, irregular, subwrinkled, dull fawn or pale ochreous; mid. pale sienna. St. splitting longitudinally, white-fibrillose, red-brown and ochreous above, pale sienna below. $G$. adnato-ascending, ventricose, brownish ; edge toothed, white.

Caspitose. Borders of fields, on the ground. Sept.-Oct. $\frac{3}{4} \times 3_{\frac{3}{4}}^{3} \times \frac{1}{15}$ in.

906. P. acuminatus Quél. (from the sharp apex of the pileus; acuminatus, sharp pointed) a $b$.

$P$. even, shining, flesh-tan ; mid. faint sienna. St. pruinose, tan above, fuscous below. $G$. adnexo-ascending, crowded, grey and black.

Dung, roadsides. Oct. - Nov. $\frac{7}{8} \times 1 \frac{1}{2} \times \frac{1}{8}$ in.

907. P. fimicola Quél. (from the usual habitat; fimus, dung, colo, to inhabit) a b $c$.

$P$. obtuse, smooth, opaque, fuliginous-grey, clay-hoary when dry: marg. with a fuscous zone. St. slightly silky-striate, whitepruinose above, dingy below. $G$. adnato-ascending, ventricose, grey and fuliginous.

Dung, rich pastures; rare. Oct. $\frac{7}{8} \times 3 \frac{1}{2} \times \frac{1}{16}$ in.

908. P. einctulus Sacc. (from the zone round the margin of the pileus : cinctus, a girdle) $a$.

$P$. even, smooth, reddish-cinnamon ; marg. zoned, dark brown. St. dull fuscous. $G$. ascending, adnexo-free, dusky- or olivaceusblack or slate.

Dunghills after rain. June-July. $2 \frac{5}{8} \times 4 \frac{1}{4} \times \frac{1}{8}$ in.

\section{PSATHYRELLA Quél.}

\section{(Diminutive of Psathyra.)}

Iril almost obsolete. Hymenophore confluent with, but heterogeneous from the cartilaginous stem. Filcus membranous, striate; margin not exceeding the gills, straight and at first adpressed to the stem. Stem central, fistulose, simple. Gills adnate or free, not variegated or becoming fuscous or purple, not deliquescent. Spores black or nearly so. (Fig. 5०.)

The species grow on rich ground, in gardens, by hedges and stumps, sometimes on wood. 
Psathyrella agrees in structure with Mycena, Nolanea, Galira and Psathyra.

Species 909-920

Pilcus conical to campanulate. Stcm tense, straight, fistulose.

$909-914$

Pileus hemispherical to campanulate or conical. Stcm Hexuous, pruinate at the apex, fistulose.

$915-920$

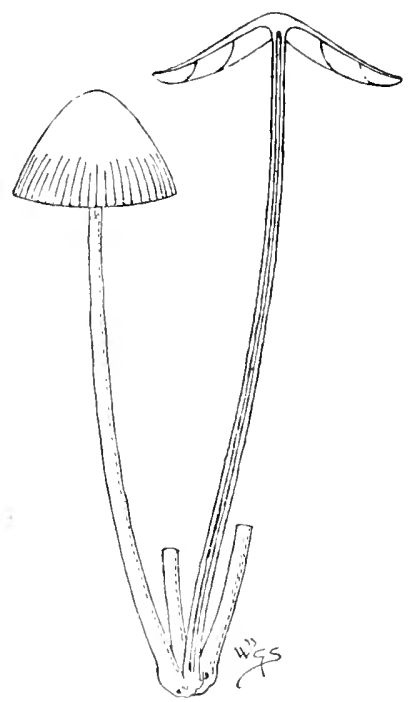

Fig. 50.-F'sathyyella subatrata Gill. One-half natural size.

Entire and in section.

909. P. subatrata Gill. (from the blackish pileus; ator, black) a b.

$P$. obtuse, subexpanded, umber-rufescent, pallid-rufescent when dry. St. smooth, whitish. $G$. adnexo-ascending, crowded, fuliginous, blackish-umber.

Gregarious. Rich ground, grassy places, woods. Sept. $2 \frac{1}{8} \times 5 \frac{3}{8} \times \frac{1}{8}$ in.

910. P. gracilis Quél. (gracilis, slender) a $b c$.

$P$. obtuse, smooth, dark- or pale-fuliginous or livid with pale shades of rose, tan when dry. St. smooth, whitish. G. adnate, cinereous-blackish ; edge rose.

Gregarious, waysides, hedgerows, etc. ; common. July-Oct. $1 \frac{7}{8} \times 4 \times \frac{1}{8}$ in.

911. P. hiascens Quél. (from the pileus opening in furrows; hiasco, to gape) $a$.

$P$. expanded, obtuse, thin, cracked-revolute, white or warm tan, becoming pale tan-yellow; mid. pale sienna. St. smooth, whitish. $G$. adnate, distant, black.

Grassy places, hedgerows, damp woods, rubbish-heaps; rare. Nov. I $\frac{5}{5} \times 3 \frac{7}{8} \times \frac{1}{8}$ in. 
912. P. arata Sacc. (from the furrowed pileus; aro, to plough) $a$.

$P$. attenuato-conical, deeply sulcate, sienna-brown. St. smooth, whitish or faint sienna-white. $G$. ascending free, purplishblack.

Hedgerows. Autumn. $\quad 1 \frac{7}{8} \times 5 \frac{1}{4} \times \frac{1}{8}$ in.

913. P. trepida Gill. (from its trembling habit ; trefidus, trembling) $a b c$.

$P$. obtuse, smooth, fuliginous; mid. date-brown. St. naked, tan-whitish. $G$. adnate, crowded, fuliginous-black.

Moist ground, bases of trunks, gardens. Oct. $\frac{7}{8} \times 3 \frac{1}{8} \times \frac{1}{15} \mathrm{in}$.

914. P. hydrophora Quél. (from its hygrophanous substance; Gr. hindor, water, phero, to carry) a $b c$.

$P$. expanded, obtuse, then cracked-revolute; mid. rufous: marg. paler. St. white, studded with drops of moisture. G. adnate, crowded, black.

Gardens. Autumn. $I^{\frac{3}{4}} \times 3 \frac{1}{2} \times \frac{1}{8}$ in.

915. P. caudata Quél. (from the tail-like base of the stem; canda, a tail) a $b c$.

$P$. gibbous then flat, smooth, sometimes areolato-cracked, atomate, date-brown or umber. St. with rooting base, undulate on surface, white. $G$. adnate, ventricose, ashy-black or dull purplish-brown.

Wooden pavement, rotten wood, rich soil, gardens, amongst dead leaves. Sept.-IJec. $2 \frac{3}{4} \times 4 \frac{5}{8} \times \frac{1}{8} \mathrm{in}$.

916. P. prona Gill. (from its frequent prostrate state; fromis, prostrate) $a b c$.

$P$. subexpanded, obtuse, subsilky, fuliginous, atomate and hoary when dry. St. smooth, whitish. $G$. adnate or adnexed, livid fuliginous, black-dotted.

Grassy places; rare. May-Oct. $\frac{5}{8} \times \mathbf{1} \frac{7}{8} \times \frac{1}{10}$ in. Var. Smithii MIass. $P$. hemispherical $\frac{1}{6}$ in. in diam.

917. P. empyreumatica Sacc. (from its odour of burning; Gr. cmpuros, scorched) a b.

$P$. hygrophanous, atomate, rufous, becoming pale. St. silkyfurfuraceous, pale sienna. $G$. adnate with a tooth, distant, veined, rufous to brown-purple; edge pallid. Flesh colour as St.

Wooden pavement. Oct. II $\times 2 \frac{1}{2} \times \frac{1}{8}$ in. Resembles 591 .

918. P. atomata Quél. (from the atomate pileus) $a b c$.

$P$. obtuse, hygrophanous, livid, tan or pale flesh-colour, sometimes reddish. St. white. G. adnate, cinereous-blackish or purplebrown.

Solitary or gregarious. Woods, hedgerows, waysides, amongst leaves; common. June-Jan. I $\times 2 \frac{5}{8} \times \frac{1}{16}$ in.

919. P. erenata Gill. (from the scalloped margin of the pileus; crenatus, scalloped) a $b$.

$P$. hygrophanous, atomate, subochreous, rufescent or fiesh-colour, then pale; mid. sometimes sienna. St. attenuate below, 
whitish. $G$. adnato-ascending, dull brownish-purple to blackish.

Amongst grass, amongst beech leaves with 1973. Nov. $1 \frac{1}{5} \times 2 \frac{1}{5} \times \frac{1}{5}$ in.

920. P. disseminata Quél. (from the scattered habit; dissemino, to scatter) $a b c$.

$P$. scurfy, then smooth, whitish or pale umber, then purple-grey and ashy; mid. grey-umber. St. subscurfy, then smooth, whitish. $G$. adnato-ascending, blackish.

Densely crowded, gregarious, cæspitose. About decayed stumps, poplar, willow, piles, in hedgerows, on brick walls, heaps of weeds and rubbish, etc. April-Nov. $\frac{1}{2} \times 2 \frac{1}{2} \times \frac{1}{16}$ in.

\section{COPRINUS Pers.}

(From the habitat; Gr. kopros, dung.)

I cil usually universal, floccose, furfuraceous or obsolete. Hymenophore distinct from the stem. Pileus at first cylindrical or ovate,

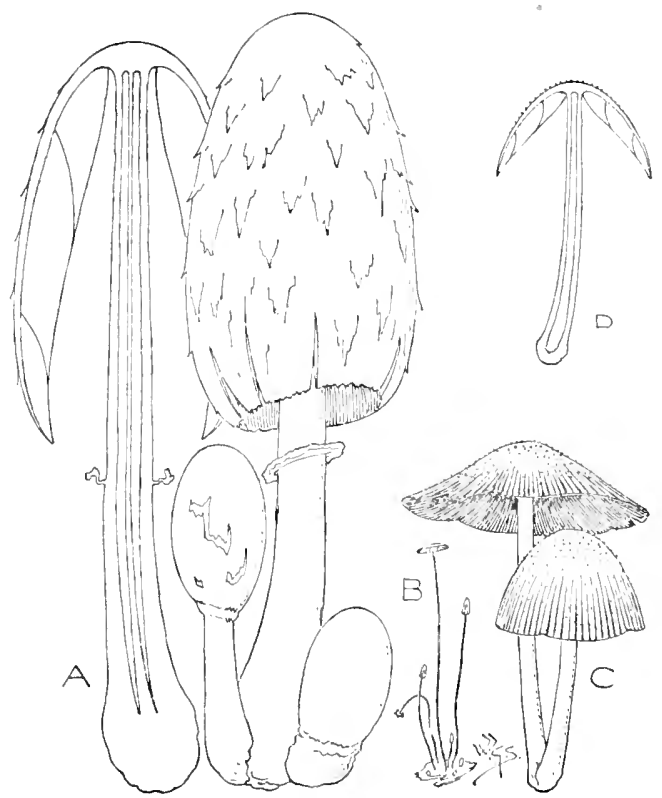

Fig. 5r.-A, Coprinus cometzus Fr., entire and in section: $B, C$. radiatus Fr, ; C, C. doncsticus Fr.; D, ditto in section. One-third natural size.

usually floccose or scurfy; margin at first straight and adpressed to the stem; flesh thin to membranous. Stem central, hollow, usually 
attenuate upwards, annulate, volvate or simple, confluent with or distinct from the hymenophore. Gills membranous, linear or ventricose, at first white and closely pressed together, cohering, scissile, at length melting into black fluid. Trama obsolete. Spores oval, even, black or dark brown. (Fig. 51.)

The species are of rapid growth, often ephemeral. They grow on rich and highly manured places in fields, parks, gardens and near human habitations, often on dung, sometimes on decaying wood, rotting straw, etc. Some are edible.

Species 921-963

Mr. George Massee, in a revision of the genus (Ann. Bot. x. ( I 896$),$ p. I 23 ), establishes new sectional characters, and the sequence of the species, as here given, is somewhat altered.

Pelliculos.e. Pileus with a pellicle not opening into furrows over the gills, but becoming torn and revolute.

a. Comate. Pileus slightly fleshy, at first ovate, the cuticle torn into large or small scales from the concrete veil; margin striate or sulcate. Stcm annulate, volvate or both. Gills free or remote.

$921-925$

b. Atramentaria. Pileus slightly fleshy, at first ovate, dotted or spotted with innate scurfy squamules from the veil. Stem imperfectly ridged-annulate at the base. Gills free.

$926-928$

c. Picacere. Pilens submembranous, at first continuous, then broken up into usually large flocculose superficial scales —warts in 932. Stem simple. Gills free.

$929-932$

d. Tomentose. Pileus membranous, at first evanescent-floccose or laxly villous from the veil, margin striate. Stim simple. Gills, except 936, free.

933-937

e. Hicacer. Pileus membranous, covered with fine, evanescent scurf and minute glittering particles from the veil, margin striate or sulcate. Stem simple, or with a slight basal annular ridge. Gills reaching the stem, adnexed in 938.

$938-941$

f. Glabratue. Pileus membranous, smooth; margin striate; veil obsolete. St'm simple, smooth.

$942-946$

Veliforutes. Pileus very thin, without a pellicle, opening into furrows over the gills and becoming plicato-sulcate. Veil present. Stem thin, fistulose. Gills melting into thin, black lines.

$947-963$

g. Cyclodece. Pileus membranous, striate. Stem annulate or volvate. Gills free.

947, 948

h. Lanatula. Pilcus submembranous to membranous, clothed with superficial, separating floccules. Stem simple. Gills free or remote. 
i. Furfurelle. Pilius micaceous or furfuraceous, striate or sulcate. St'm simple. Gills adnate, adnexed, free or remote.

j. Hentrobier. Pileus smooth. Stem simple.

$956-961$

k. Basipedes. Pilcus flocculose. Stem dilated, disc-like at the base.

\section{Pelliculos.玉.}

\section{a. Comate.}

921. C. comatus Pers. (from the shaggy pileus; coma, a hair) $a b$.

$P$. cylindrical, then expanded, white; sc. large. St. fibrillose, white. A. ragged, movable, evanescent. Vo. adnate, almost obsolete. $G$. white, through reddish and purple to black.

Often crespitose. Edible when young, used for inferior ketchup, sometimes candied with sugar, sold in American markets, at Boston, as the "English Mushroom." Gardens, parks, fields and roadsides, often near human habitations, on the ground, on rotten leaves; frequent. April-Nov. $4 \frac{1}{3} \times S_{4}^{3} \times \frac{3}{4}$ in.

922. C. ovatus Fr. (from the egg-shaped pileus; ovum, an egg) $a$.

$P$. at length expanded, densely imbricato-scaly, white; mid. pale buff. St. flocculose, rooting, scaly. $A$. ragged, movable, evanescent. $\quad V_{0}$. adnate, almost obsolete. $G$. white to umberblackish.

Usually solitary. Rich ground, pastures. $3 \times 5 \frac{1}{2} \times \frac{3}{8}$ in. Intermediate forms occur between this and $\mathbf{9 2 1 .}$

923. C. umbrinus Cooke $\&$ Mass. (from the colour of the pileus) $a$. $P$. campanulato-plane then revolute, dark umber; sc. white. St. subrooting, bulbous from the Vo., lustrous dark umber. $G$. black, edge white. Flesh pale umber.

Manured ground. Aug. $2 \times 6 \frac{1}{2} \times \frac{3}{16}$ in. Perhaps a form of $C$. stcnocoleus Lindbl.

924. C. sterquilinus Fr. (from the habitat; sterquilinium, a dunghill) $a$.

$P$. campanulato-expanded, whitish; marg. pale umber; sc. small. St. whitish. A. inferior, sometimes forming an adnate Vo. $G$. slate-purple.

Dung, cow, manured grounds, dung-heaps; rare. July-Aug. $2 \times 4^{3} \times \frac{5}{1^{5}}$ in.

925. C. oblectus Fr. (from its growing on-delighting in-dung; oblecto, to delight) $a$.

$P$. expanded, minutely scaly, then silky-smooth, white then sienna: mid. ochreous; marg. dull purplish. St. silky-white. I'o. large, free, spreading. $G$. black.

Newly-made dunghills, manure-heaps, street sweepings. Nov. $1 \frac{1}{2} \times 4 \frac{1}{2} \times \frac{1}{1}$ in. A close ally of 924. Mr. C. Crossland, who has found this plant at Italifax, says that the pileus is covered with white silky scales and always passes from white through bright flesh-colour to black, and the annulus sometimes projects nearly $\frac{1}{4}$ inch from the stem. In 924, the gills are at length ventricose, but in this plant, as described by Bolton, they are linear and slightly arcuate and pass from white through purple to black. In 924 the stem turns black when bruised, but there is no change of colour in the Halifax plant. 
925a. C. squamosus Morg. (from the scaly pileus and stem ; squama, a scale) $a$.

$P$. submembranous, ovoid then expanded, cinereous, covered with persistent reddish-brown sc, becoming split and revolute. St. hollow, almost equal, smooth and white above, scaly as P. below. $A$. median, small. $G$. free, ventricose, white, then reddish-brown and purple-brown, becoming black.

Crspitose. Base of trunks, elm. Sept. $3 \frac{1}{8} \times 4 \frac{1}{4} \times \frac{3}{8}$ in.

\section{b. Atramentaria.}

926. C. atramentarius Fr. (from the ink-like fluid into which the gills deliquesce; atramentum, ink) a b $c$.

$P$. campanulato-expanded, ribbed, sulcate, slate-white, then slate-purplish. St. fibrillose, white, pale umber at base. $A$. forming a fugacious ridge near base of St. $G$. grey-umber to umber-purple and black.

Cæspitose. Said to be edible, used for inferior ketchup, prefcrred by some to 921 . Rich soil by grassy roadsides, near stumps, usually near human habitations, amongst rubbish, has appeared in abundance on burnt coffee, rich soil, does not grow on dung; common. June-Nov. $3 \frac{1}{2} \times 6 \frac{3}{4} \times \frac{3}{8}$ in. A group of this fungus lifted a large mass of asphalt paring in Hampton Road in 1889 ; a similar occurrence took place at Dunstable in I 899 .

927. C. soboliferus Fr. (from its cæspitose habitat; soboles, an offshoot, fero, to bear) a $b$.

$P$. irregularly conical, abruptly truncate, then expanded, ribbed, grey; mid. pale sienna-brownish. St. furrowed, bluntly rooting, whitish. $A$. forming a fugacious ridge near base of St. $G$. dark brown-purplish or blackish.

Cæspitose. Base of rotten wooden palings, fences, stumps, elm. Spring and Autumn. $2 \frac{1}{5} \times S \times \frac{3}{4}$ in. The mycelium produces two crops annually. Intermediate forms between this and 926 occur. Sometimes both forms grow in one group.

928. C. fuscescens Fr. (from the dusky colour of the pileus; fuscus, dark, swarthy) $a b$.

$P$. campanulate, then revolute, somewhat ribbed, tan: mid. brownish; marg. purplish. St. whitish. A. forming a fugacious ridge near base of St. $G$. purple-umber to black.

Cæspitose. Stumps, rotten wood, wood in cellars, elm, in woods; uncommon. May-Dec. $2 \frac{3}{8} \times 4 \frac{1}{2} \times \frac{1}{4}$ in. Var. rimososquamosus Cooke. $P$. cracked into angular squamose patches.

\section{c. Picacea.}

929. C. picaceus Fr. (from the white patches on the black ground of the pileus; piar, a magpie) $a b c$.

$P$. campanulato-expanded, very smooth, then revolute. St. smooth, somewhat bulbous, biscuit, furfuraceous below. $G$. ventricose, grey, then black.

Somewhat cæspitose. Considered poisonous. Odour often fetid. Grassy places, roadsides, borders of woods, beech, rarely on rotten wood; rare. Sept.-Dec. $4 \times$ II $\times \frac{1}{1}$ in. 
930. C. aphthosus Fr. (from the pileus, like a "thrush"-diseased tongue; Gr. aphthai, the "thrush") a b.

$P$. campanulate, pale dull ochreous; sc. darker, grey-brown or dull sienna. St. fibrillose, glossy white. $G$. adnate, linear, black.

Subcrespitose. Hollow trees, wood in cellars, kitchens; rare. Autumn. $\mathrm{I} \frac{3}{4} \times 3 \frac{1}{2} \times \frac{3}{16}$ in. Var. Boltonii Mass. P. repand, olivaceous.

931. C. flocculosus Fr. (from the white-floccose scales of the pileus) $a$.

$P$. campanulate, pale purplish-grey-tan; mid. brownish; marg. striate. St. whitish. $G$. slate-purple to fuscous-black.

Usually solitary, sometimes cæespitose. Pastures, gardens; rare. June-Sept. $4 \times 5 \times \frac{3}{3}$ in.

932. C. similis B. \& Br. (from its resemblance to 930 ; similis, like) $a$.

$P$. campanulate, biscuit-white; sc. wart-like, acute, brownish at apex ; marg. striate. St. white. $G$. adnate, sublinear, brownish near edge.

Trunks of dead trees. Sept. $1 \frac{1}{2} \times 3 \times \frac{1}{8}$ in.

\section{d. Tomentose.}

933. C. exstinctorius Fr. (from the pileus, shaped like an extinguisher, exstinctor) a $b c$.

$P$. campanulate, tan- or ochreous-white; mid. livid; marg. splitting. St. subrooting, becoming smooth, whitish. $G$. lanceolate, fuscous-blackish to black.

On the ground, sandy places, about trunks, ash. Sept. $3 \times 6 \times \frac{1}{4}$ in.

934. C. fimetarius Fr. (from the habitat; finctum, a dunghill) a $b c$. $P$. campanulato-expanded, then cracked and revolute, whitefloccose, becoming grey-slate and black. St. subrooting, white villous. $G$. narrow-lanceolate, black.

Frequently clustered, sometimes solitary. Dung, horse, rich soil ; common. April-Oct. $2 \frac{5}{5} \times 8 \frac{1}{4} \times \frac{3}{16}$ in. Var. fullatus Fr. $P$. soon naked and black. Dunghills, rich meadows. Var. macrorkizus Fr. St. bulbous, rooting. Var. cinereus Fr. $P$. ashy-grey.

935. C. tomentosus Fr. (from the downy pileus; tomentum, down) $a b$.

$P$. companulato-expanded, flocculose, grey-white. St. whitevillous. $G$. narrow-lanceolate, fuscous-blackish, edge at first white.

Subcrespitose. Dung, rich pastures, gardens. Sept. $2 \frac{1}{1} \times 3 \times \frac{3}{16}$ in.

936. C. niveus Fr. (from the snow-white pileus; nix, nivis, snow) $a b c$.

$P$. at length flat, revolute, mealy-floccose or squamulose. St. white-downy, sometimes imperfectly annulate. $G$. adnexed or adnate, blackish to black.

Cæspitose. Dung, horse, deer, sometimes invading mushroom-beds. JuneDec. $2 \frac{1}{4} \times 4 \frac{1}{2} \times \frac{3}{16}$ in. There is a var. with white gills and (when present) colourless spores. 
936a. C. roseotinctus Rea (from the rose-coloured meal on the pileus and stem) $a$.

$P$. membranous, cylindrical, then expanded and revolute, umbonate, fuscous, densely powdered with deep rose-coloured meal; marg. sulcate. St. hollow, semibulbous below, when young powdered as P., less so at maturity, base white-floccose. $G$. adnexed, at first white, becoming black.

On the ground. Aug. $1 \frac{1}{8} \times 2 \frac{3}{8} \times \frac{1}{10}$ in. Allied to 936 and 946a.

937. C. cothurnatus Godey in Gill. (from the stem, sheathed with woolly squamules at the base, as if buskined; cothurnus, a buskin) $a b$.

$P$. conico-expanded, revolute, reddish-white or pale buff. St. white. $G$. narrow lanceolate, salmon to blackish.

Dung, cow. Autumn. $1 \frac{1}{4} \times 1 \frac{7}{8} \times \frac{1}{8}$ in.

\section{e. Micacere.}

938. C. micaceus Fr. (from the minute shining particles on the pileus ; mica, a shining grain) a $b c$.

$P$. campanulato-expanded, lobed, splitting, yellow-ferruginous; mid. darker ; marg. plicate, becoming purple-brown. St. whitesilky or fibrillose, with an annular ridge near base, white or pale ochreous-whitish. $G$. adnexed, purple-brown to dark slate.

Densely clustered. Amongst grass, at base of stumps and posts, gardens; common. Jan.-Nov. $2 \frac{7}{8} \times 7 \times \frac{3}{16}$ in. Two to three crops annually. Pileus sometimes white.

938a. C. truncorum Fr. (from its habitat, tree-trunks) $a$.

$P$. at first globose, then campanulate, densely covered with micaceous meal, soon naked, then tawny ochreous; marg. striate, not becoming sulcate. St. glabrous, hollow, white. $G$. free, rosy, then black.

Rotten willows, etc. $1 \frac{1}{4} \times 2 \frac{3}{8} \times \frac{1}{8}$ in. Distinguished from 938 by the P. being at first globose, with marg. striate, not plicate, and with free rosy gills.

939. C. aratus B. \& Br. (from a fancied resemblance in the sulcate pileus to furrows; aro to plough) $a b$.

$P$. campanulate then revolute, umber. St. smooth or silky, with a slight annular ridge near base, white, pale sienna-tan within. $G$. lanceolate, dark brown to purple-brown and black.

Solitary. Hollow trees, rich ground, dunghills. May-Aug. $2 \frac{3}{4} \times 6 \frac{1}{2} \times \frac{1}{4}$ in.

940. C. radians Fr. (from the radiating mycelium at the base of the stem) $a b c$.

$P$. campanulato-expanded, then revolute, tawny; mid. sienna. St. at first with an annular ridge, then smooth, white or faintly ochreous. $G$. lanceolate, reddish-purplish-brown to violaceousblack.

Subcrspitose. Damp pIaster walls, sculleries, cracks in ceilings; uncommon. Jan.-July. $\quad 1 \frac{3}{4} \times 4 \frac{1}{2} \times \frac{1}{8}$ in. The usually white or olive oclire mycelium is sometimes black, resembling radiating threads of black silk. 
941. C. papillatus Fr. (from the minute umbo on the pileus : pafilla, a teat) $a b$.

$P$. campanulato-flat, then revolute; um. prominent, ochreousumber; mid. darker. St. smooth, flocculose below, whitish. $G$. ventricose, blackish.

On the ground, amongst grass, dung. June. $\frac{1}{2} \times 1 \frac{1}{4} \times \frac{1}{16}$ in. Less fugacious than other Cofrini. Var. oxygena Fr., whitish or greyish. $P$, and St. sparingly flocculose.

\section{f. Glabratie.}

942. C. alternatus Fr. (from the stria of the pileus, alternately broad and narrow) a.c.

$P$. hemispherical, whitish; mid. umber, sometimes pale. St. whitish. $G$. adnate, narrow, purple-black to black.

Subcrespitose. On the ground. $1 \frac{3}{4} \times 4 \frac{7}{4} \times \frac{3}{16}$ in.

943. C. deliquescens Fr. (from the dissolving gills; deliquesco, to dissolve) a $c$.

$P$. campanulato-expanded, grey or livid; marg. granulosopunctate, faintly ochreous. St. shining white. $G$. free, slate to blackish.

Subcrespitose. Stumps, amongst fallen leaves, thatch ; frequent. Aug.Sept. $2 \frac{3}{4} \times 4 \times \frac{3}{16}$ in.

944. C. tardus Karst. (from its sometimes late appearance; tardus, slow, late) $a b$.

$P$. campanulato-expanded, viscid, bright brown to pale ochreoustan. St. thinly pruinose, shining white, minutely striate above, woolly below. $G$. adnexed, brown to shining black.

Densely crespitose. Odour ncne. On the ground. Oct. $2 \frac{1}{8} \times 6 \frac{1}{4} \times \frac{3}{16}$ in. Brittle, hygrophanous.

945. C. digitalis Fr. (from the finger-like young state; digitus, a finger) $a$.

$P$. conico-hemispherical, faint ochre ; mid. sienna, then yellowishgrey or olive tinted. St. faint ochreous-white. $G$, adnexed, blackish.

Tufted. On the ground, woody places. Autumn, $1 \frac{1}{4} \times 4 \frac{1}{4} \times \frac{3}{16}$ in.

946. C. congregatus Fr. (from the densely caspitose habit), $a b c$.

$P$. campanulato-expanded, viscid, ochreous; inid. sienna. St. faintly ochreous-white. $G$. adnexo-free, linear, black.

On the ground by roadsides, hothouses. Oct.-Nov. $1 \times 1 \times \frac{3}{8} \times \frac{1}{16}$ in.

\section{VELIFORMES. \\ s. Cyclodece.}

946a. C. dilectus Fr. (from its beauty; dilectus, beloved) a b.

$P$. cylindrical, then campanulate, obtuse, floccose or mealy, rosywhite, then pale, tawny; marg. finely striate, at length splitting and becoming revolute and naked. St. hollow, whitish, 
sprinkled rosy-pulverulent. Vo. reduced to whitish spreading sq. $G$. free, sublanceolate, reddish-brown, then black.

Cæspitose. On the ground, on dead and on partially buried hawthorn sticks. Nov. $\frac{7}{8} \times 2 \frac{3}{4} \times \frac{1}{10}$ in. Allied to 936a. Differing from 925 in very rudimentary volva and generally smaller size.

947. C. Hendersonii Fr. (after J. L. Henderson) $a b c$.

$P$. campanulato-expanded, minutely granular, tan ; mid. sienna ; marg. grey. St. tan. $A$. inferior, erect. $G$. subdistant, black, edge white.

Hotbeds, in fields on dung, horse, cow ; rare. Feb.-Sept. $\frac{1}{2} \times 1 \times \frac{1}{32}$ in.

947a. C. Bresadolæ Schulz. (after J. Bresadola).

$P$. subcylindrical, membranous, greyish-white, faintly brownish at mid. St. hollow, attenuate upwards, glabrous, white. $A$. deciduous. $G$. narrow, crowded, black, with a white edge.

Gregarious. Odour none. On wood, squared wood, near woods. $\frac{5}{8} \times 4 \frac{3}{4} \times \frac{1}{6}$ in. Not unlike a small 926, but whiter in colour.

948. C. volvaceominimus Crossl. (from its volva and small size) $a$.

$P$. campanulate, ashy-grey, white-squamulose; mid. darker. St. hyaline. $G$. adnexo-free, linear, blackish-purple.

Decaying straw on dunghills. $\frac{3}{16} \times \frac{7}{8} \times \frac{1}{32}$ in.

\section{h. Lanatula.}

949. C. lagopus Fr. (from the woolly stem, like a hare's foot, Gr. lagopous) $a b$.

$P$. campanulato-expanded, whitish or greyish; mid. sienna. St. densely white-woolly. G. remote, linear, black.

Sometimes cæespitose. Inodorous. Rich soil, dung, rotten wood, shady places in woods, rooting in rotten sawdust; rare. July-Oct. $2 \frac{1}{4} \times 5 \frac{1}{2} \times \frac{1}{4}$ in.

950. C. narcoticus Fr. (from the opium-like odour) $a b$.

$P$. campanulato-expanded, then revolute, villous-scaly, then naked, white or greyish; mid. pale sienna. St. villous, then smooth, faintly ochreous-white. $G$. lanceolate, blackish.

Odour very powerful, like opium, but fetid, or narcotic-alkaline, like 1211. Nanure-heaps. Oct. $I \times \mathbf{I}_{\frac{3}{4}} \times \frac{1}{16}$ in.

951. C. tuberosus Quél. (from the tuber at the base of the stem) $a$.

$P$. campanulato-expanded, then revolute, striate, white-pulverulent. St. attenuate above and below, villous-white, springing from a comparatively large sclerotium. $G$. linear, violet-black.

Dung, cow, decaying vegetable matter. Oct.-Dec. $\frac{3}{8} \times 2 \frac{3}{4} \times \frac{1}{16}$ in.

952. C. macrocephalus Berk. (from the long-headed young state; Gr. makros, long, kcphale, the head) a.

$P$. campanulate, slate; mid. brown, white-scaly. St. downy, sprinkled with fine spreading fibres, dull white. $G$. linear, black.

Subcrespitose. Putrid dung; rare. March-Sept. $I \times 2 \times \frac{1}{16}$ in. 
953. C. nyethemerus Fr. (from its length of life, a night and day, Gr. mechthemeron) a $b$.

$P$. at length flat, then revolute, furfuraceo-flocculose, then naked, grey; mid. sienna. St. flaccid, smooth, white. $G$. linear, blackish.

Subcæspitose. Dung, manured ground, decayed straw, tan; rare. July-Oct. $1 \frac{1}{8} \times 3 \times \frac{1}{32}$ in. Sometimes very small.

953a. C. cordisporus Gibbs (from the heart-shaped spores; cor, the heart, Gr. sporos, a seed) a.

$P$. cylindrico-ovate, then expanded and upturned, very thin, plicato-sulcate; marg. crenate, whitish or pallid ochraceous, furfuraceo-papillose and tawny at mid. St. filiform, glabrous, except at the densely strigoso-squamulose base, whitish hyaline. $G$. free, somewhat narrow. Spores heart-shaped, compressed.

Dung, horse, sheep, rabbit. April. $\frac{5}{16} \times I \times \frac{1}{32}$ in.

954. C. radiatus Pers. (from the radiating striæ or furrows of the pileus) $a b c$.

$P$. at length flat, downy, then naked, whitish, soon translucent; mid. pinkish-sienna. St. minutely pruinate or smooth, hyaline. $G$. few, pallid-blackish.

In troops. In fields on dung, horse, cow ; common. May-Nov, $\frac{3}{8} \times 3 \times \frac{1}{64}$ in. Often minute.

954a. C. Gibbsii Mass. \& Crossl. (after Thomas Gibbs of Sheffield, the first observer) $a$.

$P$. hemispherical, then expanded, striate, glabrous, minutely atomate, pale ochreous or very pale buff; mid. darker. St. very slender, glabrous, pellucid shining white, attached by a few white strands of mycelium. $G$. adnate, $5^{-7}$ in number.

Dung, sheep, horse. Nov. $\frac{1}{4}$ in. high. Probably the smallest agaric known; differs from 954 in its smaller size, glabrous pileus and stem, and in its circular, compressed spores.

955. C. Spraguei Berk. \& Curt. (after Charles J. Sprague) $a$.

$P$. campanulato-expanded, tomentose, grey; mid. sienna. St. pale cinnamon. $G$. few, narrow, grey-black.

Gardens. July. $\frac{3}{4} \times 2 \frac{1}{8} \times \frac{1}{16}$ in.

\section{i. Furfurella.}

956. C. domesticus Fr. (from its usual habitat; domus, a house) $a b c$. $P$. campanulato-expanded, furfuraceous, whitish, greyish or brownish; mid. sienna. St. adpressedly silky, white. $G$. adnexed, linear, reddish to fuscous-blackish.

Often cæspitose. In and near human habitations, greenhouses, gardens, cellars, cupboards, ceilings, under floors, on burnt ground, matting, damp carpets, rubbish, etc. ; frequent. April-Dec. $3 \times 3 \frac{1}{2} \times \frac{1}{4}$ in. Sometimes very light in colour. 
957. C. stercorarius Fr. (from the habitat; stercus, dung) $a b c$.

$P$. campanulato-expanded, then revolute, clad with dense white micaceous meal; mid. pale sienna; marg. faint greyish or brownish. St. pruinate, white. $G$. adnexed, narrow, black.

Rich soil, dung, roadsides. $1 \frac{1}{2} \times 4 \frac{1}{4} \times \frac{1}{8}$ in. Brefeld illustrates this plant as growing from a sclerotium. Must not be confused with 936, which is usually much smaller and with a white floccose pileus.

957a. C. stellaris Quél. (from the pileus splitting in a star-like manner) $a$.

$P$. ovoid then campanulate, striate, afterwards split in star-shape and crowned with points formed of minute diaphanous vescicles, white, becoming grey. St. filiform, fistulose, hyaline, velvety with long silky hairs, white. $G$. narrow, adnexed, attenuate, greyish then sooty-brown.

Dung, fox, man. Spring and Summer. $\frac{1}{10} \times \frac{5}{8} \times \frac{1}{40}$ in.

958. C. ephemerus Fr. (from its length of life; Gr. ephemeros, lasting for a day) $a b c$.

$P$. campanulato-expanded, delicately scurfy, white; mid. prominent, faint sienna ; marg. greyish. St. smooth, whitish. G. adnexed, linear, fuscous then black.

Dunghills, manured ground, roadsides; common. May-Oct. $\mathrm{I}_{5}^{\frac{1}{5}} \times 2_{\frac{3}{4}}^{3} \times \frac{1}{1 \frac{1}{5}}$ in. Must not be confounded with 960 , which has a depressed disc to the pileus.

958a. C. velox Godey in Gill. (from its rapid growth; z'elox, swift) $a$.

$P$. obovate, striate then plicato-sulcate, scurify between the furrows and on the mid., grey. St. white-floccose. G. narrow, grey then black.

Dung, ox, horse. $\frac{3}{8} \times 2 \frac{3}{8} \times \frac{1}{20}$ in. Must not be mistaken for 954a.

958b. C. aquatilis Peck (from its habitat, watery places; aqua, water) $a$.

$P$. ovate, then campanulate, coarsely sulcate to mid., at first somewhat furfuraceous, when young pale orange, then redbrown; mid. darker. St. somewhat swollen at base, hollow, smooth below, pulverulent above, dull white. $G$. adnexed, grey then black. Flesh in P. pale orange.

Decaying twigs, moss, Lusula sylvatica, etc., partly submerged in shaded swamps. June. $\mathbf{I} \times 2 \frac{1}{8} \times \frac{1}{12}$ in.

959. C. sociatus Fr. (from its habit, growing in groups; secio, to associate) $a$.

$P$. at length expanded, slightly scurfy, umbilicate, fuscous; mid. brown, becoming pale. St. white. $G$. adnexed, ashy-black.

Damip, loose soil, gardens, walls. Oct. $1 \frac{1}{2} \times 2$ in.

960. C. plicatilis Fr. (from the folds or furrows of the pileus; plico, to fold) $a b c$.

$P$. expanded, grey and livid-umber; mid. depressed, sienna. St. smooth, pallid to ochreous. $G$. remote, linear, slate.

Pastures, gardens, roadsides, under trees, woods; common. April-Oct. I $\frac{5}{8} \times 4 \times \frac{1}{16}$ in. Sometimes very small. 
961. C. filiformis B. \& Br. (from the thread-like stem; flum, a thread) $a$.

$P$. campanulato-expanded, grey, white-mealy; mid. sienna. St. pallid or white. $G$. linear, adnate, blackish.

On the ground in woods. Sept. $\frac{1}{8} \times \frac{5}{16} \times \frac{2}{32}$ in.

\section{j. Hemerobice.}

962. C. hemerobius Fr. (from the length of its life-one day; Gr. hemera, a day, bios, life) $a$.

$P$. campanulato-expanded, umber. St. smooth, pallid. G.adnate, linear, blackish.

Damp, shady, rich grassy places, roadsides, etc.; rare. Sept.-Oct. I $\frac{5}{8} \times 5 \times \frac{1}{5}$ in.

\section{k. Basipedes.}

963. C. Platypus Berk. in Cooke (from the basal disc; Gr. platus, broad, pous, a foot) a.

$P$. campanulato-expanded, white, then ochreous; mid. umber. St. even, whitish. $G$. free, narrow, distant, white.

Palm-stems in conservatories. $\frac{3}{16} \times \frac{5}{16} \times \frac{1}{32}$ in.

\section{BOLBITIUS Fr.}

(From a frequent habitat of the species; Gr. bolbiton, cow-dung.)

leil rudimentary, indicated by squamules on the pileus or stem, or obsolete. H!menophore distinct from the stem. Pileus moist,

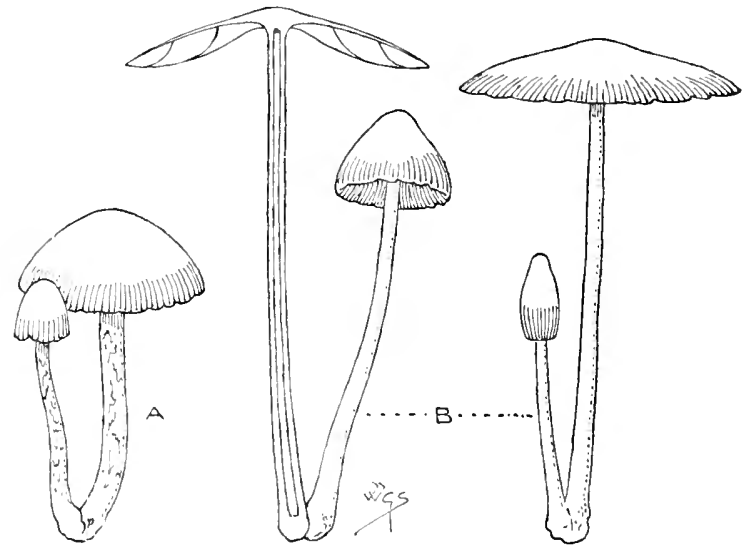

Fig. 52.-A, Bolbitius Boltonii Fr. ; B, B. fragilis Fr., entire and in section. One-half natural size.

mucid or viscid, fleshy-membranous to membranous, at first conical or ovate with the margin adpressed to the stem, usually yellow or 
salmon; margin striate or sulcate. Stem central, simple, equal or attenuate upwards, fistulose. Gills adnexed or free, membranous, becoming moist, not deliquescing. Spores somewhat ferruginous or salmon. (Fig. 52.)

A group of species resembling the Coprini in habit and short life, but not deliquescing. They chiefly grow on dung or manured ground, often near and in roads, frequently near towns, villages and human habitations. None are edible.

Species 964-973

964. B. grandiusculus Cooke \& Mass. (from its well-grown appearance; grandiusculus, well-grown) $a b$.

$P$. campanulato-expanded, papillose, ochreous-tan; mid. brownishorange; marg. whitish-salmon. St. white. $G$. linear, adnexed or free, orange or rusty-ochre.

Amongst grass. Autumn. $2 \times 4 \frac{1}{1} \times \frac{1}{8}$ in.

965. B. flavidus Mass. (flavus, light yellow) $a b$.

$P$. campanulato-expanded, glutinous, olivaceous-ochre; mid. somewhat elevated, sienna; marg. striate. St. pale yellow within and without, fibrillose below, faintly olivaceous. $V$. fugacious, white. $G$. adnexo-free, white, yellow then dusky brown.

Dunghills after rain, rotten cloth in wood. June-Aug. $2 \frac{1}{2} \times 2 \frac{1}{2} \times \frac{3}{16}$ in. "Whole plant dissolves in a brown jelly," Bolton.

966. B. Boltonii Fr. (after James Bolton of Halifax) $a b$.

$P$. flat, sulcate, viscid, sulphur-white, then dull salmon-whitish; mid. somewhat depressed. St. floccose, sulphur-white. $V$. fugacious. $G$. subadnate, white to yellow, pale dull salmon or livid brown.

Heaps of leaves and dung. May-Sept. $1 \frac{5}{8} \times 3 \times \frac{1}{8}$ in.

967. B. vitellinus Fr. (from the colour of the pileus, like yoke of egg, vitellus) $a$.

$P$. campanulate, lobed, sulcate, viscid. St. white squamulose, faint sulphur at base. $G$. slightly adnexed or free, ochreoustan.

Rich pastures, dung, horse. Autumn. $1 \frac{1}{2} \times 3 \frac{1}{8} \times \frac{3}{16}$ in.

968. B. fragilis Fr. (from its fragility) $a b c$.

$P$. flat, viscid, sulphur-white, straw or yellow. St. at first covered with fine matted down, colour as P. $G$. attenuato-adnexed, yellow, faintly olivaceous then pale cinnamon.

Road-drift and dung, horse, roadsides; common. April-Nov. $2 \frac{1}{4} \times 4 \frac{1}{4} \times \frac{1}{8}$ in.

969. B. titubans Fr. (from its tremulous habit; titubans, shaking) $a b c$.

$P$. flat, subdepressed, viscid, faint salmon-purplish; mid. faintly ochreous. St. even, shining-yellowish, sometimes white, squamulose at base. $G$. adnexed, narrow, colour as P.

Odour unpleasant. Grassy richly-manured soil, mushroom-beds, by rotten stumps; common. May-Oct. $2 \times 3^{\frac{3}{4}} \times \frac{1}{16}$ in. 
970. B. apicalis W. G. Sm. in Cooke (from the well defined apex of the pileus) $a b$.

$P$. conical, plicate to apical disc, tan-sienna or tan-umber; mid. abruptly ochreous. St. striate, minutely pruinose, salmonwhitish. $G$. free, ventricose, sienna.

Pastures, June. $\frac{1}{2} \times 1 \frac{1}{2} \times \frac{1}{16}$ in.

971. B. Niveus Mass. (from the white pileus; niveus, snowy) $a b$.

$P$. campanulato-expanded, striate halfway, subumbonate, slightly viscid; mid. cracked-areolate, faint ochre. St. clavato-bulbous, striate above, mealy-pruinose, white. $G$. free to adnate, whitish to rufescent salmon.

On earth, in paimhouse at Kew. $1 \frac{1}{1} \times 4 \frac{1}{x} \times \frac{1}{8}$ in. Very delicate and fragile.

972. B. tener Berk. (from its tender substance; tener, tender) $a b$.

$P$. smooth, even, white; mid. faintly ochreous; marg. faint salmon, sometimes wholly salmon. St. bulbous, white. $G$. adnexed, salmon.

Rich grassy ground; uncommon. Mray-Sept. $I \times \frac{3}{3} \times \frac{1}{16}$ in. Sometimes $S$ ins. high.

973. B. Rivulosus B. \& Br. (from the rivulose pileus) $a$.

$P$. campanulate, dull tan or clay. St. white. $G$. free, narrow, cinnamon.

Earth in orchid-house. July. $1 \frac{1}{2} \times 3 \times \frac{1}{8}$ in.

\section{CORTINARIUS Fr.}

\section{(From the cortina or veil.)}

Veil single, partially double or double. When single there is only an arachnoid cortina extending from the margin of the pileus to the stem, on the latter of which it often forms a more or less perfect fibrillose zone. When partially double the pileus and stem are more or less fibrous and the arachnoid cortina distinct. When double the whole plant is enveloped in infancy in a veil of gluten from which the arachnoid cortina is distinct. Hymenophore continuous with the stem. Pilezs more or less fleshy. Stem central, more or less viscid, annulate from the veil or fibrillose-zoned from the cortina; annulus and zone often evanescent. Gills usually adnate, but sometimes adnexed, free or sinuate with a decurrent tooth, membranous, persistent, arid, at first often purple or blue changing to cinnamon -from the spores. Spores the colour of iron-rust, rusty-ochre, somewhat ochreous or pale or dark cinnamon.

The species are variable in size and changeable in colour, purple and blue being common in infancy; they present a different appearance in different stages of growth and according as they are moist or dry; young as well as mature examples must therefore be studied. All are terrestrial except 1140, which sometimes grows on wood.

Some species of Pholiota and Flammula somewhat resemble the 
Cortinarii, but these usually grow upon stumps and are structurally distinct.

Species 974-1167

Subgenus r. Phlegmacium. Veil partial. Pileus viscid. Stem dry. $974-1021$

Subgenus 2. Myxacium. Teil universal. Pilezs glutinous. Stem glutinous.

$1022-1034$

Subgenus 3. Inoloma. Veil single. Pileus fleshy, silky or fibrillose, then smooth. Stem somewhat bulbous. Not hygrophanous. $\quad 1035-1057$

Subgenus 4. Dermocybe. Teil single, fibrillose. Pileus thinly fleshy, innato-silky, then smooth, dry. Stem equal. Not hygrophanous.

$1058-1085$

Subgenus 5. Telamonia. Veil universal, somewhat double. Pileus thin, moist, smooth or sprinkled with whitish fibres. Stcm annulate or peronate with scales. Hygrophanous. 1086-1128

Subgenus 6. Hydrocybe. Veil thin, fibrillose. Pilezs smooth or superficially white-fibrillose, not viscid, changing colour when dry. Stcm somewhat rigid, sometimes irregularly annulatozoned.

$1129-1167$

\section{Subgenus I. Phlegmacium.}

(From the clammy pileus; Gr. phlegma, shiny moisture.)

Vill partial, arachnoid, dry. Pilens equally fleshy, with a con-

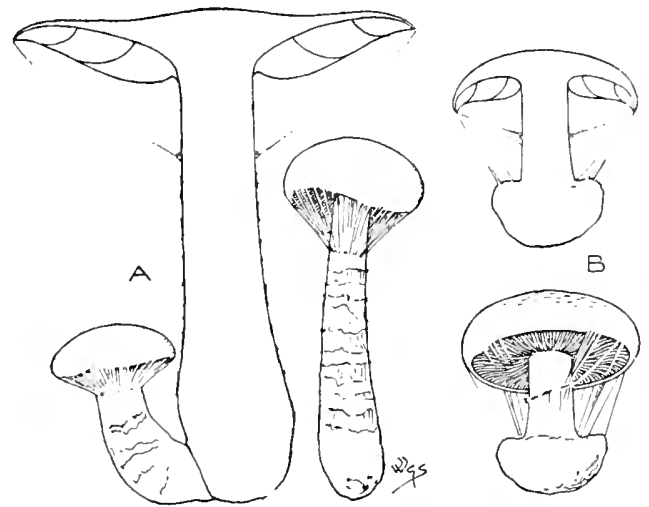

Fig. 53.-A, Cortinarius (Phlegmaciun) triumphans Fr. B, C. (Phlegmacium) fulmineus $\mathrm{Fr}$

Entire and in section. One-third natural size.

tinuous viscid pellicle. Stem firm, dry, usually solid, more or less zoned with the cortina. (Fig. 53). 
a. Cliduchiae. Partial veil depending in the form of a ring from the apex of the nearly equal or clavate stem.

Gills pallid then clay-colour.

Gills violaceous or purplish then cinnamon.

Gills yellow, cinnamon, ferruginous, not at first whitish or violaceous.

986, 987

Gills olivaceous.

$988-990$

b. Scaura. Stem fleshy-fibrous, marginato-bulbous, rounded bulbous in 988 and 1007. Cortina commonly inferior, arising from the margin of the bulb. Gills more or less sinuate.

Gills whitish, then clay-colour or pale cinnamon. 991-994. Gills violaceous, dark blue, purplish, then pale cinnamon.

Gills ferruginous, tawny or yellow.

$995-998$

Gills olivaceous.

$999-1005$

$1006-1009$

c. Elastica. Vil simple, thin, fugacious, median or inferior. Pileus usually somewhat thin, often hygrophanous. Stem commonly thin, not usually bulbous, generally attenuate upwards, rigid-elastic, externally subcartilaginous, lustrous, usually becoming hollow.

Gills whitish, then clay-colour or dull cinnamon. 1010-1014. Gills violaceous, purplish or flesh-coloured. 1015-1017 Gills usually ochre, tawny or ferruginous.

$1018-1021$

\section{a. Cliduchice.}

974. C. triumphans Fr. (from the appearance of the stem, like an enwreathed triumphal column) $a b c$.

$P$. convexo-plane, obtuse, yellow; marg. fringed with Co. St. striate, yellow, with transverse evanescent bands of sienna rings and an imperfect Z. G. emarginate, crowded. Flesh white or sulphur.

Grassy ground, woods, under birch; rare. Sept.-Oct. $5 \frac{1}{8} \times 5 \frac{1}{8} \times \frac{7}{8}$ in.

975. C. claricolor Fr. (from the clear colour; clarus, clear) $a b c$.

$P$. convexo-plane or depressed, often broken into sc.; marg. fringed with Co. St. white-scaly. $G$. emarginate or adnate, sometimes almost free, crowded.

Mixed woods, under birch, amongst heather. Ang.-Nov. $4 \frac{1}{8} \times 4 \frac{1}{8} \times \frac{3}{4}$ in.

976. C. turmalis Fr. (from growing in troops, turma) a $b c$.

$P$. convexo-plane, yellow tan; marg. fringed with Co. St. evanescent, white or faintly ochreous-woolly, zoned. $G$. variously attached, crowded, serrate.

Densely cxespitose. Mlixed woods, chiefly beech, larch. Sept.-Oct. $3 \frac{1}{4} \times 4 \frac{1}{\frac{1}{4}} \times \frac{3}{4}$ in. 
977. C. crassus Fr. (from the thick stem ; crassus, thick) $a b$.

$P$. expanded, plane or slightly depressed, subfibrillose, dull yellow or tan-brown. St. fibrillose, mealy above, whitish, with an imperfect $Z$. $G$. rounded, crowded.

Moist woods. Aug.-Oct. $6 \times 4 \frac{3}{8} \times \mathrm{I}_{\frac{1}{8}}$ in.

978. C. balteatus Fr. (from the band or zone round the stem from the veil ; balteatus, belted) $a b$.

$P$. convexo-expanded, gibbous, innato-floccose, umber; marg. lilac. St. tomentose, palest lilac. $G$. rounded or variously attached, crowded.

Chiefly pine-woods, under birch, dry places. Sept.-Oct. $4 \frac{1}{8} \times 2 \frac{1}{8} \times \frac{7}{8}$ in.

979. C. sebaceus Fr. (from its colour and clamminess, tallow-like; sebum, tallow) $a$.

$P$. campanulato-convex, then repand, smooth; marg. fringed with Co. St. subfibrillose, paler than P. with an imperfect $\mathrm{Z}$. $G$. emarginate, veined, not crowded.

Taste mild. Woods, mixed, pine. Sept.-Nov. $4 \frac{3}{8} \times 5 \frac{1}{2} \times \frac{3}{4}$ in.

980. C. lustratus Fr. (from the absence of colour; lustratus, purified, whitish) $a$.

$P$. convexo-expanded, smooth; marg. fringed with Co. St. zoned. $G$. crowded.

Sunny places, amongst heath, grass. Autumn. $2 \frac{1}{2} \times 1 \frac{3}{4} \times \frac{1}{2}$ in.

981. C. varius Fr. (from its changeable characters) $a b c$.

$P$. convexo-expanded, obtuse, yellow-tawny or umber; marg. fringed with Co. St. adpressedly flocculose, whitish, zoned. $G$. emarginate, subcrowded.

Taste somewhat nauseous. Woods, fir ; uncommon. Sept.-Nov. $4 \frac{1}{8} \times 3_{\frac{3}{4}}^{\frac{3}{4}} \times \frac{3}{4}$ in. Must not be confounded with 1015.

982. C. cyanopus Fr. (from the dark to pale purple stem; Gr. kuanos, dark blue, pons, a foot) a $b c$.

$P$. convexo-plane, obtuse, date-brown-livid, then tan. St. naked, colour as P. $G$. adnate or emarginate, edge unequal. Fles/ sometimes changing to violet.

Woods, under oak ; frequent. Aug.-Nov. $4 \times 3 \frac{3}{4} \times \frac{1}{2}$ in.

983. C. variicolor Fr. (from its variable colour) $a b$.

$P$. convexo-expanded, reddish-bay; marg. violet, fringed with Co. St. solid, grey-bluish and whitish, transversely 3-5 zoned with Co. $G$. emarginato-decurrent, crowded.

Woods, pine. Sept.-Nov. $5 \frac{1}{2} \times 6 \frac{1}{8} \times 1_{\frac{1}{4}}$ in. Var. nemorensis Fr. smaller. $P$. rivulose, yellow-bay. Si. hollow. Woods, beech.

984. C. largus Fr. (from its large size) $a b$.

$P$. convexo-plane, obtuse, tawny brick-red, sometimes deep bright purple when young; marg. pale purple, fringed with Co. St. fibrillose, pale purple above, tan below, sometimes becoming red when bruised. $G$. emarginate or adnate, crowded.

Somewhat cæspitose. Woods, pine. Sept.-Oct. $2 \frac{5}{8} \times 4 \frac{3}{8} \times \frac{5}{8}$ in. 
985. C. Riederi Fr. (after M. Rieder of St. Petersburg) $a b$.

$P$. campanulato-expanded, obtuse, ochreous; marg. fringed with Co. St. lilac-silky and tan-fibrillose. $G$. adnate or rounded, eroded, somewhat thick.

Woods, pine. Sept.-Oct. $2 \frac{1}{4} \times 4 \frac{5}{8} \times \frac{3}{8}$ in.

986. C. saginus Fr. (from its great size; sagina, corpulence) a $c$.

$P$. convexo-expanded, obtuse, repand, yellow ; mid. darker; marg. paler. Co. fugacious. St. somewhat bulbous, fibrillose, light yellowish. $G$. decurrent, eroded.

Gregarious, somewhat crespitose. Woods, larch. Oct. $5 \frac{5}{8} \times 4 \times 1 \frac{3}{8}$ in.

987. C. russus Fr. (russus, red) $a b$.

$P$. campanulato-expanded, obtuse ; marg. fibrillose. St. not bulbous, whitish. $G$. adnate or subadnate, crowded, veined.

Taste nauseous-bitter. Woods, moist places; uncommon. Sept.-Oct. $4 \frac{5}{8} \times 3 \times \frac{1}{2}$ in.

988. C. infractus Fr. (from the broken and bent margin of the pileus ; infractus, broken, incurved) $a c$.

$P$. expanded, slightly depressed, virgate, olive then yellowish; marg. at first broken, then revolute and wavy. St. adpressedly fibrillose, dull olive becoming pale. Co. fugacious. $G$. adnate, broad, crowded.

Taste nauseous-bitter. Woods, beech. Oct. $3 \times 1 \frac{1}{4} \times \frac{7}{8}$ in. Becoming olive-blackish in drying.

989. C. anfractus Fr. (from the margin of the pileus; anfractus, recurved) $a b$.

$P$. convexo-expanded, then repand, dark olivaceous; marg. thinner than 988, broken, usually sinuous and very irregular. St. fibrillose, violaceous above, dull olivaceous below. Co. superior. $G$. emarginate, distant. Flesh reddish when cut.

Woods; rare. Sept.-Oct. $3_{\frac{3}{ \pm}}^{\frac{3}{ \pm}} \times 3 \times \frac{1}{2}$ in.

990. C. Berkeleyi Cooke (after the Rev. M. J. Berkeley) $a$.

$P$. convexo-expanded, obtuse or subumbonate, silky, bright ochreous-brown, white-patched with Co. ; marg. plicato-sulcate tan-brown, lilac or violaceous, appendiculate with Co. St. fibrillose, whitish. $G$. emarginate, arcuate.

Woods. Autumn. $7 \frac{1}{8} \times 4_{\frac{3}{x}}^{\frac{3}{x}} \times \mathrm{I}_{\frac{1}{2}}$ in. In infancy the whole plant is covered with a whitish veil.

\section{b. Scaure.}

991. C. multiformis Fr. (from its varied shapes) $a b c$.

$P$. convexo-plane, slightly depressed, light yellow, clay or tawny; marg. fringed with Co. St. white or pale yellow. G. emarginate or free, serrulate, crowded, thin.

Taste and odour usually mild, odour sometimes of Stephanotis. Woods, beech; uncommon. Aug. -Nov. $3 \frac{1}{8} \times 2 \frac{5}{8} \times \frac{5}{8}$ in. Var. flavescens Cooke. $P$. tawny-yellow. $G$. yellow. Flesh tinged yellow. 
992. C. napus Fr. (from the turnip-shaped base of the stem; napus, a turnip) $a$.

$P$. convexo-plane, obtuse, fuliginous or date-brown-tawny; marg. fringed with Co. St. white, becoming yellowish below. $G$. emarginate or broadly adnate, subdistant, crisped.

Woods, pine. Oct. $2 \frac{5}{8} \times 1 \frac{1}{2} \times \frac{5}{8}$ in.

993. C. allutus Fr. (from its viscidity; allutus, bathed) $a b$.

$P$. convexo-plane, reddish-russet or orange-yellow. St. viscid, white above, reddish-striate below. $G$. adnate, subcrowded, thin, crenulate.

Woods, pine. Oct. $1 \frac{1}{2} \times 1 \frac{3}{8} \times \frac{3}{16}$ in.

994. C. talus Fr. (from the base of the stem, like swollen ankles; talus, an ankle bone) a $b$.

C. convexo-plane, dull ochre; marg. faintly olivaceous. St. smooth, tan or dull ochreous tan. $G$. adnato-emarginate. Flesh pallid whitish.

Woods. Sept. - Nov. $3 \frac{3}{8} \times 2 \frac{7}{8} \times \frac{1}{2}$ in.

995. C. glaucopus Fr. (from the pale blue stem; Gr. glankos, pale blue, pous, a foot) $a b$.

$P$. convexo-expanded, unequal, dull ochre, commonly floccososcaly with a raised dark zone at marg. St. fibrilloso-woolly and steel-blue above, base ochreous. $G$. rounded emarginate, crowded.

In troops. Woods, pine, beech; frequent. Aug.-Oct. $3 \frac{1}{4} \times 2 \frac{1}{2} \times \frac{3}{4} \mathrm{in}$. Becomes bright violet when cut or bruised.

996. C. calochrous Fr. (from its beautiful colours; Gr. kalos, beautiful, chros, colour) a $b c$.

$P$. convexo-expanded, guttate, tawny; marg. yellow. St. fibrillose, tan, yellow at base. $G$. emarginate, crowded, serrate.

Taste acrid ; odour fetid. Woods, oak, beech, pastures; frequent. Sept.Nov. $2 \frac{7}{8} \times 2 \frac{1}{4} \times \frac{3}{4}$ in.

997. C. eærulescens Fr. (from the azure-clouded pileus; cerrleus, azure) $a b c$.

$P$. convexo-plane, dull yellow, clouded pale purple, sometimes dull or bright purple or violet. St. at first fibrillose, faint or deep purple, bright or dull. $G$. adnate, thin, crowded. Flesh pale violaceous.

Taste insipid; odour almost obsolete. Mixed woods, mossy places; frequent. Aug.-Nov. $2 \frac{7}{8} \times 2 \frac{3}{8} \times \frac{3}{8}$ in. Not changing colour when broken.

998. C. purpurascens $\mathrm{Fr}$. (from the gills becoming purple when bruised) $a b c$.

$P$. convexo-plane, then repand, brown clouded with purple at the sometimes zoned marg. St. solid, fibrillose, clouded pale brown and blue. $G$. emarginate, crowded.

Single or cæspitose. Mixed woods, beech, pine; common. Sept.-Nov. $4 \frac{1}{4} \times 3 \times 1 \frac{1}{8}$ in. The purple hues have sometimes a tendency to crimson. 
Sometimes the whole plant is purple and not unlike 139. Var. subfurpurascens Fr., thinner than type, slightly virgate. $P$. tawny smoke-colour. St. hollow, pale bluish or whitish.

999. C. dibaphus Fr. (from the two colours; Gr. dibaphos, twice dipped or dyed) a. $b c$.

$P$. convexo-plane, or slightly depressed, yellow or sienna with purple or lilac marg. St. deep yellow to straw. $G$. sinuatoadnate, somewhat crowded, straw to purple-ferruginous. Flesh yellow.

Taste and odour none. Woods, beech, oak. Sept.-Oct. $4 \times 3 \frac{5}{5} \times \frac{7}{8}$ in. Wholly deep claret when dry. Var. xanthophyllus Cooke, the young state. $P$. for a long time yellow.

1000. C. turbinatus Fr. (from the shape of the base of the stem; turbo, a spinning-top) $a b$.

$P$. convexo-depressed, dull yellowish; marg. somewhat olivebrownish. St. stuffed to hollow, faintly dull yellowish. $G$. attenuato-adnate or decurrent, thin, crowded.

Woods, beech; uncommon. Sept. $5 \frac{7}{8} \times 2 \frac{7}{8} \times \frac{5}{8} \mathrm{in}$.

1001. C. corrosus Fr. (corrosus, gnawed to pieces, in reference to the late condition of the pileus) $a$.

$P$. convexo-depressed, subfloccose, ferruginous to pale tan. St. almost hollow, tan, white above. $G$. emarginate, narrow.

Woods, pine. Sept. $3 \times 1 \frac{1}{5} \times \frac{3}{4}$ in.

1002. C. fulgens Fr. (from its shining colour; fulgeo, to shine) a $b c$.

$P$. convexo-plane, silky-fibrillose, brilliant yellow. St. fibrilloso-woolly, colour as P. $G$. emarginate. Flesh yellow.

Woods, pine; rare. Sept. $3 \frac{5}{8} \times 2 \frac{1}{8} \times \frac{7}{8}$ in.

1003. C. fulmineus Fr. (from the shining pileus; fulman, lightning) $a$.

$P$. convexo-expanded, agglutinato-scaly, hard, bright yellow to tawny. St. yellow. $G$. rounded.

Shady woods, oak. Oct. $2 \frac{1}{8} \times 1 \frac{7}{8} \times \frac{1}{2}$ in.

1004. C. orichalceus Fr. (from the colour, like yellow copper ore, orichalchum) a b.

$P$. convexo-depressed, sienna-crimson ; mid. spotted-scaly; marg. dull yellow or faintly olive. St. fibrillose, sulphur to dull yellow. $G$. rounded-adnate.

Under trees, woods, pine. Sept. -0 ct. $3 \times 3 \frac{3}{8} \times \frac{5}{8}$ in.

1005. C. testaceus Cooke (from the colour; testaceus, brick-red) $a b$.

$P$. convex, flat, umbonate, vinous brick-red. St. fibroso-striate below, white above, rufous below. $G$. adnato-emarginate.

Woods, amongst leaves. Oct.-Nov. $3 \frac{3}{4} \times 3 \frac{5}{5} \times \frac{1}{2}$ in.

1006. C. prasinus Fr. (from the colour; Gr. prason, a leek) a $c$.

$P$. convexo-plane, obtuse, spotted, verdigris or tawny-fuliginous. St. greenish-pallid. $G$. emarginate, subdistant, somewhat crisped.

Commonly sporadic. Edible. Taste mild. Woods, beech. Sept.-Oct. $3 \frac{1}{8} \times 3 \frac{3}{4} \times \frac{5}{8}$ in. 
1007. C. atrovirens Kalch. (from the pileus, often dark green, atrovirens) $a b$.

$P$. convex, slightly depressed, green, sometimes olive-umber or bluish-green. St. pale-green, ferruginous below. $G$. adnate. Flesh tinged with green. Myc. sulphur or tawny.

Taste bitter; odour none. Woods, pine. Oct.-Nov. $3^{\frac{3}{4}} \times 2^{\frac{3}{4}} \times \frac{3}{4}$ in.

1008. C. seaurus Fr. (from the stem, as if with swollen ankles, scaurus) $a b$.

$P$. convex, plane, depressed, spotted, tawny-fuliginous to vinous; mid. darker. St. fibrilloso-striate, bluish-grey or pale olive, becoming white. $G$. adnate or adnexo-rounded, crowded.

Woods; rare. Sept.-Nov. $3 \frac{1}{1} \times 3 \times \frac{3}{8}$ in.

1009. C. herpeticus Fr. (from the lengthening stem; Gr. herpetikos, creeping) $a b$.

$P$. convex, obtuse, spotted, olive or yellow-olive, then tan. St. stuffed, fibrillose, greyish above, yellowish or $\tan$ below. $G$. adnate.

Woods. Sept. $2 \frac{1}{4} \times 2 \frac{1}{8} \times \frac{3}{8}$ in.

\section{c. Elasticre.}

1010. C. cumatilis Fr. (from the colour of the pileus; cumatilis, sea-coloured, blue) $a b c$.

$P$. convex, slate-grey or purple-grey; marg. fringed with Co. St. subbulbous, sometimes slightly rooting, white. $G$. attenuatoadnexed or adnate, serrate.

Sometimes subcæspitose. Woods. Sept.-Oct. $3 \frac{5}{8} \times 4 \frac{1}{4} \times \frac{3}{4}$ in.

1011. C. serarius Fr. (from the whey-like moisture of the pileus, like serum) $a$.

$P$. convexo-plane, gibbous, ochreous, buff-whitish or white. St. fibrillose, subrooting, ochre-whitish or white. $G$. broadly adnate, crowded, at first pale olive, then sienna-olive with ochre. Flesh pale yellowish.

Woods. Autumn. $3 \frac{1}{2} \times 3 \frac{1}{4} \times \frac{5}{8}$ in.

1012. C. emollitus Fr. (from its soft substance; cmollitus, soft) $a b$.

$P$. convex, obtuse, subrepand, fibrilloso-virgate, tawny ochreousyellow. St.fibrillose, white, then tinged yellow. $G$. emarginate, broad, subdistant.

Often cæspitose. Taste acrid. Woods, beech, grassy places. Sept.-Oct. $2 \frac{1}{1} \times 1 \frac{1}{1} \times \frac{1}{2}$ in.

1013. C. crystallinus Fr. (from the shining white pileus; Gr. krustallinos, crystalline) $a b$.

$P$. expanded, obtuse, at first watery-pallid, or ivory. St. fibrillose, whitish-straw, zoned. $G$. emarginate.

Taste acrid. Woods, beech, fir, oak. Oct. $1 \frac{3}{4} \times 1 \frac{7}{8} \times \frac{1}{4}$ in. 
1014. C. decoloratus Fr. (from the change of colour in the bibulous pileus, drying lighter in patches) $a b c$.

$P$. campanulato-expanded, obtuse, clay-colour or tan, corrugate when old. St. whitish. $G$. emarginate, adnate, or decurrent.

Taste acrid. Woods, birch, pine, beech. Aug.-Oct. Protean, varying large and small. Large $3 \frac{1}{4} \times 3 \frac{1}{2} \times \frac{3}{4}$ in. Small $\frac{3}{4}-1 \frac{5}{8} \times 1 \frac{3}{4} \times \frac{1}{4}$ in.

1015. C. decolorans Fr. (from the gills, soon changing colour from purple to cinnamon, but unchanging when bruised) $a b$.

$P$. convexo-flat, yellow; marg. fringed with Co. St. shiningwhite, zoned. $G$. emarginate or sinuato-adnexed, crowded, thin. Flesh white.

Woods, fir. Sept.-Oct. $22 \times 3 \frac{3}{8} \times \frac{3}{8}$ in. Must not be confounded with 981.

1016. C. porphyropus Fr. (from the purple stem; Gr. porphiureos, purple, pouss, a foot) $a b c$.

$P$. convexo-plane, innately streaked, livid brownish-clay. St. purple, becoming whitish, but again lilac or purple if bruised. $G$. adnato-emarginate or rounded, at length cinnamon, but again purple if bruised. Flesh deep purple.

Taste insipid; odour in drying sometimes of mice. Woods. Sept.-Oct. $2 \frac{1}{8} \times 2 \frac{1}{8} \times \frac{1}{4}$ in.

1017. C. croceocæruleus Fr. (from the saffron-gills and blue-purple pileus ; croceus, saffron, carnlius, azure) $a b$.

$P$. gibbous, plane or depressed. St. naked, whitish or pale purple, zoned. $G$. emarginate. Flesh pallid.

Odour disagreeable. Woods, beech. Oct. $I_{\frac{1}{2}} \times \mathrm{I}_{\frac{5}{8}} \times \frac{3}{16}$ in.

1018. C. coruscans Fr. (from the glittering pileus; coruscans, glittering) $a$.

$P$. obtuse, flat, ochreous, often spotted tawny. St. solid fibrillosostriate, white. $G$. plano-decurrent.

Taste and odour obsolete. Copses, beech. $5 \frac{1}{2} \times 4 \frac{1}{2} \times \frac{1}{2}$ in.

1019. C. papulosus Fr. (from the granular patches on the pileus; papulosizs, full of pimples) $a$.

$P$. convexo-plane, honey-tan; mid. glabrous, ferruginous-patchy when dry. St. solid, fibrillose, white. $G$. adnato-decurrent.

Woods, pine. Oct.-Nov. $2 \frac{7}{8} \times 3 \frac{3}{8} \times \frac{1}{2}$ in.

1020. C. vespertinus Fr. (from the dull colours, as of evening, vesper) a b.

$P$. convexo-plane, pale umber ; marg. fringed with Co. St. solid, fibrillose, white. $G$. emarginate, broad, crowded, umber, shaded olive.

Woods, oak. Oct. $2 \frac{5}{8} \times 4 \times \frac{1}{4}$ in.

1021. C. olivascens Fr. (from the olive-greenish pileus) $a c$.

$P$. convexo-plane, glabrous; marg. striate, fringed with Co. St. tan-white. $G$. emarginate or adfixed, olive-brown. Flesh yellowish near surface of $\mathrm{P}$. and St.

Taste acrid. Damp places amongst pines. Sept. $22 \times 3 \frac{1}{4} \times \frac{5}{16}$ in. 


\section{Subgenus 2. MyXacium.}

(From the glutinous pileus and stem ; Gr. muxa, mucus.)

Veil universal, glutinous. Pileus fleshy, glutinows, somewhat thin. Stem scarcely bulbous, viscid, more or less zoned. Gills adnate or decurrent. (Fig. 54.)

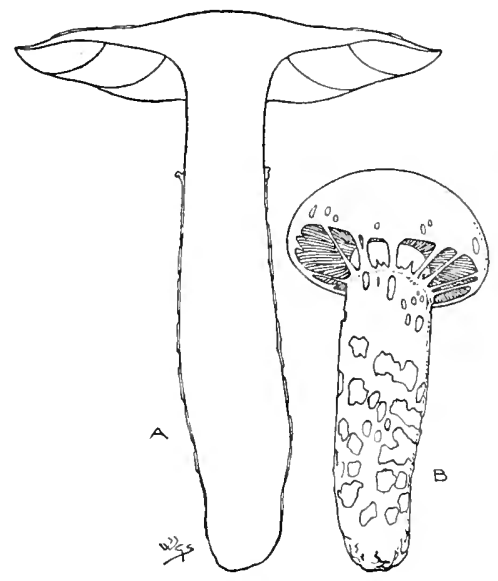

Fig. 54.-Cortinarius (Myxacizm) collinitus Fr. $A$, section of mature example; $B$, young state. One-thind natural size.

a. Collinita. Stem floccoso-peronate, the flocci at first covered with gluten.

1022-1027

b. Delibute. Veil entirely viscid. Stem not floccoso-peronate, viscid, with a varnished appearance when dry. 1028-1034 Gills whitish, then clay-colour.

Gills at first violaceous, dark blue or reddish.

$1029-1031$ Gills at first ochraceous or cinnamon.

\section{a. Collinita.}

1022. C. arvinaceus Fr. (from the viscid greasy pileus and stem; arvina, grease) $a b$

$P$. flat, slightly depressed, repand, orange-tawny; marg. striate. St. solid, equal, silky-viscid, white. Co. fugitive, forming an imperfect Z. $G$. adnato-decurrent, subdistant, crenulate, straw or ochreous-tan to bright ochreous.

Woods, beech. Oct. $3 \frac{1}{8} \times 5 \frac{1}{2} \times \frac{1}{2}$ in.

1023. C. collinitus Fr. (from the pileus and stem, besmeared with gluten ; colline, to besmear) a b $c$.

$P$. convexo-flat, obtuse, orange-tawny or umber, white-patched with Co.; marg. not striate. St. solid, equal or attenuate 
upwards, floccoso-glutinous and concentrico-scaly with the fugitive viscid-floccose Co., tan-ochreous. $G$. adnate, subcrowded, whitish-bluish-grey to cinnamon.

Woods, amongst heather; eommon. July-Nov. $4 \frac{5}{8} \times 5 \frac{1}{2} \times \mathbf{I}$ in. Var. mucusus Fr., firmer, stem even, silky. Woods, pine.

1024. C. mueifluus Fr. (from the abundant flowing gluten; micifluks, flowing with mucus) a $b$.

$P$. campanulato-expanded, obtuse, reflexed, livid-clay or shaded olivaceous, white-patched with Co. ; marg. striate. St. attenuate downwards, white, with steel-blue gluten forming zones. $G$. adnate, clay-colour then watery-cinnamon.

On the ground. Aug.-Oct. $3 \frac{1}{2} \times 5 \times \frac{5}{8}$ in.

1025. C. elatior Fr. (from its tall growth ; elatus, tall) a $b c$.

$P$. convexo-flat, subreflexed, plicato-wrinkled, light yellow, dull ochreous, deep orange, whitish, brown, grey or blackish; whitish, violaceous or olivaceous at marg. St. attenuate downwards, longitudinally fibrous, viscid-scaly, whitish, greyish or pale-purplish with a fugitive viscid Co. $G$. adnate, veined, brown-cinnamon.

Taste mild. Mixed woods, pine, beeeh; common. Aug.-Nov. $4 \frac{1}{4} \times 6 \frac{3}{8} \times 1 \frac{1}{8}$ in.

1026. C. grallipes Fr. (from the long, stilt-like stem; gralla, stilts, pes, a foot) $a b$.

$P$. flat, umbonate, repand, hygrophanous, ferruginous to ochreoustan. St. stuffed to hollow, equal, fibroso-striate, yellowishtawny to ochreous, with a fugacious, arachnoid Co. G. adnate with a decurrent tooth, crowded, clay to cinnamon.

Crespitose. Mixed woods, grassy places near trees, poplar, oak. Oct. $3 \frac{1}{8} \times 4 \frac{3}{8} \times \frac{3}{16}$ in.

1027. C. livido-ochraceus Berk. (from the livid ochre colour of the pileus) $a b$.

$P$. convexo-flat, white, with faint ochre, pale dull-lavender, or faint brown mid. or wholly brownish; lavender-purplish when young. St. violet, steel-blue or white above, brownish below, with a fugitive Co. which forms an imperfect ochre Z. $G$. rounded-adnexed, subdistant, ochre to cinnamon ; edge pale.

Odour none. Woods; uncommon. Sept.-Oct. $2 \frac{1}{8} \times 2 \frac{1}{4} \times \frac{1}{4}$ in.

\section{b. Delibute.}

1028. C. nitidus Fr. (from its shining, beautiful appearance; nitidus, bright) $a$.

$P$. convex, plane, obtuse then depressed, honey-colour, sometimes white. St. clavate, pallid-white or yellowish-tan. $G$. decurrent, crowded, serrate.

Subcæspitose. Woods, beech. Autumn. $3 \frac{1}{2} \times 3 \times \frac{1}{2}$ in. 
1029. C. salor Fr. (from its greyish colour, like the sea, salum) $a b$.

$P$. convex, obtuse or broadly umbonate; mid. grey; marg. violaceous or steel-grey. St. attenuate upwards, white, when young covered with bluish gluten. Co. forming an imperfect Z. $G$. emarginate or adnate, distant, grey or cinnamon.

Woods. Oct. $3 \times 3 \frac{3}{4} \times \frac{5}{8}$ in.

1030. C. delibutus Fr. (from the besmeared gluten; delibuo, to besmear) $a b$.

$P$. convexo-flat or subdepressed, light yellow. St. stuffed or hollow, attenuate upwards, white, sometimes pale steel-blue above. Co. forming an imperfect Z. $G$. adnate, slightly emarginate or subdecurrent, subdistant, serrulate.

Taste watery to slightly pungent. On the ground, damp grassy places. Sept.-Oct. $2 \frac{1}{4} \times 3^{\frac{3}{4}} \times \frac{5}{16}$ in.

1031. C. illibatus Fr. (from the gills, the margin entire, not serrulate, as in the last ; illibatus, entire) $a$.

$P$. convexo-plane, obtuse or subumbonate, yellow; mid. darker. St. stuffed or hollow, slightly attenuate above, white, often reddotted upwards. $G$. adnato-decurrent, crowded.

Woods, pine. Autumn. $1 \frac{7}{8} \times 2 \frac{7}{8} \times \frac{3}{16}$ in. Smaller and more slender than 1030, with no trace of violet colour.

1032. C. stillatitius Fr. (from the dripping gluten; stillo, to drip) $a b$. $P$. convexo-plane, subumbonate, hygrophanous; mid. tan-grey; marg. steel-grey and appendiculate with gluten. St. hollow, attenuate upwards, steel-grey with gluten. $C_{0}$. forming an imperfect Z. G. emarginate, subdistant.

Odour none. Mossy places, pastures, amongst dead leaves; uncommon. Oct. $2 \frac{1}{2} \times 3 \frac{1}{1} \times \frac{3}{8}$ in.

1033. C. vibratilis Fr. (from a fancied tremulous habit; vibratilis, quivering) $a b$.

$P$. plano-gibbous, hygrophanous, shining golden-yellow. St. stuffed, attenuate upwards, whitish. Co. forming a glutinous fugitive $Z$. $G$. emarginato-decurrent, thin, crowded.

Taste and odour disagreeable. Woods. Sept. $\mathbf{I}_{\frac{5}{8}}^{\frac{5}{3}} \times \mathbf{1}_{\frac{3}{4}} \times \frac{1}{4}$ in.

1034. C. pluvius (from its viscidity in rainy weather; pluo, to rain) a $P$. convexo-gibbous, hygrophanous, pale yellow-tawny; marg. striate. St. stuffed to hollow, nearly equal, naked, obsoletely viscid, white. $G$. adnexed, crowded.

Taste watery, then acrid-pungent. IVoods, pine. Sept.-Nov. $1 \frac{1}{4} \times 2 \times \frac{1}{8}$ in.

\section{Subgenus 3. Inoloma.}

(From the fibrillose or fringed veil ; Gr. is, inos, a fibre, loma, a fringe.)

Veil single, fibrillose. Pilcus equally fleshy, dry, subcompact, at first silky with innate scales or fibrils, becoming smooth, flesh 
continuous, not hygrophanous. Stem Heshy, somewhat bulbous. (Fig. 55.)

Handsome and easily distinguished.

Species $1035-1057$

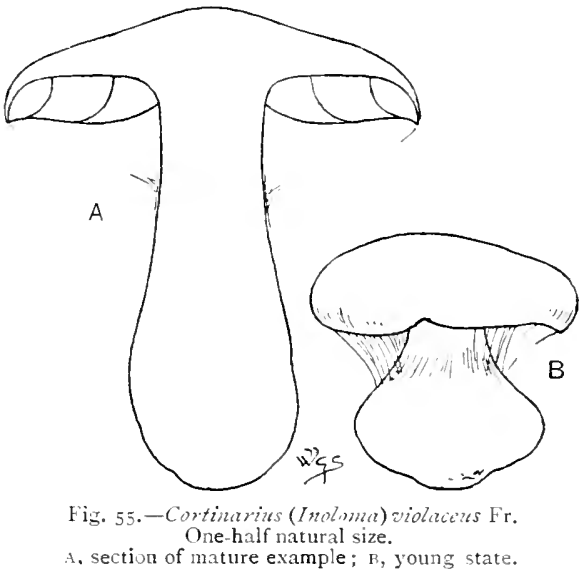

Gills at first white or pallid.

$1035-1038$

Gills, Vill and Stem violaceous.

$1039-1045$

Gills or Vil cinnamon, red or ochreous.

$1046-1052$

Gills or Veil dark, fuscous, olivaceous.

$1053-1057$

1035. C. argutus Fr. (from the graceful habit ; argtutus, graceful) a $b$.

$P$. expanded, gibbous then subdepressed, ochreous. St. solid, ventricose, fibroso-squamose, yellowish-white. $G$. adnate, rounded, subdistant, white clay-colour.

Woods, amongst leaves, oak. Oct. $4 \frac{1}{2} \times 3 \frac{1}{2} \times \frac{7}{5}$ in.

1036. C. argentatus Fr. (from the silvery white pileus and stem; arsentum, silver) a b $c$.

$P$. convexo-plane, broadly gilbbous; marg. pale lilac then dun. St. solid, slightly attenuate upwards, yellowish at base. $G$. emarginate, crowded, becoming watery-cinnamon or sienna.

Taste insipid; odour weak to strong, sweet, not feticl. Woods. Sept.-Oct. $5 \times 3 \frac{3}{4} \times \frac{3}{4}$ in. Var. ponetorum Cooke, smaller and more graceful, odour faint. Woods, pine.

1037. C. opimus Fr. (from its obese appearance; opimtzs, fruitful) $a$. $P$. convexo-plane, hard, dry, wavy-deformed, rimoso-rivulose, white, covered with short ochreous down. St. solid, hard, subturbinate, white-fibrillose with Co., base rooting. $G$. emarginate, subcrowded, whitish then tan.

solitary. Woods. Autumn, $4 \frac{1}{2} \times \mathbf{I}_{\frac{1}{2}} \times \mathbf{I}_{\frac{1}{4}}^{\frac{1}{4}}$ in, 
1038. C. turgidus Fr. (from the stem, frequently swollen or bulbous; turgidus, swollen) a.

$P$. convexo-obtuse, flat, clay-white; marg. white-silky. St. solid, bulbous, rimoso-striate, surface often undulate, colour as P., with an imperfect $Z$. $G$. emarginate, narrow, crowded, whitish to tan.

Odour strong. Woods, beech. Autumn. $3 \times 2 \frac{1}{2} \times \frac{3}{4}$ in.

1039. C. violaceus Fr. (from the violet colour) a $b c$.

$P$. convex, obtuse, flat, villous-scaly, dark purple, smeared copper-brown. St. solid, bulbous, zoned. $G$. adnate, distant, dark violaceous, clouded cinnamon. Flesh dark blue or dark purple.

Edible. Taste like that of 792. Woods and open ground. Aug.-Nov. $4 \times 4 \times \frac{7}{3}$ in. Often confounded with 138 and 139 .

1040. C. eyanites Fr. (from the dark blue colour; Gr. kuanites, dark blue) a $b$.

$P$. convex, flat, not villous-scaly, at first dark blue, then pale turquoise; marg. fringed with Co. St. solid, bulbous, colour as P., zoned. $G$. rounded, crowded, turquoise to grey-bluish. Flesh colour as P., becoming reddish, with a red juice.

Woods, mixed. Oct. $5 \times 6 \times \frac{3}{t}$ in. Var. major Fr. P. and St. compact; $G$. subdistant, cinereous, dark blue; flesh turning red more slowly than type. When bruised the blue colour changes to salmon.

1041. C. muricinus Fr. (from the purple colour, as of the mollusc Murex) a.

$P$. convex, obtuse, becoming smooth, riolaceous to reddish-liver; marg. whitish, fibrillose. St. bulbous, villous, zoned, not juicy. $G$. emarginate, subcrowded, colour as St.

Odour strong. Woods, larch, fir. Oct. $3 \frac{3}{x} \times 2 \frac{1}{2} \times \frac{5}{3}$ in.

1042. C. alboviolaceus Fr. (from the violaceous-white pileus; allus, white, violaceus, violet) a $b$.

$P$. convexo-expanded, broadly umbonate or gibbous, dry. St. solid, clavato-bulbous, white villous, zoned. $G$. adnate, subdistant, cinereous-riolaceous to cinereous-cinnamon. Flish palest azure-blue.

Taste insipid; odlour none. Woods, beech, shady places. Sept.-Oct. $3 \frac{1}{ \pm} \times 4 \times \frac{1}{2}$ in.

1043. C. malachius Fr. (from the pale purple colour, as of the mallow) $a$.

$P$. convex, obtuse, white fibrillose becoming smooth, lilac, brownish or reddish when dry. St. bulbous, bluish-lilac, then whitish, zoned. $G$. emarginate, crowded, purple to watery ferruginous. Flesh whitish.

Orlour none. Woods, fir. Sept. $2 \frac{\pi}{3} \times 2 \frac{1}{2} \times \frac{5}{5}$ in. Must not be confounded with 1133. 
1044. C. camphoratus Fr. (from its powerful odour; camphoratus, strong scented) a $c$.

$P$. convex, obtuse then flat, lilac, whitish, yellowish or tan-livid; marg. fringed with Co. St. clavato-bulbous, pale violet or lilac, zoned. $G$. emarginate or adnato-decurrent, grey-blue to purplish. Flish pale bluish.

Odour strong, fetid, penetrating, like fenugreek or curry-powder. Woods, fir. Autumn. $4 \times 4 \frac{1}{2} \times \frac{5}{8}$ in.

1045. C. hircinus Fr. (from its strong odour; hircus, a he-goat) $a$.

$P$. convex, obtuse, violet; mid. becoming ferruginous; marg. fringed with Co. St. bulbous, becoming pallid, yellowish at base, zoned. $G$. emarginate, subdistant, azure blue or violet to cinnamon. Flesh dingy, becoming yellowish.

Fetid. Woods, fir. Autumn. $2 \frac{7}{8} \times 2 \frac{3}{8} \times \frac{5}{8} \mathrm{in}$.

1046. C. traganus Fr. (from its strong odour; trarus, a he-goat) a b c .

$P$. convex, obtuse, flat, lilac-purplish, becoming yellowish. St. bulbous, spongy-solid, violaccous to whitish, zoned, subannulate. $G$. emarginate, broad, distant, thick, saffronochreous to cimmamon. Flesh yellowish.

Woods, pine, amongst ling. Taste strong, pleasant to unpleasant; odour fetid. Aug.-Sept. $4 \frac{1}{1} \times 3^{\frac{1}{2}} \times \frac{7}{8}$ in. lar. finitimus Fr., odour pleasant, peculiar, like fermenting gum.

1047. C. suillus Fr. (from its odour of the pig; suillus, relating to swine) $a$.

$P$. convex, obtuse, pallid brick-red or ochreous-cinnamon; marg. silky or floccoso-squamulose. St. clavate or equal, spongy, pallid, apex shaded light violet, darker when bruised, zoned. $G$. adnate, subdistant, fragile, connected by reins, cinnamon.

Woods, fir. Autumn. $2 \frac{3}{ \pm} \times 2 \frac{3}{4} \times \frac{3}{4}$ in.

1048. C. tophaceus Fr. (from the colour; tophus, tufa) $a b$.

$P$. hemispherico-convex, very obtuse, glittering goldentawny, with small sienna sq. St. solid, subbulbous, villososquamulose, tawny, zoned. $G$. emarginate, distant, tawny, cinnamon.

Subcrespitose or solitary. Odour sometimes strong. Woods, beech. Aus. $2 \frac{5}{8} \times 4 \times \frac{1}{2}$ in. Var. redimitus Fr. St. not bulbous or villoso-scaly.

1049. C. callisteus Fr. (Gr. kallistos, most beautiful) a b $c$.

$P$. convexo expanded, obtuse, yellow-tawny to yellow-ferruginous. St. clavato-bulbous, solid, fibrillose, yellow, rhubarbcolour at base, imperfectly zoned. $G$. adnate, subdistant, yellow-tawny.

Odour pungent or none. Woods, oak, pine ; rare. July-Oct. $2 \frac{\pi}{5} \times 4 \frac{1}{ \pm} \times \frac{1}{2} \mathrm{in}$. Resembles 580 in appearance. 
1050. C. Bulliardii Fr. (after Pierre Bulliard, French mycologist) a $b c$. $P$. convex, flat, crimson bay-brown or umber. St. solid, bulbous, fibrillose, blood-red below, pale above, irregularly zoned. $G$. adnato-subdecurrent, purplish to ferruginous, edge whitish. Flesh pale purplish-rose, flecked pale blue.

Odour strong. Woods, mixed, beech. Sept.-()ct. $2 \frac{1}{2} \times 2 \times \frac{1}{2}$ in. Sometimes approaching 1041, at other times 1064.

1051. C. vinosus Cooke (from its colour; a'inosus, like red wine) $a$.

$P$. Hat, subdepressed, shining. St. solid, marginato-bulbous, pale violet above, reddish below, zoned. $G$. adnato-subdecurrent, scarcely crowded, ferruginous-cinnamon.

Under trees. Oct. $2 \frac{3}{5} \times 2 \times \frac{1}{2}$ in.

1052. C. bolaris Fr. (from its being marked with red; bolare, to mark) $a b$.

$P$. convexo-plane, obtuse, light yellow-red, covered with siennared sc. St. stuffed to hollow, apex white, elsewhere covered with sienna-red sc., often in irregular zones. $G$. decurrent, adnate or emarginate, crowded, pale to dark cinnamon.

Sulitary or subcrespitose. Woods, beech; uncommon. Aug.-Nov. $2 \frac{1}{5} \times 2 \frac{1}{2} \times \frac{1}{4}$ in. When young P. and St. wholly sienna-crimson. Very dark when dry.

1053. C. pholideus Fr. (from the scaly pileus and stem; Gr. pholis, a scale) a b c.

$P$. convexo-plane, sometimes subumbonate, fawn-colour, covered with smaller umber and sepia sc. St. solid, pale white-purplish above, fawn below, clad with dark umber sc., often in zones, zoned cortinate above. $G$. adnexo-emarginate, violaceous or pale slate to cinnamon.

Woods. Sept.--Oct. $3 \times 3 \frac{1}{ \pm} \times \frac{1}{3}$ in.

1054. C. sublanatus Fr. (from the somewhat woolly pileus; lana, wool) a b $c$.

$P$. campanulate, expanded, umbonate, fawn, becoming ferruginous, covered with dark sq. St. solid, clavato-bulbous, apex faint azure-purplish, colour as P. below, corered with dark sc., sometimes zoned. $G$. adnate, scarcely crowded, olivaceousyellowish to cinnamon.

Odour of radish. Woods, larch, fir ; rare. Oct. $3^{\frac{3}{4}} \times 3^{\frac{1}{2}} \times \frac{1}{2} \mathrm{in}$. Intermediate between 1053 and 1084.

1055. C. phrygianus Fr. (from the colour, like gold embroidery; flirigio, an embroiderer in gold) $a$.

$P$. convex, oltuse, honey-colour, covered with small black sc. St. sulbbulbous, paler than P., black-scaly below. $G$. roundedadnate, subcrowded, dull yellow.

Odtour of radish. Sharly moist places, under beeches. Autumn. $2 \frac{3}{4} \times 2 \frac{1}{4} \times \frac{1}{2}$ in. Ilabit like that of 69. 
1056. C. arenatus Fr. (from the granulose pileus, as if sand-covered; (renu, sand) a b $c$.

$P$. convexo-expanded, sometimes umbonate, pale yellowishfuscous; mid. umber, minutely squamulose. St. usually attenuate upwards, pallid above, fuscous and dark squamulose below. $G$. adnexo-emarginate, yellowish-cinnamon or salmon

Woods; uncommon. Aug.-Oct. $2 \frac{7}{5} \times 2 \frac{3}{4} \times \frac{3}{4}$ in.

1057. C. penicillatus Fr. (from the pencilled pileus) $a b$.

$P$. campanulato-convex, subumbonate, ferruginous-fuscous, densely floccoso-scaly. St. stuffed, attenuate upwards, clothed with ferruginous-fuscous, concentric sc. $G$. rounded-adnate, dark brown. Flesh ferruginous-fuscous.

Woods, pine. Sept.-Oct. $1 \frac{1}{4} \times 2 \frac{1}{t} \times \frac{3}{15}$ in.

\section{Subgenus 4. Dermocybe.}

(From the thinly fleshy pileus; Gr. dirma, skin, kube, a head.)

Ieil single, fibrillose, forming a zone in 1064. Pileus thin, equally fleshy, at first silky, with innate villous down, becoming smooth when old, dry, not viscid or hysro(Fig. 56.) gills. phanous. Stem hollow or stuffed, except 1058, often equal or attenuate, rigid, elastic or brittle. Gills changeable in colour. Flesh when moist watery or coloured.

Polymorphous species defined with difficulty owing to the changeable colour of the

Species 1058-1085

Gills at first whitish or pallid.

$$
1058-1063
$$

Gills at first violaceous, becoming purple.

$1064-1070$

Stem and fibrillose Cortina coloured. Gills bright cinnamon, red or yellow.

$1071-1080$

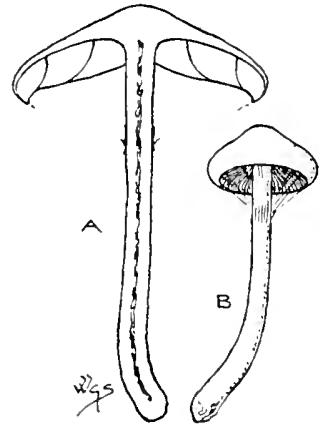

Fig. 55.-Cortinarius (Dindrcy $\left(b^{\prime}\right)$ cinnamoneus $\mathrm{Fr}$. A, section of mature example; $B$, young state.

One-half natural size.

Olivaceous. Pileus not torn into scales. Fill dull pallid or fuscous.

$1081-1085$

1058. C. ochroleucus Fr. (from the yellowish-white pileus; Gr. cchros, yellowish, leukos, white) a b.

$P$. convex, gibbous. St. solid, ventricose or subbulbous, naked, fibrillose above with Co., colour as P. G. adnexo- or adnatorounded, clay to ochreous or cinnamon.

Taste none or bitterish, not umpleasant; odour none or sweetish. Woods, mixed; rare. Sept.-Nor. $2 \frac{3}{8} \times \mathbf{I} \frac{3}{4} \times \frac{3}{5} \mathrm{in}$. 
1059. C. decumbens Fr. (from the usually decumbent stem) $\boldsymbol{a}$.

$P$. convex, gibbous or subumbonate, shining white or yellowish. St. stuffed to hollow, subclavate, smooth, white, with a fugacious Co. $G$. adnexed or adnato-rounded, white to clay and ochreous-cinnamon.

Woods and grassy places. Autumn. $\mathbf{I} \frac{3}{5} \times \mathbf{I} \frac{1}{4} \times \frac{3}{16}$ in.

1060. C. riculatus Fr. (from the cortina or veil; rica, a veil) $a$.

$P$. convexo-plane, gibbous or umbonate, atomate, buff, brown ochre or whitish; marg. adpressedly silky-floccose from Co. St. hollow, subclavate, smooth, naked, buff-white, with a slight and imperfect pallid Z. G. adnato-rounded, subcrowded, colour as P., tan to watery-ferruginous. Flesh palest yellow.

Wuods, pine. Autumn. $2 \frac{2}{2} \times 2 \frac{2}{4} \times \frac{3}{3} \mathrm{in}$.

1061. C. tabularis Fr. (from the flat pileus; tabula, a flat board) $a c$.

$P$. convex, broadly gibbous, then plane, clay or fuscous-clay, becoming pale; marg. fringed with Co. St. stuffed to hollow, equal or subclavate, white, zoned. $G$. emarginate, crowded, white to clay.

Woods; common. Sept.-Oct. $2 \frac{3}{4} \times 2 \frac{1}{4} \times \frac{1}{4}$ in.

1062. C. camurus Fr. (from the frequently crooked stem; cammms, crooked) $a b c$.

$P$. campanulato-convex, obtuse or umbonate, pallid fuscoushoary becoming pale pallid-yellowish; mid. darker; marg. fringed with Co. St. subhollow, twisted, equal, white. $G$. adnato-rounded or emarginate, grey-clay to subfuscous-waterycinnamon.

Caspitose. Taste not remarkable ; odour somewhat rancid. Woods, mixed, oak. Sept.-Oct. $2 \frac{1}{\frac{1}{4}} \times 3 \frac{3}{\frac{3}{4}} \times \frac{5}{16}$ in. Very fragile.

1063. C. diabolicus Fr. (from its doubtful position in Dermoclbe) a $c$.

$P$. hemispherical, obtuse, flat, often unequal and cracked, fuscous, crusted with grey, then smooth and fuscous-yellowish. St. attenuate downwards, white, pale bluish-grey above. $G$. adnatoemarginate, pale bluish-grey to whitish and clay.

Odour none. Woods, mixed, beech; uncommon. Aug.-Oct. $\quad 1 \frac{1}{2} \times 2 \times \frac{3}{16}$ in.

1064. C. caninus Fr. (from its commonness; canis, a dog) a b c.

$P$. convexo-plane, tan-sienna to fuscous-rufescent or tawny. St. stuffed to hollow, slightly attenuate upwards, whitish, violaceous above, zoned. $G$. emarginate, subdistant, bluish-grey or purplish to cinnamon.

Edible. Mixed woods; common. Sept.-Nov. $3 \frac{5}{5} \times 3 \frac{7}{5} \times \frac{5}{5} \mathrm{in.}$

1065. C. myrtillinus Fr. (from its chestnut-brown colour; myrtens, chestnut-brown) a $b$.

$P$. convex, gibbous, plane, fuliginous, violet or dusky-brown. 
St. clavato-bulbous, stuffed, whitish or pale violet. Co. obsolete. $G$. adnate, amethyst-azure-blue, or pale violet to dusky, not purple. Flesh watery fuscous or whitish.

Mixed woods, near trunks, beech. Sept.-Oct. $2 \frac{7}{8} \times 2 \frac{1}{2} \times \frac{3}{8}$ in. (ireatly resembles 138 and 139, but these have no arachnoid veil, etc.

1066. C. azureus Fr. (from the sky-blue pileus; asurcus, skyblue) $a b$.

$P$. expanded, obtuse, atomate, lilac, then fuscous and pallid. St. stuffed, slightly attenuate upwards, smooth, striate, bright azure then whitish, zoned. $G$. adnato-emarginate, bright bluish-violet. Flesh white in P., blue in St.

Solitary. Woods, beech, amongst moss and leaves. Autumn. $\mathrm{I}_{\frac{3}{4}}^{3} \times 2 \frac{1}{8} \times \frac{5}{15} \mathrm{in}$.

1067. C. albocyaneus Fr. (from the white pileus and blue gills; albus, white, cyancus, sea-blue) a b.

$P$. convex, plane, obtuse or umbonate, becoming yellowish, fringed with Co. St. subclavate, stuffed, whitish, zoned. $G$. emarginate, crowded, bluish-purple to subochreous.

Sometimes crespitose. Woods, beech. Sept. $2 \frac{5}{8} \times 4 \frac{1}{4} \times \frac{5}{16}$.

1068. C. anomalus Fr. (from the colour of all its parts being very changeable; anomalus, irregular) a b $c$.

$P$. convex, expanded, gibbous or subumbonate, fuliginous or olive-shaded to rufescent, then yellowish with ochreous-sienna mid. St. stuffed to hollow, attenuate upwards, fibrillose or scaly, whitish, then palest yellow, zoned. $G$. adnato-emarginate, crowded, thin, violaceous to cinnamon.

Gregarious. Odour none. Woods; common. Aug.-Oct. $2 \frac{3}{8} \times 2 \frac{1}{2} \times \frac{5}{16}$ in.

1069. C. spilomeus Fr. (from the spotted pileus and stem ; Gr. spilos, a spot) $(\%$.

$P$. convex, expanded, gibbous, rufescent to clay-colour, spotted with reddish-tawny sc. St. hollow, almost equal, white-lilac above, pale yellowish below, with many zones of reddish tawny sc. $G$. adnato- or adnexo-emarginate, crowded, thin, bluishgrey or violaceous to watery cimnamon.

Subcrespitose. Woods; uncommon. Oct. $2 \times 3^{3} \times \frac{3}{8}$ in.

1070. C. lepidopus Cooke (from the fibrillose zones round the stem; Gr. lepis, a scale, fous, a foot) a b.

$P$. convex, flat, slightly depressed, shining umber; mid. beconing rufescent ; marg. steel-grey or pale violet. St. clavate, becoming hollow, violet or steel-grey above, whitish below, with concentric biscuit-coloured zones. $G$, adnato-rounded, thin, violet to cinnamon, or olive-shaded.

Gregarious or fasciculate. Woods, amongst heath. Sept.-Oct. $2 \frac{3}{8} \times 4 \times \frac{1}{4}$ in. Perhaps a form of 1064 . 
1071. C. miltinus Fr. (from the colour, like red lead, Gr. miltos) $a b$.

$P$. expanded, gibbous or subumbonate, bay-brown-cinnamon to brick-red. St. hollow, attenuate upwards, cinnamon, reddish or crimson, salmon above and below, zoned red with Co. $G$. adnate, crowded, reddish-cinnamon to ferruginous. Flesh rose in St., pale brown in $\mathrm{P}$.

Odour none. IVoods, mixed, mossy places. Oct. $\mathbf{I}_{\frac{5}{8}}^{\frac{5}{8}} \times \mathbf{I} \frac{1}{2} \times \frac{3}{16}$ in.

1072. C. cinnabarinus Fr. (from the vermilion colour; cinnabaris, vermilion) a $b c$.

$P$. convex, obtuse or subumbonate, vivid scarlet-red. St. equal, solid, colour as P. $G$. adnate or subdecurrent, blood-red, and then blood-sienna. Flesh orange-vermilion in P., vermilion in St.

Taste mushroom-like; odour of radish. Woods, beech. Sept.-Oct. $2 \times 2 \frac{7}{8} \times \frac{1}{4}$ in. Wholly dark-brown when dry.

1073. C. sanguineus Fr. (from the wholly blood-red colour; sangzis, blood) a b c .

$P$. convex, obtuse, plane. St. equal, stuffed to hollow. $G$. adnate.

Odour of radish. Woods, pine, oak. Sept.-Nov. $2 \frac{1}{8} \times 2 \frac{1}{8} \times \frac{1}{4}$ in. Giving out a blood-red juice when pressed. Sometimes very small.

1074. C. anthracinus Fr. (from the colour of the stem and gills, like burning coal, Gr. anthra.x) a b $c$.

$P$. convex, umbonate, dark chestnut. St. hollow, equal, deep blood-red, fuscous below. $G$. rounded adnate, crowded, red to fiery-red, blood-red when bruised.

Woods, amongst moss. Aug.--iept. $1 \frac{1}{4} \times 1 \frac{3}{4} \times \frac{1}{8}$ in.

1075. C. cinnamomeus Fr. (from the cinnamon colour) $a b c$.

$P$. expanded, obtuse or subumbonate, somewhat pale or bright cinnamon. St. stuffed to hollow, zoned. $G$. adnate, crowded, blood-red, red-cinnamon, saffron, golden, or light yellow. Flesh yellowish.

Tasteless. Woods, mixed, fir ; common. Aug.-Feb. Variable in size. Large $2 \frac{7}{8} \times 3 \frac{1}{2} \times \frac{5}{16}$ in. Simall $\frac{3}{4} \times 1 \frac{3}{8} \times \frac{1}{8}$ in. Var. semisangrineus $\mathrm{Fr}$. $G$. blood-red. Var. crocens Fr., smaller than type. G. yellowish or olive. Intermediate forms occur between the type and its varieties.

1076. C. croceoconus Fr. (from the saffron colour of the conical pileus; crocus, saffron, comus, a cone) $a$.

$P$. conical, campanulate, acutely umbonate, tawny-cinnamon to yellowish-tawny. St. stuffed to hollow, yellow, zoned. $G$. adnexo-rounded, crowded, yellow to cinnamon. Flesh yellow.

Woods, pine, mossy places. Autumn. $I \times I \frac{1}{8} \times \frac{1}{16} \mathrm{in}$. 
1077. C. uliginosus Berk. (from its habitat; uligo, marshy ground) a bc.

$P$. campanulato-conical, expanded, strongly and somewhat acutely umbonate, bright brown-red. St. stuffed to hollow, paler than P., zoned. $G$. adnate with a tooth, distant, yellow to olive and cinnamon.

Woods, boggy places amongst Sfhagnum. Autumn. $\quad 1 \frac{1}{2} \times 3 \times \frac{3}{16}$ in.

1078. C. orellanus Fr. (derivation obscure) $a b c$.

$P$. convex, obtusely umbonate, orange-tawny. St. solid, sulbequal, striato-fibrillose, tawny, orange-striate. $G$. adfixed or adnate, subdistant, orange to tawny-cinnamon. Fles/l as P., reddening.

Taste mushroom-like. Woods, mixed. Aug.-Oct. $2 \frac{1}{8} \times 1 \frac{3}{4} \times \frac{3}{4}$.

1079. C. malicorius Fr. (from the colour, like the fruit of the pomegranate: malicorium, the rind of the pomegranate) $a$.

$P$. convexo-plane, obtuse, golden-tawny, or golden to fuscous or olive. St. hollow, golden. G. adnexo-rounded, crowded, goldentawny. Ficsh yellow to greenish-olive.

Taste and odour pleasant. Woods, pine. Autumn. $\mathrm{I}_{\frac{5}{8}}^{\frac{5}{3}} \times 2 \frac{7}{5} \times \frac{1}{4} \mathrm{in}$.

1080. C. infueatus Fr. (from the bright colour; fuc , to paint) $a$.

$P$. convex, obtuse, bright yellow to pale sulphur; marg. fringed with Co. St. solid, clavate, fibrillose, pallid light yellow, zoned. G. adnate, crowded, tawny to cimnamon. Filesh whitish, light yellow or pale olivaceous.

Woods. Sept.-Oct. $2 \frac{1}{2} \times 4 \frac{5}{8} \times \frac{5}{16}$ in.

1081. C. cotoneus Fr. (from the colour, like the olive, (ir. kotinos) $a b$.

$P$. campanulate, expanded, obtuse, subrepand, velvety, olivaceous. St. solid, attenuate upwards, pallid olivaceous, with one or more fuscous zones. $G$. adnexo- or adnato-rounded, subcrowded, olivaceous to cinnamon.

Odour of radish. Woods, mixed, oak. Autumn. $3 \frac{1}{2} \times 5 \frac{1}{2} \times \frac{1}{2}$ in.

1082. C. subnotatus Fr. (from its characters, hardly distinct; subnotatus, hardly marked) a b.

$P$. convex, flat, hoary-floccose, olive or shaded yellowish to dark fuscous. St. stuffed, conical to equal, naked above, yellow fibrillose below, zoned. $G$. adnate, slightly emarginate, subdistant, yellowish to olivaceous-cinnamon.

Odour strong. Under trees, beech, fir. Nov. $3 \frac{1}{2} \times 3 \frac{3}{4} \times \frac{3}{8}$ in.

1083. C. raphanoides Fr. (from the strong odour of radish; Gr. raphanos, radish, eidos, resemblance) a $b$.

$P$. campanulate, expanded, umbonate, silky-fibrillose, fuscousolivaceous to tawny. St. stuffed, slightly attenuate upwards, paler than P., yellowish above. $G$. adnate, scarcely crowded, somewhat olivaceous or orange-sienna to cinnamon ferruginous, edge paler. Flesh pale yellow or sulphur-white.

Taste acrid-bitter. Woods, mixed, beech, fir. Sept.-Cot. $2 \times 2 \frac{1}{2} \times \frac{3}{16}$ in. 
1084. C. valgus Fr. (from the frequently twisted stem; valgus, bow-legged) $a$.

$P$. convex, gibbous, olivaceous to brick-red. St. somewhat hollow, clavato-bulbous, whitish, apex pale violaceous, zoned. $G$. adfixed or rounded-adnate, yellowish to brick-red and cinnamon.

Odour none. Woods, pine, amongst moss. Oct. $3 \times 3 \frac{3}{4} \times \frac{5}{5}$ in.

1085. C. venetus Fr. (from the sea-green pileus and stem: a'enctus, sea-coloured) a $b$.

$P$. hemispherico-obtuse, convex, umbonate, becoming greenishyellow. St. stuffed or hollow abore, equal or subclavate, colour as P. G. adnate, scarcely crowded, olivaceous to orange-cinnamon. Flesh pallid yellowish.

Gregarious. Woods. Aug.-Oct. $1 \frac{3}{8} \times 1 \frac{7}{8} \times \frac{5}{15} \mathrm{in}$.

\section{Subgenus 5. Telamonia.}

(From the whitish, lint-like fibres of the veil ; Gr. telamon, lint.)

Vil universal, somewhat double. Pilizs moist, hygrophanous, at first smooth or sprinkled with the superficial, arachnoid, whitish

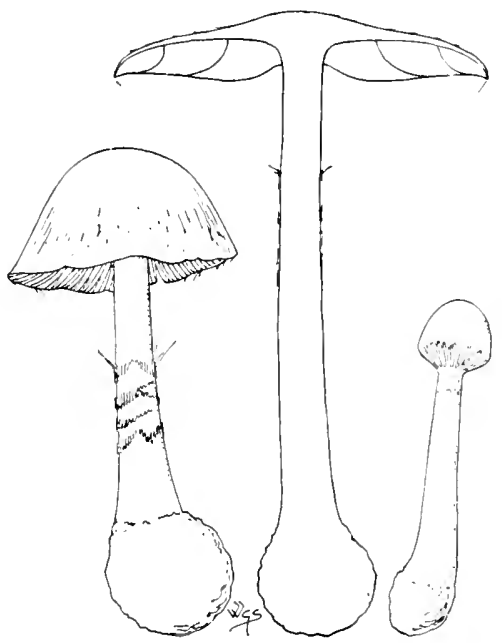

lig. 57.-Continarius (Telamonia) armillatus $\mathrm{Fr}$.

Young examples and section of a mature specimen. One-third natural size.

fibres of the veil, thin or moderately compact at the middle, thin towards margin, splitting. Stim annulate from the inferior veil, or peronate with scales, somewhat cortinate at the apex. Flish thin 
throughout or becoming so abruptly near the margin, scissile. (Fig. 57.)

The species are mostly large and handsome. Species 1086-1128 a. Platyphylle. Gills broad.

Stem and Cortina white or whitish.

$1086-1092$

Stcm violaceous. Cortina usually white-violaceous. Universal Veil white. Gills violaceous.

1093-1098

Stem and $V_{\text {iil }}$ reddish or yellow, except 1104. Gills tawny or cinnamon, neither violaceous nor becoming brown.

$1099-1107$

Stcm becoming fuscous. Vil fuscous or dull. Gills dark coloured.

$1108-1114$

b. Lefophylle. Pileus thin. Stcm externally more rigid, subcartilaginous, stuffed or hollow, often attenuate downwards. Gills more or less crowded, thin, narrow.

Stem whitish, pallid, not floccoso-scaly.

1115,1116

Stem inclining to violet.

$1117-1119$

Pileus and Stem tawny, ferruginous.

$1120-1122$

Pileus fuscous. Stem tloccoso-scaly, fuscous.

$1123-1128$

\section{a. Platspliylla.}

1086. C. macropus Fr. (from the long stem; Gr. makros, long, pons, a foot) a $c$.

$P$. convex, obtuse, flat, brick-red to ferruginous, hoary with small sc. St. solid, equal, fibrillose, dull whitish, then as P., zoned. $G$. adnexed or adnate, distant, pallid to watery cinnamon.

Woods, moist places. Oct. $3 \frac{1}{2} \times 6 \frac{1}{2} \times \frac{1}{2}$ in.

1087. C. laniger Fr. (from the woolly: pileus; lana, wool) $a$.

$P$. hemispherical, expanded, obtuse, bright tawny, clad with white sc. St. solid, equal, white, annulate or bi-annulate. $G$. rounded-adnexed, tawny-saffron.

Odour strong. Woods, larch, pine, beech, mossy places. Sept. $3 \frac{1}{2} \times 6 \times \frac{5}{5}$ in.

1088. C. bivelus Fr. (from the double veil ; bis, double, a'chm, a veil) ac .

$P$. convex, obtuse, plane, tawny-ferruginous; mid. spotted or darker. St. solid, clavato-bulbous, dull whitish, squarrose below. $G$. adnexo-rounded, bright tawny-cimnamon.

Taste mild; odour strong. Woods. Sept.-Oct. $3 \frac{3}{8} \times 3 \times \frac{1}{2}$ in.

1089. C. bulbosus Fr. (from the bulbous stem) $a b$.

$P$. campanulato-expanded, gil,bous, olivaceous, or date-brown to reddish-fuscous. St. solid, paler than P., yellow below, fugaciously zoned white. G. adnate, subdistant, cinnamon, never violet.

Odour of radish. Woods, mossy places; rare. Sept.-Oct. $3 \frac{1}{1} \times 3 \frac{3}{1} \times \frac{1}{2} \mathrm{in}$. 
1090. C. urbicus Fr. (from its habitat, near trees and human habitations; urlos, a city) a b.

$P$. hemispherical, convexo-plane, clay-whitish; marg. crenatotorn. St. solid, equal, white zoned. $G$. emarginate, crowded, watery-ferruginous.

Grassy places. Sept.-Oct. $2 \frac{1}{x} \times 2 \frac{1}{\frac{1}{4}} \times \frac{3}{y}$ in.

1091. C. licinipes Fr. (from the woolly stem; licinium, lint, pes, a foot) $a b$.

$P$. campanulate, flat, obtusely umbonate, pale yellow to pallid tan. St. stuffed to hollow, equal, whitish, fugitive floccose, villous below, annulate. $G$. adnate, subcrowded, watery cinnamon.

Odour none. Woods, fir, damp places. Oct. $2 \frac{1}{4} \times 2 \times \frac{3}{16}$ in. Var. robustiver Cooke is larger and stouter than type.

1092. C. microcyclus Fr. (from the veil, forming a small circle; Gr. mikros, small, kuklos, a circle) a b.

$P$. campanulato-expanded, reddish-fuscous; mid. darker, paler when dry. St. stuffed, attenuate upwards, whitish, zoned white. $G$. adnate, distant, lilac to cinnamon.

Under trees, pines. Oct. $1 \frac{3}{8} \times 2 \times \frac{3}{16}$ in.

1093. C. torvus Fr. (from its habit, growing in wild places as contrasted with 1090 ; torc'zs, savage) a $b c$.

$P$. convex, obtuse or subumbonate, brick-red, brown, coppery or pale brown, shaded purplish. St. solid, clavate or equal, pale violaceous above, whitish and floccoso-scaly below. $A$. white. $G$. adnate, thick, distant, becoming purplish-umber and dark cinnamon. Flesh faintly dull purplish, often much eaten by larve.

Taste insipid, not unpleasant. Odour disagreeable, like 648. Woods, mixed, beech ; frequent. Aug.-Oct. $4_{\frac{3}{4}}^{3} \times 5_{\frac{1}{1}}^{\frac{1}{2}} \times \frac{3}{4}$ in. Intermediate forms occur between this and 1094 .

1094. C. impennis Fr. (from its smoothness as contrasted with 1095 ; impennis, without feathers) a $b$.

$P$. hemispherico-convex, glabrous, brown to reddish; marg. silky and pale violet when young. St. solid to hollow, equal or subclavate, fibrillose, pallid, with a white Z. $G$. adnate or emarginate, very broad, cinnamon, shaded flesh-violet, becoming watery ferruginous. Substance soft and watery.

Taste none. Woods, pine, amongst dead leaves. Sept.-Oct. $4 \frac{3}{5} \times 5 \frac{1}{2} \times \frac{\pi}{5}$ in. Var. luconum Fr. St. sometimes tinged with violet above. G. emarginate, cinnamon slightly sbaded violet. Masiee (Eur. Fung. Fl. 192) regards this as a species.

1095. C. plumiger Fr. (from the scales on the pileus and stem; plumigry, bearing feathers) $a$.

$P$. convexo-plane, umbonate, fuscous, olive-shaded to brick-tan, white floccoso-scaly. St. solid, clavate, floccoso-scaly below, whitish. $A$. white. $G$. adnate, scarcely crowded, becoming pale cimnamon.

Woods, nixed. Sept.-Oct. $2 \frac{1}{4} \times 2 \frac{1}{2} \times \frac{5}{16}$ in. 
1096. C. seutulatus Fr. (from the stem, chequered with the veil: scutula, lozenge-shaped) a b.

$P$. hemispherico-expanded, squamuloso-rivulose or lacunosowrinkled, purple-umber or brick-fuliginous; marg. white silky. St. solid, subclavate, deep violaceous to fuscous, annulate and 4- to 7-zoned with white V. $G$. adnate, becoming cinnamon. Flesh violaceous.

Odour strong of radish. Woods, moist places. Aug.-Oct. $2 \frac{3}{8} \times 4 \frac{3}{4} \times \frac{3}{8} \mathrm{in}$.

1097. C. evernius Fr. (from the stem growing tall; icrmius, sprouting) a b c.

$P$. conico-campanulate, expanded, obtuse or subumbonate, purplebay-brown to pale pink-brown or umber with purplish marg., hoary when old. St. stuffed, slightly attemuate upwards, squamulose, violaceous or paler than P., obsoletely multi-zoned. $G$. adnate, distant, becoming cinnamon. Flesh yellowish-white in P., purplish or greyish in St.

Woods, pine, mixed, damp places; frequent. Sept.-Dec. $2 \frac{1}{4} \times 5 \frac{1}{2} \times \frac{1}{3} \mathrm{in}$.

1098. C. quadricolor Fr. (from the four colours, yellow, violaceous, cinnamon, and white) a $b$.

$P$. campanulate, expanded, umbonate, yellow to tawny. St. stuffed to hollow, fibrillose, violaceous-whitish. $A$. fugitive, white. $G$. adnate, distant, sometimes violet, becoming cinnamon, edge white.

Woods, beech. Oct. $1 \frac{7}{3} \times 3 \times \frac{1}{4}$ in.

1099. C. armillatus Fr. (from the rings or zones round the stem; armilla, a ring) a b $c$.

$P$. campanulate, flat, dry, innately fibrillose or squamulose, brickred or siemna, often cinnabar-zoned or spotted at marg. St. solid, bulbous, fibrillose below, I- to 6-zoned vermilion. $G$. rounded or sinuato-adnate, beconing dark ferruginous.

Taste agreeable or insipid ; odour of radish or of 1394, or of cold cooked potatoes. Woods, mixed, under hazel ; frequent. Aug.-()ct. $4 \frac{1}{2} \times 7 \times \frac{1}{2}$ in. The vermilion zones are permanent after drying.

1100. C. hæmatochelis Fr. (from the blood-red zone of the stem : Gr. haima, blood, chelus, the chest) a b c.

$P$. convex, gibbous, flat, pallid fuscous-brick-colour or claretsepia. St. solid, clavate, zoned rufous. $G$. rounded, adnate, crowded, pallid cinnamon.

Woods, beech. Aug.-Oct. $5 \times 4 \frac{1}{2} \times \frac{1}{2}$ in. Colour of bright red zone permanent after drying.

1100a. C. paragaudis Fr. (from the fibrillose veil; parastuda, a border or trimming of a garment) $a$.

$P$. somewhat fleshy, campanulato-expanded, umbonate, becoming somewhat fibrillose, brown, tawny, bay or tawny-tan, sometimes orange. St. hollow, twisted, soft, pallid reddish, clad 
with reddish adpressed fibrillose sc. $G$. adnexed, rentricose subdistant, opaque cinnamon.

Damp places under pines. Autumn. $2 \frac{1}{2} \times 6 \frac{1}{4} \times \frac{3}{3}$ in. Var. prestigiosus Fr. $P$. submembranous, striate, silky-fibrous towards edge, dark bay, then lignter. St. red-squamulose. $G$. distant, tawny-cinnamon. Under pines.

1101. C. croceofulvus Fr. (from the colour of the pileus; crocells saffron, fulzus, tawny) a.

$P$. campanulate, obtuse, flat, bright tawny-orange. St. hollow, equal, striate, yellow, rufous-tinged. $Z$. a bright red line. $G$. adnato-sinuate, thick, subdistant, becoming ferruginous.

Woods, fir. Sept. $2 \frac{3}{4} \times 2 \frac{1}{8} \times \frac{3}{8}$ in.

1102. C. limonius Fr. (from the lemon-coloured pileus; limonerus, lemon-coloured) $a$.

$P$. convexo-plane, obtuse, tawny lemon-yellow, sometimes orange. St. solid, equal, orange-yellow, light yellow floccoso-scaly. $G$. adnate, distant, at first light yellow. Flesh colour as P.

Odour none. Woods, pine; rare. $3^{\frac{1}{4}} \times 3^{\frac{1}{2}} \times \frac{1}{2} \mathrm{in}$.

1103. C. helvolus Fr. (from the pileus, becoming pale yellow, heliolus) a b.

$P$. conrexo-plane, obtuse or subumbonate, at first dark tawnycinnamon. St. solid, equal, fibrillose, paler than P. with a median ferruginous A. $G$. emarginate, distant, thick, somewhat veined.

Woods, mixed, wooded pastures. Sept.-Oct. $2 \frac{1}{2} \times 3 \frac{3}{2} \times \frac{5}{16}$ in.

1104. C. hinnuleus Fr. (from its fawn colour, himmleus) a b $c$.

$P$. campanulato-expanded, umbonate, often pierced-dotted, tawnycinnamon ; marg. silky. St. stuffed, slightly attenuate downwards, dull tawny; $Z$. broad, fugitive, white. $G$. emarginatoadnexed or adnate, distant, thin, veined.

Tasteless. Woods, mixed; common. Aug, - Dec. $3 \frac{1}{\frac{1}{x}} \times 4 \times \frac{3}{3}$ in. Variable in size.

1105. C. gentilis Fr. (from its relationship to its neighbours; gentilis, of the same race) $a b c$.

$P$. campanulate, expanded, obtuse, orange, tawny-cinnamon or yellowish. St. stuffed to hollow, equal, colour as P., with $3-5$ yellow or sulphur zones. $G$. adnate, very distant, thick. Flesh yellowish or pale brownish.

Gregarious. Woods, chiefly pine. Aug.-Oct. $1 \frac{5}{5} \times 6 \times \frac{1}{4} \mathrm{in}$.

1106. C. helvelloides Fr. (from a fancied resemblance to certain species of Hictella, a Discomycetous fungus) a b c.

$P$. convex, flat, obtuse or umbonate, hygrophanous, ferruginous to tawny; marg. striate. St. hollow, equal, flexuous, subferruginous, white-silky above. $Z$. broad, yellowish. G. adnate, very thick, very distant, violaceous umber to cinnamon and ferruginous.

Woods, mixed, beech, among rotten leaves; uncommon. Aus.-Oct. I $\times 4 \times \frac{1}{5}$ in. 
1107. C. rubellus Cooke (rubillus, ruddy) a.

$P$. campanulate, expanded, umbonate, rufous-orange; mid. darker. St. solid, equal or subclavate, paler than P., 3- to 6 . zoned ferruginous. $G$. adnato-sinuate, scarcely crowded, pale then bright ferruginous-red.

Swampy places. Autumn. $2 \frac{3}{4} \times 3 \frac{1}{4} \times \frac{1}{2}$ in. With the appearance of an abnormal 1099 or 1111.

1108. C. bovinus Fr. (from its stout habitat; bozinus, like a bull) a b. $P$. convex, obtuse, pallid to watery-cimnamon and tawny. St. solid, spongy-bulbous, brownish-cinnamon, whitish above the brown $Z$. $G$. adfixed or adnate, subdistant, cimmamon, becoming dark.

Woods, mixed, pine. Sept. $3 \times 2 \frac{1}{2} \times \mathrm{I}$ in.

1109. C. nitrosus Cooke (from the nitrous odour) $a b$.

$P$. convexo-expanded, obtuse, flat, becoming scaly, fawn to tawny; mid. brownish and darker. St. solid, subequal, ochreous, paler than P., marked below with squamose zones. $G$. emarginate, subdistant, violet to watery-cinnamon. Flish pale brown.

Woods, mixed. Autumn. $2 \frac{1}{4} \times 2 \times \frac{3}{3}$ in.

1110. C. brunneus Fr. (from its brownish colour ; brunnens, brown) a $b$. $P$. campanulate, flat, subumbonate, umber to dull reddish-tan. St. stuffed, subequal, brownish, white-striate. $A$. dull brownishwhite. $G$. rounded-adnate, thick, distant, purplish to brownishcinnamon.

Woods, pine, moist places, amongst grass. Sept.-Oct. $3 \frac{5}{8} \times 3 \frac{5}{8} \times \mathrm{I}$ in.

1111. C. brunneofulvus Fr. (from its colours; lintmetus, brown, fulcus, tawny) a $b$.

$P$. convexo-flat, subumbonate, fibrilloso-virgate, tawny-cinnamon ; marg. at first white fibrillose. St. stuffed to solid, subequal, fibrilloso-striate, tawny, patched cimnamon, with a fugitive dullwhite Z. $G$. adnate, ventricose, subdistant, tawny-cinnamon.

Woods, pine, oak, amongst moss. Sept. $1 \frac{3}{8} \times 2 \frac{1}{\frac{1}{4}} \times \frac{5}{16 i}$ in.

1112. C. injucundus Fr. (from its unpleasant odour; injucundus, unpleasant) a $b$.

$P$. convex, plane, obtuse, fuscous-cinnamon. St. solid, subclavate, colour as P., then tawny-yellow, faintly lilac above $Z$. $G$. emarginate, lilac-tan, then clay.

Odour musty. Woods, fir. Nor. $5 \times 4 \frac{1}{2} \times \frac{3}{4}$ in.

1113. C. glandicolor Fr. (from its colour as of the acorn, glans) a.

$P$. campanulate, expanded, umbonate, becoming dull paleochreous; marg. striate. St. hollow, subfibrillose with a fugacious white $Z$. $G$. adnexo-adnate.

Woods, pine, Sthaynum bogs, mossy places. Autumn. $\mathbf{I}^{3} \times 3 \times \frac{3}{15}$ in. Var. curtus Fr. St. I in., flexuous, peronate with white Co., cingulate; um. becoming blackish. 
1114. C. punctatus Fr. (from the minute dots or punctures on the pileus : punctutus, dotted) a.

$P$. campanulate, flat, subumbonate, tan or somewhat cinnamon. St. fistulose, fibrilloso-striate, yellow-fuscous, with a fugacious brownish Z. G. adnato-rounded, very distant, brown-cinnamon. Woods, heech, fir. Autumn. $1 \frac{3}{4} \times 2 \frac{3}{8} \times \frac{1}{8}$ in.

\section{b. Liptophylla.}

1115. C. triformis Fr. (from its assuming three forms) a, the type form, Schafferi.

$P$. flat, gibbous or subumbonate, fawn to yellowish and whitishgrey. St. stuffed, subbulbous, pallid or faintly pinkish-ochre. $A$. distant, white. $G$. emarginato-adnate, ventricose, wateryochreous to pale cinnamon.

Woods, beech. Oct. $3 \frac{3}{8} \times 3 \frac{1}{2} \times \frac{5}{8}$ in. I. Schueffiri, as above. 2. Melliopaliens. $P$. hemispherical, honey-colour, then pale tan. G. at firse yellowish. St. conically attenuate, pallid yellow. Woods, pine. 3. Fuscopallens. $I$. thin, fuscous, becoming pale. $G$. at first watery-
white. Woods, pine.

1116. C. biformis Fr. (from its resembling two subgenera; a Telamonic with the appearance of a $\left.H_{y} d r o c y b c\right) a$.

$P$. campanulate, expanded, umbonate, dark ferruginous-brown to pale bay. St. stuffed, attenuate downwards, fibrilloso-striate, paler than pileus. $A$. fugitive, white. $G$. adnato- or emarginatorounded, greyish to watery-cimnamon or whitish-umber.

Woods, mixed. Oct. $2 \frac{1}{4} \times 2 \frac{5}{8} \times \frac{1}{4}$ in. With the habit of 1140 .

1117. C. periscelis Fr. (from the garter-like annulus; Gr. periskclis, a garter) a $c$.

$P$. campanulate, convex, umbonate, lilac, greyish or brownish, white-silky. St. stuffed, colour as P., or pale steel-grey, fibrillose, multi-zoned fuscous. $G$. adnato-ascending, pallid or salmon-tan to dark ferruginous.

Eogs and under beeches. Autumn. $2 \times 3 \frac{1}{2} \times \frac{3}{16}$ in.

1118. C. flexipes Fr. (from the flexuous stem; flims, bent, pes, a foot) $a b$.

$P$. campanulate, expanded, acutely umbonate, dark-brown-fuscous, shaded violaceous to pale yellow, hoary-fibrillose. St. stuffed, pallid violaceous or steel-grey, floccoso-scaly below the white $Z$. $G$. adnate, slightly rounded, purple or umber-violaceous to cinnamon, edge whitish.

Woods, larch, fir ; frequent. Sept.-Oct. $1 \frac{3}{8} \times 3 \times \frac{1}{5}$ in.

1119. C. flabellus Fr. (from a fancied resemblance to a small fan, flabellus) a $b$.

$P$. conical, expanded, umbonate, olivaceo-fuscous to yellowumber, then tan, at first white scaly, then incised and tornfibrillose. St. stuffed, floccoso-scaly, pallid, apex violet. 
$Z$. or $A$, white or none. $G$. adnate, dark violaceous to cinnamon and olive.

In troops, gregarious. Odour strong, somewhat of radish. Woods, beech, moist places. $\frac{3}{4} \times 2 \frac{3}{4} \times \frac{1}{5}$ in.

1120. C. psammocephalus Fr. (from the furfuraceo-squamulose pileus, as if sand-covered; Gr. psammos, sand, kephale, the head) $a$. Tawny-cinnamon.

$P$. convex, plane, subumbonate, at length revolute. St. stuffed to hollow, slightly attenuate upwards, squamulose below A. $G$. sinuato-adnate, becoming umber-cinnamon.

Odour none. Woods, mixed, fir, charcoal heaps; frequent. Sept.-Oct. $2 \frac{1}{4} \times 1 \frac{5}{8} \times \frac{3}{15}$ in.

1121. C. incisus Fr. (from the cracked, incised pileus) a $b$.

$P$. convex, expanded, broadly umbonate, naked, then fibrillososcaly, brown of various shades. St. stuffed, fibrous, fibrillose, tan to ochreous and brown. $A$. white or obsolete. $G$. adnate, subdistant, salmon to deep sienna.

In troops or subcrespitose. Odour slight. Woods, pine, naked ground, dry places, dry turf bogs. Sept. $I_{\frac{1}{2}} \times I^{\frac{3}{8}} \times \frac{1}{8} \mathrm{in}$.

1122. C. iliopodius Fr. (from the colour of the stem, as if dirty; Gr. ilus, nud, pous, a foot) a b c.

$P$. expanded, umbonate, opaque cinnamon to tan. St. stuffed, equal, fuscous, pale saffron-cinnamon within. $A$. median white. $G$. adnate, subdistant, thin, salmon to cinnamon.

Taste somewhat of radish. Woods, mixed, fir, beech; common. July-Dec. $1 \frac{3}{8} \times 2 \frac{3}{4} \times \frac{3}{16}$ in.

1123. C. hemitrichus Fr. (from the partly hairy pileus and stem; Gr. hemi, half, thrix, a hair) a $b$.

$P$. convex, expanded, umbonate, grey or dark fuscous to fuscoustan, erect white-fibrillose, especially round marg.; um. dark. St. hollow, equal, white flocculose below the median white $A$. $G$. adnate, very crowded, clay to cinnamon or sienna. Fles/a sienna.

Woods, mixed, mossy places, boggy ground, amongst fallen leaves; uncommon. April-Oct. $1 \frac{7}{8} \times 22_{4}^{3} \times \frac{1}{4}$ in.

1124. C. stemmatus Fr. (from the silky marginal band of the pileus, like a wreath, Gr. stimma) $a$.

$P$. convex, expanded, subumbonate, dark to pale brown. St. stuffed to hollow, equal or slightly attenuate downwards, ferruginous-brown within and without, with grey-white zones. $G$. adnate, crowded, opaque date-brown.

Woods, moist places. Autumn. $I_{2}^{\frac{1}{2}} \times 3 \frac{33}{3} \times \frac{3}{16} \mathrm{in}$.

1125. C. rigidus Fr. (rigidus, firm) a $b$.

$P$. conico-campanulate, umbonate, cinnamon-bay to yellowishtan; marg. pellucidly striate. St. stuffed to hollow, equal, colour as $\mathrm{P}$. variegated with tan-white and umber zones. $G$. adnate, ventricose, subcrowded, salmon to cinnamon.

Odour strong. Woods, damp places. Nor. $1 \frac{3}{8} \times 2 \frac{3}{4} \times \frac{3}{16}$ in. 
1126. C. paleaceus Fr. (from the chaffy white scales of the pileus; palea, chaff) a b $c$.

$P$. convex, expanded, acutely umbonate, fuscous to greyish; mid. umber ; marg. paler or whitish. St. fistulose, floccoso-scaly, fuscous within and without. $A$. and $\mathrm{I}-7$ zones whitish. $G$. adnate, crowded, grey pallid to cinnamon.

Odour weak. Woods, beech, moist places. Sept.-Nor. $1 \frac{1}{2} \times 2 \frac{3}{4} \times \frac{1}{8}$ in.

1127. C. iris Mass. (from the rainbow-like colours; iris, the rainbow) $a$. $P$. conical, expanded, umbonate, pale ochreous-brown, densely white-fibrillose; marg. silky, splitting. St. stuffed to solid, clavate, densely fibrillose, violet, then pale above the fibrillose, bright-brown A., orange-brown at base within and without. $G$. sinuato-adnexed, subcrowded, dull ochreous to orangebrown.

Solitary, or in clusters of two to four. Woods. Oct. $1 \frac{1}{ \pm} \times 3 \times \frac{1}{8} \mathrm{in}$.

1128. C. Cookei Quél. (after Dr. M. C. Cooke) a b.

$P$. coniral, hemispherical, umbonate, tawny-yellow ; marg. woolly, white. St. stuffed, pale yellowish with $4-5$ floccose yellow zones. $G$. adnate, violet to reddish and rust-colour.

Woods, fir. $\frac{1}{2} \times 1 \frac{5}{8} \times \frac{1}{10} \mathrm{in}$.

\section{Subgenus 6. Hydrocybe.}

(From the moist or hygrophanous pileus; Gr. Inudor, water, kube, a head.)

Ieil thin, fibrillose, rarely collapsing and forming an irregular zone on the stem. Pilius smooth or only covered with white

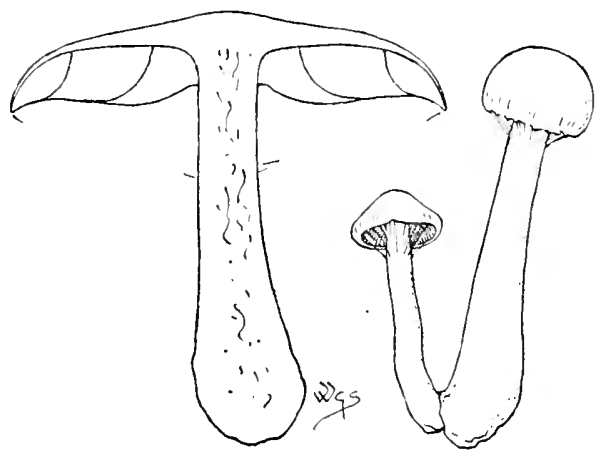

Fig. 5S.-Cortinurius (Hydrocybe) saturninus Fr., and section. One-half natural size.

superficial fibrils, not viscid, but moist when fresh, changing colour when dry; flesh very thin, scissile, rarely more compact in the middle. 
Stem slightly rigid, subcartilaginous externally, not sheathed, annulate or scaly. (Fig. 5S.)

Species $1129-1167$

a. Firmiores. Pilens subfleshy, convex, or campanulato-convex, then expanded, obtuse or at length gibbous, margin at first incurved. Stcm for the most part attenuate upwards.

Ste'm and cortina white.

$1129-1137$

St'm and gills inclining to violet.

$1138-1141$

Stem and somewhat obsolete veil yellow or rufous. 1142-1146

Stem inclining to fuscous. Cortina pallid, dull or white. Gills dark.

$1147-1150$

b. Temuiores. Pileus somewhat membranous, conical, then expanded, umbonate; umbo acute or rarely obtuse and vanishing; margin at first straight. Stem usually almost equal, or attenuate downwards.

Stem white.

$1151-1156$

Stem inclining to violet or reddish.

$1157-1160$

Stem yellowish, commonly becoming pale.

$1161-1163$

Stem inclining to fuscous.

$1164-1167$

\section{a. Firmiores.}

1129. C. firmus Fr. (from the compact substance; firmus, firm) $a$.

$P$. ferruginous to ochreous; marg. tan. St. solid, clavate, fibrilloso-striate. $Z$. ferruginous. $G$. adfixed or emarginate, pale umber to cinnamon.

Woods, mixed, grassy places. Autumn. $5 \times 3^{\frac{1}{4}} \times I_{\frac{1}{4}}^{\frac{1}{4}}$ in.

1130. C. subferpugineus Fr. (from the colour of the pileus, somewhat rust-colour, firmgo), a $c$.

$P$. umbonate, hygrophanous, dry, ferruginous to watery-cinnamon tawny or tan ; $V$. at marg. only. St. solid, attenuate upwards, adpressedly fibrillose, pallid, saffiron-yellow at base. $G$. emarginate or adnate, subcrowded, pallid to dark ferruginous.

Taste and odour unpleasant. Woods, oak, amongst rotting pine-leares. Sept.-Oct. $2 \frac{1}{8} \times 4_{4}^{3} \times \frac{3}{8}$ in.

1131. C. armeniacus Fr. (from the colour of the pileus, like the apricot, Pyrus armeniaca) a b $c$.

$P$. obtuse or subumbonate, tawny-cinnamon to ochreous; marg. substriate. St. stuffed, attenuate upwards. $Z$. white. $G$. adnate, pallid to clay-cinnamon.

Woods, pine; uncommon. Aug.-Oct. $2 \frac{1}{1} \times 2 \frac{3}{4} \times \frac{3}{8}$ in. Var. fulsarius Fr. $P$. yellow, white when dry.

1132. C. damascenus Fr. (from the colour of the pileus, like the damson) $a$.

$P$. rivulose when dry, bay-cinnamon or deep umber with a crimson shade to brick-red. St. solid, equal. $Z$. whitish. $G$. rounded-adnate, cimnamon or paler than $\mathrm{P}$.

Subcrespitose. Taste acrid. Under trees, grassy places, pastures, sept. $3 \frac{1}{ \pm} \times 3 \frac{1}{2} \times \frac{1}{2}$ in. 
1133. C. privignus Fr. (from its being fragile in a non-fragile group; frizignus, a stepson) a b.

$P$. obtusely umbonate, flat, reflexed, hygrophanous, fuscous to tan. St. stuffed to hollow, equal, silky with V. and Z. G. roundedadnate, not crowded, watery- then opaque-cinnamon.

Taste scarcely acrid. Odour strong, unpleasant. Woods, pine, oak, damp places. Oct. $2 \frac{1}{4} \times 2 \times \frac{1}{4}$ in.

1134. C. duracinus Fr. (from its firmness; durus, hard, acinus, a berry) $a b$.

$P$. obtusely umbonate, pale brick-colour or sienna to tan, or mid. sienna and marg. tan; marg. clad with V. St. solid, ventricose, attenuato-rooting, imperfectly zoned. $G$. adnate or emarginate, ventricose, pale cinnamon or whitish-sienna.

Woods, old pastures, near stumps. Ang.-Oct. $2 \frac{1}{2} \times 3 \times \frac{1}{2}$ in.

1135. C. illuminus Fr. (dark, as contrasted with the allied non-British C. candelaris; illumimus, dark) a b.

$P$. gibbous, or subumbonate, pale brick-red, or orange with mid. orange-sienna to tan. St. stuffed, equal or slightly attenuate upwards, silky-fibrillose, pallid or tan-ochre, or white above and ochre to sienna below. $Z$. ochre. $G$. adnate, subcrowded, subdistant, pallid to cinnamon or whitish-sienna.

Woods, pine. Sept. $1 \frac{1}{2} \times 2 \frac{5}{8} \times \frac{3}{16}$ in.

1136. C. tortuosus Fr. (from the somewhat twisted stem; tortuosus, twisted) a $b$.

$P$. gibbous, ferruginous-bay to brick-red, or warm-tan with mid. and marg. somewhat dark crimson-umber, purple or turkey-red when bruised. St. stuffed, equal, silvery, spotted with white imperfect zones. $G$. adnate, crowded, tawny, purple or turkeyred when bruised.

Woods, pine, damp places. Autumn. $2 \frac{1}{2} \times 4 \frac{1}{2} \times \frac{3}{8}$ in.

1137. C. dilutus Fr. (from the watery colour, dilutus) $a$.

$P$. obtuse or subumbonate, opaque bay-brown or pale brick-red to tan; marg. white-silky and fringed with V. St. stuffed, equal, silky-white. $G$. deeply emarginate, ventricose, crowded, pale cinnamon or brownish-ochre.

Odour none. Woods; rare. Nov. $1 \frac{3}{4} \times 2 \frac{3}{8} \times \frac{3}{16}$ in.

1138. C. saturninus Fr. (from its colour ; saturninus, gloomy) a $b$.

$P$. obtuse or umbonate, dark bay-brown or umber, soon pale; marg. silky and appendiculate with V. St. stuffed to hollow, attenuate upwards, deep violet or purple to pale dove-grey, partially white-zoned. $G$. rounded-adfixed or emarginate, crowded, thin, pale purple-grey to purplish and ferruginous.

Sometimes subcrespitose. Odour faint. Grassy places. Oct.-Nov. $3 \frac{1}{2} \times 2 \frac{1}{2} \times \frac{1}{2}$ in. 
1139. C. imbutus Fr. (from the moist, stained flesh; imblo, to soak) $a$.

$P$. flat, obtuse, sienna-brown to pale yellowish; marg. white hoary-fibrillose. St. solid, equal, whitish, pale violaceous above. $G$. rounded-adnate, subdistant, dark bluish-grey to watery-cinnamon. Flesh dingy pale violaceous at top of St.

Subcrespitose. Woods. Sept. $3 \frac{5}{5} \times 2 \times \frac{1}{2}$ in.

1139a. C. seiophyllus Fr. (from the dusky gills; Gr. skila, shade, fhullon, a leaf) a.

$P$. thin, convexo-expanded, obtuse, blue-purple-slate, paler towards marg.; mid. Heshy; edge narrow, silky white; $C_{0}$. white. St. solid, slightly attenuate upwards, pale lavender to violet, gradually reddish towards the white base, clad with irregular white Z. $G$. adnate, crowded, narrow, purple-brownish or dusky umber. Flesh purple-slate aloove, salmon-buff and reddish below.

Single or cæespitose. Under beeches. Autumn. $I_{\frac{3}{4}}^{\frac{3}{4}} \times 2 \frac{5}{8} \times \frac{1}{2}$ in.

1140. C. castaneus Fr. (from its colour ; castane $a$, a chestnut) a b $c$.

$P$. obtuse or subumbonate, shining fuscous- or purplish-chestnut ; mid. darker. St. stuffed, fibrillose with V., pale purplish above, purple-brownish below. $Z$. white or ochre. $G$. adnate or emarginate, thin, violaceous to ferruginous, edge whitish.

Gregarious, sometimes caspitose. Edible. Taste like that of 1394 or 792 ; odour none or fungoid. Woods, pastures, gardens, on the ground, rarely on wood; common. June-Nov. $1 \frac{5}{8} \times 2 \frac{5}{8} \times \frac{3}{16}$ in.

1141. C. bicolor Cooke (from its two prevailing colours, purple and $\tan ) a b$.

$P$. broadly and acutely umbonate, whitish or lilac shaded. $I$. fugacious, white. St. solid, equal or attenuate downwards, pallid violet or steel-grey to whitish, zoned. $G$. adnate with a tooth, subcrowded, purplish-riolet to cinnamon. Flesh whitish to bright lavender, purple at base of St.

Woods, mixed, on the ground. Aug. -Oct. $2 \frac{1}{8} \times 2 \frac{1}{2} \times \frac{3}{8}$ in.

1142. C. balaustinus Fr. (from the colour of the pileus, like the flower of the pomegranate, Gr. balaustion) a.

$P$. convex, flat, virgate, innato-fibrillose, shaded sienna-crimson or vermilion. St. solid, fibrilloso-striate, pallid to tawnyferruginous within and without, zoned red-sienna. $G$. adnate, subcrowded, ferruginous-red or paler than $P$.

On the ground, woods, mixed, beech. Oct. $2 \frac{1}{4} \times 2 \frac{3}{4} \times \frac{5}{16}$ in.

1143. C. colus Fr. (from the fibrillose stem; colus, a distaff) $a b$.

$P$. convex, obtuse or obtusely umbonate, brown-rufescent, deep bay or umber, becoming paler. St. stuffed, attenuate upwards, paler than P., base encircled by blood-red or fiery-saffron mycelium. $G$. ascending-adnate, subcrowded, pale lilac or brownish to dark cinnamon.

Woods, pine. Oct. $2 \frac{3}{8} \times 3 \frac{3}{\frac{3}{4}} \times \frac{3}{16}$ in. 
1144. C. isabellinus Fr. (from the dirty-yellow colour, like unwashed linen ; from Isabel of Austria, daughter of Philip II., who vowed not to change her linen till Ostend was taken) $a b$.

$P$. subumbonate, at first honey-colour then paler. St. stuffed, equal, striate, colour as P., or tan, imperfectly zoned. $G$. rounded-adnate, subdistant, yellow to cinnamon.

Woods, pine. Autumn. $1 \frac{3}{4} \times 3 \frac{1}{8} \times \frac{3}{8}$ in.

1145. C. renidens Fr. (from the glistening pileus; renidens, shining) $a$. $P$. expanded, flat, at first broadly and acutely umbonate, hygrophanous, ferruginous-tawny to ochreous. St. stuffed, at first bulbous, then equal, fibrillose, colour as P., imperfectly zoned yellow. $G$. rounded-adnate, subcrowded, pallid cinnamon to tawny.

Crespitose. Woods, shady places. Sept. $3 \times 3 \times \frac{3}{8}$ in.

1146. C. angulosus Fr. (from the small, pointed scales of the pileus; angulosus, full of corners) a $b$.

$P$. convex, expanded, shining tawny-yellow, variegated with small sienna or ochreous sc. $V$. fugacious, tawny. St. equal, striate, tan-orange, zoned tawny-sienna. $G$. adnate, thick, subdistant, orange-sienna.

Woods, fir. Aug.-Sept. $2 \frac{1}{2} \times 2 \frac{1}{\frac{1}{2}} \times \frac{1}{2}$ in. Var. gracilescens Mass. P. somewhat fleshy. St. hollow, base somewhat attenuate, tortuous.

1147. C. uraceus Fr. (from its mouse-colour; Gr. hurax, a mouse) $a b$. $P$. umbonate or obtuse, subfibrillose, brown, olive, red or buff. St. stuffed or hollow, equal, pale or yellowish above, fuscous or blackish below, imperfectly zoned. $G$. adnate or sinuate, distant, cinnamon.

Taste and odour not unpleasant. Woods, pine. Nov, $1_{\frac{7}{8}}^{\frac{7}{8}} \times 3 \frac{1}{3} \times \frac{3}{16}$ in.

1148. C. jubarinus Fr. (from the shining pileus; jubar, radiance) a $b c$. $P$. umbonate, often repand, rich tawny-cinnamon; marg. whitesilky with V. St. stuffed or hollow, fibrilloso-striate, pale tawny within and without. $G$. ascending-adnate, ventricose, subdistant, tawny-cinnamon.

Woods, pine, grassy places, on pine-leares. May. $2 \times 2 \frac{1}{5} \times \frac{3}{15}$ in. C. irregularis Fr. has been published as British by MIassee and Crossland (Fung. Fl. Yorks. p. 97) following Bolton (Hist. Brit. Fung. t. 13), but this figure and description differ entirely from Fries, Hym. Eurr. p. 394, and Hy'm. Sutc. il. p. 106. Thus-

In Fries- $I$. dry, glabrous. St. brick-red. G. decurrent, ferruginous. Hab. amongst pine-leaves.

In Bolton-P. covered with glutinous matter. St. pale grey. G. adnexed or free, pale dusky flesh-colour. Hab. pastures and meadows. Bolton's plant is probably an Entoloma with viscid P.

1149. C. pateriformis Fr. (from the shape of the pileus when depressed, like a patera or libation saucer) a $b$.

$P$. plane to depressed, shining dark chestnut, sprinkled with fugacious white fibrils. St. stuffed or hollow, equal, fibrillose, 
white to dusky, zoned sienna. $G$. emarginato-adnate, ventricose, brick-red to vinous-ochre.

Woods, damp grassy places. Sept.-Oct. $2 \frac{3}{8} \times 3 \times \frac{5}{16} \mathrm{in}$.

1150. C. unimodus Britz. (from its uniform colour; unimodus, of one kind) $a b$.

$P$. umbonate, brown, shaded rufous, vinous or sienna. St. stuffed, equal, paler than P., sometimes pale purple above, imperfectly 2-5-zoned. $G$. emarginato-adnate, distant, brown.

Grassy places. Sept.-Oct. $2 \times 3 \frac{1}{2} \times \frac{1}{4}$ in.

\section{b. Tenuiores.}

1151. C. dolabratus Fr. (from the shape of the stem and gills as seen in section; dolabra, a pickase) $a b$.

$P$. obtusely umbonate, smooth, brick-red to tan; marg. silky. St. stuffed or hollow, smooth, white, sometimes faintly ochreous above. $G$. rounded-adnate, distant, tawny-cinnamon or orange.

Odour strong, pungent-nauseous, stinking. Woods, pine, damp places amongst laccinium, Słhagnum, etc. Sept.-Nov. $3 \frac{1}{2} \times 3^{\frac{1}{4}} \times \frac{1}{2}$ in.

1152. C. rigens Fr. (from the rigid stem) $a b$.

$P$. obtusely umbonate, smooth, tan-clay to tan-whitish. St. stuffed, cartilaginous extemally, zoned. $G$. sinuato-adnate, watery-clay to cinnamon or sienna.

Woods, pine, mixed; uncommon. June-Oct. $1 \frac{3}{4} \times 2 \frac{1}{2} \times \frac{5}{16}$ in.

1153. C. Krombholzii Fr. (after J. V. Krombholz) $a b$.

$P$. obtusely or acutely umbonate, smooth, yellowish-brown to ochreous; mid. always dark; marg. appendiculate with V. St. fistulose, naked, whitish. $V$. membranous, fugitive, whitish. $G$. rounded-adnexed, dull purplish-brown, edge yellowish.

Oftencrespitose. Amongst moss. $2 \frac{3}{8} \times 4 \frac{1}{2} \times \frac{3}{16}$ in. Much like a Hytholoma.

1154. C. Reedii Berk. (after Miss Reed, sister of Mrs. Hussey) $a b$.

$P$. conical, expanded, strongly umbonate, brown ; mid. areolate. St. solid, bulbous, shaded pale umber. $G$. ascending, attenuatofree, ventricose, white to cinnamon.

Taste and odour none. Amongst moss and beech-mast. May. $1 \frac{1}{1} \times 1 \frac{1}{1} \times \frac{3}{16}$ in.

1155. C. leucopus Fr. (from the white stem; Gr. leukos, white, pous, a foot) $a b c$.

$P$. conical, expanded, umbonate, smooth, moist, yellowish or somewhat buff to tan. St. hollow, equal, attenuate upwards, shining, zoned. $G$. adnexed, crowded, ventricose, pallid to cinnamon.

Woods, fir, mixed; frequent. Sept.-Nov. $1 \frac{1}{4} \times 1 \frac{1}{2} \times \frac{3}{16}$ in.

1156. C. scandens Fr. (from the long wavy stem; scando, to climb) $a b$. $P$. conico-campanulate, obtusely or acutely umbonate, moist, smooth, tawny-ferruginous to honey-colour and tan; marg. 
striate; Co. white. St. fistulose, flexuous, rooting, greatly attenuate downwards, yellowish to whitish. $G$. ascendingadnate, tan to yellow and tawny-cinnamon.

Woods, pine, moist places. Oct.-Nov. $1 \frac{1}{8} \times 2 \frac{1}{2} \times \frac{3}{16}$ in.

1157. C. erythrinus Fr. (Gr. cruthres, red) $a$.

$P$. umbonate, smooth, bay-brown-rufous, or vinous-sienna to tawny; Co. white. St. stuffed to hollow, equal, smooth, striate, white, pale purple or violaceous. $G$. adnexed or somewhat adnate, pallid to pale cinnamon or sienna.

Woods, amongst leaves in damp places. Oct. $1 \frac{1}{8} \times 1 \frac{7}{8} \times \frac{1}{15} \mathrm{in.}$ Var. argyropus Fr. more slender than type. St. silvery, white-mealy above.

1158. C. decipiens Fr. (from its resemblance to several other species; decifio, to deceive) a $b c$.

$P$. acutely or obtusely umbonate, smooth, bay-brown to shining brick-red; mid. darker. St. solid, stuffed or hollow, fibrillose, pale rufescent, clouded brick-red. Z. imperfect, fugacious, white. $G$. adnate, sometimes with a tooth, crowded, thin, sienna-umber or sienna-tan.

Woods, mixed; frequent. Sept.-Oct. $2 \frac{1}{2} \times 3 \frac{1}{2} \times \frac{1}{4}$ in. Var. insignis Fr. $P$. paler; St. flexuous, glabrous; $G$. less crowded.

1159. C. germanus Fr. (from its close relationship to adjoining species; sermanus, born of the same parents) a b.

$P$. obtusely or acutely umbonate, silky, hygrophanous, fuscous to clay or tan-umber; mid. sepia; marg. whitish, or wholly tan. St. fistulose, equal, silvery-pale or somewhat lilac. Z. fugacious, ochre. $G$. adnate, slightly rounded, subdistant, waterycinnamon.

Odour powerful. Woods, pine, beech. Oct. $1 \times 2 \frac{5}{8} \times \frac{1}{8}$ in.

1160. C. ianthipes Fr. (from the violet stem; Gr. ianthios, violetcoloured, pous, a foot) a b $c$.

$P$. obtusely umbonate, fibrillose, brown; marg. yellowish. St. stuffed, smooth, equal, violaceous, base rufescent, imperfectly zoned. $G$. adnexed or adnate, subcrowded, brown olivaceous, then dark umber-sienna or deep red-brown.

Woods. Sept. $\frac{5}{8} \times 1 \frac{1}{4} \times \frac{1}{15}$ in.

1161. C. detonsus Fr. (from the smooth pileus and stem; detondeo, to shear) a $c$.

$P$. subumbonate, slightly silky to somewhat smooth, brick-red, umber or yellowish. St. fistulose to stuffed, yellowish-pallid, dull purple at mid. $G$. adnate, subdistant, yellowish brickcolour.

Woods, pine, amongst moss in damp places. Sept. $1 \frac{3}{5} \times 1 \frac{5}{5} \times \frac{1}{5}$ in.

1162. C. obtusus Fr. (from the obtuse umbo which at length disappears) $a$.

$P$. bay-brown-ferruginous, cinnamon, or orange-sienna to pale ochreous or tan-whitish; marg. striate. St. stuffed to hollow 
attenuate downwards, lighter than P.; $C_{0}$. whitish. $G$. adnate, ventricose, orange-sienna; edge white.

Gregarious. Odour strong. Woods, chiefly pine; requent. April-Oct. I $\frac{1}{2} \times 2 \frac{1}{2} \times \frac{1}{4}$ in.

1163. C. acutus Fr. (from the acute umbo) a $b c$.

$P$. honey-colour or pale yellow to whitish; marg. white fibrillose. St. fistulose, equal, white-fibrillose, colour as P., zoned. $G$. adnate, crowded, thin, white-ochre, ochreous-cinnamon or watery-brown.

Scattered, in troops, or crespitose. Odour at length nitrous. Woods, fir, mixed, moist places ; frequent. Sept.-Nov. $1 \frac{1}{4} \times 2 \frac{1}{2} \times \frac{1}{8}$ in.

1164. C. Junghuhnii Fr. (after Francis Junghuhn) a.

$P$. papillate, cinnamon or dull crimson-orange to tawny; marg. striate. St. stuffed to hollow, reddish, whitish below, adpressedly fuscous-fibrillose. $G$. adnate, subdistant, thin, reddish-ochre or olive shaded. Flesh reddish.

Odour none. Woods, mossy places. Aug. $1 \frac{1}{2} \times 2 \frac{1}{4} \times \frac{3}{16}$ in.

1165. C. depressus Fr. (from the pileus, at length depressed) $a$.

P. smooth, vinous- or ochreous-umber ; marg. at first silky then striate. St. hollow, attenuate downwards, reddish or yellowbrownish. $G$, adnate, rentricose, saffron-yellow, reddish or reddish-brown-ochre.

Odour faint of stale fish or cucumber, as in 709 , which resembles this in habit. Woods, moist places. Autumn. $1 \frac{1}{2} \times 1 \frac{3}{\frac{3}{4}} \times \frac{1}{4}$ in.

1166. C. milvinus Fr. (from the coloration, like a kite's back; milints, a kite) a $b$.

$P$. obtusely umbonate, olivaceous-fawn or olive to tan; marg. striate, white squamulose. St. stuffed, equal, pale fuscous, with white silky spots, or whitish above, tan-brown below; $C$ o. white. $G$. adnate, subdistant, ochreous olive-tan to olive.

Gregarious. Odour strong. Woods. Oct. $1 \frac{3}{8} \times 2 \frac{5}{8} \times \frac{1}{4} \mathrm{in}$.

1167. C. fasciatus Fr. (from the fibrous-splitting sten, as if in bundles, fascice) a $b$.

$P$. acutely umbonate, smooth or subsilky, brick colour or umber; mid. sepia. St. fistulose, equal, splitting longitudinally into fibres, pallid to cinnamon-fuscous, or variegated with bands of white, tan and brown. G. adnate, subrentricose, thin, distant, cinnamon.

Woods, pine, damp places, pastures under pines. Sept.-Oct. $1_{\frac{5}{9}}^{5} \times 2 \frac{5}{8} \times \frac{1}{5}$ in.

\section{GOMPHIDIUS Fr.}

(From the shape, like a large bolt or nail, Gr. gomphos.)

I'eil universal, viscoso-floccose. Pilezs at length turbinate, glutinous when moist. Stem central, continuous and homogeneous with the hymenophore, imperfectly floccoso-annulate or cortinate; 
annulus fugitive. Gills decurrent, distant, soft and somewhat branched, edge acute, composed of a mucilaginous membrane which often admits of being detached from the hymenophore and stretched

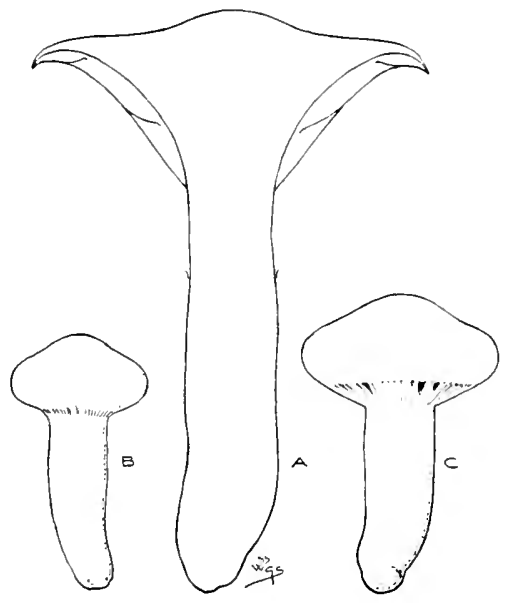

Fig. 59.-Gomphidius riscidus Fr.

A, section; $B$, young state: $C$, half-grown example showing floccose veil. One-third natural size.

out into a continuous membrane, not deliquescent. Sporcs large, fusiform, black or nearly so. (Fig. 59.)

The species grow on the ground, chiefly in pine woods, solitary, subpersistent.

The species are most nearly allied to Hysothorus, but they differ greatly in the annulate stem and the blackish spores. There is also an affinity with Cortinarius, as shown by the cortina, but the spores differ greatly in shape and colour.

Species 1168-1171

1168. G. glutinosus Fr. (from the glutinous pileus) $a b c$.

$P$. becoming flat, smooth, purple-umber or umber-crimison, at first paler. St. solid, viscid and zoned with the fugitive V., whitish above, yellowish or brownish below; $C o$. white. $G$. whitish or olive-whitish, then cinereous. Flish whitish, greyish or pale rose.

Said to be edible. Eaten at Hereford Fungus Forays, but not much approved, I S7O. Taste watery, mouldy ; odour faint. Woods, chiefly pine, larch; common. July-Nov. $4 \frac{1}{4} \times 3 \frac{3}{4} \times \frac{5}{8}$ in. Must not be confounded with 155. Var. roseus Fr. $l^{\prime}$. rose-colour, sometimes dark crimson-red. St. white above, pale rose below.

1169. G. viscidus Fr. (from the somewhat viscid pileus) $a b c$.

$P$. becoming flat, rich apricot, shaded vermilion, sometimes brick-red, often mottled bright purple. St. solid, orange, pale purplish above. $G$. olive, then crimson-purple, purplish or 
whitish-purple. Flesh yellowish, often with purple stains, rhubarb-colour at base.

said to be edible; odour not unpleasant. Woods, chiefly pine; frequent. July-Oct. $4 \frac{5}{8} \times 4 \frac{1}{2} \times \mathrm{I}_{\frac{1}{8}}$ in. Var. testacens Fr. P. brick-red. Woods, beech. Every intermediate form occurs between this and 1168, including the varieties rosens and tistaceus.

1170. G. maculatus Fr. (from the spotted pileus and stem; maculatus, spotted) a.

$P$. becoming flat and depressed, white to brownish-salmon with large umber or blackish spots; marg. striate. St. solid, slightly attenuate downwards, yellow or yellowish below, white, grey or purplish in middle, whitish above. $G$. pale slate, shaded umber. Flesh reddish.

Woods, fir. Autumn. $2 \frac{1}{4} \times 2 \frac{1}{2} \times \frac{1}{2}$ in. Var. Cookei Mass. St. whitish above, blackish at base.

1171. G. gracilis B. \& Br. (gracilis, slender) $a b c$.

$P$. becoming flat and depressed, pale vinous-brown covered with dingy-fuliginous gluten, at length black-spotted and blackbordered with the drying gluten. St. solid, slightly attenuate downwards, white-scaly above, yellow and virgate below, clouded vinous-tan. $G$. slate-white, white umber or greenishwhite.

Woods, fir ; frequent. July-Oct. $1 \frac{3}{4} \times 2 \frac{1}{x} \times \frac{1}{4}$ in. Berkeley's illustration, Outlines, t. 12, fig. 7 , does not agree with his description. Every intermediate form occurs between this and 1170 .

\section{PAXILLUS Fr.}

(From the form, like a small stake or peg, paxillus.)

Vill obsolete. Pilius fleshy, margin at first involute. Stcm central, excentric or obsolete, when present fleshy, continuous and

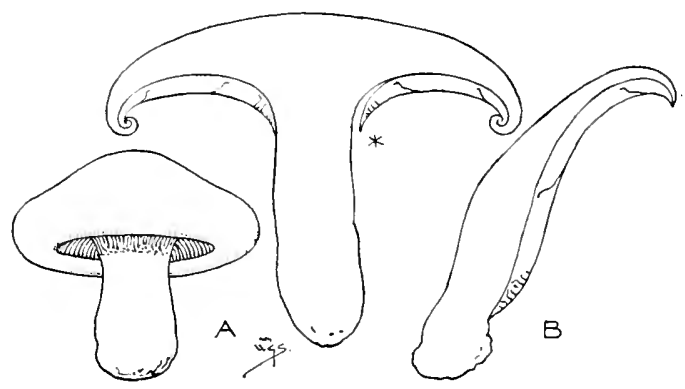

Fig. 60.-A, Parillus involutus Fr.. entire and in section; gills separating from hymenophore at $* ; \mathrm{B}$, section of $P$. panuoides Fr. One-third natural size.

homogeneous with the hymenophore. Gills decurrent, often anastomising behind and forming spurious pores as in Bolitus, readily 
separating from the hymenophore owing to the-except in 1185absence of a trama, usually separated from the flesh of the pileus by a thin differentiated horny or cartilaginous layer. Spores whitish to ferruginous. (Fig. 60.)

Fleshy, putrescent fungi, some growing on the ground, others on tree-trunks or sawdust. Some of the members of the first subgenus are allied to Tricholoma and Clitocybe amongst the white-spored Agarics; those of the second to Flammila, amongst the brown-spored Agarics.

The gills of $\mathbf{1 1 8 1}$ are sometimes wholly porous, in 1181a always wholly porous, as in Boletus.

Most of the species are said to be edible.

Species $1172-1186$

Subgenus I. Lepista. Spores dull white-reddish- or fuscouspallid in 1174-faintly ferruginous in 1176.

$1172-1179$

Subgenus 2. Tapinia. Pileus usually more or less excentric, sometimes lateral and stemless, at length depressed. Spores ferruginous.

$1180-1186$

\section{Subgenus i. Lepista.}

(From the type, 1174, P. lepistic.)

1172. P. giganteus Fr. (from its great size) a bc. Tan white.

$P$. depressed or infundibuliform, not umbonate; marg. sulcate, very involute. St. solid, smooth, stained faintly vinous. $G$. deeply decurrent, very crowded, inclined to branch and anastomose.

Odour strong, like 1394. Woods and pastures; uncommon. Aug.-Nov. $1-1 \frac{1}{2} \times 4 \frac{1}{1} \times \frac{7}{3}$ in. Very different from 190 , with which it is often confounded.

1173. P. Alexandri Fr. (from P. Alexander, who gathered the first examples described) $a b$.

$P$. plano-depressed, dry, fawn to umber-whitish; marg. striate, involute. St. colour as P., or faintly ochreous. $G$. subdecurrent, crowded, yellowish or brownish.

Amongst moss, on the ground. Sept.-Oct. $3 \frac{1}{ \pm} \times I_{\frac{3}{3}} \times \frac{7}{8}$ in.

1174. P. lepista Fr. (from the shape of the pileus when young; lepista, a pan) a b $c$.

$P$. plano-depressed, dry to subviscid; marg. involute, undulatoflexuous, rimuloso-squamulose, not striate, whitish or yellowishwhite. St. solid, whitish, sometimes inclining to fuscous below. $G$. decurrent, very crowded, whitish or pale yellowishwhite.

Solitary, sporadic, changeable in habit. Odour of rancid meal, or strong and acid. Woods, oak, fir, moist places. Oct.-Nor. $5 \frac{3}{8} \times 3 \frac{1}{1} \times 1$ in.

1175. P. extenuatus Fr. (from the thin margin of the pileus; extemuatus, made thin) a b.

$P$. campanulato-convex, broadly umbonate or obtuse, tan, clay or yellowish; marg. involute, smooth, even or striate. St. 
solid, smooth, soniewhat tuberous-rooting, colour as P. $G$. deeply decurrent, very crowded, white to mouse-colour.

Woods, fir, grassy places. Oct. $4 \times 2 \frac{1}{8} \times \frac{3}{4}$ in.

1176. P. panæolus Fr. (from the change of colour of the flesh to black; Gr. panciolos, all variegated) a b c .

$P$. convexo-plane, depressed, smooth, moist, whitish; marg. subinvolute. St. stuffed, attenuate upwards, striato-fibrillose, rufescent or as P., ochreous below. G. subdecurrent, crowded, becoming watery ferruginous or cinnamon.

Subgregarious. Woods, fir, mixed. Oct.-Nov. $1 \frac{1}{4} \times 1 \frac{1}{2} \times \frac{1}{8}$ in. Var. spilomicolus $\mathrm{Fr}$. I'. spotted with dots.

1177. P. orcelloides Cooke $\&$ Mass. (from its resemblance to 504, Clitopilus orcella) a $b$.

$P$. convexo-plane, silky, white, then clouded greyish or livid; marg. involute. St. solid, attenuate downwards, elastic, silkyfibrillose, livid-ochreous. G. adnato-decurrent, crowded, whitish to salmon-brown.

Woods, amongst grass. Sept.-Nov. $2 \frac{1}{5} \times I_{\frac{1}{4}}^{\frac{1}{4}} \times \frac{3}{15}$ in.

1178. P. lividus Cooke (from its livid colour) $\iota$.

$P$. convex, then depressed, dull whitish or livid-ochreous; marg. subinvolute. St. stuffed to hollow, attenuate downwards, fibrillose, whitish. $G$. subdecurrent, somewhat crowded, white.

In small clusters. Odour pleasant. Woods, fields. Oct. $2 \times 2 \times \frac{7}{8} \times \frac{3}{8}$ in. Allied to 1179.

1179. P. revolutus Cooke (from the, at length, slightly revolute margin of the pileus) $a$.

$P$. campanulato-obtuse, pale ochreous ; mid. darker ; marg. sometimes pale lavender. St. solid, attenuate downwards, whitish or pale lavender. $G$. arcuato-decurrent, pallid to clay.

Odour of meal. Fields. Oct. $1 \frac{5}{8} \times \mathrm{I}_{8}^{3} \times \frac{3}{8}$ in.

\section{SUbGenUs 2. TAPINIA.}

(From the pileus, at length depressed; Gr. tapininoo, to depress.)

1180. P. paradoxus Berk. ex Cooke (from its being a new, unexpected species; paradoxus, strange, unexpected) $a b c$.

$P$. convex to plane, densely tomentose, deep rufous-umber to yellowish-brown; marg. subinvolute. St. solid, attenuate downwards, sometimes rooting, yellow or reddish, or crimson to rose above, and yellow to brownish below. $G$. decurrent, distant, veined, yellow to deep yellow, reddish when bruised.

Woods, on the ground, on earth inside hollow trees, oak. Aug.-Nov. $4 \times 1 \frac{1}{2} \times \frac{5}{8}$ in. With the general appearance of a dark pileated 1472.

1181. P. involutus Fr. (from the involute margin of the pileus) a $b c$. $P$. convexo-plane, then depressed, ochreous-ferruginous; marg. very involute. St. solid, slightly enlarged downwards, dull 
yellowish, dull purplish below. $G$. decurrent, branched, sometimes forming elongate Po. near the St., pallid, pale or umber-yellow to ferruginous, changing to vinous when bruised.

Said to be edible and highly esteemed in Belgium and Russia ; tasteless when raw. Eaten at Hereford Fungus Forays, but not much approved, iS7o. Woods, by and on stumps; very common. June-Nov, $7 \times 2 \frac{3}{4} \times 1 \frac{1}{6}$ in. A yellow parasitic mould, Hypomyces chrysospermus, frequently grows on the gills. Var. excentricus Mass. St. excentric. Var. subinvolutus W. G. Sm. (Agraricus subinvolutus Batsch) margin of $P$. less involute than type.

1181a. P. porosus Berk. (from the porous hymenium) $a b$.

$P$. Heshy, viscid when moist, reddish-claret or olive-brown ; marg. thin, even, not involute. St. excentric, tough, equal or attenuate downwards, claret-brown, darker below, somewhat reticulate above with the Po. $G$. decurrent, shallow, poriform, yellow to sulphur-green, changing to pale blue and then brownish when bruised, dull green when old. $P_{0}$. round to elongate, irregular, large to small. Flesh dull pale vinousbrown, mottled and streaked, darker in stem, changing colour as St.

Tasteless. Odour very strong, unpleasant. Moist woods, under firs. Autumn. $4 \frac{3}{4} \times 3 \frac{7}{8} \times \frac{3}{4}$ in. Not unlike 1181 , to which it is closely allied, but the margin is never involute and the pores are different in colour. Small forms somewhat resemble 1468 .

1182. P. leptopus Fr. (from the thin stem; Gr. liftos, thin, pous, a foot) $a b$.

$P$. depressed, excentric, downy, then torn-villous-scaly, fuscousyellowish to yellow-olive-umber; marg. subinvolute. St. solid, attenuate downwards, yellowish. $G$. deeply decurrent, sometimes poriform at base, yellowish to full yellow or shaded olive. Flesh straw-white to yellow.

On the ground, about stumps, fir. Aug. - Sept. $3 \frac{1}{2} \times \frac{3}{4} \times \frac{3}{8}$ in.

1183. P. atrotomentosus Fr. (from the black downy pileus; atcr, black, tomentum, down) a b $c$.

$P$. convexo-plane, then depressed, deep opaque umber or deep orange-umber, somewhat orange-sienna at involute marg. St. solid, equal, somewhat rooting, excentric, rarely lateral, blackish-umber or intense crimson-umber, pale yellowish at apex. $G$. subdecurrent, branching and somewhat porous at base, yellowish to deep yellow. Flesh white or sulphur-white in P., rose-purple in St., mottled dull purplish.

Taste insipid ; odour when cut rank and strong like 2086. Stumps, pine, on the ground. Aug.-Nov. $5 \frac{3}{8} \times 2 \frac{1}{1} \times 1 \frac{3}{8}$ in.

1184. P. crassus Fr. (from the stem, thick above; crassus, thick) $a$. $P$. plane, even, ferruginous; marg. subinvolute. St. stuffed, attenuate downwards, excentric, colour as P. $G$. subdecurrent, subdistant, cinnamon.

On the ground, woods. Nov. $3 \frac{1}{8} \times 1 \times \frac{1}{4}$ in. 
1185. P. panuoides Fr. (from its shape, as in genus Pamus; Gr. cidos, appearance) a bc. Dull, opaque, yellow.

$P$. conchato-dimidiate, imbricate; marg. subinwolute. St. obsolete. $G$. decurrent to base, crowded, branched and anastomosing, trama well developed, tan-sienna or somewhat pale yellow-ochre, sometimes studded with drops.

Beech, fir, hawthorn, sawdust, in cellars, on wood; uncommon. July-Nor. Diam. 4 in. $P$. sometimes whitish-ochre wilh reeldish marg. $G$. sienna. The resupinate and cup-shaped form is Gomfthus fezizoides l'ers.

1186. P. Fagi B. \& Br. (from its habitat, stumps of beech, fagrus) a. $P$. dimidiate, crisped, becoming revolute, pallid. St. obsolete. $G$. decurrent to base, crisped, orange.

Gregarious. Autumn. Diam. $3 \frac{1}{1}$ in.

\section{HYGROPHORUS Fr.}

(From the water-bearing character ; Gr. hugros, moist, phero, to bear.)

fill universal and viscid or obsolete. Pilins more or less fleshy, glutinous, viscid or watery; margin slightly incurved. Stem central, continuous and homogenous with the hymenophore, usually examnulate. Gills furnished with a subfloccose trama, usually distant and

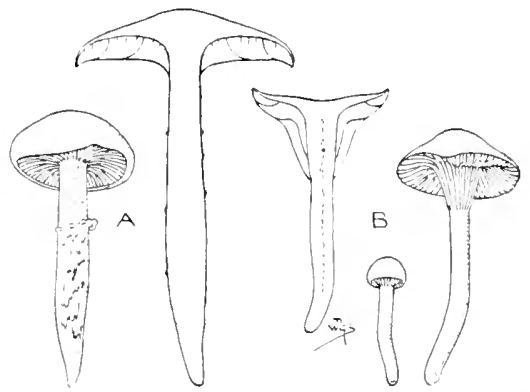

Fig. 6r.-A, Hygrophorus dieacioalizs Fr., entire and in section; $\mathrm{B}, H$. zirgineus Fr., entire and in section. Une-third natural size.

thick, but acute at the edge, not membranous, often branched and veined, the hymenium waxy and at length removable from the trama, which is of the same substance as the pileus. Spores subglobose or elliptical, smooth, white, pale umber in 1208. (Fig. 6r.)

The species are putrescent, often brilliant in colour as scarlet, orange, sulphur, green and shining-white; they all, except 1215, grow on the ground, and often appear after the first frosts of late autumn. They differ from Cortinurius, Gomphidius and Paxillus in the colour of the spores, from Lactarias in being non-milky, from Russula in not being resciculoso-rigid, and from Cantharellus in the sharp-edged gills. Many have a pleasant taste and are edible. 
Typical species of the genus are best seen in Subgenus 3, Higrecybe.

Species $1187-1228$

Subgenus r. Limacium. Pilens glutinous. Unizersal z'cil viscid, with occasionally a floccose partial veil which is annular or marginal. Stem clothed with minute scales or rough with dots above. Gills adnato-decurrent.

White or yellowish-white.

Reddish.

Tawny or light yellow.

$1187-1190$

Olivaceous umber.

$1191-1194$

Fuscous-cinereous or livid.

$1195-1198$

$1199-1202$

$1203-1206$

Subgends 2. Camarophylus. Vill none. Pileus firm, opaque, moist in rainy weather, not viscid, except 1216, 1219, 1220 and 1224, where the pileus is somewhat viscid. Stcm even, smooth or fibrillose, not rough with dots. Gills distant, arcuate.

Gills deeply and at length obconically decurrent. $\quad$ 1207-1217 Gills ventricose, sinuato-arcuate or plano-adnate.

$1218-1224$

Subgexts 3. Hygrocybe. Vill none. Piliks viscid when moist, shining when dry, rarely floccoso-scaly. Stem hollow, except 1225,1228 , stuffed in 1232, soft, not punctate. Gills soft. Whole fungus thin and watery-succulent, fragile, mostly bright coloured and lustrous.

Gills decurrent.

$1225-1234$

Gills adnexed, adnate in 1241 and 1243 , broadly emarginate in

1244, somewhat separating.

$1235-1246$

\section{Suggenus i. Limacium.}

(From the pileus and stem glutinous with the veil ; limax, a slug.)

1187. H. chrysodon Fr. (from the golden, tooth-like scales: Gr. chrusos, gold, odous, a tooth) a b c. White or sulphur-white.

$P$. convexo-plane or flat, yellow squamulose at mid. and marg. St. stuffed, equal, colour as 1', minutely yellow squamulose above, sometimes imperfectly floccoso-annulate. $G$. decurrent, distant, white with a faintly yellowish edge.

Woods, lime, beech, under trees; rare. Aug.-Nov. $2 \frac{3}{4} \times 3_{\frac{1}{2}}^{1} \times \frac{1}{2}$ in. Var. leucodon Stev., with shining-white pubescence.

1188. H. eburneus Fr. (from its ivory-white colour; cbur, ivory) a b $c$. $P$. convexo-plane, flat, repand; marg. pubescent, soon naked. St. stuffed to hollow, equal or nearly so. $G$. adnato-decurrent, distant.

Said to be edible. Odour mild, sweetish, not unpleasant or none. Woods, grassy roadsicles, pastures; frequent. Aug.-Oct. $2 \frac{3}{4} \times 3 \frac{1}{2} \times \frac{1}{2}$ in. Not unlike 115. 
1189. H. Cossus Fr. (from its odour of the goat-moth, Cossus lignipirdr) a $b$ c. Faintly yellowish-white.

$P$. convexo-plane, obtuse; mid. slightly ochreous; marg. naked. St. stuffed, slightly attenuate downwards. $G$. adnato-decurrent, distant.

Odour powerful, persistent. Woods, oak; frequent. Sept.-Nov. $2 \frac{3}{4} \times 3^{\frac{3}{4}} \times \frac{3}{4}$ in.

1189a. H. melizeus Fr. (a fanciful name to express its beauty, like the melody of a song; Gr. melizo, to sing) a. Straw colour.

$P$. fleshy, convexo-expanded, depressed, even, viscid; marg. thin, at first involute. St. stuffed, attenuate downwards, white punctato-squamulose above. $G$. decurrent, thin, distant.

Odour pleasant. Woods. Nov. $3 \frac{1}{1} \times 4 \frac{1}{2} \times \frac{3}{8}$ in.

1189b. H. discoxanthus Rea (from the yellow middle of the pileus; Gr. diskos, a disc, xanthos, yellow) a.

$P$. convex, then expanded and depressed, viscid, white then yellowish, deeper coloured at mid.; marg. becoming brownish with age. St. solid, attenuate downwards, often curved, viscid, white, soon becoming reddish-brown. $G$. somewhat distant, decurrent, veined at base, exceeding marg. of P., white, then yellowish to reddish-brown. Flesh white, becoming reddish in St.

Odour pleasant of anise. Amongst grass. Oct. $2 \times 1 \frac{3}{8} \times \frac{1}{4}$ in.

1190. H. penarius Fr. (from its edible qualities; penus, provisions) a b. Tan-whitish.

$P$. convexo-expanded, umbonate obtuse, then flat, sometimes clouded fawn-vinous; mid. palest sienna or ochre. St. solid, attenuate downwards, fusiform. $G$. adnato-decurrent, distant, thick, veined.

Taste sweet; odour pleasant. Woods, mixed. Oct. $3 \frac{3}{4} \times 3 \frac{11}{4} \times \frac{3}{x}$ in.

1191. H. pulverulentus B. \& Br. (from the powdered stem; puliris, dust) $a b$.

$P$. pulvinate, white; marg. tomentose, sometimes rose- or pale rufous-mealy. St. stuffed, attenuate downwards, fusiform, wholly powdered with rose, rose-sienna or pale rufous meal. $G$. decurrent, thick, whitish, edge obtuse.

Amongst pine-leaves. Nov.-Dec. $\frac{3}{4} \times \mathrm{I} \frac{1}{8} \times \frac{1}{5}$ in.

1192. H. erubeseens Fr. (from becoming red, crubesco) $a b c$.

$P$. gibbous, convexo-flat, adpressedly dotted-squamulose, white or pale buff, becoming red, rose-salmon or vinous-crimsonbrown; mid. sienna. St. solid, equal or attenuate downwards, red-fibrillose, red or pale dull brownish-purple, pale above. $G$. decurrent, distant, narrow, white, red-spotted or rose, or sienna-shaded. Flesh white or pale rose.

In troops, sometimes in large circles. Woods, pine. Oct. $4 \times 3 \frac{1}{3} \times \frac{3}{4}$ in. Perhaps allied to 88 . 
1193. H. pudorinus Fr. (from its flesh-colour ; pudorinus, blushing) $a b$. $P$. convex, flat, salmon-rose; mid. darker. St. solid, attenuate upwards or subventricose, white or salmon-rose above, paler or faintly ochreous below. $G$. decurrent, thick, distant, white or pale flesh-colour, edge sometimes salmon.

Odour none. Woods, fir. Oct. $4 \times 3 \times \frac{5}{8}$ in.

1194. H. glutinifer Fr. (from its gluten-bearing habit ; fero, to carry) $a b c$.

$P$. convex, obtuse, rufescent, mid. wrinkled-dotted, buff, deep buff or umber; marg. whitish. St. stuffed, ventricose downwards, white above, gradually sienna-umber below. $G$. decurrent, white or faintest olive-white. Flesh white.

Gregarious. Taste mild. Woods, grassy places. Sept.-Oct. $4 \frac{3}{4} \times 4 \times \frac{5}{8}$ in. Must not be confused with 1201 .

1195. H. arbustivus Fr. (from its habitat, plantations, arbustum) $a b$. $P$. convexo-plane, subrepand, innato-fibrillose, smooth, buff-tan, fawn-tan or sienna; marg. paler. St. solid to stuffed, equal, white above, $\tan$ at base. $G$. adnate, subdecurrent, distant, white.

Edible. Taste and odour pleasant. IVoods, birch, fir. Sept.-Dec. $2 \frac{1}{4} \times 2 \frac{1}{8} \times \frac{1}{4}$ in.

1196. H. aureus Arrh. ex Fr. (from the golden-yellow pileus; aureus, golden) $a$.

$P$. convex, flat, even. St. stuffed, equal, pale sulphur above, yellow below, sometimes annulate with the glutinous reddish $\mathrm{V}$. $G$. decurrent, distant, thin, whitish to deep sulphur.

Woods. Nov. $I_{2}^{\frac{1}{2}} \times 2 \times \frac{1}{4} \mathrm{in}$.

1197. H. discoideus Fr. (from the disc-shaped pileus) $a b c$.

$P$. convex, obtuse, flat, even, smooth, ochreous-white; mid. pale reddish-brown, sometimes dark. St. stuffed, slightly attenuate upwards, whitish, faintly ochreous or brownish below. $G$. decurrent, becoming distant, faint yellowish-tan.

Gregarious, forming rings, solitary. Woods, pine, grassy places. Oct.-Nov. $2 \frac{1}{2} \times 2 \frac{3}{4} \times \frac{1}{4}$ in.

1198. H. aromaticus Berk. (from its odour of cinnamon) $a b$.

$P$. convexo-plane, flat, cuticle netted-reticulate and separable, cinnamon. St. stuffed to hollow, rosy-cinnamon. $G$. roundedadnate to decurrent, separating from hymenophore, salmon.

Taste acrid; odour spicy. Autumn. $3 \frac{3}{3} \times 2 \frac{5}{8} \times \frac{3}{8}$ in.

1199. H. limacinus Fr. (from its slime; limax, a slug) $a b$.

$P$. convexo-plane, obtuse, umber, fuliginous or olive; marg. paler. St. solid, equal, fibrilloso-striate, white or sulphur-greenwhite, somewhat sienna below. $G$. adnato-decurrent, subdistant, thin, white or pale olive-cinereous.

Sometimes crespitose. Woods, amongst damp leaves. Oct.-Nov. $3 \frac{3}{4} \times 3 \times \frac{1}{2}$ in. 
1200. H. olivaceoalbus Fr. (from the olivaceous pileus and white gills) a b $c$.

$P$. conical, convex, subumbonate, even, smooth. St. solid, attenuate below, white above, spotted with milky drops, spotted squamulose below with fuscous V., and more or less annulate. $G$. adnato-decurrent, distant, veined, white or sulphur-greenish-white.

Taste insipid; odour none. Woods, woodland pastures, chiefly under pine, oak. Aug.-Nov. $3 \frac{1}{4} \times 4 \frac{1}{2} \times \frac{1}{2}$ in.

1201. H. hypothejus Fr. (from the sulphur-colour under the gluten; Gr. hupo, under, theion, sulphur) a $b c$.

$P$. convex, flat, depressed, gluten leaf-green or olivaceous, umber or deep madder-brown. St. stuffed to hollow, equal or slightly attenuate downwards, at first yellow-greenish, then yellow, sometimes flesh-colour, fugaciously subannulate. $G$. decurrent, distant, white to yellow, sometimes flesh-colour. Flesh pale sulphur-yellow.

Woods, pine, larch, amongst heather, etc. ; frequent. Sept.-Dec. $3 \times 3 \times \frac{1}{2}$ in. Must not be confused with 1194 .

1202. H. ceracinus Berk. (from its odour, like cherry-laurel; Prumus Lauroceresus) a b c.

$P$. convexo-expanded, obtuse or subumbonate, umber to grey; marg. minutely tomentose. St. solid, equal or attenuate downwards, white. G. adnato-decurrent, very distant, branched, white.

Somewhat gregarious. Woods, fir. Sept.-Oct. $2 \frac{5}{9} \times 2 \frac{3}{4} \times \frac{3}{8}$ in.

1203. H. fuscoalbus Fr. (from the brown pileus and white gills) $a b$. $P$. convexo-plane, obtuse, becoming cinereous; marg. whitefloccose. St. solid, equal, white above, tan below, sometimes floccoso-annulate. $G$. decurrent, somewhat thick.

Woods, mixed, amongst moss. Sept. I $\frac{3}{8} \times 2 \frac{1}{4} \times \frac{1}{4}$ in.

1204. H. agathosmus Fr. (from the pleasant odour; Gr. agathos, good, osme, scent) a b $c$.

$P$. convexo-plane, gibbous, livid grey or dull pale yellowish-olive; mid. usually darker. St. solid to hollow, equal, somewhat fibrilloso-striate, white above, as P. below. $G$. decurrent, distant, white.

Odour sweet of anise, or of fruit with spirit. Woods, fir. Sept,-Nov. $3 \frac{1}{4} \times 2 \frac{1}{8} \times \frac{3}{8}$ in.

1205. H. mesotephrus B. \& Br. (from the brown-grey middle of the pileus; Gr. mesos, middle, tephros, ash-coloured) $a b c$.

$P$. hemispherical to flat, white; mid. pale sienna or buff; marg. striate. St. stuffed, attenuate downwards, white, faint siemna or buff below. $G$. decurrent, subdistant, white.

Woods, damp places; rare. Oct. $I_{\frac{3}{4}}^{3} \times 2 \frac{1}{8} \times \frac{1}{4}$ in. 
1206. H. lividoalbus Fr. (from the livid pileus and white gills) $a b c$.

$P$. flat, buff-white or white, with white-umber marg. St. stuffed, equal, somewhat even, glabrous, white. $G$. decurrent, subdistant, white.

Woods. Oct.-Nor, $2 \frac{3}{+} \times 3 \frac{3}{3} \times \frac{1}{4}$ in.

\section{Subgenus 2. Camarophyllus.}

(From the arcuate gills; (ir. kamera, a vault, fhullon, a leaf.)

1207. H. caprinus Fr. (from the fibrillose stem, as if covered with goat's hair ; caper, a goat) a $c$.

$P$. conical, umbonate, convex, then flat or depressed, streakedfibrillose, blackish, cinereous-fuliginous or umber. St. solid, or hollow above, equal, pale umber- or grey-white. $G$. very distant, white, then glaucous.

Woods, pastures, under firs. Oct. $3 \frac{1}{4} \times 4 \times \frac{1}{2}$ in.

1208. H. leporinus Fr. (from the colour, like a hare, lepus) $a c$.

$P$. convex, gibbous, fibrilloso-floccose, ochre-sienna or umber. St. solid, rigid, subfusiform, fibrillose, pallid above, brownishtawny below. $G$. pale yellowish. Flesh brownish above and below.

Taste and odour none. Woods, downs; rare. Sept.-Oct. $2 \frac{7}{8} \times 2 \frac{1}{2} \times \frac{5}{8}$ in.

1209. H. nemoreus Fr. (from the habitat; nemus, a grove) $a b$.

$P$. convexo-expanded, gibbous, then depressed, orange, or yellowish-white-sienna. St. stuffed, squamulose, fibroso-striate, slightly attenuate downwards, pale biscuit-colour. $G$. thick, whitish to orange.

Edible. Taste mild. Woods, pastures. Oct.-Dec. $2 \frac{5}{8} \times 1 \frac{7}{8} \times \frac{1}{2}$ in.

1210. H. pratensis Fr. (from the habitat; pratum, a meadow) $a b c$. Pale buff or yellowish.

$P$. convex, obtuse or umbonate, then repand, even, smooth. St. stuffed, attenuate downwards. $G$. very distant, thick, veined.

Edible, but with little flavour. Pastures, roadsides, downs; common. Aug. -Nov. $3^{\frac{3}{7}} \times 2 \frac{3}{4} \times \frac{5}{8}$ in. Sometimes wholly white. Var. umbrinus W. G. Sm. I', olive-slate or whitish-umber. Var. cincereus Fr. P. thin, cinereous, margin striate. Var, fallidus Cooke. P. infundibuliform, pallid. Var. Meisncriensis Fr. P. skin easily separable. Tasteless or slightly insipid.

1210a. H. Karstenii Sacc. \& Cub. (after P. A. Karsten) $a_{0}$

$P$. fleshy, convexo-plane, compact at mid., glabrous, even, whitish. St. solid, attenuate downwards, wavy, white. G. deeply decurrent, distant, yellow.

Woods. Nov. $3^{\frac{1}{2}} \times 5^{3} \times \frac{3}{4}$ in. Distinguished from 1210 by its chalk. white downward tapering stem.

1211. H. fœtens Phill. (from its nauseous-fetid odour) $a b c$.

$P$. convex, plane, smooth, then squamulose, sepia. St. stuffed, attenuate downwards, paler than P. G. somewhat thick, vinous-brown-tan, somewhat slate, glaucous-pruinose.

Odour cadaverous, ordure-like, fetid-nauseous. Grassy places. Oct.-Nor. I $\times \mathbf{I} \frac{1}{4} \times \frac{1}{8}$ in. 
1212. H. virgineus Fr. (from the pure white colour) a $b c$.

$P$. convex, obtuse, Hat, depressed, at length cracked and Hoccose. St. stuffed, attenuate downwards. $G$. very decurrent, somewhat thick.

Edible. Pastures, woods, roadsides, downs. Aug.-Dec. $I_{4}^{3} \times \mathrm{I}_{4}^{3} \times \frac{1}{4}$ in. Very variable in size. Sometimes clouded with beautiful shades of clear lilac, caused by the growtl of the mould named Terticillium Marquandii. Must not be confounded with the white form of 1210 or with 1214. Var. roseipes Mass. ex Cooke. St. rose-colour or salmon within and without, sometimes strigose at base.

1213. H. ventricosus B. $\&$ Br. (from the ventricose stem) $a b$. White.

$P$. convex, unequal, lobed. St. solid or partially hollow. $G$. narrow, forked.

Amongst grass. Sept. $1 \frac{1}{4} \times 2 \frac{1}{2} \times \frac{1}{3}$ in. Often tinged with 1 ed from the growth of a parasitic Fusisporimn. which may be the cause of the hypertrophy. Greatly resembles one form of 123.

1214. H. niveus Fr. (from its snow-white colour; nizeus, snowwhite) $a b c$.

$P$. convex, flat, umbilicate; marg. striate. St. fistulose, equal or slightly attenuate downwards. $G$. thin.

Pastures, roadsides, parks, downs, etc. ; common. Sept.-Nov. $\frac{7}{8} \times 2 \times \frac{1}{16}$ in. Much like 207 in appearance. Sometimes minute. Sometimes stained lilac, as in 1212 .

1215. H. Wynniæ B. \& Br. (after Mrs. Lloyd Wynne) a b c. Semitransparent lemon, faintly greenish, sometimes tan or white.

$P$. conves, umbilicate, or subinfundibuliform; marg. striate. St. stuffed, equal, or attenuate downwards. $G$. narrow, thin.

On chips, twigs, old stumps, etc. Oct. $1 \times 1 \frac{3}{8} \times \frac{1}{5}$ in. The habitat points to Mycena or Omfratia.

1216. H. pussocoriaceus Berk. \& Mill. (from the odour of Russian leather; coriucius, leathery) a $b c$. White.

$P$. convex, expanded, obtuse, slightly viscid. St. solid, attenuate downwards, smooth. $G$. thick, distant, few.

Odour varying of Potentilla atrosanguinea. Pastures; rare. Sept.-Dec. $\mathrm{I} \times \mathrm{I} \frac{1}{5} \times \frac{3}{16} \mathrm{in}$. Odour sometimes persistent in dried examples for more than twenty years. Must not be confounded with 207.

1217. H. micaceus B. \& Br. (from the shining mica-like particles on the pileus) a $c$.

$P$. hemispherical, Hat, slightly depressed, wrinkled, light yellow to cinereous. St. solid, equal, colour as P., then brown below. $G$. pallid umber to clay-sienna.

Clayey soil. Oct. $\frac{5}{5} \times \frac{5}{5} \times \frac{1}{16}$ in. Whole plant turns brown in drying.

1218. H. fornicatus $\mathrm{Fr}$. (from the pileus, at first convex; formiatus, arched) a $b c$.

$P$. becoming expanded and obsoletely broadly umbonate, viscid, white or pale livid; mid. brown. St. solid, hollow or stuffed. 
equal, smooth or subscaly, whitish to greyish. $G$. adnexed or sinuato-adnexed, ventricose, distant, white.

Pastures. Oct.-Nov. $3 \times 2 \frac{5}{8} \times \frac{3}{8}$ in. Var. clizalis Fr., white. $P$. thin, fragile ; marg. incurved, striate, not viscid. St. short, fragile, attenuate downwards. $G$. rather thick.

1219. H. distans Berk. (from the very distant gills) a b.

$P$. convex, plane, subdepressed or umbilicate, viscid, white or faintly brownish. St. solid, attenuate downwards, white, cinereous or pale ochre-tan below. $G$. plano-decurrent, few.

Sometimes fragrant of essence of almonds, pleasant, sweet. Woods; rare. Oct. $2 \times \mathbf{J} \frac{1}{2} \times \frac{1}{t}$ in.

1220. H. Clarkii B. \& Br. (after I. Aubrey Clark) a $b$.

$P$. obtuse, convexo-plane, viscid, opaque umber or lividcinereous; marg. even, white. St. solid, stuffed or hollow, equal or attenuate downwards, viscid and white-squamulose above, pale umber-scaly below. $G$, adnate, distant, broad, thick, veined, ivory-white.

Woods, pastures. Sept.-Oct. $3 \frac{3}{4} \times 2 \frac{5}{8} \times 1 \frac{1}{8}$ in. H. latitabundus Britz. is this species, as is Kalchbr. Hy'men. Hung. t. 24, f. 1 , incorrectly named H. limacinus $\mathrm{Fr}$.

1221. H. metapodius Fr. (from the stem, sometimes thick above and attenuate below; Gr. meta, upside down, pous, a foot) a $b c$.

$P$. convex, plane, obtuse, cinereous-fuscous or pale rose-shaded sepia. St. stuffed, unequal, smooth, cinereous. G. adnate, broadly emarginate, distant, veined, grey-white. Flesh pallidgrey, reddish when broken.

Sometimes in clusters and deformed. Taste mild, insipid to sweet; odour of new meal. Old mossy pastures. Oct.-Jan. $2 \frac{1}{4} \times 1 \frac{5}{8} \times \frac{5}{8}$ in. At maturity becoming black or deep sepia within and without. Analogous with 1306 and 1307.

1222. H. ovinus Fr. (from the woolly pileus; oris, a sheep) a $b c$.

$P$. campanulate, expanded, subumbonate, revolute, incised, subviscid, then obscurely squamulose, dull-sienna or yellow; mid. sienna-slate; marg. tan. St. somewhat stuffed, almost equal, smooth, tan-grey. G. emarginate or arcuato-adnate, veined, whitish-grey then rufescent.

Odour of new meal. Woods, pastures, mossy places; uncommon. Sept.Dec. $2 \frac{1}{4} \times 2 \frac{3}{8} \times \frac{1}{2} \mathrm{in}$. Sometimes changing to black.

1223. H. subradiatus Fr. (from the radiato-striate pileus) $a b$.

$P$. convex, expanded, subumbonate, pale yellowish; mid. sienna and umber; marg. sienna, sometimes lilac. St. stuffed to hollow, equal, smooth, often twisted, whitish or pale lilac above, tan-brownish below. $G$. decurrent, somewhat thin, distant, veined, white to pale yellowish.

Old pastures, heathy ground. Sept.-Oct. $3 \frac{1}{2} \times 2 \times \frac{1}{2}$ in. Var. lacmus Fr. wholly or almost wholly shining-lavender.

1224. H. irrigatus Fr. (from its watery texture; irrigo, to wet) a. $P$. convex, obtuse, flat, smooth, viscid, pale fawn; mid. brown; 
marg. striate. St. hollow, equal, smooth, very viscid, colour as P., but with a pale yellowish tinge. $G$. adnate with a tooth, subdistant, somewhat thick, veined, white, grey, or tan.

Woods, fir, grassy places. Oct.-Nov. $2 \times 2 \frac{3}{8} \times \frac{1}{4} \mathrm{in}$.

\section{Subgenus 3. Hygrocybe.}

(From the moist pileus; Gr. hugros, moist, kube, a head.)

1225. H. Colemannianus Blox. ex Berk. (after W. H. Coleman) $a b c$. $P$. convexo-expanded, umbonate, dark reddish-umber; marg. striate, paler. St. solid, nearly equal, somewhat silky, salmonwhitish, shaded pale brownish. $G$. distant, veined, paler than P. to whitish.

Grassy places, lawns. Oct.-Nov. $\mathbf{r}_{\frac{1}{8}} \times \mathbf{r}_{\frac{3}{4}}^{\frac{3}{4}} \times \frac{1}{4}$ in.

1226. H. seiophanus Fr. (from its delicacy as compared with 1210 ; Gr. skia, a shadow, phaino, to appear) $a$.

$P$. hemispherical, expanded, obtuse, deep tawny, brown, red or vermilion to white; marg. striate, paler. St. equal, smooth, tawny-yellowish to white. $G$. adnate to plano-decurrent, ventricose, distant, colour as P., or inclining to rose or yellow.

Densely gregarious. Grassy and mossy places. Autumn. $\mathbf{I}_{\frac{1}{4}}^{\frac{1}{4}} \times 1 \frac{7}{8} \times \frac{1}{8} \mathrm{in}$.

1227. H. lætus Fr. (from the bright colour ; letus, pleasing) $a b c$.

$P$. convex, flat, slightly depressed, slippery-smooth, deep yellow, tawny, vermilion-orange or salmon; marg. striate. St. fistulose, equal, slippery-smooth, tawny or yellow. $G$. plano-decurrent, distant, thin, veined, flesh-colour, whitish, fuliginous or shaded brownish. Flesh in P., yellow and orange, in St., tan-yellow.

In troops. Pastures, downs; frequent. Oct.-Nov, $1 \frac{1}{4} \times 2 \frac{1}{4} \times \frac{1}{8}$ in.

1228. H. Houghtonii B. \& Br. (after the Rev. Wm. Houghton) $a b c$.

$P$. convex, flat, umbilicate, glutinous, rugose to mid., flesh-colour with sienna marg., pale vermilion shaded darker or wholly cinnabar, sometimes very pale. St. fistulose, equal, very glutinous, pale salmon above, pale or dark yellow below, sometimes wholly cinnamon. $G$. plano-decurrent, thick, pale yellowish or salmon. Flesh in $\mathrm{P}$. Hlesh-colour or orange, in St. salmon above, tan below.

Tasteless; odour strong, foxy. Amongst grass. Oct. $1 \frac{3}{8} \times 2 \frac{7}{8} \times \frac{1}{8}$ in.

1229. H. vitellinus Fr. (from the egg-yellow colour, within and without ; vitellus, yolk of egg) a b.

$P$. convexo-expanded, smooth, varying lemon-yellow with a suggestion of olive, becoming white; marg. plicato-striate. St. equal, even, smooth, becoming white. $G$. decurrent, subdistant.

Woods, pastures. Oct.-Dec. $1 \times 2 \frac{1}{8} \times \frac{1}{8}$ in.

1230. H. ceraceus Fr. (from the colour, like yellow wax; ccra, wax) $a b c$.

$P$. convexo-plane; marg. striate, not becoming white; mid. sometimes vermilion. St. equal to unequal, even, smooth. $G$. adnato-decurrent.

In troops. Woods, pastures; common. Sept.-Dec. $1 \frac{3}{9} \times 3 \frac{1}{1} \times \frac{1}{8}$ in. 
1231. H. coceineus Fr. (from its scarlet colour, coccinens) $a b c$.

$P$. hemispherical, convex, plane, smooth, not floccoso-scaly, crimson-scarlet or yellow to orange, clouded and blotted carmine, becoming whitish or yellowish. St. even, not slipperysmooth, unequal, scarlet above, yellow below, sometimes rose or purplish, becoming whitish or yellowish. $G$. adnate, sometimes with a decurrent tooth, distant, veined, yellow below, purplish above, edge glaucous. Flesh crimson, orange or sulphur, external layer of St. orange-vermilion.

Pastures ; very common. June-Nov, $2 \frac{3}{4} \times 2 \frac{5}{8} \times \frac{1}{2}$ in.

1232. H. miniatus Fr. (from its colour; minium, vermilion) a $b c$.

$P$. convex, umbilicate, even, smooth then squamulose, becoming rose or orange. St. stuffed, slightly attenuate downwards, smooth, shining. $G$. adnate or slightly decurrent, distant, rather thick, yellow or vermilion-shaded. Flesh of P. and external layer of St. scarlet.

Open and boggv places, woods, moist banks, pastures. June-Oct. I $\times 2 \frac{1}{1} \times \frac{1}{8}$ in. Sometimes very small. Does not adhere to paper in drying.

1233. H. turundus Fr. (from the flocculose pileus; turmada, lint) a b c c.

$P$. convex, plane, depressed or umbilicate, yellow to orange; mid. vermilion; sq. cinereous-fuscous ; marg. sometimes crenate. St. equal, even, lustrous, tawny-reddish or orange above, sulphur below, base white. $G$. slightly to deeply decurrent, white to orange.

liogs amongst Hydrocotyle z'ulguris, peaty soil, grassy places. July-Oct. $1 \frac{5}{8} \times 2 \frac{1}{2} \times \frac{3}{16}$ in. Adheres to paper in drying. Var. mollis B. $\mathcal{S}$ Br. $P$. golden-yellow-squamulose.

1234. H. mucronellus Fr. (from the shape of the pileus, acutely conical when young; mucro, a sharp point) $a$.

$P$. becoming campanulate and repand, umbonate, scarlet, bright red, orange or yellow, then hoary; marg. striate when moist. St. equal, even, smooth, yellow or scarlet, white below. $G$. subdecurrent, yellow, orange above.

Fields. Dec. $\frac{3}{4} \times \mathrm{I}_{\frac{1}{8}} \times \frac{1}{16} \mathrm{in}$.

1235. H. puniceus Fr. (from the colour; punicus, blood-red) a $b c$.

$P$. convex, obtuse, expanded, subumbonate, scarlet-crimson, sometimes clouded orange, sometimes orange-brown. St. equal or ventricose, striate; colour as P., white, yellowish or sulphur at base. $G$. ventricose, thick, distant, white-yellow, often reddish above. Flesh deep orange and sulphur in P., sulphur in St.

Pastures, downs, mossy places, sometimes woods; frequent. June-Nov. $4 \frac{1}{2} \times 5 \times \frac{7}{5}$ in.

1236. H. obrusseus Fr. (from the golden colours; olrussa, the assaying of gold by fire) a bc.

$P$. campanulate, expanded, obtuse or umbonate, golden sulphuryellow or golden to orange; mid. somewhat vermilion. St. 
compressed, unequal, hollow, smooth, even, light or dark sulphur, base tawny or sulphur-white. $G$. ventricose, distant, white to sulphur and yellow-orange.

Woods, grassy places. Aug.-Oct. $2 \frac{1}{3} \times 4 \frac{1}{1} \times \frac{3}{8}$ in.

1237. H. intermedius Pass. (from its resemblance to adjoining species) $a$.

$P$. convex, obtuse, flat, fibrilloso-silky, golden-yellow becoming cinereous; mid. orange. St. compressed, fibrilloso-striate, sulphur to orange. $G$. ventricose, distant, whitish then yellowish.

Odour of meal. Roadsides, grassy places, damp ground. Autumn. $2 \frac{3}{8} \times 2 \frac{5}{8} \times \frac{3}{8}$ in. Like 1236, but becoming grey.

1238. H. conicus Fr. (from the conical pileus) $a b c$.

$P$. often acute, then expanded and cracked, smooth, bloodscarlet, orange, sulphur-greenish, sulphur or smoky light yellow. St. equal, fibroso-striate, twisted, sulphur or yellow, reddish at base. $G$. ventricose, thin, subcrowded, white, yellowish or sulphur.

Pastures, downs, roadsides, etc. ; common. July-Nov. $2 \frac{7}{8} \times 3 \frac{1}{2} \times \frac{3}{8}$ in. Usually, but not invariably, becoming wholly jet black at maturily.

1239. H. calyptræformis B. \& Br. (from the pointed hood-shaped pileus ; calyptra, a hood) a $b c$.

$P$. acutely conical, expanded, repand, splitting, minutely innatofibrillose, salmon-rose, purplish-rose or white; mid. ochreous or salmon. St. somewhat ventricose, smooth, substriate, white. $G$. ventricose, rose to pallid, or as P.

Said to be edible. Pastures, lawns, etc.; uncommon. July-Oct. $3 \times 4 \frac{1}{1} \times \frac{3}{8}$ in. Var. nizeens Cooke, wholly white.

1240. H. chlorophanus Fr. (from the pale yellow colour; Gr. chloros, greenish-yellow, phaino, to appear) $a b c$.

$P$. hemispherical, expanded, obtuse, plane, smooth, cracked, viscid, sulphur, yellow, orange or scarlet; mid. sometimes vermilion; marg. striate. St. equal, viscid when moist, rich light yellow. $G$. emarginato-adnexed, ventricose, thin, distant, yellow. Flesh yellowish.

Woods, grassy and mossy places; common. July-Oct. $\quad 1 \frac{7}{8} \times 3 \frac{1}{1} \times \frac{1}{4}$ in.

1241. H. psittacinus Fr. (from the yellow or red and green colours; psittacus, the ringed green parrot) $a b c$.

$P$. campanulate, expanded, umbonate, yellow-reddish or whitish, smeared with green gluten. St. equal, even, colour as P. $G$. adnate or with a decurrent tooth, ventricose, thick, subdistant, yellow, green above.

Pastures, downs, roadsiles, etc. ; common. Aug.-I)ec. $2 \frac{1}{4} \times 2 \frac{3}{4} \times \frac{1}{4}$ in. When young wholly green from the gluten. 
1242. H. spadiceus Fr. (from the brown pileus; spadicens, datebrown) $a$.

$P$. conical, subacute, campanulate, fibrillose, virgate, covered with olive-bay gluten. St. equal, dry, fibrillose, tawny or yellow, white below. $G$. ventricose, distant, lemon or clear yellow.

Mossy and grassy places, on the ground. July. $2 \frac{3}{8} \times 2 \frac{1}{2} \times \frac{3}{8}$ in. Not turning black.

1243. H. unguinosus Fr. (from the glutinous pileus and stem; unguinosus, oily) a b.

$P$. campanulato-convex, obtuse, sepia, umber, fuliginous or yellowish-brown. St. equal or slightly attenuate above and below, colour as P. G. adnate with a tooth, ventricose, white.

Taste and odour none. Woods, pastures, moist places; frequent. Aug.-Oct. I $\frac{7}{8} \times 3 \frac{13}{8} \times \frac{1}{4}$ in.

1244. H. nitratus Fr. (from the nitrous odour) $a b c$.

$P$. convex, obtuse or depressed, at first viscid, then flocculososquamulose and rimosely incised, fuscous-cinereous, blotted, becoming pale, or deep umber with whitish marg. St. equal, smooth, lustrous, grey-whitish. $G$. broadly emarginate, distant, veined, whitish to glaucous.

Odour very strong and disagreeable, like compounds of nitrogen andi oxygen. Woods, pastures; uncommon. Aug.-Nov, $\frac{15}{8} \times 2 \frac{3}{4} \times \frac{1}{4}$ in. Must not be confounded with 111. There is a form larger than type. Var. glauconitens Fr., rigid. $P$. dark-olive or sooty, becoming pale. $G$. becoming glancous. Persoon and others, including myself, regard the variety as a distinct species. Berkeley, I believe, looked upon it as a Tricholoma near 111 .

\section{LACTARIUS Fr.}

(From the milky juice ; lac, milk.)

Hymenophore confluent and homogeneous with the stem. I'eil more or less obsolete, present in the ragged-appendiculate or pubescent margin of the pileus in some species and in the gluten of others. Pileus Heshy, somewhat rigid, the texture floccose or vesciculose, not fibrous, often zoned, margin at first involute, milky. Stem usually central, not corticate, exannulate, milky. Gills adnatodecurrent, adnate in 1256, 1264, 1270, 1290 and 1302, often branched, unequal, membranous-waxy, subrigid, edge acute, trama vesiculose, milky. Spores subglobose, minutely echinulate, white, rarely yellowish. (Fig. 62.)

Every part of the plant contains numerous anastomosing lactiferous cells filled with densely granular latex or milk which is usually white, but in some instances the colour changes on exposure 
to the air; in a few cases the milk is coloured before its escape from the cells; it varies in taste from mild to intensely acrid, and is of a resinous nature.

. The species, except 1305, grow on the ground. The nearest allies

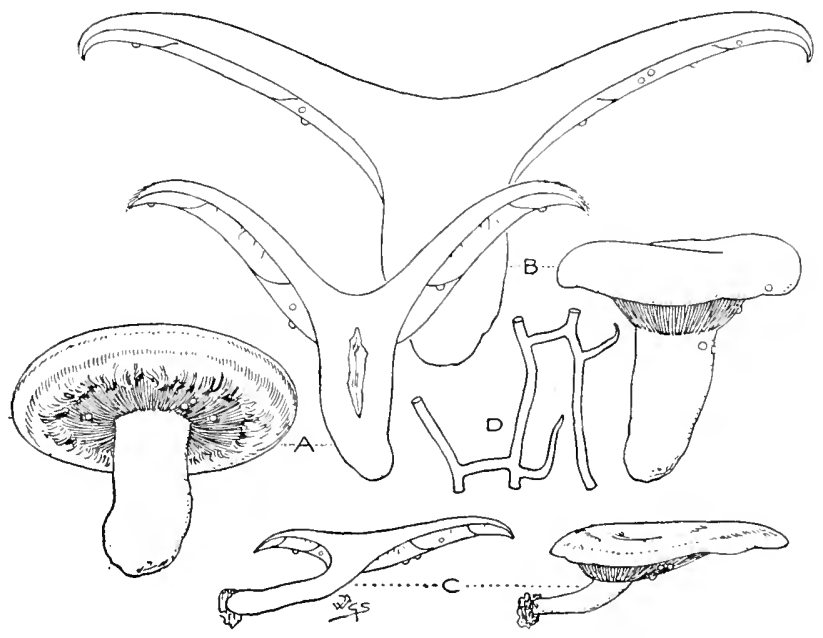

Fig. 62.-A, Lacturins torminosus Fr. ; B, L. piperatus Fr.; C, L. obliqun. Fr. : entire and in section. One-third natural size. $\mathrm{D}$, Lactiferous tubes of $L$. sudulcis $\mathrm{F}$. $\times$ roo.

are found in Russulu, which has no latex. Some are edible, others are poisonous.

The Lactarii of France and Italy are popularly termed "cows" or "goats"; the acrid species are called "pepcrone" and "poire blanc."

The sclerotia and perfect plants of 248 sometimes grow from the gills of Lactarii. Sphceronema vitrenm (Spheropsidece) frequently grows on the Lacturii and Russule. The mould Panicillium macrosporam occurs on Lactarius.

Species 1245-1305

Piperites. Stom central. Gills not changing colour, not pruinose. Nilk white, usually acrid.

a. Tricholomoidece. Pileus viscid when moist; margin at first involute and tomentose.

$1245-1253$

b. Limacince. Pileus viscid when moist, pelliculose, margin usually naked.

$1254-1261$

c. Piperate. Pilcus without a pellicle, dry, usually not shining.

$1262-1274$

Dapetes. Stem central. Gills naked. Milk from the first deeply coloured. 
Russulares. Stem central. Gills pallid, then changing colour to darker, glancing when turned to the light, at length white pruinose. Milk at first white, mild or from mild turning acrid. Pileus at first viscid.

$1277-1283$

Pileus not lustrous, squamulose, villous or pruinose.

Pilens lustrous, smooth.

$1284-1294$

$1295-1304$

Pleuropode. Stcm excentric or lateral. Growing on trunks. 1305

PIPERITES.

\section{a. Tricholomoidece.}

1245. L. scrobiculatus Fr. (from the pitted stem; scrobis, a trench) a b c .

$P$. convex, then infundibuliform, villous-downy; marg. at first appendiculate, yellowish or buff. St. stuffed to hollow, equal, light yellowish or white above, $\tan$ below. $G$. thin, crowded, whitish to yellowish. Milk white, then sulphur-yellowish.

Taste very acrid; odour not unpleasant. Woods, on the ground, damp places. Sept.-Oct. $9 \times 2 \frac{1}{2} \times \mathbf{I} \frac{1}{2}$ in. l'ileus sometimes $\mathbf{I} 2 \mathrm{in}$. in diam.

1246. L. intermedius B. \& Br. (from its position between 1245, 1247 and 1248) $a b$.

$P$. convex, then infundibuliform, smooth, ochreous or brownishyellow, somewhat zoned; marg. at first ragged-tomentose. St. solid to hollow, equal, pitted as in 1245 , yellowish. $G$. whitish. Flesh white then pale sulphur. Milk white, then yellow-white.

Somewhat acrid. Woods. $3 \frac{3}{8} \times 1 \frac{1}{2} \times \frac{3}{4}$ in. Perhaps the same as 1248 .

1247. L. torminosus Fr. (from its painful acridity; tormina, gripes) at b $c$.

$P$. convex, then infundibuliform, becoming even, somewhat zoned, pallid flesh-colour; marg. ragged-fibrillose, white. St. stuffed to hollow, equal or attenuate downwards, even or obsoletely pitted, white, palest rose above, ochre or tan below. $G$. very thin, crowded, paler than P. or tan. Milk white.

Eaten with oil and vinegar in Russia after being preserved in salt. Very acrid; odour none. Mixed woods, on the ground, fields, amongst grass,

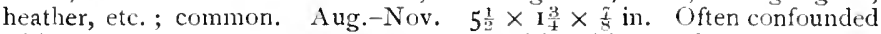
with 1276. Hypomyces torminosus, a parasitic white mould, changing to yellow and dark-brown is frequent on the gills.

1248. L. cilicioides Fr. (from the tomentose pileus; Gr. kilikion, goat's-hair cloth, eidos, appearance) $a b c$.

$P$. convex, then infundibuliform, wholly tomentose, Hesh-colour to yellowish or fuscous; marg. fibrillose-woolly. St. solid to hollow, equal or attenuate downwards, even, pallid. $G$. crowded, branched, white to yellowish. Flesh yellowish-white. Milk white to yellowish-white.

Wouds, pine, on the ground; frequent. Aug.-Nov. $6 \frac{1}{2} \times 2 \frac{1}{2} \times 1 \frac{3}{3}$ in. Intermediate between 1247 and 1250 . 
1249. L. lateritioroseus Karst. (from the colour ; lateritins, brickred, roseus, rosy) a.

$P$. convex, then subinfundibutliform or umbilicate, wavy, usually zoneless, sometimes flesh-colour, becoming pale or lavendersalmon; mid. granuloso-squamulose. St. stuffed to hollow, unequal, slightly flocculose, colour as P. or paler. $G$. subdistant, furcate, veined, colour as St., becoming yellowish. Milk white.

Acrid. Woods. Autumn. $3 \frac{1}{4} \times 2 \times \frac{3}{4}$ in.

1250. L. turpis Fr. (from its ugly appearance; turpis, base) a $b c$.

$P$. convex, then depressed, rigid, usually zoneless, umber or olivaceous. St. solid to hollow, equal or attenuate downwards (sometimes upwards), pallid or dark olivaceous or shaded pale slate. $G$. thin, crowded, furcate, white straw-colour or tan. Flesh white or shaded pale slate. Milk white.

Poisonous. Gregarious. Woods, fir, mixed, roadsides, etc.; common. Sept.-Nov. $\delta \times 2 \frac{1}{2} \times \mathbf{1} \frac{1}{8}$ in. Habit of 1181. Var. plumbeus Cooke. $P$. blackish-brown.

1251. L. controversus Fr. (from the pileus, at first convex then infundibuliform) $a b c$.

$P$. convex, then infundibuliform, rigid, flocculose to smooth, white, spotted and zoned blood-red, varying flesh-colour. St. solid, equal, sometimes excentric, viscid, white or spotted as P. $G$. thin, very crowded, branched, pallid, white-fleshcolour. Mill white.

Taste very acrid and pungent; odour weak, pleasant. Woods, pastures, under poplars, chestnuts; uncommon. Aug. -Nov. $S \times 3 \frac{1}{\frac{1}{2}} \times \mathbf{I} \frac{7}{8}$ in. Said by Dr. Badham to be eaten at Lucca under the name of "Lucchese Goat."

1252. L. pubescens Fr. (from the pubescent margin of the pileus) $a b$. $P$. convex, then broadly infundibuliform, zoneless, even, dry, shining, whitish to flesh-colour, rose, ochreous or faint brown. St. stuffed, attenuate downwards, even, colour as P. G. crowded, pale flesh-colour or yellowish to salmon. Mith white.

Taste very acrid ; odour none or pungent. Pastures, grassy places, amongst pebbles. Sept.-Oct. $4 \times 1 \frac{5}{8} \times \frac{3}{4}$ in.

1253. L. aspideus Fr. (from the shield-like shape of the pileus ; aspis, a shield) a $b$.

$P$. gibbous-convex then depressed, straw-colour, pale yellowish, or faintly lilac-shaded; marg. with a tomentose, deciduous, white zone. St. solid to stuffed, equal or attenuate downwards, whitish. $G$. somewhat thick, whitish, faintly shaded lilac. Flesh colour as G. Milk white then lilac.

Taste sweet. Damp pastures, moist places. Sept. $2 \frac{3}{4} \times I_{4}^{\frac{1}{4}} \times \frac{3}{8}$ in. 


\section{b. Limacince.}

1254. L. insulsus Fr. (from its indifferent resemblance to 1276 ; insulsus, insipid) a $b c$.

$P$. umbilicate then deeply infundibuliform, rigid, smooth, yellow or orange; mid. sienna-yellow, usually zoned, spotty-palesienna. St. solid to hollow, whitish or faint yellowish. $G$. decurrent, very crowded, forked at base, colour as St. Milk white.

Taste acrid. Mixed woods and their borders, pastures; common. Aug.Oct. $3 \frac{5}{8} \times 1 \frac{1}{2} \times \frac{7}{8}$ in. Pileus sometimes 7 in. in diam.

1255. L. zonarius Fr. (from the zoned pileus) $a b c$.

$P$. convexo-plane, depressed, even, smooth, 4 - to 7 -zoned near the minutely ruguloso-flocculose marg., pallid orange or pale yellowish; mid. sometimes umber. St. solid, smooth, dry, white then yellowish. $G$. thin, subcrowded, branched, whitish to dull yellowish, dingy when bruised. Milk white.

Taste very acrid, strong, disagreeable. Woods and their borders, grassy places; rare. Aug.-Oct. $4 \frac{1}{8} \times 1 \frac{3}{8} \times \frac{7}{8}$ in.

1256. L. utilis Fr. (from its edible qualities; utilis, useful) $a$.

$P$. convexo-plane, obtuse, then infundibuliform, even, smooth, tan, pale dull ochre or livid. St. solid to hollow, even, colour as P. G. adnate, crowded, pallid. Milk white.

Highly esteemed as an esculent in Russia. Taste mild to slightly acrid. Woods, on the ground. Autumn. $58 \times 2 \times 1 \frac{1}{4}$ in. Sometimes olive shaded.

1257. L. blennius Fr. (from the slimy pileus; Gr. blennos, mueus) a b c .

$P$. plano-depressed, pallid olivaceous, æruginous-grey or indigosepia, shining with gluten; mid. dark; marg. at first slightly downy. St. stuffed to hollow, subequal or attenuate downwards, even, viscid, pallid above, pallid grey or pale slate. $G$. thin, crowded, white, cinerous when bruised. Milk white.

Poisonous. Taste very acrid. Woods, beech, fields near woods, on the ground, rarely on trunks; common. July-Nov, $3 \frac{5}{8} \times 1 \frac{7}{4} \times \frac{7}{8}$ in.

1258. L. hysginus Fr. (from the colour of the pileus; Gr. Mus rinon, a crimson dye) $a b c$.

$P$. umbilicate, depressed, plane, even, red flesh-colour, reddishbrown or dark brownish sienna-salmon, somewhat zoned pale umber. St. solid to hollow, attenuate downwards, smooth, warm sienna-yellowish, somewhat spotted. $G$. thin, crowded, white to primrose or yellow-ochreous. Milk white.

suspected poisonous. 'Taste acrid. Woods, under beech, amongst grass; rare. Aug.-Nov, $2 \frac{3}{4} \times 2 \times \frac{3}{4}$ in.

1259. L. trivialis Fr. (from its commonness; trizialis, common) a $b c$.

$P$. convex, sometimes umbonate, then depressed and infundibuliform, even, smooth, zoneless, tan-umber-lavender, dull orange- 
buff or dark to pale lurid. St. hollow, even, smooth, slippery, palest tan-grey, white, yellowish or paler than P. G. somewhat thin, crowded, whitish or pale yellowish. Milk white.

Taste very acricl. Woods, chiefly pine, moist mossy places; uncommon. Aug.-Oct. $6 \times 3 \times \frac{3}{4}$ in.

1260. L. circellatus Fr. (from the zones on the pileus; circellus, a small ring) $a b c$.

$P$. convexo-plane then depressed, umbilicate, even, smooth, rufous, fuscous or umber becoming pale, zoned grey or claretbrown. St. solid, equal, or attenuate downwards, even, smooth, pale yellowish, shaded faintly brownish or steel-whitish. $G$. thin, crowded, forked, whitish to pale yellowish. Milk white.

Taste stinging-acrid ; odour not unpleasant. Woods, amongst grass; rare. Sept.-Oct. $4 \times 2 \frac{1}{4} \times \frac{5}{8}$ in. Must not be confonnded with 1262 or 1263 .

1261. L. uvidus Fr. (from the moist, clammy pileus ; uvidus, moist) $a b c$.

$P$. convex, plane, then depressed, even, smooth, usually zoneless, pale lilac-umber on a yellowish ground, blotted lilac. St. hollow, smooth, viscid, whitish or light-yellowish, sometimes spotted yellow-ferruginous or lilac. $G$. thin, crowded, unequal, somewhat veined, whitish or yellowish, lilac when bruised. Milk white, becoming bright lilac.

Taste insipid to acrid; odour weak, nauseous-pungent, somewhat aromatic. Woods, moist places; uncommon. Aug.-Nov. $3 \frac{7}{5} \times 2 \frac{7}{8} \times \frac{1}{2}$ in.

\section{c. Piperate.}

1262. L. flexuosus (from the frequently undulate margin of the pileus; flcxus, a bending) $a b c$.

$P$. hard, plane, depressed, subinfundibuliform, smooth to rivulososcaly, dull, zoned or zoneless, lead-grey, violet-grey, violetbrown or purplish-umber. St. solid, equal or attenuate downwards, whitish or greyish, base yellowish. G. thick, distant, branched, light yellowish to whitish flesh-colour. Milk white.

Said to be edible. Taste when raw very acrid. Woods, pine. July-Oct. $5 \times 3 \frac{3}{8} \times 1 \frac{1}{3}$ in. Var. roseszonatus Fr. P. rosy flesh-colour with darker z.

1263. L. pyrogalus Fr. (from the very acrid milk; Gr. pur, fire, gala, milk) $a b c$.

$P$. convex, plane, depressed, smooth, cinereous-grey, dull yellow or umber-tan, zoned sienna or madder-brown. St. stuffed to hollow, usually attenuate downwards, dull pallid-whitish or paler than P. and G. $G$. thin, subdistant, light wax-yellow, tan-ochre or shaded reddish-salmon or straw-colour. Milk white.

Poisonous. Odour sometimes of apple. Woods, fir, pastures; frequent. Aug. -Nov. $3 \frac{1}{2} \times 1 \frac{3}{8} \times \frac{1}{2}$ in. Sometimes very small.

1264. L. squalidus Fr. (from the dingy colouring; squalidus, dirty) $a b$.

$P$. convex, umbilicate, depressed, smooth, tan-olive-green with darker mid. or yellowish-olive-umber. St. solid, equal, smooth, 
whitish or pale brownish. $G$. adnate, narrow, yellowish or shaded faint olive or tan. Milk white.

Taste mild to sweet. Woods, beech, moist mossy places. Oct. $3 \frac{3}{4} \times 3^{\frac{1}{4}} \times \frac{1}{2}$ in.

1265. L. capsicum Schulz. ex Fr. (from the peppery taste ; capsicum, red-pepper) $a$.

$P$. convex, obtuse, pulvinate, dark chestnut or deep sienna ; mid. paler. St. solid, equal, whitish with tawny or rufescent fibres, paler above. $G$. subcrowded, tawny, somewhat orange or ochreous-sienna. Flesh yellowish, becoming pale sienna when broken. Milk white.

Woods, under birches, grassy places. Sept. $2 \frac{1}{4} \times 1 \frac{3}{4} \times \frac{5}{8} \mathrm{in}$.

1265a. L. glaucescens Crossl. (glaucescens, becoming sea-green) a b c .

$P$. Hleshy, rigid, convex, umbilicato-depressed, smooth, glabrous, dry, zoneless, white then cream-colour, slightly spotted ochreous-white; marg. involute. St. solid, attenuate downwards, smooth, compact, colour as P. $G$. adfixed, densely crowded, somewhat forked, narrow, colour as P. Milk copious, white, then pale glaucous-green.

Taste acrid. Woods. Aug. - Sept. $2 \frac{1}{2} \times 1 \frac{7}{8} \times \frac{5}{8}$ in.

1266. L. ehrysorrheus Fr. (from the gold-coloured milk; Gr. chrusos, gold, rheo, to flow) $a b c$.

$P$. umbilicate then infundibuliform, pale yellow-pink, brownishsalmon, apricot or whitish, zoned reddish-brown. St. stuffed to hollow, equal, even, white or shaded yellowish-salmon. $G$. very thin, crowded, slightly furcate, pallid-yellowish, salmon or tan-straw colour. Flesh white, bright sulphur when broken. Milk white.

Very acrid. Woods, beech, mixed ; frequent. Aug.-Oct. $2 \frac{7}{8} \times 2 \frac{1}{8} \times \frac{3}{8}$ in.

1267. L. acris Fr. (from the acrid taste) $a b$.

$P$. convex, plane, sometimes subumbonate, at length depressed, scarcely zoned, umber or deep umber. St. stuffed to hollow, slightly attenuate downwards, tan, shaded umber. $G$. adnatorounded to subdecurrent, thin, pallid to yellow flesh-colour or pale ochre, sparingly-spotted sienna-pink. Milk white, then reddish.

Odour strong, stinking. Woods; rare. Aug.-Nov. $4 \times 2 \times \frac{1}{2}$ in. Often grows in company with 1289.

1267a. L. violascens Fr. (from the change of colour in the flesh and milk to pale violet) a $c$.

$P$. fleshy, depressed, glabrous, ashy; 2. smoky. St. solid, sometimes becoming hollow, equal, ashy- or greyish-white. $G$. somewhat crowded, white. Milk white, then violaceous or pale lavender. Flesh changing as milk.

Poisonous. Taste sweet. Woods; rare. Autumn. $3 \frac{3}{4} \times 2 \frac{3}{4} \times \frac{3}{4}$ in. 
1268. L. umbrinus Fr. (from the colour of the pileus) $a b c$.

$P$. convexo-plane, depressed, rivuloso-floccose, umber to deep sepia or dark rufous-brown, zoneless or obscurely zoned darker. St. solid, white to greyish, or as P. G. crowded, pallidyellowish, grey-spotted. Milk white, becoming greyish.

Acrid. Pine woods, damp places. Autumn. $3 \frac{1}{5} \times 1 \frac{1}{2} \times \frac{3}{4}$ in.

1269. L. plumbeus Fr. (from the colour; plumbcus, leaden) $a b c$.

$P$. convex, then depressed and infundibuliform, greyish-brown, sooty-sepia with a shade of olive or tan-olive; mid. darker. St. solid, equal, paler than P. or tan-ochre. $G$. crowded, yellowish grey-white. Milk white.

IVoods. Nov. $9 \frac{1}{2} \times 3 \frac{1}{1} \times 1 \frac{1}{\frac{1}{8}}$ in.

1270. L. pergamenus Fr. (from its parchment colour ; pergamena, parchment) $a b$. White or pale yellowish.

$P$. convex, plano-depressed, smooth, slightly wrinkled, zoneless. St. stuffed, smooth, equal or slightly attenuate downwards. $G$. adnate, very crowled, becoming straw-colour. Milk white.

Taste very acricl. Woods. Aug.-Oct. $3 \frac{1}{2} \times 3 \frac{1}{\frac{1}{4}} \times \frac{5}{8}$ in.

1271. L. piperatus Fr. (from the peppery taste; piper, pepper) a $b c$. White.

$P$. convex, then depressed and infundibuliform, rigid, even, smooth, zoneless, often spotted faint umber or grey. St. solid, attenuate downwards, subpruinose. $G$. crowded, branched, becoming spotted dull, pale brownish-yellow. Milk copious, white.

Said to be edible; poisonous when raw. Odour none. Mixed woods; common. July-Oct. $7 \frac{1}{ \pm} \times 3 \times \mathrm{r}_{\frac{3}{4}}$ in. Sometimes clouded yellowish within and without.

1272. L. vellereus Fr. (from the pubescent pileus; vellus, Heece) a $b c$. White.

$P$. convex to depressed, dry, zoneless, rigid, becoming pale yellowish or spotted pale brownish or yellowish. St. solid, equal or attenuate downwards, thinly pubescent, pale sulphur within at base. $G$. somewhat thick, subdistant, branched. Flesh white, changing to pale sulphur. Milk sparing, sometimes absent, white, sometimes pale sulphur, changing to white.

Poisonous. Taste bitter-acrid. Woods, beech; common. Aug.-Dec. $8 \times 6 \times 2 \frac{1}{4}$ in.

1273. L. seotieus B. \& Br. (found first in Scotland) $a$.

$P$. convex to slightly depressed, pubescent, pale yellowish or faint brownish. St. solid, equal, pale flesh-colour. $G$. thin, broad, scarcely branched, pallid-white. Milk white.

Taste very acrid ; odour pungent. Amongst moss. Autumn. $2 \frac{1}{5} \times 1 \frac{1}{5} \times \frac{3}{8} \mathrm{in}$. 
1274. L. involutus Sopp. ex Cooke (from the involute margin of the pileus) $a b$. White or faintly ochreous.

$P$. convex to slightly depressed, smooth, even; marg. silky. St. solid, equal. $G$. very crowded, narrow, sometimes furcate. Milk white.

Taste very acrid. Woods, on the ground. Autumn. $1 \frac{3}{x} \times 1 \frac{1}{8} \times \frac{1}{4}$ in. Very firm and rigid as in 1272 .

\section{DAPETES.}

1275. L. deliciosus Fr. (from its flavour when cooked) $a b c$.

$P$. convex to infundibuliform, smooth, subviscid, dull orangeyellow, zoned orange-sienna, sometimes clouded green. St. stuffed to hollow, equal, colour as P., usually spotted orangereddish. $G$. crowded, saffron, green when bruised. Flesh orange, becoming green when cut. Milk red-saffron, then green.

Gregarious. Edible. Sometimes acrid when raw ; odour aromatic. Woods, pine. July-Nov. $5: \times 2 \frac{1}{4} \times \frac{7}{8}$ in. This and 1295 are the "vegetable sheeps-kidneys" of French cooks. The gills are often attacked by a reddish parasitic fungus, Hypomyces lateritius. Epicoccum micropus grows upon this species when decaying. The deep orange milk of this plant must not be confounded with the sulphur-coloured milk of 1280 .

1276. L. sanguifluus Fr. (from the blood-coloured milk; sanguis, blood, fluo, to How) $a b$.

$P$. convexo-depressed, smooth, sometimes zoned, blood-red. St. narrowed upwards, smooth, pallid. G. thin, white. Milk blood-red, changing to green.

Taste mild. Oct. $2 \frac{1}{2} \times 2 \times \frac{3}{4}$ in. Closely allied to 1275 , but known by its blood-red, not orange, milk.

\section{Russulares.}

1277. L. pallidus Fr. (from its pale colour) $a b c$. Clay flesh-colour or dull buff-whitish.

$P$. convex to depressed, smooth, zoneless. St. stuffed to hollow, equal, even, smooth. $G$. somewhat thin, crowded, slightly furcate. Milk white.

Taste somewhat mild, almost sweet. Woods, beech, mixed; uncommon. Sept.-Oct. $4 \times 2 \frac{1}{4} \times \frac{7}{8}$ in.

1278. L. quietus Fr. (from its mild taste; quietus, mild) a $b c$.

$P$. convex to depressed, rich sienna, clouded, splashed and somewhat zoned darker. St. stuffed, smooth, rufescent or as P. $G$. somewhat furcate at base, whitish to yellowish sienna. Fles/l white to rufescent. Milk white.

Taste sweet, somewhat of walnuts; odour oily, bug-like. Woods, moist; common. July-Nov, $4 \times 2 \frac{3}{4} \times \frac{5}{8}$ in. Not unlike 202.

1279. L. aurantiacus Fr. (from the bright orange-coloured pileus and stem ; aurantiacus, orange-coloured) $a b$.

$P$. plano-depressed, even, zoneless. St. stuffed, smooth. $G$. crowded, very decurrent, paler than P. Milk white.

Taste slowly acrid. Woods, on the ground amongst moss. Sept. $2 \frac{1}{2} \times 2 \times \frac{3}{8}$ in. 
1280. L. theiogalus Fr. (from the sulphur-coloured milk ; Gr. theion, brimstone, salar, milk) a $b c$.

$P$. convex to infundibuliform, sometimes papillate, even, smooth, somewhat rufous-tawny with a shade of liver; marg. crenulate. St. stuffed to hollow, equal, or nearly so, even, rufous-salmon within and without. $G$. adnato-decurrent, thin, somewhat distant, faintly ochreous to deep yellow or rufescent. Milk white, slowly becoming sulphur.

Taste mild, then slowly acrid; odour none. Woods, mixed, pine, oak, amongst heather, sometimes on dead root-stocks of P'teris aquilina ; frequent. Aug.-Nov. $2 \frac{5}{5} \times 2 \frac{3}{8} \times \frac{3}{8}$ in. The yellow milk of this species must not be confounded with the deep orange milk of 1275 .

1281. L. cremor Fr. (from the thick milk; cremor, thick juice) $a b$. Thin, fragile.

$P$. convex to plano-depressed, minutely punctulate, tawny, zoneless; mid. darker; marg. striate. St. hollow, equal, colour as P. G. adnate, subdistant, pallid, or as P. Milk somewhat transparent white.

Taste mild or slowly acrid. Woods, larch, beech, etc. Sept. $4 \frac{1}{4} \times 1 \frac{1}{2} \times \frac{5}{4}$ in. Var. pauper Karst., smaller. P. 3 in. in diam.

1282. L. vietus Fr. (from its appearance; zictus, shrivelled) $a b c$.

$P$. convex, then depressed, somewhat papillate, even, smooth, flesh-colour or livid-grey, becoming whitish-grey; mid. pale umber. St. stuffed to hollow, equal or attenuate downwards, pale rose-brown or as P. $G$. somewhat crowded, white to yellowish. Milk white, then pale lavender-grey.

Taste mild, becoming slowly acrid; odour faint but pungent. Woods, mixed, damp, grassy places, on the ground. Sept.-Oct. $3 \frac{1}{1} \times 1 \frac{3}{8} \times \frac{3}{8}$ in.

1283. L. eyathula Fr. (from the cup-shaped pileus; cyathula, a little cup) $a b$.

$P$. convexo-plane, depressed, sometimes papillose, rimoso-rivulose, Hesh-colour, pale yellowish or ivory, lavender-shaded, zoned, yellowish-brown or zoneless. St. stuffed, equal, even, smooth, whitish or yellowish, lavender-shaded. $G$. very crowded, thin, whitish, then yellowish-salmon. Milk white.

In troops. Taste acrid ; odour at first none, then strong of bugs. Woods, birch, damp ground. Aug.-Oct. $2 \frac{1}{8} \times 2 \frac{1}{4} \times \frac{1}{4}$ in.

1284. L. rufus Fr. (from the reddish colour) a $b c$.

$P$. plane to infundibuliform, usually umbonate, deep crimsonsienna, sometimes paler, zoneless or with one zone; marg. sometimes sulcate. St. stuffed, equal, rufescent, paler than 1'. $G$. ochreous to rufescent. Milk white.

l'oisonous. Taste very acrid-l)itter; odour none. Woods, pine, etc., dry places. June-Nov. $3 \frac{7}{5} \times 2 \frac{1}{2} \times \frac{1}{2}$ in.

1285. L. helvus Fr. (from the colour; hclucus, pale yellowish) a bc.

$P$. convex to slightly depressed, sometimes subumbonate, granuloso-floccose, sometimes sienna-biscuit, sometimes faintly I- to 2-zoned. St. stuffed to hollow, equal, pale buff. 
$G$. crowded, whitish, pale yellowish or pale salmon. Mitk watery-white.

Taste mild, sweet then slightly acrid ; odour faint, sweetish. Woods, fir, mossy places, swampy ground. Aug.-Oct. $3 \frac{1}{4} \times 2 \frac{1}{4} \times \frac{5}{5}$ in.

1286. L. tomentosus Cooke (from the woolly tomentose pileus) $a b$.

$P$. at first umbonate, then infundibuliform, dull salmon to deep sienna. St. stuffed to hollow, equal, naked, smooth, pallid or salmon above, ochreous below red-streaked at mid. $G$. yellow flesh-colour or ochre, or white-ochre near edge and red above. Milk whitish.

Taste mild to slightly acrid; odour pleasant. On the ground, damp places. $4 \frac{1}{8} \times 1 \frac{3}{4} \times \frac{1}{2}$ in. Sometimes confused with 1285 .

1287. L. mammosus Fr. (from the papillate pileus; mamma, a breast) $a b$.

$P$. convex to depressed, at first acutely umbonate, dry; greyfuscous or dull pale indigo-greenish, zoneless or faintly zoned; marg. white pubescent. St. stuffed to hollow, equal, pubescent, faint yellowish or brownish. $G$. crowded, whitish or pale yellowish to pallid ferruginous. Milk white.

Taste slowly acrid; odour none. Woods, pine, on the ground. Oct. $3 \frac{1}{2} \times 2 \frac{1}{2} \times \frac{3}{4}$ in. Var. monstrosis Cooke. Larger than type.

1288. L. glyciosmus Fr. (from the sweet smell; Gr. glukus, sweet, osme, smell) a b $c$.

$P$. convex to slightly depressed, acutely papillate, yellowish, shaded over with pale lavender-purple, zoned pale purplish. St. stuffed to hollow, pubescent, whitish or light yellowish, tawny when bruised. $G$. crowded, thin, white to strawochreous. Milk somewhat scanty, white, unchanging or changing to pale straw-green.

Taste milı, then slowly acricl; odour strong, pungent-sweet, fragrantaromatic. Woods mixed, chiefly pine, on the ground. Sept.-Nov. $2 \frac{7}{8} \times 2 \frac{1}{2} \times \frac{3}{8}$ in.

1289. L. fuliginosus Fr. (from the smoky colour; fuligo, soot) a $b c$.

$P$. convex to subinfundibuliform, dry, full velvety ochreous- or smoky-brown; marg. paler. St. stuffed, equal or attenuate downwards, white, tan or pale tan-umber. $G$. somewhat distant, white, then pale ochreous, pale indian-red when bruised. Milk white, sometimes watery, changing to rose and saffron. Flesh changing to vinous when cut.

Probably poisonous. Taste mild, becoming very acrid-nanseous; odour faint nauseous-pungent. Shady woods; frequent. Aug.-Oct. $4 \frac{1}{2} \times 2_{4}^{3} \times$ I in. Often growing in company with 1267.

1290. L. picinus Fr. (from the black pileus; ficinus, pitch-black) a $b$. $P$. convex, obtuse or subumbonate, minutely velvety, umber to blackish-umber. St. stuffed, equal, smooth, paler than P. or almost white, brownish below. $G$. adnate, thin, very crowded, pale ochreous to rufous tan. Mill white.

Taste acrid. Woods, pine. (lried turfy swamps. Aug.-Oct. $2 \frac{1}{2} \times 2 \times \frac{3}{8}$ in. 
1291. L. lignyotus Fr. (from the woody substance; lirmum, wood, (ir. ous, an ear) $a$.

$P$. convex to subinfundibuliform, umbonate, plicato-rugulose, velvety, deep umber-purplish or opaque sooty-umber. St. solid to stuffed, equal, striate above, colour as P., yellowish or whitish at base. $G$. adnexed to decurrent, somewhat crowded, whitish or yellowish, reddish when bruised. Flesh white, changing to ochreous, saffron and ferruginous. Mille white, scanty, changing through reddish to saffron.

Poisonous. Tasle mild, sweet, pleasant ; odour slight. Amongst moss, in damp places under firs, beech. Oct. $3 \frac{1}{4} \times 3 \times \frac{1}{2}$ in.

1292. L. lilacinus Fr. (from the lilac pileus) a $b$. Fragile.

$P$. convex to depressed, papillate, floccose, rosy-lilac, zoneless; marg. tan. St. stuffed to hollow, pale yellowish-brown or dull rose, lilac-shaded, or as P., white mealy. G. subdistant, pallid flesh-colour or yellowish-white. Milk white, copious.

Taste acrid. Woods, damp places. Aug.-Oct. $2 \frac{1}{4} \times 1 \frac{1}{8} \times \frac{1}{4}$ in.

1293. L. retisporus Mass. (from the reticulate spores) a $b$.

$P$. convex, then plane and depressed, minutely velvety, dark smoky-brown. St. equal, solid, even, glabrous, paler than P. or pale sepia or greyish. $G$. subdistant, deeply sinuate, slightly adnexed, pale ochreous with darker spots. Flesh dull yellowish or whitish-salmon, becoming reddish when cut. Milk sweet, white, changing to brown and becoming thick and tenacious.

Under beeches, on the ground. Sept. $3 \frac{3}{4} \times 2 \frac{1}{4} \times \frac{5}{5}$ in.

1294. L. spinosulus (quél. (from the spines on the pileus; sfinosulus, full of little spines) $a$.

$P$. convex to depressed, acutely umbonate, clad with minute, erect spines, brick-red to rosy-lilac, often zoned and spotted. St. stuffed to hollow, rugose, granulate, shining, colour as $\mathrm{P}$. $G$. thin, yellowish flesh-colour to yellowish. Milk white.

Taste acrid. On the ground. Sept. $I \frac{1}{2} \times I \frac{\frac{1}{2}}{2} \times \frac{5}{16}$ in.

1295. L. volemus Fr. (from its resemblance to a certain large pearthe "Red Warden" or baking-pear-volema-pira) a b $c$.

$P$. plano-convex to subdepressed, compact, bright rufous-tawny or golden, becoming pale; mid. deep-sienna, zoneless. St. solid to stuffed, equal, pruinose, colour as P. $G$. thin, crowded, white to yellowish or yellow, orange-umber when wounded. Milk white, sometimes changing to yellow.

Edible. Taste sweet, pleasant when raw. Woods, mixed. (fct. $4 \times 4 \times 1 \frac{7}{2}$ in. The "Golden Brötling" of Germany and IIungary. With 1275 the "vegetable sheeps-kidneys" of French cooks.

1296. L. ichoratus Fr. (from the watery milk; Gr. ichor, lymph) a b.

$P$. plane to depressed, dry, tawny-reddish; mid. brown-sienna; marg. sienna-yellow, sometimes zoned. St. solid, equal, colour 
as P. G. scarcely crowded, white to tan-yellow or ochreous. Milk white, unchangeable.

Taste sweet; odour strong. Woods. Cct. $2 \frac{1}{2} \times 2 \frac{5}{4} \times \frac{1}{2} \mathrm{in}$.

1297. L. serifluus Fr. (from the watery milk; scrum, whey, fluc, to flow) $a b c$.

$P$. plane to depressed, dry, brown-tawny or orange-reddish, sometimes crimson-purplish, zoneless or with one faint zone. St. solid, yellowish, red-salmon or paler than I. $G$. crowded, colour as St., yellowish or ochre-brownish. Milk whitish, especially thin in examples from wet places.

Taste almost mild; odour disagreeable, bug-like. Woods, mixed, damp places; frequent. July-Nov. $3 \times 2 \frac{1}{4} \times \frac{1}{2}$ in. Resembling 193.

1298. L. mitissimus Fr. (from its mild taste; mitissimus, very mild) $a b c$.

$P$. convex to depressed, at first papillate, even, bright goldentawny or bright orange, zoneless or faintly zoned. St. stuffed to hollow, even, smooth, colour as P. $G$. thin, paler than P., often rufous-spotted. Milk copious, white.

Taste mild to somewhat bitterish or slightly acrid-disagreeable. Woods, mixed, pine, hedge-banks, etc. ; common. Aug.-Nov. $2 \times 2 \frac{3}{4} \times \frac{3}{16}$ in. Like a small 1295.

1299. L. subduleis Fr. (from the somewhat sweet taste; dulcis, sweet) $a b c$.

$P$. convex to depressed, at first papillate, even, dry, rufescent or orange-sienna, usually zoneless. St. stuffed to hollow, equal, subpruinate, colour as P. $G$. crowded, colour as P., or paler. Flesh rufescent. Milk white.

Taste mild or sweet, then slightly bitter-acrid; odour none or like cedarwood. Woods, mixed, fir. July-Nov. $3 \times 2 \times \frac{1}{2}$ in. Resembling 201. Form concavus Fr. P. concavo-depressed; colours of 1284. Form sphagneti Fr. Marg. of $P$. reflexed, crenate, shining as if varnished, red-bay.

1300. L. camphoratus Fr. (from the strong odour, sometimes of camphor) a b $\mathrm{C}$.

$P$. convex to depressed, dry, brownish-red to sienna-orange, paler when young, somewhat zoned or zoneless. St. stuffed, equal, colour as P., paler above. $G$. yellow-reddish, paler than P., or salmon. Fles/l somewhat ochre or sienna. Milk watery, white.

Taste mild; odour sometimes of melilot, permanent in dried examples. Woods, chiefly pine; frequent. Aug.-Nov. $2 \frac{5}{8} \times 2 \frac{1}{4} \times \frac{1}{4}$ in. Resembling 194. Var. Torrei Cooke. P. $\frac{1}{2}$ in. in diam. corrugate, depressed, baybrown. St. colour as P., clad with orange-down.

1301. L. cimicarius Mass. (from the odour; cimex, a bug) $a$.

$P$. convex to subinfundibuliform, deep sienna, shaded deep umber. St. stuffed to hollow, lighter than P., paler above. $G$. dusky ochre, sienna or rufous. Flesh grey to yellowish. Milk watery, white.

Taste somewhat acrid; odour when fresh, strong, heavy, oily, bug-like, fugitive. Woods, on the ground. Autumn. $2 \frac{1}{8} \times \mathbf{5}_{3}^{5} \times \frac{1}{4}$ in. 
1302. L. subumbonatus Lindgr. (from the somewhat umbonate pileus) $a b$.

$P$. convex to depressed, acutely papillate, rugose, punctate, dark cinnamon, zoneless. St. stuffed, equal or attenuate downwards, rufescent, crimson-umber below. $G$. adnate, flesh-colour or reddish. Flesh pale brown. Milk watery, white.

Taste mild; odour none when fresh, fetid in drying. Woods, on the ground. Sept. $1 \frac{3}{8} \times I \times \frac{3}{16}$ in.

1303. L. obnubilis Fr. (from the clouded pileus ; obnubilo, to overcloud) $a b$.

$P$. convex to depressed, at first papillate, brown-fuliginous or umber, zoneless: marg. somewhat striate. St. stuffed to hollow, equal, paler than P. G. somewhat crowded, yellowish. Milk white.

Taste mild, somewhat sweet. Woods, damp places under alders, pastures. Sept.-Nov. $2 \frac{1}{8} \times 1 \frac{7}{8} \times \frac{1}{4}$ in. Var. crencatus Mass. $P$. coarsely and regularly sulcate. Usually larger than type. Fir woods.

1304. L. minimus IV. G. Sm. (from its very small size; minimus, very small) $a b c$. Pallid clay-colour.

$P$. pulvinate, obtuse, or subumbonate. St. usually excentric. $G$. furcate, moderately distant, slightly darker than P. Milk white.

Taste mild. Woods, pastures. Oct. $\frac{5}{16} \times \frac{3}{16} \times \frac{1}{16}$ in.

\section{Pleuropode.}

1305. L. obliquus Fr. (from the oblique stem) a. White, changing to yellowish.

$P$. plano-depressed, lobate, silky, zoned grey. St. stuffed to hollow. $G$. crowded, white. Milk white.

Odour strong. Trunks, beech, burnt stumps, banks. Oct. $2 \frac{7}{8} \times \mathbf{I} \times \frac{1}{4}$ in. Pileus sometimes 6 in. or more in diam.

\section{RUSSULA Fr.}

(From the frequently reddish colour of the pileus ; russulus, reddish.)

Veil obsolete. Hymenophore confluent and homogeneous with the stem. Pilcus fleshy, rigid, regular, at length depressed. Stcm central, stout, rigid, without cartilaginous bark, shining, exannulate. Gills adnate, rigid, fragile, often equal in length or furcate, edge thin, acute, sometimes exuding watery drops, especially in rainy weather, but not milky as in Lacturius; trama, vesicular. Spores minutely verruculose or echinulate, white or pale yellowish. (Fig. 63.)

'Terrestrial fleshy rigid and brittle putrescent fungi appearing in late summer and autumn. A few species are reputed edible, but most are noxious, and there is some danger of confusing the esculent with the poisonous. Odour none or unpleasant. 
Russula agrees with Lactarins in size and some other characters, but differs in the absence of milk.

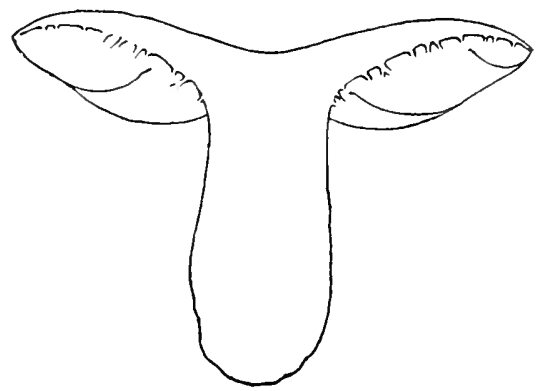

Fig. 63. - Section of Russula lepida Fr. One-half natural size.

The sclerotia and perfect plants of 248 sometimes grow from the gills of 1306 and 1307.

Spharonema aitrem frequently grows on species of Russula and Lacturins. Species 1306-1367

a. Compacte. Pilius fleshy, margin bent inwards, without stria, without a distinct viscid pellicle, colour only changing with age and the state of the atmosphere. Stem solid, fleshy. Flesh compact, firm. Gills unequal.

$1306-1313$

b. Furate. Pileus compact, firm, covered with a thin closely adnate pellicle, which at length disappears, margin abruptly thin, at first inflexed, acute, even. Stcm at first compact, then spongy-soft within. Gills somewhat forked, with a few shorter ones intermixed, commonly attenuate at both ends, thin, and normally narrow.

$1314-1324$

c. Riridce. Pilens without a viscid pellicle, dry, rigid, the cuticle breaking up into flocci or granules, margin straight, never involute or striate. Stem solid, at first hard, then softer or spongy. Flesh thick, compact, firm, ending short of the margin of the pileus. Gills, a few dimidiate, others divided, rigid, dilated in front, running out with a broad, rounded front, whence the margin of the pileus becomes obtuse. Handsome, somewhat rare.

$1325-1334$

d. Hitcophylle. Pileus fleshy, firm, with a thin, at first infleved margin, then, except 1340, expanded and striate, covered with a thin adnate pellicle. Stem solid, stout, spongy within, sometimes becoming hollow. Gills consisting of many shorter, mixed with longer ones, others furcate.

$1335-1346$

e. Fraciles. Pilens more or less fleshy, covered with a pellicle which is always continuous, and in wet weather viscid and 
somewhat separable, margin membranous, at first convergent and not involute, in full grown plants commonly sulcate and tubercular, not striate in 1348, 1356 and 1363. Stcm spongy, at length wholly soft and hollow. Gills almost all equal, simple, broadening in front, free. Fles/ commonly floccose, lax, friable. Several doubtful forms occur; 1357 is specially variable in colour.

Gills and spores white.

$1347-1355$

Gills and spores white, then bright lemon-yellow. 1356-1361 Gills and spores ochreous.

$1362-1369$

\section{a. Compactu.}

1306. R. nigricans Fr. (from its changing to jet-black in decay; nigricans, becoming black) a $b c$.

$P$. convex, depressed; streaky umber-tan, sometimes oliveshaded; mid. almost black. St. attenuate downwards, siennatan, steel-greyish or faint purplish. $G$. rounded-adnexed, very thick, distant, pale yellowish. Flesh white, then reddish or pale purplish, at length black.

Odour none. Woods, mixed, etc.; common. June-Nov. $5 \frac{1}{2} \times 2 \frac{1}{2} \times 2$ in. Becoming wholly black and in this character analogous with 1221 . 1389 grows on decaying examples of this species.

1307. R. adusta Fr. (from its scorched appearance; adustus, scorched) a $b \mathrm{c}$.

$P$. convex to depressed or subinfundibuliform, smooth, whitish to pallid yellowish, at length clouded olive-sepia. St. equal, colour as young $\mathrm{P}$. $G$. adnate to decurrent, thin, crowded, white then dull. Flesh unchangeable or becoming grey or pale sooty.

Taste mild to acrid. Woods; frequent to rare, local. Aug.-Oct. $3 \frac{1}{2} \times 2 \frac{1}{4} \times 1 \frac{1}{5}$ in. Not becoming wholly black. 1390 grows upon this species and on 1341. Var. carnulescens Fr. becomes blue when broken or cut.

1308. R. albonigra Fr. (from the colours; albus, white, niger, black) a $b$. Tery rigid.

$P$. convex to infundibuliform, viscid, whitish; marg. smoky. St. dusky, or white above, pale grey-ochreous towards base, spotted-sooty. $G$. decurrent, crowded, thick, unequal, connected by veins, dimidiate, dusky whitish or yellowish. Flesh white, then black or sooty, not red.

Taste somewhat acrid and unpleasant to mild; odour none. Grassy places under trees. Aug. $3 \frac{3}{8} \times \mathbf{I}_{t}^{\frac{3}{3}} \times \mathbf{I}_{8}^{3} \mathrm{in}$.

1309. R. densifolia Cooke (from the crowded gills, as compared with 1306 ; densus, thick, folium, a leaf) a b.

$P$. convex to depressed, smooth, whitish, becoming streakyfuliginous, grey or brownish; mid. black. St. smooth, whitish to grey, then blackish. $G$. adnato-decurrent, unequal, thin, 
white to rosy, red when bruised. Flesh white, reddish when bruised, becoming black.

Taste mild to slowly acrid. Woods, under trees. Aug.-Sept. $4 \frac{3}{8} \times 2 \frac{1}{4} \times \frac{7}{8}$ in.

1310. R. semicrema Fr. (from the white stem becoming cinereous and black when broken; scmi, half, cremo, to burn) $a$. White or faintly yellowish.

$P$. convex to depressed, even. St. equal or slightly attenuate upwards. $G$. decurrent, crowded, thin. Flesh in P. white.

Taste mild; odour none. Woods, mixed, amongst leaves. Aug.-Sept. $4 \frac{1}{3} \times 2 \frac{3}{4} \times \frac{7}{8}$ in.

1311. R. delica Fr. (from its resembling a milkless 1272 in appearance ; dclicus, weaned) a $b c$. White or whitish.

$P$. convex to depressed, even, smooth. St. even, smooth. $G$. decurrent, thin, distant, unequal, exuding small watery drops in wet weather.

Woods, mixed, usually pine; uncommon. Sept.-Oct. $5 \times 2 \times 1$ I in

1311a. R. chloroides Bres. (from the greenish-yellow colour of the gills; Gr. chloros, greenish-yellow, cidos, appearance) $a b c$.

$P$. fleshy, plane to infundibuliform ; marg. incurved, pubescent, whitish, whitish-buff or livid. St. solid, equal or attenuate downwards, thinly pubescent, whitish. $G$. decurrent, somewhat crowded, furcate, veined, verdigris-white, greenish-white, or turquoise-white, shaded emerald-green-white. Flesh thick, whitish to snow-white.

Acrid. Woods, pine. Aug. -Oct. $4 \times 2 \frac{1}{2} \times \mathrm{I}$ in.

1312. R. elephantina Fr. (from Fries thinking, in error, that Bolton's obese Agricus elephantinus might be one form of this comparatively slender species) $a b$.

$P$. convexo-umbilicate, fuscous-tan ; marg. undulate, paler, dark wash-leather colour when dry. St. hard, even, smooth, paler than P. $G$. adnate or sinuato-adnate, then subdecurrent, arcuate, somewhat crowded to distant, thin, obtuse, divided behind, unequal in front, spotted pale yellowish when touched, dull pale salmon when dry.

Odour none. Woods. Sept. $3 \times 2 \frac{1}{3} \times \mathrm{I}$ in. Habit of 1341 , but inodorous. A. elephantinus Iolton (Hist. Fung. t. 28 ) is undoubtedly 1306 , as is shown by the remote gills and the change of colour to black.

1313. R. mustelina Fr. (from the colour of the pileus; mustela, a weasel) a $b$.

$P$. convex to depressed, opaque yellow-ochre or sienna with yellow to full burnt sienna. St. white, whitish, or faintly shaded yellowish, brownish or salmon. $G$. adnexed, adnate or rounded, crowded, veined above, somewhat branched.

Woods. Oct. $3 \times 2 \frac{1}{2} \times \frac{3}{4}$ in. 


\section{b. Furcate.}

1314. R. olivascens Fr. (from the pileus, more or less olive) $a b$.

$P$. gibbous to slightly depressed, olive, ochreous-olive or olivebrownish; mid. yellow to olive-brown. St. equal, white or shaded pale yellowish or olive-tan. $G$. adnate, crowded, almost equal, colour as St.

Taste mild, becoming slightly acrid, gills acric. Woods, bushy shady places. Aug. $5 \times 2 \frac{1}{6} \times \mathrm{I}$ in.

1315. R. furcata Fr. (from the forked gills; furcatus, forked) $a b c$. $P$. gibbous to depressed, even, smooth, not breaking into warts, æruginous-greenish, umber-greenish, olive-tan, dark indigo-olive, slate, or dull reddish-purple, sometimes clouded dull rose. St. equal, white. $G$. adnato-decurrent, rather thick, somewhat distant to crowded, white. Filesh white, often pale rose under olive pellicle of pileus.

Taste mild to more or less bitter. Woods, grassy places under trees, meadows; common. Nlay-Oct. $5^{\frac{1}{2}} \times 2 \frac{3}{4} \times 1 \frac{1}{x}$ in. Var. pictipes Cooke. St. rosy above, greenish below. Var. ochroviridis Cooke. P. marg. ochreous, mid. olivaceous or fuliginous. Flesh fuliginous when cut.

1316. R. sanguinea Fr. (from the blood-coloured pileus; sangzis, blood) a b c.

$P$. convex to depressed, shining, even, mid. thick ; marg. becoming pale, sometimes white-clouded. St. equal, slightly striate, white or reddish. $G$. decurrent, sometimes adnate, crowded and veined, white. Flesh white, carmine under pellicle of pileus.

Poisonous. Taste acrid, peppery. Woods, chiefly fir, grassy places; uncommon. Aug.-Sept. $4 \times 2 \frac{1}{4} \times \mathbf{I}$ in. Must not be confounded with 1329, which differs in the solid firm flesh and the form of the gills.

1317. R. rosacea Fr. (from the rose-coloured pileus) $a b$.

$P$. convex to flat, even, pellicle viscid and separable in wet weather, white, salmon, pale rose to red-rose and darker at the sometimes faintly-ochreous mid., sometimes white, red-spotted. St. even, smooth, white to reddish. $G$. adnate, thin, crowded to distant, white. Flesh white, rose-colour under pellicle of $\mathrm{P}$.

Taste mild, becoming slowly acrid, gills acrid. Woods, mixed grassy places; frequent. Aug.-Oct. $3 \frac{3}{8} \times 2 \frac{1}{8} \times \frac{7}{8}$ in.

1318. R. maculata Quél. (from the spotted pileus and stem ; maculatıs, spotted) $a b$.

$P$. hemispherical to depressed, viscid, reddish flesh-colour to pallid, then discoloured and spotted purple or brown; marg. striate, often dark. St. reticulato-striate, white or rosy then spotted ochre, not becoming grey. $G$. adnate, pallid-sulphur to somewhat peach-colour.

Taste somewhat acrid. Sept. $3 \frac{1}{4} \times 1 \frac{1}{2} \times \frac{5}{8} \mathrm{in}$. 
1319. R. sardonia Fr. (from the acrid taste like that of a certain plant, Herba Sardoa, Raminalus sceleratus) a b c.

$P$. convex to plane or slightly depressed, viscid in wet weather, reddish-purple or pale lemon with mid. orange-yellow, often partially white; marg. sometimes striate and somewhat tuberculose. St. equal, even, white, yellowish or reddish. $G$. adnate, crowded, white, spotted yellowish from exuded drops in wet weather. Flesh white.

Woods, chiefly fir, larch, near paths; uncommon. Sept.-Nov, $4 \frac{1}{2} \times 2 \frac{1}{2} \times I$ in.

1320. R. depallens Fr. (from the pileus becoming pale; difalleo, to be pale) $a b$.

$P$. convex to plane or depressed, often undulate or irregular, reddish, red-crimson, fuscous, purplish-rose or dark purplecrimson, the hues becoming pale to yellowish or white, the slightly striate marg. sometimes cobalt-purple. St. equal, white to pale greyish. $G$. adnexed, scarcely crowded, white to pale yellowish.

Edible. Taste mild to bitter or acrid ; odour none. Woods, beech, pastures, etc.; uncommon. June-Oct. $4 \times 1 \frac{7}{3} \times \frac{3}{4}$ in.

1321. R. purpurea Gill. (from the purple pileus) $a b$.

$P$. hemispherical to depressed, rugoso-plicate, deep purple; mid. darker ; marg. even, then faintly striate. St. striate, white, or white above, rosy in middle, yellowish below. $G$. rounded, white to yellowish. Flesh yellowish, red beneath the pellicle.

Taste mild. Uniler larches, oak, amongst grass. Aug.-Oct. $3 \frac{1}{\frac{1}{4}} \times \mathbf{I} \frac{7}{8} \times \frac{5}{5} \mathrm{in}$.

1322. R. cœrulea Fr. (from the partially azure-blue colour ; co'rulens, azure) $a b$.

$P$. convex or umbonate to slightly depressed, shining, deep redpurple or bluish-purple; marg. even, somewhat cobalt, sometimes with a white line round edge; mid. bluish-purple or buff. St. white, sometimes rose at base. $G$. adnate, white to yellowish. Flesh white, deep purple under pellicle.

Taste mild. Woods, pine, etc., grassy places. Aug.-Oct. $3 \frac{3}{4} \times 2 \frac{3}{4} \times \frac{1}{2}$ in.

1323. R. drimeia Cooke (from the pungent taste; Gr. drimus, pungent) a $b$.

$P$. hemispherical to depressed, scarcely viscid, bright deep-purple or deep brownish-red-purple; marg. even. St. equal, tinged with purple. $G$. adnexed, pale sulphur to pale yellow.

Intensely acrid. Under larches. Sept.-Nov. $3 \frac{7}{8} \times 2 \frac{5}{5} \times \frac{3}{4}$ in. Colour and habit of 1345 , differing in yellow gills and intense acridity.

1324. R. virginea Cooke $\&$ Mass. (from its wholly white colour) a $b$. $P$. convex to depressed, smooth, even, viscid when moist, lustrous when dry : marg. even. St. solid, slightly attenuate upwards, finely rugulose. $G$. decurrent, crowded, furcate, narrow.

Taste mild. Terrestrial. Under trees, beech. Oct. $2 \times 2 \frac{1}{2} \times \frac{1}{2}$ in. If ust not be confounded with other Russulue bleached white with age. 


\section{c. Rigida.}

1325. R. lactea Fr. (from its milk-white colour; lac, milk) a b.

$P$. convex, sometimes faintly spotted cream-buff; mid. biscuitbuff. St. nearly equal, even, white, sometimes rose at base. $G$. free to adnate, thick, distant, furcate at base and apex, ivory.

Said to be edible, but suspicious. Taste mild; odour of meal. Woods, mixed, fir ; uncommon. Aug.-Oct. $3 \frac{1}{4} \times 2 \frac{1}{4} \times \frac{3}{4}$ in. Var. incarnata Cooke. $P$. shaded with pink-buff or dull purple-rose, with lighter and a few pale sienna spots.

1326. R. virescens Fr. (from the frequently green pileus; virco, to be green) $a b c$.

$P$. pulvinate to depressed, innato-flocculose, without a pellicle, surface soon broken up into patches or warts, verdigris-green, varying olive, dull ochre with mid. verdigris, reddish-purple and other shades. St. somewhat rivulose, often clouded pale verdigris. $G$. free, white to biscuit. Flesh white, verdigris under pellicle.

Edible. Taste mild, like hazel-nuts when raw, sometimes slowly and slightly acrid ; odour of 1394. Woods; frequent. July-Oct. $4 \frac{1}{8} \times 2 \frac{3}{x} \times I$ in. The "Champignon des Dames" of French authors.

1327. R. cutefracta Cooke (from the broken cuticle); cutefractus, with the skin broken) a $b$.

$P$. pulvinate to depressed, even, cuticle cracking as in 1326, surface green, purple, bluish-purple, dull red, claret-red, etc., purple beneath cuticle. St. equal, smooth, white, often clouded rose-purplish. $G$. adnexed or nearly free, somewhat crowded, white or pale yellowish.

Taste mild. Woods. Sept. $3 \frac{1}{1} \times \mathrm{I}_{\frac{3}{4}}^{\frac{3}{4}} \times \frac{3}{1}$ in. Probably a form of 1326 ; intermediate forms occur.

1328. R. lepida Fr. (from its appearance; lepidus, elegant) $a b c$.

$P$. convex to slightly depressed, often rimoso-squamulose, reddish-rose with deep red-rose mid. or sienna-ochreous with deep red mid., which at length becomes whitish. St. even, equal, white to rose. $G$. adnato-rounded, somewhat thick, somewhat crowded, white or pale yellowish, often rose at edge.

Edible. Taste mild. Woods, mixed, amongst grass; frequent. Aug.-Oct. $4 \frac{1}{8} \times 2 \frac{1}{8} \times \mathbf{I}$ in. Becomes green in cooking.

1329. R. rubra Fr. (from the red pileus; nuber, red) $a b c$.

$P$. hemispherical to plane or slightly depressed, dry, becoming lustrous, often rivuloso-rimose when old, intense crimson; mid. almost black or deep purple, becoming pale. St. even, equal, or slightly attenuate upwards, white, sometimes clouded rose or purplish. $G$. adnate, somewhat crowded, whitish to yellowish, often red at edge. Flesh reddish under cuticle.

Poisonous. Flesh mild, gills very acrid. IVoods, mixed, grassy places; frequent. Aug.-Nor. $4 \frac{1}{1} \times 4 \frac{1}{2} \times \mathrm{I}$ in. Must not be confounded with 1316 . 
1330. R. Linnæi Fr. (in honour of Linnæus) $a$.

$P$. convex to plano-depressed, even, smooth, dry, dark purple or blood-red to light rose. St. sometimes hollow, subventricose, deep blood-red to rose. $G$. adnato-decurrent, rather thick, not crowded, white to pale yellowish.

Taste mild, sweet. Woods. Oct. $5 \times 3 \frac{3}{4} \times 1 \frac{1}{4}$ in. Sometimes white.

1331. R. xerampelina Fr. (from the colour of the pileus, like dying vine-leaves; Gr. xerampelinai, of the colour of dry vineleaves) $a b c$.

$P$. convex to depressed, dry, even, at length finely granulate, rosy-purplish or dark rosy-red, clouded buff; mid. becoming yellowish-white. St. sometimes hollow, equal or slightly attenuate upwards, white or reddish. $G$. adnexed, somewhat crowded, whitish to yellowish. Flesh whitish.

Taste mild; odour strong of crab. Woods, fir, grassy places. July-Oct. $4 \frac{3}{8} \times 3 \frac{1}{2} \times \frac{7}{3}$ in.

1332. R. olivacea Fr. (from the colour of the pileus, at length olive) $a b$.

$P$. hemispherical to slightly depressed, slightly silky-squamulose, dull purple to olivaceous or fuscous-olivaceous, not lustrous. St. equal or ventricose, pallid, rose-colour or clouded pale purple or orange-crimson, crimson and ochre at base. $G$ adnexed, yellow.

Edible. Taste mild. Woods, pine, grassy places. Aug. $4 \times 3 \times \frac{3}{8}$ in.

1333. R. serotina Quél. (from its sometimes late appearance; serotinus, backward) $a b$.

$P$. hemispherical to flat, pruinose, sooty-purplish or olive, or clouded-slate and dull olive ; marg. lilac or purplish-reddish. St. equal or attenuate downwards, wrinkled, mealy, white. $G$. adnexed, crowded, yellowish-white.

Taste acrid. Under beeches. Aug.-Sept. $1 \frac{1}{4} \times I \times \frac{3}{16}$ in.

1334. R. Duportii Phill. (after the Rev. Canon J. M. Du Port) a b.

$P$. convex to depressed, smooth; mid. rufous, dull purplish, brown, or flesh-red; marg. becoming indistinctly striate and tuberculate, dull bluish or greenish. St. minutely striate, glabrous, white or dull sienna-white. $G$. rounded, broad, distant, white or faintly yellowish. Flesh reddish-brown when cut.

Taste mild, pleasant ; odour of crab. Woods. Sept. $2 \frac{5}{8} \times 2 \frac{1}{2} \times \frac{5}{8}$ in.

\section{d. Hetcrophylla.}

1335. R. vesea Fr. (from its edible qualities; vescus, eatable) $a b c$.

$P$. convex to depressed, viscid, slightly wrinkled, rose-purple, red-flesh-colour, liver, tan-buff umber or ochre, or clouded purple and buff; mid. usually darker, not becoming pale. 
St. wrinkled, equal or attenuate downwards, white, sometimes shaded pale rose, purple or ochre. G. adnate, crowded, white or yellowish.

Edible. Taste mild, pleasant to unpleasant ; odour of hoy's lard or somewhat like crab, becoming like lobster or crab when cooked. Woods, mixed ; frequent. Aug.-Oct. $4 \frac{3}{8} \times 2 \frac{1}{2} \times \frac{7}{8}$ in.

1336. R. lilacea Quél. (from its lilac colours) $a$.

$P$. convex to depressed, viscid, violet, purple or rosy-purple; marg. at length almost white. St. attenuate downwards, pruinose above, white, sometimes rosy at base. $G$. adnate, distant, white. Flesh violet under pellicle.

Taste mild. Woods, moist places, Aug. $3^{\frac{1}{2}} \times 2 \frac{3}{8} \times \frac{3}{4}$ in.

1337. R. azurea Bres. (from the azure-blue pileus) $a b$.

$P$. convex to depressed, minutely granulose, dry, bright blue, deep purple, dark indigo or greenish-slate; mid. sometimes ochre or brown; marg. scarcely striate, sometimes lilac, becoming dilute cobalt and indigo. St. rugulose, equal, ventricose or subclavate, white or clouded pale rose. $G$. adnexed, crowded, white.

Edible. Taste mild, sometimes bitter. Woods, fir, grassy places under trees. Aug.-Sept. $3 \frac{3}{4} \times \mathbf{I}_{\frac{3}{4}}^{3} \times \frac{3}{x}$ in.

1338. R. cyanoxantha Fr. (from the colours; Gr. kuanos, blue, xanthos, yellow) $a b$.

$P$. hemispherical to depressed, viscid, lilac, purplish, olivaceousgreen, green-slate, purplish-rufous, pale rose, purplish-slate or buff; mid. becoming pale or yellowish. St. equal, smooth, shining, hollow when old, white, $G$. rounded, somewhat crowded, white. Flesh white, reddish or purplish beneath pellicle.

Said to be edible. Taste mild, pleasant. Moist places in woods, mixed: common. June-Oct. $3 \frac{5}{8} \times 2 \frac{1}{2} \times \frac{3}{4} \mathrm{in}$.

1338a. R. mitis W. G. Sm. (from the mild taste; mitis, mild) $b$.

$P$. convex, then plano-expanded and depressed, viscid, then smooth, with a separable pellicle, striate and in old examples, tuberculate; mid. pale yellowish, surrounded by purplishmouse-colour or pale rose and purple; marg. yellowish or biscuit. St. stuffed, then hollow, slightly attenuate downwards, rugulose, white. $G$. adnate, branched, veined above, white, then faint straw-colour. Flesh white, tinging to rusty, pale ochre beneath pellicle.

Taste mild. Woods. Aug. $3 \times 2 \times \frac{3}{t}$ in.

1339. R. heterophylla Fr. (from the gills, of different lengths; Gr. heteros, one of two, plullon, a leaf) a $b c$.

$P$. hemispherical to depressed, even, shining, usually apple-green or indigo-apple-green; mid. darker or pale apple-green, clouded brownish or sienna, never reddish or purple. 
St. equal, even, white. $G$. thin, attenuate behind and reaching the stem, crowded, white.

Edible. Taste mild, sweet, nutty, odour of hog's lard, but like lobster or crab when cooked. Woods, etc. ; common. July-Oct. $4 \times 2 \frac{1}{4} \times \mathrm{I}$ in. Var. salochroa Fr. $P$. white to whitish-apple-green.

1340. R. consobrina Fr. (related to 1341 ; consobrinus, a cousin) $a b$.

$P$. campanulate to depressed, viscid, dark cinereous to fuscousolivaceous, tan-umber, tan-sepia, sooty-sepia or yellowishumber ; mid. darker; marg. even. St. even, smooth, shiningwhite, then cinereous. $G$. adnate, crowded, shining-white. Flesh white, ashy under pellicle.

Taste of flesh acrid, gills intensely acrid : not fetid as in 1341. Mixed woods, pine, beech. July-Oct. $3 \frac{5}{8} \times 1 \frac{5}{8} \times \frac{5}{8}$ in. Var. sororia Fr., margin striate. Var. intermedia Cooke. $P$. becoming striate. St. usually attenuate downwards. $G$. dull white.

1341. R. fœtens Fr. (from the fetid odour) a $b c$.

$P$. bullate to expanded, sometimes depressed, viscid in wet weather, sienna- or brown-yellow ; marg. striato-ribbed, at length tuberculate. St. becoming hollow, whitish or yellowish. $G$. adnexed, crowded, exuding watery drops, whitish.

Saicl to be poisonous. Taste acricl, rarely mild; odour fetid-burnt, empyreumatic, very strong in mature examples in wet weather, sometimes slight. Woods; very common. July-Oct. $4^{\frac{1}{4}} \times 3_{\frac{1}{4}} \times \mathrm{I}_{\frac{1}{8}}$ in. 1390 grows on this species.

1342. R. subfœtens W. G. Sm. (from the somewhat fetid odour) $a b$. $P$. convex to plane and subdepressed, somewhat viscid, siennayellowish or buff, paler at the striato-tuberculate marg. St. equal, even, whitish or faintly buff. $G$. thick, branched, distant, yellowish-white.

Taste slightly acrid and bitter; odour somewhat disagreeable. Grassy places. Aug.-Sept. $3 \frac{1}{\frac{1}{4}} \times 2 \times \frac{3}{4}$ in. Whole plant tough, elastic.

1343. R. fellea Fr. (from the bitter-acrid taste; fellens, full of gall) a $b c$.

$P$. hemispherical to convex, smooth, lustrous, straw-colour to ochre or buff; mid. brownish. St. equal, even, white to straw or pale yellowish, or shaded pale buff. $G$. adnate, more or less crowded, thin, exuding drops in wet weather, pale yellowish or faint buff.

Poisonous. Taste intensely bitter and acrirl; odour none. Woods, beecl, open places; common. Aug. -Nov. $4 \frac{1}{4} \times 2 \frac{1}{2} \times \frac{3}{4}$ in.

1344. R. elegans Bres. (clegans, pretty) $a b$.

$P$. hemispherical to depressed, glutinous and densely granulate, bright rosy flesh-colour, soon ochreous at marg. or pale crimson ; mid. purple-crimson or rose-buff, spotted crimson or purplish-crimson; marg. tuberculose. St. equal, becoming hollow, subrugulose, white, ochre-buff below. $G$. adnexed or rounded-adnate, crowded, rarely furcate, whitish, ochre above, becoming orange-ochre with age. Flcsh very firm, white, ochre-buff with age.

Woods, moist. Sept. $3 \frac{5}{5} \times 2 \times \frac{3}{7}$ in. 
1345. R. Queletii Fr. ex Quél. (after Dr. Lucien Quelet) a $b c$.

$P$. convex to infundibuliform, even, viscid, intense blackishcrimson or purple-crimson, sometimes paler at marg. or wholly intense purple, blue-purple or slate. St. equal or attenuate downwards, mealy, dark or pale-purple to faint rose, sometimes paler above. $G$. adnexed, exuding drops, white to biscuit, at length bluish-ashy or cinereous spotted. Flesh white, colour as P. under pellicle.

Taste acrid. Woods, fir ; common. Auy.--Nov. $2 \frac{3}{4} \times 2 \frac{1}{2} \times \frac{1}{2}$ in.

1346. R. expallens Gill. (from its losing colour; expallens, becoming pale) a $b$.

$P$. convex to depressed, viscid, deep bright crimson-purple or deep claret-purple; mid. darker, sometimes almost black, losing colour and becoming livid-cream, with mid. dull pale purplish. St. equal, mealy, rose-purple, paler or somewhat buff at base. $G$. adnexed, pallid yellowish or somewhat lemon. Flish purple, pale yellowish or white.

Taste intencely acrid. Under trees. Sept.-Oct. $3 \frac{3}{4} \times \frac{31}{\frac{1}{4}} \times \frac{3}{4}$ in.

$$
\text { e. Fragiles. }
$$

1347. R. emetica Fr. (from its acting as an emetic) $a b$.

$P$. convex to plane or slightly depressed, lustrous, blood-red to rose, sometimes bleaching to almost white or purplish, tawny when old. St. equal, even, longitudinally rugulose, white or reddish. $G$. adnexo-free, somewhat distant. Fles/ white, rose-colour under the separable pellicle.

Poisonous. Taste very acrit. Woods and open damp places ; common. July-Lec. $\quad 4 \frac{1}{t} \times 3 \frac{1}{\frac{1}{t}} \times \frac{i}{5}$ in. Var. Clusii Fr. Flesh white, turning yellowish. Var. fallar Fr. is a form of 1354.

1347a. R. luteotacta Rea (from the change of colour to light yellow when bruised; luteus, yellow, tactus, a touch) $b$.

$P$. hemispherical, then plane and subumbonate, ruguloso-rivulose, carmine-scarlet, becoming whitish at the striate marg. St. equal, or slightly attenuate downwards, rugulose, white, shaded pale lilac, yellowish when bruised. $G$. furcate, adnate, very light straw-colour, yellow when bruised. Flesh white, colour as $\mathrm{P}$. under pellicle, tinging yellowish when bruised or slug-eaten.

Taste of flesh acrid, of gills very acrid. On the ground, woody places. Aug. $1 \frac{5}{8} \times 1 \frac{7}{8} \times \frac{1}{3}$ in.

1348. R. fingibilis Britz. (from its seeming distinctness; fingibilis, inaginary) $a$.

$P$. convex to depressed, viscid, yellow, with orange or brownish mid. ; marg. not striate. St. equal, white or faintly yellowish. $G$. somewhat crowded, thin. Flesh white.

Taste mild; odour none. Amongst grass under trees. July, $2 \frac{1}{2} \times 1 \frac{7}{8} \times \frac{1}{2}$ in. 
1349. R. pectinata Fr. (from the sulcate margin of the pileus, like the teeth of a comb ; pecten, a comb) $a c$.

$P$. convex to flat or slightly depressed, rigid, viscid, yellowishumber or dull ochre, with a dark or purplish mid., becoming pale; marg. tuberculoso-sulcate. St. equal, spongy-stuffed, rigid, slightly striate, white or faintly greyish. $G$. attenuatofree, subcrowded.

Suspected poisonous. Taste somewhat acrid; odour weak, nauseous, somewhat like 1341. Woods, mixed. Aug. $3 \times 1 \times 5 \times \frac{5}{8}$ in.

1350. R. ochroleuca Fr. (from the usually yellowish-white pileus; Gr. ochros, pale yellow, latkos, white) a b c.

$P$. convex to slightly depressed, lustrous, deep or light yellow, becoming pale, never reddish: marg. striate when old. St. equal, slightly reticulato-wrinkled, white to lemon-white, becoming pale steel-grey. $G$. rounded, white or pale yellowish.

Taste of flesh mild, gills acrid ; odour none. or pleasant. Woods, chiefly fir; frequent. July-Nor. $3 \frac{5}{8} \times 2 \frac{1}{4} \times \frac{3}{4}$ in. Var. claroflas'ta Cooke. $P$. deep chrome-yellow. $G$. white, becoming pale lemon then somewhat ochraceous.

1351. R. granulosa Cooke (from the, at length, minutely granular pileus and stem) $a b$.

$P$. convex to subinfundibuliform, pale or ochreous yellow; mid. darker; marg. paler. St. equal, white above, as P. or somewhat sienna below. $G$. adnexed or adnate, rarely furcate, somewhat crowded.

Taste of flesh mild, gills acrid; odour sour. Under trees. Aug.-Sept. $3 \frac{3}{4} \times 2 \frac{1}{2} \times \frac{3}{4}$ in.

1352. R. æruginea Fr. (from the verdigris-green pileus; aruginens, like verdigris) a $b$.

$P$. convex to slightly depressed, even, somewhat dry, sometimes olive or umber-yellowish, blotted with olive: mid. darker. St. equal, even, smooth, white. $G$. attenuate behind and slightly adnexed, somewhat distant.

Taste mild, pleasant. Woods. Aug. $4 \frac{3}{2} \times \mathrm{I}_{\frac{7}{5}} \times \mathrm{I}_{\frac{1}{5}}$ in.

1353. R. citrina Gill. (from the citron-coloured pileus) $a b$.

$P$. convex to depressed, smooth, sulphur-lemon with mid. brassyyellow, sometimes faintly greenish with mid. becoming pale ochreous. St. equal, striate, white to faintest lemon. $G$. attenuate and furcate behind, adnexed. Flesh white, brassyyellow under pellicle.

Taste sweet to acrid; odour almost obsolete. Woods, mixed. Aug.-Oct. $3 \frac{1}{4} \times I_{\frac{3}{4}} \times \frac{5}{3}$ in.

1354. R. fragilis Fr. (from the brittle substance) a b $c$.

$P$. convex and sometimes umbonate to depressed, crimson, purple, rose-flesh-colour to buff or white, often clouded, changing colour; mid. darker or fuscous, not red under the 
pellicle. St. equal, slightly striate, white. G. adnexed, thin, crowded, ventricose.

Taste very acrid. Moist places in woods; common. Aug.-Oct. $2 \frac{5}{5} \times 1 \frac{7}{3} \times \frac{3}{8}$ in. Var. nizer Cooke. $P$. white. Var. violacea Cooke. $P$. violet. Var. fallax Mass. $P$. dirty reddish, or variously coloured as mid. pale greyishslate and marg. rose, or mid. brown and marg. rose and white. Included under 1347 by Fries.

1355. R. punctata Gill. (from the dotted pileus; punctatus, dotted) $a$. $P$. convex to depressed, rosy or purple, punctate with dark rufous pointed tubercles, becoming pale; mid. darker; marg. sometimes cream. St. attenuate downwards, colour as P., base whitish, non-punctate. $G$. adnexed, white to yellowish, edge often reddish.

Taste mild, sweet. Woods, grassy places. July. $2 \frac{1}{2} \times I \frac{1}{2} \times \frac{3}{3}$ in. Var. lencopus Cooke. St. white.

1356. R. veternosa Fr. (from its uncertain affinities; i'eternosus, languid) $a b$.

$P$. hemispherical to slightly depressed, deep to pale rose or purplish; mid. usually yellowish or whitish; marg. plane. St. equal, even, white. $G$. adnexed to adnate, crowded, white then straw-colour.

Suspected poisonous. Taste acrid. Woods, amongst grass under trees. July-Sept. $2 \frac{3}{4} \times 2 \frac{3}{8} \times \frac{5}{5}$ in.

1357. R. integra Fr. (from its frequently perfect form; integer, entire) $a b c$.

$P$. convex to depressed, viscid, red, purplish-crimson or rose with marg. buff, or tan, clouded crimson, or dull yellowish, clouded pale crimson-brown, or pale yellow with ochre mid. and purple marg., or umber, slate-purple or almost black; marg. sulcate and tubercular. St. somewhate ventricose, white, sometimes faintly rose or pale yellowish. $G$. adnexo-free, very broad, subdistant, white, sometimes rose or pale purple with ochreous base. Pileus sometimes bleaching, almost white.

Taste mild to astringent. Woods, chiefly pine, oak; common. June-Nor. $5 \times 23 \times I_{4}^{\frac{1}{4}}$ in. Var. alba Cooke. Whole plant, except gills, creamwhite.

1358. R. decolorans Fr. (from the changeable colour of the pileus; dicolorans, changing colour) $a b$.

$P$. spherical to slightly depressed, orange-red becoming light yellow, sometimes olive-ochre or variegated pale yellow, orange or vermilion. St. rugoso-striate, white, shaded steelgrey or faintest yellow-olive. $G$. rounded-free, white then yellowish.

Taste mild. Woods, pine, mixed; uncommon. Aug.-Sept. $3 \frac{1}{2} \times 3 \frac{3}{3} \times \frac{3}{\frac{1}{4}}$ in.

1359. R. aurata Fr. (from the golden-yellow pileus; anmm, gold) a $b c$.

$P$. hemispherical to plane: mid. scarlet, orange or red: nıarg. lemon; sometimes mid. sulphur; marg. vermilion. St. 
obsoletely striate, white to lemon. $G$. rounded-free, whitish to light yellow, bright lemon at edge. Flesh white, as P. under pellicle.

Said to be edible, but suspicions. Taste mild to slightly acrid; odour pleasant, sweet. Woods, oak; uncommon. June-Oct. $4 \frac{3}{4} \times 2 \frac{1}{8} \times \frac{7}{8}$ in.

1360. R. Barlæ Quél. (after J. B. Barla) a b.

$P$. convex to depressed, even, peach-colour, yellow, tinged with orange-red or clouded ochre, dull crimson, reddish or coppercolour; mid. darker. St. equal, silky-pruinose, white to faintly yellowish or reddish. $G$. white, then pallid-ochreous, sometimes orange.

Taste mild, sweet; odour of crab, or melilot. Amongst grass under trees. July-Oct. $3 \frac{3}{5} \times 1 \frac{5}{8} \times \frac{5}{8} \mathrm{in}$.

1361. R. nitida Fr. (from the shining pileus; nitidus, shining) $a b c$.

$P$. convex to slightly depressed, bay-brown-purplish, purplishcrimson, crimson-brown, crimson, or purple-white; mid. darker ; marg. tuberculose. St. equal, even, white or faintly clouded rose. $G$. adnexed, crowded, bright lemon.

Suspected poisonous. Taste mild to nauseous or somewhat fetid; odour strong, unpleasant, sometimes obsolete. Woods, fir ; uncommon. JulySept. I $\times 1 \frac{1}{2} \times \frac{3}{8}$ in. Var. cuprea V. G. Sm., Agaricus cupreus Kromb. $P$. copper-colour. Var. pulchralis W. G. Sm., Russula pulchralis Britz. $P$. spotted red or purple at mid., ochreous at marg., or the reverse.

1362. R. alutacea Fr. (from the colour of the gills; aluta, tanned leather) $a b c$.

$P$. hemispherical to depressed, even, crimson, red, rose, purple, black-purple, black, olive, greenish, rarely æruginous, becoming pale; mid. often darker; marg. tubercular. St. equal or slightly clavate, white, usually clouded red, rose or purplish, sometimes deep crimson. $G$. adnexed or adnate, light yellow to bright deep ochreous. Flesh white to pale yellowish.

Edible. Taste mild, pleasant, acrid when old. Woods, beech, mixed; common. July-Oct. $5 \frac{1}{4} \times 3 \times I_{\frac{1}{8}}$ in.

1363. R. armeniaca Cooke (from the colour of the pileus and the odour; armeniacus, of the colour of the apricot) $a b$. Very fragile.

$P$. convex to depressed, smooth, even, orange, ochre, shading into sienna, sometimes lemon-colour. St. equal or slightly attenuate upwards, smooth, white. $G$. adnato-rounded, somewhat distant, yellowish to ochre and egg-yellow.

Taste mild; odour sweet of apricot. Woods, amongst grass under trees. Aug. $2 \frac{1}{4} \times 2 \frac{1}{4} \times \frac{5}{16}$ in.

1364. R. puellaris Fr. (from its small size and grace; puellaris, girlish) $a b$.

$P$. hemispherical, obtuse to depressed, purplish, purple, rose or pink-orange; mid. darker, sometimes almost black, becoming pale, not shining; marg. tuberculate. St. equal, fragile, white, 
becoming yellowish or clouded pale rose. $G$. adnate or adnexed, thin, crowded, white, then pallid yellow.

Taste mild. Woods, birch, pine, waysides, amongst Sthagmum. Aug.Oct. $I_{4}^{3} \times 2 \frac{3}{8} \times \frac{5}{16}$ in. Sometimes turning pale, foxy and yellow where bruised. Yar. intensior Cooke. $P$. deep purple; mid. nearly black. Var. roseipes Cooke. $P$. rose, rosy-orange, becoming blanched. St. white, partially rosy, not changing to sienna where bruised. All the forms grow in company.

1365. R. ochracea Fr. (from the ochreous pileus) $a$.

$P$. hemispherical to infundibuliform, shining; marg. sulcate. $S t$. equal, striate, yellow, pale yellowish or white. $G$. roundedadnexed, scarcely crowded, furcate at base, ochreous. Flesh white to ochreous.

Taste mild. Woods, fir, mixed, grassy places. Aug. $3 \frac{1}{4} \times \mathbf{1}_{\frac{3}{4}}^{\frac{3}{4}} \times \frac{3}{4}$ in.

1366. R. lutea Fr. (from the yellow pileus; luteus, yellow) a $c$.

$P$. convex to plano-depressed, becoming pale, sometimes white, varying orange with scarlet mid.; marg. striate. St. equal, even, white, shaded greyish, never reddish. $G$. adnexo-free, crowded, all equal, egg-yellow.

Taste mild; odour pleasant of apricot, or almost obsolete. Woods; uncommon. July-Oct. $22^{\frac{3}{4}} \times 2 \times \frac{3}{8}$ in.

1367. R. nauseosa Fr. (from the nauseous taste and odour) a b $c$.

$P$. hemispherical to depressed, viscid, bright yellow or purplish to livid, becoming pale or whitish, never red or reddish, with mid. deep ochre; varying orange or yellow with mid. darker; marg. tuberculoso-striate. St. slightly striate, equal or subclavate, whitish. G. adnexo-free, ventricose, white or yellowish.

Tasle acrid, sometimes mild ; odour fetid to feeble, strong, sour, like decaying oal-cake. Woods, chiefly pine. Sept.-Oct. $2 \frac{1}{1} \times 2 \frac{1}{8} \times \frac{3}{x}$ in. Often resembling 1341. Var. flavida Cooke. $P$. clear primrose-yellow.

1368. R. vitellina Fr. (from its egg-yellow colour; witellus, yolk of egg) a $c$.

$P$. convex to plane, light yellow to pallid; marg. striato-tuberculate. St. equal, white. $G$. adnexo-free, equal, distant, somewhat thick, connected by veins, saffron.

Taste mild ; odour strong. Woods, fir, mixed ; uncommon. Aug.-Sept. I $\times$ I $\times \frac{1}{4}$ in. There is a large form with P. $2-3$ in. in diam.

1369. R. chamæleontina Fr. (from its changing colour, chameleonlike) $a b$.

$P$. convex to slightly depressed, flesh-colour, changing to yellow at mid., then wholly yellow, sometimes purple, olive with slate mid., scarlet, crimson, pale rose or vermilion with yellow mid. St. equal, slightly striate, white. $G$. adnexed, thin, crowded, equal, somewhat forked, yellow.

Taste mild, sweet; odour none. Woods, pine, beechl, downs; rare. Sept. Oct. $\quad I_{\frac{1}{2}}^{\frac{1}{2}} \times I_{\frac{1}{4}} \times \frac{1}{4}$ in. 


\section{CANTHARELLUS Juss.}

(From the cup-shaped pileus of some of the species;

Gr. kantharos, a cup.)

Veil obsolete. Hymenophore continuous and homogeneous with the stem, when latter is present. Pileus fleshy to membranous. Stem

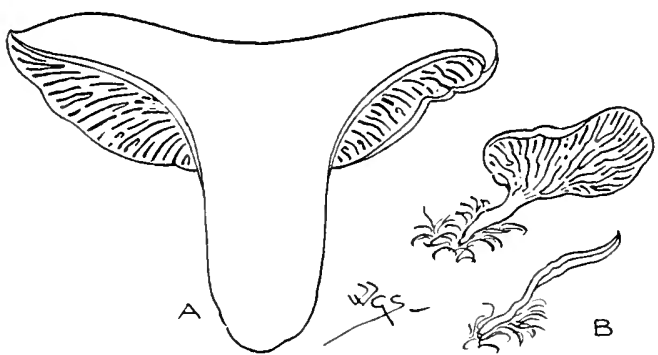

Fig. 64.-A, section of Cantharellus cibarius Fr. One-half natural size. B, C. muscigenus Fr, entire and in section. Natural size.

central, excentruc or obsolete, exannulate, without cartilaginous bark. Gills decurrent, thick, fleshy-waxy, fold-like, somewhat branched, edge obtuse, trama floccose. Spores white. (Fig. 64.)

The species grow on the ground and on mosses, rarely on wood. Some are edible, others are said to be poisonous. Sporodinia Aspersillus sometimes grows on Cantharelli. Species 1370-1388

a. Mesopoda. Stem central.

Pileus entire, solid. Stcm fleshy. 1370-1376

Pileus somewhat membranous. Stem tubular, shining.

$1377-1384$

b. Pleuropode. Dimidiate. Stem lateral.

1385,1386

c. Resupinate. Resupinate.

1387,1388

\section{a. Misopode.}

1370. C. cibarius Fr. (from its use as food; cibaria) a b c. Eggyellow.

$P$. convex to depressed, turbinate, smooth; marg. often repand and irregular. St. solid, attenuate downwards. $G$. shallow, narrow, much swollen.

In troops, clusters or rings. Edible. Taste pleasant; odour when mature strong of apricots or ripe greengages, sometimes heavy or earthy and somewhat pungent. Woods, chiefly beech, oak, chestnut; common. July-Dec. $+\times 1 \frac{3}{4} \times \frac{7}{8}$ in. Sold in Italian markets under the name of Gallinaccio; at Cordova under the name of Encina; in French markets in a dried state on strings. Var. ruffices Gill,, red at base of stem. There is a white and a yellowish-white var. 
1371. C. Friesii Quél. (after Elias Fries) a $b c$.

$P$. convex to infundibuliform, Heshy, villous, somewhat orange. St. solid, attenuate downwards, yellowish-white or pale orange. $G$. narrow, yellow or orange.

Woods, on elder. Aug.-Nov, $1 \frac{1}{2} \times \frac{7}{5} \times \frac{3}{16}$ in. Intermediate between 1370 and 1372.

1372. C. aurantiacus Fr. (from its golden yellow colour; aurantiacus, orange-yellow) a $b c$.

$P$. plane to infundibuliform, somewhat thin, subtomentose; marg. undulate, at first involute, orange-ochreous; mid. sienna. St. stuffed to hollow, attenuate downwards, ochreous or reddish, sienna or black at base. $G$. crowded, thin, deep orange or orange-vermilion.

Gregarious. Said to be poisonous. Taste unpleasant. Woods, chiefly pine, heaths, on elder, on grass-roots. Oct.-Dec. $3^{\frac{1}{2}} \times 2 \times \frac{7}{\frac{1}{4}}$ in. Possibly a Clitocybe rather than a Cantharellus. There is a white and a yellowish-white var. with white gills. A mould, Dactylium dendroides, the conidial stage of Hypomyices rosillus, is common on the gills of this fungus.

1372a. C. hypnorum Brond. (from its habitat, moss, Hypmum).

$P$. campanulato-convex then expanded and slightly depressed; marg. incurved, minutely downy, pale primrose-yellow to pale ochre. St. slender, slightly flexuous, almost glabrous, yellow, sometimes darker than $P$. near base. $G$. thin, edge acute, somewhat crowded, branched, decurrent, yellow. Flesh thin, whitish.

Cudworth, Yorks. Sept. 1905. I I $\frac{1}{2} \times 1$ in. Allied to 1372, but differing in colour, $P$. less tomentose and spores smaller.

1373. C. Brownii B. \& Br. (after J. Brown) a b. Ochreous-white.

$P$. convex, obtuse or umbonate, thin, obscurely silky. St. stuffed, equal, somewhat furfuraceous. $G$. subdecurrent, linear, narrow, subdistant, sometimes branched.

Amongst grass. Autumn. $5 \times 1 \frac{1}{2} \times \frac{1}{16} \mathrm{in}$.

1374. C. carbonarius Fr. (from its habitat, burnt wood and earth; carbo, charcoal) a $b c$.

$P$. convexo-umbilicate to infundibuliform, striato-squamulose, date-brown to dark purple-slate and black. St. solid, attenuate downwards, often deeply rooting, yellowish-white above, as P. below. $G$. narrow, white.

Often fasciculate. Charcoal beds, burnt ground. July-Dec. $2 \frac{3}{3} \times \mathbf{I} \times \frac{1}{2}$ in. ; rooting stem $2 \frac{1}{2}$ in. Often grows in company with 183 and 277 , to which small examples of 1374 are not unlike.

1375. C. umbonatus Pers. (from the sometimes umbonate pileus, a $b c$.

$P$. convex and umbonate to depressed, even, dry, floccoso-silky, cinereous-blackish, somewhat pale slate or umber-slate. St. 
stuffed, equal or slightly attenuate upwards, cinereous. G. thin, crowded, white to faintly brownish.

Gregarious. Taste and odour almost obsolete: Woods, amongst mosses; rare. April-Oct. $\mathbf{I} \frac{1}{4} \times 3 \times \frac{1}{5}$ in. Sometimes becomes reddish when bruised.

1376. C. albidus Fr. (from the whitish colour; allus, white) a $c$. Whitish to faintly yellowish.

$P$. subinfundibuliform, irregular, splitting, tough, somewhat faintly zoned. St. solid, equal or attenuate downwards, smooth. $G$. thin, subcrowded, branched.

Taste and odour heavy, earthy. Pastures, mossy places, amongst larch and fir. Sept.-Oct. $2 \times \frac{7}{5} \times \frac{1}{4}$ in. A form occurs with the gills distant and thick.

1377. C. leueophæus Nouel (from its white gills; Gr. leukos, white, fhaino, to appear) a $C$.

$P$. depressed to deeply infundibuliform, smooth, tough, umber. St. slightly attenuate upwards, even, colour as P., dilrker at base. $G$. distant.

On the ground. Autumn, $1 \frac{3}{5} \times \mathrm{I} \frac{1}{\times} \times \frac{1}{5}$ in.

1378. C. Houghtonil Phill. ex Cooke (after the Rev. William Houghton) $a b$.

$P$. umbilicato-depressed, smooth, dull white, somewhat fleshcolour; marg. waved, iregular. St. attenuate downwards, delicately fibrillose, rooting, colour as P. G. narrow, scarcely forked, pallid flesh-colour to salmon.

Single or subcrespitose. On the ground. Autumn. $2 \frac{1}{2} \times 3 \times \frac{1}{4}$ in.

1379. C. tubæformis Fr. (from the trumpet-shaped, mature pileus; tuba, a trumpet, forma, form) a $b c$.

$P$. umbilicato-convex to deeply infundibuliform, flocculose, umber or yellowish-umber, sometimes faintly olive-shaded, sometimes perforate to base of St.; marg. wared. St. compressed, lacunose, smooth, orange-yellow, paler above and below. $G$. distant, much branched, rellow-fuliginous or yellowishumber.

Gregarious. Woods, often on fallen branches, twigs, rotten wood, and on the ground; common. Aug. $-\mathrm{Nov} .4 \frac{3}{\frac{3}{4}} \times 4 \frac{\frac{1}{3}}{5} \times \frac{3}{4}$ in. Intermediate forms occur between this and 1380. Var. lutescens Fr. St. pale yellowish.

1380. C. infundibuliformis Fr. (from the shape; infunditulum, a funnel, forma, form) a $b$.

$P$. convex, umbilicate to infundibuliform, sometimes pervious to base of St., floccoso-wrinkled, yellow-cinereous or fuliginous, paler when dry, sometimes pale purplish-lavender; mid. deep siemna-umber; marg. undulate. St. even, smooth, light or bright yellow, sometimes pale purplish-lavender above. $G$. light yellowish to cinereous.

Gregarious; somewhat crespitose. Suspected poisonous. Woods, chips, rotten wood, on the ground; common. July-Jan. $2 \frac{1}{5} \times 2 \frac{3}{8} \times \frac{3}{16}$ in. Intermediale forms occur between this and 1379 . 
1381. C. cinereus Pers. (from the cinereous gills) a b $c$.

$P$. infundibuliform, often pervious to base of St., villoso-squamulose; marg. lobed, waved and very irregular, hoary-fuliginous or streaky umber. St. attenuate downwards, smooth, fuliginousblackish or gradually dark umber to base. $G$. somewhat pale lavender.

Woods, beech, on the ground. Sept.-Nor. $2 \times 2 \times \frac{3}{8}$ in. Must not be confounded with 1782 .

1382. C. cupulatus Fr. (from the cup-shaped pileus; cufa, a cup) $a b c$.

$P$. when young strongly umbonate, then plane to cup-shaped, with um. sometimes at bottom of cup, smooth to flocculose, pallid fuscous, pale and slightly rufescent when dry, or pale umber or brownish-buff; marg. striate. St. equal, tough, smooth, pale buff or paler than P. $G$. very distant, much branched, grey, lavender or yellowish-brownish.

Waste places, on the ground; rare. Oct. $\frac{5}{8} \times \frac{3}{7} \times \frac{1}{16}$ in. Must not be confounded with 1448 .

1383. C. Stevensonii B. \& Br. (after the Rev. John Stevenson) a $c$.

$P$. convexo-umbilicate, smooth, buff-white. St. delicately pulverulent, white to buff-white. $G$. pallid, becoming fuscous in front.

Rotten stumps and wood amongst moss. Nar.-April. $\frac{3}{8} \times \frac{3}{4} \times \frac{1}{32}$ in.

1384. C. replexus Fr. (from the margin of the pileus, bent back, repand) a $b$.

$P$. membranous, convex to depressed, subumbonate, fuscous to cinereous or umber-yellowish; marg. striate. St. smooth, equal or slightly thickened above, white above, lavender below. $G$. adnate to decurrent, hoary-white, cinereous or pale purple.

On the ground, amongst grass, moss and leaves. Oct. -Nov. $\frac{5}{8} \times \frac{7}{3} \times \frac{1}{16}$ in. With the habit of a mj'cena. Var. devixus Fr. P. hood-shaped.

\section{b. Plimopode.}

1385. C. muscigenus Fr. (from the habitat; muscus, moss, grgno, to bear) a b $c$.

$P$. membranous, spathulate, smooth, undulate, fuscous, cinereouswhitish when dry, or umber-yellowish or buff. St. horizontal and continuous with P., colour as P., villous at base. $G$. distant, branched, colour as P.

On larger mosses, Hypnum, Tortula, sometimes on the ground: uncommon. June-Nov. $\frac{3}{4} \times \frac{1}{8} \times \frac{1}{16}$ in.

1386. C. glaucus Fr. (from the sea-green colour; glaums, sea-green) a $b c$. Grey or indigo-whitish.

$P$. membranous, spathulate, silky, zoneless. St. pruinate. $G$. distant, dichotomous.

On the ground, sandy slopes. Autumn. $\frac{1}{2} \times \frac{3}{16} \times \frac{1}{16}$ in. 


\section{c. Resupinatie.}

1387. C. retirugus Pers. (from the reticulate gills; rite, a net, ruga, a wrinkle) $a b c$.

$P$. sessile, inferior, membranous, irregular, repando-lobed, cinereous-fuliginous, or pale fuliginous over biscuit $G$. superior, radiating from a central or lateral point, whitish-fuliginous or paler than $P$.

On sticks and mosses in bogs, Hytnum; uncommon. April-May. P. ${ }_{4}^{3}$ in. in diam. Sometimes wholly salmon or biscuit.

1388. C. lobatus Fr. (from the lobed pileus) $a b c$. Dark fuscous, pale when dry.

$P$. sessile, inferior, membranous, irregular. $G$. superior, radiating from a central or lateral point, somewhat distant, branched.

On mosses in bogs, Hypmum cuspidatum, H. revolens, Sphag'num, mossy soil; uncommon. Spring to winter. P. $\mathrm{I} \frac{1}{8}$ in. in diam.

\section{NYCTALIS Fr.}

(From the habit, growing in dark places; Gr. mux, night.)

Fill obsolete or indefinite. Hymenophore continuous and homogeneous with the stem. Pilcus fleshy. Stem central, exannulate,

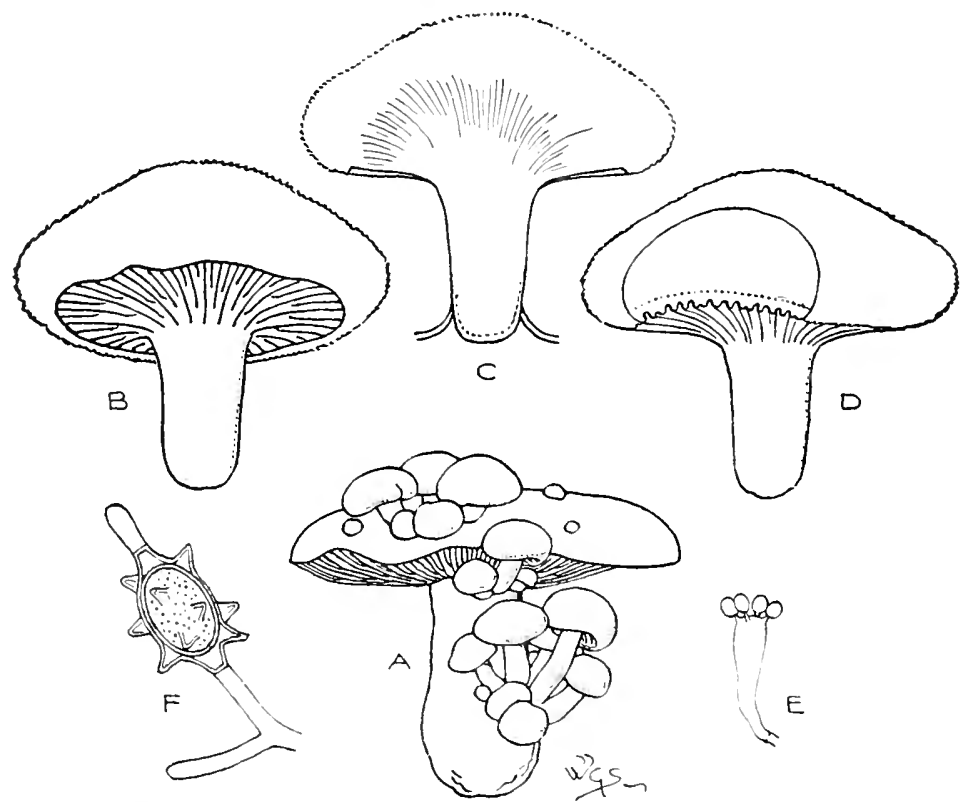

Fig. 65.- Vyctalis asterophon Fr. A, plants growing on Russula adusta. Ore-half natural size. $B, C$, entire and in section; $D$, with slice removed from edge, showing transverse section of vein-like gills. $\times_{2}$. E, basidia and spores; F, conidium. $\times_{4}$ oo. 
without cartilaginous bark. Gills fleshy, thick, juicy, obtuse at the edge, distant, adnate. Spores elliptical, subglobose, colourless. Reproduction is also effected by conidia borne on the pileus. (Fig. 65.)

Parasitic on the Agraricacee, gregarious and fasciculate.

Species 1389, 1390

1389. N. asterophora Fr. (from the minute stellate conidia borne on the pileus; aster, a star, fero, to bear) $a b c$.

$P$. hemispherical, floccoso-pulverulent, white to fawn. St. solid, equal, whitish to brownish. $G$. very shallow, thick, grey-white.

Gregarious or subcespitose. Parasitic on 229, 1306, 1307 and a few other species of Agaricacea ; frequent. July-Nov. $\frac{3}{7} \times \frac{3}{4} \times \frac{3}{16}$ in. The stellate conidia on the pileus have been named Hypomyces asterofhorus.

1390. N. parasitica Fr. (from its parasitic habit) a b c.

$P$. conico-campanulate to expanded, obtuse or umbonate, pruinose, whitish fuscous to pale, or whitish-grey with mid., faint sienna. St. fistulose, slightly attenuate upwards, pubescent, white. $G$. somewhat broad, thick, distant, very pale rose-brown.

Gregarious or cespitose. Parasitic on 1306, 1307, 1311 and 1341; common. Aug. - Nov. I $\times \mathbf{I} \frac{7}{8} \times \frac{1}{8}$ in. Often wholly lavender-steel colour. The pileus produces large elliptical, smooth conidia.

\section{MARASMIUS Fr.}

(From the habit, drying or shrivelling, not becoming putrid; Gr. maraino, to wither or shrivel.)

I cil obsolete except in one non-British species-M. subannulatus and a slight indication in the young of 1404. Hymenophore con-

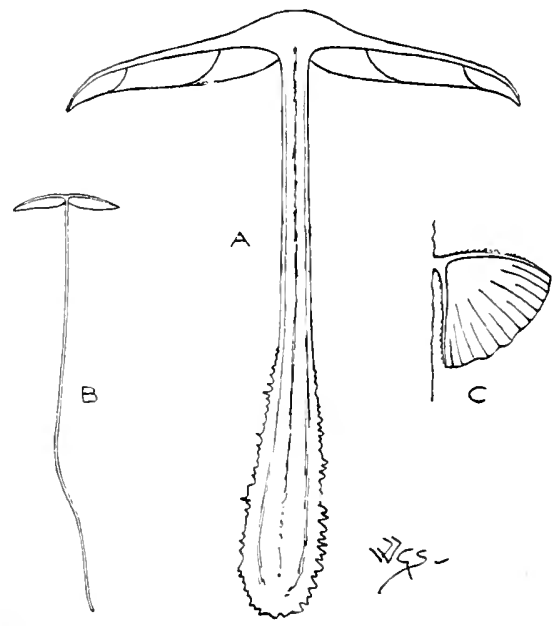

Fig. 66.-A, section of 1/arasmius peronatus Fr. One-half natural size. $\mathrm{F}$, section of H. epiphyllus Fr.: c, ditto, .Y. spodoleucus B. \& Br. Natural size. 
tinuous with but heterogeneous from the stem. Pileus arid, pliant, more or less thin. St'm central, cartilaginous or horny, absent in 1431. Gills usually simple, pliant, somewhat distant, edge acute, entire. Spores white. (Fig. 66.)

The species are commonly small, thin and epiphytal, those which appear to grow on the ground are generally on putrid leaves or roots of grasses; many have a strong odour, sometimes fetid, or of garlic, never of meal. Marasmins is closely allied to Collybia, but the true Marasmii shrivel in dry weather and revive in rain. Some are edible, others are esteemed for flavouring, 1391 is dangerous.

Species 1391 - 1431

A. Pileus fleshy-pliant, at length somewhat coriaceous, sulcate or wrinkled; margin at first involute. St'm somewhat cartilaginous.

a. Scrotex. Stem solid or stuffed, then hollow, internally fibrous, externally cartilaginous and covered with villous down which is easily removed. Gills separating-free.

Stem woolly or strigose at the base.

$1391-1393$

Stcm naked at the base, often composed of twisted fibres.

$1394-1396$

b. Tirgine. Pileus thinner than a in section, hygrophanous, even or striate. Stem rooting, tubular, cartilaginous, not fibrous. Gills separating-free.

Stem woolly downwards, smooth upwards.

Stem wholly pruinato-velvety:

$1397-1400$

$1401-1405$

c. Calopodes. Pileus convexo-involute, then plane or depressed. Stcm curt, not rooting, inserted, often with a basal floccose tubercle. Gills adnate or subdecurrent.

Stem smooth and shining upwards, base simple. 1406-1410 Stem velvety or pruinate, base somewhat tubercular.

$1411-1414$

B. Pilius somewhat membranous, campanulate then expanded; margin at first straight and adpressed to the stem. Stem horny, fistulose, sometimes stuffed, tough, arid. Myclinm rhizomorphoid, corticate.

d. Chordales, Pileus campanulate or convex. Stem rigid, rooting or adnate by a dilated disc.

1415 - 1417

c. Rotula. Pileus soon becoming somewhat plane or umbilicate.

Stem filiform, flaccid, inserted at the base. Growing on leaves.

Stem very smooth, shining.

St'm velvety or pilose.

$1418-1422$

$1423-1430$

c. Pilcus sessile, resupinate. 
A.

a. Scrotice.

1391. M. urens Fr. (from its burning taste: uro, to burn) a $b c$.

$P$. convex to expanded, even or slightly squamulose or rimosorivulose, pale rellowish or brownish: mid. darker. St. solid, slightly attenuate upwards, white or yellowish, wholly whitefloccose. $G$. free to remote, pale yellowish-brown to brownish.

(iregarious, somewhat ceespitose. Poisonous. Taste becoming very pungent. Woods, mixed, grassy places, on leaves, twigs, etc. : frequent. May-Sept. $2 \frac{1}{4} \times 4^{\frac{3}{4}} \times \frac{1}{8}$ in.

1392. M. peronatus Fr. (from the base of the stem, as if booted with strigose down; fere, a kind of boot) a $b c$.

$P$. hemispherical to expanded, slightly wrinkled; marg. striate, whitish-umber. St. stuffed to hollow, attenuate upwards, light yellow or pale brownish, densely woolly-sheathed at base with whitish or yellowish strigose down. $G$. adfixed to free, crowded, whitish, yellowish or pale rufescent.

Taste acrid. Woods, vak, amongst leaves, twigs, etc., bramble, beech-nuts ; common. July-Dec. $3 \frac{1}{2} \times 4 \frac{\frac{1}{2}}{2} \times \frac{3}{15}$ in.

1393. M. porreus Fr. (from its odour of garlic; porrum, the leek) a b $c$.

$P$. hemispherical to flat, coriaceo-membranous, dull yellowish or pale dull brownish ; mid. darker : marg. striate. St. stuffed to hollow, equal, pubescent, whitish or brownish above, deep purple to almost black below. $G$. adnexo-free, distant, light yellowish.

Odour strong, disagreeable, alliaceous. Woods, chiefly amongst oak-leaves, fir : frequent. sept.-Dec. $2 \frac{1}{4} \times 3 \frac{3}{7} \times \frac{1}{5}$ in. Dried examples more than one hundred years old retain the garlic odour.

1394. M. oreades Fr. (from its growing in fairy rings; Gr. orcias, a mountain nymph) a bc .

$P$. fleshy, hemispherical to plane, obtuse or umbonate, even, smooth, whitish, pale yellowish or brownish; mid. darker. St. solid to stuffed, equal, whitish. G. free, broad, distant, whitish or pale yellowish.

Gregarious. Edible. Taste pleasant; odour agreeable, stronger when dry. Pastures, lawns, roadsides; common. May-Oct. $2 \frac{1}{2} \times 2 \frac{5}{8} \times \frac{1}{8}$ in. A colourless ketchup can be made from this. Often pickled or dried for winter use.

1395. M. plancus Fr. (from the-at length flat-pileus; plancus, flat) a $c$.

$P$. convex to plane and depressed, even ; mid. dark rosy-sienna; marg. rufescent or lightish, becoming pale. St. hollow, attenuate downwards, white above, yellowish to brownish below. $G$. free, distant, yellowish or brownish.

Taste mild, sweet. Woods, shady places, on the ground. Autumn. $1 \frac{3}{4} \times 1 \frac{3}{3} \times \frac{3}{16}$ in. 
1396. M. scorteus Fr. (from its leathery substance; scorteus, leathery) a $c$. Whitish or white.

$P$. convex, somewhat obtuse. St. somewhat fistulose, equal, smooth, fuscous when dry. $G$. free, broad, rentricose.

Taste mild. Woods, moist places, amongst grass, under trees. Aug.-Jan. $\frac{3}{4} \times 2 \times \frac{1}{16}$ in. Habit that of a small 1394 .

\section{b. Tersince.}

1397. M. prasiosmus Fr. (from its odour of leek or garlic; Gr. prason, a leek, osme, a scent) a bc.

$P$. campanulate to flat, obtuse, rugulose, pale buff; mid. pale siemna. St. fistulose, pallid above, pale rufous or fuscous below. $G$. adnexed, somewhat crowded, whitish.

Odour strong. Amongst leaves. July-Oct. I $\times 2 \frac{1}{4} \times \frac{1}{8}$ in.

1398. M. varicosus Fr. (from the blood-red juice, as if from a swollen vein, a'aria) a $c$.

$P$. campanulate to plane, umbonate, even, dark rose- or reddishbrown, darker when dry: marg. paler. St. slightly attenuate upwards, claret-brown, almost black below, filled with bloodred juice. $G$. very crowded, umber-fuscous or somewhat dark dull-purple, darker when dry.

Solitary. Odour none. Damp mossy places. Sept.-Oct. $1 \frac{1}{4} \times 2 \times \frac{1}{16} \mathrm{in}$.

1399. M. fuseopurpureus Fr. (from the dark purplish colours) $a b c$. Juiceless.

$P$. hemispherical to plane, slightly umbilicate, somewhat wrinkled, dark to pale purple-brown or tan. St. slightly attenuate upwards, rufous or dark purple, strigoso-downy and rubiginous at base. $G$. distant, rufescent, purple-brown or slate-purple.

Solitary, crespitose or gregarious. Odour none. Woods, on sticks, dead leaves, beech, dead grass, etc.; frequent. Aug.-Oct. $I_{\frac{1}{4}} \times I_{\frac{1}{2}} \times \frac{1}{8}$ in.

1400. M. terginus Fr. (from the leathery substance; torgum, hide, leather) $a b c$. Juiceless.

$P$. campanulate to plane or slightly depressed, shining, fleshcolour to white; marg. striate. St. slightly attenuate upwards, shining, pallid or sulphury above, reddish below. $G$. somewhat crowded, pale yellowish, sulphury or salmon.

Solitary or in pairs. Odour none. Amongst leaves. beech, moss. Oct.Nov. $1 \frac{1}{2} \times 2 \frac{1}{4} \times \frac{1}{5}$ in. This is M. Sterensii B. \& Br.

1401. M. Wynnei B. \& Br. (after Mrs. Lloyd Wynne) a b c.

$P$. convexo-plane, subumbonate, lilac-brown, purplish-lavender or purplish-salmon, clouded pale slate. St. attenuate downwards, colour as P., or yellowish above to brown and brownblack at base within and without. $G$. adnexed or adnate, lilac, lavender or salmon.

Gregarious or caspitose. Odour none. Amongst leaves, twigs, etc.. on the grouncl. Aug.-Dec, I考 $\times 3 \frac{3}{8} \times \frac{3}{15}$ in. 
1402. M. erythropus Fr. (from the red stem; Gr. cruthros, red, pous, a foot) a b c. Juiceless.

$P$. campanulate to flat, obtuse, slightly wrinkled, pale buff with sienna mid., paler at marg., becoming almost white. St. smooth, equal, dark red. $G$. veined, not crowded, whitish to pale salmon.

Single or somewhat crespitose. Odour none. Woods, anıongst dead leaves, beech, beech-mast, near and rarely on stumps; frequent. Sept.-Oct. $2 \frac{1}{5} \times 4 \frac{3}{5} \times \frac{1}{8}$ in. Sometimes becoming purple-black in drying.

1403. M. archyropus Fr. (perhaps an error for arsyropus: Gr. arcuros, silver, pous, a foot, in reference to the white-pruinose stem) a $b c$.

$P$. hemispherical to plane or slightly depressed, smooth, tan or salmon-shaded, becoming pale. St. pale yellowish or pallid rufescent, white pruinose. $G$. crowded, narrow, pallid.

Crespitose. Odour none. Amongst leaves: rare. Oct. $I_{4}^{\frac{1}{4}} \times 3 \times \frac{1}{16}$ in.

1404. M. torquescens Quél. (from the twisted stem; torquen, to twist) a $b$.

$P$. convexo-plane, rugoso-striate, pallid-whitish; mid. tawny or fulvous. St. white ahove to brown or deepest brown below. $G$. thin, finely serrulate, distant, white or whitish.

Woods, amongst onk-leaves, twigs, etc., in moist places. Autumn. $\frac{1}{2} \times 2 \frac{1}{ \pm} \times \frac{1}{16}$ in.

1405. M. impudicus Fr. (from the odour; imfudicus, disgusting) a b c.

$P$. convex to plane, depressed or subumbonate, striato-plicate, bay-brown-rufous or rose-umber: nid. dark umber, becoming pale. St. equal or attenuate downwards, velvety, rufous or violaceous-purple, white downy, white when dry. $G$. free, veined, whitish flesh-colour or salmon. Flesh rose-vinous.

Gregarious. Odour strong, disgusting, like burnt flannel and fetid, more powerful when cut or broken. On and about rotten pine-trunks. on pineleaves. Sept.-Oct. I $\times \mathbf{I} \frac{1}{2} \times \frac{1}{8} \mathrm{in}$.

\section{c. Calopodes.}

1406. M. scorodonius Fr. (from the odour of garlic; Gr. skordion, a plant with the odour of garlic) a $b c$.

$P$. arid, wrinkled, crisped, rufous or yellowish-sienna; mid. vinous, becoming pale to whitish. St. fistulose, equal, horny, rufous, paler above, darker or blackish below. $G$. adnate, veined, whitish.

Usually gregarious. Edible, esteemed for flavouring. Odour strong. Heaths and dry pastures, on twigs and leares; rare. Oct. $1 \frac{3}{8} \times 2 \frac{3}{y} \times \frac{1}{16}$ in.

1407. M. calopus Fr. (from the beautiful stem ; Gr. kalos, beautiful, pous, a foot) a b $c$.

$P$. rarely depressed, even, smooth, whitish. St. fistulose, slightly attenuate upwards, deep rich brown, paler above. $G$. slightly emarginate, white.

Odour none. On twigs, grass-roots, etc. Autumn. $\frac{3}{4} \times \mathrm{I}_{\frac{1}{6}} \times \frac{1}{32} \mathrm{in}$. 
1408. M. Vaillantii Fr. (after Leon Vaillant, French botanist) $a b c$. $P$. tough, arid, plicato-rugose, whitish or pale yellowish. St. stuffed, attenuate downwards, date-brown, paler or whitish above, blackish below. $G$. adnate, subdecurrent, broad, distant, white.

Odour none. On dead wood, fallen twigs, leaves. Sept.-Oct. $\frac{7}{3} \times 1 \frac{1}{1} \times \frac{1}{16}$ in.

1409. M. angulatus B. \& Br. (from the angular folds of the pileus) $a b$. $P$. plicato-angular, fuscous-whitish or yellowish; mid. pale brownish. St. slightly enlarged above and below, base hairy, rufescent-grey or white above, brown-black below. $G$. adnexed, distant, whitish.

On grass, submerged rushes, roots, on sandhills. Aug. $\frac{1}{4} \times I \times \frac{1}{32}$ in.

1410. M. languidus Fr. (from its weak substance; languco, to be weak) $a$.

$P$. slightly fleshy, umbilicate, flocculose, rugoso-sulcate, whitish, light yellowish, or somewhat flesh-colour. St. stuffed, attenuate downwards, naked, pailid to fuscous, white villous at base. $G$. adnate to decurrent, veined, white.

Gregarious. Odour none. On dead grass, twigs. $\frac{5}{8} \times \frac{3}{4} \times \frac{1}{16}$ in.

1411. M. fœtidus Fr. (from its fetid odour) $a b c$.

$P$. convex to Hat, umbilicate, striato-plicate, bay-brown-tawny or yellowish-sienna; mid. alnost black. St. fistulose, attenuate downwards, sometimes with a small floccose tubercle at base, deep brown, almost black below. $G$. adnexed to subdecurrent, distant, veined, yellowish-rufescent or salmon. Flesh claretbrown, becoming pale.

Odour strong of burnt flannel or horse-radish, very fetid. Dead twigs, rotten branches, etc.; rare. Aug. -Jan. $1 \frac{1}{3} \times 1 \frac{1}{2} \times \frac{1}{16}$ in.

1411a. M. lagopinus Post ex Fr. (from the squamulose base of the stem ; Gr. lagopous, hare's foot) $a$.

$P$. convex, then plane, even, glabrous, pallid. St. slender, equal, whitish, upper half glabrous, lower half with white spreading sq. $G$. adnate, slightly ventricose, straw-colour.

Gregarious. Dead branches. Oct. $\frac{5}{8} \times \frac{1}{2} \times \frac{1}{20}$ in.

1412. M. amadelphus Fr. (from its nearness to 1413 ; Gr. $a n$, together, adelphos, a brother) a $c$. Pale buff.

$P$. arid, convex to plane, umbilicate, striate; mid. darker. St. stuffed, equal, sometimes with an almost obsolete tubercle. $G$. alnato-decurrent, distant.

Gregarious. Odour none. Dead branches, twigs; rare. Sept.-Dec. $\frac{3}{8} \times \frac{1}{1} \times \frac{1}{32} \mathrm{in}$.

1413. M. ramealis Fr. (from its habitat; ramus, a branch) a $b c$.

$P$. arid, convex to plane, obtuse or depressed, white; mid. rufescent. St. stuffed, whitish, rufescent at base, sometimes with a small tubercle. $G$. adnate, subdistant, whitish.

Densely gregarious. Odour none. Dead twigs, branches, burnt sticks, juniper, willow, bramble; common. May-Jan. $\frac{1}{2} \times \frac{1}{2} \times \frac{1}{16}$ in. 
1414. M. eandidus Fr. (from its white colour : candidus, shining white) at $b i$.

$P$. campanulate to expanded or depressed, becoming sulcatorugulose, wholly white or with mid. pale brown. St. stuffed, base floccose, white with a brownish base or wholly white. $G$. adnexel, ventricose, distant.

Gregarious, twigs, rotten stems, pine, cedar, leaves. Sept. - Nor. $3 \times \frac{1}{2} \times \frac{1}{32}$ in.

B.

\section{d. Choridules.}

1415. M. alliaceus Fr. (from its odour of garlic, allinm) $a b c$.

$P$. campanulate to expanded, subumbonate, smooth, dry, striate, white or faintly ochre to fuscous. St. fistulose, minutely velvety, blackish or deep dull purple, paler above. $G$. adnexed, ventricose, subdistant, whitish or fuscous-whitish.

Odour strong, persistent. Woods, leaves, rotten wood: frequent. Aug.Ocl. I I $\times 4 \times \frac{1}{15}$ in.

1416. M. caulicinalis Fr. (from its habitat-growing on stalks and stems: canlis, a stem) a b c.

$P$. striate, bright or dull yellow or yellowish-sienna. St. fistulose, equal, floccoso-villous, sulphur above, dark siemna below. $G$. adnato-decurrent, subdistant, veined, pallid light yellow or pale sulphur.

Taste very bitler. On fir-cones, leaves, etc.; rare. Aug.-Nov. $I \times I \frac{7}{3} \times \frac{1}{15}$ in.

1417. M. torquatus Fr. (from the sometimes twisted stem; torquce, to twist) a $b$.

$P$. membranous, campanulate, obtuse, plicato-sulcate, whitish; mid. bright siemna. St. hollow, smooth, shining, base dilatedtuberculose, ivory. G. adnate, distant, thick, connected by veins, forming a collar near St., white. Flesh in I'. pale sienna, elsewhere white.

stems and leaves. Autumn. $3 \frac{1}{2} \times 1 \frac{1}{8} \times \frac{1}{3}$ in.

$$
\text { i: Rotulie. }
$$

1418. M. rotula Fr. (from the resemblance of the pileus-seen from below - to a little wheel; rota, a wheel) a b c.

$P$. arid, hemispherical, umbilicato-depressed, whitish or brownish ; marg. undulato-crenulate. St. fistulose, horny, often branched, sometimes barren, blackish, paler above. $G$. adnate to a free collar, broad, few, distant, white.

Gregarious or caspitose, with root-like branches. Dead twirs, stumps, old roots, branches, grass-roots, yew, elm, maple, oak; common. June-Jan. $\frac{1}{2} \times 2 \frac{1}{4} \times \frac{3}{6}$ in.

1419. M. graminum B. \& Br. (from its usual habitat, leaves and stems of grass, samen) a b c.

$P$. campanulate to expanded, subumbonate, sulcate, pale, rufousbrown or salmon; mid. darker. St. shining, hair-like, black, 
whitish above. $G$. adnate to a free collar, ventricose, distant, veined, white or pale yellowish.

On grass, leaves, twigs, etc. ; uncommon. July-Feb. $3 \frac{3}{8} \times 2 \frac{1}{4} \times \frac{1}{67}$ in.

1420. M. androsaceus Fr. (derivation uncertain) a $b c$.

$P$. arid, convex to expanded, subumbilicate, smooth, striate, white pale buff or somewhat fuscous. St. fistulose, horny, black, paler above. $G$. adnate, without a collar, distant, whitish to salmon.

Woods, leaves, twigs, briar, beech, oak, acorn-cups, rushes, fern-stems, fir, juniper, larch ; common. April-Dec. $\frac{1}{2} \times 2 \times \frac{1}{64}$ in. Sometimes growing from entangled masses of black horsehair-like mycelium. Sometimes non-pileate stems only produced.

1421. M. splachnoides Fr. (from the gut-like stem; Gr. splagchna, intestines, eidos, appearance) $a b c$.

$P$. convex to flat, umbilicate, smooth, striate, white; mid. pale yellowish. St. fistulose, horny, usually red, becoming fuscous or pale above and sienna below, or dark brown. $G$. subdecurrent, crowded, anastomosing.

Odour none. Amongst pine-leaves. Oct. $\frac{3}{4} \times 2 \times \frac{1}{64}$ in.

1422. M. Curreyi B. \& Br. (after Frederick Currey) $a$.

$P$. convex to expanded or plane, sulcate, pallid rufous; mid. fuscous or yellowish-sienna. St. black, white above. $G$. attached to a free collar, few, subventricose, veined, pale yellowish.

On grass. Aug. $\frac{3}{8} \times 1 \frac{1}{4} \times \frac{1}{67}$ in.

1423. M. perforans Fr. (from the habit, the stem perforating the leaves on which it grows) $a c$.

$P$. hemispherical to convexo-plane, not umbilicate, white or whitish, becoming pale rufescent. St. fistulose, tough, equal, bay-brown to black. $G$. adnate, subdistant, whitish.

Odour very feticl, not of garlic. Leares, fir, larch; rare. Aug.-Oct. $\frac{3}{8} \times 2 \frac{1}{4} \times \frac{1}{67}$ in.

1424. M. insititius Fr. (from the habit-the stem ingrafted on the host-plant; insero, to insert) a $b c$.

$P$. arid, convexo-plane, subumbilicate, plicato-sulcate, white or purplish-salmon-whitish. St. fistulose, horny, attenuate downwards, rufous to fuscous or sienna, paler above. $G$. adnate, distant, white or pale salmon-purplish.

Gregarious. Odour none. Leaves, oak, decayed grass, etc. ; rare. Aug.Oct. $\frac{5}{8} \times 2 \times \frac{1}{32}$ in.

1424a. M. selepotipes Bres. (from the hard sclerotium at the foot of the stem ; pes, a foot) $a$.

$P$. membranous, convex, then plane, umbilicate, ruguloso-striate, subflocculose; marg. reflexed, white; mid. yellow. St. filiform, pruinose under a lens, pallid rufescent, apex whitish, base 
adnate to a rufescent yellowish sclerotium. $G$. distant, adnate, white.

On the ground, swampy places. $\frac{1}{4} \times \frac{7}{8} \times \frac{1}{20}$ in. Autumn. Sclerotium $\frac{1}{5}$ in. long.

1425. M. Hudsonii Fr. (after William Hudson, author of "Flora Anglica ") $a b c$.

$P$. hemispherical, usually sulcate, pale fuscous-rufescent, rosepurple to white with sienna mid. beset with erect purple hairs. $S t$. horny, dark purple, paler above. $G$. adnexed, white.

Odour none. On fallen holly-leaves; frequent. Mar.-Dec. $\frac{1}{4} \times 1 \frac{3}{4} \times \frac{1}{64}$ in.

1426. M. epichloe Fr. (from its habit, growing on grasses; Gr. epi, upon, chloe, grass) a $b$.

$P$. plano-convex, somewhat papillate, not striate, whitish or pale brownish-yellow; mid. darker. St. striate, bay-brown. $G$. adnexo-rounded, somewhat crowded, pale yellowish, faintly brown.

Base of dead grasses, spines of Robinia. Aug. -Oct. $\frac{3}{8} \times 1 \frac{1}{2} \times \frac{1}{32}$ in.

1427. M. actinophorus B. \& Br. (from the brownish star on the pileus; Gr. aktis, a ray of light, phero, to bear) a $b c$.

$P$. convex, umbilicate, bay-brown or pale sienna, clouded with an indefinite brownish star at mid. St. pallid or brown, paler above. $G$. few, adnato-decurrent, white or pale yellowish.

Fallen twigs, sometimes terrestrial. Aug. $\frac{1}{4} \times \frac{7}{3} \times \frac{1}{6 \pm} \mathrm{in}$.

1428. M. saccharinus Fr. (from the pileus, as if powdered with white sugar, saccharent) a b $c$.

$P$. campanulate to convexo-expanded, papillate, sulcate, white. St. Hocculose to smooth, reddish, pale above. $G$. adnate, thick, distant, reticulato-wrinkled, whitish.

Dear twigs, leaves; rare. Aug. $\frac{3}{8} \times \frac{3}{4} \times \frac{1}{3.2}$ in. Pileus sometimes marked as in 1427 .

1429. M. epiphyllus Fr. (from the habit-growing on leaves; Gr. epi, upon, pluallon, a leaf) a b $c$.

$P$. convex to plane, umbilicate, plicato-rugose, white. St. fistulose, subhorny, date-brown or sienna, white above. $G$. adnate, distant, veined and branched, white.

Gregarious. Odour none. Dead leaves, especially ivy, twigs, etc., oak, beech, poplar; common. Sept.-Dec. $\frac{3}{8} \times 2 \frac{1}{8} \times \frac{1}{6 x}$ in. Pileus sometimes marked as 1427 , sometimes without gills, or gills replaced by wrinkles.

1430. M. polyadelphus Cooke (from its growing in crowded patches; Gr. polus, many, adclphos, a brother) a. White.

$P$. hemispherical, sulcate, flocculose. St. tough, floccose below. $G$. subdecurrent, almost fold-like.

Densely gregarious. Dead leaves, etc. Autumn. $\frac{3}{16} \times \frac{1}{2} \times \frac{1}{6+}$ in. 
C.

1431. M. spodoleueus B. \& Br. (from its grey pileus and white gills; Gr. spodos, wood-ash, leukos, white) a $b c$.

$P$. at first flat, resupinate, then campanulate, at length pendulous, pulverulent or subfurfuraceous, cinereous or pale umber. $G$. inside bell, few, short, leaving a naked space at apex of bell, interstices even. Flesh umber.

Dead twigs, elm. Nov.-Mar. Diam. $\frac{3}{4}$ in. The same as .iT. Broomei Berk. Sometimes not unlike 1449.

\section{LENTINUS Fr.}

(From the tough, pliant substance; lentus, tough or pliant.)

Veil ragged-pendulous at the margin of the pileus and more or less fugitive-annulate in the first section, in the other-except 1439-

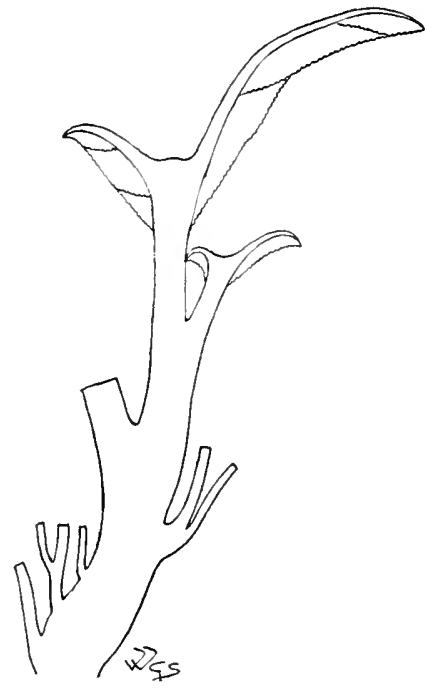

Fig. 67.-Section of Lentinus cochleatus Fr.

One-half natural size obsolete. Pilens fleshy-coriaceous, pliant or hard, persistent. Stcm hard, central, lateral or obsolete, continuous with the hymenophore when present. Gills concrete with the hymenophore, thin, nembranous, unequal, tough, edge acute, of ten serrate, crenate or irregular; trama none. Spores white. (Fig. 67.)

Growing on wood, rarely terrestrial, polymorphous, somewhat irregular. Distinguished from Plcurotus by the hardness of the flesh and the serrate gills. Species 1432-1441 Mesopode. Pileus nearly entire, margin at first inrolled. Stem distinct.

a. Lepidece. Pilius scaly. More or less veiled. 1432, 1433

b. Pulierulente. Pileus villous or pulverulent. 1434-1436

c. Cochleate. Pilens smooth.

1437

Pleurote. Dimidiate, sessile or furnished with a somewhat lateral stem.

$1438-1441$

MESOPOD瓜.

a. Lefidece.

1432. L. tigrinus Fr. (from the tiger-like markings of the pileus) $a b c$. $P$. convex, umbilicate to infundibuliform, pale yellowish, clad with umber sc.; mid. sepia. St. solid, equal or attenuate 
downwards, central to excentric, fugitive-torn-annulate, pale yellowish. $G$. decurrent, crowded, white or pale pallid yellowish.

Often subgregarious or cxspitose. Tasteless; odour strong, acrid or acid. Stumps, oak, ash, poplar, fir ; rare. April-Oct. $2_{\frac{3}{4}} \times 1 \frac{1}{8} \times \frac{1}{4}$ in. Var. Dunalii Fr. $P$. with adpressed separating, spot-like sc., then even.

1433. L. lepideus Fr. (from the scaly pileus; Gr. lepis, a scale) a b c. $P$. convex to depressed, pale yellowish, clad with umber sc.; mid. darker. St. solid, almost woody, irregular, usually somewhat excentric and attenuate downwards, colour as P., dark sienna-umber at base, fugitive-torn-annulate. $G$. decurrent or sinuate, crowded, pale yellowish.

Solitary or subcrespitose. Odour strong, from pleasant to that of rotten cheese. On pine wood, trunks, stumps, fallen pines, elm, railway sleepers, wooden bridges, in houses, cellars, railway tunnels. Mar.-Oct. $5 \times 5 \frac{1}{4} \times \mathbf{I}$ in. Often abnormal and Clavaria-like. Sometimes phosphorescent. Var. contigutus Fr. P. thinner, plano-depressed, even. St. slender, even.

\section{b. Pulicrulente.}

1434. L. leontopodius Schulz. ex Fr. (from a fancied resemblance in the shape and colour of the pileus to a lion's foot ; Gr. leon, a lion, pous, a foot) $a$.

$P$. expanded, depressed, boldly lobed, tan-clay colour, cinnamon or salmon-umber. St. woody, equal, excentric, pulverulent, pale chestnut or umber-salmon, blackish downwards. $G$. decurrent, veined, white.

Gregarious or cæespitose in small clusters. Taste pleasant, sweet. Decayed willow. Autumn. $7 \times 3 \times 1 \frac{1}{2}$ in.

1435. L. pulverulentus Fr. (from the pulverulent pileus) $a$.

$P$. convex, yellow, ochreous-cinnamon or umber-yellowish, lighter at marg., white-pulverulent. St. solid, excentric, velvety dark umber above, brownish-yellow below. $G$. crowded, subdecurrent, white or pale yellowish.

Somewhat caspitose, sometimes connato-branched and deeply rooting. Dead trunks, pines. Oct. $\mathbf{I}_{\frac{3}{4}} \times 3 \times \frac{1}{2}$ in. Var, resinaceus Fr. $P$. somewhat gummy villous.

1436. L. adhærens Fr. (from the sticky gluten of the pileus and stem) $a$.

$P$. convex, subumbonate, then depressed to subinfundibuliform, sulcate, umber-tan or umber-yellowish. St. somewhat hollow, rooting, white, then as P. $G$. sinuato-decurrent, thin, white.

Taste at length astringent. Woods, pine; rare. Autumn. $1 \frac{3}{4} \times 2 \frac{1}{2} \times \frac{1}{ \pm}$ in.

1436a. L. suffrutescens Fr. (from the somewhat branching habit: frutex, a shrub) a.

$P$. tough, convex, then infundibuliform, unequal, glabrous, whitish, becoming brown stained. St. elongated, somewhat 
branched, woody, pubescent, colour as P. G. crenato-torn, yellowish.

Squared wood in cellars. Feb. $3 \frac{1}{\frac{1}{4}} \times 6 \times \frac{1}{2}$ in. Often abnormal in habit.

\section{c. Cochleate.}

1437. L. cochleatus Fr. (from the shape of the pileus, somewhat shell-like; cochlea, a snail-shell) a $b c$.

$P$. convexo-umbilicate, subinfundibuliform, at length irregularly lobed, brownish-yellow; mid. darker. St. usually branched, proliferous and irregularly indented, central to almost lateral, colour as P., sienna-umber at base. $G$. decurrent, crowded, lighter than P., or white Hesh-colour.

Solitary to crespitoso-proliferous. Said to be edible. Odour strong of anise, spicy, often scenting the air for long distances. Trunks, stumps and on the ground; frequent. Aug.-Oct, $2 \frac{1}{2} \times 5 \times \frac{1}{2}$ in. Changes to rich brown or blackish in drying.

\section{Pleurote.}

1438. L. scoticus B. \& Br. ex Fr. (from its first observed place of growth-Scotland) $a b c$.

$P$. convexo-umbilicate to expanded and depressed, pallid to brownish or yellowish-buff, hygrophanous; marg. lobed, sinuate or plicate. St. solid, darker than P., or yellowish-sienna. $G$. decurrent, subdistant, white.

Solitary or crespitose. Odour none. Decayed stumps and rotten wood, furze, birch, spruce. Nov.-Jan. $2 \frac{3}{4} \times \frac{3}{4} \times \frac{1}{4}$ in. Stem sometimes absent and pileus reniform.

1439. L. fimbriatus Curr. (from the fugitive fimbriate annulus in infancy) a $c$.

$P$. convex to deeply depressed, floccose-scaly, fawn-colour or umber-salmon ; marg. substrigose. St. solid, lateral, rough with reflexed sc., brown-white. $G$. subdecurrent, pale brown or salmon-white.

Imbricate. Stumps, posts. Sept. $\frac{3}{t} \times \frac{1}{4} \times \frac{1}{5}$ in.

1440. L. vulpinus Fr. (from its strong, often foxy, odour : z'ulpes, a fox) $a b c$.

$P$. sessile, reniform-conchate, surface rough and corrugate with raised radiating ribs or adnate teeth, warm yellow-brownish; marg. rose flesh-brown, base darker. $G$. extended to base, crowded, brownish, pale yellow or pale yellowish-rose.

Imbricate. Odour sometimes of field-mint. Trunks, stumrs; rare. Mar.-Oct. Diam. 5 in. Becomes black in clrying.

1441. L. flabelliformis Fr. (from the fan-shaped pileus; flabcllum, a small fan, forma, form) a $c$.

$P$. sessile, reniform ; marg. corrugate, at length curled backwards, smooth, fawn-colour or yellowish-brown, darker at base. St. 
rudimentary. $G$. extended to base, subdistant, somewhat thick, whitish or faintly shaded as P.

Imbricate. Old trees, stumps, trunks; rare. Feb. Diam. $2 \frac{3}{4}$ in.

\section{PANUS Fr.}

(A name given to an arboreal fungus by Pliny.)

Veil obsolete. Pileus fibrous, Heshy-coriaceous. Stem lateral or none, when present confluent with the hymenophore. Gills entire,

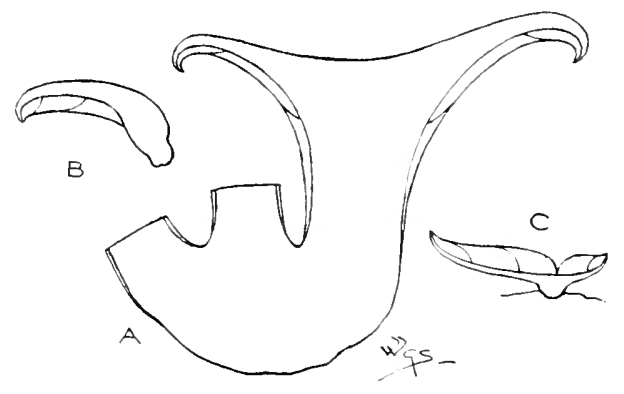

Fig. 68.-A, section of Panus torulosus Fr. One-half natural size. $B$, section of $P$. stypticus Fr.: $\mathrm{C}$, ditto, P. patellaris Fr. Natural size.

concrete with the hymenophore, at length coriaceous, edge acute, not serrate. Spor's white. (Fig. 68.)

Growing on stumps and lasting long.

Species $1442-1447$

Pileus irregular. Stem excentric.

1442,1443

Stem lateral.

1444,1445

Pilezs resupinate, sessile or extended behind.

1446,1447

1442. P. conchatus Fr. (from the shape, like certain shells; concha, a shell) a b $c$.

$P$. more or less dimidiate, deeply depressed, fleshy, pliant, thin, cimnamon or brownish-yellow, pale sienna-scaly in old examples. St. solid, equal or attenuate downwards, brownish-yellow. $G$. deeply decurrent, narrow, whitish or pale flesh-colour, at length yellowish.

Caspitose, often imbricate. Trunks, stumps, beech, poplar, lime; rare. June-Oct. $4 \frac{1}{ \pm} \times \frac{3}{4} \times \frac{1}{2}$ in. Stem sometimes branching.

1443. P. torulosus Fr. (from the hairy down on the stem; torulus, a tuft of hair) $a b c$.

$P$. entire, plane to infundibuliform, compactly fleshy, even, smooth, brownish-yellow, buff or rufescent-livid, sometimes minutely sienna-scaly. St. solid, equal, oblique, grey, violaceous or buff-downy. $G$. deeply decurrent, subdistant, reddish, tan or yellowish.

Crespitose. Old trunks, stumps, ash, birch, willow, oak; frequent. May-Aug. $3 \frac{3}{4} \times 1 \frac{1}{4} \times \frac{3}{4}$ in. 
1443a. P. pudis Fr. (from its rough appearance; rudis, rough) $a b$.

$P$. becoming corky, thin, unequal, bristling with hairs, ochreousfawn or reddish, lilac shaded: marg. incurved. St. short, unequal, shaggy-velvety, ochreous-fawn, hairs lilac. $G$. very decurrent, very crowded, narrow, whitish-pink, then pale ochreous-fawn, sometimes lilac shaded. Flesh white.

Crespitose. Odour pleasant of anise. Trunks, beech. May. $2 \frac{7}{8} \times 2 \frac{1}{2} \times \frac{1}{2}$ in.

1444. P. stypticus Fr. (from its astringent taste; stypticus, astringent) a $b c$.

$P$. arid, thin, reniform, sometimes minutely white-furfuraceous scaly, tan or cinnamon, becoming pale. St. attenuate downwards, pruinose, paler than G. $G$. adıate, thin, crowded, salmon or ochreous-salmon to cinnamon.

Gregarious, caspitose, usually densely imbricate. Taste hot, pungent. On decaying stumps, trunks, dead trees, etc., fir, alder, beech, oak; common. Feb.-Dec. $\mathbf{I} \frac{1}{2} \times \frac{3}{4} \times \frac{1}{4}$ in. Becoming wholly rich orange when dry.

1445. P. farinaceus Fr. (from the scurfy pileus; farina, meal) a $b c$.

$P$. somewhat coriaceous, flexuous, reniform, cinnamon-umber with pale grey scurf. St. colour as P. G. adnexed, distinct, paler than P. or yellow ochre. Flesh whitish-grey.

Trunks, branches, fir. Dec. $I \times \frac{1}{5} \times \frac{1}{16}$ in. Not unlike 1444 or 439 in appearance. Var. albidotomentosus Cooke \& Mass. $P$. clad with short relvety-whitish down. St. short or absent. Often in imbricate tufts. P. $\frac{2}{3}-\frac{1}{2}$ in. in diam.

1446. P. patellaris Fr. (from the shape, like a small dish, fatilla) a.

$P$. sessile, resupinate, coriaceous, at first viscid, umber or dark sepia to buff; marg. white. St. obsolete or rudimentary. $G$. concurrent to a subcentral point, arid, yellowish to somewhat pale cinnamon.

Branches, cherry, beech. Mar. $\frac{3}{4}$ in. in diam.

1447. P. Stevensonii B. \& Br. (after the Rev. John Stevenson).

$P$. spathulate, olivaceous light yellow. St. attenuate downwards, convex, slightly hispid, golden. G. narrow, entire.

Oak. rept. - oct.

\section{XEROTUS Fr.}

(From the dry substance and ear-like shape:

Gr. xeros, dry, ons, an ear.)

I'cil obsolete. Pilius coriaceo-membranous. Stim central, contimuous with the hymenophore, which descends into the trama. Gills adnato-decurrent, coriaceous, broadly fold-like, dichotomous, edge entire, obtuse. Sforcs white. (Fig. 69.) 
Rigid, persistent, analogous with Cantharellus, but differing in its leathery substance.

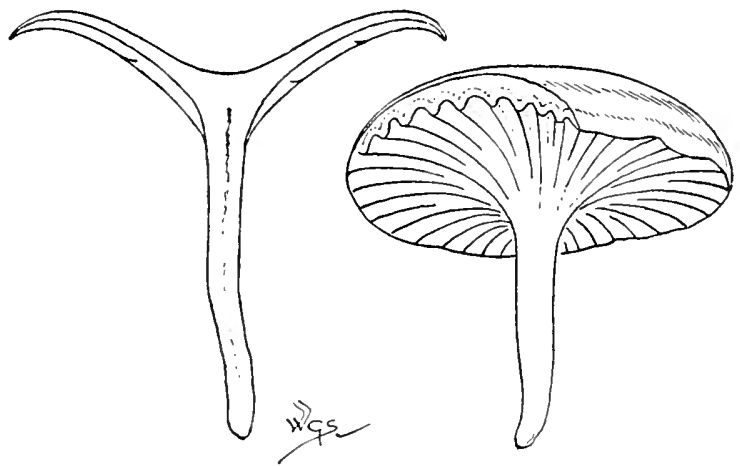

Fig. 69.- Jerotus degentr. Fr. in section and entire with portion removed to show the gills. Natural size.

1448. X. degener Fr. (from its ill-developed condition in comparison with other species of Xortus; degener, degenerate) a $b$.

$P$. thin, very tough, plano-infundibuliform, somewhat zoned, striate when moist, flocculose when dry, brown-grey to grey, cinnamon, salmon or pale yellowish-brown. St. solid to stuffed, attenuate downwards. $G$. very distant, whitish-grey, yellowish or salmon-white.

Peaty ground, amongst short grass in sterile gravelly places. Jan. $\mathbf{I} \frac{3}{4} \times \mathbf{I} \frac{1}{4} \times \frac{1}{8}$ in.

\section{TROGIA Fr.}

(After Jacob Gabriel Trog, Swedish botanist.)

Pileus membranous, soft, flaccid, arid, persistent, texture fibrillose. Stem lateral or none. Gills fold-like, venose, edge in the only

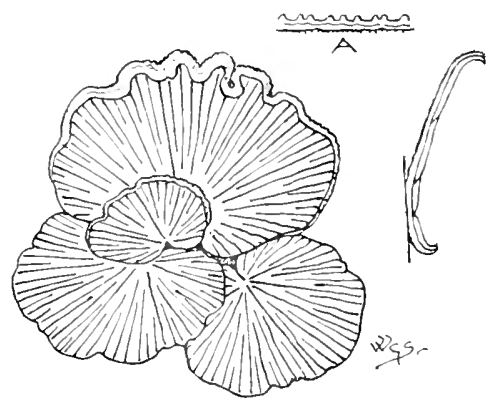

Fig. 7o.-Trogia crispa Fr., entire and in section. Natural size. A, section across gill. 
European species obtuse, crisped, not channelled as in non-European species. Spores white. (Fig. 70.)

Growing on wood, becoming dry and reviving with moisture.

1449. T. crispa Fr. (from the crisped gills) $a b c$.

$P$. cup-shaped, expanded, resupinate, light-yellowish-rufescent, zoned rufous; marg. whitish, sometimes almost white. St. obsolete or rudimentary. $G$. vein-like, dichotomous, narrow, white to steel-grey.

Gregarious, imbricate. Logs, branches, twigs, beech, birch; uncommon. Jan.-Dec. Diam. $I_{\frac{1}{8}}^{1}$ in. Sometimes almost wholly white.

\section{LVI1. SCHIZOPHYLLUM Fr.}

(From the gills, split longitudinally at the edge;

Gr. schiso, to split, fhullon, a leaf.)

Vill obsolete. Pilcus thin, arid. Stem lateral or none. Gills coriaceous, chamnelled longitudinally with edges revolute. Spores white. (Fig. 7 I.)
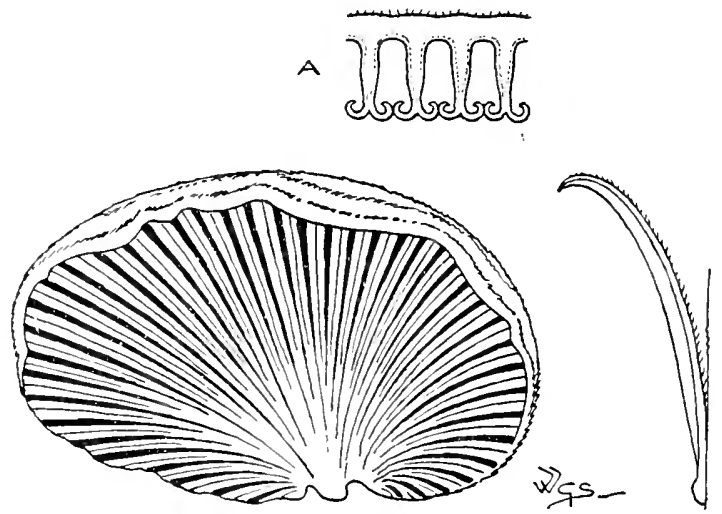

Fig. 71.-Schizophyllum commune Fr., entire and in section.

Natural aize. A, section across gills. $x$ ro.

1450. S. commune Fr. (from its being common in certain countries; communis, common) a $b c$.

$P$. arid, at first cup-like, then expanded, reniform or excentric, resupinate, downy, white or umber-white, commonly zoned greyish. St. rudimentary or none. $G$. splitting, the edges revolute, fuscous-grey to purplish, cinereous or clay-white to tan-salmon.

Said to be edible. Sold in a dry state in China for soup; Soo lbs were exported from Auckland, New Zealand, to China in IS72. Dead wood, rotten trunks, logs, water-butts, beer-casks, wood in greenhouses, horsechestnut, beech, alder, dry hay in silos; rare in Britain. May-Jan. Diam. 2 in. 


\section{LENZITES Fr.}

\section{(After Harold Othmar Lenz, German botanist.)}

T'il obsolete. Pilitus corky or coriaceous, texture arid, floccose, dimidiate, sessile. Stem lateral or none. Gills coriaceous, firm,

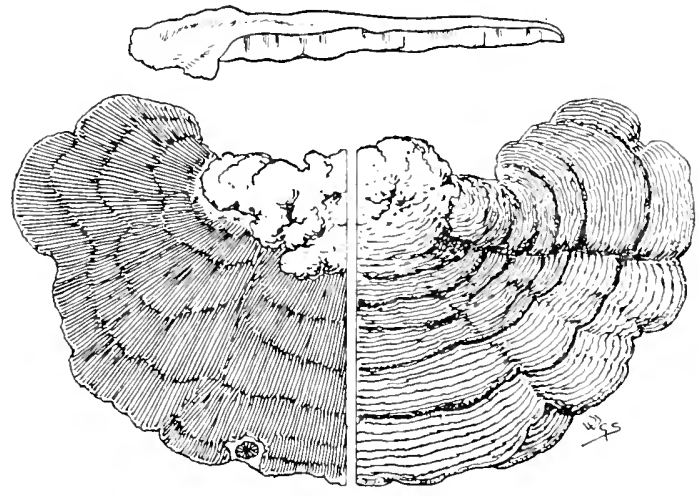

Fig. 72.-Lenites betulina Fr., entire and in section. Lower surface on left, upper on right. One-third natural size.

sometimes simple and unequal, sometimes anastomosing and forming pores behind, edge obtuse to acute; trama floccose, similar to the pileus. (Fig. 72.)

Growing on wood, persistent. Allied most nearly to Trametes and Dedalea amongst the Polyporacce. Species 1451-1455 On wood of deciduous trees.

1451,1452

On coniferous wood.

$1453-1455$

1451. L. betulina Fr. (from its frequent habitat, birch trunks; betula, birch) $a b c$.

$P$. firm, rigid, tomentoso-woolly, flat, lobed, white, greyish, buff or umber-whitish; marg. and $\mathrm{z}$. darker. $G$. reaching the base, simple, branched or anastomosing, edge acute. Flesh white.

Often imbricate. Stumps, logs, gate-posts, birch, oak, bird-cherry, alder ; perennial, common. Jan.-Dec. Diam. $\oint_{\frac{1}{4}}$ in. When young small, resupinate, with gills radiating from a central point. Often green with unicellular Algie. Surface and coloration of pileus like that of 2001.

1452. L. flaceida Fr. (from the flaccid substance) $a b c$.

$P$. thin, easily bent, flat, lobed, whitish to dingy, zoned same colour. $G$. reaching the base or an excentric point, broad, crowded, simple or branched towards base, not anastomosing, white to salmon. Flesh white.

Stumps, beech. Nar.-Jan. Diam. 4 in. Pileus often green as in 1451. 
1453. L. sæpiaria Fr. (from the habitat, fences; sapis, a hedge) $a b c$.

$P$. thin, flat, lobed, strigoso-tomentose to squamulose, zoned; tawny-yellow to brown, black when old. $G$. reaching to and anastomosing at the base, rigid, firm, yellowish to umber. Flesh tawny to dark sienna.

Often imbricate. Trunks, fences, pine. Jan.-Dec. Diam. 5 in.

1454. L. abietina Fr. (from its hahitat, firs, abies) $a b c$.

$P$. thin, flat, tomentose, becoming smooth, umber or yellowishrich-brown or deep umber-sienna, zoned dark umber ; narg. bright sienna-yellow, or wholly vinous. $G$. reaching the base, distant, simple, unequal, not anastomosing but torn into teeth at the base, salmon or yellowish-brown.

Imbricate. Chiefly dressed fir, deal, trunks, branches. Oct. Diam. $3^{\frac{3}{4}}$ in. Sometimes remaining resupinate.

1455. L. heteromorpha Fr. (from its varied shapes; Gr. heteros, different, morthe, shape) $a b c$.

$P$. gibbous, rugose, thin to thick, yellowish- or buff-white, or colour of washleather. $G$. reaching the base, branched, crowded, often broken into teeth, sometimes effused, colour as $\mathrm{P}$.

Imbricate. Fences, sticks, chips, bark, pine. Oct. Diam. 3 in., sometimes effused to 7 in. or more.

\section{FAM. II. POLYPORACEÆ.}

Hymenium inferior, in resupinate species superior, consisting of tubes with poriform orifices which are round or angular, sometimes sinuous or torn.

Fleshy, coriaceous or woody fungi, intermediate between Agaricacece and Hydnacie, connected with the former by Trametes and Dedaled, and with the latter by Fistulina. Species 1456-1686

The sequence of the genera and species is almost identical with the latest views of Fries as expressed in his Hymcnomycetes Eurofici, I874. An exception is made in the genus Polyforus, which is here broken up into four genera-Polyporus, Fomes, Polystictus and Poria, as given by Cooke in I 885 and $\mathrm{I} 886$, in his Precursores ad Monosraphic Polypororum, in Greaillea, xiii., pp. So and I I4, xis., pp. I7, 77 and 109 , and $x v$, p. I9, with additions and corrections on p. 50. Cooke based his work on the Nore Symbole Mycologica of Fries, published in I $85 \mathrm{I}$, but in this Fries only suggested that one section of Polyporus should be elevated to generic rank, viz. Polystictus. Saccardo, in his Sylloge Fungrum, rii., I 888 , immediately adopted Cooke's views. It is in deference to the laborious work of Cooke that the old genus Polporus is here broken up into four. 


\section{Key to the Genera.}

Terrestrial.

Tubes easily separable from the hymenophore. Stem central

Tubes not separable, very large with transverse dissepiments. Stem central

59 Boletus.

60 Strobilomyces.

Mlostly growing on trunks, stumps or wood.

Stipitate to sessile. Tubes small, without dissepi. ments. substance fleshy, hard, tough or somewhat soft . Sessile.

Substance fleshy, juicy

62 POlyporus.

Substance woody

6 I Fistulina.

63 Fones.

Substance coriacens, spongy or membranous.

Pores round

64 Polystictus.

Pores oval or elongate

Pores sinuous....

Sessile to resupinate. Substance cartilagino-soft to subgelatinous............................. Resupinate.

Substance thin, woody or waxy

66 Tranetes.

67 D.EDALEA.

65 ilerulius.

65 PORIA.

69 Porothelium.

\section{BOLETUS Dill.}

(From the clod-like shape of the pileus; Gr. bolos, a clod.)

Fil universal, forming an annulus, perfect to imperfect or obsolete. Pileus Heshy. Stim central, fieshy, continuous and homo-

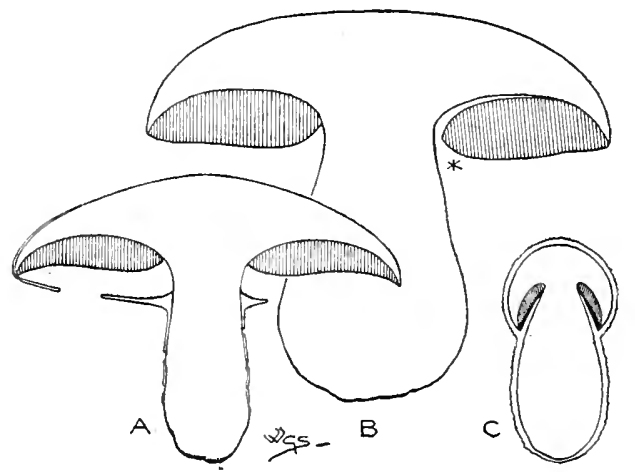

Fig. 73.-A, section of Beletus luteus L., showing gelatinous membranous veil. E, section of $b$. e'dulis Bull., showing at tubes separating from hymenophore. C, section of $B$. cy'anescens Bull., young example showing universal floccoso-scaly veil. One-third natural size.

geneous with the hymenophore, annulate to simple. Tubes distinct from the hymenophore, connected in a stratum, the surface of which 
is dotted with their orifices or pores, easily separating from the hymenophore and from one another; the mouths of the tubes or pores are round or angular, in the subgenus Gyrodon sinuous or gyroso-plicate; trama none. Spores usually fusiform, rarely oval or roundish, white, rosy, yellowish or brown. (Fig. 73.)

Terrestrial, fleshy, putrescent fungi; mostly edible, a few poisonous.

Species $1456-1515$

Subgenus i. Euboletus. Pores round or angular, not sinuous or gyroso-plicate. Trama not descending between the tubes.

$1456-1513$

Euchroæ. Tubes usually yellow, not white or grey.

a. Viscipelles. Stem solid, not bulbous or reticulate, except 1458. Tubes adnate, decurrent in 1457, 1467 and 1468. Pores rarely sinuate, of one colour. Sfores usually yellowish. $1456-1468$

b. Subtomentose. Pileus villous when young, rarely at length becoming smooth. Stem not bulbous or reticulate; here and there rugose or striate. Flesh rarely changing colour. Tulis adnate, of one colour.

$1469-1477$

c. Subpruinisa. Pileus pruinose to smooth. Stem equal, even, not bulbous or reticulate. Tulles adnate, yellowish.

$1478-1481$

d. Calopodes. Stem stout, at first bulbous, reticulate; reticulations usually red.

$1482-1488$

e. Edules. Stem stout, bulbous, not reticulate or dotted red except 1489, 1490, 1491 and 1493. Tubis somewhat free, adnate in 1494. Pores not red. Flish scarcely changing colour. Taste pleasant.

$1489-1497$

f. Luride. Pileus pulvinate, compact then soft. Stem stout at first, curt and bulbous, then elongate and clavate to nearly equal, somewhat reticulate or dotted. Tubes rounded-free. Pores red to red-brown. Flesh juicy. Suspected poisonous. Growing chiefly in woods of deciduous trees. 1498-1500

Tephroleuce. Tubis at first white or grey.

g. Foriose. Tubes large, angular, unequal, often shortened near the stem, adnate, not rounded-free. Spores brown.

$1501-1504$

h. Versifelles. Tubes free, ventricose. Pores minute, round, equal. Stores ferruginous.

$1505-1508$

i. Hyporhodice. Tubes whitish to pale salmon. Spores rosy to white flesh-colour.

1509,1510

j. Cariosa. Stem not reticulate, stuffed to hollow. Tubes white to light yellowish. Pores minute, round. Spores white.

$1511-1513$

Subgenus 2. Gyrodon. Thles short, somewhat adnate. Pores sinuous or gyroso-plicate.

1514, 1515 
Subgenus 3. Boletinus. Trama descending somewhat between the tubes, the hymenophore is therefore not smooth after their removal; in this respect Boletimus resembles Strobilomyces. The relationship between Bolitus and Boletimus is similar to that between Polyorus and Trametes.

$1515 a$

\section{Subgenus i. EubOletus.}

(From being typical examples of the genus; Gr. cu, well.)

\section{EUCHROÆ.}

a. Viscipell's.

1456. B. luteus L. (from the yellow tubes; luteus, yellow) a $b c$.

$P$. gibbous, pulvinate to convex, deep vinous brown, covered with dark purplish gluten which is at first continuous over the tubes, forming a V. St. equal, dull light yellowish. A large, membranous, white to dark purplish. $T$. yellow or brownishyellow. Po. minute.

Edible. Woods, pine; common. July-Nov. $5 \times 2 \frac{3}{4} \times \frac{7}{8}$ in.

1457. B. elegans Schum. (from its elegance) a b $c$.

$P$. convex, dark rich yellow, shaded orange. St. sulphur to orange above $\mathrm{A}$., darker below. $A$. broad, fugacious, sulphur. $T$. decurrent, sulphur to bright yellow. Po. minute.

Woods, chiefly larch, oak, pine; common. June-Oct. $4 \frac{1}{5} \times 2_{4}^{3} \times \frac{7}{8}$ in.

1458. B. flavus With. (from the yellow pileus; flar'us, light yellow) a b $c$.

$P$. convex or pulvinate, full luminous yellow; covered when young with claret-brown gluten. St. equal, reticulate, yellow above, brownish-yellow below. A. membranous, fugacious, white to yellow-white. $T$. adnate, bright yellow or brownishyellow. Po. larger than in 1456 and 1457.

Woods, mixed ; common. Aug. - Nov. $4 \frac{1}{4} \times 3 \frac{1}{3} \times \frac{7}{3}$ in.

1459. B. flavidus Fr. (from the yellowish pileus; flavidus, light yellowish) a b $c$.

$P$. convex, obtuse, brownish-yellow or pale yellowish-buff. St. equal, whitish-yellow or straw-colour, granular above the brownish viscid A. T. adnate to slightly decurrent, yellow, somewhat sulphur. Po. angular. Flesh pale ochre.

Woods, pine. July-Oct. $2 \frac{1}{8} \times 2 \frac{1}{ \pm} \times \frac{1}{ \pm}$ in.

1460. B. collinitus Fr. (from the gluten-besmeared pileus : collino, to besmear) $a b c$.

$P$. pulvinate to expanded and slightly depressed, brownish-buff, sometimes pale, covered with rich vinous-brown gluten. St. attenuate downwards, rooting, white, becoming brownish. T. adnate to subdecurrent, pallid to yellow. Flesh white.

Solitary. Edible. Woods, fir. Nov. $3 \frac{3}{4} \times 2 \frac{1}{2} \times 1 \frac{1}{4}$ in. When young resembles 1456 . 
1461. B. granulatus L. (from the granulate pores and stem) $a b c$.

$P$. convex, obtuse, luminous- or cinnamon-yellow, covered with claret-brown, vinous or rich siemma gluten. St. white-sulphur above, brownish below, scabrous with honey-coloured granules. $T$. pale sulphur to orange. Po. simple, exuding a thin gummy juice which dries in granules. Flesh pale sulphur.

Commonly gregarious, sometimes crspitose. Odour rank, heavy, disagreeable, like that of 410 . Woods, fir ; rare. July-Nov. $3 \frac{3}{4} \times 2 \frac{1}{4} \times \frac{7}{8}$ in. Sometimes springing from yellow mycelium. Much eaten by squirrels.

1462. B. tenuipes Mass. (from the slender stem; tener, slender, pes, a foot) a b.

$P$. convexo-pulvinate, smooth, becoming minutely fibrillose, slightly to very viscid, red- or rose-purplish over yellow, or red-buff, shaded Indian red. St. attenuate downwards, somewhat striate above, slightly to very viscid, yellow, vinous in mid., becoming rufescent, or paie yellow above to gradually vinous below. T. large, angular, bright yellow. Flesh pale vinous under pellicle, white in mid., pale buff-vinous in St.

Taste mild. Woods. Oct. $\quad \mathbf{I}_{\frac{3}{4}}^{3} \times \mathbf{2 \frac { 3 } { 4 }} \times \frac{3}{8} \mathrm{in}$.

1463. B. bovinus L. (from the buff-reddish colour of the pileus, as of the ox, bos) $a b c$.

$P$. convex to expanded, buff, rose-buff or orange-buff, membrane of P., involute and overlapping the tubes. St. equal, even, ochre-rose or pale buff, rhubarb tinted at base. $T$. decurrent, ochre, olive-ochre or rose- or yellow-buff. Po. compound. Flesh pale buff, sometimes changing to cobalt.

Gregarious, subcespitose. Taste mild; odour pleasant. Woods, chiefly pine ; uncommon. Sept.-Nov. $4 \frac{1}{2} \times 2 \frac{3}{4} \times \frac{1}{2}$ in.

1463a. B. pusio Howse ex B. \& Br. (from its small size; pusio, a little boy) $a$.

$P$. hemispherical, pulvinate, pulverulent, reddish-buff. St. slightly thickened downwards, plicate, pulverulent, dull yellowish-white, white above, becoming blackish. $T$. dull sulphur-white.

1464. B. badius Fr. (from the colour; badius, bay-brown) $a b c$.

$P$. pulvinate to flat, villoso-viscid, claret-brown, umber or dark Indian-red-brown. St. equal or subclavate, pruinate, minutely granular, lighter than P. and streaky. $T$. adnate to slightly sinuate, whitish dull olive. Po. angular. Flesh rose under pellicle of P., cobalt over T.

Single or crespitose. Woods, chietly pine: frequent. Aug.-Nov. $5 \times 4 \times 1$ 负 in.

1465. B. paludosus Mass. (from growing in bogs, pathudes) a b.

$P$. convex to flat, glabrous, dark burnt-sienna or vinous redsienna; mid. darker, paler when dry. St. attenuate downwards, smooth, paler than P., or burnt-sienna with crimson, or dull 
dark sienna. T. adnate to subdecurrent, yellow to olive-green or whitish-green. Po. large, angular, compound. Flesh unchanging, salmon-white.

Gregarious, not crespitose. Bogs, amongst Sphagnum. Sept. $4 \frac{1}{1} \times 5 \times \frac{1}{2}$ in. Resembles a slender 1464 , but flesh does not change colour.

1466. B. rutilus Fr. (from the reddish pileus ; rutilus, golden-red) $a$. $P$. pulvinate, glabrous, brownish-red or umber, not dark. St. equal, glabrous, sulphur above, pale claret-brown below. $T$. sinuato-adnate, sulphur. Po. minute.

Woods, oak. Oct. $1 \frac{5}{8} \times 1 \frac{5}{8} \times \frac{1}{4}$ in. Must not be confounded with 1472 or 1474 .

1467. B. aurantiporus Howse ex Cooke (from the golden-yellow pores; aurantius, orange) $a b$.

$P$. convex, expanded, sometimes umbonate, ferruginous, orangesienna, claret-brown or buff. St. equal, orange above, with large purple-crimson reticulations, sulphur and pale yellow below. $T$. deeply decurrent, yellow to bright orange, becoming brownish when bruised. Po. broad angular. Flesh usually pale rose, sometimes pale yellowish, changing to pale orange-red.

Under or near trees, yews. Aus.-Oct. $3 \frac{1}{2} \times 3^{\frac{1}{2}} \times \frac{5}{5}$ in. Howse considered this plant to be allied to 1469 .

1468. B. piperatus Bull. (from the peppery taste; piper, pepper) $a b c$. $P$. pulvinate to expanded, dull ochreous-orange or yellow-ochre. St. equal or attenuate downwards, sienna-orange. T. decurrent, sienna with ochre and vermilion, or rich sienna-scarlet. Flesh deep sulphur, orange in stem.

Sometimes crespitose. Poisonous. Taste very acrid. Woods, mixed, under limes; frequent. Aug.-Nov. $2 \frac{1}{5} \times 2 \times \frac{5}{15}$ in. Often small. Springing from yellow mycelium.

\section{b. Subtomentosce.}

1469. B. variegatus Swartz (from the different colours of stem, pileus and tubes) $a b c$.

$P$. pulvinate, yellowish-sienna or yellowish-brown, clouded sienna or vinous; marg. extended beyond T., inrolled. St. equal, bright yellow to dull ochre, shaded orange, burnt-sienna or vinous. $T$. yellow, or orange- or olive-brown, not dark. Po. minute. Flesh yellowish, changing sparingly or wholly to blue, sometimes unchanging.

Tasteless; odour unpleasant. Woods, chiefly pine, heaths; frequent. July-Nov. $5 \frac{3}{8} \times 2 \frac{1}{3} \times 1 \frac{1}{3}$ in.

1470. B. sulphureus Fr. (from the sulphur-colour) a b c. Wholly sulphur-colour.

$P$. hemispherical to expanded-obtuse. St. ventricose, with rooting red base. $T$. adnato-decurrent, at length greenish. Po. minute, compound. Flesh becoming blue.

Cæspitose, sometimes in clusters of fifty or more. Tasteless. Sawlust, stumps. Sept.-Nor. $4 \times 2 \frac{1}{8} \times I_{\frac{5}{5}}^{5}$ in. Sometimes changing to reldish 
where touched. Mycelium woolly, matting sawdust, sulphur. Pileus sometimes 9 in. in diam.

1471. B. stpiæpes Secr. (from the striate stem; stria, a line, pes, a foot) a $b$.

$P$. pulvinate to expanded, orange-buff, shaded umber; marg. paler or whitish. St. slightly ventricose, velvety, sulphur-white above, umber below, broadly striate with small brown dots. $T$. yellow, yellow-greenish, sulphur or apricot-sulphur. Po. minute. Flesh white, or faintly shaded pale yellowish or brownish, sparingly changing to blue.

Woods; rare. Oct. $2 \frac{3}{8} \times 2 \frac{1}{2} \times \mathrm{I}$ in.

1472. B. chrysenteron With. (from the golden-yellow flesh; Gr. chrusos, gold, entera, inward parts) a $b c$.

$P$. convex to plane or slightly depressed, yellowish, umber or crimson-brownish over tan, often cracked, interstices crimsonred. St. equal, fibroso-striate, tan-white or buff, speckled crimson or sulphur above, spotted and streaked below, base umber. $T$. sulphur to yellow-olive. $P$ o. somewhat large, angular. Flesh yellow-white, pale rose under pellicle of P., pale blue near $\mathrm{T}$.

Edible. Woods, mixed, beech, hedges, open ground; common. July-Nov. $3 \frac{1}{\frac{1}{2}} \times 2 \frac{\frac{7}{5}}{5} \times \frac{1}{2}$ in. Var. namus Mass. P. olive. St. yellow above, crimson below. Flish changing to red when cut. P. I in. in diam.

1473. B. sanguineus With. (from the blood-red pileus; sangris, blood) $a b$.

$P$. pulvinate, dry, almost velvety. St. equal, rose above, sometimes reticulated crimson, tan or orange below, clouded crimson. T. adnate to subdecurrent, dull ochre to bright or olive-yellow. Flesh yellowish-white, changing to blue.

Woods; rare. July-Sept. $4 \times 3 \frac{1}{4} \times \frac{7}{5}$ in. Pileus sometimes 9 in. in diam., sometimes very small.

1474. B. subtomentosus L. (from the somewhat tomentose pileus) a bc.

$P$. pulvinato-expanded, umber or yellowish-umber, sometimes with a red line at marg., often cracked, interstices yellow. St. equal, ribbed-sulcate, slightly scabrous, bright yellow, sometimes clouded crimson. $T$. rounded-adnate, bright yellow, shaded pale olive-umber. Po. large, angular. Flesh whitish-yellow, sparingly changing to blue.

Woods, mixed, oak, beech, sometimes from interior of beech-nuts; common. July-Nov. $3 \frac{3}{4} \times 3 \frac{7}{1} \times \frac{7}{3}$ in. Pileus sometimes $7 \frac{1}{2}$ in. in dian. Var. radicans Mass. P. olive-ochre. St. fusiform, colour as P. T. colour as P. Taste insipid to bitter.

1475. B. spadiceus Fr. (from the date-brown colour of the pileus; spadix, a palm branch) $a b$.

$P$. pulvinate, rich yellowish-sienna, clouded red-brown or vinous. St. equal to clavate, flocculoso-furfuraceous, whitish-or pale luminous-yellow, olive-shaded above, clouded umber and 
rinous below. $T$. sinuato-adnate, pale yellow-olive, shaded greenish. Po. minute, somewhat round. Flesh pale sulphur or buff-white, with a faint blue line over $\mathrm{T}$.

Taste insipid or pleasant, with a peculiar lasting acid flavour. Woods, mixed. July-Nov. $4^{\frac{7}{5}} \times \mathbf{2}_{4}^{3} \times \mathbf{I}_{\frac{3}{4}}^{3}$ in.

1476. B. radicans Pers. (from the rooting stem ; radix, a root) $a b$.

$P$. pulvinate, yellowish-umber to umber or olive, nembrane of $P$. overlapping T. and involute. St. usually attenuate downwards, pale primrose to apricot-yellow, and dark ochre, pruinate, rose and sienna clouded where touched. $T$. adnate, colour as St. Po. unequal, large. Flesh yellowish-white, sometines changing to pale blue or slate.

Taste somewhat bitter. Woods. Sept. $3 \frac{1}{3} \times 2 \frac{1}{\frac{1}{x}} \times \frac{5}{3} \mathrm{in}$. Often confused with 1472 .

1477. B. rubinus W. G. Sm. (from the ruby-coloured tubes) a b c .

$P$. pulvinate to flat, whitish orange-umber or yellow-fuscous. St. equal or slightly attenuate downwards, yellow clouded crimson. $T$. adnato-decurrent, carmine. $P o$. compound. Flesh unchanging, vivid yellow.

Taste mild. Woods, roadsides, under trees, oak, beech. Aug.-Sept. $2 \frac{3}{4} \times 1 \frac{1}{2} \times \frac{3}{4}$ in.

\section{c. Sulpruinose.}

1478. B. versicolor Rostk. (from the change of colour in the flesh) $a b$. $P$. pulvinate to convexo-plane, bright deep crimson-purple. St. slightly pruinose, smooth, colour as P. or orange-brown, lighter above and below, sometimes sulphur above. $T$. adnatorounded, orange. Po. large, angular. Flesh whitish-yellow, changing to cobalt and vinous.

Said to be edible. Taste slightly disagreeable or none; odour strong and rank, like 2086. Woods, beech, gardens. Aug.-Sept. $2 \frac{3}{4} \times 2 \frac{1}{\frac{1}{4}} \times \frac{5}{5} \mathrm{in.}$

1479. B. pruinatus Fr. (from the pruinose pileus) $a b$.

$P$. convex to flattish, rigid, yellowish vinous-umber. St. somewhat ventricose or clavate, even, smooth, yellow, finely lined with crimson. T. yellow. Po. minute, round. Flesh whitish, sparingly changing to blue.

Grassy places. Autumn. $2 \frac{7}{5} \times 2 \times \frac{3}{3}$ in.

1480. B. purpuraseens Rostk. (from the purple pileus and stem) $a$.

$P$. convex to plane, dry, glabrous, deep brownish-purple or deep purple-crimson. St. attenuate downwards, rooting, even, glabrous, colour as P., sometimes yellowish above and below. T. dull yellow. Po. minute. Flesh dull grey or yellowish, streaked or marbled.

Woods, pine. Autumn. $3 \frac{1}{5} \times 2 \times \frac{3}{4} \mathrm{in}$.

1481. B. parasiticus Bull. (from the parasitic habit) $a b c$.

$P$. obtuse, plane, silky to smooth, yellowish-brown. St. slightly attenuate downwards, colour as P., or lighter, with pale vinoussienna streaks. $T$. adnate to subdecurrent, colour as St., 
sienna-shaded. Po. compound. Flesh yellowish-white or pale vinous, orange-brownish at base of St.

Parasitic on 2086 and 2087. Often orerlooked, as the colour of P. agrees with that of the peridium of host. Sept.-Oct. $2 \frac{1}{2} \times 2 \times \frac{\frac{1}{2}}{2}$ in.

\section{d. Calopodes.}

1482. B. appendiculatus Schæff. (from the membrane of the pileus overlapping at the margin) $a$.

$P$. pulvinate, expanded to plane, pubescent, crimson, bay-brownrose, indian-red or vinous-buff. St. subventricose, somewhat rooting, yellowish-white, buff below, sometimes crimson in middle. $T$. adnate, sulphur-greenish, clouded pale indigo where touched. Po. minute, angular. Flesh pale sulphur, becoming pale blue.

Edible. Woods; uncommon. Oct. $4 \frac{1}{3} \times 3 \frac{\pi}{5} \times \mathrm{I}$ in.

1483. B. variecolor B. \& Br. (from the varied colours) $a b$.

$P$. pulvinate, subtomentose, olivaceous; marg. involute. St. clavato-bulbous, pubescent and rufescent above, yellowish downwards. T. rounded-free, yellow. Po. minute. Flesh pale yellowish, marbled, dark purple under membrane of $\mathrm{P}$.

Wroods; uncommon. Aug.-Sept. $1 \frac{5}{8} \times 2 \frac{3}{5} \times \frac{1}{2}$ in.

1484. B. ealopus Pers. (from the beautiful stem; Gr. kalos, beautiful, pous, a foot) a b c .

$P$. globose to pulvinate, subtomentose, umber, rosy-umber or olive-umber. St. ventricose or nearly equal, somewhat rooting, reticulate and carmine above, dark umber below, or wholly: crimson or yellow above, crimson in middle, yellow and brown at base. $T$. rounded-adnate, whitish-orange. Po. minute, angular. Flesh white, changing to bright blue, or variegated blue, green, carmine and umber, crimson at base.

Taste bitter. Woods, mixed; frequent. July-Nor. $4 \frac{1}{2} \times 2 \frac{3}{4} \times 1 \frac{1}{4}$ in.

1485. B. olivaceus Schæff. (from the olive-coloured pileus) $a b$.

$P$. convex, even, becoming smooth, yellowish olive-umber to olive. St. subclavate, salmon, sometimes white at apex, yellow-brown below, with salmon-pink or rose granular streakings or reticulations. $T$. adnate, greenish-yellow. Po. minute, unequal. Flesh salmon-umber-whitish, sometimes changing to blue.

Almost tasteless ; odour somewhat strong and disagreeable. Woods, beech; uncommon. Aug.-Sept. $2 \frac{3}{4} \times 2 \times \frac{3}{4} \mathrm{in}$.

1486. B. regius Kromb. (from the regal aspect) $a b$.

$P$. globose to pulvinate, minutely tomentose, bright rose, pale purple, pale claret, buff or vinous-yellow-umber. St. subventricose to almost equal, pale or bright yellow, dull purple, red-crimson, orange or olive at base. T. rounded-adnate, deep 
golden-yellow. Po. minute. Flcsh yellow and pale salmon, sometimes changing to pale blue.

Solitary or gregarious. Edible. Open places in woods. Aug. $5 \times 5 \times 1 \frac{3}{4}$ in.

1487. B. crassus Mass. (from the stout stem ; crassils, thick) $a b$.

$P$. convex to expanded, irregularly waved, densely tomentose, white, then pale yellow-brown or fawn colour. St. at first almost globose, paler than P., white-reticulate above, base almost white. $T$. free, or nearly so, white then primrose. Flesh white, then pale primrose or pale biscuit.

Taste sweet, nutty; odour strong, acid. Under trees in pastures, beech. Aug. $4 \times 4 \times 2 \frac{1}{8}$ in. Compare 1490 and 1496 .

1488. B. pachypus Fr. (from the thick stem; Gr. pachus, thick, pous, a foot) a $b c$.

$P$. pulvinate, pale warm umber, umber or yellowish-umber or biscuit-colour. St. equal to clavate or bulbous, pale salmonrose above and below, rose in middle or yellow above, and umber and whitish-yellow below. $T$. rounded-adnate, bright yellow to green. Flesh whitish, pale vinous under membrane of pileus and at base of stem, changing sparingly and slowly to blue or unchanging.

Taste not unpleasant. Woods, chiefly pine. Sept.Oct. $4 \frac{1}{1} \times 3 \frac{1}{1} \times 1_{1}^{\frac{1}{4}}$ in.

\section{e. Edules.}

1489. B. candicans Fr. (from the white pileus and stem; candicans, whitish, shining-white) $a b c$.

$P$. convex, brownish- or yellowish-white, or almost white. St. obese, clavate or bulbous, reticulate to smooth, ochre- or primrose-white, pale umber below. $T$. adnato- or adnexorounded, sulphur to primrose. Flesh ochre-white, changing to bright blue or indigo.

Solitary or 2-3 connate. Open places, under oaks, in company with 1499. June-Oct. $5 \frac{1}{2} \times 3^{\frac{1}{2}} \times \mathbf{1}_{\frac{1}{2}}$ in. This is $B$. elephantinus Schrff.

1490. B. edulis Bull. (from its edible properties) $a b c$.

$P$. pulvinate to expanded and flat, pale umber, yellowish- or purplish-umber or tan, rarely sooty; marg. usually lighter or white. St. obese to clavate and equal, pale umber-white, buff-white or white above and tan below, white-reticulate above. $T$. adnexo-rounded, white to greenish-yellow. Flesh unchanging white, sometimes salmon-white under pellicle of $\mathrm{P}$.

Woods, especially beech; common. June-Nov. $8 \frac{1}{2} \times 5 \times 1 \frac{7}{5}$ in. The cèpe of French markets, the Fungo porcino, Porcino Boli porcin or Ferre of Italian markets. The "suillos" of Mart. Ep. iii. 60. Sometimes attacked by a yellow parasitic mould, Hypomyces chrysospermus, and then not edible. Var. licaipes Mass. St. even, white. There is also a wholly white var.

1491. B. æreus Bull. (from the copper-coloured stem; ces, copper) $a b c$.

$P$. pulvinate, smooth, umber, somewhat olive or blackish. St. subclavate to equal, somewhat reticulate, pale buff or yellow 
above, rosy-umber below. $T$. sulphur-primrose. Po. minute. Flesh pale sulphur, vinous under pellicle of $\mathrm{P}$.

Woods. Autumn. $4 \frac{7}{5} \times 4 \frac{1}{\frac{1}{x}} \times \mathrm{I}_{\frac{1}{2}}^{\frac{1}{2}}$ in.

1492. B. carnosus Rostk. (from the fleshy pileus; caro, flesh) $a$.

$P$. pulvinate, smooth, crimson-brown. St. bright yellow above, orange below or warm brown, somewhat finely streaked red and crimson. T. ochre-greenish or dark yellow. Po. somewhat large, angular. Flesh pale ochre.

Woods. Autumn. $4 \times 2 \frac{1}{2} \times 1 \frac{1}{8}$ in.

1493. B. vaceinus Fr. (from the reddish colour of the pileus; a'acca, a cow) a b.

$P$. convex, expanded, minutely tomentose, rich orange-umber, brighter at marg. or deep chestnut. St. yellowish, reddish or brownish, not dark. $T$. adnato-rounded, white to sulphur and bright yellow. Po. minute. Flesh white to pale siennayellowish, vinous under pellicle of $\mathrm{P}$., becoming pale blue.

Woods, beech. Autumn. $2 \frac{5}{5} \times 2 \times \frac{3}{4}$ in.

1494. B. Rostkovii Fr. (after Friedrich Wilhelm Rostkovius) $a b c$.

$P$. convex to plano-expanded, minutely tomentose, ochre, orange, or dull olive-brown or rufous. St. attenuate downwards, obconic, warm umber, buff, or pale reddish-yellow. T. adnate, yellow, sulphur or yellow-green. Po. irregular, compound. Flesh buff- or vinous-white, becoming reddish-brown under pellicie of $\mathrm{P}$., here and there blue.

Under trees, beech. Aug. $3 \frac{T}{5} \times I_{\frac{1}{x}}^{\frac{1}{x}} \times \mathrm{I}$ in.

1495. B. fragrans Vitt. (from the pleasant odour) $a$.

$P$. pulvinate, subtomentose, yellowish rosy-umber. St. somewhat clavate, sometimes fusiform-rooted, yellowish, pale vinousclouded below. $T$. cream-yellowish. Po. minute, round. Flesh buff- or yellow-whitish, changing to pale biue.

Single or crespitose. Woods, oak. Sept.-Oct. $5 \frac{1}{\frac{1}{3}} \times 3 \frac{1}{\frac{1}{2}} \times \mathrm{I}_{\mathrm{s}}^{\frac{5}{5}}$ in.

1496. B. impolitus Fr. (from the dull colour of the pileus; impolitus, unpolished) $a b$.

$P$. pulvinate, sometimes cracking into areolæ, flocculose, dull warm- or dull yellowish-umber. $S t$. clavate, pubescent, yellowish- or olive-white above, brown or olive-brown below. $T$. rounded-free, sulphur to yellow and dull olive. $P_{0}$. minute. Flesh dull biscuit-colour, sparingly changing to blue.

Edible. Taste and odour pleasant. Woods, mixed : rare. June-Sept. $5^{\frac{1}{2}} \times 3 \frac{T}{5} \times I_{\frac{1}{2}}$ in. Pileus sometimes 9 in. in diam.

1497. B. æstivalis Fr. (from its time of growth ; estiz'alis, pertaining to summer) $a b$.

$P$. convex to expanded, almost white, shaded pale buff, yellowish or brownish: marg. often waved. St. clavate, even, smooth, pale yellow or pale buff above, vinous-brown below. $T$. pale to bright sellow then sulphur-greenish. Flesh white to pale 
yellowish, sometimes with a pale blue line over the T., sometimes vinous at base.

Edible. Taste and odour pleasant. Woodland pastures, roadsides, fieldsides. June-July. $5_{\frac{1}{2}}^{\frac{1}{2}} \times 3^{\frac{3}{4}} \times 2$ in. Pileus sometimes I ft. 2 in. in diam.

\section{f. Lurida.}

1498. B. satanas Lenz (from its fancied bad qualities) $a b c$.

$P$. subviscid, smooth, tan-white, shaded grey-white. St. carmine above, dark carmine reticulate, pale yellowish, dull brownish or crimson below. $T$. sulphur-ochre. Po. minute, carmine. Flesh whitish, clouded rose and bright blue.

Said to be poisonous, probably harmless. Taste mild. IVoods, mixed, oak, woody and chalky places; rare. July-Oct. $6 \times 3 \frac{1}{4} \times 2 \frac{3}{4}$ in.

1499. B. luridus Schæff. (from the lurid colours) $a b c$.

$P$. subviscid, tomentose, dark umber or dark olive-brown. St. scabrous, dull ochreous-orange, speckied crimson. $T$. pale yellow. Po. round, dark dull crimson or dull dark red. Flesh pale ochre, changing to dark dull blue, stem streaked blue and red, crimson-streaked at base.

Said to be poisonous. Taste pleasant, sweetish; odour not unpleasant. Woods, mixed, woody places; common. June-Nov. $4 \frac{1}{3} \times 4 \times 1 \frac{5}{3}$ in. Var. erythropus Fr., smaller than type, stem within and without blood-red.

1500. B. purpureus Fr. (from the sometimes purple pileus, stem and flesh) $a b$.

$P$. pulvinate, subvelvety, dry, clouded dull claret-purple, and red or brown. St. stout, yellow, base deep claret-purple, sometimes wholly ochre, variegated with purple veins and dots, or redreticulate above. $T$. adnato-rounded, yellow. Po. crimson. Flesh yellow changing to blue, deep claret-purple, or wholly crimson in St.

Taste mild. Woods, oak. June-Oct. $4 \frac{3}{8} \times 3^{\frac{3}{4}} \times 1 \frac{3}{\frac{3}{3}}$ in.

\section{TEPHROLEUCE.}

\section{g. Forose.}

1501. B. laricinus Berk. (from the habitat, larch plantations; larix, larch) $a b c$.

$P$. convex, rarely umbonate, somewhat squamulose with $V$, viscid, olive-white, shaded pale olive, pale ochre, or pale slatepurple; marg. white at first, appendiculate with V. St. equal, annulate, cribrose above A., pitted below, whitish, pale slatepurple above, pale sepia-streaky below, or pale claret-brown above and pale buff below, or pale claret-crimson-patchy. $T$. adnato-decurrent, nearly white, pale whitish-purple. $P$ o. compound. Flish white. unchangeable.

Frequent. July-Sept. $3 \frac{1}{4} \times 2 \frac{1}{ \pm} \times \frac{3}{5}$ in. 
1502. B. rubiginosus Fr. (from the reddish-brown pileus; rubigo, rust) $a b$.

$P$. conrex, pubescent to glabrous, red-brown. St. subclavate, wholly reticulate, whitish to pale brownish-biscuit. T. adnate, unequal, white. Flesh unchanging white.

scattered. Under beeches. Aurumn. $3 \frac{1}{2} \times 2 \frac{3}{5} \times \mathrm{I}$ in.

1503. B. viscidus I. (from its riscidity) $a b c$.

$P$. pulvinate, smooth, pale yellowish-brown or pale buff, clouded pale greenish or umber; marg. sometimes appendiculate with V. St. equal, vaguely reticulate above, somewhat annulate, pale yellowish-brown, slightly streaked, somewhat specked above. T. adnate, pale grey then umber and sienna, shaded unber or livid.

Woods, larch; rare. July-Aug. $5 \times 2 \frac{3}{4} \times \frac{1}{2}$ in. Intermediate forms occur between this and 1501 .

1504. B. porphyrosporus Fr. (from the purplish spores; Gr. porphureos, purple, sporos, seed) $a b$.

$P$. convex, minutely velvety, dark olive, yellowish-umber or umber, blackish when bruised. St. equal, minutely punctate, brown-yellowish, shaded olive, not dark. T. adnate, brownishyellow, faintly salmon or grey, brown when bruised. Flesh white, becoming blue near $\mathrm{T}$.

solitary or subcrespitose. Odour strong of fish. Woods, mixed, open grassy places under trees. Aug. -Sept. $5 \times 4 \frac{3}{8} \times \mathrm{I}_{\frac{1}{4}} \mathrm{in}$.

\section{h. Virsipelles.}

1505. B. versipellis Fr. (from the changeable colours of the pellicle; $z^{\prime}$ 'rto, to turn, pellis, the stem) a $b c$.

$P$. pulvinate, dry, tomentose, becoming even, red-orange, rufousbrown or fuliginous; marg. appendiculate with the V. St. attenuate upwards, yellowish-white, rough with small brown scabrous sc. $T$. adnato-rounded to free or sinuate, yellowishor pale brownish-white. Flesh whitish, becoming clouded pale grey.

Edible. Woods, heaths : frequent. July-Oct. $4 \frac{1}{2} \times 6 \times 1 \frac{1}{2}$ in. This is the "Rosetto" of Italian markets. (rathered for food by Italians in Epping Forest as the "Rossett."

1506. B. scaber Bull. (from the rough stem; scaber, rough) $a b c$.

$P$. pulvinate, smooth, viscid when moist, at length rugulose or rivulose, usually umber, but sometimes olive-umber, red, orange, fuliginous, cinereous-blackish or white. St. attenuate upwards, white or yellowish-white, striated and sprinkled with minute brownish or grey sc., sometimes imperfectly granuloso-amnulate. $T$. adnato-rounded or sinuate, white or yellowish-white, becoming dingy brownish. Flesh whitish, changing to pale slate-colour.

Edible. Woods ; common. June-Nov. $4 \frac{1}{2} \times 7 \frac{1}{2} \times \mathbf{1}$ in. This is the Forcinello or Allarello of Italian markets. Var. niz'tus Fr., white. Some- 
times attacked by a parasitic mould, Hyponyees chrysosformus, and then not edible.

1506a. B. rugosus Fr. (from the wrinkled stem; rusa, a wrinkle) a $b$.

$P$. pulvinate, even, glabrous, dry; marg. naked, brown. St. somewhat hollow, costato-rugose, slightly scabrous, somewhat ventricose, almost equal, paler than $\mathrm{P}$. $T$. free, ivory, with a faintly brownish shade. Po. round, minute. Flesh changing from white to brown or sometimes black.

Autumn. $2 \frac{5}{5} \times 4 \frac{3}{4} \times 1 \frac{5}{5}$ in. Sowerby's t. 421 does not agree with his original drawing, and the section behind is from another drawing, not of this species.

1507. B. duriuseulus Schulz. ex Fr. (from the harsh and rough stem ; duriusculus, somewhat harsh and rough) $a b$.

$P$. globose to pulvinate, smooth, viscid when moist, whitishtawny, dull chestnut, olivaceous, yellowish-brown or vinousumber. St. ventricose, attenuato-rooting, whitish or yellowish, densely brown or grey punctato-squamulose. $T$. adnexo-free, white then yellowish or yellow to olive-brownish. Po. bright yellow. Flesh white, becoming rose-colour, coppery-red, rosy- or red-slate or violet-greyish.

Edible. Woods. Aug.-Oct. $3 \frac{3}{4} \times 6 \frac{1}{2} \times 1 \frac{1}{6}$ in.

1508. B. eruentus Vent. (from the change of colour in the flesh to blood-red; criwor, gore) a $b$.

$P$. convex to plane, smooth to rugulose, brown-yellowish or olive, salmon-red or vinous where bruised. St. clavate, attenuate to a rooting base, longitudinally scabrous, yellowishwhite or yellow below and orange above. T. adnate, pale yellow. Flesh pale yellowish or pale sulphur changing to rose or vinous, darker at base of St.

Odour fetid. Under beeches. Aug. $3 \frac{13}{ \pm} \times 3 \frac{7}{8} \times 1 \frac{1}{8}$ in.

\section{i. Hyporhodice.}

1509. B. felleus Bull. (from the bitter taste; $f_{c} l$, gall) $a b c$.

$P$. pulvinate to expanded or slightly depressed, at first slightly mealy, then smooth, even, pale umber, clouded darker. St. clavate, brown-reticulate above, paler than P. T. long, adnate to adnexo-rounded. Po. angular. Flesh white, more or less changing to salmon.

Said to be poisonous. Woods, fir, beech. July-Dec. $4 \frac{3}{8} \times 4 \times 1$ in.

1510. B. alutarius Fr. (from the colour of the pileus; aluta, tanned leather) $a b$.

$P$. pulvinate to expanded, velvety then glabrous, umber-yellowish, not dark, clouded slightly darker. St. clavate, somewhat even, rugulose above, colour as $\mathrm{P}$. $T$. short, sinuato-adnate, clouded brownish. Po. round. Flesh yellowish-white, almost unchangeable.

Taste watery, mild. Wuodland pastures; rare. Oct. $2 \frac{3}{8} \times 3 \frac{3}{3} \times \frac{5}{8} \mathrm{in}$. 


\section{j. Cariosa.}

1511. B. cyanescens Bull. (from the change of colour in the flesh to blue ; cyantus, dark blue) a b c.

$P$. convex, floccoso-scaly, brownish-ochre. St. equal to clavate, spongy-cavernous, dry, cracking, slightly annulate when young, squamose, corticate, colour as P. T. adnato- or adnexorounded, yellowish-white or faintly-shaded olive. Flesh yellowish- or brownish-white, changing to blue.

Taste none. Woods, mixed; rare. July-Sept. $3 \frac{1}{\frac{1}{4}} \times 3^{\frac{1}{2}} \times \mathrm{I}_{\frac{1}{4}}$ in. Whole plant dry like 1516, drying in decay, not decomposing. Wholly veiled in infancy by a thick floccose coat. Changes to sulphur-yellow when dry.

1512. B. castaneus Bull. (from the colour of the pileus; castanea, the chestnut) $a b c$.

$P$. convex, expanded, velvety, sometimes becoming repand, yellowish-white or vinous-cream, then cinnamon. St. clavate, velvety, corticate, cracking transversely, stuffed to hollow, colour as P. T. free, whitish-sulphur to pale primrose, becoming clouded or spotted pale brownish. Flesh white.

Woods, pastures under trees, amongst brambles; rare. Aug.-Oct. $3 \frac{1}{2} \times 2 \frac{3}{8} \times \frac{3}{4}$ in.

1513. B. fulvidus Fr. (from the yellowish-brown or tawny pileus; fulans, tawny) a b.

$P$. convex to plane, smooth, shining, rigid, yellowish- or brownish-orange. St. equal to subclavate, cracking, stuffed to hollow, colour as P., paler below. T. free, white to sulphuryellowish. Flesh white to yellowish.

Taste sweet, nutty. Under trees, oak. Aug.-Sept. $2 \frac{3}{4} \times 2 \frac{3}{8} \times \frac{5}{8}$ in.

\section{Subgenus 2. Gyrodon.}

(From the gyroso-toothed tubes ; Gr. odous, a tooth.)

1514. B. eæspitosus Mass. (from the cæspitose habit) $a$.

$P$. hemispherical, tomentose, dry olivaceous-umber; marg. pale rose. St. solid, even, glabrous, yellow, base dull red. T. very short. Po. yellow. Flesh firm, yellow, changing to dark blue or bluish-black, then rufous fading to dull white, dingy-red at base.

Densely fasciculate, stems more or less connate at base. Under trees, amongst grass. Aug. $2 \frac{1}{8} \times 2 \frac{1}{8} \times I$ in.

1514a. B. Sistotrema Fr. (from the resemblance in the tubes to those of Sistotrema) $a$.

$P$. fleshy at mid., thin at marg. where it is flattened above and below, glabrous, dry, rufous- or yellowish-brown. St. equal or slightly enlarged below, even, pallid rufous or as T., tinged pale brown. T. adnate, short, unequal, yellow-tawny or pale yellowish-sulphur. Po. becoming gyroso-plicate. Flesh white clouded pale sulphur.

Dry woods. Autumn. $2 \frac{5}{8} \times 2 \frac{3}{4} \times \frac{3}{8}$ in. Var. brachyforus IV. G. Sm. (Boletus brachyforzs Kostk.) P. white. St. white, pale sulphur below. $T$. pale sulphur. Flesh sulphur-white. Changing to pale green where touched. 
1515. B. MeWeeneyi WV. G. Sm. (after Dr. Edmunds Mclleeney), Gyrodon rubellum Mclleeney, $a$.

$P$. convex, even, smooth, dry, red; mid. purplish; marg. yellowish or clouded and streaked bright crimson on a bright yellow ground. St. equal, smooth, even, solid, bright yellow. T. pale yellow. Po. bright yellow. Flesh unchanging bright yellow.

On the ground amongst moss. Oct. $\frac{3}{4} \times \frac{3}{4} \times \frac{3}{16}$ in.

\section{Subgenus 3. Boletinus.}

(Diminutive of Boletus.)

1515a. B. cavipes Opat. (from the hollow stem ; cavus, hollow, pes, a foot) $a$.

$P$. convex, umbonate, dry, floccoso-scaly, dull yellow, yellow or pale buff. St. hollow, Hoccose and pale buff below white A., white above. $T$. decurrent, somewhat large, compound, pale sulphur. Flesh white, clouded pale buff.

Autumn. $3 \frac{1}{5} \times 3 \frac{3}{8} \times \frac{5}{5}$ in.

\section{STROBILOMYCES Berk.}

(From a fancied resemblance in the pileus to a pine-cone;

Gr. strobilos, a pine cone, mukts, a fungus.)

$V_{\text {cil }}$ white, floccose, appendiculate at the margin of the pileus and forming a fugitive annulus on the stem. Pileis fleshy, tough,

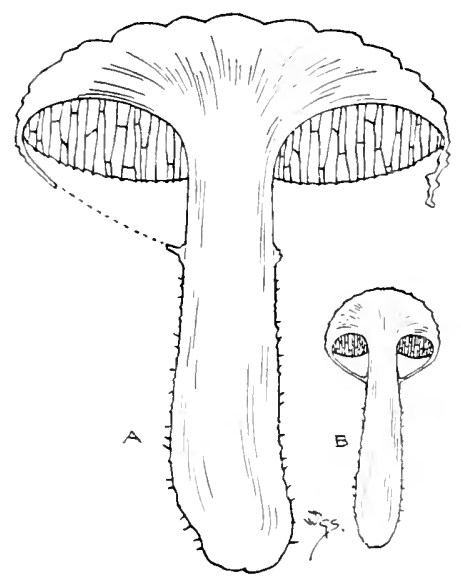

Fig. 74.-Strolilonyce's strolilaceus Berk.

$A$, section, showing ruptured veil; $B$, ditto, young example showing veil intact. One-third natural size.

broken into scales, hymenophore continuous and homogeneous with the stem. Stem solid. Tulbes very large, anastomosing. (Fig. 74.) 
1516. S. strobilaceus Berk. (from the top of the pileus, clad with pine-cone-like scales: Gr. strobilos, a pine cone) a $b c$.

$P$. globose to pulvinate, broken into large thick scales; sc. dark umber on a pale greyish ground. St. clavate, sulcato-reticulate above, coarsely fibrillose, white above, vinous-brown below. $T$. adnate, with transverse dissepiments, white, shaded dull brownish. Flesh sienna-brown.

Woods, under fir, oak, lime, amongst bracken, open grassy places. Aug.Oct. $6 \times 7 \frac{3}{8} \times \mathrm{I}_{\frac{1}{4}}^{1} \mathrm{in}$.

\section{FISTULINA Bull.}

(From the pipe-like character of the tubes; fistula, a pipe.)

Vil obsolete. Pilcus fleshy, juicy. Stim obsolete. Tubes cylindrical, free from each other as are the spines in Hydnaceer, but adnate with the substance of the pileus. (Fig. 75.)

Growing on wood. Intermediate between Polyporacece and Hydnacce.

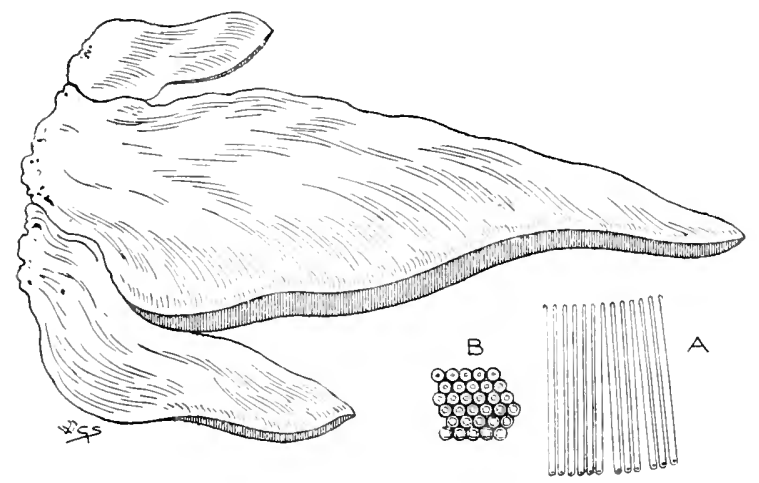

Fig. 75.-Fistalina hipatica Fr. One-third natural size. $A$ tubes. $\times 3$. B, orifices of ditto. $\times 6$.

1517. F. hepatica Fr. (from the liver-like appearance; Gr. hapatikos, of the liver) $a b c$. Imbricate.

$P$. roundish, dimidiate or subspathulate, rough with minute points, dull pale purplish-red, red, blood-red, liver-colour or chocolate, becoming blackish. T. ochre-whitish, then yellowishred. Po. distinct. Flesh thick, juicy, red-streaked like beetroot, distilling a red juice.

Edible when quite ripe. Taste somewhat acid. Trunks, oak, ash, walnut, willow, beech, chestnut, hornbeam, elm. Aug.-Oct. Diam. I ft. 4 in. Sometimes attaining a great size with a weight as much as $19 \mathrm{lbs}$.: unlike many Poly,foracea it perishes in about three weeks after its first appearance. The "liver" or "beef-steak" fungus. Said by Hartig to be the cause of a deep red-brown decomposition in oak. Cirionyyces (Pty'chogaster) hepaticus is said by Saccardo to be an imperfect state of this. 


\section{POLYPORUS Mich.}

(From the many pores or mouths of the tubes of the hymenium; Gr. polus, many, poros, a pore.)

Pilcus woody, corky or somewhat soft, not zoned, flesh thick. Stem long, short or none, when present simple. Hymenophore descending and forming a trama between the tubes. Tubes connate

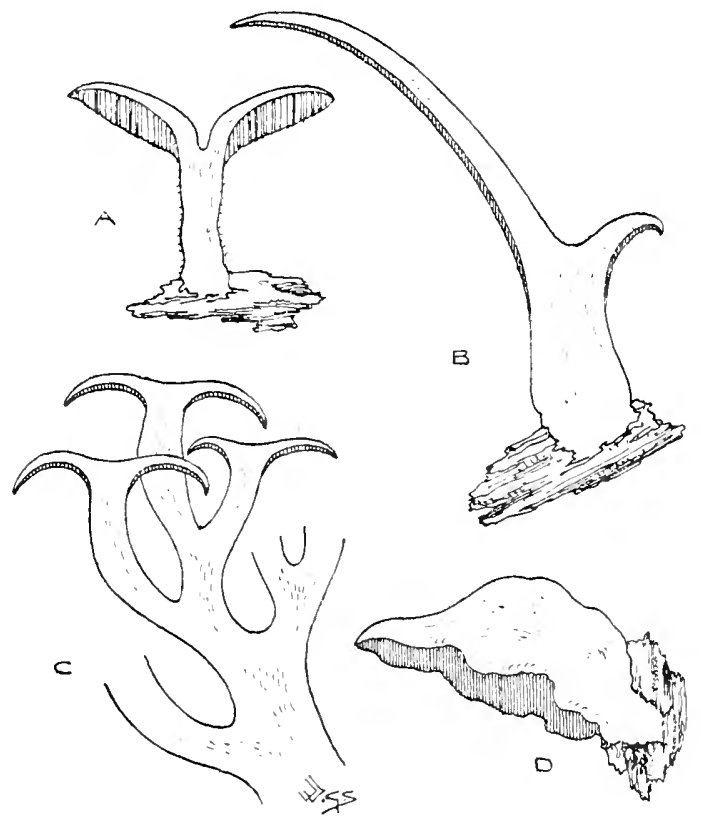

Fig. 76.-Sections of A, Folyponuslentus Berk.; B. P. Rostkonii Fr.; C, F. umbellatus Fr.; D, F. cassius Fr. One-half natural size.

with the substance of the pileus and not separable from it or from each other, extended with round, angular or torn pores, not normally stratose. (Fig. 76.)

Persistent fungi, mostly growing on wood. Odour, if any, slightly acid. A few are said to be edible. Species 1517a-1575 Mesopode. Pileus entire or excentric. Stem distinct, vertical, central, simple, self-coloured, not blackish at the base.

a. Carnose. Pilens fragile or pliant, dull in colour, scaly or floccose, not coriaceous, zoneless. Spores white, growing on the ground. Autumnal. Edible. 
b. Lente. Pileus fleshy-pliant, then coriaceous, zoneless. Spores white. Growing on trunks. Persistent.

Pilens lustrous, scaly or villous.

1519,1520

Pileus even, smooth.

1521,1522

c. Spongiose. Pilus spongy-soft, hygrophanous, tomentose, then corky or coriaceous, regular, irregular, lobed or dimidiate. Stem short, irregular. Pores irregular, changing colour. Spores white. Mostly terrestrial, lasting through the winter in a dead state, not peremnial.

1523,1524

d. Subcoriacee. Pileus at first indurated, arid; when full grown corky or coriaceous, tomentose, velvety. Stem definite, rarely lateral. Pores at first sprinkled over with a white bloom. Substance ferruginous. Terrestrial or on trunks; more regular, rigid and persistent than Spongiose. Not reviving.

$1524 a-1526$

Pleuropode. Pileus pliant or corky, horizontal, not circular. Stem simple, lateral or excentric, black at the base. Growing on wood.

a. Linte. Pileus fleshy-pliant, zoneless. Stcm excentric, blackish at the base. Tuhes short. Substance somewhat fibrous, pallid.

$1527-1534$

b. Suberosolignose. Pileus thick, hard zoneless. Stem stout, vertical, of the same colour at the base. Tubes elongated.

1535

Merismatæ. With many branching stems proceeding from a common trunk, each as a rule bearing a pileus or pileolus. The largest of fungi are found in this group.

a. Carnosa. Tufts central, stipitate, springing from a common, more or less concrete base. Pileus fleshy firm floccosofibrous, zoneless, not coriaceo-indurate. Tubes separating from the pileus. Spores white. Partly terrestrial. Autumnal. Edible.

$1536-1539$

b. Lentie. From the tough pileus. Tufts lateral, substipitate, in many imbricate layers. Pilcus fleshy-pliant, then subcoriaceous, fragile when old, more or less zoned, fibrous within. Stems more or less connate, or growing from a common tuberous base. Growing at the base of trunks. Spores white. Autumnal, not lasting till the following spring. Not edible. 1540-1542

c. Cascosa. Tufts sessile on the stems of trees, commonly dimidiate, but in horizontal positions, central and expanded on all sides. Springing from a tubercular base inito numerous pileoli. Pilezs cheesy, at first soft and juicy, then arid, without a pellicle, zoneless. Tubes separating. Growing in spring and summer, soon decaying. Acid. 
d. Suberose. Tufts somewhat sessile, lateral or central. Pilei or Pilioli corky or coriaceous, persistent, tough, free at the base, not effused. Substance floccose, somewhat soft, suitable for tinder. Tulies adnate. On trunks close to the ground. Not edible.

1547,1548

ApoD E. Pileus sessile, adnate by a thickened, dilated base, dimidiate or entire, attached behind, commonly by an umbo; nore rarely attenuate and sessile. On wood. Polymorphous.

$1549-1575$

a. Anodermee. Fileus somewhat fleshy, without a cuticle, the surface broken into flocci or fibres, zoneless, the substance transversely zoned within and more or less fibrous. Annual, not reviving.

Pileus cheesy, at first watery-soft, fragile, flocculose, not bristlyhispid; when fully grown, soft to hard, but soon putrefying, not lasting through the winter. Tubes white.

Pores round, entire, obtuse, without small teeth. Not becoming hard. 1549-1553

Pores elongate, Hexuous, acute and torn. 1554-1559 Pileus tough, fleshy-pliant, soft, elastic, villoso-tomentose. Tules somewhat adnate, coloured. Lasting till the following spring.

Substance coloured.

$1560-1562$

Substance white or slightly brownish.

$1563-1568$

At first moist, spongy, when dry firm, elastic, usually bristlyhisped, internally fibrous. Autumnal, annual. The finest examples growing on living trunks. Must not be confounded with the section Stuppose of Poly'stictus, where the members are dry and rigid.

Substance coloured.

1569,1570

Substance and spores white.

1571,1572

b. Placodirmete. Pileus clothed with a continuous crust, zoneless or concentrically sulcate. Persistent.

Substance coloured.

1573

Substance white, not zoned. Spores white.

1574,1575

MESOPOH:.

a. Carnosi?.

1517a. P. flavovirens Berk. \& Rav. (from the yellowish-green colour of the pileus; flavus, light yellow, vireo, to be green) $a$.

$P$. soft, pulvinate or depressed, irregularly lobed, subtomentose, dull yellowish-green, yellowish-olive or olive. St. slightly attenuate downwards, pallid, paler than P., or shaded white and yellowish. T. slightly to very decurrent, short, irregular, 
toothed, at length torn as in Irpex, white, yellow, then greenish. Flesh white, clouded pale sulphur.

Woods, mixed, under Pinus sylvestris. Sept. $3 \frac{1}{4} \times 2 \frac{3}{4} \times \frac{7}{5} \mathrm{in}$.

1518. P. leucomelas Fr. (from the colours; Gr. leukos, white, melas, black) $a$.

$P$. expanded, thick, subfragile, slightly silky-squamulose, dark olive-umber or blackish fuliginous. St. equal to unequal, subtomentose, yellow-umber or as P. $T$. decurrent $P o$. large, short, unequal, white to cinereous, black when dry. Flesh white, changing to faintest rufous-purplish or black.

Woods, fir. Sept. $5 \frac{1}{\frac{1}{4}} \times \frac{7}{5} \times \frac{7}{5}$ in.

\section{b. Lente.}

1519. P. lentus Berk. (from the tough-pliant substance; lentus, tough) $a b c$.

$P$. umbilicate, thin, minutely scaly, buff-white, shaded buff, ochreous or reddish-brown. St. equal, hispid or furfuraceous, colour as P., shaded sienna-buff. $T$. decurrent, long, large, unequal, yellowish-white. Po. toothed.

Dead roots and branches, furze, hawthorn; frequent. April-Sept. $2 \times 1 \times \frac{1}{4} \mathrm{in}$.

1520. P. brumalis Fr. (from the time of growth; bruma, winter) $a b c$. $P$. convexo-expanded, very thin, villous to squamulose and smooth, rich cinnamon to umber or slate-umber; marg. paler. St. equal, minutely dark-umber-floccose on a pale brown ground, when young brown-ochre above, ochre below. $T$. decurrent, very shallow, white, becoming yellow. Po. toothed. Flesh white.

Woods, on dead branches, sticks, hazel, birch, beech, sometimes on the ground; frequent. $2 \times 2 \frac{1}{2} \times \frac{3}{16}$ in.

1520a. P. arcularius Fr. (arcularius, a casket-maker; in reference to the fulvous pores, covered by the pileus-as if in a casket) $\boldsymbol{t}$.

$P$. pliant, then coriaceous, somewhat umbilicate, zoneless, orangebrown, in the first year covered with fuscous sq., in the second glabrous with a yellow strigose margin. St. somewhat squamulose. greyish-fuscous or sooty-brown. 7. oblong. Po.rhomboidal, thin, rather large, entire, whitish then fulvous. Flesh white.

Dead twigs. Sept. Varying from $\frac{3}{8} \times \frac{1}{2} \times \frac{1}{1 \frac{1}{6}}$ in. to $2 \frac{7}{5} \times 1 \frac{1}{2} \times \frac{1}{4}$ in. Distinguished from 1520 by the shape of the pores, the strigose margin and fuscous squamules of the pileus.

1521. P. fuscidulus Fr. (from the somewhat fuscous pileus) $a$.

$P$. convexo-plane, rery thin, leathery, pale yellowish-brown or pale olive-brown. St. equal, smooth, pale yellowish-olive. $T$. adnato-decurrent, somewhat long, warm yellowish-white.

Woods, chips ; rare. $2 \frac{1}{4} \times 1 \frac{3}{4} \times \frac{1}{4}$ in. 
1522. P. leptocephalus Fr. (from the thin pileus: Gr. liptos, thin, kiphale, the head) a b c.

$P$. convexo-plane, slightly depressed or stibumbilicate, thin, leathery, pale yellowish-buff or shining yellowish. St. smooth, colour as P., sometimes black at base. $T$. adnate. Po. minute, colour as P. or whitish. Flesh white.

Woods, on trunks, lead birch; rare. Oct.-1lar. $2 \frac{1}{4} \times \mathbf{I} \frac{3}{8} \times \frac{3}{10}$ in.

\section{c. Sponiriosa.}

1523. P. Schweinitzii Fr. (after Ludwig David von Schweinitz) a bc . $P$. expanded, rugged-uneven, strigoso-tomentose, thick, deep orange-sienma to sienna-umber, irregularly zoned brown; mid. almost black. St. rugged-uneven or obsolete, sulphur, umberclouded below, dark umber at base. $T$. decurrent, large, sulphur to green-brownish-sulphur. Po. torn. Flesh deep sienna.

Woods, about roots, fir, pine, larch. July-Oct. $6 \frac{1}{\frac{1}{4}} \times \mathrm{I}_{\frac{3}{4}}^{\frac{3}{4}} \times \mathrm{I}_{\frac{3}{8}}^{\frac{3}{3}}$ in. Hartig states that this fungus has an effect on living wood similar to that of the "Dry-Rot" 1680.

1524. P. pufescens Fr. (from the rufescent colour) a $b c$.

$P$. depressed to infundibuliform, somewhat thin, strigose, pale vinous-umber : mid. darker: marg. irregular. St. equal, uneven, sometimes lateral or absent, colour as P., darker and clouded below. $T$. decurrent, pale yellowish-rinous-umber. Po. torn. Flesli colour as P.

On and about stumps, ash, elm. Sept.-Jan. $5 \times 2 \frac{1}{5} \times \mathrm{I}$ in. Sometimes wholly biscuit-buff.

\section{d. Subcoriace.}

1524a. P. tomentosus Fr. (from the minutely velvety pileus; tomentumi, wool) at $c$.

$P$. plane, corky, becoming hard, often lobed and irregular, tawny ; marg. thin, straight, sometimes yellowish. St. solid, attenuate upwards, sometimes a mere base or central point, sometimes rooting, steel-grey. $T$. subdecurrent, short. $P o$. very minute, entire, pale- or greyish-yellow, at first delicately whitish-pruinose. Flesh pale tawny.

On the ground. $2 \times \frac{7}{5} \times \frac{1}{4}$ in. This with $1524 \mathrm{~b}, 1525$ and 1526 have been placed by Cooke, Saccardo and Massee in Poly'tictus.

1524b. P. Montagnei Fr. (after J. F. C. Montagne) $a$.

$P$. cyathiform, irregular, zoneless, uneven, ferruginous, becoming smooth; marg. thin. St. unequal, pubescent, colour as $\mathrm{P}$. $T$. short. Po. large, round, edge entire, yellowish-white, then ferruginous. Flesh tawny-cinnamon.

Distinguished from 1525 by the zoneless pileus and large entire pores. Autumn. $1 \frac{1}{2} \times 1 \frac{1}{1} \times \frac{3}{15}$ in. 
1525. P. perennis Fr. (from its peremial habit) $a b c$.

$P$. plano-infundibuliform, velvety to smooth, thin, pale yellowishbrown, zoned darker; marg. fimbriate. St. uneven, attenuate upwards, velvety, vinous-umber, pale sienna below. $T$. decurrent, short, pale umber. $P_{0}$. small, torn, at first with a white bloom. Flesh pale rinous-umber.

On and about stumps, beech, burnt ground; frequent. July-Jan. $2 \frac{3}{8} \times 1 \frac{1}{4} \times \frac{1}{4}$ in.

1526. P. cinnamomeus Sacc. (from the cinnamon colour of the pileus) a b.

$P$. plano-depressed or subinfundibuliform, velvety to smooth, thin, leathery, flaccid, bright sienna-cimnamon, zoned deep rich brown. St. usually attenuate downwards, base sometimes tuberous, colour as P. T. adnate to adnato-decurrent, short, brownish-cinnamon, fulvous when dry. Po. large. Flish rich siemna-cinnamon.

Solitary or in rows to subcrspitose. On the ground, under trees, burnt places. Oct. $3 \frac{1}{4} \times \frac{3}{4} \times \frac{1}{4}$ in. Pilens sometimes almost jet hlack.

\section{PleuroponÆ.}

$$
\text { a. Lentic. }
$$

1527. P. squamosus Fr. (from the scaly pileus; squama, a scale) a b $c$.

$P$. fan-shaped, flat, pale brownish, sienna-brown-scaly. St. yellowish-umber, black-spotted, blackish at base. T. decurrent, short. Po. toothed, yellowish-white, like the pores of tripe. Flesh white.

Single to crespitose or imbricate. Odou rank. Trunks and stumps, ash, mulberry, walnut, maple, wych elm; common. Sometimes $2 \mathrm{ft}$. 6 in. in diam. and $34 \mathrm{lbs}$, in weight. April-Nor. I ft. 4 in. $\times I_{\frac{3}{4}}^{\frac{3}{4}} \times \mathrm{I}_{\frac{3}{4}} \mathrm{in}$. The flesh makes good razor-strops, but is often larva pierced. In the British Museum collection of drawings Bolton illustrates an antler-like form of this species, growing from a dark brown sclerotioid base.

1528. P. Michelii Fr. (after Pier' Antonio Micheli) $a$.

$P$. spathulate, slightly silky, yellowish-white with darker sq. St. equal, rough, white, becoming fuscous at base. $T$. decurrent, short. Po. large, entire, white.

Somewhat crespitose. Stumps, willow. $2 \frac{3}{4} \times \frac{3}{4} \times \frac{1}{2}$ in.

1529. P. melanopus Fr. (from the black stem; Gr. melas, black, pois, a foot) a $b c$.

$P$. subinfundibuliform, delicately flocculose to smooth, somewhat thick, pale yellowish. St. attenuate upwards, somewhat velvety, umber, rich brown or black. T. decurrent, short, white. Po. minute.

Somewhat cespitose. Dead wood, roots; uncommon. Aug.-Oct. $2 \frac{7}{8} \times 1 \frac{1}{2} \times \frac{3}{8}$ in. 
1530. P. Rostkovii Fr. (after Friedrich Wilhelm Gottlieb Rostkovius) a b $c$.

$P$. dimidiato-infundibuliform, somewhat thick, yellowish-sienna, deep umber at mid. and behind. St. equal, reticulate, deep umber to black. $T$. decurrent, short, white to dull yellowish. Po. large, angular, toothed.

Somewhat crespitose. Woods, on stumps, trunks, ash; rare. July-Jan. $4 \frac{7}{8} \times 1 \frac{1}{4} \times \frac{3}{1}$ in.

1531. P. picipes Fr. (from the pitch-black stem; pix, pitch, pes, a foot) $a b c$.

$P$. somewhat depressed at mid. or behind, red-brown or chestnut. St. equal or attenuate downwards, velvety to naked. $T$. decurrent, somewhat short, whitish to yellowish. Po. round, small. Flesh white.

Odour somewhat sweet. Trunks, willow ; frequent. July-Dec. $6 \frac{5}{8} \times 1 \frac{1}{8} \times \frac{3}{8}$ in. Like a large 1533 , var. mummularius.

1532. P. varius Fr. (from its variable size, colour and form) $a b c$.

$P$. depressed at mid. or behind, somewhat virgate, yellowishbrown, shaded palish vinous-umber; marg. undulate. St. even, smooth, cinereous blackish downwards. $T$. decurrent, short, white to yellowish. Po. minute. Flesh white.

Single to subcrespitose. Sometimes sessile. Trunks, stumps, ash, willow, elder, birch; frequent. Aug. $-\mathrm{Feb} .3 \frac{3}{4} \times 1 \frac{1}{4} \times \frac{5}{5}$ in.

1533. P. elegans Fr. (from its elegance) $a b c$.

$P$. convex to slightly depressed, usually excentric or dimidiate, becoming woody, ochreous, orange or reddish. St. even, smooth, black, abruptly pallid at apex. $T$. adnate or slightly decurrent, yellowish-white, brownish-yellow or" slightly orange. Po. minute.

Single to subcrespitose. Trunks, birch, willow, beech ; frequent. July-Feb. $2 \mathbf{2}_{+}^{3} \times \mathbf{1} \frac{3}{4} \times \frac{3}{8}$ in. Var. mummularius $\mathrm{Fr}$. Smaller than type, $P$. almost regular. $I$ in. in diam.

1534. P. petaloides Fr. (from a fancied resemblance in its shape and thinness to a leaf; Gr. petalon, a leaf, cidos, appearance) $a b$.

$P$. spathulate, thin, rugose, zoneless, dark rich chestnut. St. smooth, whitish, sometimes dark brown below. $T$. decurrent, somewhat short, white. Po. minute.

Old stumps. Jan. $2 \times \frac{3}{4} \times 3$ in.

\section{b. Suberosolignosa.}

1535. P. lucidus Fr. (from the shining pileus and stem; lucidus, bright) $a b c$.

$P$. fan-shaped or spathulate, sulcato-rugose, corky to woody, lustrous, deep rich blood-chestnut. St. lateral, irregular, uneven, lustrous, deep purple-chestnut. T. adnate, somewhat 
long, rarely stratose, yellowish-white, clouded pale brownish. Flesh tan-brownish.

Single to crespitose. On and abont stumps, hornleam ; frequent. Oct.-April. $7 \times 6 \times 1 \frac{1}{4}$ in. Sometimes sessile. Occurs in peat-beds in the fens of the Eastern counties and in the lake-side pile-dwellings of switzerland and Italy. The varnished appearance of the pileus is due to the presence of resin. This species has been placed in Fomes by Cooke and Saccardo.

\section{MERISMAT 玉.}

\section{a. Carnosie.}

1536. P. umbellatus Fr. (from the many stems and pileoli forming an umbel) $a$.

$P i$ very numerous, entire, umbilicate, fibrous-fleshy, warm-umber, or yellowish-reddish- or slate-umber, rarely white. St. white to yellowish-white. $T$. short, subdecurrent, colour as St. Po. minute.

Said to be edible. Woods, near and on stumps. July. Mass $9_{9}^{3} \times 9_{\frac{3}{4}}^{3}$ in. Pi. $1 \frac{1}{2} \times 1 \frac{1}{1} \times \frac{1}{4}$ in.

1537. P. frondosus Fr. (from the branching habit; frons, a leafy branch) a $c$.

Pi. numerous, dimidiate, rugose, convex to recurved, somewhat thin and tough, yellowish-umber to pale purple-slate. St. yellowish-white. $T$. decurrent, somewhat short, yellowishwhite. Po. small.

Stumps, trunks, roots, oak; rare. Oct. Nass $10 \frac{1}{2} \times 9 \frac{3}{4}$ in. Pi. $3 \frac{3}{4} \times 1 \frac{3}{\frac{3}{4}} \times \frac{3}{4}$ in.

1538. P. intybaceus Fr. (from a fancied resemblance to succory, Cichorium Intybus) a bc.

Pi. numerous, dimidiate, fragile, sepia, not dark, lighter at mid. St. white. T. decurrent, very short, pale sepia.

Edible. Taste usually pleasant, sometimes slightly astringent when raw; odour of mice, in decay like japanner's varnish. Slumps, trunks, oak; rare. Sept.-Dec. MIass $12 \frac{1}{2} \times 7 \frac{1}{2}$ in. Pi. $3 \times 1 \frac{3}{4} \times \frac{1}{2}$ in. The cauliflowerlike stems are the edible parts, not the pileoli. The "cock of the woods" (grey grouse-hen) of the Vosges and Arclennes.

1539. P. cristatus Fr. (from the crested appearance; crista, a crest) a $c$.

$P i$. numerous, dimidiate, somewhat thick, fragile, bright yellow or greenish, sometimes clouded reddish. St. irregular. $T$. very short, whitish. Po. minute, angular, torn. Flesh white, becoming ochre.

Woods, beech; rare. Mass $7_{\frac{3}{4}}^{3}$ in. in diam. Pi. $3 \frac{1}{2}$ in.

\section{b. Lente.}

1540. P. giganteus Fr. (from its great size) a b c.

$P i$. several, dimidiate, thick, umber : $z$. darker. St. short, pale yellowish-white, brownish or ashy where bruised. $T$. somewhat 
short. Po. minute, colour as St. Flesh cheesy, rinouswhite.

Taste astringent, disagreeable; odour strong, oppressive, like rotten cheese. stumps, elm, oak, beech, acacia. Beconing blackish in drying. JulyJan. Jass $I \mathrm{ft} .3$ in. Pi. $8 \frac{3}{4}$ in.

1541. P. osseus Kalch. (from its colour, like bone, os) a $c$.

White, shaded grey or buff-white.

$P i$. several, thick, convex to flat, smooth. St. short. T. decurrent, short. Po. minute, shallow.

Odour acid. Stumps, larch. Oct. Mass $6 \frac{3}{4}$ in. Pi. $4 \frac{3}{8}$ in.

1542. P. acanthoides Fr. (from a fancied resemblance to Acanthus; Gr. cidos, appearance) a b $c$.

Pi. numerous, dimidiate, thin, pliant, yellowish-sienna ; z. sienna ; marg. paler. St. pale brownish. Po. lamelloso-sinuate, toothed, colour as St. Flesh faintly rufous.

Trunks, roots, buried wood. Sept. Nass $S_{ \pm}^{3}$ in. Pi, $4 \frac{1}{2}$ in.

\section{c. Caseose.}

1543. P. sulphureus Fr. (from the sulphur-colour) $a b c$.

$P$. few, thick, juicy-cheesy, sulphur-yellowish, somewhat clouded ochreous-reddish or salmon. T. short. Po. minute, colour as P. Flesh thick, pale sulphur, somewhat vinous above.

Imbricate. Said to be edible. Taste slightly astringent, somewhat acid; odour fragrant to disagreeable, acid, foxy, pleasant if burnt when dry. Living trees, stumps, oak, yew, chestnut, willow, cherry, alder, poplar, walnut, pear, apple, larch, Kobinia, ash, pine. May-Oct. Mlass $9 \frac{1}{2}$ in. P. $7 \frac{1}{t}$ in. Pileus covered with crystals of oxalate of lime. One of the fungi used in past times for tinder. Usually rots the middle of a treetrunk, leaving the outside sound. Sometimes phosphorescent. Cerionyces (Ptychugaster) aurantiacus is said by Saccardo to be an imperfect state of this species.

1544. P. imbricatus Fr. (from the imbricate habit) $a b c$.

$P$. few to many, firmer than 1543 , thin, rugose, subviscid, buff. T. long, pale yellowish. Po. lustrous. Flesh thin, white.

Odour strong, like seed-cake. Trunks, oak; rare. June-Nov. Mass II in. P. 6 in.

1545. P. spongia Fr. (from the spongy substance) $a b$.

$P$. few, thick to thin, often rugose, yellowish- chestnut- or umbersienna, darker at base: $z$. indistinct ; marg. sometimes sulphur or pale dull ochre. $T$. somewhat short. Po. minute, torn, pale primrose, faintly reddish where bruised. Flesh pale opaque sienna.

Subimbricale. Trunks, pine. Uct.-Nor. IIass I I in. P. 6 in. Boletus Herbergii Rostk. is a form of this. 
1546. P. alligatus Fr. (from its binding or enveloping habit; alligo, to bind to) a $b c$.

$P$. many, villous, white; marg. pale umber. T. short. Po. minutely labyrinthiform, colour as P. Flesh biscuit-whitish.

Imbricato-connate. Roots, etc. ; rare. Sept. Mass 5 in. P. $2 \frac{3}{2}$ in. Sometimes faintly shaded, vinous.

\section{d. Sulberosa.}

1547. P. heteroclitus Fr. (from its irregular, leaning habit; Gr. heteros, one of two, klino, to lean) a $c$.

$P$. few or many, thin, shaggy-villous, zoneless, yellow, siemna behind ; marg. lobed. $T$. somewhat short. Po. pale primrose to golden-yellow. Flesh white.

Imbricate. Stumps, partly buried, oak; rare. Jan. Mass 7 in. P. $3 \frac{1}{2}$ in. 1548. P. salignus Fr. (from a frequent habitat; salix, willow) $a b c$.

$P$. few to many, thin, adpressedly villous, whitish; marg. crested. $T$. somewhat short. Po. intricately flexuous, whitish. Flesh white.

Imbricate. Odour none. Trunks, willow, ash, poplar, oak, apple ; uncommon. Oct.-Feb. Mass $7 \frac{1}{ \pm}$ in. P. $3 \frac{3}{4}$ in. A dark line separates the tubes from the flesh of the pileus as seen in section.

\section{APODE.}

\section{a. Anodermede.}

1549. P. epileucus Fr. (from its whitish colour; Gr. cpi, upon, leukos, white) a bc.

$P$. few, pulvinate, villous-rugged, thick, cheesy-soft, yellowishwhite, clouded greyish, rarely faintly zoned. $T$. long, biscuitprimrose or brownish-white. Flesh buff-white.

Subimbricate. Stumps, trunks, elm, fir, willow, birch: rare. Sept.-Nov. Mass $S \frac{3}{3}$ in. $P .5 \frac{1}{2}$ in.

1550. P. alutaceus Fr. (from its colour, like tanned leather, aluta) $a$. Whitish-ochre.

$P$. few, tough, reniform-dilated, slightly velvety; marg. acute, even. Po. small.

Imbricate. Hecayed stumps, pine, beech. Sept.-Oct. Mlass $5 \frac{3}{\frac{3}{4}}$ in. P. 3 in.

1551. P. pallescens Fr. (from the colours becoming pale; fallesco, to grow pale) a $b c$.

$P$. few, corky, even to rugose, pale buff; marg. lighter. $T$. small, pale yellowish, pale vinous towards base. Flesh buff-white.

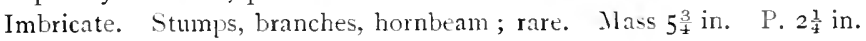

1552. P. chioneus Fr. (from its snow-white colour; Gr. chion, snow) a b c. Pearly or biscuit-white.

$P$. few, soft, fragile, smooth, thin, zoneless or faintly zoned. $T$. long, but varying in length.

single to 3 -imbricate. Taste astringent; odour acid. Stumps, larch, fir, birch; uncommon. June-Dec. P. $2 \frac{1}{6}$ in. 
1553. P. cerebrinus B. $\& \mathrm{Br}$. (from its brain-like appearance; corcbrum, the brain) a b. White.

$P$. pulvinate, delicately tomentose, then smooth; marg. crenate. T. rounded, entire, dissepiments of the large Po. thick, obtuse.

Fir. Aug. P. I in. Resupinate; not unlike 1657.

1554. P. lacteus Fr. (from the milk-white colour; lac, milk) a $c$. White.

P. I-3 connate, gibbous, thick, flesh-fibrous, pubescent, zoneless. $T$. long. $P o$. toothed, at length labyrinthiform and torn.

Imbricato-comnate. Taste astringent; odour none. Dead bark, leaves, laburnum, birch, beech ; rare. Siept.-Nov. I'. 3 in.

1555. P. fragilis Fr. (from its fragile substance) $a b c$.

$P$. convex to plano-depressed, rugose, thick, whitish, pale foxy-red where bruised. $T$. long. $P$ o. minute, intricate, colour as $P$.

single to 7 -imbricale. Stumps and branches, pine, covering moss on pinebark, larch; uncommon. Sept.-Nov. P. $2 \frac{1}{t}$ in.

1556. P. mollis Fr. (from its softness; mollis, soft) a $c$.

$P$. effuso-reflexed, rugose, fleshy fibrous, pale flesh-colour, livid or brownish-biscuit, sometimes slightly zoned darker; marg. acute, foxy where bruised. $T$. long, unequal, soft, white or salmon-white, foxy where bruised.

Single to 3 -imbricate, or connate. Dead pine. Sept. P. $2 \frac{1}{2}$ in.

1557. P. eæsius Fr. (from its bluish-grey clouding; cesins, bluishgrey) a b c. Ivory-white, becoming pale turquoise or bluishgrey where bruised.

$P$. thick, fleshy, lobed, unequal, silky. T. long. $P o$. torn into teeth.

single to 3 -imbricate or connate. Dead fir, larch, dead fir-leaves, roots, trunks, posts, sticks. Mar.-Nov. P. $4 \frac{1}{x}$ in.

1558. P. trabeus Rostk. (from its frequent habitat, the wood of ships ; trabs, timber) a $c$. White.

$P$. thick, firm, effuso-reflexed, transversely elongate, zoneless, pallid. T. short. Po. minute, toothed. Flesh hyaline when moist.

Single. Wood, yew, pine. Ocl. P. $3^{\frac{3}{4}}$ in. Commonly confounded with 1559 , from which it is distinguished by being more regular and brighter in colour.

1559. P. destructor Fr. (from its destructive effect on wood) $a b c$. Ivory-white.

$P$. watery-fleshy, rugose; marg. pale brownish. $P$ o. toothed or torn.

Connato-imbricate. Worked wook, which it softens and destroy's, stumps, branches, fir, larch, fir-leaves, willow; common. Mlay-1)ec. P. $3 \frac{5}{8}$ in. Var. undulatus Sacc. Broadly expanded, marginate, pale foxy-brown. Cirionyces (Ptychograster) albus (fig. 77) is very commonly associated with this species and 1572. Berkeley consiters Ptychosaster to be an imperfect conidial slate of one or both of these Polypores, whilst others regard it as a parasite. It has also been made a species of Polyporns, viz. P. Ftychogaster 
Ludw., and as such is placed after 1559 by Saccardo. The plant grows on rotten fir-trunks and on the ground, involving fir-leaves.
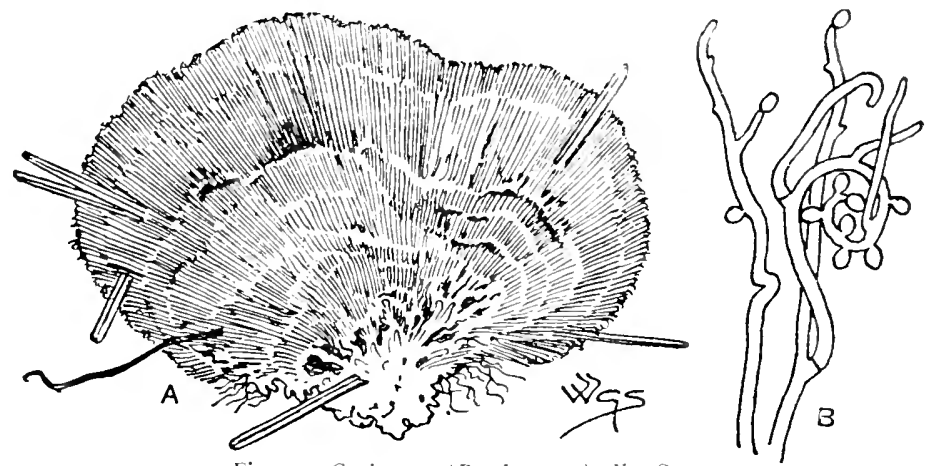

Fig. 77.-Cerinnuces F'tycluggester albus Sacc. A, section, three-quarters natural size; $B$, threads and conidia. $\times 370$.

1560. P. nidulans Fr. (from the nest-like shape; nidus, a nest) a $b c$. Whitish-sienna and umber, or slightly orange.

$P$. single, pulvinate, villous, zoneless, thick, soft. $T$. long, unequal, angular.

Single, usually in small troops. Fragrant when dry. Dead wood, branches, wych elm; uncommon. Jan.-Dec. P. I in. At first resupinate and from 3 to 7 in. in diam.

1561. P. putilans Fr. (from its reddish colour; mtilo, to be reddish) a b $c$.

$P$. thin, villous to smooth, zoneless, ivory-whitish, then brownish, clouded vinous. $T$. long. Po. minute, equal, cimnamon or vinous. Flesh pale ochre, vinous towards T.

Single to 2-imbricate or connate. Odour powerful and pleasant of anise. Logs, branches, sticks, aak; uncommon. A pril-Dec. P. I $\frac{3}{4}$ in. At first resupinate. Perhaps this and 1560 are one species. See 1607.

1562. P. gilvus Fr. (from its colour; gilizı, pale yellowish) a b $c$.

$P$. thick, zoneless, pale siemna-yellow or sienna-umber; marg. velvety, becoming smooth, lighter than $P$., or dull orange. $P$ (') minute with barren patches, dark orange-brown: marg. barren. Flesh bright orange-brown.

Single to 3-imbricate or connate. Trunks, willow, oak; rare. Jan. P. 3 in. Boletus imfuber Sow. t. I95. At first effuso-resupinate.

1563. P. fumosus (from its smoky colour ; fumosus, smoky) a b $c$.

$P$. firm, zoneless, somewhat thin, silky to smooth, pale buff, pale biscuit or slate-umber, sometimes shaded vinous. $T$. whitish to ivory-umber, or smoky-livid, with a smoky tinge, darker where bruised, often with harren patches. $P$ '. round, entire. Flesh very pale buff or brownish.

2-I6 imbricate and connale. Stumps, logs, poplar, willow, birch, horsechestnut ; common. July-Feb. P. 2 in. Jlr. Carleton Rea has met with a very large example on a wooden grass-roller. 
1563a. P. fragrans Peck (from the fragrance).

$P$. Heshy, effuso-reflexed, imbricate, sometimes thickened at the base, velvety, with minute tomentum, pale reddish-grey or alutaceous ; marg. thin. T. $\frac{1}{12}$ in. long. Po. minute, unequal, angular, whitish, becoming darker with age, blackish where bruised. Flesh slightly fibrous, zoned, concolorous.

stumps, elm. $4 \times 2$ in. Often mistaken for 1563 , but distinguished by the sweet odour.

1564. P. adustus Fr. (from its scorched appearance; aduro, to burn) a $b c$.

$P$. somewhat thin, buff-ivory, with 4-6 darker z.; marg. becoming blackish. $T$. somewhat short. $P$ o. minute, warm irory-brown, becoming ragged and tinted slate-colour. Flesh whitish to buff-ivory.

Single to S-imbricate. Stumps, alder, oak, willow, birch, poplar, hornbeam, dead leaves ; frequent. Jan.-Dec. l'. $2 \frac{3}{4}$ in.

1565. P. crispus Fr. (from the crisped margin of the pileus) $a c$.

$P$. rugose, tuberculose behind, thin, ivory-buff, brownish behind; marg. umber-white. $T$. somewhat short. $P o$. large, unequal, labyrinthiform, slate-whitish or brown-white; marg. barren. Flesh buff-white.

About 4-9 connate. Old stumps; uncommon. May-Jan. Mass $3 \frac{1}{ \pm}$ in. P. $1 \frac{1}{4}$ in.

1566. P. amorphus Fr. (from its shapelessness; Gr. a, without, morthe, form) a b $c$.

$P$. thin, silky, somewhat tuberculose behind, orange to white. $T$. somewhat short. Po. unequal, pale orange-rermilion or pale ivory-vermilion : marg. barren.

Multi-imbricate. Stumps, leares, fir ; uncommon. Sept.-Mlar. Mass 3 in. P. I in. Cooke refers 1622 to this, but the spores are different in shape and size. Often in part resupinate. Cerionyces (Ftychogaster) citrinus is said by Saccardo to be an imperfect state of this.

1567. P. adiposus B. \& Br. (from its fat-like substance; adips, fat) a $c$.

$P$. effused, waxy-soft, shortly reflexed, thin, obscurely tomentose, bright red-brown. T. short. Po. small, salmon; marg. salmonorange.

On the ground near stumps, on mosses, Marchantia; uncommon. Dec. llass $1 \frac{1}{2}$ in. P. $\frac{1}{2}$ in.

1568. P. Keithii B. \& Br. (after the Rev. Dr. James Keith) a b.

$P$. bell-shaped, decurrent behind, rough with rigid, tooth-like processes, bright red-brown. $P_{0}$. pallid, deep vinous when dry, dissepiments lacerate.

Single to 2 -imbricate. Sticks, naked wood. P. $\frac{1}{2}$ in.

1569. P. hispidus Fr. (from the hispid pileus) $a b c$.

$P$. thick, dimidiate, pulvinate, hispid, rich red-brown; marg. sienna-ivory: T. long. Po. minute, ivory to pale brown-ochre. Flish juicy, tender, elastic, spongy, dull orange to siemn.

Single to 3-imbricate. Living trunks, pine, ash, larch, apple, mulberry, 
walnut, lime, oak, elm, Euonymus; common. May-Feb. P. Iol in. Close to the bark of the tree the pubescence often resembles Ozonizm auricomum; see 237. Sometimes 18 in. in diam. Becomes dense and fibrous in age. Tubes sometimes distilling drops of water; becoming wholly llack in age.

1570. P. euticularis Fr. (from its thimness, skin-like, as compared with 1569 ; cuticula, a thin external skin) a $b c$.

$P$. thin, subrugose, hairy or tomentose, obscurely zoned, yellowbuff to deep vinous-brown. $T$. somewhat long. Po. minute, vinous- or orange-brown, not dark. Flesh vinous-brown, not dark.

Single to 3 -imbricate. Trunks, beech, fir. Nov.-Dec. 1'. $5^{\frac{3}{4}}$ in. Hearing the same relationship to 1569 as 1544 does to 1543 .

1571. P. spumeus Fr. (from its early condition of frothy-softness; sfumer, froth). Ivory or ochre-white.

$P$. pulvinate, gibbous, somewhat thick, rugoso-hispid to plane. $T$. somewhat short. $P_{0}$. minute.

Single to 3 -imbricate. Trunks and stumps, hornbean, elm, apple, ash, willow, beech, birch, vak. July-1) $\mathrm{cl}$. P. $7 \frac{1}{ \pm}$ in.

1572. P. borealis Fr. (from its being common in northem latitudes; borealis, northern) a $c$. White to yeilowish-buff.

$P$. subpulvinate, bairy, somewhat thick, fibrous. Po. small, sinuoso-flexuous, torn ; mare. barren.

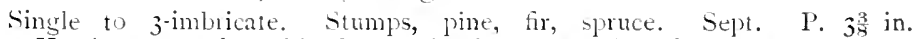
Hartig states that this fungus is the cause of "white-rot" in spruce. berkeley considers that $C_{i}$ riomyces (Ptychogaster) albus may be an imperfect state of this species or of $\mathbf{1 5 5 9}$.

\section{Placidermete.}

1573. P. dryadeus Fr. (from its habitat, oak, (ir. drus) a b $C$.

$P$. pulvinate, thick, rugged to smooth, somewhat ferruginous, becoming fuscous. $T$. long, pale ferruginous. Flesh vinoussienna, not dark, streaked and zoned darker.

Single to 2 -imbricate. Taste somewhat acid or astringent. Trunks, oak, beech; frequent. July-Uct. l'. 9 in. Exuding drops all over, wlich become black in drying.

1574. P. betulinus Fr. (from its habitat, birch, bitula) $a b c$.

$P$. hoof-shaped, zoneless, smooth, thick, the vertex in the form of an um. or short stem, pellicle thin, cracking, pale unberwhitish, becoming darker. $T$. very short. Po. minute, white.

Trunks, brancles, birch ; common. May-Jan. P. $S$ in. Pores sometimes stained by Hyfomyces roselhus and Hyfocrea ochracea. Used by rustics in past times for razor-strops. Saicl by Hartig to cause the "red-rot" of birch.

1574a. P. benzoinus Fr. (from its odour of benzoin) a.

$P$. woody, conchiform, but constricted at the base, fuscousrubiginous, somewhat zoned, rugose when dry, and becoming darker. $T$. long. Po. at first whitish, then ferruginous. Flish yellowish-white.

Subimbricate. Fallen celars. Jan. P. 7 in. S-Sweet-scented, odour retained in dryins. Allied to Tramete's. 
1575. P. quepcinus Fr. (from its habitat, oak, querus) a b c.

$P$. pulvinate, very thick, soft to hard, ivory-or sulphur-whitish, clouded and marrined reddish. $T$. short, minute, whitish, clouded as P. Flesh irory-white, zoned, orange-red and pale sulphur.

single to 3 -imbricate. Taste intensely bitter. Old oaks, oak-logs, often in company with 1517, which it somewhat resembles. May-Dec. Mass 6 in. P. $3 \frac{1}{2}$ in. (Iften harren : sometimes I ft. in liam.

\section{FOMES Karst.}

(From the use in kindling fire in past times: fomes, touch-wood, tinder).

Pilens hard and woody from the first, texture consisting of interwoven hyphæ, covered with a hard, rigid, crustaceous cuticle, zone-
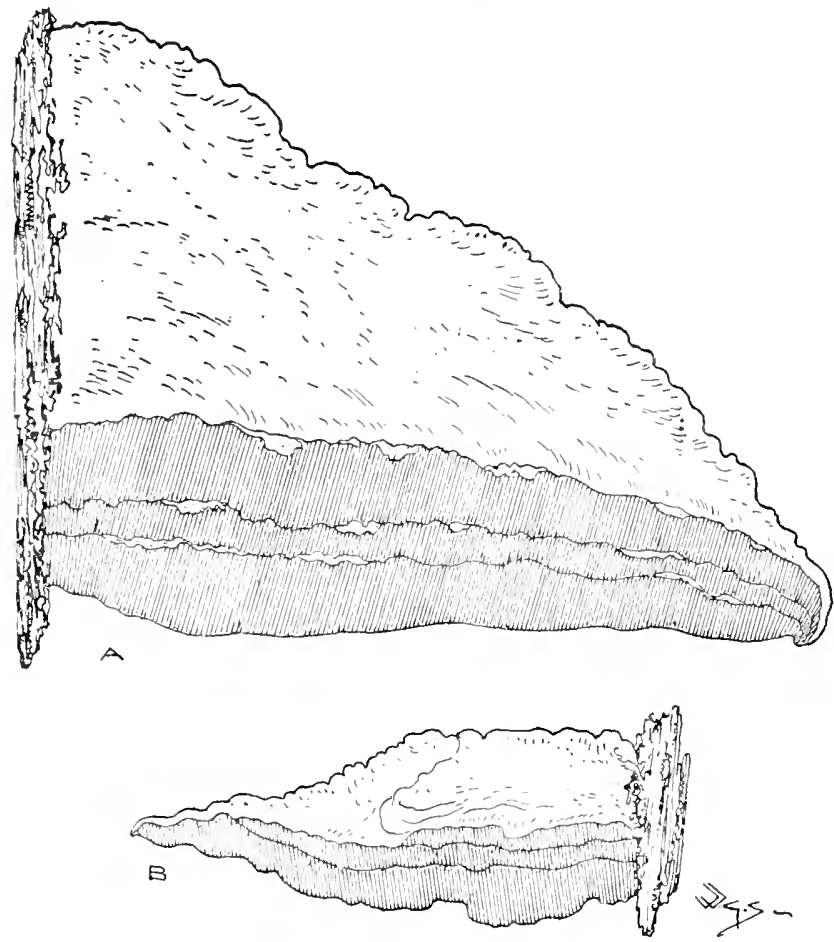

Fig. 7s.-A, section of Fones foncutarius Karst. : B, ditto F. annosus Cooke. (ine-half natural size.

less but often concentrically sulcate, perennial, forming successive strata. (Fig. 78 .) 
a. Fomentarice. Pilcus pulvinate, juiceless, not fleshy or spongy, floccose, covered with a hard, horny crust. Tubes stratose after the first year. Perennial, lasting for twenty years or more, adding new strata annually. Growing on wood.

Substance of tubes umber or fuscous.

Substance of tubes somewhat ferruginous.

$1577-1585$ Substance white or pallid.

$1586-1589$

b. Lignose. Pilizs hard, woody, juiceless, covered with a thin, somewhat lustrous crust, smooth or becoming so, somewhat effused at the base. Flatter than Fomentarice or effusoreflexed.

$1590-1594$

\section{a. Fomentarie.}

1576. F. vegetus Karst. (from its vigorous growth, a'tectus) a $c$.

$P$. concentrically and radiato-corrugate or rugose, deep oliveslate, dark brown behind: marg. sometimes brown. $T$. somewhat long, single to 5-stratose. Po. minute, ivory-brownish, surface brown-white; marg. barren. Flesh thin, floccoso-lax, yellow-brown or vinous warm-brown.

Single to 3 -imbricate. Trunks, lime, elm; rare. P. $7 \frac{3}{t}$ in. diam. Polytorns Stevenii Leveill., according to Bresadola.

1577. F. applanatus Karst. (from its flattened shape; flano, to level; $a b c$.

$P$. flattened, tuberculose, vaguely rugged-sulcate-zoned, smooth, cuticle crustaceous, fragile, somewhat shining, cinnamon or brown, becoming greyish with age. $T$. long, single to 6-stratose, subferruginous. Po. roundish, very minute, white, brownish when bruised. Flesh thick, floccoso-soft, rich brown.

Sometimes imbricate. Odour acid. Trunks, ash, beech, fir, oak, birch, poplar ; uncommon. July-Mar. I liam. I ft. According to Bresadola Folyforus Invenge Fr. and Polyforus roburneus Fr. are both forms of this. Much softer than 1578. Sometimes weighing 4-5 lbs.

1578. F. fomentarius Karst. (from its former use as tinder, fomentum) a b $c$.

$P$. hoof-shaped, more or less triangular in section, concentrically rugged-sulcato-zoned, smooth, cuticle thick, hard, yellowishbrown-reddish, fuliginous- or dingy-brown; marg. paler, at first with a white bloom. $T$. long, single to 9 -stratose, ferruginous. $P^{\prime}$. subangular, white, then ferruginous or ivory-brown. Flish Hoccoso-soft, orange-brown.

Trunks, oak, birch, beech, lime, hornbeam ; common. Jan.-Dec. Diam. $S$ in. Formerly used as tinder in is soft natural state. Generally destroys exterior of trunk for one quarter of circumference and from base to $20 \mathrm{ft}$. up. Said by Hartig 10 cause "white-rot" of beech and oak. Occurs in peat-beds in the eastern counties and in the lake-side piledwellings of Switzerland and Italy. Formerly used as a hæmostatic. The ashes are used by the Ostiaks and Kamtschatkins as snteff. 
1579. F. nigricans Karst. (from its black pileus: niger, black) a $c$.

$P$. hoof-shaped or pulvinate, very thick, deeply and concentrically sulcato-zoned, cuticle very hard, shining, black; marg. very obtuse, ferruginous. $T$. somewhat long, single to $3^{3}$-stratose, ferruginous. Po. very small, obsoletely angular, dark yellowishgrey. Flesth very hard, yellowish-brown.

Living and dead birch, beech, hornbeam : uncommon. Nay-Uct. Diam. $5^{\frac{1}{2}}$ in. A var. of 1578 according to Bresadola.

1580. F. igniarius Karst. (from its former use as tinder; ignis, fire) a b $c$.

$P$. tuberculoso-globose, immarginate, then hoof-shaped, deeply sulcato-zoned, uneven, smooth, cuticle very hard, ochre to reddish- and blackish-brown; marg. rounded. $T$. long, single to 9-stratose. Po. very small, pale tawny, at length cimnamon, when old filled with white mycelium. Flesh very hard, zoned, ferruginous.

Trunks, ash, poplar, willow, cherry, plum, plane, apple, oak, fir, beech, alder, maple, birch ; common. Mar.-Nov. Diam. $6 \frac{1}{2}$ in. Formerly used as a hremostatic. The ashes are used by the Ostiaks and Kamtschatkans as snuff. Only used as tinder after baking, boiling and beating. Hartig considers this to be a parasite. Occurs in peat-beds in the eastern counties and in the lake-side pile-dwellings of Switzerland and Italy. The form on willow has been named Polyforns pomaceus Pers.

1581. F. fulvus Karst. (from the tawny pileus; fuli'us, tawny) a b $c$. $P$. ventricose, hoof-shaped, triangular in section, at first villosohairy, then even, rich tawny. $T$. short, ochre or brown-biscuit. Po. minute, colour as P. Flesh woody-corky, tawny.

Somewhat caspitose and imbricate. Decayed trunks, beech, silver-fir, spruce; rare. Oct.-MIar. Diam. $3 \frac{3}{4}$ in. Pores not always distinctly siratose. According to Bresadola the same as Polyporns cinnamomens Trog.

1582. F. pectinatus Cooke (from the comb-like markings on the pileus; ficten, a comb) a b $c$.

$P$. flattened, thin, concentrically lamellato-plicate and tomentososcaly, whitish-brown, salmon or deep umber-sienua. $T$. short, yellow-brown; marg. barren. Po. very small, somewhat pale salmon-umber.

Imbricate. Trunks. Group $6 \frac{1}{8}$ in. diam. P. $4 \frac{1}{2}$ in. diam. A form of 1584, according to Bresadola.

1583. F. eonchatus Karst. (from its shape; concha, a shell) $a b c$.

$P$. flattened, thin, concentrically sulcate, somewhate corrugate, tomentose, deep yellow-brown or sienma-brown; marg. acute. $T$. short to long. $P o$. very small, yellow-orange or brownish, sometimes olive-shaded. Flesh thin, hard, corky, yellowishbrown.

Imbricate. Trunks, willow, beech; frequent. Feb.-Oct. Group $4 \frac{1}{2}$ in. diam. P. $2 \frac{7}{3}$ in. diam. Often wholly resupinate. A form of 1585 according to bresadola. 
1583a. F. Euonymi W. G. Sm., Polyporus Eucnymi Kalchb. (from its host plant, Euculmus curopicus) a c.

$P$. fleshy, rugose, cinnamon, darker with age, salmon at the marg., edge white. T. short, minute, cimnamon. Po. minute, salmon or whitish-cinnamon. Flesh cinnamon.

Mass $3^{\frac{3}{4}} \times 3^{1}$ in., projection 2 ins. Tubes sometimes multi-stratose, with flesh of pileus reduced to $\frac{1}{10}$ in. thick. Closely allied to F. Lonicerta.

1584. F. Ribis Cooke (from its habitat, currant and gooseberry bushes; ribes, currant) a b $c$.

$P$. thick, somewhat sulcato-zoned, effused amongst branches, somewhat even, indistinctly zoned, velvety, orange- or brownsienna, becoming slate-brown behind. $T$. short, colour as flesh. Po. very small, yellow-sienna becoming sepia-cinnamon. Flesh corky-coriaceous to soft, brownish siemna-orange.

Connato-imbricate. Perennial. Common. April-July. Group $3 \frac{3}{4}$ in. diam. P. $2 \frac{1}{5}$ in. diam. Substance suitable for tinder. The same as 1582 and 1583a according to Bresadola.

1585. F. salicinus Karst. (from its habitat, willow, salix) a $c$.

$P$. undulate, whitish-brown, yellowish-brown or black, $3^{-8}$ zoned, dark-umber. $T$. deep umber-sienna. $P_{0}$. very small, ferruginous-cinnamon; marg. barren. Flish woody, very hard, whitish- or yellowish-brown.

Caspitoso-imbricate, for the most part or wholly resupinate. Common. Group $3 \frac{7}{8}$ in. in diam. P. I $\frac{3}{4}$ in. diam. A form of 1583, according to Bresadola. Cerionyces (Ptychograster) spongria is said by saccardo to be an imperfect state of this.

1586. F. roseus Cooke (from its rose colour) a $b c$.

$P$. thin, somewhat flattened, even, subrugose, shining dull rose or rose-red, dark zoned; marg. dark. T. somewhat long; marg. barren. Po. minute, colour as P. Flesh corky-woody', floccoso-fibrous, colour as P. or paler.

Imbricate, caspitose. Stumps, apple, dressed wood. Oct. P. $2^{\frac{3}{t}}$ in. diam. Colour permanent in drying. The same as $F$. rufotallidus and 1592 according to Bresadola.

1587. F. ulmarius Cooke (from its habitat, elm, ulmus) a b $c$.

$P$. thick, convex, tubercular, uneven, becoming smooth, white to leather-buff, blackish in old examples; marg. white, becoming tawny. $T$. single to 6 -stratose, ivory, bright salmon or buffvinous, brown in decay. Flesh corky-woody, very hard, white.

Connato-imbricate. Single. Odour disagreeable. Inside old elms. Sept.Feb. P. 65 in. diam.

1588. F. eytisinus Cooke (from one of its habitats, laburnum, Cytisus) a b.

$P$. thick, convex, smooth, whitish, biscuit, or pale brownish. $T$. 
somewhat short, white-salmon, marg. barren. Po. biscuit or with a sulphur shade. Flesh thick, close, velvety-fibrous, white. Single 10 imbricate. Stumps, lressed wood; rare. P. 61 in. diam. Bresadola considers this and 1590 lo be the same.

1589. F. connatus Cooke (from its connate habit) a $b c$.

$P$. somewhat thick, villous, whitish-buff, brown shaded. $T$. somewhat short, colour as P.; marg. barren. Po. minute, white to cream. Flish corky-woody, pale buff to full buff.

Effuso-reflexed, connato-imbricate. Trunks, lime, apple, elm, maple; frequent. Oct.-Jan. P. $6 \frac{1}{5}$ in. diam. See 1631.

\section{b. Limnose.}

1590. F. fraxineus Cooke (from its habitat, ash, fraximus) a b $c$.

$P$. thick, flat, concentrically sulcato-plicate, zoneless, white or ivory to rich brown: marg. whitish. $T$. decurrent, moderate in length, white, cinnamon- or pale vinous-white. Flesh whitishcinnamon.

Solitary to connate. Perennial. Odour strong, penetrating. Frequent. Jan.-Dec. P. $5 \frac{1}{2}$ in. diam. Brevalch considers this and 1588 to be the same.

1591. F. variegatus Cooke (from the variegated pileus, orange and bay-brown) a $b$.

$P$. thin, somewhat plane, even, smooth, shining tawny-orange, darker or bay-brown behind. $T$. decurrent, moderate in length, colour as P. Po. minute, unequal, tom, pale yellowishwhite, shaded biscuit: marg. barren. Flish pale buff.

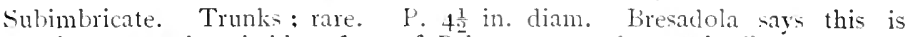
unknown, and probably a form of Polytorus ungulatus schatif.

1592. F. carneus Cooke (from its Hesh-colour; aro, flesh) a $c$.

$P$. somewhat thick, convex, rugose, smooth, salmon-buff to reddish-salmon, varying deep rich brown behind, pale at marg. $T$. shallow to moderate, full opaque-salmon. Flesh pale bright salmon or red-salmon, varying rose-brown: marg. barren.

Connalo-imbricate. L'erennial. Trunks, stumps, juniper, Ficat. Nor. P. $2 \frac{3}{s}$ in. diam. A form of 1586 according to Bresadola. Said to be the cause of "red-rot" in Red Ceclar, Junferus virginiuna.

1592a. F. castaneus Cooke (from the colour: castantus, chestnut) $a$.

$P$. coriaceo-woody, flattened, smooth, glabrous, zoneless, chestnutcolour. Po. small, yellowish. Flish white.

Imbricato-connate. 1Black poplar. $I_{\frac{7}{8}}^{\frac{7}{8}} \times \mathrm{I}_{\frac{3}{4}}$ in. $\frac{7}{8}$ in. projection.

1593. F. annosus Cooke (from its growing in yearly strata; ammis, a year) a $b c$.

$P$. convex, rugged, radiato-sulcate, sulcato-zoned, at first silky, then crusty, cinnamon-ochre when young, then deep rich vinous- or sienna-brown; marg. almost white. T. moderate in 
length, stratose: whitish-buff. Po. medium, salmon-white. Flesh colour as T.

Imbricate. About stumps, posts, roots, squared timber, pine, spruce, fir, larch, juniper, birch, beech, Sequoia gigantea: very common. Jan.Dec. l'. 73 in. diam. Sometimes wholly resupinate. Some forms of this are Trametes raticiperda, said to cause "red-rot" and piping in larch, the mycelium growing from the roots to the trunk. Sometimes phosphorescent in mines and seen 20 yards off. The white mycelium so often seen running over the hymenium belongs to Verticillium microspermum, the conidial condition of Hypomyes Brommeanus.

1594. F. populinus Cooke (from its habitat, trunks of poplar, populus) a b c.

$P$. rigid, zoneless, rugose, villous, warm-biscuit. $T$. deeply decurrent, not stratose, shallow. Po. minute, colour as P. Flesh colour as P.

Nearly solitary to subconnate or imbricate, for the most part resupinate. Aug.-Jan. P. $2 \frac{1}{4}$ in. diam. Polyports connatus Weinm. (not Fr.) and 1631 are forms of this according to Bresadola ; see 1631 .

\section{POLYSTICTUS Fr.}

(From the pores or punctures on the hymenium; Gr. polus, many, stiktos, punctured.)

Pileus coriaceous, membranaceous or somewhat spongy, cuticle thin and loosely fibrous. Tubes first appearing in the centre and successively developing towards the margin, at first superficial and

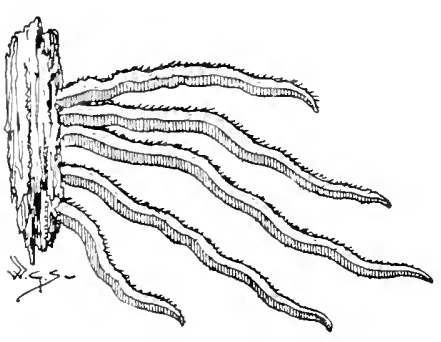

Fig. 79.-Section of Polystictus versicolor Fr. One-half natural size. distinct, then becoming deeper and more crowded, not stratose. (Fig. 79.) Species 1595-1608

1595, 1595a and 1596 are placed in Polystictus by Cooke and Massee, in Poria by Saccardo.

a. Stuppose. Pilius flocculose, or adpressedly villous or smooth, uneven, zoneless, texture fibrous.

Substance coloured.

$1595-1597$

Substance white. 1598,1599

b. Coriacee. Pileus conaceous, villous, zoned, usually in different colours.

$1600-1605$

\section{a. Stupposa.}

1595. P. radiatus Cooke (from the radiato-rugose pileus) a b $c$.

$P$. velvety to smooth, deep rich brown to dull orange; marg. zoned bright sienna. T. colour as flesh. $P$ o. minute, greyish- 
salmon, silvery-glistening, at length ferruginous. Flesh rigid, corky, leathery, dull orange.

Imbricate. Not pærennial. Trunks, beech, birch, oak, hazel, alder, gorse ; frequent. Oct.-April. P. $4^{\frac{1}{ \pm}}$ in. diam. List not be confounded with 1603.

1595a. P. nodulosus Cooke (from the nodular pileus) a.

$P$. triquetrous, nodular, corky, woody, not zoned, villous, rugose, rough, fulvous then rust-colour. $T$. somewhat long. $P_{0}$. roundish, unequal, acutely torn, light cinnamon with a silvery sheen. Flesh very hard, lighter than $\mathrm{P}$.

Imbricate. Dead beech. Oct. $2 \frac{1}{\frac{1}{x}} \times \frac{3}{8}$ in. Distinguished from 1595 by the at length confiuent pores and by the absence of the brightly coloured margin of pileus.

1596. P. polymorphus Cooke (from its many forms; Gr. pclus, many, morphe, form) a.

$P$. smooth, somewhat pale vinous-brown, dark shaded; marg. crisped. $T$. deeply decurrent. Po. somewhat large, angular, torn, colour as flesh. Flish coriaceous, salmon.

Connato-imbricate. Branches, worked wood, fir. Aug. Group $2 \frac{5}{8}$ in. diam. P. I in. diam. Sometimes resupinate.

1597. P. cryptarum W. G. Sm., Boletus cryptarum Bull. (from its frequent habitat-cellars; crypto, a vault) $a b c$.

$P$. corrugate, zoneless, adpressedly silky, pale yellowish-vinousbrownish. T. deeply decurrent, long. $P$ c. minute, colour as flesh. Flesh corky-cottony, paler than P.

Connato-imbricate. Fir ; frequent. Group $4^{\frac{3}{3}} \mathrm{in}$. diam. Transferred to Fomes by Cooke and Saccardo. The same as 1608 according to Massee,

1597a. P. ravidus Cooke (from the grey or tawny pileus; ravus, grey) $a$.

$P$. coriaceo-corky, flattened, zoned, villoso-rugose, ochreous, slightly tinged brown-olive, base darker, sienna. T. somewhat short. Po. larger at mid., then smaller and absent at marg., biscuit-white. Flesh ivory-white.

Imbricate. Stumps of old willows. Spread $5 \frac{3}{x}$ in., I in. thick. Colour variable, usually yellowish.

1598. P. gossypinus Cooke (from the white-cottony pileus; gossypium, cotton) a $c$.

$P$. tomentose, zoneless; marg. slightly scaly, white. $T$. long, but varying. $P$ o. labyrinthine, then angular, pallid cinereous.

Somewhat connato-imbricate. Trunks, furze. Jan. Diam. $2 \frac{1}{2}$ in.

1599. P. fibula Fr. (from its button shape; fibula, a button) $a b c$.

$P$. velvety-hairy, zoneless, sometimes radiato-rugose, white. $T$. varying in length. $P_{0}$. small at length torn, white, sometimes faintly sulphur.

Single to subimbricate or in troops. Wood in cellars, forests, elm, oak. Oct.-Jan. P. 2 in. diam. Sometimes shield-like, attached behind a 
centre of pileus, sometimes pendulous, at other times hoof-shaped and triangular in section.

\section{b. Coriacere.}

1600. P. hirsutus Fr. (from the hairy pileus; hirsutus, hairy) a $b c$. $P$. becoming plane, depressed behind, zoned with furrows, varying white, pale slate-brown or brassy-yellow; z. yellowishbrown. T. long, deep, ochreous-white. Po. tom, whitishsalmon. Flesh thin, corky-coriaceous, colour as $\mathrm{T}$.

Single to imbricate, effused behind. Dead trunks, branches, willow, oak,

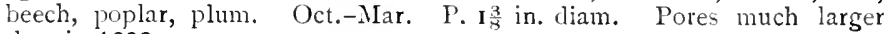
than in 1603.

1601. P. velutinus Cooke (from the velvety pileus; velluctum, velvet) $a b c$.

$P$. plane, slightly zoned, white to ivory-ochreous; marg. white. $T$. short, white. Po. minute, white to yellowish; marg. barren. Flesh corky-coriaceous, white.

Single to connato-imbricate. Stumps, branches, etc., willow, beech, fir, birch; uncommon. Nov. Group $2 \frac{3}{4}$ in. diam. P. $x^{\frac{1}{2}}$ in. diam.

1602. P. zonatus Fr. (from the zoned pileus) a b $c$.

$P$. convex, tubercular-gibbous behind, villous; $z$. unicolorous to varying ivory, ochre, brown, rufous, sienna, etc., or whitishyellow, graduated and zoned sienna. Po. minute, biscuit. Flcsh corky-coriaceous, yellowish-white.

Single to subimbricate. Trunks, elm, poplar, birch. June-Oct. P. $3 \frac{1}{\text { ta }}$ in. diam.

1603. P. versicolor Fr. (from its variable colours) $a b c$.

$P$. even, velvety, shining pale smalt-blue, sooty-slate, or of various tints of ochre, ivory, brown or greenish. $T$. shallow. Po. minute, ivory; marg. barren. Flesh thin, rigid-coriaceous, ivory.

Imbricate. Dead wood, fences, branches, encircling twigs, back of an old

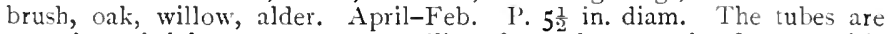
sometimes bright orange or vermilion from the growth of a parasitic mould, Hyponyces aurantius. When growing on sticks, if the stick is inverted, a new hymenial surface with pores will appear on the downward pileus. Pores sometimes wholly eaten away by insects. 1774 cometimes grows on this. Var. fuscatus MIass. P. brown. Po. torn into teeth, yellow.

1604. P. abietinus Cooke (from its habitat, fir-trunks; Abics, fir) a $b c$. $P$. villous, white, zoned dove-grey; marg. sometimes purplish. $T$. very shallow. $P$ o. unequal, pale slate, becoming pale brown to vinous- or purplish-sienna. Flesh very thin, pale brown.

Connato-imbricate, effuso-reflexed. Fir, cedar, cypress; common. Jan.-Nov. Group $3 \frac{1}{ \pm}$ in. rliam. Pileus frequently overgruwn by green algre. Sometimes wholly resupinate with a broad white woolly margin. II ust not be confounded with 1610 . 
1605. P. Wynnei Cooke (after Mrs. Lloyd Wynne) $a b c$.

$P$. membranous, marked with silky raised lines, faintly brownish, yellow-white, sometimes orange or tan. $T$. varying in length. Po. small, whitish.

Effuso-reflexed, almost wholly resupinate. Running over twigs, grass, etc., around stems of Carer paniculata. Sept.-Dec. Group 3 in. diam. Pore surface with barren places in middle and at margin.

\section{PORIA Pers.}

(From the porous hymenium.)

Resupinate, forming more or less extended patches or thin membranous expansions. Pores forming a continuous stratum, springing from a thin, often rudimentary subiculum. (Fig. 8o.)

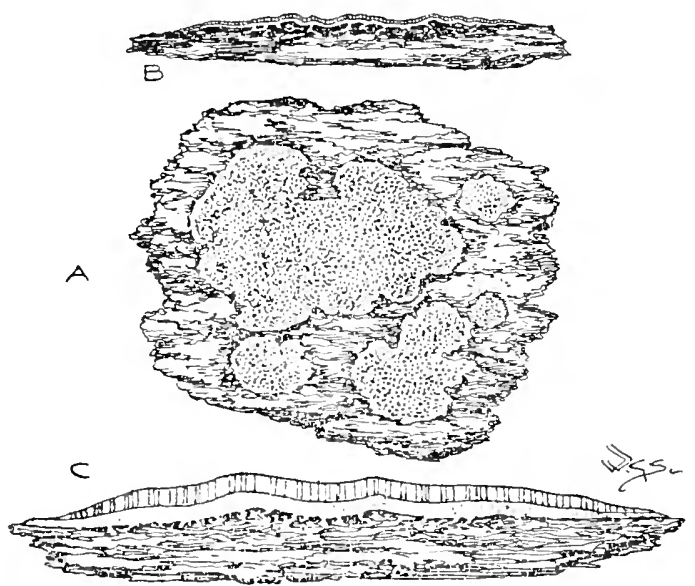

Fig. 8o.-Foria zaporizir Cooke.

$A$, entire; $B$, in section. One-half natural size. C, section. $X_{4}$.

This is almost entirely the section of Polyporus named Resupinate by Fries, the sequence adopted is that of the Hymonomycetes Enropai.

Pores ferruginous or cinnamon.

Species $1605 a-1648$

Pores purple or violaceous.

$1605 a-1609$

Pores red.

1610,1611

Pores yellowish.

$1612-1615$

Pores at first white, changing colour.

$1616-1620$

Pores usually persistently white, minute crowded, round, equal.

$1628-1635$

Pores persistently white, unequal, angular, crowded, commonly somewhat large.

Pores white, superficial, distant, punctiform.

$1636-1643$

Of uncertain affinity. 
1605a. P. obliqua Achar. (from the oblique growth of the tubes) a $c$. Sub. very thin; marg. barren, sometimes recurved, forming a narrow $\mathrm{P}$. T. more or less elongate owing to oblique growth, usually minute, $\frac{1}{10}$ to $\frac{1}{5} \mathrm{in}$. long when not oblique, orangebrown or cinnamon, sometimes of two strata. Flesh colour of Po.

Trunks, logs, rotting wood. $4 \times 2 \frac{1}{t} \times \frac{1}{16}$ in. Regarded as a species of Fomes by Cooke and Saccardo.

1606. P. umbrina Cooke (from its brown colour; umbrimus, browncoloured) $a$.

Undulato-tubercular, full to dingy umber; marg. smooth salmonor vermilion-white, paler than pore-surface. Suth. thin but variable. T. somewhat long, varying. Po. minute, roundish, unequal ; sienna.

Trunks. $3 \frac{1}{5}$ in. Fomes zumbrina Saccardo.

1607. P. resupinata W. G. Sm., Boletus resufinatus Bolt. (from its resupinate habit) $a$.

Undulato-tubercular, spongy-coriaceous, umber, yellowish vinousumber or ferruginous. Sult. thin fibrous. $T$. long, varying, sometimes stratose. Po. minute, pore surface nodulose to plane, umber.

Broadly effused. Trunks, branches, hazel, Feb. $3^{\frac{3}{z}}$ in. This may be a resupinate form of 1560 or 1561 . Compare 1605a. Fomes resufinata Massee.

1608. P. fepruginosa Karst. (from its rusty colour; ferrum, iron) a b $c$.

Undulato-tubercular, ferruginous or cinnamon; marg. barren, sometimes reflexed as a narrow P., ochre or rust colour, with white mycelium at edges of new growths. Sut. thin but varying. $T$. long, varying, sometimes 2 -stratose or reduced to $\frac{t}{3} \frac{1}{2}$ in. Po. medium to minute, brown. Myc. white to ochreous.

Imbricate, broadly effused. Trunks, branches, posts, sticks, abele, beech, oak, pine, alder, bramble, sometimes on dead leaves ; common. Oct.May. $5 \frac{1}{2}$ in. At first appearing as a confervoid stratum. Fomes forruginosit Massee.

1609. P. contigua Karst. (from its close investment of sticks, etc.; contiguns, continuous) a $c$.

Somewhat plane, cinnamon or yellowish-sienna, dingy when old; marg. barren. Sub. thin, from $\frac{1}{5}$ in. to a membrane. T. short. Po. somewhat large, brownish. My'c. ochreous.

Effused, less so than in 1608. Trunks, branches, sticks, rotten wood, etc., pines, sweet-gale, maple, hornbeam; uncommon. 3 in. Sometimes wholly investing branches.

1610. P. violacea Cooke (from its violet colour) $a c$.

Plane, purple with marg. paler or whitish, or deep purplish to crimson-brown, with marg. light or ivory, clouded rufous. 
Sub. a film. $T$. short. Po. round or wrinkled as in Mirulius.

Effused, determinate. Stumps, trunks, poles, fir; frequent. Aug.-May. 4 in. Allied to Merulius. Must not be confounded with 1604, in which the pores are deeper and torn and the margin reflexed.

1611. P. purpurea Cooke (from its purple colour) $a c$.

Plane, purple-lilac; marg. barren, paler. Sub. a film. $T$. short, Po. minute. Myc. mucedinous, flocculose, white.

Stumps, willow, beech. $33^{3}$ in. The violet colour disappears in drying.

1612. P. incarnata Cooke (inarmatus, flesh-colour) a $c$.

Plano-undulate, coriaceous, marginate or immarginate, dark fleshcolour or orange, becoming dark purple-brown-crimson or purple-blackish; marg. broad, white or ochreous, sometimes vinous-clouded, sometimes shortly reflexed as a narrow $P$. Sub. a film. Po. short, unequal.

Effused. Fir; rare. June-Nov. $6 \frac{1}{2}$ in. Sometimes with barren patches. Must not be confounded with 1604 .

1613. P. rufa Cooke (from its reddish colour; rufus, red) a $c$.

Plano-undulate, umber, vinous-umber, blood-red-rufous or deep reddish; marg. barren, byssoid when young, greyish. Sub. a film. Po. short.

Effused. Trunks, sticks, branches, etc., broom, beech, bird-cherry. $3 \frac{1}{5}$ in.

1614. P. rhodella Cooke (from its rose-colour; Gr. rhadon, a rose) a. Plane, soft, white, flesh-colour, vermilion-salmon or Indian-redsalmon; marg. determinate, salmon. Sub. a film. Po. short, minute, not torn.

Effused. Trunks, fir, beech. Aug. $t_{ \pm}^{\frac{3}{t}}$ in. Thinner than 1613.

1615. P. micans Ehrb. (from its glittering pores; mico, to glitter) a $c$. Plane, often with rain-drop-like barren depressions on surface, whitish-flesh or biscuit-colour; marg. flaxy, byssoid, white. $S u l$. a membrane. $T$. short. $P_{t}$. minute, irregular, angular.

Effused, orbicular, confluent. Dead wood, rotten trunks, ash. Nov. $3 \frac{3}{8}$ in. Fugacious.

1616. P. nitida Pers. (from its shining pores; nitidus, shining) a $c$.

Plane, bright yellow to pale sienna-yellow; marg. villosodeterminate. Sub. a membrane. T. short. Po. round, minute.

Effused, subadnate. Deal wood; rare. Mar. $3 \frac{1}{2}$ in.

1617. P. Læstadii Cooke (after C. P. Lrestadius) a b.

Plano-tubercular, brittle, separable, bright lemon-yellow, whitishsulphur or ivory-salmon when dry; marg. not barren, edge minutely and narrowly fibrillose. Sub. thin, white. T. short, of different depths. Po. very minute, sinuous.

Effused, sometimes stratose as if perennial. Deal boards, sometimes in greenhouses. Aug. $3 \frac{1}{2} \mathrm{in}$. 
1618. P. bombycina Cooke (from the silky membranous subiculum; bonty $x$, silk) a b c .

Sub. very thin, translucent; marg. byssoid. Po. large, angular, sinuous; marg. arachnoid-velvety, pale buff or white.

Effused. Rotten wood: rare. Sept. 3 in. The grain of the wood some. times shows through the extremely thin sub:tance.

1619. P. aneirina Cooke (from the margin not being woolly as in 1618 ; Gr. an, not, cirinces, woolly) a c.

Sul. thin, coriaceous, somewhat flaxy ; marg. byssoid. Po. shallow, large, waxy, fulvous, tawny, warm biscuit-colour or bright orangeyellow ; marg. flaxy, becoming waxy, white. Myc. buff-white.

Effused. Dead wood, branches, poplar, pine under the bark; rare. Oct. $4 \mathrm{in.}$ Contracting and becoming torn in drying.

1620. P. ramentacea Cooke (from one of its habitats, chips, ramenta) $a b$.

Sub. thin, tomentose, white. Po. somewhat large, hexagonal, honey-colour; marg. almost obsolete, irregular, warm-ivory.

Somewhat orbicular. Dead branches, fir. Sept. $\mathbf{I} \frac{1}{2} \times \mathbf{I}$ in. Cartilaginous and horny when dry. Barren patches occur on the hymenium.

1621. P. eineta Cooke (from the encircling, erect strigose fibres; cingo, to encircle) $a$. White to ivory.

Sub. membranous, sometimes barren, and with lacunæ; marg. byssoid. Po. extremely minute.

Old deal boards, rotten planks; rare. $3 \frac{3}{2}$ in. Patches of vertical strigre become confluent and form a subiculum.

1621a. P. fuscocarnea Cooke (from the brownish flesh-colour ; fuscus, dark brown, aro, flesh).

Sub. thin; marg. definite. $T \cdot \frac{1}{15}$ to $\frac{3}{16}$ in. long. Po. minute, fawn-colour when fresh or vinous-brown, graduated paler.

Rotten wood, putiid trunks. $3 \frac{1}{2} \times 3$ in.

1622. P. armeniaca IT. G. Sm., Polyporus armeniacus Berk. (from its apricot-colour: armeniacum, apricot) a $b$.

Sul. membranous to $\frac{\mathrm{I}}{4}$ in. or more thick; marg. thin, well defined, minutely downy or byssoid. Pe. shallow, to $\frac{1}{8}$ in. deep, rounded, somewhat irregular, minute, white, then bright buff, or glancing with the light, ochreous on side view, bright foxy-brown on full view.

broadly effused, suborbicular, confluent. Bark, pine. Nov-April. $3 \frac{1}{2} \times 1 \frac{1}{2}$ in. Sometimes a rugged, free pileus is formed. Cooke refers this to 1566 , but the spores are different in shape and size.

1623. P. Rennyi Cooke (after James Renny) a b.

Sub. thin to somewhat thick, pulvinate, at first somewhat frothy, then dry and pulverulent: marg. byssoid. Po. minute, few, irregular in depth, here and there elongated, white, becoming lemon or pale buff. MYyc. gelatinous, partly byssoid.

Odour pungent. On the ground, rotten wood, running orer Harchantia, fir. Nov. $2 \frac{1}{2} \times \frac{3}{4}$ in. Pores sometimes absent or subiculum with large barren patches. 
1624. P. subfuscoflavida Cooke (from the pale brownish-yellow colour ; subfuscus, somewhat dusky, flazidus, yellow) a $c$.

Sul. thin, coriaceous, dry ; marg. thin, byssoid, determinate, white. Po. minute, irregular, white, then yellowish-brown or glancing white and grey-brown.

Broadly effused, confluent. Dead oak wood, planks, etc. $2_{8}^{3}$ in.

1625. P. viridans Cooke (from its pale æruginous colour; virido, to become green) a $c$.

Sub. thin. Po. minute, shallow, white, ivory or pale buff, then pale verdigris; marg. pulverulento-tomentose.

Effused, crustaceo-adnate. Rotten wood; rare. Sept. $2 \frac{3}{3}$ in. Sometimes a mere thin, pale reruginous film without pores.

1626. P. teprestris Cooke (from its terrestrial habit) a $c$.

Sub. very thin; marg. arachnoid flaxy, fugacious with a byssoid white edge. Po. shallow, minute, angular, becoming smaller and scattered towards the wide, barren marg., white, becoming pale umber or faintly rufescent.

Effused. Black earth, red clay, rotten wood; uncommon. May-Nov. 4 in.

1627. P. subgelatinosa Cooke (from the somewhat gelatinous subiculum) $a b$.

Orbicular, forming small pulvinate masses with an obtuse raised border, at first tomentose and pallid, becoming black and subgelatinous. $P$ o. somewhat large, others small, variable in size, pale grey.

On dead wood, on $1566 . \quad 15$ in.

1628. P. medullapanis Cooke (from its colour like pith or bread; medulla, pith, panis, bread) a bc.

Somewhat undulate, firm: marg. barren, whitish, sometimes upturning and separating from matrix. $T$. somewhat long. Po. medium size.

Decayed branches, mulberry, oak, hornbeam, on the ground; uncommon. Sept.-Feb. 4 $4_{\frac{7}{y}}$ in. Sometimes in certain positions $4^{-6}$ stratose.

1629. P. mucida Pers. (from its mucid substance) $a c$.

Sub. somewhat thick, soft, elastic; marg. barren, indeterminate, flaxy. Po. shallow, medium size, unequal, torn, white to ochre-white. $\quad H_{y} c$. spreading and often forming small orbicular, transparent, barren patches.

Effused in long patches. I lecayed fir. $3 \frac{1}{6}$ in. Thicker than 1634.

1630. P. vitrea Pers. (from its shining glassy appearance as if wet; z'itrum, glass) a $c$.

Sub. somewhat thick. $T$. somewhat long, translucent, whitish. $P_{0}$. minute, entire, roundish, whitish, becoming buff-white or vinous-buff-whitish.

Broadly and unequally effised. Dead wood, fir, on the ground under beech; uncommon. Aug.-Mar. $3 \frac{1}{3}$ in. With the appearance of shining wax. 
1631. P. obducens Cooke (from the covered-over or superimposed strata ; obduco, to cover over) a $c$.

Sub. a hard, almost obsolete, inseparable film. $T$. somewhat long: $\frac{1}{16}$ to $\frac{1}{s}$ in. Po. minute, white, becoming pale dull salmon.

Effused. Rotten trunks, pine, oak, beecli; rare. July-Feb. $3 \frac{1}{\text { in }}$. Bresadola says this is a resupinate form of 1594, Massee of 1589 . During the first year it resembles 1633. Irregular in section, perennial by strata for six or more years.

1632. P. callosa Cooke (from the hard subiculum; callum, hardened skin) a c.

Sull. a firm, somewhat thick, leathery membrane, separable from the matrix, white, becoming umber. $T$. somewhat long. $P o$. round, equal, entire, white becoming biscuit; marg. white.

Eroadly effused. Dead wood, oak, maple. April. $3 \frac{1}{2}$ in. Surface sometimes with small depressions as if from rain-drops. Annual, not stratose.

1633. P. vulgaris Cooke (from its commonness; zulgaris, common) ac. Sub. a membrane, closely adnate to the matrix, almost obsolete. Po. somewhat shallow, white to biscuit or faintly ochreous, sometimes with a white, irregular barren marg., attached to thin, white, cloud-like growths of mycelium; marg. soon even and smooth.

Broadly effused. Dead wood, branches, pine, oak, beech ; common. JulyIar. $5 \frac{1}{2}$ in. Patches often very large, sometimes wholly investing branches, sometimes with vacuoles. Annual not stratose. Appearance wax-like.

1634. P. mollusea Cooke (from its softness; molluscus, soft) a $b c$.

Sub. a membrane. Po. somewhat shallow, large in middle, smaller towards marg., white becoming pale biscuit or brownishwhite; marg. broad, barren, white, radiate with white strands of mycelium.

Broadly effused. Dead wood, dead leaves, furze, poplar, ash; common. Jan.-Dec. $4^{\frac{1}{t}}$ in. Sometimes proliferous or 2 -stratose.

1635. P. collabefacta Cooke (from the pores which appear to arise from the collapsing substance; collaticfio, to be brought to ruin) a $b$.

Sub. a nembrane. Po. minute, shallow, white to ivory; marg. obtuse, barren, with a few barren places on surface.

Effused. Dead wood. Oct. $3 \frac{1}{2}$ in. Running over the matrix like a thin Corticium.

1636. P. sanguinolenta Cooke (from its changing to blood-red where bruised; sanguis, blood) a b c.

Sul. a membrane. T. somewhat shałlow. Po. small, irregular, becoming torn, white to yellowish-white.

At first nodulose, soon confluent and effused. Decayed trees and branches, rails, dead wood, running over earth, clay and moss; uncommon. Aug.Nov. $2 \frac{3}{4} \mathrm{in.}$ 
1637. P. radula Pers. (from the small teeth, as of a comb, where the pores are elongated; radula, a scraper) ac.

Sub. a membrane, separable from the matrix. $T$. shallow, somewhat long in middle, sometimes 2 -stratose. Po.tooth-like or comb-like, spinous near the sometimes barren marg., white to biscuit, not turning red.

Effused. Trunks, branches, fir, oak, hornbeam. Surface with small depressions, sometimes with barren places. Aug.-Jan. $3 \frac{3}{8}$ in.

1638. P. vaporaria Cooke (from the warm, moist air, in which it luxuriates; a'aporarimm, a stove) a bc.

Sub. a thin, firm, inseparable membrane. $P$. shallow, large, torn, white to ivory.

Broally effused. Moist woods on dead branches, decaying wood, rails, pine, oak, poplar, alcler, bamboo, rumning orer dead leaves, on root wounds, barked and injured places, squared timber in buildings; very common. Jan.-Dec. 4 in. Hartig states this fungus to be as destructive as the "dry-rot" fungus 1680 on living spruce and pine. Ceriomyces (Itychogaster) rubescens is said by Saccardo to be an imperfect state of this. Var. scicrmibilis Mass., separating from the matrix; white, then honey-colour; fir-leaves under moss.

1639. P. hibernica Cooke (from the place where first observed, Wicklow, Ireland) $a$.

Sub. a membrane, not separable from matrix. Po. small, shallow, polygonal, not fimbriato-toothed, white; marg. narrow, thin, radiato-byssoid.

At first small, orbicular, then confluent and broadly extended. Decorticated fir branches. Sept. $3 \frac{1}{2}$ in.

1640. P. Gordoniensis Cooke (after the Marchioness of Huntly) a $c$.

Sub. a thin, extremely delicate film, separable from matrix. $P_{0}$. minute, fimbriato-toothed, shining white, becoming faintly yellowish-white; marg. broad, barren, white.

somewhat effused. Fir poles. Feb. $2 \frac{3}{4}$ in. Closely resembling 1639 in general appearance.

1641. P. Vaillantii Cooke (after Leon Vaillant, French botanist) $a b c$. Sub. a thin film, here and there traversed by ribs. Po. rather large, shallow, produced here and there in clusters, white or slightly rufescent: marg. broad, barren, bordered by broad barren radiating growths ending in strands of mycetium.

Broadly effused. Deal wood. April-Oct. 6 in.

1642. P. farinella Cooke (from the pulverulent margin; farina, meal) ac $c$.

Sutb. a very delicate, thin film. Po. shallow, with a ragged, toothed appearance: marg. broad, barren, unwoven, edge pulverulent.

Broadly and irregularly effused. Old timber, dead wood, beech, oak, pine,

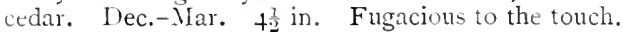


1643. P. hymenocystis Cooke (from the cyst-like pores of the hymenium) $a b$.

Sub. very thin, arachnoid. Po. large, shallow, irregular, dissepiments scarious, collapsing, white, becoming pallid; marg. narrow, byssoid.

Black, rotten wood. 4 in.

1644. P. blepharistoma Cooke (from the eyelid-like appearance of the pores; Gr. blepharon, an eyelid, stoma, a mouth) $a b$.

Sub. a thin film. $P_{0}$. small, dissepiments thin, edge finely toothed, white; marg. barren, edge minutely byssoid, somewhat mealy. Myc. arachnoid.

Dead wood, decorticated branches, leaf-mould. July-Nor, 4 in.

1645. P. corticola Cooke (from its growing on bark: cortex, bark, colo, to inhabit) $a$.

Sub. a film, firm. Po. very minute, often obsolete, or in small irregular patehes, white to biscuit ; marg. radiate, byssoid.

Poplar, birch, horse-chestnut, fir. $3 \frac{1}{2}$ in.

1646. P. reticulata Cooke (from the net-like hymenium; rete, a net) $a c$.

Sul. a thin film. Po. cup-like, somewhat large, white; marg. barren, radiato-floccose or byssoid.

Orbicular, confluent. Kotten wood. Sept.-Feb. 4 in. Very delicate, soon disappearing.

1647. P. bathypora Cooke (from the deep cup-like pores; Gr. bathus, deep, poros, a pore) a.

Sub. a thin film. $P^{\prime}$. cup-shaped, somewhat large, dissepiments toothed, white, becoming brownish : marg. flaxy.

Effused. Dead oak, beech. $3 \frac{1}{2}$ in. Sometimes 2-stratose. Resembling the resupinate form of 1660 .

1648. P. hybrida Cooke (from the possibility of its being a monstrous form of 1559) a b $c$.

Sul. a thick felt-like membrane, white, sometimes branched, sometimes forming small inflexed superimposed white pilei. Po. very minute to large, shallow, to $\frac{2}{t}$ in. deep, torn, white to biscuit; marg. irregularly radiato-branched.

On oak in ships, etc. causing "dry-rot." Jan.-Dec. 7 in. Sometimes a mere radiato-branched white membrane with a few small patches of pores or forming pendulous masses from horizontal beams, with tubes beneath.

\section{TRAMETES Fr.}

(From the generic distinction depending on the trama-the substance which grows between the membranes of which the dissepiments of the pores are composed.)

Trama continuous and homogeneous with the flesh of the pileus. Pilicus, when present, woody, corky-woody, corky or soft. Stem 
none. Tubes unequal in length, rarely stratose. Pores subround, more or less elongated radially; not labyrinthiform, entire, often unequal in depth, dissepiments somewhat thick. (Fig. SI.) $_{\text {I. }}$
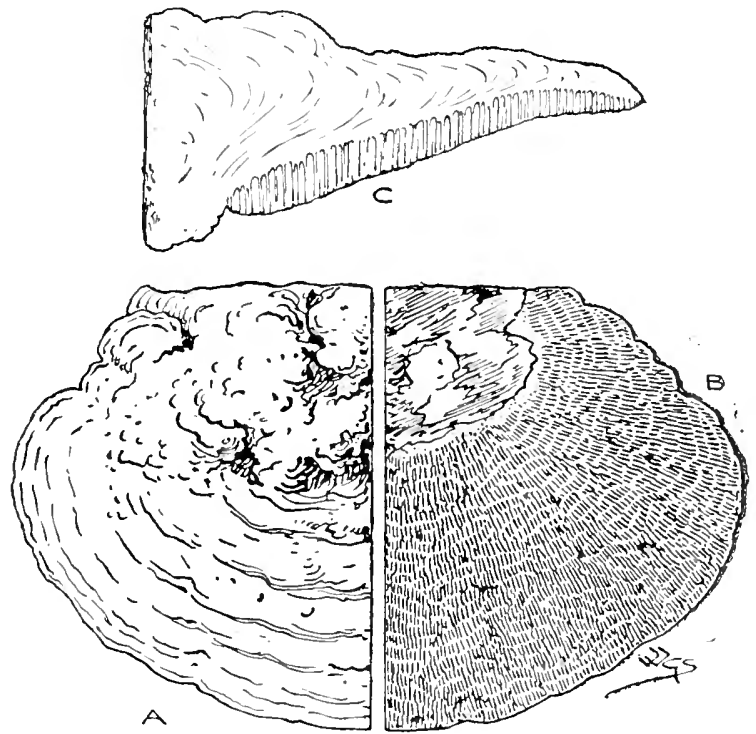

Fig. 8r.-Tramctes giblosa Fr. One-half natural size. A, upper surface; $B$, lower sufrace: C, section.

Several species are fragrant of anise, never acid. Growing on wood.

Intermediate between Polyorus and Dradalia.

Species 1649-1657a

a. Apoda. Without a stem.

Substance coloured.

Substance white or whitish.

1649

$1650-1654$

b. Resupinato. Resupinate.

$1655-1657 \mathrm{a}$

a. Apode.

1649. T. Pini Fr. (from its habitat, pine) $a b c$.

$P$. pulvinate, sulcato-rugged, sienna to deep sepia; marg. yellowish. $T$. somewhat long, brown-yellowish to bright ferruginous, rarely stratose. Pि. large irregular, oval. Flesh corky-woody, brownish to deep sienna.

Odour faint, pleasant. Trunks, pine, fir, larch; rare. Sept.-Oct. $5 \frac{5}{8}$ in. Often covered with alge and lichens. Hartig states that this fungus is the cause of the diseases known as "bark-shake" and "ring-shake" or " heartshake." The same as Polyforus Demidotfii Lév. according to Bresadola. 
1649a. T. cinnabarina Fr. (from the red-vermilion colour of the pileus; Gr. kimnabari) a.

$P$. fleshy, corky, convexo-plane, pubescent to glabrous, somewhat zoned, rugulose, red-rermilion, not dark. $T$. and $P o$. somewhat crimson.

Connate. Trunks, birch, beech, etc. Oct. $3 \frac{1}{4} \times \frac{3}{4}$ in.; projection 2 in.

1649b. T. Trogii Berk. ex Trog. (after J. G. Trog) $a$.

$P$. fleshy, convex, somewhat zoned, pilose, buff-white to pale buff. T. white-buff. Po. variable in size, subangular, toothed. Flesh white.

Connato-imbricate. Dead aspen. Oct. $5 \times 2 \frac{1}{2}$ in. ; projection $\mathrm{I}_{2}^{\frac{1}{2}}$ in.

1650. T. gibbosa Fr. (from its thickness and convexity; sibbus, gibbous) a $b c$.

$P$. pulvinate, velvety, somewhat rugged behind, obsoletely zoned and tuberculate, whitish-ochre, greyish at the obtuse marg. T. long, faintly brownish-yellow. Po. round, oval, colour as P. Flesh corky, compact, yellowish-white.

Sometimes imbricate. Odour slight of anise when fresh. Stumps, posts, rails, oak, beech, willow, poplar; not common. Oct.-Feb. 6 in. Not always gibbous, sometimes thin.

1650a. T. pubescens Fr. (from the pores becoming sienna-red) $a$.

$P$. dimidiate, corky, thin, smooth, at length rugoso-zoned, white to rose-salmon and olive-lorown, densely pulverulent. Po. roundish, irregular, white-salmon, clouded rose-colour.

Salix caprea. Oct. $1 \frac{3}{4} \times 1 \frac{1}{\frac{1}{4}} \mathrm{in}$. Pores and flesh changing from white to crimson on being scratched.

1651. T. Bulliardii Fr. (after Pierre Bulliard) a $c$.

$P$. pulvinate, whitish, yellowish or brownish, sometimes zoned dark brown; marg. greyish or pale ochre. T. long, vinousyellowish. Po. large, irregularly roundish or sinuous. Flesh faintly brownish-white, becoming fuscous.

Fragrant. Dead wood. Sept.-Oct. $5^{\frac{3}{4}}$ in. Closely allied to Dicidalea.

1652. T. suaveolens Fr. (from its fragrance; suavis, sweet) $a b c$.

$P$. pulvinate, villous, sometimes rugged, zoneless, whitish. $T$. long, sometimes irregular or short, whitish. Po. roundish, whitish, becoming fuscous. Flesh corky-soft, whitish or dull straw-colour.

Often imbricate. Odour of anise till dry. Trunks, willow, lime, etc.; uncommon. Oct.-Dec. $6 \frac{3}{4}$ in. Often enveloping grass and twigs.

1653. T. odora Fr. (from its fragrance, odor) $\iota$.

$P$. pulvinate, uneren to smooth, zoneless, ivory-white or greywhitish. $T$. shorter than in 1652, white or pale buff-white. Po. minute, roundish, half the size of 1652. Flesh corkyelastic, white.

Willow; rare. Jan. $3 \frac{1}{2}$ in. 
1654. T. inodora Fr. (from its being scentless, inodorus) a.

$P$. expanded, thin, tomentose, smooth, zoneless, white or greyishwhite. $T$. long, unchanging white. $P$ o. minute, roundish. Flesh corky-firm, white.

Stumps, beech. $2 \frac{1}{\frac{1}{4}} \mathrm{in}$.

\section{Resupinate.}

1655. T. mollis Fr. (from its soft substance; mollis, soft) $a b c$.

Sub. somewhat membranous, whitish. $T$. shallow. $P o$. large, cup-shaped, irregular, yellowish- or brownish-white, becoming fuscous, then black; marg. broadly barren, determinate, then revolute, pubescent beneath.

Often broadly effused. Dead wood, beech; common. Jan.-Dec. $4 \frac{\frac{1}{2}}{\text { in. }}$ Probably lasting for two years.

1656. T. Terryi B. \& Br. (after Michael Terry) $a$.

Sul. pulvinate, corky, suborbicular, I in. thick, whitish ; marg. determinate, undulate. $T$. shallow, 4 -stratose in examples first found. Po. irregular, with thin walls. Flish dry, with 4-5 annual growths, white.

Odour none. Beech. 4 in.

1657. T. serpens Fr. (from its frequent sinuous creeping habit; serpens, creeping) a b c .

Sub. somewhat pulvinate, $\frac{1}{16}-\frac{1}{5}$ in. thick, arid, white. $T$. shallow to somewhat elongate. Po. large, somewhat round, unequal, ivory-white; marg. barren, determinate, pubescent.

At first orbicular, soon confluent. Hornbeam, privet, oak, oak chips in tan pits, squared oak timber, beech. Aug.-Jan. $5 \frac{1}{3}$ in. Polyponus Stevensii B. \& Br. Must not be confounded with 1553.

1657a. T. purpurascens B. \& Br. (from the hymenium becoming purple).

Sub. somewhat leathery, subtomentose, chestnut. $T$. rigid, becoming purple. Po. small.

Dead willow. $\frac{3}{4}$ in.

\section{DÆDALEA Pers.}

(From the Cretan labyrinth made by Dadalos-in reference to the intricate, labyrinthine pores.)

Agreeing with Frametes, except in the firmer and sometimes thicker dissepiments of the pores, which, when fully grown are irregularly sinuous or labyrinthine, the floccose substance of the hard pileus descending unchanged into the trama. (Fig. 82.)

Inodorous.

Corky-woody, dimidiate, sessile. 
Coriaceous, dimidiate, sessile.

1662,1663

Resupinate.

1664,1665

Resupinate or effuso-reflexed, of uncertain affinity.
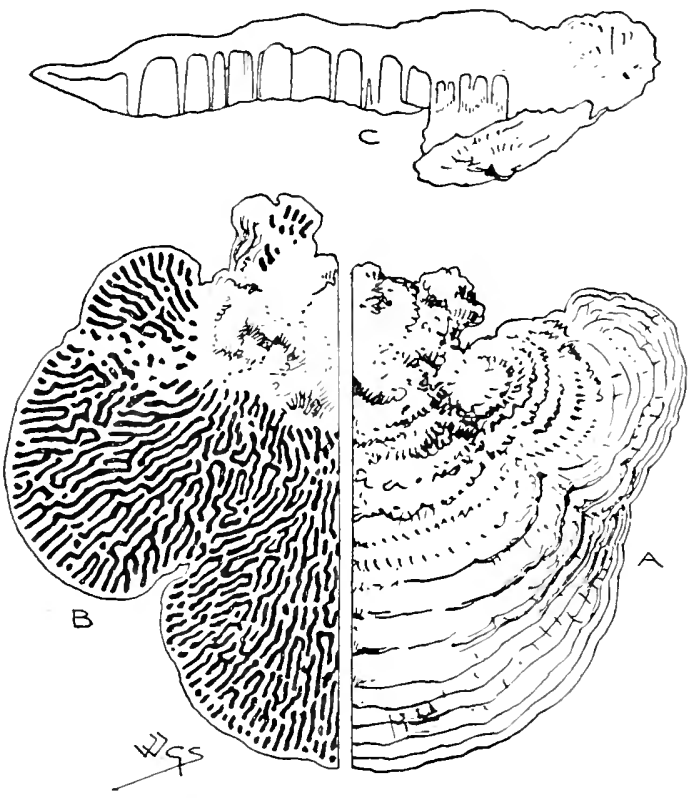

Fig. 82.-Dätalea guevcinat Pers. One-half natural size. A, upper surface; B, lower surface; C, section.

1658. D. quepeina Pers. (from its habitat-oak, quercus) $a b c$.

$P$. somewhat zoned, rugulose, uneven, somewhat smooth, buffbrown, darker behind. $T$. long. Po. at first simple, then labyrinthine, edge obtuse, whitish-salmon. Flesh pale sienna.

single or imbricate; perennial. Sometimes on squared timber. Jan.-Dec. $7 \mathrm{in}$. Sometimes resupinate. Occurs in peat-beds in the eastern counties and in the lake-side pile-dwellings of Switzerland and Italy.

1659. D. aurea Fr. (from its golden colours; aurum, gold) a c.

$P$. somewhat zoned, rugulose, uneven, coarsely velvety, orangeor yellowish-brown. T. somewhat long. Po. simple then sublabyrinthine, opaque sienna-orange. Flesh sienna-white.

Single to imbricale. Dead oals. $2 \frac{3}{4}$ in. Pores usually rounder than 1658.

1660. D. confragosa Pers. (from the rugged pileus; confragus, rough) $a b$.

$P$. roughly rugulose and zoned, buff- or liver-brown. $T$. somewhat long. Po. moderately large, chiefly round and oblong, or 
sublabyrinthine, pale umber, becoming darker. Flesh pale sienna.

Single, rarely subimbricate. Oak, willow, poplar, beech, service. Oct.Feb, $5 \frac{1}{2}$ in. In drying smells of stinking fish.

1661. D. einerea Fr. (from its ashy pileus; cincrits, ash-colour) $a c$.

$P$. undulate, zoned, tomentose, whitish-umber to greyish; $z$. darker. $T$. somewhat long. $P o$. round to oblong and labyrinthine, whitish to ashy or biscuit. Flesh white to biscuit.

single to imbricate; perennial. Dead trunks. Jan.-Dec. $4 \frac{7}{8}$ in.

1662. D. polyzona Pers. (from the many-zoned pileus; Gr. polus, many) a c.

$P$. tomentose, yellowish-brown, darker or reddish behind; $z$. dark brown. T. somewhat long, sometimes in two strata. Po. sublabyrinthine, buff-white. Flesh somewhat thin, buff-white.

Imbricate. Jan.-Dec. $5^{\frac{1}{ \pm}} \mathrm{in}$.

1663. D. unicolor Fr. (from its single colour; unus, one, color, colour) a b c.

$P$. somewhat rugged, villoso-strigose, zoned, yellowish-brown. $T$. moderately long. Po. labyrinthiform, sometimes of two strata, pale buff. Flesh pale buff.

single to imbricate, sometimes resupinate and effused with small development of pileus. Stumps, rails, birch, beech, acacia, maple, oak, willow, chestnut, hornbeam; common. Jan.-Dec. $4 \frac{1}{2} \mathrm{in}$. Flesh sometimes much eaten by larve.

1664. D. latissima Fr. (from its broadly spreading habit; latus, broad) a $b c$.

Sub. from a film to $\frac{\mathrm{r}}{4} \mathrm{in}$. thick, undulate. $P o$. of varying depths, small, round to sublabyrinthine, narrow, distant, colour of washleather; marg. barren.

Deal wood, branches, beech; rare. 5 in. Sometimes effused for $2 \mathrm{ft}$.

1665. D. vermicularis Pers. (from the colour, or the worm-like shape of the pores; a'imis, a worm) a.

Sub. thin to $\frac{1}{\mathrm{i}} \mathrm{in}$. Po. shallow, partly elongated to $\frac{1}{\mathrm{~s}} \mathrm{in}$. deep, sinuous, round near the barren marg., very pale salmon, or partly white and partly pale Hesh-colour.

On the ground, in contact with wood and earth. Aug. $5 \frac{1}{ \pm}$ in.

1666. D. ferruginea Schum. (from its colour of iron-rust; ferrum, iron) $a$.

$P$. at first white-villous, becoming yellowish-sienna or yellowishbrown; z. $2-3$, umber. $T .{ }_{s}^{1}$ in. deep, round to sublabyrinthine, tawny or yellowish-sienna; marg. barren, white-villous. Flesh $\frac{1}{s}$ in., pale yellowish-sienna.

Imbricato-connate. Dead wood. $\mathbf{I}_{4}^{\frac{3}{4}} \mathrm{in}$. 


\section{MERULIUS Halle.}

(From the common colours of the hymenium, yellow or orange, or partially black, the colours of the beak and plumage of the blackbird, merula.)

Resupinate. Pileus, when present, effuso-reflexed. Stem none. Hymcnophore a soft, woven, mucedinous mycelium, covered with the

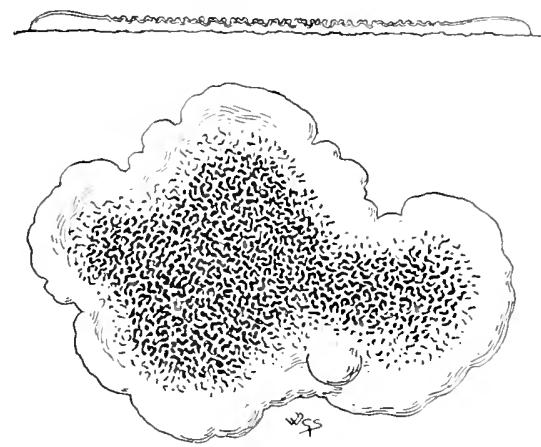

Fig. 83.-17emlins Lacrymans Schum., entire and section above. One-third natural size.

waxy-soft, porous, gyrose or obsoletely toothed hymenium. Spores white or ferruginous. On wood, sawdust, leaves, mosses, etc., rarely on the ground. (Fig. $8_{3}$.)

Species 1666a-1681

a. Leptospore. Sfores white.

Pilezs effuso-reflexed, circumference determinate.

$1666 \mathrm{a}-1669$

Resupinato-effused, flaxy-membranous, separating, flaxy beneath and at the circumference. 1670-1674

Crustaceo-adnate, somewhat flaxy at the circumference.

$1675-1679$

b. Coniophora. Stores ferruginous. Hymenium at length pulverulent with spores.

1680,1681

$$
\text { a. Leptospora. }
$$

1666a. M. confluens Schwein. (from the habit, small growths becoming confluent into large patches) $a$.

Resupinate, longitudinally effused, somewhat fleshy, vinous biscuit-colour ; marg. free, inflexed, subtomentose, biscuitcolour. Po. very small, uneven, reticulate.

When the small patches become confluent, the lines of junction become raised as irregular hexagons. Branches, alder. Aug. Snall growths papillate, about $\frac{3}{8}$ in. diam. Confluent masses about 3-4 in. 
1667. M. tremellosus Schrad. (from the tremulous substance) $a b c$.

$P$. dentato-squarrose near marg., yellowish-white, salmon shaded. Po. pale ochre to yellowish-white, brownish-ochre at base; marg. barren, white. Flesh fleshy-tremellose, approaching Tremella, very pale blue-grey and pale salmon.

Connato-imbricate. Odour strong, fungoid. Decayed trees, wood, beech, birch, oak; rare. Aug.-Feb. 6 in. At first resupinate. Hymenium usually rich vinous-brown when dry.

1668. M. aurantiacus Berk. (from the colour; aurum, gold) $a$.

$P$. tomentose, sometimes zoned, dull yellowish-white, or ashy. Po. somewhat poriform, minute, dull orange; marg. broad, barren. Flesh fleshy-coriaceous.

Dead trunks, beech ; rare. May. I $\frac{3}{4}$ in.

1669. M. corium Fr. (from the thin leather-like subiculum; corinm, skin, leather) a b $c$.

$P$. becoming slightly reflexed, paper-like, zoned, villous-hairy, separating, white. Po. small, gyrose or pore-like, flesh-colour, pale tan, orange-yellow, orange or sienna-orange ; marg. broad, barren, white. Flesh soft.

Dead wood, trunks, branches, hornbeam, oak, furze, moss, sometimes on old beams, etc., in houses, sometimes pendulous, like a small inverted cup ; common. Mar.-Jan. $6 \frac{x}{ \pm}$ in.

1670. M. læticolor B. \& Br. (from the colour; letus, bright) $a$.

Po. at first obsolete, then plicato-rugose, distant, bright orange; marg. broad, barren, white.

Coarse sawdust and leaves, oak, pine, mountain-ash. Nov. 3 in.

1671. M. aureus Fr. (from the colour; aureus, golden) $a c$.

Po. large, walls thin, plicato-porous, orange or golden-yellow; marg. broad, barren, sulphur.

Pine boards, pine-leares and cones. Oct. 2t in. Hymenium dark brown when dry.

1672. M. himantioides Fr. (from the thong-like shape of the folds of the hymenium; Gr. himas, a thong, cidos, appearance) a b c.

Po. porous to gyrose, folds tooth- or thong-like, yellow to dull ochre or yellow-olivaceous ; marg. barren, white or sulphur.

Dead wood, fir, cherry, club-moss; rare. Sept.-Dec. $7 \frac{3}{x}$ in. Hymenium olive or dark brown when dry.

1673. M. molluscus Fr. (from the soft substance; molluscus, soft) $a b c$.

Po. large, round, poroso-gyrose, flesh-colour, deep carnation or ochre to rich orange; marg. broad, barren, white. Hym. rufous or vinous-brown when dry.

Wood, branches, fir, sometimes spreading over and conjoining fallen twigs; uncommon. Sept.-Feb. 8 in.

1674. M. fugax Fr. (from its collapsing hymenium and fugitive habit; fugio, to flee) a. b c . Byssoid to membranous, very thin.

Po. reticulate, feebly developed and soon collapsing, white to 
yellowish-white; marg. very broad, barren, white. Hym. brown when dry.

Bark, pine, alder. Nov.-Jan. $3 \frac{1}{2}$ in. Pores sometimes not developed.

1675. M. porinoides Fr. (from the poriform folds of the hymenium) a $b c$.

Po. large, round, dissepiments feeble, pale dull yellowish or brownish-ochre; marg. broad, barren, white to yellowish-white.

Dead wood, chips, bark, fir, on the ground; rare. Sept. $4 \frac{1}{2}$ in.

1676. M. rufus Pers. (from the reddish hymenium; rufus, reddish) a b c. Waxy-soft.

Po. regular, round, medium to small, red-sienna, or light to dark vinous-brown; marg. obsolete, or narrow and irregular, barren, colour as Po.

Often effused. Dead wood, oak, willow; uncommon. May-Feb. $3^{\frac{1}{2}}$ in. Vinous-blackish when dry. (ireatly resembling a resupinate Poly'forzus.

1677. M. serpens Tode (from its wary, creeping habit; serpo, to creep) $a b c$. Inseparable, thin.

Po. wrinkled to round, angular, entire, dissepiments sometimes feeble, pale to ochreous-salmon or red; marg. broad, barren, white. Hym. dark vinous- or rufous-brown when dry.

Fir, lime, ash, cedar, cones; rare. Oct.-Mar. $6 \frac{1}{8} \mathrm{in}$.

1678. M. pallens Berk. (from its pale colour; pallco, to be pale) a $b c$. Inseparable, somewhat gelatinous.

Po. round, but variable, minute to somewhat large, feebly developed, pale reddish- or brownish-salmon, sometimes orange-brown ; marg. indeterminate, barren. Hym. pale purpleumber to deep vinous-brown when dry.

Fir, oak; rare. June-Dec. $3 \frac{3}{8}$ in.

1679. M. Carmichælianus Berk. (after Capt. Dugald Carmichael) a $c$. Confluent, very thin.

Po. large to small, often hexagonal, very shallow, with feeble dissepiments, white; marg. narrow, obsoletely punctate, edge finely laciniate.

Bark. $3 \frac{1}{2}$ in. Brown when dry.

\section{b. Coniophore.}

1680. M. lacrymans Schum. (from the watery drops on the fully developed hymenium; lachryma, a tear) $a b c$.

Spongy-fleshy, arachnoid-velvety below, sometimes reflexed with a small extended rugose whitish more or less imperfect $P$. Po. large, round to gyroso-toothed, sienna-orange, yellowish towards the barren tomentose white or salmon marg. Flesh grey-whitish-flesh colour or vinous-brown. Hym. dark brown, sometimes almost black when dry.

Odour very strong, fungoid, penetrating. Dressed wood in buildings, spreading over brickwork, plaster, mortar, carpets, etc. Does not grow out of houses in this country. Jan.-Dec. I ft. $5 \frac{1}{2}$ in. The fungus of "dryrot." The flesh gives a red stain to paper like 1517. Var. fulverulentus 
Fr., a membranous form, with a somewhat more feeble development of pores than in type. Must not be confused with 1884, a plant which has no pores.

1681. M. squalidus Fr. (from its squalid appearance) $a b c$. Smooth, with extensive barren places.

Po. large to small, somewhat sinuous, hyaline flesh-colour; marg. broad, barren, paler, fibrillose at edge. Hy'm. dull, dark vinous-umber or purple slate-black when dry.

Ilornbeam, posts, dressed wood. Feb. $3 \frac{1}{8}$ in.

1681a. M. Guillemotii Boud. (after M. Guillemot) $a$.

$P$. dimidiate, imbricate, smooth, white, becoming greyish with age. Po. gyrose, subgelatinous, fulvous to brassy-orange, slightly olive, pulverulent with spores. Flesh white to pale lavender.

On posts, palings, willow. June-Sept. $4 \frac{3}{4} \times 2 \frac{3}{4}$ in. ; projection $2 \frac{3}{4}$ in.

\section{POROTHELIUM Fr.}

(From the perforate tubercles, spines or teat-like teeth;

Gr. poros, a pore, thele, a teat.)

Subiculum more or less membranous, bearing obtuse, or elongate, distinct papille, which are at first closed, then open in the form of pores or tubes. On dead branches, etc. (Fig. 84.)

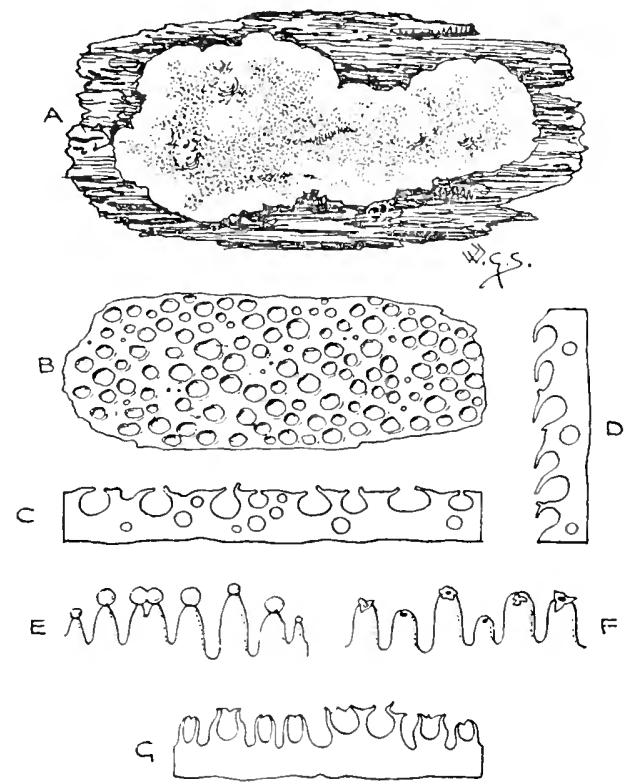

Fig. 84.-A, Porothelium fimbriatum Fr., one-half natural size. B, pores; c, section when grown horizontally; D, ditto when grown vertically. $x$ ro. E, P. Friesii Mont., papilla from a damp position; $F$, ditto from a dry position; $G$, section. $\times$ ro. 
Closely allied to Fistulina, with which it agrees in the fimbriate mouths of the free tubercles. or tubes. The tubes frequently distil drops of fluid in damp positions. The appearance commonly somewhat resembles effused groups of minute $\angle$ Ecidia.

Nassee places this genus in Hydnacee.

Species $\mathbf{1 6 8 2 - 1 6 8 6}$

Fries has made Solenia follow Porothelium, but in Solcnia each cup or cylinder is a complete plant, not a mere tube. To find a place in the Polyporacee each cup should be furnished with tubes. The true place for Solenia is after Cyphella in the Thelephoracte.

1682. P. fimbriatum Fr. (from the fimbriate margin of the subiculum) ac .

Sub. separable. $P a$. hemispherical, immersed, small, biscuitcolour ; marg. broad, barren, fimbriate, white.

Birch, beech. $3^{\frac{3}{4}} \mathrm{in}$. General appearance much like the pore surface of a minutely pored resupinate Poly'torns. Pa. sometimes distant. Often with extensive barren places on subiculum.

1683. P. Friesii Mont. (after Elias Fries) $a b c$.

Sub. less fimbriate than 1682. Pa. hemispherical, immersed, somewhat larger than in 1682, varying from minute to larger, ivory or pale brown.

Pine, juniper. Sept.-Feb. 3 in.

1684. P. Stevensonii B. \& Br. (after the Rer. Dr. John Stevenson) a. Sub. somewhat thick, gelatinous. $P a$. elongate, whitish or pale yellowish, each bearing a diaphanous, yellow globule; marg. rather coarsely hispid, then more or less denuded.

Pine, squared pine. $3 \frac{3}{3}$ in. The mycelium imparts a sweet scent to the wood.

1685. P. Keithii B. \& Br. (after the Rev. Dr. James Keith).

$S u b$. adnate, gelatinous in centre. $P a$. short, collapsing, pallid; marg. very thin, at first somewhat gelatinous.

Dead fir. April.

1686. P. confusum B. \& Br. (from its having been confounded with other members of the genus).

Sul. adnate, at first floccoso-pulverulent. $P a$. very small, pallid; marg. thin, arachnoid.

Fir sticks. June-Sept. 


\section{FAM. III. HYDNACEÆ.}

Hymenium inferior in the stipitate and dimidiate species, superior in the resupinate series, bearing from the first persistent spines, teeth, tubercles, papillæ or wart-like folds.

Basidia tetrasporous except in Knciffic, where they are monosporous; Mucronella, which has been described as monosporous, is sometimes tetrasporous.

Species $1687-1780$

\section{Key to the Genera.}

Pileate.

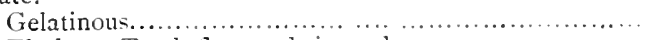

Fleshy. Teeth flattened, irrestular ....................

7I TREMELLODON

72 SISTOTREMA.

Pileate or resupinate. Fleshy. Spines acute, distinct at base 70 Hydvum.

Resupinate.

Hymenium bearing somewhat acute teeth which spring from folds or ridges............................

Hymenium bearing teeth in the form of coarse deformed tubercles ............................... 74 Radulun.

Hymenium covered with folds or wrinkles ............. 75 Phlebia.

Hymenium covered with granulesApices excavated

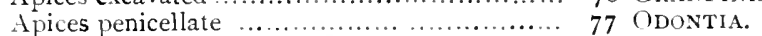

Hymenium covered with minute acute spicules .........
Hymenium bearing teeth in the form of long scattered 73 IRPEX. 74 Raduluis
75 Phlebia.

76 Grandinia. 77 OdONTIA. usually distant hristles. Subiculum nearly obsolete.

79 Mucronella.

\section{HYDNUM L.}

(From the Greek name for a truffle, hydnon, appropriated without reason by Linnaus for this genus.)

Hymenimm inferior in the first four series, superior in the fifth. Spini's awl-shaped, acute, distinct at the base. (Fig. 85.)

Species $1687-1745$

Miesopode. Stem central. Terrestrial, chiefly in pine woods.

a. Carnose. Pilizs fleshy, somewhat fragile, stem solid. Mostly edible.

Pilezs scaly or tomentose. Spines changing colour.

Pilius even, smooth. Spinis changing colour. 1691-1693 Spine's unchangeable, unicolorous, whitish, or pale shades of ochre, buff or brownish. 
b. Lignose. Pilens corky or coriaceous.

Spines changing colour, and, as well as the spores, somewhat fuscous.

$1698-1700$ Spines changing colour, and, as well as the spores, ferruginous. 1701,1702 Sfints unchangeable, spores whitish.

$1703-1706$

\section{Pleuropode.}

St'm lateral, somewhat dimidiate.

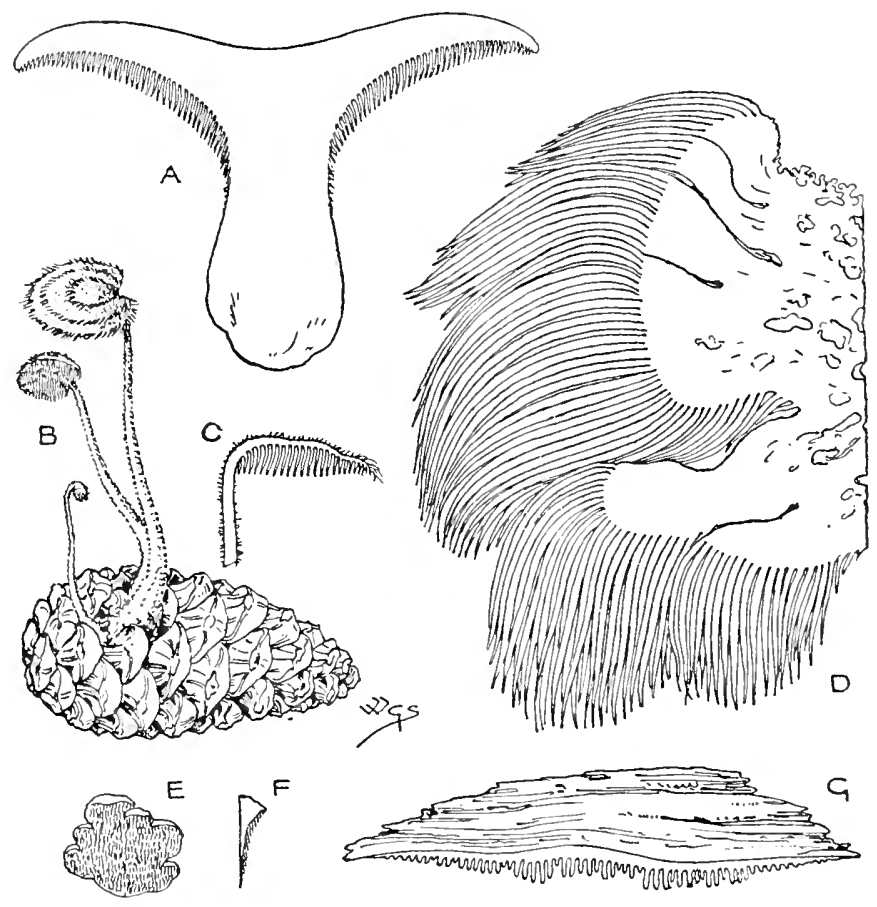

Fig. 85.-A, Hydnum repandunt L., section, one-half natural size. B, H. anriscalfium L., entire, one-half natural size. C, ditto, section through pileus, natural sizt. D, H. erinaccus Bull., section, one-half natural size. E, H. ochraccum Pers., entire, one-half natural size. F, ditto, section, one-half natural size. G. $H$. squalinum Fr., section, one-half natural size.

Merismate. Branched.

Very much branched.

Simple, as seen without cutting, tuberculiform, immarginate, but slightly branched as seen in section.

1709,1710

Apone. Stem absent.

Pilerus fleshy, soft.

$1711-1713$

Caspitose. Pili imbricate, commonly pliant, tough.

Simple. Pilezs coriaceous or corky. 
Resupinati. Pilezs none. Stines vertical or oblique according to situation.

Spines fuscous, ferruginous.

$1716-1723$

Spines yellowish, greenish.

$1724-1736$

Stines flesh-colour, lilac, rufescent.

1737,1738

Spines white, or light yellowish when old.

$1739-1745$

\section{MLSOPODE.}

\section{a. Carnosie.}

1687. H. imbricatum L. (from the imbricate scales of the pileus) $a b c$.

$P$. at length depressed, rich, dark claret-brown, the innate sc. exposing the paler flesh. St. even, whitish- or yellowish-claretbrown, base as P. Sp. decurrent, pale brownish-biscuit. Flesh pale claret-brown in upper part of P., white elsewhere.

Usually single. Siaid to be elible. Taste when cooked like tough liver, bitter, disagreeable. Amongst firs : rare. Sept. - Nor. $9 \frac{1}{2} \times 2 \frac{1}{2} \times 1 \frac{1}{2}$ in.

1688. H. squamosum Scheff. (from the scaly pileus; squama, a scale) $a$.

$P$. at length depressed, breaking up into rough irregular sc., brownish- or vinous-salmon; sc. brownish. St. equal or attenuate downwards, somewhat scaly, pale- or brownish-buff, sometimes darker below. Sp. decurrent, purplish-salmon. Flesh pale purplish-salmon, almost white.

Often connate. Amongst mosses in pine woods. Sept.-Oct. $2_{4}^{\frac{3}{4}} \times 1 \frac{1}{2} \times \frac{5}{4} \mathrm{in}$.

1689. H. scabrosum Fr. (from the rough, scaly pileus; scabrosus, rough) $a b$.

$P$. turbinate, then plane, usually uneven, squamulose, pale vinousumber ; mid. darker; marg. white; sc. umber. St. attenuate downwards, vinous, umber or cinereous, blackish at base. Sp. decurrent, buff-irory or ferruginous with a grey fuscous effect. Flesh salmon-ivory.

Usually single. Woods, pine. Sept. $4 \times \mathbf{I} \frac{1}{4} \times \mathbf{I}$ in.

1690. H. molle Fr. (from the downy pileus; mollis, soft) a $c$. White. $P$. at length depressed, tomentose. St. almost equal. St. decurrent. Flesh pale, brownish-white.

Woods, pine. $4 \frac{1}{2} \times \mathrm{r}_{\mathrm{5}}^{\frac{1}{5}} \times \mathrm{r}$ in.

1691. H. lævigatum Swartz (from the smooth pileus; laz'is, even) a b. $P$. at length depressed, pale yellowish-umber. St. equal, whitish above, colour as I'. below. St, decurrent, pale brown.

Woods, pine. Aug. -1 No. $4 \frac{1}{2} \times I_{\frac{5}{8}} \times 1$ in. Sometimes occurs three times the size of drawing. Hecoming more or less black in drying. 
1692. H. fragile Fr. (from its fragility) $a b$.

$P$. at length depressed, at first pubescent, then smooth and subsquamulose, yellowish-brown to reddish-brown-buff, zoned darker. St. unequal, greyish, brownish-grey or as P., sometimes vinous-brown below. $S p$. decurrent, ivory-grey or dull pale grey-ochre. Flesh pale brown or pale vinous, darker at base of St.

Odour of curry powder. Woods, pine, amongst heath; rare. Sept.-Nov. $4 \frac{3}{8} \times 2 \frac{3}{4} \times 1$ in.

1693. H. fusipes Pers. (from the fusiform stem ; fusis, a spindle, pes, a foot) a.

$P$. fibrous, tough, lobed, becoming subdepressed, cold umber to deep claret-brown; marg. paler, sometimes with a narrow white edge. St. colour as P., usually paler. Sp. decurrent, very short, salmon- or brown-whitish. Flesh white or shaded, pale salmon or brownish.

Woods, pine. Sept. $2 \frac{1}{4} \times 1 \frac{1}{4} \times I_{\frac{1}{4}}^{1}$ in.

1694. H. repandum L. (from the repand pileus in old examples) a $b c$.

$P$. somewhat smooth, rarely floccoso-pruinose, pale buff. St. almost equal, paler than P. Sp. decurrent, colour as St. Flesh white, beconing as $\mathrm{P}$.

subgregarious. Sometimes in rings. Edible, but requires stewing slowly for four hours. Woods; common. Aug. - Nov. $4 \frac{7}{8} \times 1_{\frac{3}{4}}^{3} \times \mathbf{I}$ in. Becoming wholly ochre or orange in drying.

1695. H. rufescens Pers. (from the rufescent colour) $a c$.

$P$. thin, somewhat lobed, pulvinate to plane, pubescent, buff, orange-brown or brown. St. almost equal, frequently excentric, pale yellowish, yellow- or bright-cinnamon. Sp. adnatodecurrent, pale ochre to bright cinnamon. Flesh white to pale rufescent.

Sometimes connate. Woods; frequent. Sept. $3 \frac{3}{8} \times 3 \frac{1}{4} \times \frac{1}{2}$ in.

1696. H. acre Quél. (from the acrid taste) $a$.

$P$. turbinate, flattish, pale yellowish, olive shaded; mid. darker. St. equal, colour as P. or darker. Sp. decurrent, $\frac{1}{16}$ in., colour as P. Flesh pale yellowish, greyish or pale brownish.

Poisonous. Taste bitter-acrid. Woods, pine, chestnut, sandy places. $5 \times 1 \frac{1}{4} \times 1$ in.

1697. H. fuligineoalbum Schmidt (from the smoky-whitish stem; fulligo, soot, albus, white) a $c$.

$P$. convex, becoming depressed, repand, squamulose; sc. brownish on an umber-white ground. St. scaly, dull salmon. Sp. decurrent, pale vinous-brown. Flish white, clouded pale rosy-brown or pale sienna.

Woods, pine, pastures near woods. $4 \frac{1}{\frac{1}{4}} \times 1 \frac{3}{4} \times \frac{7}{5}$ in. Becoming dull vinousbrown in drying. 


\section{b. Lignose.}

1698. H. compactum Pers. (from its compact substance) a $b c$.

$P$. irregular, plane, tuberculose, orange-sienna or umber, sometimes ivory, shaded grey. St. irregular, tuberculose, orangesienna or umber. Sp. decurrent, salmon-brown. Flesh zoned brown and bright blue, or ivory and pale blue, the blue gradually becoming indigo.

Often confluent and misshapen. Amongst firs, heather, etc. Aug.-Nov. $3 \frac{3}{8} \times I_{\frac{1}{2}} \times I_{\frac{1}{4}}^{\frac{1}{4}}$ in. Becomes blackish in drying.

1699. H. aurantiacum A. \& S. (from the colour; aurum, gold) a $b c$. $P$. convex or turbinate, beconing flat, rugged, yellowish-orange or yellowish-sienna, buff when young. St. sienna-orange above, deep sienna below. Sp. decurrent, white to ivoryochre, sometimes orange near St. Flish pale dull orange.

Inodorous. Woods, pine. Sept.-Nov. $2 \frac{3}{4} \times 1 \frac{1}{\frac{1}{4}} \times \frac{5}{8}$ in. Becoming vinousbrown in drying.

1700. H. ferpugineum Fr. (from the rust-of-iron colour; fermm, iron) a $c$.

$P$. flat or slightly depressed, rugged, sienna-umber, at first downy, exuding blood-red drops. St. unequal, pale ochre, umber or slate. Sp. decurrent, reddish-salmon. Flesh pale yellow, vertically brown-streaked.

Often confluent, woods, involving fir-leaves, grass, etc. Frequently connate. Woods, pine, under ling. Aug.-Oct. $4 \frac{1}{5} \times \mathbf{I}_{\frac{3}{4}}^{3} \times \mathbf{I}$ in.

1700a. H. einereum Bull. (from the grey pileus and spines; cinis, ashes).

$P$. corky to woody, becoming infundibuliform, covered with small elevations, villous, white to grey, varying vinous or umber. St. somewhat ventricose, vinous-ivory, somewhat brown below. Sp. decurrent, short, white to grey and pale olive-brown. Flesh white, vinous-buff below.

Often connate. Woods. Sept. $2 \frac{3}{4} \times 2 \times \frac{5}{4}$ in.

1701. H. scrobiculatum Fr. (from the pitted pileus; scrobis, a trench) $a b c$.

$P$. rugged, tuberculose, downy; mid., tubercles and marg. deep sienna on a salmon ground. St. rugged, unequal, smooth, often rooting, vinous-salmon above, vinous-blackish below, or umber. Sp. decurrent, colour as P. or lighter. Flesh vinoussalmon, zoned vinous-brown.

Involving pine-leaves, elc. Often connate. Woods, fir, amongst ling. Aug.-Nov, $1 \frac{3}{4} \times 2 \frac{1}{4} \times \frac{3}{4}$ in.

1702. H. zonatum Batsch (from the frequently zoned pileus) $a b c$.

$P$. depressed; mid. rugged-squarrose; marg. radiato-rugose, $\mathrm{I}-\mathrm{I} 2$ Loned, z. sometimes nearly obsolete, ferruginous. St. unequal, rugged, floccose, colour as P. Sp. decurrent, small, slender, pallid to ferruginous. Flesh rich vinous-brown.

Commonly involving moss, leaves, etc., sometimes full of embelded firleaves; fragrant. Woods, fir, oak; rare. Aug.-Nov. $2 \frac{1}{1} \times 1 \frac{3}{8} \times \frac{3}{4}$ in. 
1702a. H. Queletii Fr. ex Quél. (after Dr. Lucien Quelet) $a$.

$P$. fleshy, coriaceous, plane, then depressed, zoneless, or with one zone, somewhat rugoso-radiate, vinous-sienna, darker at mid. ; marg. white. St. colour as P., paler above. Flesh pale salmon-umber.

Often connate. On the ground in woods. Oct. $2 \times 1 \times \frac{3}{16}$ in.

1703. H. nigrum Fr. (from the blackish colour; niger, black) $a b c$.

$P$. turbinate, rugged-tubercular, tomentose, lavender-grey, purple or blackish-blue ; z. 2-5, deep purple. St. unequal, lavenderumber, shaded umber, to black. $S p$. decurrent, lavenderwhitish, greyish or purple. Flesh slate or deep purple.

Often connate. Woods, pine. Sept.-Nor. $2 \frac{3}{4} \times 1 \frac{1}{2} \times \frac{3}{4}$ in.

1704. H. graveolens Fr. (from its strong odour; grazis, strong, oleo, to smell) a b $c$.

$P$. thin, zoneless, smooth, rugose, depressed, pale to dark umber or black-fuscous; marg. paler or whitish. St. equal, smooth, colour as P. Sp. decurrent, grey. Flesh umber.

Usually crespitose and confluent, rarely solitary. Odour heavy, spicy-sweet, like curry powder. Woods, fir, oak; rare. Sept.-Nov. $I_{\frac{3}{4}}^{3} \times 2 \times \frac{1}{5}$ in. Varied in shape and size, sometimes spathulate. Examples gathered in I 55 retained their odour in $\mathbf{1 9 0 I . ~ S t e m ~ s o m e t i m e s ~ v e r y ~ s h o r t . ~}$

1705. H. melaleucum Fr. (from the black pileus and white spines; Gr. melas, black, liukos, white) ac .

$P$. thin, plane, depressed, slate-blackish or slate-brownish or dark brown-grey ; z. darker; marg. whitish; when young pale umber with white marg. St. smooth, ashy-grey to slate-blackish. Sp. adnato-decurrent, white or whitish. Flesh umber-white to slate.

Sometimes crespitose. Odour none. Amongst pines. Sept. $1 \frac{3}{4} \times 1 \frac{1}{5} \times \frac{3}{16} \mathrm{in}$.

1706. H. cyathiforme Schæeff. (from the pileus, sometimes cupshaped; cyathus, a cup, forma, form) a bc.

$P$. thin, plano-infundibuliform, tomentose, somewhat rugged at mid., brown-whitish to dark claret-brown; marg. sometimes white-tomentose; z. 3-II, darker, when dry very glossy. St. unequal, smooth, sometimes almost obsolete, colour as P. or darker. $S p$. adnato-decurrent, dull salmon or salmon-grey.

Sometimes connate. Woods, fir. Ang. - Nor. $2 \frac{3}{4} \times I_{8}^{3} \times \frac{3}{9}$ in. With the exception of the spines, sometimes much like 1525 .

\section{Pleuropode.}

1707. H. auriscalpium L. (from the shape of small examples; auriscalpium, an ear-pick) a b c.

$P$. thin, coriaceous, reniform, hispid-hairy, brownish-salmon or brown, sometimes blackish; z. darker. St. rooting, hispid, often branched, colour as P. Sp. white, salmon or brown. Flesh pale umber or salmon-white.

Sometimes springing from thick rhizomorphoid growths with stems sometimes without pilei. Fir-cones, fir-leaves. Nay-Dec. $\frac{f}{5} \times 2 \times \frac{1}{15} \mathrm{in}$. 


\section{MERISMAT王.}

1708. H. coralloides Scop. (from its appearance, like branched white coral ; Gr. kerallion, coral, cidos, appearance) a b c.

Much and narrowly branched, wholly white, becoming faintly buff or yellowish. $S_{p} \cdot \frac{1}{16}$ to $\frac{5}{5} \mathrm{in}$. long.

Trunks, beech, birch, ash, oak, etc.; rare. Oct. $\mathbf{I} 2 \times 7 \frac{1}{2}$ in. Sometimes dark brown when dry.

1709. H. epinaceus Bull. (from a fancied resemblance, when young, to a hedgehog, crinacuss) a b $c$.

Slightly and broadly branched, wholly white, becoming pale yellowish. Sf. $\mathrm{I}_{2}^{\frac{1}{2}}$ to $2_{\frac{3}{4}} \mathrm{in}$. long, pendulous in large tufts. Flesh branched, cancellate.

Taste mild. Trunks, oak, beech, hornheam, alder, etc.; rare. Sept.-Nov. $13 \times 13$ in. Sometimes dark brown when dry. Sometimes very large and heavy, weighing $22 \mathrm{lbs}$. or more.

1710. H. caputmedusæ Pers. (from a fancied resemblance to Medusa's head; cafut, a head) at $c c$.

Sp. in connate, tuberculiform groups, $\frac{1}{2}$ to $1 \frac{1}{\mathrm{in}}$. long, growing in various directions in matted locks as of hair, substipitate, wholly white or ivory, becoming grey.

Trunks; rare. Diam. 63 in.

1710a. H. Schiedermayeri Heuf. (after K. B. Schiedermayer) $a$.

Fleshy, immarginate, ivory-white, becoming buff when old. Sf. $\frac{3}{s}$ to $\frac{T}{2}$ in. long. Flesh somewhat cancellate, pale sulphur, becoming buff below when old.

Connato-imbricale. Growing from cracks in bark of old apple trunks. In connato-imbricate groups of varions sizes, each example $1 \frac{1}{2} \times I$ in. Groups $6 \times 3$ or $4 \mathrm{in}$.

\section{APODE.}

1711. H. cirrhatum P'ers. (from the pileus, clad with small curls, like little locks of hair : cirrus, a curl) a $b c$.

$P$. pulvinate, expanded, dark biscuit-colour ; marg. fimbriate. St. $\stackrel{3}{3}$ to $\frac{1}{2}$ in. long, paler than P. Flish paler than $\mathrm{P}$.

Imbricate or simple. Trunki, beech. Ang. Group 7 in. Diam. $5 \frac{1}{2}$ in. sometimes dark brown when dry.

1712. H. diversidens $\mathrm{Fr}$. (from the spines, differing from the scalelike growths on the pileus : dizirsus, diverse, dens, a tooth) a bc. Wholly biscuit-colour, pale to dark.

$P$. somewhat lobed, thick, beset with small rariously shaped sc.

Substipitate, obscurely branched. lrregularly imbricate. Trunks, hornbeam, oak, beech, birch. Oct. Dian. $5 \frac{1}{4}$ in. One cause of "white-rot."

1713. H. puleherrimum B. \& Curt. (from its appearance: fulchirrimus, very beautiful) a $c$.

P. pulvinate, fleshy-fibrous, spongy-soft, dimidiate, expanded, 
stiffly-downy, white, shaded, pale tawny; marg. thin, lobed. $S p$. variable in size, $\frac{1}{5}$ to $\frac{1}{4}$ in., tawny.

Subimbricate. Oct. Diam, $1 \frac{3}{8}$ in.

1714. H. multiplex Fr. (from the many folds of the pileus; multus, many, plecto, to twist) $a c$.

$P$. thin, leathery, deeply folded, spathulate, velvety, rich brown. Sp. $\frac{1}{16}$ in. long, crowded, very slender, grey, shaded brownish. Flesh bright brown, paler than $\mathrm{P}$.

Imbricate. Group $4 \frac{1}{2}$ in. in diam.

1715. H. ochraceum Pers. (from the ochreous colour) $a b c$.

Sub. thin. $P$. when present, thin, pale ochreous, zoned, usually resupinate or with marg. slightly upturned and forming more or less perfect connate pilei. Sp. very small, somewhat blunt, ochreous flesh-colour, orange-buff, sometimes fawn, rarely dull crimson.

Trunks, oak, branches, fir-leares, bramble, apple, moss; common. Oct.A pril. Diam. 5 in. Readily separating from matrix.

\section{RESUPINATÆ.}

1716. H. squalinum Fr. (from a fancied resemblance in the spines to sharks' teeth; squalus, a shark) a $c$.

Suh. adnate, coriaceous, pale yellowish-fawn. Sp. entire, stout, compressed, rounded at apex, $\frac{3}{16}$ in. long, colour as Sub.

Trunks, beech. Diam. $2 \frac{5}{8}$ in.

1717. H. fuscoatrum Fr. (from the dusky-black clouding ; fuscus, dusky, ater, black) a $c$.

Sub. a thin film, glaucous, flocculoso-pruinose, salmon-umber; mid. larker; or dark brown shading into sienna and brownochre at marg. Sp. short, conico-subulate, acute, colour as Sub.

Rotten wood, birch, beech, alder. Diam. $2 \frac{1}{2}$ in.

1718. H. membranaceum Bull. (from the membranous subiculum) $a b c$.

Sub. an effused waxy-membranous film, pale vinous sienna; marg. lighter. Sp. awl-shaped, crowded, acute, short, darker than Sub.

Fallen sticks, pear ; frequent. Jan. Diam. $2 \frac{7}{5}$ in.

1719. H. molluscum Fr. (mollis, soft, in reference to the thin subiculum).

Sub. membranous, dry, readily separable from the matrix, whitish. $S p$. short, slender, reddish.

Wood. Distinguished from 1718 by the dry white subiculum, separating from the matrix.

1720. H. Weinmannii Fr. (after J. A. Weinmann, mycologist) $a b c$.

Sub. effused, waxy-membranous, agglutinate, fawn-grey, pale slate, or umber and white. $S p$. minute, acute, somewhat 
distant, sometimes collected in small groups or fascicles, slate, somewhat umber, apex white.

Fallen branches, ash, oak. Oct.-April. Diam. $4^{\frac{1}{4}}$ in. When dry, cracking and becoming full grey or fawn to deep brown or almost błack.

1721. H. crinale Fr. (from the slender hair-like spines; crinis, a hair) $a$.

Sub. effused, villoso-interwoven, thin, sienna-ochre. St. $!$ in. long, crowded, very slender, sienna-umber or rufous-brown.

Dead wood; rare. Diam. 3 in. The hair-like spines resemble those of Mucronilla.

1722. H. ferruginosum Fr. (from the rust-of-iron colour; firm, iron) $a c$.

Sub. effused, submucedinous, separable from matrix, tawny, ferruginous. St. crowded, conico-awl-shaped, acute, colour as Sub.

Decayed wood, especially under bark, rarely on the ground. July-Oct. Diam. 2 in. Sometimes mere conidial forms of other fungi, as Isaria amongst the Hyphomycetes, or 1780 are mistaken for this plant.

1723. $\mathrm{H}$. variecolop $\mathrm{Fr}$. (from the varying colours) $a b c$.

Sub. effused, adnate, forming a furfuraceous crust, white, becoming cream, siemna and sienna-umber in drying, sometimes resembling white miniature clouds; marg. finely white-fimbriate. Sp. crowded, conical, short, or minute with a ragged appearance, but here and there longer and more typically $H_{y} d m u m-$ like, commonly adpressed, colour as Sub.

Dead stumps, oak; rare. Oct. Diam. $3_{\frac{3}{4}}^{\frac{3}{1} \text {. }}$

1724. H. aureum Fr. (from the colour ; anrmm, gold) $\alpha$.

Sub. somewhat cartilaginous, jagged-radiate; marg. irregular, narrow, byssoid. $S p$. often developed in small patches, awlshaped, colour as Sub.

Dead branches. Dec. Diam. $5 \frac{1}{ \pm}$ in. Appearance merutioill. Mycetium permeating wood and causing defined yellowish flesh-coloured spots.

1725. H. clenticulatum Pers. (from the slightly toothed spines; dens, a tooth) $a$.

Sub. thin, somewhat fleshy, mealy, shining light-yellow-ochreous or olive-ochre, sometimes clouded pale brownish-rose. Sp. crowded, equal, minutely $2-3$ toothed at the whitish apex.

Rotten wood. June. Dian. 25 in.

1726. H. alutaceum Fr. (from the colour, like tanned leather, aluta) $a . b c$.

Sub. crustose, adnate, very thin, pallid ochreous, pale brownishyellow or biscuit. $S p$. acute, very minute and short, here and there a little longer and typically $H_{y}$ d $m$ m $m$-like, colour as Sub.; marg. barren.

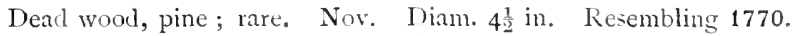


1727. H. sulphureum Schwein. (from the sulphur-colour) $a$.

Sub. effused, adnate, Membranous, colour paler at marg. and gradually fading into the colours of the bark: marg. barren, byssoid. Sp. minute, few, subulate.

Dead wood, birch. Diam. 4 in. The barren spineless form can hardly be distinguished from 1890.

1728. H. sordidum Weinm. (from the dingy colour ; sordidus, dirty) a. Sub. effused, thin, mucid, easily separating, dingy yellow; marg. gyroso-porous, sulphur-yellow. Sp. very crowded, somewhat gelatinous, compressed, incised, subacute, colour as Sub.

Dead wood. Nor. Diam. $3 \frac{1}{2}$ in. Becoming dull-reddish in drying.

1729. H. viride Fr. (from the colour; itridis, green) $a$.

Sub. effused, thin, softly velvety, somewhat pale leaf-green, becoming yellowish; marg. in places slightly upturned. Sp. stout, short, somewhat thick, irregular, somewhat incised, colour as Sub.

Rotten wood, alder. Dirm. $3^{\frac{3}{8}}$ in. Sometimes effusel for 8 to 10 in.

1730. H. limonicolor B. \& Br. (from the lemon colour; Citrus Limonum, the lemon) a.

Sul. adnate, scanty. Sp. crowded, short, acute. Myc. scanty or obsolete, when present shining-white.

On stone buried amongst pine-leases. Oct. Diam. 3 in. Distinguished from 1736 by the scanty subiculum and the absence of a persistent barren white margin.

1731. H. pinastpi Fr. (from the habitat, pines, pinus) a $c$.

Sub. effused, membranous, fleecy or minutely hispid, separable, light yellow, ivory or biscuit; marg. lighter, villous beneath. Sp. equal, subulate, acute, in groups, oblique in different directions, pale brown.

Mossy bark, wood, pine. Diam. $4 \frac{1}{\frac{1}{x}}$ in.

1732. H. spathulatum Fr. (from the spathulate spines) $a$.

Sub. effused, membranous, separable, yellowish; marg. fimbriate, villous beneath. Sp. very small, oblique, obtuse, orange.

Dead wood, Robinia ; rare. Jan.-Dec. Diam. $3 \frac{1}{2}$ in. Often in cracks in bark.

1733. H. multiforme B. \& Br. (from its many forms; multus, many, forma, form).

Sub. inseparable, at first wholly barren, then here and there fertile, ochreous-white, texture floccoso-mealy; marg. coarsely fibrillose. Sp. very acute, crowded, becoming pallid and fimbriate.

Dead wood. Very variable, sometimes almost tow-like with margin inflexed. Perhaps a Corticium, as the spines when present are barren.

1734. H. anomalum B. \& Br. (from the anomalous spines) $a b$.

Sub. thin, gelatinous, pallid, light-yellow. St. minute, at first in the form of granules, then stipitate and obtusely divided, few, but here and there more, colour as Sub.

Rotten wood, ash. Mar. Diam. $2 \frac{1}{2}$ in. With immersed ovate or circular 
veacicles. Brown when lry. Near Mucronella and perhaps not a true Hydnum.

1735. H. melleum B. \& Br. (from the honey-colour; mat, honey) a $c$. Suh. thin, effused, pulverulent; marg. delicately flaxy. Sp. acute, minute, sometimes divided at apex with a tendency to grow in groups, partially pulverulent.

A doubtful Hydzumn. On rails on the ground. Dian. $4 \frac{1}{1}$ in.

1736. H. sepultum B. \& Br. (from its habitat; scpultus, buried) a.

Sulb. golden; marg. white. St. acute to obtuse, minute to medium size.

On wood, bark and stones buried amongst pine-leaves. Sept. $\frac{1}{8}$ to $\frac{5}{5}$ in. diam. Forming little scattered patches. Pale ochreous when dry.

1737. H. udum Fr. (from its moist substance; $u d u s$, moist) $a b c$.

Sub. effused, thin, subgelatinous, agglutinate to matrix, fleshcolour to watery-yellowish, sometimes bright sulphur. Sp. acute, 2-3 forked, small, but varying in length, crowded, arranged in groups, very small near the barren marg., colour as Sub.

Dead branches, willow, oak, alder ; frequent. Sept.-April. Diam. $5 \frac{1}{2}$ in. Forming large patches.

1738. H. bicolor A. \& S. (from the white subiculum and brown spines; bis, two, color, colour) a $c$.

Sub. widely effused, tomentose, adpressed, thin, white; marg. broadly barren. Sp. minute, white-villous, naked at acute apex, pale umber to rufous-brown.

Bramble, fir. March. Diam. 2 in.

1738a. H. Hollii Fr. (after F. Holl) a.

Sub. adnate, effused, waxy membranous, whitish-brown; marg. finely powdery. Sp. very minute, blunt, deformed.

On naked wood. Oct. $4 \times 2 \frac{1}{2}$ in.

1739. H. nodulosum Fr. (from the nodulose subiculum) $a$.

Sub. broadly effused, crustaceous, nodules (less in size than a pea) sterile above, furnished with spines below, whitish to pallid yellowish. $S p$. free and pendulous below nodules, adnate and elongate on the interstices, colour as Sub.

Trunks, stumps, fir. Oct. Diam. 4 in.

1740. H. Stevensonii B. \& Br. (after the Rev. Dr. John Stevenson) a c. Suth. effused, mealy beneath, here and there flaxy, white. Sp. minute, crowded, cylindrical, obtuse, pulverulent at apex, colour as Sub.

Dead wood, somelimes spreading and encrusting mosses, leaves, etc. March. Diam. I $\frac{1}{5}$ in. Having the appearance of a resupinate Polyporzs.

1741. H. plumosum Duby (from the plumose apex of the spines) $a c$. Sub. very delicate, downy, white. Sp. very small, divided, colour as Sub.

Dead wood, pine; rare. Diam, $1 \frac{3}{4}$ in. Cracking when dry and becoming ivory-brown. 
1742. H. niveum Pers. (from the snow-white colour; nix, niz'is, snow) $a b c$.

Sub. effused, thin, membranous, lustrous, adnate ; marg. fibrillosoflaxy. Sf. at first granulose, then acutely pointed, smooth.

Dead wood, leares, pine, birch, beech; unconmon. Nov. - April. Diam. $2 \frac{1}{2}$ in. Persistent. Ochreous or sulphur-white when dry.

1743. H. farinaceum Pers. (from the mealy subiculum; farina, meal) $a b c$.

Sub. effused, indeterminate, adnate, white; marg. subflocculose. Sp. thin, subdistant, acute, interstices finely farinose, colour as Sub.

Dead wood, fir, oak. Sept.-May. Diam. 5 in. Varying pale brownish, pale yellowish, or sulphur- or ochre-white.

1744. H. argutum Fr. (from the acute spines; argatus, acute) a b $c$.

Sul. effused in scattered patches, tomentoso-interwoven, spongy, adnate, white. Sf. acute, awl-shaped, unequal, slightly serrate, colour as Sub.

Wood and bark, alder, willow. Sept. Diam. $2 \frac{1}{t}$ in. The hymenium has a finely mossy appearance from the unequal, divergent spines. Becoming colour of coffee with milk when dry.

1745. H. stipatum Fr. (from the crowded spines; stipatus, crowded) $a c$. Sub. widely effused, Hocculoso-farinaceous, forming an adnate crust, white to brown-white or light yellowish. Sf. at first granulose then forming obtuse teeth, colour as Sub.

Rotten wood. Dec.-Jan. Diam. $3 \frac{1}{\ddagger}$ in. Has the appearance of a resupinate Polyporas.

\section{TREMELLODON Fr.}

(From its resemblance to a Tremclay; Gr. odous, a tooth.)

Gelatinous, pileate, hymenium inferior, covered with acute awlshaped spines. Basidia ovate, somewhat obliquely $2-4$ divided

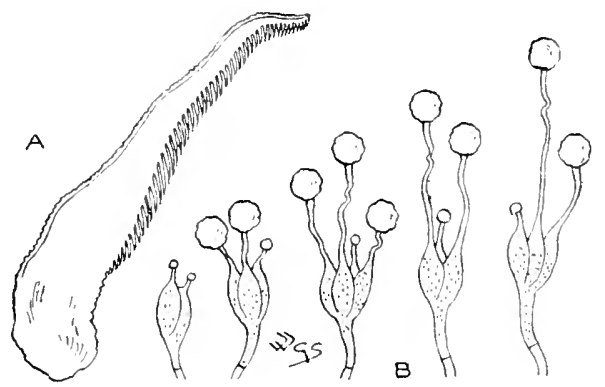

Fig. 86.-Trentlodon gelatinosum: $\mathrm{Fr}$.

A, section, one-half natural size. B, basidia, stericmata and spores. $\times 500$.

almost to the base, sometimes undivided for twice the length of the spore, sterigmata I-5 lengths of spore. (Fig. 86.) 
Mr. Fred. Currey (Journ. Limn. Soc. vol. v. p. is I, r86I) was the first to call attention to the cloven basidia in this species, and to point out the resemblance in the fruit to some of the Tremellinacee. Mr. George Massee (British Fumgus Flore, vol. I. p. 65) has placed Tremillodon at the end of the Tremillinacea, but the nature of the basidium and sterigmata is an insufficient reason for the removal, and if adopted many other removals would have to be made.

1746. T. gelatinosum Fr. (from the gelatinous substance) a $b c$. Wholly translucent, grey-white.

$P$. fan-shaped, Heshy, dimidiate, substipitate, white-frosted with shining particles. St. when present, white-frosted above.

Taste pleasant. Rotten pine, sawdust; rare. Sept.-Nov. $3 \frac{1}{ \pm} \times 3 \frac{1}{2}$ in.

\section{SISTOTREMA Pers.}

(From the irregular arrangement of the spines and their interstices, as if displaced; Gr. seistos, shaken, trema, a pore.)

Pilcus fleshy, irregular or dimidiate. Hymenium inferior. Teth resembling broken, jagged gills, not radiate, readily separating from
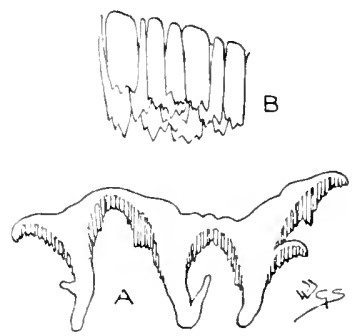

Fig. 87.-A, section of Sistotrema contucns Pers., one-half natural size; $B$, teeth of ditto, $\times 3$.

the hymenophore. Stim central, excentric, flattened or almost obsolete. Basidia 4 -spored. (Fig. S7.)

Closely allied to Irpex, but the latter is not fleshy and is usually sessile or resupinate and seldom terrestrial.

1747. S. eonfluens Pers. (from the confluent habit) $a b c$. Wholly white or whitish, becoming pale yellowish or brownish.

$P$. horizontal, subdepressed, villous. St. attenuate downwards. Sp. decurrent.

Gregarious. On the ground, investing sticks, leaves, moss, grass, etc., under pines; uncommon. Sept.-Nov. Group $2 \frac{5}{5} \times 1 \frac{5}{5} \mathrm{in.}$ 


\section{IRPEX Fr.}

(From a fancied resemblance in the fruiting surface to the teeth of a harrow, irpit.)

Hymenium inferior, toothed, teeth firm, somewhat coriaceous, acute-spathulate in 1753-concrete with the pileus or subiculum,
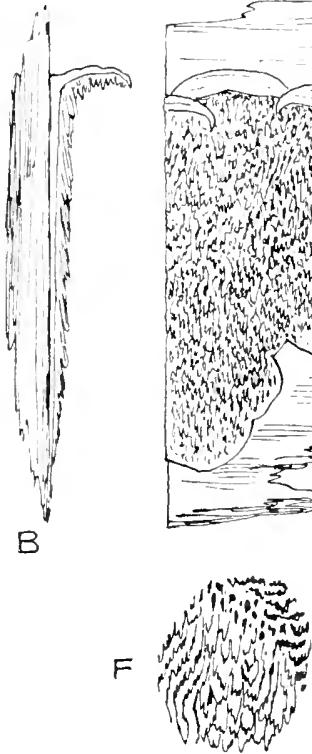
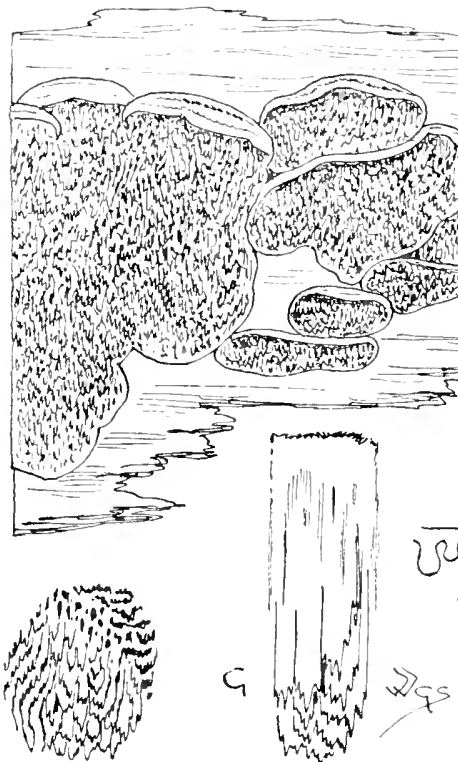
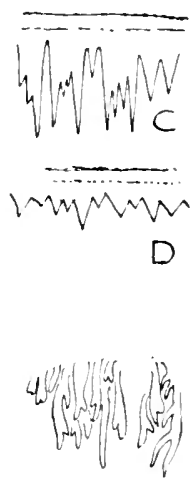

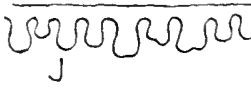

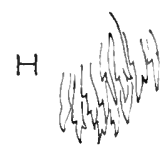

Fig. 88.-A, Irfex fuscoviolacens Fr. : B, section of same; two-thirds natural size. C, n, teeth of ditto; $\times$ 3. E, I. fendulus Fr., teeth enlarged. F, I. cbliquzs Fr., ditto: G, ditto; $\times 6$. $\mathrm{H}, I$. deformis Fr., teeth enlarged. J, I, spathulatus Fr., teeth ; $\times 3$.

arranged in irregular rows, like broken gills or broken honeycomb, connected at the base by veins or folds. Basidia 4 -spored. (Fig. 88.)

Generally growing on wood, somewhat sessile or resupinate, approaching Lenzitis and Daduled.

Pendulous, with the pileus extended behind.

Species $1748-1756$

Effuso-reflexed, marginate.

1748

Resupinate.

1748. I. pendulus Fr. (from the pendulous habit) a $c$.

$P$. membranous, slightly rugose or plicate, adpressedly squamuloso-pilose, light yellow to white-brown. Te. in rows, large, incised, white.

sticks, pine, larch, sprealing on 10 moss; rare. $1 \frac{5}{5}$ in. ; group $2 \frac{1}{4}$ in. Must not be confounded with 1757 . 
1749. I. fuscoviolaceus $\mathrm{Fr}$. (from the brown-violet colour of the teeth; fuscus, brown) a b $c$.

$P$. coriaceous, silky, zoned, brown-white to ochre-white, zoned brownish. $T t$. in rows in the form of much incised plates, of the purple-slate-whitish tint seen in the gills of some Pratella.

Single to imbricate. Pine, beech; rare. $2 \frac{5}{8}$ in. ; group $4 \frac{1}{2}$ in. Sometimes there is a considerable development of pileus.

1750. I. lacteus Fr. (from the milk-white colour; lac, milk) $a b c$. White.

$P$. coriaceous, villous, concentrically sulcate. $T e$. crowded, gyrose in rows, acute, somewhat incised. Irregularly porous at marg., becoming ragged at middle and base.

Sometimes subimbricate. Mountain-ash, birch, fir, beech. Nov. Group $3 \frac{1}{8}$ in.

1751. I. hypogæus Fück. (from its sometimes growing in and under earth; Gr. hupo, under, $s_{c}$, the earth) $a b c$.

Myc. dispersed, white to yellowish. Sub. marg. determinate, compactly byssoid, white, then pallid brown-ochreous to dark brown; hymenium often barren or exhibiting barren patches. $T e$. irregular, somewhat labyrinthiform, lax, variable in size, straight, incised 2-6 $\mathrm{mm}$. long, base usually compressed, colour as P.

Woods, pine, investing pine-leares, twigs, grass, earth, pebbles; rare. Oct. Group $4 \frac{1}{5}$ in.

1752. I. Johnstonii Berk. (after Dr. George Johnstone) $a$.

Effused, coriaceo-membranous, separable from matrix; marg. naked, reflexed all round, white. $T e$. compressed, unequal, arranged in rows, resembling the spines of $H y d m u m$, but seated upon fine folds.

Dead beech. Mas be a form of $1750 . \mathbf{I}_{\frac{1}{8}}$ in. ; group $3^{\frac{1}{x}} \mathrm{in}$.

1752a. I. candidus Weinm. (from the shining white colour; candidus, white) $a$. Snow white.

Broadly effused, thin, separable, arachnoid, flaxy at the marg. $T i$. subulate, or compressed, thin, toothed.

Dead wood, pine. Feb.

1753. I. spathulatus Fr. (from the shape of the teeth; spatha, a spatula) $a b c$.

Effused, membranous, flaxy, then smooth, white to biscuit. $T i$. entire, somewhat large, reticulato-connected with obsolete veins. Marg. sometimes obsolete, at other times broadly barren, with other barren places on hymenium. Never porous.

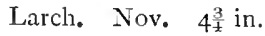

1754. I. obliquus Fr. (from the oblique teeth) $a b c$.

Effused, forming an adnate crust, white, clouded and shaded pale sienna ; marg. broadly barren ; circ. flaxy. $T e$. extended from 
a base resembling small honeycomb, compressed, unequal, incised. Often with large pores near the marg.

Stumps, dead branches, fir, larch, beech, birch, palms in nurseries. Oct.NIay. $4^{\frac{3}{4}}$ in.

1755. I. deformis Fr. (from the misshapen teeth; deformis, misshapen) $a c$.

Effused, crustaceous, thin, white; circ. pubescent, somewhat flaxy. $T i$. awl-shaped, growing from a minutely porous base, thin, somewhat incised, unevenly and partially spread over the subiculum, matrix seen between the groups of teeth.

Wood. $2 \frac{1}{5}$ in. Allied to the Polyporacia.

1756. I. carneus Fr. (from its flesh-colour; caro, flesh) a b c.

Effuso-gelatinous, membranous, adnate, collapsing, reddish; marg. barren. Te. awl-shaped, entire, united at the base.

Wood and bark, beech. Autumn. $2 \frac{1}{5}$ in. Flaced in Radulum by Fuckel. Must not be confounded with 1720 .

\section{RADULUM Fr.}

(From the root-like appearance of the processes of the hymenium; radix, a root.)

Hymenium resupinate, tubercular. Tubercles distinct at the base, coarse, irregular in shape, commonly elongate, obtuse, mostly
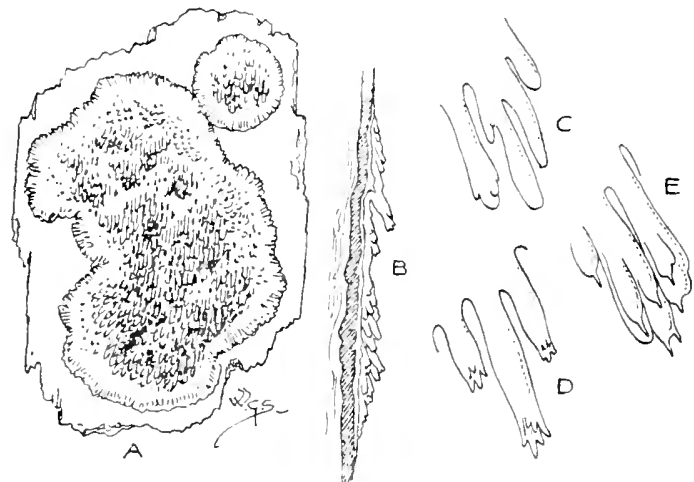

"w. Fig. So.-A, Radulum muerinum Fr., one-half natural size; E, ditto, section, natural size: C, tubercles of $R$. orficulare Fr. : D, ditto of $K$. quercinum Fr. : $E$, ditto of $R$. fugineum Fr. ; enlarged.

waxy, scattered or fasciculate. Basidia 4-spored. Growing on wood and on and under bark. (Fig. 89.) Species 1757-1764

1757. R. pendulum Fr. (from the pendulous habit) a $c$.

Longitudinally effused, thick, softly coriaceous, whitish or ivory, upper marg. shortly reflexed and rugose, lower marg. barren Tul. elongate, somewhat lax, irregular.

Alder, birch, pine. 5 in. Must not be confounded with 1748. 
1758. R. opbiculare Fr. (from its frequent orbicular shape) $a b c$.

Orbicular, confluent ; circ. flaxy, becoming waxy-fleshy, white to ochre-ivory; mid. faintly vinous; marg. white. Tul. short to long and broad to narrow, mere granules near the barren marg. Birch, oak, larch, cherry ; frequent. Oct.-Mar. $2 \frac{1}{2}$ in. ; group $4 \frac{3}{8}$ in. Ends of tubercles usually crested.

1759. R. quereinum Fr. (from its habitat, oak, quercus) a $b c$.

Roundish, broadly confluent, innate, crustaceous, white to ochrewhite. Tub. round, stout, obtuse, scattered or fasciculate, irregular in shape, floccoso-villous at apex; marg. barren ; edge pure white.

Oak, sometimes on old squared oak timber. Nor.-Mar. $3^{\frac{1}{2}}$ in. Sometimes confounded with 1716, 1723 and species of Irtex.

1760. R. tomentosum Fr. (from its downy appearance, especially at the margin ; tomentum, down) a b c.

Effused, innate, somewhat thick, opaque or translucent white; circ. swollen or erect, tomentose. Tub. short, crowded, angular, obtuse, smooth, confluent, resembling white tomentum, smaller at marg.

Willow, mountain-ash, pine, sawdust. Dec. $5 \frac{1}{\frac{1}{5}} \mathrm{in}$.

1761. R. deglubens B. \& Br. (from its habit of peeling from the matrix; deslubo, to peel off) $a$.

Orbicular, ferruginous, somewhat diaphanous. Tut. erect, somewhat cylindrical, irregular, scattered, interstices eren, pulverulent with white spores.

Naked wood, bark, ash. Jan. $\frac{1}{2}$ in.; group $5 \frac{1}{4}$ in. Growing in troops of small patches.

1762. R. corallinum B. \& Br. (from its coral-like appearance) $a$.

Somewhat effused, very thin, pelliculose, shining white. $T u h$. fasciculate, fascicles $\frac{1}{4} \mathrm{in}$. or more across, surrounded by barren sub., divided, obtuse, coralloid, white.

On partially lichen-covered lranches, oak. Sept. Fascicles I in,; group 6 ir.

1763. R. epileueum B. \& Br. (from the hymenium growing upon a white subiculum; Gr. chi, upon, lakkos, white) a b.

Effused. Hym. waxy, edge slightly upturned, pale, honey-colour. Sub. forming a powdery white marg. Tub. scattered, distant, cylindrical, short, under a lens fimbriate at apex, deciduous exposing the sub.

Decorticated wood. Aug. $S \frac{1}{5}$ in.

1764. R. fagineum Fr. (from the habitat, beech, fagus) a b.

Effused, somewhat fleshy, white or pale yeilowish-brown; marg. radiate with ivory-white myc. Tub. enlongate, round, varied, entire, sometimes crested, white to sienna-white.

Chiefly on bark, rarely underneath, sometimes on birch. Nov.-Mar. $5 \frac{3}{3}$ in.

[Radulum aterrimum Fr. is not a Basidiomycete but apparently one of the Dimatiea. Under the micruscope the appearance is that of conceptacles of Rhizoitonia cromm, but it bears $\mathbf{I}-4$-septate brown spores after the manner of Cladesforimm; the fruiting branches are compacted togetber and take the form of short, obtuse teeth, as in the compacted form of 
Penicillium named Coremium. It grows on naked birch wood or bark. Sowerby named his example Hydnum crectum; this grew upon a Polyporus. There is no drawing by Sowerby in the British Museum collection. It has also been named Spharonema hydnoidium Fr. and Hydnum aterrimum Fr.]

\section{PHLEBIA Fr.}

(From the reined appearance of the hymenium; Gr. phleps, a vein.)

Resupinato-effused, amphigenous, waxy-soft or subgelatinous, from the first covered with crowded interrupted persistent veins which
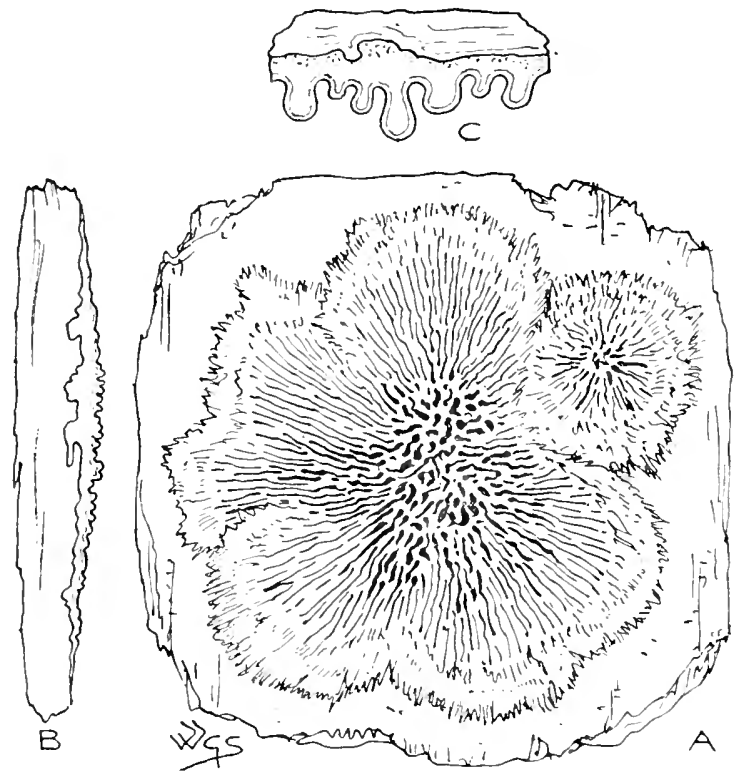

Fig. go.-A, Phlebia nerismoides Fr. : B, section of ditto ; two-thirds natural size; $c$, veins enlarged.

are entire, not jagged, at the edge, rarely anastomosing and forming pores. Becoming cartilaginous or horny when dry. Basidia tetrasporous. (Fig. 90.)

Dead wood, stumps and branches.

Species $1765-1769$

1765. P. merismoides Fr. (from the resemblance of the wrinkled hymenium to that of the non-British section Merismate of Sterim) a b c.

Whitish-orange or whitish-vermilion; mid. livid, pale purplish ; marg. strigose, vermilion-white. $V_{e}$. simple or slightly branched, straight, crowded, never reticulate or porous.

Stumps, branches, naked wood, bark, leaves, moss, grass, oak, beech, holly vlum, cherry. Aug. - Ian. $3 \frac{3}{4} \mathrm{in}$. 
1766. P. padiata Fr. (from the radiate veins) $a b c$.

Orbicular, vermilion-orange or vermilion; mid. darker; marg. vivid orange-whitish. $V_{\ell}$. straight.

Dead wood, birch, mountain ash; rare. $2 \frac{1}{4} \mathrm{in.}$; group $5 \frac{1}{2} \mathrm{in.}$ When in perfect condition forming somewhat large, conjoined, more or less hexagonal growths as in the carapace of a tortoise. Thinner and brighter in colour than the other British species.

1767. P. contorta Fr. (from the intricate veins; contorqueo, to twist) $a b c$.

Yellowish, sienna or brownish or dull red flesh-colour or redvermilion. $\quad V e$. conglomerate, branched, flexuous, much smaller than 1765-6, or as in Dadalca on a reduced scale.

Decayed wood, furze; rare. $\frac{1}{2}$ in. ; mass $2 \frac{5}{8}$ in. Forming more or less conjoined circular patches.

1768. P. vaga Fr. (from the wandering appearance of the veins; $c^{\prime} a g u s$, wandering) $a b c$.

Adnate, waxy, dull yellowish, central parts sometimes purplish or grey; marg. flaxy-fibrillose and powdery. $V e$. forming intricate wrinkles which often terminate in a granulose papilla.

Dead wood ; common. Jan. 63 in.

1769. P. lirellosa B. \& Br. (from the veins, like small furrows ; lira, a ridge or furrow) $a b$.

$H y m$. veined, interstices resembling small, round or elongate pores, umber-grey to white.

Dead branches, poplar. $3^{\frac{3}{4}}$ in. Purple-blackish in drying. The interstices resemble the pores of Trametes. Sometimes confused with Dadalea.

\section{LIXVI. GRANDINIA Fr.}

(From the granular appearance of the hymenium; srando, hail.)

Crustaceous, effused, thin, soft. Hy'menizm resupinate, amphigenous, waxy, not dry as in Odontia. Gramules small, persistent,

B
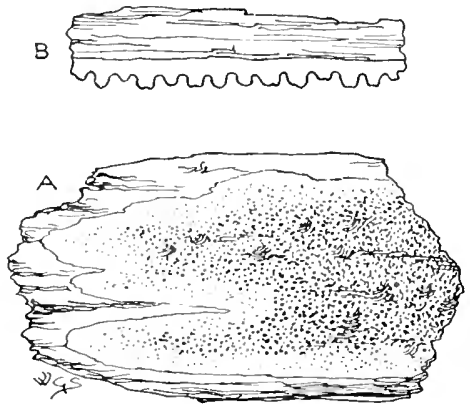

Fig. 9x.-A, Grandiniu crustosa Fr., natural size; B. section of ditto. $\times$ so. 
entire, smooth, crowded; apices not pointed as in Hydmum, usually indented or excarated as in Porothilinm. Basidia 4-spored. (Fig. 9r.)

The hymenial surface is often spuriously papillose from Spharia on the bark beneath.

Species $1770-1774$

1770. G. granulosa Fr. (from the minutely granular hymenium) $a b c$. Agglutinate, $\tan$ to sienna-irory; marg. determinate, ivory. $G r$. small, crowded in groups, smaller at marg., hemispherical to somewhat elongate.

Dead branches, pine, My'rica Gale. Oct.-May. 5 in. Cracking when dry.

1771. G. mueida Fr. (from its waxy mucid substance) $a$.

Subinnate, somewhat gelatinous, biscuit; marg. determinate, subradiate, minutely villous, ivory-white. Gr. large, unequal, soft, scattered or in groups.

Rotting wood, pine, birch ; rare. May-Nov. $4 \frac{1}{x}$ in. Cracking when dry.

1772. G. ocellata Fr. (from the minute eyelet, sometimes seen at the apex of the granules; ocellus, a little eye) $a b$.

Waxy, livid, rufous flesh-colour or warm brownish-salmon; marg. indeterminate, sterile, yellowish. Gr. crowded, subconical, obtuse, somewhat scattered or in groups.

Dead trunks. Aug.-Mar. $6 \frac{1}{2}$ in. Cracking when dry.

1773. G. papillosa Fr. (from the minute teat-like granules; papilla, a teat) a $c$.

Membranous, somewhat round, separating, white, becoming slightly sienna-ivory ; marg. furfuraceous. Gr.minute, crowded, equal, roundish.

Sticks, pine, juniper, bird-cherry. 3 in. Cracking when dry.

1774. G. erustosa Fr. (from its crust-like appearance) $a b c$.

Adnate, irregularly effused, floccoso-mealy, white to ivory, becoming pale brownish-yellow; marg. indeterminate. $G r$. crowded, obtuse, roundish, minute, regularly distributed, but with barren lacunæ.

Branches, willow, pine, 1603. 63 in. Nol cracking when dry.

\section{ODONTIA Pers.}

(From the minute teeth which invest the hymenium;

Gr. odens, odentes, a tooth.)

Resupinate, effused, arid, not waxy as in Grandinit. Hymenium spreading from and between interworen branched fibres, covered with minute teeth or warts which are crested or penicillate at the apex. (Fig. 92.)

1775 tends towards $H_{y} d m u m$. 
1775. 0 . barba-Jovis Fr. (from the minute orange fringe at the apex of each tooth, a fanciful comparison with the beard of Jupiter, barba Jozis) ac.

Flaxy membranous, whitish to yellowish-orange, faintly shaded brownish; marg. determinate, broadly barren, white. Te. papillose, then conical, white with an orange fimbriate crest.

I ecayed wood, oak, birch. $6 \frac{1}{1} \mathrm{in}$. The hymenium usually exhilits barren patches.

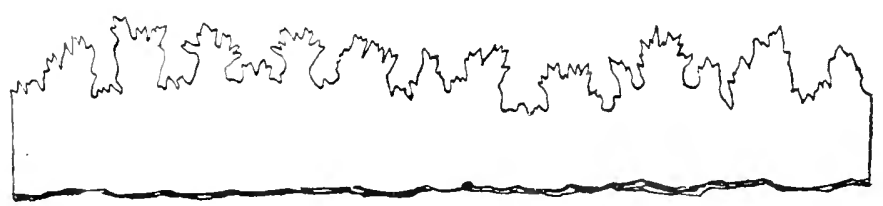

B

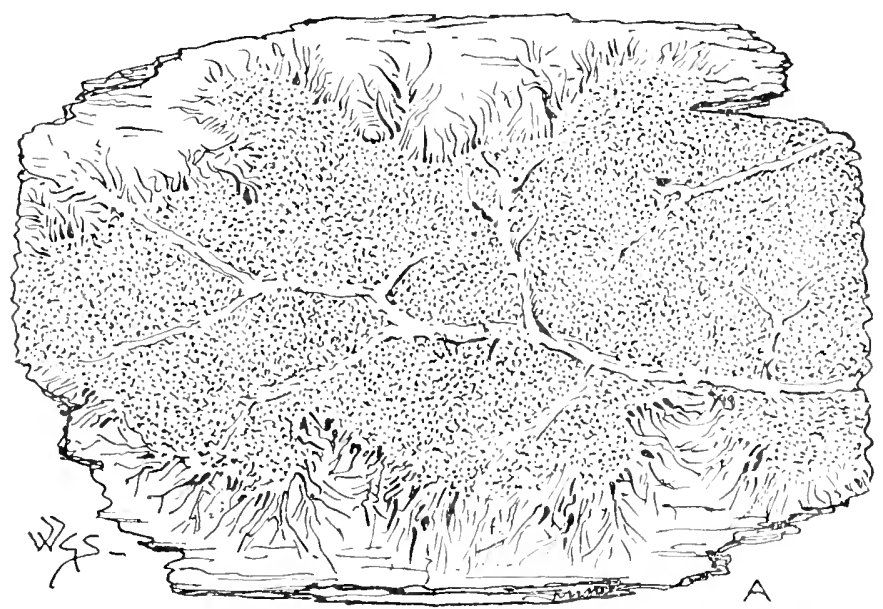

Fig. 92.-A, Odontic fimbriata Pers., natural size: B, section of ditto, enlarged.

1776. 0. fimbriata Pers. (from the fibrillose or fringed margin; fimbrice, a fringe) a b $c$.

Membranous, separating, traversed by prominent root-like branching threads continuous with the fringed marg., pallid or yellowish, suffused with dull salmon or pale fawn.

Dead wood, willow, plane, dog-wood, bark, moss. Aug.-April. $7 \frac{1}{4}$ in. Extensive barren patches sometimes nccur on the hymenium. Fawncolour when dry, sometimes becoming deep rich brown.

1777. 0. cristulata Fr. (from the minutely crested teeth) a b c.

Crustaceo-adnate, thin, not ribbed, pale warm brown or brownishwhite; marg. broadly barren, here and there finely downy.

Rotten branches, hirch. Feb, $5_{\frac{3}{7}}^{3}$ in. Cracking all orer when dry as in Corticintm. 
1777a. 0. Pruni Lasch. (from its host plant, sloe, Prunus spinosa) a.

Crustaceo-adnate, effused, thin, white, becoming pallid; marg. byssoid, warts minute, rounded.

\section{KNEIFFIA Fr.}

\section{(After Friederich Gotthard Kneiff, mycologist of Baden.)}

Resupinate, effused, thin. Hymenimm strigose and roughened with rigid, scattered or fasciculate bristles which are barren outgrowths from the fertile hymenial surface. Laxly fleshy, soft, collapsing and becoming flocculose when dry. Basidia monosporous. (Fig. 93.)
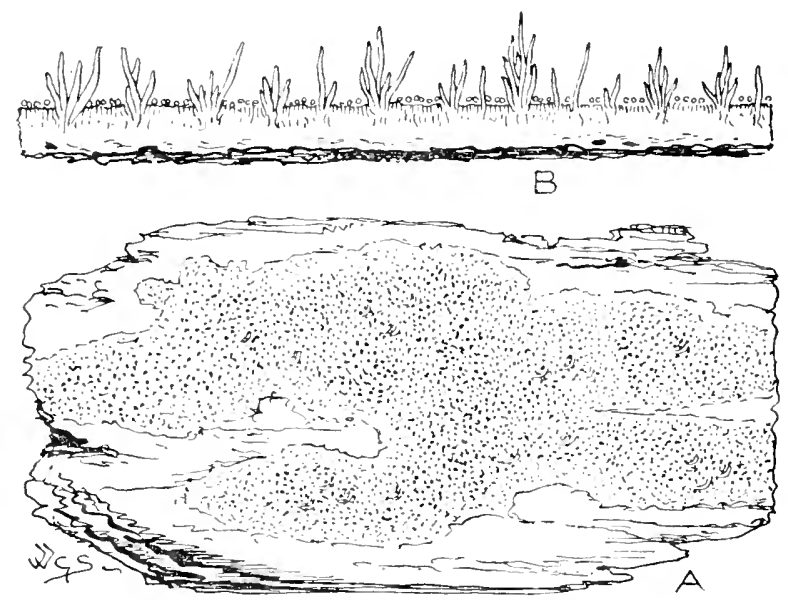

Fig. 93.-A, Kneiffir setigera Fr., natural size; B, section, enlarged.

The most perfect condition, where the growth is hemispherical and three inches in diameter, has not been observed in Britain.

Must not be confounded with Piniophora, where the basidia are 4 -spored and the cystidia, which are bristle-like, are covered with minute particles of oxalate of lime.

Species 1778,1779

1778. K. setigera Fr. (from the minute hairs or bristles borne on the hymenium ; seter, a bristle, gero, to carry) a $b c$.

Sub. white, becoming ochreous-ivory, sometimes pale grey; marg. sometimes pulverulent. Br. minute, single or several connate, more or less scattered, hyaline; the basidia occur between the barren bristles.

Dead wood, blackberry, pine, juniper, magnolia, grass. Jan.-Dec. $4 \frac{1}{5}$ in. Sometimes the spuriously tuberculose hymenium resembles that of $\mathbf{1 7 7 0}$ or 1839. 
1779. K. subgelatinosa B. \& Br. (from the subgelatinous substance) $a b$.

Hyaline yellowish, ivory or pale buff, surface very finely granuloso-crested.

Stumps and cut surfaces of fir. 4 in. Sometimes accompanied by a green alga which penetrates the tissue of the fungus. Cracking when clry as in Corticium.

\section{MUCRONELLA Fr.}

(From the sharp-pointed spines; diminutive of mucro, a sharp point.)

Subiculum an extremely thin film, which soon becomes obsolete, spines subulate, simple, acute, glabrous, scattered or fasciculate, and more or less connate at the base. Basidia I-4-sporous. (Fig. 94.)

Mucronella was at first placed under Isariu (I. calia) by Fries, but its fruit shows it to be a true Basidiomycete, and its fugitive

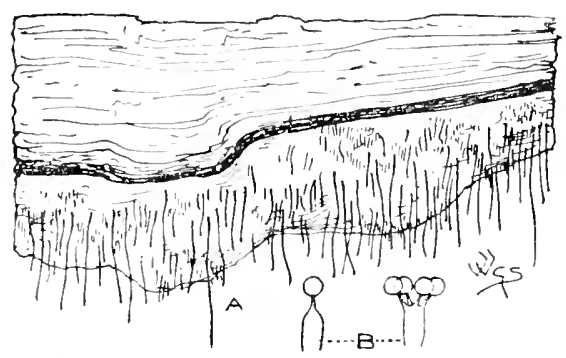

Fig. 4.-A, ifucronella calva Fr, one-half natural size; $B$, basidia and spores, $\times 500$.

subiculum to be one of the Hydnacie. Fries seems not to have observed the feeble subiculum, sometimes distinct in M. fascicularis.

1780. M. calva Fr. (from the habit, a fanciful comparison with an almost bald head, bearing a few scattered short hairs; calz' $\alpha$, the bald scalp) $a$.

Sub. obsolete or nearly so. Sp. slender, smooth, even, sharppointed, covered except at the barren tips with $\mathrm{I}-4$-sporous basidia, whitish, then grey. The growth of the spines is in a downward direction; dry examples revive with moisture.

Scattered or gregarious. Rotten wood, pine. Spines vary in size from a minute granule to $\mathbf{I}$ in. long. 


\section{FAM. IV. THELEPHORACEF}

Erect and stipitate, cup-shaped, effused, with the upper portion free and reflexed or resupinate. Hymenium inferior or amphigenous, coriaceous, waxy or gelatinous, even to rugulose, ribbed or papillose, the surface glabrous, finely pulverulent or minutely velvety, with projecting sete. Basidia clavate, I-4-spored, normally tetrasporous. Spores usually aseptate, 3 -septate in the non-British Heterobasidium chlorascuns. The papillæ are for the most part accidental, generally arising in the resupinate species from Stharice, growing on the bark beneath and not existing when the fungus grows on a surface free from Spharia. True papilla, however, sometimes occur on the pileate, terrestrial species. Some species are occasionally phosphorescent, as 1861. In the gelatinous species the aseptate and tetrasporous basidia distinguish them from certain members of Tremellinacce, where the basidia are longitudinally and cruciately septate, or if the spores are aseptate the basidia are deeply bifurcate and bisporous. Hypomyces rosellus often stains the hymenium of Thelephory, Corticium, and other genera of Thelephoracee a bright purple-rose colour.

Nassee has published a Monomraph of the Thelephoracee in Joum. Linn. Soc. (Bot.) xxv. ( $S_{89}$ ) and xxvii. (I 890 ). The sequence of the genera adopted here is that of P. A. Saccardo, Sylloge Fungorum, vol. vi., which is a slight modification of the order as given in Fries's Hymenomycet's Europiei.

Species 1781-1925

\section{KeY TO THE GeNerA.}

Large.

Erect, funnel-shaped; hymenium glabrous.

So Craterellus.

Erect to resupinate; hymenium rugulose ........

Effuso-reflexed ; hymenium even, glabrous......... $\delta_{3}$

Horizontal, attached by a small base behind ......

I THELEPHORA.

3 STEREUTI.

Resupinate.

Hymenium smooth-setulose

Cladoterris.

Hymenium rough-setulose....

$\mathrm{S}_{4}$ HyneNochete.

Hymenium glabrous

S6 PENiophora.

Hymenium pulverulent

S5 Corticilis.

$S_{7}$ CONIOPHORA.

Smail, cup-shaped, usualiy single.

S9 Cyphella.

Minute, cup-shaped, crowded

9 SOlenia.

Parasitic on living leaves and stems

SS ExOBASIUIU⿴囗十 


\section{CRATERELLUS Pers.}

(From the shape, like a small crater, cup or bowl.)

Hymenium waxy-membranous, distinct, but adnate to the bymenophore, inferior, continuous, smooth, even, or rugose. Pilus

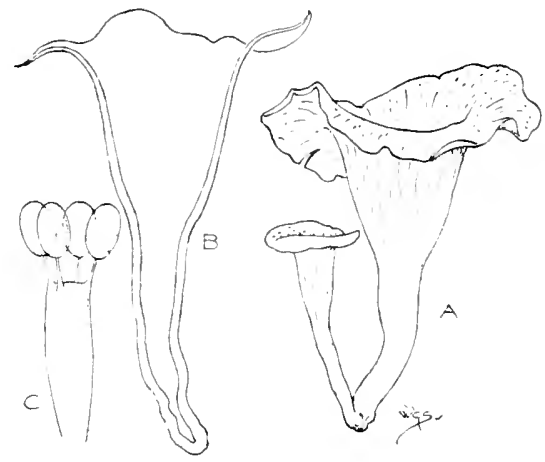

Fig. 95.-A, B, Craterillus cornucopioides Pers., entire and in section, one-third natural size. C, basidium and spores, $X_{500}$.

entire, stipitate, fleshy or membranous. Spores white. (Fig. 95.)

Usually large, erect and fumnel-shaped. Growing on the ground. Autumnal. Allied to Cantharellus.

Species $1781-1785$

Tubiform, pervious to the base of the stem. 1781, 1782

Infundibuliform, stem stuffed.

1783,1784

Irregularly shaped, pileus and stem fleshy.

1781. C. lutescens Fr. (from the yellowish colour; lutescons, yellowish) $a$.

$P$. thin to submembranous, undulate, flocculose, warm vinous, orange-brown or dull ochre. St. smooth, ochre or salmon, or as P. Hym. rugoso-veined, ochre-cream, golden-sulphur or salmon, sometimes greyish. Flesh white-sulphur-ochre.

Sometimes craspitose. Odour strong as of spirit. Moist woods; rare. Sept.Oct. $\mathbf{2}_{t}^{3} \times 4 \times \frac{3}{4}$ in. Sometimes turning black in decay. Easily confounded with 1379.

1782. C. cornucopioides Pers. (from the shape, like a cornucopia) a bc.

$P$. thin to submembranous, undulate, squamulose, umber-sepia. St. pale grey-slate, often blackish at base. Hym. even, at length slightly wrinkled, dull purplish-grey.

Solitary to crespitose. Edible. Taste and odour agreeable. Woods, mixed, fir. Aug.-Oct. $3 \frac{1}{2} \times 5^{\frac{1}{2}} \times \frac{5}{8}$ in. Never insect-eaten. Sometimes confounded with 1381, in which there are thick gills. This is the Italian "Trombetta di morte." MIrs. Hussey recorls the sale of this species in Covent Garden market. 
1783. C. sinuosus Fr. (from the undulate pileus; sims, a curve) $a b c$. $P$. subfleshy, floccose, pale warm umber, pale ochre-umber or fuscous-grey. St. pallid cinereous or umber, becoming sootyumber. $H y ' m$. even, at length veined, folds broad, sometimes rather distant, pallid, pale ochre-umber, almost white or dull in hue. Flesh colour as St.

Odour strong, musky. Woods, fir, mixed; frequent. Aug.-Nov. $1 \frac{3}{4} \times 2 \frac{7}{8} \times \frac{1}{4}$ in. Small forms are often confounded with 1789.

1784. C. erispus Fr. (from the crisped or curled pileus) $a b c$.

$P$. fleshy-membranous, brownish-ochre, fuliginous or fuscous. $S t$. pallid, pale warm- or yellowish-umber. Hym. even, pallid, pale ochre-umber, almost white or pale dull ochre. Flesh pale ochre-umber.

Edible; odour strong, musky. Woods, mixed; frequent. Aug.-Nor. $2 \frac{1}{2} \times 2 \times \frac{1}{4}$ in. Perhaps a variety of 1783 . Small forms are almost identical with 1789 . They may, with 1733 , be forms of one species.

1784a. C. pusillus Fr. (pusillus, very small) a c.

$P$. convex, umbilicate, thin, rugose, villous, cinereous. St. stuffed, somewhat compressed, grey. $H_{y^{\prime} m}$. smooth or slightly wrinkled, pruinose, bluish-grey.

Single to crespitose. Bare soil under beeches. Aug. $\frac{3}{8} \times \frac{5}{8} \times \frac{1}{8}$ in.

1785. C. clavatus Fr. (from the club shape when young; claris, a club) $a c$.

$P$. turbinate, truncate or depressed, dull pale yellowish; mid. pale purplish-salmon. St. attenuate downwards, colour as P. Hym. even, then corrugate, dull brown-salmon, becoming lavender-white or brown-purplish. Flesh pale dull yellowish or pale purple-salmon.

Solitary or caspitose, sometimes branched. Edible. Woods, heech, fir. Oct. $\quad 6 \frac{1}{4} \times 3 \times I_{\frac{1}{2}}$ n. Sometimes strongly resembles certain of the larger Lactarii.

\section{THELEPHORA Ehrh.}

(From the sometimes papillose hymenim: Gr. thel, a teat, fhero, to bear.)

Hymcnimm inferior or amphigenous, continuous with and similar to the hymenophore, even, rugulose or ribbed, ribs sometimes more or less papillose. Without the fibrillose stratum present in Stirum. Basidic 4-spored. Sfores warted or echinulate. (Fig. 96.)

Coriaceous, dry, fibrous, destitute of a cuticle, varied in form, pileate, clavate or resupinate. Terrestrial or on stumps, branches, leaves, earth and stones; sometimes investing seedlings and living plants. Some species nore or less resemble brown flowers with 
laciniate petals; 1794 and 1801 are fetid. The sequence of species is that of Fries, Berkeley, Stevenson and Saceardo.

Species $1786-1804$

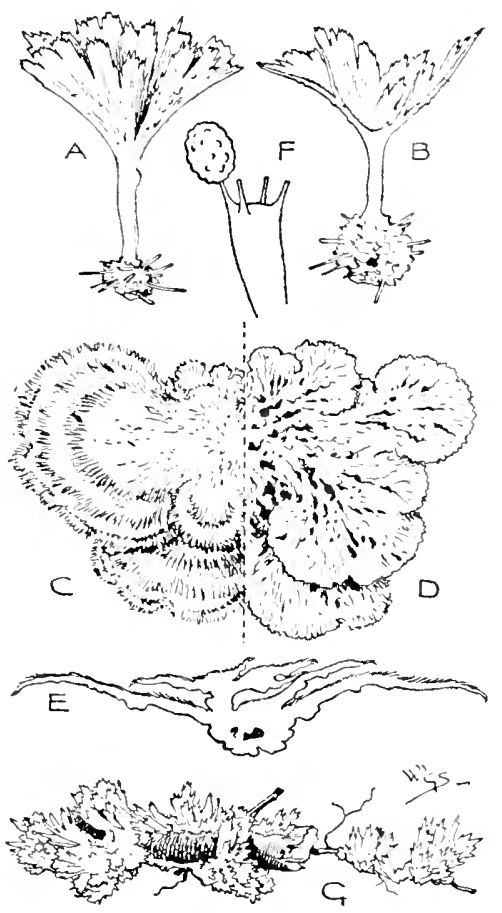

Fig. g6.-A, B, Thelephora caryophyllea Pers., encire and tin section, one-half natural size. C, D, E, 1. laciniata Pers., upper and under surface and section, one-half natural size; $F$. basidium and spore, $\times 750$. G, $T$. firstidiose $\mathrm{Fr}$., one-half natural size.

Erect, pileus entire or branched.

$1786-1794$ Pileate, dimidiate, horizontal, subsessile or effuso-reflexed.

1795-1799

Resupinate, for the most part incrusting, of various forms; hymenium even. Soft, subgelatinous when moist and with a compact, not strigose pileus ; forming effused patches or dendritic masses on twigs, grass, etc.

$1800-1804$

1786. T. Sowerbei Berk. (after James Sowerby) a b. White, becoming yellowish.

$P$. infundibuliform, more or less laciniate and toothed, rough with radiating projecting processes. St. attenuate downwards. Hy'm. plain, setulose.

On the ground amongst moss, under beech; rare. Oct.-Nor. $2 \times 2^{3} \mathrm{in}$. Sometimes occurs with a tuberous base as in 1791 . 
1786a. T. vitellina Plowr. (from the colour, like yolk of egg ; ritellus, egg-yellow) $a$.

$P$. flabelliform, expanded upwards; marg. slightly incurved. St. attenuate downwards, but enlarged at base. Hy'm. rugulose, zoneless.

Gregarious. Dead fir-branches amongst moss, interior of hollow firs. Sept. I $\times 2 \times \frac{1}{5}$ in. Differs from 1786 in its habit and pale egg-yellow colour ; sometimes pale sulphur with marg. brighter and base of stem luminous yellow.

1787. T. pallida Pers. (from the pallid colour) a $c$. Pale yellowishbrown.

$P$. infundibuliform, strigoso-squamose, more or less laciniate and toothed. St. short or obsolete. Hym. costate, setulose.

Caspitose. Woods, pine, beech. Nov. $I_{\frac{3}{x}}^{3} \times 2 \frac{1}{2}$ in. Intermediate forms occur between this and $\mathbf{1 7 8 6}$.

1788. T. multizonata B. \& Br. (from the many-zoned pileus; multus, many) a b.

$P$. infundibuliform, formed from confluent lobes and stems; marg. lobed, crenulate, bright rufous flesh-colour, zoned, darker. St. obsolete. $H_{y^{\prime} \prime \prime}$. slightly ribbed, smooth, paler than P.

Caspitose. Woods, on the ground; rare. Oct. $6 \frac{1}{2} \times 5$ in. Simple fanshapel forms occur, with the substance split.

1789. T. undulata Fr. (from the undulate pileus) a $c$. Pale yellowbrownish.

$P$. coriaceo-membranous. depressed, even, somewhat smooth; marg. entire. St. central, equal or attenuate downwards, villous. Hym. ribbed, somewhat setulose.

On the ground. Oct. $\frac{1}{2} \times \frac{3}{3}$ in. Often confounded with small forms of 1783 and 1784 .

1790. T. caryophyllea Pers. (from a fancied resemblance to a carnation, Dianthus Caryophyllus) a c. Deep claret-brown or purple-brown.

$P$. subcoriaceous, depressed, fibrous-torn. St. equal. Hy'm. smooth, somewhat even. Flesh very dark.

Odour none. On the ground, cliefly about the roots of fir ; rare. Sept.Nov. $2 \frac{1}{2} \times \frac{3}{4} \times \frac{1}{3}$ in.

1791. T. tuberosa Fr. (from the tuberous base) a. Brownish salmonwhite.

$P$. subcoriaceous, divided to top of stem into compressed branches, infundibuliform. St. obscurely furrowed or lacunose, bulbous. $H y m$. smooth. Flesh dark brown with a whitish cortex.

Scattered. On the ground; rare. $3 \frac{1}{x} \times \frac{1}{4} \times \frac{1}{8}$ in.

1792. T. anthocephala Fr. (from a fancied resemblance to a flower;

Gr. anthos, a flower, kiphale, the head) a b c.

$P$. coriaceous-soft, pubescent, divided into large and small divisions, 
apices usually fimbriate, yellowish- or claret-brown. St. short or obsolete, soon furcate, colour as $\mathrm{P}$.

Odour none. Woods, on the ground. Aug.-Nov. $2 \frac{\pi}{3} \times 3$ in. Somewhat resembles a Claz'aria or Aylaria hypoxylon in habit. Very variable in the form of the laciniz.

1793. T. elavularis Fr. (from the nail-like branches; cluz'ula, a small nail) $a$. Claret-brown or rufous-fuscous.

$P$. coriaceous-soft, much and irregularly branched, branches round, attenuate, even, smooth, apices acute, pubescent, whitish or pale yellowish. St. short, thick, somewhat tuberous.

Otlour none. On the ground. $I_{\frac{3}{4}}^{3} \times \frac{1}{2} \times \frac{1}{2}$ in. Habit as 1792 .

1794. T. palmata Fr. (from a fancied resemblance to the palm of the hand) a $b c$.

Coriaceous-soft, much branched, pubescent, dark yellowish-brown or dark claret-brown, apices cottony-fringed, brownish-white. St. branched, flattened even, larger lobes wedge-shaped, the smaller sometimes in form of small infundibuliform pilei.

Odour fetid, carrion-like. Woods, fir, maple. Aug.-Nov. $3^{\frac{3}{4}} \times 4^{\frac{3}{8}}$ in. Habit as $\mathbf{1 7 9 2 .}$

1795. T. intybacea Pers. (from a fancied resemblance to the flowers of chicory, Cichorum Intyluss) a b c.

$P$. fibrous, somewhat jagged and toothed, slightly laciniate, zoned or zoneless, rufous-ferruginous, deep sienna or deep claretbrown; marg. white. Hym. papillose, subfloccose, veined near marg., brownish-vinous, pale vinous at marg. Tufts central and obconic, lateral and subtriangular or spathulate.

Gregarious, imbricate. Pine-woods, pine-twigs, Taccinium MIyrtillus. Nov. $2 \times \frac{1}{2} \times \frac{1}{5}$ in. Intermediate forms occur between this and 1796 and 1797 .

1796. T. terrestris Pers. (from the terrestrial habit) $a b c$.

$P$. soft, fibrous-strigose, lobed, usually zoneless, extended into a somewhat lateral, almost obsolete stem or base, deep vinousbrown; z. when present darker; narg. pale brownish-white, often with a narrow white-pubescent edge. Hy'm. radiatorugose, claret-brown-yellowish or salmon.

Gregarious, imbricate. Woods, fir. July-Oct. Diam. I $1 \frac{3}{4}$ in.

1797. T. laciniata Pers. (from the fringed margin of the pileus; lacinic, a flap) a b c.

$P$. coriaceous-soft, fibrous-scaly, effuso-reflexed, somewhat zoned, deep sienna, somewhat claret; marg. fibrous-fringed, whitish. St. when present incrusting branches as a thick membrane, paler than P. Hym. veined and papillose, colour as St.

Imbricate, growing in strata, perennial. Woods, pine, larch, branches, twigs, leaves, oak, heather, Laccinium llyrtillus, earth, often on compacted masses of fir-leaves. Aug.-Nov. Diam. $2 \frac{5}{8}$ in. At first forming a resupinate, yellowish or salmon-brown film.

1797a. T. atra Weinm. (from the black colour; ater, black) a. Soft. $P i$. springing from a tuberous base, purple-black above and below, sometimes cinereous; mid. paler. Hy'm. smooth, setulose, 
black, white-pruinose. St. attenuate downwards, deformed sooty-black.

Crespitose. Dead logs. $3 \times 2 \frac{1}{4} \times \frac{1}{4} \mathrm{in}$.

1798. T. biennis Fr. (from its period of growth. two years, bionnis) a bc.

Coriaceous-soft, broadly incrusting, cinerous-fuscous. $P$. narrowly reflexed, tomentose, pale claret-brown : marg. fringed. $H y m$. in part resupinate, smooth, rery slightly bristly, more or less plicate, the resupinate parts white-edged.

Subimbricate. Stumps, fir, on the ground amongst wood, leaves and stones; common. Aug.-Nor. Resupinate lyymenium $4 \frac{1}{1}$ in. Pilei $\mathbf{I}$ in.

1799. T. mollissima Pers. (from its very soft substance; mollis, soft) a bc.

$P$. subtomentose, white to pale or dark claret-brown with a broad white tomentose marg. Hym. smooth, even, claret-brown or pale snuff-colour. Flish claret-brown, clouded and streaked darker to almost black.

Subimbricate. On the ground, or on twigs, leaves, etc., oak, beech; frequent. Sept.-Oct. $5_{\frac{3}{*}}^{3}$ in. Very variable, wholly effused, palmate or like a soft stemless imbricate Polyturns : sometimes forming a thin faint brown-ochre membrane with a paler margin. Short stems are sometimes developed.

1800. T. eristata Fr. (from the short, fringe-like crest; crista, a crest) $a b c$. Pallid or ivory-colour.

Incrusting, slightly tough, becoming branched, the apices crested or fringed. Hym. papillose.

On the ground in woods or amongst or on moss, dead leaves and grass; frequent. Oct. 5 in.

1801. T. fastidiosa Fr. (from the disgusting odour ; fastidiosus, disgusting) $a b c$. White.

Effused, incrusting, confluent, soft, irregular in shape, shortly branched and toothed. Hym. papillose, white or biscuit.

On the ground, involving twigs, rootlets, branches, leaves, etc., sloe, ivy, beecl, beech-mast, oak, acorns, moss. Sept.-Nov. Group $5 \frac{1}{2}$ in. Odour the same as 1794 .

1802. T. sebacea Pers. (from its tallow-like appearance and consistency; scha, tallow) a $b c$. White or pale yellowish-white.

Effused, fleshy-waxy, becoming hard, incrusting, irregular in shape like melting and running tallow; marg. determinate. Hym. collapsing, flocculoso-pruinose.

Involving twigs, grass, moss, leaves, cones, clover, ivy, Hydumm auriscalpium, etc. Jan.-Oct. 5 in.

1803. T. erustacea Schum. (from its forming a crust) $a b c$. Fuscousbrown.

Broadly effused, somewhat fleshy, undulate and tubercular ; marg. white-floccose. Hym. papillose, somewhat bristly.

On the ground, involving grass, leaves, etc., and on rotten sawdust, which it resembles in colour. Feb.-Nov. $3 \frac{1}{2}$ in. 
1804. T. cæsia Pers. (from its colour; cesius, bluish-grey) $a b c$. Cinereous bluish-grey.

Effused, soft, continuous, often minutely veined or folded; marg. determinate. Hym. even or slightly papillose, slightly bristly.

On the ground, stumps, etc., involving earth, bark, twigs, etc. Aug. $3 \frac{1}{2} \mathrm{in}$.

\section{CLADODERRIS Fr.}

(From the branched and coriaceous structure; Gr. klados, a young branch, deris, a leathern coat.)

Coriaceous. Hymenium woody, maiate or rugulose with branched ribs, or narrow greatly ramified folds, persistent, at length roughened with warts. Horizontal and attached by a small base behind. (Fig. 97.)

Typical species of Cladoderris resemble Lentinus in appearance, with a squarroso-hispid pileus of the colour and texture of leather and a veined hymenium. The species are usually stemless, but sometimes there is a pervious stem as in 1782. The plants are not usually small although small examples occur. Cladoderris dendritica varies from 7 in. to $\frac{3}{4}$ in. in diam. The prevailing size is from 4 to $6 \mathrm{in}$. in diam. Some species are large, infundibuliform and alnost stemless.

The species chiefly grow in Australia, Cuba and the Philippines.

1805. C. minima B. \& Br. (from its very small size; minimus, the least) $a b$. White.

Flabelliform, springing from a stem-like or obsolete base, resupinate. $P$. tomentose, some- . what zoned. $H^{\prime} m$. radiate on branched ribs.

Birch. Dec. Diam. $\frac{3}{16}$ in. Resembling a dwarfed 1806.
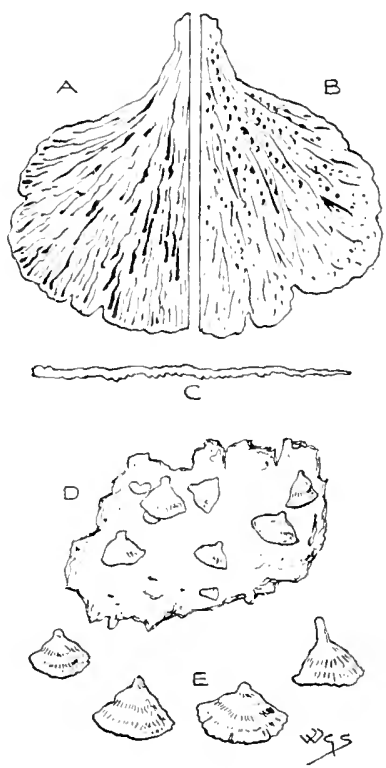

Fig. 97.-A, B, C, upper and lower surface and section of Cladoderris australica Berk., drawn as a type of the genus, one-half natural size of C. minima B. \& Br., natural size. E, ditto, $\times 2$.

\section{STEREUM Pers.}

(From its hard substance; Gr. stereos, hard.)

Hymenium inferior, coriaceous, separated from the inodermous velvety-silky or fibrous-coated pileus or subiculum by an indeter- 
minate fibrillose stratum, even, smooth, composed of basidia and hymenial cells compactly arranged side by side and of uniform height. Basidia 4-spored. Spores minute, roundish-ovate, white or coloured. (Fig. 98.)
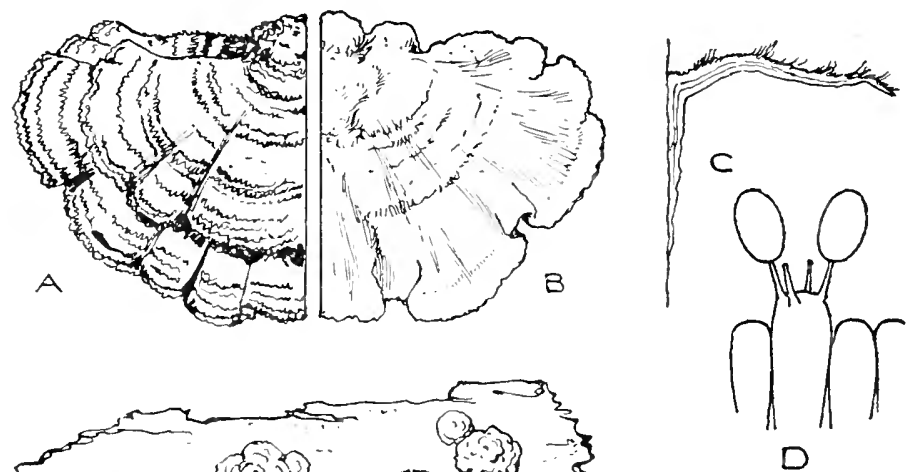

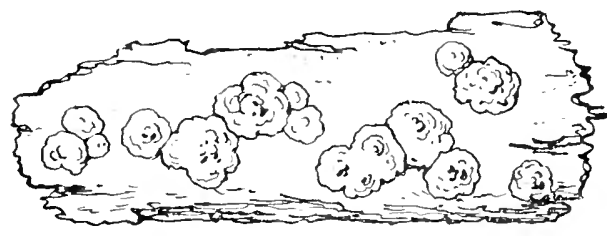

E

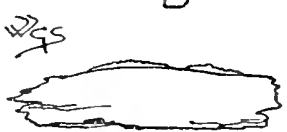

$F$

Fig. 98.-A, B, C, Stereun hirsutum Pers., upper and lower surface and section, two-tbirds natural size. D, basidium and spores, $\times 660$. E, F, S. Pini Fr., upper surface and section, twothirds natural size.

Coriaceous or woody, mostly perennial, somewhat zoned, entire, definite in form, effuso-reflexed. Species $1806-1818$

a. Apoda. Pileus dimidiate, sessile or at first resupinate, then effusedly refiexed, marginate.

$1806-1814$

b. Resupinate. Agglutinate, effused; marg. not free, or scarcely so.

$1815-1818$

\section{a. Apodie.}

1806. S. hirsutum Pers. (from the hairy pileus; hirsutus, hairy) $a b c$. Coriaceous, rigid.

$P$. hispid-zoned, effused, refiexed, lobed and crisped, usually ivory-buff varying dull primrose, orange or brownish; marg. subobtuse, white or whitish. Hy'm. even, smooth, obscurely zoned, juiceless, usually warm-biscuit.

Imbricate. Stumps, oak, beech, birch, poplar, laburnum. Jan.-Dec. 4 in. Said to cause "fly"-wood and "yellow- and white-piped" wood. Pileus often green at the base from the growth of algie. A deep rose-coloured stuin is frequent on this plant cansed by a parasitic mould, Hypomyces rosellus. Tar. subcastatum Mass. Hym. costate or rugose. Tar. cristulatum Quél. P. strigose, grey. 
1807. S. ochroleucum Fr. (from the yellowish-white pileus; Gr. ochros, pale yellow, leukos, white) a c. Coriaceous-membranous.

$P$. reflexed, expanded, flaccid, silky, zoned, hoary-yellowish, shaded pale brownish. Hy'm. even, smooth, cracking, sellowish or greyish.

Wood, fir, maple, oak, hornbeam. Jan. $2 \frac{1}{4}$ in.

1808. S. purpureum Pers. (from the purple hymenium; furfureus, purple) $a b c$. Coriaceous-soft.

$P$. effuso-reflexed, subimbricate, zoned, vilioso-tomentose, pale yellowish. Hy'm. even, smooth, bright rose- or dark-purple, clouded, salmon. Flesh sometimes I in. thick, bright rosepurple, 5-7 zoned.

Dead wood, horse-chestnut, sycamore, hornbeam, fir, poplar, willow, birch, elm, twigs, grass. May-Dec. $3 \frac{1}{2}$ in. Said by Prof. J. Percival to be the cause of "silver-leaf" disease in plum, peach, apricot and sloe. Var. atromarginatum W. G. Sm. A beautiful and apparently distinct plant, illustrated with drawings by P'urton and Sowerby, in the Jiritish Inseum collection; it has been named Auricularia elesans Sow. t. 4 I2, f. I. Berkeley regarded the drawings as a variety of Stereum furfureum Pers. $P$. woolly or frosted, white, with a narrow black zone near the white margin. On a moss-covered tree.

1809. S. sanguinolentum Fr. (from the habit of the hymenium, bleeding when bruised; sanguis, blood) $a b$. Coriaceous-thin.

$P$. effuso-reflexed, substriate, adpressedly silky, pale brownishyellow ; marg. acute, white. Hy'm. even, smooth, pruinose when old, brown-whitish or ashy, rarely as $P$.

Wood, branches, twigs, pine ; common. Jan.-Dec. $3^{\frac{1}{2}}$ in.

1810. S. conchatum Fr. (from the shell-like shape; conchat, a shell) $a$. Coriaceous-thin.

$P$. conchato-reflexed, subcrisped, rugose, glabrous, obscurely zoned, dull yellowish or brown-buff-whitish. $H_{y^{\prime} m}$. even, smooth, dry, brown.

Subimbricate. Fir. $1 \frac{1}{2}$ in.

1811. S. spadiceum Fr. (from the date-brown hymenium; spadix, date-brown) $a b$. Coriaceous.

$P$. effuso-reflexed, hispid-zoned, very hispid at base, pale yellowish or orange-brownish. $H_{y} m$. even, smooth, brown-vinous over pale yellow, not dark, or claret-brown, exuding red juice when bruised.

Stumps and branches, ash, aak; common. Jan.-Dec. $4^{\frac{3}{4}}$ in.

1812. S. rugosum Pers. (from the commonly wrinkled hymenium; musa, a wrinkle) a $b c$. Coriaceous-rigid.

$P$. effuso-reflexed, shortly reflexed, obtusely marginate, pruinose, then smooth, buff, paler behind, zoned sienna. $H_{y} \prime m$. pruinose, sellowish-brown or cream-buff, shaded pale livid, becoming pale sinous where bruised.

Stumps. fir, hornbeam, oak, hazel; very common. Jan.-Dec. $6 \frac{1}{\mathrm{z}} \mathrm{in.}$ Var. Lauro-cerasi Stev. grows on cherry laurel, Prumus Lauro-cerasus. 
1813. S. vorticosum Fr. (from a fancied resemblance in the habit to waves in a vortex or whirlpool) a $b$. Coriaceous.

$P$. effuso-reflexed, imbricate, obscurely zoned, rugose, strigose, crisped, pale buff; marg. white. Hym. smooth, somewhat ribbed, salmon, or purplish-salmon, sometimes orange shaded.

Beech. $4^{\frac{3}{4}}$ in. Intermediate between 1806 and 1808.

1814. S. rufum Fr. (from the rufous pileus) $a b c$. Rigid, coriaceocartilaginous.

$P$. effuso-reflexed, rugose-rough, deep vinous-biown, with pale yellowish marg. and white edge. Hym. minutely cracked, grey-pruinose.

Dead pine, ash, poplar. Sept.-Mar. $3 \frac{1}{2}$ in. Effused and immarginate when young.

\section{b. Resupinate.}

1815. S. Pini Fr. (from the habitat, pine bark) $a b c$.

Coriaceous-cartilaginous, adnate as a shield, marginate, smooth benedih, at length bullate. Hym. noduloso-rough, purple flesh-colour, then fuscous, deep sienna or deep vinous-brown.

On Scotch fir. Nov. $3 \frac{1}{ \pm}$ in. Sometimes identical in colour with the pine bark and easily overlooked.

1816. S. frustulosum Fr. (from the habit, breaking to fragments; frustrum, a piece) ac.

Woody, tubercular, thick, crowded and subconfluent, breaking to fragments, smooth beneath, and at the obsoletely marginate circumference. Hym. convex, pruinose, blackish-brown, cinnamon, becoming salmon.

Wood and bark, oak, beech, branches, hard to rotten sticks. $2 \frac{1}{4}$ in. ; frustules $\frac{1}{8} \times \frac{3}{16}$ in.

1816a. S. quercinum Potter (from the habitat, oak, quercus) $a$.

Coriaceous, at first filling in between crevices of bark. Hym. slightly papillose, young examples with a central papilla, pale buff, becoming cinnamon-whitish; marg. slightly raised from matrix, dark brown, finely zoned beneath, edge lighter. Abundant white mycelium in and under bark.

Oak bark. Jan.-Nov. $2 \frac{3}{ \pm} \times 1 \frac{1}{8}$ in.

1817. S. acerinum Fr. (from its usual habitat, maple branches; accr, maple) a $b c$.

Crustaceous, adnate, effused, even, usually smooth, sometimes minutely nodulose or rugoso-rough, often transversely cracked, white, pale brownish-white or ivory.

Maple, fir, juniper, elm. Dec. $4 \frac{1}{4}$ in. A slight repand hirsute pale-yellowish margin or pileus is sometimes developecl.

1818. S. stratosum B. \& Br. (from the stratose habit) $a$.

Stratose, the pallid strata at length separating, effused, smooth, here and there wrinkled, bright ochreous-white, becoming yellow.

$3 \frac{3}{4}$ in. 


\section{LXIXIV. HYMENOCHÆTE Lév.}

(From the bristly hymenium ; Gr. lumm, a membrane, chaite, a bristle.)

Pileus coriaceous, variable in form. Hymenium plane to papillose, velvety, with minute, rigid, persistent, bright brown setæ or modified

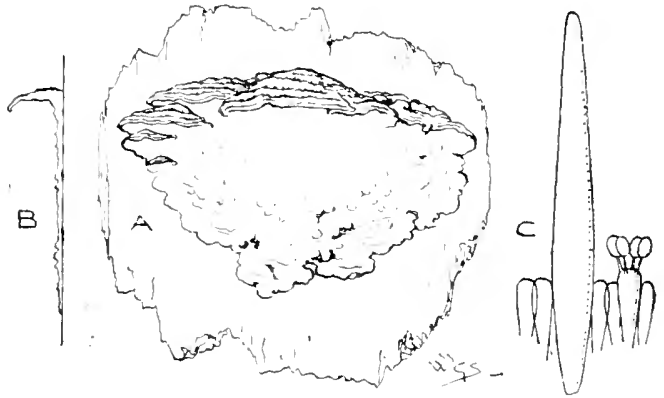

Fig. 99.-A, E, Hymenochate mbiginosa I.év., entire and in section, one-half natural size. C, part of bymenium, showing cystidium, basidium and spores, $X 500$.

cystidia, three or four times the length of the basidia; in 1830 the setre at first very pale. Basidia tetrasporous. Sfore's white or olive. (Fig. 99.)

Allied to Pinithora.

Species $1819-1831$

a. Afoda. No stem. Spores white, cystidia acuminate, plane.

1819,1820

b. Resupinate. With a resupinate habit, except 1827.

Sporis white, cystidia acuminate, plane or rough. 1821-1824.

Spores olive.

$1825-1827$

Cystidia subclarate, sometimes rough.

$1828-1831$

$$
\text { a. Apode. }
$$

1819. H. pubiginosa Lér. (from the iron-rust colour of the hymenium; rubiso, rust) a b $c$.

$P$. effused, reflexed, somewhat imbricate, zoned, relvety, deep orange-sienna; marg. orange. Hy m. papillose, ferruginous or umber, sometimes deep claret-browm.

Hard wood, twigs, oak, alder, sometimes sprealing to moss. Jan.-Dec. 6 in.

1820. H. Avellana Lév. ex Cooke (from the habitat, hazel, Corylus Arellana) a $c$.

$P$. effused, reflexed, zoned, villous, bay-brown; marg. obtuse, free, narrowly reflexed. Hym. papillose, pale ferruginous or pale brown, here and there bleeding, sometimes minutely cracked all over.

Hazel, beech, fir, etc. $\frac{1}{2}-1$ in. 


\section{b. Resufinate.}

1821. H. nigrescens Mass. (from the at length blackish hymenium; nigrico, to be blackish) a b.

$P$. circular, flattened; marg. free, a little reflexed. $H_{y} m$. papilloso-rough, brownish-or greyish-umber, becoming blackish, with irregular black zones. Flesh indigo-umber.

Solitary, gregarious or confluent. Dry wood. MIay. $3^{\frac{1}{2}}$ in.

1822. H. Stevensonii B. \& Br. (after the Rer. Dr. John Stevenson) a b. $H y m$. pallid, fawn-colour, or ivory-salmon with an obtuse slightly elevated paler marg.

Yew. Sept. $2 \mathrm{in}$. This is Stereum mofohistidum Ster.

1823. H. leonina B. \& C. (from the tawny colour; Gr. leon, a lion) a $i$. $H_{y} \cdot m$. somewhat papillose, ferruginous, saffron, orange or orangesienna, sometimes dark, with a few lighter spots; marg. tomentose, lighter.

Dead wood, holly. $3^{\frac{1}{x}}$ in.

1824. H. fuliginosa Lév. (from the smoke-coloured bymenium; fuligo, soot) a $c$.

Hym. somewhat papillose, dark smoky bay-brown or opaque claret-brown; marg. lighter.

Wood. $2 \frac{1}{2}$ in.

1825. H. corpugata Lév. (from the wrinkles or folds on the bymenium; corrugo, to wrinkle) a $c$.

Somewhat effused, closely adnate, soon grumous. Hy'm. papillose, red, red-pale- or vinous-brown, or deep claret-sienna, sonetimes darker at marg.

Bark, alder, maple, oak, blackberry, - hawthorn, holly, ling, taccinium, Myrica. Mar.-Dec. $5 \frac{1}{\frac{1}{2}}$ in. Twigs, leares, etc., are commonly agglutinated to the hymenium. Becomes much cracked in drying.

1826. H. eroceoferruginea Mass. (from the orange-brown hymenium; crocus, saffron, firrugo, iron-rust) a.

Effused, broadly encrusting, very thin. Hym. papillose, saffron, yellow-sienna, tawny, pale sienna or yellowish-chocolate; marg. paler or yellow.

Dead dog.rose. $3^{\frac{1}{2}} \mathrm{in}$. IIymenium cracking.

1827. H. tabacina Lév. (from the tobacco colour) a b $c$.

Somewhat coriaceous, thin, flaccid, effused, imbricate. P. silky, then smooth, sienma-orange, pale or deep siemna or purplishumber, zoned sienna, base blackish-umber; marg. retlexed, ivory. Hy'm. ferruginous, deep- or crimson-siemn, with a white, ivory, yellow or orange marg.

Somewhat uncommon. Trunks, branches, etc., willow, hazel, plum, onk, birch, lilac, blackberry, cotoneaster, rhododendron, Antromelda, l'accinium, sometimes spreading on to moss. Oct.-Mar. $3 \frac{1}{2}$ in.

1828. H. erassa Cooke (from the thick substance ; rassus, thick) $t$.

$P$. velvety, pallid-rufous; marg. thick, at length free. $H_{j} \prime \prime$. unequal, colour as $P$.

Trunks. $4 \frac{1}{2}$ in. 
1829. H. abietina Mass. (from the habitat, trunks of fir, abics) a b.

$P$. effused, tomentose, sienna-brown. Hym. papillose, fuscousferruginous, dull yellowish-purple, brownish-salmon or claretbrown, clouded ivory-ochre or purple-lilac, becoming pale.

Decaying fir, spruce-fir. $2 \frac{1}{4}$ in. Cracking wlien dry.

1830. H. disciformis W. G. Sn., Thelephora disciformis DC. (from its frequent disc shape) a. White to ivory-white.

$P$. disciform, thinner towards the definite free naked marg. Hy'm. unequal.

Trunks, oak. $\mathbf{1} \frac{1}{4}$ in. This is Sterem disciforme Fr. Cooke has placed it in Peniophorie, and has illustrated the plant with granular setie. Saccardo follows Cooke. My plants and those in British Museum have smooth setie.

1831. H. Boltonii Cooke (after James Bolton of Halifax) a.

$P$. villous, white to ochre or pale lavender, zoned brown or black. Hym. white to pale brown, becoming ferruginous-fuscous or dark red; marg. crisped, free, thin.

Bird cherry. Feb. $\mathbf{I}_{\frac{3}{x}}^{3}$ in. Cracking when dry.

\section{LXXXY. CORTICIUM Pers.}

(From the usual habitat, bark, cortcx.)

Resupinate. Hymcnium often broadly effused, in a fer species the margin is slightly upraised, amphigenous, even or tuberculose,

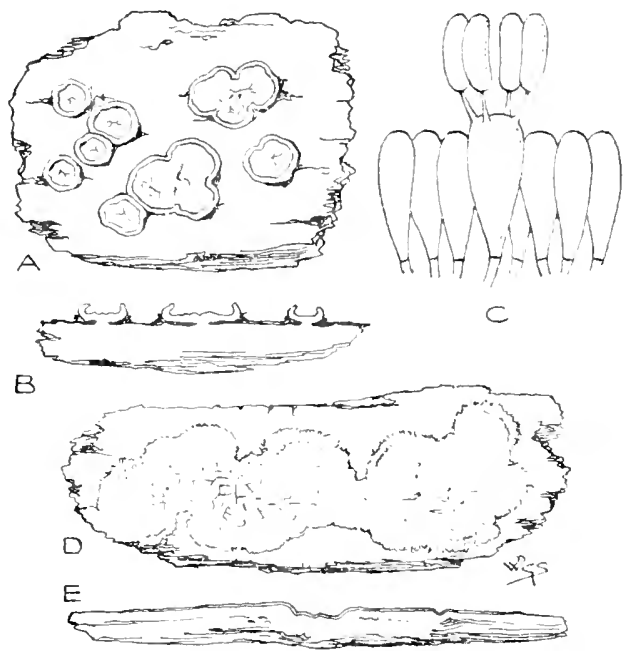

Fig. 100.-A, B, Corticium salicinzm Fr., entire and in section, one-half natural size. C, hymenium with basidia and spores, $x$ 500, D, E, C. calcenum Er., entire and in section, one-half natural size.

waxy or fleshy-soft, usually contracting and cracking when dry, smooth, arising immediately from the mycelium without an intermediate 
fibrillose stratum; without setæe or modified cystidia. Spores white. (Fig. 100.)

Usually growing on bark and wood, sometimes on sawdust, earth, frr-cones, stems of herbaceous plants, leaves, moss, etc. Some species are phosphorescent. Young examples of some species of Corticinm may readily be confounded with Pesize. The hymenium of some species occasionally exhibits pale rose-coloured to deep crimson stains caused by the presence of a parasitic mould, Hypomyces rosellus.

Species $1832-1866$

Marginate, margin free, determinate.

$1832-1837$

Margin usually indefinite, byssoid, strigose, pulverulent or mucedinous-clouded.

Hy'menium white, or ochreous.

$1837 \mathrm{a}-1851$

Hymenium brightly coloured.

$1852-1864$

Amphigenous, very thin, innate, throwing off the bark.

1865,1866

1832. C. salicinum Fr. (from the habitat, willow, salix) $a c$.

Coriaceous, soft, rigid when dry, small, fixed by centre. $H y^{\prime} m$. somewhat rugulose, becoming finely but sparingly cracked, deep claret-crimson; marg. reflexed all round, villous, white or pale rose.

Gregarious. Willow, rarely poplar. Aug.-Sept. $\frac{1}{4}-\mathbf{I}$ in. Resembles a Fĩiza.

1833. C. amorphum Fr. (from the supposed ambiguous structure of the hymenium ; Gr. a, without, morphe, form) a $b c$.

Somewhat coriaceous, small, waxy-pliant, cup-shaped then flattened. $H_{y \prime m}$. white- grey- or yellowish-umber; marg. white.

Gregarious, silver fir, larch. Aug.-Nov, $\frac{1}{8}-\frac{5}{8}$ in. Kesembles a Peziza. This is Pesiza amortha lers., Thelephora amorpha Fr. It grows in company with Prsisa calycina, with pallid examples of which it may be easily confounded. When dry the Corticium and Pesiza resemble each other closely.

1834. C. evolvens Fr. (from the habit, as of new plants emerging from the margins of older ones; carolie to unroll) a bl $c$.

Soft, cup-shaped to effuso-reflexed, gregarious, becoming confluent. $H_{y}^{\prime} m$. somewhat rugose or tubercular, cracking, pale sienna, clouded darker or pale yellowish-brown.

Bark, cherry, willow, poplar ; frequent. Oct.-April. $\frac{1}{4}-1 \frac{3}{4} \mathrm{in}$.

1835. C. poposum B. \& C. (from the pore-like markings on parts of the hymenium) $a$.

White, becoming pale ochre; marg. free, reflexed.

Wood. $\frac{3}{4}-3$ in. The "pores" somewhat resemble the true pores of Porothelium, they are of differenl sizes and appear as thin dried circular lines, such as are lefi by minute drops of moisture in drying. 
1836. C. populinum Fr. (from the habitat, poplar, fotulus). Soft, tuberculiform, soon confluent and effused, at length involute. $H_{1} \prime m$. uneven, ferruginous-cinerous, whitish-tomentose beneath. l'oplar.

1837. C. Lycii Cooke ex Mass. (from the usual habitat, Lycium) a. Effused, thin. $H_{y} \cdot n$. papillose, lilac or white-salmon; marg. free, somewhat byssoid, pallid. Lycium, lilac. $\frac{3}{4}-3$ in.

1837a. C. seriale Fr. (from the habit, growing in long, gregarious rows; series, a row $)$.

Elongato-effused, adfixed to matrix, thin. $H_{y} \mathrm{y}^{\prime}$. rimose, thin, papillose, dull ochreous, white pruinose; marg. fibrillose whitish. Wood, pine.

1838. C. calceum Fr. (from the chalk-white colour; calx, lime) $a b c$. Broadly effused, agglutinate, waxy. Hym. even, smooth, rigid, cracking, white or pallid, becoming pale dull salmon; marg. finely pulverulent, white.

Wood, pine, blackberry, lilac, willow, lime, hornbeam, birch, Myrica, leaves, earth. Nay-Aug. $\frac{1}{4}-5$ in. This is $C$. cretacin Pers.

1839. C. serum Fr. (from its time of growth-winter; serus, late, growing in winter) $a b c$.

Broadly effused, incrusting, fleshy to thin. $H_{y} \prime m$. smooth to pruinose, crowded with papille, splitting into flocci, white; marg. fading in a mucedinous manner into the wooden matrix, sometimes forming finely pulverulent cloud-like growths.

Trunks, birch, alder, hornbeam. $\frac{1}{2}-3 \frac{1}{2}$ in. This is Hypochnus Sacc., Lycomyces Karst. Uften resembling spluttered clrops, as from a wax candle.

1840. C. scutellare B. \& C. (from the salver-like form it sometimes assumes; scutclly, a salver) a $c$.

Broadly effused. Hym. cracking into minute areole, with intermediate finely-folded rugose patches, whitish, becoming warm biscuit-buff; marg. forming thin clond-like growths in mature examples.

Rotting logs, furze, oak, cedar, deal herbaceons stems. 6 in.

1841. C. fœitum B. \& Br. (from its fetid odour) $a$.

Effuserl. Hym. cracking, white to ivory and ochreous, clouded pale grey and faint sienna.

Sawdust. $\frac{1}{4}-2 \frac{1}{2}$ in.

1842. C. lacteum Fr. (from the milk-white colour; lac, milk) a $b c$.

Froadly effused, submembranous. Hym. waxy, cracking and parting when dry, white to pale biscuit with mid. darker, sometimes pale straw or pale orange-colour; sub. and marg. loosely fibrillose, often fringed with long branching strands of white mycelium, sometimes with pilose or farinose cloud-like growths.

Trunks and beneath bark, chestnut, beech, holly, lime; frequent. Nov.Feb. 6 in. 
1843. C. læve Pers. (from the even hymenium; lae'is, even) $a b i$.

Effused, membranous, separating from matrix. $H_{1} m$. even, smooth, rarely papillose, commonly with vacuoles exposing the wood, cracking, white to sienna-yellowish, flesh-colour, livid, salmon or pale red; marg. sometimes broad, edge pilose, ivory to white, sometimes with radiating strands of white mycelium.

Wood, bark, sticks, pine, poplar, birch, alder, oak; very common. Nov.May. $\quad$ S $\frac{1}{2}$ in. Some conditions resemble 1770.

1844. C. confluens Fr. (from the habit-forming confluent patches) $a b c$.

Agglutinate, submembranous. Hym. even, naked, cracking, somewhat concentrically rugose when young, hyaline becoming white, lavender-brown-white, yellowish-brown, pale brownish or pale rose-purple; marg. spreading in a dentate manner.

Bark, beech, alder, ash, willow. Mar.-Aug. $\frac{5}{5}-3 \frac{5}{5}$ in.

1845. C. nudum Fr. (from the hymenium becoming bare, mans) $a b c$.

Waxy, agglutinate, very thin, becoming rigid. Hym. flesh-colour, white-salmon, umber-ivory or yellowish-buff, clad with fugacious white meal, cracking when dry; marg. determinate, smooth.

Dead wood, bramble, aller, yew, willow. Aug.-April. $\frac{1}{ \pm}-4 \frac{1}{2} \mathrm{in.}$

1846. C. arachnoideum Berk. (from the mycelium-like spider-rreb; Gr. arachnt, a spider) a $b c$.

Very thin, effused, delicately flaxy. Hym. waxy, continuous or somewhat papillose, somewhat cracked when dry, white or faintly clouded sulphur, yellowish or brownish, floccose beneath; marg. white-fibrillose.

Wood, bark, oak, pine, twigs, leaves, grass, lichens, etc. Oct.-Mar. $7 \frac{1}{2}$ in.

1847. C. radiosum $\mathrm{Fr}$. (from the radiate habit) $a b c$.

Membranous, subrotund with radiate lobes. Hym. smooth, glabrous, irregularly papillose, cracking when dry, primrosewhite to dark ochreous or buff, adpressedly fibrillose beneath; marg. fibrilloso-radiate, often with long strands of mycelium.

Eim, birch, pine, hormbeam, naked wood. Oct.-Feb. $4 \frac{1}{2}$ in.

1848. C. Typhre Fück. (from the usual habitat, bulrushes; Typha, a bulrush) a $b c$.

Longitudinally effused, thin, at first forming white byssoid spots, smooth then mealy, cracking when dry, ivory to tan; marg. usually ragged or ill-defined.

Dry leaves of Tytha latifolia and species of Carex. I $\frac{1}{2} \mathrm{in}$.

1849. C. Sambuci Pers. (from the habitat, elder, Sambucus) a b $c$. Broadly effused, indeterminate, encrusting, somewhat innate, cracking and breaking into floccules when dry, white.

Very common. Aug.-May. 7 in. Sometines forming hyaline-whitish spotty cloud-like growths. 
1850. C. lactescens Berk. (from its habit of exuding a watery milk-like fluid; lac, milk) a b $c$.

Waxy-soft, agglutinate, undulate or rugose at length cracked, pale flesh-colour or pale salmon; marg. shortly byssoid.

Dead wood, ash, willow, Oct.-Feb. 6 in. Interstices of cracks silky. Superficially resembles 1893.

1850a. C. strigosum Pers., var. filamentosum IV. G. Sm., Thelephora byssoides DC. (from the thread-like habit; filum, a thread).

Sul. web-like, filamentous, string-like, dull yellowish, externally pulverulent.

On Amaryllis.

1850b. C. violeum W. G. Sm., Hjpochnus ziolens Quél. (from the colour).

Byssoid, thin, orbicular. $H_{y} \prime m$. velvety-floccose varying lilac and rose ; marg. thin pallid.

Dead branches, pear, apple.

1851. C. lacunosum B. \& Br. (from the hymenium, full of openings; lacano, to hollow out) a.

Broadly effused, soft, pulverulent, lacunose, exposing matrix beneath, tawny, ochreous or cinnamon, clouded pale umber. Myc. woolly, tawny.

Wood, branches. $5 \mathrm{in}$.

1852. C. roseolum Mass. (from the rose-coloured hymenium) a $b$.

Broadly effused, indeterminate, very thin, waxy. Hym. smooth, continuous, not cracking, pale rose or salmon; marg. whitish, silky.

Worlied wood, palings. Nov. 7 in.

1853. C. aurora B. \& Br. (from the rosy colour, like the dawn, alcrora) a b.

Effused, thin, agglutinate, rosy, becoming pale salmon with a lighter indeterminate marg.

Dead leaves and stems of Carex, C. paniculata. Feb. $2 \frac{1}{4}$ in.

1854. C. anthochroum Fr. (from the rosy flower-like cloudings; Gr. anthos, a flower, chros, colour) a b $c$.

Broadly effused, membranous, floccoso-velvety, clouded brick-red or rose on a pale brownish ground.

Investing dry bark, twigs, etc., sycamore, birch, spreading to earth; rare. Nov.-MIar. $6 \frac{1}{t}$ in. Sometimes forming small, dark vinous patches.

1855. C. molle Fr. (from its soft substance; mollis, soft) $a c$.

Irregularly rounded, floccoso-fleshy, loosely interwoven. $H_{y}^{\prime} m$. waxy, papillose, cracked when dry, pallid, pale sienna to reddish-buff; marg. naked, paler, sometimes byssoid.

Fir. $3 \frac{1}{2}$ in.

1856. C. polygonium Pers. (from the hynenium, often covered with small polygons, from the confluence of small growths) $a b c$.

Adnate, determinate, soon hardened, somewhat grumous. Hy'm. 
pruinate, at length more or less cracked, lavender-white or flesh-colour, red bencath.

Dead branches, hornbeam, poplar. sloe, lilac, elm, Rohinia; frequent. Mar.-April. $\frac{1}{5}-3 \frac{1}{2}$ in. Growing in small round patches from the ostiola of Sphcric, at length confluent and forming large patches which become rugged with age.

1857. C. maculæforme Fr. (from the rose-coloured spots on the bymenium: macula, a spot, forma, form) a.

Orbicular, confluent, indurate, thin. $H_{y} m$. spuriously papillose, purple with rose-coloured spots and smaller purple spots; marg. determinate, pale purple.

Dead wood. $2 \frac{1}{2} \mathrm{in}$.

1858. C. sanguineum Fr. (from its blood-red colour; sangliis, blood) a b c.

Broadly effused, indeterminate, even, smooth, loosely adherent, arachnoid beneath, slightly cracking when dry, bright deep cinnabar-crimson, clouded purple and black-crimson: marg. sometimes broadly floccose with cinnabar-white mycelium, sometimes forming cimnabar-white cloud-like growths.

Wood, squared wood, pine, larch, spreading to leares. Oct.-Feb. Io $\frac{1}{2}$ in. Deeply staining wood a permanent blood-red colour. Rarely fertile.

1859. C. Carlylei Mass. (after Dr. David Carlyle of Carlisle) a.

Effused in elongate patches, agglutinate, between waxy and soft. $H y^{\prime} m$. even, smooth, dull orange; marg. white.

Oak bark. $3 \frac{1}{4} \times \frac{7}{3}$ in.

1860. C. flaveolun Mass. (from the yellowish hymenium ; fluz'ts, light yellow) a.

Effused, membranous, loosely adhering. $H_{1} m$. pallid yellowish, or very pale lemon with a determinate white marg.

Trunks of tree-ferns. $3 \mathrm{in.}$

1861. C. cœruleum Fr. (from its frequent azure-blue colour ; chrmlenm, azure-blue) a $b i$.

Broadly effused, adnate. $H_{1}^{\prime} \prime \prime$. waxy-soft, papillose, tomentose, slightly cracking, deep smalt or Prussian-blue, sometimes almost black or clouded deeper and lighter azure-blue; marg. determinate, bordered with a fine white line or narrowly flaxy and pale blue, sometimes upturned.

Sticks, dressed wood, dead wood, gates, rails, fir, chestnut, oak, alder ; common. Dec,-April. 6 in. Sometimes phosphorescent. Sometimes black when dry.

1862. C. violaceolividum Fr. (from its violet and livid clouding) $a c$.

Somewhat effused, adnate, indurate. $H_{y^{\prime} \prime \prime}$. spuriously corrugate, tuberculose, mealy, cracking all over when dry, livid purple, clouded darker or indigo-white, clouded pale brown; marg. paler or yellowish.

Dead wood, willow, lilac. $4^{\frac{3}{4}}$ in. 
1863. C. lividum Pers. (from the livid colour) $a$ b $c$.

Effused, closely adnate, between waxy and soft. $H_{y} m$. even, sometimes rugose or tubercular, somewhat viscid when moist, cracking when dry, variable in colour, clouded pale buff, pale liver or livid azure-blue and fuscous-purplish : marg. pulverulent, granular or determinate, paler or white.

Dead birch, elm ; rare. Oct.-June. 5 in.

1864. C. atrovipens Fr. (from the colour, blackish, shaded greenish; atir, black, irire, to be green) it.

Irregularly effused, tomentose beneath. $H_{1} m$. waxy, smooth, pruinose, somewhat pale dull indigo with a greenish shade; marg. tomentose.

Rotten wood, leaves, sticks; rare. 3 in.

1865. C. nigrescens Fr. (from its blackish colour; nimrico, to be blackish) a $c$.

Effused, interrupted, waxy, agglutinate. $H_{y} m$. here and there papillose, cracking when dry, yellowish then blackish or brown-whitish to brown-slate, densely powdered with large spores.

Branches, slicks, under the bark, oak. 3 in.

1866. C. comedens Fr. (from its habit of destroying the bark, when very thin, under which it grows; comide, to consume) $a b c$.

Effused, innate, exposed only when the bark splits. Hym. even, sometimes papillose or finely granular, slightly viscid when moist, cracking when dry and peeling from matrix, flesh-colour, lilac or variegated pale dull buff and pale to somewhat dark purplish.

Sticks, branches, hazel, alder, hawthorn, chestnut, pine, oak, hornbeam ; very common. Aug.-Mar. $5 \frac{1}{2}$ in. Never found above the bark.

\section{PENIOPHORA Cooke.}

(From the shuttle-like setre, metuloides, or modified cystidia borne on the hymenium; Gr. panion, a shuttle, phero, to bear.)

Resupinate, effused, coriaceous or subcarnose. Hymmimm, as seen under a pocket lens, setulose, cystidia projecting, fusiform, colourless, hyaline at first smooth then verruculose above with minute particles of oxalate of lime, which are derived from water containing this substance in solution. The cystidia give the hymenium a velvety appearance, but they sometimes break away and leave the hymenium smooth; in this condition care must be taken not to confuse the species with Corticim. Basidia tetrasporous. Spores white, hyaline. (Fig. IOI.) 
Growing on wood, bark, leaves, etc. Young examples of some species may be readily confused with $P_{\text {eisiale. Species } 1867-1882}$

Margin free, more or less incurved.

$1867-1870$

Margin adpressed, often indeterminate.

$1871-1881$

Setæe aggregate in fascicles.

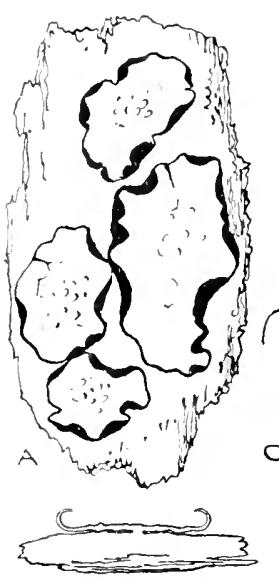

B

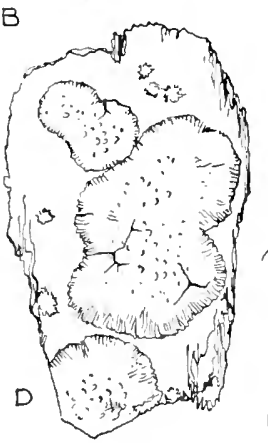

E
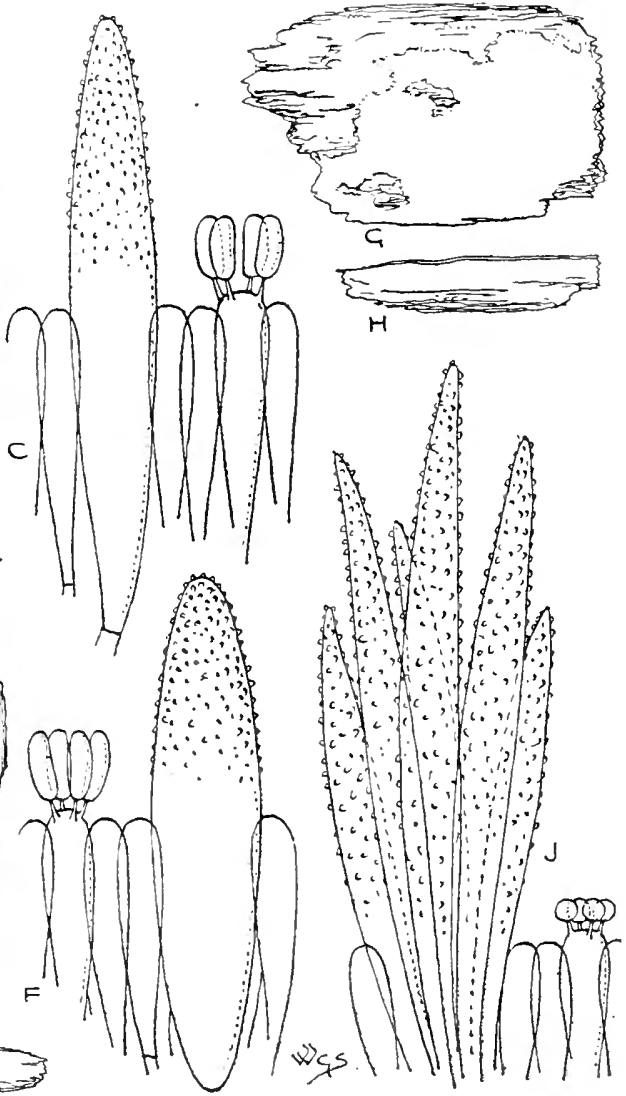

Fir ror-A, E, Pemiothora mercinu Cooke, entire and in section, one-half natural size. C, basidium, spores and cystidium of ditto, $\times 500$. D. E, $P$. resca Mass, entire and in section, one-half natural size. F, basidium, spores and cystidium of ditto, $\times 500$. $G, H, P$. hydrocides Cooke $\&$ Mass, entire and in section, one-half natural size. $\mathrm{J}$, basidium, spores and cystidium of ditto, $\times 500$, is:

1867. P. quereina Cooke (from the usual habitat, oak, quercus) a b $c$. Cartilaginous, membranous, at first agglutinate, then fixed at centre, rigid, smooth, slightly cracking when dry, turning black beneath. Hym. papillose, or irregularly rugose, sometimes 
rugged all over, flesh-colour, pale purplish-rose, pale yellow-buff, dull salmon or liver-whitish.

Oak, birch, lilac, lime, hazel, ash, chestnut. Aug.-April. 7 in.

1868. P. pezizoides Mass. (from its resemblance to Piriza; Gr. cidos, resemblance) $a$.

Somewhat leathery, cup-shaped, then flattened, fixed at centre, externally pallid villous. Hym. continuous, ochreous or somewhat pale umber; mid. darker ; marg. white.

Branches, horse-chestnut. $\frac{1}{4}-\frac{3}{8}$ in.

1869. P. gigantea Mass. (from its large size, gigantenc) a $b c$.

Broadly effused, swelling when moist, fleshy-tough, waxy, cartilaginous when dry, slightly cracking; marg. free, incurved. $H y^{\prime} m$. even, continuous, partly granular, white to ivory, shaded greyish ; marg. rugose, grey-ivory beneath.

Stumps, trunks, branches, fir, larch, sometimes investing fir-leaves, moss and grass; common. Oct.-April. $8 \frac{1}{ \pm}$ in.

1870. P. Crosslandii Mass. (after Charles Crossland of Halifax).

Effused, thin, soft when moist, wholly separating from matrix when dry. Hym. pale grey, faintly ochreous when dry.

Wood and bark, fir. Differing from 1869 in the smaller spores and shorter cystidia.

1871. P. limitata Cooke (from the margin limited by a black line) $a$. Somewhat orbicular, grumous, indurated, smooth. Hym. lurid, lavender-brown, shaded brown.

Wood and bark, broom. $3^{\frac{3}{4}}$ in.

1872. P. posea Mass. (from the rose-coloured hymenium) $a b c$.

Effused, very thin, slightly cracking, showing grain of wood beneath. $H_{y^{\prime} m}$. minutely papillose in middle, rose or rosesalmon becoming pale, sometimes clouded pale brown; marg. often finely fimbriate or rugoso-radiate and paler, sometimes tinted pale sulphur.

Wood, bark, birch, poplar, willow, walnut, rose, often spreading over grass, lichens, etc. Ocl.-Mar. 4妾 in. Beconing rufous-salmon when dry. Illosporium roseum is probably a young condition of this or of 1873 .

1873. P. inearnata Mass. (from the flesh-coloured hymenium; incarnatus, flesh-coloured) $a b c$.

Somewhat waxy, agglutinate, thin, cracking when dry. $H_{y} \prime m$. dull pale red, pale vermilion, red-orange, salmon- or vermilionwhite, or salmon, when young sometimes white; marg. radiate, varying darker or paler.

Wood, bark, bramble, gorse, birch, beech, laburnum, alder, plane, fir. Dec.-April. $5^{\frac{1}{ \pm}}$ in. Illosporium carnethn is probably a young state of this or of 1872 .

1874. P. ochracea Mass. (from the ochreous-yellow hymenium) a $c$.

Broadly effused, cracking when dry. Hym. pallid ochreous, at first sprinkled with scattered shining gold-like atoms, sometimes pale vinous.

Wood, bark, ash. $5 \frac{3}{8}$ in. This must not be confounded with 1895 . 
1875. P. einerea Cooke (from the ash-coloured hymenium; cinis, ashes) $a b c$.

Waxy, becoming rigid, confluent, cracking when dry. Hym. minutely papillose or granular, cinereous or lurid, umber-white or white-lavender, often clouded umber, here and there pale salmon-white; marg. usually definite.

Wood, bark, herbaceous stems, pine, oak, poplar, lime, ash, honeysuckle, ivy, bramble, Lycium, Viburmum. Aug.-June. 6 in.

1876. P. velutina Cooke (from the velvety hymenium; vellus, fleece) a $c$.

Broadly effused, adnate, very delicate, cracking when dry. Hy'm. even, flesh-colour, rose-salmon, ivory, scarlet-white, sometimes rose-lavender, paler at the somewhat broad, fleecy, usually fimbriate marg., extending in radiate strands of mycelium.

Wood, bark, pine, bracken, sometimes involving moss. Oct.-May. 6 in.

1877. P. rimosa Cooke (from the cracked hymenium; rima, a cleft) $a$.

Broadly effused, agglutinate, cracking into areolæ with silky interstices. Hym. cinereous, pallid fawn, or somewhat pale brown-sienna-ochre.

Bark, wood. 4 in. Differing from 1876 principally in the larger spores.

1878. P. scotica Mass. (from the first place of finding, Scotland) $a$.

Broadly effused. Hy'm. cinnamon or pale brownish-ochre, obscurely vinous and clouded opaque cinnamon-brown ; marg. fibrillosely-radiate.

Inside bark. $3 \frac{1}{2}$ in. The irregularities of the inner side of the bark show through the substance.

1879. P. phyllophila Mass. (from its habitat, leaves; Gr. phullon, a leaf, philos, loving) $a$.

Broadly effused, membranous. $H_{y} m$. continuous, ivory, sometimes faintly sulphurous or ochreous; marg. fibrillose or indeterminate.

Dead leaves. $4 \frac{3}{8}$ in. Must not be confounded with the non-British Corticium efiphyllum, from which it is distinguished by its setze.

1880. P. pubera Sacc. (from the downy hymenium ; $p u b e$; downy) $a c$. Broadly effused, closely adnate, very delicate, cracking. $H_{y} \cdot m$. even, white, clay-colour or colour of milky coffee; marg. determinate to indeterminate but minutely ragged, sometimes slightly pulverulent.

Oak, beech, mountain ash, rough pieces of rotten wood. Jan,-Feb. $3 \frac{1}{2}$ in.

1881. P. terrestris Mass. (from its occasional place of growth, the ground, terra) a $b$.

Effused, very thin. Hym. cinereous, pallid fawn or lavender-slatewhite.

Earth, twigs, branches, leaves. 5 in. Resembles pale dull lavender clouds. 
1881a. P. Chrysanthemi W. G. Sm., Corticium Chrysanthemi Plowr. (from the habitat, base of living chrysanthemum stems) $a$.

Effused, incrusting, indeterminate, uneven from the irregularities of the matrix. Sub. filamentous. $H_{y} m$. indeterminate, white. Autumn. 5 in.

1882. P. hydnoides Cooke \& Mass. (from the resemblance of the hymenium to certain resupinate species of Hydum; eidos, appearance) $a$.

Broadly effused, thin, somewhat innate. Hym. cinereous or dull lavender-white, clouded.

Bark. Nov. $5 \frac{1}{4}$ in. Somewhat resembles Grandinia.

\section{CONIOPHORA DC.}

(From the dust or spore-powdered hymenium;

Gr. konis, dust, phoreo, to carry.)

Resupinate, broadly effused, margin defined or indeterminate, closely attached to the matrix. Hymenium even, powdered with

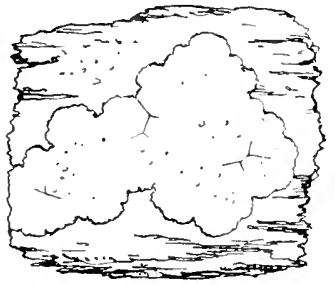

A
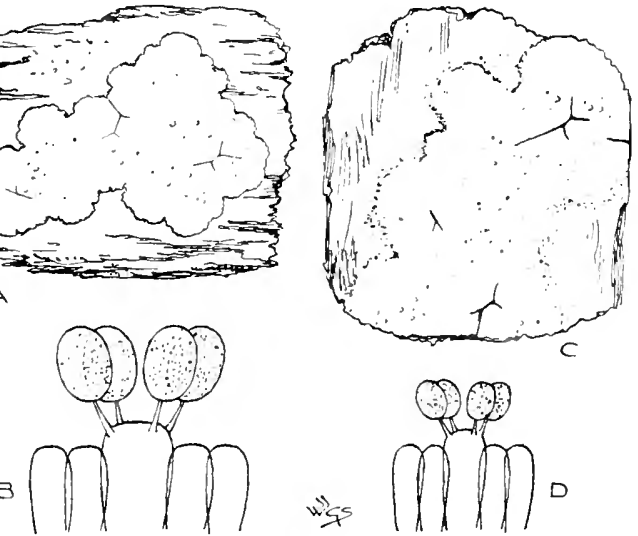

Fig. ro2.-A, Coniophora olivacea Karst., one-half natural size. B, basidium and spores of ditto, $\times 500$. C, C. ochracea Mass., one-half natural size. $\mathrm{D}$, basidium and spores of ditto, $\times 500$.

spores, without setæ. Basidia tetrasporous. Spores smooth, pale brownish or pale dull ochreous. (Fig. I02.).

Growing in broadly expanded patches on dead wood or bark.

Species $\mathbf{1 8 8 3 - 1 8 9 8}$

Spores large.

$1883-1893$

Spores small.

$1894-1896$

Substance soft, subgelatinous.

1897,1898

1883. C. olivacea Karst. (from the olivaceous colour) $a b c$.

Membranous, broadly effused, orbicular or elongate. Hy'm. dull 
olive, sparkling with oxalate of lime crystals when fresh; marg. fimbriate, whitish. Spores pale dull ochre.

Trunks and wood, pine; uncommon. Oct.-Nov. $5 \frac{1}{2}$ in.

1884. C. pulverulenta Sacc. (from the pulverulent hymenium) $a$.

Effused, dry, cracking. $H_{y} m$. brown, ferruginous- or yellowishbrown, clouded and mottled darker; marg. membranous, white or pale yellowish-brown. Spores pale dull yellow.

Wood. 7 in. Has been considered a form of 1680 , var. fulierulentus.

1885. C. puteana Karst. (from its often growing in cellars, humid greenhouses, etc.; putcanum, a well) a c.

Broadly effused, fleshy, fragile, somewhat thick, compact, almost waxy, usually with open spaces exposing the matrix, sometimes cracked and showing the fibrillose substratum. Hym. subundulate, pallid, yellowish or buff to olivaceous-brown or brown-sienna ; marg. mucedinous, white, sometimes indefinite. Spores tawny olive.

Bark and wood, cypress, dead wood, rotten trunks, running orer dead leaves, damp walls in cellars, greenhouses, etc. : frequent. Oct.-Nov. $5_{t}^{3}$ in.

1886. C. cinnamomea Mass. (from the cinnamon colour) a b $c$.

Effused, confluent, irregular, fleshy, soft, somewhat thick, cracking and exhibiting the fibrilloso-strigose Sub. $H_{y} \cdot m$. pale cinnamon to dark rich sienna-umber or chocolate, sjotted or mottled darker. Spores pale dull yellow or very pale cimnamon.

Wood, bark, plum, hazel, hornbeam, birch, oak; rare. Nov. 4 in.

1887. C. umbrina Karst. (from the brown colour, umber) a b c.

Effused, confluent, fleshy-soft, somewhat thick. Sub. villous. $H y m$. tuberculose then collapsing, varying pale to dark brown or sooty- or yellowish-umber, often slightly olive; marg. shortly fimbriate. Spores pale, dull brown.

Dead wood, branches, sticks, etc. Sometimes on the ground. Feb. 6 in. Not easily separating from the matrix. Surface sometimes rugosopapillose all over.

1888. C. inerustans Mlass. (from the incrusting habit) a $b$.

Effused, thin, indeterminate, almost waxy when perfectly developed. Hym. waxy-pulverulent, pallid to pale yellowish. Spere's pale yellow.

Enveloping leares, twigs, etc., with a film. 6 in. Thinner and les= nodulose than 1898.

1889. C. arida Karst. (from its dry substance: aridus, dry) a b c.

Effused, membranous, adnato-agglutinate, continuous, cracking. $H y$ m. even to slightly tuberculose, sulphur-yellow, becoming ferruginous-umber, clouded ; marg. broadly radiato-byssoid, sometimes pulverulent, sulphur-white, sometimes zoned ochre and sulphur near marg. Sfores pale dull yellow.

Logs, woods, cellars, pine, fir, juniper ; common. Oct.-Feb. 7 in.

1890. C. sulphurea Mass. (from the sulphur colour) $a b c$.

Effused, fibrilloso-flaxy, somewhat thick, when perfect thick and waxy-soft, cracking, often imperfect and spongy, passing into 
radiate, cord-like, branching sulphur-coloured strands. Hym. minutely papilloso-rugose or granulose, bright sulphur-yellow, or clouded and mottled sulphur and orange on an ivory ground, fading to yellow-umber, sometimes rich sienna-orange with a pale somewhat byssoid sulphur marg. Stores olive.

Dead wood, bark, twigs, leaves, stipules, etc., pine, oak, beech, sometimes on earth. Oct.-Jan. $5 \frac{1}{2}$ in. Var. ochroidea Mass. Hym. ochreous witi a cream or sulphur margin. Spores larger than type, probably specifically distinct.

1891. C. subdealbata Mass. (from the whitish colour ; sub, somewhat, dealbatus, whitened) a $b$.

Broadly effused, thin, determinate, cracking. $H_{y} m$. shiningwhite, thickly powdered, pallid fawn, or pale ochreous-whitish, mottled brownish-ochre or shaded olive, often with paler barren patches. Spores ochreous or pale dull yellow.

Bark, pine. Dec. $4^{\frac{3}{4}}$ in.

1892. C. stabulapis Karst. (from its being first recorded from an ox-stall ; stabulum, a stall) a $c$.

Effused, flaxy when young, fleshy-soft. $H_{y} \cdot m$. tubercular, tubercles collapsing, white, whitish-yellow, pale yellowish-brown or vinous-fuscous, white-pruinose; marg. definite flaxy or pulverulent, white.

Odour felid from its habitat. Squared wood, fir. $4 \frac{1}{4}$ in.

1893. C. Berkeleyi Mass. (after the Rev. M. J. Berkeley) a b c.

Effused, thick, cracked; marg. determinate, sometimes minutely byssoid. Hym. vinous-brown, somewhat yellowish, somewhat paler at the pallid marg. Sporis tawny.

Bark, decorticated wood. $t$ in. Superficially resembles 1850.

1894. C. Cookei Mass. (after Dr. M. C. Cooke) $a$.

Effused, fibrilloso-nembranous. Hym. ferruginous-olive or yellowish-brown, clouded darker; marg. byssoid, pallid. Spores pale dull yellow.

Rotting wood. $+\frac{1}{2}$ in.

1895. C. ochracea IIass. (from the ochreous colour) $a b$.

Broadly effused, submembranous, indeterminate, cracking. Hym. with a woolly appearance, subpapillose, sulphur-white to ochre, salmon-white when dry, or pale vinous-brown with a yellowish tinge; marg. broad, pulverulent, pale yellowish. Spores yellowish or pale dull yellow.

Wood, bark, elm. July. 5 in. Kesembles a thin form of 1890 . Must not be confoundel with 1874 or the var. ochroidea of 1890 .

1896. C. membranacea DC. (from the somewhat membranous substance) $a$.

Somewhat orbicular or effused, fragile, cracking and peeling off the host. Hym. dull ferruginous or opaque-yellowish, siennaumber ; mid. somewhat dark, constantly paler at the fibrillose marg. Spores ochre.

Forming patches a fool or more in diam. on walls, wood, paper, etc. $9^{\frac{3}{1}}$ in. 
1897. C. gelatinosa W. G. Sm., Aldridged gelatinosa Mass. (from its gelatinous substance) $a$.

Broadly effused, fleshy, subgelatinous, sometimes $\frac{1}{8}$ in. thick, becoming collapsed and rigid. $H_{y} m$. pallid becoming purplebrown; marg. determinate. Spore's olive.

Sawdust, extending for several inches, the irregularities of the hymenium due to the substratum. 5 in.

1898. C. byssoidea Karst. (from its, at first, flaxy condition; Gr. byssos, fine flax, cidos, appearance) $a c$.

Irregularly effused, at first grey, then compact; mid. fleshy. $H^{\prime} y^{\prime}$. light to ferruginous yellow, or colour of coffee and milk; marg. narrow, somewhat arachnoid or flaxy, whitish.

Forming a biscuit-coloured film sometimes a foot broad on fir-leaves, moss, earth, etc. ; frequent. $4 \frac{1}{2}$ in.

\section{EXOBASIDIUM Woron.}

(From the habit, the spores growing from basidia only with no true sporophore; $e x$, out of.)

Entophytes parasitic on living leaves and stems, which they deform and hypertrophy. Basidia crowded, cylindrico-clavate,
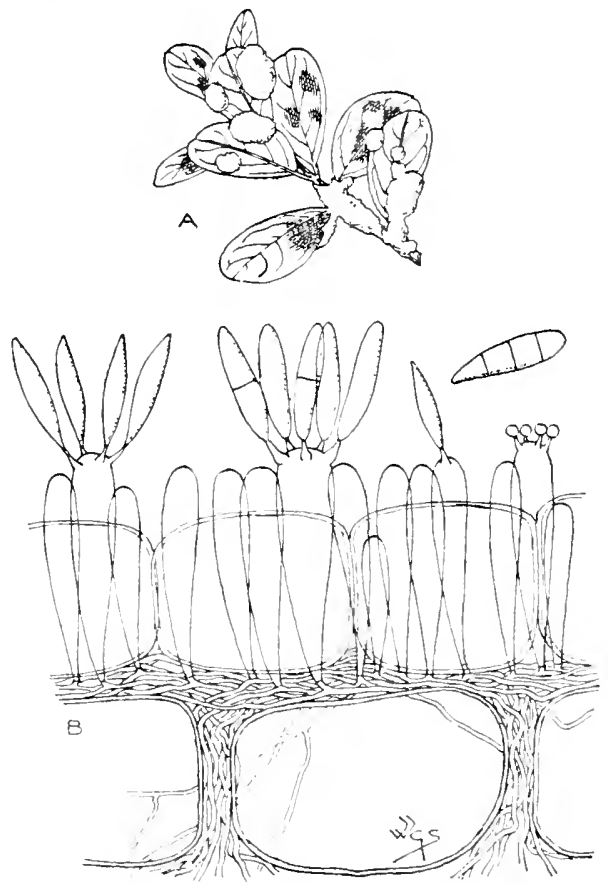

Fig. ro3.-A, Exobasidium Íaccinii Woron., one-half natural size. B, section through hymenium of ditto, showing basidia and spores, $\times 500$. 
normally tetrasporous, but spores sometimes varying in number from one to five. The hymenial cells are borne on vegetative hyphæ which grow in the cellular tissue of the host and form a loose stratum on its external surface. Spores ovate to elongate-oval, continuous, rarely becoming septate, colourless. (Fig. ro3.)

Most nearly allied to Corticium, but differing in the absence of a sporophore or subiculum and in being parasitic. Exoascus, an Ascomycetous fungus, sometimes grows in company with Exobasidium.

Species 1899, 1900

1899. E. Vaccinii Woron. (from its growth on Vaccinium and its allies) $a b c$.

Innate, effused, usually orbicular or elongate, often confluent. $H_{y} \cdot m$. on under surface of leaves and round stems, pale rose, covered with a white bloom.

June-Aug. $\frac{1}{2}$ in. Red or purple patches occur on the upper surface of the leaves, opposite to the portion occupied by the fungus beJow.

1900. E. Rhododendri Cramer (from its growth on Rhododendron) a $c$. Forming gall-like bullate excrescences, at first pale, then reddish and shining on stems, petioles and under surface of leaves.

On Rhododendron ferrugineum, $R$. hirsutum, $R$. Wilsonii. July. I in. Insects, mites, etc., are often found in the gall-like swellings of Exobasiatium.

\section{CYPHELLA Fr.}

(From the usual shape, like a small goblet, Gr. kuphos.)

Usually small, sometimes almost minute, somewhat membranous, cup-shaped, rarely plane, adnate from a central or oblique point, sometimes with a stem and pendulous. Hymenium within the cup or disc. Basidia tetrasporous, rarely bisporous. Spores subovate. (Fig. r 04. )

Closely allied to Solenia. C. perpusilla is intermediate. Owing to the strong external resemblance in many Cyphelle to certain small species of $P e z i z a$, a genus of Ascomycetous fungi, it is necessary to examine microscopically the hymenium of some of the species for the decisive characters of basidia or asci. C. faginca Lib. is British, but it is an Ascomycete.

Species 1901-1922

Stipitate. $1901-1905$

Sessile or subsessile. Spores hyaline. Spores coloured.
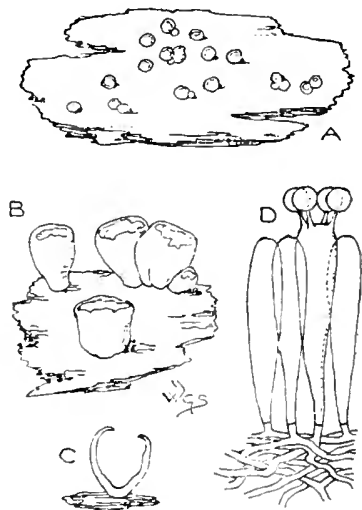

Fig. 104.-A, Cyphella brunnea Phill., $\times 2$. $E$, ditto with $C$, section, $\times$ ro. D, basidium and spores,
$\times 500$. 
1901. C. Pimii Phill. (after Greenwood Pim, mycologist) $a b$.

Fasciculate, cyathiform, erect or pendent, nembranous, soft, fragile, pubescent, white or pale yellow. Stem somewhat slender, crooked, enlarged upwards ; marg. somewhat incised.

Dead herbaceous stems in water, nettle, butter-bur. Feb. $\frac{1}{6} \times \frac{1}{8}$ in.

1902. C. capula Fr. (from its resemblance to a small bowl, capula) a $b c$.

Membranous, obliquely campanulate, smooth; marg. sinuate, irregular. Hym. even, whitish to yellowish, rarely greyish, brownish, or wholly white.

Dead herbaceous stems, twigs, branches, nettles, fennel, moss, strawberry, comfrey; frequent. Oct.-Aug. $\frac{1}{t}$ in.

1903. C. eernua Mass. (from the habit, bending downwards; cernuus, nodding) $a$.

Thin, obliquely campanulate, glabrous, stem elongate, equal, clear primrose-yellow, yellowish-white, somewhat sulphur or very pale yellow.

Bark, elder. $\frac{1}{4}$ in. Perhaps the same as 1902, but Massee considers it distinct in its more erect habit and larger spores.

1904, C. cuticulosa Fr. ex Berk. (from the membranous substance; cuticula, the outer skin) $a b$.

Membranous, at first oblong, then cup-shaped, smooth externally, diaphanous white.

Dead grass stems; rare. Feb. $\frac{1}{12}-\frac{1}{6}$ in.

1905. C. lacera Fr. (from the cleft margin ; lacerus, torn) a b c.

Membranous, cup-shaped, vertical or pendulous, torn into clefts, slightly striate and black fibrillose above. $H_{j^{\prime} \prime \prime}$. slightly wrinkled and whitish, grey externally.

Dead twigs. $\frac{1}{ \pm} \times \frac{1}{\frac{1}{x}}$ in.

1906. C. galeata Fr. (from its resemblance to a metal skull-cap or helmet; galea, a helmet) a c.

Membranous soft, subsessile, even; marg. entire, then torn. Hym. slightly wrinkled, white to grey-white or pale rose-brown.

Mosses; uncommon. Dec. $\frac{1}{4}-\frac{1}{12}$ in.

1907. C. Goldbachii Weinm. (after Carl Ludwig Goldbach) $a b c$.

Membranous, cup-shaped, sessile, lobed or cleft, externally villous. $H_{y} \prime m$. even, pallid, white, ivory, or white-ochre.

Dead leaves of Aira caspitosa, Carex paniculata. Feb. $\frac{1}{6} \times \frac{1}{12} \mathrm{in}$.

1908. C. museigena Fr. (from its habit, borne on mosses; muscus, moss, gigno, to bear) $a b c$.

Membranous soft, becoming .plane, subdimidiate, externally silky under a lens. Hym. slightly wrinkled, shining-white, becoming pale brown.

Various mosses; uncommon. Sept.-Jan. $\frac{3}{3}$ in. Varied in form, fanshaped, spathulate, etc. Sometimes stipitate, sometimes $\frac{3}{x}$ in. across. This is Cantharellus lazis Fr. 
1909. C. eatilla W. G. Sm. (from its shape, like a small bowl, catillus) a.

Submembranous, expanded; marg. crisped, undulate. Hym. veined, pale grey or faint claret-brown.

Often imbricate. Moss and dead leaves. Nov, $\frac{3}{\frac{3}{x}}$ in. Closely allied to 1908 .

1909a. C. griseopallida Weinm. (from the pallid grey colour; sriseus, grey) a.

Somewhat membranous, globose, then campanulate, sessile, floccose externally. Hym. even, smooth.

Elm, elder, honeysuckle, lime, pine. Dec. $\frac{1}{10}$ in. A supposed variety of this, found on dead Carex faniculata and described by Berkeley, is 1916.

1910. C. fulva Berk. \& Rar. (from the tawny colour; fuli'us, tawny) a $b c$.

Membranous, cup-shaped, deflexed, externally hispid-tomentose, rich tawny-brown.

Dead bark, alder, elm. Jan. $\frac{1}{1: 2}$ in.

1911. C. brunnea Phill. (from the brown colour; brunneus, brown) $a b$. Subgelatinous, sessile, cupulate, grey-white-pruinate at the incurved lacerate marg., dull brown or somewhat vinous umber, mouth oblique, base very dark. Hym. smooth, discoloured brown.

Wocd and bark, old elder trees. $\frac{1}{2}$ in.

1912. C. stuppea B. \& Br. (from the coarsely hispid coat, as if clad with tow : stuppius, made of tow) a.

Erumpent, sessile, cupulate, brownish, becoming white. Hy'm. fuscous.

Broom. Mar. $\frac{1}{2}$ in.

1913. C. alboviolaseens Karst. (from the pallid violet hymenium; albus, white) at $\dot{b} c$.

Cupulate, sessile or nearly so, densely villous, white. Hy'm. even, pallid, tinged with violet.

Gregarions, often proliferous. Wood, bark, twics, burdock, nettle, thistle, holly, broom, lilac, sloe, oak, rose, poplar, ferns, chips. Oct.-June. $\frac{1}{27}$ in.

1914. C. villosa Karst. (from the villose coating) a $b c$.

Sessile, spherical with a contracted mouth, whitish.

Stems, leaves, branches, potato, vine, elder, burdock, sow-thistle, butter-bur, log-wod, fennel, strawberry, Lathyrus fratusis, Chinopodium. Oct.-June. $5 \frac{1}{4} \mathrm{in}$. This and 1913 are much alike, both very while-villous externally, with hymenium often wholly hidden. Distinguished by the colour of the hymenium.

1915. C. dochmiospora B. \& Br. (from the oblique spores; Gr. dochmos, oblique).

Minute, cupulate, villous, mouth subconnivent, white.

Twigs, stems of herbacions plants, etc. Oct. $\frac{1}{2}-\frac{1}{45}$ in. 
1916. C. Berkeleyi Mass. (after the Rev. M. J. Berkeley) $a$.

Sessile, globose, then expanded, at length campanulate, minutely pilose, reddish-grey as is the even hymenium.

Dead Carex paniculata. Jan. $\frac{1}{12}-\frac{1}{24}$ in. Berkeley considered this to be a form of 1909a. Massee has made it a species, owing to a difference in size of the spores and the different habitat.

1917. C. Bloxamii Berk. \& Phill. (after the Rev. A. Bloxam) a b.

Floccoso-membranous, crenato-lobed, white villous externally. $H_{y} \prime m$. white, then pale yellow.

Furze. Mar.-April. Minute to $\frac{1}{16}$ in.

1917a. C. eyclas Cooke \& Phill. (from the shape, like the bivalve shell, Cyclas) a b.

Conchiform, dimidiate, attached on one side, pendulous, clad with flexuous hairs, whitish. Hym. smooth, very pale rosecolour.

Dearl wood. $\frac{1}{2}$ in.

1918. C. pallida B. \& Br. (from the pallid colour) $a b c$.

Sessile, at first orbicular, then irregularly lobed, plane, densely tomentose to slightly hispid. $H y m$. at length wrinkled, pallid ochreous.

Old stems of Clematis litalba. Nov.-April. $\frac{1}{12}$ to $\frac{1}{24}$ in.

1919. C. fraxinicola B. \& Br. (from its habitat, bark of ash, Fraximus; colo, to inhabit) $a b c$.

Orbicular, shortly villous, white. $H_{y} m$. light yellow, becoming fuscous. Spores pale olive.

Proliferous. Dec.-Feb. Winute to $\frac{1}{50}$ in.

1920. C. muscicola Fr. (from its growing on mosses; muscus, moss, colo, to inhabit) a $b$.

Membranous, subsessile, cupulate, nodding, externally slightly fibrilloso-striate; marg. slightly downy, repand, torn. Hym. even, whitish, rarely cinereous.

Mosses; rare. Dec.-Mar. $\frac{1}{4}$ in.

1921. C. ochroleuea B. \& Br. (from the yellow-white colour; Gr. ochros, pale yellow, leukos, white) a $b$.

Membranous, cupulate, villous above; marg. at length split. Hym. even, pale ochre, brighter than exterior.

Sticks, bramble; rare. July-Oct. $\frac{1}{8}$ in. Rarely stipitate.

1922. C. fuscospora Curr. ex Cooke (from the colour of the spores; fuscus, dusky).

Sessile, white tomentose; marg. connivent. Hym. becoming yellow. Spores subglobose, punctulate.

Bark. $\frac{1}{4}-\frac{1}{5} \mathrm{~mm}$. 


\section{SOLENIA Hoffm.}

(From the short pipe-like shape; Gr. solen, a pipe.)

Cylindrical, more or less contracted at the mouth, the external hairs in some species rough with particles of oxalate of lime, cavity covered with the hymenium. Basidia tetrasporous. (Fig. 105.)

The species are all minute, rarely exceeding $\frac{1}{1}$ in. in height. They grow, usually in crowded masses, on rotten wood. They are closely allied to Cyphiclla, but are distinguished by the densely crowded habit and by the cylinders never expanding.

Fries has placed this genus in the Poly'foracece, but each cylinder in Solmia is a distinct Peziza-like plant and not comparable with a mere tube of one of the Polyporaces. To be allied to the Polyporacce each cylinder should be lined with minute basidia-bearing tubes.

\section{Species 1923-1925}

Externally white.

1923, 1924

Externally ochreous.

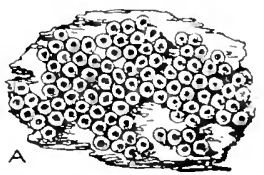

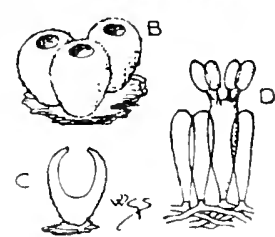

Fig. I05.-A, Solenic anomala $\mathrm{Fr} ., \times 5$. B, ditto with $C$, section, $\times 20$. D, basidium and spores, $\times$ 500. 1925

1923. S. maxima Mlass. (from the comparatively large size of the spores; maximus, largest) $a$.

Gregarious or subfasciculate, not crowded, subcylindrical, slightly contracted at base, externally villous, with slender aseptate hyphæ rough with minute particles of oxalate of lime, whitish or pale buff, ochreous when dry.

Forming patches $\frac{1}{2} \times \frac{1}{\frac{1}{x}}$ in. May. $\frac{1}{12} \times \frac{1}{24}$ in. Spores $5 \times 3 \mu$. This is 1925, var, cinerea Cooke.

1924. S. fasciculata Pers. (from its growing in groups or fascicles) $a b c$.

Gregarious, usually fasciculate, cylindrico-clavate, externally minutely silky and almost smooth, sometimes faintly shaded, brownish-yellow.

Willow, pine. Nov. - April. $\frac{1}{2+} \times \frac{1}{2 \pi}$ in. Spores $4 \mu$.

1925. S. anomala Fr. (from its superficially anomalous or perhaps scattered character) a $b \mathrm{c}$.

Usually densely crowded, pyriform, forming effused scattered patches; marg. incurved, pilose externally, varying from dull ochreous to ferruginous.

IBirch, willow, vine, oak, beech, dog-wood. Aug.-May. $\frac{1}{24} \times \frac{1}{24}$ in. Spores $7 \times 4 \mu$. Var. ochracea Mass. is somewhat smaller in size than type. 


\section{FAM. V. CLAVARIACEÆ}

Hymenium not distinct from the hymenophore. Amphigenous somewhat fleshy fungi, vertical, branched or simple, never coriaceous or incrusting. The branched species of Claz'aria must not be confounded with certain coriaceous species of Thelephori.

Species $1926-2000$

Key to THE Genera.

Branched, sponge-like. Branches compressed, flat or leaf-like gi SiPARAssis. Branched to simple.

Terete.

Stem not distinct from club

Stem distinct from club, not usually springing from a sclerotium

92 Clavaria.

Usually springing from a sclerotium.......... 96 PISTILLAK1.

Gelatinoso-cartilaginous, viscid ......................... 93 CALOCERA.

Hair-like ............................................. 94 Pterula.

\section{ICI. SPARASSIS Fr.}

(From its appearance, as if torn to pieces; Gr. sparasso, to tear to pieces.)

Fleshy, with flat leaf-like branches, composed of two plates, fertile on both sides. Basidia tetrasporous. Spores continuous, hyaline. (Fig. ro6.)

Species 1926, 1926a

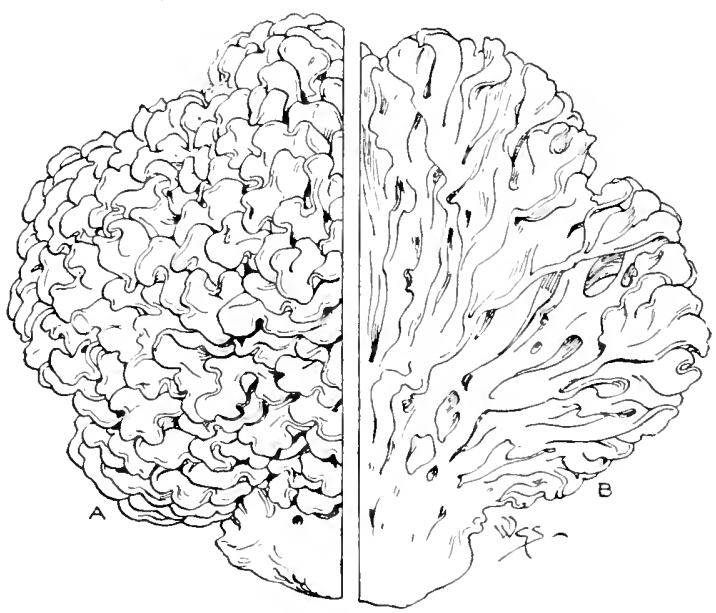

Fig. 106.-A, B, Sparassis crista Fr., entire and in section. One-half natural size.

1926. S. crispa Fr. (from its crisped or curled appearance) $a b c$. Whitish to pale yellowish. Very much branched, branches 
recurved, intricate at apex, the whole forming a large spongelike mass; stemless or with a short stout stem.

Edible. Odour very sweet of anise. Beside fir-trees and stumps, on dead fir-leaves, terrestrial on heaths. Aug.-Nov. I ft. I in. $\times$ I ft. I in. Sometimes attaining a diameter of I 8 in. and a weight of 2 or 3 lbs.

1926a. S. laminosa Fr. (from the leaf-like plates or layers of the entire plant ; lamina, a thin leaf) $a$.

Very much branched, branches laminar, spreading, yellowish straw-colour.

Woods. Sept. ro $\frac{1}{\frac{1}{4}} \times 7 \frac{1}{2}$ in. Stronger smelling and softer than 1926, never fimbriate at edges, lamine large, loose.

\section{ClaVARIA L.}

(From the club-like shape of some typical species; claz'a, a club.)

Fleshy, erect, branched or simple, branches somewhat round, with or without a distinct stem. Hymenium continuous, dry, even, homogeneous. Basidia tetrasporous. Spores coloured or uncoloured. For the most part terrestrial. (Fig. I07.)

Species 1927-1975

RAMARIE. Branched, branches attenuate upwards.

a. Lencospora. Spores white or nearly white.

Red, yellow or violet.

1927-1933

White or grey. 1934-1942

b. Ochrosporre. Spores pale yellow, ochreous or cinnamon.

Yellow, pinkish-yellow or dull ochreous.

1943-1949

Whitish, grey or riolet.

Growing on wood.

1950,1951

1952,1953

Syncoryne. Clubs almost simple, tufted at the base.

Reddish.

Yellowish or white.

1954-1956

$1957-1963$

Smoky or blackish. 1964

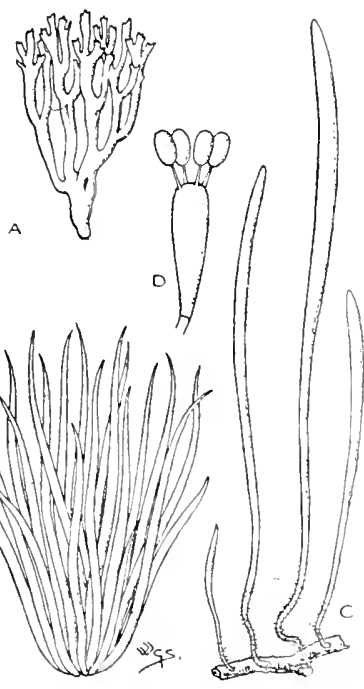

Fig. ro7.-A, Clavariz coralloidt's L.: B, C. Ecrmicularis Fr.; C, C. fistulosa Holmsk., one-third natural size. D, $C$. pistillaris $\mathrm{L}$., basidium and spores, $\times 33^{\circ}$.

Holocorine. Clubs entire or simple or nearly so, distinct at the base.

Whitish.

Yellow or pallid, becoming darker. 
There are analogous species in both sections of Ramaria which resemble each other in form and colour, but the Leucospore are somewhat brittle-soft and several of the species are considered edible, whilst the Ochrospore are spongy-tough and mostly bitter and unfit for food. The spore-colour, the taste and the substance, whether soft or tough, are salient characters.

\section{RAMARIE.}

a. Leucospore.

1927. C. flava Schæef. (from the frequent light yellow colour ; flav'us, light yellow) a $c$.

Very much branched; branches cylindrical, vertical, even, obtuse, yellow. Trunk thick, fleshy, white.

Edible. Woods, pine. $6 \frac{1}{5} \times 5^{\frac{3}{2}}$ in. Fragile. Nust not be confounded with 1943.

1928. C. botrytis Pers. (from the deep vinous purple colour of the ultimate branchlets; Gr. botrus, a cluster of grapes) $a b c$.

Wholly white, buff, salmon, yellow, brownish, etc., sometimes more or less claret-colour. Much branched; branches swollen, unequal, somewhat wrinkled, rose or brown-crimson at the apices. Trunk thick, unequal.

Odour very pleasant. Woods, mixed, pine, beech; uncommon. Ang.-Nov. $5 \times 5^{1}$ in.

1929. C. amethystina Pers. (from the amethyst colour) $a c$.

Very much branched; branches round, even, obtuse, equal, purple, bright purple with a bluish shade or purple-lavender. Said to produce violent colic.

Grassy woods and pastures; uncommon. Aug.-Oct. $2 \times 2 \frac{3}{x}$ in. Nyc. lavender-whitish or pale cobalt.

1930. C. fastigiata L. (from the parallel clustered and erect branches; in some cases the branches decrease in height outwardly, giving a sloping, obconic or gable-shape to the plant ; fastigin m, a slope, a gable) a $b$ c.

Very much branched; branches short, divaricate, slightly tough, brittle towards base, egg-yellow, apices light yellow to fuscous.

Usually cespitose. Slightly fragrant. Pastures; very common. Aug.Nar. $I^{7} \times 2 \frac{3}{4} \mathrm{in}$.

1931. C. muscoides L. (from a fancied resemblance to a tuft of moss; muscus, moss; Gr. cidos, appearance) $a b c$.

Twice or thrice forked, becoming slender, branchlets crescentshaped, acute, slightly tough, base tomentose, yellow.

Solitary. Pastures; frequent. Aug.-Oct. $3 \times 3 \frac{1}{3}$ in.

1932. C. kewensis Mass. (from its first observed place of growth, Royal Gardens, Kew) a.

Branches numerous, repeatedly and dichotomously divided, axils rounded, trunk very short, dull ochreous.

Odour strong, spicy, resembling 168 or 1437 . Rotten stumps, under holly. April. $1 \frac{7}{8} \times 2 \frac{3}{4}$ in. 
1933. C. curta Fr. (from its short stature; curtus, short) $a b$.

Very much branched, adpressed; branches short, crowded, obtuse; stem none, but there is usually a fleshy base, greenishyellow.

On the ground, on fir-leaves. Oct. $\frac{1}{4}$ to $\frac{3}{4}$ in.

1934. C. coralloides L. (from its appearance, like white coral; Gr. cidos, appearance) a b $c$.

Branches unequal, dilated upwards; branchlets crowded, acute; trunk somewhat thick, repeatedly and irregularly much branched, base not downy, somewhat fragile, hollow within.

Commonly cespitose. Edible : sold for food in markets of Italy. Shady woods; rare. Oct.-Nov. $5 \frac{1}{2} \times 5 \frac{3}{8}$ in. Sometimes faintly shaded buff.

1935. C. cinerea Pers. (from its ashy colour ; cinis, ashes) $a b c$.

Branches and branchlets thickened, irregularly shaped, somewhat wrinkled, subobtuse; trunk somewhat thick, short, very much branched, fragile, stuffed.

Woods, mixed, fir ; frequent. Aug.-Nov. $5 \frac{5}{8} \times 4 \frac{7}{8}$ in.

1936. C. umbrina Berk. (from the colour, usually pale brown, umbrinus) a.

Slightly branched; branches and branchlets cylindrical, obtuse, forked, pale umber to salmon-yellowish.

Woods, lawns, etc. July-Oct. $2 \frac{5}{\frac{5}{8}}$ in.

1937. C. cristata Pers. (from the crested apices of the branchlets; crista, a crest) $a b c$.

Branches dilated above, acutely incised, crested, tough, even, stuffed, white, crests at length becoming pale buff.

Somewhat crespitose. Woods, mixed, pine; frequent. June - Nov. $3 \frac{1}{8} \times 3 \frac{1}{8}$ in.

1937a. C. gigaspora Cotton (from the large spores; Gr. gigars, a giant) $a$.

Irregularly branched; branches sometimes palmate, erect, terete or compressed, much compressed at the acute axils, ultimate branches attenuate, apices blunt, greyish with a shade of yellow.

Caspitose, but distinct at the base, or solitary. Amongst moss on rocky heathy slopes. Nov. $1 \frac{1}{2} \times \frac{7}{3}$.

1938. C. rugosa Bull. (from the wrinkled stem; $r u g a$, a wrinkle) $a b c$.

Sparingly branched to simple, thickened upwards, irregularly shaped, tough, white.

Edible. Woods, beech, pastures; very common. Aug.-Dec. 5 in. Var. macrospora W. G. sm., C. macrospora Britz. Spores longer and somewhat larger than type. Type 7-10 $\mu$. Var. S-10 $\times$ I2-I4 $\mu$.

1939. C. Krombholzii Fr. (after Julius Vicenz von Krombholz) $a b c$. Slightly branched, even; branches somewhat compressed, obtuse, not wrinkled, fragile, white.

Crespitose. Old pastures. Sept. $2 \frac{3}{4}$ in. 
1940. C. crassa Britz. (from the thickness of the clubs; crassus, thick) $a$.

Much branched; branches obtuse, club-shaped, compressed. Base attenuate downwards, riolet.

Solitary. Not unlike 193S and 1939 in habit, but differing in colour and in the attenuate base. 3 in.

1941. C. Kunzei Fr. (after Karl Sebastian Heinrich Kunze) a b c.

Very much branched from the thin caspitose base; branches elongate, crowded, repeatedly forked, fastigiate, even, equal, axils compressed, somewhat fragile, white.

Woods; rare. Aug. - Sept. $2 \frac{1}{5} \mathrm{in}$.

1941a. C. subtilis Pers. (from its small and slender habit; subtilis, fine, slender) $a$.

Tough, thin, glabrous at base, of equal thickness throughout; branches few, dichotomously forked and somewhat fastigiate, white becoming yellowish.

Amongst grass. Sept. I $\frac{1}{5}$ in.

1942. C. pyxidata Pers. (from the shape of the palmate ends of the branchlets in typical examples, like a small box, frivis) a $c$.

Trunk thin, smooth, branched in an umbellate manner in typical examples, pallid to tan, somewhat rufescent, varying ivory and sulphur-white; branchlets taking a cup-like shape at the cuspo-radiate apices.

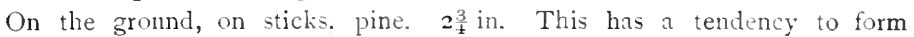
primary and secondary umbels, the latter with from 5 to 9 rays; when the branchlets are short the apices have a fancied resemblance to little boxes.

\section{b. Odhospore.}

1943. C. aurea Schæff. (from the golden colour; an $u m$, gold) a b $c$. Trunk thick, elastic, soft, divided into stout tense straight very dichotomous round obtuse somewhat toothed branches, buff, pale yellow, pale reddish, or pale salmon. Fleshy stem sometimes absent.

Woods, mixed, fir, beech. Aug.-Oct. $5 \times 5^{\frac{3}{4}}$ in. M Yust not le confounded wilh 1927.

1944. C. rufescens Schæff. (from the red apices of the branchlets; rufesco, to become red) a $b c$.

Trunk thick, elastic, tan or pale salmon, much branched; branches multifid, crowded, somewhat fastigiate, even, tan, pale salmon or pale vermilion, with red, crimson or vermilion apices.

On wood. Sept. $4 \frac{1}{x} \times 4$ in.

1945. C. formosa Pers. (from its beauty; formosus, beautiful) a $b c$.

Trunk thick, elastic, ivory, pale buff or pale olive-brown, darker below, much elongato-branched; branchlets obtuse, luminous 
yellow, salmon or salmon-buff, apices sometimes orange or sulphur.

Odour pleasant. Downs, commons, woods, pine; rare. July-Oct. $4 \stackrel{3}{\frac{3}{4}} \times 6 \mathrm{in}$.

1946. C. spinulosa Pers. (from the attenuate spine-like branchlets; spinulosus, spined) $a b c$.

Trunk short, somewhat thick, pale yellow; branches crowded, tense, straight, attenuato-pointed, yellow-orange, dull yellow or warm brown.

Woods, fir, beech, mixed. Sept. $5 \times 6$ in.

1947. C. abietina Pers. (from the habitat, under fir, abies) a b c .

Trunk short, somewhat thick, very much branched; branches crowded, branchlets tense, straight, acute, olive-ochre or olive-sienna-ochreous, becoming green where touched.

Taste bitter. Under fir, larch ; common. Aug.-Dec. $3 \times 3$ in.

1948. C. flaceida Fr. (from its flaccid habit) $a b c$.

Stem thin, very short, very much branched; branches crowded, even, unequal, acute, flaccid, often drooping, ochreous or buff. Amongst leaves and moss in woods, pine; rare. Oct. $3 \times 2 \frac{1}{1} \mathrm{in}$.

1949. C. erocea Pers. (from the saffron colour ; crocils, saffron) $a b$.

Ninute, thin; stem naked, pallid or yellow; branches and branchlets orange-scarlet.

Downs, gardens, on peat. Sept.-Feb. $\frac{3}{x} \times \frac{1}{2}$ in. The general appearance is not that of a true Basidionycete; it has somewhat the appearance of a slender minute branched Calocera.

1950. C. grisea Pers. (from the grey colour; sriseus, grey) a b c.

Trunk somewhat thick, firm, often rooting, whitish; branches and branchlets attenuate, somewhat wrinkled, fuliginouscinereous.

Woods; rare. Sept.-Oct. $4 \times 5$ in. Sometimes confounded with 1935, in which the spores are white.

1951. C. condensata Fr. (from the closely adpressed branchlets; condenso, to press close together) $a$.

Tufts very dense, usually without a trunk, very much branched from the base, smooth, tan-rufescent or salmon; branches tense and straight, crowded, even, fastigiate, twice or thrice-toothed, yellow.

On the ground, under trees. $3 \frac{1}{2} \times 4$ in.

1952. C. stricta Pers. (from the tense, straight habit ; stringo, to draw tight) $a b c$.

Trunk short, thin, very much branched; branches and branchlets tense and straight, even, crowded, adpressed, somewhat acute, pallid yellowish or whitish-yellow to buff-tan, fuscous where bruised.

Woods, pine, on and about stumps, sawdust; uncommon. Aug.-Nov. $4 \frac{1}{2} \times 3 \frac{1}{2}$ in. Often springing from cord-like mycellium. 
1953. C. exispula Fr. (from the slightly curled branchlets; crispulus, slightly curled) $a b c$.

Trunk very thin or obsolete, very much branched; branches flexuous, multifid, branchlets divaricate, tan to ochreous, buff or ochre, almost white below.

Base of trees, ash, elder; rare. Oct.-Dec. $3 \frac{1}{2} \times 2_{4}^{\frac{3}{4}} \mathrm{in}$.

\section{SYNCORYNE.}

1954. C. purpurea Müll. (from the colour ; purpureus, purple) a $c$.

$\mathrm{Cl}$. elongate, hollow, then compressed, acute, fragile, opaque, claret-brown and slightly purplish to purple.

Crespitose. Under pine; rare. Aug.-Oct. $3 \frac{5}{8}$ in.

1955. C. rufa Pers. (from the reddish colour; rufus, red) a $c$.

$\mathrm{Cl}$. stuffed, thickened, fusiform, sometimes bifid, rarely trifid, pale warm brown, rarely bright red or orange-vermilion, apices darker with age.

Cæspitose. Odour somewhat strong. Sept. $2 \frac{1}{x}$ in.

1956. C. rosea Dalman (from the rose colour) $a b c$.

$\mathrm{Cl}$. stuffed, fragile, pale to claret-pink or full rose-colour, apices attenuate or rounded, becoming yellowish with age.

Somewhat fasciculate. Pastures, amongst moss; rare. $2 \frac{1}{1}$ in.

1957. C. fusiformis Sow. (from the fusiform clubs) $a b c$.

Cl. somewhat fusiform, apex acute, sometimes bifid, trifid or toothed, firm, soon hollow, bright yellow, apices fuscous. Spores even.

Cæespitoso-connale. Amongst grass, woods, pastures, etc. ; common. Sept.Nov, 5 in. Sometimes intensely bitter. Often confounded with 1958 and 1959. Var. ceranoides W. G. Sm., Claziria ceranoides Pers., unequal, divided above, branched, upper part often antler-like.

1957a. C. Iuteoalba Rea (from colour of the club, yellow below, white above ; luteus, yellow, albus, white) $a$.

Cl. simple, stuffed, attenuate to base, apex acute to blunt, deep rich yellow, apex constantly white.

Fasciculate or scattered. Amongst grass and bracken. Sept.-Oct. $2 \frac{1}{8} \mathrm{in}$.

1958. C. dissipabilis Britz. (from the scattered habit; dissipabilis, dissipated, scattered) $a$.

Cl. elongato-clavate, apex rounded, rarely subacute, round or compressed and channelled, solid, collapsing, fragile, even, glabrous, deep orange yellow. Sfores spinulose.

Solitary or in small clusters. Short grass in pastures, roads, woods; common. Oct. $2 \frac{7}{5}$ in. Often confounded with 1957 and 1959.

1959. C. inæqualis Müll. (from the clubs being often unequal in diameter and more or less unevenly compressed ; inaqualis, unequal) a $b c$.

Cl. variable, usually simple and rounded above, narrow or 
inflated, fragile, stuffed, rarely bifid, yellow, usually paler than 1957 and 1958. Spores even.

Somewhat fasciculate or gregarious. Amongst grass, woods, pastures; very

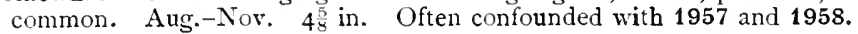

1960. C. argillacea Pers. (from the colour of clay, argilla) $a b c$.

$\mathrm{Cl}$. usually simple, variable, sometimes short and obese, rarely with short rounded obtuse branchlets, apex rounded, sometimes in hammer-form, whitish-yellow to brownish-buff or buffivory, rarely sulphur, base yellowish.

Usually fasciculate. Woods, amongst moss, under firs. Aug.-Nov. $4 \frac{5}{8} \mathrm{in}$.

1961. C. vermicularis Fr. (from a fancied worm-like shape; z'ermis, a worm) $a b$.

$\mathrm{Cl}$. stuffed, cylindrical, flexuous, acutely pointed, fragile, white, apices at length somewhat yellowish, base often faintly brownish.

Cæespitose. Amongst grass, pastures, roadsides; very common. May-Oct. $4 \frac{1}{4}$ in.

1962. C. fragilis Holmsk. (from its brittleness ; fragilis, fragile) a $b c$. $\mathrm{Cl}$. soon hollow, obtuse and rounded above, rarely forked, white, sometimes ivory, yellowish or pale brownish, white below.

Fasciculate. Pastures, roadsides; common. Aug.-Nov. 3 in.

1962a. C. Michelii Rea (after Piero Antonio Micheli) $a$.

$\mathrm{Cl}$. thin, cylindrical, hollow, apex acute, very fragile, yellow, white at the base.

Fasciculate. On the ground under trees, cherry. Sept. This plant should not have been named Michelii, as Micheli's plant, Nova Plant. Genera, t. 87, figs. 6, IO, is stout, obtuse and white. According to Fries this is C. fragilis.

1963. C. striata Pers. (from the striate clubs) $a$.

Cl. elongate, compressed, somewhat tortuoso-flexuous, somewhat longitudinally striate, subfuliginous or pale dull to dark brown.

Crespitose. Woods, amongst grass; rare. Sept. $3 \frac{5}{8}$ in.

1964. C. fumosa Pers. (from the smoky colour ; fimus, smoke) $a b$.

$\mathrm{Cl}$. even, usually tense and straight, somewhat compressed, fistulose, fragile, a pices rounded or acute, fuliginous or yellowish dull umber.

Fasciculate. Pastures: uncommon. Aug.-Sept. $4 \frac{1}{4}$ in.

\section{HOLOCORYNE.}

1965. C. canaliculata Fr. (from the small longitudinal channels on the clubs; canaliculus, a small channel) $a$.

Cl. rarely somewhat branched, slightly tough, hollow, very smooth, at length compressed or split longitudinally, white or shaded faintest salmon.

Solitary, sometimes in pairs, rarely in threes. On the ground. $3^{\frac{3}{4}} \mathrm{in}$. 
1965a. C. tenerima Mass. \& Crossl. (from its tender substance; tener, tender).

Cl. cylindrical, flexuous, somewhat attenuate above, base not narrowed, hyaline white.

Gregarious bit distinct at base. Amongst short grass. $1 \frac{1}{2}$ in. Resembles 1962 in habit.

1966. C. acuta Sow. (from the sharp pointed clubs; acutus, sharp) a $b c$.

Tense, straight with a translucent stem distinct from the opaque club, white.

Solitary. Sides of damp ditches, in garden pots; rare. Sept.-Nov. I in. Hardly a Clavaria. Sowerby's original drawing has the appearance of a Torrubia growing on a dead chrysalí, perhaps a Pistillaria.

1967. C. uncialis Grev. (from its usual length, about one inch, uncia) $a b c$.

Cl. stuffed, tough, tense and straight, obtuse, smooth, white, sometimes shaded faintest rose, becoming pale amber.

Dead stems of herbaceous plants, umbellifers; uncommon. May-Nor. I in.

1968. C. pistillaris L. (from the pestle shape; pistillum, a pestle) $a b c$.

Fleshy, stuffed, ovato-clavate, obtuse, light yellow to brownishtan or rufescent.

Solitary. Taste bitter; odour not unpleasant. Woods, amongst ferns and leaves, roadsides under hedges; uncommon. Sept.-Nov. I I $\frac{3}{8}$ in.

1969. C. ligula Schaeff. (from the shape, like a small tongue or strap, ligula) ac $c$.

Spongy-fleshy, elongato-clavate, obtuse, rarely slightly forked, villous at base, yellowish to pallid rufescent, rarely pale red.

Gregarious. Woods, fallen twigs and branches, amongst fir-leaves, moss. $4 \frac{1}{4}$ in.

1970. C. contorta Holmsk. (from the usually twisted shape; contortus, twisted) $a b c$.

Contorted, wrinkled, obtuse, sometimes attenuate upwards or broadly spathulate, rarely bifid, spongy-fleshy, stuffed, wateryyellowish to somewhat pale olive-yellow.

Solitary or crespitose. Wood, twigs, alder ; rare. Sept.-Nov. $\frac{3}{x}$ in.

1971. C. fistulosa Holmsk. (from the hollow clubs ; fistula, a pipe) $a c$. Slender, tense, straight, hollow, light yellowish, yellow, ivory-buff or buff to orange and rufescent, rooting and villous below.

Twigs, branches, etc. $9_{\frac{3}{4}}^{3}$ in.

1972. C. tuberosa Sow. (from the tuberous base) $a b$.

Tough, even, aculeate, hollow, tuberous and solid below, held to the matrix by flocci, ochre to somewhat pale salmon-umber.

Caspitose. Sticks, birch, bursting through the bark. Nov. 3 in.

1973. C. juncea Fr. (from the rush-like shape; juncus, a rush) $a b c$.

Filiform, flaccid, hollow, acute, pallid to rufescent or yellow, pale ochre or pale orange, base creeping, fibrillose.

Gregarious. Woods, amongst leaves, twigs and moss, fir, poplar, beech, oak. Sept.-Nov. 5 in. Sometimes hair-like in thinness, the base 
spreading over leaves. Sometimes certain individuals spring from a small orange-brown sclerotium as in Typhula or a white base 1 in. in diam. Must not be confounded with 1986 or 1992.

1974. C. ardenia Sow. (after Lady Arden) $a b c$.

Apex of the very hollow inflated $\mathrm{Cl}$. acute or rounded, then more or less collapsing and becoming depressed, brown-ochre with an olive shade or date-brown, base creeping, white tomentose.

Fallen branches, amongst fir-leaves, under laurels, on rotting hazel sticks; rare. Oct.-Dec. $S_{\frac{1}{2}}$ in.

1975. C. incarnata Weinm. (from the flesh colour; incarnatus, fleshcoloured).

Solid, cylindrical, flesh-colour, internally purple.

Gregarious. On the ground.

\section{CALOCERA Fr.}

(From the shape and colour, like beautiful horns; Gr. kalos, beautiful, keras, a horn.)

Gelatinoso-cartilaginous, horny when dry, somewhat cylindrical, simple or branched, viscid, without a distinct stem. Hymenium

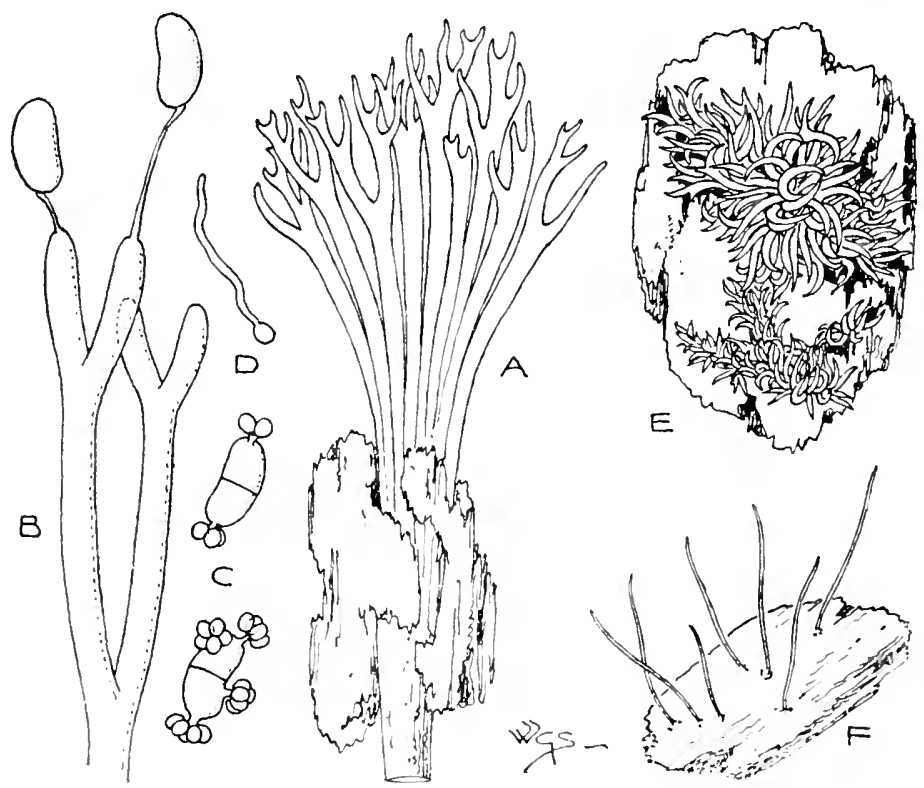

Fig. 108.-A, Calocera ziscose Fr., two-thirds natural size. B, basidia and spores of ditto: $\mathrm{C}$, spores germinating and producing sporidiola: $\mathrm{D}$, sporidiolum germinating: $\times 600$. E, C. comea Weinm.; F, C. stricta Fr. ; two-thirds natural size. 
amphigenous. Basidia furcate or bilobed, each lobe bearing a single one-spored sterigma. Spores oblong, curved, septate on germination, producing heads of ellipsoid sporidiola as in the Tremellinacee. (Fig. I08.)

Growing on wood.

Species 1976-1982

Branched.

Cæspitose.

1976, 1977

Simple, solitary.

1978, 1979

$1980-1982$

1976. C. viscosa Fr. (from its viscidity) $a b c$.

Branches more or less vertical, repeatedly dichotomous, orange, the rooting base paler.

Rotten wood, stumps, chiefly pine; very common. July-Dec. $2 \frac{7}{2}$ in. Base $2 \frac{1}{2}$ in.

1977. C. palmata Fr. (from the palmate branching) a $c$.

Tremelloso-tough, compressed, dilated upwards and more or less horizontally palmato-divided, orange-yellow or orange.

Wood. ${ }_{4}^{3}$ in.

1978. C. cornea Weinm. (from the horn-like shape; corm, a horn) $a b c$.

Simple, awl-shaped, sometimes bifid rarely trifid, connate at base, rooted, even, orange.

Dead wood, pine, oak, beech, willow, birch, old deal boards; frequent. June-Feb. $\frac{5}{8}$ in.

1979. C. corticalis Fr. (from the habit, piercing through bark, corti $x)$ a $c$.

Awl-shaped. Cl. somewhat distinct, soft, pellucid, pallid fleshcolour.

Dead bark. Dec.-Jan. $\frac{1}{6}$ in.

1980. C. stricta Fr. (from the habit, tense and straight, strictus) a b c. Linear, sometimes slightly spathulate, yellow, sometimes brownish below.

Ash, naked wood. Oct. I in.

1981. C. striata Fr. (from the strix on the clubs) $a b$.

Lanceolato-clavate, tough, acute, striate when dry, yellow.

Wood, prostrate trunks. March. $\frac{1}{4}$ in.

1982. C. glossoides Fr. (from the tongue-like shape; Gr. slossa, the tongue, eidos, appearance) $a b c$.

Clavate, subtremellose, obtuse, compressed, stem round, light yellow.

Oak. Dec. $\frac{1}{2}$ in. 


\section{PTERULA Fr.}

(From a fancied resemblance to a little wing; Gr. pteron, a wing.)

Subcartilaginous, filiform, arid, stemmed or stemless, branched in the two British species, subulate, apices attenuato-acute; branches

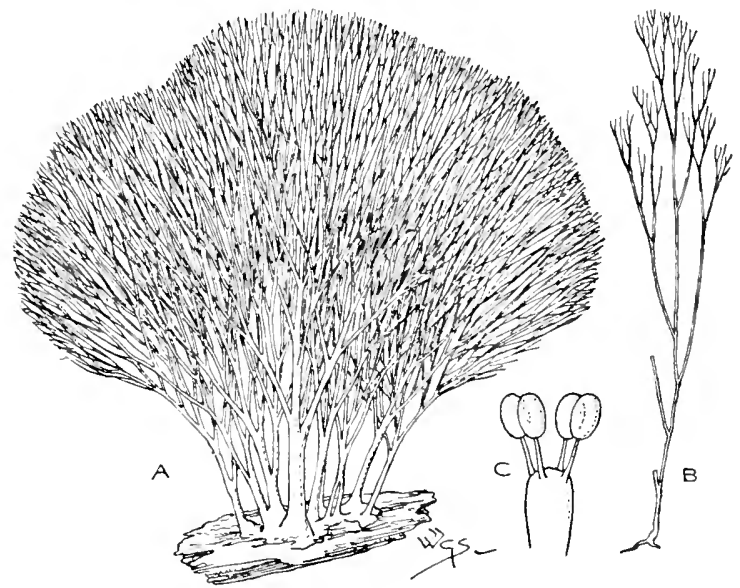

Fig. rog.-A, Pterula subulatı Fr., natural size; B, single branch of ditto. C, basidium and spores of $P$. multifida $\mathrm{Fr}, \times 500$.

somewhat anastomosing, pubescent then smooth. Basidic tetrasporous. When young the stems and branches are considerably thicker than when mature. (Fig. I09.)

The cartilaginous substance indicates a relationship with Tremellinacea and Calocera; the basidia and spores are typical of the Clazariacece.

Species 1983, 1984

1983. P. subulata Fr. (from the awl-shaped branches; subulu, an awl) a $c$.

Densely crowded, sparingly branched, tense, equal, the threadlike smooth branches multifid at the acute apices, whitishcinereous, becoming yellowish.

On wood, amongst pines, gardens. $\mathbf{I}_{4}^{\frac{3}{4}}$ in.

1984. P. multifida Fr. (from the many branchlets; multus, many, findo, to cleave) $a b c$.

Very much branched, very delicate, flaccid, branches tense, straight, usually hair-like, variable in thickness, apices attenuate, pointed, base sometimes thick or with several confluent stems, at first pallid whitish then dull pale yellowish or sulphurybrown.

Branches, leaves, bases of dead ferns, etc. Sept.-Nov. 2 in. 


\section{TYPHULA Fr.}

(From a fancied resemblance in some of the species to a minute $T y$ pha or reed-mace.)

Small thread-like fungi with a long or short filiform-stem, heterogeneous from the linear or slightly inflated club, often springing from a small sclerotium. Hymenium waxy. (Fig. i Iо.)

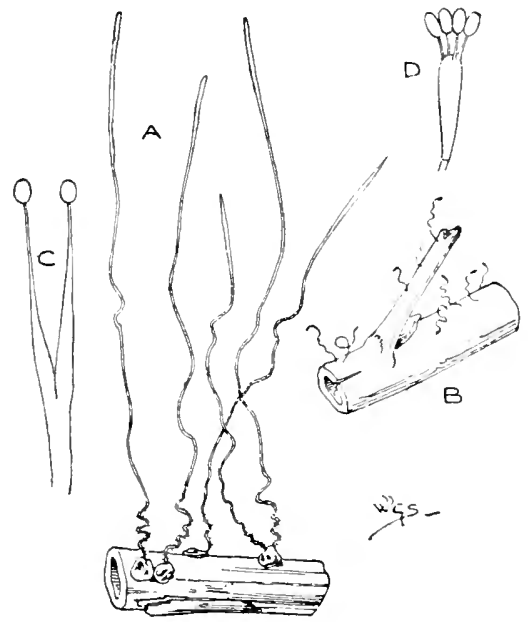

Fig. r10.-A, Typtula phacom-hiza Fr., one-half natural size. B, T. gracillina White, one-half natural size. C, basidium and spores of $T$. sracilis Berk. \& Desm. ; D, ditto of $T$. Erythropus Fr.; $\times 500$.

Growing on twigs, leaves, etc., rarely terrestrial, but the sclerotia will readily grow in earth if planted.

Species $1985-1995$

a. Phacorrize. Springing from a sclerotium. 1985-1989

b. Leptorrhize. Sclerotium absent.

$1990-1995$

The characters as given by Fries are far from permanent; all the species of $T_{y}$ phula may grow with or without a sclerotium. A sclerotium sometimes occurs in the allied genera, Claviaria and Pistillaria.

\section{a. Phacorrhiza.}

1985. T. erythropus Fr. (from the red stem; Gr. cruthros, red, pous, a foot) a $b c$.

Cl. linear or nearly so, cylindrical, smooth, white. St. elongate, filiform, usually bent or variously twisted, finely pubescent under a lens, crimson-brown. Sil. $\frac{1}{20}$ in. or less, depressed, 
sometimes hidden beneath a thin bark or membrane and easily overlooked, brown, dark-brown when dry, sometimes absent.

Leaves, sticks, poplar, alder, ash, sunflower; common. Sept.-Nor. I in. The sclerotium is Scleretium scutellatum A. \& $\mathrm{S}$.

1986. T. phacorrhiza Fr. (from the lentil-like base; Gr. phakos, a lentil, rhiza, a root) a $b c$.

Cl. linear, cylindrical, pallid to ivory and orange. St. linear, confluent with club, elongate, flexuous, yellowish, pilose at the sometimes fuscous base. Scl. $\frac{1}{5}$ in. or less, pale brown or buff, reddish-brown when dry.

Woods, hedgerows, gardens, herbaceous stems, leares, moss, plane, hazel, alder, ash, apple, sometimes on earth; uncommon, but sometimes found in considerable abundance in patches. Oct.--Nov. $4 \frac{1}{1} \mathrm{in}$. Must not be confounded with 1973 or 1992 . The sclerotium is Sclerotiun scutellatum A. \& S. and S. complanatum Tode.

1987. T. incarnata Fr. (from the flesh colour; incarnatus, fleshcoloured) a $c$.

$\mathrm{Cl}$. cylindrical, obtuse, rarely forked, flesh-colour or white, more or less tinged with salmon-pink above. St. continuous with the club, pilose downwards. Scl. $\frac{1}{5}$ in. or less, compressed, fuscous.

Dead herbaceous stems; uncommon. 3it in. Closely allied to 1986. The sclerotim is Sclerotizm fulzum A. \& S.

1988. T. gyrans Fr. (from the twisted habit; gyro, to turn in a circle) a $b c$. White.

Cl. cylindrical. St. filiform, gyroso-undulate, pubescent. $\mathrm{Scl}$. $\frac{1}{30}$ in., pallid to orange and brown, becoming fuscous.

Straw, twigs, leaves, alder, poplar ; uncommon. Oct. $\frac{1}{2}$ in. The sclerotium is Sclerotium comflanatum Tode.

1989. T. muscicola Fr. (from its habit, living on mosses; muscus, moss, colo, to inhabit) a c. White.

Cl. elongate, cylindrical, obtuse. St. thin, smooth. Scl. $\frac{1}{16}$ in., even, pallid.

Larger mosses; uncommon. $\mathbf{I} \frac{1}{8}$ in.

\section{Leptorrhize.}

1990. T. Grevillei Fr. (after Dr. Robert Kaye Greville) a $b c$. White.

Cl. thickened, obtuse. St. capillary, pilose white.

Dead leaves: frequent. Oct. $\frac{1}{1}$ in. Only clistinguished from 1988 by the absence of a sclerotium.

1991. T. gracillima White ex B. \& Br. (from the very slender habit; gracilis, slender). White.

Cl. elongate. St. very slender, curved, smooth.

Various herbaceous plants. $\frac{1}{t}$ in. 
1992. T. filiformis Fr. (from the thread-like stem; filum, a thread, forma, form) $a b$ c.i

$\mathrm{Cl}$. elongate, thickened, smooth, whitish. St. often branched, decumbent, date-brown.

Dead leaves, holly, Comptonia astlinifolia, matting; rare. Nov. $2 \frac{3}{g}$ in. Sometimes springing from a very small sclerotium. II ust not be confounded with 1973 or 1986.

1992a. T. tenuis Fr. (from its attenuation; tenuis, thin): $a$.

Cl. simple, glabrous, hair-like, thickening upwards, brown-black.

Gregarious. On rotting wood in cellar. $\frac{1}{5}$ to $\frac{1}{x}$ in. "It resembles a little black hair thickening upwarls," Sowerby.

1993. T. gracilis Berk. \& Desm. (from the slender habit; sracilis, slender) a b. Pallid.

Cl. simple or forked, acute, pubescent. St. short, distinct, smooth or as club.

Putrid leaves; uncommon. Dec. $\frac{3}{15}$ in. Has the appearance of Isaric fuberula, but bears bisporous basidia.

1994. T. pusilla Schrcet. (from its very small size; pusilhıs, very small) a. White.

Cl. linear, usually drooping. St. linear.

Horse-tail, plane, willow, wych-elm, alder. $\frac{1}{1}$ in. Leaves often covered with minute sclerotia, from which the Typhulu sometimes springs.

1995. T. translucens B. \& Br. (from its translucent substance) $a$. Pellucid white.

$\mathrm{Cl}$. irregular, somewhat obovate. St. short, thickened upwards.

Terrestrial. Oct. "Not a fungus," Massee, Brit. Fung. Fl. vol. i. p. 9 I. "Resembling somewhat a prematurely dried Myxogast, but a true Basidiomycete," B. \& Br. in Ann. Magr. Nat. Hist. ser. 4, vol, xvii. p. I3s.

\section{PISTILLARIA Fr.}

(From the form, like a pestle, pistillum.)

Small, club-shaped or attenuate downwards into a short continuous stem, rarely forked, cartilaginous and rigid when dry, sometimes springing from a sclerotium. (Fig. I I I.) Species 1996-2000 1996. P. tenuipes Mass. (from the thin stem; tenuis, thin, pes, a foot) $a$.

Cl. inflated, obovate, wrinkled, pallid clay-colour or ochre. St. slender, flexuous, somewhat distinct from the club, ivory.

Bare heathy ground, charcoal heaps. Nov.-Jan. I in. Clavaria tenuipes B. \& Br.

1997. P. micans Fr. (from its glistening surface; mico, to glisten) $a b c$.

Cl. inflated, obovate, obtuse, smooth or slightly pruinose, glistening rose-colour, rarely purplish. St. short, attenuate, white.

Dead stems and leaves, thistles, nettles, spurge, Artemisia ; rare. July. $\frac{1}{8}$ in. 
1997a. P. fulgida Fr. (from its shining colour ; fulgeo, to shine) $a$.

Cl. lanceolato-clavate, subacute, white or pale orange below, yellow or orange above.

"On the bractere of Dipsacus filosus," Sowerby. $\frac{1}{8} \mathrm{in}$.

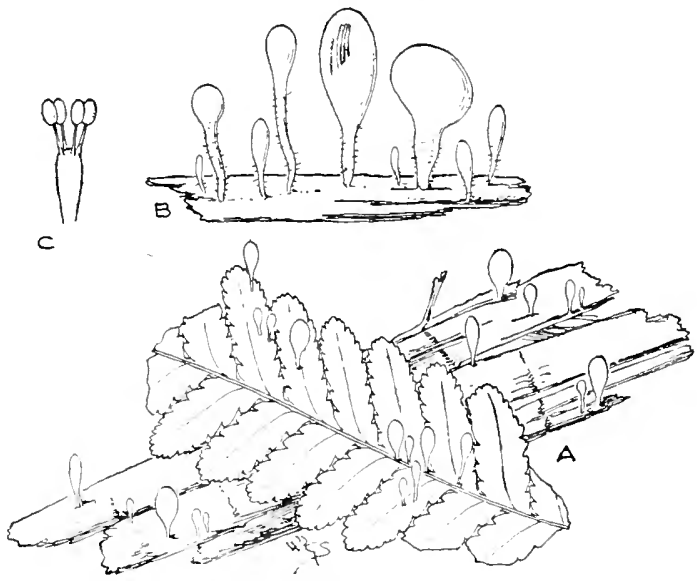

Fig. I1 1.-A, Pistillaria fubcrula Perk, natural size. B, ditto, $\times 4$. $c$, basidium and spores, $\times 500$.

1998. P. eulmigena Fr. ex Mont. (from its habitat, grass-stems; culmus, a haulm, signo, to bear) it $b c$. White.

Cl. ovato-clavate, obtuse, hyaline-pellucid. St. distinct, very short, cartilaginous when dry.

stems and leaves of grasses, Secale, Triticum, Dactylis glomerata. Oct.Nov. $\frac{1}{8}$ in.

1999. P. quisquiliaris Fr. (from its habitat, sweepings, rubbish, etc., quisquilice) a $b c$. Whitish.

Cl. ovato-clavate, somewhat compressed, rarely bifid, soft when fresh, rigid when dry. St. short, often springing from a sclerotium.

Fern-stems, oak, wych-elm, grass, willow-herb, Chworophyllum. April-Nov. $\frac{1}{5}$ in.

1999a. P. ovata Fr. (from the ovate shape) $a$.

Cl. obovate, ventricose, subdepressed, hollow, white. St. very short, glabrous, pellucid.

Dead stems and leaves, birch, bramble, poplar, elm, willow. $\frac{1}{5}$ in.

2000. P. puberula Berk. (from the tomentose stem; pubes, downy) a $b$ c. White.

Cl. ovate, ventricose. St. short, distinct, attenuate upwards, pellucid, fibrous, tomentose.

Ferns, stems and leaves of herbaceous plants ; rare. Sept.-Oct. $\frac{1}{12}$ in.

2000a. P. pusilla Fr. (from its very small size; pusillus, small) $a$.

Small, linear, attenuate upwards, stem scarcely distinct, white.

Dead leaves. Feb. $\frac{1}{1-\frac{3}{8}}$ in. 


\section{FAM. VI. TREMELLINACE $Æ$}

Entire fungus homogeneous, gelatinous, collapsing when dry, retaining its form when moistened, traversed internally by branched hyphæ which terminate in basidia at the periphery. Basidia variable in form, elongate or fusoid, transversely septate or continuous, undivided or forked at the apex, or subglobose and longitudinally divided and bearing 2 to 4 sterigmata. Spores hyaline, from globose to sausage-shaped and curved, continuous or septate, often becoming variously septate on germination and producing sporidiola of different forms.

Species 2001-2041

\section{Key to the Subfamilies and Genera.}

Basidia elongate or fusoid, simple, transversely septate ...

Attached broadly.

Subfamily I. AURICULARIOIDEE.

Attached by a point

97 AURICULARIA.

Basidia subglobose at maturity, longitudinally quadripartite ..........................Subfamily II. EutrenellinoideÆ.

Cup-shaped, truncate or irregularly lobed ... 99 ExiniA.

Pulvinate, gyrose $\ldots \ldots \ldots \ldots \ldots \ldots \ldots \ldots \ldots \ldots$ IOO Ulocolla.

Brain-like or lobed ........................... IOI TREMELLA.

Convex, with a central hard nucleus ........ IO2 NÆ.1ATELIA.

Erect, spathulate ........................... 103 Gyrocephalus.

Basidia cylindrical or clavate .............. Subfamily III. DACRYOMYCETOIDEÆ. Small, pulvinate, gyrose ....................... IO 4 DACRYOMYCES.

Irregularly cup-shaped, sessile ................ IO4A FEMSJoxia.

Versiform, substipitate ..................... 105 GUep1N1A.

Stipitate, hymenium on expanded apex ..... I06 DITlOLA.

\section{Subfamily I. AURICULARIOIDEA}

Basidia elongate or fusoid, simple, transversely septate.

XCVII. AURICULARIA Bull.

(From a fancied resemblance in some of the species to an ear, auricula.)

Subcoriaceous or gelatinous when moist, broadly attached. Hymenium remotely and reticulately costate or plicate, collapsing when dry. Basidia cylindrical, transversely tri- quadri- or quinqueseptate, each joint or cell producing a single, slender sterigma from 
its apex. Spores oblong, curved, producing on germination a branched promycelium bearing several strongly curved sporidiola. (Fig. 1 I 2.)

A genus of fungi greatly resembling Stereum in habit, and perhaps more nearly allied to the Thelephoracee than the Tremcl-

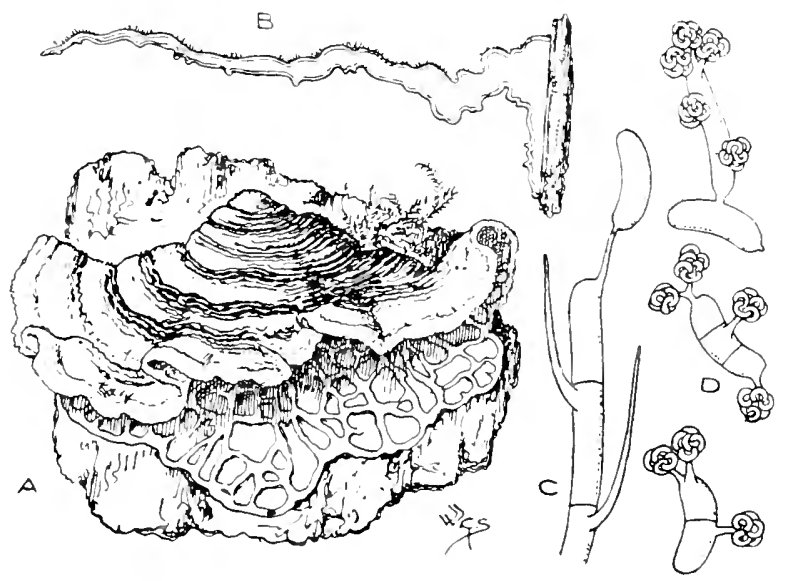

Fig. I12.-A, B, Auricularia mescnteriau Pers, entire and in section. One-half natural size. $C$, basidium and spore; $D$, spores germinating and producing sporidiola. $\times 500$.

linacce; the fructification is, however, very different from that of Thclephora, while the strigose cartilaginous pileus differs from typical Tremellinacea. The next genus Hirneola is clearly allied to Auricularia in habit, substance and fructification.

Species 2001, 2002

2001. A. mesenterica Pers. (from a fancied resenblance to the mesenteric membrane) $a b c$.

$P$. resupinate, then reflexed, entire or slightly lobed, villous, yellowish, often green at base from algal growths; $z$. brownish. Hyr. costato-plicate, brownish-violet, livid-purple or grey-liver.

Taste insipid; odour none. Trunks, stumps, beech, elm, walnut, vine, chestnut, furze, broom, yew ; frequent. Jan.-Nov, $\mathcal{S}_{2}^{1}$ in. Sometimes terrestrial, springing from buried wood, and then sometimes not unlike Sticta pulmonaria in appearance.

2002. A. lobata Sommerf. (from the lobed pileus) $a b c$.

$P$. effuso-reflexed, much lobed, strigose or velvety, warm yellowishbrown; z. darker, often green at base from algal growths. $H y m$. broadly reticulato-ribbed, fuscous-livid.

Bark of trees, elm; not uncommon. $4 \frac{5}{8}$ in. Closely allied to, if not identical with the last; the fruit is the same. 


\section{HIRNEOLA Fr.}

(From the jug-shape when young; hirnce, a jug.)

Gelatinous or cartilagineo-gelatinous, soft and tremelloid when moist, cup- or ear-shaped. Hy'menium gelatinous, even or plicate, barren under-surface minutely velvety, attached by a point, coriaceous

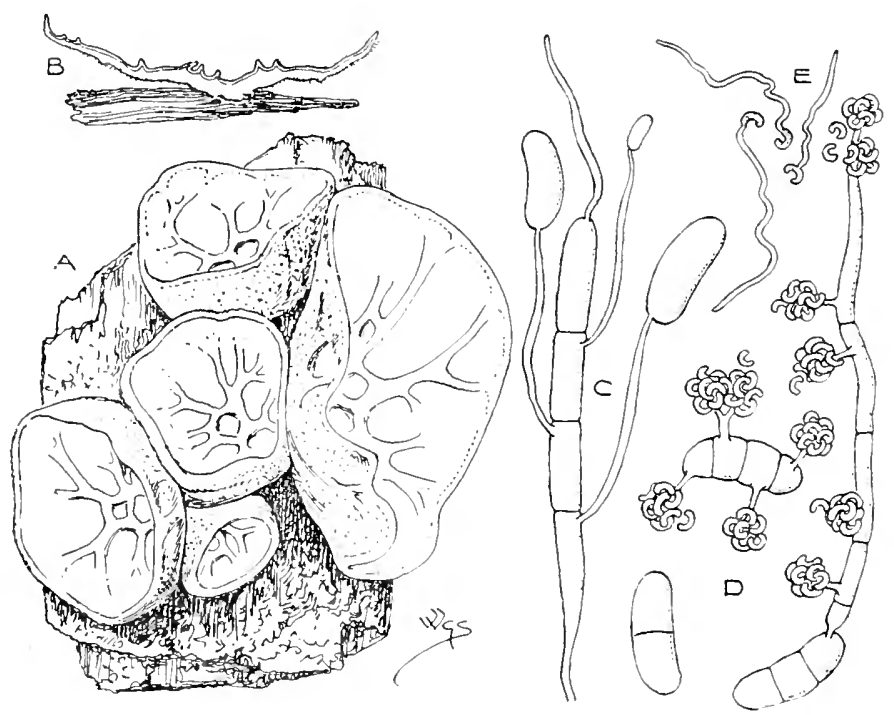

Fig. I13.-A, B, Hirneola Auricula-Fude Eerk, entire and in section. One-half natural size. C, basidium and spores; D, spores germinating and producing sporidiola: E, sporidiola germinating. $\times 500$.

and membranous when dry, reviving with moisture. Basidia rodshaped or fusoid, transversely septate, each cell bearing a single monosporous sterigma. Spores oblong, curved, becoming $\mathrm{I}-3$ septate and on germination producing minute sporidiola, sometimes on septate promycelium. (Fig. II3.)

2003. H. Auricula-Judæ Berk. ("Jew's-ear") a $b c$.

$P$. thin, concave, flexuous, somewhat veined, tomentose, brown flesh-colour to reddish-brown or blackish. Hy'm. venosoplicate, glossy salmon, warm brown or reddish, purple-black when dry.

Crspitose. Trunks, elder, elm, willow, walnut, oak, holly, Robinia; frequent. Jan.-Dec. $3 \mathrm{in.}$.I Iuch like Fesiat vesicalosa in appearance when young. Formerly used as a remedy for dropsy. Eaten by inhabitants of Ukraine. 


\section{Subfamily II. EUTREMELLINOIDEÆ}

Basidia subglobose, at maturity longitudinally quadripartite and producing at the apex two to four elongate sterigmata.

Species 2004-2029

\section{EXIDIA Fr.}

(From the exuding receptacle; Gr. cxidio, to exude.)

Gelatinously distended, tremelloid, irregularly gyrose and lobed, submarginate or effused, often papillose, when young cup-shaped and truncate. Basidia globose or oroid, typically longitudinally four-

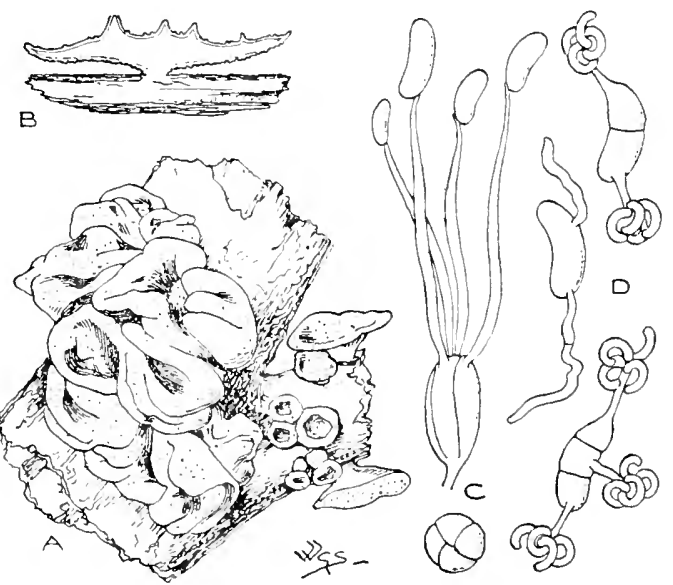

Fig. I14.-A, B, Exidiz glandulosa Fr., entire and in section. One-half natural size. C, basidium and spores; D, spores germinating and producing sporidiola. $\times 500$.

partite, bearing four elongate stont sterigmata. Sfores reniform, for a long time continuous, becoming $\mathbf{I}$ - to many-septate on germination, each cell or loculus of the spore giving origin to a very short promycelium bearing a crown of strongly-curved sporidiola. (Fig. I I 4.)

a. Auriculine, Pezizoid.

Species 2004-2007

b. Spicularice. Somewhat plane, not lustrous beneath, glandular, here and there gyrose.

2006, 2007

\section{Auriculina.}

2004. E. truneata Fr. (from its appearance, as if cut short; trunco, to maim, shorten) a $c$. Soft, distended, shining black.

Disc truncato-plane, glandular, at length cavernous, rough with dots beneath. St. very short.

Bursting through bark, lime, willow. I)e. $1 \frac{7}{3}$ in. Like 2006 on a small scale. 
2005. E. recisa Fr. (from its appearance as if cut back; recido, to cut short) $a b c$.

Very soft, warm yellow-brownish or brownish-vinous. Disc truncato-plane, ribbed, papillose, somewhat repand, rough with dots beneath. St. very short excentric.

Branches, willow, poplar, plum; common. Sept.-Dec. II in.

\section{b. Spicularice.}

2006. E. glandulosa Fr. (from the glandular hymenium) $a b c$.

Effused, thick, somewhat plane, undulate, sometimes pendulous, and 8 to 10 inches long, the upper hymenial surface papillose and somewhat veined, the lower surface next to the matrix crape-like to the touch and lustreless, black, blackish, purpleslate or olive-blackish.

Tasteless. Branches, oak, willow, Ailanthus, lime, alder, walnut. Ang.-Mar. $5 \frac{1}{2}$ in. When young somewhat cup-shaped and like one of the Fesizc or Bulsaria inquinums in appearance. Translucent when quite fresh. "Witch's Butter."

2007. E. albida Bref. (from the whitish colour; alblls, dead white) $a b c$.

Tough, undulate, subgyrose, pruinose, white or faintly shaded ivory or lavender.

Branches, birch, beech, alder, pine, hawthorn. Sept.-IIay. $1 \frac{1}{2}$ in.

\section{Ulocolla Bref.}

(From its glue-like consistency and appearance;

Gr. oulos, shaggy, kolla, glue.)

Sporphore convex, pulvinate, gyrose, cerebriform, gelatinous, rather large. Basidia globose, soon longitudinally quadri-partite.
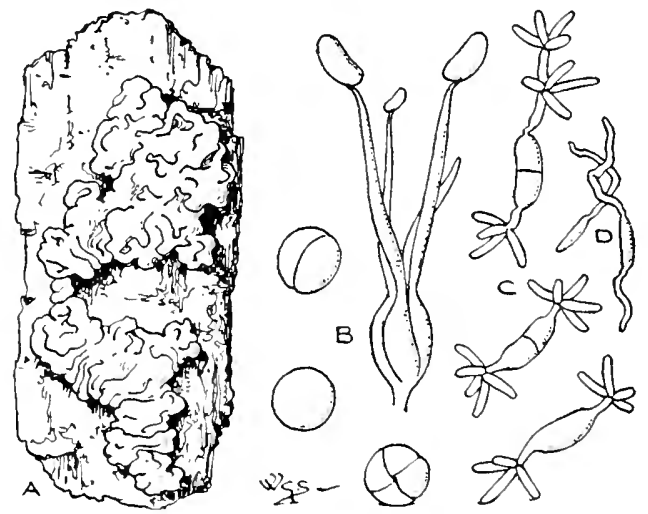

Fig. 115.-A, Llocolla saccharina Bref. One-half natural size. $B$, basidia and spores; C, spores germinating and producing sporidiola; D, sporidiola germinating. $\times 500$. 
Sterigmata elongate, thick. Spores for a long time continuous, then uniseptate, reniform, each loculus on germinating giving origin to a very short promycelium bearing at its apex straight rod-like sporidiola. (Fig. II 5.)

Species 2008, 2009

2008. U. saccharina Bref. (from the colour, like one kind of sugar, saccharon) a bc.

Effused, tubercular, gyroso-undulate, thick, sprinkled with a few minute obtuse evanescent papillæ, tawny cinnamon.

Branches, larch, pine ; rare. Nov. 3 in.

2009. U. foliacea Bref. (from a fancied leafy or lamellate appearance; folizm, a leaf) $a b c$.

Flaccid, even, diaphanous, undulate, plicate at base, cinnamonsalmon or vinous-buff, sometimes shaded yellowish.

Cæspitose. Stumps, logs, rails, etc., pine, oak, amongst fir-leaves, spreading to the ground. Aug.-Jan. $3 \frac{1}{1}$ in.

\section{TREMELLA Dill.}

(From the jelly-like tremulous substance.)

Pulvinate or effused, brain-like or lobed, gelatinous, tremelloid, immarginate, generally smooth. Basidia globose, sometimes bifid or
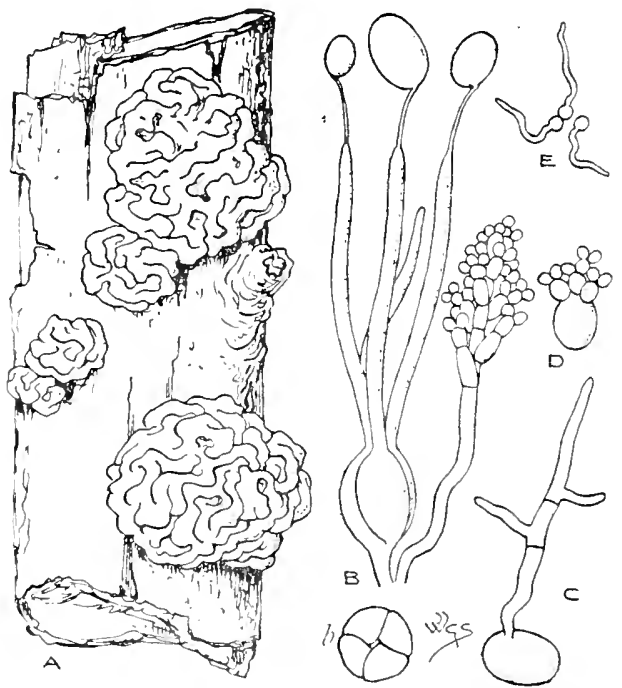

Fig. I16.-A, Tremella lutescens Pers. One-half natural size. $B$, basidium, with surface view, $b$, spores and conidia; $c$, spore germinating and producing hyphæ; D, ditto, producing sporidiola; $\mathrm{E}$, sporidiola germinating. $\times 500$.

trifid, but usually longitudinally quadripartite, each quadrant of the basidium elongating into a long, stout sterigma. Spores subglobose, 
continuous; on germination a tube is formed that is covered with broadly elliptical sporidiola. Conidia when present, as in 2012, 2013 and 2021, subglobose, racemose in sporiferous conceptacles in the sporophore. (Fig. I 6. .)

Species 2010-2024.

a. Mesentcriformes. Cartilagineo-gelatinous; lobes large, foliaceous, not pruinose with spores, cæspitose.

$2010-2012$

b. Cerebrina. Lobes short, contorted, brain-like, pruinose with white spores.

c. Crustacia. Effused, applanate.

$2013-2015$

d. Tuberculiformes. Small, suberumpent.

$2016-2018$

$2019-2024$

\section{a. Mesenteriformes.}

2010. T. fimbriata Pers. (from the somewhat fimbriate edges of the lobes) $a b$.

Erect, corrugate, lobes flaccid, blackish-olive to brown-black; marg. undulato-fimbriate or incised.

Dead branches; rare. $4_{ \pm}^{3}$ in.

2011. T. frondosa Fr. (from a fancied leaf- or lamella-like appearance in the lobes; frondosus, leafy) $a b c$.

Even, gyroso-undulate, plicate at base, diaphanous, pale yellowishbuff, shaded salmon or vinous in places, base brownish-vinous.

Stumps, roots of living trees, oak, beech, hornbeam. July-Nov. 9 in.

2012. T. lutescens Pers. (from the yellowish colour; Intens, yellow) ac.

Tremulous, soft, inclining to fluid, undulato-gyrose, lobes crowded, entire, at first whitish then pale yellowish.

Stumps, oak; frequent. Aug.-Feb. $\mathbf{I}_{\frac{3}{8}}^{3}$ in.

\section{b. Cercbrine.}

2013. T. mesenterica Retz. (from a fancied resemblance to the mesenteric membrane) $a b c$.

Expanded, somewhat tough, plicato-undulate, gyrose, changeable in form, smooth, vivid yellow to rich orange.

Dead branches, sticks, rails, oal, alder, beech, furze, broom, iry; common. Jan.-Dec. $3^{\frac{1}{2}}$ in.

2014. T. intumescens Sm. (from the swollen habit; intumesco, to swell up) $a b c$.

Rounded or conglomerate, somewhat tortuous and lobed, obsoletely punctate, soft, brown.

Subcrspitose. Fallen trunks in a wet state, beech; rare. Jan. $2 \frac{1}{2}$ in. Becoming a thin black membrane when dry.

2015. T. vesicaria Sm. (from the swollen, bladder-like lobes; vesica, a bladder) a $b c$.

Erect, firm, gelatinous, gyrose, much waved and wrinkled, gelatinous within, dull biscuit.

On the ground; rare. Sept. $4 \frac{5}{8}$ in. Often much inflated and soon falling over. 


\section{c. Crustacie.}

2016. T. viseosa Berk. (from the viscid substance) $a c$.

Effused, thin, resupinate, obscurely veined, undulato-uneven, following inequalities of matrix, white, becoming hyaline or translucent yellowish-white.

Dead branches, rotten wood, oak; common. Jan.-Dec. 4 in. Collapses completely in drying, leaving only a purple-grey stain on the bark.

2017. T. epigæa B. \& Br. (from its habitat, the earth; Gr. exi, upon, $g e$, the earth) $a b c$.

Effused, thin, gelatinous, obscurely gyroso-plicate, hyaline, then white.

Cosering twirs, moss, beech-nuts, etc., and spreading on to earth; rare. Aug.-Nov. 4 in.

2018. T. violacea Relh. (from the violet colour) $a$.

Erumpent, firm, somewhat compressed, gyrose, violaceous.

Gregarious. Trunks, pear; rare. $\frac{3}{8}$ in. Sometimes confounded with 2020.

\section{a. Tuberculiformes.}

2019. T. indecorata Sommf. (from its dull colour ; indecorus, ugly) $a$. Sessile, rounded, convex, plicate, moist, opaque dull livid or olivaceous, becoming dark brownish or slate-colour.

Branches, birch, oak, willow, hazel, poplar ; rare. Oct.-Nov. Length of connate group $3 \frac{1}{x}$ in. ; width $1 \frac{1}{5}$ in.

2020. T. moriformis Sm. (from a fancied resemblance in the shape and colour to a mulberry; morns, a mulberry, forma, form) $a b c$.

Conlobate, sinuous, somewhat gelatinous but firm, mulberryblack. Flesh purple-crimson-blackish, base black.

Naple, chestnut, Robinia, elm, plane; rare. June-Feb. Common size about I in. $\times \frac{5}{8}$ in. Sometimes confounded with 2018.

2021. T. tubercularia Berk. (from its appearance, like a little tuber, tuberculum) a b.

Erumpent, substipitate, pileate, stem round, dull white.

Bark, wood, oak, on old decayed Corticium. Oct.-Dec. $\frac{1}{10}$ in. Tubercularia albida B. \& Br., Brit. Fl. vol. v. p. 354. "Coryne sp." Berk MS. on original example. "Certainly not a Tremella, but the conidial form of some species," Massee, Brit. Funs. Fl. vol. i. p. 64.

2022. T. versicolor B. \& $\mathrm{Br}$. (atersicolor, changeable in colour) a $b c$.

Orbicular, tear-like, pallid when young, then orange, at length brownish-ochre.

On 1845, ash, briar; uncommon. Feb.-May. $\frac{1}{16}$ in.

2023. T. atrovirens Sacc. (from the blackish-green colour; ater, black, rirens, green) a $b c$.

Erumpent, disciform, very minutely papillate and rugose.

Gregarious or confluent, srowing through cracks in bark. Dead branches, broom. Oct. $\frac{1}{16}$ to $\frac{1}{5}$ in. 
2024. T. clavata Pers. (from its somewhat clavate shape) $a$.

Incrassate, fleshy-red, becoming black at base.

Solitary, simple or connate. Branches. Average size of single growth $\frac{5}{8} \times \frac{1}{2}$ in. Possibly a form of $T$. sarcoides With., and, if so, a condition of Ombrofhila sarcoides Jacq., one of the Discomycetes.

[T. sarcoides With. is an early condition of Ombrophila scarcoides Jacq. T. folizcola Fuck. is not a Trentella; it forms small selatinous tufts or spots on the leaves of Ruhus fruticosus in company with Phrasmidium. T. brassicacola B. \& Br. is probably a form of Hypocrea rufa Fr.; it grows on and in cabbage stumps.]

\section{NÆMATELIA Fr.}

(From the gelatinous substance which surrounds the nucleus;

Gr. naima, gelatine, eileo, to roll or wrap round.)

Subgelatinous, firm, convex, with a firm fleshy central solid portion called the nucleus, not shrivelling when dry. Basidia subglobose, longitudinally quadripartite. Sfores broadly elliptical, continuous. (Fig. I I 7.)

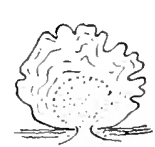

$B$

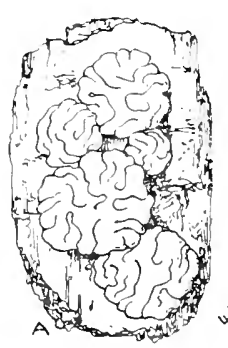

Fig. Ir7.-A, B, Fanatelia enceptala Fr., entire and in section. One-half natural size. $c$, basidium with surface view, $b$, and spores; $\mathrm{D}$, spore germinating and producing sporidiola. $\times 500$.

Readily distinguished by the presence of the central solid nucleus, which is composed of interwoven hypha and covered with a thick gelatinous spore-bearing stratum. Growing on wood, sometimes on moss, rarely terrestrial.

Species 2025-2028

2025. N. encephala Fr. (from the contained nucleus; Gr. $c n$, within, kephale, the head) a $b c$.

Somewhat sessile, pulvinate, plicato-rugose, pallid flesh-colour, salmon or salmon-whitish, nucleus hard, large, white.

Solitary to connate. Branches, rails, pine, larch; frequent. Sept.-Mar. I in. Conmate for 4 in. 
2026. N. pubiformis Fr. (from a fancied resemblance to a small fruit of Rubus, bramble) a $b$.

Somewhat sessile, orbicular, gyroso-tuberculose, yellow.

Dead branches, twigs, sometimes on moss, spreading to the ground. Oct. $\frac{3}{16}$ in.

2027. N. nucleata Fr. (from the nucleus) $a \cdot b c$.

Sessile, flattened, somewhat gyrose, whitish, then tawny yellow, nucleus white, about $\frac{1}{5}$ in. in diam.

Rotten wood, sticks, lime, plane, oak; rare. MIar. Single plants $\frac{3}{16}$ in. in diam. Connate to $\mathbf{I} \frac{1}{t}$ in. II Tust not be confounded with 2007.

2028. N. vireseens Corda (from the greenish colour; aireo, to be green) $a b$.

Sessile, suborbicular, depressed, gyroso-tubercular, somewhat pale leaf-green. Forming green gelatinous masses.

Furze, ivy: common. Oct.-June. Single plant $\frac{3}{16}$ in. Connate chains I $\frac{1}{2}$ in.

\section{GYROCEPHALUS Pers.}

(From the globular shape; Gr. guros, round, kephale, the head.)

Sporophore erect, substipitate, flattened, subspathulate, irregular, tremelloso-cartilaginous. Hymenizm confined to one surface.

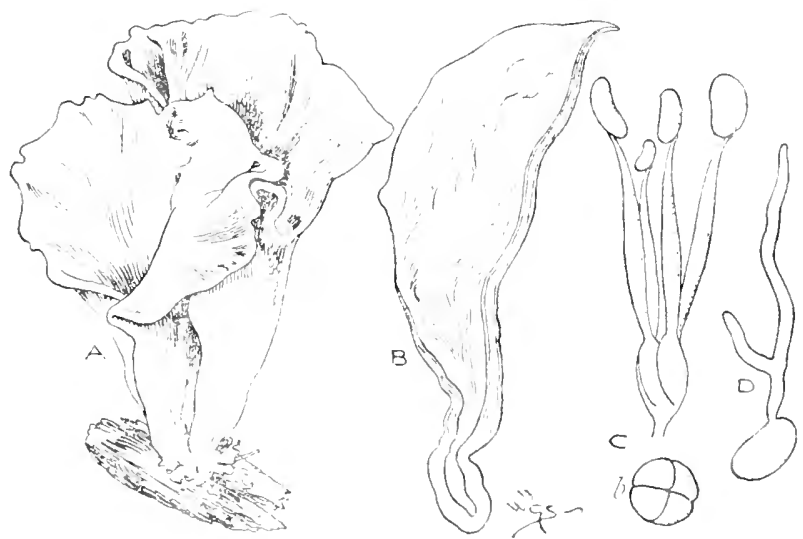

Fig. 11S.-A, B, Gyrecephatus mufus Fref., entire and in section. One-half natural size. $c$, basidiun with surface view, $b$, and spores; $D$, spore germinating. $\times 500$.

Busidia globose, soon longitudinally quadripartite. Sterigmatic 2-4, elongate, thick. Spores ovato-pyriform, continuous. (Fig. I I S.)

2029. G. rufus Bref. (from the red colour; rufus, red) $a b c$.

Erect, cartilagineo-gelatinous, substipitate, subspathulate,-sometimes stemless, variable, orange to red or dull scarlet above and orange below. Hymenimm inferior, smooth.

Terrestrial, under pines, on rotten trunks, fragments of wood. $3 \frac{3}{4} \times 3$ in. 


\section{Subfamlit III. DACRYOMYCETOIDE $Æ$}

Basidia cylindrical or clavate, divided at the apex into two long sterigmata, not septate, except in Femsjonia, where the basidia and sterigmata become septate with age.

Species 2030-2041

\section{DACRYOMYCES Nees.}

(From the tear-like habit; Gr. dakru, a tear, mikes, a fungus.)

Small, pulvinate, gelatinous, homogeneous, somewhat plicatogyrose, everywhere covered with the hymenium. Basidia terminating normal hyphæ, tereti-clavate, bifurcate at the apex when mature.

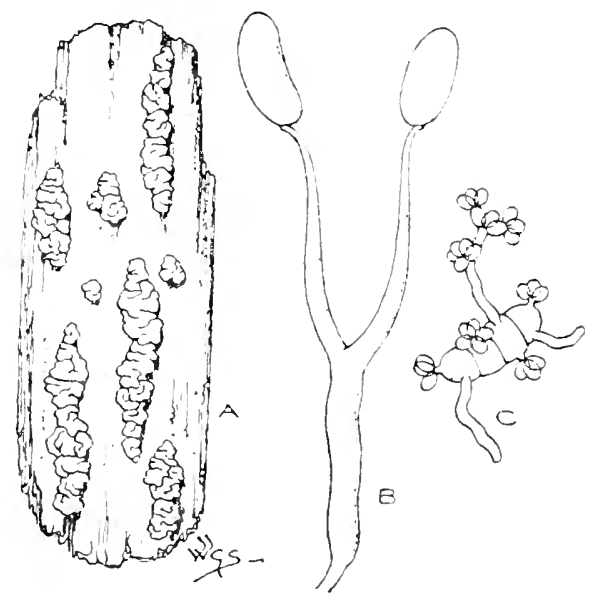

Fig. Iry-A, Dacryonyces deliquescens Duby: One-half natural size. $B$, basidium and stores; $C$, spore germinating and producing sporidiola. $\times 500$.

Spores cylindric-oblong, curved, at maturity or during germination variously septate or muriform. In the conidia-bearing sporophores the conidia, which more or less resemble the spores in size and form, are produced in chains. (Fig. I I9.)

The species grow on dead wood.

Rose-coloured.

Orange, yellow or yellowish.

Species 2030-2036 2030

Pallid or fuscous. 2034

2030. D. macrosporus B. \& Br. (from the long spores; Gr. makros, long) $a b c$.

Gelatinous, tuberculate, minutely gyroso-granular.

Branches, in cracks, on remains of Sfhuria stigma. Dec.-Apr. Connate chains $2 \times \frac{1}{8}$ in. 
2031. D. deliquescens Duby (from its appearance as if dissolving; deliquesco, to dissolve) a b $c$.

Somewhat round, rooted, convex, immarginate, at length twisted, hyaline-yellowish, varying to sulphur, rarely to orange.

Dead wood, cut wood, pine, holly, ash, oak. Dec.-Mar. Connate chains $1 \frac{1}{8} \times \frac{1}{5}$ in.

2032. D. stillatus Nees (from the drop-like habit; stillo, to drop) $a b c$.

Somewhat round, convex, at length plicate, yellow to orange or scarlet.

Rotten wood, fir-poles, cut wood, pine, fir. Jan.-Dec. Connate chains $I_{\frac{3}{4}} \times \frac{1}{8}$ in. Distinguished from 2031 by being ustrally smaller in size and brighter and more persistent in colour.

2033. D. ehrysocomus Tul. (from the colour as of golden hair; Gr. chrusos, gold, kome, hair) a $b c$.

Spherical, orbicular, immarginate, soon collapsing, pezizoid, at length flattened, even, bright golden-yellow, varying greenishor buff-yellow or orange.

Rotten wood, fir ; rare. $\frac{1}{16}$ in. Small in size, usually in large numbers scattered over wood or bark.

2034. D. sebaceus B. \& Br. (from a fancied resemblance to droppings of tallow, sebum) a. $b c$.

Somewhat round, cup-shaped, steel-grey.

Branches, ash, willow, pea-sticks. Jan.-April. Single plants about $\frac{1}{8}$ in. in diam. Curved chains $1 \frac{1}{4}$ in.

2035. D. tortus Fr. (from the twisted habit; torque, to twist) $a b c$.

Round, depressed, gyroso-tuberculate, substipitate, yellow to orange, sometimes vermilion.

Dead wood, rotten powdery lark, oak. Oct.-June. Single plant $\frac{1}{8}$ in. Connate groups $\frac{7}{5}$ in.

2036. D. vermiformis B. \& Br. (from a fancied resemblance to certain small worms; z'rmis, a worm, forma, form) $a c$.

Minute, worm-shaped, grey.

Rotten wood. April-Sept. Single plants $\frac{1}{2 \frac{7}{4}}$ in. Vermiform chains $\frac{3}{8}$ in.

[D. succinius $\mathrm{Fr}$, appearing as minute dots on pine leaves has been shown by Phillips and Plowright to be the conidial stage of Hymenoscypha clictrina Phill. \& Plowr.]

\section{CIVA. FEMSJONIA Fr.}

(From Femsjo, the name of a place.)

Cup-shaped, distended with firm jelly, different on the two sides, disc thick, heterogeneons, even, not glandular. Sporophores immersed, 
globose. Spores oblong, curved, becoming septate, at first colourless, then orange-brown. (Fig. I 20.)

Growing on wood, erumpent, brightly coloured.
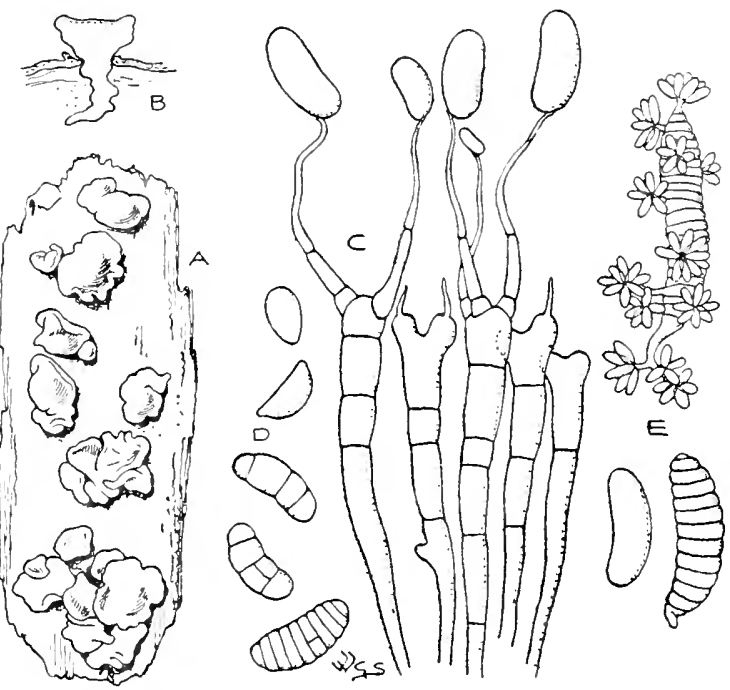

Fig. I20.-A, B, Fonsionia lutcoalba Fr., entire and in section One-half natural size. C, basidia and spores: D, detached spores, the mature spores are septate, $\times 500$. E, Gutpinia Fensjoniana Olsen. Three spores, one serminating and producing sporidiola. $\times 500$.

2036a. F. luteoalba Fr. (from the yellow disc and white exterior; lutens, yellow, albus, white) a b.

At first cup-shaped, becoming disc-shaped and plane, at length convex and folded, disc bright brassy-yellow, with a narrow white marg., under surface at first white-tomentose to brassywhite, the tomentum soon passing away, rooting, erumpent.

Branches, birch, oak. Sept. 55 in. in diam. Looks like 2035 on a large scale. Guepinia Femsjoniana Olsen is perhaps the same as this.

\section{GUEPINIA Fr.}

\section{(After Jean Pierre Guepin.)}

Unequally cup-shaped, substipitate, versiform, cartilagineogelatinous, the two surfaces diverse in structure. Hymenimm unilateral. Basidia linear, deeply bifurcate. Spores curved. Chains of conidia produced on the tips of hyphe springing from the surface opposed to the hymenium. (Fig. I 2 I.) 
2037. G. Peziza Tul. (from the Peziza shape) a $b$.

Cup-shaped, cup oblique, sessile or stipitate, yellow.

Dead branches, wood. $\frac{3}{4} \times \frac{1}{2}$ in.

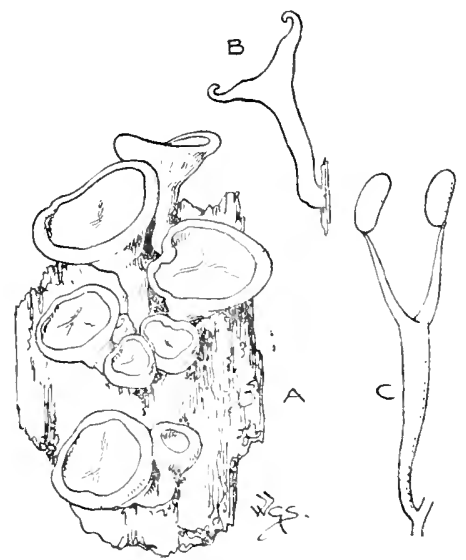

Fig. 121.-A, B, Gutpinitr Feciza Tul., entire and in section. $\times 2$. $c$, basidium and spores. $\times 500$.

2038. G. obliqua Mass. (from the oblique habit) $a$.

Ninutely hairy on reverse side of Hym. Hy'm. slightly concave, oblique, passing into a very short stem-like base, glabrous, deep bright orange when moist, tinged with pink when dry.

Gregarious. Nov. $\frac{1}{6}$ in. high.

\section{DITIOLA Fr.}

(From the down-like universal veil ; Gr. dittos, double, ioulos, down.)

Veil universal, down-like. Stroma firm, more or less stem-like. Hymenium discoid, gelatinous. Basidia furcate. Spores at first contimuous then septate. (Fig. I 22.)

Nassee has removed 2040 and made it the sole type of a new genus, Dacryopsis. It is undesirable to break up such a small and natural genus as Ditiola. Dacryopsis is said to be distinguished from Ditiola by having the hymenial surface at first covered with conidiophores and conidia as in Tuberculerite: but this is hardly a distinction of generic value as conidia are present in some species of Trmell and not in others.

Species 2039-2041

2039. D. radicata Fr. (from the rooting base; radix, a root) a $c$.

Stipitate, rooting, cups nearly plane, disc golden-yellow. St. thick, white.

Wood, fir, amongst pine-leaves. $\frac{1}{4} \times \frac{1}{4}$ in. No conidia recorded. 
2040. D. nuda B. \& Br. (from the absence of a veil; mudus, naked) $a$.

Hcad subpileiform, corrugate, sometimes umbilicate, goldenyellow. St. somewhat thick, not rooting, cylindrical, whitetomentose. Fruiting surface at first covered with conidiophores and conidia.

Stumps, fir. Sept. $\frac{1}{8} \times \frac{1}{4}$ in.

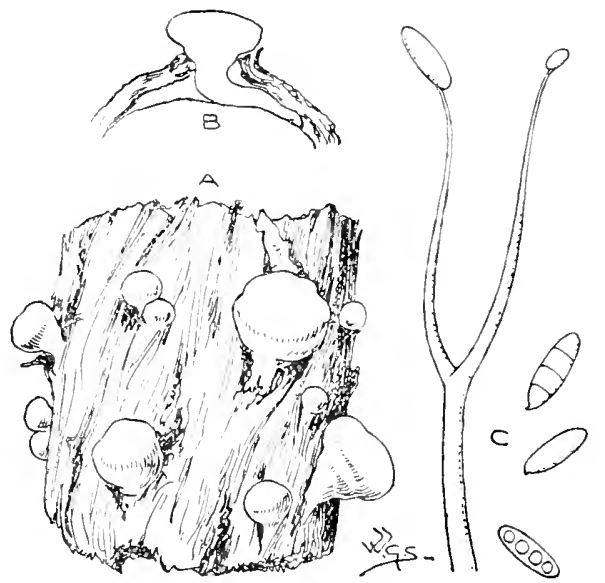

Fig. I22.-A, B, Diliola Ulicis Plowr, entire and in section, $X_{2}$. $c$, basidium and spores. $\times 500$.

2041. D. Ulicis Plowr. (from the habitat, gorse, Ulex europans) a $c$. is Hicad globose, then flattened and wrinkled, pale lemon-yellow, slightly white-villous. St. firm, paler than head, sometimes absent or extending only from the wood through the bark.

Dead stems; very common. Jan. $\frac{1}{2} \times \frac{1}{1}$ in. No conidia recorded.

$\therefore$ [The genus Apyrcminm Fr. is omitted. A. lignatile Fr. is the conidial stage of Hypocrea mifa Fr. A. armeniacum B. \& Br. is the same condition of $H$. gclatinosa Fr. Hymomula punctiformis B. \& Br. is an imperfect form of some fungus not belonging to the Tremellinacea.] 


\section{ORDER II. GASTEROMYCETES}

\section{FAMr. VII. PHALLOIDACEÆ}

Pileus and stem or latticed receptacle at first enclosed in a universal volva of two membranes with a niddle gelatinous stratum ; volva at length opening at the apex. Gleba mucilaginous, at length elevated from the volva on a stem or lattice. Spores minute, ellipticoblong, continuous, at first immersed in olive-brown mucilage.

Species 2042-2045

Key to the Genera.

Pileus free, reticulate

IO7 Ithyphallus.

Pileus alnate

IOS MUTINUS.

Pileus divided in vertical lobes

I IO LYSLRUS.

Receptacle forming a hollow lattice of large meshes

rog Clathrus.

CVII. ITHYPHALLUS Fisch.

(From a fancied resemblance to the Ithuthalles, carried at the festivals of Bacchus.)

Pileus reticulate, attached to the apex of a hollow, perforate spongy, stem-like receptacle. $V_{\epsilon i l}$ forming a thin film--sometimes a feeble white membrane-over the sporiferous mucous and slightly attached to the stem. (Fig. I 23.)

2042. I. impudicus Fisch. (from its phallic resemblance; impudicus, shameless) a b $c$.

$P$. white, at first covered with olive-brown sporiferous mucous. Rec. elongato-fusiform, spongy-cellular, white. M/yc. cordlike.

Highly fetid. Woods, thickets, gardens, generally amongst rotten tree-roots and stumps, by gates, in and by summer-houses, sheds, porches, often near to and in dwellings. May-Aug. Height ro in. Var. iosmos Sace. Ioorders of retieulations and pileus serrate, sometimes resldish-grey in eolour, said to be violet-seented. 


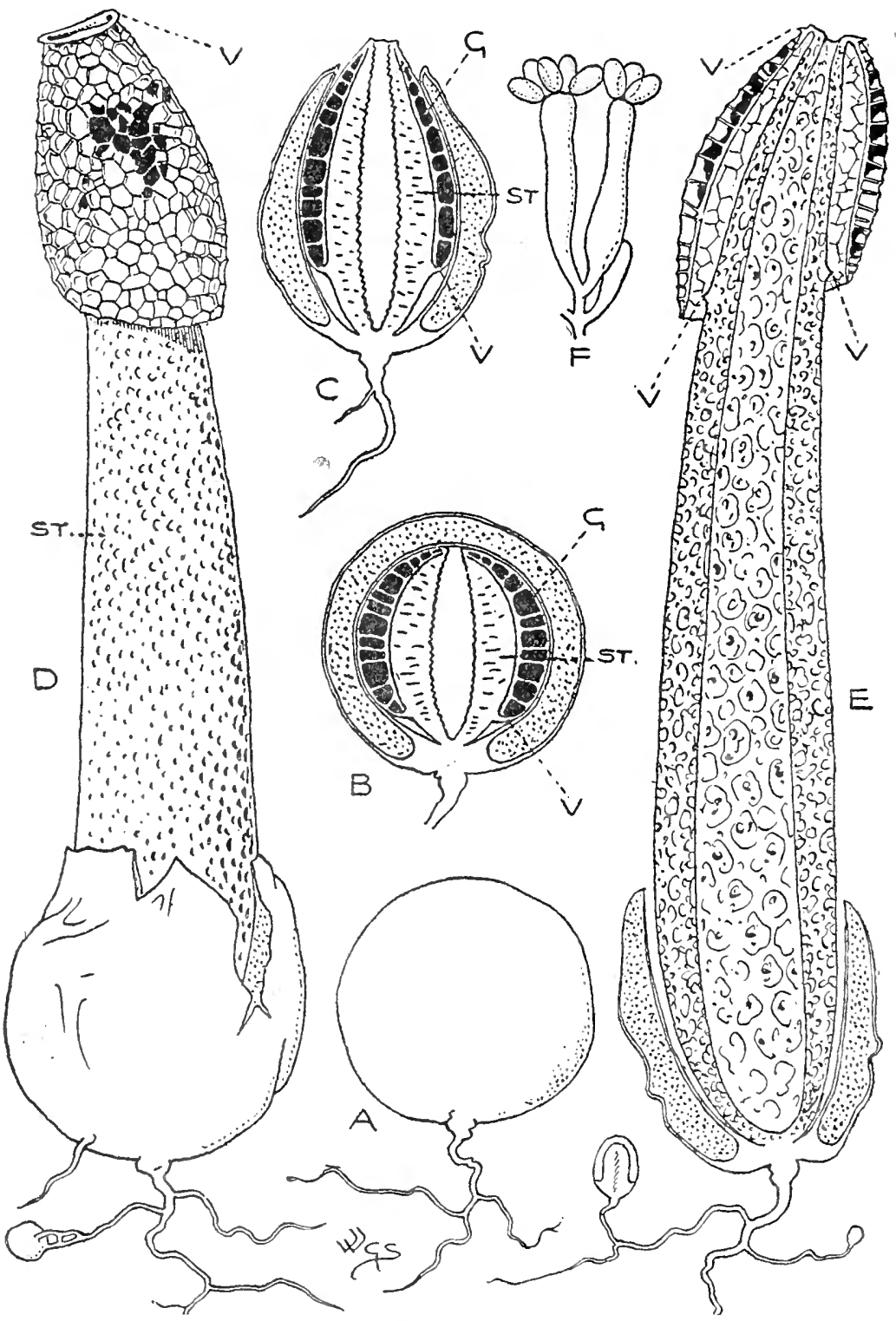

Fig. 123.-lthyplallus itzpulizus Fisch. Two-thirds natumal size.

A, young plant. 1 , section of clitto, showing veil at $v$. C, dito, showing pileus emerging from volv, veil at $v$. D, mature plant, remains of veil at $v$. E, section of ditto. F, basidium and spores, $X$ rooo. r, gleba: ST, stem or receptacle. 


\section{MUTINUS Fr.}

(An appellation of Priapus.)

Pileus adnate to the hollow, perforate or imperforate spongy stem, at first immersed in olive-brown sporiferous mucus. Icil

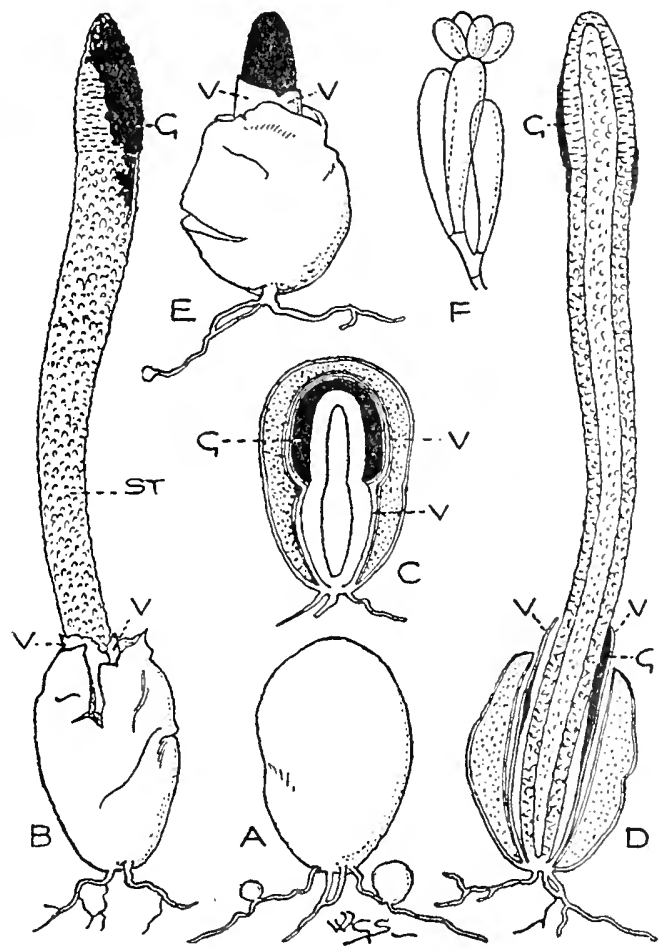

Fig. 124,-Mutinats caninus Fr. Two-thirds natural size.

A, young plant. B, mature plant. C, section of young plant. D, section of mature plant. E, pileus emerging from volva. F, basidiun and spores, $\times$ I 000 . G, gleba: ST, stem or receptacle; v, veil.

distinct, springing from the base of the stem within the volva, white. (Fig. I 24.)

2043. M. caninus Fr. (canis, a $\operatorname{dog}) a b c$.

$P$. short, subacute, rugulose, red. $R e$. elongato-fusiform, cellular, white or rosy. When the receptacle first emerges from the volva it is covered with the white veil.

Woods, bushy places, rotten stumps, pine, ash, decayed leaves in woods and hedges. June-Dec. Height $5 \frac{1}{4}$ in. 
2044. M. bambusinus Fisch. (from its original place of growth, at the base of bamboos, bambusa) $a$.

$P$. long, acutely conical, tuberculose or rugose, dull purple. Rec. elongato-fusiform, imperforate, spongy-cellular, pallid rubiginous or rosy.

Very fetid. Hleight $5 \frac{1}{\frac{1}{x}} \mathrm{in}$.

\section{CLATHRUS Nich.}

(From the latticed receptacle; clathrus, a lattice.)

Receptucle hollow, globose, latticed, the meshes at first filled and covered inside by olive-brown sporiferous mucus. (Fig. 125.)

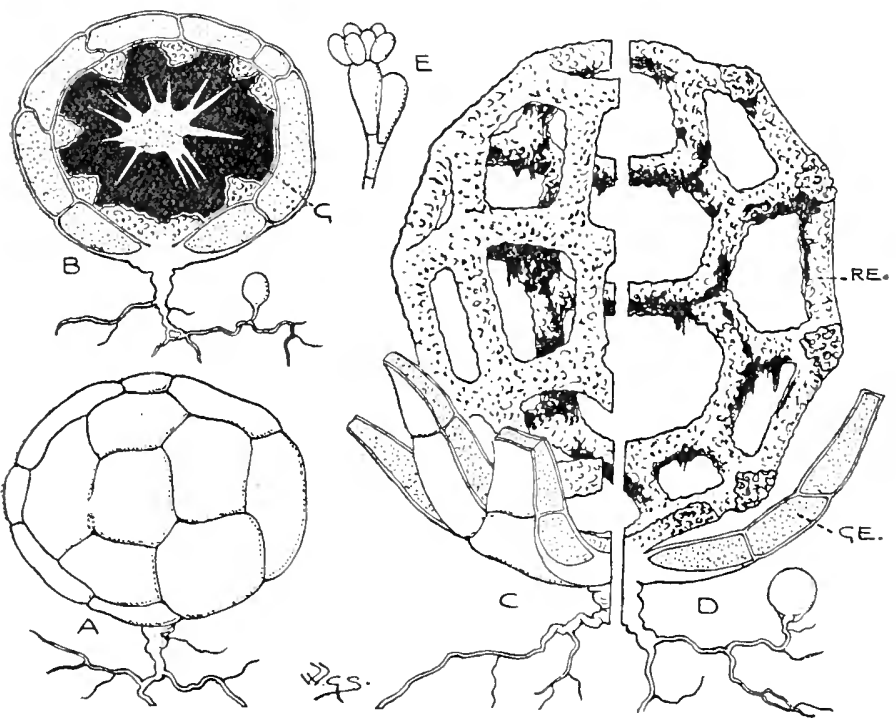

Fig. 125.-Clathrus cancellatus Tournef. One-half natural size.

A, young plant. B, ditto in section. C, mature plant, left half entire. $D$, ditto in section. $E$, basidium and spores, $\times 750$. G, gleba; GE, gelatinous stratum of volva; $K E$, receptacle.

2045. C. cancellatus L. (from the lattice-like receptacle; cancellatus, latticed) $a b c$.

Rec. sessile, globose or ovate, the branches triangular in section with a flat face outwards, orange-vermilion outside, vermilioncrimson inside, inner sporiferous mucus dark olive, enclosing a central mass of soft dark gelatine. Vo. of two white membranes, marked internally and externally with lines corresponding with the lattice within. Myc. cord-like.

single or catspitose. Highly fetid. Plantations, gardens, stoves. Oct.-Nov. $4^{\frac{3}{4}} \times 3^{\frac{1}{2}}$ in. The interior of the receptacle at length drips with olivebrown mucus. 


\section{LYSURUS Fr.}

(From the free tail-like lobes of the mature receptacle;

Gr. Lwo, to loosen, our a a tail.)

Stipitate, pileus divided in vertical lobes, free at the apex, distinct from the stem. Sporiferous mucus on the interior of the lobes. (Fig. I 26.)
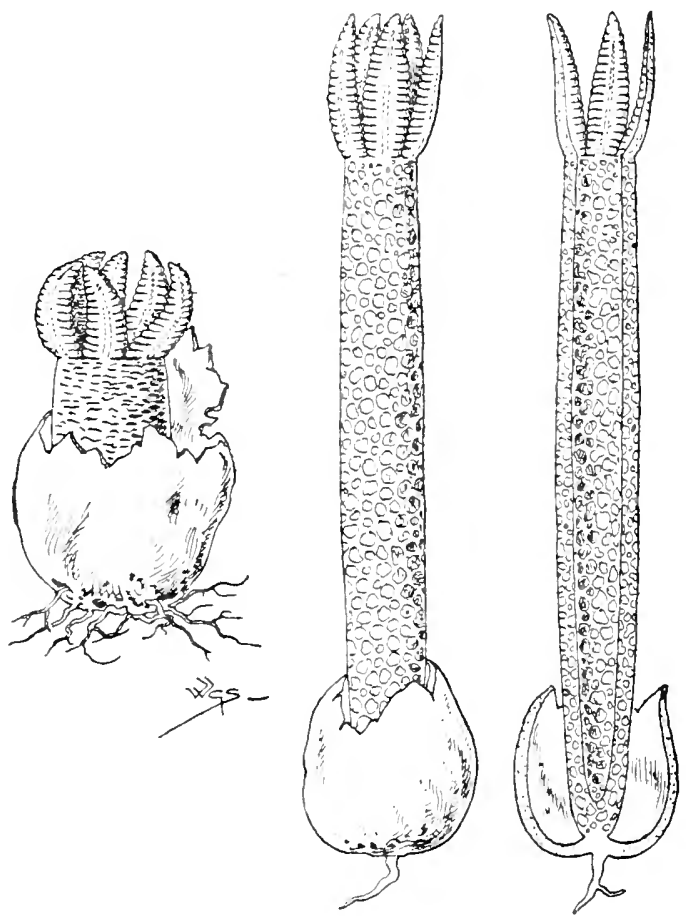

Fir. 126.-Lysurus austrulicnsis Cooke \& Mass. One-half natural size. Young plant on. left, mature plant and section on right.

2045a. L. Australiensis Cooke \& Mass. (from its native countryAustralia).

$P$. for the most part 5-lobed, lobes attenuate upwards, at first connivent, then somewhat reflexed, with a longitudinal depression along the middle, transversely rugose, tawny. Rec. cylindrical, hollow cellular, whitish. Vo. globose, lobed, white.

Refuse of wheat, probably Australian, Kidderminster, Worcester. I4 Nov., 1902. Carleton Rea. 63 $\times \frac{3}{4}$ in. 


\section{FAMr. VIII. LYCOPERDACEÆ}

Peridium double, rarely single, gelatinous stratum absent. Spores forming a powdery mass at maturity, mixed with a well-developed capillitium. A very feeble membrane separating the gleba from the double outer peridium, may be detected in immature examples.

Species 2046-2084

\section{Key to the Genera.}

Peridium vertically compressed. Stoma apical.

I I BATTARREA.

Peridium subglobose.

Stoma apical

II 2 Tulostoma.

Stoma none

I 3 QUele'tia.

Exoperidium splitting in a stellate manner

Exoperidium breaking up in the form of warts or spines. Base sterile

Exoperidium flaking off in patches. Sterile base absent ...

II4 GEASTER.

I I 5 LYCOPERDON. II6 Borista.

\section{BATTARREA Pers.}

\section{(After Antonio Battarra.)}

Volva universal, central stratum gelatinous. Peridium vertically compressed, pileus-like, bursting through the volva and at length raised on a tall stem. (Fig. I 27.)

2046. B. phalloides Pers. (from a fancied resemblance to Ithyphallus; Gr. eidos, appearance) $a b$.

$P_{\epsilon}$. convex, bearing the spores and capillitium, orange-brown. St. hollow with a central pith of silky threads, wall of three layers, the outer broken up into coarse fibres pointing downwards, orange-brown. $\%$. ovate formed of two white membranes with an internal gelatinous olive-yellow layer.

In and near decayed trees, ash, on sandhills. Dec. $12 \frac{1}{2} \times 2$ in. When in the young state sometimes buried several inches deep in sand or decayed wood. Sometimes 14 in. high with a pilens $2 \frac{1}{2}$ in. in diam. 


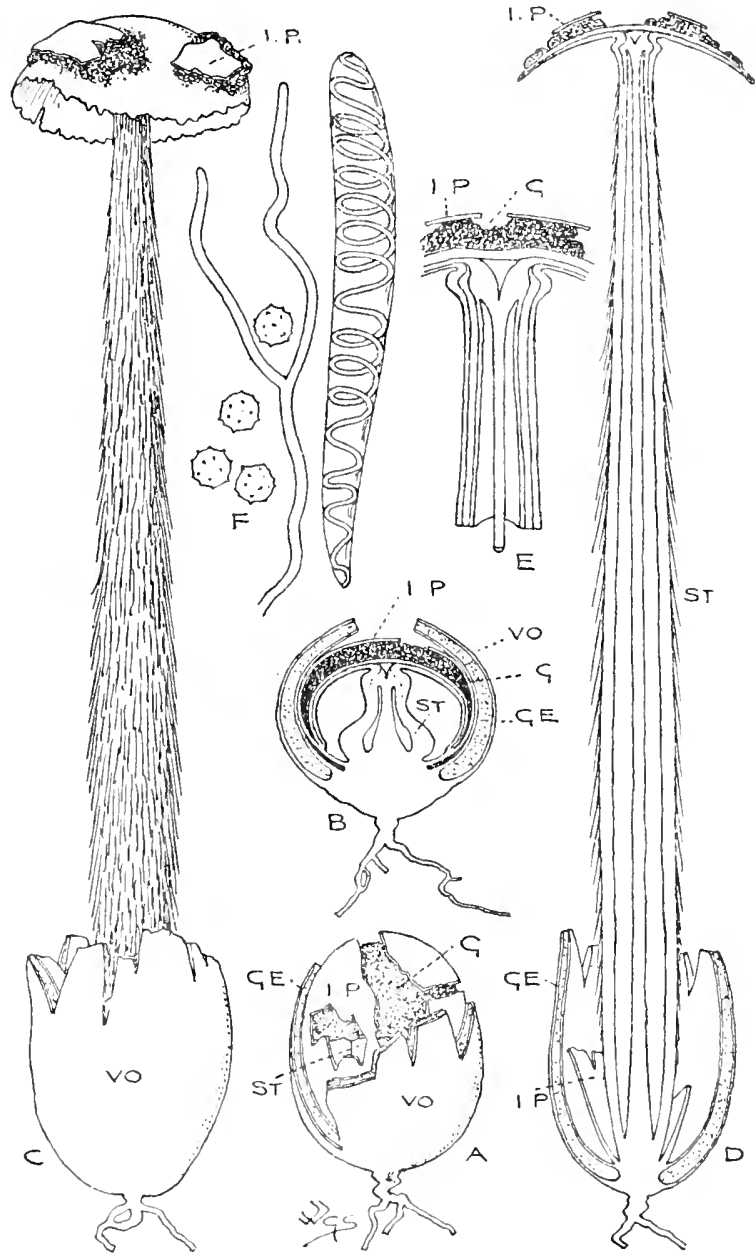

Fig. 127.-Battarrea phalloides Pers. One-half natural size.

A, young plant, bursting outer peridium or volva, vo. B, section of ditto. c, mature plant. D. section uf ditto. E, section of upper part of stem. Natural size. F, threads of capillitium and spores. $\times 75 \circ$. G, gleba, with capillitiun and spores; G $\mathrm{E}$, gelatinous stratum; I 1 , inner peridium; ST, stem; vo, volva. 


\section{TUlostoma Pers.}

(From the structure of the mouth of the peridium;

Gr. tulos, cartilage, stoma, a mouth.)

Periditm subglobose, dehiscing by an apical stoma, outer coat, veil or cortex, deciduous, distinct from the at length elongate stem. Threads of the capillitium swollen at the septa. (Fig. I 28.)
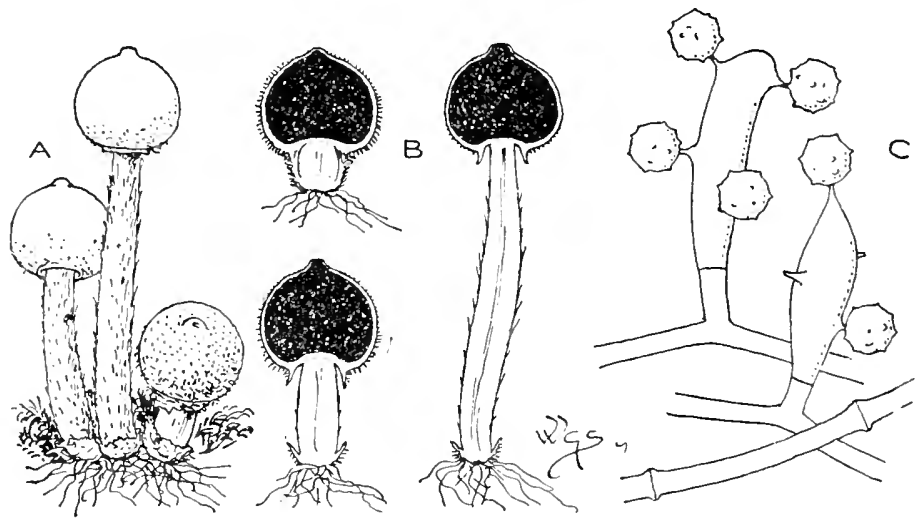

Fig. 128.-Tulostoma mammosum Fr. Two-thirds natural size.

$A$, entire plant. E, sections. C, basidia, spores and thread of capillitium. $\times$ rooo.

2047. T. mammosum Fr. (from the breast-like peridium ; mamma, a breast) $a b c$.

$V$. universal, distinct at the base of Pe. and at the lower part of St. Pi. globose, at first sessile or nearly so, stoma mamniform, entire, ochre. St. equal, squamoso-fibrillose, ragged at the base with remains of $\mathrm{T}$., somewhat pale vinous-brown.

Amongst moss on old walls, dry banks, sandhills, etc. Oct.-Feb. $2 \frac{1}{2} \times \frac{5}{5}$ in. There are two forms, brown, with a stout stem, and yellowish-white, with a slender stem. Intermediate forms occur.

\section{QUELETIA Fr.}

\section{(After Dr. Lucien Quelet.)}

Peridium subglobose, simple, stoma none, the wall breaking up irregularly at maturity. Stcm at length long, squarrose, the base of the peridium breaking at the apex and forming a collar or veil. Spores borne on tetrasporous basidia. (Fig. I29.)

2048. Q. mipabilis Fr. (from its unusual appearance; mirabilis, wonderful) $a$. 
$P \epsilon$. thin, even, glabrous, rigid, fragile, breaking away in irregular patches at maturity, whitish, then dull greyish. St. subclavate, fibroso-carnose, externally densely squarrose, whitish, brownish towards base.

Gregarious. $6 \frac{1}{ \pm} \times 2 \frac{1}{ \pm}$ in. At first wholly subterranean.
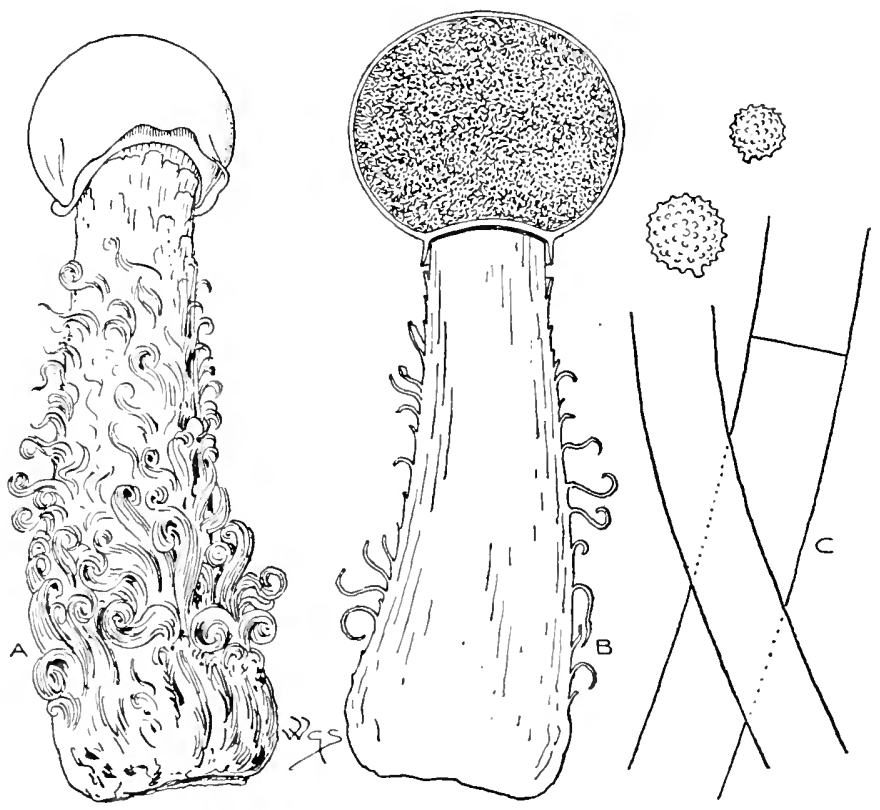

Fig. 129.-O Ouletic nirabilis Fr. One-half natural size. A, entire plant.

I., section. C, threads of capillitium and spores. $\times 750$.

\section{GEASTER Mich.}

(From the star-like appearance of mature plants;

Gr. se, the earth, aster, a star.)

Peridinm at first continuous, consisting of three layers, the two outermost-exoferidium-splitting from the apex in a stellate manner, the inner layer-indopidinm-sessile or pedicellate, furnished at the apex with one orifice, rarely more. Columella prominent, rudimentary or obsolete. Cafillitimm dense. 'Terrestrial. (Fig. I30.)

Species 2049-2062

a. Myriostomate. Endoperidium with many orifices. 2049

b. Monostomatie. Endoperidium with a single orifice.

Endoperidium pedicellate.

$2050-2054$

Endopiridium sessile. 


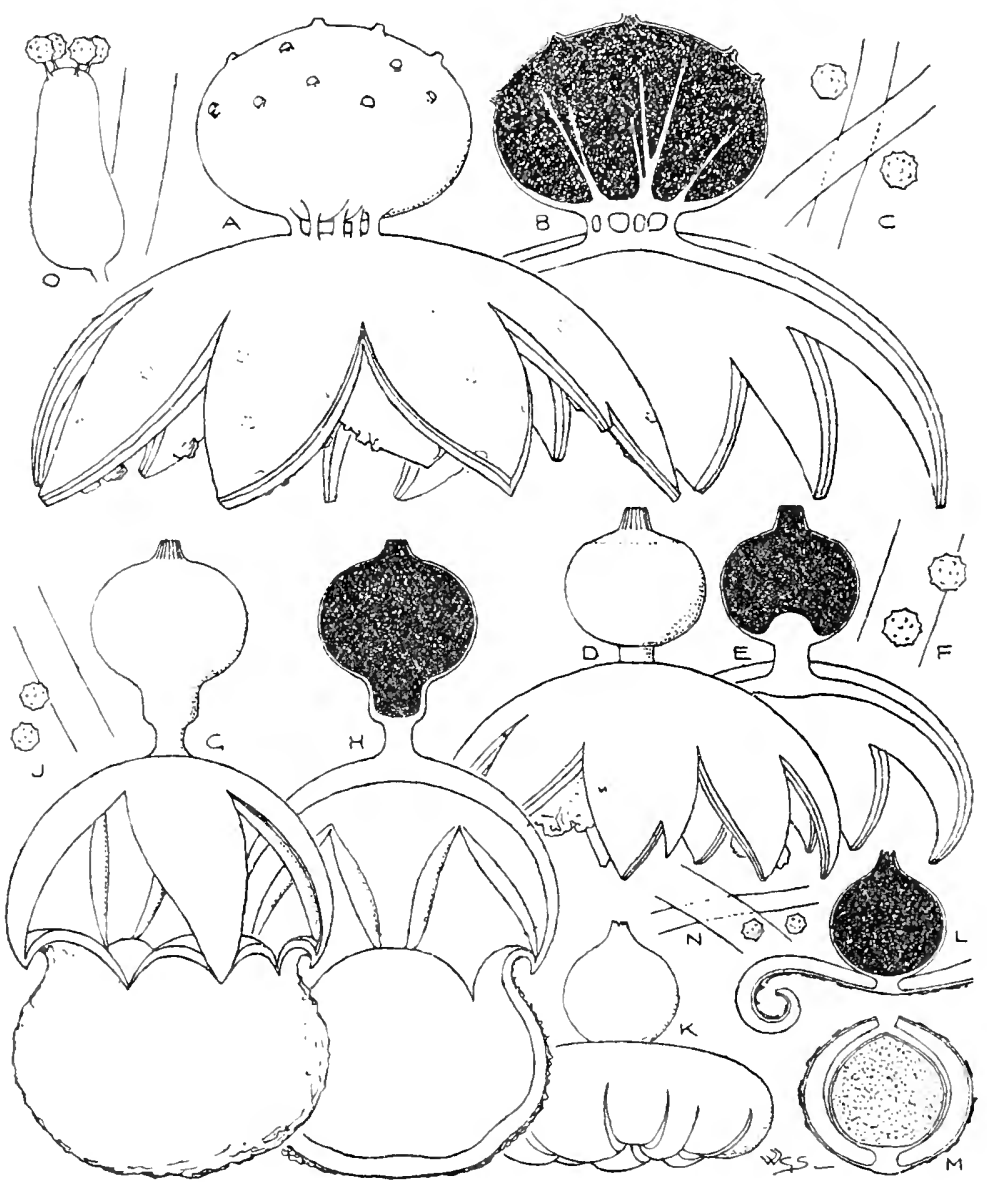

Fig. 130.-A, B, Geaster coliformis Pers, entire and in section. One-half natural size. $c$, threads of capillitium and spores. $\times 750 . \quad \mathrm{D}, \mathrm{E}, G$. Berktcki Mass., entire and in section. One-half natural size. F, thread of capilltium and spores, $\times 750, G, \mathrm{H}, G$, fomicatus Fr., entire and in section. One-half natural size. I, thread of capillitim and spores. $\times 750$. $\kappa, L, G$. fimbriatus $\overrightarrow{F r}$., entire and in section; $M$, young plant in section. One-half natural size. $N$, threads of capillitium and spores. $\times 750$. $O$, basidium with spores and thread of capillitium of $G$. mésciens Pers. 


\section{a. Myriostomate.}

2049. G. coliformis Pers. (from a fancied resemblance in the endoperidium to a colander, colum a $a b c$.

Ex. 7- to 10-segmented, unequal, pale umber. End. subglobose, scabrous, supported on numerous pedicels, pale slate. $\mathrm{Col}$. usually obsolete or occurring as continuations of the pedicels. Gl. umber.

Sandy banks. Oct. Diam. of expanded Ex. $5 \frac{1}{2}$ in.

\section{b. Monostomatis.}

2050. G. Bryantii Berk. (after Charles Bryant) a b $c$.

Ex. 8- to r z-segmented, becoming involute, pale umber to warm brown. End. subglobose or obpyriform, with a distinct groove between End. and Ped., dark purple-slate to warm umber, more or less whitish-farmose. Per. elongate, conical, sulcate. Col. hemispherical. Gl. dark brown.

Amongst leaves, under hedges, sandy places, on wood, dry marly banks, on the ground. July-Feb. Diam. of expanded Ex. $3 \frac{3}{4}$ in. Tar. minor Mass., under one inch when expanded.

2051. G. Schmidelii Vitt. (after Casimir Schmidel) a $c$.

Ex. 5- to 8-segmented, revolute, white to ivory. End. subglobose, lead-colour to pale salmon. Per. prominent, plicate, apex fimbriate, whitish. Col. large.

On the ground in open places. Diam. of expanded Ex. 2 in.

2051a. G. umbilicatus Fr., var. Smithii IV. G. Sm., G. Smithii C. (x. Lloyd (after Worthington (ieorge Smith) $a$.

Ex. 7 - to 8-segmented, unequal, sublanceolate, pale umber-ochre, shaded darker, limbs slightly vinous-sienna, externally pale umber. End. sessile, globose, or depressed-globose, colour as Ex. with a tinge of olive. $P(r$. conical plicato-striate in a circular depressed marginate umbilicus. Gl. deep vinous-umber.

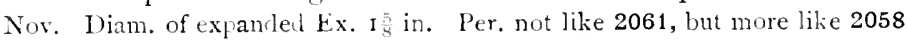
or 2057.

2052. G. Berkeleyi Mass. (after the Rer. M. J. Berkeley) $a$.

Ex. 7- to 9-segmented, unequal, vinous-brown when dry, externally buff and umber. End. broadly ovate, shortly and stoutly pedicellate, coarsely farinose, pale brown. Per. prominent, conical, coarsely sulcate inside and out, surrounded by a. smooth slightly depressed silky zone. Col. short, hemispherical. Gl. umber.

On the ground, amongst fir-leaves. Oct. Diam. of expanded Lx. 33 in. According to MIassee this species was confused by leerkeley with 2051 and 2055 ---see the dried examples in the Kew herbarium.

2053. G. limbatus Fr. (from the fringed or silky, fimbriato-ciliate peristome; limbatus, bordered, as with another colour) a b c. Ex. multifid, unequal, vinous, scurfy and umber beneath. End. subpyriform to subglobose, shortly pedicellate, blackish-brown 
to deep vinous-umber. Pir. slightly depressed, subacute, fimbriato-ciliate, surrounded by a small pale silky disc. Col. almost obsolete. $G l$. dark umber.

Woods, hedgebanks, amongst firs, leaves. Sept.-Nov. Diam. of expanded Ex. 4 in.

2054. G. fornicatus Fr. (from the arched inner layer of the exoperidium ; fornicatus, arched over) $a b c$.

Ex. 4-(rarely j-) segmented, the two layers separating, outer cuplike, scurfy and brown externally, tan within, inner arched and attached to outer by tips of segments, umber, blackish-umber or rufous, tan within. End. obpyriform, shortly pedicellate, dark brown. Per. conical, ciliato-striate. Col. slender, somewhat clavate.

Meadows, pastures, heaths, amongst firs. Mar.-Nov. Diam. of expanded Ex. $2 \frac{3}{4}$ in. $5 \frac{3}{4}$ in. high.

2055. G. striatus DC. (from the sulcato-striate peristome) $a b c$.

Ex. 6- to 9-segmented, coriaceous, thin, tan, darker externally. End. subsessile, globose, often scabrous, umber-white to leadcolour. Per. prominent, conical, sulcato-striate. Gl. brown.

On the ground, sandhills amongst dead leaves, amongst trees, firs. Mar.Nov. Diam. of expanded Ex. 2 in.

2056. G. Michelianus W. G. Sm. (after Piero Antonio Micheli) a $b c$.

Ex. 4- to 6-segmented, unequal, thick, warm or pale vinous, outside much cracked, brown. Enl. subglobose, tan-umber. $P r$. mammiform, surrounded by a silky zone. Col. clavate. Gl. brown.

On the ground, amongst rhododendrons. Oct. Diam. of expanded Ex. 5 in. 1)iffers from 2061a, for which it has sometimes been mistaken. At maturity this plant will somelimes throw itself elastically some 9 inches from its place of growth.

2057. G. lagenæformis Vitt. (from a fancied resemblance to a narrow-mouthed bottle or jar; lasena, a flask, forma, shape) a $b c$.

Ex. 7- to 9-segmented, attenuate, subequal, salmon-white to ivory, pale rose or vinous. End. subglobose or ovate, pale buff, umberwhite or faintly vinous. $P e r$. broadly-conical, sides incurved, silky, striate, surrounded by a disc. Col. clavate. Gl. brown, somewhat olive.

On the ground. April-Nov. I liam. of expanded Ex. $2 \frac{1}{4} \mathrm{in}$.

2058. G. saccatus Fr. (from the sac or pouch-like shape when young; saccus, a bag) a b c.

Ex. 7- to 9-segmented, pale dove-colour, very pale yellowishlavender or pale buff, externally umber and ochre. Ent. globose, colour as Ex. Por. broadly-conical, sides incurved, stoma fimbriate, silky, surrounded by a silky disc. G\%. dark brown.

On the ground, amongst stones and earth, in hedges, sometimes on sticks with profuse Myc. Aug. Diam. of expanded Ex. 3 in in. 
2059. G. mammosus Chev. (from the breast-like endoperidium; mammosus, full-breasted) $a b c$.

Ex. 7- to ro-segmented, hygrometric, strongly incurved when dry, vinous-brown to umber, ochreous or glossy yellow-white-umber outside. End. globose, white-brown. Per. broadly and acutely conical, fimbriato-ciliate to striate, surrounded by a pale silky disc. $G l$. vinous or dark brown.

On the ground. Feb.-Dec. Diam. of expanded Ex. $I_{5}^{\frac{5}{8}}$ in.

2060. G. rufescens Pers. (from the at length rufescent colour) $a b c$.

$E x$. 6- to 9-segmented, thick, becoming deeply cracked, brownish flesh-colour or red-salmon, clouded rufescent, externally dark sienna or sienna-buff. End. globose, pale to dark umber, stoma slightly mammiform, dentate. Col. obsolete or short. Gl. dark brown.

Woods, pastures. Sept.-Dec. I) iam. of expanded Ex. $4 \frac{1}{2}$ in. Var. minor Pers., $5^{-} S$ segmented, less than half the size of type.

2061. G. fimbriatus Fr. (from the fimbriate mouth of the peristome) a $b c$.

F.x. 5- to I 5-segmented, Haccid, somewhat thick, buff- or umberwhite with white cracks. End. subglobose, pale umber, stoma indeterminate, piloso-fimbriate. Col. obsolete. Gl. brown.

Woods, pine, on the ground amongst leaves. Aug.-Dec. Diam. of

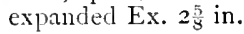

2061a. G. triplex Jungh. (from the superficial appearance, as of three peridia) $a$.

Ex. 5- to 6-segmented, the fleshy inner layer often becoming detached and forming a spurious peridium at the base of the End., vinous-brown. End. globose, with a definite even stoma, yellow-brown. Col. prominent, persistent.

Woods, fields. Diam. of expanded Ex. $4 \frac{1}{2}$ in. When young this plant is pouch-like with an acute apex as in 2058, and greatly differs in this respect from 2056, where also the colours of the peridia are the reverse of 2061 a.

2062. G. hygrometricus Pers. (from its absorption of moisture from the air; (ir. Inucros, moisture, metron, a measure) a $b c$.

Ex. 7 - to 20 -segmented, harsh and horny, vinous-brown, yellowishbrown outside, rigidly inflexed when dry, and often cracked all over. End. ovato-globose in section, floccose, crimson-brown or purple-lavender, stoma irregularly torn. Col. obsolete. Gl. crimson-brown.

On the ground. Woods, pine. Feb.-Dec. Diam. of expanded Ex. $2 \frac{1}{4}$ in.

\section{LYCOPERDON L.}

(From an old belief that puff-balls grew from the dung of the wolf; Gr. lukos, a wolf, perdon, dung.)

Piridium double, external layer or cortex becoming broken up into spine-like tufts or warts, inner layer smooth dehiscing by a small 
apical stoma; the whole of the upper portion being sometimes evanescent. Sterile base usually stem-like. (Fig. I3 3 .)

The species are remarkable for their variability in size, very small mature examples of all the species being frequent. They are popularly named "Puff-balls" and "Devil's snuff-boxes."

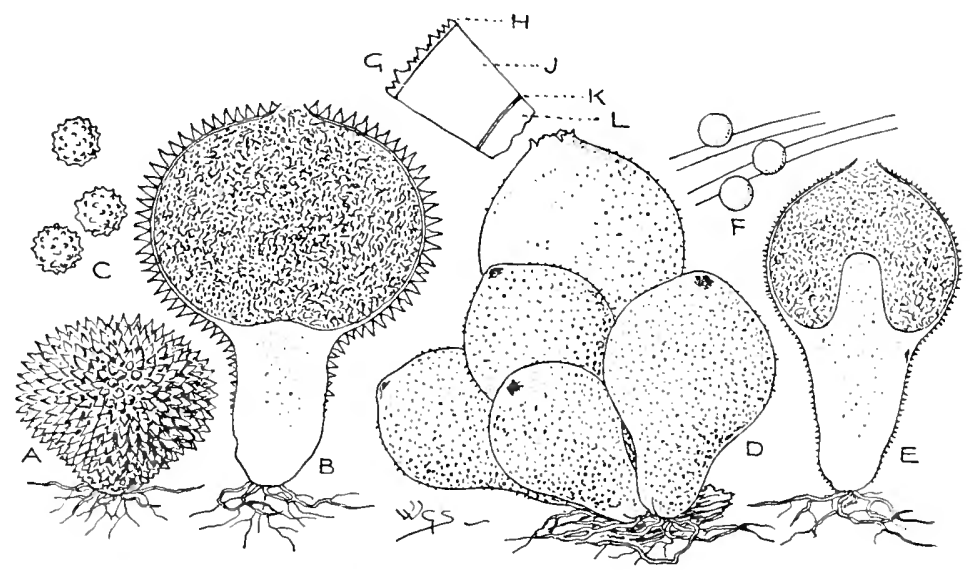

Fig. 1331.-A, B, Lycoperdon echinatum Pers., young plant entire and mature plant in soction. One-half natural size. C, spores. $\times 750$. I, E, L. pyriforme Schæff, entire and in section. One-half natural size. F, spores and thread's of capillitium. $\times 750$. G, section through cortex of L.gemmatum Batsch $\times 5$; H, onter scurfy coat; $J$, inner coat; $k$, thin metubrane over gleba; L, gleba.

Some of the species are dangerous if eaten in a raw state; rapid inflammation of the throat and a greatly swollen tongue have been known to ensue. This is probably also true of species of Bozista.

Sfores rough.

Species 2063-2077

Spores smooth.

$2063-2068$

$2069-2077$

2063. L. echinatum Pers. (from the hedgehog-like spines of the peridium ; cchimus, a hedgehog) a $b c$.

$P e$. globose, usually passing into a stout stem, sometimes stemless, at first covered with large pyramidal floccose spines, vinous-brown varying buff, stoma small, irregularly torn, base usually clad with white thread-like Myc. St. spongy-cellular within. Cap. and Spores deep vinous-brown.

Usually solitary, Amongst trees, beech. Mar.-Oct. Diam. $2 \frac{2}{4}$ in. Sometimes made a var, of 2068.

2064. L. Hoylei B. \& Br. (after Mr. Hoyle, who first brought the plant to the notice of the Rev. M. J. Berkeley) $a b c$.

$P$ e. subglobose, passing into a short stem, at first covered with pyramidal floccose spines, purple-brown, stoma small, irregularly 
torn, base usually clad with white thread-like Myc. St. lacunose, spongy-cellular and olivaceous within. Cap. and Spores light in colour, pale purplish-brown, but described by Berkeley as lilac.

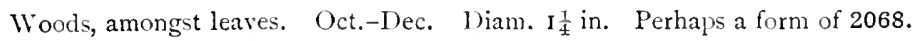

2064a. L. velatum Vitt. (from the external layer of the peridium forming a universal veil; ichlum, a covering) $a b$.

$P$ e. pyriform or subglobose, slightly umbonate, outer layer tomentose, breaking up into evanescent star-shaped rosettes and forming a false ring-like appearance at the apex of the stem, at length almost completely disappearing, snow-white, inner layer furfuraceous and furnished with very thin short spines, white then delicate flesh-colour, finally greyish. St. with white cord-like Myc., white. Caps. and Spores white, then fulvous, finally ashy.

Amongst leaves. Oct. $2 \frac{1}{2} \times 1 \frac{7}{5}$ in.

2065. L. atropurpureum Vitt. (from the blackish-purple capillitium and spores; ater, black) $a b c$.

$P_{\ell}$. subglobose or pyriform, at first covered with slender spines, warm yellowish-brown or rufous, stoma small, irregular. St. plicate below, spongy-cellular and pale dull purple within. Cat. and Spores dull- to blackish-purple.

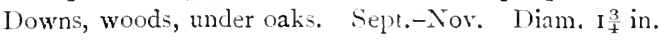

2066. L. excipuliforme Pers. (from a fancied resemblance to a basin, excipula) a b c .

$P i$. subglobose, usually passing into a long, stout stem, rarely almost stemless, at first covered with slender spinose warts, white to ivory. St. even, somewhat plicate below, sometimes lacunose, spongy-cellular and pale olive within. Cat. and Spores dull olive or yellowish-brown.

l'astures, woods, under firs. May-Nov. 1)iam. $3^{\frac{1}{5}}$ in. Sometimes $7 \frac{1}{2}$ in. high. Sometimes confused with or made a var. of 2068,2070 or 2071 , the two latter have smooth spores.

2067. L. saceatum Fr. (from a fancied resemblance to an inverted sac or bag, saccus) a b $c$.

$P_{i}$. subglobose, passing into a long stout stem, plicate below, at first covered with spinulose warts, brownish-ivory, stoma small, irregular. St. often more or less lacunose, spongy-cellular and yellowish-olive within, brownish below. Cap. and Spores yellowish-brown.

Often in rings of very large size, individuals a foot apirt. Open places, near pines, in and near thickets. Aug.-Oct. 1 siam. 4 in. Often 8 in. or more high.

2068. L. gemmatum Batsch (from the peridium covered with pointed warts; gcmma, a young bud) a $b c$.

$P$ i. subglobose, usually passing into a stem, rarely stemless, at first covered with small pointed spines, pale brown. St. 
tapering downwards, coarsely spongy cellular and faintly olive within. Cap. and Spores olivaceous- to dark-umber.

Woods, shady places, pastures, from the decayed interior of an acorn. Sept.-Nov. $2 \frac{5}{5} \times 3 \frac{7}{8}$ in. Sometimes confused with 2071 , of which 2068 has been considered a variety, but in 2071 the spores are smooth, while in this they are minutely warted or spiny.

2069. L. lacunosum Bull. (from the pits or depressions on the stem; lacuna, a hole or cavity) $a$.

$P_{c}$. subglobose, passing into a long stout stem, covered with small pointed spines, pale buff. St. tapering downwards covered with small lacunæ. Cap. and Spores olive-brown.

Woods. Autumn. $1 \frac{3}{4} \times 4 \frac{1}{1}$ in.

2070. L. pyriforme Schæff. (from its pear-like shape; pyrus, a pear, forma, shape) $a b c$. Subumbonate.

$P_{c}$. thin, flaccid, at first covered with minute pointed warts, brown, stoma small, torn, base sometimes forming a Col., slightly cellular. Cap. and Spores olive.

Commonly cæespitose, often held together by coarse strands of white $\mathrm{M}_{\mathrm{y}} \mathrm{c}$. On the ground in fields and woods, on rotten stumps and branches. pollards, oak. July-Nov, $1 \frac{5}{8} \times 2 \frac{1}{4}$ in. Col. not a permanent character.

2071. L. perlatum Pers. (from the great width of the peridium in comparison with the slender stem; pcrlatus, very wide) $a b c$.

$P$ e. subglobose or flattened, often passing into a somewhat slender stem, umbonate, often plicate below, at first covered with stout obtuse short spines, base sometimes forming a Col., pale brownish-salmon, stoma small. St. slightly spongycellular and in section convex above. Cap. and Spores olivaceous.

Woods, thickets, amongst trees, firs. Sept.-Nov. $2 \frac{1}{4} \times 5$ in. Col. not a permanent character. Sometimes made a var. of 2068, or 2068 is sometimes made a var. of this, but the spores are different.

2072. L. molle Pers. (from its softness or soft scurfy surface; mallis, soft).

Turbinate, base broad, abrupt. $P_{i}$. papyraceous, at first furfuraceous, collapsing, umber to olive, stoma small, irregular. Catp. collapsing, ochreous-olive. Sterile base well developed, slightly spongy-cellular.

On the ground, woods, oak. $1 \frac{5}{8} \times 2 \frac{1}{4} \mathrm{in.}$ 1)iffering from 2068 in the smocth spores.

2072a. L. cruciatum Rost. (from the somewhat cross-shaped spines of the cortex) $a$.

$P$ e. subglobose to broadly pyriform, narrowed below into a short stout sterile stem-like base, $\mathrm{I}_{4}^{\frac{1}{4}}-\mathrm{I}_{2}^{\frac{1}{2}}$ in. high by $\frac{3}{4}-\mathrm{r} \frac{1}{4} \mathrm{in}$. broad. Cor. whitish, formed of groups of minute, more or less pyramidal spines, breaking away in large flakes and exposing the inner yellowish-brown minutely granulated peridium; dehiscence by a small irregularly torn apical stoma. $\mathrm{Gl}$. umber. Spore's globose, smooth, almost hyaline, 5-6 $\mu$ in diam.; 
threads of capillitium mostly unbranched, slender, $4-5 \mu$ thick, ends tapering, very slightly coloured yellow-brown. The lacunose sterile base not projecting into the Gl. as a Col.

On the ground among hazel bushes. $I_{\frac{3}{4}}^{\frac{3}{4}} \times 1_{\frac{1}{x}}$ in. Whitish outer wall of peridium peeling off in large flakes as in species of Boorista, exposing the brown, minutely granulated inner Pe.

2073. L. hiemale Bull. (from its time of growth, winter, hicms) a b c.

Iyriform, rarely almost sessile and globoso-depressed. $P t$. membranous, flaccid, white then grey, yellowish or rufescent, stoma irregularly lacerate. Cap. and Spores smoky-yellow to olivebrownish. Sterile base well developed, at first verrucosoaculeate, elastic-tough with a thick bark.

Fields, downs, amongst dead leaves. Sept. -Jan. $2 \frac{1}{4} \times 3_{\frac{3}{8}}$ in.

2074. L. cælatum Bull. (from the large conical warts of the peridium as if embossed or carved in bas-relief; cclum, a sculptor's chisel) $a b c$.

At first subglobose or pyriform, then depressed, flaccid and ruptured above, contracted below into a short stout tapering stem and more or less covered with large conical warts, whitish or irory. Cap. and Spores ochreous-olive or opaque yellowgreen. Sterile base spongy-cellular.

Odour disagreeable, musty, mouse-like. Fields, woods, grassy roadsides. Iay-Oct. $7 \frac{1}{2} \times 6 \frac{3}{4}$ in.

2074a. L. depressum Bonard. (from the depressed peridium) $a$.

$P$ e. obconical, at first rounded above then flattened, base somewhat plicate, yellowish-white, then greyish-yellow, finally brownish, covered with spinulose warts. Stoma at first well defined, greatly extending with age. Sterile base well developed, separated from the olive Gl. by a diaphragm.

Commons, woods. Aug.-Oct. $1 \frac{1}{\frac{1}{4}} \times 1 \frac{1}{\frac{1}{4}} \mathrm{in}$.

2075. L. Bovista L. (from the resemblance to Borista) $a b c$.

Globose, sessile, usually plicate at base, at first subtomentose then smooth and glossy, white, stoma large, irregular. Cap. and Spores dull olive or olive-umber.

Sometimes grows in rings $30-50 \mathrm{ft}$. in diam. Edible when young and perfectly white within. Rich pastures, orchards, gardens, roadsides, grassy places. Aug.-Oct. $12 \times 11$ in. P'opular names "Puff-ball" and "I evil's snuff-box." Hodern Greek name "kranion," like a skull. A drawing in British Museum shows a diam. of IS in. A weight has been recorded of 20 lbs. An American example has been described with a larger diam. of $5 \mathrm{ft} .4 \mathrm{in}$. and a lesser of $4 \mathrm{ft} .6 \mathrm{in}$., height $9 \frac{1}{2} \mathrm{in}$.

2076. L. furfuraceum Schæff. (from the branny or scurfy peridium ; furfur, bran) a b $c$.

$P_{\ell}$. subglobose, passing into a short, stout stem, flaccid above, floccose, white then pale olive-yellowish, base white. Cap. and Spores olivaceous. Sterile base spongy-cellular, white.

Fields, fallow ground, old walls, about stumps. Sept. $1 \frac{3}{4} \times \mathbf{I}_{\frac{5}{5}}$ in. 
2077. L. Cookei Mass. (after Dr. M. C. Cooke) $a c$.

Subglobose or flattened below and hemispherical, abruptly contracted into a short stem, minutely furfuraceous, smoky-brown, umber-salmon or umber with a faintly purplish shade. St. pale spongy-cellular within. Cap. and Spores yellow- to olive-umber.

Gregarious. Sept. $I \frac{1}{5} \times I$ in. Like a small form of 2071 .

\section{CAVI. Bovista Pers.}

(From the German popular name, bofist, a fairy ball.)

Piridium double, external layer or cortex smooth, fragile, deciduous, not remaining as adnate warts, except in 2 c81 and 2083.

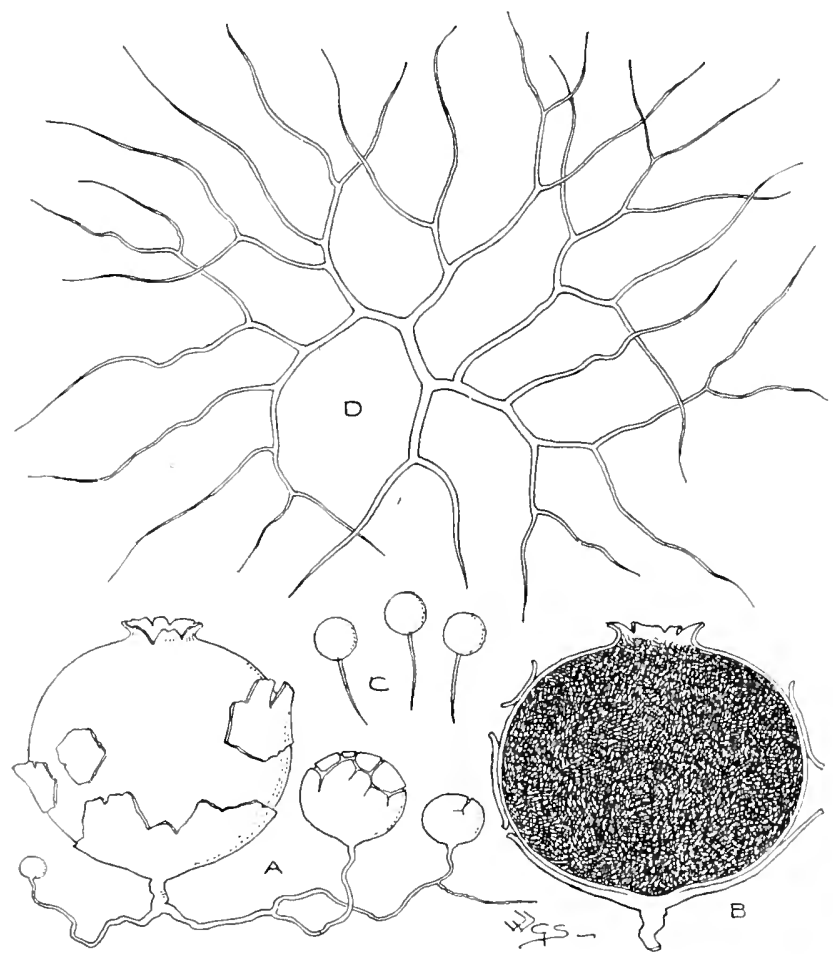

Fig. 132.-A, B, Fovista nigrescens Pers., entire and in section. One-half natural size. C, spores. $\times 750,1$, thread of capillitium. $\times 100$.

usually falling away in flakes, inner layer smooth, dehiscing by a definite or irregularly torn apical stoma. Sterile base absent. Spores globose or elliptical. (Fig. I 32.) Species 2078-2084

Spores globose.

Spores elliptical. 
2078. B. plumbea Pers. (from the lead-coloured inner layer of the peridium ; flumbeus, leaden) a $b c$.

Globose. Cor. thin, whitish, persistent towards lase, imner layer tough, thin, dehiscing by a small irregular somewhat prominent stoma, lead-colour, slate-whitish or purplish-slate. Cap. and Stores brown.

Dry grasiy and heathy places, downs, rarely on stumps. July-Nov. $1 \frac{5}{5} \times I_{8}^{5}$ in.

2079. B. nigrescens Pers. (from the at length black or blackish colour of the inner layer of the peridium; migrescins, turning black) $a b c$.

Subglobose. Cor. papery, whitish, inner layer thin, tough, dehiscing by an irregular somewhat prominent stoma, blackish, brown-, slate-, olive-brown- or blue-black. Cap. and Spores olive-brown then purplish-brown.

Dry pastures, heathy places. Jan.-Dec. $2 \frac{1}{2} \times 2 \frac{1}{1}$ in.

2080. B. olivacea Cooke $\&$ Mass. (from the at length olive-coloured capillitium and spores) a $c$.

Globose. Cor. thin, fugacious, whitish or ochreous, inner layer thick, soft, becoming brittle and breaking away in patches above, white or ochreous. Cap. and Sporcs citrine then olive.

On the ground. Sept. $2 \times 2$ in.

2081. B. ammophila Lév. (from the habitat, sandy places; Gr. ammos, sand, frilco, to love) a.

Broadly obovate, plicate below and tapering into a long stout root-like growth. Cor. broken up into tomentose warts, whitish, inner layer thin, dehiscing by a small irregularly torn stoma, pallid. Cap. and Spores olive.

Sancly places. Sept. $I_{\frac{3}{y}} \times I_{\frac{3}{4}}^{3}$ in. without root.

2082. B. cepæformis Mass. (from the shape, like a flat onion; cefa, an onion, forma, shape) $a$.

Subglobose, flattish, somewhat plicate below. Cor. minutely. furfuraceous, whitish to very pale buff, inner layer dehiscing by a small tom stoma, whitish or pale buff. Cat. and Sfores eitrine. Myc. rooting, cord-like.

(On the ground. $1 \frac{5}{8} \times 1 \frac{1}{8}$ in.

2083. B. pusilla Pers. (from the very small size; fusillus, small) a b. Subglobose. Cor. breaking up into minute scurfy squamules, pale yellowish-brown, inner layer flaccid, dehiscing by a small stoma, pale yellowish-brown or olivaceous ochre. AIyc. cordlike, rooting.

Pastures, hedgebanks. $\frac{3}{4} \times \frac{5}{5}$ in.

2084. B. ovalispora (Cooke \& Mass. (from the oval spores) $a b$.

Subglobose. Cor. very thin, subpersistent towards base, whitish or ochreous, inner layer thin, flaccid, smooth, dull lead-colour, dehiscing by an irregular stoma. Cap. and Spores brown.

On the ground. IVian. 2 in. Formerly inclueled with 2078 and 2079, but differing in the oval spores and thinner cortex. 


\section{FANI. IX. SCLERODERMACE \&}

Peridium thick with a well-defined base, sometimes forming a stem, opening at the apex in an irregular manner, feebly developed with a sterile base. Capillitium absent or scanty. Species 2085-2089

\section{Key to the Genera.}

Gleba composed of numerous distinct cells containing peridiola

I 7 Polysaccum.

Gleba cellular, cells without peridiola II 8 SCLERONERMA.

\section{POLYSACCUM DC.}

(From the many small cells within the peridium:

Gr. polus, many, saccos, a bag.)

Peridium irregularly globose, corky, attenuate downwards into a more or less elongated stem-like base, dehiscing by an irregular

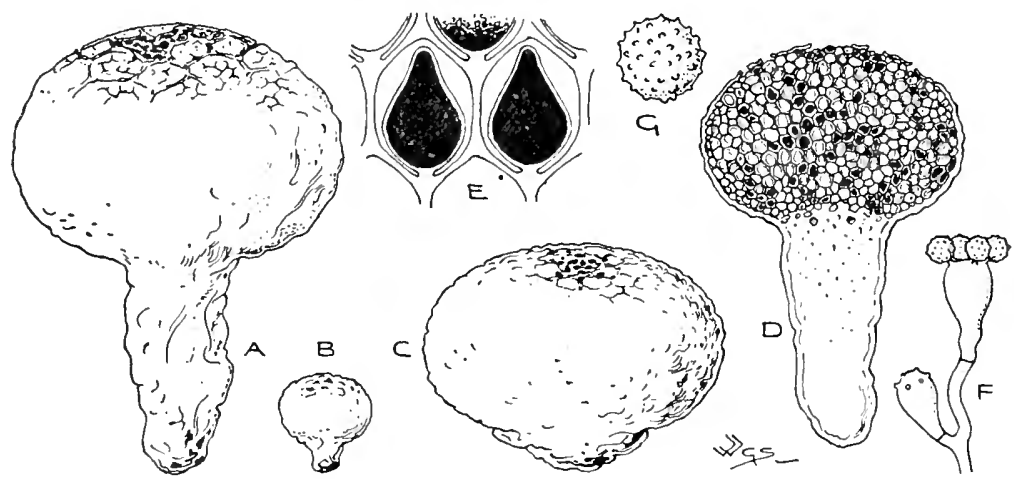

Fig. 133.-A, B, C, D, Folysaccun pisocarpium Fr., entire and in section. One-half natural size. E, section through part of gleba, showing carities and enclosed peridiola. $\times 5 . \quad F$, basidia and spures. $\times 250$. G, spore. $\times 750$.

rupture of the apex. Gleba consisting of numerous cells, each containing a peridiolum. Piridiole at first saccate or irregularly ovoid, sometimes filling the cell and becoming irregularly polygonal. (Fig. 133.)

2085. P. pisocarpium Fr. (from the pea-like peridiola; fisum, a pea, carpellum, a fruit) a $b$.

$P$. irregularly globose or ovate, smooth, subtuberculose, corky, attenuate downwards into a more or less stem-like base, or 
sessile or nearly so, pale to olive-umber, base spongy-cellular within. Gl. composed of numerous cells, each containing an olive-umber Peri. Spores dark brown.

On the ground. May, $25 \times 3 \frac{5}{8} \mathrm{in}$.

\section{SCLERODERMA PerS.}

(From the hard skin or peridium; Gr. skleros, hard, derma, skin.)

Peridium firm, cortex persistent in the form of warts, scales or granules, indehiscent or breaking up in an irregular or more or less

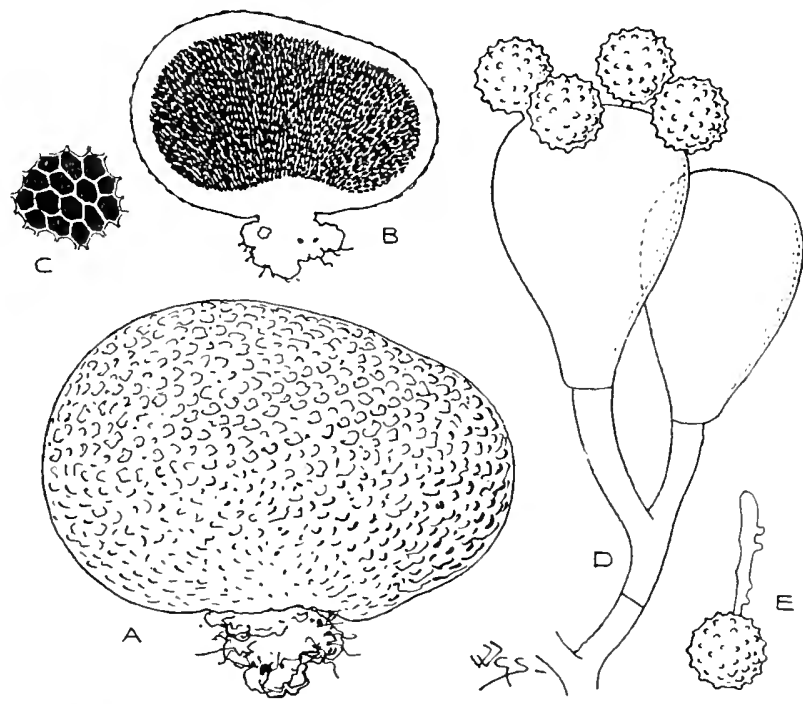

Fig. 134.-A, F, Sclcroderma zulgare Hornem., entire and in section. One-half natural size.

C, cells of gleba. $\times 5$. D, basidia and spores; E, spore germinating. $\times 750$.

stellate manner, convex internally at base. Glibr cellular, subpersistent, without peridiola. Trama in the cell walls. (Fig. I34.)

The peridium is frequently and extensively perforated by larve which eat the spore-mass and help to disseminate the spores.

Species 2086-2089

2086. S. vulgare Hornem. (from its commonness: a'ulgaris, common) $a b c$.

Subsessile, often lobed below and usually slightly depressed above, ovate, reniform in vertical section. $P$. thick, corky, verrucose or adpressed-scaly, pale warm-brown, scales darker. Gl. dark slate-colour. Trema white.

Often crespitose. Under the name of "vegetal)le tripe" it has been eaten when quite young and found not poisonous. Used for the adulteration of pâté de 
foie gras, sausage and poulard truffé in place of the French truffle, Tuber macrosportm. Odour rank, strong, disagreeable, sulphureous-alliaceous. Open places near trees. Aug.-Nov. Diam. $3^{\frac{3}{ \pm}}$ in. Often closely. resembles horse-droppings. Sometimes attacked by 1481 , which in turn is not infrequently attacked by Hyfomyces lutioririns. I'igs in search for truftles and pig-nuts will not eat Sclerodermn. Sometimes $5 \frac{1}{2}$ inches in diam. Var. cepa W. G. Sm., Scleroderma cepa Pers. Flattish, onion-shaped. Tar. levigatum Fuick. nomen. Even, smooth, often confounded with var. cervinum (Bolt. Hist. Funs. t. I16). Var. aurantiacum IV. G. Sm., Ly'copernon anrantiacum Bull. Brassy-yellow. Var. spadicenm IV. G. Sm., Scleroderma spadicenm l'ers. Wate-brown. Var. cercinum IV. G. Sm., Scleroderma corinum lers. Usually less than an inch in diam., hard and granular all over, resembling Elafhomyces granulatus, of which species it is sometimes, withont examination, placed in herbaria as a var. Fir woods. Probably a distinct species.

2087. S. verpucosum Pers. (from the warted peridium; acrucosus, full of warts) $a b c$.

Subsessile to stipitate. Pe. subglobose, thin, fragile and breaking up irregularly above, externally finely squarrose or minutely warted, when stipitate continued into a more or less elongate and sometimes lacunose stem, ochreous or dull brownish. $G l$. at first umber, then slate-colour to blackish. Trama whitish. Often furnished with a mass of Myc. at base.

Sandy ground, commons, open places, near lushes. July-Nov. $3 \frac{1}{ \pm} \times 5$ in. Sometimes attaclied by 1481 .

2088. S. Bovista Fr. (from its resemblance to a Bovista) a b c.

Subsessile, globose. $P_{\ell}$. thin, pliant, breaking up irregularly above, becoming nearly smooth, yellowish. Gl. yellowish- or olive- to purplish-brown. Trama yellow. Usually furnished with a mass of Myc. at base.

sandy ground near bushes. Sept. Diam. $2 \frac{1}{4}$ in.

2089. S. Geaster Fr. (from a fancied resemblance to a Geastcr) a b c. Sessile, globose. $P_{i}$. thick, almost smooth, splitting and expanding in an irregularly stellate manner, dull yellowish-brown. Gl. umber to pale and then dark slate. Trama whitish. Sometimes with a mass of Myc. at base.

Sandy places, stubble fields, woods, under oaks. Aug.-Oct. Diam. 3 in.

\section{FAII. X. NIDULARIACEÆ}

Spores produced in the interior of one or more indehiscent globose or compressed peridiola or sporangia which are at first contained within a peridium.

Species 2090-2098 
Key to the Gexera.

Peridiola several.

Peridiola attached by a funiculus or cord to the wall of the peridium.

Peridium of three layers, cup-shaped ................. II9 Cyathus,

Peridium of two layers, onter thick and felt-like, inner thin ; cup-shaped

i 20 CRucibulum.

Peridiola not attached by a funiculus to the wall of the peridium. Peridium of a single membrane,

globose $\ldots \ldots \ldots \ldots \ldots \ldots \ldots \ldots \ldots \ldots \ldots \ldots \ldots \ldots \ldots \ldots \ldots \ldots$
Peridiolum solitary, ejected from the peridium. Peridium globose, subglobose or urceolate, sessile ......... I22 SРнÆrobolus.

\section{CYATHUS Haller.}

(From the cup-shape; Gr. Kuathos, a wine cup.)

Peridium cup-shaped, composed of three distinct, closely connected layers, apex at first closed with a white membrane or epiphragm,

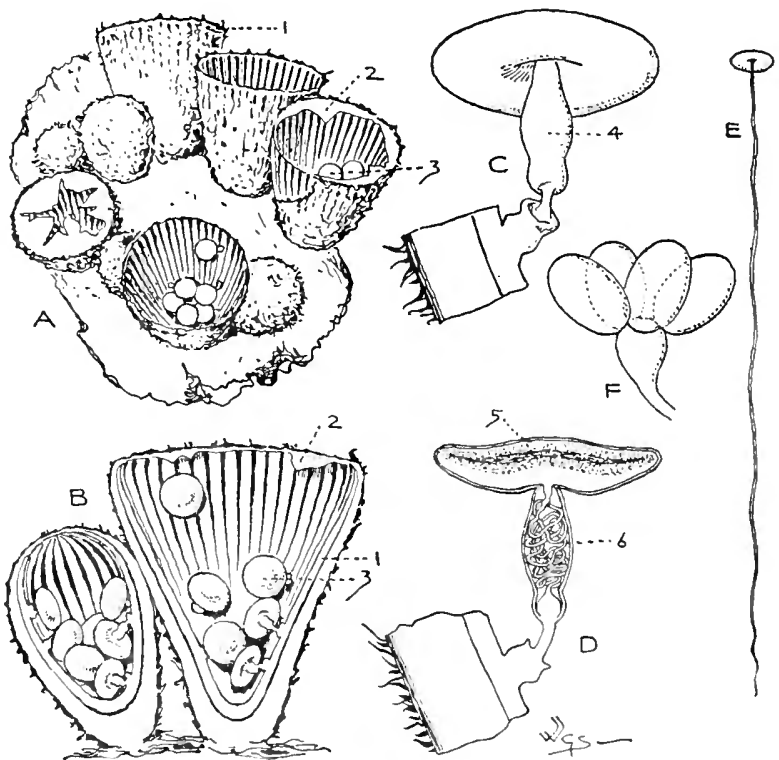

Fig. I35.-Cynthus striatus Hoffm.

A, group of plants. Natural size. I, section of young and mature plants. $\times 3$. c, peri. diolum; $D$, section of ditto. $\times$ ro. E, peridiolum with the filiform appendage 6 of fig. I $)$ uncoiled and drawn out of the funiculus. $\times 3 . \mathrm{F}$, basidia and spores. $\times 500.1$, peridium; 2 , epiphragm; 3. peridiola; 4, funiculus; 5 , hymenial layer, lining peridiolum; 6, filifurm appendage coiled within the funiculus.

becoming broadly open. Peridiola compressed, umbilicate, attached to inner wall of peridium by an elastic cord or funiculus. (Fig. I 35.) 
The British species grow in fields and gardens, generally on chips, twigs and rotten wood; some foreign species grow on dung of horse and cow, and on bagging, matting, coffee-chaff, etc. Species 2090, 2091 2090. C. striatus Hoffm. (from the striate inner surface of the peridium) a $b c$.

Obconical, truncate at base and apex, striate and shining leadcolour within, hirto-tomentose and ferruginous without. Peri. subcircular, biconvex, lead-colour. Funn. whitish, containing a long filiform appendage within. Springing from coarse brown Myc.

Fasciculate. Fields, woods, gardens, decaying twigs, wood, old willow, fircones, coco-nut fibre. Feb.-Nov, $\frac{3}{x} \times \frac{5}{5}$ in.

2091. C. vernicosus DC. (from the silky-shining outer surface of the peridium; z'ernicosus, shiny as though varnished) a $b c$.

Campanulate, undulate, tapering downwards to a narrow base, inside smooth, lead-colour or brownish, outside minutely silky, ferruginous, ochreous or greyish. Peri. circular, biconvex, blackish or blackish-brown. Funn. white.

On the ground, stubble fields, gardens, flower-pots, flower-boxes, rotten vrood, chips. Feb.-Sept. $\frac{1}{4} \times \frac{1}{4}$ in.

\section{CRUCIBULUM Tul.}

(From a fancied resemblance to a small melting-pot, crucibulum.)

l'eridium cup-shaped, consisting of two layers, the outer thick and felt-like, the inner a thin membrane which is at first continuous over

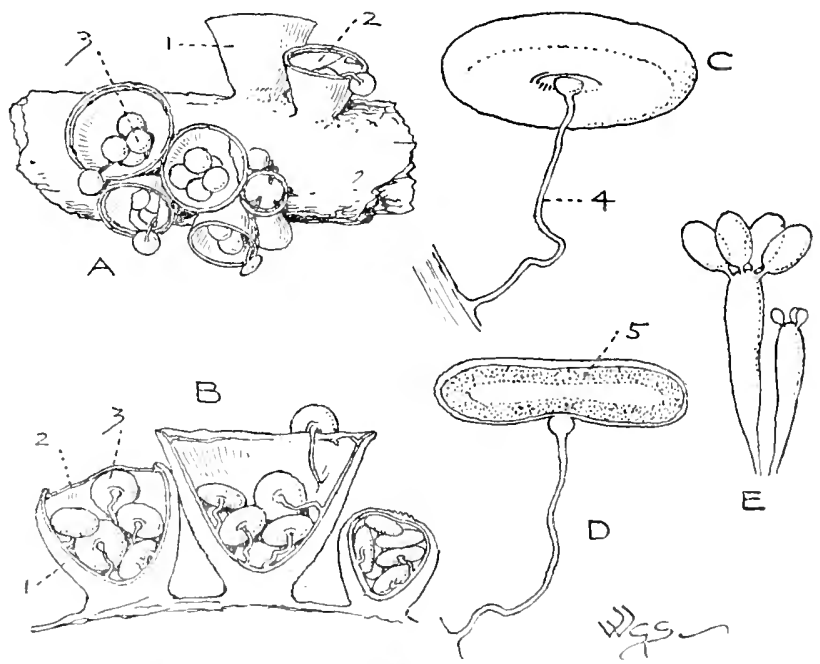

Fig. I36.-A, Crwibulum zulgar Tul. $\times \frac{4}{3}$. E, section showing young and mature examples. $\times$ 3. C, peritiolum; 1 , section of ditto. $X$ I2. E, basidia and spores. $\times 660 . \quad$, peridium; 2, epiphragm; 3 , peridiola ; 4, funiculus ; 5 , hymenial layer lining peridiolum. 
the apex as a flat epiphragm. Peridiola numerous, attached to the inner wall of the peridium by a long cord which originates from a depression in a central nipple-like tubercle on the under side of the peridiolum. (Fig. $\mathbf{1}_{3} 6$.)

2092. C. vulgare Tul. (from its commonness; anlgaris, common) $a b c$.

$P_{\ell}$. thick, sienna-white, cinnamon or greyish, externally minutely tomentose, internally smooth and shining. Peri. circular, biconvex, pale ochreous or sienna-white.

Rotten branches, planks, twigs, chips, cones, herbaceous stems, grass, rotten straw, dead fern-fronds. Oct.-Nov. $\frac{3}{8} \times \frac{1}{4}$ in.

\section{NIDULARIA Bull.}

(From a fancied resemblance to a nest, nidus.)

Peridium globose, consisting of a single membrane, at first closed then opening by a more or less regular mouth or becoming irregularly

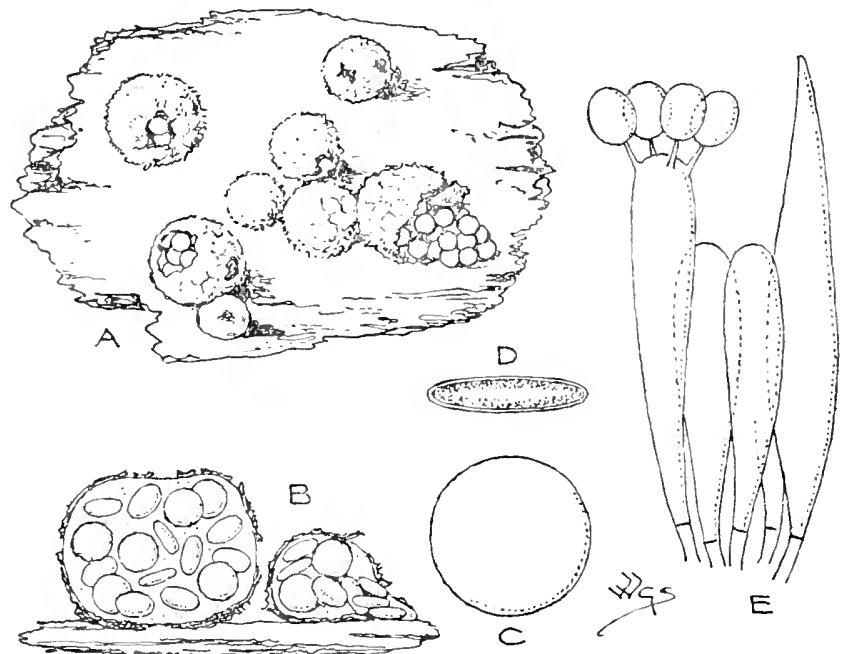

Fig. 137.-A, Vidularia fisiformis Tul. $\times \frac{4}{3} . \quad B$, sections showing peridiola embedded in mucus. $\times 3 . \mathrm{C}, \mathrm{D}$, peridiolum and section. $\times$ I2, E, basidium with spores, cystidium and the smaller cells of hymenial surface. $\times 660$.

ruptured, containing numerous peridiola enveloped in mucus, not attached to inside by a funiculus. The mucus arises from disintegration of hyphæ. (Fig. I37.)

Species 2093-2095

2093. N. pisiformis Tul. (from the pea-like shape; pisum, a pea, forma, shape) $a b c$.

$P_{\ell}$. turbinate, strigose, tuberculose, splitting irregularly, cinnamon, pale buff or salmon. Peri. subrotund, biconvex, smooth, shining, brown.

Gregarious or solitary. Dead branches, chips, leaves, pine, sometimes on the 
ground, woods. May-Oct. $\frac{5}{16}$ in. Spores $7-8 \times 8-9 \mu$. Var. Broomti IIass., on pine wood. Spores Io $\times 4-5 \mu$.

2094. N. Berkeleyi Mass. (after the Rev. M. J. Berkeley) $a$.

$P_{\ell}$. subglobose, thick, hirto-tomentose, bright cinnamon. Piri. orbicular, biconvex, smooth, shining, bright brown.

Solitary or 2-3 together. Twigs, woods. $\frac{5}{16} \mathrm{in}$.

2095. N. confluens Fr. (from the confluent habit) $a b c$.

$P e$ subglobose, thin, villous, whitish or buff-white. Peri. orbicular, compressed, pale yellowish-umber to chestnut.

Putrid leaves, twigs, chips, wood, rarely on the ground. Oct.

\section{SPHÆROBOLUS Tode.}

(In reference to the ejection of the peridiolum from the peridium;

Gr. sphaira, a ball, ballo, to throw.)

Peridinm globose, subglobose or urceolate, sessile; peridiolum solitary, ejected from the peridium. (Fig. 138.) Species 2097, 2098

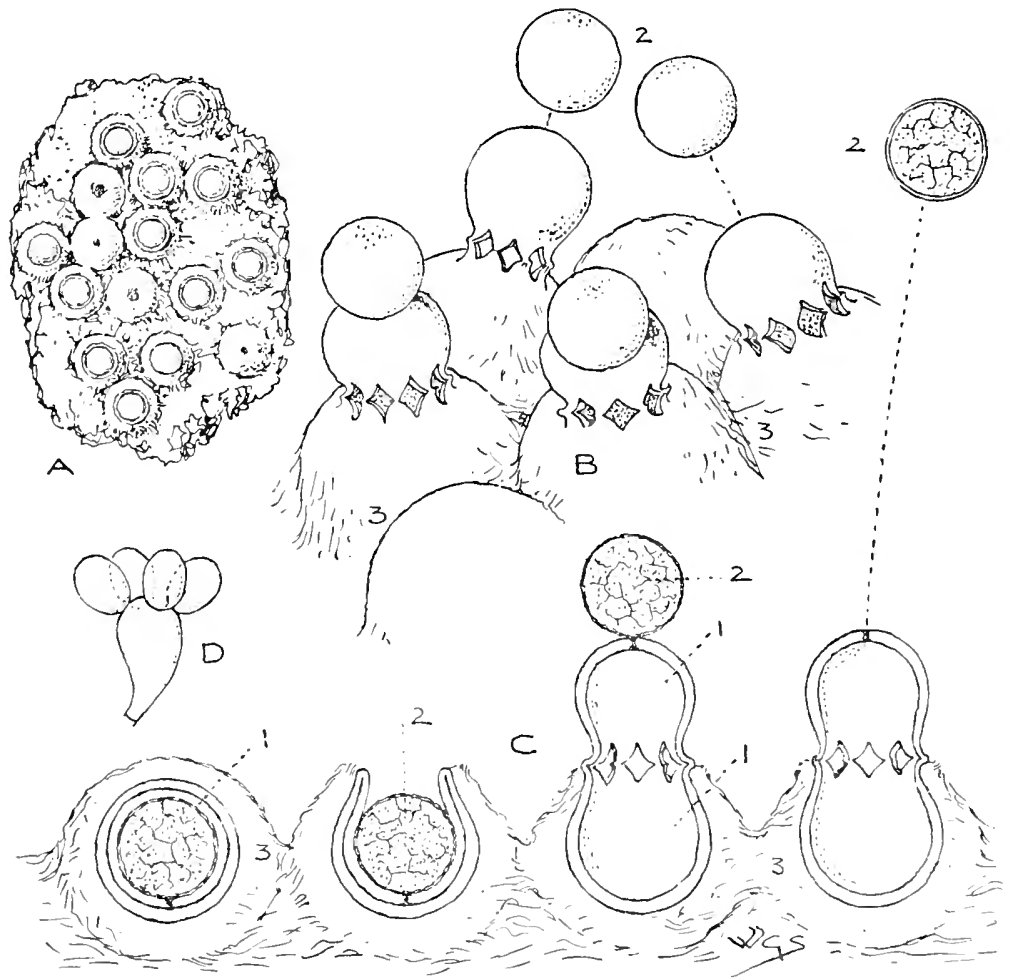

Fig. I38.-A, Spharobolus stellatus Tode. $x_{3}$. B, group of ditto; c, sections of young and mature examples. $\times$ i2. D, basidium and spores, $\times 660 .$, , peridium of two coats; 2 , peridiola $\cdot 3$, mycelium, 
Peridizm globose, of two layers, the inner at length extruded elastically and ejecting a single peridiolum.

2096, 2097

Pcridium homogeneous, sessile on a broad base, subrotund then urceolato-ventricose; peridiolam extruded from the apex of the peridium.

2098

2096. S. stellatus Tode (from the stellate mouth of the peridium) $a b c$.

$P e$. minutely tomentose, at first immersed in a thick web of white Myc., opening above in a 5-to 7 -stellate manner, elastically extruding the inner layer and ejecting a globular yellowish or orange peri.; pale yellow or pale yellowish-buff, darker or orange within. The peri. which encloses basidia and spores is ejected for 8 or more inches with a slight clicking sound.

Usually crowded in troops. Rotten wood, sawdust, earth of old stumps, twigs, leaves, rotten grass. Often in stoves and greenhouses. Jan.-Dec. Dian. $\frac{1}{10}$ in.

2097. S. dentatus W. G. Sm. (from the toothed peridium; dentatus, toothed) $a$.

$P e$. finely pilose with erect hairs, springing from scanty brownish Myc., opening above in a $4^{-}$to 7 -stellate manner, the rays clad with long white hairs, which at first converge over the opening, outside pale livid-buff to vinous-brownish, pale brown or brown, inside white, ivory, white-greyish or faint olive-ivory, ejecting a reddish-brown peri.

Scattered. Dead elder. Failand, Bristol, Aug. ISS8, R. Baker. Diam. $\frac{1}{16}$ in.

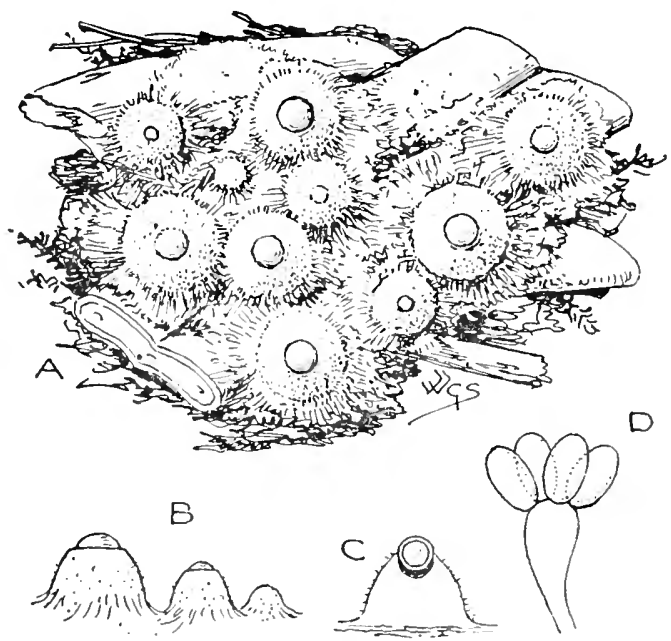

Fig. 13.-Spharobolus terrestris W. G. Sm.

A, seen from above; 1 , seen from side; $c$, section. $\times 12.1$, basidium and spores. $\times 660$. 
2098. S. terrestris W. G. Sm., Thelebolus terrestris A. \& S.-not of Tode-(from the frequent habitat, the earth; terrestris, pertaining to the earth) $a b c$.

$P_{t}$. at first hemispherical, saffron-yellow honey-colour or sienna, seated on a dense tomentose Sub. of buff-white here and there brownish Myc. Peri. spherical, colour as Pe.

Crowded in troops. Earth, leaves, pine, moss, rotten wood, oak. Mar.Nov. Diam. $\frac{1}{32}$ in.

\section{FAM. XI. HYMENOGASTRACEE}

Subterranean. Peridium indehiscent. Capillitium absent. Spores $2-7$. Species 2099-2122

Key to THE Genera.

Spores globose warted.

Sterile base of peridium well developed.............. I23 OCtaviania.

Sterile base of peridium absent ...................... I25 HVDnangium. Spores smooth.

Sterile base of peridium absent. Spores elliptical ... I24 Melanogaster.

Gleba cartilagineo - glutinous. Spores minute, elliptical .....................................

Peridium with root-like fibres on the surface $\ldots . . . . .$.
es large, elliptical or fusiform, rugulose or nodulose.

Sterile base of peridium well developed............. I $2 S$ HyMenogaster.

CXXIII. OCTAVIANIA Vitt.

(After Dr. Vincent Ottaviani.)

Pridium continuous or cracked, cottony, running down into a well developed sterile base. Trama byssoid, easily divisible, cells at first empty, peripheral cells small, spherical, central cells much larger and irregular. Spores $2-7$, globose, warted. (Fig. I 40.)

Distinguished from Hydnanginm by the presence of a sterile basal stratum and from Mclanogaster in the cavities of the gleba being at first empty and the trama byssoid. Species 2099-2101

2099. 0. asterosperma Vitt. (from the star-shaped spores; Gr. aster, a star, spermat, a seed) a $b c$.

Globose, regular or irregular. $P e$. continuous or cracked into areolæ, minutely downy, at first whitish-buff, then rosy-salmon, clouded umber. $G l$. salmon, slightly buff, cells umber.

Generally near the surface, often partially exposed. Odour sweet, of Ocymum basilicum, sometimes of new cheese or pungent. Adhering to buried 
twigs and leaves. Oct. Diam. $\mathbf{I} \frac{1}{2}$ in. Changing to turquoise-blue and black where bruised. Commonly attacked by a bright orange mould, sepedonium chrysospermum.

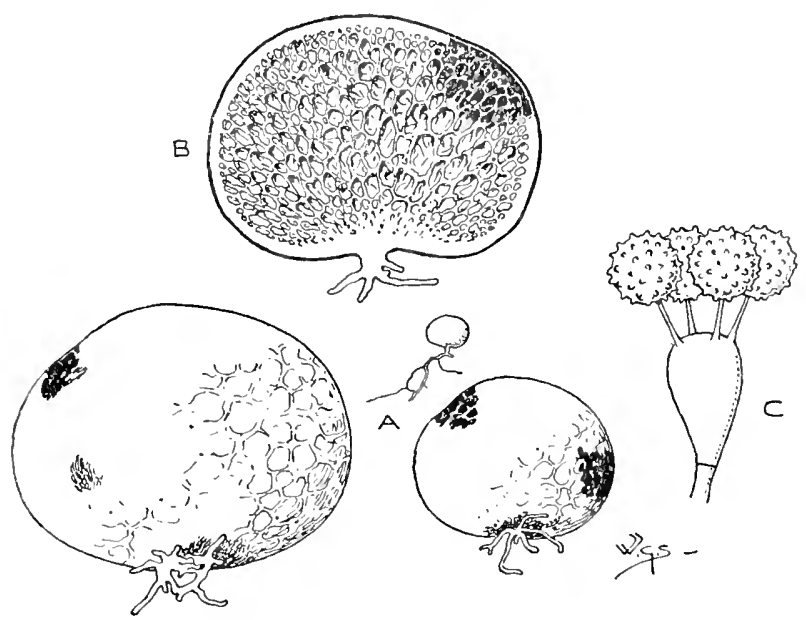

Fig r fo.-A, в, Octarizinia asterosperna Vitt., entire at different ages, and in section. Natural size. C, basidium and spores, $\times 500$.

2100. 0. Stephensii Tul. (after H. O. Stephens) $a \cdot b c$.

Irregularly globose or nodular-oblong, usually irregular in shape, base rugoso-plicate, cribrose, furnished with branched fibrous root-like growths. $P_{\epsilon}$. continuous, pale red to rufous, clouded darker. Gl. white then pale salmon, cells small, pale sienna.

Odour disagreeable like 1280. About one-quarter of the plant is usually seen above ground, but often wholly superficial, covered with dead leaves, etc. Woods under lime-trees with Nostoc. Aug.-Dec. Diam. $1 \frac{1}{8}$ in. Exuding a white aromatic milky juice.

2101. 0. compacta Tul. (from the compact sulstance) $a b c$.

Irregularly subglobose, minutely cottony. $P e$. continuous or slightly granular, white to ochreous-white. $G l$ salmon-white, cells pale salmon-buff.

Gregarious. Wholly or half-buried, involved in rooting masses of white Myc. Amongst root., grass, earth and mycelium. Oct.-Nov. Diam. $\frac{3}{4} \mathrm{in}$.

\section{CXiviv. MELANOGASTER Corda.}

(From the black interior; Gr. melers, black, sastir, the belly.)

Piridium without a distinct base, branched fibres springing from every part of its surface. Cells of gleba more or less rounded, large in the centre, smaller towards the periphery, walls of cells thick, 
continuous with the peridium. Spores $3 \mathbf{5}$, subsessile on the basidia, smooth, usually coloured. (Fig. I 4I.)

The absence of a distinct rooting base, the branched fibres

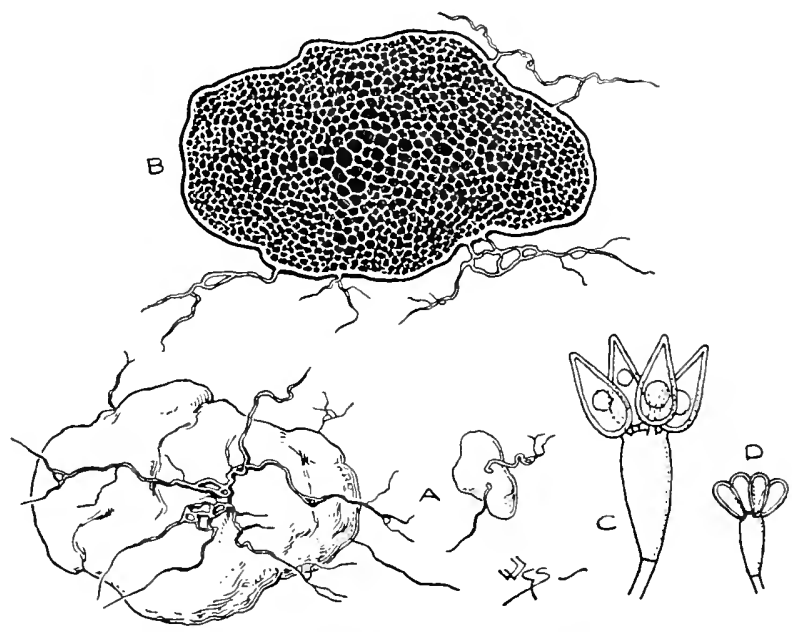

Fig. I41.-A, B, Melanogaster ambigans Tul., entire and in section. Natural size. $c$, basidium and spores. $\times 500 . \mathrm{D}$, basidium and spores of 11 . variegitus Tul. $\times 500$.

traversing the whole surface of the peridium, and the smooth spores are characteristic. Species 2102, 2103 2102. M. variegatus Tul. (from the variegated gleba) a $b c$.

Irregularly globose or nodular-oval. $P \epsilon$. adpressedly tomentose, at first yellow or ochreous, then dark reddish-ferruginous. $G l$. blackish with indigo-black cells. Trama white, whitish or bright orange.

More or less exposed above ground dnring growth, covered with leaves and twigs. Taste sweet and approved by many when cooked; odour sharp, strong, aromatic, agreeable, not unlike bitter almonds. Under beech, poplar, oak. June-Nov, $2 \frac{1}{4}$ in. Spores Io $\times 5 \mu$, elliptic-oblong. Eaten on the Continent, harmless but hardly pleasant. Sold half a century or more ago as an esculent in Bath under the name of Red Truffle. Var. Broomenanus Tul. Trama dull white or yellowish, never bright yellow or orange. Usually gregarious, some two or three inches beneath the surface or half exposed. Scentless when young but soon acquiring a sweet, treacley odour, or a scent of decaying pears.

2103. M. ambiguus Tul. (from its being doubtfully a true species; ambiguzus, doubtful) $a b c$.

Irregularly globose or oval-nodular. $P e$. nearly even, pale olive then reddish-brown. Gl. jet- or slate-black. Trama buffwhite or white.

Iighly fetid of assafaetida. Under beech, poplar, oak, fir. April-Oct. I in. Spores $\mathbf{I} \mathfrak{j}^{-1} 5 \times 7-S \mu$ ovate, papillate. Var. intermedius Tul. has a velinwisb trama which becomes red in drying. 
CXXV. HYDNANGIUM Wallr. ex Klotzsch.

(From the tuberous form; Gr. Indnon, a tuber or truffle, aggeion, a vessel.)

Peridium fleshy or membranous, smooth or silky ; sterile base not developed. Trama vesicular, cells minute, unequal, at first empty then filled with globose or ovate, echinulate spores. Sfores 2-4. (Fig. 142.)
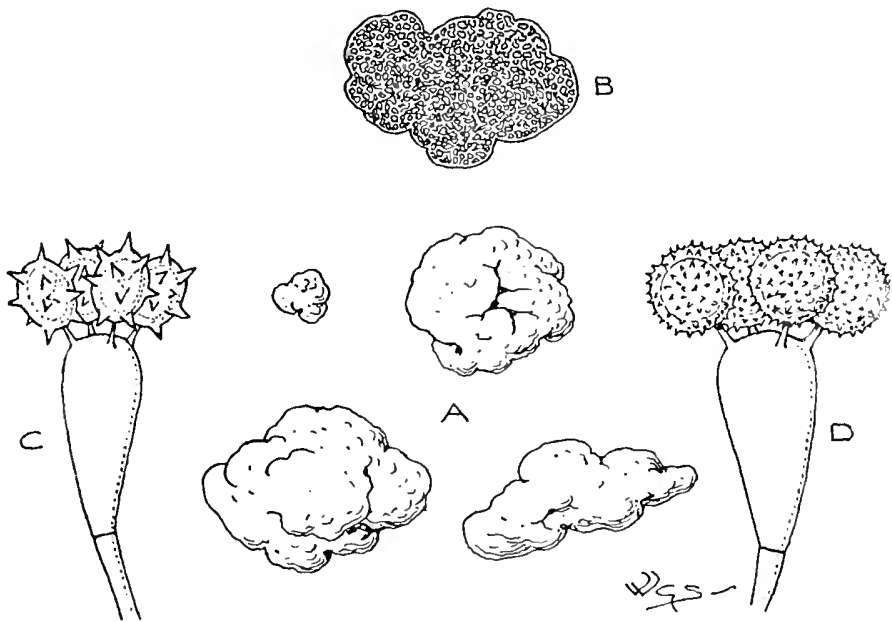

Fig. I42, A, L, Hydnangizm carotacolor Berk., entire and in section. $\times \frac{4}{3}$.

C, basidium and spores. $\times 660$. D, basidium and spores of $H$. camcum Wallr. $\times 660$.

The globose or ovate, usually small spores and the absence of a sterile base separate Hydnangium from its nearest ally Octaviania.

Species 2104, 2105

2104. H. carotæcolor Berk. (from the carrot-like colour; carota, carrot) $a b c$.

Globoso-oblong or oblong-rounded, nodular, rootless. $P$ e. thin, slightly tomentose, carrot-red, becoming dark orange-brownish when dry. $G l$. orange.

Sometimes half-exposed, often superficial. Woods, downs, under trees. Aug.-Jan. I Hiam. $1 \frac{1}{8}$ in.

2105. H. carneum Wallr. ex Klotzsch (from the flesh-colour; carneus, of flesh) $a b c$.

Irregularly subglobose or rounded-elongate, rontless; P'. very thin, at first covered with white fugacious down, flesh-colour, becoming ochreous-salmon when dry.

Subterranean or half-exposed, often superficial. Above the roots of Eucalyptus, etc. Oct.-I)ec. I liam. 5 in. 


\section{HYSTERANGIUM Vitt.}

(From the shape; Gr. hustora, the womb, agrcion, a vessel.)

Peridium more or less separating from the gleba in drying. Gleba cartilagineo-glutinous, cavities at first empty. Spores 2-3, seldom nore, smooth. (Fig. I43.)

Characterised by the elongate elliptical smooth spores and the cartilaginous-nucous consistency of the gleba, which resembles

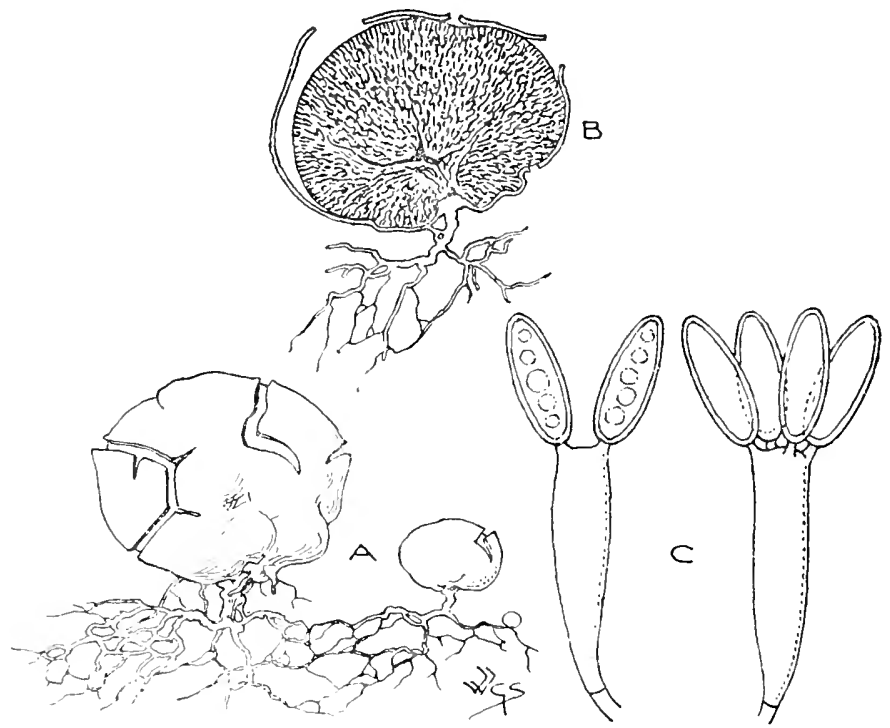

Fig. ${ }^{1}$ 43.-A, D, Hysterangium nephriticum Berk., entire and in section. $\quad \times \frac{4}{3}$. $c$, basidia and spores. $\times 660$.

that of Ithyphallus and Clathrus in the young state. There is frequently a copious development of mycelium. Species 2106, 2107 2106. H. nephriticum Berk. (from a fancied resemblance to a kidney when seen in section; Gr. nephros, the kidneys) $a b c$.

Irregularly globose, more or less indented. $P e$. somewhat thick, firm, elastic, tomentose, white, becoming pale rufous when bruised. Gl. pale blue or grey, here and there greenish, cells irregular, minute, radiating from the base.

Commonly S-9 $_{-9}$ in. deep in the ground. Odour at first slight, then like Hypericum, at last like decaying puff-balls. Woods. Sept.-Feb. $\frac{3}{t}$ in.

2107. H. Thwaitesii B. \& Br. (after George Henry Kendrick Thwaites) $a b c$.

Irregularly globose, more or less indented. $P \boldsymbol{e}$. membranous, slightly silky, white, becoming rufous when bruised.

Commonly 8-9 in. deep in the ground. Woods. Aug.-Oct. $\frac{7}{5}$ in. 


\section{RHIZOPOGON Fr.}

(From the tuberous growth with the attached fibres;

Gr. rhiza, a root, pogon, a beard.)

Pcridim thick, subcoriaceous or submembranous, continuous or cracked and somewhat evanescent, with vein-like branched fibres

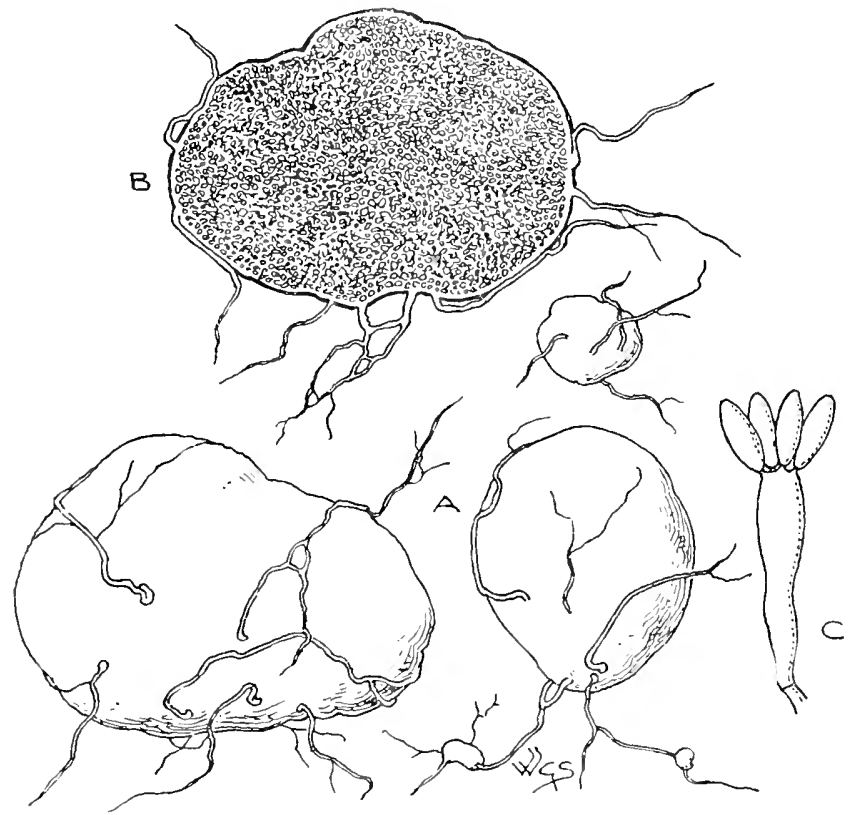

Fig. 144.-A, $\mathrm{B}$, Rhisopogm rubecens Tul, entire and in section. $\times \frac{4}{3}$. c, basidium and spores. $\times 660$.

traversing and partially adnate to the surface; substance of gleba firm, cavities at first empty. Spores 2-7. (Fig. I 44.)

2108. R. pubescens Tul. (from its becoming vinous rose-colour when bruised; rubescens, becoming red) a $b c$.

Irregularly subglobose or ovate. $P_{c}$. silky, white, when mature yellow, olive or warm-brown. $G l$. at first white, then yellowish, greenish or brownish.

Somewhat superficial but often some inches deep. Odour acid, when young like sour ham, at length nauseous-feticl. Sandy fir-woods, plantations in very light peaty or fibrous soil, amongst pines. Oct.-Dec. Diam. I 3 in. Var. Berkeleyanus IV. G. Sm., Melanogaster Berkileyanus Br., smaller and highly fetid. 
2109. R. luteolus Fr. (from the dull yellowish colour when halfmature; huteolus, yellowish) $a b c$.

Irregularly subglobose or ovate. $P e$. thick, subcoriaceous, whitish to dull yellow and olive- or nut-brown. Gleba black with white septa.

Solitary or gregarious. Subterranean or partly exposed. Taste insipid; odour at first weak then strong and offensive. Sandy fir-woods, under beech. Sept.-Oct. Diam. I $\frac{5}{8}$ in.

\section{HYMENOGASTER Vitt.}

(From the usually thin peridium; Gr. humen, a membrane, gaster, the belly.)

Globose or irregular. Peridium fleshy or thin, simple, homogeneous, running down into a sterile base, except 2116. Cavities of gleba at first empty, radiating from the base or irregularly scattered.

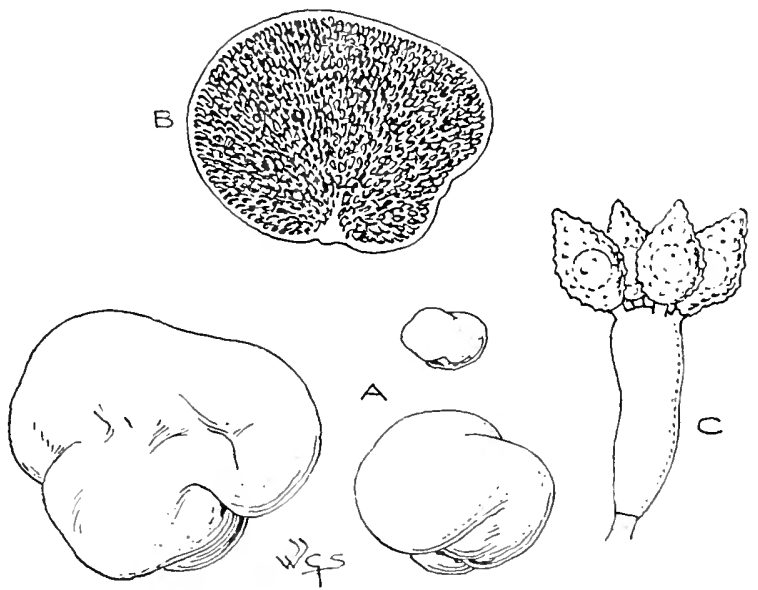

Fig. 145.-A, E, Hymenogaster tener Berk., entire and in section. $\times \frac{4}{3}$. c, basidium and spores. $\times 660$.

Trama composed of elongate celis, not of byssoid flocci, not easily separable. Spores I-4. (Fig. I 45.)

All the species are nearly superficial in growth, none are edible. Species $2110-2122$

2110. H. Klotzschii Tul. (after Johamn Friedrich Klotzsch) a b c.

Irregularly globose to kidney-shaped. $P t$. adpressedly downy, fibrillose at base, dull white becoming yellowish. Gl. pallid, becoming rufous-ochre.

Sandy soil. I)ec. I)iam. 
2111. H. muticus B. \& Br. (from the absence of a base; muticus, curtailed, blunt) a $b$.

Globose, scarcely lobed. $P_{t}$. at length much cracked, white then brownish. Gl. almost destitute of a sterile base, somewhat firm and dry, cells loose, pale yellowish-brown.

Odour slight. Nov, Diam. $\frac{5}{8}$ in.

2112. H. luteus Vitt. (from the yellow gleba ; lutus, yellow) $a b c$.

Irregularly globose or ovate, sometimes deformed. $P_{\ell}$. very thin, soft, silky, white to brownish. Gl. bright yellow.

Almost scentless or slightly sweet, becoming fetid. Sept.-Mar. Diam. I in.

2113. H. lycoperdineus Vitt. (from the shape, like a small puff-ball, Lycoperdon) a b c.

Subglobose, somewhat deformed, varied in form, plicate at base. $P_{\ell}$. smooth, white then pale whitish-salmon to brownish. $G l$. soft, elastic, with somewhat large, irregular cavities, pallid to pale fuliginous.

Gregarious. Odour strong, onion-like, unpleasant when dry. In earth and clay. Nov. Diam. $\mathbf{1}_{\mathrm{z}}^{\mathrm{3}}$ in.

2114. H. decorus Bull. (decorus, seemly) a b c.

Irregularly globose or oval, sometimes slightly deformed. $P e$. dull white becoming yellowish in places. Gl. lilac-brown at length blackish.

Woods, tan-pits. Oct.-Nov. Diam. $\mathbf{I}_{8}^{3}$ in.

2115. H. vulgaris Tul. ex B. \& Br. (from its commonness; z'ulgaris, common) $a b c$.

Subrotund, irregular, often indented, somewhat soft. $P_{\epsilon}$. white, becoming discoloured. Gl. with rather large irregular cavities, dull white then dark brown.

Gregarious or subcæspitose. Loose soil. July-Oct. Diam. $\frac{5}{8}$ in.

2116. H. pallidus B. \& Br. (from the pallid peridium) $a b$.

Rounded, depressed. $P i$. nearly smooth, somewhat soft, white, then dull tan-colour. $G l$. without a sterile base, white, yellow, then pale brown.

Odour weak. Fir plantations. Oct. Diam. $\frac{3}{8} \mathrm{in}$.

2117. H. citrinus Vitt. (from the colour ; citrcus, lemon-yellow) a $b c$. Irregularly globose or ovate, lobed, indented, deformed. $P c$. shining as if silky, greenish-lemon when young, then lemon, golden-yellow, bright brassy-yellow or sulphur-cream, becoming rufous-black.

Used as food in past times by country folk in the truffe districts of England. Odour, which communicates itself to anything near, strong of cheese. May-Lec, Diam. I $\frac{1}{4}$ in.

2118. H. olivaceus Vitt. (from the colour of the gleba, like a ripe olive) a $b c$.

Irregularly globose or ovate, lobed, indented, deformed. $P e$. at first silky, whitish or faintly lemon, becoming rufescent when 
touched. Gl. whitish, passing through buff to rufous olive. Trama white.

Odour spicy. Woods. Nay-Nov. Diam. I I in. A form between this and 2117 has been named var. modestus $\mathbf{B}$. \& Br. This is $H$. populetorum B. \& Br. non Tul.

2119. H. tener Berk. (from the tender substance; tencr, tender) $a b c$. Globose, irregular, more or less indented, silky or obscurely scaly, soft, tender. Pe. thin, dull white. Gl. with a conspicuous sterile base, pale rose, then greyish-brown.

Odour strong, pungent, like the odour of acrid Lactarii, in decay like sewergas. Woods, under oak-leaves. Sept.-Jan. Diam. I in. This is $H$. lilacinus B. \& Br. non Vitt.

2120. H. Thwaitesii B. \& Br. (after George Henry Kendrick Thwaites) $a c$.

Irregularly ovate or elongate to globose, firm. $P e$. dull white, here and there discoloured. Gl. brown.

Sept.-Oct. Diam. $\frac{1}{2}$ in.

2121. H. griseus Vitt. (from the grey gleba ; grisens, grey) $a$.

Globose, somewhat irregular or depressed. $P c$. brown, at first covered with evanescent whitish down. $G l$. with minute grey cells which become mottled-blackish.

Odour sweet of lily-of-the-valley. In leaf soil. Diam, $\frac{1}{2}$ in.

2122. H. pusillus B. \& Br. (from the small size; pusillıs, small) $a b$.

Obovate or subdepressed, white. $G l$. with a comparatively large sterile base, cells comparatively large, dull white.

Almost scentless. Mossy ground. Oct. Diam. $\frac{1}{8}$ in. Yellowish-brown when dry and then closely resembling Sclerotium complanatum.

\section{RECENT ADDITIONS}

52a. Lepiota serena Gill. (seremıs, clear, bright). White.

$P$. campanulate, glabrous; mid. sometimes slightly darkened. St. slender, subbulbous. $A$. deciduous.

Amongst grass. Carlisle, Miss Decima Graham, Sept. 190S. $1 \frac{1}{8} \times 2 \frac{3}{8} \times \frac{1}{8}$ in.

121a. Tricholoma earneolum Karst. (from the colour, caro, flesh).

$P$. hemispherical to convexo-plane, pale flesh-colour. St. partly stuffed, rigid, subpruinose. $G$. finally very broad, very crowded, white.

Grassy places in woods. Yorkshire, Sept. IgoS. $I_{\frac{1}{2}} \times \mathbf{I}_{4}^{\frac{1}{4}} \times \frac{1}{3} \mathrm{in.}$

154b. Clitocybe obscurata Cooke in litt. (olscurus, obscure).

$P$. plane, then infundibuliform, moist, greyish-umber, paler towards marg. St. solid, smooth or slightly striate, slightly attenuate 
downwards and never clavate, colour as P. G. decurrent, subdistant, white.

Odour of meal. Amongst grass and dead leaves in company with 154. Carlisle, Miss Decima Graham, Sept. IgoS. $2 \times \mathbf{I}_{2}^{\frac{1}{2}} \times \frac{1}{4}$ in.

187a. Clitocybe connata Gill. (from the connate stems at the tuberous base). White.

$P$. Heshy, thin, conical, then expanded with an evanescent umbo, unequal, naked. St. hollow, thick, smooth. $G$. unequally decurrent, narrow, crowded, subarcuate.

Moist woods. Carlisle, Miss Decima Graham, Sept, 19oS. $2 \frac{1}{4} \times 3 \times \frac{1}{3}$ in.

262. Collybia dryophila Quél. Var. alícaris Cooke in litt. Large, coarse.

$P$. shaped like a bee-hive, rufescent at mid., 3 in. diam. St. very hollow.

Amongst dead leaves. Carlisle, Miss Decima Graham, Sept. 190 S.

593a. Pholiota soporia Karst. (from its relationship to 593, soror, a sister).

$P$. fleshy, convexo-expanded, striate, squamulose, fulvous $\cdot$ cinnamon. St. fistulose, equal, flexuous, with white fibrillose sq., apex furfuraceous. G. sinuato-adnate, crowded, pallidcinnamon.

On wood-shavings. Yorkshire, Sept. IgoS.

640a. Inocybe commixta Bres. (from being confused with 640, commixtus, mixed together).

$P$. Heshy, conico-campanulate, expanso-umbonate, white or whitecinereous, silky-fibrillose, dry; marg. often fissile. St. solid, white, slightly pruinose, apex furfuraceous, base turbinatobulbous. $G$. crowded, free, white to grey-cimnamon.

Pine-woods. Yorkshire, Sept. IgoS.

\section{GENERA OMITTED}

Cenococcum Fr., one of the Tubericece.

Pilacre Fr., placed by Lindau (in Engler and Prantl, P. flinzinfimiticn, vol. i. pt. I***, p. 86) near Auricularia, is of doubtful position.

Polyangium Link, an insect production.

Thelebolus Tode, one of the Ascomycetes. 


\section{CORRIGENDA}

Page I IO, No. 505a, for C. Sarnicus rial C. sarnicus.

,, I7I, No. 788, for P. Elvensis read P. elvensis.

," 317 , after BOLETUS for Dill. rad L.

,, 333, after POLYPORUS for Mich, rad Haller.

,, 344, No. 1563, after fumosus aid Fr.

, $36 \mathrm{~S}$, after MERULIUS for Halle rad Haller.

, 436, No. 1965a, for tenerima rial tenerrima.

,$\quad 449$, after TREMELLA for Dill. rad L.

", 462, after CLATHRUS for Mich, riad L.

,, 467, after GEASTER for Mich. real Pers. 


\section{GLOSSA R Y}

Adnate (adnascor, to grow to), of gills broadly attached to the stem.

Adnexed (ad, to, nicto, to join), of gills slightly joined to the stem.

Ecidia (Gr. oikidion, a little house), small cup-shaped fungi.

Eruginous (crugo, rust of copper), the blue-green colour of verdigris.

Estival (iestiz'alis), belonging or peculiar to summer.

Agglutinate (asglutino, to glue), glued to a surface.

Algal (alga, a sea-weed), relating to Alsa, a group to which belong the seaweeds and many fresh-water plants, including microscopic green plants which grow in water and damp places.

Alveolate (alveus, a hollow), in the form of small depressions like a shallow honeycomb.

Amphigenous (Gr. amphi, around, sennaro, to begret), not confined to onc surface, all round, as in the hymenium of Clavaria.

Anastomosing ( $\mathrm{Gr}$. anastomosis, a bringing to a point), united by running together irregularly.

Annular, in the form of a ring.

Annulate, bearing a ring on the stem.

Annulus (annulus, a ring), the ring round the stem.

Apiculate (afex, the top), terminating in a small point.

Appendiculate (appendicula, a small appendage), hanging in small fragments.

Applanate (al, to, planatus, made flat), flattened.

Approximate ( $a d$, to, proximo, to approach), of gills which approach but do not touch the stem.

Arachnoid (Gr. arachn', a spider's web, eidos, resemblance), like a cobweb.

Arcuate (arcuatus, bent like a bow), of gills, how-shaped.

Areolate (area, a plot, a space), divided into small areas or patches.

Ascus (Gr. askos, a wine-skin), the swollen end of a hyphal branch, in which spores are borne in Ascomycetes.

Atomate, sprinkled with small particles.

Basidium (basidizm, a little perlestal), the mother-cell which bears the spores in Hy'menomycetes and Gastiromycetes.

Bifid (bifulus, twice-cleft), divided half-way into two.

Bullate (bulla, a bubble), furnished with a boss or stud.

Byssoid (byssus, fine flax), composed of fine threads.

Cæspitose (cuspes, a clump), growing in tufts.

Calyptra (calyftrc, a hood), any extinguisher-shaped covering.

Campanulate (campana, a bell), bell-shaped.

Cancellate (cancellatus), latticed, as in Clathrus.

Capillitium (capillus, a hair), the dense mass of sterile fibres mixed with the spores in the gleba of the Gastironycetes. 
Cerebriform (cerebrum, the brain), having an irregular brain-like appearance, like the kernel of a walnut.

Ciliate (cilinm, an eye-lash), fringed with hairs.

Cinereous (cinis, ashes), ash-coloured.

Cingulate (cingulum, a girdle), bearing a ring or annulus on the stem.

Clavate (clava, a club), club-shaped.

Columella (columella, a small pillar), the small columnar base of barren tissue in Lycoperdon, Geaster, etc.

Conceptacle (conceptaculum, a receptacle), a hollow case enclosing reproductive bodies.

Conchiform (concha, a shell), shell-shaped.

Concrete (concretus, growing together), as when the scales adhere to the flesh of the pileus.

Conidia (Gr. konis, dust), simple asexual spores.

Connate (con, together, nascor, to be born), united by growing together.

Connivent (conniveo, to wink), arching over to meet.

Coriaceous (corium, leather), leathery.

Cortex (bark or rind), the periderm in the Gasteromy'cetes.

Corticate, with a bark-like covering.

Cortina (cortina, a veil), a veil of spider-web structure as the veil in Cortinarius.

Cortinate, furnished with a veil.

Costate (costatus, ribbed), ribbed or veined.

Crenate (crenc, a notch), with rounded notches on the margin.

Crenulate, with small rounded notches.

Cribrose (cribrum, a sieve), pierced with many holes.

Cupulate (cupula, a little cup), cup-shaped.

Cuspidate (cusfis, a point, spear), with a sharp spear-like point.

Cuticle (cuticula, the onter skin), the outermost skin.

Cyathiform (Gr. kuathos, a wine-cup), cup-shaped.

Decumbent (decumbo, to recline), reclining but with summit ascending.

Decurrent (decum, to run down), of gills and tubes running down the stem in the Asaricacea, Polyforacee, etc.

Dehiscing (dehisco, to yawn), opening when ripe.

Deliquescent (deliquescens, melting away), becoming fluid when mature.

Denticulate (denticulatus, minutely toothed), as in the minute teeth on the edge of the gills in certain Agaricacete.

Depressed (depressus, sunk down), slightly hollowed.

Determinate (detcrimino, to limit), ending definitely, as in the margin of some species of Corticium, etc., not powdered or clouded off indefinitely.

Diaphanous (Gr. dia, through, phaino, to appear), more or less transparent.

Dichotomous (Gr. dichotomeo, to cut in two), divided into two, forked.

Dimidiate (dimidio, to halve), as when one part of an organ is smaller than the other as in the pilei of many fungi.

Disciform (discus, a quoit), circular and flat.

Dissepiments (dissefimentum, a partition), dividing walls.

Distant, of gills in the Agricacea with a wide distance between them.

Divaricate (divarico, to spread asunder), very divergent, separating at an obtuse angle.

Echinulate (echinus, a hedgehog), with short bristles.

Effused ( $e f f u n i b$, to pour out), spread over without regular form. 
Emarginate (e, out of, margo, the margin), of gills with a sudden curve as if scooped ont at the point of attachment to the stem.

Endoperidium (Gr. endon, within), the inner layer of the peridium.

Endophyte, Entophyte (Gr. entos, within, phuton, a plant), growing within another plant.

Epiphyte (Gr. epi, upon, fhuton, a plant), growing upon another plant.

Erumpent (erumpens, breaking through), bursting through the epidermis.

Evanescent (ex'anescens, disappearing), lasting only a short time.

Excipulus (excipula, a basin), the rim round the base of the apothecium.

Exoperidium (Gr. exo, without), the outer layer of the peridium.

Farinose (farina, meal), mealy.

Fasciate ( fascia, a band or girtl), zoned as with coloured bands.

Fasciculate ( $f a s c i s$, a bundle), growing in small bundles.

Fastigiate (fastiginm, a slope or gable), with branches parallel, clustered and erect, sometimes decreasing in height outwards and giving a gable shape.

Ferruginous ( fermm, iron), of the colour of iron-rust.

Fibrillose ( fibra, a fibre), clothed with small fibres.

Filiform ( $f ! n m$, a thread), thread-like.

Fimbriate (fmbria, a fringe), fringed.

Fissile (fissitis, tending to split), capable of being split.

Fistulose (fistula, a pipe), hollow like a pipe.

Flocci (floccus, a lock of wool), woolly locks.

Floccose, woolly.

Flocculent, diminutive of floccose.

Flocculose, covered with small flocci.

Free, of gills which reach the stem, but are not attached to it.

Fuliginous (fuligineus, sooty), soot-coloured.

Funiculus ( funis, a rope), a cord of hyphre in Nidularia which attaches the peridiolum to the inner surface of the wall of the peridium.

Furcate (furcatus, forked), as in the branching gills common in Russula.

Furfuraceous ( $f u r f u r$, bran), with branny scales or scurf.

Fuscous (fuscus, dusky), of a dingy brownish colour.

Fusiform (fusus, a spinclle), somewhat thick but tapering towards each end.

Fusoid, somewhat spindle-shaped.

Gibbous (gibbus, hump-backed), of a pileus with a swelling or broad umbo, or convex above and flat beneath.

Gills, the plates of an agaric on which the hymenium is situated.

Glabrous (slaber, without hair), not bearing hairs.

Glaucous (Gr. glankos, bluish-grey'), covered with a bluish-grey bloom.

Gleba ( $g l e b a$, a clod), the contents of the peridium in the Gasteromycetes.

Greaved, of a stem clothed as if with a greave.

Gregarious (gresarius, belonging to a flock), growing in company, but not cæspitose.

Grumous (grumus, a little heap), clotted ; of flesh composed of little clustered grains.

Guttate (gutto, a tear), with tear-like spots.

Gyrose (Gr. stros, round), curved backward and forward in turn.

Habit (habitus, appearance), the general appearance.

Habitat (hatitatio, dweiling), the locality. 
Heterogeneous (Gr. hetcros, one of two, sinnao, to beget), not uniform in structure.

Hispid (hispidus, bristly), beset with rough hairs or bristles.

Homogeneous (Gr. homos, one and the same, schnao, to beget), uniform in structure.

Hyaline (hualos, clear), colourless, translucent.

Hybernaculum, hibernaculum (hiberno, to winter), applied to forms in which certain fungi pass the winter, sclerotia.

Hygrometric (Gr. hugros, moist, metron, a measure), influenced by moisture.

Hygrophanous (Gr. husros, moist, fhaino, to appear), of a watery appearance when moist.

Hymenium (Gr. humen, a membrane), the spore-bearing surface.

Hymenophore (Gr. humen, a membrane, phero, to bear), the structure which bears the hymenium, as the under surface of the pileus to which the grills are attached in $A_{s}$ aricacee, the sporophore.

Hypertrophy (Gr. huper, above, trophe, food), an abnormal enlargement of an organ by clisease.

Hypha (Gr. huphe, a web), pl. hyphæ, the thread-like element of which a fungus is made up.

Imbricate (imbricatus, covered with tiles), overlapping as the tiles on a roof.

Immarginate (in, negative, margo, a margin), without a distinct border.

Immersed, sunk in the matrix.

Inferior (infirior, lower), of the annulus on the stem, far down.

Infundibuliform (infundibulum, a funnel), funnel-shaped.

Innate (in, into, nascor, to be born), adhering by growing into.

Involute (in, in, volvo, to roll), rolled inwards.

Isabelline or Isabella colour, dirty-tawny, as of soiled linen.

Laciniate (lacinia, a lappet, flap), divided into flaps.

Lacunæ (lacma, a hole or cavity), holes or depressions.

Lacunose, marked with small hollows.

Lamellæ (lamella, a thin plate), the gills in Asaricaicu.

Lanceolate (lancea, a lance or spear), lance-shapel; narrow and tapering to both ends.

Linear (linea, a line), narrow and straight, several times longer than wide.

Loculus (loculus, a little compartment), a cell of a septate spore.

Mammiform (mamma, a breast), breast-shaped.

Marginate (marso, a margin), having a distinct border.

Matrix (matrix, the womb), the substance on which a fungus grows.

Medial (medium, the middle), of the annulus, when at the middle of the stem.

Merismoid (Gr. merizo, to divide, eidos, form), having a branched or laciniate pileus.

Merulioid, resembling the hymenium of Merulius.

Mitrate (Gr. mitra, a liead-dress), with thick rounded pileus.

Mucedinous (mucidus, mouldy), of the nature of the group Mucedines, nakedspored moulds.

Multifid (multifidus, many cleft), divided half-way into many lobes.

Multipartite (multifartitus, many times divided), divided many times and more deeply than multifid.

Muricate (murex, a sharp stone, a bit with sharp spikes, a spiked ball), rough with short hard tubercular excrescences. 
Muriform (mumus, a wall), with cells resembling bricks in a wall.

Mycelium (Gr. mukes, a fungus), spawn of fungi, the vegetative portion, composed of hyphe.

Mycology (Gr. mukes, a fungus, logos, a discourse), the study of fungi.

Nuclear (mucleus, a kernel), pertaining to a kernel-like growth as in Ncmatcliu.

Obconic (ob, inversely, comus, a cone), inversely conical.

Obovate (ob, inversely, $o c^{\prime} t \mathrm{~m}$, an egg), inversely egg-shaped.

Obtuse (obtusus), blunt or rounded at the end.

Ochreous (ochra, yellow-earth), yellow, not red-ochre.

Operculum (oferculum, a lid), a cover.

Ostiolum (ostiolum, a little door), the aperture through which the spores escape in the Gasteromycetes.

Ovate (oc'um, an egg), egg-shaped.

Papilla (fapilla, a nipple), a nipple-like elevation.

Papillate, Papillose, with nipple-like elevations.

Paraphyses (Gr. fara, beside, fluusis, growth), sterile filaments in a hymenium.

Partial, of a veil, clothing the stem and reaching to the edge of the pileus, but not extending beyond it.

Patelliform (fatella, a small dish), dish-shaped.

Pectinate (fecten, a comb), like the teeth of a comb.

Pedicel (ficticulus, a small foot), a support or stalk, as the small column which supports the inner peridium in some species of Geaster.

Pedicellate, born on a pedicel.

Pellicle (fillicula, a small skin), a delicate superficial membrane.

Pelliculose, furnished with a pellicle or delicate distinct skin.

Peltate (felta, a target), target-shaped.

Pencilled, with pencil-like hairs either on the tip or border.

Penicillate (fenicillum, a little brush), pencil-shaped.

Peridiola, diminutive of peridium.

Peridium ( $\mathrm{Gr}$. feridion, a little pouch), the outer enveloping coat enclosing the gleba in the Gasteronycetes.

Peristome (Gr. feri, about, stoma, a mouth), the fringe or its homologue round the orifice of the peridium in Giaster, Tulostona, etc., amongst the Gastero. my'cte's.

Perithecium (Gr. feri, about, theke, a case), a receptacle enclosing spores.

Peronate (fero, a kind of high boot), sheathed, e.g. of a stem which has a woolly covering like a legged boot.

Pervious (ferrius, passable), having an open passage-way; hollow to the base, as in some stems.

Pezizoid (pidos, resemblance), cup-shaped, like a Peziza, a genus of Ascomylcet's.

Pileate, with a cap.

Pileoli, secondary pilei, arising from the division of a primary pileus.

Pileus (filizs, a cap), a part of the receptacle of a fungus, $c \cdot s$. the cap-like head in Asaricaceet.

Pilose (filus, a hair), covered with hairs.

Plane (flanus), level, even, that.

Plasmodium (Gr. flasma, formed substance), the naked nucleated protoplasm of the Mycetozoa.

Plicate (flico, to fold), folled into plaits. 
Plumose (flumosus, feathered), downy.

Polymorphic (Gr. polus, many, morthe, a change), with several or various forms. Poriform, in the form of pores.

Porous, furnished with pores.

Potential (potentia, a force), existing in possibility, rather than in actuality.

Proliferous (proles, offspring, fero, to bear), applied to an organ which gives rise to secondary organs of the same kind.

Promycelium (fro, for, mycelium, the vegetative portion of the thallus composed of hyphi), the short-lived product of germination of a spore which bears spores of a different nature from the mother-spore.

Protoplasm (Gr. protos, first, flasma, formed substance), the living substance of plants and animals.

Pruinose (pruina, hoar-frost), covered with frost-like bloom.

Pruniform (przuns, a plum), plum-shaped.

Pubescent (fuber, downy), slightly hairy.

Pulverulent (fulverulentus, dusty), powdered as if dusted over.

Pulvinate (pulvinus, a cushion), cushion-shaped.

Punctate (functus, a point), dotted with points.

Pyriform (fyrus, a pear), pear-shaped.

Racemose (racemus, a bunch of grapes), borne in a bunch.

Radiate (radius, the spolis of a wheel), spreading from a centre.

Receptacle (receftaculum, a reservoir), an axis bearing one or more organs, as the stcm upon which the hymenium is elevated in the Phalloidacet.

Remote, of gllls which do not reach the stem, but leave a free space round it.

Reniform (renes, the kidneys), kidney-shaped.

Repand (refando, to throw open), bent backwards.

Resupinate (resupino, to throw on the back), with hymenium upwards.

Reticulate (rete, a net), netted.

Revolute (rezolio, to roll back), rolled backwards; of the margin of a pileusthe opposite of involute.

Rhizomorphoid, rhizomorphous (Gr. thiza, a root, morphi, shape), like a rhizomorph, a root-like branched strand of mycelial hyphæ.

Rimose (rima, a crack), craclied.

Rimulose, diminutive of rimose.

Ring, a part of the veil adhering to the stem in the form of a ring or annulus.

Rivulose (rivula, a small stream), marked with lines like rivulets.

Rounded, applied to gills and tubes, rounded near point of contact with the stem.

Rubiginous (muligo, rust), colour of iron-rust.

Rufescent (rufescens), becoming reddish.

Rufous (rufus), reddish.

Rugose (ma, a wrinkle), wrinkled.

Rugulose, somewhat wrinkled.

Scabrous (scaber, rough), rough on the surface.

Scales, applied to the broken up epidermis of the pileus and stem.

Scarious (Gr. skaros, a kind of sea-fish), applied to a shrivelled membrane.

Scissile (scindo, to cleave), capable of being cleft asunder; of two plates, e.s. lying together, but capable of being separated.

Sclerotioid, sclerotium-like.

Sclerotium (Gr. skleros, hard), a compact mass of hyphe in a dormant state. 
Scrobiculate (scrobis, a trench), marked with small pits.

Separating, becoming detached, as gills from a stem, or resupinate fungi from the matrix.

Septate, divided.

Septum (seftum, a hedge), a division.

Serrate (sir a, a saw), toothed like a saw.

Serrulate, minutely toothed.

Sessile (sideo, to sit), seated without a stem.

Setæ (seta, a bristle), bristles.

Setulose, resembling a fine bristle.

Simple, applied to a stem without ring or volva.

Sinuate, waved; of a pilens with a wavy or curved margin; of gills with a sudden wave, curve or sinus where they reach the stem.

Sinus (sinzes), a curve.

Smooth, applied to a surface which is destitute of hairs or other protuberances.

Spathulate (Gr. spathi, a spatula), spoon-like.

Spawn, see Mycelium.

Sphæriæ, small spherical black fungi common on branches.

Spiculæ (sficulum, a small needle), fine erect acutely pointed spines.

Spinulose (sfina, a thorn), bearing fine spines.

Sporangium (Gr. spora, a seed, asgeion, a vessel), a spore-bearing receptacle.

Spores ((ir. spora, a seed), the analogues of seeds of flowering plants.

Sporidia, diminutive of spores-small spores.

Sporophore (Gr. sfora, a seed, fhoric, to carry), the part which bears the hymenial surface.

Squamose (squana, a scale), scaly.

Squamule, a small scale.

Squamulose, covered with small scales.

Squarrose (squarrosus, rough, scurfy), rough with scales.

Sterigma (Gr. sterigma, a prop), pl. sterigmata, the slender thread which carries a ipore.

Stipes (stifis), a stalk.

Stipitate, stemmed.

Stoloniferous (stolc, a short sucker or rumer, fero, to bear), stolon-bearing.

Stoma (Gr. stoma, a mouth), the mouth through which the spores escape in certain fungi, as Geraster, Tulostoma, etc.

Striate (stria, a furrow, flute of a column), marked with lines in the form of channels.

Strigæ (stris' a swathe), small straight hair-like scales.

Strigose, rourh with sharp-pointed hairs, hispid.

Stroma (Gr. stroma, a mattress), a cushion-like body in which the perithecia of sone fungi are immersed.

Stuffed, of a stem filled with substance of a different texture from its walls.

Subiculum (subiculum, an under layer), the felted or byssoid mycelium of a resupinate fungus, forming the unter layer bearing the hymenium.

Sulcate (sulcus, a furrow), marked with grooves.

Superior (sufir, above), applied to the annulus when it is near the apex of the stem.

Terete (teres, rounded), circular in transverse section.

Tessellate (tessellatus, squared stones), checquered. 
Tetrasporous (Gr. tetras, four, sfora, a seed), bearing four spores.

Thallus (Gr. thallos, a sprout), the whole of the body which does not serve fur reproduction.

Tomentose (tomentum, wool, etc.), downy.

Trama (trama, the weft or filling of a web), the substance between the hymenial layers in Agaricacce and Polyporace.

Tremelloid (tremo, to tremble), of the consistency of jelly.

Truncate (trunco, to main), ending abruptly as if cut short.

Tubercle (tuberculum, a little tuber), a small wart-like excrescence.

Tuberculate, with small warty projections or excrescences.

Tuberculiform, resembling a little tuber.

Tubiform (tuba, a trumpet), trumpet-shaped.

Turbinate (turbinatus, cone-shaped), top-shaped.

Umbilicate (umbilicus, the navel), with a small central depression.

Umbonate (umbo, the boss of a shield), with a central boss-like elevation.

Uncinate (uncinus, a hook), hooked.

Universal, of the veil or volva which entirely envelops the fungus when young.

Urceolate (urceola, a pitcher), pitcher-like, hollow and contracted at the mouth like an urn or pitcher.

Veil (velum, a covering), a covering of various texture more or less completely enwrapping a fungus when young.

Veliform (velum, a covering), of a thin veil-like covering.

Venose (venosus, veiny), having veins.

Ventricose (venter, the belly), swollen in the middle.

Vermiform (vernis, a worm), worm-shaped.

Verrucose (e'erruca, a wart), warty.

Versiform (zersiformis, changing shape), of clifferent shapes, or altering in shape with age.

Villous (cillus, a tuft of hair), downy with soft hairs.

Vinous, of the colour of claret, dark or dilute.

Virgate (zirsa, a twig, stripe), streaked.

Volva (c'olva, a wrapper), a covering or sac enveloping the young fungus.

Volvate, having a volva.

Zone, a circle of hairs, asperities, colour, etc.

$\mu$, a micromillimetre or $\frac{1}{1000}$ of a millimetre; used for spore measurements. 


\section{NDEX}

\begin{tabular}{|c|c|c|c|c|c|c|}
\hline & & & $\Gamma \Lambda G E$ & & & $I^{\prime} A G E$ \\
\hline ACETABULARIA & . & . & I 20 & AMANITA & . & I 4 \\
\hline acetabulosa & . & . & I 2 I & arida & . & 17 \\
\hline AGARICACE & . & . & I I & aspera . & . & 17 \\
\hline Agaricus affricatus & . & . & $\mathrm{S}_{5}$ & aureola. & . & 15 \\
\hline arvensis & . & . & I 7 I & cariosa. & . & I 6 \\
\hline aureus. & . & . & . 122 & citrina. & . & I 6 \\
\hline blattarius & . & - & - 123 & excelsa. & - & 16 \\
\hline campestris & . & . & 171,172 & junquillea & . & 17 \\
\hline caferatus & . & . & - 122 & lenticularis & . & 17 \\
\hline causetta. & . & . & 29 & magnifica & . & I 7 \\
\hline cinerascons & - & . & 55 & mappa . & . & I 5 \\
\hline cryptarmm & . & . & . $17 \mathrm{I}$ & megalodactyla & . & 17 \\
\hline cufreus. & . & . & . 292 & muscaria & . & $\mathbf{I} 6$ \\
\hline durus. & . & . & . 123 & nitida & . & 17 \\
\hline erebius. & . & . & . $\quad 122$ & niz'alis . & . & IS \\
\hline fodiens. & . & . & . $6 \mathrm{I}$ & pantherina & . & 16 \\
\hline fulvus . & . & . & IS & phalloides & . & 15 \\
\hline geoplinllus & - & . & . $\mathrm{I}+\mathrm{I}$ & porphyria & - & 15 \\
\hline innuetus & . & . & . $\quad 176$ & recutita. & . & I 5 \\
\hline laricinus & . & . & $3^{\circ}$ & rubescens & . & 16 \\
\hline lenticularis & . & . & 17 & solitaria & . & 16 \\
\hline loluatus. & 。 & . & 54 & spadicea & . & IS \\
\hline molliscorinm . & . & . & . 123 & spissa & . & 16 \\
\hline ombrophilus. & . & . & . 123 & strobiliformis & . & $\mathbf{I} 6$ \\
\hline fellosfermus & . & . & . $19 \mathbf{I}$ & verna & . & 15 \\
\hline feromatus & - & . & . $\quad$ I 73 & virosa & . & 15 \\
\hline $\operatorname{srcecox}$ & . & . & . 124 & AMANITOPSIS . & . & $1 \mathrm{~S}$ \\
\hline mosiofhyllus & . & . & . $\quad$ I 74 & adnata & . & I 9 \\
\hline Smithii. & . & . & . IIS & fulva & . & IS \\
\hline sphale'romorph & ins & . & . $\quad 124$ & strangulata & . & $\because 9$ \\
\hline subine'olutus & . & . & 53 & vaginata & . & IS \\
\hline suferbus & . & . & $19 \mathrm{I}$ & ANELLARIA & . & I) 4 \\
\hline Terreyi. & . & . & 24 & fimiputris & . & I95 \\
\hline terrisenus & . & . & 122 & scitula & . & I95 \\
\hline tomlaris & . & . & 123 & separata & . & 195 \\
\hline Tohlii. & . & . & . 122 & ANNULARIA & . & 99 \\
\hline vaporarius & . & . & I $7 \mathrm{I}$ & lævis & . & 99 \\
\hline virsiculor & . & . & 30 & ARMILLARIA & . & $2 \mathrm{~S}$ \\
\hline xanthodermus & & . & . $\quad 172$ & aurantia & . & 29 \\
\hline Aldridgea grelatinosa & - & . & . 422 & bullbiger & . & 28 \\
\hline
\end{tabular}


ARMILLARIA (continute)

PAGE

caligata

citri . . . 30

constricta $\quad . \quad$. 29

denigrata . . 30

focalis . . . . 29

hiematites . . . 29

Jasonis . . . . 29

mellea . . . . jo

mucida . . . 30

ramentacea . . . 29

robusta . . . . 29

subcava . . . 30

AURICLLARIA . • • . 444

lobata. . . . $4+5$

mesenterica . . $\$ 45$

AUKICULARIOIDEE . . $4+4$

Battarkea . . . . $4^{6} 4$

phalloides . . 464

íolbitius . . . . 211

apicalis. . . . 213

Boltonii . . . 212

Havidus . . . 212

fragilis . . . $2 \mathrm{I} 2$

grandiusculus . $\quad 2 \mathrm{I} 2$

niveus . . . . $2 I_{3}$

rivulosus . . . 213

tener . . . . $2 I_{3}$

titubans . . . 212

vitellinus . . . 212

Boletinus . . . . 331

BOLETUS . . . . 317

areus . . . . $\quad 325$

astivalis . . . 326

alutarius . . . 329

appendiculatus . $\quad 324$

aurantiporus . . . 321

badius . . . . 320

bovinus . . . 320

brachyforus . . . 330

crespitosus . . . 330

calopus. . . . 324

candicans . . . 325

carnosus . . . 326

castaneus . . . 330

cavipes . . . . $33 \mathbf{I}$

chrysenteron . . . 322

collinitus
BOLETUS (continuad)

PAGE

\begin{tabular}{|c|c|}
\hline crassus . & \\
\hline cruentus. & - \\
\hline crytarum & - \\
\hline cyanescens & • \\
\hline duriusculus & . \\
\hline edulis . & . \\
\hline elegans. & - \\
\hline felleus . & . \\
\hline Haviılus. & - \\
\hline flavus . & - \\
\hline fragrans. & - \\
\hline fulvidus . & - \\
\hline granulatus & . \\
\hline impolitus & • \\
\hline impuber. & • \\
\hline laricinus. & . \\
\hline
\end{tabular}

luridus . . . . 327

luteus . . . . 319

McWeeneyi . . . 33I

olivaceus . . . 324

pachypus . . . 325

paludosus . . . 320

parasiticus . . . 323

piperatus . . . $32 \mathrm{I}$

porphyrosporus . . $32 \mathrm{~S}$

pruinatus . . . 323

purpurascens . . . 323

purpureus . . . 327

pusio . . . . 320

radicans. . . 323

regius . . . . 324

resufinatus . . . 356

Rostkovii . . . 326

rubiginosus . . . $32 \mathrm{~S}$

rubinus . . . . 323

rugosus . $\quad . \quad . \quad . \quad 329$

rutilus . . . . $32 \mathrm{I}$

sanguineus . . . 322

satanas . . . . 327

scaber . . . . $32 \mathrm{~S}$

Sistotrema . . $33^{\circ}$

spadiceus . . . 322

strixpes . . . 322

subtomentosus . . 322

sulphureus . . . $32 \mathrm{I}$

tenuipes. . . . 320

vaccinus . . $\quad 326$

variecolor . . . 324 


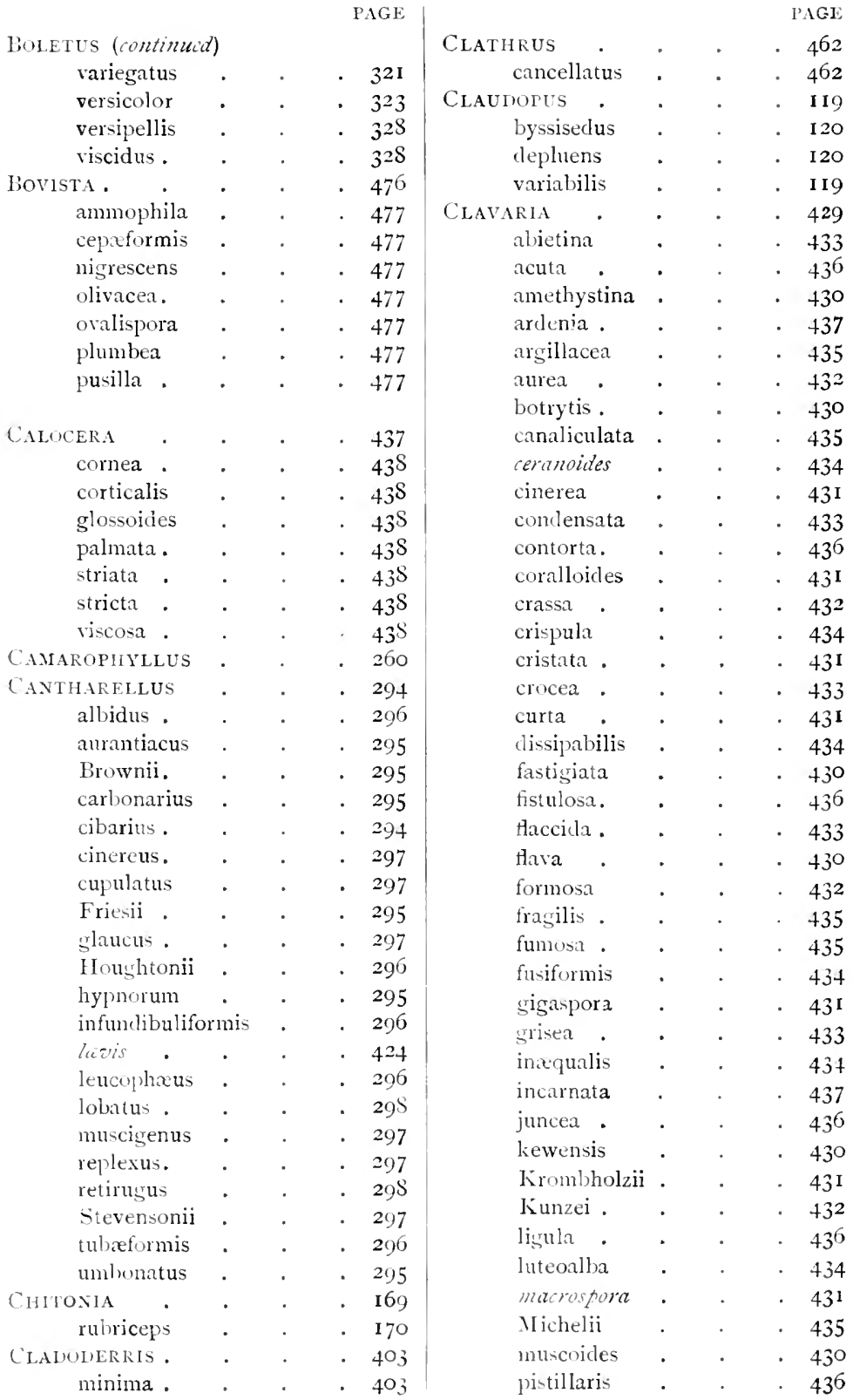


Clavaria (continued)

\begin{tabular}{|c|c|c|c|}
\hline purpurea & & & 434 \\
\hline pyxidata & * & & $43^{2}$ \\
\hline rosea . & • & - & 434 \\
\hline rufa & • & - & 434 \\
\hline rufescens & $\cdot$ & - & $43^{2}$ \\
\hline rugosa . & • & - & $43 I$ \\
\hline spinulosa & • & 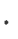 & - 433 \\
\hline striata . & - & - & - 435 \\
\hline stricta & . & - & . 433 \\
\hline subtilis . & . & $\cdot$ & - 432 \\
\hline tenerrima & . & - & - 436 \\
\hline tenuipes. & . & - & . 442 \\
\hline tuberosa & . & $\cdot$ & . $43^{6}$ \\
\hline umbrina & - & - & - $43 \mathrm{I}$ \\
\hline uncialis & . & . & - 436 \\
\hline vermicularis & . & $\cdot$ & - 435 \\
\hline$\checkmark A R I A C E A$ & . & . & - $42 \mathrm{~S}$ \\
\hline CYBE & - & . & . $\quad 45$ \\
\hline aggregata & • & . & $5 \mathrm{I}$ \\
\hline amara. & . & . & $4 \mathrm{~S}$ \\
\hline amarella & . & - & $4 S$ \\
\hline ampla . & - & - & 50 \\
\hline angustissima & . & . & 57 \\
\hline bella .. & . & - & 57 \\
\hline brumalis & . & . & $5^{6}$ \\
\hline candicans & . & . & $5^{\circ}$ \\
\hline catinus . & . & - & 54 \\
\hline cerussata & . & . & 49 \\
\hline cinerascens & • & - & 55 \\
\hline clavipes & $\cdot$ & . & 47 \\
\hline comitialis & • & · & 47 \\
\hline concava & • & . & 56 \\
\hline connata & - & • & 495 \\
\hline cryptarum & • & . & 52 \\
\hline curtipes & $\cdot$ & . & $4 S$ \\
\hline cyanophrea & $\cdot$ & . & $4^{S}$ \\
\hline cyathiformis & - & · & 55 \\
\hline dealbata & . & · & 50 \\
\hline decastes & - & $\cdot$ & $5 \mathrm{I}$ \\
\hline diatreta. & - & · & 56 \\
\hline ditopus. & - & . & $5^{6}$ \\
\hline ectypa . & • & • & 57 \\
\hline elixa . & . & - & 51 \\
\hline ericetorum & & $\cdot$ & 55 \\
\hline expallens & $\cdot$ & & 55 \\
\hline flaccida. & & & 54 \\
\hline $\begin{array}{l}\text { agrans. } \\
\text { imosa. }\end{array}$ & & & \\
\hline
\end{tabular}

\section{Clitocybe (continued)}

PAGE

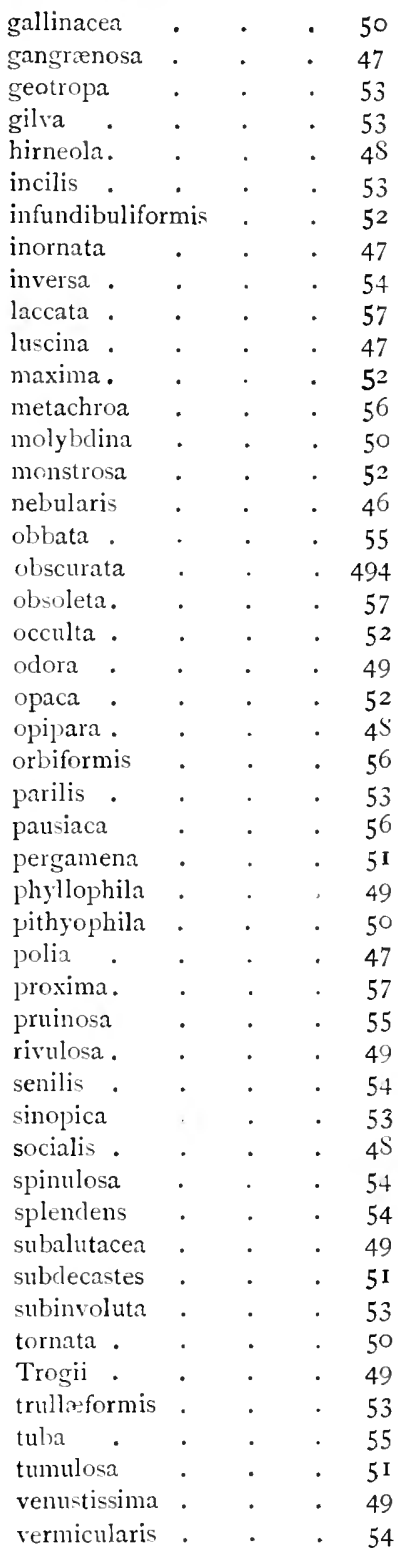




\section{Clitocye (continuci)}

vernicosa

Clitopilus

zygophylla . . . $4^{\mathrm{S}}$

cancrinus . . . I IO

carneoalbus . . . I IO

cretatus. . . . I IO

mundulus . . . I IO

orceila . . . . IO9

popinalis . . . IIO

prunulus . . . IO9

Sarnicus . . . I IO

stilbocephalus . . I I I

straminipes . . . I I I

undatus. . . . I IO

vilis . . . I I I

Collybia . $. \quad . \quad 58$

acervata . . . 65

ambusta . . . 68

aquosa . . . 66

atrata . . . 68

bibulosa . . . $6 \mathbf{I}$

butyracea . . . $6 \mathbf{I}$

caldarii . . . . 69

cirrhata. . . . 63

clavus . . . 66

clusilis . . . . 68

collina . . . . 64

confluens . . $\quad 63$

conigena . . . 63

coracina. . . 67

distorta. . . $6 \mathbf{I}$

Dorotheæ . . . 69

dryophila . . 65,495

eriocephala . . $\quad 59$

esculenta . . . 65

eustygia . . . 67

exsculpta . . . 66

extuberans . . . 66

floccipes . . 62

fodicns . . . 6 I

fusipes . . . 60

hariolorum . . . 63

Henriettæ . . . 59

ingrata . . . . 63

inolens . . . 67

lacerata. . . 68

lancipes . . 60

laxipes . . . 62
Collybia (continued)

leucomyosotis . . 66

longipes . . . 59

macilenta . . . 66

maculata . . . 60

mephitica . . . 67

mimica . . . . 62

murina . . . $6 \mathrm{~S}$

muscigena . . . 66

nitellina , . 64

nummularia . . $\quad 65$

ocellata. . . . 66

ozes . . . . 67

planipes . . . 65

platyphylla . . . 60

plexipes. . . . 67

prolixa . . . 61

protracta . . . 68

psathyroides . . . 64

pulla . . . . $6 \mathbf{I}$

racemosa $. \quad . \quad .63$

radicata. . . . 59

rancida. . . 67

retigera. . . 59

semitalis . . . 60

Stevensonii . . . 64

stipitaria . . . 62

stridula . . . . $6 \mathbf{I}$

succinea $\quad . \quad \cdot \quad \cdot 65$

tenacella . . . 65

tesquormm . . . 68

thelephora . . . 64

tuberosa $\quad . \quad \cdot 63$

tylicolor . . . 68

velutipes . . . 62

ventricosa . . . 64

vertirugis . . . 62

xanthopus . . . $6_{4}$

xylophila . . 62

CONIOPHORA . . . . 419

arida . . . 420

Burkeleyi . . . $42 \mathrm{I}$

byssoidea . . . 422

cinnamomea . . . 420

Cookei . . . $42 \mathrm{I}$

gelatinosa . . . 422

incrustans . . 420

membranacea . . . $42 \mathrm{I}$

ochracea . . $42 \mathrm{~J}$ 
CONIOPHORA (continuta)

COPRINus (continuta)

olivacea

pulverulenta . . . 420

puteana. . . 420

stabularis . . . 42 I

subdealbata . . . 421

sulphurea . . . 420

umbrina . . . 420

COPRINARII . . . 194

COPRINUS . . . . 201

alternatus . . 207

aphthosus . . . 205

aquatilis . . 2 IO

aratus . . . . 206

atramentarius. . . 204

Bresadolie . . . 208

comatus . . . 203

congregatus . . . 207

cordisporus . . . 209

cothurnatus . . . 206

deliquescens . . 207

digitalis. . . $\quad 207$

dilectus. . . 207

clomesticus . . . 209

ephemerus . . . 210

exstinctorius . . . 205

filiformis . . $2 \mathrm{II}$

fimetarius . . $\quad 205$

flocculosus . . . 205

fuscescens . . . 204

Gibbsii . . . 209

hemerobius . . . 2 I I

Hendersonii . . . 208

lagopus . . . . 208

macrocephalus . . $20 \mathrm{~S}$

micaceus . . 206

narcoticus . . 208

niveus . . . . 205

nycthemerus . . 209

oblectus . . . 203

ovatus . . . . 203

papillatus . $\quad .207$

picaceus . . 204

platypus . . 2 I I

plicatilis . . . 210

radians . . . . 206

radiatus. . . . 209

roseotinctus . . . 206

similis . . . 205

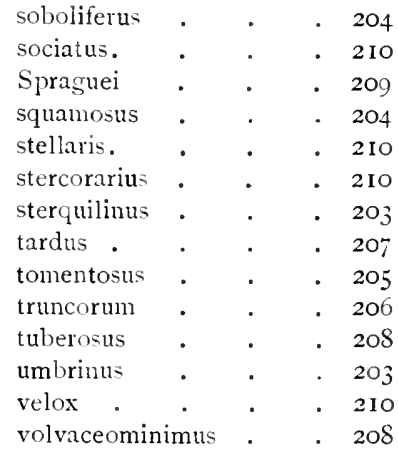

Corticium . . . . 409

amorphum . . 410

anthochroum . . . 413

arachnoideum . . 412

atrovirens . . . 4 15

aurora. . . . $4 \mathbf{I} 3$

calcenm, . . . $41 \mathrm{I}$

Carlylei . . $4 \mathbf{I} 4$

Chrysanthe'mi. . . 419

cœrulenm . . . 4I4

comedens . . . $4 \mathbf{I} 5$

confluens . . . 412

critaceun . . . $4 \mathrm{II}$

evolvens . . . 7 IC

flaveolum . . . $4 \mathrm{I} 4$

fotidum . . . 4 I I

lactescens . . . 413

lacteum. . . . $4 \mathrm{II}$

lacunosum . . . 413

leve. . . 412

lividum. . . $\quad 4 \mathrm{I} 5$

Lycii . . . . 4II

maculæforme. . . 414

molle . . . . $4 \mathrm{I} 3$

nigrescens . . . 4I5

nudum. . . . 4I2

polygonium . . . 413

populinum . . $\quad 4 \mathbf{I}$

porosum . . . $4 \mathrm{IC}$

radiosum . . . 412

roseolum . . . $44^{13}$

salicinum . . . 410

Sambuci . . . $4 \mathrm{I} 2$

sanguineum . . . $4 \mathbf{I} 4$ 


\section{CORTICIUM (continuid)}

scutellare

seriale

serum

strigosum

Typhre.

violaceolividum

violeum.

Cortinarius .

acutus

albocyaneus

alloviolaceus .

$\begin{array}{lcccc}\text { allutus . } & \cdot & \cdot & \cdot & 2 \mathbf{I S} \\ \text { anfractus } & \cdot & \cdot & \cdot & 2 \mathbf{I} \\ \text { angulosus } & \cdot & \cdot & \cdot & 246 \\ \text { anomalus } & \cdot & \cdot & \cdot & 23 \mathrm{r} \\ \text { anthracinus } & \cdot & \cdot & \cdot & 232 \\ \text { arenatus } & . & . & . & \mathbf{2 2 9}\end{array}$

argentatus . . . 225

argutus . . . . $\mathbf{2 2 5}$

armeniacus . . . 243

armillatus . . . 237

arvinaceus . . . 222

atrovirens . . . 220

azureus . . . . $23 \mathrm{I}$

balaustinus . . . 245

balteatus . . . 216

Berkeleyi . . $2 \mathbf{I} 7$

bicolor. . . . 245

biformis. . . 240

bivelus . . . . 235

bolaris . . . . 228

bovinus. . . . 239

brunneofulvus . . 239

brunneus . . . 239

bulbosus . . . 235

Bulliardii . . . $22 S$

cærulescens . . . 2 IS

callisteus . . . 227

calochrous . . . $21 \mathrm{~S}$

camphoratus . . . 227

camurus. . . .230

caninus . . . 230

castaneus . . . 245

cinnabarinus . . . 232

cinnamomeus. . $\quad 232$

claricolor . . . 215

collinitus . . . $\mathbf{2 2 2}$

colus . . . 245
Cortixirius (continuad)

PAGI:

Cookei . . . 242

corrosus . . . 219

coruscans . . . $22 \mathrm{I}$

cotoneus . . . 233

crassus . . . . 216

croceocæeruleus . . $22 \mathrm{I}$

croceoconus . . $\quad 232$

croceofulvus . . . $23 \mathrm{~S}$

crystallinus . . . 220

cumatilis . . . 220

cyanites. . . . 226

cyanopus . . . 216

damascenus . . . 243

decipiens . . . $24 \mathrm{~S}$

decolorans . . . 221

decoloratus . . . 221

decumbens . . . 230

delibutus . . . 224

depressus . . . 249

detonsus . . $24 \mathrm{~S}$

diabolicus . . 230

dibaphus . . . 219

dilutus. . . . $24+$

dolabratus . . . 247

duracinus . . . 244

elatior . . . 223

emollitus . . . 220

erythrinus . . . 248

evernitss . . 237

fasciatus . . . 249

firmus . . . . 243

flabellus . . . 2.40

flexipes. . . 240

fulgens. . . . 219

fulmineus . . . 219

gentilis . . . $\quad{ }_{23} \mathrm{~S}$

germanus . . . $24 \mathrm{~S}$

glandicolor . . . $\quad 239$

glaucopus . . $21 \mathrm{~S}$

grallipes . . . 223

hrmatochelis . . . 237

helvelloides . . . 235

helvolus. . . $\quad 23 \mathrm{~S}$

hemitrichus . . . 241

herpeticus . . . 220

linnuleus . . $\quad 235$

hircinus. . . . 227

ianthipes . . . 248 


\section{Cortinaries (continued)}

iliopodius

illibatus.

ilhuminus

imbntus.

impennis

incisus.

infractus

infucatus

injucundus

iris

isabellinus

jubariuus

Junghulanii

Krombholzii .

laniger .

lasus .

lepidopus

leucopus

licinipes

limonius

Jivido-ochraceus

lustratus

macropus

malacnius

malicorius

microcyclus

miltinus

milvinus

mucifluus

multiformis

muricinus

myrtillinus

nipus

nitidus .

nitrosus.

obtusus .

ochroleucus

olivascens

opimus .

crellanus

orichalceus

paleaceus

papulosus

paragaudis

pateriformis

penicillatus

periscelis

pholideus
PAGE

\section{CORTINARILS (continulid)}

phrygianus . . . $22 \mathrm{~S}$

plumiger . . . 236

pluvius . . . 224

porphyropus . . . 221

prasinus . . . 219

privignus . . . 244

psammocephalus . . $24 \mathrm{I}$

punctatus . . . 240

purpurascens . . . 2 IS

quadricolor . . . 237

raphanoides . . . 233

Reedii . . . . 247

renidens . . . 246

riculatus . . . 230

Riederi. . . . $2 \mathrm{I} 7$

rigens . . . . 247

rigidus . . . . $24 \mathrm{I}$

rubellus . . . 239

russus . . . . 217

saginus . . . . 217

salor . . . . 224

sanguinens . . . 232

saturninus . . . 244

scandens . . . 247

scaurus . . . 220

sciophyllus . . . 245

scutulatus . . . 237

sebacens . . . 216

serarius . . . 220

spilomens . . . 231

sternmatus . . . $24 \mathbf{I}$

stillatitius . . . 224

subferrugineus . . 243

sublanatus . . . $22 \mathrm{~S}$

subnotatus . . . 233

suillus . . . . 227

tabularis . . . 230

talus . . . . $2 \mathrm{IS}$

testaceus . . . 219

tophaceus . . . 227

tortuosus . . . 244

torvus . . . . 236

traganus . . . 227

triformis . . . 240

trimphans . . . 215

turbinatus . . . 2 I9

turgidus . . . 226

turmalis . . . 215 
CuRtixarits (continutid)

uliginosus

unimodus

uraceus

urbicus

valgus

variicolor

varius

venetus.

vespertinus

vibratilis

rinosus .

violaceus

Craterellus .

clavatus.

cornucopioides

crispus.

lutescens

pusillus.

sinuosus.

Crepidotus .

alveolus.

applanatus

crlolepis

chimonophilus

epibryus

epigreus .

haustellaris

mollis

palmatus

pezizoides

Phillipsii

proboscidens

putrigenus

Ralfsii.

Kubi

Crathus vulgare .

striatus .

vernicosus

Cyphella

alboviolascens.

Berkeleyi

Bloxamii

brunnea.

capula

catilla

cernua
PAGE

233

247

246

236

234

216

216

234

$22 \mathrm{I}$

224

- $22 \mathrm{~S}$

226

397

398

397

398

397

398

398

I67

I 67

I68

I6S

I68

I 68

169

I6S

I67

167

I68

$16 \mathrm{~S}$

168

169

169

168

482

${ }_{4} S_{3}$

$48 \mathrm{I}$

$4 S_{2}$

482

423

425

426

426

425

424

425

424
Cyphella (continuid)

cuticulosa . . . 424

cyclas . . . . 426

dochmiospora. . . 425

fraxinicola . . 426

fulra . . . . 425

fuscospora . . . 426

galeata . . . . 424

Goldbachii . . . 424

griseopallida . . $\quad 425$

lacera . . . . 424

muscicola . . . 426

muscigena . . . 424

ochroleuca . . . 426

pallida . . . . 426

Pimii . . . . 424

stuppea. . . . 425

villosa . . . . 425

DACRYOMYCES . . . 454

chrysocomus . . . 455

deliquescens . . 455

macrosporus . . . 454

sebaceus . . 455

stillatus . . 455

tortus . . . 455

vermiformis . . . 455

DACRYOMYCETOIDEE • 454

DEDALEA . . . . 365

aurea . . . .366

cinerea. . . .367

confragosa . . . 366

ferruginea . . . 367

latissima . . $\quad 367$

polyzona . . . 367

quercina . . . 366

unicolor . . . 367

DERMINI $\cdot 367$

DeRMoCibE . 。 . . 229

DitTOLA . . . . 457

nuda . . . $45 \mathrm{~S}$

radicata. . . 457

Ulicis . . . $45^{\mathrm{S}}$

ECCIlia . . . . II

acus . . . . 119

atrides . . . IIS

atropuncta . . . IIS

carneogrisea . . . I 8

flosculus . . . 119 


\section{Eccilia (continued)}

griseorubella . nigrella parkensis rhodocylix Smithii .

Entolona .

ameides

ardosiacim . . . 105

Batschianum . . I04

Bloxamii . . . 105

clypeatum . . . 107

Cookei. . . 107

costatum . . 108

erophilum . . 104

Farrahii . . . 105

fertile . . . 106

griseocyaneum . . 106

helodes . . . 104

jubatum. . . . 106

liquescens . . . I05

lividum. . . 103

madidum . . . 105

majale . . . . I07

nidorosum . . . IoS

nigrocinnamomeum. . 107

Persoonii . . . 108

placenta . . . 104

pluteoides . . . 107

porphyrophæum . . 104

prunuloides . . . I04

pulvereum . . . 106

repandum . . . 104

resutum. . . . 106

rhodopolium . . . 107

Rozei . . . 106

Saundersii . . . 106

sericellum . . . $\mathbf{I 0 6}$

sericeum . . IOS

sinuatum . . . 103

speculum . . . IoS

Thomsonii . . . 107

tortipes . . . IOS

Wynnei . . . IOS

Euboletus . . . $\quad 3 \mathbf{I 9}$

EUTRENELLINOIDEÆ • 447

EXIDIA . . . . 447

albida . . . . 448

grandulosa . . . 448
Exidia (continuid)

PAGE

recisa . . . 448

truncata. . . . 447

Exobasidium . . . 422

Rhododendri . . . 423

Vaccinii . . 423

Fenisjoxia . . . . 455

luteoalba . . . 456

Fistulist . . . 332

hepatica . . .332

Flaingla . . . . 147

Aldridgei . . 148

alnicola. . . . 15 I

apicrea . . . 152

astragalina . . . I5 I

carbonaria . . . 150

clitopila . . . $\quad \mathbf{I 4 9}$

conissans . . . 152

decipiens . . . $\mathbf{I} 49$

filia . . . 150

filicea . . . . 153

flavida . . . . I 5 I

floccifera . . . I49

fusus . . . . I I I

gummosa . . . I 50

gymnopodia . . . 148

helomorpha . . . I53

hybrida. . . . 152

inaurata $. \quad . \quad 151$

inopus . . . . 152

juncina . . . . 150

lenta . . . 149

liquiritia . . $\quad 152$

lubrica . . . . $\mathbf{I} 49$

lupina . . . . $\mathbf{1} 49$

mixta . . . . 150

nitens . . . . 149

ochrochlora . . . 153

picrea . . . 153

purpurata . . . 148

rubicundula . . . $\mathbf{I}_{5} \mathbf{I}$

sapinea . . . . 152

scamba . . . I53

spumosa . . . 150

vinosa. . . 148

Fones . . . . . 347

annosus . . . $\quad 35 \mathrm{I}$

applanatus . . . $34 \mathrm{~S}$ 


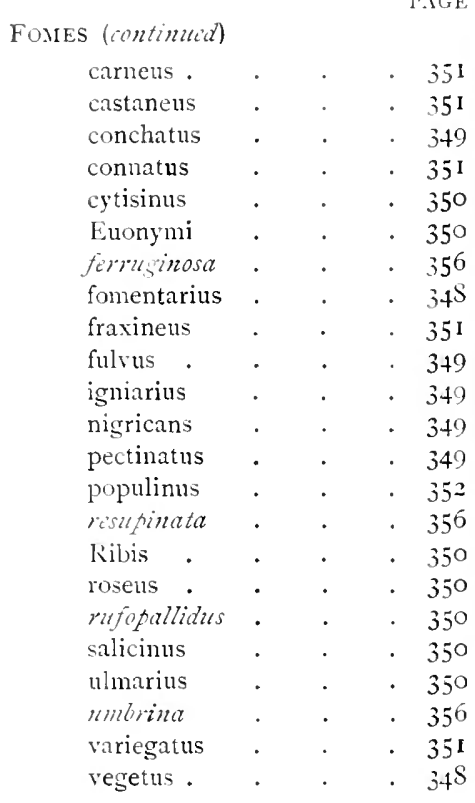

Galeri .

antipus. 163

apala . . . 162

conferta . . . 163

hypnorum . . . $16_{4}$

lateritia. . . . $\mathbf{1} 62$

minuta . . . . 164

mniophila . . . 164

mycenopsis . . . I64

ovalis. . . $\mathbf{I} 6_{3}$

pygmaeaffinis . . I $6_{3}$

ravida . . . $\mathbf{I} 64$

rubiginosa . . . I 64

siliginea . . . 162

spartea. . . . 163

spicula . . . . $1 \sigma_{3}$

tenera . . . I62

vittæformis . . 163

GASTEROMYCETES . . 459

GeAster

Terkeleyi . .467

Bryantii . . . 469

coliformis . . . 469

fimbriatus . . $47 \mathbf{I}$
GeAsTer (continut)

PAGF:

fornicatus . . 470

hygrometricus. . . $47 \mathrm{I}$

lagenzeformis . . . 470

limbatus . . . 469

mammosus . . . $47 \mathrm{I}$

Michelianus . . . 470

rufescens . . . $47 \mathrm{I}$

saccatus . . 470

Schmidelii . . . 469

Smithii. . . . 469

striatus . . . 470

triplex . . . . $47 \mathbf{I}$

umbilicatus . . . 469

Gomphidils . . . . 249

glutinosus . . . 250

gracilis . . . 25 I

maculatus . . . $25 \mathrm{I}$

viscidus. . . . 250

Grandinia . . . . $39 \mathrm{I}$

crustosa . . . . 392

granulosa . . . 392

mucida . . . . 392

ocellata . . . 392

papillosa . . . 392

Guepinia . . . . 456

Finsjoniana . . . 456

obliqua . . . . 457

Peziza . . . . 457

Gyrocephilus . . . 453

rufus . . . . 453

Gyronon $\cdot \quad \cdot \quad \cdot 33^{\circ}$

mebllum . . . $33 \mathrm{I}$

HEBELOMA . . . . $\mathbf{I} 42$

capniocephalum . . 146

claviceps . . . I +4

crustuliniforme . . $\mathbf{1} 45$

elatum . . . . 145

fastibile. . . 143

firmum . . . . 144

glutinosum . . . 143

ischnostylum . . . 146

longicaudum . . . 146

lugens . . . . 146

magnimamma . . I 17

mesophrum . . . 144

musivum . . . 143

nauseosum . . . 146 
Hebeloma (continuil)

PAGE

nudipes

petiginosum . . . 147

punctatum . . . 144

senescens . . . 145

sinapizans . . . 145

sinuosum . . . 143

strophosum . . . 144

subcollariatum . . 145

subsaponaceum . . I45

testaceum . . . 143

truncatum . . . 146

versipelle $\cdot$. $\quad 144$

Eyrei $\cdot \begin{array}{lllll}\cdot & \cdot & \cdot & \cdot & 26 \\ & \cdot & \cdot & 27\end{array}$

grangei . . . $\quad 27$

lycoperdoides . . 27

Wynnex . . . 27

IIIRNEOLA . • . . 446

Auricula-Judæ . . 446

HYDNACEE . . . 373

Hydiangium . . . . 4 SS

carneum . . . 4\$9

HYDNUM

carotacolor . . . $4 \mathrm{~S}_{9}$

$\cdot . \quad 373$

alutaceum

argutum $\cdot \quad \cdot \quad \cdot 3_{4}$

aurantiacum . . 377

aureum . . . . ${ }_{3} \mathrm{~S}_{1}$

auriscalpium . $\quad \cdot 37 \mathrm{~S}$

bicolor . $\cdot \quad \cdot \quad 3_{3}$

caputmedusæ . . . 379

cinereum . $\cdot 377$

cirrhatum . . $\quad 379$

compactum . . . 377

coralloides . . $\quad 379$

crinale ..$\quad \cdot 3^{\mathrm{S}}$

cyathiforme . . $\quad 37 \mathrm{~S}$

clenticulatum . $\quad . \quad 3 \mathrm{~S}$

diversidens . . $\quad 379$

erinacens . . $\quad 379$

farinaceum . $\quad . \quad 3 S_{4}$

ferrugineum . . $\quad 377$

ferruginosum . $\cdot{ }_{3} \mathrm{~S}_{\mathbf{I}}$

fragile . . . $\quad 376$

fuligineoalbum . $\quad .376$

fuscoatrum . . . 3 So
HYDXuM (continuted)

PAGE

fusipes . . . . 376

graveolens . . $37 \mathrm{~S}$

Hollii . . . . $3_{3}$

imbricatum . . . 375

lævigatum . . . 375

limonicolor . . . $\mathrm{j}_{2}$

melaleucum . . . 378

mellemm . . . $3 \mathrm{~S}_{3}$

membranaceum . . $3^{\mathrm{So}}$

molle . . . . 375

mollnscum . . . 3 So

multifurme . . . $3_{3} \boldsymbol{2}$

multiplex . . 3So

nigrum . . . $37 \mathrm{~S}$

niveum . . . $\quad 3_{4}$

nodulosum . . . $\quad 3_{3}$

ochraceum . . . $3 \mathrm{So}$

pinastri . . . $\quad 3^{\mathrm{S} 2}$

plumosum . . . $3_{3} 3$

pulcherrimum. . . 379

Queletii. . . $37 \mathrm{~S}$

repandum . . . 376

rufescens . . .376

scabrosum . . . 375

Schiedermayeri . . 379

scrobiculatum. . . 377

sepultum $\cdot \quad \cdot \quad \cdot{ }_{3} S_{3}$

sordidum ..$\quad 3^{\mathrm{Sz}}$

spathulatum . . . $3 S 2$

squalinum . . 3 So

squamosum . . . 375

Stevensonii . . $\cdot{ }_{3} S_{3}$

stipatum . . . $3^{S_{4}}$

sulphureum . $\cdot{ }_{3}{ }_{2}$

udum . $\quad . \quad \cdot \quad 3_{3}$

variecolor . . . $\quad 3 \mathrm{SI}$

viricle..$\quad .3 \mathrm{Sz}$

Weinmannii . . 3 So

zonatum . . . 377

IIYDROCYBE . . . . 242

Hygroctie . . . . 263

HigrophuRUS . . . 255

agathosmus . . $\quad 259$

arbustivus . . $25 \mathrm{~S}$

aromaticus . . $25 \mathrm{~S}$

aureus . . $\quad 25 \mathrm{~S}$

calyptreformis . . 265

caprinus . . 260 
INDEX

HYGROPHORL's (continuid)

ceraceus. . .

ceracinus $\quad \cdot \quad 203$

chlorophanus . . . 265

chrysodon . . . 256

Clarkii . . . . 262

coccineus . $\quad . \quad 264$

Colemannianus , . $\quad 263$

conicus . . . 265

Cossus . . . 257

discoideus . . . $25 \mathrm{~s}$

discoxanthus . . $\quad 257$

distans . . . . 262

eburneus . . . 256

erubescens . . . 257

fcetens. . . . 260

fornicatus . . , 261

fuscoalbus . . 259

glutinifer . . $.25 \mathrm{~S}$

Houghtonii . . . 263

hypothejus . . . 259

intermedius . . . 265

irrigatus. $\quad . \quad$. 262

Karstenii . . . 260

læetus . . . . 263

latitabundus . . . 262

leporinus . . 260

limacinus . . 258

lividoalbus . . . 260

melizeus . . . 257

mesotephrus . . . 259

metapodius . . . 262

micaceus . . . $26 \mathbf{1}$

miniatus . . . 264

mucronellus • . . 264

nemoreus . . . 260

nitratus . . . . 266

nivells . . . . $26 \mathbf{I}$

obrussens . . . 264

olivaceoalbus . . $\quad 259$

ovinus . . . . 262

penarius . . . 257

pratensis . . 260

psittacinus . . . 265

pudorinus . . . $25 \mathrm{~s}$

pulverulentus . . . 257

puniceus . $\quad . \quad 264$

rusiocoriaceus . . $26 \mathbf{I}$

sciophanus . $\quad . \quad 26.3$
HYGROPHORUS (continuid)

PAGE

$\begin{array}{lllll}\text { spadiceus } & \cdot & \cdot & \cdot & 266 \\ \text { subradiatus } & \cdot & \cdot & \cdot & 262 \\ \text { turundus } & \cdot & \cdot & \cdot & 264 \\ \text { unguinosus } & \cdot & \cdot & \cdot & 266 \\ \text { ventricosus } & \cdot & \cdot & \cdot & 26 \mathbf{I} \\ \text { virgineus } & \cdot & \cdot & \cdot & 26 \mathbf{I} \\ \text { vitellinus } & \cdot & \cdot & \cdot & 263 \\ \text { Wynniæe } & \cdot & . & . & 26 \mathbf{I}\end{array}$

Hranechete . . . 407

abietina. . . 409

Avellana . . . 407

Boltonii . . . 409

corrugata . . . $40 \mathrm{~S}$

crassa. . . . 408

croceoferruginea . . 408

disciformis . . . 409

fuliginosa . . 408

leonina . . . 408

nigrescens . . . 408

rubiginosa . . . 407

Stevensonii . . 408

tabacina . . . 408

HyMenogatier . . . 492

citrinus . . . . 493

decorus. . . . 493

griseus . . . . 494

Klotzschii . . . 492

lilacinus . . . 494

luteus . . . . 493

lycoperdineus . . 493

muticus . . 493

olivaceus . $\cdot 493$

pallidus . . . 493

populetorum . . . 494

pusillus . . . . 494

tener . . . . 494

Thwaitesii . . . 494

vulgaris . . . 493

HYMENOGASTRACEA . 486

HYMENOMYCETES • • II

Hyтholoma • • . . 179

appendiculatum . $\quad \mathbf{I}_{3}$

Candolleanum . $\quad \mathrm{I}_{3}$

capnoides . . . ISo

cascum . . . . $\quad \mathrm{I}_{3}$

dispersum . . . $\mathbf{I} \mathbf{S}_{\mathbf{I}}$

egenulum . $\quad . \quad \mathbf{I} 8_{4}$

elroodes . . . $\mathbf{I} S \mathbf{I}$ 


\section{IypHOLOMA (continued)}

epixanthum . . . ISo

fasciculare . . . $\mathbf{I} \mathrm{S}_{\mathbf{I}}$

felinum . . . $\mathrm{I}_{4}$

hydrophilum . . . $\mathbf{1} \mathrm{S}_{4}$

incomptum . . . ISI

instratum . . . $I_{\mathbf{I}} \mathbf{I}$

lacrymabundum . . $\mathbf{I S}_{2}$

lanaripes . . . $\mathrm{I}_{3}$

leucotephrum . . $\mathbf{I} \mathrm{S}_{4}$

œdipus . . . . I $S_{2}$

pilulaforme . . . $\mathrm{IS}_{4}$

pseudostorea . . . IS2

punctulatum . . . $\mathrm{I}_{3}$

pyrotrichum . . . $\mathbf{I} \mathbf{S}_{2}$

silaceum . . . ISo

sublateritium . . . ISO

velutinum . $\quad . \quad \mathbf{I} 2$

Hypochnus violeus . . . . 413

HYPORHOIII . . . 97

IIYSTERANGIUM . . . 490

nephriticum . . 490

Thwaitesii . . 490

INOCYBE. . . . . $13 \mathrm{I}$

adequata . . . 135

asterospora . . . $13 \mathrm{~S}$

Bongardii . . . 135

brunnea. . .. $\mathrm{I}_{3} \mathrm{~S}$

Bucknallii . . . 139

cæsariata . . . $\mathbf{I} 40$

calamistrata . . . $\mathbf{1 3 3}$

calospora . . . 133

carpta . . . 136

cervicolor . . . 136

cincinnata . . . 134

Clarkii . . . I 140

commixta . . . 495

corydalina . . . I 40

Curreyi. . . . 137

deglubens . . . 136

descissa. . . . 139

destricta . . $\quad 139$

dulcamara . . . I 33

duriuscula . . . $\mathbf{1 3 9}$

eutheles . . $\mathrm{I}_{3} \mathrm{~S}$

fasciata . . . 134

fastigiata . . 137

fibrosa . . . . 137

flocculosa . . $\quad 135$
INOCYBE (continuta)

fulvella . . . I4I

geophylla . . I40

Godeyi . . . 139

hremacta . . . $\mathbf{I 3 4}$

hirsuta . . . 133

hiulca . . . . $\mathbf{1 3 7}$

hystrix . . . 132

incarnata . . . $\mathbf{1 3 4}$

lacera . . . . 135

lanuginosa . . . $\mathbf{I} 33$

lucifuga . . . 140

margarispora . . . $\mathbf{I}_{3} \mathrm{~S}$

maritima . . . 135

mimica . . . 133

mutica . . . . 135

obscura . . . 136

perbrevis . . . 139

perlata . . . 137

phrocephala . . . I37

plumosa . . . I33

pratervisa . . 138

proximella . . . 137

pyriodora . . . I34

relicina. . . $13^{2}$

Rennyi . . . . 136

rimosa . . . . 137

sambucina . . . 140

scabella . . . I4I

scabra . . . . $\mathbf{1} 35$

schista . . . 136

sindonia . . . 140

subrimosa . . . ${ }_{13} 8$

trechispora . . . I4I

Tricholoma . . . 142

Trinii . . . 139

vatricosa . . . 141

violaceofusca . . . 141

Whitei . . . . I4I

INOLOMA $. \quad . \quad . \quad .224$

IRPEX . . . . . 356

candidus . $\quad . \quad{ }_{3} S_{7}$

carneus. . . . $3 S S$

deformis . . $35 S$

fuscoviolaceus $\quad . \quad{ }_{3} S_{7}$

hypogzus . . $\quad{ }_{3} \mathrm{~S}_{7}$

Johnstonii . . . ${ }_{3} S_{7}$

lacteus . . . . 387

obliquus . . . $3^{87}$ 
I'AGE

IRPEX (continued)

pendulus . . . 386

spathulatus . $\quad \cdot{ }_{3}^{87}$

ITHYPHALLUS . $\quad$. $\quad$. 459

impudicus . . . 459

KNEIFFIA • . . . 394

setigera . . 394

subgelatinosa . . $\quad 395$

LACTARIUS . . . . 266

acris . . . . 272

aspideus . . . $\quad 269$

aurantiacus . . . 274

blennius . . . 270

camphoratus . . $\quad 27 \mathrm{~S}$

capsicum . . . 272

chrysorrheus . . . 272

cilicioides . . . 268

cimicarius . $\quad .27 \mathrm{~S}$

circellatus . . . $27 \mathbf{I}$

controversus . $\quad . \quad 269$

cremor . . . . 275

cyathula . . . 275

deliciosus . . . 274

flexuosus . . $\quad 27 \mathbf{I}$

fuliginosus . . . 276

glaucescens . . . 272

glyciosmus . . . 276

helvus . . . . 275

hysginus . . . 270

ichoratus . . $\quad 277$

insulsus . . . . 270

intermedius . . . 268

involutus . . . 274

lateritioroseus . . 269

lignyotus . . . 277

lilacinus . . . 277

mammosus . . . 276

minimus . . . 279

mitissimus . . . $27 \mathrm{~S}$

obliquus . $\quad . \quad \cdot 279$

ubnubilis . . . 279

pallidus . . . . 274

pergamenus . . . 273

picinus . . . 276

piperatus . . . 273

plumbeus . $\quad . \quad 273$

pubescens . . . 269

pyrogalus
LACTARIUS (continued)

I'AGE

quietus . . . . 274

retisporus . . . 277

rufus . . . . 275

sanguifluus . . $\quad 274$

scoticus . . . . 273

scrobiculatus . . . 268

serifluus. . . . $27 \mathrm{~S}$

spinosulus . . . 277

squalidus . . . $27 \mathrm{I}$

subdulcis . . . $27 \mathrm{~S}$

subumbonatus. . . 279

theiogalus . . . 275

tomentosus . . . 276

torminosus . . . 268

trivialis . . . 270

turpis . . . . 269

umbrinus . . . 273

utilis . . . . 270

uvidus . . . . $27 \mathbf{I}$

vellereus . . . 273

vietus . . . . 275

violascens . . . 272

volemus. . . . 277

zonarius. . . 270

Lentinus . . . $30 \mathrm{O}$

adhærens $\quad . \quad . \quad 309$

cochleatus . . . 310

fimbriatus . . . 310

flabelliformis . . . 310

leontopodius . . 309

lepideus. . . . 309

pulverulentus . . $\quad$. 309

scoticus . . . 3 IO

suffrutescens . . . 309

tigrinus. . . . 308

vulpinus. . . . 310

LENZITES . . . . 315

abietina. . . 316

betulina. . . . 315

flaccida . . . . 315

heteromorpha $\quad . \quad 3 \mathbf{I} 6$

sepiaria . . . 316

LEIJOTA. . . . . 19

acutesquamusa . . 2 I

amianthina . . . 25

atrocrocea . . 24

Badhami . . . $2 \mathrm{I}$

biornata. . . 22 
LEPIOTA (continued)

Bucknalli

carcharias

cepæstipes

cinnabarina . . . 24

citrophylla . . . 24

clypeolaria . . . 22

cristata . . . . 23

delicata. . . 26

emplastrum . . . 21

erminea. . . . 23

excoriata . . $2 \mathrm{I}$

felina. . . 22

Friesii . . . 2 I

Georginæ . . 26

glioderma . . . 26

gracilenta . . . $\mathbf{2 I}$

sranulosa . . . 24

hispida . . . . 22

holosericea . . . 23

ianthina. . . . 25

illinita . . . 26

licmophora . . . 24

martialis . . 25

mastoidea . . . 21

meduilata . . . 26

meleagris . . . 22

mesomorpha . . . 25

metulæspora . . . 23

micropholis . . . 23

naucina . . . . 23

nigromarginata . . 22

nympharum . . . 23

parvannulata . . . 25

permixta . . 20

polysticta . . . 25

procera . . . 20

prominens . . 20

rachodes . . . 20

seminuda . . 25

serena. . . 494

sistrata . . . . 25

submarasmioides . . 26

Vittadinii . . . 23

LEPISTA . . . . 252

LEPTONIA . . . . III

athiops . . . $\mathbf{I}_{\mathbf{I}} \mathbf{3}$

anatina . . . II 2

asprella . . . . II4
LEPTONIA (continted)

chalybæa . . II3

chloropolia . . II4

euchroa . . . II 3

formosa. . . 113

incana. . . 113

lampropus . . 112

lappula . . . I I 2

lazulina. . . I 13

nefrens . . . . II 4

placida. . . I I 2

serrulata . . . II 3

solstitialis . . . II 3

LEUCOSPORI . . . $\mathrm{I} 4$

LIMACIUM . . . . 256

LYCOPERDACEÆ . . 464

LYCOPERDON · . . . $47 \mathrm{I}$

atropurpureum . . 473

aurantiacum . . . 480

Bovista . . . 475

cælatum . . . 475

Cookei . . . 476

cruciatum . . . 474

depressum . . 475

echinatum . . . 472

excipuliforme. . . 473

furfuraceum . . . 475

gemmatum . . . 473

hiemale. . . 475

Hoylei . . . . 472

lacumosum . . . 474

molle . . . . 474

perlatum . . . 474

pyriforme . . . 474

saccatum . . 473

velatum . . . 473

Lysurus . . . 463

australiensis . . 463

MarasmiUs . $\quad . \quad . \quad 299$

actinophorus . . 307

alliaceus . . $\quad 305$

amadelphus . . 304

androsaceus . . 306

angulatus . . $\quad 304$

archyropus . . $\quad 303$

Broomei. . . . 308

calopus . . . . 303

candidus . . 305 


\section{M.ıR.Asmius (continute)}

caulicinalis

Curreyi .

epichloe

epiphyllus

erythropus

fœetidus

fuscopurpureus

graminum

Hudsonii

impudicus

insititius

lagopinus

languidus

oreades .

perforans

peronatus

plancus .

polyadelphus .

porreus

prasiosmus

ramealis

rotula.

saccharinus

sclerotipes

scorodonius

scorteus

splachnoides

spodoleucus

Stevensii

terginus.

torquatus

torquescens

urens

Vaillantii

varicosus

Wynnei .

Melanogaster

ambiguus

Birkeleyanus

variegatus

\section{Merulius}

aurantiacus

aureus . . . $\quad 369$

Carmichalianus . $\quad 370$

confluens . . $\quad 368$

corium . . . . 369

fugax . . . . 369

Guillemotii . . . $37 \mathrm{I}$
MERUlius (contimute)

PAGE

himantioides . . . 369

lacrymans . . . 370

laticolor . . . 369

molluscus . . . 369

pallens . . . 370

porinoides . . 370

rufus . . . . 370

serpens . . . . 370

squalidus ..$\quad .37 \mathbf{1}$

tremellosus . . . 369

Mucronella . . . . 395

calva . . . . 395

Mutinus $\cdot$. $\quad . \quad \cdot 461$

bambusinus . . . 462

caninus . . . $46 \mathrm{I}$

MYCENA. . . . . 69

acicula . . . . 79

Adonis . . . . 73

retites . . . 77

alkalina. . . . 76

amicta . . . $7 \mathrm{~S}$

ammoniaca . . . 76

atroalba. . . . 75

atrocyanea . . . 76

aurantiomarginata . . 7 I

balanina . . . $7 \mathrm{I}$

Berkeleyi . . . 74

capillaris . . . $\mathrm{S}_{2}$

carneosanguinea . . $7 \mathrm{I}$

chelidonia . . . 79

cinerea. . . . ${ }_{7} 8$

citrinella . . . 80

clavicularis . . . 80

codoniceps . . . 75

cohærens . . . 74

collariata . . . 78

consimilis . . . 77

corticola . . . $8 \mathbf{I}$

crocata . . . . 79

cruenta . . . . 79

debilis . . . $7 \mathrm{~S}$

discopus . $\quad . \quad 8$ I

dissiliens . . . 76

elegans . . . . 72

epipterygia . . . So

excisa . . . 74

farrea $. \quad . \quad . \quad .73$

flopes . . . . 77 


\section{Mricena (convinued)}

flavipes .

flavoalba

galericulata

galopus .

gypsea

hæematopus

hiemalis.

iris

juncicola

lactea . . . . 73

leptocephala . . . 76

leucosala . . . So

lineata . . . . 73

luteoalba . . . 73

marginella . . . $7 \mathbf{I}$

metata . . . . 76

mirabilis . . . 7 I

olivaceomarginata . . 72

parabolica . . . 75

paupercula . . . 76

pelianthina . . 70

pelliculosa . . . So

peltata . . . . 77

plicatocrenata. . . $8 \mathbf{I}$

plicosa . . . . 77

plumbea . . . 78

polygramma . . . 75

prolifera . . . 74

psammicola . . . 74

pseudopura . . . 72

pterigena . . . $8 \mathbf{I}$

pullata . . . . 76

pura . . . . 72

rorida . . . . $8 \mathrm{I}$

rosella. . . . 72

rubromarginata . . 72

rugosa . . . . 74

saccharifera . . . $S_{\mathbf{I}}$

sanguinolenta. . . 79

setosa . . . . $\quad$ S2

speirea . . . . $7 \mathrm{~S}$

stannea. . . . 77

strobilina $\quad . \quad . \quad . \quad 72$

stylobates . . . $\quad$ SI

sudora . . . . 74

tenella . . . . 79

tenerrima . . . $\mathrm{SI}$

tenuis . . . . 77
MrCENA (continued)

PAGE

tintinnabulum. . . 75

vitilis . . . $\quad 78$

vitrea . . . . . 77

vulgaris . . . . 80

zephira . . . . 73

MYXACIUM $\quad . \quad . \quad . \quad .222$

Nematelia . . . . $45^{2}$

encephala . . $45^{2}$

nucleata. . . . 453

rubiformis . . . 453

virescens . . . 453

NaUCORia . . . . 153

abstrusa. . . . 156

anguinea . . 156

arvalis . . . . 158

badipes . . . 158

carpophila . . . 160

centuncula . . . 156

cerodes . . . . $\quad$. 157

cidarls . . . . $\mathbf{I} 55$

conspersa . . . 160

Cucumis . . . 155

echinospora . . . 156

erinacea. . . . 160

escharoides . . . 160

festiva . . . . 155

glandiformis . . . 157

graminicola . . . I6I

hamadryas . . . $\mathbf{1 5 5}$

horizontalis . . . 156

innocua. . . . 157

latissima . . . 159

lugubris. . . . 155

melinoides . . . $\mathbf{1 5 7}$

Myosotis . . . 159

nasuta . . . . 158

nucea . . . . 157

obtusa . . . . 155

pediades . . . $15 \mathrm{~S}$

porrigrinosa . . . 159

pusiola . . . . I 57

rimulincola . . . 156

rubricata . . . 156

scolecina . . . 157

semiflexa . . . 156

semiorbicularis . $\quad$ I 59

sideroides 
Nitucoria (continutid)

siparia . . . $\mathbf{I} 60$

sobria . . . . 160

striapes. . . . $\mathbf{I} 57$

subglobosa . . . 155

tabacina . . . 159

temulenta . . . 159

tenax . . . . 159

triscopus ..$\quad 15^{8}$

vervacti. . . . 158

Nidularia $\quad . \quad \cdot \quad \cdot \quad 4 S_{3}$

Berkeleyi . . . $4 S_{4}$

confluens . . . $4 \mathrm{~S}_{4}$

pisiformis . . $\quad{ }_{4} S_{3}$

NIDULARIACE E . . 4 SO

NOLANEA . . . . II4

Babingtonii . . . II5

ccelestina . . . II

exilis . . . . I I 7

fulvostrigosa . . . I I6

icterina. . . . 116

infula . . . . I 16

juncea . . . . II 5

mammosa . . . II5

nigripes. . . . I 16

papillata . . . II5

pascua . . . . 115

picea . . . . I I6

pisciodora . . . II6

rhodospora . . . II7

rubida . . . . II 7

rufocarnea . . . $1 \pm 6$

verecunda . . . $1 \mathbf{I} 7$

Nyctalis . . . 298

asterophora . . . 299

parasitica . . . 299

(ICTAVIania . . . . 486

asterosperma . . . 486

compacta . . . $4{ }_{7} 7$

Stephensii . . . $4 \$ 7$

Odontia . . . 392

barba-Jovis . . . 393

cristulata . . $\quad 393$

fimbriata . . . 393

Pruni . . . . 394

UMPHALIA $\quad \cdot \quad \cdot \quad \cdot \quad \mathrm{S}_{2}$

abhorrens . . . $\quad S_{7}$

alutacea . . . 56
Onphalia (continuca)

I'AGF:

Bellixe . . . . S9

buccinalis . $\quad . \quad$. $\quad \mathrm{S}_{9}$

bullula . . . . $\quad$ S9

caspitosa . . . $\quad \$_{5}$

campanella . . . 87

camptophylla . . $8 S$

chrysophylla . $\quad . \quad S_{4}$

demissa . . . $\$ 6$

detrusa . . . . $\$_{3}$

directa. . . . SS

epichysium . . . $\quad S_{5}$

fibula . . . SS

glaucophylla . . . $\$ 6$

gracilis . . . . . $\quad \mathrm{S}_{9}$

gracillima . . . $\quad$ S9

grisea . . . . 88

griseopallida . . . $\quad \$_{7}$

hepatica . . . S6

hydrogramma. . $\quad . \quad \mathrm{S}_{3}$

infumata $. \quad . \quad . \quad 87$

integrella . . . $\quad S_{9}$

leucophylla $. \quad . \quad . \quad \mathrm{S}_{4}$

Luffii . . . . $\mathrm{S}_{5}$

maura . . . $\quad S_{t}$

muralis . . . 86

Neville. . . . S9

offuciata $. \quad . \quad S_{4}$

onisca . . . . $\quad \mathrm{S}_{5}$

philonotis . . . $\mathrm{S}_{5}$

picta . . . . Ss

Postii . . . . $\mathrm{S}_{4}$

pseudoandrosacea . . $\quad S_{7}$

pseudodirecta . . $S S$

pyxidata $\quad . \quad . \quad . \quad \mathrm{S}_{4}$

retosta. . . . 87

rustica . . . . $\$ 6$

sphagnicola . $\cdot \quad \cdot \quad 85$

stellata. . . . $\quad S_{7}$

striepileus . . . $\quad S_{4}$

telmatica . . . $\mathrm{S}_{5}$

umbellifera . . . 86

umbilicata . $\quad . \quad . \quad \$_{3}$

umbratilis . . . $\$ S$

velutina . . $\quad . \quad S_{7}$

PANEOLUS . . . . 196

acuminatus . . 198

caliginosus . . . 198 


\begin{tabular}{|c|c|c|}
\hline \multicolumn{3}{|l|}{ H'IN.EOLUS (continued) } \\
\hline campanulatus & $\cdot$ & 197 \\
\hline cinctuius . & . & - I9S \\
\hline egregins & . & . $\quad 197$ \\
\hline fimicola & . & - 198 \\
\hline leucophanes. & . & . $\quad 197$ \\
\hline papilionaceus & . & . $\quad 197$ \\
\hline phalænarum. & . & . $\quad 197$ \\
\hline retirugis . & . & . $\quad 197$ \\
\hline sphinctrinus & . & 197 \\
\hline subbalteatus & . & 198 \\
\hline Panus . . . & . & $3^{I I}$ \\
\hline conchatus & . & - 3 II \\
\hline farinaceus & . & - $\quad 312$ \\
\hline patellaris & . & - $3^{12}$ \\
\hline rudis & . & - $31 \mathbf{I 2}$ \\
\hline Stevensonii & $\cdot$ & - 312 \\
\hline stypticus & . & - 312 \\
\hline torulosus & . & - $3 I I$ \\
\hline Paxillus & . & . $\quad 25 \mathrm{I}$ \\
\hline Alexandri & . & . $\quad 252$ \\
\hline atrotomentosus & as & - $\quad 254$ \\
\hline crassus . & - & . 254 \\
\hline extenuatus & . & - $\quad 252$ \\
\hline Fagi . & - & . $\quad 255$ \\
\hline giganteus & . & . $\quad 252$ \\
\hline involutus & . & . 253 \\
\hline lepista . & . & - 252 \\
\hline leptopus & . & . 254 \\
\hline lividus . & . & . 253 \\
\hline orcelloides & . & . 253 \\
\hline panæolus & - & . $\quad 253$ \\
\hline panuoides & . & . 255 \\
\hline paradoxus & . & . $\quad 253$ \\
\hline porosus & . & . $\quad 254$ \\
\hline revolutus & . & . $\quad 253$ \\
\hline PENIOPHORA & . & . $4^{15}$ \\
\hline Chrysanthemi & . & . 419 \\
\hline cinerea. & . & . $4 \mathrm{IS}$ \\
\hline Crosslandii & . & - $\quad 4 \mathrm{I} 7$ \\
\hline gigantea & · & - $4 \mathrm{I} 7$ \\
\hline lnydnoides & $\cdot$ & . $\quad 419$ \\
\hline incarnata & - & - 417 \\
\hline limitata. & $\cdot$ & - $\quad 417$ \\
\hline ochracea & . & 417 \\
\hline pezizoides & . & . $4 \mathrm{I} 7$ \\
\hline phyllophila & . & - $4 \mathrm{IS}$ \\
\hline pubera. & . & - 418 \\
\hline quercina & . & . 416 \\
\hline
\end{tabular}

Peniophora (continued)

PAGE

\begin{tabular}{|c|c|c|c|}
\hline rimosa. & - & - & 418 \\
\hline rosea. & . & . & 417 \\
\hline scotica. & . & - & $4 \mathrm{IS}$ \\
\hline terrestris & - & . & $4^{I S}$ \\
\hline velutina & . & . & $4 \mathrm{IS}$ \\
\hline Pexiza amorpha & . & - & 410 \\
\hline PHALLOIDACE $A$ & & . & - 459 \\
\hline Phlebia. & . & - & 390 \\
\hline contorta & . & . & - $\quad 39 \mathrm{I}$ \\
\hline lirellosa & - & - & - 391 \\
\hline merismoides & . & . & - 390 \\
\hline radiata & - & . & . $39 \mathbf{I}$ \\
\hline vaga . & - & - & $39 I$ \\
\hline PhLEgmaCiUm & - & . & $2 \mathrm{I}_{4}$ \\
\hline PHOLIOTA & - & . & 125 \\
\hline adiposa & . & . & . $\quad 128$ \\
\hline ægerita. & . & . & . $\quad 127$ \\
\hline aurivella & . & . & I 27 \\
\hline capistrata & . & • & 127 \\
\hline comosa & . & . & 127 \\
\hline confragosa & - & . & 130 \\
\hline Cookei . & . & . & 130 \\
\hline cruentata & . & . & 129 \\
\hline curvipes & . & . & 129 \\
\hline dissimulans & . & . & 130 \\
\hline flammans & . & . & I 29 \\
\hline grandis . & . & . & $12 \mathrm{~S}$ \\
\hline heteroclita & - & - & 127 \\
\hline Junonia. & . & . & 129 \\
\hline leochroma & . & . & I 26 \\
\hline lucifera & . & . & 128 \\
\hline luxurians & . & . & 127 \\
\hline marginata & . & . & 130 \\
\hline mustelina & . & . & I 30 \\
\hline mutabilis & . & . & 130 \\
\hline mycenoides & . & . & $\mathbf{I}_{3} \mathrm{I}$ \\
\hline Paxillus & - & . & 129 \\
\hline pudica. & - & . & $\mathbf{1 2 6}$ \\
\hline pumila. & - & . & $I_{3} I$ \\
\hline radicosa & . & . & 126 \\
\hline sororia & - & - & 495 \\
\hline spectabilis & . & . & $12 S$ \\
\hline squarrosa & - & . & $12 \mathrm{~S}$ \\
\hline sublutea & . & . & $\mathrm{I}_{3} \mathrm{O}$ \\
\hline subsquarrosa & • & - & 128 \\
\hline tuberculosa & • & . & 129 \\
\hline unicolor & 。 & . & $13^{\circ}$ \\
\hline
\end{tabular}




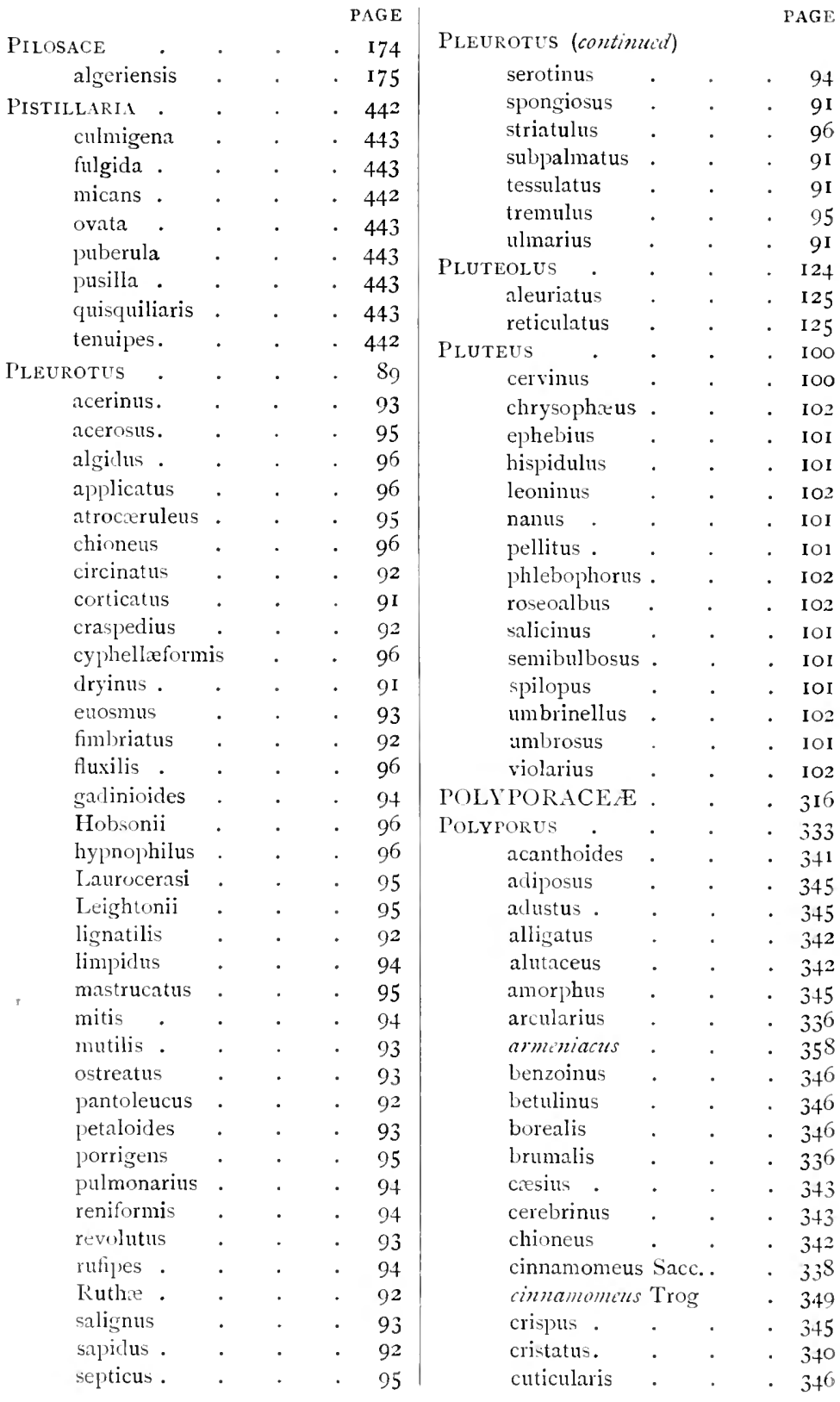




\section{POLYPORUS (contimuel')}

\begin{tabular}{|c|c|c|c|}
\hline destructor & . & - & . 343 \\
\hline dryadeus & . & • & . $34^{6}$ \\
\hline elegans. & . & - & . 339 \\
\hline epilencus & . & . & - 342 \\
\hline Euony'mi & - & - & - 350 \\
\hline flavovirens & - & • & . 335 \\
\hline fragilis . & . & . & . 343 \\
\hline fragrans. & . & . & . 345 \\
\hline frondosus & . & . & . 340 \\
\hline furnosus. & - & - & . 344 \\
\hline fuscidulus & . & . & - 336 \\
\hline giganteus & . & . & . 340 \\
\hline gilvus . & - & · & . 344 \\
\hline heteroclitus & . & . & . 342 \\
\hline hispidus. & . & . & . 345 \\
\hline imbricatus & . & . & . $34 \mathrm{I}$ \\
\hline intybaceus & . & • & . 340 \\
\hline Keithii . & . & • & . 345 \\
\hline lacteus . & . & . & . 343 \\
\hline lentus & . & • & . 336 \\
\hline leptocephalus & . & . & . 337 \\
\hline leucomelas & . & • & - 336 \\
\hline lucidus . & . & . & 339 \\
\hline melanopus & . & . & . $\quad 33 \mathrm{~S}$ \\
\hline Nijchelii & . & . & - $33 \mathrm{~S}$ \\
\hline mollis . & . & . & 343 \\
\hline Montagnei & . & . & . $\quad 337$ \\
\hline nidulans & . & . & . 344 \\
\hline osseus . & $\cdot$ & . & - $34 \mathrm{I}$ \\
\hline pallescens & . & . & . 342 \\
\hline perennis & . & . & - $33^{S}$ \\
\hline petaloides & • & . & 339 \\
\hline picipes . & • & . & 339 \\
\hline pomaceus & · & r & 349 \\
\hline quercinus & . & r. & 347 \\
\hline Rostkovii & . & . & 339 \\
\hline rufescens & . & . & 337 \\
\hline rutilans. & · & r. & $3+4$ \\
\hline salignus. & . & . & . 342 \\
\hline Schweinitzii & . & . & 337 \\
\hline spongia . & - & . & - $34 \mathrm{I}$ \\
\hline spumeus & - & . & . 346 \\
\hline squamosus & - & . & $33^{S}$ \\
\hline Steannii. & • & - & . 348 \\
\hline Sterensii & - & - & . $\quad 365$ \\
\hline sulphureus & - & . & 341 \\
\hline tomentosus & - & & 337 \\
\hline rabeus . & . & . & . 343 \\
\hline
\end{tabular}

I'AGE

Polyporus (continuid)

umbellatus . . 340

varius . . . 339

Polysaccum . . . 478

pisocarpium . . $47 \mathrm{~S}$

Polystictus . $\quad . \quad \cdot \quad \cdot 352$

abietinus . . . 354

cryptarum . . . 353

fibula . . . . 353

gossypinus . . $\quad 353$

hirsutus . . . 354

nodulosus . . . 353

polymorphus . . . 353

radiatus. . . . 352

ravidus . . . . 353

velutinus . . . 354

versicolor . . . 354

Wynnei . . 355

zonatus . . . . 354

PORIA . . . . . 355

aneirina. . . $\quad .35 \mathrm{~S}$

armeniaca . . $\quad 358$

bathypora . . . 362

blepharistoma. . . 362

bombycina . . 358

callosa . . . . 360

cincta. . . $.35 \mathrm{~S}$

collabefacta . . . 360

contigua . $\quad 356$

corticola . . . 362

farinella . . . $36 \mathbf{I}$

ferruginosa . . . 356

fuscocarnea . $.35^{S}$

Gordoniensis . . . $3^{61}$

hibernica . . $36 \mathbf{I}$

hybrida . . 362

hymenocystis . . $\quad 362$

incarnata . . . 357

Lastadii . . . 357

medullapanis . . . 359

micans . . . . 357

mollusca . . . 360

mucida . $\quad . \quad$. 359

nitida . . . . 357

obducens . . . 360

obliqua . . . . 356

purpurea . . . 357

radula . . . . $36 \mathbf{I}$

ramentacea . . $35^{\mathrm{S}}$ 
INDEX

PORIA (continued)

Psathyra (continucel)

Rennyi .

resupinata . . . 356

reticulata $\quad . \quad$. 362

rhodella . . . 357

rufa . . . . 357

sanguinolenta . $\quad 360$

subfuscoflavida . $\quad 359$

subgelatinosa . $\quad 359$

terrestris . . . 359

umbrina . . . 356

Vaillantii . . . $36 \mathrm{I}$

vaporaria . . . $36 \mathbf{I}$

violacea . . . 356

viridans . . . $\quad 359$

vitrea . . . . 359

vulgaris . . . 360

Porotheliula. $\quad . \quad$. $37 \mathrm{I}$

confusum . . $\quad 372$

fimbriatum . . . 372

Friesii . . . . 372

Keithii . . . . 372

Stevensonii . . . 372

PRATELLA . . . . . 169

Psalliota . . . . 170

arvensis . . . I7 I

augusta . $\quad . \quad \cdot$ I I I

campestris . . . 172

comtula. . . . 173

cretacea. . . . I72

dulcidula . . . I 74

echinata . . . $\mathbf{I} 74$

Elvensis. . . . I7 I

hæmorrhoidaria . . 173

peronata . . . 173

perrara . . . . $17 \mathrm{I}$

pratensis . . . 172

rusiophylla . . . 174

sagata . . . . 174

silvatica. . . . 173

subgibbosa . . . 173

villatica. $. \quad . \quad 172$

xanthoderma . . . I 72

PSATHYka . . . . 190

fibrillosa . . . 193

frustulenta . . . 192

glareosa. . . . I9I

Gordonii . . . 193

gossypina . . . 194

gyroflexa . . . I92

helobia . . . . 19.3

Loscosii. . . . 193

mastigera . . . I9I

microrrhiza . . . 194

neglecta. . . . 192

noli-tangere . . . 194

obtusata. . . 192

pellosperma . . . I9I

pennata. $. \quad . \quad .193$

semivestita . . . 193

spadiceogrisea $\quad . \quad$. 192

urticrecola . . . 194

Psathyella. . . . I $9 S$

arata . . . . 200

atomata. . . . 200

caudata . . . 200

crenata. . . . 200

disseminata . . . 201

empyreumatica . . 200

gracilis . . . . 199

hiascens . . . 199

hydrophora . . . 200

prona . . . 200

subatrata . . . 199

trepida . . . . 200

Psilocybe . . . . . $\mathrm{IS}_{4}$

agraria . . . . IS6

ammophila . ..$~ I \$ 7$

areolata. . . . IS6

atrorufa. . . . IS7

bullacea. . . . IS7

callosa . . . . IS9

canobrunnea . . . 188

canofaciens . . . IS6

catervata $\quad . \quad . \quad . \quad \mathbf{I} 99$

cernua . . . . . ISg

chondroderma. $\quad . \quad$ IS7

clivensis. . . . $\mathbf{I S 9}$

compta . . . . ISS

coprophila . . . $\mathrm{IS}_{7}$

ericæa . $. \quad . \quad$. $\mathbf{I} \$ 6$

fonisecii . . . IS9 
Psilocybe (contimued)

hebes

helvola.

nuciseda . . . ISS

physaloides . . . I $\mathrm{S}_{7}$

sarcocephala . . . I 55

scrobicola . . . I $S 7$

semilanceata . . I ISS

spadicea . . . ISS

squalens. $\quad . \quad$. $\quad$ I $\mathrm{S} 9$

subericæa . . . IS6

ula . . . . IS6

virescens . . . IS6

Pterula $\quad \cdot \quad \cdot \quad \cdot \quad \cdot \quad \cdot 439$

multifida . . . 439

subulata . . . 439

Queletia • . . . 466

mirabilis . . . 466

RADULUNI . . . . $3 S S$

corallinum . . . $3 \mathrm{~S}_{9}$

deglubens . . . 3 S9

epileuctm . . . 359

fagineum . . . 389

orbiculare . . . $3^{S 9}$

pendulum . . . $3 S S$

quercinum . . . 359

tomentosum . . . $3{ }_{3} \mathrm{Sa}$

RHIzOPOGON . . . . 49 I

luteolus. . . . 492

rubescens . . . $49 \mathrm{I}$

Russula $\quad \cdot \quad \cdot \quad \cdot \quad \cdot \quad 279$

adusta . . . . 2 SI

aruginea . . . 290

albonigra . . . $2 \$ \mathbf{I}$

alutacea. . . . 292

armeniaca . . . 292

aurata . . . $29 \mathrm{I}$

azurea . . . $2 S_{7}$

Barle . . . . 292

chameleontina . . 293

chloroides . . . $2 \$ 2$

citrina . . . . 290

cœrulea. . . . $2 \mathrm{~S}_{4}$

consobrina . . $2 S S$

cutefracta . . . $2 S_{5}$

cyanoxantha . . . $2 \mathrm{~S}_{7}$

decolorans
Russula (continued)

PAGE

delica . . . $2 \mathrm{~S}_{2}$

densifolia . . . $2 \mathrm{~S}_{\mathrm{I}}$

depallens . . . $2 S_{4}$

drimeia . . . $\quad 28_{4}$

Duportii . . . 286

elegans . . . . $28 S$

elephantina . . . $2 S 2$

emetica . . . . $2 S_{9}$

expallens . . . $2 \mathrm{~S} 9$

fellea . . . . $2 S S$

fingibilis . . . 289

fotens. . . . $2 S S$

fragilis . . . 290

furcata . . . . $2 S_{3}$

granulosa . . . 290

heterophylla . . . $2 \mathrm{2S}_{7}$

integra . . . . $29 \mathrm{I}$

lactea . . . . $2 S_{5}$

lepida . . . . $2 S_{5}$

lilacea . . . . 287

Linnei . . . 286

lutea . . . . 293

lu victa . . . 289

macula a . . $2 S_{j}$

mitis . . . $2 S_{7}$

mistelina . . . $2 \mathrm{~S}_{2}$

nauseosa . . . 293

nigricans . . . $2 S 1$

nitida . . . . 292

ochracea . . 293

ochroleuca . . 290

olivacer . . 286

olivascens . . $\quad 2 S_{3}$

pectinata . . . 290

puellaris . . . 292

fulchralis . . . 292

punctata . . . $29 \mathrm{I}$

purpurea . . . $2 S_{4}$

Gueletii. . . . $2 \mathrm{~S}_{9}$

rosacea. . . . $2 S_{3}$

rubra . . . 285

sanguinea . . . $2 \mathrm{~S}_{3}$

sardonia . . . $2 \mathrm{~S}_{4}$

semicrema . . $2 \mathrm{~S}_{2}$

serotina. . . . 256

subfotens . . . $2 S S$

vesca . . . . 286

veternosa . . . $29 \mathbf{I}$ 


\begin{tabular}{|c|c|c|c|}
\hline \multicolumn{4}{|l|}{ Russula (centinued) } \\
\hline virescens & • & . & $2 S_{5}$ \\
\hline virginea. & . & . & 284 \\
\hline vitellina. & . & . & 293 \\
\hline xerampelina & . & . & 286 \\
\hline
\end{tabular}

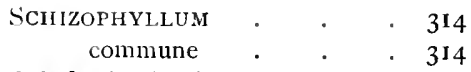

Schulatria Eyrei . . . $\quad 27$ grangei. . . . 27 lycoperdoides. . . 27

Scleroderma $\quad . \quad$. 479 Bovista . . . 480 cepa . . . . 480 cervinum . . . $48 \mathrm{O}$ Geaster . . . 480 sfadicenm . . . 480 verrucosum . . . 480 vulgare. $\quad . \quad$. 479

SCLERODERMACEA . . 478

Sistotrema . . . . $38_{5}$

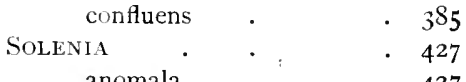

fasciculata $\cdot \cdot 427$

maxima . . $\quad 427$

Sparassis . . . $\quad 428$

crispa . . . . 428

laminosa . . . 429

Spherobolus . . . 484

dentatus . . . 485

stellatus . . . $4{ }_{5}$

terrestris . . . 486

Stereum . . . . 403

acerinum . . . 406

conchatum . . . 405

disciforme . . . 409

frustulosum . . . 406

hirsutum . . . 404

ochroleucum . . . 405

Pini . . . . 406

purpureum . . . 405

quercinum . . . 406

rufohispidum. . . 408

rufum . . . . 406

rugosum . . . 405

sanguinolentum . . 405

spadicem . . . 405
STEREum (continued)

PAGE

(

Strobilomyces . . . $33 \mathbf{I}$

stratosum . . . 406

vorticosum . . . 406

STROPHARIA . . . . I75

æruginosa . . . 176

albocyanea . . . 176

caput-medusæ . . 178

coronilla . . . 177

hypsipus . . . I 79

inuncta. . . . 176

Jerdonii . . . $\quad$ I 79

luteonitens . . . 177

melasperma . . . 177

merdaria . . . 178

obturata . . . 177

Percevalii . . . $\mathbf{I} 77$

scrobinacea . . 178

semiglobata . . . 178

spintrigera . . . $\mathbf{I} 79$

squamosa . . , 177

squamulosa . . . 176

stercoraria . . . 178

ventricosa . . . 178

Worthingtonii . . 177

TAlinia . $\quad$. $\quad . \quad 253$

Telamonia $. \quad . \quad . \quad .234$

Thelebolus terrestris . • • 486

TIILLEPHORA . . . . 398

amorpha . . 410

anthocephala. . 400

atra . . . . 401

biennis . . . . 402

byssoides . . . 413

casia . . . . 403

caryophyllea . . . 400

clavularis . . . $40 \mathrm{I}$

cristata . . . . 402

crustacea . . . 402

disciformis . . . 409

fastidiosa . . . 402

intybacea . . . $40 \mathbf{I}$

laciniata . . . 40I

mollissima . . . 402

multizonata . . . 400

pallida . . . 400

palmata . . . $40 \mathrm{I}$ 


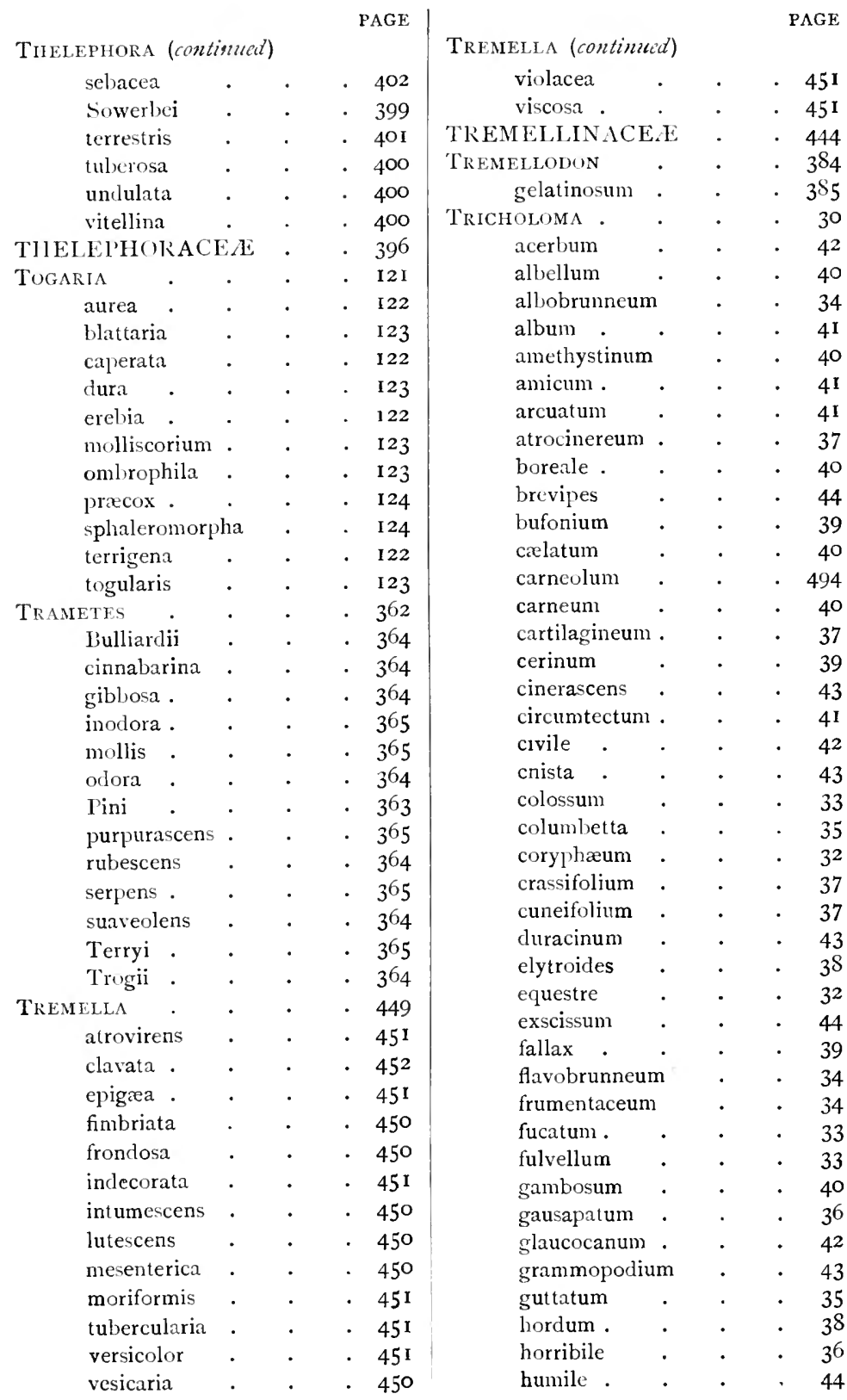




\section{Tricioloma (continuta)}

imbricatum . . . 35

immundum . . . 35

inamonum . . . 39

inodermum . . . $3^{6}$

ionides . . . . 39

lascivum . $\quad . \quad 39$

leucocephalum . . 42

lixivium. . . . 44

loricatum . . . 37

luridum . . . . 35

macrorhizum . . . 36

melaleucum . . . 43

militare . . . . 42

murinaceum . . . 38

nictitans . . . 33

nuclum . . . . 43

onychinum . . $\quad 39$

opicum . . . $\quad{ }_{3} 8$

oreinum. . . . 4 I

pedidum . . . 44

pancolum . . . 43

patulum . . . 4 I

personatum . . . 42

pes-capre . . . 40

pessundatum . . . 34

portentosum . . . 33

puticlum . . . 45

quinquepartitum . $\quad 33$

resplendens . . . 33

Russula . . . 34

rutilans . . . . 34

sxvum . . . . 42

saponaceum . . . 36

scalpturatum . . . 35

Schumacheri . . . 41

sejunctum . . . $\quad 32$

sordidum . . . 44

spermaticum . . . 33

squarrulosum . . . 36

stans . . . . 34

subpulverulentum . . 44

sudum . . . . 37

sulphureum . . . $3 S$

tenuiceps . . . 37

terreum . . . . 36

tigrinum . . . 40

tumidum . . $\quad 38$

ustale . . . . 34

vaccinum . . $\quad 35$
Tricholoma (continued)

PAGE

(1)

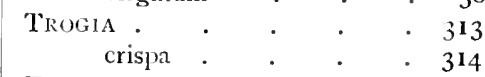

Tubaria $\quad . \quad . \quad . \quad$ I64

autochthona . . . 166

crobulus . . . $\mathbf{1 6 6}$

cupularis . . . 165

embolus . . . I66

furfuracea . . . 165

inquilina . . . $\mathbf{1 6 6}$

muscorum . . . $\mathbf{1 6 6}$

paludosa . . . $\mathbf{1} 66$

pellucida . . . 166

stagnina . . . $\mathbf{I 6 6}$

Tubircularia albida . . . 451

Tulustuma . . . . 466

mammosum . . . 466

Tyfilula $\quad . \quad . \quad .440$

erythropus . . . 440

filiformis . . . 442

gracilis . . . . 442

gracillima . . . $44 \mathrm{I}$

Grevillei . . . 44 I

gyrans . . . . $44 \mathbf{I}$

incarnata . . . $44 \mathrm{I}$

muscicola . . . $44 \mathbf{I}$

phacorrhiza . . . $44 \mathbf{I}$

pusilla . . . . 442

tenuis . . . . 442

translucens . . . 442

UloCOLla $\cdot \quad \cdot \quad \cdot 448$

foliacea . . . . 449

saccharina . . 449

Volvaria $\quad \cdot \quad$. $\quad .97$

bombycina . . . 98

gloiocephala . . . 98

Loveiana . . . $9 \mathrm{~S}$

media . . . . 99

prarvula . . . . 99

speciosa. . . . 98

Taylorii. . . . 98

temperata . . . 98

volvacea $\quad . \quad . \quad 98$

Xerotus . . . 312

degener. . . $3^{1} 3$ 
LONIBUN :

PRINTED BY WILLIAM CLOWES AND SONS, LIMITED,

DUKE STREET, STAMFORD STREET, S.E., AND GREAT WINDMILL STIFET, W. 
SER.1. LEUCOSPORI.

+ Hymenophore distinct from the fieshy stem.

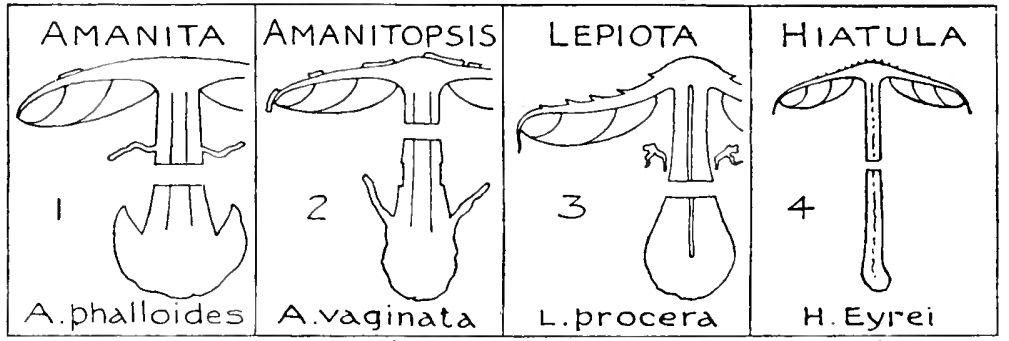

++ Hymenophore confluent and homogeneous with the fleshy stem.

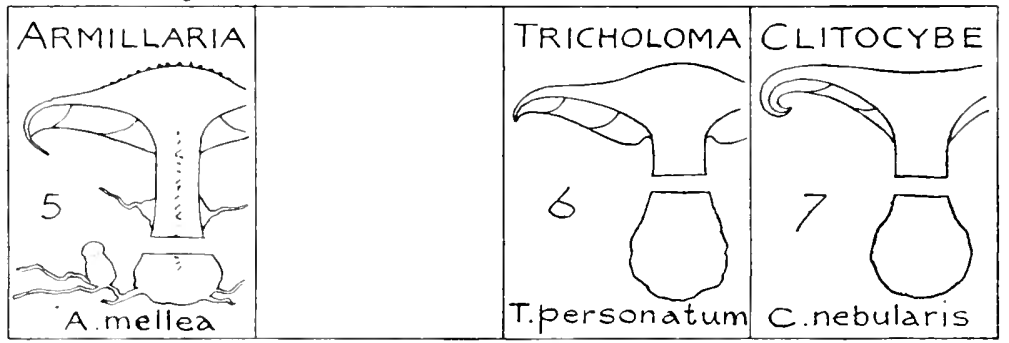

+++ Hymenophore confluent with but heterogeneous from the cartilaginous stem.

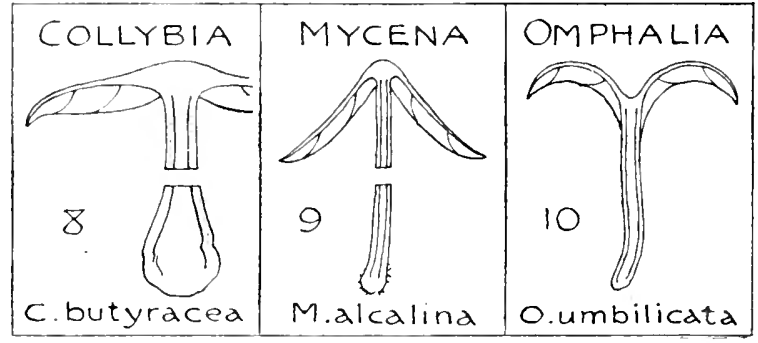

+..+ Hymenophore confluent \& homogeneous with the fleshy excentric stem.

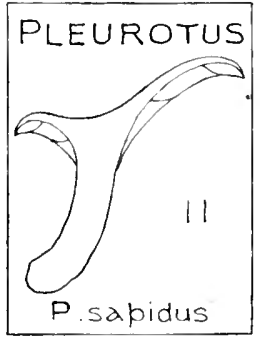



SER. II. HYPORHODII

+ Hymenophore distinct from the fleshy stem

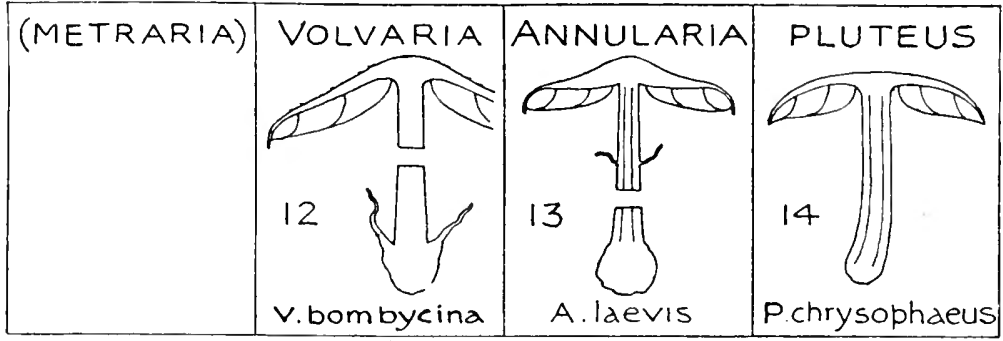

++ Hymenophore confluent and homogeneous with the fleshy stem.

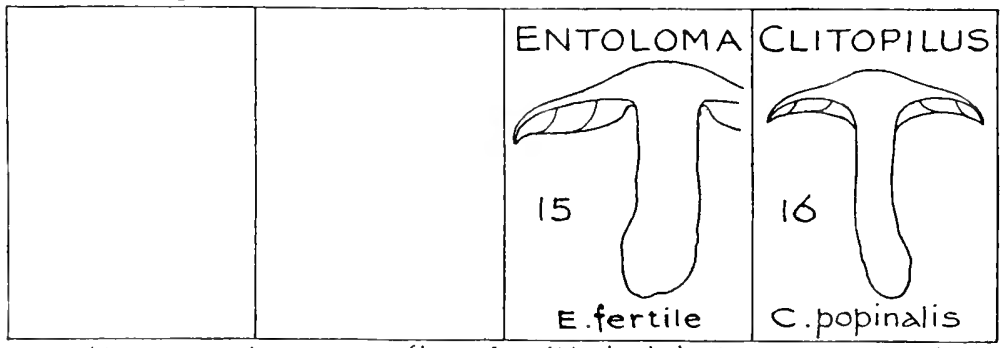

+++ Hymenophore confluent with but heterogeneous from the cartilaginous stem.

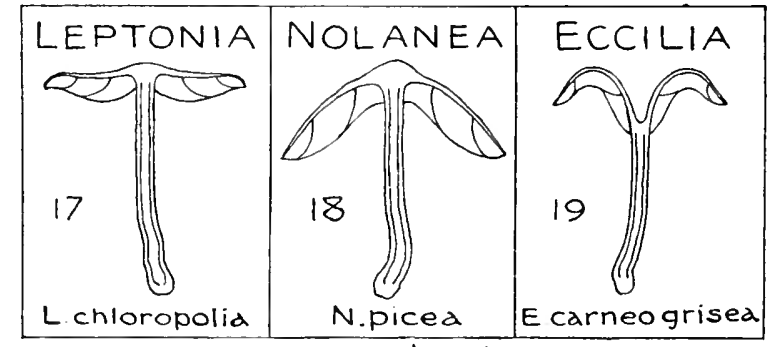

+++ Hymenophore confluent \& homogeneous with the fleshy excentric stem.

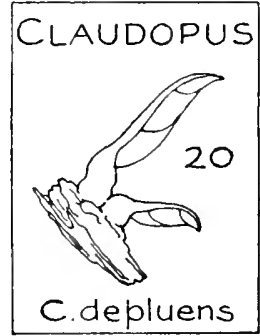



Pe. III

SER. III. TERMINI.

+ Hymenophore distinct from the fleshy stem.

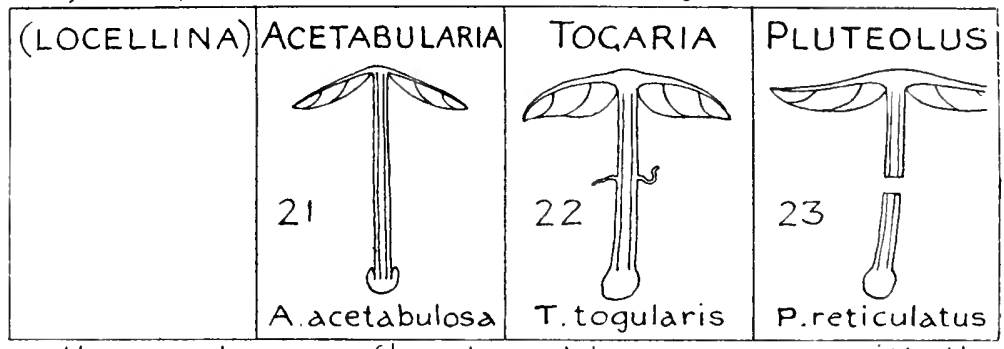

++ Hymenophore confluent and homogeneous with the fleshy stem.

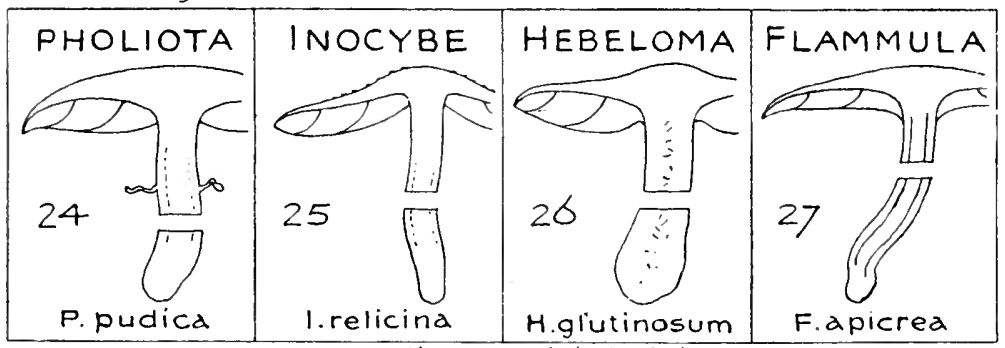

+++ Hymenophore confluent with but heterogeneous from the cartilaginous stem.

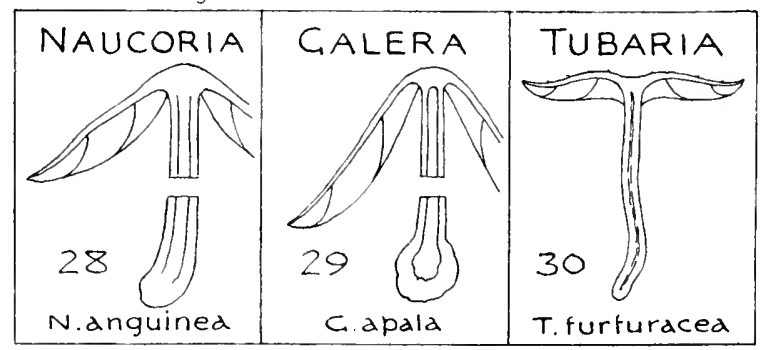

+++ Hymenophore confluent \& homogeneous with the fleshy excentric stern.

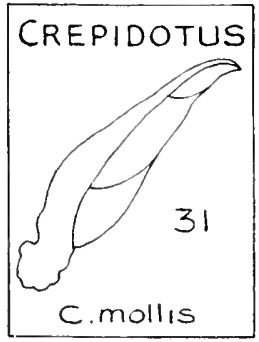



SER IV. PRATELLI.

+ Hymenophore distinct from the fleshy stem.

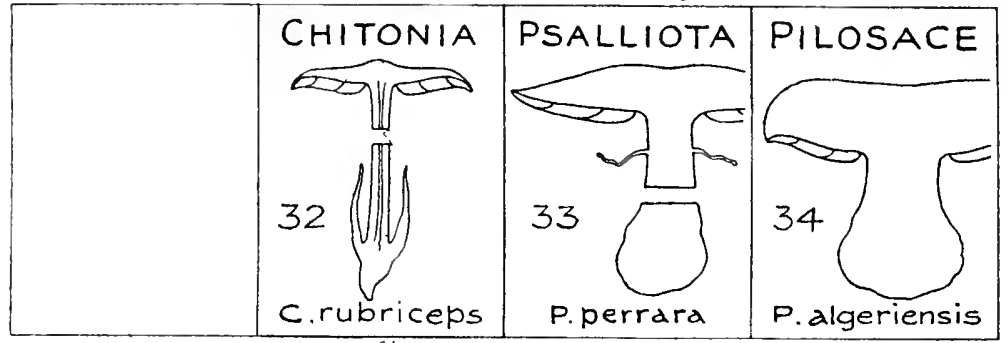

++ Hymenophore confluent and homogeneous with the fleshy stem.
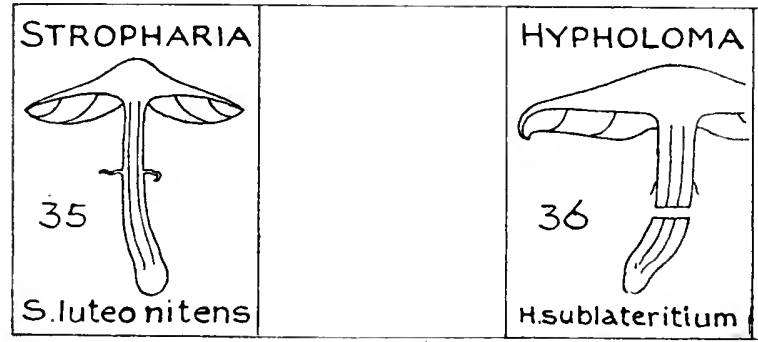

+++ Hymenophore confluent with but heterogeneous from the cartilaginous stem.

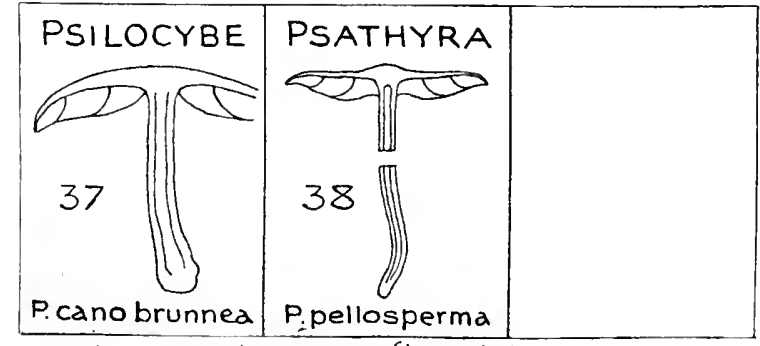

+.+. Hymenophore confluent \& homogeneous with the fleshy excentric stem 



\section{SER. V.COPRINARII.}

+ Hymenophore distinct from the fleshy stem.

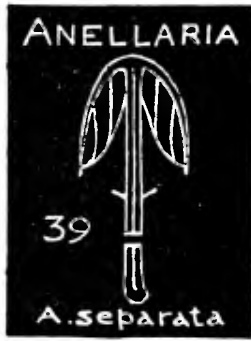

++ Hymenophore confluent and homogeneous with the fleshy stem.

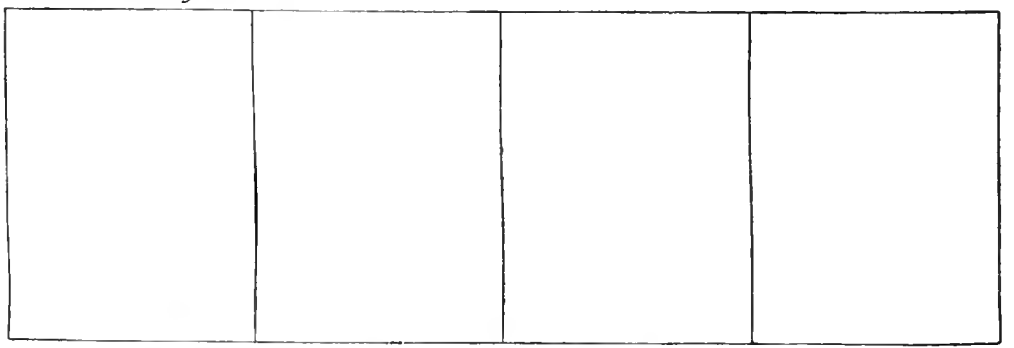

+++ Hymenophore confluent with but heterogeneous from the cartilaginous stem.

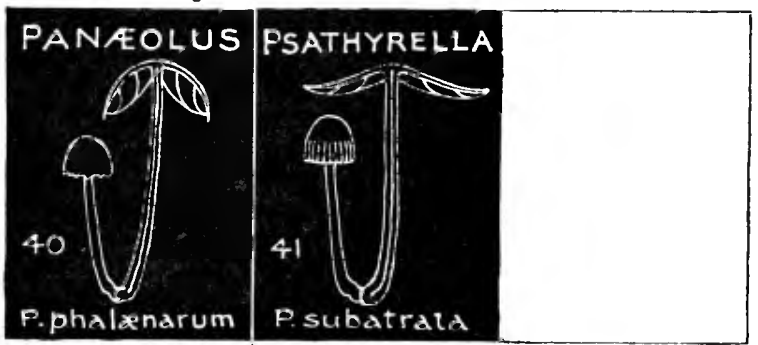

+++ Hymenophore confluent \& homogeneous with the fleshy excentric stem. 



\section{NATURAL HIS'TORY PUBLICATIONS OF THE TRUSTEES OF THE BRITISH MUSEUM.}

The following publications can be purchased throngh the Agency of Messrs. Longmans \& Co., 39, Patemoster Row, London, E.C.; Mr. QUaRITCH, 11, Grafton Street, New Bond Street, $W_{.}$; and Messrs. Dulau \& Co., 37, Soho S'quare, $W . ;$ or at the Natural History Museum, Cromwell Road, London, S.W.

The History of the Collections contained in the Natural History Departments of the British Museum :-

Vol. I. Libraries; Botany ; Geology ; Minerals. Pp. xvii., 442. 1904, Svo. $15 s$.

Vol. II. Separate historical accounts of the several collections included in the Department of Zoology. Pp. 732. 1906, 8vo. 30s.

Catalogue of the Books, Manuscripts, Maps, and Drawings in the British Musenm (Natural History). Compiled by B. B. Woodward, Assistant in charge of the General Library, with some clerical assistance :-

Vol. I. A-D. Pp. viii., 500. 1903, 4to. 20y.

Vol. II. E-K. Pp. 501-1038. 1.904, 4to. 20 s.

Catalogue of the Specimens and Drawings of Mammals Birds, Reptiles, and Fishes of Nepal and Tibet. Presented by B. H. Hodgson, Esq., to the British Museum. 2nd edition. By John Edward Gray. Pp. xii., 90. [With an account of the Collection by Mr. Hodgson.] 1863, 12 mo. $2 s .3 d$.

Catalogue of the Mammalia and Birds of New Guinea in tho Collection of the British Musemn. [With list of Species of New Guinea Birds, and those of the neighbouring Localities.] By John Edward Gray, Ph.D., F.R.S., and George Robert Gray, F.L.S., \&c. Pp. 63. Woodcuts. 1859, Svo. 1s. $6 d$. 
Report on the Zoological Collections made in the IndoPacific Ocean luring the voyage of H.M.S. "Alert," 1881-2. Pp. xxv., 684. 54 Plates. 18\$4, 8vo. 1l. 10 s.

\begin{tabular}{|c|c|c|c|}
\hline Summary of the & Voyage... & & By Dr. R. W. Coppinger. \\
\hline Manmalia ... & $\ldots \quad \ldots$ & $\ldots$ & ... " o. Thomas. \\
\hline Aves... $\quad \ldots$ & $\ldots$ & $\ldots$ & ... , R. B. Sharpe. \\
\hline Reptilia, Batrach & ia, Pisces & $\ldots$ & $"$ A. Ginther. \\
\hline Mollusca $\ldots$ & $\ldots \quad \ldots$ & ... & , E. A. Smith. \\
\hline Echinodermata & $\cdots$ & $\ldots$ & "F. J. Bell. \\
\hline Crustacea ... & $\ldots$ & $\ldots$ & " F. J. Miers. \\
\hline Coleoptera ... & $\ldots$ & ... & "C. O. Waterhouse. \\
\hline Lepidoptera & $\ldots \quad \ldots$ & ... & "A. G. Butler. \\
\hline Alcyonaria and $S$ & Spongiida & $\ldots$ & „S. O. Ridley. \\
\hline
\end{tabular}

Report on the Collections of Natural History made in the Antarctic Regions during the Voyage of the "Southern Cross." Pp. ix., 344. 53 Plates. 1902, Royal 8vo. 22l.

Mammalia $\ldots \quad \ldots \quad \ldots$ By Capt. G. E. H. Barrett-Hamilton.

Notes on Antaretic Seals ... , E. A. Wilson, M.B.

Extracts from the "Diary" ! of the late Nicolai Hanson. $f$

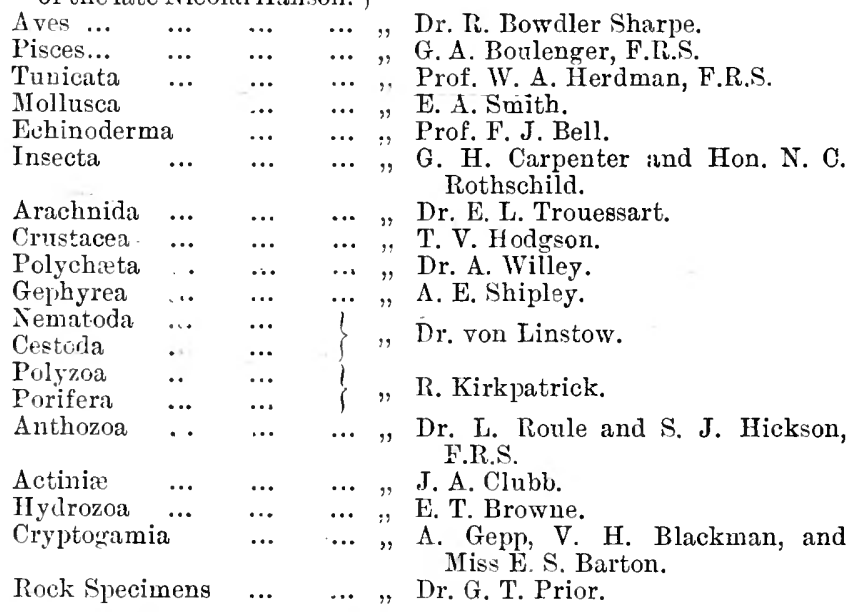

National Antarctic Expedition, 1901-1904. [Discovery Report.] Natural History :-

Vol.1. Geology (Field-Geology: Petrography). Pp. xii., 160 : 10 plates, 72 text-figures, 2 maps. [With Index.] 1907, 4to. 1l. 10s.

Field-Geology $\quad$... ... By II. T. Ferrar, MI.A., F.G.S.

Rock-Specimens $\ldots . \quad \ldots$, G. T. Prior, M.A., D.Sc., F.G.S.

Vol. II. Zoology (Vertebrata : Mollusca : Crustacea). Pp. xiv., 355 : 33 plates (17 coloured), 146 text-figures,

1 map. 1907, 4to. 37.

Miammalia (pp. $69: 5$ p's. [3 col.], 36 figs.).

Aves (1.j). $121: 13$ pls. col., 46 figs.)

Anatomy of Penguins (pp. $24: 1 \mathrm{pl}$., .. W. P. Pycraft. 8 figs.). 


\section{National Antarctic Expedition, 1901-1901-continued.} Vol. II-continued.

Fishes (pp. $5 ; 2$ pls.) $\quad \ldots \quad \ldots \quad \ldots \quad$ By G.A. Boulenger, F.R.S. Cephalodisens (pp. $67: \ddot{7}$ pls. $[\ddot{1}$ col. $]$, "W. G. Ri lewood, D.Sc. 17 figs.).

Mollusca. I.-Cephalopoda (pp. 2: "W. E. Hoyle, D.Se. 1 fig.).

$" \quad$ II.-Gastropoda $\left\{\begin{array}{c}(\text { pp. 12, I : } \\ 2 \text { pls. })\end{array}\right\}$, E. A. Smith, I.S.O.

" IV.-Nudibranchiata (p. 28: "Sir Chas. Eliot,

"1 pl., 30 figs.). "K.C.M.G., LL.D.

, V.-Lamellibranchiata (pp.7:)

Blichiopoda (pp. $2: 4$ figs.) $\quad \ldots \quad \ldots\}$ 1 pl.). $\left.\begin{array}{cccc}\text { Crusticea I.-Decapoda (pp. 7) } & \ldots & \ldots \\ , & \text { II.-Cumacea (pp. 6 6: } & 1 & \text { pl., }\end{array}\right\}$, W. T. Calman, D.Sc.

Tol. III. Zoology and Botany (Invertebrata: Marine Algæ, Musci). Pp. vi., 273: 51 plates, 8 text-figures, and 1 chart. 1907 , 4to. 2l. $10 \mathrm{~s}$.

On Collecting in Antarctic Seas (pp.10) By T. V. Ifodgson, F.L.S.

Mollusea. VI.-F'teropoda (pp. 15: "Sir Charles Eliot, 2 pls.).

Crustacea. III.-Amphipoda (pp. 39 : , A. O. Walker, F.L.S. 13 pls.).

IV.-Leptostraca (lp. 2: "Dr. J. Thiele. 2 figs.)

V.-Ostracoda (pp.9:3 pls.) "Prof. G. S. Brady,

VI.-Cirripedia (pp. 4: 1 pl.) " Prof. A. Gruvel.

Pyenogonida (pp. 72: 10 pls.) ... $\quad \ldots \quad$ ". T. V. Hodgson, F.L.S.

Aeari (pp. $6: 1$ pl.) $\quad \ldots \quad \ldots \quad \ldots \quad$,... Dr. E. L. Trouessart.

Chrotognatha (pp. $6: 1$ chart) $\ldots \quad \ldots \quad$ ".. "Dr.G.H.Fowler, F.L.S.

Nematoda (pp. $4: 1$ pl.) $\quad \ldots \quad$... $\quad$, Dr. O. von Linstow.

Cestoda (pp. $6: 1$ pl.) $\quad \ldots \quad \ldots \quad \ldots \quad \ldots \quad$ ", A. E. Shipley, F.R.S.

Coelentera. I-Alcyonaria (pp. 15: ,. Prof. S. J. Hiekson, 2 pls.). F.R.S.

II.-Hydroid Zoophytes , Prof. S. J. Hickson, (pp. $34: 4$ pls.).

III.-Tentacles of a Siphonophore (pp. $3: 5$ figs.).

Porifera. I.-Hexactinellida (pp. 2\%: ; pls.).

Marine Alga. I.-Phrophycer and Floridea (p). 15: 4 pls.).

$" \quad$ "II.-Corallinaceie (1p. 2 : , M. Foslie.

Musci (pp. $6: 2$ pls.) $\quad \ldots \quad$... $\quad \ldots \quad$, ,J. Cardot. F.R.S., and F. H. Gravely.

"Dr. J. Rennie.

, R. Kirkpatrick.

A. Gepp and Mrs. E. S. Gepp.

ol. IV. \%oology (various Invertebrata). Pp. iv., 281 : 65 plates, 1 text-figure. 1908,4 to. $12.15 \mathrm{~s}$.

Mullusea. VII.-Solenogastres (pp. 13 : By Dr. H. F. Nierstrasz. 2 pls.).

Insecta.-Aptera (pp. 5: 1 pl.)

G. HI. Carpenter, B.Se.,

M.R.I.A.

Crustacea. VII.-Sehizopolla (pp. 42: "W.M.Tattersall, M.Sc. 8 pls.).

VIII.-Copepoda (pp. 44: "R. Norris Wolfenden,

9

7 pls.).

M.D. 
National Antarctic Expedition, 1901-1904-continued. Vol. IV-continued.

Echinoderma (pp. 16 : 5 pls.) ... $\quad$... By F. Jeffrey Bell, M.A.

Echinoderm Larva (pp. 9: 1 pl.) $\quad$... $\quad$ E. W. McBride, M.A., F.R.S., and J. C. Simpson, B.Sc.

Myzostomidæ (pp. $26: 1$ pl. and 1 figure) "Dr. Rudolf Ritter von Stummer-Traunfels.

Sipunculoidea $(p p .6) \quad \ldots \quad \ldots \quad \ldots \quad$, W. F. Lanchester, M.A. Coelentera. IV.—Actiniæ (pp. $1 \dddot{2}: 3$ pls.) " "J. A. Clubb, M.Sc.

Porifera. II.-Tetraxonida (pp. 56: "R. Kirkpatrick. 19 pls.).

III.-Calcarea (pp.52: 12 pls.) „, C. F. Jenkin, B.A.

A Monograph of Christmas Island (Indian Ocean) : Physical Features and Geology by C. W. Andrews, B.A., B.Sc., F.G.S., with descriptions of the Fauna and Flora by numerous contributors. Pp. xv.,337: 22 plates (7 coloured), a map, and 27 illustrations in text. [With Index.] 1900, 8vo. $20 s$.

First Report on Economic Zoology. By Fred. V. Theobald, M.A., \&e. [With an Introduction, containing a Classification of Animals from the point of view of Economic Zoology, by Prof. E. Ray Lankester, LL.D., F.R.S.] Pp. xxxiv., 192. 18 Woodents. 1903, Roj. 8vo. 6s.

Second Report on Economic Zoology. By Fred. V. Theobald, M.A., \&e. Pp. x., 197. 29 Illustrations. 1904, Roy. 8vo. tis.

\section{MAMMALS.}

Catalogue of the Bones of Mammalia in the Collection of the British Museum. By Edward Gerrard. Pp. iv., 296. 1862, 8ro. $5 s$.

Catalogne of Monkeys, Lemurs, and Fruit-eating Bats in the Collection of the British Museum. By Dr. J. E. Graty, F.R.S., \&c. Pp. viii., 137. 21 Woodents. 1870, svo. $4 s$.

Catalogue of Carnivorous, Pachydermatous, and Edentate Mammalia in the British Musenm. By John Edward Gray, F.R.S., \&c. Pp. vii., 398. 47 Woodcuts. 1869. Svo. 6is. 6d.

Catalogue of Seals and Whales in the British Musenm. By John Elward Gray, F.R.S., \&c. 2nd Edition. Pp. vii., 402. 101 Woodcuts. 1866, svo. 8s.

Supplement. By John Edward Gray, F.R.S., \&c., Pp. vi., 103. 11 Woodcuts. 1871, Svo. 2s. $6 d$.

List of the Specimens of Cetacea in the Zoological Depart. ment of the British Museum. By William Henry Flower, LI.D. F.R.S., \&c. [With Systematic and Alphabetical Indexes.] Pp. iv., 36. 1885 8vo. 1s. 6d. 
Catalogue of Ruminant Mammalia (Pecora, Linnæus) in the British Museum. By John Edward Gray, F.R.S., \&c. Pp. viii., 102. 4 Plates. 1872, 8vo. 3s. $6 d$.

Catalogue of the Marsupialia and Monotrematia in the Collection of the British Museum. By Oldfield Thomas. Pp. xiii., 401. 4 Coloured and 24 plain Plates. [With Systematic and Alphabetical Indexes.] 1888, Svo. $11.8 s$.

\section{BIRDS.}

Catalogue of the Birds in the British Museum :-

Vol. VII. Catalogue of the Passeriformes, or Perching Birds, in the Collection of the British Museum. Cichlomorphase: Part IV., containing the concluding portion of the family Timeliidx (Babbling Thrushes). By R. Bowdler Sharpe. Pp.xvi.,698. Woodents and 15 colonred Plates. [With Systematic and Alphabetical Indexes.] 1883, 8vo. $11,6 s$.

Vol. VIII. Catalogue of the Passeriformes, or Perching Birds, in the Collection of the British Museum. Cichlomorphes: Part V., containing the families Paridæ and Laniidæe (Titmice and Shrikes); anrl Certhiomorphe (Creepers and Nuthatches). By Hans Gadow, M.A., Ph.D. Pp. xiii., 386. Woodents and 9 coloured Plates. [With Systematio and Alphabetical Indexes.] 1883, 8ro. $17 \mathrm{~s}$

Vol. X. Catalogne of the Passeriformes, or Perching Birds, in the Collection of the British Musem. Fringilliformes: Part I., containing the fanilies Dicæidæ, Hirmdinidæ, Ampelidæ, Mniotiltidæ, and Motacillidæ. By R. Bowdler Sharpe. Pp. xiii., 68\%. Woodcnts and 12 coloured Plates. [With Systematic and $\mathrm{Al}$ phabetical Indexes.] 1855, 8vo. 11.2s.

Vol. X1. Catalogue of the Passeriformes, or Perehing Birds, in the Collection of the British Musenm. Fringilliformes: Part II., containing the families Cœrebidæ, Tanagridæ, and Icteridæ. By Philip J utley Sclater, M.A., F.R.S. Pp. xvii., 431. Woolcuts and 18 coloured Plates. [With Systematic and Alphalbetical [ndexes.] 1886,8 vo. 1 l.

Vol. XII. Catalogue of the Passeriformes, or Percling Birds, in the Collection of the British Muselin. Pringilliformes: Part III., containing the family Fringillidæ. By R. Bowdler Sharpe. Pp. xv., s7i. Woodcuts and 16 coloured Plates. [With Systematic and Alphabetical Inclexes.] 1888, svo. 17. Ss.

Vol. XIII. Catalogue of the Passeriformes, or Perching Birds, in the Collection of the British Museum. Sturniformes, containing the families Artamidr, 
Catalogue of the Birds in the British Museum-continued. Siturnidæ, Ploceidæ, and Alaudidæ. Also the families Atrichiidæ and Menuridæ. By R. Bowdler Sharpe. Pp. xvi., 701. Woodcuts and 15 coloured Plates. [With Systematic and Alphabetical Indexes.] 1890, 8vo. 17. 8 s.

Vol. XIV. Catalogue of the Pdsseriformes, or Perching Birds, in the Collection of the British Museum. Oligomyode, or the families Tyrannid?, Oxyrhamphidæ, Pipridæ, Cotingidæ, Phytotomidæ, Philepittidæ, 1'ittidæ, Xenicidæ, and Eurylæmidæ. By Philip Jutley Sclater, M.A., F.R.S. Pp. xix., 494. Woodents and 26 coloured Plates. [With Systematic and Alphabetical Indexes.] 1888, svo. 1l. 4s.

Vol. XV. Catalogue of the Passeriformes, or Perching Birds, in the Collection of the British Museum. Tracheophonce, or the families Dendrocolaptidæ, Formicariidæ, Conopophagidæ, and Pteroptochidæ. By Philip Lutles Sclater, M.A., F.R.S. Pp. xvii., 371. Woodcuts and 20 colonred Plates. [With Systematic and Alphabetical Indexes.] 1890, 8vo. $1 l$.

Vol. XVI. Catalogue of the Picariæ in the Collection of the British Museun. Unupe and Trockili, by Osbert Salvin. Coracice, of the families Cypselidæ, Caprimulgide, Podargidæ, and Steatornithidæ, by Ernst Hartert. Pp. xvi., 703. Woodcuts and 14 coloured Plates. [With Systematic and Alphabetical Indexes.] $1892,8 \mathrm{vo}, 17.16 s$.

Vol. XVII. Catalogue of the Picariæ in the Collection of the British Museum. Coracice (contin.) and Halcyomes, with the families Leptosomatidw, Coraciidr, Meropidæ, Alcedinidæ, Momotidæ, Totidæ and Coliidæ, by R. Bowdler Sharpe. Bucerotes and Trogones, by W. R. Ogilvie Grant. Pp. xi., 522. Woodcuts and 17 coloured Plates. [With Systematic and Alphabetical Indexes.] 1892, Svo. 1l. 10s.

Vol. XVIII. Catalogue of the Picaria in the Collection of the British Musenm. Scansores, containing the fanily Picida. By Edward Hargitt. Pp. xv., 597. Woodents and 15 coloured Plates. [With Systematic and $A l_{1}$;habetical Indexes.] 1890, 8vo. 1l. $6 \mathrm{~s}$.

Vol. XIX. Catalogne of the Picaria in the Collection of the British Museum. Scansores and Coccyges: containing the families Rhanphastidæ, Galbulidæ, and Bucconidie, by P. L. Sclater'; and the families Indicatoridæ, Capitonidæ, Cuculidæ, and Musophagidæ, by G. F. Shelley. Pp. xii., 484: 13 coloured Plates. [With Systematic and Alphabetical Indexes.] 1891, Svo. 1l. 5s. 
Catalogne of the Birds in the British Musenm-continued.

Vol. XX. Catalogue of the Psittaci, or Parrots, in the Collection of the British Musenm. By T. Salvalori. Pp. xvii., 658. Woodcuts and 18 coloniel Plates. [With Systematic and Alphabetical Indexes.] 1891. S̀vo. 1l. $10 \mathrm{~s}$.

Vol. XXI. Catalogue of the Columbre, or Pigeons, in the Collection of the British Museum. By T. Salvadorf Pp. xvii., 676. 15 coloured Plates. [With Systematic and Alphabetical Indexes.] 1893, Svo. 1l.10s.

Vol. XXII. Catalogue of the Game Birds (Pterocletes, Gallina, Opisihocomi, Heminodii) in the Collection of the British Musenm. By W. R. Ogilvie Grant. Pp. xvi., 585. E colomred Plates. [With Systematic and Alphabetical Indexes.] 1893, 870.1l.6s.

Vol. XXIII. Catalogue of the Fulicarise (Rallidie and Heliornithidæ) and Alectorides (Aramidæe, Hurypygidæ, Mesitidæ, Rhinochetidæ, Gruidæ, Psophiidre, and Otidide) in the Collection of the British Museum. By R. Bowdler Sharpe. Pp. xiii., 353. 9 coloured Plates. [With Systematic and Alplabetical Indexes.] 1894,8 vo. $20 s$.

Vol. XXIV. Catalogne of the Limicolre in the Collection of the British Museum. By R. Bowdler Sharpe. Pp.xii., 794. Woodents and 7 coloured Plates. [Wjth Systematic and Alphabetical Indexes.] 1896, 8vo. 1l. $5 s$.

Vol. XXV. Catalogue of the Gaviæ and Tubinares in the Collection of the British Ifusenm. Gavix ('Tern, Gulls, and Skuas), by Howard Saunders. Tubinares (Petrels and Albatrosses), by Osbert Salvin. Pp. xv., 475. Woodcuts and 8 coloured Plates. [With Systematic and Alphabetical Indexes.] 1896, 8vo. 1l. 1s.

Vol. XXVI. Catalogue of the Platalea, IIerolions, Steganopoles, Pygopodes, Alcæ, and Imponnes in the Collection of the British Museum. Platalex (lbises and Spoonbills) and Herodiones (Herons and Storits), by R. Bowller Sharpe. Steganopodes (Cormorants, Gamnets, Frigate-birds, Tropic-birds, and Pelicans), Pygopodes (Divers and Grebes), Alcæ (Auks), and Impennes (Penguins), by W. R. Ogilviu-Grant. Pp. xvii., 687. Woodcuts and 14 colonred Plates. [With Systematic and Aiphabetical Indexes.] 1898, 3vo. 1l.5s.

Vol. XXVII. Catalogue of the Chenomorphix (Palamedeæ, Phoenicopteri, Anseres), Crypturi, and Ratitæ in the Collection of the British Museum. By T. Salvadori. Pp. xv., 636. 19 coloured Plates. [With Systematic and Alphabetical Indexes.] 1895, Svo. 1l. $12 s$. 
A Hand-list of the Genera and Species of Birds. [Nomenclator Avium tum Fossilium tum Viventium.] By R. Bowdler Sharpe, LL.D. :-

Vol. I. Pp. xxi., 303. [With Systematic Index.] 1899, 8vo. $10 s$.

Vol. II. Pp. xv., 312. [With Systematic Index, and an Alphabetical Index to Vols. I. and II.] 1900, 8vo. $10 s$.

Tol. III. Pp. xii., 367. [With Systematic and Alphabetical Indexes.] 1901, 8vo. 10s.

Vol. IV. Pp. xii., 391. [With Systematic and Alphabetical Indexes.] 1903, 8vo. 10 s.

List of the Specimens of Birds in the Collection of the British Museum. By George Robert Gray :-

Part III., Sections III. and IV. Capitonidæ and Picidæ. Pp. 137. [With Index.] 1868, 12mo. 1s. 6d.

Part IV. Columbæ. Pp. 73. [With Index.] 1856, 12mo. 1s. $9 d$.

Part V. Gallinæ. Pp. iv., 120. [With an Alphabetical Index.] 1867, 12mo. 1s. $6 d$.

Catalogue of the Birds of the Tropical Islands of the Pacific Ocean in the Collection of the British Museum. By George Robert Gray, F.L.S., \&c. Pp. 72. [With an Alphabetical Index.] 1859, 8vo. 1s. 6d.

Catalogue of the Collection of Birds' Eggs in the British Museum (Natural History) :-

Vol. I. Ratitæ. Carinatæ (Tinamiformes-Lariformes). By Eugene W. Oates. Pp. xxiii., 252. 18 Coloured Plates. [With Systematic and Alphabetical Indexes.] 1901, 8vo. 30s.

Vol. II. Carinatæ (Charadriiformes-Strigiformes). By Eugene W. Oates. Pp. xx., 400. 15 Coloured Plates. [With Systematic and Alphabetical Indexes.] 1902, svo. 30 s.

Vol. III. Carinatæ (Psittaciformes - Passeriformes). By Lugene W. Oates and Capt. Savile G. Reid. Pp. xxiii., 319. 10 Coloured Plates. [With Systematic and Alpliabetical Indexes.] 1903, 8vo. 25s.

Vol. IV. Carinatæ (Passeriformes continued). By Eugene W. Oates, assisted by Capt. Savile G. Reid. Pp. xviii., 352. 14 Coloured Plates. [With Syste. matic and Alphabetical Indexes.] 1905, 8vo. 30s. 


\section{REPTILES.}

Catalogue of the Tortoises, Crocodiles, and Amphisbænians in the Collection of the British Musenm. By Dr. J. E. Gray, F.R.S., \&c. Pp. viii., 80. [With an Alphabetical Index.] 1844, 12mo. $1 s$.

Catalogue of Shield Reptiles in the Collection of the British Museum. By John Edward Gray, F.R.S., \&c.:-

Appendix. Pp. 28. 1872, 4to. 2s. 6d.

Part II. Emydosaurians, Rhynchocephalia, and Amphisbænians. Pp. vi., 41. 25 Woodeuts. 1872, 4to. $3 s .6 d$.

Hand-List of the Specimens of Shield Reptiles in the British Museum. By Dr. J. E. Gray, F.R.S., F.L.S., \&c. Pp. iv., 124. [With an Alphabetical Index.] 1873, 8vo. 4s.

Catalogue of the Chelonians, Rhynchocephalians, and Crocodiles in the British Museum (Natural History). New Edition. By George Albert Boulenger. Pp. x., 311. 73 Woodcuts and 6 Plates. [With Systematic and Alphabetical Indexes.] 1889, 8vo. 15s.

Catalogue of the Lizards in the British Museum (Natural History). Second Edition. By George Albert Boulenger :-

Vol. I. Geckonidæ, Eublepharidæ, Uroplatidæ, Pygopodidæ, Agamidæ. Pp. xii., 436. 32 Plates. [With Systematic and Alphabetical Indexes.] 18s5, 8vo.20s.

Vol. II. Iguanidæ, Xenosauridæ, Zonuridæ, Anguidæ Anniellidæ, Helodermatidæ, Varanidæ, Xantusiidæ Teiidæ, Amphisbænidæ. Pp. xiii., 497. 24 Plates [With Systematic and Alphabetical Indexes.] 1885, 8 vo. 20 s.

Vol. III. Lacertidæ, Gerrhosauridæ, Scincidæ, Anelytropidæ, Dibamidæ, Chamæleontidæ. Pp. xii., 575. 40 Plates. [With a Systematic Index and an Alphabetical Index to the three volumes.] 1887, 8vo. 1l. (is.

Catalogue of the Snakes in the British Museum (Natural History). By George Albert Boulenger, F.R.S., \&c. :-

Vol. I., containing the families Typhlopidæ, Glauconiidæ, Boidæ, Ilysiidæ, Uropeltida, Xenopeltidæ, and Colubridæ aglyphæ (part). Pp. xiii., 448 : 26 Woodcuts and 28 Plates. [With Systematic and Alphabetical Indexes. 7 1893, 8vo. 1l. 1s. 
Catalogue of the Snakes in the British Museum-continued. Vol. II, containing the conclusion of the Colubridæ aglyphæ. Pp. xi., 382: 25 Woodcuts and 20 Plates. [With Systematic and Alphabetical Indexes.] 1894, 8vo. $17 s .6 d$.

Vol. III., containing the Colubridæ (Opisthoglyphr and Proteroglyphæ), Amblycephalidæ, and Viperidæ. Pp. xiv., 727 : 37 Woodeuts and 25 Plates. [With Systematic Index, and Alphabetical Index to the 3 volumes.] 1896, Svo. 1l.6s.

Catalogue of Colubrine Snakes in the Collection of the British Museum. By Dr. Albert Giinther. Pp. xvi., 281. [With Geographic, Systematic, and Alphabetical Indexes.] $1858,12 \mathrm{mo} .4 \mathrm{~s}$.

\section{BATRACHIANS.}

Catalogue of the Batrachia Salientia in the Collection of the British Musenm. By Dr. Albert Günther. Pp. xvi., 160. 12 Plates. [With Systematic, Geographic, and Alphabetical Indexes. 7 1858, 8vo. 6 s.

\section{FISHES.}

Catalogue of the Fishes in the British Museum. Second edition. Vol. I. Catalogue of the Perciform Fishes in the British Museum. Vol. I. Containing the Centrarcbidie, Percidæ, and Serranitre (part). By George Albert Boulenger, F.R.S. Pp.xix.,394. Woodcuts and 15 Plates. [With Systematic and Alphabetical Indexes.] 1895, Svo. $15 s$.

Catalogue of Fish collected and described by Lanrence Theodore Gronow, now in the British Museum. Pp. vii., 196. [With a Systematic Index.] 1854, 12mo. 3s. 6d.

Catalogue of Lophobranchiate Fish in the Collection of the British Museum. By J. J. Kanp, Ph.D., \&c. Pp. iv., 80. 4 Plates. [With an Alphabetical Index.] 1856,12mo. $2 \mathrm{~s}$.

\section{MOLLUSCA.}

Guide to the Systematic Distribution of Mallusca in the British Musenm. Yart I. By John Edward Gray, Ph.D., F.R.S., \&c. Pp. xii., 230. 121 Woodcuts. 1857, 8vo. ว̌s. 
Catalogue of the Collection of Mazatlan Shells in the British Museum, collected by Frederick Reigen. Described by Philip P. Carpenter. Pp. xvi., 552. 1857, 12mo. 8s.

Catalogue of Pulmonata, or Air Breathing Mollusca, in the Collection of the British Museum. Part I. By Dr. Louis Pfeiffer. Pp. iv., 192. Woodcuts. 1855, 12mo. 2s. 6d.

Catalogue of the Auriculidæ, Proserpinidre, and T'runcatellidæe in the Collection of the British Museum. By Dr. Louis Pfeiffer. Pp. iv., 150. Woodcuts. 1857, 12mo. 1s. 9d.

List of the Mollusca in the Collection of the British Museum. By John Edward Gray, Ph.D., E.R.S., \&c. :-

Part II. Olividæ. Pp. 41. 1865, 12mo. 1s.

Catalogue of the Conchifera, or Bivalve Shells, in the Collection of the British Museum. By M. Deshayes:-

Part I. Veneridæ, Cyprinidæ, Glauconomidæ, and Petricoladæ. Pp. iv., 216. 1853, 12mo. 3s.

Part JI. Petricoladæ (concluded); Corbiculadæ. Pp. 217-292. [With an Alphabetical Index to the two parts.] $1854,12 \mathrm{mo} .6 \mathrm{~d}$.

\section{BRACHIOPODA.}

Catalogue of Brachiopoda Ancylopoda or Lamp Shells in the Collection of the British Musemm. [Issued as "Catalogue of the Mollusca, lart IV."] ]p. iv., 128. 25 Woodents. [With an Alphabetical Index.] 1853, 12mo. $3 s$.

$$
\text { POLYZOA. }
$$

Catalogue of Marine Polyzoa in the Collection of the British Nuseum. Part III. Cyclostomata. By George Busk, F.R.S. Pp. viii., 39. 38 Plates. [With a Systematic Index.] 1875, 8vo. $5 \mathrm{~s}$.

\section{CRUSTACEA.}

Catalogue of the Specimens of Amphiportous Crustacea in the Collection of the British Musenm. By C. Spence Bate, F.R.S., \&c. Pp. iv., 399. 58 Plates. [With an Alphabetical Index.] 1862, 8vo. 1l. $5 s$. 


\section{ARACHNIDA.}

Descriptive Catalogue of the Spiders of Burma, based upon the Collection made bs Eugene W. Oates and preserved in the British Museum. By 'J'. 'Thorell. Pp. xxxvi., 406. [With Systematic List and Alphabetical Index.] 1895, Svo. 10 s. $6 d$.

\section{INSECTS.}

\section{Coleopterous Insects.}

Nomenclature of Coleopterous Insects in the Collection of the British Museum :-

Part VII. Longicornia, I. By Adam White. Pp. iv., 174. 4 Plates. 1853, 12mo. 2s. $6 d$.

Part VIII. Longicornia, II. By Adam White. Pp. 237. 6 Plates. $1855,12 \mathrm{mo} .3 s .6 d$.

Illustrations of Typical Specimens of Coleoptera in the Collection of the British Museum. Part I. Ljcidæ. By Charles Owen Waterhouse. Pp. x., 83. 18 Coloured Plates. [With Systematic and Alphabetical Indexes.] 1879, 8vo. 16 s.

Catalogue of the Coleopterous Insects of Madeira in the Collection of the British Museum. By T. Vernon Wollaston, M.A., F.L.S. Pp. xvi., 234 : 1 Plate. [With a Topographical Catalogue and an Alphabetical Index.] 1857, Svo. 3s.

Catalogue of the Coleopterous Insects of the Canaries in the Collection of the British Musenm. By T. Vernon Wollaston, M.A., F.L.S. Pp. xiii., 648. [With Topographical and Alphabetical Indexes.] 1861, Svo. 10s. 6d.

Catalngue of Halticidx in the Collection of the Britısh Museum. By the Rev. Hamlet Clark, M.A., F.L.S. Physapodes and Edipodes. Part I. Pp. xii., 301. Frontispiece and 9 Plates. 1860, 8vo. $7 \mathrm{~s}$.

Catalogue of Hispidæe in the Collection of the British Museum. By Joseph S. Baly, M.E.S., \&c. Part I. Pp. x., 172. 9 Plates. [With an Alphabetical Index.] 1858, Svo. $6 s$.

\section{Hymenopterous Insects.}

Catalogue of Hymenopterous Insects in the Collection of the British Museum. By Frederick Smith. 12mo. :-

Part I. Andrenidæ and Apidæ. Pp. 197. 6 Plates. $1853,2 s .6 d$. 
Catalogue of Hymenopterous Insects in the British Musenm-continued.

Part II. Apide. Pp. 199-465. 6 Plates. [With an Alphabetical Index.] 1854. $6 s$.

Part III. Mutillidæ and Pompilidæ. Pp.206. 6 Plates. 1855. $6 s$.

Part IV. Sphegidæ, Larridæ, and Crabronidæ. Pp. 207497. 6 Plates. [With an Alphabetical Index.] 1856. $6 s$.

Part V. Vespidæ. Pp.147. 6 Plates. [With an Alphabetical Inclex.] 1857. 6s.

Part VI. Formicidae. Pp. 216. 14 Plates. [With an Alphabetical Index.] 1858. 6s.

Part VII. Dorylidæ and Thynnidæ. Pp. 76. 3 Plates. [With an Alphabetical Index.] 1859. 2s.

List of Hymenoptera, with descriptions and figures of the Typical Specimens in the British Museum. Vol. I., Tenthredinidæe and Siricidæ. By W. F. Kirby. Pp. xxviii., 450. 16 Colonred Plates. [With Systematic and Alphabetical Indexes.] 1882, Svo. 1l. $18 s$.

\section{Dipterous Insects.}

A Monograph of the Culicidæ, or Mosquitoes. Mainly compiled from the Collections received at the British MInsenm from various parts of the world in connection with the Investigation into the canse of Malaria conducted by the Colonial Office and the Royal Society. By Fred. V. Theobald, M.A., \&c. :-

Vol. I1I. Pp. xvii., 359: 17 plates, 1 diagram, and 193 illustrations in text. 1903, Svo. 1l. 1s.

Vol. IV. Pp. xix., 639: 16 plates and 297 text-figures. [Witl Index.] 1907, 8vo.1l.12s.6d.

A Monograph of the Tsetse-Flies (Genus Glossina, Westwooll), based on the Collection in the British Museum. By Ernest Edward Austen. With a chapter on Mouth-parts by H. J. Hansen, Phil. Doc. Pp. ix., 319: 9 plates (7 coloured), 16 woodcuts, 1 map. 1903 , Roy. 8vo. 15 s. 


\section{Lepidopterous Insects.}

Catalogue of the Lepidoptera Phalænæ in the British Museum. By Sir George F. Hampson, Bart. :-

Vol. I. Catalogue of the Syntomidæ in the Collection of the British Museum. Pp. xxi., 559 : 285 woodents. [With Systematic and Alphabetical Indexes.] 1895, Svo. $15 s$.

- Atlas of 17 Coloured Plates, 8vo. 15s.

Vol. II. Catalogue of the Arctiadæ (Nolinæ, Lithosiana) in the Collection of the British Museum. Pp. xx., 589 : 411 wondeuts. [With Systematic and Alphabetical Indexes.] 1900, ชัvo. 18s.

—Atlas of 18 Coloured Plates (xviii.-xxxv.), 8vo. $15 \mathrm{~s}$.

Vol. III. Catalogue of the Arctiadæ (Arctianæ) and Agaristidre in the Collection of the British Museum. Pp. xix., 690: 294 woodcuts. [With Systematic and Alphabetical Indexes.] 1901, 8 vo. $15 s$.

Atlas of 19 Coloured Plates (xxxvi-liv.), 8vo. 16 s.

Vol. IV. Catalogue of the Noctuidæ [Agrotinæ]. Pp. xx., $689: 125$ woodcuts. [With Systematic and Alphabetical Indexes.] 1903, 8vo. 15s.

—Atlas of 23 Coloured Plates (lv.-lxxvii), 8vo. $16 s$.

Vol. V. Catalogue of the Noctuidæ [Hadeninæ]. Pp. xvi., 634: 172 woodcuts. [With Systematic and Alphabetical Indexes.] 1905, Svo. 15s.

-Atlas of 18 Coloured Plates (lxxviii.-xcv.), 8vo. $15 \mathrm{~s}$.

Vol. VI. Catalogue of the Noctuidæ [Cucullianæ]. Pp. xiv., 532: 172 woodcuts. [With Systematic and Alphabetical Indexes.] 1906, 8vo. 15s.

- - Atlas of 12 Coloured Plates (xcvi.-crii.), 8vo. $10 s$.

lllustrations of Typical Specimens of Lepidoptera Heterocera in the Collection of the British Museum :-

Part V. By Arthur Gardiner Butler. Pp. xii., 74. 78-100 Coloured Plates. [With a Systematic Index.] 1881, 4to. 2l. 10s.

Part VI. By Arthur Gardiner Butler. Pp. xv., 89. 101-120 Coloured Plates. [With a Systematic Index.] 1886, 4to. $2 l .4 s$.

Part VII. By Arthur Gardiner Butler. Pp. iv., 124. 121-138 Coloured Plates. [With a Systematic List.] 1889, 4to. $2 l$.

Part VIII. The Lepidoptera Heterocera of the Nilgiri District. By George Francis Hampson. Pp. iv., 144. 139-156 Coloured Plates. [With a Systematic List.] 1891, 4to. $2 l$. 
Illustrations of Typical Specimens of Lepidoptere Heterocera in the Collection of the British Museum-continued.

Part IX. The Macrolepidoptera Heterocera of Ceylon. By George Francis Hampson. Pp. v., 182. 157-176 Colonred Plates. [With a General Systematic List of Species collected in, or recorded from, Ceylon.] 189:3 4to. $2 l .2 s$.

Catalogue of the Collection of Palrearctic Butterflies formed by the late John Henry Leech, and presented to the Trustees of the British Museum by his Mother, Mrs. Eliza Leech. By Richard South, F.E.S. Pp. vi., 228. 2 Coloured Plates. With a Portrait and Biographical Memoir of $\mathrm{Mr}$. Leech. 1902, 4to. 17.

Calalogue of Diurnal Lepidoptera described by Fabricins in the Collection of the British Musenm. By Arthur Gardiner Butler, F.L.S., \&c. Pp. iv., 303. 3 Plates. 1869, 8vo. 7 s. 6d.

List of the Specimens of Lepidopterous Insects in the Collection of the British Museum. By Francis Walker. 12mo.:-

Part XXIII. Geometrites. Pp. 756-1020. 1861. 3s. 6d. Part XXV. Pp. 1281-1477. 1862. 3s.

Part XXVI. —— Pp. 1478-1796. [With an Alphabetical Index to Parts XX.-XXVI.] 1862. 4s.6d. Part XXVII. Crambites and Tortricites. Pl. 1-286. 1863. 4s.

Part XXVIII. Tortricites and Tineites. Pp. 287-561. 1863. 4s.

Part XXIX. Tineites. Pp. 562-835. 1864. 4s.

Part XXX. —— Pp. 836-1096. [With an Alphabetical Index to Parts XXVII.-XXX.] 1864. 4s. Part XXXI. Supplement. Pp. 1-321. 1864. 5s. Part XXXII.— Part 2. Pp. 322-706. $1865.5 s$.

Part XXXIII.

Part 3. Pp. 707-1120.

1865. $6 s$.

Part XXXIV.

1865. 5s. $6 d$.

Part XXXV.

[With an Alphabetical Index to Parts XXXI.XXXV.] 1866 . $7 s$.

\section{Neuropterous Insects.}

Catalogne of the Specimens of Neuropterous Insects in the Collection of the British Museum. By Dr. H. Hagen. Part I. Termitina. Pp. $34.1858,12 m$. 6d. 


\section{Orthopterous Insects.}

Catalogue of Orthopterous Insects in the Collection of the British Museum. Part I. Phasmidæ. By John Obadiah Westwood, F.L.S., \&c. Pp. 195. 48 Plates. [With an Alphabetical Index.] 1859, 4to. $3 l$.

Catalogue of the Specimens of Blattariæ in the Collection of the British Museum. By Francis Walker, F.L.S., \&c. Pp. 239. [With an Alphabetical Index.] 1868, 8vo. 5s.6d.

Catalogue of the Specimens of Dermaptera Saltatoria in the Collection of the British Museum. By Francis Walker, F.L.S., \&c. :-

Part 1I. Locustidx (continued). Pp. 225-42? [With an Alphabetical Index.] 1869, 8vo. 4s. $6 d$.

Part III. Locustidæ (continued).-Acrididæ. Pp. 425604. [With an Alphabetical Index.] 1870, 8vo. 4s.

Part IV. Acrididæ (continued). Pp. 605-809. [With an Alphabetical Index.] 1870, 8vo. $6 s$.

Part V. Tettigidæ.-Supplement to the Catalogue of Blattariæ.-Supplement to the Catalogue of Dermaptera Saltatoria (with remarks on the Geographical Distribution of Dermaptera). Pp. 811-850; 43; 116. [With Alphabetical Indexes.] 1870, 8vo. 6s.

Synonymic Catalogue of Orthoptera. By W. F. Kirby :-

Vol. I. Orthoptera Euplexoptera, Cursoria, et Gressoria. (Forficulidæ, Hemimeridæ, Blattidæ, Mantidæ, Phasmidæ.) Pp. x., 501. [With Index.] 1904, 8 vo. $10 s$.

Vol. II. Orthoptera Saltatoria. Part I. (Achetidæ et Phasgonuridæ.) Pp. viii., 562. [With Index.] 1906, 8ro. 15 s.

\section{Hemipterous Insects.}

Catalogue of the Specimens of Heteropterous Hemiptera in the Collection of the British Museum. By Francis Walker, F.L.S., \&c. 8vo. :-

Part IV. Pp. 211. [With Alphabetical Index.] 1871. 6s. Part V. Pp. 202. [With Alphabetical Index.] 1872. $5 s$. Part VI. Pp. 210. [With Alphabetical Index.] 1873. 5s. Part ViI. Pp. 213. [With Alphabetical Index.] 1873. $6 s$. Part VIII. Pp. 220. [With Alphabetical Index.] 1873. 


\section{Homopterous Insects.}

A Synonymic Catalogue of Homoptera. Part I. Cicadidæ. By W. L. Distant. Pp. 207. [Index.] 1906, 8vo. 5s.

\section{VERMES.}

Catalogue of the Species of Entozoa, or Intestinal Worms, contained in the Collection of the British Mnseum. By Dr. Baird. Pp. iv., 132. 2 Plates. [With an Index of the Animals in which the Entozoa mentioned in the Catalogue are found, and an Index of Genera and Species.] 1853, 12mo. 2s.

\section{ANTHOZOA.}

Catalogue of Sea-pens or Pennatulariida in the Collection of the British Museum. By J. E. Gray, F.R.S., \&c. Pp. iv., 40. 2 Woodcuts. 1870, 8vo. 1s. 6 d.

Catalogue of Lithophytes or Stony Corals in the Collection of the British Museum. By J. E. Gray, F.R.S., \&c. Pp. iv., 51. 14 Woodeuts. 1870, 8vo. 3s.

Catalogue of the Madreporarian Corals in the British Museum (Natural History) :-

Vol. I. The Genus Madrepora. By George Brook. Pp. xi., 212. 35 Collotype Plates. [With Systematic and Alphabetical Indexes, and Explanation of the Plates.] 1893, 4to. 1. $4 \mathrm{~s}$.

Vol. II. The Genus Turbinaria ; the Genus Astræopora. By Henry M. Bernard, M.A. Cantab., F.L.S., F.Z.S. Pp. iv., 106. 30 Collotype and 3 Lithographic Plates. [With Index of Generic and Specific Names, and Explanation of the Plates.] 1896, 4to. $18 s$.

Vol. III. The Genus Montipora; the Genus Anacropora. By Henry M. Bernard, M.A., \&c. Pp. rii., 192. 30 Collotype and 4 Lithographic Plates. [With Systematic Index, Index of Generic and Specific Names, and Explanation of the Plates.] 1897, 4to. 17. $4 s$.

Vol. IV. The Family Poritila. I.-The Genus Goniopora. By Hemry II. Bernard, M.A. Pp. viii., 206. 12 Collotype and 4 lithographic l'ates. [With Index of Generic and Specific Names, and Explanation of the Plates.] 1903, 4to. $1 /$.

Vol. V. The Family Poritidæ. II.--The Genus Porites. Part I.-Porites of the Indo-Pacific Region. By Henry M. Bernard, M.A. P1. vi., 303. 35 Plates. [With Index of Generic and Specific Names. and Explanation of the Pates.] 1905, 4to. £1 $15 \mathrm{~s}$. 
Catalogue of the Madreporarian Corals in the British Museum-continued.

Vol. VI. The Family Poritilæ. II.-The Genus Porites. Part II.--Porites of the Atlantic and West Indies, with the Euroyean Fossil Forms. The Genus Goniopora, a supplement to Vol. IV. By Henry M. Bernard, M.A. Pp. vi., 173. 16 Collotype and 1 Lithographic Plates. [With Index of Generic and Specific Names, and Explanation of the Plates.] 1906,4 to. $£ 1$.

\section{BRITISH ANIMALS.}

Catalogue of British Birds in the Collection of the British Museum. By George Robert Gray, F.L.S., F.Z.S., \&c. Pp. xii., 248. [With a List of Species.] 1863, 8vo. 3s.6d.

Catalogue of British Hymenoptera in the Collection of the British Museum. Second edition. Part I. Andrenirle and Apidæ. By Frederick Smith, M.E.S. New Issue. Pp. xi., 236. 11 Plates. [With Systematic and Alphabetical Index.] 1891, Sro. 6s.

Catalogue of British Fossorial Hymenoptera, Formicidx, and Vesplike in the Collection of the British Museum. By Frederick Smith, V.P.E.S. Pp. 236. 6 Plates. [With an Mlphabetical Index.] 1858, 12mo. 6s.

Illustrations of British Blood-sucking Files, with notes by Ernest Edward Austen, Assistant, Department of Zoology British Museum (N.H.). Pp. 74. 34 Coloured Plates. $1 ! 06$, roy. 8vo. £1 5 s.

A Catalogue of the British Non-parasitical Worms in the Collection of the British Musenm. By George Johnston, M.D., Edin., F.R.C.L., Ed., Lu.D., Marischal Coll., Aberdeen, \&c. Pp. 365. Woodcuts and 24 Plates. [With an Alphabetical Index.] 1865, 8vo. $7 \mathrm{~s}$.

Catalogue of the British Hchinoderms in the British Museum (Natural History). By F. Jeffrey Bell, M.A. Pp. xvii., 202. Woodcuts and 16 Plates (2 Coloured). [With Table of Contents, Tables of Distribution, Alphabetical Index, Description of the Plates, \&c.] 1892, Svo. 12s. 6d.

List of the Specimens of British Animals in the Collection of the British Museum; with Synonyma and References to figures. 12mo.:-

Part V. Lepirloptera. By J. F. Stephens. 2nd Fdition. Revised by H. T. Stainton and E. Shepherd. Pp. iv., 22t. 1856 . $1 s .9 d$.

Part VI. Hỵmenoptera. By E. Smith. Pp. 134. 3851. 2s. 
List of the Specimens of British Animais in the Collection of the British Museum-continued.

Part VII. Mollusca, Acephala and Brachiopoda. By Dr. J. E. Gray. Pp. iv., 167. 1851. 3s. 6d.

Part VIII. Fish. By Adam White. Pp. xxiii., 164. (With Index and List of Donors.) 1851. 3s. $6 d$.

Part IX. Eggs of British Birds. By George Robert Gray. Pp. 143. 1852. 2s. 6d.

Part XI. Anoplura, or Parasitic Insects. By H. Denny. Pp. iv., 51. 1852. $1 s$.

Part XII. Lepidoptera (continued). By James F. Stephens. Pp. iv., 54. 1852. 9d.

Part XIII. Nomenclature of Hymenoptera. By Frederick Smith. Pp. iv., 74. 1853. 1s, 4 .

Part XIV. Nomenclature of Neuroptera. By Adam White. Pp. iv., 16. 1853. 6d.

Part XV. Nomenclature of. Diptera, I. By Adam White. Pp. iv., 42. 1853. $1 s$.

Part XVI. Lepidoptera (completed). By H.'T. Stainton. P1. 199. [Witl an lndex.] 1854. is.

\section{PLANTSE.}

Illusirations of Australian Plants conlected in 1770 duriug Captain Cook's Voyage round the World in H.M.S. "Endeavour." By the Right Hon. Sir Joseph Banks, Bart., K.B., P.R.S., and Dr. Daniel Solander, F.R.S. [Being a series of lithographic reproductions of copperplates engraved after paintings by F. P. Nodder, James Miller, J. F. Miller, and John Cleveley.] With Introduction and Determinations by James Britten, F.L.S., Senior Assistant, Department of Botany, British Museum :-

Part I.-101 Plates, with 31 pages of descriptive text. 1900, fol. $£ 15 s$.

Part II.-142 Plates (pls. 101-243), with 41 pages of descriptive text (pp. 35-75). 1901, fol. $\$ 115 s$.

Part III.-77 Plates (pls. $244-318,45 \mathrm{~A}$, and 122), with 26 pages of descriptive text, including Index to the whole work (pp. $77-102)$, and 3 maps. 1905 , fol. £1 58.

Catalogue of the African Plants collected by Dr. Friedrich Welwitsch in 1853-61:-

Vo. I. Dicotyledons. By William Philip Hiern, M.A., F.L.S., \&.c. :-

Part I. [Ranunculaceæ to Rhizophoraceæ.] Pp. xxvi., 336. [With Portrait of Dr. Welwitseh. Introduction, Bibliography, and Index of Genera ] 1896. 8vo. 7 s. $6 d$. 
Catalogue of the African Plants collected by Dr. Friedrich Welwitsch in 1853-61-continued.

Vol. I-continued.

Part II. Combretaceæ to Rubiaceæ. Pp. 337-510. [With Index of Genera.] 1898, 8vo. 4s.

Part III. Dipsaceæ to Scrophulariaceæ. Pp. 511784. [With Index of Genera.] 1898, 8vo. 5 s.

Part IV. Lentibulariaceæ to Ceratophylleæ. Pp. 7851035. [With Index.] 1900, 8vo. 5s.

Vol. II. Monocotyledons, Gymnosperms, and Cryptogams :-

Part I. Monocotyledons and Gymnosperms. By Alfred Barton Rendle, M.A., D.Sc. F.L.S., Assistant, Department of Botany. Pp. 260. [With Index of Genera.] 1899, 8vo. 6s.

Part II. Cryptogamia. Pp. 261-566. [With Table of Errata, and General Index to the whole work.] 1901, 8vo. $6 s$.

\begin{tabular}{|c|c|c|c|c|c|}
\hline \multicolumn{3}{|c|}{ Vascular Cryptogams } & ... & By & William Carruthers, F.R.S. \\
\hline Mosses & $\ldots$ & ... & $\cdots$ & $"$ & Antony Gepp, M.A., F.L.S. \\
\hline Hepatics & $\ldots$ & .. & $\cdots$ & & F. Stephani. \\
\hline Marine $\mathrm{Al}_{\mathrm{g}}$ & & $\ldots$ & $\cdots$ & " & Ethel S. Barton. \\
\hline Freshwate & Algæ & $\ldots$ & $\ldots$ & $\because$ & $\begin{array}{l}\text { W. West, F.L.S., and G. S. } \\
\text { West, B.A. }\end{array}$ \\
\hline Diatomace & & $\cdots$ & $\cdots$ & "g & Thomas Comber, F.L.S. \\
\hline Lichenes & $\ldots$ & ... & ... & & E. A. Wainio. \\
\hline Fung: & ... & ... & $\cdots$ & :" & Annie Lorrain Smith. \\
\hline Mycetozoa & $\ldots$ & $\ldots$ & $\ldots$ & & Arthur Lister, F.R.S, \\
\hline
\end{tabular}

Synopsis of the British Basidiomycetes: a Descriptive Catalogue of the Drawings and Specimens in the Department of Botany, British Museum. By Worthington George Smith, F.L.S. Pp. 531. 5 Plates and 145 Figures in Text. [With Index.] 1908, 8ro. 10s.

A Monograph of Lichens found in Britain : being a Descriptive Catalogue of the Species in the Herbarium of the British Museum. By the Rev. James M. Crombie, M.A., F.L.S., F.G.S., \&c. Part I. Pp. viii., 519: 74 Woodcuts. [With Glossary, Synopsis, Tabuiar Conspectus, and Index.] 1894, 8vo. 16s.

List of British Diatomacer in the Collection of the British Museum. By the Rev. W. Sinith, F.L.S., \&c. Pp. iv., 55. $1859,12 \mathrm{mo} .1 \mathrm{~s}$. 


\section{FOSSILS.}

Catalogue of the Fossil Mammalia in the British Musenm (Natural History). By Richard Lydekker, B.A., F.G.S.:-

Part I. Containing the Orders Primates, Chiroptera, Insectivora, Carnivora, and Rorlentia. Pp. xxx., 268. 33 Woodents. [With Systematic and Alphabetical Indexes.] 1885, 8 vo. $5 s$.

Part II. Containing the Order Ungulata, Suborder Artiodactyla. Pp. xxii., 324. 39 Woodents. [With Systematic and Alphabetical Indexes.] 1885, 8 vo. 6s.

Part III. Containing the Order Ungulata, Suborders Perissodactyla, Toxodontia, Condylarthra, and Amblypoda. Pp.xvi., 186. 30 Woodents. [With Systematic Index, and Alphabetical Index of Genera and Species. inclnding Synonyms.] 1886, 8vo. $4 s$.

Part IV. Containing the Orter Ungulata, Suborter Proboscidea. Pp. xxiv., 2:55. 33 Woodents. [With Systematic Index, and Alphabetical index of Cenera and species, including Synonyms.] 1856, Svo. 5s.

Part V. Containing the Gromp Tillorlontia, the orrers Sirenia, Cetacea, Elentata, Marsupialia, Monotremata, and Supplement. Pl. xxxv., 345. 55 Woodents. [With Systematic Index, and Alphabetical Index of Genera and Species, inclucling Synonyms.] 1887, \$vo. 6 s.

Catalogue of the Fossil Birds in the British Musenm (Natural History). By Richard Lydekker, B.A. Pp. xxvii., 365. 75 Woodents. [With Systematic Index, and Alphabetical Index of Genera and Species, inclnding Synonyms.] 18:1, $8 \nabla 0.10 s .6 d$.

Catalogue of the Fossil Reptilia and Amphibia in the Britisls Musenm (Natural History). By Richard Lydekker, B.A., F.G.S. :-

Part I. Containing the Orders Ornithosanria, Crocodilia, Dinosauria, Squamata, Rhynchocephalia, and Proterosanria. Pp. xxviii., 309. 69 Woodcuts. [With Systematic Index, and Alphabetical Index of Genera. and Species, including Synonyms.] 1888, 8vo. 7s. 6\%.

Part II. Containing the Orders Ichthyopterygia and Sauropterygia. Pp. xxi., 307. 85 Woodcuts. [With Systematic Index, and Alphabetical Index of Generis and Species, including Synonyms.] 1889, 8vo. $7 \mathrm{s.6}$.

Part III. Containing the Order Chelonia. Pp. xviii., 23:9. 5:3 Woodcuts. [With Systematic Inclex, and Alphabetical Index of Genera and Species, including Synonyms.] 1889, 8vo. $7 \mathrm{~s} .6 \mathrm{~d}$. 
Catalogne of the Fossil Reptilia and Amphibia in the British Museum-continued.

Part IV. Containing the Orders Anomodontia, Ecandata, Candata, and Labyrinthodontia; and Supplement. Pp. xxiii., 295. 66 Woodcuts. [With Systematic Index, Alplabetical Indes of Genera and Species, including Synonyms, and AIphabetical Index of Genera and Species to the entire work.] 1890, 8vo. 7s. $6 \mathrm{~d}$.

Catalogue of the Fossil Fishes in the British Musenm (Natural History). By Arthur Smith Woodward, LL.D., F.R.S., F.G.S., \&c. :-

Part I. Containing the Elasmobranchii. Pp. xlvii., 174. 13 Woodcuts and 17 Plates. [With Alphabetical Index, and Systematic Index of Genera and Species.] 1889,8 vo. 21s.

Part II. Containing the Elasmobranchii (Acanthodii), Holocephali, Ichthyodnulites, Ostracodermi, Dipnoi, and Teleostomi (Crossopterygi: and Chondrostean Actinopterygii). Pp. xliv., 567.58 Woodents and 1i) Plates. [With Alphabetical Index, and Systematic index of Genera and Apecies.] 1831, Svo. $21 \mathrm{~s}$.

Part III. Containing the Actinopterraian Teleostomi of the Order's Chondrostei (conclnded), Protospondyli, Aetheospondyli, and Isospondyli (in part). Pp. xlii., 544. 45 Woodents and 18 Plates. [With Alphabetical Index, and Systematic Index of Genera and Species.] 1895, 8vo. 2!s.

Part IV. Containing the Actinopterggian Teleostomi of. the Suborters Isospondyli (in part), Ostariophysi, Apodes, Percesoces, Homibranchii, Acanthopterygii, and Anacanthini. Pp. xxxix., 636. 22 Wondeuts and 19 Plates. [With Alphabetical Index, and Systematic Index of Genera and Species.] 1901, Svo. $21 s$.

A clescriptive Catalogue of the Tertiary Vertebrata of the Fayûm, Fgypt. Based on the Collection of the Egyptian Government in the Geological II usenm, Cairo, and on the Collection in the British Mnsem (Natural History), London. By C. W. Andrews, D.Sc. Pp. xxxvii., 324: 98 Text Fignres and 26 Plates. [With Systematic and Alphabetical Indexes.] 1!06, tto. 11.15s.

Systematic List of the Edwards Collection of British Oligocene and Eocene Mollusea in the British Musenm (Natural History), with references to the type-specimens from similar horizons contained in other collections belonging to the Geological Department of the Museum. By Richard Bullen Newton, F.G.S. Pp. xxviii., 365. [With table of Families and Genera, Bibliography, Correlation-table Appendix, and Alpbabetical Index.] 1891, 8vo. 6 s. 
Catalogue of Tertiary Mollusca in the Department of Geology, British Nusenm (Natural History). Part I. The Australasian Tertiary Mollusca. By George F. Harris, F.G.S., \&c. Pp. xxvi., 407. S Plates. [With Table of Families, Genera, and Sub-Genera, and Index.] 1897, 8vo. 10s.

Catalogue of the Fossil Cephalopoda in the British Museum (Natural History) :-

Part I. Containing part of the Suborder Nautiloidea, consisting of the families Orthoceratidæ, Endoceratidæ, Actinoceratidæ, Gomphoceratidæ, Ascoceratida, Poterioceratidæ, Cyrtoceratidæ, and Supplement. By Arthur H. Foord, F.G.S. Pp. xxxi., 344. 51 Woodents. [With Systematic Index, and Alphabetical Index of Genera and Speeies, including Synonyms.] 1888, Svo. 10s. $6 d$.

Part II. Containing the remainder of the Suborler Nautiloidea, consisting of the families Lituitidx, Trocloceratidæ, Nantilidie, and Supplement. By Arthur H. Foord, F.G.S. Pp. xxviii., 407. 86 Woodcuts. [With Systematic Index, and Alphabetical Index of Genera and Species, including Synonyms.] 1891, 8vo. 15 s.

Part 1II. Containing the Bactritidx, and part of the Suborder Ammonoidea. By Arthur H. Foord, Ph.D., F.G.S., and George Charles Crick, A.R.S.M., F.G.S. Pp. xxxiii., 303. 146 Woodents. [With Systematic Index of Genera and Species, and Alphabetical Index.] 1897, 8vo. 12s. 6d.

List of theTypes and Figured Specimens of Fossil Cephalopoda in the British Muserm (Natural History). By G. C. Crick, F.G.S. Pp. 103. [With Index.] 1898, Svo. 2s. 6d.

A Catalogue of British Fossil Crustacea, with their Synonyma and the Range in Time of each Genus and Order. By Henry Woodward, F.h.s. Pl. xii., ]5\%. [Wiiu an Alphabetical Index.] 1877, 8vo. 5́s.

Vatalogue of the Fossil Bryozoa in the Department of Geology, British Mnsenm (Natural History):-

The Jnrassic Bryozoa. By.J. W. Gregory, D.Sc., T.G.S., F.Z.S. Pp. [viii., 239: 20 Wondents and 11 Plates. [With I.ist of Species and ] Bistribution, Biblinguphy, Index, and Explanation of l'lates.] 18.16, 85o. 16s.

The Cretaceous Bryozoa. Vol. I. By J. W. Aregory, D.Sc., F.G.S., F.Z.S. Pl. xiv., 457 : it Woodeuts and 17 Plates. [With Index and Lxplanation of Plates.] 1899, svo. 16s. 
Catalogue of the Blastoidea in the Geological Department of the British Mnseum (Natural History), with an account of the morphology and systematit position of the gronp, and a revision of the genera and species. By Robert Etheridge, jun., of the Department of Geology, British Museum (Natural History), and H. Herbert Carpenter, D.Sc., F.R.S., F.L.S. (of Eton College). Pp. xv., 322. 20 Plates. [With Preface by Dr. H. Woodward, Table of Contents, General Index, Explanations of the Plates, \&c.] 1886 4to. $25 \mathrm{~s}$.

The Genera and Species of Blastoidea, with a List of the Specimens in the Britisk. Museum (Natural History). By F. A. Bather, M.A., F.G.S., of the Geological Department. Pp. x., 70. 1 Woodcut. 1899, 8vo. 3s.

Catalogue of the Palæozoic Plants in the Department of Geology and Palæontology, British Museum (Natural History). By Robert Kidston, F.G.S. Pp. viii., $28 \%$. [With a list of works quoted, and an Index.] 1886 8 vo. 5 s.

Catalogue of the Mesozoic Plants in the Department of Geology, British Museum (Natural History). By A. C. Seward, M.A., F.R.S., F.G.S., University Lecturer in Botany and Fellow of Emanuel College, Cambridge :-

Part I. The Wealden Flora. Part I. ThallophytaPteridophyta. Pp. xxxviii., 179. 17 Woodcuts and 11 Plates. [With Alphabetical Index, Explanations of the Plates, \&c.] 1894,8 vo. 10s.

Part II. The Wealden Flora. Part II. Gymnosperme. Pp. viii., 259. 9 Woodents and 20 Flates. [With Alphabetical Index, Explanations of the Plates, \&c.] 1895,8 vo. $15 s$.

Part 11I. The Jurassic Flora. Part I. The Yorkshire Coast. Pp. xii., 341. 53 Woodcuts and 21 Plates. [With Alphabetical Index, Explanations of the Plates, \&c.] 1900,8 vo. $20 \mathrm{~s}$.

Part 1V. The Jurassic Flora. Part II. Liassic and Oolitic Fioras of England (excluding the Inferior Oolite Plants of the Yorkshire Coast). Pp. xv., 192. 20 Woodcuts and 13 Plates. [With Alphabetical Index, Explanations of the Plates, \&c.] 1904, Svo. I0s.

Catalogue of the Fossil Plants of the Glossopteris Flora in the Department of Geology, british Museum (Natural History). Being a Monograph of the Permo-carboniferons Flora of India and the Southeru Jiemisphere. By E. A. Newell Arber, M.A., F.L.S., T.G.S. Pp. lxxiv., 255: 51 'Text Figures and $S$ Plates. [With Bibliography and Alphabetical Index.] 1905, 8vo. 12s. 6d. 


\section{GUIDE-BOOKS, ETC.}

A General Guide to the British Ninseum (Natural History), Cromwell Road, London, S.W. 11th Edition. With 58 woodents, 2 plans, 2 views of the building, and an ilinstrated cover. Pp. 125. 1906, Svo. 3d.

Guide to the Specimens illustrating the Races of Mankind (Antliropology), exhibited in the Department of Zoology, British Museum (Natural History). [By R. Lyclekker, F.R.S.] Illustrated by 16 Eigures. Pp.31. 1908, \$vo. 4d.

Guide to the Galleries of Mammals (other than Ungulates) in the Department of Zoology of the British Museum (Natural History). Sth Edition. Pp. 101. 52 Woodents and 4 plans. Index. $190 \dot{0}, 8 v 0.6 d$.

Guide to the Great Game Animals (Ungulata) in the Department of Zoology, British Museum (Natural History). Pp. 93. 53 Text and other figures. With list of Horns, Antlers and 'Tusks, and Index. 1907, 8vo. 1s.

Guide to the Elephants (Recent and Fossil) exhibited in the Department of Geology and Palæontology in the British Museum (Natural History). [By Dr. C. W. Andrews, F.R.S.] Illustrated by $3 \mathrm{I}$ text-figures. Pp. 46. 1908, 8vo. 6id.

Guide to the Specimens of the Horse Family (Equidr) exhibited in the Department of Zoology, British Museum (Natural History). [By R. Lydekker, F.R.S.] Pp. 42. 26 Higures. 1907, 8vo. 1 s.

Guide to the Domesticated Animals (other than Horses) exhibited in the Central and North Halls of the British Museum (Natural History). [By R. Lyctekker, F.R.S.] Illustrated by $2 \pm$ Figures. Pp. 55. [With table of Contents, List of Illustrations, and Index.] 1908, 8vo. 6d.

Guide to the Gallery of Birds in the Department of Zoology of the British Museum (Natural History). [By W. R. Ogilvie Grant.] Pp. iv., 2:8. 24 1'lates, and 7 Illustrations in text. With Index. 1905, roy. svo. 2s. 6d.

Guide to the Gallery of Birds in the Department of Zoology, British Museum (Natural History). Part I. Generd Series. [By W. R. Ogilvie Grant.] Pp. 149. With Index. 1905 , roy. Svo. $6 d$.

Guide to the Nesting Series of British Birts. Being Part II. of the Guile to the Gallery of Birds in the Department of Zoology, British Musem (Natural Il istory). [By W. R. Orgilvits (irant.] I'p. 62. 4 l'lates. Index. 190, roy. Sovo. $t \%$.

Guide to the Gallery of Reptilia and Amphibia in the Department of Zoology of the British Museum (Natural History). [By R. Isydekker, F.R S.] Illustrated by 76 text and other Figures. P'p. iv., 75. [With Table of Contents.] 1906, 8vo. bil. 
Guide to the Gallery of Fishes in the Department of Zoology of the British Mnseum (Natural History). [By Dr. W. G. Ridewood.] Illustrated by $96^{\circ}$ Fignres. Pp. จ., 209. [With Preface by Sir E. Ray Lankester, Table of Classification, and Index.] 1908, 8vo. 1s.

Guide to the exhibited Series of Insects, Zoological Depart. ment (Insect Section), British Musemm (Natural History). By C. O. Waterhouse. Pp. 57:62 text and full-page Illustrations. [With Table of Contents and Index.] 1908, Sro. 1s.

Guide to the Shell and Starfish Galleries (Mollusca, Polyzoa, Brachiopoda, Tunicata, Echinoderma, and Worms). Department of Zoology, British Museum (Natural History), Fifth Edition. Pp. iv., 133. 125 Woodcuts, Plan and Indexes. 1908, 8vo. 6d.

Guide to the Coral Gallery (Protoza, Porifera or Sponges, Hydrozoa, and Anthozoa) in the Department of Zoology, British Musenm (Natural History). Second Edition. Pp. [iv. 8] 73. 90 Illustrations, Plan and Index. 1907, 8vo. 1s.

A Guide to the Fossil Mammals and Birds in the Department of Geology and Paleontology in the British Musenm (Natural History). Sth Edition. [By A. S. Woodward, LL.D., F.R.S.] Pp. xvi., 100. 6 Plates, 88 Woodents. [With List of Illustrations, Table of Stratified Rocks, and Index.] 1904, 8vo. 6d.

A Guide to the Fossil Reptiles, Amphibians, and Fishes in the Department of Geology and Palæontology in the British Museum (Natural History). 8th Elition. [By A. S. Woodward, LL.D., F.P.S.] Pp. xviii., 110. 8 Plates and 116 Text-Figures. [With List of Illustrations, Geological Time-Scale and Index.] 1905, 8vo. 6 $d$.

A Guide to the Fossil Invertebrite Animals in the Department of (Xeology and Paleontology in the British Musenm (Natural History). [By F. A. Bather, D.Sc.] Pp. ix., 182. 7 Plates and 6 Text-Figures. [With List of Illustrations, Geological Time-scale, and Index.] 1907, Svo. 1 s.

List of British Seer-plants and Ferms exhibited in the Deprartment of Botany, British Museum (Natural History). With table of Sequence of Orlers, and Index of Genera. P1. 41. 1907, svo. 4d.

Guide to Sowerby's Molels of British finngi in the Depurtment of Botany, Britisln Museum (Natual IHistory). Second Elition, revised. By Worthington G. Smith, F.L.S. Pp. \$3. 91 Woolcuts. With Table of Diagnostic Character's, Glossary, and Index. 1908, 8vo. $4 d$. 
Guide to the British Mycetozoa exhibited in the Department of Botany, Britisl Mrsenm (Natural History). By Arthur Lister, F.R.S. Second Ellition, reviserl. Pp. 48. 45 Wooleuts. Index. 190\%, svo. 23.

A Guide to the Mineral Gallery of the British Musemm (Natural History). 10th Elition. [By L. Fleteher, M.A., F.R.S.] Pp. 32. Plan. 1918, svo. 1d.

The Sturent's Index to the Collection of Minerals, British MLsenm(Natural IIistory). 23rd Edition. [By L. Fletcher, M.A., I.R.S.] Pp. 36. With a Plan of the Mineral Gallery. 1208, sro. 2d.

An Introdnction to the Study of Minerals, with a Guide to the Mineral Gallery of the British Nuseum (Natural History). By L. Hletch(P, M.A., F.R.S. 12th Edition. Pp. 12?. 41 Wookents. With Plan of the Mineral Gidlery and Index. 190R, Bro.tid.

An introduction to the Strily of Rocks. I. Fletcher, M.A., F.R.A. Pp. 11R. [Wib Plan of the Mineral trallery, 'Fable of Contents, and lindex.] 1898, 8ro. 6c\%.

An Introduction to the Study of Meteorites, with a list of the Meteorites represented in the Collection. By L. Flotoler, MI.A., F.K.G., \&c. 'Tenth Elition. Pp. 120. "With a Plan of the Minerai Gallery and an Index to the Metoorites representer in the collection.] $190 \mathrm{~s} .5 \mathrm{ro}, \mathrm{b} r$.

\section{SPeCIAL Guides.}

No. 1.-Guide to an Fxhibition of Old Natural History Books, illustrating the origin and prugress of the Study of Natural History up to the time of Linneus. [By B. B. Woodward.] Pp. 27. 1905, 8vo. 37.

No. 2.-Books and Portraits illustrating the History of Plant Classification exhibited in the Department of Botany. [By A. B. Kendle, MI.A., D.Sc.] 1'p. 10. I Plates. 1906, Svo.4d.

No. 3.-Memorials of Linnæus: a collection of Portraits, Manuscripts, Specimens, and Books exhibited to commemorate the Bicentenaly of his Birth. [By A. B. Rendle, M.A., D.Se.] l'p. 16. 2 Plates. 1907, Svo. : d. 
Handbook of Instructions for Collectors, issued by the British Museum (Natural History). With Illustrations. Third Edition. Pp. 144. Inrtex. 1906, Svo. 1s. 6d.

Instructions for Collectors :-

No. 1.-Mammals. 3rel Edition. Pp. 1:. Text illust. $1905,8 \mathrm{ro} .3 d$.

No. 2.-Birds. 4th Edition. Pp. 10. 5 figures in text. 1908,8 ro. $3 d$.

No. 3.-Reptiles, Batrachinians, and Fishes. [3̈rd Edition.] Pp. 12. 1903, 8ro. 4d.

No. 4.-Insects. 4th Edition. Pp. 11. Text illust. 1907,8 vo. $3 d$.

No. 5.-Diptera (Two-winged Flies). 3rd Edition. Pp 16. Text illust. 1908, 8vo. 3d.

No. 6.-Mosquitoes (Culicida). [3rd Edition.] Pp. 8. 1 Plate, 1 figure in text. 19(4), šro. $3 d$.

No. 7.-Blood-sucking Elies, Ticks, \&c. By E. K. Austen.

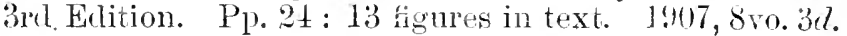

No. 8.-Spiders, Centipedes, \&c. 2nd lidition. Pp. 4. 1906, svo. 3d.

No. 9-Soft-bodied Invertebrate Animals; Shells of Molluses. [2nd Edition.] Pp. 15. 1902, 8จo. 4

No. 10.-Plants. [3rd Edition.] P. 8 : 2 figures in text. $1903,8 v 0.4 d$.

No. 11.-Fossils and Minerals. Brd Edition. I’p. 1906, svo. $3 d$.

British Museum (Natural History),

Cromwell Roakl, Londion, S.W.

October 24th, 1908.

L O N D ON :

PRINTED FOR HIS MAJESTY'S STATIONERY OFFICE, By DARLING \& SON, LTd., 34-40, Bacon Street, E. 
$A 2: 00$ 


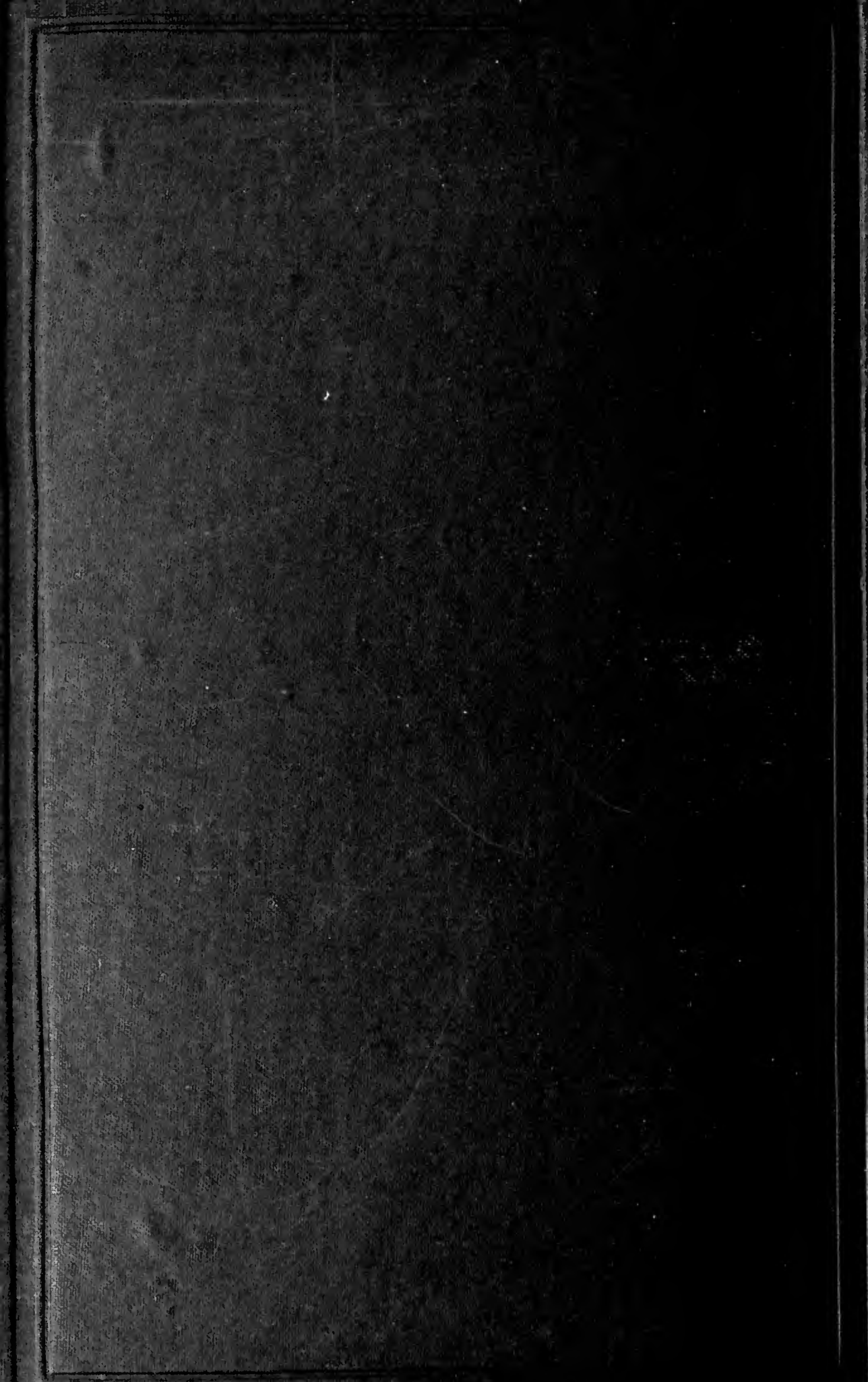

